\title{
Ferguson, Gray, and Davis With De Sousa, Tuggle, and Harrison Too: \\ An Analysis of Recorded Crime Incidents and Arrests in Baltimore City, March 2010 to March 2020
}

\author{
Stephen L. Morgan \\ Johns Hopkins University \\ Rhiannon N. Miller \\ Johns Hopkins University
}

May 15, 2020

This document can be downloaded from this link:

https://osf.io/preprints/socarxiv/fcm7t/

The original report (dated March 15, 2016) can be downloaded from this link: https://osf.io/preprints/socarxiv/nshme/

The updated report (dated November 15, 2016) can be downloaded from this link: https://osf.io/preprints/socarxiv/6gdra/ 


\section{Table of Contents}

1. Motivation for This Final Report ..............................................................................................1

2. Tough Times for Baltimore City and the Baltimore Police Department, 2016-2020......................2

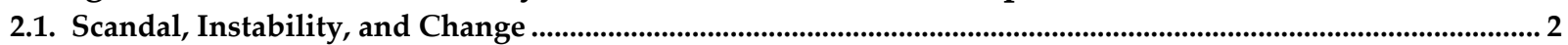

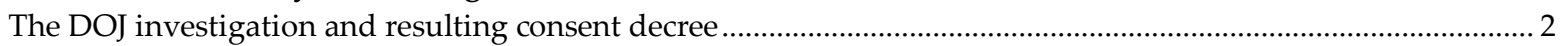

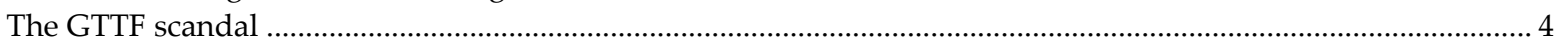

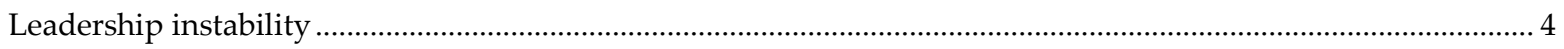

Population change ..................................................................................................................................... 6

2.2. Changes in the Policy and Practice of Policing, From the Streets to 8,500 Feet .................................................... 7

Misinformation and the consent decree.......................................................................................................

The 2019 crime plan and its reception......................................................................................................... 10

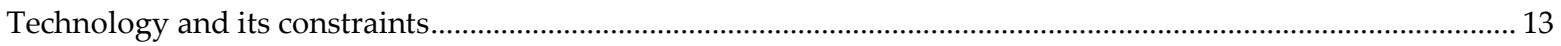

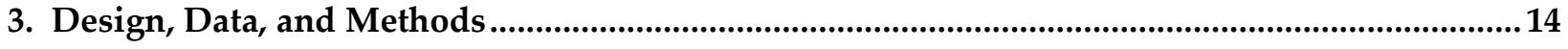

3.1. Time Intervals for the Analysis....................................................................................................................... 14

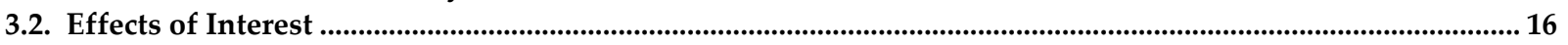

3.3. Data Sources and Measures …………………….............................................................................................. 18

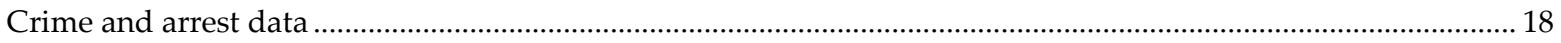

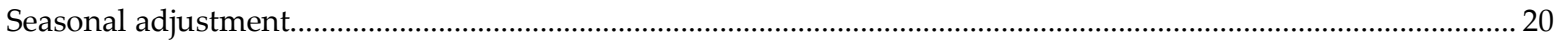

More refined measures of non-school days....................................................................................................... 20

3.4. Methods and Models................................................................................................................................................ 23

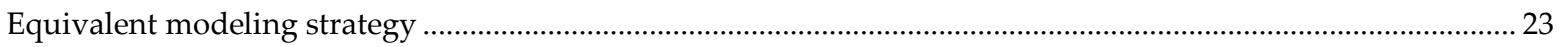

Slight changes to the specifications .............................................................................................................. 23

4. Minor Revisions to the Conclusions of the 2016 Reports ...............................................................25

4.1. Revisions to the Estimates and Conclusions of the Original Report (March 15, 2016) .................................... 25

4.2. Revisions to the Estimates and Conclusions of the Updated Report (November 15, 2016)................................ 29

5. Results for Crime Incidents and Arrests That Include Data Through March 1, 2020 ..................31

5.1. Pre-Ferguson Models That Are Used to Generate Counterfactual Trajectories ................................................... 31

Specifications of the two models.................................................................................................................... 36

5.2. Changes in Shootings and Homicides ....................................................................................................................... 39

5.3. Percentage Changes in the Prevalence of Crime Incidents, Disaggregated by Type ............................................ 46

Changes in crime incidents through Davis' first year as police commissioner ....................................................... 46

Changes in crime incidents through Harrison's first year as police commissioner ................................................. 48

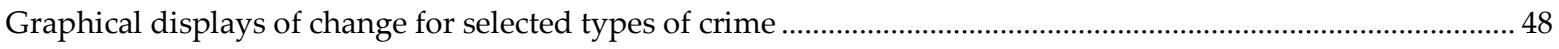

5.4. Percentage Changes in the Prevalence of Arrests, Disaggregated by Type .......................................................... 58

Changes in arrests through Davis' first year as police commissioner.......................................................................... 58

Changes in arrests through Harrison's first year as police commissioner ……………............................................ 60

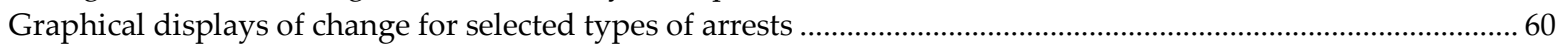

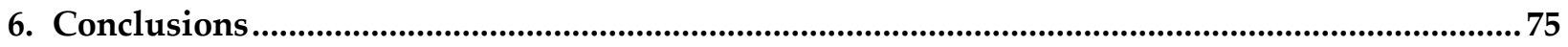

6.1. The Ferguson and Gray Effects on Crime ……................................................................................................. 75

6.2. The Ferguson and Gray Effects on Arrests ......................................................................................................... 78

6.3. From Davis Through Harrison, 2016-2020 ............................................................................................................ 80 


\section{Motivation for This Final Report}

In the midst of a debate on twenty-first-century policing in the US, and in the aftermath of a breakdown in police-community relations in Baltimore, we released a report sponsored by the $21^{\text {st }}$ Century Cities Initiative at Johns Hopkins University as

Morgan, Stephen L. and Joel A. Pally. 2016. “Ferguson, Gray, and Davis: An Analysis of Recorded Crime Incidents and Arrests in Baltimore City, March 2010 through December 2015." A Report Prepared for the 21st Century Cities Initiative at Johns Hopkins University. Baltimore, MD: Johns Hopkins University. (URL: https://osf.io/preprints/socarxiv/nshme/)

The original report was prepared to enhance the local dialogue on policing in Baltimore, primarily by providing objective and credible analysis to local media outlets. With this same rationale, an updated report was released six months later as

Morgan, Stephen L. 2016. “Fall Update for Ferguson, Gray, and Davis: An Analysis of Recorded Crime Incidents and Arrests in Baltimore City, March 2010 through October 2016." Baltimore, MD: Johns Hopkins University. (URL: https://osf.io/preprints/socarxiv/6gdra/)

Through subsequent citations by others, the original report has entered into the scholarly literature on policing and crime. Because the original report has proven useful for the broader research literature, we decided to produce this final report to update others on the patterns of crime incidents and arrests in Baltimore through the beginning of March 2020.

We intend to continue with research on crime and arrest patterns in Baltimore, but this report will be the final one that uses as primary motivation the debate on the Ferguson effect from 2014 and how any such Ferguson effect shaped the causes and consequences of the unrest in spring 2015 that followed the death of Freddie Gray while in police custody. That focus was wholly appropriate for our reports from 2016, given the debates that were of interest to media outlets and researchers at the time. It is still a reasonable motivation for continued analysis. But, as we move forward to new modeling efforts, and into a new phase of policing in Baltimore as the BPD attempts to fully implement reforms, Ferguson-effect framing is less useful for the scope of the new analysis that will be needed. To begin to appreciate why, we next offer background details on the depth of the institutional challenges faced by Baltimore since 2016. 


\section{Tough Times for Baltimore City and the Baltimore Police Department, 2016-2020}

In this final report, we offer conclusions based on an analysis that includes an additional 40 months of data, covering the three full "crime seasons" from March to November of 2017, 2018, and 2019. During these 40 months, the city has suffered from considerable institutional instability, and all of the instability is entangled with the concern over elevated rates of violent crime. Before proceeding to our full analysis of crime incidents and arrests, we provide in this section relevant background on these changes in Baltimore City.

\subsection{Scandal, Instability, and Change}

We first characterize federal interventions into crime and policing in Baltimore, and we then consider how these developments have interacted with changes in the leadership of the BPD and city government. We conclude with the attendant population decline in the city, which may be a response to the erosion in police-community relations alongside a prolonged period of elevated violent crime and reduced public safety.

The DOJ investigation and resulting consent decree. Following the death of Freddie Gray in 2015, the federal government launched an investigation of the BPD. The investigation was welcomed by the city's political leadership, and it received considerable support from the community as well.

In August of 2016, the Civil Rights Division of the US Department of Justice offered a 163-page report of its investigation, alleging a pattern of policing at odds with constitutional standards and well below DOJ's best-practice benchmarks for twenty-first-century policing. Baltimore City subsequently signed a consent decree with the federal government on April 7, 2017, pledging to reform the police department under the guidance of a court-appointed monitoring team accountable to the Chief Justice of the US District of Maryland, James K. Bredar.

The scope of the requirements of the consent decree are broad, and at the outset full compliance was not expected for at least five years. The core reforms agreed to were centered on the need to restructure all street-level policing so that it would align with the constitutional standards articulated by the federal government. In order to do so, investigative stops would need to be based on reasonable and articulable suspicion of involvement in criminal activity, as carefully defined in new department policies. Dispersals of groups of individuals would need to be justified similarly by new policies, typically in response to a call from a business or residence and then based on an officer's own observation that action is authorized by existing policy. At the same time, police officers would be required to document more completely their interactions with members of the community, and the department would be required to upgrade its technology and modeling capacity to analyze the data on all such interactions. With new data, and a twenty-first-century records management system, the department would be required to enact accountability-based management while also evaluating regularly whether its 
policing met the constitutional standards defined in its consent-decree-approved policies. To gain the trust of the community, and thereby promote cooperation in the fight against crime, a new model of community policing would need to be developed and then deployed. Finally, the department would also be required to overhaul its internal affairs and public accountability functions in order to more effectively prevent police misconduct.

Since April of 2018, the presiding judge has convened all parties to the agreement - the city (including the police department), attorneys from the DOJ, and his own monitoring team for quarterly public hearings. The transcripts of these hearings (URL here) indicate that progress has been slower than expected. The first stage of scheduled reform was to develop new BPD policies and then to train officers to adhere to these policies. ${ }^{1}$ While the rewriting of department policy has proceeded largely on schedule, in coordination with the DOJ and the court's monitoring team, the department's training materials and staff were still being developed through the end of 2019.

The slow progress has made policing even more difficult. In August of 2019, the Baltimore Sun, which has played an important role in arguing for police reform and accountability, lamented on its editorial page that

Officers face new standards on the use and reporting of force but have not been thoroughly trained on what they are or how to do their jobs well within them. They are being burned out by mandatory overtime. They are being told to build relationships in the community but face so many demands that such interactions are perfunctory. They are stuck with outdated technology and facilities. And they see a revolving door in the commissioner's post, resulting in a department with no consistent leadership and direction. ${ }^{2}$

Not unrelated to these challenges, over the same time period the BPD has declined in size, losing more officers than it has been able to hire. While undertaking more fundamental reforms, the BPD has also had to overhaul its recruitment program in order to attempt to maintain its standing capacity.

\footnotetext{
${ }^{1}$ As part of the agreement, the city agreed that, in the initial period of reform, the BPD would also use its existing data resources to establish baselines for street-level policing (e.g., "hit rates" after investigative searches, broken down by the race of the individual stopped). As the presiding judge gradually learned over the course of the first two years of consent-decree monitoring, the BPD had no capacity to provide such baseline estimates. Since then, and in spite of commissioning a thorough technology and data plan by outside consultants, progress has stalled. As of this writing, the monitoring team estimates that a fully implemented, field-based records management system will not be deployed until 2021 at the earliest. Thus, the BPD will not be able to produce the interim reports on progress toward meeting constitutional standards, using any data from the field before 2022, and only if additional resources are provided by the city and state. With the budget crises created by the coronavirus pandemic, such resources seem less likely to be provided.

2 "Baltimore's consent decree is hurting police officer morale. It's also the solution." Editorial board, Baltimore Sun, August 29, 2019.
} 
The GTTF scandal. The consent decree process, while the most important set of activities for the future of policing in Baltimore, has been overshadowed for the past three years by a more dramatic form of federal intervention. Around the same time that the DOJ launched its investigation of the BPD, the FBI launched a specific investigation of corruption and abuse by a plainclothes tactical unit of the BPD named the Gun Trace Task Force (GTTF). The investigation became public in March 2017 when seven Baltimore police officers were indicted on a range of charges from racketeering to robbery and extortion. During the investigation, enough evidence of criminal conduct was uncovered that twelve long-serving Baltimore police officers were convicted and sent to federal prison. ${ }^{3}$ The revelations from the cases slowed over the course of 2019, but federal prosecutors indicated in February of 2020 that they were "not finished."

While the consent decree process and the convictions of police officers have been welcomed by the community, we have seen no evidence that the community has interpreted these developments as evidence that the BPD has been "cleaned up." Many of Baltimore's residents appear to hold the view that corruption within the BPD is endemic and that the GTTF investigation has only scratched the surface of the problems. Some of these same residents, if media portrayals are accurate, also appear to believe that the only solution is to cut funding to the BPD by enough that the police presence in the city will decline noticeably. In response, communities, it is argued, will then develop their own strategies to control low-level deviance, cooperating with what remains of the police force to combat violent crime when necessary.

Other residents, of course, do not take this view. They appear to regard the federal interventions as necessary catalysts that will result in a better police department that will earn back a higher level of legitimacy. These residents do not advocate less policing, only more effective policing. They may suspect that the GTTF investigation has not uncovered all of the corruption problems in the department, but they appear to believe that "crooked cops" are comparatively rare. This group also appears to lay more blame at the feet the city's leadership, which we discuss next.

Leadership instability. Mayor Stephanie Rawlings-Blake chose not to seek re-election in 2016, following pronounced criticism from all directions of her leadership during the unrest that followed the death of Freddie Gray. Catherine Pugh, a sitting Maryland State Senator from Baltimore, won the 2016 election. She took leadership alongside a shaken-up Baltimore City Council, with nine new members joining five re-elected incumbents.

After signing the consent decree with the federal government on April 7, 2017, Mayor Pugh fired the sitting police commissioner Kevin Davis eight months later on January 19, 2018, noting her "impatience" with his inability to reverse the increase in violent crime since 2016.

\footnotetext{
${ }^{3}$ It is hard to overstate fallout of the GTTF trials. For example, the Baltimore State's Attorney has had to toss nearly 800 cases in which the convicted officers participated, given the evidence presented in federal court of how easily the GTTF officers were willing to plant false evidence and provide misleading testimony in court.
} 
She elevated Deputy Commissioner Darryl De Sousa to Acting Commissioner on the same day, and she directed him "to reduce the numbers and to reduce them quickly."

De Sousa pledged to surge officers to violent hotspots and reduce crime quickly. After securing confirmation from the city council on a 14-to-1 vote, he was sworn in as police commissioner on February 28, 2018. Apparently, De Sousa was well-liked by BPD officers because of his long career in the department. Yet, with the consent decree implementation process just beginning in earnest, De Sousa seemed a puzzling choice to many outside of the department. Some community leaders wondered whether he could introduce the sweeping reforms that the mayor had agreed to on behalf of the city.

Whether De Sousa could lead reform effectively would never become known. Less than three months after his confirmation, he was charged by federal prosecutors with failure to file tax returns for three years. After a brief period during which Mayor Pugh continued to express support for him, De Sousa was forced to resign. When he entered into a plea deal, the full extent of his tax fraud was publicly revealed. As the Baltimore Sun noted, even for the years when he did file tax returns, "He also claimed deductions he was not entitled to, including for unreimbursed employee expenses that he did not incur, mortgage interest and local property taxes when he did not have a mortgage or own any property, and business losses when he didn't operate a business." ${ }^{5}$ De Sousa was sentenced to 10 months in federal prison.

When De Sousa resigned on May 15, 2018, Gary Tuggle was appointed by Mayor Pugh as interim commissioner. He had just been hired by De Sousa in March as a deputy commissioner, having served as a young BPD officer in the 1980s before taking various postings in the Drug Enforcement Agency (including, for a few years, as the head of the Baltimore DEA office). Shortly after his appointment as interim commissioner, Tuggle expressed interest in the permanent position. He later announced in October 2018 that he was no longer interested in being a candidate for commissioner, following several months of pronounced violence in the city that appeared to have eroded support for his candidacy among members of the city council.

After a protracted national search, with multiple restarts and disagreements with members of the city council, including the public dithering of Interim Commissioner Tuggle, Mayor Pugh turned to a genuine outsider. Michael Harrison was the superintendent of the New Orleans Police Department, and he had experience implementing reforms from a consent decree while in that position. He was appointed as acting commissioner on February 11, 2019, and he was sworn in as commissioner on March 12, 2019 after confirmation by the city council. The judge presiding over the consent decree reforms echoed the praise and hope of others, repeating the claim that the city had finally attracted the sort of leader who could take charge and lead reform of the BPD.

\footnotetext{
4 "Press conference transcript: Baltimore Mayor Catherine Pugh, new police chief Darryl De Sousa." Wesley Case, Baltimore Sun, January 19, 2018.

5 “Plea deal sheds new light on former Baltimore policy commissioner De Sousa's tax crimes." Tim Prudente, Baltimore Sun, December 18, 2018.
} 
One month later, Mayor Pugh withdrew from service as mayor under a cloud of suspicion, created by Baltimore Sun accounts of corruption while in office (a truly bizarre kickback scheme, involving fictitious "sales" of a children's book that she had written and selfpublished). She resigned office on May 2, 2019, later pleading guilty to tax evasion and conspiracy. She was sentenced to three years in federal prison. In retrospect, Mayor Pugh's initial support of Darryl De Sousa's defense against charges of tax evasion seemed less surprising than was the case at the time.

The chair of the City Council, Bernard Young, was elevated to mayor in May of 2019, but he appears unlikely to win a full term in the November 2020 election. The two leading contenders, as of this writing, are (1) the former BPD spokesman T.J. Smith, hired by thenCommissioner Kevin Davis, and (2) the former Mayor Sheila Dickson, who was herself convicted of "fraudulent misappropriation" while in office 2008. None of the candidates who can claim meaningful separation from past city politics and Baltimore policing has yet gained enough support to suggest that a genuine "outsider" will be able to offer a fresh form of city leadership. As a result, few residents of Baltimore seem optimistic that more effective political leadership will emerge.

Population change. The population of Baltimore City grew very slightly before the death of Freddie Gray - from an estimate of 620,862 in 2010 to an estimate of 623,165 in 2014. This growth was touted as evidence of a genuine renaissance for the city. Unfortunately, following the unrest in 2015, the city's population resumed its prior decline, falling to 593,490 by 2019. Thus, from the 2014 peak, the city's population declined by an estimated 4.8 percent. We do not yet have enough data to determine whether the decline differs by age, race, or neighborhood. ${ }^{6}$

Nonetheless, it seems plausible, as highlighted by local media portrayals, that some portion of the decline is an individual-level response to the elevated rate of violent crime in the city since 2015. Other factors must be important as well, including a general level of dissatisfaction with the city's leadership and the evolving structure of economic opportunity. Responses to changes in economic opportunities are especially complicated to assess. On the one hand, it is possible that the strength of the regional labor market between 2015 and 2019 boosted net family income, which in turn fueled residential mobility to surrounding areas where schools and other social services are thought to be of higher quality. On the other hand, the realized pattern of economic opportunity may be a function of patterns of violent crime,

\footnotetext{
${ }^{6}$ Table 1 of the original report (Morgan and Pally 2016, page 17) offers the US Census Bureau's total population estimate for Baltimore City as 642,300, and this was based on our analysis of the American Community Survey, which tends to suggest higher population counts (for reasons related to how the group quarters population is handled). We performed that analysis so that we could decompose the results across census tracts and attempt to estimate the demographic composition of each BPD district. We have not undertaken a similar but updated analysis for this final report.
} 
which may have affected business investment and the willingness of those outside of the city to move into the city (or even simply travel into the city for recreation and leisure).

\subsection{Changes in the Policy and Practice of Policing, From the Streets to 8,500 Feet}

In our analysis below, we will offer detailed models of trends in arrests on different charges, and these findings suggest a continuing decline in police activity when measured by this metric alone. We do not have much additional systematic data on "street-level" change in the activity of BPD police officers, but a number of developments allow for a characterization of some types of change.

In this section, we discuss first how the consent decree itself has shifted the environment that the police confront. Then, we present the 2019 dispute over Commissioner Harrison's crime plan to demonstrate both some recent on-the-ground changes, as well as opposition to them from the police union. Finally, we discuss changes in technology, and lack thereof, that constrain the options for policing in Baltimore.

Misinformation and the consent decree. As we discussed above, rather few reforms from the consent decree process have been implemented since 2017. Yet, as the Baltimore Sun quotation above suggests, it is likely that policing practice has changed in response to the existence of the consent decree. The environment in which officers must do their work is different than it was, and almost certainly more challenging.

Consider Judge Bredar's lengthy statement, offered during a public hearing in January of 2020, on the challenges that misinformation about the consent decree creates for reform efforts, as well as those who must still police their posts:

While I believe that the parties themselves are crystal clear on what the consent decree does and does not require, there are additional interested entities with whom the parties and the monitoring team interact, and they may misunderstand some of the decree's many provisions, particularly its more subtle ones.

I first want to address the topic of investigative stops. Under the United States Supreme Court's 1968 opinion deciding the case of Terry v. Ohio, a police officer in Baltimore, or any other American city, for that matter, is authorized to stop and question a person when that officer has a reasonable and articulable suspicion that the person is involved in, is about to be involved in, or just immediately was involved in criminal activity. If, during the course of that stop, the officer develops a further reasonable suspicion that the individual is armed and presently dangerous, then that officer is allowed to pat the person down, searching for weapons or other items that could reasonably pose a danger to the officer.

Sometimes these police/citizen encounters are, in my view erroneously, referred to as stops and frisks. This is bad terminology because the Terry opinion never authorized a frisk in every instance where there was a stop. Stops and frisks are 
separate concepts. First the stop, then maybe a patdown, depending on what suspicions reasonably arise in the officer's mind.

Nothing in the consent decree forbids Baltimore officers from conducting appropriate investigative stops. As has always been true under federal constitutional law, though, the stop must always be preceded by the development of a truly reasonable suspicion, not a hunch, not a guess that someone is involved in criminal activity, but a solidly reasonable and articulable suspicion of the same.

So to the extent that some in our community are suggesting that Baltimore officers are no longer permitted to make investigative stops, Terry stops, that's just plain incorrect.

As part of the consent decree implementation process, Baltimore officers are being retrained, though, on what constitutes reasonable suspicion. As I said, a mere hunch or a guess that someone is involved in criminal activity is not enough. The officer has to know something, have seen something, have heard something, something concrete that gives rise to a reasonable suspicion in him or her, and any other reasonable police officer or commander had they been present, that criminal activity is afoot, something concrete and describable.

To the extent that Baltimore officers previously stopped and searched persons on a hunch that they were carrying a gun, on an educated guess that they were carrying illegal drugs for sale, yes, those days are over. Those hunches and guesses didn't amount to reasonable suspicion. So they by themselves could not support a stop or a patdown.

Stopping persons on less than reasonable suspicion is destructive to the essential relationship between the police and the community. It's that sort of police practice that led us to where we are now, in the midst of a crime wave during which many in the community don't trust the police and, therefore, won't help them to do the work necessary to stop the shootings and the drug dealing.

As we meet here today, Baltimore officers are being retrained, trained to lean into the crime fight while simultaneously respecting constitutional rights. Constitutional policing and effective policing go together. You don't get one without the other.

This sort of reform has been accomplished in other cities, L.A., New Orleans, Seattle, and it will happen here as well. The community demands it. The parties - the City, the Police Department and the Department of Justice - are all acting to deliver it. Most of all, the law, in the form of the consent decree, requires it.

Accordingly, as noted so many times previously, this Court intends to ensure that the reforms are accomplished exactly as the decree specifies. It's the law of the land and, as a judge in this court, that's where I get my orders.

Let's deal with some other misinformation. Some individuals and entities with whom the Court's monitoring team regularly interact have suggested that Baltimore 
police officers no longer have the authority to prevent congregations of people form blocking access to businesses, no authority to interfere with persons who are blocking others who simply wish to go about their daily activities, passing in and out of shops and stores and other business establishments. This is incorrect.

While there have been serious questions in the past about whether the Baltimore Police Department was properly enforcing ordinances that forbid activities such as loitering, and while the Department's new policies in training reflect the best practices of policing in 2020, where concepts like clearing corners give way to more sophisticated methods, emphasizing more careful analysis of street dynamics and who's doing exactly what, it nevertheless remains true that when officers receive a call for service wherein a business or property owner or tenant is complaining that congregated individuals are impeding access to their business or property, the officers may act to correct that situation, provided their own observations corroborate those of the caller.

Whenever there's a significant change in a large organization such as the Baltimore Police Department, it's common that certain mythologies develop, a sort of separate set of truths that lots of people believe in but, upon close examination, aren't rooted in anything and aren't true at all. It's the responsibility of the Police Department, as part of this implementation process, to root out and expose those myths, and then dispel them through good internal and external communication strategies, and through training.

Nothing in the consent decree forbids investigative stops. No provision prevents officers from clearing congregations that are blocking access to a business when the business requests that service. On the most general level, the court detects nothing in the consent decree directing police officers in this city to pull back from the difficult job of enforcing our state's and our nation's criminal statutes.

The task in 2020 under this consent decree and in the midst of this crime wave is to embrace modern best practices of policing, respect the Constitution, and then lean in, using all appropriate police powers, investigative strategies, apprehension techniques, communities engagement, and trust-building methods to help secure this city.

The Baltimore Police Department is capable of coming into compliance with the decree and simultaneously making progress in the crime fight. The experiences of other cities, again, L.A., New Orleans, Seattle, even Cleveland, teach this. Reforms in policing bring a reduction in crime, not the opposite. The data's there.

Police departments that embrace reform earn back the trust of their communities, and people who trust the police drop the dime. They tell the police what they know and who's up to what. They don't stand by and tolerate the kicking of a police officer who's on the ground, trying to make a lawful arrest. Instead, they assist that officer and they readily report on others who help the resistor.

There will always be lawbreakers. But in Baltimore in 2020, the fight is to regain for the police the support of the people in the middle, those that aren't offenders, but 
also now don't trust the cops. Compliance with the decree will help achieve this. Most significantly, this is the strategy that this city has embraced. It's set out in the consent decree. It carries with it the force of law. ${ }^{7}$

It is not so important to fully understand which "individuals and entities" may be spreading misinformation, whether they be community members resisting the police, or even police officers looking for an excuse for not acting when they know they should. What is important is that the judge overseeing the reforms recognizes that confusion prevails, and to infer from it that this is a common feeling among parties to the agreement. This confusion must make policing even more challenging than it would otherwise be.

The 2019 crime plan and its reception. After the leadership volatility in the BPD from January of 2017 through February of 2019, Commissioner Harrison introduced a crime plan in June of 2019, responding to calls from the city's leadership that a crime plan be developed. The plan was released in June of 2019 as two documents: a "five-year vision," titled "Crime Reduction and Departmental Transformation Plan" (URL $\underline{\text { here) }}$ ) as well as a plan for immediate implementation, the "Baltimore Police Department Crime Reduction Strategy" (URL here). An example page from the vision document follows on the next page as an image in Figure 1.

We will discuss more details of Harrison's 2019 crime plan when presenting the pattern of results for 2016-2020 below, and in our concluding section. The primary tactical change was to propose additional hotspot targeting and new proposals for surge patrol in particular neighborhoods at particular times of the day. In addition, the plan proposed returning more sworn officers into patrol positions, rather than allowing them to serve in positions that could be staffed effectively by civilians. The plan retained some important changes implemented by prior commissioners, including a decrease in reliance on plainclothes tactical units, more restrictions on overtime work by officers, and a change in shift structure that was negotiated with the police union during the collective bargaining process at the end of 2018.

The crime plan was well received by the city's political leadership, but rather less well by the police union (i.e., Baltimore City Lodge \#3 Fraternal Order of Police). Moving beyond critique-via-twitter, in October of 2019 the FOP leadership released its own glossy report, "The Mismanagement of the Baltimore Police Department and Its Impact on Public Safety" (URL here). And, at the end of 2019, the elected president of the union offered a blistering press release, displayed below as Figure 2 (see the following page). We cannot determine how much of the union's criticism is genuine and how much is posturing in the hopes of influencing future negotiations. It is possible that Harrison's crime plan has more support among the "rank and file" than the FOP claims that it does.

\footnotetext{
${ }^{7}$ James K. Bredar, Chief Judge, US District of Maryland in the Transcript of Proceedings (pages 4-10) for a public hearing held on January 22, 2020 (Civil Case No. JKB-17-0099, United states of American v. Baltimore Police Department, et al.). (URL here.)
} 
The Commissioner's vision for the Department is built around seven core focus areas:
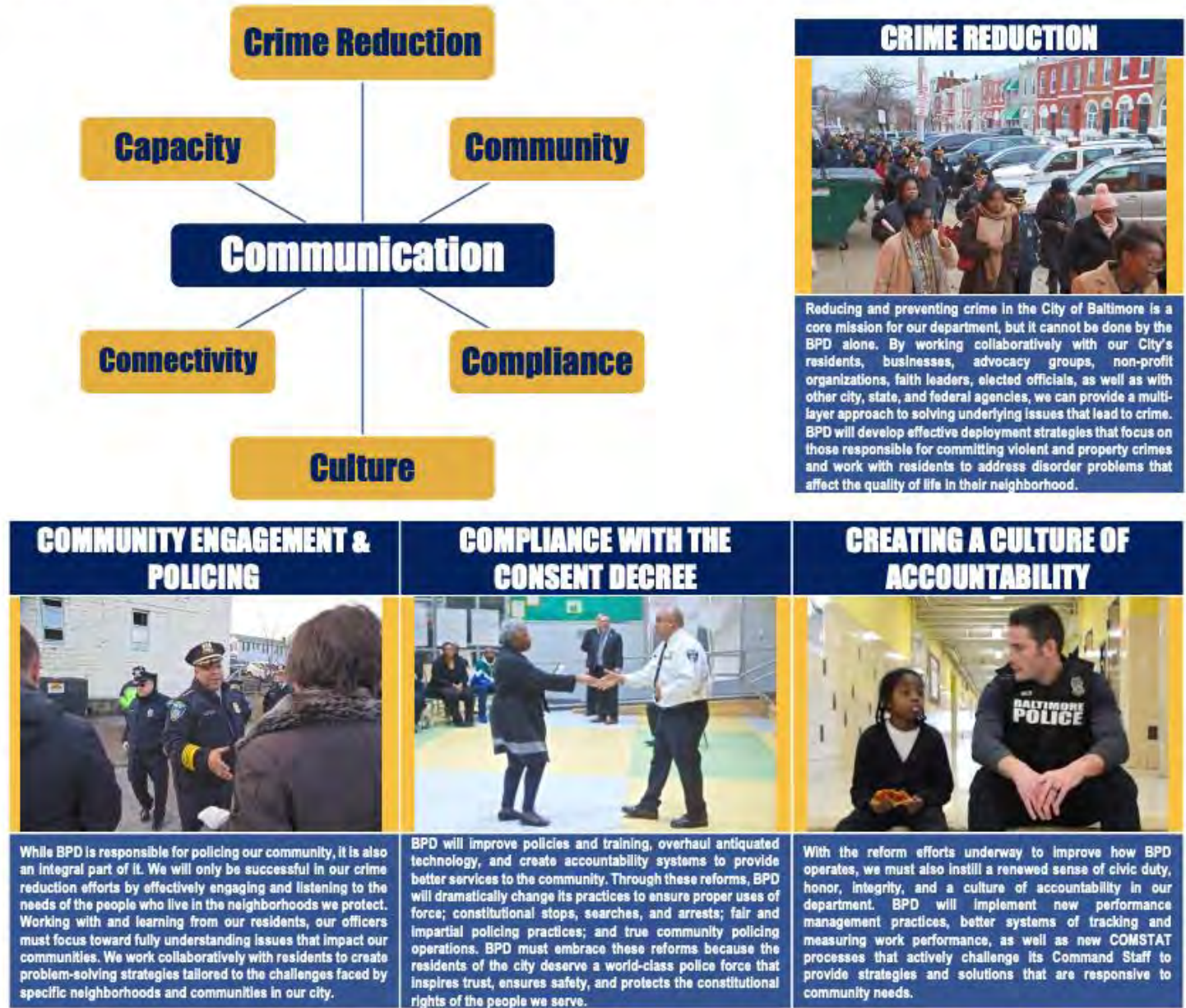

BPD will Improve policies and training, overhaul antiquated
technology, and creato accountability systoms to provide better scrvices to the communily. Through these reforms, BPD will dramatically change its practices to insure proper uses of

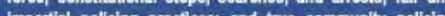
Impartial pollcing practices; and true community policing realdente of the city deserves a worldelases pollos fores that

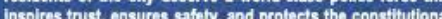
inspires trust, ensures sately,
rights of the people wo sorve.
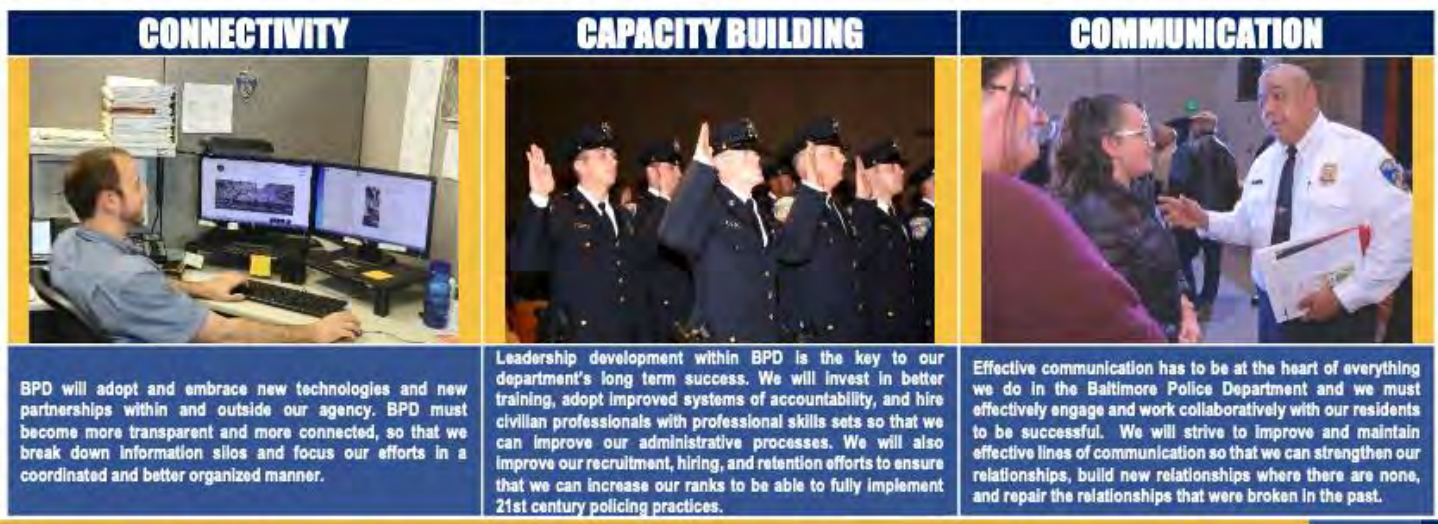

PG.4 | Baltimore Police Department

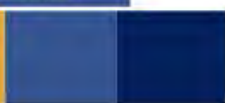

Figure 1. Overview of the vision for Harrison's 2019 crime plan (page 4 from the document) 


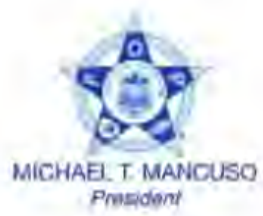

Prosidon't

\author{
3 \\ BALTIMORE CITY LODGE No. 3 \\ 3920 BUENA VISTA AVENUE: \\ BALTIMORE, MARYLAND 21211 \\ Phone: (4,0) 243-9141 \\ Fax: (410) 467-1643 \\ Twitter: @FOP3
}

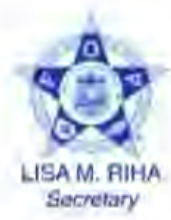

\section{FOR IMMEDIATE RELEASE}

December 31, 2019

Yesterday's Mayoral press conference is just another example of how out of touch this administration

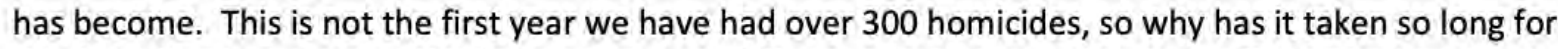
this "plan" to be presented? The idea for a citywide coordination to assist in the fight on crime is not new and has been promoted by law enforcement experts, and others, for years. It's not rocket science and to see it announced as though a light bulb suddenly went off in the minds of both the Mayor and Police Commissioner is terribly disconcerting. This was just another Jedi mind trick perpetrated on the citizens and media of Baltimore. At this point, 347 people are dead and there have been almost another 1000 failed murders this year, all a result of the complete ineffectiveness of the team at the top of the fight to protect the public's safety.

As for Commissioner Harrison's suggestion that the FOP leadership does not speak for the rank and file members of the BPD, we can say, without question, that claim is patently untrue. It is laughable when the PC says things like the men and women of the department tell him the opposite of what you hear from FOP leadership. Commissioner Harrison, the rank and file know this is a paramilitary organization and fear retribution if they tell you exactly how they feel about your crime fight. We hear the truth from them, and it is contrary to your claims of support. Unlike the PC, our FOP leadership listens to our members every day. We hear directly from them - their problems, their complaints, and their struggles.

The rank and file of the BPD are stretched thin! Our patrol ranks remain hundreds of officers short and our detectives are handling caseloads that would make law enforcement experts gasp if they knew the volume. The Commissioner's plan to solicit help from both the Federal and State governments has, unfortunately, become his only option. He cannot recruit or retain BPD officers because of the working conditions, the unrealistic expectations of the Consent Decree, and the consistent fear of unwarranted prosecution against our members. Currently, the 2019 numbers reflect a deficit of 25 officers when factoring the number hired versus the number resigning or retiring.

We ask that the citizens of Baltimore know that the men and women of the BPD understand how difficult these times are for them. We continue to work every day to provide as much protection as possible. Sadly, though, we do not have the support of the City's leadership or the current BPD administration. Each day another policy or plan is announced that effectively neuters our ability to help you! We believe that Baltimore will, someday, turn around but not with the current leadership team.

Figure 2. The response of the local FOP to statements by the mayor and commissioner 
Technology and its constraints. The title of this subsection is only partly figurative. One innovation, to be introduced for the 2020 crime season, is to supplement Baltimore's policing with the flights of three Cessna propeller planes at 8,500 feet, outfitted with cameras, for approximately 12 hours per day. As a pilot program, the BPD's new unit, Aerial Investigation Research, will attempt to capture all movement in Baltimore at a resolution that enables examination of the routes that individuals and cars in the vicinity of violent crime scenes take after the conclusion of each incident. Activist groups, and some civil liberties watchdogs, have opposed the "spy planes" as both an invasion of privacy and another form of over-policing. Court battles over how the images can be used have already begun. ${ }^{8}$

Three lower-altitude changes were implemented earlier. From 2017 onward, the city has invested resources to repair and extend its network of 750 fixed-position, closed-circuit television cameras. Consistent with national trends, body cameras have also been introduced. Finally, in 2018, the department introduced a ShotSpotter audio detection system, which can determine the approximate location of gunshots based on a web of microphones across the city (along with some risk of false positives, it is believed, from backfiring dirt bikes, motorcycles, and cars).

At altitudes between the planes and the cameras and microphones, the city continues to use its helicopters to assist regular patrol as well as tactical operations. A favorite of police radio enthusiasts, the various helicopters, referred to as "Foxtrot" when airborne, remain actively engaged in the crime fight in the city, whether assisting officers on the ground attempting to make arrests or directing patrol cars and tactical units toward the expected routes of packs of dirt bike riders.

The BPD continues to rely very heavily on car patrol, coordinated by traditional districtbased dispatch through police radio. Most cars do not contain computers, and the 2019 crime plan proposed that within a number of years, in alignment with consent-decree-related reforms, most patrol cars will contain computers that enable immediate field-based input into a new records management system. These changes are on the distant horizon, and thus they have not affected policing practice during the period considered by our analysis below. The odds of full implementation are difficult to assess, given that the consent-decree process has estimated that launching the new plan will require at least $\$ 65$ million in new investment. Many residents of the city favor budget cuts for the BPD, and thus it seems only likely to be provided if the State of Maryland allocates the necessary resources. However, as noted above, the budget crises at both the city and state levels, generated by the coronavirus pandemic, suggests this level of supplemental funding is unlikely to be provided.

\footnotetext{
${ }^{8}$ After test flights and demonstrations since 2016, a contract was signed by Baltimore City and Persistent Surveillance Systems, LLC, to put the planes in service beginning in spring 2020 for six months. The contract is funded by private philanthropy (Arnold Ventures). The program has already been challenged in federal court by the ACLU, which seeks to prevent the usage of any images taken. See details here. As of this writing, the courts have allowed the planes to fly, with the first flights apparently on April 27, 2020. The full flight schedule began on May 1, 2020.
} 


\section{Design, Data, and Methods}

The core design strategy in this final report is the same as in the original report, which is described in detail there (see Morgan and Pally 2016, pages 11-22). In brief, we seek to determine how the patterns of recorded crime incidents and arrests by the BPD have evolved between 2010 and 2020, using interrupted time series models.

Since the original reports were written in 2016, we have updated the data and underlying modeling strategy. For this final report, we re-estimated all findings from the prior reports, implementing two types of changes: (1) changes that improve the original analysis without changing the core estimation strategy and (2) changes that deliver an analysis of the 2010-2016 interval that are fully consistent with the requirements of the new analysis in this final report of the 2016-2020 interval. For the most important example of the first type of change (explained further below), we have used a more refined set of measures to take account of the types of days when school is out of session. This change was implemented after we came to understand how much weekly arrest patterns are a consequence of whether Thanksgiving or Christmas falls within the week. For the most important example of the second type of change, we improved our categorization of arrests. This change was necessary because of the growth in types of arrests for the additional 40 months of data as well the need to standardize how nonviolent, drug-related arrests are best characterized over a longer time interval that includes data from 2013 through 2020.

In the remainder of this section, we present the focal time intervals for our analysis in this final report, which now include periods for the 40 months of data that were not considered for the reports written in 2016. We also present the data sources and specific measures that we use in the subsequent analysis to adjust for the seasonality of recorded crime incidents and arrests. In conclusion, we provide a few details of the models that we will estimate for this report, holding off on providing many of the details until we present the results in subsequent sections.

\subsection{Time Intervals for the Analysis}

We consider how trends in both recorded crimes and arrests changed in nine periods, after a baseline period that ended in early August of 2014. The first five periods of change, demarcated in weeks that begin with Mondays, were analyzed in the two reports from 2016:

1. the Ferguson Period:

August 11, 2014-April 19, 2015

The interval beginning with the nationally broadcast protests in Ferguson, Missouri and ending before substantial protests began in Baltimore in response to the arrest of Freddie Gray 
2. the Gray Period:

April 20, 2015 - July 12, 2015

The interval beginning with substantial protests following the death of Freddie Gray and ending with the week of the firing of Baltimore Police Commissioner Batts

3. the Unrest Week:

April 27, 2015 - May 3, 2015

The week that began with a riot/unrest/uprising, was followed by large-scale peaceful protest activity, and was accompanied by the deployment of the National Guard and the imposition of a night-time curfew

4. the Davis Transition:

Luly 13, 2015 - October 18, 2015

The interval beginning with the first full week during which Interim Police Commissioner Davis was appointed and ending with his confirmation as Police Commissioner Davis

5. the Davis First Year:

October 19, 2015 - October 16, 2016

The interval beginning with the first full week during which Kevin Davis was confirmed as Police Commissioner Davis through the last full week of his first year as police commissioner

For this final report, we consider four additional periods of change:

6. the Davis Final 15 Months:

October 17, 2016 - January 21, 2018

The interval beginning with the first full week of Davis' second year as police commissioner, through the signing of the consent decree in April of 2017, and ending with his firing by the mayor three months into his third year as police commissioner

7. the De Sousa Spring:

January 22, 2018 - May 13, 2018

The interval beginning with the first full week during which Acting Police Commissioner Darryl De Sousa was appointed, through his confirmation as 
police commissioner in February of 2018, and ending when he took a leave of absence because of allegations of tax fraud (after which he subsequently resigned)

8. the Tuggle Interregnum:

May 14, 2018 - February 10, 2019

The interval beginning with the first full week during which Interim Police Commissioner Gary Tuggle was appointed, ending with the appointment of Acting Police Commissioner Michael Harrison

9. the Harrison First Year:

February 11, 2019 - March 1, 2020

The interval beginning with the first full week during which Acting Police Commissioner Michael Harrison was appointed, through his confirmation as Police Commissioner in March of 2019, and continuing through the end of his first full year (and concluding just before Governor Larry Hogan declared a State of Emergency because of the approach of the coronavirus pandemic)

Note that all intervals are sequential, except for interval 3 which lies within interval 2. For intervals 1 to 5, we will re-estimate the effects already presented in the original 2016 reports (and only small changes will result). For intervals 6 to 9 , we will provide new estimates. As noted in the next subsection, none of these estimated effects have simple interpretations from a counterfactual perspective.

\subsection{Effects of Interest}

As explained in the original report, our initial interest was in the assessment of changes in response to the events in Ferguson, the emergent national dialogue on police conduct, and the arrest of Freddie Gray and events that followed it. We were also interested in whether the appointment of the new Police Commissioner Kevin Davis would restore confidence in the BPD. These effects were featured in the title of the original report, "Ferguson, Gray, and Davis ..."

For the original report, we explained the complexity of defining these effects from a counterfactual perspective. For example, consider this excerpt:

The Ferguson Effect. A Ferguson effect on crime in Baltimore would exist, by our reasoning, if the number of crime incidents recorded after the beginning of the Ferguson period differs from the number that would have been recorded if the events in Ferguson had not set off a shift in the national dialogue on policing. The Ferguson effect for arrests would have the same basic structure and would not necessarily align with the Ferguson effect for crime. Indeed, there is good 
reason to expect substantial divergence. Consider the following possibilities. Suppose that police officers, because of concerns about additional monitoring and the need to document the appropriateness of their conduct, take a more deliberate approach to routine police activity. Or suppose that they decide to refrain from some discretionary police activity in order to minimize the odds that they will find themselves in encounters that are hard to control. In either case, the arrest count would decrease, especially for discretionary arrests, and a Ferguson effect on arrests would emerge. This decrease could occur regardless of changes in the pattern of crime incidents. Suppose, however, that individuals inclined to commit crimes anticipate that the police may be about to back off from aggressive policing, or, more plausibly, observe a decline in discretionary arrests in their communities. In these circumstances, crime incidents might increase, and such a change would qualify, by our reasoning, as a Ferguson effect on crime. This effect on crime could accompany or follow a Ferguson effect on arrests.

The structure of these sorts of effects is established through a comparison of what is observed to an expectation of what would have been observed in the absence of the cause, which in this case is the shift in the national dialogue on police conduct that emerged following the protests in Ferguson. In the language of academic research, the assessment of such effects requires the modeling of counterfactual values: what would have happened in the absence of something that actually did happen. In order to make any progress in an empirical assessment of effects such as these, the analyst needs to develop a model of unobserved counterfactual trajectories that yield comparison values.

The Gray Effect. If this type of analysis were not challenging enough already, additional complications immediately arise when considering the onset of effects in response to the arrest of Freddie Gray. As with the Ferguson effect, we can start by defining a Gray effect using a simple counterfactual: the difference in crime incidents or arrests between what is observed and what would have been observed if Freddie Gray had not been arrested and had not died from the injuries he sustained while in police custody. The complication with this definition is the possible presence of a preexisting Ferguson effect during the post-Ferguson, pre-Gray time interval, labeled above as the "Ferguson period." There is no good reason to believe that a preexisting Ferguson effect would vanish the moment a Gray effect begins. Rather, it would be most reasonable to assume that the existence of any ongoing Ferguson effect would then shape the nature of a subsequent Gray effect, either for crime incidents, or arrests, or both.

Moreover, the Gray effect itself has two components, each of which is clearly observable in the raw data that we will analyze below: (1) changes during the dramatic events of the week of unrest that began on April 27, 2015 and ended when the National Guard stood down seven days later, and (2) the more 
encompassing period delineated above, which began after the death of Freddie Gray on April 19th, and which included substantial journalistic coverage of both peaceful and violent protests. Furthermore, it is reasonable to assume that substantial discussion of Freddie Gray's arrest would have been present in the ranks of the Baltimore police before April 27th, given that he was arrested on April $12^{\text {th }}$ and his death was widely reported on April $19^{\text {th }}$. Indeed, the officers involved in his arrest were suspended on April 21 ${ }^{\text {st }}$, which was six days before the riot/uprising began.

Altogether, our strategy is to interpret changes that are observed in the Gray period relative to the Ferguson period in counterfactual terms, but with the stipulation that the changes produced by a Gray effect are not cleanly separable from any preexisting Ferguson effect. We will not attempt to estimate complex counterfactuals, such as the Gray effect that would have occurred if the Ferguson protests and all associated events had not occurred in the months prior to Freddie Gray's arrest. Similarly, we will not attempt to evaluate the Ferguson effect in the Gray period, as if Freddie Gray had not been arrested or had been arrested but had not died. Rather, we will allow all change in the Gray period to be attributed to a nominal Gray effect. But we will then discuss in our subsequent interpretations how the Ferguson and Gray effects may be fundamentally entangled because of how the size of the Gray effect may depend on the nature of an underlying Ferguson effect.

Finally, even though we will model the nominal Gray effect in two pieces, generating estimates that allow us to separate the piece of the Gray effect that is attributable to the Gray period as a whole from the additional spike that occurred during the week of unrest, this separation is artificial as well, since the overall Gray period cannot be fundamentally separated into these two pieces. The pattern of crime and arrests in the weeks following the unrest would not have been the same if the week of unrest had not occurred. (Morgan and Pally 2016, pages 13-15)

In this final report, we remain interested in these entangled effects, and we will re-estimate them just as we did in the original report. The new analysis that we offer in this report is based only on the final four periods detailed above: from Davis' final 15 months through Harrison's first full year. The same cautions from the original report apply to effects defined by these four periods as well. However, as we will show below, the estimated effects for these later periods are similar enough that our inability to cleanly separate them in a counterfactual framework is less consequential for overall interpretations and conclusions.

\subsection{Data Sources and Measures}

Crime and arrest data. The BPD releases data, over the citywide Open Baltimore data portal, on a regular basis. In this analysis, we consider 487,762 recorded crime incidents from March 1, 2010 through March 1, 2020. For arrests, we use all data that are publicly available to 
us: 198,360 arrests of individuals from January 1, 2013 through March 1, 2020. We bin these crime incidents and arrests into weeks beginning on Monday and ending on Sunday in order to cumulate rare events into meaningful counts. Accordingly, all of the outcomes we consider are weekly counts. ${ }^{9}$

For types of crime, we use BPD's own released categories. One of these categories, assaults by threat, has been discontinued since our analysis in 2016. In fact, the BPD changed the data on assaults by threat that we analyzed for our prior two reports. As shown in Appendix 2 (see section 45), and unlike in our original report, assaults by threat declined abruptly to zero in the pre-Ferguson period and remained at zero through March 1, 2020. We have handled this change in the data by allowing assaults by threat, which number only about 10 per week when categorized for the first portion of the time series, to continue to count as a category within the total crime incident count that we analyze. We then also offer models for assault by threat in Appendix 2 (see section 45) in order to show when the abrupt decline to zero appears. We do not include conclusions about assaults by treat in the main document of this report, and we do not include assaults by threat in the rows of the summary tables below, except insofar as they are included within the total weekly counts of crime incidents when they were a recognized crime category by the BPD and reported in the data that the BPD released. ${ }^{10}$

All other measurement issues for crime incidents are the same as was the case for the original report. And the caveats from the original report still apply, notably that crime is almost surely under-reported and that the under-reporting is not random.

For arrests, we use an updated version of our hierarchical keyword-based coding of types of arrests. We detail the coding procedure and coding results in Appendix 1, which shows how we categorized more than 16,000 types of charge descriptions into 25 meaningful groups for analysis. For this final report, we updated the severity ranking of types of charges, which determines how arrests on multiple charges are categorized by our coding. We also then combined groups of arrests in slightly different fashion for the analysis. Most importantly, in the original report, non-violent drug arrests with intent to distribute were categorized as substantially more serious than drug possession alone, and both types of arrests were then analyzed separately. For this final report, we combined non-violent drug distribution and drug possession for subsequent analysis because we doubt that charges that include "intent to distribute" are comparable over the full time period, given how the policing of drug crime has evolved in the city. Note that this change only matters for arrests that do not contain charges that our categorization deemed more serious. For example, an individual who was arrested for a handgun violation and drug possession is placed in our "deadly weapon" group (which is $7^{\text {th }}$ in severity, after murder, attempted murder, rape, arson, robbery, and aggravated assault).

\footnotetext{
${ }_{9}^{9}$ Our analysis is based on data that we downloaded on April 18, 2020, combined with data that are no longer available online, but which we had archived previously.

${ }^{10}$ We have been unable to verify the rationale or exact contours of the change, but it appears that incidents of assault by threat were reclassified by BPD as civil disputes rather than crime incidents. It does not appear that assaults by threat were reclassified as either common assaults of aggravated assaults.
} 
Seasonal adjustment. We use the same external data sources as in the original report to account for temperature, daylight, snowfall, and precipitation. The values for the four measures are presented in Figure 3 (see next page), with the horizontal axis representing time as the index and with the measurements in the panels representing weekly averages of the variables detailed on the vertical axes (see also the legend).

More refined measures of non-school days. For the original report, we used an adjustment variable for the proportion of the week that school was in session, as determined using the school calendar of the Baltimore City School District. In subsequent analysis, we determined that arrest counts were more variable across weeks with different types of nonschool days, especially for the weeks that include Thanksgiving or Christmas. Figure 4 (see the page after the next page) shows the five variables that we use simultaneously to adjust for the mix of days in each week in this final report. 


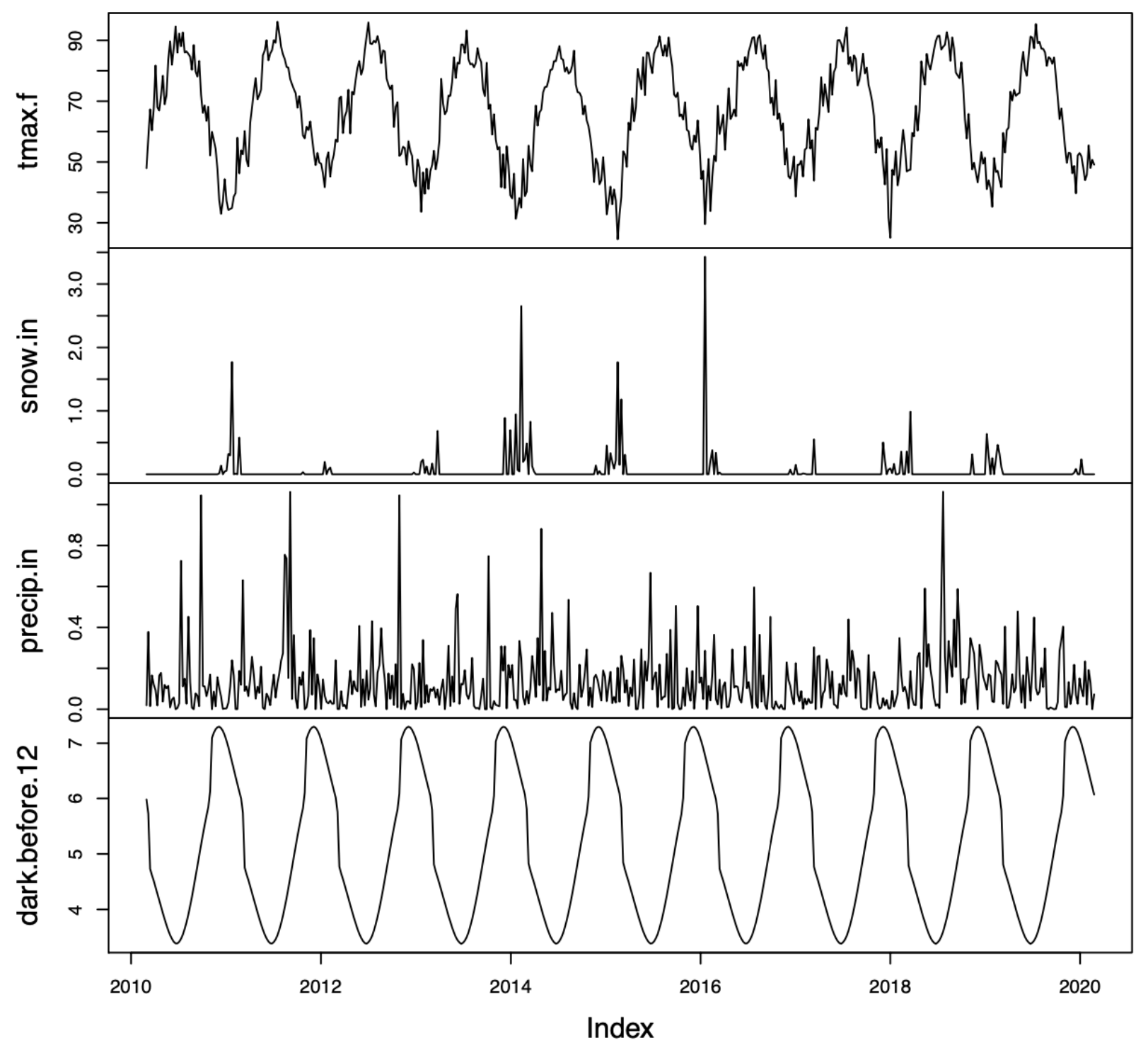

- tmax.f is the weekly average of the daily maximum temperature, measured in degrees Fahrenheit

- snow.in is the weekly average of total daily snowfall, measured in inches

- precip.in is the weekly average of total daily precipitation, measured in inches

- dark.before.12 is the weekly average of daily hours between sunset and midnight

Figure 3. Seasonal adjustment variables for the analysis 

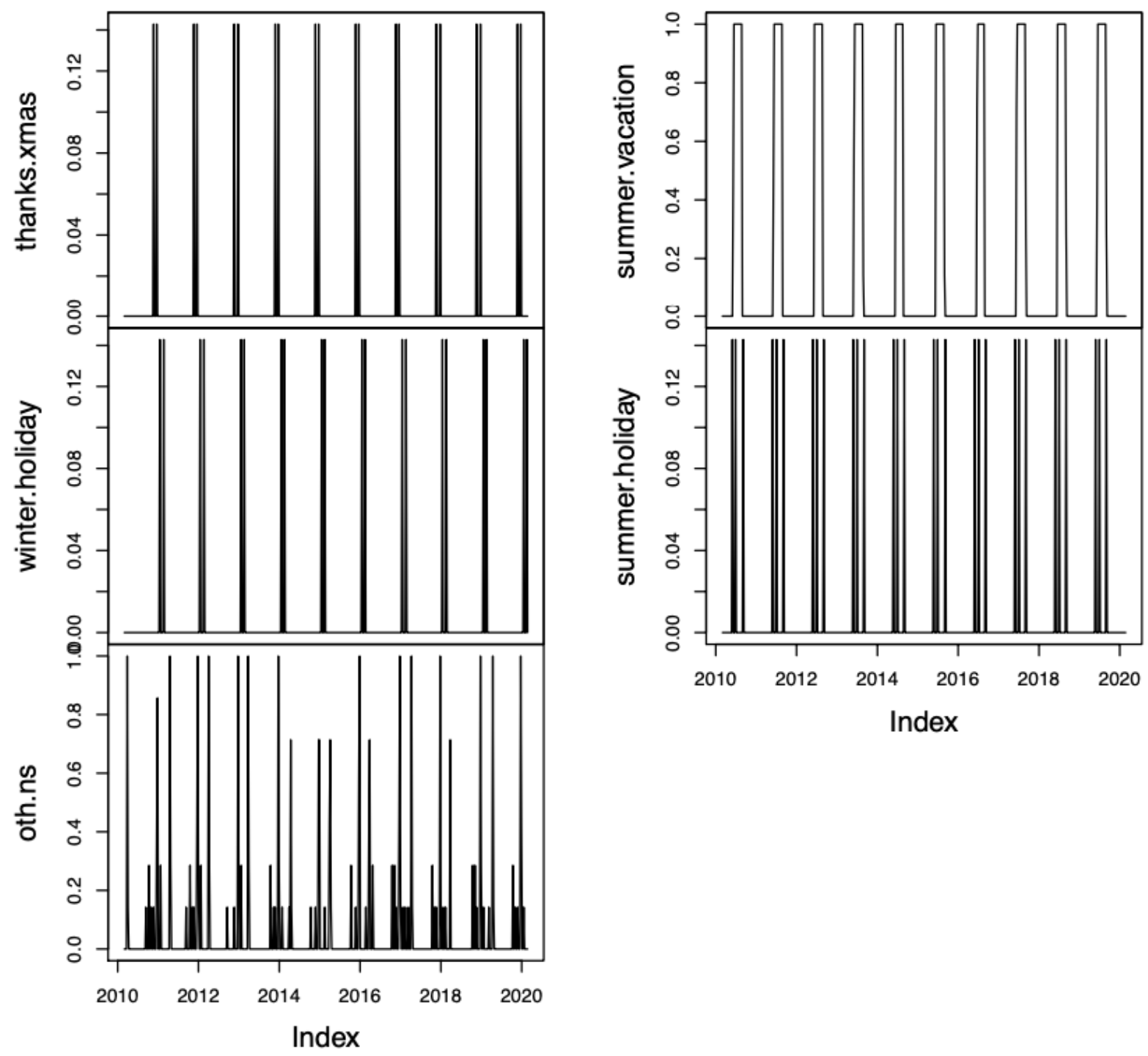

- thanks.xmas is the proportion of days of the week that are either Thanksgiving or Christmas

- winter.holiday is the proportion of days of the week that are a winter holiday other than Thanksgiving or Christmas

- other.ns is the proportion of days of the week that are other types of non-school days for the Baltimore City Schools (e.g., professional development days and winter vacation days)

- summer.vacation is the proportion of days of the week that are summer vacation for the Baltimore City Schools

- summer.holiday is the proportion of days of the week that are a summer holiday (e.g., 4 th of July)

Figure 4. Adjustment variables for the proportion of each week with different types of nonschool days.

Note: The scales of the vertical axes differ. Summer holiday weeks and non-school-day weeks are the only ones that can reach weekly values of 1 . 


\subsection{Methods and Models}

We use the same basic modeling approach as in the original 2016 reports. We explain the approach briefly in this section for those who are familiar with the original reports. For those who are new to our approach, the following paragraphs will read as unreasonably obtuse. If so, we recommend skipping ahead to the results, where the approach will be explained as it is demonstrated in use.

Equivalent modeling strategy. As for the original reports, we first estimate preFerguson models for crime incidents and arrests (after settling on a core right-hand-side specification of predictors, based on an analysis only of total crime incidents and total arrests). We then use coefficients estimated from models for each outcome (but still using the core righthand-side specification of predictors to protect against overfitting) in order to generate counterfactual trajectories. These counterfactual trajectories are used for graphical displays in our figures.

For our preferred models of change, we estimate period-specific change in the same interrupted time series design, after de-trending the outcome for the full time series (using the observed predictor values in each time period along with the estimated coefficients from each outcome-specific, pre-Ferguson model). We then offer interpretations of these estimates, often as percentage change, scaled by the count of the outcome variable in the last year of the preFerguson period.

Slight changes to the specifications. For this final report, we have improved the preFerguson prediction models slightly. In the case of crime incidents, we have also used them in a more constrained way to structure counterfactual trajectories. The main improvement is the more variegated specification of types of non-school days presented above in Figure 4 . We implemented this improvement for both crime incidents and arrests.

We have also changed the specifications of the underlying time trajectories. For the models for arrests, we removed the baseline time counter altogether. As a result, the counterfactual trend after the pre-Ferguson period is determined only by seasonality and types of days. We implemented this change because the pre-Ferguson time period for arrests is comparatively short, resulting in estimated underlying time trends that were more of a distraction than an aid to interpretation (because they were either zero, and thus uninformative for the analysis, or something other than zero, and therefore suspect because of the amount of data they were based on). With several more years of data, it is now clear that the pattern for arrests is easily interpretable without the need to fit an underlying time trend to help structure a counterfactual trajectory.

For the models for crime incidents, we kept the baseline time counter as a predictor variable in the pre-Ferguson model. As with the original reports, we still believe that we have enough data from the pre-Ferguson period to be able to offer sufficiently precise estimates of 
underlying time trends for crime incidents. As a result, these estimated time trends can be used to structure the counterfactual trend through the post-Ferguson intervals.

However, for the final four intervals considered in this report, we made the decision that the underlying time trend should be constrained. Following Davis' first year as commissioner, the cyclical pattern of the counterfactual trend is determined only by seasonality, weather variation, and types of days. The consequences of this change are best seen in figures for types of crime incidents where substantial pre-Ferguson trajectories are present, such as for aggravated assault. As can be seen in the relevant figure in Appendix 2 (see section 11), the counterfactual trend line in red slopes downward through Davis' first year but then flattens for the remainder of the time series, net of the cyclicality modeled throughout. As we will explain below, we implemented this constraint because we believe that crime patterns settled into a new quasi-equilibrium from the second year of Davis' term as commissioner onward. 


\section{Minor Revisions to the Conclusions of the 2016 Reports}

To introduce the results from the new analysis in this report, we begin by offering in this section updated conclusions for the original reports. We regard the analysis in this final report as providing definitive estimates of the Ferguson, Gray, and Davis effects that we presented previously in 2016. The latest results leave the core conclusions unaltered, as we show below, with only minor changes in the magnitudes of some of the supporting evidence.

\subsection{Revisions to the Estimates and Conclusions of the Original Report (March 15, 2016)}

This final report offers updated full results for the 2010-2016 interval below, and the new analysis continues to support the conclusions of the original report. To demonstrate, we paste below the conclusions from the Executive Summary of the original report. ${ }^{11}$ We show how the results presented below in subsequent sections of this final report provide a very slightly different set of supporting findings for the same overall conclusions. We use crossed out text for deletions and underlined text for additions.

\section{Conclusions for the Time Period Before the Arrest of Freddie Gray}

1. Adjusting for seasonality, evidence of a Ferguson effect on crime is very weak for the eight months after the Ferguson protests were widely publicized and before Freddie Gray was arrested.

- Demarcating the post-Ferguson, pre-Gray period as the interval from August 11, 2014 through April 19, 2015, many categories of crime decreased slightly relative to the expected seasonal trend, such as homicide (down $3 \%$ 5\%), automobile theft (down $7 \%$ 10\%), common assault (down $13 \% \underline{12 \%}$ ), and larceny (down 12\% 10\%). Other categories of crime were unchanged, such as street robbery and burglary. Small increases were recorded for larceny from automobiles (up $3 \% \underline{6 \%}$ ) and aggravated assault (up 6\% $\underline{5 \%}$ ). Against such stability and what we regard as trendless fluctuation, the only substantial increases in recorded crime were themselves modest in comparison to the changes that unfolded from April 20, 2015 onward. These increases included shootings (up 13\% 14\%) and commercial robbery (up 19\% 20\%).

- Overall, we conclude that it is unlikely that the full profile of change in recorded crime in this period reflects any substantial response to protest events in Ferguson, or a reaction to any other police conduct that received national press coverage before the arrest of Freddie Gray.

2. During this same time period, changes in arrest rates are consistent with a Ferguson effect on police conduct. Arrests decreased substantially for

\footnotetext{
${ }^{11}$ The original text is drawn from pages 2-5 of Morgan and Pally (2016).
} 
many areas of less serious crimes, and these declines occurred during a period characterized by typical fluctuation in recorded crime incidents.

- The total arrest count, which is not subject to under-reporting, fell by 19\% 22\% between August 11, 2014 through April 19, 2015 in comparison to the prior 52-week period, and after adjustments for seasonality. In particular, the average weekly total of $800 \underline{792}$ arrests declined by $152 \underline{174}$ arrests per week. These changes unfolded alongside a mostly stable period in recorded crime incidents (see, above, for our first conclusion on crime incidents).

- Although the charges associated with each arrest are very difficult to categorize, the declines are most substantial for categories of less serious crime, such as property destruction (down $34 \%$ 27\%), driving violations (d3\%), prostitution (down 33\% 34\%), police noncompliance (down 38\%), non-violent drug-related charges (down

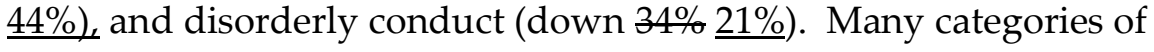
arrest for violent crimes, such as murder, attempted murder, and robbery, and aggravated assault did not decrease over the same time period, suggesting that the declines in arrests were in areas where police have discretionary alternatives to arrest.

Taken together, these two conclusions for the period before Freddie Gray was arrested suggest that the only evidence in support of a "Ferguson effect" in Baltimore is for the pattern of arrests. Recorded crime varied as expected, but arrests declined, nonetheless. Baltimore's police officers may have used discretionary alternatives to arrest more frequently in this time period, possibly out of concern that the appropriateness of their conduct could become the subject of controversy.

\section{Conclusions for the Time Period After the Arrest of Freddie Gray}

3. The heavily reported spike in shootings and homicides that emerged in the three months following the arrest of Freddie Gray occurred alongside large increases in other types of crime.

- Adjusting for the seasonality of crime, and removing the week of unrest from the calculations, shootings and homicides increased by $140 \% 138 \%$ and $92 \%$ 91\%, respectively, in the interval from April 20, 2015 through July 12, 2015. Over the same time period, street robbery increased by $31 \%$ 33\%, commercial robbery increased by $82 \% \underline{89 \%}$, carjacking increased by $33 \% \underline{44 \%}$, and automobile theft increased by $53 \%$ 36\%.

4. Arrests declined further from April 20, 2015 through July 12, 2015, even after removing the complex pattern of arrests that emerged during the week of unrest. These declines are consistent with the widely discussed 
conjecture that the Baltimore police pulled back from some routine policing in response to a perceived lack of support from the city's leadership. These declines are also consistent with other narratives, such as the onset of a period of recovery following the overtime work of the police during the week of unrest, an accentuation of a Ferguson effect on arrests that had already emerged during the post-Ferguson, pre-Gray period, and a decline in cooperation from the community that made police work more challenging.

- The total arrest count declined by an additional $30 \%$ 31\% between April 20, 2015 and July 12, 2015, excluding the disrupted pattern of arrests during the week of unrest that began on Monday, April 27th, 2015.

- Among the areas of arrest that declined during the post-Ferguson, pre-Gray period and that may reflect a Ferguson effect on police activity, many of these areas of arrest continued to decline. Arrests for driving violations police non-compliance, prostitution, and disorderly conduct declined by an additional $44 \%$ 38\%, 40\%, and $49 \%$ $58 \%$, respectively.

- Some areas of arrest that did not decline substantially during the postFerguson, pre-Gray period contributed to the $30 \%$ 31\% decline in the total arrest count during this period. Arrests for burglary, larceny, driving violations, and trespassing declined by $37 \%$ 19\% $39 \%$, $41 \%, 47 \%$, and $74 \%$, respectively, while arrests for bro that we label "police noncompliance" declined by a further $58 \%$. Arrests for murder and attempted murder decreased by $30 \% \underline{28 \%}$, and arrests for robery decreased by $18 \%$ $\underline{29 \%}$.

5. It is fundamentally unclear whether the crime spike after April 20, 2015 should be regarded as evidence in support of a Ferguson effect on crime in Baltimore.

- It is notable, as detailed above for our first conclusion, that crime incidents evolved as expected over the eight months that followed the protests in Ferguson and that preceded the arrest of Freddie Gray. At the same time, arrests declined in many areas of less serious crime. In this sense, there is little or no evidence of a Ferguson effect on crime in Baltimore for many months when there could have been such evidence.

- It is undeniable that the unrest and its aftermath were shaped deeply by the media coverage of Freddie Gray's arrest, including the available cellphone video of it. These influential journalistic treatments drew connections to the nationwide focus on police conduct. In view of these explicit connections, it would be 
unreasonable to conclude that the week of unrest was an inwardfocused event, disconnected from the emergent national discourse on police conduct.

- One reasonable interpretation is that the crime spike is a Ferguson effect that might have remained dormant had it not been ignited by a localized Gray effect. Nonetheless, the size and duration of the crime spike is almost certainly attributable to particular features of the unrest and its aftermath, possibly including an increase in gangrelated conflict over drug distribution as well as a police pullback in protest of the city's leadership. These accelerants have little or no connection to the core narrative of the Ferguson effect, although they may have been shaped to some extent by the cumulative events of spring 2015, which appear to include a decline in community cooperation with the police.

6. [Prefatory note: The larger number of changes for this conclusion arises from the shift in the time periods adopted for the updated report and which have been carried forward for the analysis in this final report.] Arrests increased during the remainder of 2015, after the appointment of a police commissioner the transition period when Kevin Davis served as interim police commissioner and before he was confirmed as permanent commissioner on October 19th 2015 . During the same time period, recorded crime incidents declined.

- Adjusting for seasonality, the weekly total arrest count increased by $20 \% 16 \%$ between July 13, 2015 and the end of the year October 19th, $\underline{2015}$, bringing the count back up to $77 \% \underline{64 \%}$ of the weekly total arrest count that prevailed in the year before the Ferguson protests.

- These increases in arrests surely reflect a natural response to types of crime that increased in the summer of 2015, such as arrests for murder and attempted murder, which increased by $43 \% 33 \%$, and arrests for deadly weapons violations, which increased by $39 \% \underline{47 \%}$.

- Arrests in other areas that had declined before Interim Commissioner Davis was appointed also increased. Arrests for burglary, larceny,

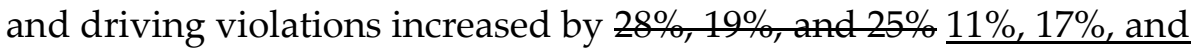
37\%, respectively. Arrests for our ategory of "police noncompliance" increased by $25 \%$ as

- With the exception of carjacking, which continued to increase, Recorded crime incidents decreased during the remainder of 2015 transition period. Shootings and homicides decreased by $64 \% \underline{62 \%}$ and $29 \% \underline{23 \%}$, respectively, leaving net increases at the end of the year of $76 \% \underline{90 \%}$ and $63 \% \underline{64 \%}$ relative to the period before Freddie Gray was arrested. Street robbery, commercial robbery, and automobile 
theft declined by $12 \%, 68 \%$, and $28 \% 16 \%, 66 \%$, and $11 \%$, leaving net increases of $19 \%, 14 \%$, and $26 \% 17 \%, 42 \%$, and $16 \%$.

The main text of the original report explains these conclusions in considerable detail. The models that we offer in this final report continue to support these core conclusions, even though some changes in modeling decisions have altered the fine detail of the supporting results. For example, as discussed above, the arrest coding algorithm shifted some arrests out of the "disorderly conduct" category into the "police non-compliance" category, but this shift does not change the overall conclusion about the trajectories of levels of arrests for incidents where police officers have substantial discretion in how to proceed.

\subsection{Revisions to the Estimates and Conclusions of the Updated Report (November 15, 2016)}

The updated report, released in November 2016, included additional analysis of the first 10 months of 2016, and it subdivided the Davis effect into two pieces (an estimated effect for the transition period while Davis served as interim commissioner and an estimated effect for his first full-year as the city-council-confirmed commissioner with a five-year contract). As with the original report, the additional conclusions of the updated report are also supported by the more definitive results we offer in this final report. To demonstrate, we paste the conclusions of the updated report below, again using crossed out for deletions and underlined text for additions to incorporate the results offered in subsequent sections of this final report. ${ }^{12}$

1. During the period between the appointment of Kevin Davis as Interim Police Commissioner in July 2015 and his confirmation as the $38^{\text {th }}$ Police Commissioner of Baltimore in October 2015, crime rates decreased substantially from the levels that prevailed in the months following the April 2015 death of Freddie Gray while in police custody.

- Adjusting for the seasonality of crime, shootings and homicides decreased by $64 \% \underline{62 \%}$ and $26 \% \underline{23 \%}$, respectively, between Monday, July 13, 2015 and Sunday, October 18, 2015. Street robbery, commercial robbery, and aggravated assault declined by $16 \%, 66 \%$, and $11 \%$.

- In this same period, most categories of property crime changed only modestly. Although automobile theft declined by $15 \%$ 11\%, burglary and larceny from automobiles declined by only $2 \%$, while other types of larceny increased by $4 \%$.

2. During the first full year of Kevin Davis' five-year term as the $38^{\text {th }}$ Police Commissioner of Baltimore, progress on reducing both violent and property crime was substantial in many categories but uneven overall.

\footnotetext{
12 The original text is drawn from pages 1-2 of Morgan (2016).
} 
- Homicides declined by an additional $23 \%$ for the one-year interval beginning on October 19, 2015, in comparison to the transition period before it, and after adjusting for seasonal differences. However, in this same time period, shootings did not decline. In addition, street robbery and residential robbery increased by $\% \underline{10 \%}$ and $18 \% \underline{20 \%}$ while carjacking soared by $117 \% \underline{120 \%}$.

- In contrast, property crime declined consistently. Automobile theft declined by $25 \%$ 5\%, burglary by $5 \% \underline{4 \%}$, larceny from automobiles by $15 \% \underline{17 \%}$, and other types of larceny by $11 \% \underline{8 \%}$.

3. Even after the progress in many categories of crime during the first year of Kevin Davis' appointment, violent crime remained much higher than before the national dialogue on policing began in the summer of 2014. The absolute level of violent crime presents a continuing challenge for Baltimore, its police, and its residents.

- Averaged over a year from October 19, 2015 onward, and in comparison to levels of crime that prevailed before the national dialogue on policing began in the summer of 2014, violent crime rates remained much higher. Homicides remained at $140 \% \underline{141 \%}$ of prior levels, and shootings at $190 \%$ 193\%. Street robbery, commercial robbery, and aggravated assault stood at 127\%, $137 \% 140 \%$, and $132 \%$ of prior levels. And, finally, carjacking soared to $313 \% \underline{321 \%}$ of prior levels.

4. The fall-off in arrests of $31 \%$ during the period following the death of Freddie Gray was turned around by an increase of $23 \% 16 \%$ during the transition period when Kevin Davis was Interim Police Commissioner. Levels of arrests then changed very modestly during the first full year of Kevin Davis' term as the $38^{\text {th }}$ Police Commissioner of Baltimore, declining by $4 \% \underline{3 \%}$ during the year. As of the end of his first year, the arrest level stood at $67 \% \underline{60 \%}$ of its prior level before the national dialogue on policing began, with most of the decline explained in the original report.

While the results we will offer below are still consistent with these conclusions, in retrospect the tone of the conclusions in the updated report now feels a bit too naïve, and perhaps a bit too sanguine as well. It is clear, as shown below, that the limited improvements achieved through the first 10 months of 2016 were not sustained. We turn next to a full reporting of analysis we have undertaken for this final report. 


\section{Results for Crime Incidents and Arrests That Include Data Through March 1, 2020}

We first explain the updated prediction models for the pre-Ferguson time periods, along with graphical displays of total counts for crime incidents and arrests. We then offer summary estimates and additional graphical displays for types of crime incidents and types of arrest.

\subsection{Pre-Ferguson Models That Are Used to Generate Counterfactual Trajectories}

Like the original report, all results in this report are structured by two omnibus pre-Ferguson prediction models, which are then used subsequently to model types of crime incidents and types of arrests. In this section, we present the right-hand-side specifications of these two models. Before doing so, we demonstrate their fit to the pre-Ferguson raw data as well as their subsequent usage over the full time series to generate the key counterfactual trajectories.

Before detailing the model specifications and coefficient estimates, we offer four summary figures (see the next four pages). The vertical dimension of Figures 5 and 6 is the weekly count of total crime incidents per week, and the horizontal dimension is weeks from March 1, 2010 through March 1, 2020. Figures 7 and 8 are the analogous figures for the weekly count of total arrests, with the horizontal dimension over the more limited time interval from January 1, 2013 through March 1, 2020.

For Figures 5-8, the vertical bars demarcate the time intervals of interest, as defined above. ${ }^{13}$ For Figures 5 and 7, the gray dots are the observed weekly totals. For Figures 6 and 8 the gray dots are replaced by jagged blue lines, which are the three-week moving averages of the weekly totals for crime incidents and arrests, respectively. The jagged black lines are predicted values from the pre-Ferguson models detailed below, based on a fit to the data (i.e., gray dots) for the pre-Ferguson period only. The jagged red lines are then counterfactual predictions, which are formed using the coefficients from the pre-Ferguson models along with the values of the same predictor variables observed from the Ferguson period onwards. The differences between the gray dots and the red lines are the sources of the interval estimates of change, as explained fully in the original report and below.

\footnotetext{
${ }^{13}$ The unrest period is not depicted because it is embedded within the Gray period that begins to the right of the line labelled "Great Protests Begin." In some subsequent figures, the unrest week is easy to identify as an outlier gray dot (e.g., for the figure for arrests for disorderly conduct).
} 


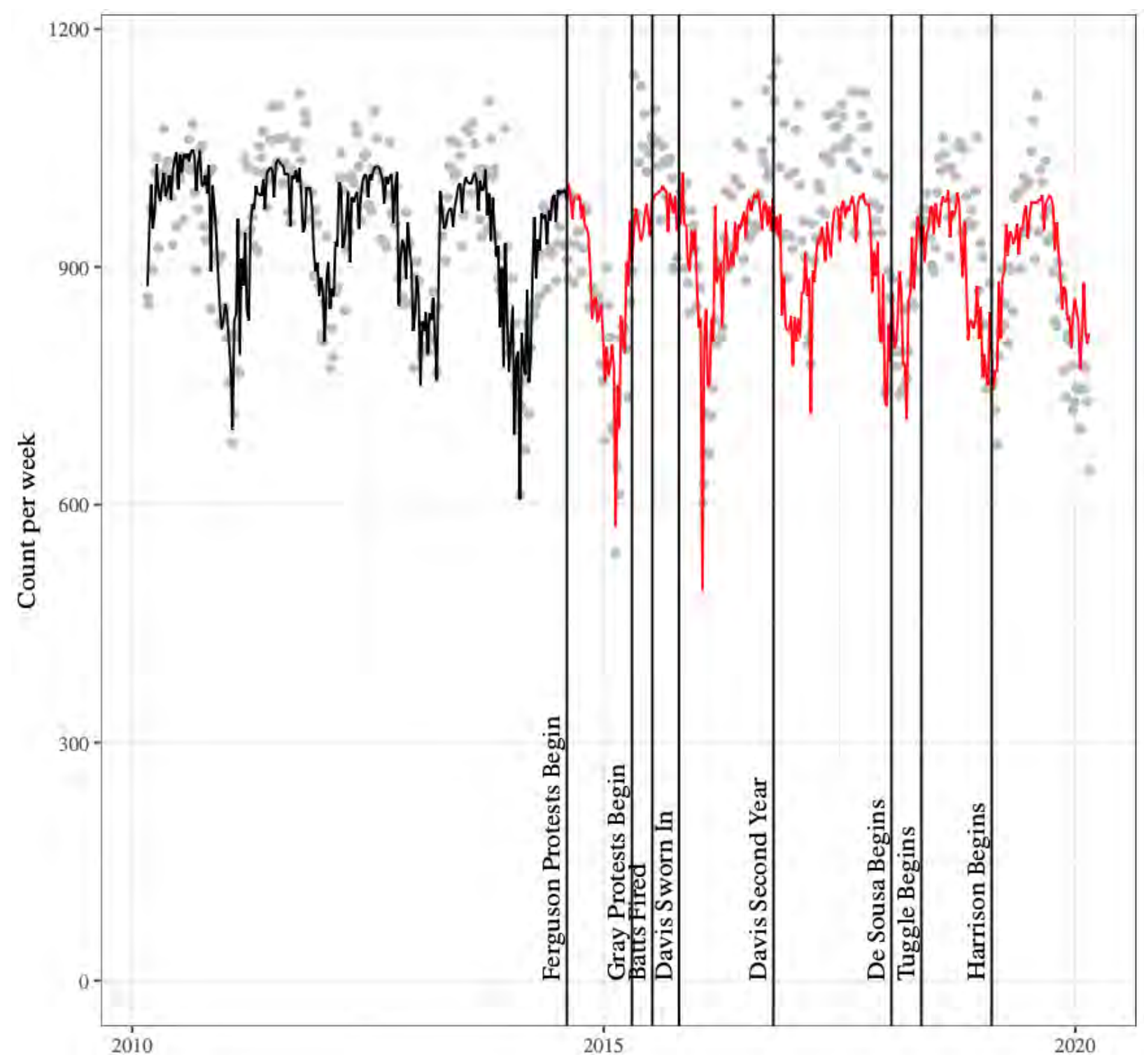

Figure 5. Total weekly recorded crime incidents (gray dots) with predicted values from the model before the Ferguson protests began (black line) and extrapolated counterfactual values after the Ferguson protests began (red line) 


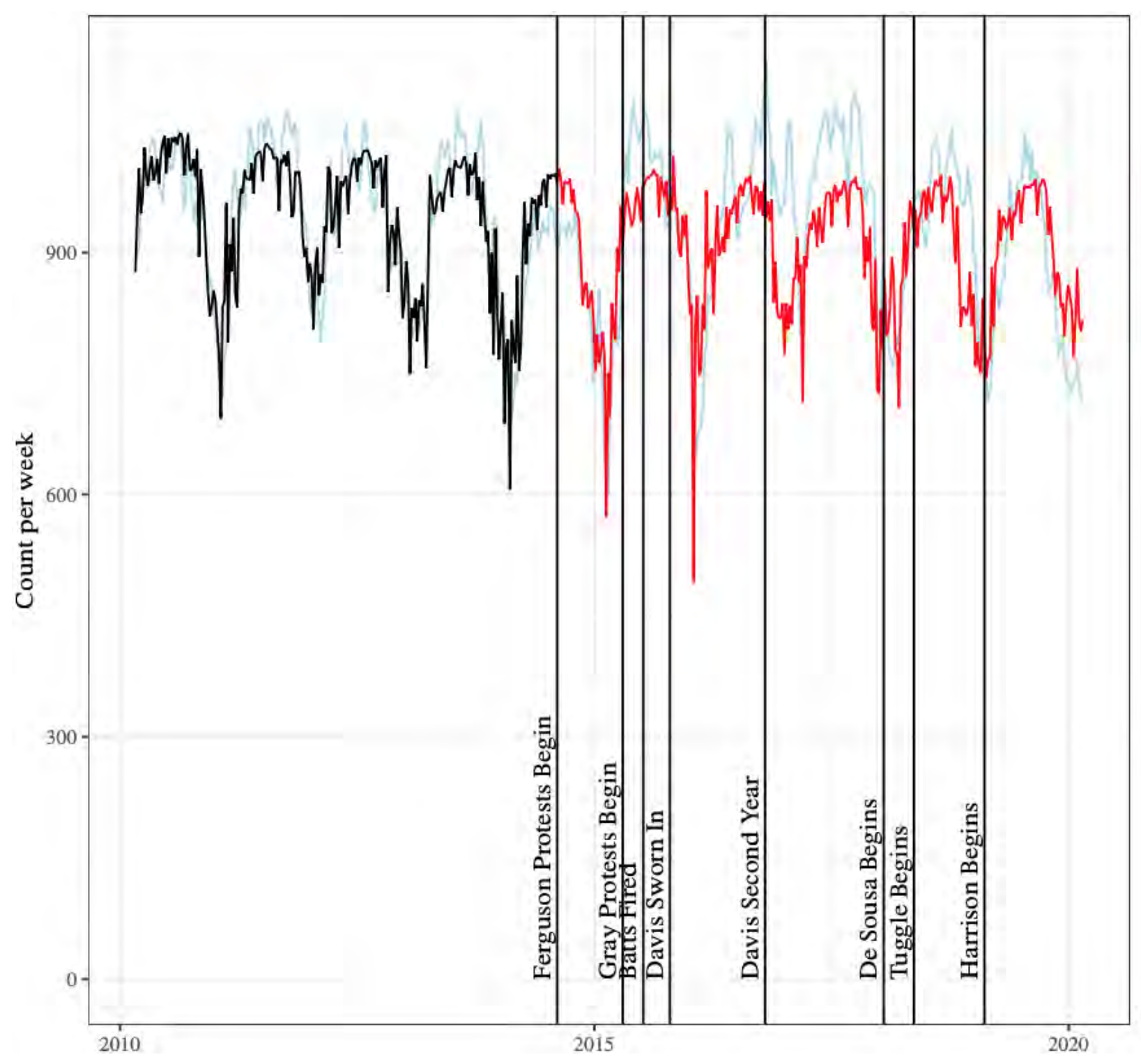

Figure 6. Total weekly recorded crime incidents (three-week moving average, blue line) with predicted values from the model before the Ferguson protests began (black line) and extrapolated counterfactual values after the Ferguson protests began (red line) 


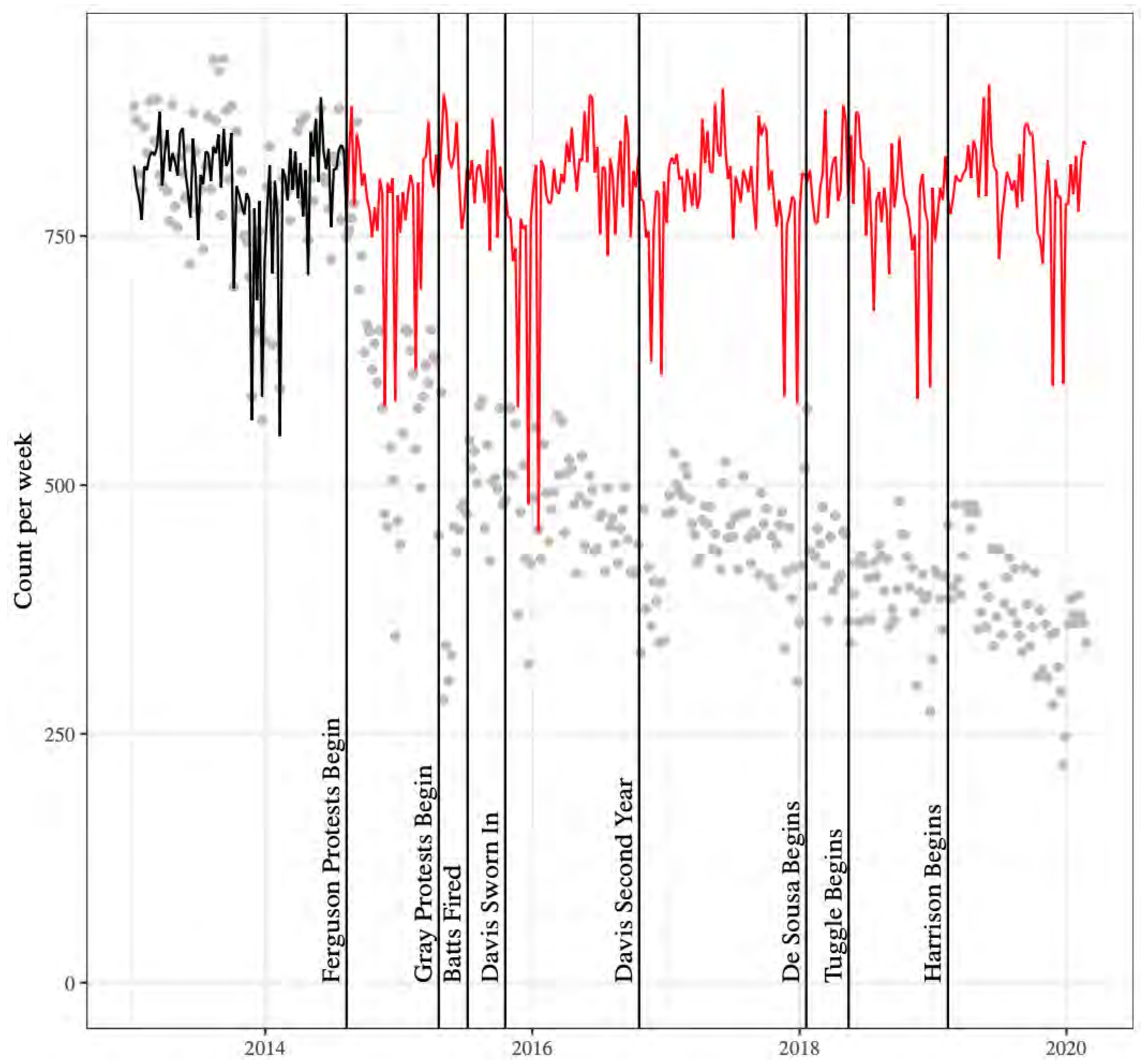

Figure 7. Total weekly arrests (gray dots) with predicted values from the model before the Ferguson protests began (black line) and extrapolated counterfactual values after the Ferguson protests began (red line) 


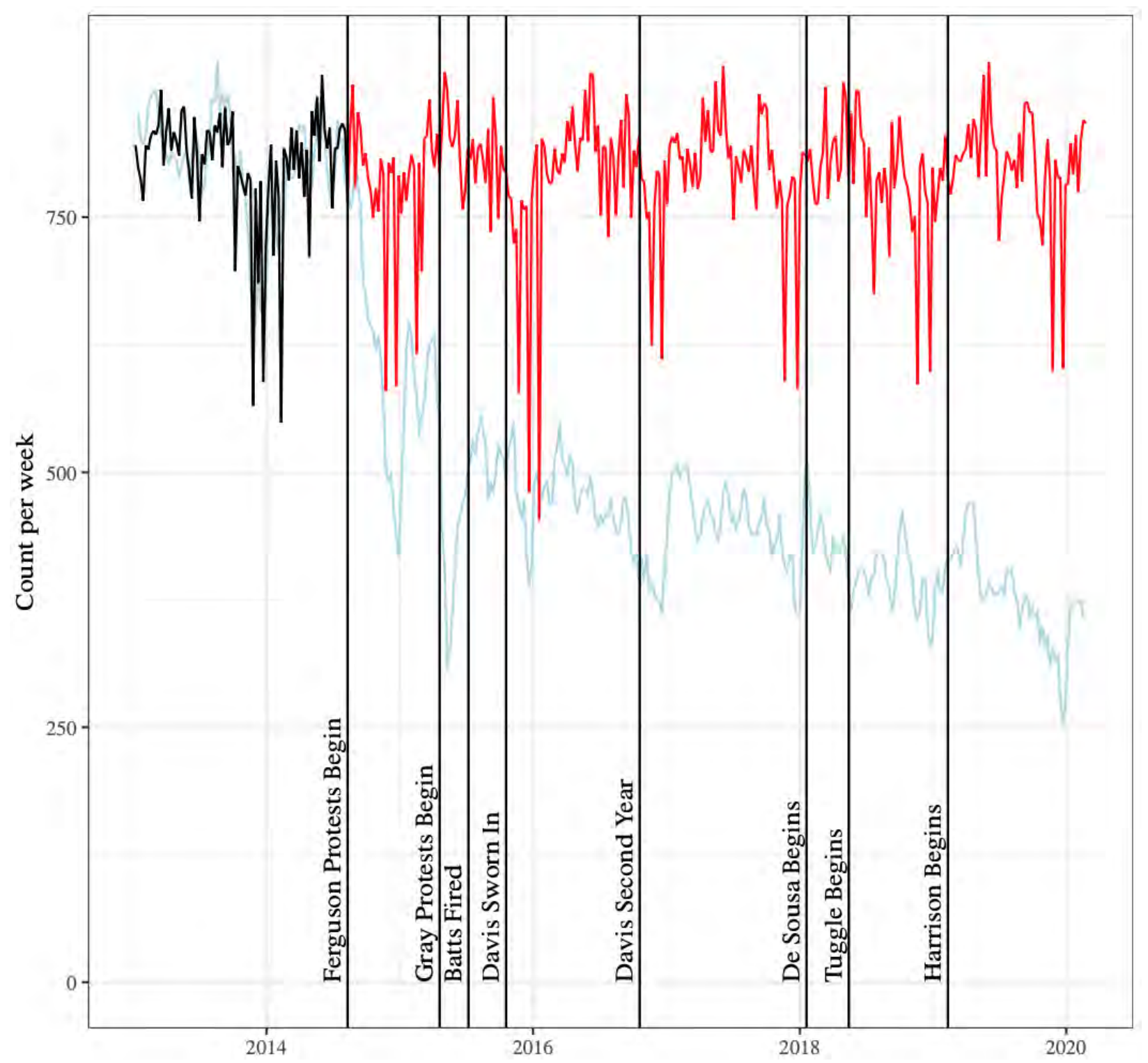

Figure 8. Total weekly arrests (three-week moving average, blue line) with predicted values from the model before the Ferguson protests began (black line) and extrapolated counterfactual values after the Ferguson protests began (red line) 
Specifications of the two models. The right-hand-side specifications of the two preFerguson prediction models were selected using only the weekly total crime incidents and weekly total arrests displayed in Figures 5-8. We then used the same specifications for the comprehensive sets of models that are presented in Appendix 2 for types of crime incidents and Appendix 3 for types of arrests.

Consider first Table 1, which offers results from three models for total crime incidents. The first column presents coefficients for the model that generates the predictions plotted as the jagged black line in Figure 5. The predictor variables for the model are presented in the row labels, and they collectively generate a model that explains 70 percent of the variance of weekly crime totals in the pre-Ferguson period from March 1, 2010 through August 10, 2014. Although we caution against attaching too much meaning to any single coefficient, since most of these predictor variables move together in seasonal fashion, it is clear that temperature, snowfall, precipitation, and hours of darkness are strongly predictive. The nonlinear partial relationship between temperature and crime is notable, with the effects appearing to accelerate with changes in the 50s, before leveling off in the 70s and then further in the 80s and above. These associations, however, are net of snowfall and precipitation, which themselves are temperature related. Overall, it is clear that the black jagged line in Figure 5 traces the core of the variation in the gray dots very well. For Table 2 , the same model does nearly as well in predicting arrests. But, for arrests, holidays are particularly important. In Figure 7, these are the downward spikes in the jagged black line that reach down to the corresponding gray dots.

The coefficients in the second column of both Tables 1 and 2 represent our preferred estimates for the effects of change over time, and they provide the values that, when scaled by an appropriate baseline count for the outcome, determine the percent-change findings reported below. The coefficients are calculated by first "de-trending" the outcome variables, which in this case is equivalent to transforming the weekly totals by subtracting from each week's value the corresponding value in that week for the value on the jagged line (the black line before the Ferguson period began and the red line afterwards). As a result, for this model, the outcome variable is the difference between the raw observed total crime count (the gray dots) and the modeled value represented by the black/red line. The coefficients for the model in the second column are then determined by fitting coefficients for indicator variables for each time period, with the periods switching on from 0 to 1 when each period begins (and remaining on at 1 through the end of the time series). One period is handled differently. The indicator variable for the week of unrest switches on from 0 to 1 during its single week, and it then switches off from 1 to 0 for all subsequent weeks. This "spike" parameterization for the week of unrest results in a Gary-period estimate that nets out the anomalous pattern of crime for that week, during which the National Guard was deployed to enforce a nighttime curfew. 
Table 1. Three models for total weekly recorded crime incidents

\begin{tabular}{|c|c|c|c|}
\hline Predictor variable & $\begin{array}{c}3 / 1 / 2010- \\
8 / 10 / 2014 \\
\text { model }\end{array}$ & $\begin{array}{c}\text { Preferred } \\
\text { model }\end{array}$ & $\begin{array}{c}\text { Alternative } \\
\text { model }\end{array}$ \\
\hline Time counter (in weeks) & -0.19 & & -0.20 \\
\hline After Ferguson protests begin (week of $8 / 11 / 14$ onward) & & -37.35 & -34.85 \\
\hline After Gray protests begin (week of $4 / 20 / 15$ onward) & & 110.13 & 107.27 \\
\hline Unrest and National Guard (during week of 4/27/15) & & 126.39 & 139.87 \\
\hline After Batts fired (week of 7/13/15 onward) & & -46.13 & -46.39 \\
\hline After Davis confirmed (week of 10/19/15 onward) & & -24.64 & -20.69 \\
\hline After Davis first year (week of 10/17/16 onward) & & 88.41 & 94.98 \\
\hline After De Sousa begins (week of 1/19/18 onward) & & -114.06 & -104.99 \\
\hline After Tuggle begins (week of 5/14/18 onward) & & 77.84 & 76.09 \\
\hline After Harrison begins (week of 2/11/19 onward) & & -88.03 & -75.08 \\
\hline Average maximum temperature to 50 degrees & 4.49 & & 5.94 \\
\hline Plus degrees in the $50 \mathrm{~s}$ & 5.35 & & 0.95 \\
\hline Plus degrees in the $60 \mathrm{~s}$ & -2.89 & & 1.28 \\
\hline Plus degrees in the $70 \mathrm{~s}$ & -4.90 & & -4.00 \\
\hline Plus degrees Greater Than 80 & -1.02 & & -2.35 \\
\hline Snowfall (inches) & -65.58 & & -47.26 \\
\hline Precipitation (inches) & -28.96 & & -23.24 \\
\hline Darkness before midnight (hours) & 23.87 & & 26.38 \\
\hline Thanksgiving/Christmas (proportion of week) & -32.28 & & -44.45 \\
\hline Winter holiday (proportion of week) & -211.39 & & -269.33 \\
\hline Other non-school days (proportion of week) & -27.91 & & -54.92 \\
\hline Summer vacation (proportion of week) & 20.88 & & 1.81 \\
\hline Summer holiday (proportion of week) & -261.19 & & -222.81 \\
\hline Observations (weeks) & 232 & 522 & 522 \\
\hline Proportion of variance explained (R-squared) & 0.70 & 0.30 & 0.71 \\
\hline
\end{tabular}

Notes: The outcome variable for the first and third columns is the same - the total weekly recorded crime count - but the Rsquared values cannot be compared across the two columns because the weeks for the model differ. Relatedly, the outcome for the second column is a residualized total weekly crime count, and as such the R-squared value of 0.30 cannot be meaningfully compared to either 0.70 or 0.71 in the first and third columns, even though the models in the second and third columns are estimated for the same weeks. 
Table 2. Three models for total weekly arrests

\begin{tabular}{|c|c|c|c|}
\hline Predictor variable & $\begin{array}{c}1 / 1 / 2013- \\
8 / 10 / 2014 \\
\text { model }\end{array}$ & $\begin{array}{c}\text { Preferred } \\
\text { model }\end{array}$ & $\begin{array}{c}\text { Alternative } \\
\text { model }\end{array}$ \\
\hline After Ferguson protests begin (week of $8 / 11 / 14$ onward) & & -170.07 & -171.84 \\
\hline After Gray protests begin (week of $4 / 20 / 15$ onward) & & -245.64 & -237.88 \\
\hline Unrest and National Guard (during week of 4/27/15) & & 169.59 & 160.30 \\
\hline After Batts fired (week of 7/13/15 onward) & & 127.73 & 119.17 \\
\hline After Davis confirmed (week of 10/19/15 onward) & & -25.87 & -32.95 \\
\hline After Davis first year (week of 10/17/16 onward) & & -37.50 & -30.51 \\
\hline After De Sousa begins (week of 1/19/18 onward) & & -20.94 & -14.51 \\
\hline After Tuggle begins (week of 5/14/18 onward) & & -15.30 & -21.99 \\
\hline After Harrison begins (week of 2/11/19 onward) & & -37.18 & -32.82 \\
\hline Average maximum temperature to 50 degrees & 1.83 & & 2.55 \\
\hline Plus degrees in the $50 \mathrm{~s}$ & -9.51 & & -3.43 \\
\hline Plus degrees in the $60 \mathrm{~s}$ & 9.32 & & 1.66 \\
\hline Plus degrees in the 70s & 3.27 & & -1.79 \\
\hline Plus degrees Greater Than 80 & -8.33 & & -1.73 \\
\hline Snowfall (inches) & -88.87 & & -30.07 \\
\hline Precipitation (inches) & -143.98 & & -91.09 \\
\hline Darkness before midnight (hours) & -23.89 & & -13.17 \\
\hline Thanksgiving/Christmas (proportion of week) & $-1,396.50$ & & -765.85 \\
\hline Winter holiday (proportion of week) & -1.88 & & 237.57 \\
\hline Other non-school days (proportion of week) & -5.46 & & -38.26 \\
\hline Summer vacation (proportion of week) & -46.77 & & 10.16 \\
\hline Summer holiday (proportion of week) & -468.88 & & -148.99 \\
\hline Observations (weeks) & 83 & 373 & 373 \\
\hline Proportion of variance explained (R-squared) & 0.66 & 0.87 & 0.91 \\
\hline
\end{tabular}

Notes: The outcome variable for the first and third columns is the same - the total weekly recorded arrest count - but the R-squared values cannot be compared across the two columns because the weeks for the model differ. Relatedly, the outcome for the second column is a residualized total weekly arrest count, and as such the R-squared value of 0.91 cannot be meaningfully compared to either 0.66 or 0.87 in the first and third columns, even though the models in the second and third columns are estimated for the same weeks. 
We offer one additional model in each table, which is an alternative to our preferred model. It fits coefficients for weather, hours of darkness, and types of days per week simultaneously with coefficients for the period indicator variables. The advantage of the model is that it uses additional data on the seasonal adjustment variables, which could provide better estimates of the underlying seasonal trend. The disadvantage is that the periods of interest are not randomly distributed relative to the adjustment variables, and thus the model is forced to simultaneously estimate all coefficients at once, which may generate misleading estimates of the period effects. Also, in the case of crime in Table 1, we do not have quite as much control because the baseline time counter is specified to be constant over all weeks. We cannot detrend the data for the alternative model, as we did for the preferred model, by suppressing the effect of the time counter after Davis' first year (although we could re-specify to do so). This is one reason why the coefficients for period differ between the preferred and the alternative model. However, for arrests in Table 2, the coefficients for the periods are very similar, suggesting little difference over the years on this pattern of cyclicality.

\subsection{Changes in Shootings and Homicides}

To explain how the models in Tables 1 and 2 are utilized, we first present results for the topic of greatest concern to many in the city: changes over time in shootings and homicides. Figures 912 (see next four pages), which are drawn from Appendix 2, present graphical displays of the results for shootings and homicides. Several patterns are immediately clear from the figures.

First, shootings and homicides are low frequency events, and thus the scale of the vertical dimensions in these figures is much different than for Figures 5-8 above. Also, as a consequence, week-to-week variability is more notable. For this reason, it is easier to interpret Figures 10 and 12, which represent the raw outcomes (the gray dots from Figures 9 and 11) as the blue lines that are three-week moving averages of shootings and homicides.

Second, the spread around the jagged black line appears more substantial than for total crime incidents (see Figure 5 above for comparison). As can be seen in Appendix 2, this pattern is genuine. The pre-Ferguson model, with the right-hand-side specified exactly as the one presented above in the first column of Table 1, has a proportion of variance explained (the model fit R-squared) of 0.26 for shootings and 0.12 for homicides. As shown in Table 1, the Rsquared for the model for total crime incidents is 0.70 . Some of the difference is attributable to variability across weeks, which is amplified for comparatively rare events. But this is only part of the story. The seasonality of shootings and homicides is less pronounced than for other types of crime, and these other types are collectively captured by the total crime incident count analyzed for Figures 5 and 6.

Third, the overall pattern is clear for the city as a whole. The jagged blue lines move above the counterfactual red line after the Gray period begins. For both shootings and homicides, they remain above the counterfactual red line for almost all weeks through 2020. 


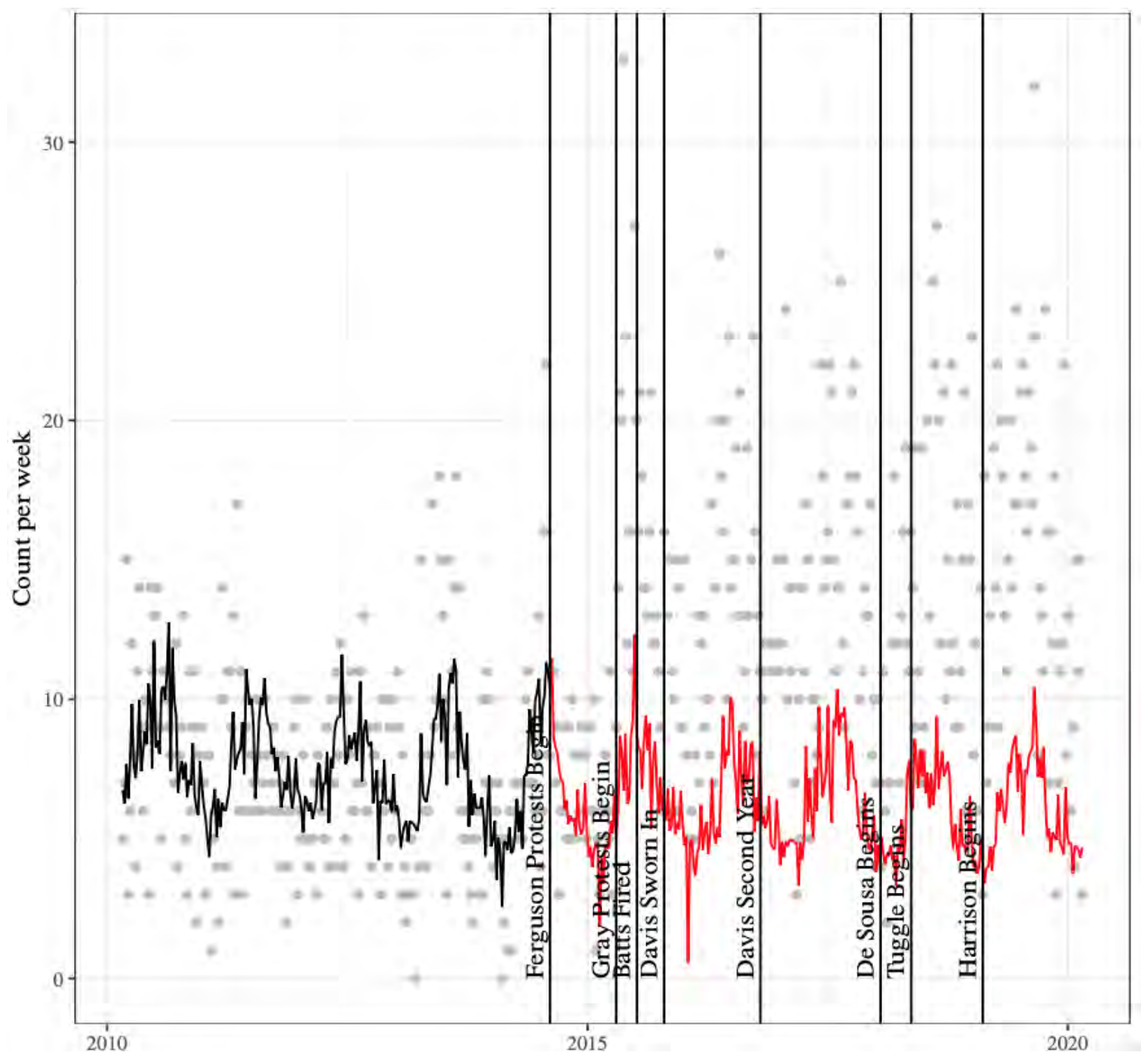

Figure 9. Total weekly shootings count (gray dots) with predicted values from the model before the Ferguson protests began (black line) and extrapolated counterfactual values after the Ferguson protests began (red line) 


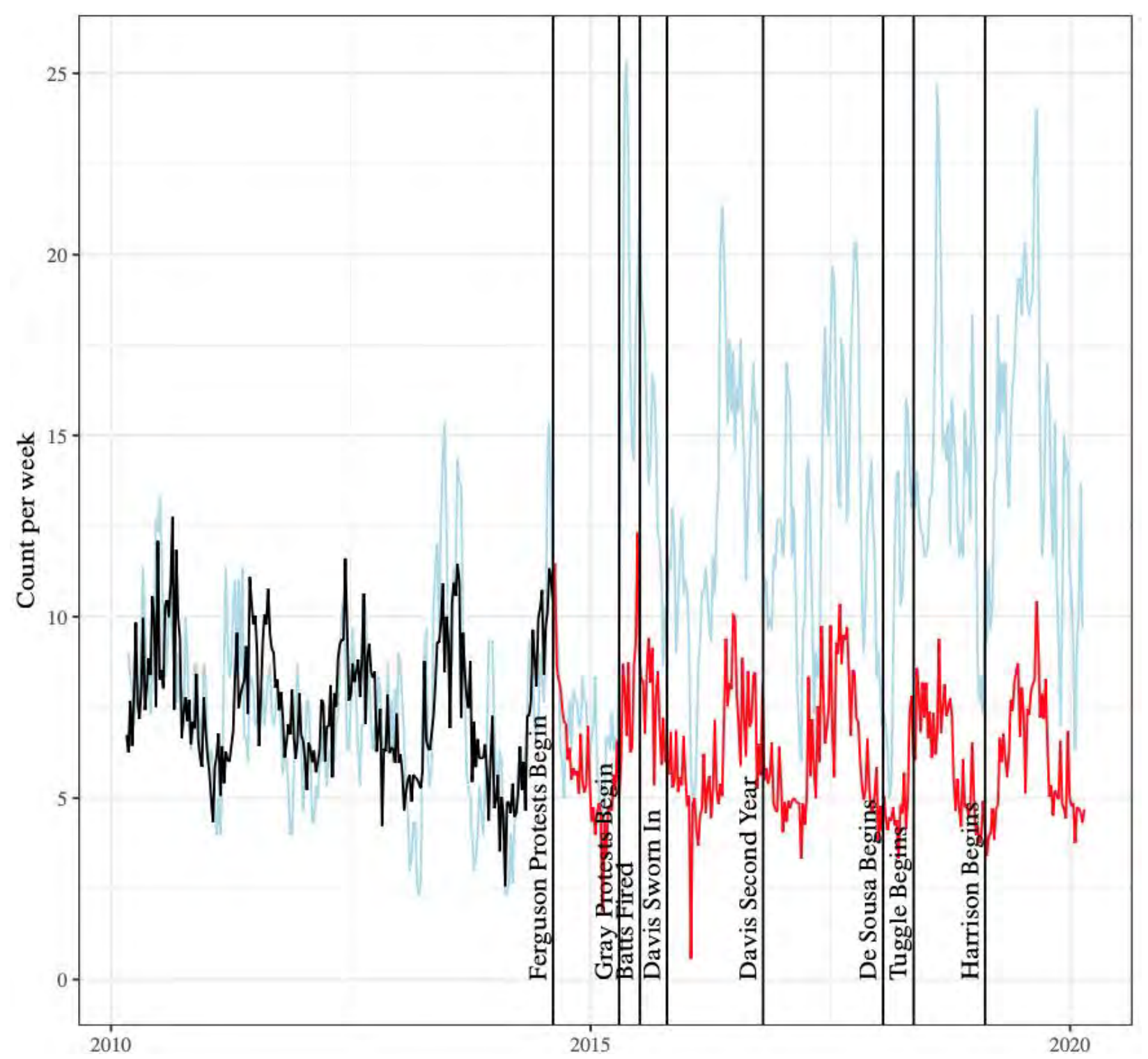

Figure 10. Total weekly shootings count (three-week moving average, blue line) with predicted values from the model before the Ferguson protests began (black line) and extrapolated counterfactual values after the Ferguson protests began (red line) 


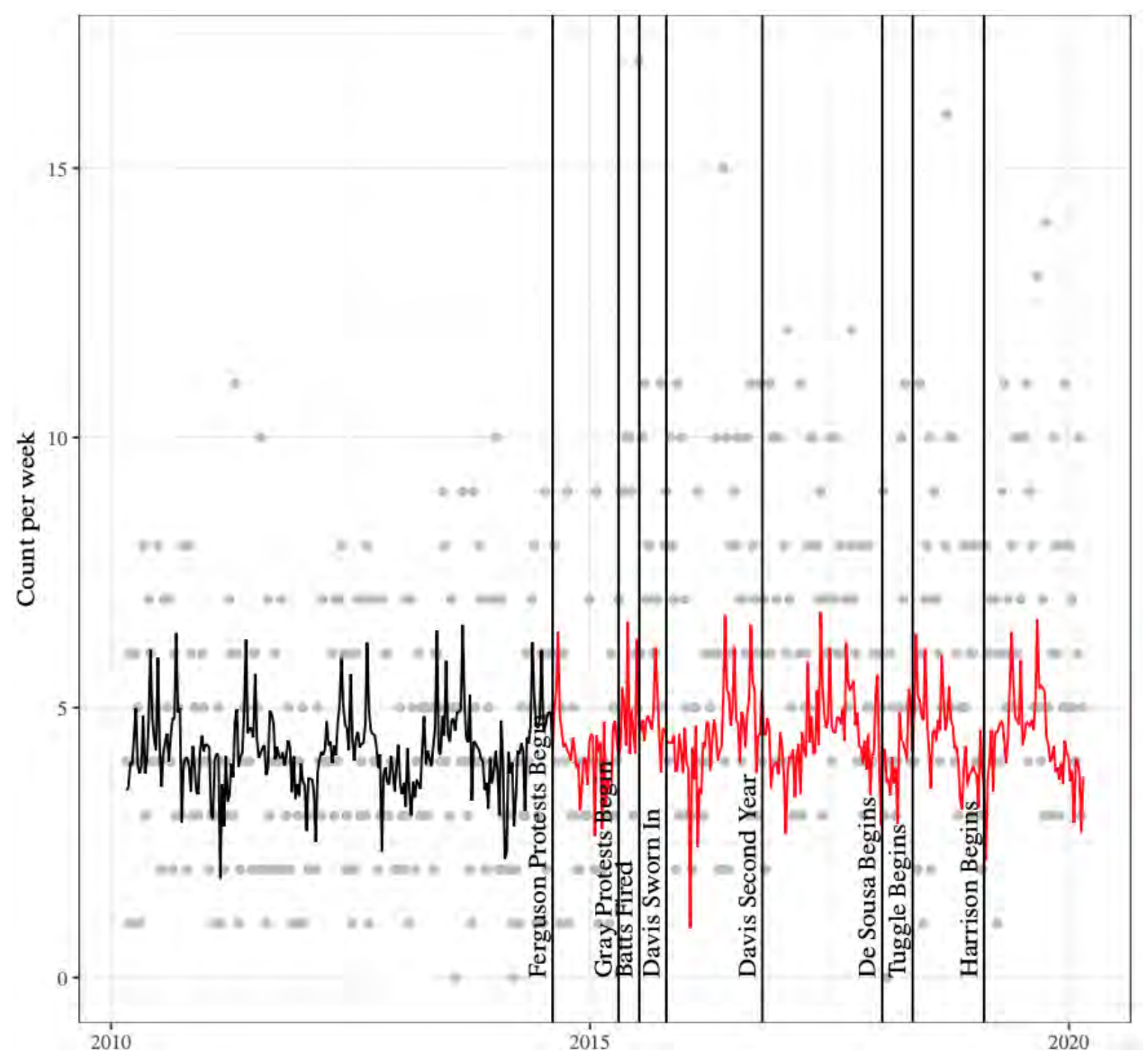

Figure 11. Total weekly homicide count (gray dots) with predicted values from the model before the Ferguson protests began (black line) and extrapolated counterfactual values after the Ferguson protests began (red line) 


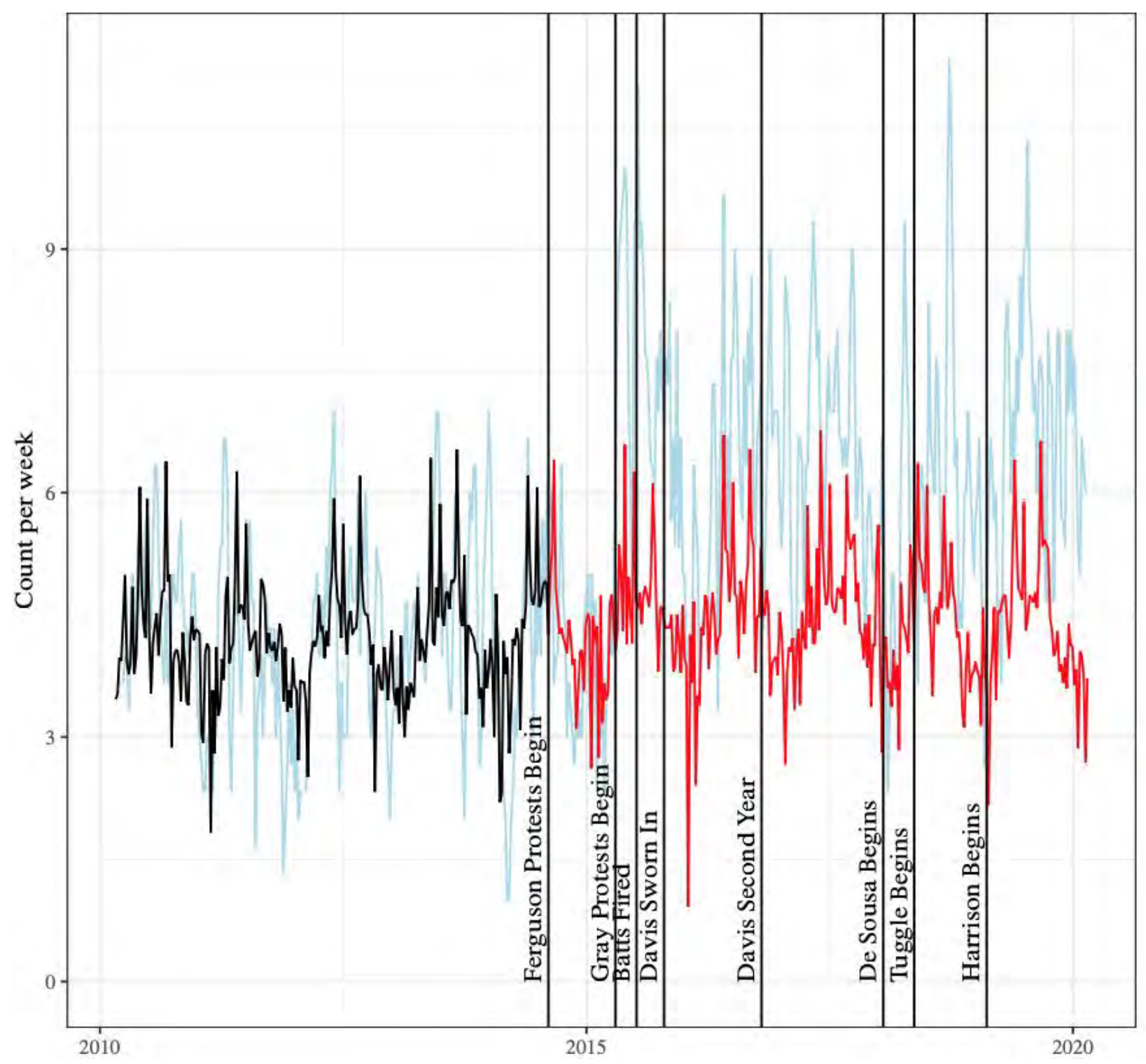

Figure 12. Total weekly homicide count (three-week moving average, blue line) with predicted values from the model before the Ferguson protests began (black line) and extrapolated counterfactual values after the Ferguson protests began (red line) 
The pattern of findings suggested by a visual inspection of Figures 9-12 can be estimated precisely using underlying models analogous to those presented in Table 1. The specific models that do so are presented in detail in Appendix 2 (see sections 4 and 5). Table 3 (see next page) combines estimated coefficients from these models in order to produce period-specific estimates of shootings and homicides for the city as a whole. In addition, Table 3 also offers results for each police district, using estimated coefficients from eighteen subsequent models in Appendix 2 (see sections 27-44).

For Table 3, the "baseline count" in the first column is the number of shootings and homicides for the 52 weeks of the year immediately before the Ferguson period (i.e., early August 2013 through early August 2014). The numbers in subsequent columns are the estimated average weekly totals, adjusted for seasonality, weather, and types of days. Because these periods differ in length, and because they start and end at different points in each year, the estimated average weekly totals are not simple averages of the raw values for shootings and homicides. With reference to Figures 9 and 11, the period-specific estimates in Table 3 are not the averages of the gray dots in these two figures. Instead, the estimates are the average differences between the gray dots and the jagged red line in each period, added to the appropriate estimated baseline count (and removing the contribution of the unrest week).

For a guide to interpretation, consider some comparisons in Table 3. In the year before the Ferguson period began, the city as a whole averaged 7.1 shootings per week. This average increased to a high of 17.9 shootings per week during the Gray period. During the periods when Davis was commissioner, the shooting rate declined to less than 14 per week. This level was still far above the baseline of 7.1 per week. Davis was then fired, according to the mayor (see above), because the shooting rate was still too high. Finally, shootings have increased slightly since Tuggle and Harrison took control of the BPD. As of March 1, 2020, the prior year's weekly average shooting rate was 15.4 per week, more than twice the 7.1 average observed for the baseline year before the Ferguson period. The other rows of the first panel show that the increases in shootings were widespread across police districts, with the largest relative increases as of 2020 occurring in the Central, Eastern, and Southeastern districts. The high point of shootings, during the Gray period, was more localized in the Western and Eastern districts. (For a map of the districts, see page 16 of Morgan and Pally 2016 or Figure 34 below.)

The homicide estimates in the second panel of Table 3 reveal similar patterns. The overall rate of increase is lower, and the spatial distribution is slightly different. However, homicides are elevated in nearly all districts in every period. And, as shown in the final column, the elevated level of homicides as of March 2020 is strongly related to the elevated level of shootings. One specific comparison should be offered. In the baseline year, the estimated homicide count was 224 (e.g., 52.14 weeks x 4.3 homicides per week). For the most recent time period since Harrison became commissioner, the estimated homicide count was 344 homicides (e.g., 52.14* 6.6). This matches the actual raw count for 2019 very closely, which ended with 348 homicides. Thus, the current level of elevation in homicides is approximately 120 deaths per year. 
Table 3. Adjusted estimates of weekly shootings and homicides by time period for the city as a whole and for each BPD police district separately

\begin{tabular}{|c|c|c|c|c|c|c|c|c|c|}
\hline & \multirow{2}{*}{$\begin{array}{c}\text { Baseline } \\
\text { count } \\
\text { per week }\end{array}$} & \multicolumn{8}{|c|}{ Adjusted number for eight sequential periods: } \\
\hline & & $\begin{array}{l}\text { Ferguson } \\
\text { period }\end{array}$ & $\begin{array}{l}\text { Gray } \\
\text { period }\end{array}$ & $\begin{array}{l}\text { Davis } \\
\text { transition }\end{array}$ & $\begin{array}{l}\text { Davis } \\
\text { first year }\end{array}$ & $\begin{array}{l}\text { Davis last } \\
15 \text { months }\end{array}$ & $\begin{array}{c}\text { De Sousa } \\
\text { spring }\end{array}$ & $\begin{array}{c}\text { Tuggle } \\
\text { interregnum }\end{array}$ & $\begin{array}{l}\text { Harrison } \\
\text { first year }\end{array}$ \\
\hline \multicolumn{10}{|l|}{ Shootings } \\
\hline Northwestern & 0.7 & 1.2 & 1.2 & 1.6 & 1.3 & 1.9 & 1.3 & 2.2 & 1.7 \\
\hline Northern & 0.4 & 0.5 & 0.6 & 1.6 & 0.9 & 0.8 & 0.9 & 0.9 & 0.8 \\
\hline Northeastern & 0.9 & 0.9 & 1.7 & 1.3 & 1.7 & 1.7 & 1.6 & 1.6 & 2.0 \\
\hline Western & 1.2 & 1.7 & 4.7 & 1.9 & 2.5 & 2.5 & 2.3 & 2.3 & 2.4 \\
\hline Central & 0.5 & 0.2 & 1.2 & 0.3 & 0.9 & 0.9 & 0.8 & 1.1 & 1.5 \\
\hline Eastern & 1.1 & 1.1 & 4.2 & 1.9 & 2.3 & 1.8 & 2.0 & 2.4 & 2.7 \\
\hline Southwestern & 1.0 & 1.2 & 1.9 & 2.6 & 1.8 & 1.5 & 1.9 & 1.6 & 1.5 \\
\hline Southern & 0.7 & 0.7 & 1.7 & 1.7 & 1.6 & 2.0 & 1.1 & 1.6 & 1.5 \\
\hline Southeastern & 0.5 & 0.5 & 0.8 & 0.7 & 0.8 & 0.7 & 1.0 & 1.3 & 1.4 \\
\hline Total shootings & 7.1 & 8.1 & 17.9 & 13.5 & 13.7 & 13.7 & 12.8 & 14.9 & 15.4 \\
\hline \multicolumn{10}{|l|}{ Homicides } \\
\hline Northwestern & 0.7 & 0.6 & 0.9 & 0.7 & 0.5 & 0.7 & 0.5 & 0.5 & 0.6 \\
\hline Northern & 0.4 & 0.3 & 0.4 & 0.7 & 0.3 & 0.5 & 0.5 & 0.4 & 0.5 \\
\hline Northeastern & 0.5 & 1.0 & 1.3 & 1.1 & 1.0 & 1.0 & 0.8 & 1.0 & 1.2 \\
\hline Western & 0.6 & 0.4 & 1.4 & 1.5 & 1.1 & 0.8 & 0.8 & 0.9 & 1.1 \\
\hline Central & 0.3 & 0.3 & 0.2 & 0.4 & 0.3 & 0.4 & 0.3 & 0.4 & 0.2 \\
\hline Eastern & 0.6 & 0.5 & 1.2 & 0.9 & 1.1 & 1.0 & 0.8 & 1.0 & 1.0 \\
\hline Southwestern & 0.6 & 0.6 & 1.4 & 0.8 & 0.7 & 0.7 & 0.6 & 0.9 & 0.8 \\
\hline Southern & 0.4 & 0.3 & 0.8 & 0.6 & 0.7 & 0.7 & 0.8 & 0.8 & 0.7 \\
\hline Southeastern & 0.3 & 0.2 & 0.2 & 0.3 & 0.5 & 0.5 & 0.4 & 0.2 & 0.6 \\
\hline Total homicides & 4.3 & 4.1 & 8.0 & 7.0 & 6.0 & 6.3 & 5.5 & 5.9 & 6.6 \\
\hline
\end{tabular}




\subsection{Percentage Changes in the Prevalence of Crime Incidents, Disaggregated by Type}

In this section, we provide tabular summaries of period-specific estimates of change in the prevalence of crime incidents. We follow these summaries with graphical depictions of types of crime incidents that are of particular interest. For the tabular summaries, we first present updated estimates of types of changes reported in the original reports. Then, we offer wholly new estimates for the four intervals from 2016 through 2020. We scale all estimates by percentages to facilitate comparisons in the trajectories of low-prevalence crimes, such as homicides and carjackings, to high-prevalence crimes, such as common assaults and larceny.

Changes in crime incidents through Davis' first year as police commissioner. Table 4 (see next page) presents period estimates for all types of crime incidents for the Ferguson period through the first Davis period. The estimates are expressed as percentage change and as a percentage of the count in the baseline reference year (in particular, the weekly average for the 52 weeks before the Ferguson protests began). For both sets of numbers, the percentage scale is set by the baseline reference year. These numbers are presented in the first column of the table.

To understand the scaling, consider homicides again. For the Ferguson period, the weekly rate of homicides fell by 4.6 percent for the Ferguson period and then increased by 91.6 percent for the Gray period. The second panel then presents the rate of homicides as a percentage of the rate in the baseline reference year. For example, the 4.6 percent decline for the Ferguson period rendered the Ferguson period's homicide rate as 95.4 percent of the homicide rate for the baseline reference year. Then, the 91.6 percent increase for the Gray period rendered the Gray period's homicide rate as 186.9 percent of the homicide rate for the baseline reference year. Note that relationships among these latter numbers match those among the unscaled numbers from the bottom row of Table 3 above, subject to slight differences from rounding. ${ }^{14}$

The first three columns of estimated change in Table 4 were also estimated for the original report, with slightly different specifications and without BPD's edited data (see Table 2 in Morgan and Pally 2016). All of the estimates in Table 4 were also re-estimated for the updated report (see Tables 2 and 3 in Morgan 2016). While we regard the estimates in Table 4 of this final report as improved for the reasons discussed above, the improvements are small enough that the core conclusions from these periods for crime incidents do not require any meaningful revisions. See the prior section for these conclusions, which plug in values from Table 4 to demonstrate why we stand by the conclusions from 2016.

\footnotetext{
14 The value of 4.1 in the bottom row of Table 3, when divided by 4.3 , is 0.953 . The value of 8.0 , when divided by 4.3 , is 1.860. If the raw numbers in Table 3 were not rounded to the first decimal place, such division would deliver the exact same numbers as in Table 4, after multiplying by 100 to place them on a percent-change scale.
} 
Table 4. Baseline and percentage change in the weekly count of recorded crime incidents from the Ferguson period through Davis' first year

\begin{tabular}{|c|c|c|c|c|c|c|c|c|c|c|}
\hline & \multirow{2}{*}{$\begin{array}{c}\text { Baseline } \\
\text { count } \\
\text { per week }\end{array}$} & \multicolumn{5}{|c|}{ Adjusted percentage change from prior period } & \multicolumn{4}{|c|}{ Adjusted percentage of baseline during period } \\
\hline & & $\begin{array}{l}\text { Ferguson } \\
\text { period }\end{array}$ & $\begin{array}{c}\text { Gray } \\
\text { period }\end{array}$ & $\begin{array}{l}\text { Unrest } \\
\text { period }\end{array}$ & $\begin{array}{c}\text { Davis } \\
\text { transition }\end{array}$ & $\begin{array}{c}\text { Davis } \\
\text { first year }\end{array}$ & $\begin{array}{l}\text { Ferguson } \\
\text { period }\end{array}$ & $\begin{array}{c}\text { Gray } \\
\text { period }\end{array}$ & $\begin{array}{c}\text { Davis } \\
\text { transition }\end{array}$ & $\begin{array}{c}\text { Davis } \\
\text { first year }\end{array}$ \\
\hline Homicide & 4.3 & -4.6 & 91.6 & 23.5 & -23.0 & -23.2 & 95.4 & 186.9 & 163.9 & 140.8 \\
\hline Shooting & 7.1 & 13.6 & 138.3 & -40.4 & -61.9 & 2.9 & 113.6 & 252.0 & 190.1 & 193.0 \\
\hline Rape & 5.3 & -7.1 & 29.7 & 4.1 & -36.9 & 20.9 & 92.9 & 122.5 & 85.7 & 106.6 \\
\hline Robbery (carjacking) & 2.6 & 65.9 & 43.8 & 377.4 & -8.4 & 120.0 & 165.9 & 209.7 & 201.3 & 321.3 \\
\hline Robbery (street) & 51.5 & 0.4 & 33.0 & -31.4 & -16.4 & 9.8 & 100.4 & 133.4 & 117.0 & 126.8 \\
\hline Robbery (commercial) & 11.0 & 20.0 & 88.5 & -7.7 & -66.2 & -2.2 & 120.0 & 208.5 & 142.3 & 140.0 \\
\hline Robbery (residence) & 9.8 & -10.2 & 21.7 & 9.4 & -28.9 & 19.5 & 89.8 & 111.5 & 82.6 & 102.1 \\
\hline Aggravated assault & 82.3 & 5.4 & 30.5 & 59.0 & -11.6 & 7.3 & 105.4 & 135.9 & 124.4 & 131.7 \\
\hline Common assault & 157.4 & -12.4 & 1.5 & -29.7 & -3.0 & 6.5 & 87.6 & 89.1 & 86.1 & 92.5 \\
\hline Burglary & 139.0 & 0.3 & 13.3 & 187.3 & -2.1 & -4.3 & 100.3 & 113.6 & 111.5 & 107.2 \\
\hline Larceny from auto & 125.6 & 6.2 & 15.6 & -61.4 & -2.3 & -16.6 & 106.2 & 121.8 & 119.5 & 102.9 \\
\hline Larceny & 226.7 & -9.9 & -13.7 & -25.5 & 3.6 & -8.2 & 90.1 & 76.4 & 80.0 & 71.8 \\
\hline Auto theft & 79.3 & -9.7 & 36.4 & -23.4 & -10.6 & -8.7 & 90.3 & 126.7 & 116.1 & 107.4 \\
\hline Arson & 4.3 & 18.0 & 23.1 & 646.3 & 9.0 & -3.2 & 118.0 & 141.1 & 150.1 & 146.9 \\
\hline Total & 913.2 & -4.1 & 12.1 & 13.8 & -5.1 & -2.7 & 95.9 & 108.0 & 102.9 & 100.2 \\
\hline
\end{tabular}

Notes: The unrest period is modeled with a "spike" specification, and this modeling choice removes it from the calculation of cumulated change in the second panel. 
Changes in crime incidents through Harrison's first year as police commissioner. Table 5 (see next page) offers analogous estimates for the final four time periods considered: from Davis' last 15 months as commissioner through Harrison's first year as commissioner. The same baseline count is used to scale the estimated percentages.

Much could be written on the patterns in Table 5, and we will hold our overall conclusions for the final section of this report. Nonetheless, it should be clear from the final four columns of Table 5 that all categories of violent crime remain substantially above the baseline reference year before the Ferguson protests and the unrest in Baltimore. The variation across police commissioners from late 2016 through early 2020 is small, whether one considers homicides, shootings, carjackings, street robbery, or aggravated assault.

In contrast, property crimes - burglary, larceny from auto, other types of larceny, and auto theft - have trended lower, especially during Harrison's first year. The decline in incidents of property crime is consistent with the decline in commercial robbery during Harrison's first year as well. It is therefore possible that the 2019 crime plan, which has shifted resources even more heavily toward particular hotspots, has reduced property crime. Nonetheless, during the same period, violent crime did not decline meaningfully, except perhaps for street robbery. Thus, it would be inappropriate to infer that violent crime has been reduced just because of the overall 9.6 percent decline in total crime incidents during Harrison's first year (see the last row of Table 5). While it is true that the total number of crime incidents is now lower than in the baseline year, that decline does not reflect what city residents, and its leadership, have good reason to care most about.

Finally, it is possible that the decline in property crime is an artefact. It could be that police officers have pulled back from some types of routine policing, and thus fewer incidents of larceny from auto, for example, have been recorded. According to this line of thinking, fewer genuine incidents of property crime have been noticed and recorded by officers patrolling their posts. It is also possible that fewer incidents have been reported to the police because the legitimacy of the department has eroded further as the GTTF scandal has unfolded. We will return to these matters of interpretation in the concluding section of this report.

Graphical displays of change for selected types of crime. Figures 13-20 (see subsequent pages) offer times series plots for types of crime incidents, analogous to Figures 6, 8, 10, and 12 above. These figures are again drawn from Appendix 2, where crime-incident-specific analogs to Table 1 can be found. Figure 13 is the most dramatic, insofar as it shows the relentless increase in carjackings. Other figures present trends for street robbery, aggravated assault, common assault, burglary, larceny from automobiles, and larceny. Recall also that similar figures for shootings and homicides were presented above in Figures 10 and 12. Altogether, the figures are an alternative way to understand the changes that are summarized in a targeted fashion in Tables 3, 4, and 5. Both types of representations should not be over-interpreted. Inherent variability is prominent, especially for low-prevalence types of crime. 
Table 5. Baseline and percentage change in the weekly count of recorded crime incidents from Davis' second year through Harrison's first year

\begin{tabular}{|c|c|c|c|c|c|c|c|c|c|}
\hline & \multirow{2}{*}{$\begin{array}{c}\text { Baseline } \\
\text { count } \\
\text { per Week }\end{array}$} & \multicolumn{4}{|c|}{ Adjusted percentage change from prior period } & \multicolumn{4}{|c|}{ Adjusted percentage of baseline during period } \\
\hline & & $\begin{array}{l}\text { Davis last } \\
15 \text { months }\end{array}$ & $\begin{array}{c}\text { De Sousa } \\
\text { spring }\end{array}$ & $\begin{array}{c}\text { Tuggle } \\
\text { interregnum }\end{array}$ & $\begin{array}{l}\text { Harrison } \\
\text { first year }\end{array}$ & $\begin{array}{l}\text { Davis last } \\
15 \text { months }\end{array}$ & $\begin{array}{c}\text { De Sousa } \\
\text { spring }\end{array}$ & $\begin{array}{c}\text { Tuggle } \\
\text { interregnum }\end{array}$ & $\begin{array}{l}\text { Harrison } \\
\text { first year }\end{array}$ \\
\hline Homicide & 4.3 & 5.2 & -17.9 & 9.9 & 16.6 & 146.0 & 128.1 & 138.0 & 154.6 \\
\hline Shooting & 7.1 & -0.4 & -13.2 & 30.5 & 6.4 & 192.6 & 179.4 & 209.9 & 216.4 \\
\hline Rape & 5.3 & 25.2 & 4.2 & -15.1 & -23.2 & 131.8 & 136.0 & 120.9 & 97.7 \\
\hline Robbery (carjacking) & 2.6 & 136.3 & -100.2 & 41.5 & 73.0 & 457.6 & 357.4 & 398.9 & 471.9 \\
\hline Robbery (street) & 51.5 & 12.0 & -22.4 & 10.0 & -13.2 & 138.9 & 116.4 & 126.4 & 113.2 \\
\hline Robbery (commercial) & 11.0 & 39.6 & -48.2 & 29.9 & -44.1 & 179.6 & 131.4 & 161.2 & 117.2 \\
\hline Robbery (residence) & 9.8 & 16.6 & 7.6 & -22.5 & -6.1 & 118.7 & 126.3 & 103.8 & 97.7 \\
\hline Aggravated assault & 82.3 & 23.6 & -12.1 & 13.5 & -5.7 & 155.3 & 143.2 & 156.7 & 151.0 \\
\hline Common assault & 157.4 & 19.4 & -6.7 & 5.5 & -4.1 & 111.9 & 105.3 & 110.8 & 106.7 \\
\hline Burglary & 139.0 & 8.8 & -21.0 & -4.1 & -11.4 & 116.0 & 95.0 & 90.9 & 79.5 \\
\hline Larceny from auto & 125.6 & 1.3 & -11.0 & 21.1 & -21.5 & 104.2 & 93.1 & 114.2 & 92.8 \\
\hline Larceny & 226.7 & -0.1 & -8.9 & 14.6 & -6.8 & 71.7 & 62.8 & 77.4 & 70.6 \\
\hline Auto theft & 79.3 & 8.5 & -10.5 & -5.9 & -9.5 & 115.8 & 105.4 & 99.4 & 90.0 \\
\hline Arson & 4.3 & -0.1 & -43.6 & -21.2 & -1.9 & 146.8 & 103.1 & 82.0 & 80.0 \\
\hline Total & 913.2 & 9.7 & -12.5 & 8.5 & -9.6 & 109.9 & 97.4 & 105.9 & 96.3 \\
\hline
\end{tabular}




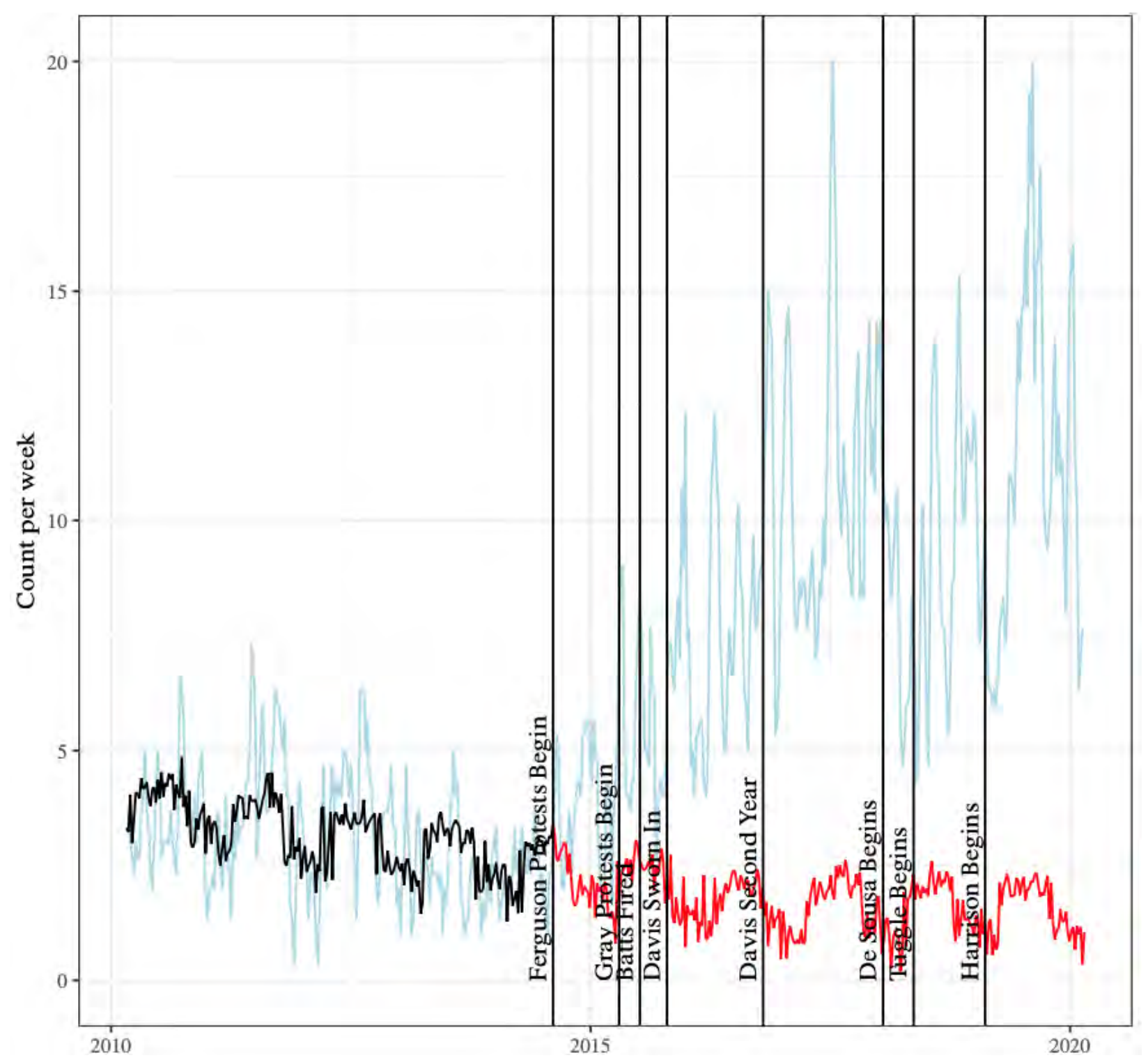

Figure 13. Total weekly carjacking count (three-week moving average, blue line) with predicted values from the model before the Ferguson protests began (black line) and extrapolated counterfactual values after the Ferguson protests began (red line) 


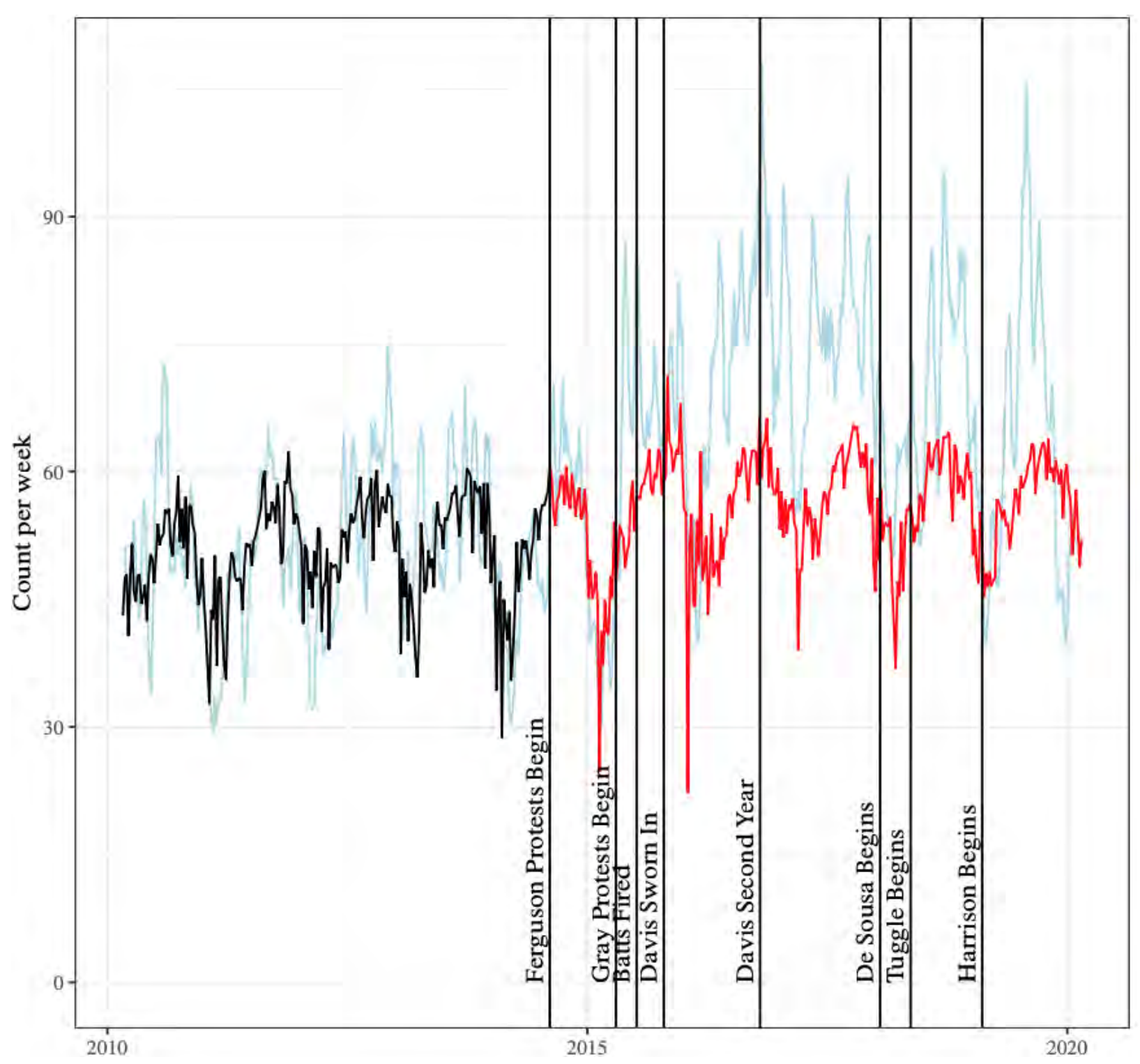

Figure 14. Total weekly street robbery count (three-week moving average, blue line) with predicted values from the model before the Ferguson protests began (black line) and extrapolated counterfactual values after the Ferguson protests began (red line) 


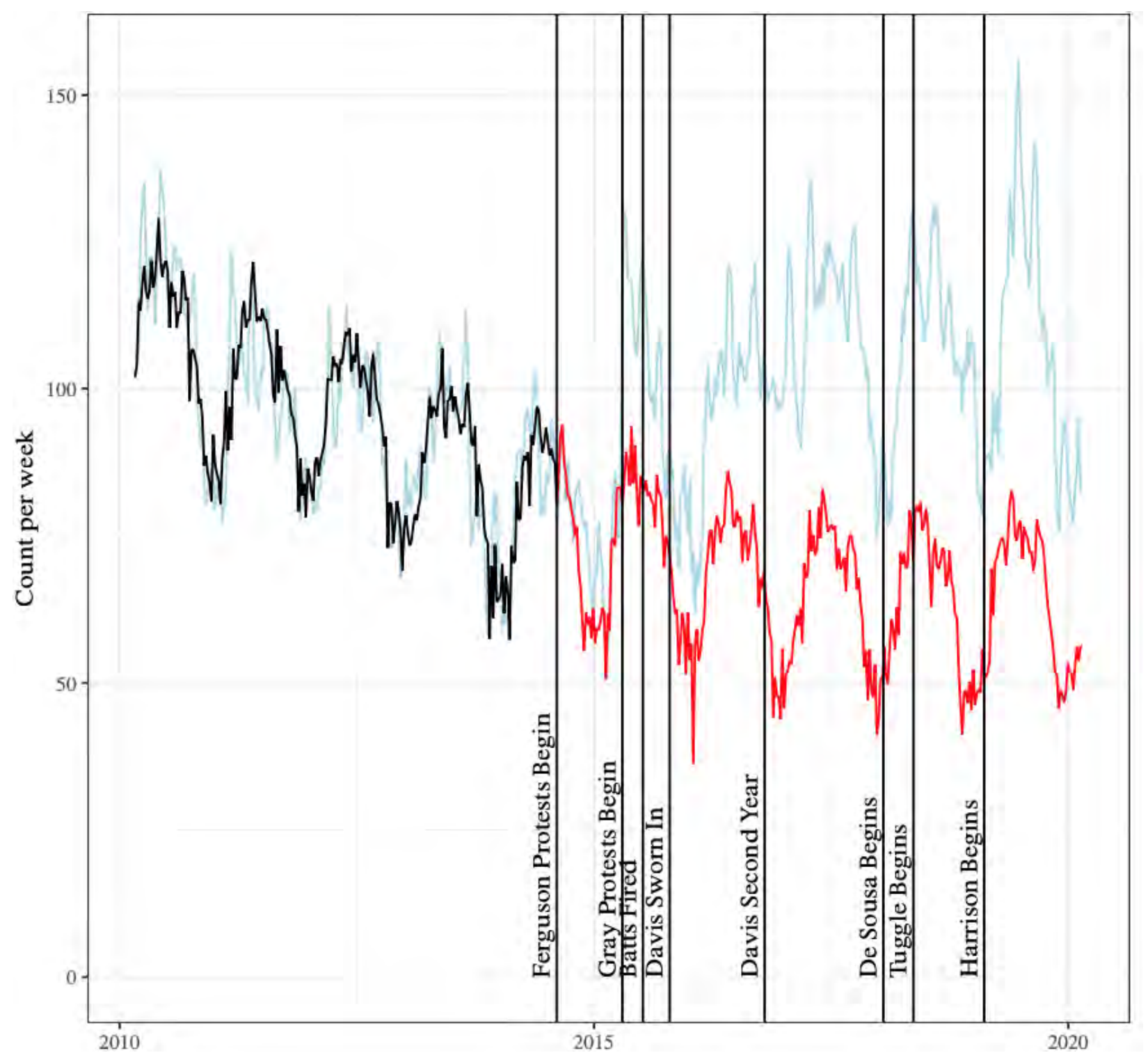

Figure 15. Total weekly aggravated assault count (three-week moving average, blue line) with predicted values from the model before the Ferguson protests began (black line) and extrapolated counterfactual values after the Ferguson protests began (red line) 


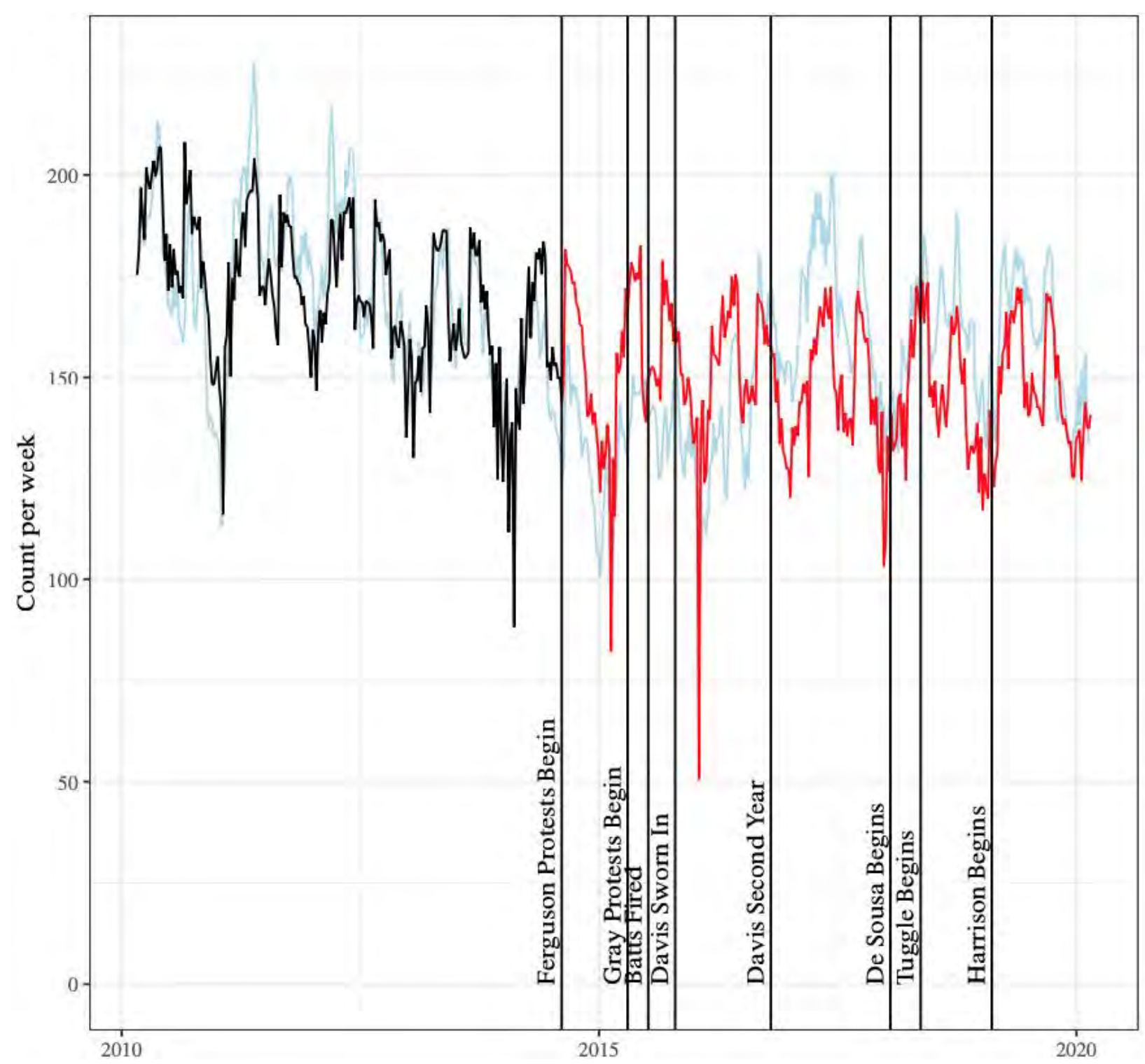

Figure 16. Total weekly common assault count (three-week moving average, blue line) with predicted values from the model before the Ferguson protests began (black line) and extrapolated counterfactual values after the Ferguson protests began (red line) 


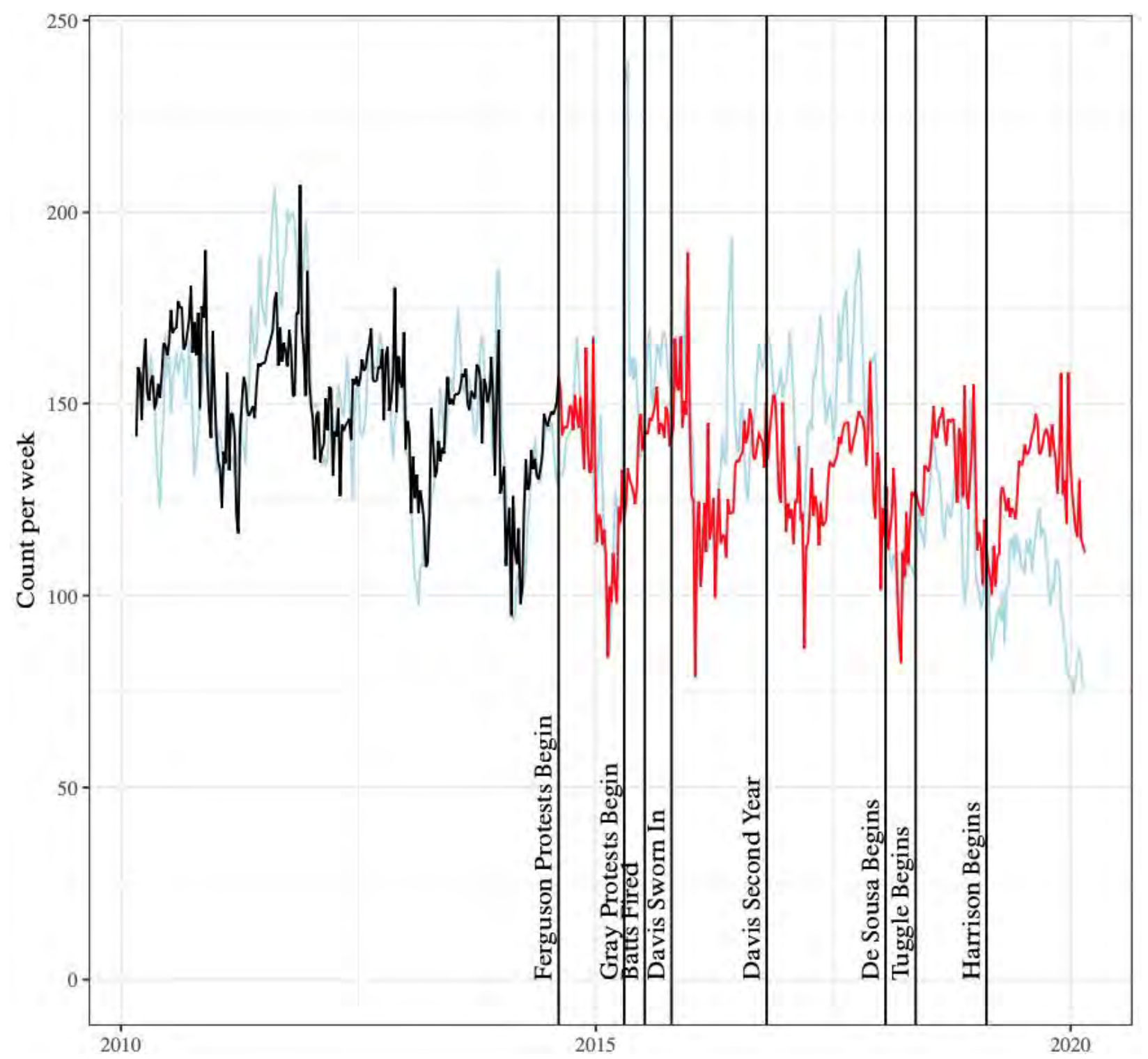

Figure 17. Total weekly burglary count (three-week moving average, blue line) with predicted values from the model before the Ferguson protests began (black line) and extrapolated counterfactual values after the Ferguson protests began (red line) 


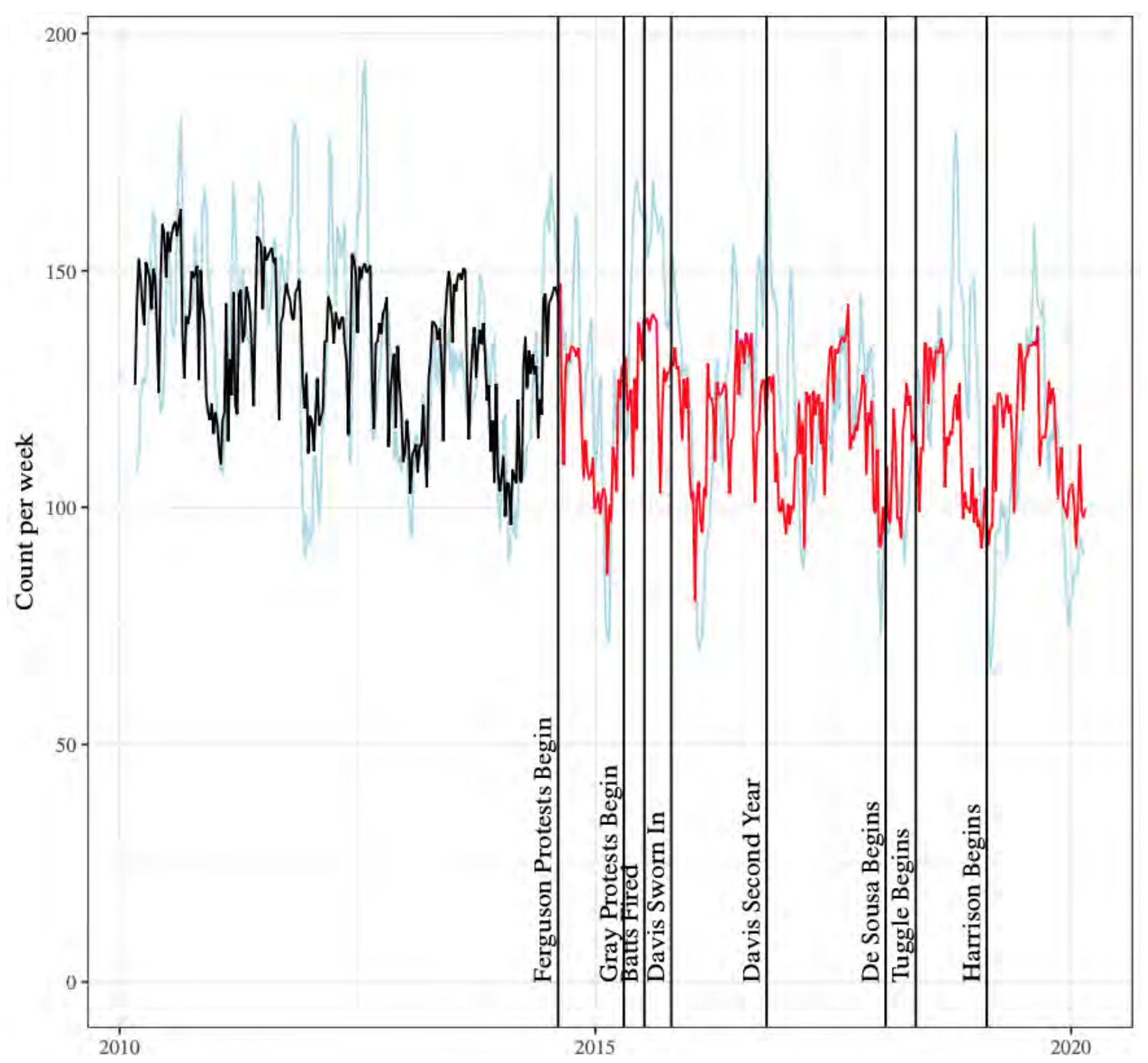

Figure 18. Total weekly larceny from automobiles count (three-week moving average, blue line) with predicted values from the model before the Ferguson protests began (black line) and extrapolated counterfactual values after the Ferguson protests began (red line) 


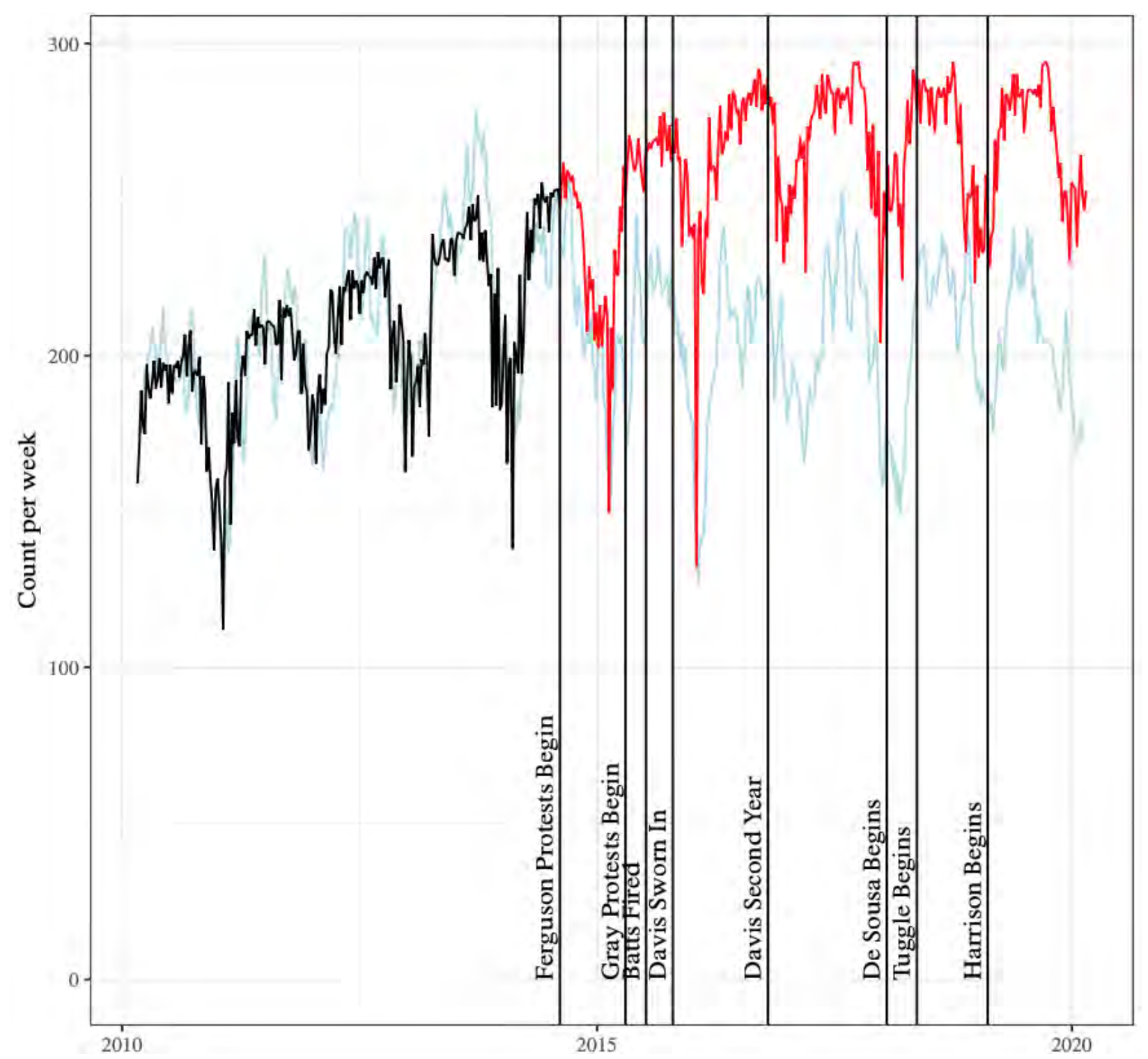

Figure 19. Total weekly larceny count (three-week moving average, blue line) with predicted values from the model before the Ferguson protests began (black line) and extrapolated counterfactual values after the Ferguson protests began (red line) 


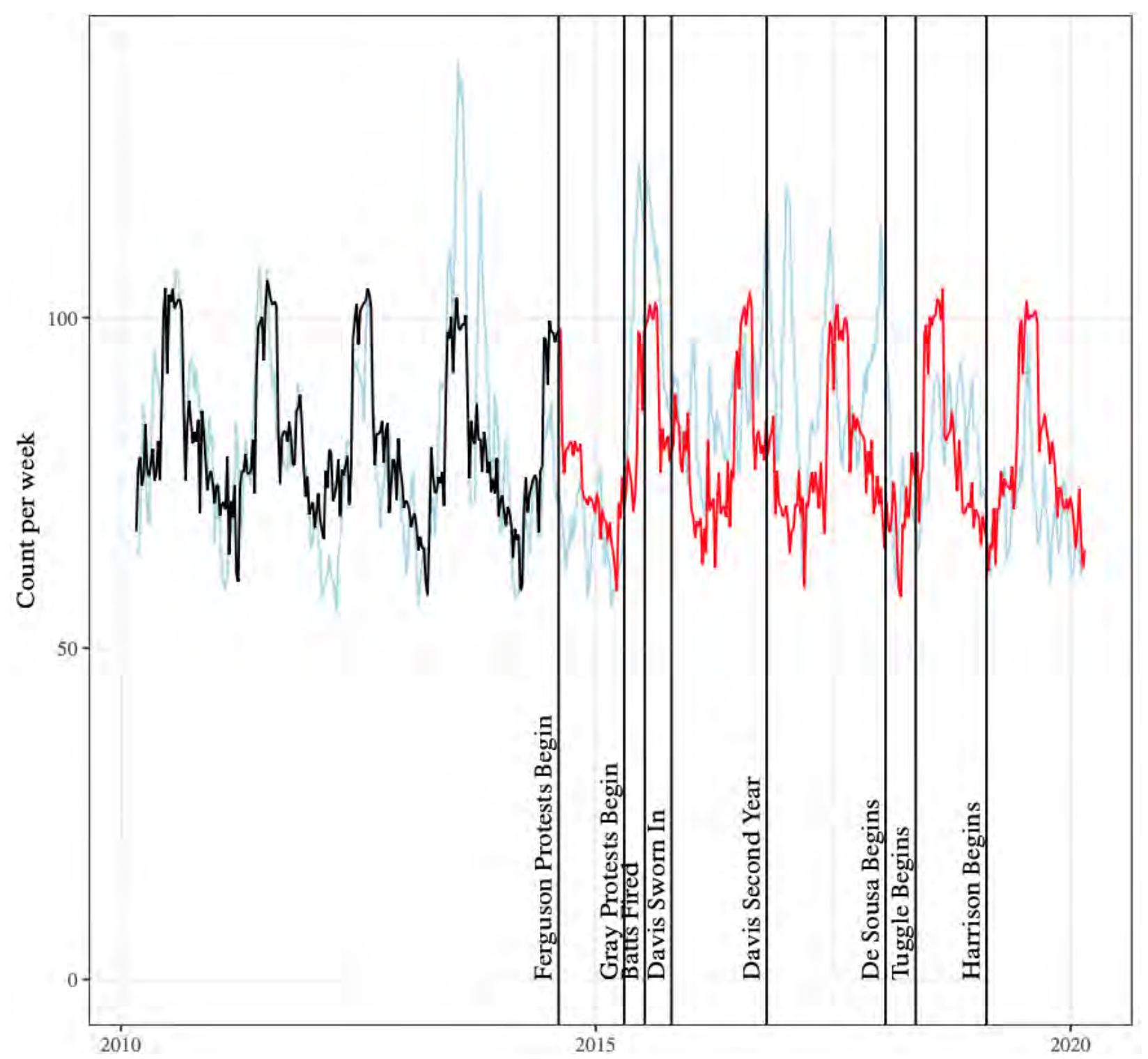

Figure 20. Total weekly automobile theft count (three-week moving average, blue line) with predicted values from the model before the Ferguson protests began (black line) and extrapolated counterfactual values after the Ferguson protests began (red line) 


\subsection{Percentage Changes in the Prevalence of Arrests, Disaggregated by Type}

In this section, we provide parallel tabular summaries of period-specific estimates of change in the prevalence of arrests, again followed by graphical displays of types of arrests that are of particular interest. We follow the same order as was the case for our presentation of types of crime incidents. We will not repeat the guidance on how to interpret the percentage changes.

Changes in arrests through Davis' first year as police commissioner. Table 6 (see next page) presents period estimates for all types of arrests for the Ferguson period through the first Davis period. The first three columns of estimated change in Table 6 were estimated for the original report (see Table 5 in Morgan and Pally 2016), but with slightly different specifications, without BPD's edited data, and using a slightly different coding of arrests (explained above). These type-specific effects were not re-estimated for the updated report in 2016 (but see Tables 6 and 7 in Morgan 2016 for estimates of changes in arrest totals).

As with crime incidents, we regard the estimates in Table 6 of this final report as improved for the reasons discussed above. The improvements are small enough that the core conclusions from these periods do not require any meaningful revisions. See the prior section for these conclusions, which plug in values from Table 6 to demonstrate why we stand by the conclusions we formulated in 2016. 
Table 6. Baseline and percentage change in the weekly count of arrests from the Ferguson period through Davis' first year

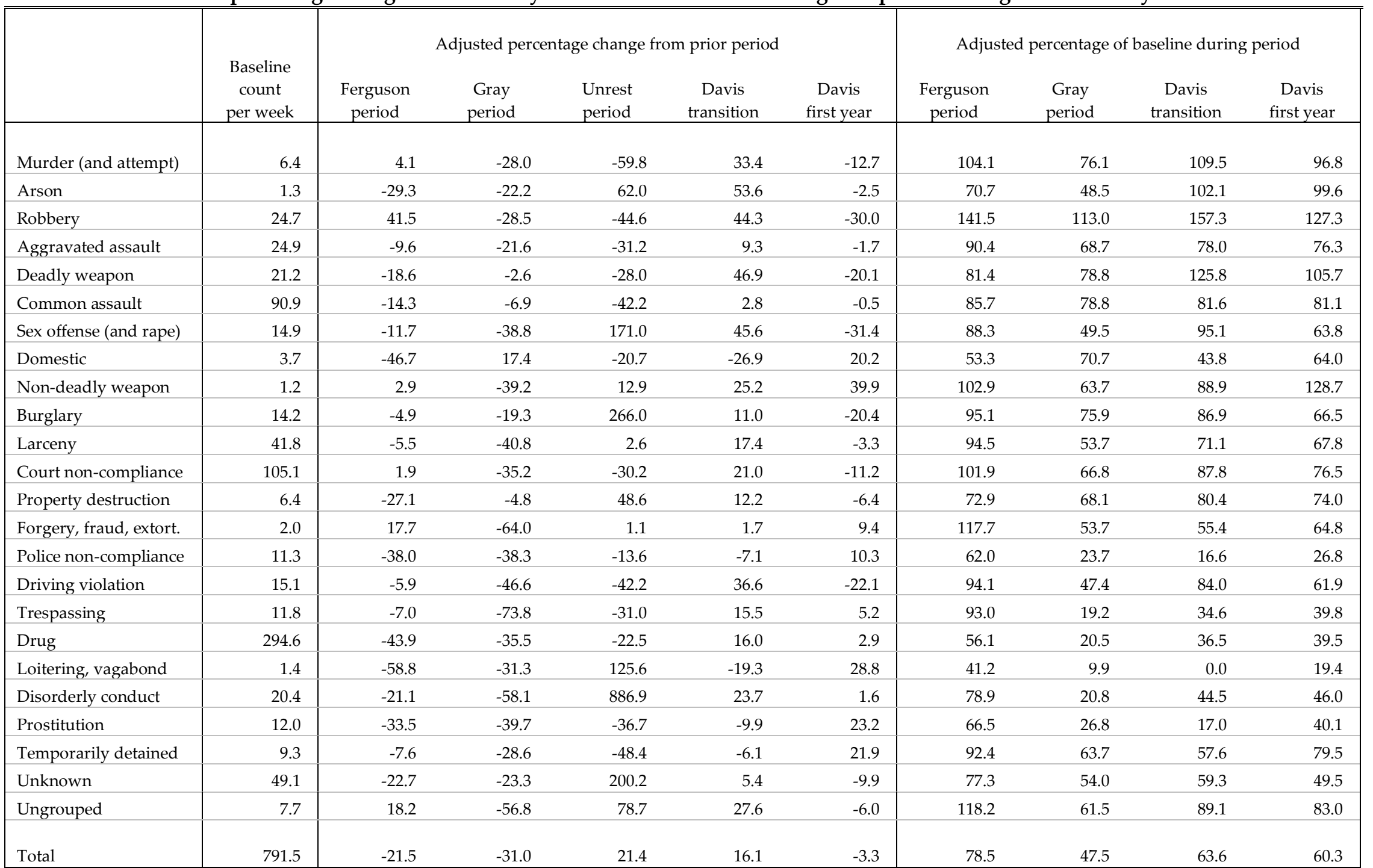

Notes: The unrest period is modeled with a "spike" specification, and this modeling choice removes it from the calculation of cumulated change in the second panel. 
Changes in arrests through Harrison's first year as police commissioner. Table 7 (see next page) offers analogous estimates for the final four time periods considered. As with crime incidents, much could be written on the patterns in Table 7. We will continue to hold our overall conclusions for the final section of this report. Nonetheless, it should be clear from the final four columns of Table 7 that arrests continue to decline progressively, with the rate of overall decline accelerating into Harrison's first year.

Some types of arrests, which used to be common, have fallen dramatically, such as for police non-compliance and disorderly conduct. They were only 12.5 and 19.2 percent, respectively, of the levels in the baseline reference year. It may be reasonable to conclude, as we have in prior reports, that these declines reflect a greater propensity for policing in Baltimore to adhere to constitutional standards. Other declines in arrests are the result of explicit policy change, such as lightening up on arrests for drug possession (and presumably inferring substantially less "intent" to distribute). Here, the very large number of arrests from the baseline reference year (294.6 per week!) has declined to only 18.2 percent of that level.

Two other factors should be noted. If crimes were being "solved," then in periods of rising crime arrests should also be rising. It is hard to see positive trends in this regard for violent crime, such as murder, attempted murder, robbery, and aggravated assault. Declines in the homicide clearance rate, which may reflect over-worked investigative units, could be a crucial factor for why arrests in our murder group declined (see Figure 2 above for the relevant FOP claim). It is also possible, as has been widely reported in the media, that these types of arrests have declined because police legitimacy has declined, reducing the willingness of witnesses to support the BPD's investigations.

Overall, it would be inappropriate to regard the decline in the total arrest count as either uniformly positive or uniformly negative. As with the distinction between violent crime incidents and property crime incidents in the last section, disaggregation is key to understanding the trends.

Graphical displays of change for selected types of arrests. Figures 21-33 (see subsequent pages) offer times series plots for types of arrests. These figures are drawn from Appendix 3, where arrest-specific analogs to Table 2 can be found. The types of arrests presented first are murder and attempted murder, robbery, aggravated assault, deadly weapon, common assault, burglary, larceny, and property destruction. These patterns, as implied by Table 6 and 7, are much less dramatic than those that follow for police non-compliance, driving violations, trespassing, non-violent drug-related arrests, and disorderly conduct. As with crime incidents, these figures are an alternative way to understand the changes that are summarized in a targeted fashion in Tables 6 and 7. And, again, both types of representations should not be over-interpreted: inherent variability is prominent, especially for low-prevalence types of arrests. 
Table 7. Baseline and percentage change in the weekly count of arrests from Davis' second year through Harrison's first year

\begin{tabular}{|c|c|c|c|c|c|c|c|c|c|}
\hline & \multirow{2}{*}{$\begin{array}{c}\text { Baseline } \\
\text { count } \\
\text { per Week }\end{array}$} & \multicolumn{4}{|c|}{ Adjusted percentage change from prior period } & \multicolumn{4}{|c|}{ Adjusted percentage of baseline during period } \\
\hline & & $\begin{array}{l}\text { Davis last } \\
15 \text { months } \\
\end{array}$ & $\begin{array}{c}\text { De Sousa } \\
\text { spring }\end{array}$ & $\begin{array}{c}\text { Tuggle } \\
\text { interregnum }\end{array}$ & $\begin{array}{l}\text { Harrison } \\
\text { first year }\end{array}$ & $\begin{array}{c}\text { Davis last } \\
15 \text { months } \\
\end{array}$ & $\begin{array}{c}\text { De Sousa } \\
\text { spring }\end{array}$ & $\begin{array}{c}\text { Tuggle } \\
\text { interregnum }\end{array}$ & $\begin{array}{l}\text { Harrison } \\
\text { first year }\end{array}$ \\
\hline Murder (and attempt) & 6.4 & 8.2 & -29.0 & 12.8 & -11.4 & 105.0 & 75.9 & 88.7 & 77.3 \\
\hline Arson & 1.3 & -19.1 & -13.0 & 16.5 & -13.0 & 80.5 & 67.5 & 84.0 & 70.9 \\
\hline Robbery & 24.7 & -10.4 & -19.5 & 10.3 & -3.6 & 116.9 & 97.4 & 107.6 & 104.0 \\
\hline Aggravated assault & 24.9 & 7.1 & -2.0 & -5.5 & 12.7 & 83.4 & 81.4 & 75.9 & 88.6 \\
\hline Deadly weapon & 21.2 & -17.3 & 16.4 & -15.0 & -6.4 & 88.3 & 104.7 & 89.8 & 83.4 \\
\hline Common assault & 90.9 & 8.4 & -0.3 & -6.0 & 3.1 & 89.5 & 89.2 & 83.2 & 86.2 \\
\hline Sex offense (and rape) & 14.9 & 1.8 & -31.6 & 26.1 & -12.2 & 65.6 & 34.0 & 60.1 & 48.0 \\
\hline Domestic & 3.7 & 18.5 & -9.9 & -39.5 & 34.8 & 82.5 & 72.6 & 33.1 & 68.0 \\
\hline Non-deadly weapon & 1.2 & -34.6 & -20.3 & 22.3 & -31.2 & 94.1 & 73.8 & 96.1 & 64.8 \\
\hline Burglary & 14.2 & 6.9 & 5.3 & -6.6 & -7.3 & 73.4 & 78.7 & 72.1 & 64.8 \\
\hline Larceny & 41.8 & -6.8 & -0.5 & -7.6 & -4.9 & 61.0 & 60.5 & 52.9 & 47.9 \\
\hline Court non-compliance & 105.1 & -3.8 & 3.7 & 1.4 & -1.4 & 72.7 & 76.5 & 77.9 & 76.5 \\
\hline Property destruction & 6.4 & -6.3 & -15.6 & 19.9 & -14.5 & 67.6 & 52.1 & 72.0 & 57.5 \\
\hline Forgery, fraud, extort. & 2.0 & -11.6 & 0.2 & -13.5 & -2.4 & 53.1 & 53.3 & 39.8 & 37.4 \\
\hline Police non-compliance & 11.3 & -12.7 & 9.3 & -4.0 & -7.0 & 14.2 & 23.5 & 19.5 & 12.5 \\
\hline Driving violation & 15.1 & -29.0 & -16.4 & 12.9 & -4.3 & 33.0 & 16.6 & 29.5 & 25.1 \\
\hline Trespassing & 11.8 & -10.1 & -2.5 & -17.0 & 3.7 & 29.7 & 27.3 & 10.2 & 14.0 \\
\hline Drug & 294.6 & -5.3 & -5.0 & -0.6 & -10.4 & 34.2 & 29.1 & 28.6 & 18.2 \\
\hline Loitering, vagabond & 1.4 & -3.3 & -0.1 & -17.0 & 7.2 & 16.1 & 16.1 & 0.0 & 6.3 \\
\hline Disorderly conduct & 20.4 & -19.0 & -6.8 & 0.4 & -1.4 & 27.0 & 20.2 & 20.6 & 19.2 \\
\hline Prostitution & 12.0 & -10.2 & 67.2 & -45.1 & -11.7 & 29.9 & 97.1 & 52.0 & 40.3 \\
\hline Temporarily detained & 9.3 & -8.2 & 18.9 & -30.5 & -2.1 & 71.2 & 90.1 & 59.6 & 57.5 \\
\hline Unknown & 49.1 & -11.1 & -13.1 & 1.6 & -0.4 & 38.4 & 25.3 & 26.9 & 26.5 \\
\hline Ungrouped & 7.7 & -13.4 & -5.7 & -1.6 & -0.4 & 69.7 & 63.9 & 62.3 & 61.9 \\
\hline Total & 791.5 & -4.7 & -2.6 & -1.9 & -4.7 & 55.6 & 53.0 & 51.0 & 46.3 \\
\hline
\end{tabular}




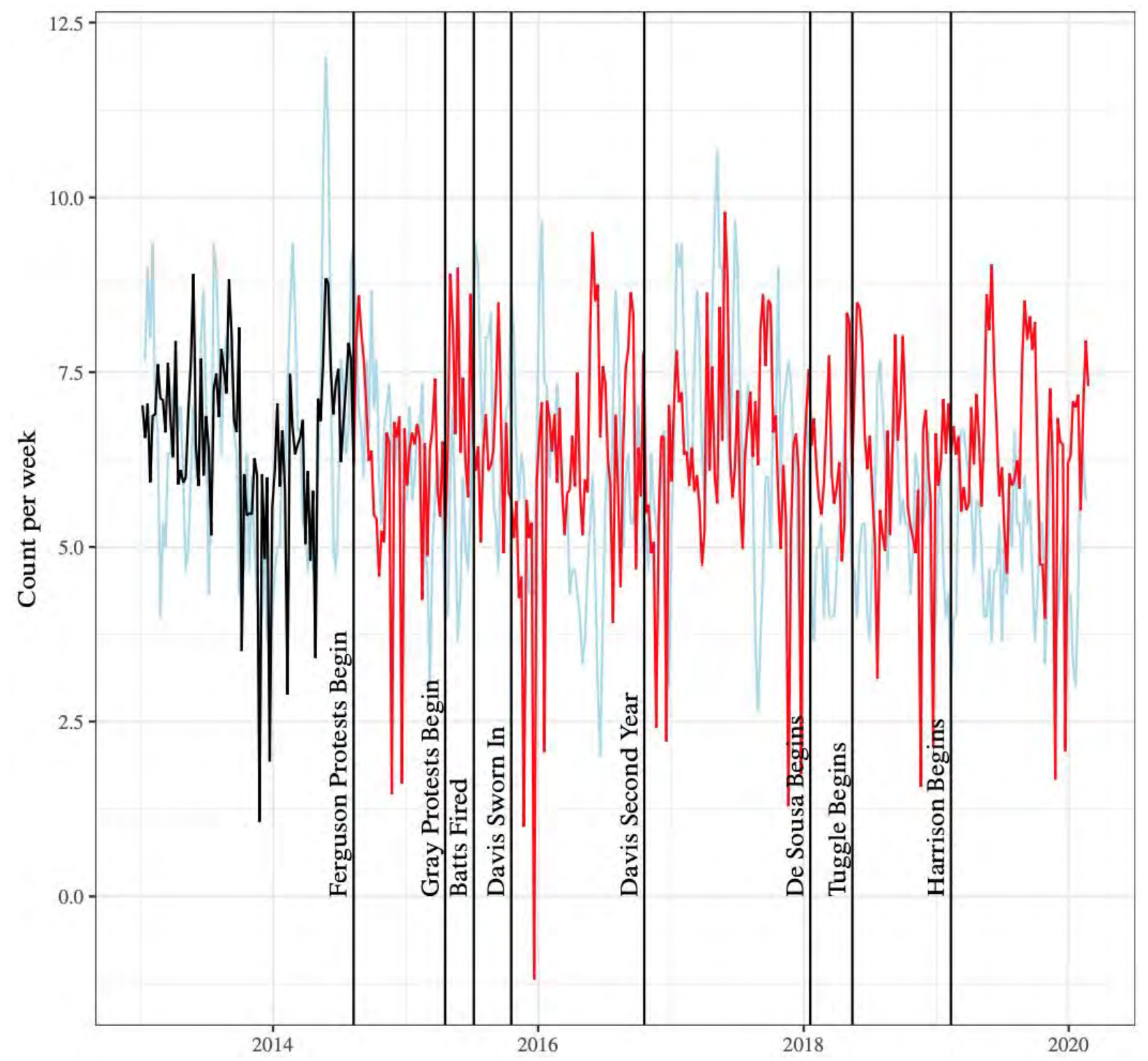

Figure 21. Total weekly arrest counts for murder and related charges (three-week moving average, blue line) with predicted values from the model before the Ferguson protests began (black line) and extrapolated counterfactual values after the Ferguson protests began (red line) 


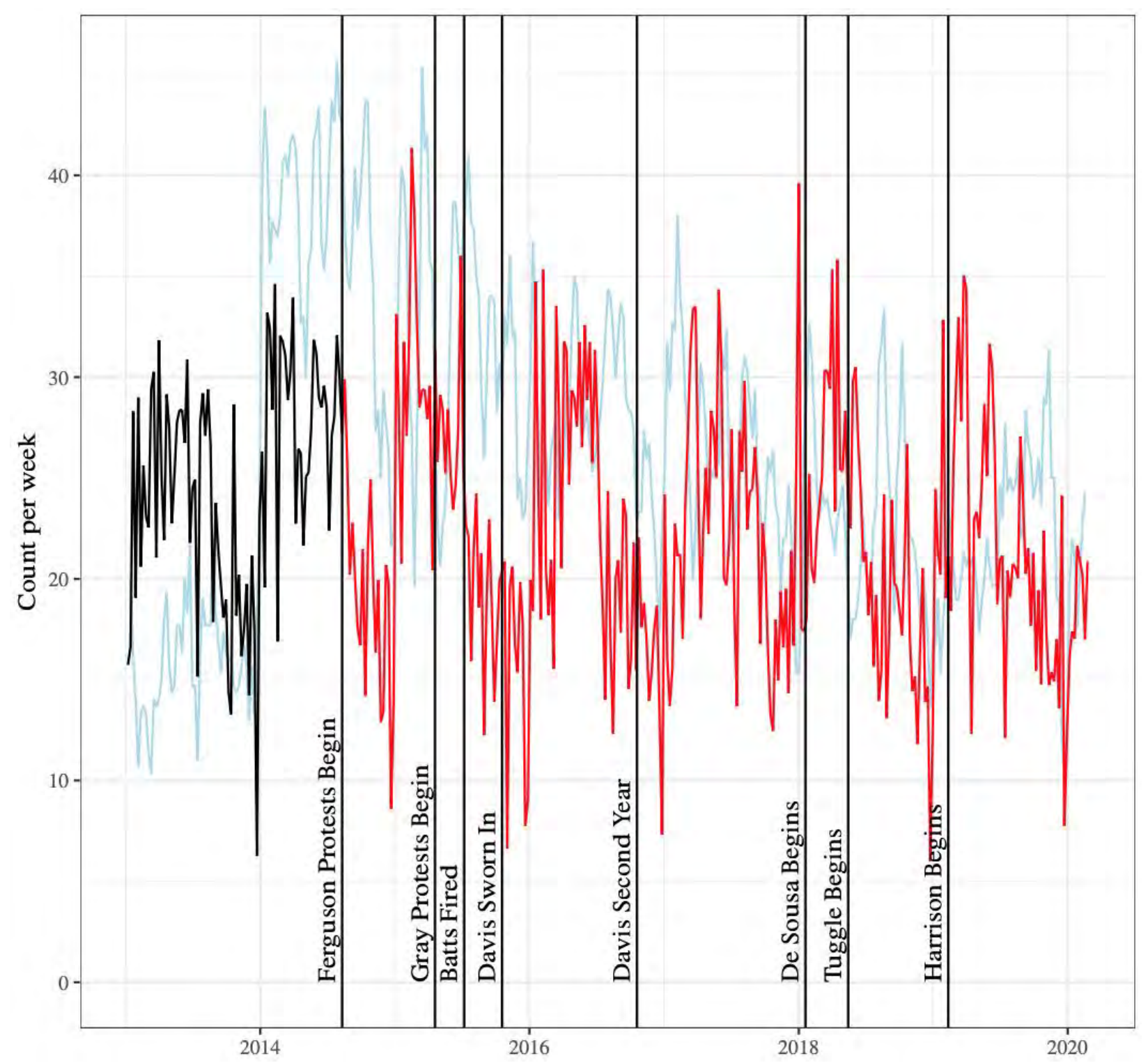

Figure 22. Total weekly arrest count for robbery (three-week moving average, blue line) with predicted values from the model before the Ferguson protests began (black line) and extrapolated counterfactual values after the Ferguson protests began (red line) 


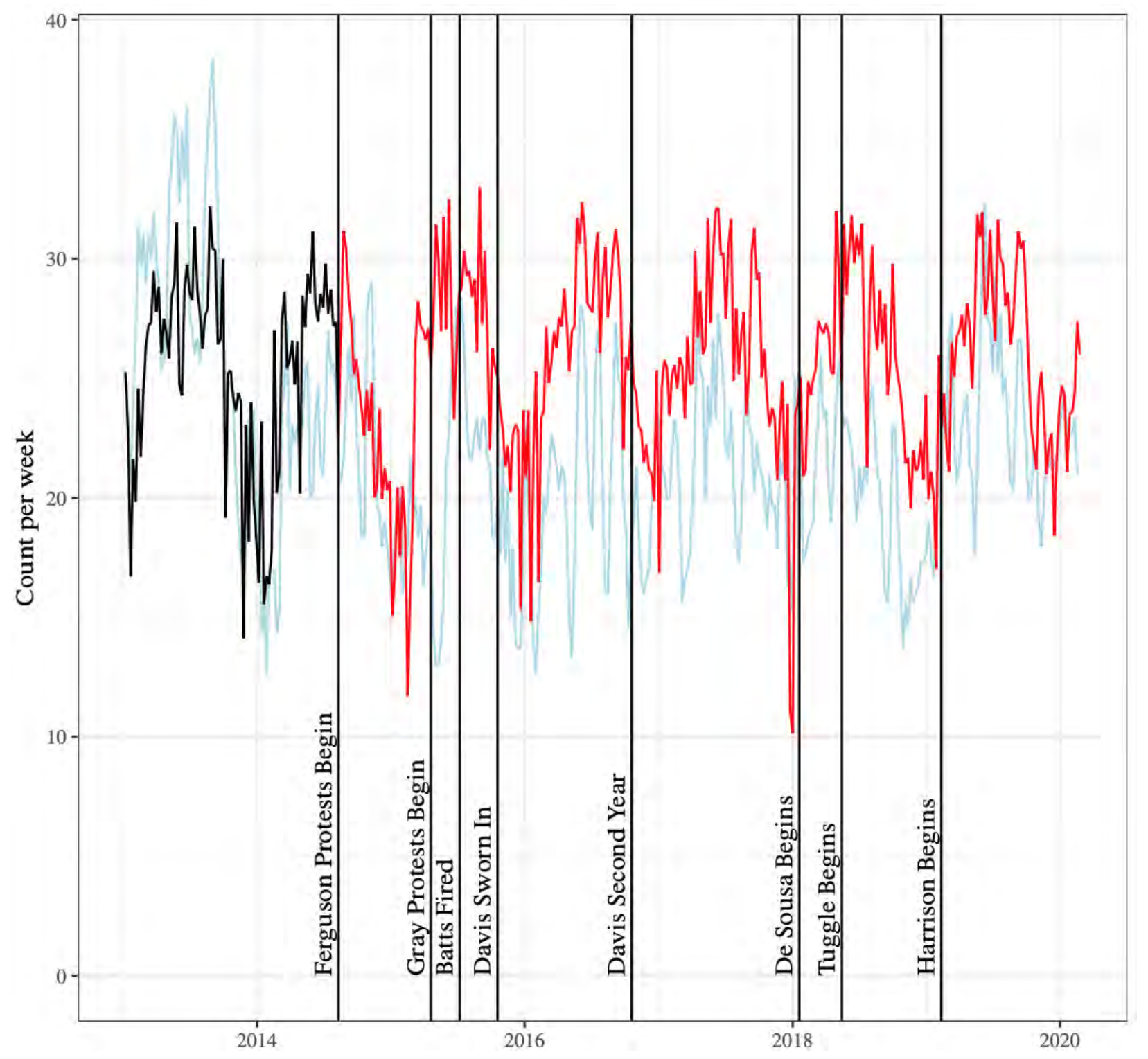

Figure 23. Total weekly arrest count for aggravated assault (three-week moving average, blue line) with predicted values from the model before the Ferguson protests began (black line) and extrapolated counterfactual values after the Ferguson protests began (red line) 


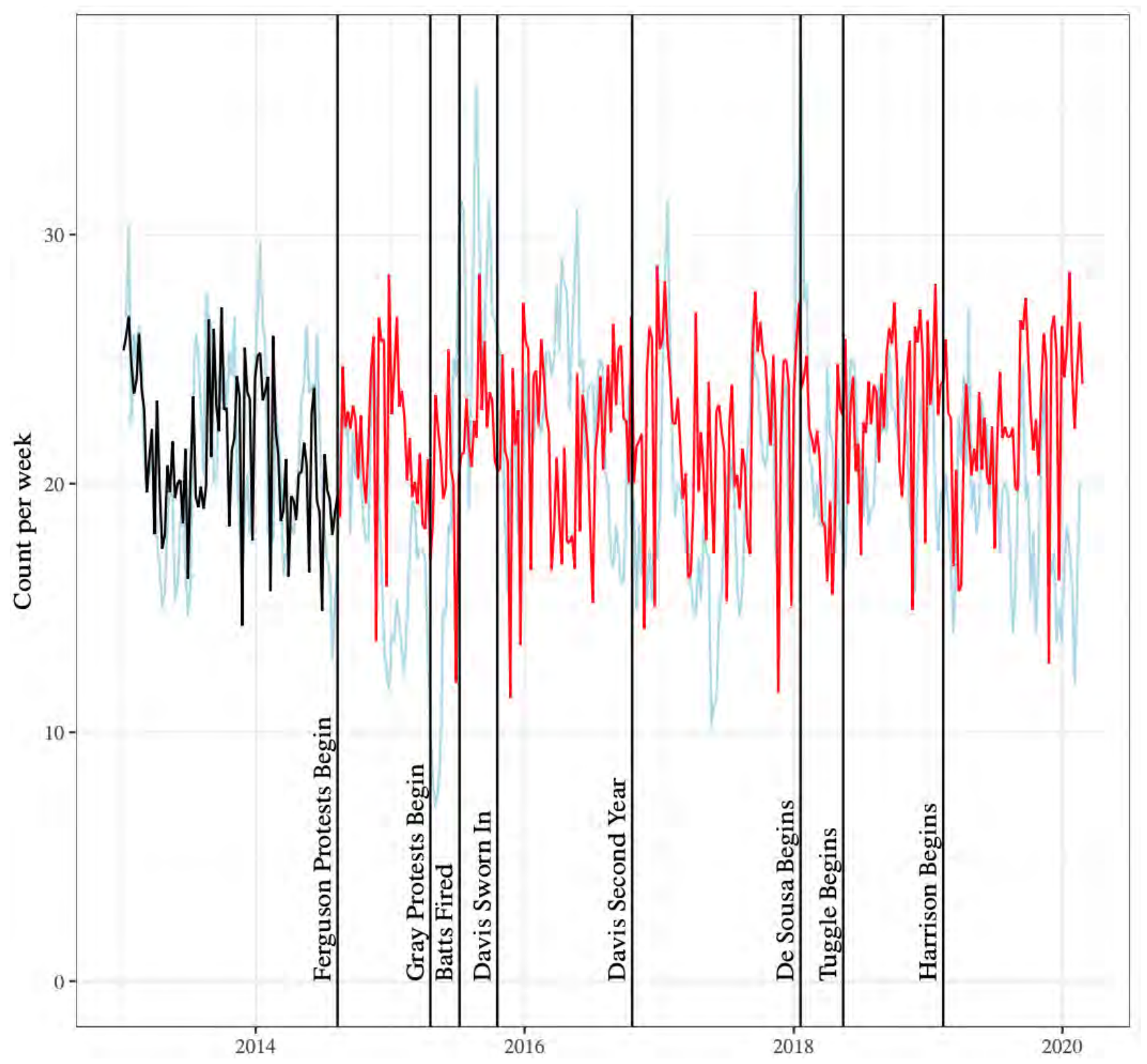

Figure 24. Total weekly arrest count for a deadly weapon charge (three-week moving average, blue line) with predicted values from the model before the Ferguson protests began (black line) and extrapolated counterfactual values after the Ferguson protests began (red line) 


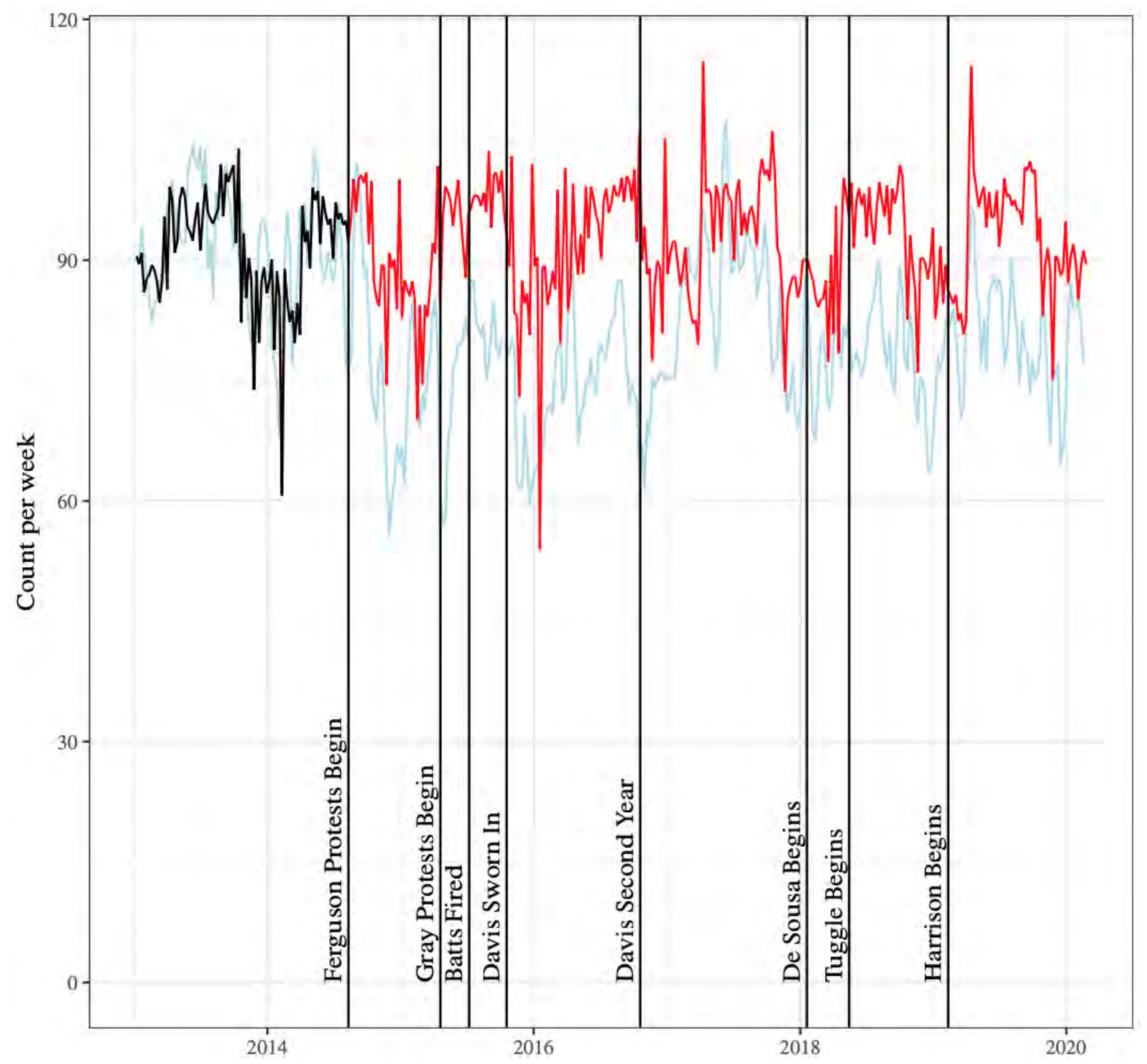

Figure 25. Total weekly arrest count for common assault (three-week moving average, blue line) with predicted values from the model before the Ferguson protests began (black line) and extrapolated counterfactual values after the Ferguson protests began (red line) 


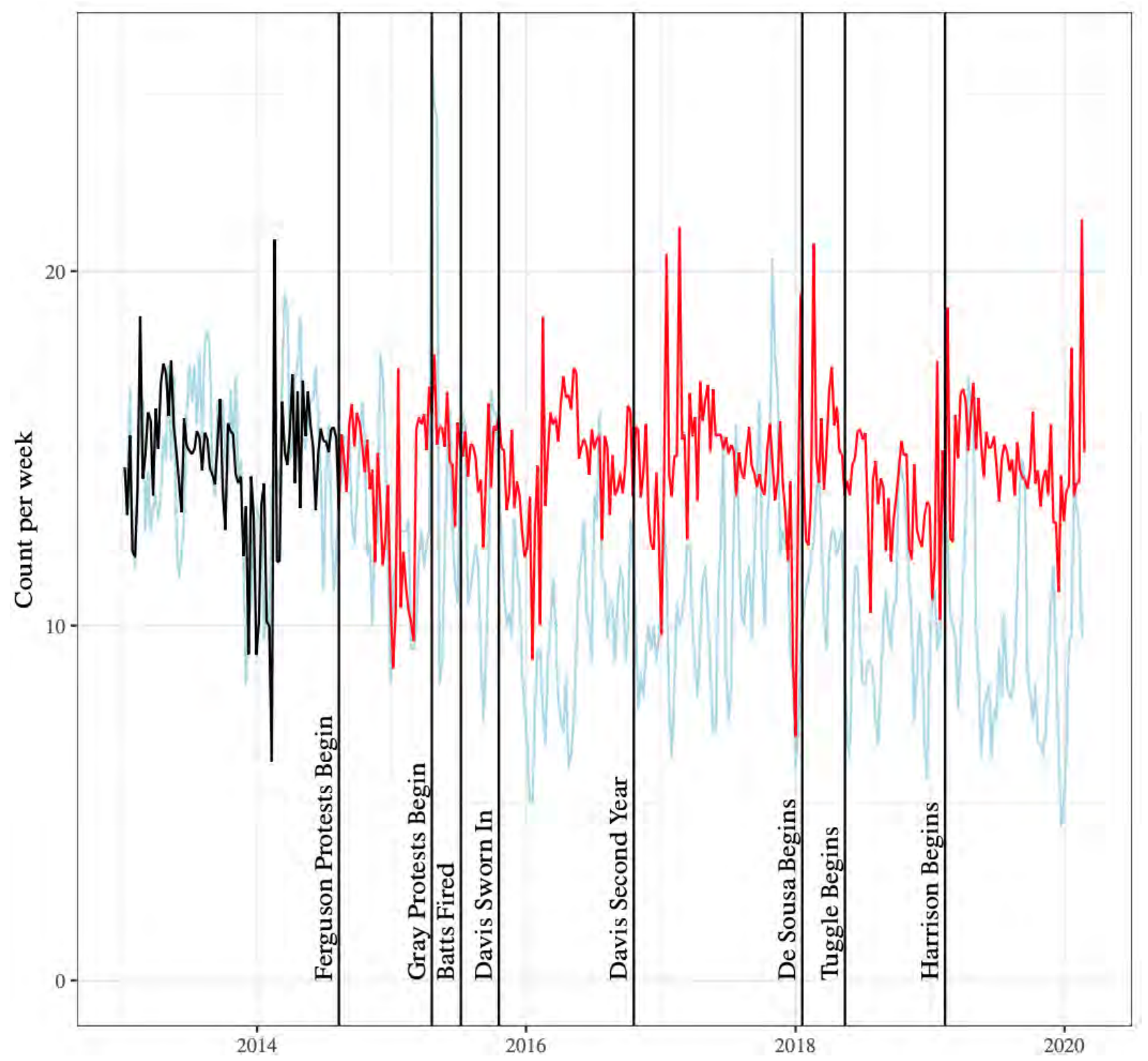

Figure 26. Total weekly arrest count for burglary (three-week moving average, blue line) with predicted values from the model before the Ferguson protests began (black line) and extrapolated counterfactual values after the Ferguson protests began (red line) 


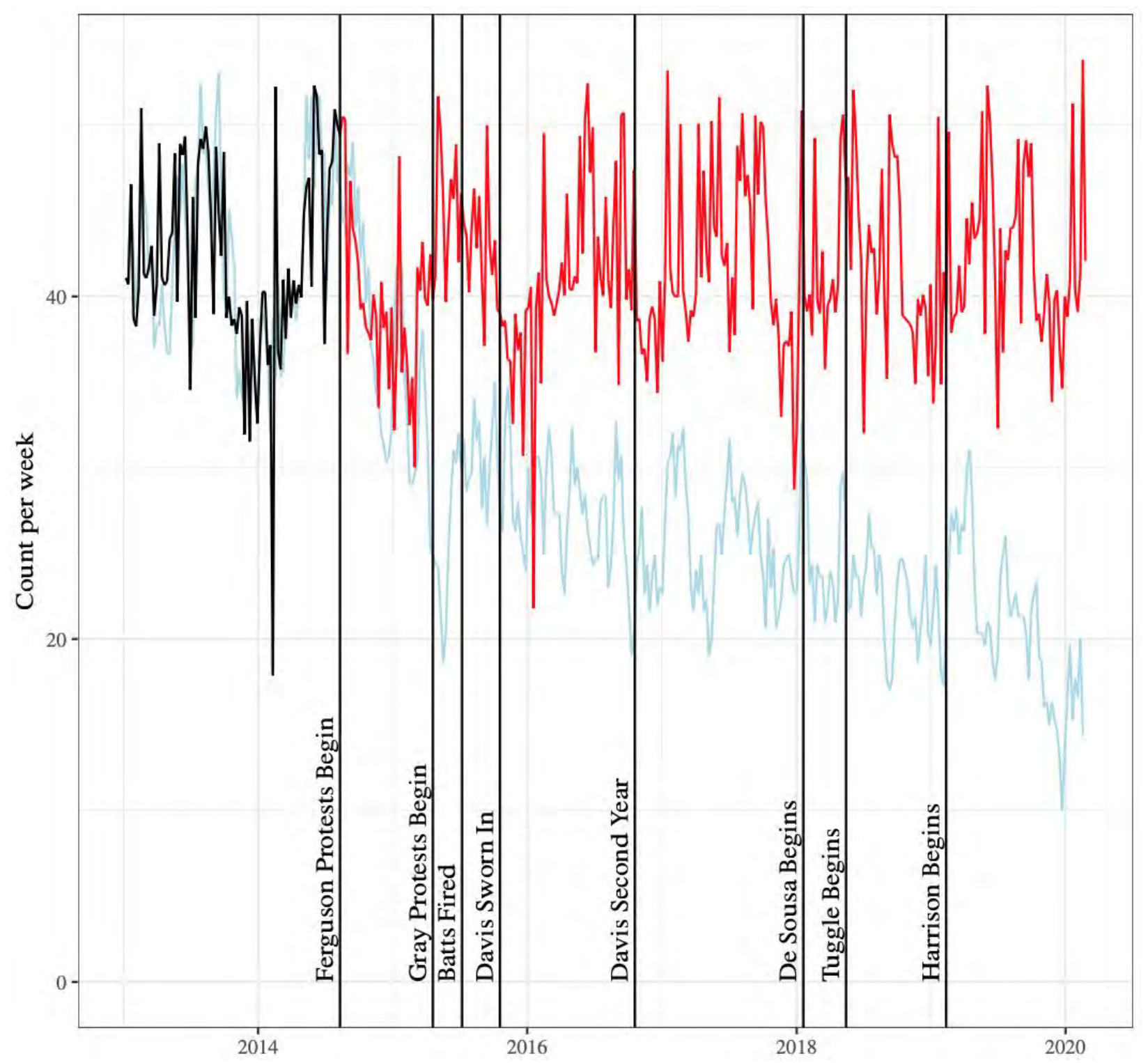

Figure 27. Total weekly arrest count for larceny (three-week moving average, blue line) with predicted values from the model before the Ferguson protests began (black line) and extrapolated counterfactual values after the Ferguson protests began (red line) 


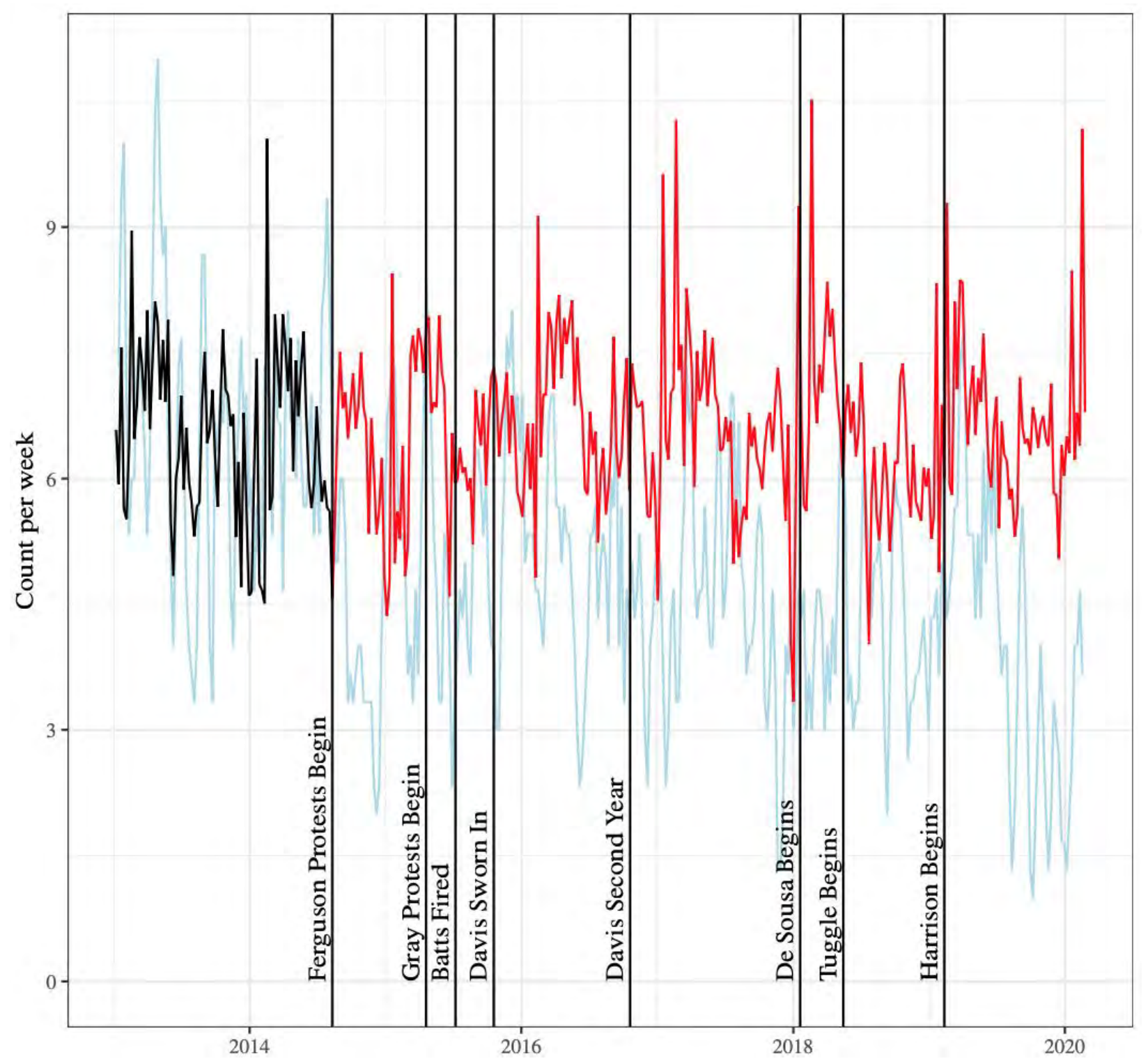

Figure 28. Total weekly arrest count for property destruction (three-week moving average, blue line) with predicted values from the model before the Ferguson protests began (black line) and extrapolated counterfactual values after the Ferguson protests began (red line) 


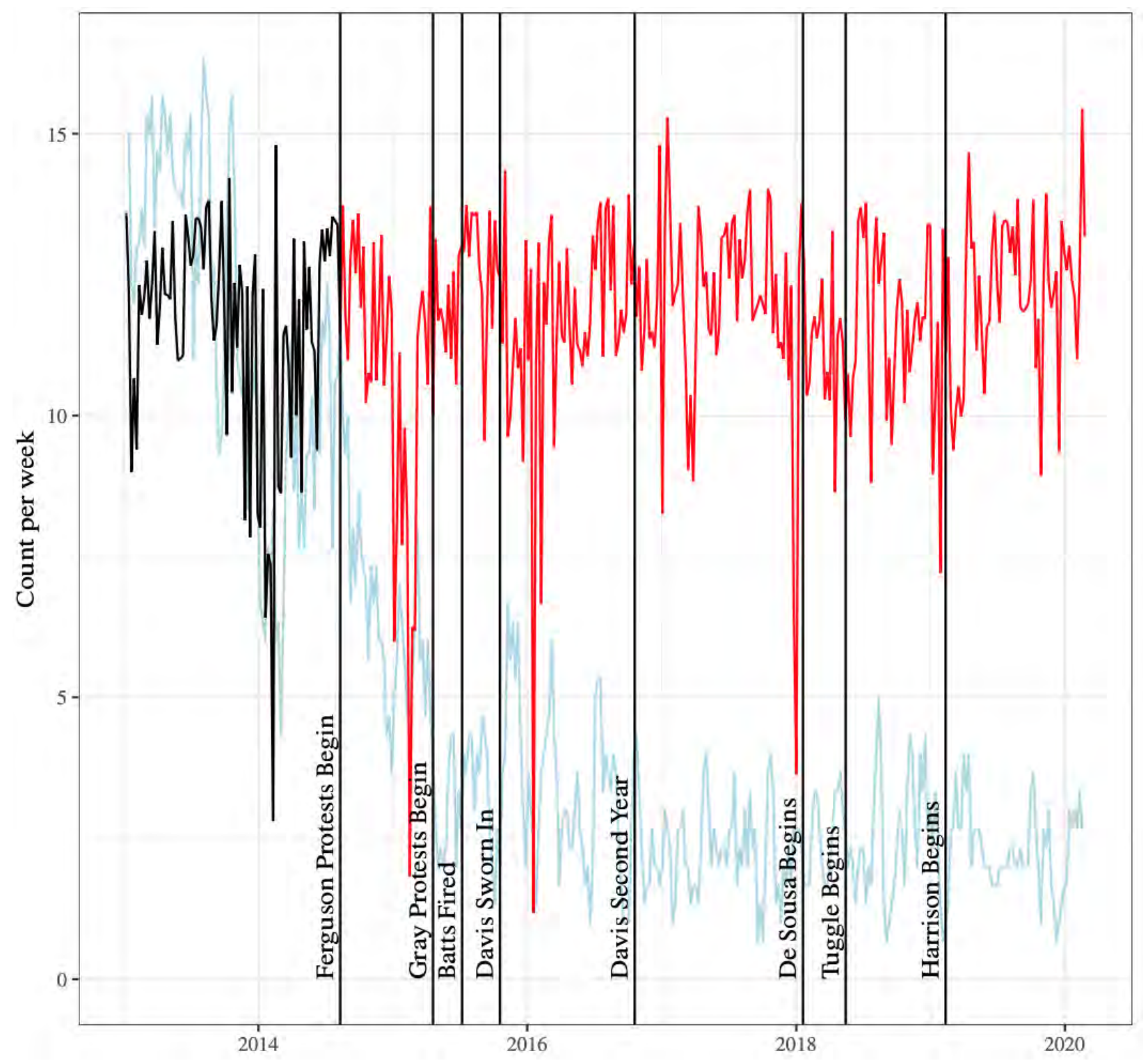

Figure 29. Total weekly arrest count for police non-compliance (three-week moving average, blue line) with predicted values from the model before the Ferguson protests began (black line) and extrapolated counterfactual values after the Ferguson protests began (red line) 


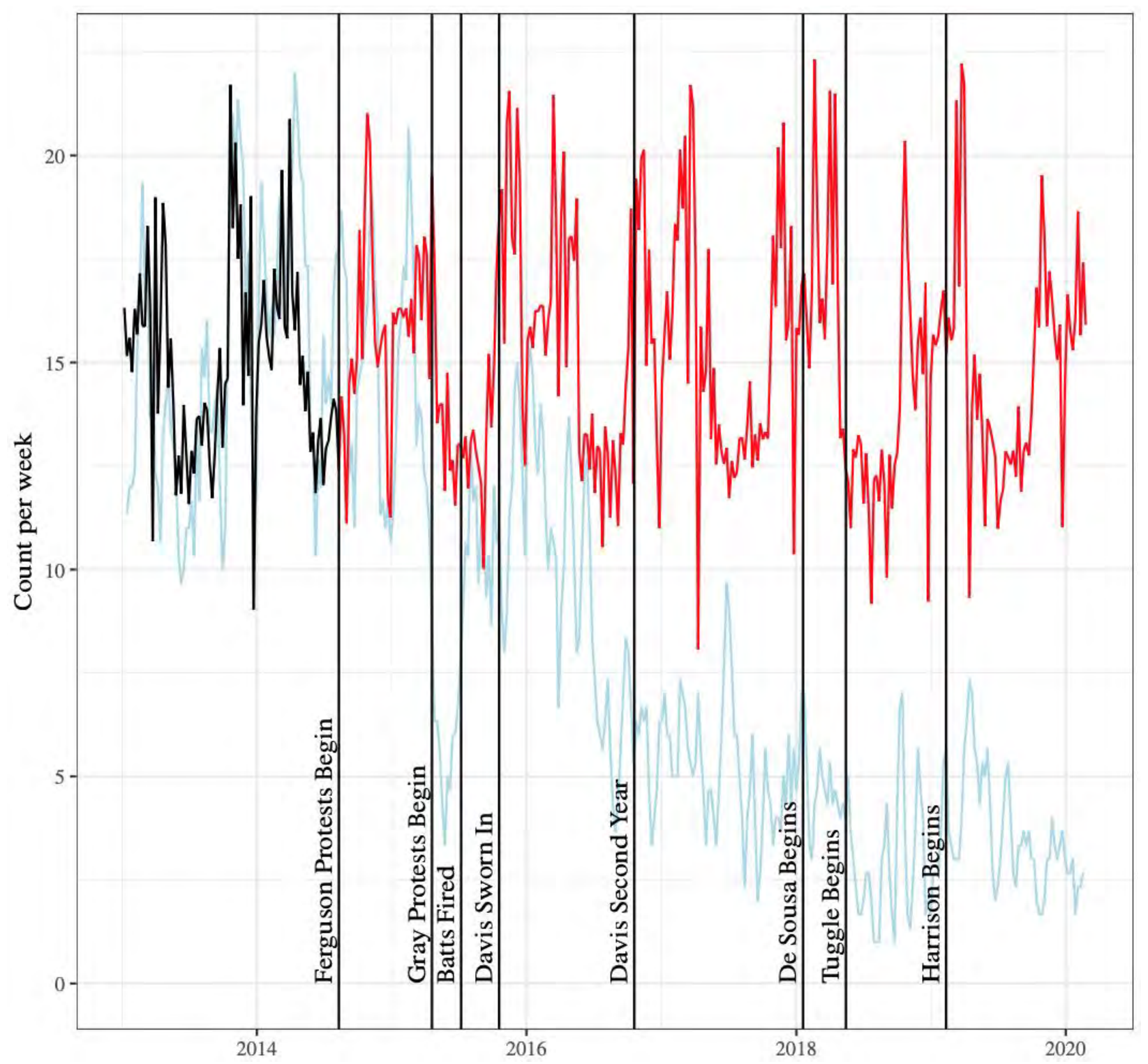

Figure 30. Total weekly arrest count for a driving violation (three-week moving average, blue line) with predicted values from the model before the Ferguson protests began (black line) and extrapolated counterfactual values after the Ferguson protests began (red line) 


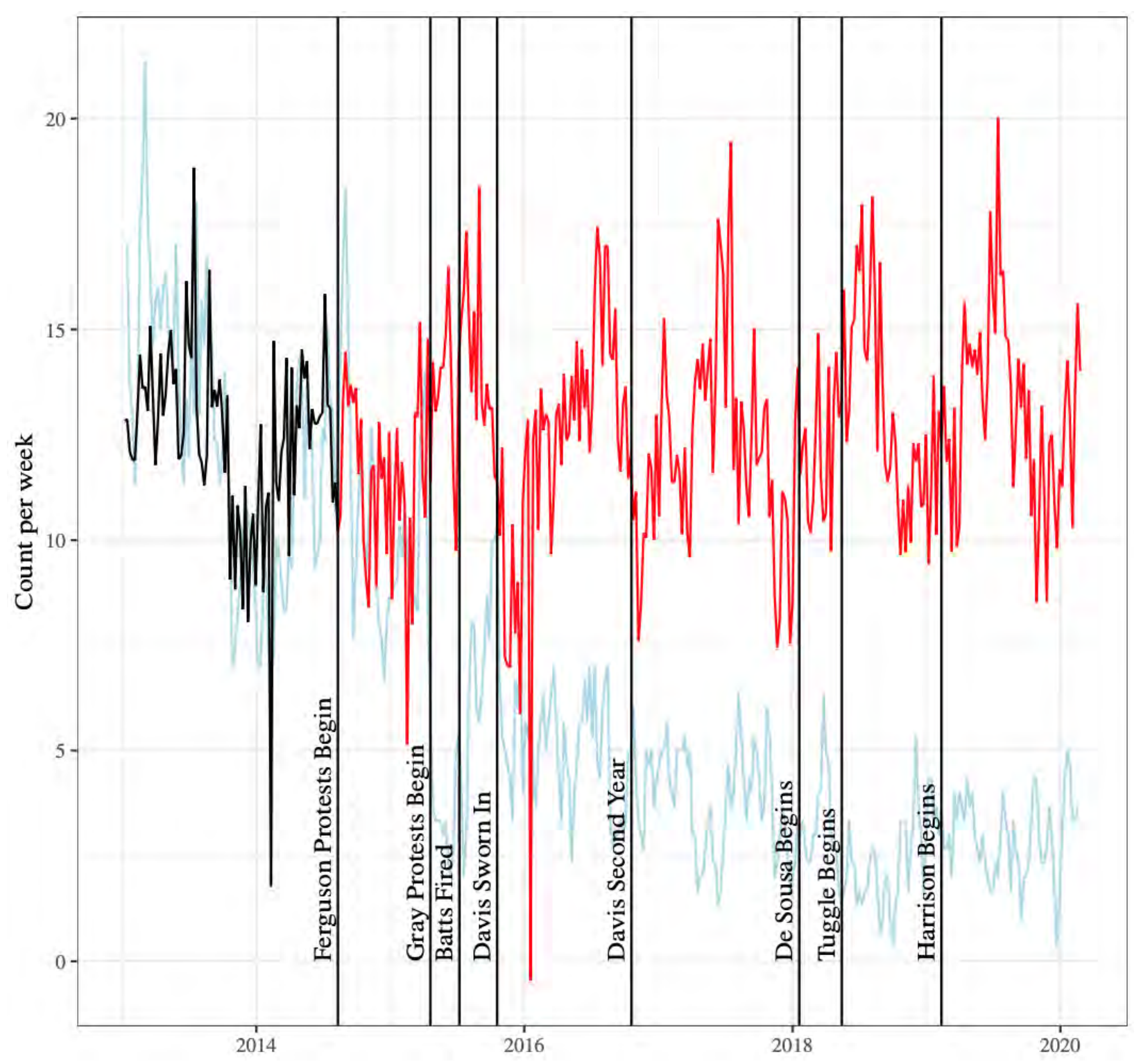

Figure 31. Total weekly arrest count for trespassing (three-week moving average, blue line) with predicted values from the model before the Ferguson protests began (black line) and extrapolated counterfactual values after the Ferguson protests began (red line) 


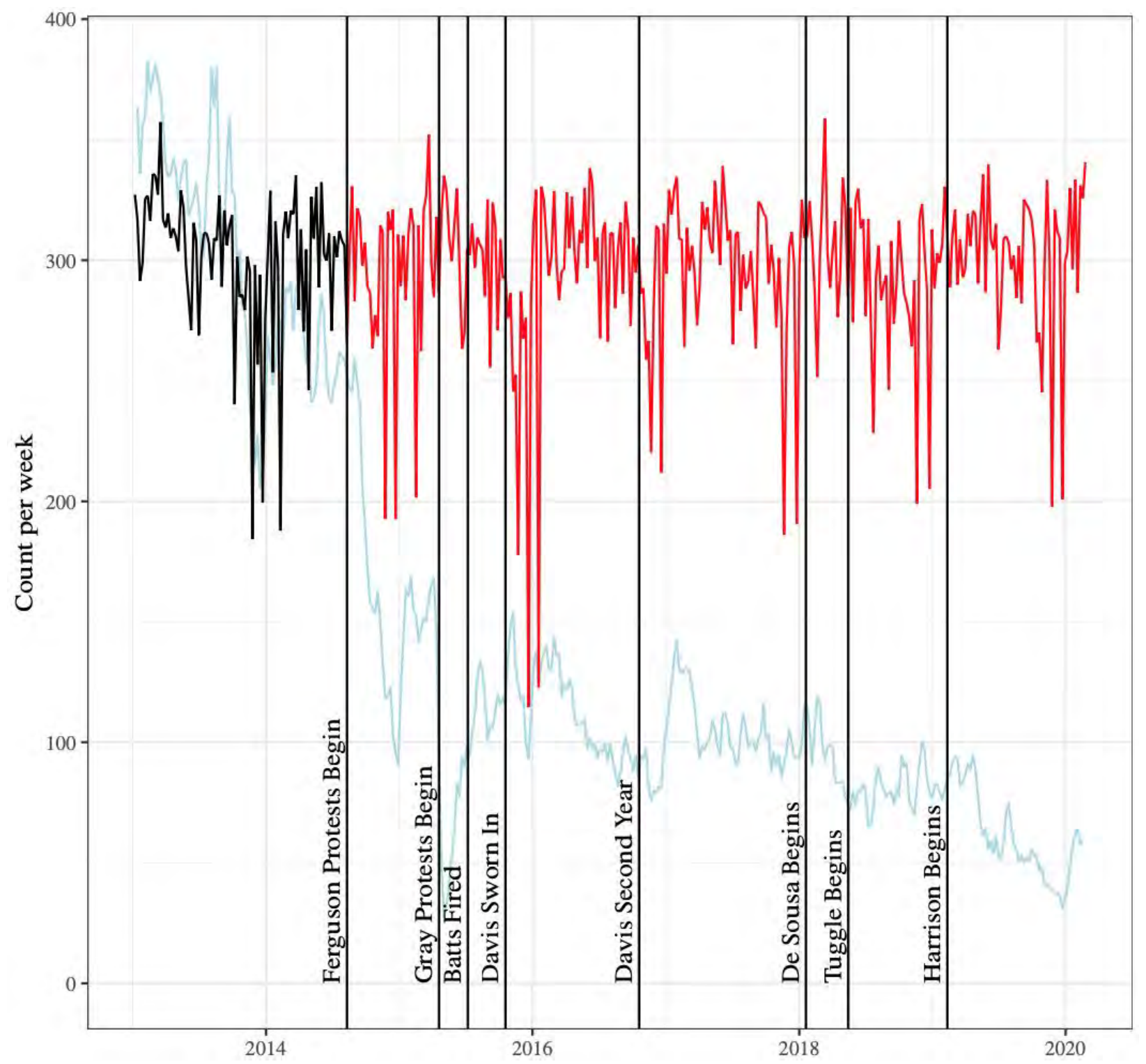

Figure 32. Total weekly arrest count for drug possession and possession with intent to distribute (three-week moving average, blue line) with predicted values from the model before the Ferguson protests began (black line) and extrapolated counterfactual values after the Ferguson protests began (red line) 


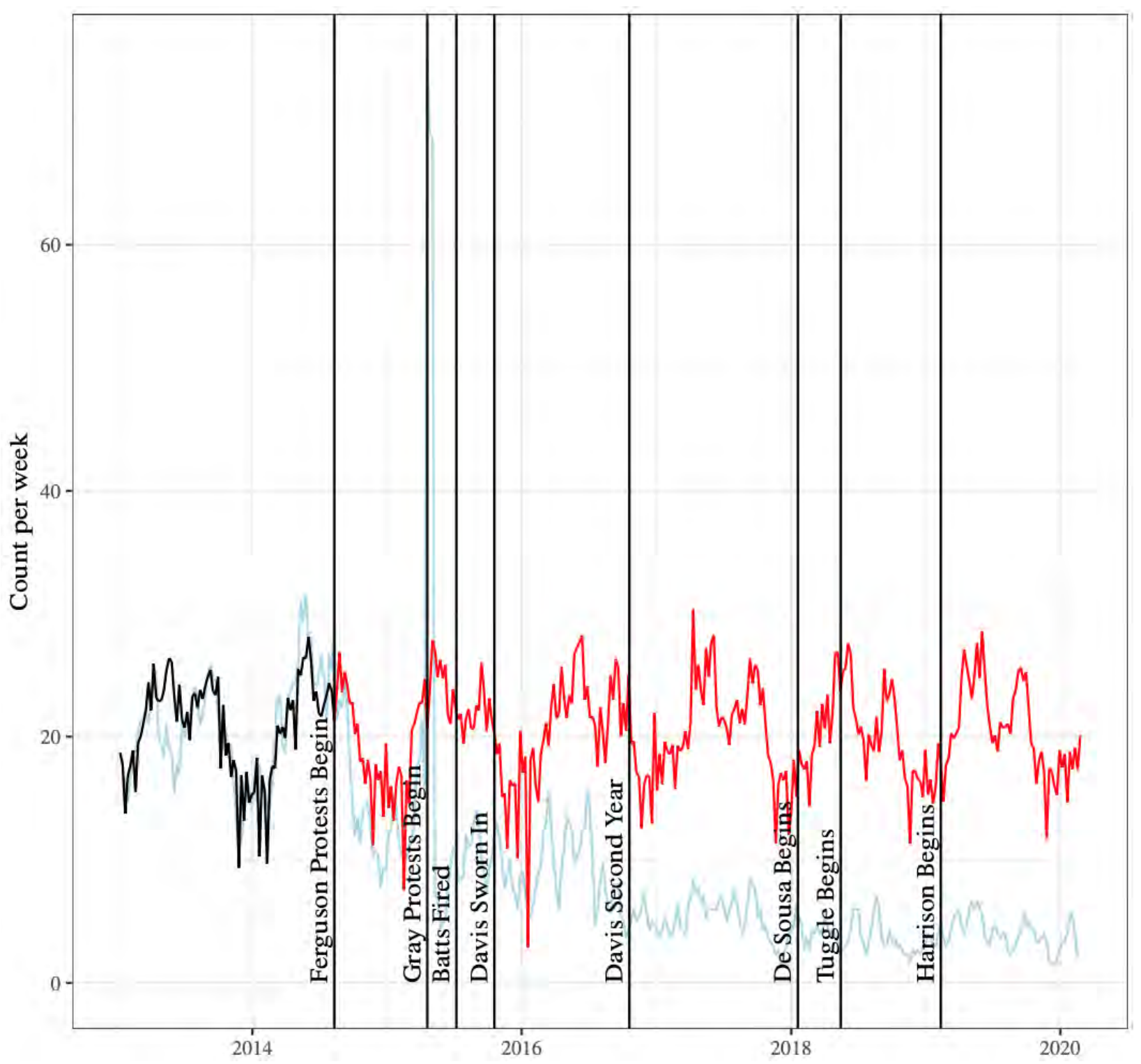

Figure 33. Total weekly arrest count for disorderly conduct (three-week moving average, blue line) with predicted values from the model before the Ferguson protests began (black line) and extrapolated counterfactual values after the Ferguson protests began (red line) 


\section{Conclusions}

With the hindsight of 2016-2020, as well as a refined analysis of 2010-2016, it is possible to offer conclusions with greater clarity. What looked in the 2016 reports as a complex tapestry of subtle associations, punctuated by the dramatic months surrounding the Baltimore unrest, no longer looks as difficult to interpret.

\subsection{The Ferguson and Gray Effects on Crime}

Consider first the baseline period before the national dialogue on policing reached CNN viewers and roused opinion writers. In Baltimore between 2010 and 2014, the prevalence of different types of crime was either holding steady or falling consistently, net of a basic pattern of cyclicality that is attributable to seasonal effects, broadly construed. The two most heavily watched types of crime were not changing much at all, with homicides trending little from 2010 to 2014 and shootings declining only very slightly. Robbery was also mostly unchanged, across all four types categorized. Nonetheless, other types of less violent crime were declining noticeably, including aggravated and common assault. Property crimes, especially burglary, were also trending downward. ${ }^{15}$ Altogether, levels of crime were declining, but the most violent types of crimes were not.

The Ferguson protests, and their coverage in the news media, did not change this pattern much at all. It is possible that, in Baltimore, the effects of the Ferguson protests on crime were gathering force before the arrest of Freddie Gray approximately eight months later. These months included only a few of the months when crime tends to be highest, and thus associational patterns in the city may have held any latent Ferguson effect on crime in abeyance. We can, however, rule out the possibility that the events in Ferguson led to a clearly noticeable, near-term effect on crime levels. Thus, as stated in the original report, adjusting for seasonality, evidence of a Ferguson effect on crime is very weak for the period before Freddie Gray was arrested.

After Freddie Gray died while in police custody, nearly all types of crime increased over the next three months, not just those that had been holding steady, but also those that were declining. Homicides and shootings received the most media attention, as they should, but the breadth of the crime increases in Baltimore was astounding.

How should we interpret the relationship between what would appear to be a small or nonexistent Ferguson effect on crime before Freddie Gray was arrested and a large and dramatic Gray effect on crime that emerged after Freddie Gray died while in police custody?

\footnotetext{
${ }^{15}$ Only larceny was clearly increasing from 2010 to 2014, and this pattern might be complicated by off-setting categorizations of crime. At the same time larceny was increasing, larceny from automobiles and thefts of automobiles were both decreasing. In this regard, it is also noticeable that larceny was the only type of crime that fell during both our Ferguson period and Gray period (see Table 3), which again prompts us to wonder about the BPD's method of categorization. Unlike arrest records, where we have a lot of detail on the charges associated with each arrest, for the crime incidents of larceny we have almost no detail.
} 
As noted above, and in the original report, we do not believe that a definitive answer can be provided that will satisfy all readers, beyond noting the connections that emerged in media commentaries. In this regard, the original report stated:

It is undeniable that the unrest and its aftermath were deeply shaped by the media coverage of Freddie Gray's arrest, including the available cellphone video of it. These influential journalistic treatments drew connections to the ongoing nationwide focus on police conduct. As a result, the Gray effect is fundamentally entangled with the Ferguson effect narrative, and it is not surprising therefore that the debate on the Ferguson effect picked up momentum shortly after the unrest in Baltimore. (Morgan and Pally 2016, page 46)

These connections are still undeniable. And, with hindsight and four more years of perspective, we feel more comfortable in this final report discussing why we believe these connections were advanced by others, as well as disagreeing with the standard narrative of how they should be considered to be connected.

It has become much clearer in the last few years that dissatisfaction with the BPD was deep at the time Freddie Gray was arrested, that it had been so for years, and that many members of the public had good reason to feel that at least some BPD officers were crooked and abusive. As a result, we cannot rule out entirely the possibility that the Ferguson protests were irrelevant and, thus, that the Gray effect on crime would have emerged just as it did, even in the absence of the Ferguson protests and/or the national dialogue on policing that the Ferguson protests made more salient. However, as we will explain below, we do now see a stronger case for a type of Ferguson effect on crime after the Gray unrest subsided. Taken together, we are comfortable arguing that a Gray effect of some type would have occurred in the absence of the Ferguson protests, but the particular Gray effect on crime that Baltimore experienced was amplified by the Ferguson protests and the national dialogue on policing.

The crucial question for us is this: Did the protests in Ferguson, and their portrayal in the media, shape the arrest of Freddie Gray, then contribute to the unrest in Baltimore following Freddie Gray's arrest, and finally, by this indirect route, contribute to a Gray effect on crime? We see three matters to consider in providing an answer to this question.

First, it is sometimes argued that the threat of being recorded on cellphone video causes police officers to step back from both effective and ineffective policing. If such a dynamic had emerged in the BPD following the Ferguson protests, it was not a factor in Freddie Gray's arrest or death. His apprehension was recorded in broad daylight, in front of Baltimore residents who recorded the events while offering commentary on them, just as they had in the recent past for other incidents. ${ }^{16}$ Likewise, the "rough ride" that Freddie Gray may have received while in the

\footnotetext{
${ }^{16}$ One video taken in full view of the officers restraining Gray before he was first placed in the van has a man remarking loudly, "We recordin' it. ... I've been recording it," and then, after being asked to move back off the street by an officer, "I sure the fuck will. But that ain't gonna stop me from usin' this phone" (see URL here). And, from a
} 
police van occurred in view of Baltimore's network of CCTV cameras. Thus, we conclude that the officers involved were surely aware that their actions were observed and recorded. They acted in the way that they did with this awareness.

Second, did the Ferguson protests play a role in generating the unrest? It is quite likely that the Ferguson protests, as well as the extensive media coverage that they received, further legitimized confrontational street-level protest among some residents of Baltimore (and, given the paramilitary equipment used so proudly, but ineffectively, by the Ferguson police, made it seem to be a valiant cause). Because no one has been able to document claims that the BPD provoked Baltimore residents and thus instigated street-level conflict on the most dramatic day of the unrest, we believe that it is reasonable to maintain that, in the absence of the national dialogue on policing, including the protests in Ferguson, the unrest that enveloped Baltimore may still have occurred, but, if so, it very likely would have been substantially diminished. ${ }^{17}$

Third, and in view of these first two conclusions, can any portion of the elevated crime in three months after the Baltimore unrest be attributed to the protests in Ferguson? Yes, we now think a case be made that this effect has support in Baltimore, but not by the mechanisms most often asserted by others who allege a Ferguson effect. The unrest itself grievously damaged the BPD's leadership and the authority of the city government. The mayor at the time did appear to encourage protests, even if her goal was only to encourage peaceful protests that could de-escalate tension and foster dialogue. Police officers had legitimate complaints that they did not have enough equipment or training to protect themselves in a riot, if that is what occurred. Then, when local prosecutors announced very quickly that all of the officers involved in Freddie Gray's arrest would be charged with very serious crimes, and with little or no additional evidence provided at the time to justify such serious charges, at least some police officers must have assumed that Baltimore's residents wanted them to police less and that it was in their interests to do so.

Altogether, therefore, we see the Gray effect that unfolded as harrowing elevated crime of all types as, at its core, a product of deliberate de-policing in an environment in which it was encouraged. Before the national dialogue on policing was underway, most observers believe that the BPD had relatively low legitimacy in the city, and that its low legitimacy was to a large extent grounded in its own ineffectiveness. The BPD was policing aggressively (indeed, by standards that were clear in 2013 and 2014, unconstitutionally), but the BPD had not truly brought violent crime under control while doing so. As a result, the BPD confronted the disorder that followed the unrest from a very weak position. And the approach taken appears

\footnotetext{
video taken at a greater distance, a woman remarks, "They makin' me mad, y'all. That's how they been round here" and then later, yelling toward the officers, as they picked Gray up, "Hey, his leg look broke!” (see URL here).

17 Small-scale protest activity has been fairly common in Baltimore in the last few decades, and these protests have sometimes followed episodes of contention between the police and community members. Only in the aftermath of Freddie Gray's death did a diverse array of residents join together for a full week of street activity, one day of which led to enough property destruction that it could be considered a riot. The deployment of the National Guard brought the property destruction under full control and then supported peaceful day-time protests that lowered tensions.
} 
clearly to have been what most everyone wanted: police officers stepping back from proactive policing. Police Commissioner Anthony Batts saw the de-policing as a problem, and he tried to get his officers to re-engage, later offering interviews with the Baltimore Sun in which he said his officers "took a knee" just after the unrest and allowed the crime rate to rise. ${ }^{18}$ Batts was fired within three months by a mayor who was in the process of destroying her own legitimacy.

To accept this conclusion requires accepting that de-policing occurred and that it was the product of decisions by the rank and file, not simply reduced capacity for policing in the aftermath of a period of unrest that taxed resources. We believe that our analysis of arrests is perhaps the best evidence that we have that de-policing really did occur. ${ }^{19}$ And, here, the story is complex, but not unclear, as we show in the next section.

To conclude this section, we see little or no evidence that a Ferguson effect on crime emerged before Freddie Gray was arrested. However, a Gray effect on crime is strongly supported by our analysis, and we are willing to more strongly conclude in this final report that a type of Ferguson effect played a role in amplifying it.

\subsection{The Ferguson and Gray Effects on Arrests}

Our analysis of arrests provides substantial evidence of both Ferguson and Gray effects on policing, but the weight of the evidence is compromised by the more limited data on arrests that are available to analyze. While we have crime data from 2010 onwards, our arrest data begin only in 2013.

Although we do not have as much baseline information on arrest patterns, it is clear from a variety of sources that changes were occurring in how non-violent, drug-related crime was being policed. In the summer months before the Ferguson protests, arrests on drug charges without other charges related to violence were declining noticeably. As shown above, while arrests for police non-compliance and disorderly conduct all rose in spring through early August of 2014 in the expected cyclical pattern (see Figures 29 and 33 ), arrests on drug charges continued to decline from the levels observed for 2013 (see Figure 32).

After the Ferguson protests, this decline in drug-related arrests accelerated and widened, leading to an overall seasonally adjusted decline in all types of arrests of 21.5 percent in the eight months just before Freddie Gray was arrested (see Table 6). As noted in the original report, we see this decline as "consistent with" a Ferguson effect on police conduct, wherein Baltimore's police officers more frequently used discretionary alternatives to arrest because of a concern that the appropriateness of their conduct could become the subject of controversy. If

\footnotetext{
18 "Anthony Batts says police 'took a knee' after Baltimore riots," Colin Campbell, Baltimore Sun, September 2, 2015. For additional context, see also "Arrests in Baltimore plummet, and residents are fearful," Doug Donovan and Colin Campbell, Baltimore Sun, June 13, 2015

${ }^{19}$ It would be preferable to be able to do a more complete analysis, considering how similar calls for police service, and patterns of on-view policing, changed for spring and early summer of 2015, in comparison to 2013 and 2014 . A credible analysis at that level of detail is not possible, given the data that are available.
} 
the decline in arrests had been more gradual, and there was more evidence that it took hold earlier in the summer of 2014, then we might have been tempted to conclude that a forwardlooking policy change was responsible, possibly a reduction in other types of arrests accompanying changes in how drug crime was being policed. In other words, reducing the number of stops and investigative searches, with the explicit goal of no longer arresting Baltimore residents for minor drug possession, could have reduced all arrests that could follow stops and investigative searches. Our reading of the evidence, however, does not support this conclusion, particularly when consulting the patterns for police non-compliance and disorderly conduct. These types of arrests only declined appreciably at the end of the summer of 2014, unlike non-violent, drug-related charges that were already falling before the Ferguson protests took place.

Regardless of whether we are willing to move beyond a weak "consistent with" assertion about a Ferguson effect on arrests, it is difficult to argue that these changes were not positive for Baltimore. They were not accompanied by any substantial increases in crime, and thus we stand by the conclusion in the original report:

The decline in arrests that is interpretable as a Ferguson effect in the period of time before the arrest of Freddie Gray can be considered a positive development for the Baltimore community. This conclusion would follow from the position that a decline in discretionary arrests is a desired goal, following a period in Baltimore during which many residents and their local leaders concluded that the robust policing of struggling communities is not helpful for their redevelopment. Moreover, this beneficial change may reflect a turn away from broken-windows policing and stop-and-frisk policing that would have occurred even in the absence of the national dialogue on police conduct that first captured headlines in the summer of 2014.

However, this same decline can also be considered a negative outcome for the Baltimore community if the decline in discretionary arrests encouraged the subsequent crime spike that emerged after the unrest receded. This conclusion would follow from the position that (a) the unrest itself was made more likely because of the decline in discretionary arrests and/or (b) the crime spike represents opportunistic crime that would have emerged even if Freddie Gray had not been arrested and the resulting unrest had not occurred.

On balance, we reason that the positive outcome scenario is more likely, although we concede that we are not immune from hopeful thinking. We believe that the composition of the crime spike that began in May 2015 is more likely attributable to the particular features of the unrest and how it was handled than by the decline in discretionary arrests that preceded it. (Morgan and Pally 2016, pages 68) 
We continue to support this reasoning, although we wish we had even more direct evidence in support of it.

The Gray effect on arrests was even more dramatic, with an additional 31 percent decline overall, on top of the 21.5 percent decline that occurred in the preceding eight months. The decrease was again more substantial for arrests that have a discretionary element, such as police non-compliance and disorderly conduct, but arrests for both violent crime and property crime decreased as well. We continue to think that these declines provide evidence of genuine de-policing. In this regard, it is also notable that arrests increased by 16 percent during the transition period after Kevin Davis was hired as an interim to replace the fired police commissioner, Anthony Batts. Davis was able to boost the arrest rate, and crime fell from its highest levels. It did not come down enough, and thus we conclude that his initial efforts to mitigate de-policing did not eliminate the crime rise that de-policing facilitated only a few months prior.

\subsection{From Davis Through Harrison, 2016-2020}

We have interpreted the 31 percent decline in total arrests during the three months following the unrest in Baltimore as evidence of deliberate de-policing, primarily because it occurred abruptly and alongside dramatic increases in crime, and secondarily because the police commissioner at the time, Anthony Batts, later expressed to local media outlets that his officers "took a knee." As just noted above, when Kevin Davis was hired as interim police commissioner in July of 2015, he appears to have been able to reverse some of the de-policing that he inherited. In his three-month probationary period from mid-July through mid-October, arrests increased by 16 percent while most categories of violent crime declined meaningfully (see Table 4). Because Davis pulled back some of the sharp increase in crime, he won the support of the city council to become the $38^{\text {th }}$ Police Commissioner of Baltimore.

Unfortunately, after being confirmed as commissioner, he was unable sustain the rate of improvement. During his first full year as commissioner, he reduced homicides further, but they still remained 41 percent above the baseline level observed for the year before August 2014. Other types of violent crime increased slightly, including the heavily reported crimes of street robbery and carjackings, but also the less heavily reported crime of aggravated assault. Then, in his second year on the job, almost all categories of crime increased further. Homicides crept upwards, and shootings remained 90 percent higher than the baseline year before August of 2014. Street robbery and carjackings soared to all-time highs. Davis was fired that winter.

Even the picture of arrests became muddled. While Davis had reversed some of the decline that we have argued above is evidence of de-policing, thereafter changes in arrests followed a complex pattern. Arrests for violent crimes bounced around. Arrests on deadly weapons charges (mostly hand-gun violations) fell substantially in both years. It seemed unlikely to most observers that unlawful hand-gun possession had actually decreased. At the same time, arrests for police non-compliance and disorderly conduct fell almost continuously, so that by the end of Davis' term, they stood at only 14 and 28 percent, respectively, of their 
2014 levels. Finally, arrests for non-violent, drug-related charges resumed their decline in his second year, ending at only 34 percent of the 2014 level.

For members of the community whose primary goal was to eliminate unconstitutional policing, and who felt that the DOJ's investigation of the BPD had given evidence that it needed to be eliminated, the decline in arrests during Davis' first two years was a positive development. Still, the declines are not easily interpreted, and they may reflect a number of causes unrelated to standards of constitutional policing. Regardless of the complexity, Davis handed over a police department to his replacement, Darryl De Sousa, that was arresting many fewer people. The declines were largest in areas most responsive to the changes called for by the consent decree, and also the areas least likely to be related to shootings and homicides.

By the time of Davis' handover to De Sousa in early 2018, local attention to the Ferguson protests in 2014 and even the unrest that followed Freddie Gray's arrest in spring 2015 had almost disappeared. The elevated rates of homicides, shootings, street robberies, and carjackings were enough by themselves to sustain alarming news coverage. The federal interventions that led to the GTTF scandal and the signing of the consent decree in 2017 were the core of the narrative that justified the need for top-to-bottom reform of the BPD, not how the unrest was handled three years prior, or even more remotely, whether the national dialogue on policing had any special relevance for Baltimore.

Without much media coverage in 2018 and early 2019, arrest rates continued to fall gradually under the leadership of De Sousa, and then Tuggle as well. As we have shown in this report, the declines were still disproportionately in types of arrests where police discretion was an alternative. Thus, the decline in arrests continued to be patterned in a way that was consistent with calls for reform.

To the extent that the falling arrest levels were noticed and reported upon by local commentators, and without the sort of categorization that we have offered in this report, some alternative conclusions were offered. For some commentators, the BPD was not being proactive enough. For others, taking the lead of the FOP, the BPD was demoralized and losing officers, with the result that the department could not place enough officers in patrol to keep order. Either way, lower arrests seemed to be attributed to dysfunction within the BPD, especially failures of management, and not at all evidence that officers were already responding positively to calls for reformed police conduct. Contrary to these views, we would like to argue that some of the decline in arrests in this time period reflects at least some officers trying to meet what they understood to be new standards for police practice, but we do not have any direct evidence to offer. The truth likely falls somewhere in the middle of all of these interpretations.

Finally, a new commissioner was hired from the outside, Michael Harrison. He had prior experience managing department-wide reform in response to a consent decree with the DOJ. Supported by staffing and IT studies completed in cooperation with the consent-decreemonitoring team, he tackled management challenges and hired a new leadership team. Within 
a few months, he introduced a crime plan, which the city's leadership had called for. The core strategy is presented in Figure 34 (see next page), and it is a variant on hotspot targeting that many others believe has been effective in other cities.

Harrison's first year has been surprising in many ways, not the least of which was the fierce opposition to his crime plan from the police union (see the letter in Figure 2 above). Most surprising, homicides, shootings, and carjackings all increased according to our models, even though street robbery, commercial robbery, and many types of property crime decreased. The temping conclusion is that hotspot targeting displaced the most violent crime, but it was effective in reducing at least some robberies and in protecting property. This mixed improvement was achieved while arrests continued to fall in almost all categories.

Overall, the pattern from 2016 through early 2020 is fairly clear. Homicides and shootings remain much higher than from 2010 to 2014, with only modest variation across the years. While robberies have declined somewhat in the last year, they still remain higher than they were in 2013 and 2014. Property crime has gradually been brought under better control, if one believes that property crime is reported and recorded at the same rate and with the same fidelity. Finally, arrests on charges that we consider more discretionary than other types of charges have continued to trend lower since 2016.

With the exception of the most violent crimes, an optimistic story can be pieced together for 2019 and the first part of 2020. The police are taking a lighter touch, arresting fewer Baltimore residents than they did in the past, and they have reduced property crime and robberies. If homicides, shootings, and carjackings can be reduced in the remainder of 2020 and then in 2021, then Baltimore will have turned a corner to a better future than seemed possible only one year ago. 


\section{STRATEEIC OBJEGTIVES AND ROLES}

For the BPD to efficiently and effectively achieve sustainable reductions in violent crime, we are leveraging research and data to develop a comprehensive and evolving strategy at discrete micro-geographies. Our goal is to create tailored solutions for each zone that respond to the needs of that specific target area.

Using crime history on all gun-related incidents (homicides, aggravated assaults, robberies, and non-fatal shootings) for the past five years, BPD has established new focused patrol areas and district action team (DAT) activity zones where the levels of these crimes have been highest.

BPD has assigned each sector patrol officer to cover specific deployment zones that cover no more than a four-square block area. In addition, there will be larger areas designated for DATs assigned to each police district. Overall, these zones comprise roughly $5 \%$ of the city's geography but account for approximately $33 \%$ of the all the city's gun violence in the past five years. A narrow and more structured focus by the DATs along with directed patrol enables BPD to be more agile, targeted, and efficient in our ability to reduce, deter, and prevent crime.

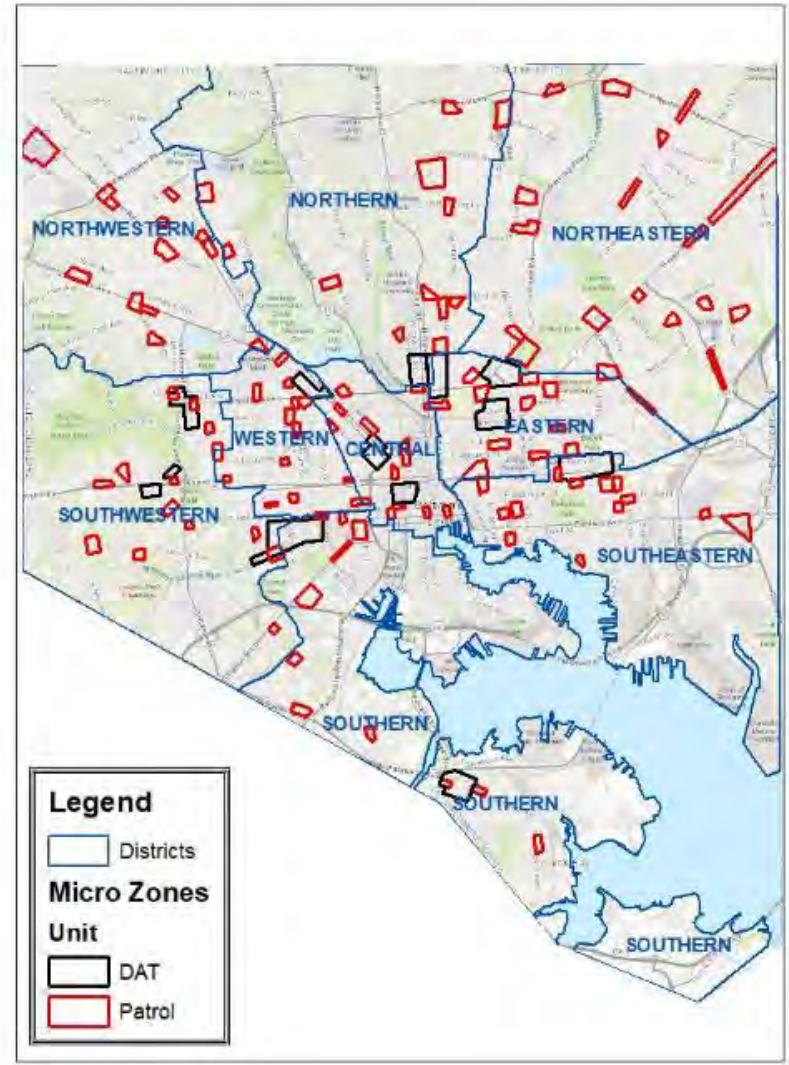

\section{PG. 5 | Baltimore Police Department}

Figure 34. The hotspot design of Harrison's June 2019 crime reduction strategy (page 5 from the document) 


\section{Appendix 1 \\ Coding of Arrest Groups}

\author{
Stephen L. Morgan \\ Johns Hopkins University
}

\author{
Rhiannon N. Miller \\ Johns Hopkins University
}

April 19, 2020

Details on the Coding of Raw Arrest Records Into Groups for Analysis ..................................................... 1

Arrest Groups (Listed in Order of Severity) ......................................................................................... 3

Murder (Subsequently Combined with Attempted Murder) ................................................................... 3

Attempted Murder (Subsequently Combined with Murder) ……………................................................ 4

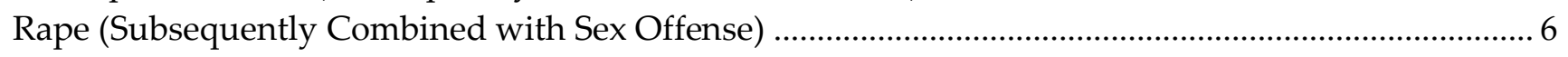

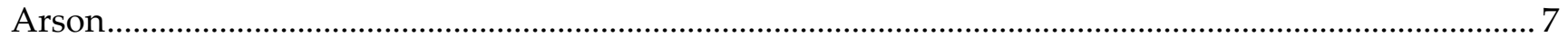

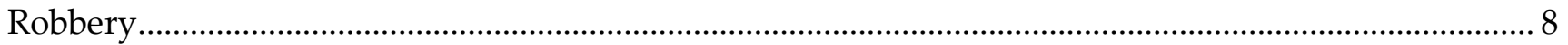

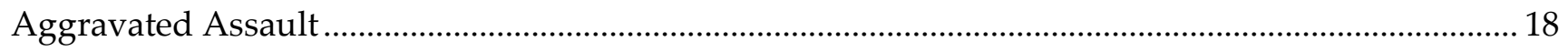

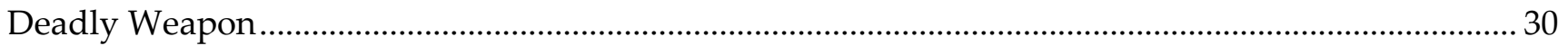

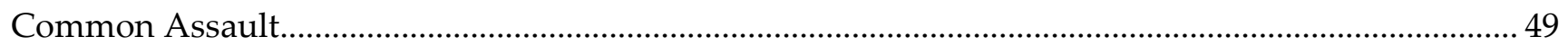

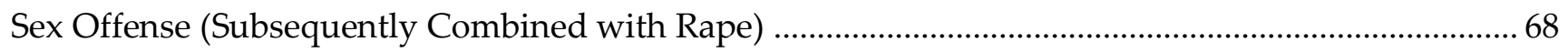

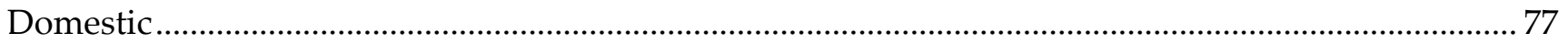

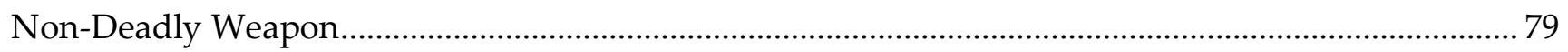

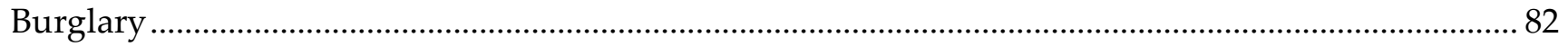

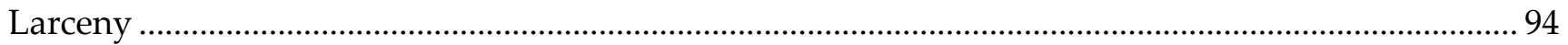

Drug Distribution (Subsequently Combined with Drug Possession) ................................................... 117

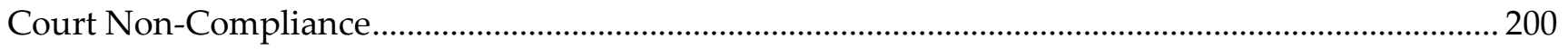

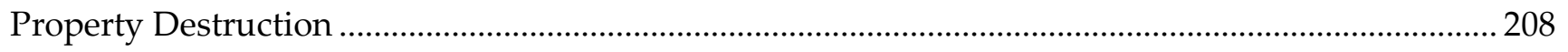

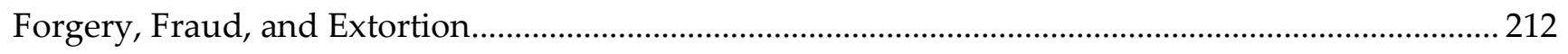

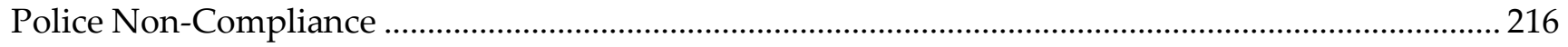

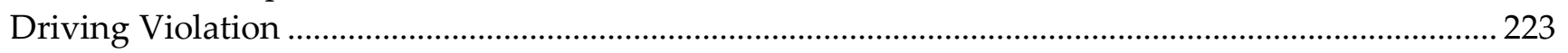

Gambling (Subsequently Demoted to the Category "Ungrouped") ................................................... 235

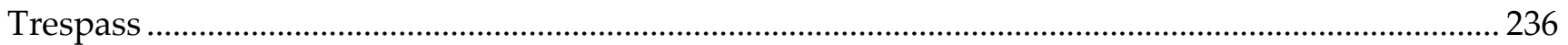

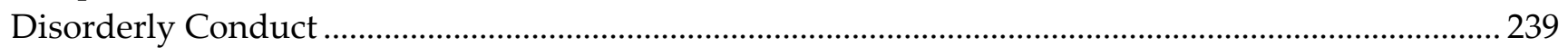

Drug Possession (Subsequently Combined with Drug Distribution) ………………………………........ 246

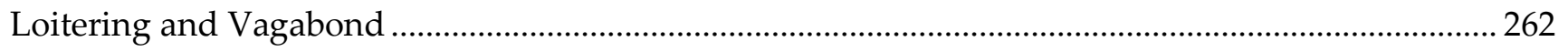

Bike and Dirtbike (Subsequently Combined with Disorderly Conduct)................................................ 263

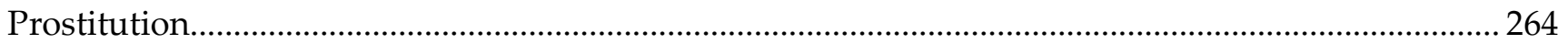

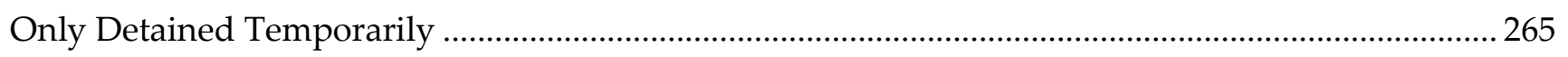

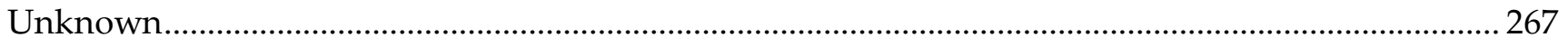

Ungrouped (Subsequently Incorporates Gambling) ……………………………………………...... 267 


\section{Details on the Coding of Raw Arrest Records Into Groups for Analysis}

The raw BPD arrest data available for download from the Open Baltimore data portal contains abundant information on the charges associated with each arrest. We developed an algorithmic coding scheme to create 29 meaningful categories for arrests that are small enough in number, but large enough in size, to enable comparative analysis of trends in types of arrest. We collapsed these groups to the 25 analyzed in the report (e.g., combining murder and attempted murder into a murder group).

Coding by Severity. Our first decision was to categorize arrests with multiple charges by the most serious charge identified, placing each arrest in one and only one category. Unfortunately, no consensus exists on how to define the severity of charges, even though some local orderings are not controversial (e.g., murder is more severe than aggravated assault; robbery is more severe than larceny). We used the P-1 offenses of the FBI Uniform Crime Reporting program as a general guide, but some of our decisions, especially for the least severe types of charges, were based only on our reasoning. Our decisions were also shaped by our desire to generate a manageable number of final categories that could be used to consider police activity.

Creating the Data Elements to be Categorized. All arrest records in the raw data - 199,659 arrests for the data downloaded through 4/18/2020 - have two fields that detail the arrest charges: a textual description of the charges and a single charge code for each arrest. In some cases, one field is empty, and in others both fields are empty (or contain entries such as "Unknown charge").

To carry out our categorization of arrests, we first created a data element for each arrest record that is a concatenation of the fields for the charge description and the charge code, resulting in arrest data elements such as these:

Att 1St Deg. Murder, Attempt 1St. Degree Murder I| 20910

Asslt-Sec Degree, Armed Robbery Commercial I| 11415

Asslt-Sec Degree, Attempt Rape I| 11415

Asslt-First Degree, Poss Heroin || 11420

Arson-Trash Containers, Malicious Burning In The Sec || 22003

Discharge Firearm I I

Con-Mal Dest Prop/Valu - \$500, Destruction Of Proper II 3C4025

Driving On Suspended Lic / Pri I I

HANDGUN/CDS VIOLATION || 20696

Theft: Less \$1,000 Value, 4Th Degree Burglary || 10621

RIDING A DIRT BIKE I ।

Dis.Erly Conduct, Disturbing The Peace || 20050

POSS W INT HEROIN \& COCAINE | | 30233

Disturb Peace - Loud Noise, Failure To Obey I| 20065

4TH DEG BURGLARY/CDS I | 43550

Trespass: Private Property, Loitering/Tresspassing || 22220 
The portion of the data element before $\mathrm{II}$ is the charge description, and the portion after is the charge code (which is missing for three of the arrests above). For the 199,659 arrest records available as of $4 / 18 / 2020$, the number of unique data elements is 16,368 .

Categorization Method. Our categorization is based on both the textual charge descriptions and the charge codes, mostly because the charge codes were often missing or could not be mapped to any documentation that we could find. Notice also that some of the data elements above contain redundant information, and other data elements contain more than one charge. In comparison to many of the data elements listed below in this appendix, these charge descriptions are easy to interpret.

We used the same hierarchical keyword-based strategy for categorization, which is explained in Appedix 1 of the original report from 2016. However, for this final report we altered the hierarchy of arrest groups slightly. We also needed to develop the keyword pattern coding substantially because the number of unique arrest data elements increased by 60 percent since the end of 2015 .

The Resulting Categories and Subsequent Groups for Analysis. In subsequent sections of this appendix, we provide a full categorization of all types of arrests, listing the 16,638 distinct data elements that were categorized into 29 initial categories. These were then collapsed to the 25 final groups used for the analysis in the main report.

The final two categories of arrests are "unknown" and "ungrouped" (both beginning below on page 267). The latter contains a hodgepodge of elements, from animal abuse through telephone misuse, as well as some badly misspelled items. Mispelled and incomplete items could have been manually coded but constituted such a small number that we decided to rely only on our structured keyword categorization. In the report, we analyze these two groups in order to show that the "unknown" arrests trend downward, much like the total arrest trend (but with a spike upward during the week of the 2015 unrest). The "ungrouped" arrests trend lower as well, although to a smaller degree.

We do not claim that our categorization is as accurate as a comprehensive and skilled one-byone manual coding could achieve, but the following lists demonstrated to us a level of accuracy that exceeded our tolerance for error. 


\section{Arrest Groups (Listed in Order of Severity)}

\section{Murder (Subsequently Combined with Attempted Murder)}

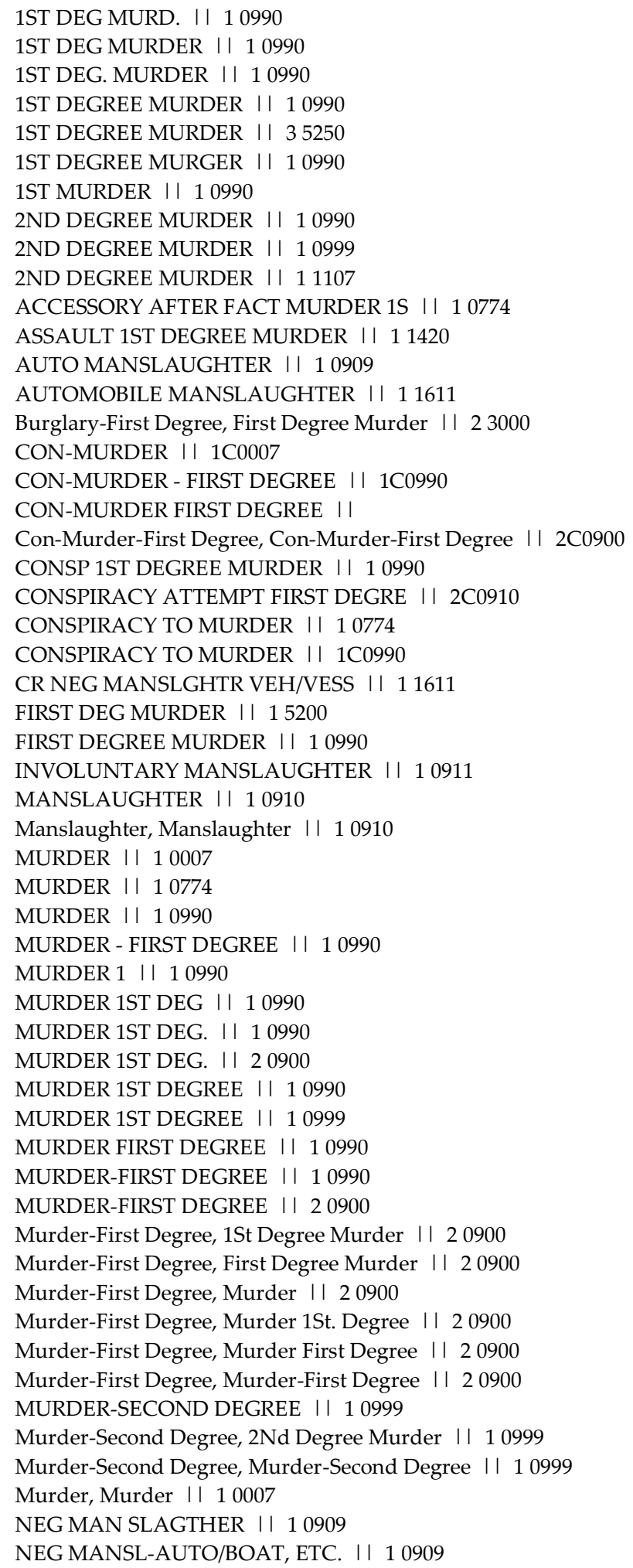


POISON:ATTEMPT || 20999

VEHICULAR MANSLAUGHTER || 11611

\section{Attempted Murder (Subsequently Combined with Murder)}

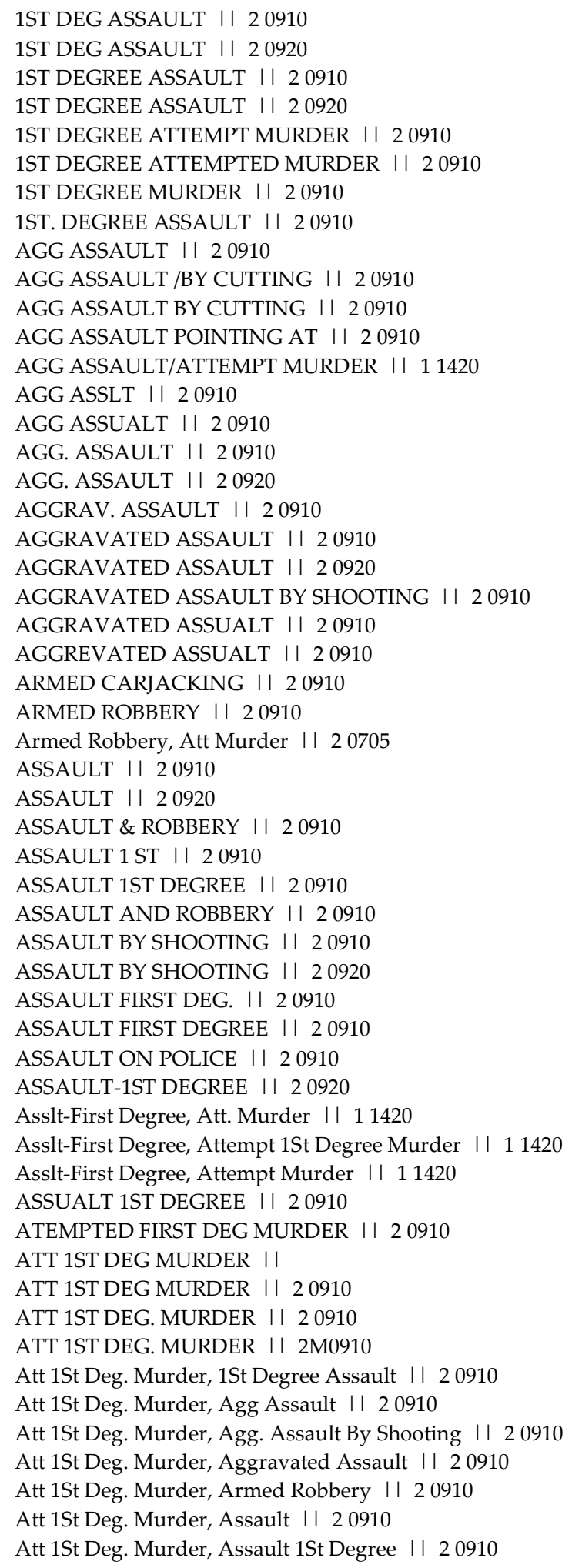


Att 1St Deg. Murder, Assault-First Degree I| 20910

Att 1St Deg. Murder, Att 1St Deg. Murder I| 20910

Att 1St Deg. Murder, Att 1St Degree Murder II 20910

Att 1St Deg. Murder, Att Murder I| 20910

Att 1St Deg. Murder, Att. 1St Degree Murder I| 20910

Att 1St Deg. Murder, Att. Murder I| 20910

Att 1St Deg. Murder, Attemp Of Murder II 20910

Att 1St Deg. Murder, Attempt 1St. Degree Murder I| 20910

Att 1St Deg. Murder, Attempt Murder I | 20910

Att 1St Deg. Murder, Attempted 1St Degree Murder I| 20910

Att 1St Deg. Murder, Attempted Murder I| 20910

Att 1St Deg. Murder, First Degree I| 20910

Att 1St Deg. Murder, Robbery || 20910

ATT 1ST DEGREE MURDER । |

ATT 1ST DEGREE MURDER I I 20910

ATT 1ST MURDER || 20910

ATT 1ST. DEGREE MURDER || 20910

ATT 2ND DEG. MURDER I| 20920

Att 2Nd Deg. Murder, 1St Degree Assault I| 20920

Att 2Nd Deg. Murder, Att 2Nd Deg. Murder I| 20920

Att 2Nd Deg. Murder, Attempted Murder II 20920

ATT FIRST DEGREE MURDER I | 20910

ATT IST DEG MURDER I।

ATT MUDER 1ST I| 20910

ATT MURDER I| 11137

ATT MURDER II 20910

ATT MURDER 1ST DEGREE || 20910

ATT MURDER, AGG ASSAULT || 11420

ATT-MURDER I| 1A0007

ATT-MURDER I| 20705

ATT-MURDER-FIRST DEGREE || 2A0900

ATT-MURDER-SECOND DEGREE I I 1A0999

Att-Murder-Second Degree, Att-Murder-Second Degree II 1A0999

ATT. 1ST DEG. MURDER I I 20910

ATT. 1ST DEGREE MURDER I| 20910

ATT. FIRST DEGREE MURDER I 20910

ATT. MURDER I| 11420

ATT. MURDER || 20910

ATT. ROBBERY || 20910

ATTEMP MURDER I| 20910

ATTEMPT MURDER II 20910

ATTEMPT 1 DEG. MURDER || 20910

ATTEMPT 1ST DEG MURDER | | 20910

ATTEMPT 1ST DEG. MURDER I | 11420

ATTEMPT 1ST DEGREE MURDER I।

ATTEMPT 1ST DEGREE MURDER | | 20910

ATTEMPT FIRST DEGREE MURDER | | 20910

ATTEMPT MURDER I। 11285

ATTEMPT MURDER || 11415

ATTEMPT MURDER ।| 11420

ATTEMPT MURDER I| 15200

ATTEMPT MURDER I| 20910

ATTEMPT MURDER I| 20920

ATTEMPT MURDER 1ST DEGREE || 11420

ATTEMPTED 1ST DEG. MURDER I I 11420

ATTEMPTED 1ST DEGREE MURDER I | 20910

ATTEMPTED 1ST DEGREE MURDER || 23000

ATTEMPTED FIRST DEGREE MURDER | | 20910

ATTEMPTED HOMICIDE I| 20910

ATTEMPTED MURDER || 11420 
ATTEMPTED MURDER || 20910

ATTEMPTED MURDER (2 COUNTS) || 2M0910

CAR JACKING II 20910

Cds: Possession-Marihuana, Attempt 1St Degree Murde I| 10573

COMMON ASSAULT || 20910

CON-ATT 1ST DEG. MURDER I| 2C0910

Con-Att 1St Deg. Murder, Con-Att 1St Deg. Murder II 2C0910

CON-ATT 2ND DEG MURDER I| 2C0920

CON-ATT MURDER I| 20910

CONSPIRACY ATTEMPT 2ND MURDER ।| 11480

FIRST DEG ASSAULT I| 20910

FIRST DEGREE ASSAULT | | 20910

FIRST DEGREE ASSAULT || 20920

FIRTS DEGREE ASSAULT । | 20920

HANDGUN VIOLATION || 20910

HANG GUN VIOLATION | | 20910

HGV || 20910

Murder-1St Deg/Arson-Att, Att-Murder-1St Deg/Arson II 1 A0912

Murder-Att, Att-Murder I| 1A0007

RECKLESS ENDANGERMENT || 20910

ROBBERY || 20910

\section{Rape (Subsequently Combined with Sex Offense)}

1ST DEG RAPE || 11102

1ST DEG. RAPE || 11102

1ST DEGREE RAPE || 11102

1ST DEGREE RAPE || 21103

1ST RAPE || 11102

2ND DEG RAPE || 21103

2ND DEGREE RAPE || 11102

2ND DEGREE RAPE || 21103

2ND DEGREE RAPE || 43600

2ND DEGREE RAPE ATTEMMPT || 21110

2ND RAPE || 21103

ASSAULT 1ST/RAPE || 11102

ASSAULT-W/INTENT RAPE || 11397

Asslt-First Degree, Rape I| 11420

Asslt-Sec Degree, 1St Deg Rape I I 11415

Asslt-Sec Degree, Attempt Rape II 11415

Asslt-Sec Degree, Rape I| 11415

ATT 1ST DEG RAPE || 21120

Att 1St Deg Rape, Att 1St Deg Rape I| 21120

Att 1St Deg Rape, Att.Rape I| 21120

Att 1St Deg Rape, Attempt Rape I| 21120

ATT 2ND DEG RAPE || 21110

Att 2Nd Deg Rape, Att 2Nd Deg Rape I| 21110

Att 2Nd Deg Rape, Attempted Rape I| 21110

Att-Rape 2Nd Deg - See 2 1110, Att-Rape 2Nd Deg || 2A1103

ATTEMPT 2ND DEG RAPE | | 53550

ATTEMPT RAPE || 21110

ATTEMPTED RAPE || 11420

ATTEMPTED RAPE | | 21110

ATTEMPTED RAPE || 21120

CONSP. COMMIT RAPE/HUMAN TRAFF || 31005

RAPE || 11102

RAPE || 11338

RAPE || 21103

RAPE || 23600 
RAPE || 33600

RAPE 1ST DEG I।

RAPE 1ST DEG || 11102

RAPE 1ST DEGREE || 11102

RAPE 2ND || 21103

RAPE 2ND DEG || 21103

RAPE 2ND DEGREE || 21103

RAPE FIRST DEGREE I ।

RAPE FIRST DEGREE || 11102

Rape First Degree, 1St Deg. Rape I| 11102

Rape First Degree, 1St Degree Rape I| 11102

Rape First Degree, Rape I| 11102

Rape First Degree, Rape 1St Degree || 11102

Rape First Degree, Rape First Degree || 11102

Rape First Degree, Robbery || 11102

RAPE SECOND DEGREE || 21103

Rape Second Degree, 2Nd Degree Rape I| 21103

Rape Second Degree, Rape I| 21103

Rape Second Degree, Rape 1St Degree I| 21103

Rape Second Degree, Rape 2Nd Degree || 21103

Rape Second Degree, Rape Second Degree II 21103

Sex Offense Second Degree, Statutory Rape II 23600

Sex Offense Third Degree, Rape 2Nd Degree II 33600

Sex Offense Third Degree, Statutory Rape II 33600

STATUTORY RAPE II 43600

\section{Arson}

1ST DEGREE ARSON I| 16501

A.H. ARSON || 11415

ARSON II 10692

ARSON II 11415

ARSON II 16500

ARSON || 16501

ARSON II 16505

ARSON I| 1A6500

ARSON (1ST DEGREE) I | 16500

ARSON 1ST DEGREE || 16500

ARSON 1ST DEGREE || 16501

ARSON 2ND DEGREE I I

ARSON 2ND DEGREE || 16501

Arson 2Nd Degree, Arson 2Nd Degree || 16501

ARSON THREAT I| 11415

ARSON THREAT I| 16505

ARSON THREAT I I 1A6500

ARSON THREAT || 22220

ARSON THREAT I| 23030

Arson Threat Explode, Arson Threat Explode II 16506

ARSON-FIRST DEGREE || 16500

Arson-First Degree, Arson II 16500

Arson-First Degree, Arson 1St Deg I| 16500

Arson-First Degree, Arson 1St Degree II 16500

Arson-First Degree, Arson-First Degree I| 16500

ARSON-TRASH CONTAINERS I| 22003

Arson-Trash Containers, Malicious Burning In The Sec I| 22003

ARSON/THREAT I I

ARSON/THREAT || 16505

Arson/Threat, Arson Threat II 16505

Arson/Threat, Arson/Threat I| 16505 
Arson/Threat, Bomb Threat || 16505

Arson/Threat, Disorderly || 16505

Arson/Threat, Threat Of Arson II 16505

Arson/Threat, Tresspassing || 16505

ATT-ARSON 2ND DEGREE || 1A6501

ATT-ARSON-FIRST DEGREE || 1A6500

Att-Arson-First Degree, Assault 1St Degree II 1 A6500

Att-Arson-First Degree, Att-Arson-First Degree II 1A6500

Att-Arson-First Degree, Attempt Arson II 1A6500

ATT-ARSON/THREAT I I 1A6505

ATT-MALICIOUS BURNING/1ST DEG | | $1 \mathrm{~A} 6502$

ATT. ARSON || 16503

ATT.ARSON || $1 \mathrm{~A} 6500$

ATTEMPT ARSON || 11415

ATTEMPT ARSON I| 16500

ATTEMPT ARSON || 16503

ATTEMPT ARSON || 16505

ATTEMPT ARSON I| 1A6500

ATTEMPT. ARSON I| 22220

ATTEMPTED ARSON || 10720

ATTEMPTED ARSON || 1A6500

ATTEPTED ARSON II 16500

Con-Arson-First Degree, Con-Arson-First Degree II 1C6500

LARSENCY/THEFT || 10690

LARSONY I I 10521

Mal Dest Prop/Valu - \$500, Threat Of Arson II 34025

MAL DEST/BURNING || 34025

MALICIOU BURNING || 16503

Malicious Burn/Fraud, Malicious Burn/Fraud II 16504

MALICIOUS BURNING || 16503

MALICIOUS BURNING || 22003

MALICIOUS BURNING || 34030

MALICIOUS BURNING I| 43550

MALICIOUS BURNING/1ST DEG || 16502

Malicious Burning/1St Deg, Arson First Degree || 16502

Malicious Burning/1St Deg, Malicious Burning/1St Deg || 16502

MALICIOUS BURNING/2ND DEG | | 16503

Malicious Burning/2Nd Deg, Arson 2Nd Degree II 16503

Malicious Burning/2Nd Deg, Malicious Burning/2Nd De || 16503

Malicious Burning/2Nd Deg, Malicious Burning/2Nd Deg || 16503

THEFT LESS THAN 100 DOLLARS || 10521

THREAT OF ARSON I| 10356

THREAT OF ARSON ।| 16505

THREATS-ARSON I| 10465

Threats-Arson, Threats-Arson II 10465

\section{Robbery}

1ST DEGREE ASSAULT || 20705

A \& R COMMERIAL I| 20705

A+R || 20705

AGG ASSAULT || 20705

AGG ASSAULT BY CUTTING | | 20705

AGG. ASSAULT || 20705

AGGRAVATED ASSAULT | | 20705

AGGRAVATED ASSAULT A\&R || 20705

AGGRAVATED ASSAULT/ROBBERY || 20700

ARM ROBBERRY || 20705

ARM ROBBERY || 20705 
ARMED ROBBERY (COMMERCIAL) I | 20705

ARMED ASSAULT \& ROBBERY || 20705

ARMED ASSAULT AND ROBBERY | | 20705

ARMED BANK ROBBERY || 20700

ARMED BANK ROBBERY || 20705

ARMED CAR JACKING ।

ARMED CAR JACKING || 10826

ARMED CAR JACKING I | 11119

ARMED CAR JACKING || 11609

ARMED CAR JACKING II 20480

ARMED CAR JACKING || 20705

ARMED CARJACKING ||

ARMED CARJACKING || 10825

ARMED CARJACKING || 10826

ARMED CARJACKING || 11119

ARMED CARJACKING || 11136

ARMED CARJACKING || 11415

ARMED CARJACKING || 11609

ARMED CARJACKING || 15212

ARMED CARJACKING || 15299

ARMED CARJACKING || 20480

ARMED CARJACKING | | 20705

Armed Carjacking, Armed Car Jacking || 10826

Armed Carjacking, Armed Carjacking || 10826

Armed Carjacking, Armed Robbery II 10826

Armed Carjacking, Stolen Auto I| 10826

ARMED CARJACKING/HGV || 10622

ARMED COMM ROBBERY || 10621

ARMED COMMERCIAL ROBBERY | | 11415

ARMED COMMERCIAL ROBBERY | | 20700

ARMED COMMERCIAL ROBBERY | | 20705

ARMED HOME INVASION | | 20705

ARMED ROBBBERY/COMM । I 20700

ARMED ROBBBRY || 20705

ARMED ROBBERRY || 20705

Armed Robbery II

ARMED ROBBERY I |

ARMED ROBBERY || 10493

ARMED ROBBERY || 10521

ARMED ROBBERY | | 10573

ARMED ROBBERY || 10621

ARMED ROBBERY || 10826

ARMED ROBBERY || 11106

ARMED ROBBERY || 11111

ARMED ROBBERY | | 11137

ARMED ROBBERY | | 11202

ARMED ROBBERY | | 11285

ARMED ROBBERY || 11338

ARMED ROBBERY || 11415

ARMED ROBBERY || 11420

ARMED ROBBERY | | 11455

ARMED ROBBERY || 11609

ARMED ROBBERY || 15200

ARMED ROBBERY || 15212

ARMED ROBBERY || 15285

ARMED ROBBERY I | 15299

ARMED ROBBERY || 1A0826

ARMED ROBBERY I I 1C0826

ARMED ROBBERY I | 20480

ARMED ROBBERY | | 20700 
ARMED ROBBERY || 20705

ARMED ROBBERY || 23000

ARMED ROBBERY || 2A0700

ARMED ROBBERY | | 2A0705

ARMED ROBBERY || 2C0700

ARMED ROBBERY || 2C0705

ARMED ROBBERY || 31005

ARMED ROBBERY I| 43550

ARMED ROBBERY COMMERCIAL || 11415

ARMED ROBBERY W/HG I। 20705

Armed Robbery, 1St Degree Assault II 20705

Armed Robbery, Agg Assault I | 20705

Armed Robbery, Agg. Assault || 20705

Armed Robbery, Aggravated Assault I| 20705

Armed Robbery, Armed Assault Robbery II 20705

Armed Robbery, Armed Carjacking II 20705

Armed Robbery, Armed Commercial Robbery || 20705

Armed Robbery, Armed Robbbery I| 20705

Armed Robbery, Armed Robber I I 20705

Armed Robbery, Armed Robbery I| 11202

Armed Robbery, Armed Robbery || 20705

Armed Robbery, Armmed Robbery II 20705

Armed Robbery, Assault And Robberry II 20705

Armed Robbery, Assault And Robbery || 20705

Armed Robbery, Assault And Roberry II 20705

Armed Robbery, Assault Armed Robbery II 20705

Armed Robbery, Att Robbery || 20705

Armed Robbery, Att. Armed Robbery || 20705

ARMED ROBBERY, ATTEMPT II

Armed Robbery, Attempt (Unarmed Robbery) I| 20705

Armed Robbery, Attempt Armed Robbery II 20705

Armed Robbery, Attempt Strong Armrobbery | | 20705

Armed Robbery, Attempted Armed Robbery II 20705

Armed Robbery, Attemted Armed Robbery || 20705

Armed Robbery, Comm Armed Robbery II 20705

Armed Robbery, Comm. Armed Robbery I| 20705

Armed Robbery, Himil Robbery II 20705

Armed Robbery, Robbery I| 20705

Armed Robbery, Robbery Armed || 20705

Armed Robbery, Unarmed Robbery || 20705

ARMED ROBBERY/HGV || 11202

ARMED ROBBERY/CAR JACKING || 10826

ARMED ROBBERY/CARJACKING || 10826

ARMED ROBBERY/HGV I I 20705

ARMED ROBBEY || 20705

ARMED ROBBGERY || 20705

ARMED ROBERRY | | 20705

ARMED ROBERY | | 10826

ARMES ROBBERY || 20705

ASLT ROBBERY || 11415

ASS. FIRST DEG || 20705

ASSAULT || 20705

ASSAULT || 2A0705

ASSAULT / ROBBERY I| 11420

ASSAULT / ROBBERY I I 20700

ASSAULT / UNARMED ROBBERY || 11415

ASSAULT /ROBBERY || 20700

ASSAULT \& ROBBERY || 11415

ASSAULT \& ROBBERY || 11420

ASSAULT \& ROBBERY || 15299 
ASSAULT \& ROBBERY || 20700

ASSAULT \& ROBBERY || 20705

ASSAULT \& ROBBERY CDS || 43550

ASSAULT + ROBBERY || 20700

ASSAULT + ROBBERY I| 20705

ASSAULT 1RST || 20705

ASSAULT 1ST DEGREE || 20705

ASSAULT AND ARMED ROBBERY I | 2C0705

ASSAULT AND ROBBERY || 10621

ASSAULT AND ROBBERY || 10690

ASSAULT AND ROBBERY | | 11299

ASSAULT AND ROBBERY || 11415

ASSAULT AND ROBBERY || 15299

ASSAULT AND ROBBERY || 20700

ASSAULT AND ROBBERY I I 20705

ASSAULT AND ROBBERY ARMED || 20705

ASSAULT BY POINTING || 20705

ASSAULT ON POLICE || 20705

ASSAULT ROBBERY || 11415

ASSAULT ROBBERY || 20700

ASSAULT ROBBERY || 20705

ASSAULT SEC/UNARMED ROBBERY || 20700

ASSAULT/ ROBBERY || 20700

ASSAULT/ROBBERY || 11415

ASSAULT/ROBBERY || 11420

ASSAULT/ROBBERY || 1A0826

ASSAULT/ROBBERY || 20700

ASSAULT/ROBBERY || 20705

ASSAULT/ROBBERY || 2A0700

ASSAULT/UNARMED ROBBERY || 20700

ASSAULT\&ROBBERY || 20700

Asslt-First Degree, Armed Robbery II 11420

Asslt-First Degree, Robbery II 11420

Asslt-Sec Degree, Agg. Assault/Robbery II 11415

Asslt-Sec Degree, Armed Robbery || 11415

Asslt-Sec Degree, Aslt Robbery || 11415

Asslt-Sec Degree, Assault \& Robbery | | 11415

Asslt-Sec Degree, Assault Robbery I| 11415

Asslt-Sec Degree, Assault/Robbery || 11415

Asslt-Sec Degree, Assault/Unarmed Robbery II 11415

Asslt-Sec Degree, Assult And Robbery II 11415

Asslt-Sec Degree, Att Armed Robbery II 11415

Asslt-Sec Degree, Attempt Commercial Robbery I| 11415

Asslt-Sec Degree, Attempt Commercial Unarmed Rob || 11415

Asslt-Sec Degree, Attempt To Unarmed Robbery II 11415

Asslt-Sec Degree, Attempt Unarmed Commercial Rob I| 11415

Asslt-Sec Degree, Attempt Unarmed Robbery || 11415

Asslt-Sec Degree, Attempted Robbery I| 11415

Asslt-Sec Degree, Comerical Robbery II 11415

Asslt-Sec Degree, Comm Armed Robbery || 11415

Asslt-Sec Degree, Commercial Armed Robbery II 11415

Asslt-Sec Degree, Commercial Robbery I| 11415

Asslt-Sec Degree, Commercial Unarmed Robbery II 11415

Asslt-Sec Degree, Commerical Armed Robbery I| 11415

Asslt-Sec Degree, Domestic Assault / Robbery II 11415

Asslt-Sec Degree, Robbery II 11415

Asslt-Sec Degree, Robbery And Assualt I| 11415

Asslt-Sec Degree, Ua Robbery II 11415

Asslt-Sec Degree, Unarm Robbery II 11415

Asslt-Sec Degree, Unarmed Assault And Robbery II 11415 
Asslt-Sec Degree, Unarmed Assault/Robbery || 11415

Asslt-Sec Degree, Unarmed Commercial Robbery || 11415

Asslt-Sec Degree, Unarmed Robbery II 11415

ASSUALT \& ROBBERY || 11415

Att - Theft: Less \$1,000 Value, Robbery || 1A0621

ATT ARM ROBBERY || 20705

ATT ARMED PERSON I| 20705

ATT ARMED CAR JACKING | | 11415

ATT ARMED CARJACKING || 1 A0826

ATT ARMED CARJACKING I| 20705

ATT ARMED ROBBERY || 11415

ATT ARMED ROBBERY || 11420

ATT ARMED ROBBERY | | 1 C0826

ATT ARMED ROBBERY | | 20705

ATT ARMED ROBBERY I | 2A0700

ATT ASSAULT AND ROBBERY || 20700

ATT COMM ARMED ROBB || 20705

ATT COMMERCIAL ROBBERY | | 20700

ATT ROBBERY || 11111

ATT ROBBERY || 11137

ATT ROBBERY || 11415

ATT ROBBERY || 1A0621

ATT ROBBERY || 1A0825

ATT ROBBERY || 1A1202

ATT ROBBERY || 1C0621

ATT ROBBERY || 20700

ATT ROBBERY || 20705

ATT ROBBERY || 2A0705

ATT ROBBERY (ARMED) I। 20705

ATT ROBBERY/ CDS I| 20705

ATT UNARMED ROBBERY || 11415

ATT UNARMED ROBBERY || 20700

ATT UNARMED ROBBERY I | 43550

ATT- ARMED CARJACKING || 1A0826

ATT- ROBBERY || 2A0700

Att- Robbery, Att Robbery I I 2A0700

Att- Robbery, Att- Robbery I| 2A0700

Att- Robbery, Att-Commercial Robbery II 2A0700

Att- Robbery, Home Invasion I| 2A0700

Att- Robbery, Robbery I| 2A0700

ATT-ARMED CARJACKING || 1A0826

ATT-ARMED ROBBERY | | 11420

ATT-ARMED ROBBERY || 1A1202

ATT-ARMED ROBBERY I | 2A0705

Att-Armed Robbery, Att-Armed Robbery II 2A0705

Att-Armed Robbery, Attempt Assault \& Robbery || 2A0705

Att-Armed Robbery, Attempted Robbery II 2A0705

ATT-CARJACKING I।

ATT-CARJACKING || 1A0825

ATT-ROBBERY || 1A0023

ATT-ROBBERY || 1A1299

Att-Robbery, Att-Robbery I| 1A1299

Att-Theft Less Than \$100.00, Att Robbery II 1A0521

ATT. ARMED CARJACKING || 1A0826

ATT. ARMED COMMERCIAL ROBBERY || 10521

ATT. ARMED ROBBERY | | 11415

ATT. ARMED ROBBERY || 20705

ATT. ARMED ROBBERY I| 2A0700

ATT. CAR JACKING || 1A0826

ATT. COMM ARMED ROBBERY || 20705 
ATT. COMMERCIAL ROBBERY || 11415

ATT. ROBBERY || 11415

ATT. ROBBERY || 20700

ATT. ROBERY || 23000

ATT. UNARMED ROBBERY || 11415

ATT. UNARMED ROBBERY | | 20700

ATT.UNARM ROBBERY || 11415

ATTEMPT ARMED ROBBERY I 20700

ATTEMPT ARMED CAR JACKING || 11415

ATTEMPT ARMED CARJACKING I| 1A0826

ATTEMPT ARMED COMMERCIAL ROBBE I| 20700

ATTEMPT ARMED COMMERCIAL ROBBE || 20705

ATTEMPT ARMED ROBBERY || 10521

ATTEMPT ARMED ROBBERY || 11420

ATTEMPT ARMED ROBBERY || 20700

ATTEMPT ARMED ROBBERY || 20705

ATTEMPT ARMED ROBBERY | | 2A0700

ATTEMPT ARMED ROBBERY | | 2A0705

ATTEMPT ARMED ROBERRY | | 20705

ATTEMPT BANK ROBBERY || 20700

ATTEMPT BANK ROBBERY | | 20705

ATTEMPT CAR JACKING || 11313

ATTEMPT CAR JACKING || 1 A0826

ATTEMPT CARJACKING || 20700

ATTEMPT COMM ARMED ROBBERY || 20705

ATTEMPT COMMERCIAL ARMED ROBBE || 1A1137

ATTEMPT COMMERCIAL ARMED ROBBE || 20705

ATTEMPT COMMERCIAL UNARMED ROB || 11415

ATTEMPT COMMERICAL ARMED ROBBE । । 20705

ATTEMPT ROBBERY || 11415

ATTEMPT ROBBERY || 1A0521

ATTEMPT ROBBERY || 1A1136

ATTEMPT ROBBERY || 1A1137

ATTEMPT ROBBERY || 20700

ATTEMPT ROBBERY || 20705

ATTEMPT ROBBERY | | 2A0705

ATTEMPT ROBBERY I| 31005

ATTEMPT ROBBERY || 34025

ATTEMPT ROBBERYH || 11415

ATTEMPT UNARMED ROBBERY | | 11415

ATTEMPT UNARMED ROBBERY | | 20700

ATTEMPT UNARMED ROBBERY I | 2A0700

ATTEMPTED ARMED BANK ROBBERY || 20700

ATTEMPTED ARMED ROBBERY I I 11415

ATTEMPTED ARMED ROBBERY || 11420

ATTEMPTED ARMED ROBBERY || 20705

ATTEMPTED ARMED ROBBERY || 2A0705

ATTEMPTED CAR JACKING | | 1A0826

ATTEMPTED CARJACKING I | 11415

ATTEMPTED COMM ARMED ROBBERY || 20705

ATTEMPTED COMMERCIAL ROBBERY | | 20705

ATTEMPTED COMMERCIAL UNARMED R | | 20705

ATTEMPTED ROBBERY I | 11420

ATTEMPTED ROBBERY | | 11609

ATTEMPTED ROBBERY I | 20700

ATTEMPTED ROBBERY | | 20705

ATTEMPTED ROBBERY || 2A0700

ATTEMPTED UNARMED ROBBERY || 20700

ATTEMPTED UNARMED ROBBERY | | 2A0700

ATTEMTED ARMED ROBBERY || 11202 
ATTEPMPTED UNARMED ROBBERY || 2A0700

BANK ROBBERY || 20700

BANK ROBBERY || 20705

Burglary-First Degree, Armed Robbery I| 23000

CAR JACKING || 10825

CAR JACKING || 10826

CAR JACKING || 11136

CAR JACKING | | 11420

CAR JACKING || 20480

CAR JACKING || 20705

CAR-JACKING || 20700

CARJACKING || 10622

CARJACKING || 10623

CARJACKING || 10825

CARJACKING I| 10826

CARJACKING || 11420

CARJACKING || 1A0825

CARJACKING II 20480

CARJACKING || 20705

CARJACKING || 43550

Carjacking, Armed Car Jacking I| 10825

Carjacking, Armed Carjacking I| 10825

Carjacking, Carjacking II 10825

CARJACKING/ROBBERY W/DEADLY WE || 10826

CDS I| 20705

CDS DISTROBUTION || 2A0696

CDS/ROBBERY || 43550

COM ARMED ROBBERY || 20705

COM. ARMED ROBBERY | | 20705

COMM ARMED ROBBERY | | 11415

COMM ARMED ROBBERY || 20705

COMM ROBBERY I| 20705

COMM. ARMED ROBBERY || 20700

COMM. ARMED ROBBERY || 20705

COMM. ARMED ROBERY || 20705

COMMERCIAL AND ROBBERY | | 20705

COMMERCIAL ARMED ROBBERY | | 10175

COMMERCIAL ARMED ROBBERY | | 10521

COMMERCIAL ARMED ROBBERY || 10621

COMMERCIAL ARMED ROBBERY | | 10692

COMMERCIAL ARMED ROBBERY | | 10826

COMMERCIAL ARMED ROBBERY | | 11415

COMMERCIAL ARMED ROBBERY || 1F0521

COMMERCIAL ARMED ROBBERY I | 20700

COMMERCIAL ARMED ROBBERY || 20705

COMMERCIAL ARMED ROBBERY || 23010

COMMERCIAL ARMED ROBBERY || 43550

COMMERCIAL ROBBERY | | 10521

COMMERCIAL ROBBERY || 10621

COMMERCIAL ROBBERY || 11137

COMMERCIAL ROBBERY | | 11415

COMMERCIAL ROBBERY | | 20700

COMMERCIAL ROBBERY || 20705

COMMERCIAL UNARED ROBBERY || 11415

COMMERCIAL UNARMED ROBBERY | | 10826

COMMERCIAL UNARMED ROBBERY | | 11415

COMMERCIAL UNARMED ROBBERY || 20700

COMMERCIAL UNARMED ROBBERY | | 20705

COMMERCILA ARMED ROBBERY | | 20705

COMMERIAL ARMED ROBBERY || 20705 
COMMERICAL ARM ROBBERY || 20705

COMMERICAL ARMED ROBBERY || 11415

COMMERICAL ARMED ROBBERY I | 15299

COMMERICAL ARMED ROBBERY | | 20705

COMMERICAL ATT ARMED ROBBERY || 20705

COMMERICAL ROBBERY || 10521

COMMERICAL ROBBERY | | 11415

COMMERICAL ROBBERY | | 20705

COMMERICAL UNARMED ROBBERY || 11415

COMMISSION ROBBERY/BURGLARY || 23050

COMMON ASSAULT II 20705

COMMON ROBERY || 20705

Con- Theft: Less $\$ 1,000$ Value, Armed Robbery || 1C0621

Con- Theft: Less $\$ 1,000$ Value, Robbery II 1C0621

CON-ARMED CARJACKING | | 1C0826

Con-Armed Robbery II

CON-ARMED ROBBERY || $1 \mathrm{C} 1202$

CON-ARMED ROBBERY || 2C0705

Con-Armed Robbery, Armed Robbery I I 2C0705

Con-Armed Robbery, Attempt Armed Robbery II 2C0705

Con-Armed Robbery, Con-Armed Robbery II 1 C1202

Con-Armed Robbery, Con-Armed Robbery II 2C0705

Con-Armed Robbery, Robbery II 2C0705

Con-Burglary-4Th Degree Theft, Armed Robbery I| 2C3040

CON-ROBBERY I| 1C0023

CON-ROBBERY I| 1C1299

CON-ROBBERY || 2C0700

Con-Robbery, Unarmed Robbery I| 2C0700

CON. ARMED CARJACKING I| 10826

CON.ARMED ROBERY || 20705

CON.UNARMED ROBBERY || 11415

CONSP. ARMED ROBBERY || 20705

CONSPIRACY ARMED ROBBERY || 10621

CONSPIRACY TO ROB WITH DEADLY | | 10521

CONSPIRACY-ASSAULT AND ROBBER || 20700

Conspiracy, Armed Robbery II 17399

Deadly Weapon-Conceal, Armed Assault And Robbery I| 15202

Deadly Weapon-Conceal, Armmed Robbery II 15202

DOMESTIC ASSAULT \& ROBBERY || 11415

DOMESTIC ASSAULT AND ROBBERY | | 11415

FAILURE TO FOLLOW PROBATON II

False Imprisonment, Unarmed Robbery | | 10042

FIRST DEGREE ASSAULT || 20705

Handgun In Vehicle, Armed Robbery II 10175

Handgun On Person, Armed Robbery I| 15212

HGV || 20705

Hgv Use/Fel-Viol Crime, Armed Robbery || 15299

Hgv Use/Fel-Viol Crime, Robbery II 15299

HOME INVASION || 20705

Kidnapping, Robbery || 31005

LARCENY I| 20705

Mal Destr Prop Value + \$500, Unarmed Robbery II 34030

Motor Veh/Unlawful Taking, Armed Carjacking II 20480

Reg Firearm:Illegal Possession, Armed Robbery || 11106

Robbery $1 \mathrm{I}$

ROBBERY II

ROBBERY || 10023

ROBBERY || 10521

ROBBERY || 10621

ROBBERY || 10825 


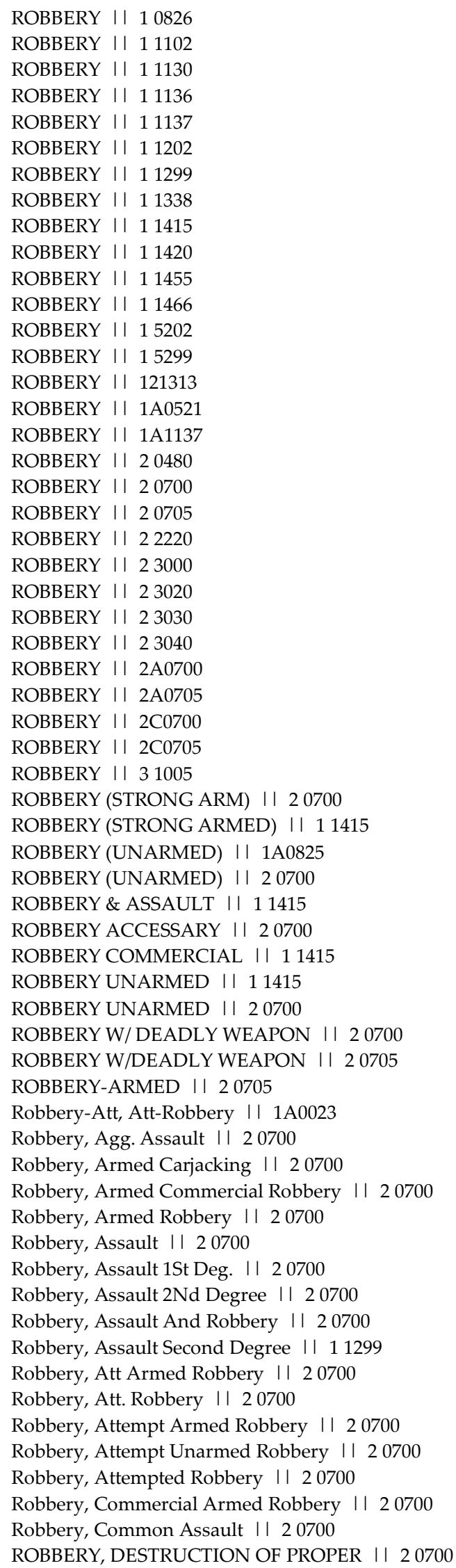


Robbery, Home Invasion I I 20700

Robbery, Larceny || 20700

Robbery, Robbery I| 10023

Robbery, Robbery II 11299

Robbery, Robbery I| 20700

Robbery, Roberry I| 20700

Robbery, Theft I| 20700

Robbery, Un Armed Robbery I I 20700

Robbery, Unarm Robbery I| 20700

Robbery, Unarmed Bank Robbery II 20700

Robbery, Unarmed Carjacking I| 20700

Robbery, Unarmed Commercial Robbery I| 20700

Robbery, Unarmed Robbery I| 20700

Robbery, Unarmed Robbery And Assault I| 20700

ROBBERY/THEFT I I 11130

ROBBERY/AGG ASSAULT । | 20700

ROBBERY/ASLT I| 20705

ROBBERY/ASSAULT I| 20700

ROBERRY || 11415

ROBERY || 20705

SECOND DEGREE ASSAULT/ ROBBERY | | 11415

SODOMY/ARMED ROBBER || 21102

STOLEN AUTO || 20705

STOLEN AUTO/ARMED CARJACKING || 10826

STOLEN AUTO/CARJACKING | | 11106

STRONG ARM ROBBERY || 11415

STRONG ARM ROBBERY || 20700

THEFT ।| 20705

THEFT $<\$ 1000$ || 20705

Theft Less Than $\$ 100.00$, Robbery | | 10521

Theft Less Than \$100.00, Unarmed Comm Robbery II 10521

Theft Less Than \$100.00, Unarmed Commercial Robbery II 10521

Theft Less Than \$100.00, Unarmed Commerical Robbery II 10521

THEFT OVER $\$ 1,000.00$ || 20705

Theft: \$1,000 To Under \$10,000, Unarmed Robbery || 10622

Theft: Less \$1,000 Value, Armed Robbery I| 10621

Theft: Less \$1,000 Value, Assault \& Robbery II 10621

Theft: Less \$1,000 Value, Assault Armed Robbery II 10621

Theft: Less \$1,000 Value, Commercial Robbery || 10621

Theft: Less \$1,000 Value, Robbery || 10621

Theft: Less \$1,000 Value, Unarmed Robbery || 10621

U/A ROBBERY || 20700

UARMED ROBBERY I 20700

UN ARMED ROBBERY I| 11415

UN ARMED ROBBERY || 20700

UN-ARMED ROBBERY || 20700

UNAMRED ROBBERY | | 10621

UNARM ROBBERY || 11415

UNARM ROBBERY || 20700

UNARMED ASSAULT \& ROBBERY || 10621

UNARMED ASSAULT \& ROBBERY || 11415

UNARMED ASSAULT \& ROBBERY || 20700

UNARMED ASSAULT AND ROBBERY || 11420

UNARMED ASSAULT AND ROBBERY | | 20700

UNARMED CAR JACKING || 10825

UNARMED CARJACKING || 10825

UNARMED CARJACKING || 11415

UNARMED COMM. ROBBERY | | 11415

UNARMED COMMERCIAL ROBBERY | | 11415

UNARMED COMMERCIAL ROBBERY || 23090 


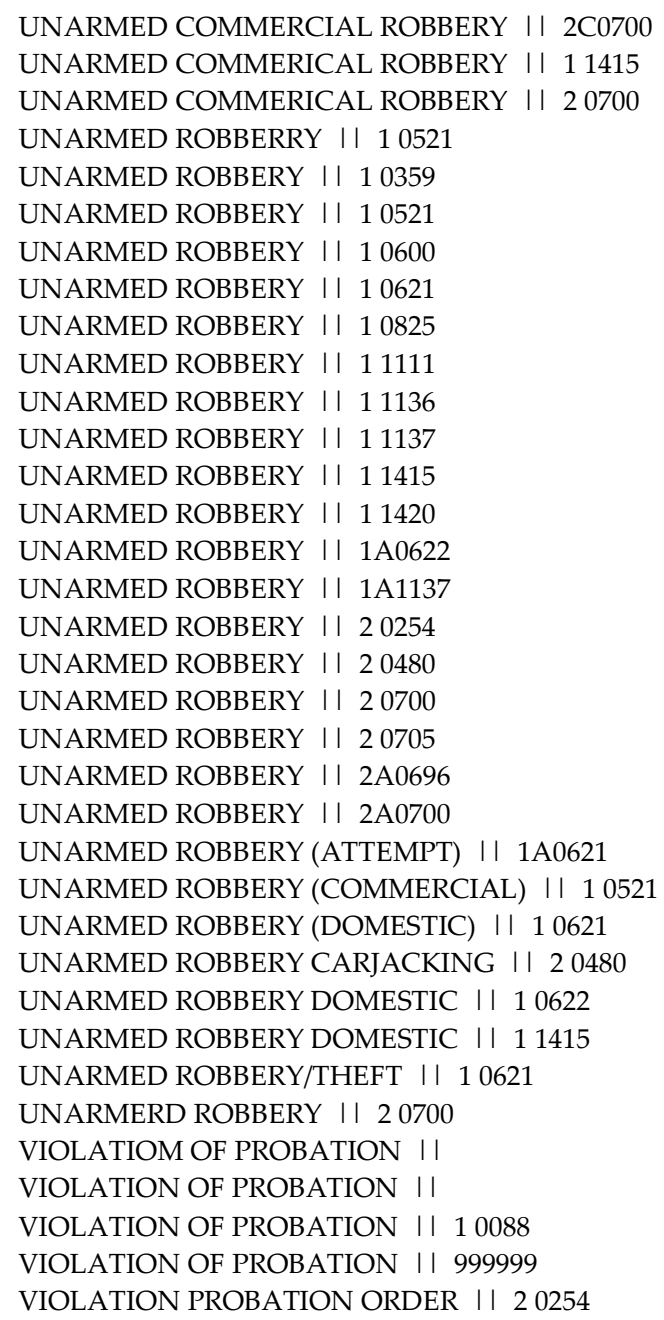

\section{Aggravated Assault}

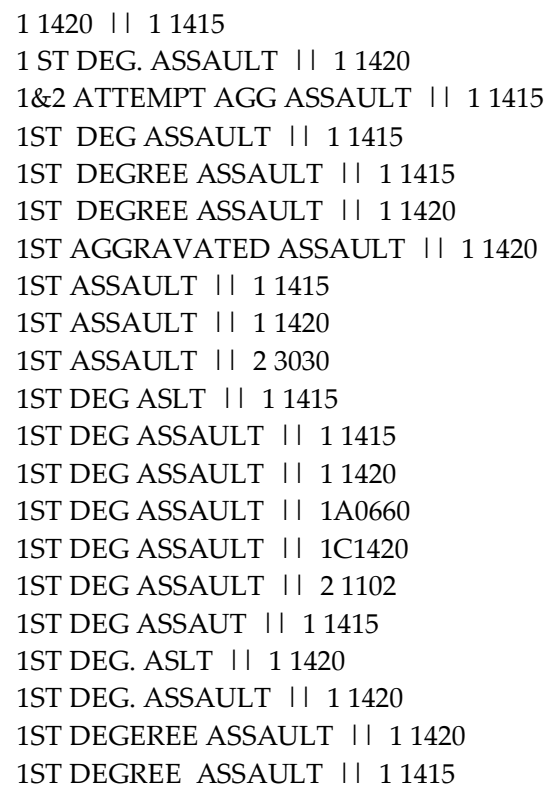




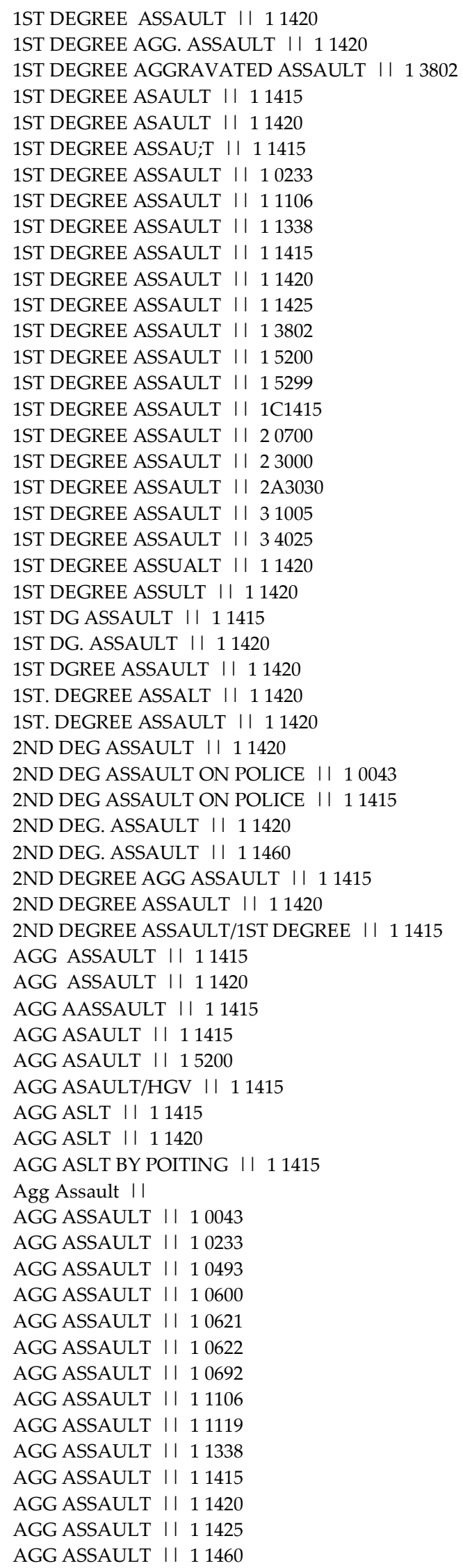


AGG ASSAULT || 11564

AGG ASSAULT || 11609

AGG ASSAULT || 13802

AGG ASSAULT || 15200

AGG ASSAULT || 15202

AGG ASSAULT || 15212

AGG ASSAULT || 15299

AGG ASSAULT || 16505

AGG ASSAULT || 20105

AGG ASSAULT | | 20238

AGG ASSAULT | | 20254

AGG ASSAULT | | 20700

AGG ASSAULT || 21102

AGG ASSAULT || 22220

AGG ASSAULT || 23000

AGG ASSAULT || 23030

AGG ASSAULT || 23090

AGG ASSAULT || 25200

AGG ASSAULT || 25212

AGG ASSAULT || 31005

AGG ASSAULT | | 35399

AGG ASSAULT - CUTTING || 13802

AGG ASSAULT (CUTTING) || 11420

AGG ASSAULT (DV) || 11415

AGG ASSAULT (POINTING) | | 11609

AGG ASSAULT (STABBING) I। 11420

AGG ASSAULT / 1ST DEG ASSAULT || 11415

AGG ASSAULT / SELF INFLICTED S | | 11425

AGG ASSAULT 1ST DEG | | 11420

AGG ASSAULT 1ST DEGREE I |

AGG ASSAULT 1ST DEGREE | | 10439

AGG ASSAULT 1ST DEGREE | | 11415

AGG ASSAULT 1ST DEGREE | | 11420

AGG ASSAULT 2ND || 11415

AGG ASSAULT 2ND DEGREE | | 11415

AGG ASSAULT BY CUTING | | 11420

AGG ASSAULT BY CUTTING | | 11415

AGG ASSAULT BY CUTTING || 11420

AGG ASSAULT BY DEADLY WEAPON || 11415

AGG ASSAULT BY POINTING । |

AGG ASSAULT BY POINTING | | 10692

AGG ASSAULT BY POINTING || 11106

AGG ASSAULT BY POINTING || 11415

AGG ASSAULT BY POINTING I | 11420

AGG ASSAULT BY POINTING || 15299

AGG ASSAULT BY POINTING || 30233

AGG ASSAULT BY SHOOTING AT I | 11420

AGG ASSAULT BY STABBING | | 11415

AGG ASSAULT BY STABBING || 11420

AGG ASSAULT BY THREAT | | 11415

AGG ASSAULT BY VEHICHLE | | 11415

AGG ASSAULT DOMESTIC | | 11415

AGG ASSAULT DOMESTIC || 16503

AGG ASSAULT ON POLICE || 11415

AGG ASSAULT POINTING || 11415

AGG ASSAULT VIA THREAT | | 11415

AGG ASSAULT WITH A DEADLY WEAP || 11415

AGG ASSAULT. II 11420

AGG ASSAULT/ 1ST DEGREE | | 11415

AGG ASSAULT/ HGV || 11119 
AGG ASSAULT/1ST DEGREE ASSAULT | | 11415

AGG ASSAULT/CDS || 11420

AGG ASSAULT/HGV || 11420

AGG ASSSAULT || 11420

AGG ASSUALT || 11420

AGG ASSULT || 11420

AGG ASUALT BY POINTING | | 11415

AGG DOMESTIC ASSAULT || 11415

AGG- ASSAULT || 20700

AGG-ASSAULT | | 11415

AGG, ASSAULT || 11415

AGG. ASSAULT || 11415

AGG. ASSALUT BY VEHICLE || 11420

AGG. ASSAULT || 10439

AGG. ASSAULT || 10825

AGG. ASSAULT || 11285

AGG. ASSAULT || 11338

AGG. ASSAULT || 11415

AGG. ASSAULT || 11416

AGG. ASSAULT || 11420

AGG. ASSAULT || 11425

AGG. ASSAULT || 11564

AGG. ASSAULT || 15200

AGG. ASSAULT || 15202

AGG. ASSAULT || 15299

AGG. ASSAULT || 1C1420

AGG. ASSAULT || 20700

AGG. ASSAULT || 25200

AGG. ASSAULT || 2A0700

AGG. ASSAULT || 2C0700

AGG. ASSAULT || 30233

AGG. ASSAULT || 35399

AGG. ASSAULT (CUTTING) | | 11415

AGG. ASSAULT BY CUTTING | | 11415

AGG. ASSAULT BY CUTTING || 11420

AGG. ASSAULT BY POINTING | | 11415

AGG. ASSAULT BY POINTING || 11420

AGG. ASSAULT BY SHOOTING || 11420

AGG. ASSAULT BY SHOOTING AT । | 11420

AGG. ASSAULT BY THREAT | | 11415

AGG. ASSAULT CUTTING | | 11420

AGG. ASSAULT ON POLICE || 11415

AGG. ASSAULT ON POLICE || 11420

AGG. ASSAULT W/ GUN || 12801

AGG. ASSAULT/DES OF PROPORTY || 11415

AGG. ASSUALT || 11420

AGG. ASSULT || 11420

AGG.ASAULT BY POINTING | | 11415

Agg.Assault II

AGG.ASSAULT || 10487

AGG.ASSAULT || 11415

AGG.ASSAULT I| 11420

AGG.ASSAULT || 15200

AGG.ASSAULT BY CUTTING | | 11415

AGG.ASSAULT BY POINTING | | 11420

AGG/ASSAULT | | 11415

AGG/ASSAULT || 11420

AGG/ASSAULT (DV) I | 11415

AGGARTED ASSAULT | | 11415

AGGARVATED ASSAULT || 11338 
AGGARVATED ASSAULT || 11415

AGGARVATED ASSAULT || 11420

AGGASSAULT | | 11415

AGGAVATED ASSAULT | | 11415

AGGERVATED ASSAULT | | 11415

AGGERVATED ASSAULT | 11420

AGGG ASSAULT I | 11415

AGGR ASSAULT I| 11415

AGGRATED ASSAULT || 11415

AGGRATED ASSUALT BY CUTTING || 11420

AGGRAV ASSAULT DV || 11415

AGGRAV ATED ASSAULT | | 11415

AGGRAVAED ASSAULT || 11420

AGGRAVATE ASSAULT । 11415

AGGRAVATE ASSAULT | 11420

AGGRAVATE ASSAULT-CUTTING || 11415

AGGRAVATED || 11420

AGGRAVATED ASSAULT | 11415

AGGRAVATED AASSAULT || 11415

AGGRAVATED ASAAULT || 11420

AGGRAVATED ASASULT || 11420

AGGRAVATED ASLT | 11420

AGGRAVATED ASSAUIT || 11415

AGGRAVATED ASSAUKT | | 11420

AGGRAVATED ASSAUL | | 11415

AGGRAVATED ASSAULLT || 15212

Aggravated Assault II

AGGRAVATED ASSAULT | |

AGGRAVATED ASSAULT | | 10043

AGGRAVATED ASSAULT || 10173

AGGRAVATED ASSAULT | | 10334

AGGRAVATED ASSAULT | | 10493

AGGRAVATED ASSAULT I | 10600

AGGRAVATED ASSAULT | | 10692

AGGRAVATED ASSAULT || 10990

AGGRAVATED ASSAULT | 11111

AGGRAVATED ASSAULT | | 11338

AGGRAVATED ASSAULT || 11415

AGGRAVATED ASSAULT || 11420

AGGRAVATED ASSAULT | | 11425

AGGRAVATED ASSAULT | | 11609

AGGRAVATED ASSAULT | | 11661

AGGRAVATED ASSAULT | | 12903

AGGRAVATED ASSAULT | | 13605

AGGRAVATED ASSAULT | | 13802

AGGRAVATED ASSAULT || 15200

AGGRAVATED ASSAULT | | 15202

AGGRAVATED ASSAULT || 15212

AGGRAVATED ASSAULT || 15299

AGGRAVATED ASSAULT | | 1A0521

AGGRAVATED ASSAULT | | 1A1137

AGGRAVATED ASSAULT I| $1 \mathrm{C1420}$

AGGRAVATED ASSAULT || 20254

AGGRAVATED ASSAULT || 20350

AGGRAVATED ASSAULT | | 20480

AGGRAVATED ASSAULT || 21120

AGGRAVATED ASSAULT || 23020

AGGRAVATED ASSAULT || 23030

AGGRAVATED ASSAULT || 23090

AGGRAVATED ASSAULT || 2A0700 
AGGRAVATED ASSAULT || 2A3000

AGGRAVATED ASSAULT || 30233

AGGRAVATED ASSAULT || 31005

AGGRAVATED ASSAULT | | 34025

AGGRAVATED ASSAULT | | 34030

AGGRAVATED ASSAULT || 43550

AGGRAVATED ASSAULT (CUTTING) | | 11415

AGGRAVATED ASSAULT (DV) | | 11415

AGGRAVATED ASSAULT /DEADLY WEA || 11420

AGGRAVATED ASSAULT BY CUTTING || 11415

AGGRAVATED ASSAULT BY CUTTING | | 11420

AGGRAVATED ASSAULT BY CUTTING || 34030

AGGRAVATED ASSAULT BY POINTING || 10175

AGGRAVATED ASSAULT BY POINTING || 11415

AGGRAVATED ASSAULT BY POINTING || 11420

AGGRAVATED ASSAULT BY POINTING || 11610

AGGRAVATED ASSAULT BY POINTING || 15299

AGGRAVATED ASSAULT BY SHOOTING I| 11420

AGGRAVATED ASSAULT BY STABBING || 11420

AGGRAVATED ASSAULT BY THREAT || 10621

AGGRAVATED ASSAULT BY THREAT || 11415

AGGRAVATED ASSAULT BY THREAT I | 11420

AGGRAVATED ASSAULT CUTTING || 11420

AGGRAVATED ASSAULT ON POLICE || 11415

AGGRAVATED ASSAULT ON POLICE || 11420

AGGRAVATED ASSAULT. I I 11420

AGGRAVATED ASSAULT/CDS || 11415

AGGRAVATED ASSAULY || 11415

AGGRAVATED ASSAUT || 11415

AGGRAVATED ASSUALT || 11420

AGGRAVATED ASSULT BY POINTING || 11420

AGGRAVATED SSAULT || 15200

AGGRAVAVTED ASSAULT I| 11415

AGGRAVET ASSAULT - CUTTING || 11415

AGGRAVETD ASSAULT || 11420

AGGRAVETED ASSAULT || 11415

AGGRAVETED ASSAULT || 11420

AGGRAVTED ASSAULT || 11415

AGGRAVTED ASSAULT || 11420

AGGREGATE ASSAULT | | 11415

AGGREGATED ASSAULT | | 11420

AGGREGATED ASSAULT | | 15200

AGGRESSIVE ASSAULT | | 11415

AGGREVATED ASSAULT | | 11415

AGGREVATED ASSAULT | | 11420

AGGRIVATED ASSAULT || 11415

AGGRIVATED ASSAULT || 11420

AGGRIVATED ASSAULT BY CUTTING || 11420

AGGRVATED ASSAULT || 10521

AGGRVATED ASSAULT || 11420

AGRAVATED ASSAULT || 11420

AGRRAVATED ASSAULT | 11420

AHH ASSUALT CUTTING || 11420

Alc. Bev./Intox:Endanger, Agg Assault || 14200

Alc. Bev./Intox:Endanger, Agg. Assault || 14200

Alc. Bev./Intox:Endanger, Aggravated Assault || 14200

ASLT I| 11420

ASLT ON POLICE || 11415

ASSALT 1ST DEGREE I| 11420

ASSAULT || 11420 


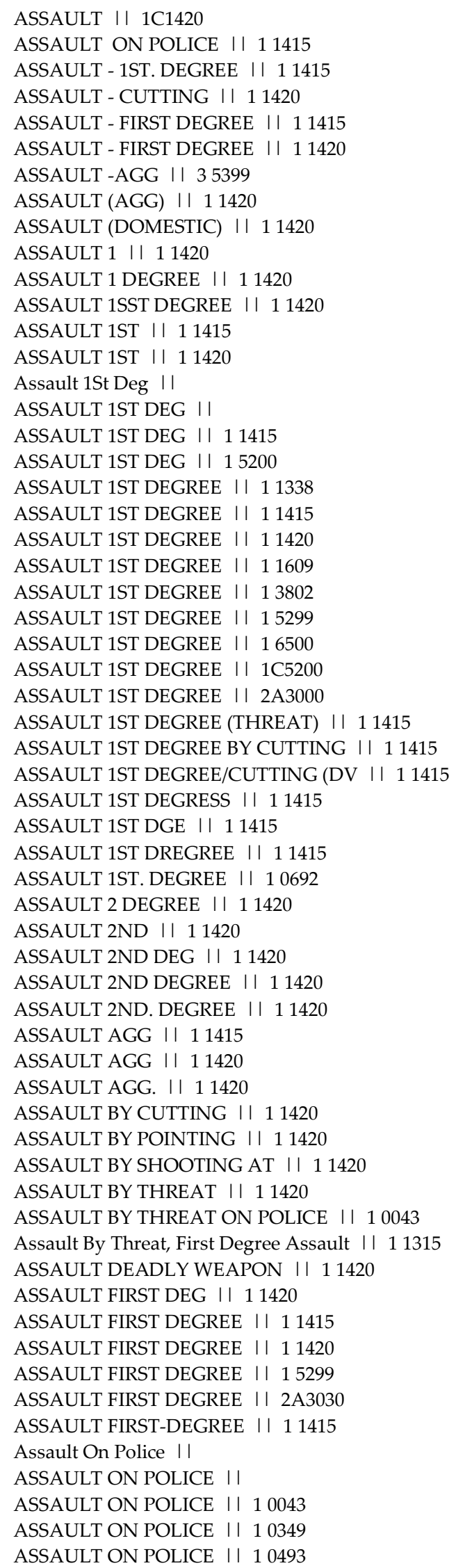




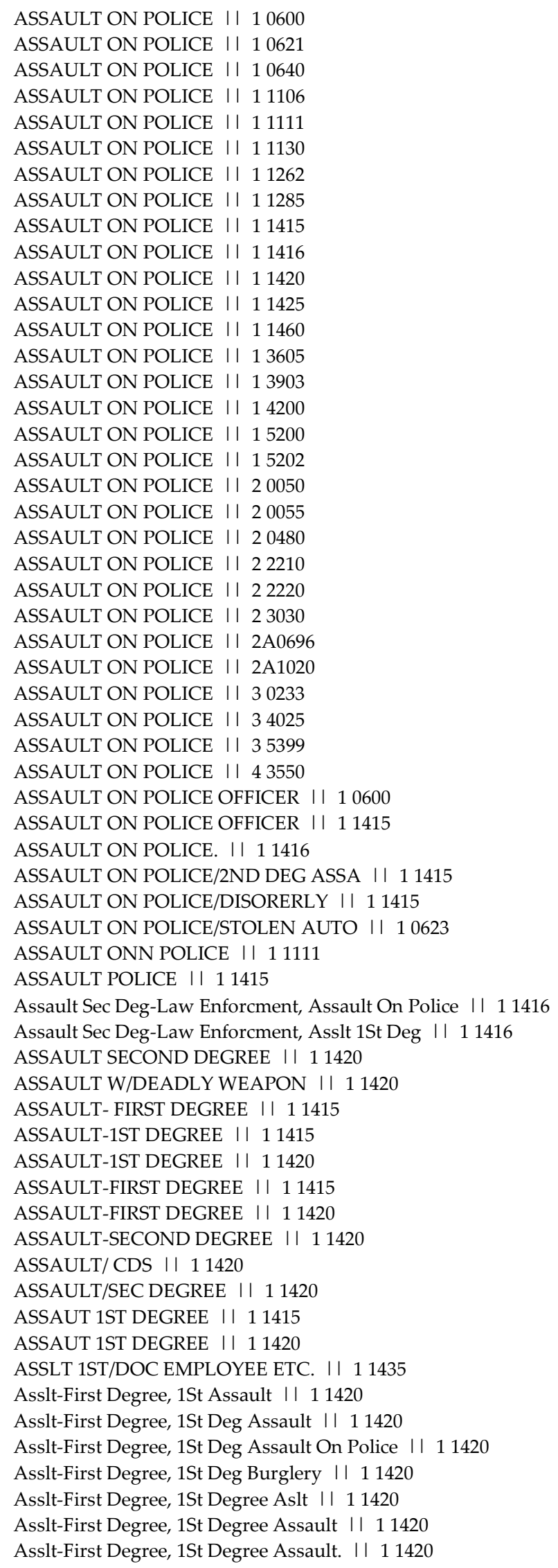


Asslt-First Degree, 1St Degree Burglary || 11420

Asslt-First Degree, 1St Degree Child Assault/Abuse || 11420

Asslt-First Degree, 2Nd Degree Assault || 11420

Asslt-First Degree, 2Nd Deg Assault || 11420

Asslt-First Degree, 2Nd Degree Assault I| 11420

Asslt-First Degree, Agg Asaault || 11420

Asslt-First Degree, Agg Assault II 11420

Asslt-First Degree, Agg Assault (1St Degree) II 11420

Asslt-First Degree, Agg Assault By Cutting II 11420

Asslt-First Degree, Agg Assault By Pointing || 11420

Asslt-First Degree, Agg Assault Pointing || 11420

Asslt-First Degree, Agg Assault(Cutting) I| 11420

Asslt-First Degree, Agg Assault/Cutting || 11420

Asslt-First Degree, Agg Asssault || 11420

Asslt-First Degree, Agg Assualt I| 11420

Asslt-First Degree, Agg, Assault I| 11420

Asslt-First Degree, Agg. Assault || 11420

Asslt-First Degree, Agg. Assault || 11420

Asslt-First Degree, Agg. Assault By Pointing II 11420

Asslt-First Degree, Agg. Assault By Stabbing II 11420

Asslt-First Degree, Agg. Assault On Police II 11420

Asslt-First Degree, Agg.Assault || 11420

Asslt-First Degree, Aggravated Assault I| 11420

Asslt-First Degree, Aggravated Aslt II 11420

Asslt-First Degree, Aggravated Assalt || 11420

Asslt-First Degree, Aggravated Assault || 11420

Asslt-First Degree, Aggravated Assault By Cutting II 11420

Asslt-First Degree, Aggregated Assault || 11420

Asslt-First Degree, Aggrevated Assault || 11420

Asslt-First Degree, Aggrivated Assault || 11420

Asslt-First Degree, Assault || 11420

Asslt-First Degree, Assault 1St Deg || 11420

Asslt-First Degree, Assault 1St Deg. II 11420

Asslt-First Degree, Assault 1St Degree II 11420

Asslt-First Degree, Assault 2Nd Degree || 11420

Asslt-First Degree, Assault Agg || 11420

Asslt-First Degree, Assault By Pointing At || 11420

Asslt-First Degree, Assault First Deg I| 11420

Asslt-First Degree, Assault First Degree || 11420

Asslt-First Degree, Assault On Police II 11420

Asslt-First Degree, Assault Sec Degree || 11420

Asslt-First Degree, Assault-1St Deg || 11420

Asslt-First Degree, Assault-First Degree I| 11420

Asslt-First Degree, Assault-Sec Degree I| 11420

Asslt-First Degree, Assault, 1St Degree I| 11420

Asslt-First Degree, Attemt. Agg Assault || 11420

Asslt-First Degree, Common Assault I| 11420

Asslt-First Degree, Cutting || 11420

Asslt-First Degree, Dest Of Property || 11420

Asslt-First Degree, Felony Possession Of A Handgun I| 11420

Asslt-First Degree, First Degree Assault || 11420

Asslt-First Degree, First Degree Assault || 11420

Asslt-First Degree, Frist Degree Assault || 11420

Asslt-First Degree, Handgun Violation I| 11420

Asslt-First Degree, Hgv II 11420

Asslt-First Degree, Ist Deg Assault I| 11420

Asslt-First Degree, Ist Degree Assault || 11420

Asslt-First Degree, Physical Child Abuse || 11420

Asslt-First Degree, Poss Heroin II 11420

Asslt-First Degree, Second Degree Assault || 11420 
Asslt-First Degree, Stalking || 11420

Asslt-Sec Degree, 11420 || 11415

Asslt-Sec Degree, 1St And 2Nd Degree Assault || 11415

Asslt-Sec Degree, 1St Assault I| 11415

Asslt-Sec Degree, 1St Deg Aslt I| 11415

Asslt-Sec Degree, 1St Deg Assault || 11415

Asslt-Sec Degree, 1St Degree Assault I | 11415

Asslt-Sec Degree, Agg Assault || 11415

Asslt-Sec Degree, Agg Assault Attempt II 11415

Asslt-Sec Degree, Agg Assault By Cutting || 11415

Asslt-Sec Degree, Agg Assault Domestic I| 11415

Asslt-Sec Degree, Agg Asslt I I 11415

Asslt-Sec Degree, Agg Assualt I| 11415

Asslt-Sec Degree, Agg Assualt/ Pan Handling II 11415

Asslt-Sec Degree, Agg Assult I| 11415

Asslt-Sec Degree, Agg. Assault II 11415

Asslt-Sec Degree, Agg. Aslt || 11415

Asslt-Sec Degree, Agg. Assault || 11415

Asslt-Sec Degree, Agg. Assault/Cutting II 11415

Asslt-Sec Degree, Agg. Assault By Cutting II 11415

Asslt-Sec Degree, Agg. Assault Knife II 11415

Asslt-Sec Degree, Agg. Assualt I| 11415

Asslt-Sec Degree, Agg.Assault || 11415

Asslt-Sec Degree, Aggravated Asault | | 11415

Asslt-Sec Degree, Aggravated Assault II 11415

Asslt-Sec Degree, Aggravated Assault On Police I| 11415

Asslt-Sec Degree, Aggravated Assauly II 11415

Asslt-Sec Degree, Aggravated Asslt I| 11415

Asslt-Sec Degree, Aggravated Assualt I| 11415

Asslt-Sec Degree, Aggravated Assult || 11415

Asslt-Sec Degree, Aggrevated Assault I| 11415

Asslt-Sec Degree, Aggrevated Assult || 11415

Asslt-Sec Degree, Aggrivated Assault I| 11415

Asslt-Sec Degree, Aggrvated Assault || 11415

Asslt-Sec Degree, Aslt 1St Degree I| 11415

Asslt-Sec Degree, Aslt On Police II 11415

Asslt-Sec Degree, Aslt/Police || 11415

Asslt-Sec Degree, Assault On Police I| 11415

Asslt-Sec Degree, Assault - 1St Degree || 11415

Asslt-Sec Degree, Assault 1St || 11415

Asslt-Sec Degree, Assault 1St Deg || 11415

Asslt-Sec Degree, Assault 1St Degree || 11415

Asslt-Sec Degree, Assault 1St Dgr || 11415

Asslt-Sec Degree, Assault 1St. Degree I| 11415

Asslt-Sec Degree, Assault 2Nd Degree On Police II 11415

Asslt-Sec Degree, Assault Agg | | 11415

Asslt-Sec Degree, Assault First Deg | 11415

Asslt-Sec Degree, Assault First Degree I| 11415

Asslt-Sec Degree, Assault On Police I| 11415

Asslt-Sec Degree, Assault On Police Officer || 11415

Asslt-Sec Degree, Assault On Police/2Nd Degree A I| 11415

Asslt-Sec Degree, Assault-First Degree || 11415

Asslt-Sec Degree, Assault/Police II 11415

Asslt-Sec Degree, Assualt On Police I| 11415

Asslt-Sec Degree, Assult On Police Officer I| 11415

Asslt-Sec Degree, Att Agg Assault | | 11415

Asslt-Sec Degree, Att Agg. Assault || 11415

Asslt-Sec Degree, Att Agg.Assault | | 11415

Asslt-Sec Degree, Att. 1St Degree Assault II 11415

Asslt-Sec Degree, Att. Agg. Assault I| 11415 
Asslt-Sec Degree, Att. Aggravated Assault I| 11415

Asslt-Sec Degree, Attempt Agg Assault || 11415

Asslt-Sec Degree, Attempt Aggravated Assault I| 11415

Asslt-Sec Degree, Attempted Agg Assault I| 11415

Asslt-Sec Degree, Attempted Aggravated Assault II 11415

Asslt-Sec Degree, Cds/Assault On Police II 11415

Asslt-Sec Degree, Common Assault On Police II 11415

Asslt-Sec Degree, Cutting - Agg Assault I| 11415

Asslt-Sec Degree, Cutting-Agg | 11415

Asslt-Sec Degree, Domestic Agg Assault II 11415

Asslt-Sec Degree, Domestic Aggravated Assault || 11415

Asslt-Sec Degree, First Degree Assault I| 11415

Asslt-Sec Degree, Second Degree Assault On Polic || 11415

ASSUALT || 11420

ATT 1ST DEG ASSAULT I I 11415

ATT AGG ASSAULT || 11415

ATT AGG ASSAULT ।| 15200

ATT AGGRAVATED ASSAULT | | 11415

ATT AGGRAVATED ASSAULT | | 11420

ATT AGGRAVATED ASSAULT || 15200

ATT DIST/ ASSAULT ON POLICE I| 2A3550

ATT-ASSAULT-FIRST DEGREE I I 1A1420

Att-Asslt-First Degree, Att-Assault-First Degree II 1A1420

Att-Burglary-Fourth Degree, Agg. Assault || 2A3030

Att-Prostitution-General, Agg Assault || 1A1093

ATT. AGG. ASSAULT || 11415

ATTEMP AGG. ASSAULT || 11415

ATTEMPT AGG ASSAULT || 10042

ATTEMPT AGG ASSAULT || 11415

ATTEMPT AGG ASSAULT || 11420

ATTEMPT AGG ASSAULT || 15200

ATTEMPT AGG. ASSAULT || 11415

ATTEMPT AGG. ASSAULT I 11420

ATTEMPT AGGRAVATED ASSAULT I| 11415

ATTEMPT AGGRAVATED ASSAULT || 11420

ATTEMPT AGGRAVATTED ASSAULT | | 11415

ATTEMPT ASSAULT ON POLICE I| 11415

ATTEMPTED AGG ASSAULT || 11415

ATTEMPTED AGG. ASSAULT || 15200

ATTEMT AGG. ASSAULT || 11415

Burglary-First Degree, 1St Degree Assault || 23000

Burglary-First Degree, Agg. Assault || 23000

Burglary-First Degree, Assault I| 23000

Burglary-Fourth Degree, Aggravated Assault I| 23030

Burglary-Fourth Degree, Assault On Police I| 23030

CDS I| 11420

CDS POSS I| 11460

CDS POSSESSION || 11460

CDS VIOLATION || 11420

CDS VIOLATION || 11460

Cds: Poss Marihuana L/T 10 G, Cds/ Assault On Police II 11635

Cds: Possession-Marihuana, Assault On Police II 10573

Cds: Possession-Marihuana, Cds Poss/Assault On Polic || 10573

Cds:P W/I Dist:Narc, Assault On Police I| 30233

Cds:Possess-Not Marihuana, Aggravated Assault II 43550

Cds:Possess-Not Marihuana, Assault On Police I| 43550

Cds:Possess-Not Marihuana, Cds Poss./Assault On Poli || 43550

Cds:Possess-Not Marihuana, Cds/Assault On Police II 43550

CDS/ASSAULT ON POLICE I| 11415

CHILD ABUSE || 11420 
CHILD ABUSE PHYSICAL || 11420

Child Abuse:Parent, Agg. Assault || 13802

Child Abuse:Parent, Aggrevated Assault || 13802

Child Abuse:Parent, Assault 1St Degree || 13802

COMMON ASSAULT || 11420

COMMON ASSAULT || 11460

COMMON ASSAULT I| 1A1420

COMMON ASSAULT (DV) I 11420

COMMON ASSAULT (POLICE) | | 11415

COMMON ASSAULT 2ED DEGREE I | 11420

COMMON ASSULT I| 11420

CON-ASSAULT-FIRST DEGREE || 1 C1420

Con-Asslt-First Degree, Con-Assault-First Degree I| 1 C1420

CUTTING || 11420

DEADLY WEAPON || 11420

Deadly Weapon-Conceal, Aggraveted Assault || 15202

Deadly Weapon-Int/Injure, 1St Degree Assault || 15200

Deadly Weapon-Int/Injure, Agg Assault I| 15200

Deadly Weapon-Int/Injure, Agg. Assault || 15200

Deadly Weapon-Int/Injure, Agg. Assault 1St Degree || 15200

Deadly Weapon-Int/Injure, Aggravated Assault || 15200

Deadly Weapon-Int/Injure, Aggrevated Assault I| 15200

Deadly Weapon-Int/Injure, Aggrivated Assault I| 15200

Deadly Weapon-Int/Injure, Assault 1St Degree I| 15200

Deadly Weapon-Int/Injure, Assault Agg I| 15200

Deadly Weapon-Int/Injure, Assault First Degree I| 15200

Deadly Weapon-Int/Injure, Att. Agg Assault || 15200

Deadly Weapon-Int/Injure, Domestic Agg Assault || 15200

Deadly Weapon-Int/Injure, First Degree Assault I| 15200

DEST OF PROPERTY I 11420

DEST OF PROPERTY/AGG. ASSAULT || 11415

Dis.Erly Conduct, Aggravated Assault || 20050

Dis.Erly Conduct, Assault On Police I| 20050

Dis.Erly Conduct, Common Aslt. On Police I| 20050

DISARM LAW OFFICER | 11460

Disarm Law Officer, Assault On Police II 11460

Disarm Law Officer, Cds I| 11460

Disarm Law Officer, Disarm Officer || 11460

Disarm Law Officer, Fto || 11460

Disturb The Peace, Assault On Police I| 20060

DOEMESTIC || 11420

DOMESTIC AGG ASLT । | 11609

DOMESTIC AGG ASSAULT || 11415

DOMESTIC AGG ASSAULT | | 11420

DOMESTIC AGG. ASSAULT || 11415

DOMESTIC AGGRAVATED ASSAULT || 11415

DOMESTIC ASSAULT I I 11420

DOMESTIC COMMON ASSAULT || 11420

DRIVING STOLEN AUTO || 11420

Fail Obey Renble/Lawfl, Assault On Police || 20055

False Imprisonment, Agg. Assault || 10042

False Stmt To Peace Officer, Assault On Police II 10690

FIRST DEGREE ASSAULT I| 11420

FIRST DEG ASSAULT । I 11415

FIRST DEG ASSAULT I| 13802

FIRST DEGREE ASSALT | | 11415

FIRST DEGREE ASSAULT | | 11338

FIRST DEGREE ASSAULT | | 11415

FIRST DEGREE ASSAULT । | 11420

FIRST DEGREE ASSAULT || 15212 
FIRST DEGREE ASSAULT || 23030

FLEEING/ELUDING/CDS VIOLATION || 11420

HAND GUN VIOLATION II 11420

Handgun On Person, 1St Degree Assault || 15212

Handgun On Person, Agg Assault By Pointing I| 15212

Handgun On Person, First Degree Assault || 15212

HANDGUN POSSESSION | | 11420

HANDGUN VIOLATION || 11420

HANGDUN VIOLATION || 11420

HGFV || 11420

HGV || 11420

HGV || 1C1420

HGV AGG ASSAULT || 11420

Hgv Use/Fel-Viol Crime, 1St Degree Assault II 15299

Hgv Use/Fel-Viol Crime, Agg Assault II 15299

Hgv Use/Fel-Viol Crime, Agg. Assault By Pointing I| 15299

Hgv Use/Fel-Viol Crime, Aggravated Assault II 15299

HIT \& RUN | | 11460

HIT AND RUN || 11420

HOME INVASION || 11420

IST COMMON ASSAULT || 11420

IST DEGREE ASSAULT | | 11420

LARCENY | 11420

Mace/Chem Device-Int/Injure, Agg Assault I| 25200

Mal Dest Prop/Valu - \$500, Agg Assault II 34025

Mal Dest Prop/Valu - \$500, Aggravated Assault II 34025

Mal Destr Prop Value + \$500, Agg Assault II 34030

Mal Destr Prop Value + \$500, Assault On Police II 34030

Obstructing \& Hindering, Assault On Police II 10043

PHYSICAL CHILD ABUSE || 11420

POSS DEADLY WEAPON || 11420

RECKLESS DRIVING || 11420

Reckless Endangerment, 1St Deg Assault II 11425

Reckless Endangerment, 1St Degree Assault || 11425

Reckless Endangerment, Agg Assault I| 11425

Reckless Endangerment, Agg. Assault || 11425

Reckless Endangerment, Aggravated Assault I| 11425

Reckless Endangerment, Assault First Degree II 11425

Resist/Interfere With Arrest, Assault On Police || 10600

Rifle/Shotgun:Unregistered, Aggravated Assault || 25212

SECOND DEGREE ASSAULT I| 11420

SECOND DEGREE ASSAULT || 1C1420

Sex Offense First Degree, Assault Sec Degree II 21102

STOLEN AUTO || 11420

THEFT I| 1C1420

Theft: $\$ 1,000$ To Under $\$ 10,000$, Assault On Police I| 10622

Theft: Less \$1,000 Value, Agg. Assault I| 10621

THEFT/AGG ASSAULT I| 11415

Trespass: Private Property, Assault On Police I| 22220

TRESPASSING/AGG ASSAULT | | 11415

TRESSPASSING/ AGG ASSAULT I | 11415

UNAUTHORIZED USE I| 11420

WRECKLESS ENDANGERMENT | | 11420

\section{Deadly Weapon}

4TH DEGREE BURGLARY || 15212

ALETERED SHOTGUN I 25212

ARMED PERSON || 25212 
ASS. W/DEADLY WEAPON || 11415

ASSAULT || 10175

ASSAULT BY POINTING || 15212

ASSAULT BY THREAT I| 10439

ASSAULT DEADLY WEAPON I| 11415

Assault Sec Deg-Law Enforcment, Handgun Violation I| 11416

ASSAULT W/DEADLY WEAPON I | 11415

ASSAULT WITH DEADLY WEAPON II 11415

ASSAULT WITH DEADLY WEAPON INT I I 11415

ASSAULT/HGV || 11609

Asslt-Sec Degree, Aslt W/Deadly Weapon I I 11415

Asslt-Sec Degree, Assault Deadly Weapon II 11415

Asslt-Sec Degree, Assault With A Deadly Weapon I| 11415

Asslt-Sec Degree, Assault With Deadly Weapon II 11415

Asslt-Sec Degree, Concealed Deadly Weapon I| 11415

Asslt-Sec Degree, Deadly Weapon || 11415

Asslt-Sec Degree, Deadly Weapon Int. Injure I| 11415

Asslt-Sec Degree, Firearm I| 11415

Asslt-Sec Degree, Handgun I| 11415

Asslt-Sec Degree, Handgun Violation I| 11415

Asslt-Sec Degree, Hgv II 11415

Asslt-Sec Degree, Hgv 11075 || 11415

Asslt-Sec Degree, Poss Deadly Weapon II 11415

Asslt-Sec Degree, Possession Deadly Weapon Inten || 11415

Att-Cds Manuf/Dist., Hgv/Cds II 2A3550

ATT-DANGEROUS WEAPON-INT/INJUR I| 1A5200

Att-Reg Firearm-Unlawful Sale/Trans, Hgv || 1 A0642

ATTEMPT DISTRIBUTION || 11609

B \& E || 11609

BREAKING AND ENTERING || 10175

BURGLARY || 15212

CARRY DANGEROUS WEAPON II

CARRY DANGEROUS WEAPON I| 15200

CARRYING A LONG RIFLE I I

CARRYING CONCEALED WEAPON || 15202

CARRYING LONG BARREL FIREARMS । ।

CARRYING RIFLE LONGER 14 INCH I | 10439

CARRYONG A LONG RIFLE I।

CDS || 10175

CDS || 10439

CDS || 10692

CDS || 11609

CDS || 15212

CDS || 35255

CDS - POSS OF FIREARMS । 11610

CDS / HANDGUN VIOLATION | | 10175

CDS / HANDGUN VIOLATION || 10233

CDS / HANDGUN VIOLATION I | 11106

CDS / HGV || 10493

CDS / HGV || 11609

CDS / HGV || 30233

CDS / HGV II 43550

CDS/HANDGUN || 11111

CDS HEROIN/HGV || 15212

Cds Manuf/Dist-Narc, Hand Gun Violation II 20696

Cds Manuf/Dist-Narc, Handgun Violation I| 20696

Cds Manuf/Dist-Narc, Hgv II 20696

Cds Manuf/Dist., Handgun Violation I I 23550

Cds Manuf/Dist., Hgv II 23550

CDS PODD HEROIN/ DEADLY WEAPON । | 43550 
CDS POSS || 11609

CDS POSS HERION I| 10439

Cds Poss W/Int To Dist, Cds Violation\Hgv | 10233

Cds Poss W/Int To Dist, Cds/Handgun I| 10233

Cds Poss W/Int To Dist, Handgun Violation I 10233

Cds Poss W/Int To Dist, Hgv I| 10233

Cds Poss W/Int To Dist, Hgv / Cds II 10233

Cds Poss W/Int To Dist, Hgv Violation I I 10233

Cds Poss W/Int To Dist, Hgv/Cds || 10233

Cds Poss W/Int To Dist, Hgv/ Drug Trafficking Crime I| 10233

Cds Poss W/Int To Dist, Hgv/Cds II 10233

Cds Possess - Lg Amt, Hgv Cds I| 10881

CDS PWI COCAINE/ HGV | 10493

CDS PWI/HGV || 11106

CDS PWID || 11609

CDS PWID \& HGV | | 30233

CDS PWID/HGV || 11111

CDS PWID/HGV || 11119

CDS PWID/HGV || 10881

CDS VIOALTION || 11609

CDS VIOLATION || 10692

CDS VIOLATION ॥ 11609

CDS VIOLATION || 15212

CDS VIOLATION/ HGV || 10493

CDS VIOLATION/ HGV || 11609

CDS VIOLATION/HANDGUN | | 10487

CDS VIOLATION/HGV || 10487

CDS VIOLATION/HGV || 10493

CDS VIOLATION/HGV || 10692

CDS VIOLATION/HGV I| 11454

CDS W/ CONCEALED WEAPON । | 10573

CDS W/ HGV II 30233

CDS-HGV I| 11609

CDS-POSS OF FIREARMS || 10692

Cds-Poss Of Firearms, Cds || 10692

Cds-Poss Of Firearms, Cds Violation II 10692

Cds-Poss Of Firearms, Cds-Poss Of Firearms || 10692

Cds-Poss Of Firearms, Firearm Violation I| 10692

Cds-Poss Of Firearms, Hand Gun I| 10692

Cds-Poss Of Firearms, Hand Gun Violation I| 10692

Cds-Poss Of Firearms, Handgun On Person I| 10692

Cds-Poss Of Firearms, Handgun Violation I I 10692

Cds-Poss Of Firearms, Hgv II 10692

Cds-Poss Of Firearms, Hgv W/ Cds II 10692

Cds-Poss Of Firearms, Poss Intent Cocaine I| 10692

Cds-Poss Of Firearms, Poss Of Firearms || 10692

CDS: DISTR ETC. W/FIREARM I 10487

Cds: Distr Etc. W/Firearm, Dist Heroin II 10487

Cds: Distr Etc. W/Firearm, Hgv II 10487

Cds: Poss Marihuana L/T 10 G, Handgun Violation || 11635

Cds: Poss Marihuana L/T 10 G, Hgv II 11635

Cds: Poss Marihuana L/T 10 G, Weapons Violation I| 11635

Cds: Possession-Marihuana, Cds Marijuana/Hgv I| 10573

Cds: Possession-Marihuana, Concealed Deadly Weapon II 10573

Cds: Possession-Marihuana, Hand Gun Violation II 10573

Cds: Possession-Marihuana, Handgun || 10573

Cds: Possession-Marihuana, Handgun In Vehicle I| 10573

Cds: Possession-Marihuana, Handgun Violation I| 10573

Cds: Possession-Marihuana, Hgv || 10573

Cds: Possession-Marihuana, Hgv / Cds Pwid II 10573 
Cds: Possession-Marihuana, Hgv/Cds II 10573

Cds:P W/I Dist:Narc, Cds Dist Cocaine/ Hgv || 30233

Cds:P W/I Dist:Narc, Cds/ Hgv I| 30233

Cds:P W/I Dist:Narc, Cds/Handgun I| 30233

Cds:P W/I Dist:Narc, Cds/Hgv || 30233

Cds:P W/I Dist:Narc, Hand Gun Violation I| 30233

Cds:P W/I Dist:Narc, Handgun On Person I| 30233

Cds:P W/I Dist:Narc, Handgun Violation I| 30233

Cds:P W/I Dist:Narc, Hgv I| 30233

Cds:P W/I Dist:Narc, Hgv / Pwi Cds II 30233

Cds:P W/I Dist:Narc, Hgv/ Cds II 30233

Cds:P W/I Dist:Narc, Hgv/ Drug Trafficking I| 30233

Cds:P W/I Dist:Narc, Hgv/Cds || 30233

Cds:P W/I Dist:Narc, Hgv/Cds Drug Trafficking || 30233

Cds:P W/I Dist:Narc, Hgv/Drug Trafficking I| 30233

Cds:P W/I Dist:Narc, Hgv/With Drug Trafficking II 30233

Cds:P W/I Dist:Narc, Poss Of Handgun I | 30233

Cds:Poss Para, Cds/Hgv I| 53550

Cds:Poss Para, Dangerous Weapon II 53550

Cds:Poss Para, Deadly Weapon II 53550

Cds:Poss Para, Hgv I| 53550

Cds:Possess-Not Marihuana, Cds Violation/Hgv II 43550

Cds:Possess-Not Marihuana, Cds/Hgv I| 43550

Cds:Possess-Not Marihuana, Deadly Weapon I| 43550

Cds:Possess-Not Marihuana, Firearm Violation II 43550

Cds:Possess-Not Marihuana, Hand Gun Violation I| 43550

Cds:Possess-Not Marihuana, Handgn Violation II 43550

Cds:Possess-Not Marihuana, Handgun I| 43550

Cds:Possess-Not Marihuana, Handgun On Vehicle II 43550

Cds:Possess-Not Marihuana, Handgun Violation II 43550

Cds:Possess-Not Marihuana, Handgun Violation W/Cds II 43550

Cds:Possess-Not Marihuana, Hgv II 43550

Cds:Possess-Not Marihuana, Hgv / Cds II 43550

Cds:Possess-Not Marihuana, Hgv-Cds II 43550

Cds:Possess-Not Marihuana, Hgv/ Drug Trafficking Cri || 43550

Cds:Possess-Not Marihuana, Hgv/Drug Traffic Crime II 43550

Cds:Possess-Not Marihuana, Hgv/Drug Trafficking Crim I| 43550

Cds:Possess-Not Marihuana, Hgv/With Cds Trafficking I| 43550

Cds:Possess-Not Marihuana, Weapon Violation I| 43550

CDS/ HGV II 10493

CDS/HGV || 10692

CDS/HGV I| 11609

CDS/ HGV I| 30233

CDS/ HGV II 43550

CDS/CONCEALED DEADLY WEAPON || 10573

CDS/DEADLY WEAPON I| 43550

CDS/FIREARM II 10493

CDS/FIREARM VIOLATION I| 43550

CDS/HANDGUN || 10692

CDS/HANDGUN || 11106

CDS/HANDGUN || 11119

CDS/HANDGUN VIOL I 10692

CDS/HANDGUN VIOLATION || 10487

CDS/HGV || 10233

CDS/HGV II 10487

CDS/HGV || 10493

CDS/HGV || 10573

CDS/HGV || 10692

CDS/HGV || 11106

CDS/HGV || 11111 


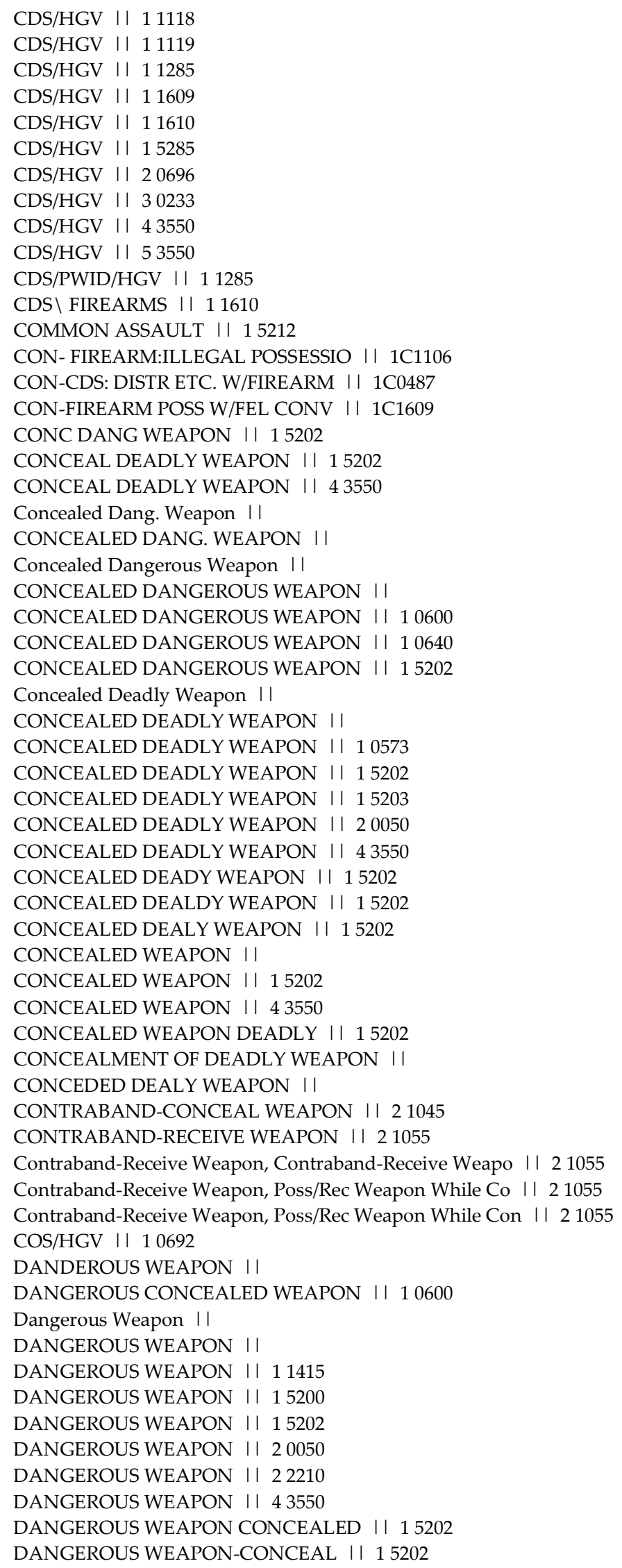


DANGEROUS WEAPON-INT/INJURE || 15200

DANGEROUS WPN ON SCHOOL PROP || 15203

DEADLEY WEAPON || 15202

DEADLY WEAPON || 15202

DEADLY CONCEALED WEAPON । 20050

Deadly Weapon II

DEADLY WEAPON I।

DEADLY WEAPON || 11415

DEADLY WEAPON || 13903

DEADLY WEAPON || 15200

DEADLY WEAPON || 15202

DEADLY WEAPON || 20050

DEADLY WEAPON || 22210

DEADLY WEAPON I| 22220

DEADLY WEAPON || 25200

DEADLY WEAPON || 2A3550

DEADLY WEAPON || 35399

DEADLY WEAPON || 43550

DEADLY WEAPON I| 53550

DEADLY WEAPON \& POSS MARIJUANA || 10573

Deadly Weapon Conceal II

DEADLY WEAPON W/INT TO INJUR I | 11415

DEADLY WEAPON WITH INTENT | | 11415

Deadly Weapon-Conceal, 2Nd Degree Assault || 15202

Deadly Weapon-Conceal, Armed Person I| 15202

Deadly Weapon-Conceal, Assault By Threat I| 15202

Deadly Weapon-Conceal, Bb Gun Violation I| 15202

Deadly Weapon-Conceal, Carry Concealed Weapon I| 15202

Deadly Weapon-Conceal, Carrying Concealed Deadly We I| 15202

Deadly Weapon-Conceal, Cds I| 15202

Deadly Weapon-Conceal, Cds Distribution II 15202

Deadly Weapon-Conceal, Cds Poss II 15202

Deadly Weapon-Conceal, Cds Violation I| 15202

Deadly Weapon-Conceal, Cds-Poss Marijuana II 15202

Deadly Weapon-Conceal, Con Deadly Weapon I| 15202

Deadly Weapon-Conceal, Conceal Dangerous Weapon I| 15202

Deadly Weapon-Conceal, Conceal Deadly Weapon II 15202

Deadly Weapon-Conceal, Concealed Dangerous Weapon I| 15202

Deadly Weapon-Conceal, Concealed Deadly Weapon I| 15202

Deadly Weapon-Conceal, Concealed Weapon II 15202

Deadly Weapon-Conceal, Concealing Deadly Weapon I| 15202

Deadly Weapon-Conceal, Conceled Deadly Weapon I| 15202

Deadly Weapon-Conceal, Concenled Deadly Weapon I| 15202

Deadly Weapon-Conceal, Consealed Deadly Weapon II 15202

Deadly Weapon-Conceal, Dangerous Weapon I| 15202

Deadly Weapon-Conceal, Dangerous Weapon-Conceal || 15202

Deadly Weapon-Conceal, Deadly Weapon II 15202

Deadly Weapon-Conceal, Deadly Weapon Conceal I| 15202

Deadly Weapon-Conceal, Deadly Weapon Knife II 15202

Deadly Weapon-Conceal, Deadly Weapon/Switch Blade || 15202

Deadly Weapon-Conceal, Disorderly || 15202

Deadly Weapon-Conceal, Failure To Obey I| 15202

Deadly Weapon-Conceal, Handgun Violation II 15202

Deadly Weapon-Conceal, Hgv I| 15202

Deadly Weapon-Conceal, Poss - Marijuana II 15202

Deadly Weapon-Conceal, Poss Cds || 15202

Deadly Weapon-Conceal, Poss Deadly Weapon || 15202

Deadly Weapon-Conceal, Poss Heroin I| 15202

Deadly Weapon-Conceal, Poss Marijuana I| 15202

Deadly Weapon-Conceal, Poss Of Deadly Weapon I| 15202 
Deadly Weapon-Conceal, Poss Pellet Gun II 15202

Deadly Weapon-Conceal, Poss. Of Deadly Weapon I| 15202

Deadly Weapon-Conceal, Poss.Switch Black Knife II 15202

Deadly Weapon-Conceal, Possession Deadly Weapon I| 15202

Deadly Weapon-Conceal, Possession Of Bbgun || 15202

Deadly Weapon-Conceal, Possession Of Dangerous Weapo I| 15202

Deadly Weapon-Conceal, Possession Of Deadly Weapon I| 15202

Deadly Weapon-Conceal, Spring Assist Blade II 15202

Deadly Weapon-Conceal, Tresspassing || 15202

Deadly Weapon-Int/Injure, 1St Deg B/E I| 15200

Deadly Weapon-Int/Injure, 2Nd Deg Assault II 15200

Deadly Weapon-Int/Injure, Assault I| 15200

Deadly Weapon-Int/Injure, Assault 2Nd Degree I| 15200

Deadly Weapon-Int/Injure, Assault By Threat I| 15200

Deadly Weapon-Int/Injure, Assault W/A Deadly Weapon II 15200

Deadly Weapon-Int/Injure, Attempt Assault II 15200

Deadly Weapon-Int/Injure, Child Abuse I| 15200

Deadly Weapon-Int/Injure, Common Assault I| 15200

Deadly Weapon-Int/Injure, Dangerous Weapon- Int/Inju || 15200

Deadly Weapon-Int/Injure, Dangerous Weapon-Int/Inju || 15200

Deadly Weapon-Int/Injure, Dangerous Weapon-Int/Injur || 15200

Deadly Weapon-Int/Injure, Deadly Weapon II 15200

Deadly Weapon-Int/Injure, Destruction Property || 15200

Deadly Weapon-Int/Injure, Disorderly Conduct II 15200

Deadly Weapon-Int/Injure, Domestic Assault I| 15200

Deadly Weapon-Int/Injure, Poss Deadly Weapon I| 15200

Deadly Weapon-Int/Injure, Possession Deadly Weapon I| 15200

Deadly Weapon-Int/Injure, Violation Of Prot Order || 15200

DEADLY WEAPON; DESTRUCTION OF I। 34025

DEADLY WEAPON/2ND ASSAULT I | 11415

DEADLY WEAPON/ASSAULT || 11415

DEADLY WEAPON/ASSAULT BY THREA I।

DEADLY WEAPON/CDS I I 43550

DEADLY WEAPON/DISORDERLY I | 20050

DEADLY WEAPON/SWITCHBLADE || 15202

DIRT BIKE VIOLATION || 15212

Dis.Erly Conduct, Concealed Deadly Weapon I| 20050

DISCHARGE FIREARM । ।

DISCHARGE HANDGUN IN CITY । |

DISCHARGE OF A FIREARM । |

DISCHARGE OF FIREARM | | 11425

DISCHARGE. OF A FIRE ARM || 11609

DISCHARGING || 15212

DISCHARGING - HGV । ।

DISCHARGING A FIREARM । |

DISCHARGING FIREARM । |

DISCHARGING FIREARM || 11425

DISCHARGING OF A FIREARM । ।

DISCHARGING OF FIREARM | | 11285

DISCHARGING OF FIREARM || 11425

DISCHARGING OF FIREARM || 11455

DRIVING ON SUSPENDED LICENSE || 11609

DRV W/O LICENSE I I 10175

Dw/Switch Blade II

EXECUTION OF SGS WARRENT (HGV) I। 43550

FAIL REG GUN I ।

FAIL REG GUN OFFENDER । |

FAIL REG-GUN OFFENDER । ।

FAIL REG. GUN OFFENDER । ।

FAIL TO REGISTER HANDGUN । । 
FAIL/REG GUN OFF/ UPDATE INFO | |

FAILREG GUN OFFENDER I ।

FALSE IMPRISONMENT || 11609

FALSE INFO-APP FOR FIREARM || 20642

FALSE PRETENSE II 10175

FELON IN POSSESSION FIREARM | | 53550

FELON IN POSSESSION OF FIREARM || 10692

FELON/POSS OF HANDGUN || 11106

FELONY HANDGUN || 10487

FIREARM II

FIREARM || 10175

FIREARM || 11106

FIREARM || 11610

FIREARM - POSS W/FEL CONV | 11609

FIREARM AND DRUG TRAFFICKING || 10692

FIREARM CHARGES || 10439

FIREARM DRUG TRAFFICING || 11610

FIREARM DRUG TRAFFICKING I | 10487

FIREARM DRUG TRAFFICKING || 11455

FIREARM POSS I।

FIREARM POSS || 11285

FIREARM POSS I| 11609

FIREARM POSS W/ FEL CONVICT । ।

FIREARM POSS W/FEL CONVICT $\| 11609$

Firearm Poss W/Fel Convict, 2Nd Deg Assault I I 11609

Firearm Poss W/Fel Convict, Assault By Pointing || 11609

Firearm Poss W/Fel Convict, Burglary || 11609

Firearm Poss W/Fel Convict, Cds || 11609

Firearm Poss W/Fel Convict, Cds/Hgv || 11609

Firearm Poss W/Fel Convict, Felony Poss/Handgun II 11609

Firearm Poss W/Fel Convict, Firearm Poss W/Fel Conv || 11609

Firearm Poss W/Fel Convict, Firearm Poss W/Fel Convi || 11609

Firearm Poss W/Fel Convict, Hand Gun On Person II 11609

Firearm Poss W/Fel Convict, Hand Gun Violation I| 11609

Firearm Poss W/Fel Convict, Handgun || 11609

Firearm Poss W/Fel Convict, Handgun On Person I| 11609

Firearm Poss W/Fel Convict, Handgun Viol. II 11609

Firearm Poss W/Fel Convict, Handgun Violation I| 11609

Firearm Poss W/Fel Convict, Handgun W/ Drug Traffick II 11609

Firearm Poss W/Fel Convict, Hgv I| 11609

Firearm Poss W/Fel Convict, Hgv Violation I | 11609

Firearm Poss W/Fel Convict, Hgv/Cds II 11609

Firearm Poss W/Fel Convict, Poss Handgun II 11609

Firearm Poss W/Fel Convict, Poss Of Handgun I| 11609

Firearm Poss W/Fel Convict, Poss. Handgun I I 11609

Firearm Poss W/Fel Convict, Poss. Of A Handgun I| 11609

Firearm Poss W/Fel Convict, Possession Handgun I| 11609

Firearm Poss W/Fel Convict, Regulated Firearm: Illeg || 11609

FIREARM POSSESION || 10233

FIREARM POSSESSION || 11610

FIREARM POSSESSION WITH FELONY | | 11609

FIREARM PROHIBITED || 10493

FIREARM USE/FEL-VIOL CRIME || 15299

Firearm Violation II

FIREARM VIOLATION I ।

FIREARM VIOLATION || 10233

FIREARM VIOLATION || 10439

FIREARM VIOLATION I| 10487

FIREARM VIOLATION | | 10500

FIREARM VIOLATION || 10692 
FIREARM VIOLATION || 11314

FIREARM VIOLATION || 11415

FIREARM VIOLATION || 11609

FIREARM VIOLATION II 11610

FIREARM VIOLATION || 11612

FIREARM VIOLATION || 25212

FIREARM VIOLATION II 30233

FIREARM VIOLATION I| 43550

FIREARM W/ DESTROYED SERIAL \# | | 15212

FIREARM-POSS W/FEL CONV || 10692

FIREARM-POSS W/FEL CONV II 22030

Firearm: Alter Etc Id Number, Hand Gun Violation I I 35210

FIREARM: STOLEN/SELL I| 35250

FIREARM/DRUG TRAF CRIME || 10493

FIREARM/DRUG TRAF CRIME || 20696

Firearm/Drug Traf Crime, Cds || 10493

Firearm/Drug Traf Crime, Cds / Hgv I| 10493

Firearm/Drug Traf Crime, Cds Pwi / Hgv || 10493

Firearm/Drug Traf Crime, Cds Violation II 10493

Firearm/Drug Traf Crime, Cds/Hgv I| 10493

Firearm/Drug Traf Crime, Distribution Cocaine II 10493

Firearm/Drug Traf Crime, Firearm/Drug Traf Crime II 10493

Firearm/Drug Traf Crime, Firearms Violation II 10493

Firearm/Drug Traf Crime, Hand Gun I| 10493

Firearm/Drug Traf Crime, Hand Gun Violation I| 10493

Firearm/Drug Traf Crime, Handgun I| 10493

Firearm/Drug Traf Crime, Handgun On Person I| 10493

Firearm/Drug Traf Crime, Handgun Vioaltion II 10493

Firearm/Drug Traf Crime, Handgun Violation II 10493

Firearm/Drug Traf Crime, Hgu Violation II 10493

Firearm/Drug Traf Crime, Hgv I| 10493

Firearm/Drug Traf Crime, Hgv/Cds II 10493

Firearm/Drug Traf Crime, Hgv/ Cds Felony II 10493

Firearm/Drug Traf Crime, Hgv/Cds II 10493

Firearm/Drug Traf Crime, Hgv/Cds Possession I| 10493

Firearm/Drug Traf Crime, Poss Cocaine II 10493

Firearm/Drug Traf Crime, Poss Marijuana W/I Dist I I 10493

Firearm/Drug Traf Crime, Poss W/I Marijuana II 10493

Firearm/Drug Traf Crime, Poss W/Int Cocaine I| 10493

Firearm/Drug Traf Crime, Poss W/Int Marijuana II 10493

Firearm/Drug Traf Crime, Possession W/Int Heroin II 10493

Firearm/Drug Traf Crime, Possession With Intent I| 10493

Firearm/Drug Traf Crime, Pwi Cocaine II 10493

Firearm/Drug Traf Crime, Pwid II 10493

Firearm/Drug Traf Crime, Pwid Cocaine II 10493

Firearm/Drug Traf Crime, Pwid Cocaine/ Hgv || 10493

Firearm/Drug Traf Crime, Pwid Marijuana II 10493

FIREARM/PROHIBITTED | | 10233

FIREARMS || 10493

FIREARMS || 11106

FIREARMS / CDS VIOLATION || 11609

FIREARMS DRUG TRAFFICKING || 10233

Firearms Violation II

FIREARMS VIOLATION II

FIREARMS VIOLATION II 10439

FIREARMS VIOLATION || 10493

FIREARMS VIOLATION || 10573

FIREARMS VIOLATION || 10692

FIREARMS VIOLATION || 11106

FIREARMS VIOLATION || 11285 
FIREARMS VIOLATION || 11610

FIREARMS VIOLATION || 25212

FIREARMS VIOLATION || 35250

FIREARMS VIOLATION || 43550

FIREARMS VIOLATION/CDS || 10573

Firearms-Access By Minors, Handgun Violation I| 20239

Firearms-Access By Minors, Hgv || 20239

FTO || 11609

FUGITIVE FROM PHILADELPHIA | ।

GUN OFFENDER FAIL REGISTER । ।

GUN OFFENDER FAIL TO REG । ।

GUN OFFENDER FAIL TO REGISTER I ।

GUN OFFENDER, FAIL TO REGISTER । ।

HAND GUN || 10175

HAND GUN || 11106

HAND GUN || 11119

HAND GUN || 11454

HAND GUN I| 15212

HAND GUN || 30233

HAND GUN VIO || 15212

HAND GUN VIOLAATION || 11111

Hand Gun Violation I I

HAND GUN VIOLATION I |

HAND GUN VIOLATION | 10175

HAND GUN VIOLATION I | 10233

HAND GUN VIOLATION | | 10439

HAND GUN VIOLATION || 10487

HAND GUN VIOLATION | | 10493

HAND GUN VIOLATION | | 10692

HAND GUN VIOLATION | | 10908

HAND GUN VIOLATION || 11106

HAND GUN VIOLATION | | 11130

HAND GUN VIOLATION | | 11285

HAND GUN VIOLATION | | 11415

HAND GUN VIOLATION | | 11454

HAND GUN VIOLATION I | 11455

HAND GUN VIOLATION | | 11564

HAND GUN VIOLATION | | 11609

HAND GUN VIOLATION | | 11610

HAND GUN VIOLATION | | 11612

HAND GUN VIOLATION | | 15212

HAND GUN VIOLATION || 15285

HAND GUN VIOLATION | | 22030

HAND GUN VIOLATION | | 22210

HAND GUN VIOLATION | | 30233

HAND GUN VIOLATION | | 43550

HAND GUN VIOLATION/CDS || 30233

HAND GUN VIOLTION I ।

HANDGN VIOLATION || 15285

HANDGUN || 10175

HANDGUN || 10233

HANDGUN I| 10493

HANDGUN || 10690

HANDGUN || 10692

HANDGUN || 11106

HANDGUN || 11119

HANDGUN || 11285

HANDGUN || 11454

HANDGUN || 11455

HANDGUN || 11609 
HANDGUN || 11612

HANDGUN || 15212

HANDGUN || 15285

HANDGUN || 30233

HANDGUN || 43550

HANDGUN VIOLATION | 15285

HANDGUN VIOLATION I| 20254

HANDGUN ARREST || 10175

HANDGUN FIREARMS VIOLATION || 11609

HANDGUN GUN VIOLATION || 11106

HANDGUN IN CAR I | 11454

HANDGUN IN VECHILE || 10175

HANDGUN IN VEHICLE || 10175

HANDGUN IN VEHICLE || 10692

HANDGUN IN VEHICLE || 11285

HANDGUN IN VEHICLE | | 30233

Handgun In Vehicle, Cds Violation I | 10175

Handgun In Vehicle, Cds/Hgv || 10175

Handgun In Vehicle, Firearms Violation I| 10175

Handgun In Vehicle, Gun Violation I I 10175

Handgun In Vehicle, Hand Gun Violation II 10175

Handgun In Vehicle, Handgun I| 10175

Handgun In Vehicle, Handgun In Vehicle I| 10175

Handgun In Vehicle, Handgun On Person || 10175

Handgun In Vehicle, Handgun Possession I| 10175

Handgun In Vehicle, Handgun Violation I| 10175

Handgun In Vehicle, Hangun || 10175

Handgun In Vehicle, Hangun Violation | | 10175

Handgun In Vehicle, Hgv || 10175

Handgun In Vehicle, Hgv/Poss Marijuana I| 10175

Handgun In Vehicle, Poss Handgun || 10175

Handgun In Vehicle, Poss. Of Marijuana II 10175

Handgun In Vehicle, Regulated Firearm: Illegal Pos II 10175

HANDGUN ON PERSON I।

HANDGUN ON PERSON || 10175

HANDGUN ON PERSON I| 10233

HANDGUN ON PERSON || 10487

HANDGUN ON PERSON || 10493

HANDGUN ON PERSON || 10692

HANDGUN ON PERSON || 11106

HANDGUN ON PERSON || 11285

HANDGUN ON PERSON || 11454

HANDGUN ON PERSON || 11455

HANDGUN ON PERSON I| 11609

HANDGUN ON PERSON || 11610

HANDGUN ON PERSON || 15212

HANDGUN ON PERSON || 15285

HANDGUN ON PERSON || 15299

HANDGUN ON PERSON || 1A1118

HANDGUN ON PERSON || 20696

HANDGUN ON PERSON || 2A3550

HANDGUN ON PERSON || 30233

Handgun On Person, Cds Poss/Intent || 15212

Handgun On Person, Cds Violation I| 15212

Handgun On Person, Common Assault II 15212

Handgun On Person, Dirtbike I| 15212

Handgun On Person, Hand Gun On Person II 15212

Handgun On Person, Hand Gun Violation II 15212

Handgun On Person, Handgun I| 15212

Handgun On Person, Handgun On Person I| 15212 
Handgun On Person, Handgun Possession I| 15212

Handgun On Person, Handgun Violaion || 15212

Handgun On Person, Handgun Violation I| 15212

Handgun On Person, Hangun Violation I| 15212

Handgun On Person, Hgv II 15212

Handgun On Person, Hgv/Cds || 15212

Handgun On Person, Hgv/ Poss Of Marijuana II 15212

Handgun On Person, Hgv/Cds II 15212

Handgun On Person, Poss Firearm Hgv I| 15212

HANDGUN POSS || 11609

HANDGUN POSSESSION I |

HANDGUN POSSESSION || 10175

HANDGUN POSSESSION | 10493

HANDGUN POSSESSION || 11106

HANDGUN POSSESSION | | 15212

HANDGUN POSSESSION || 15285

HANDGUN POSSESSION PROHIBITED || 11609

HANDGUN PROHIBITED | | 11285

HANDGUN USED IN COMMISSION OF || 11455

HANDGUN VILOATION || 15202

HANDGUN VIO. I I 11106

HANDGUN VIOALTION || 10468

HANDGUN VIOALTION || 10493

HANDGUN VIOALTION || 11285

HANDGUN VIOATION || 11609

HANDGUN VIOL I। 11106

HANDGUN VIOL || 11609

HANDGUN VIOL || 11610

HANDGUN VIOL || 15212

HANDGUN VIOLAION | 11285

HANDGUN VIOLATATION || 10175

HANDGUN VIOLATIOIN | | 30233

Handgun Violation II

HANDGUN VIOLATION I।

HANDGUN VIOLATION || 10175

HANDGUN VIOLATION || 10233

HANDGUN VIOLATION || 10439

HANDGUN VIOLATION || 10487

HANDGUN VIOLATION || 10493

HANDGUN VIOLATION || 10521

HANDGUN VIOLATION || 10573

HANDGUN VIOLATION || 10642

HANDGUN VIOLATION | | 10692

HANDGUN VIOLATION || 10826

HANDGUN VIOLATION || 10881

HANDGUN VIOLATION || 10908

HANDGUN VIOLATION || 11106

HANDGUN VIOLATION || 11111

HANDGUN VIOLATION || 11118

HANDGUN VIOLATION || 11119

HANDGUN VIOLATION || 11130

HANDGUN VIOLATION || 11285

HANDGUN VIOLATION || 11415

HANDGUN VIOLATION || 11425

HANDGUN VIOLATION || 11454

HANDGUN VIOLATION || 11455

HANDGUN VIOLATION | | 11564

HANDGUN VIOLATION || 11609

HANDGUN VIOLATION || 11610

HANDGUN VIOLATION || 11612 
HANDGUN VIOLATION || 12801 HANDGUN VIOLATION || 15212 HANDGUN VIOLATION || 15285 HANDGUN VIOLATION || 15299 HANDGUN VIOLATION || 1 A0483 HANDGUN VIOLATION || 20239 HANDGUN VIOLATION I | 20480 HANDGUN VIOLATION || 20696 HANDGUN VIOLATION || 22210 HANDGUN VIOLATION || 22411 HANDGUN VIOLATION I| 23000 HANDGUN VIOLATION || 25212 HANDGUN VIOLATION || 2A0696 HANDGUN VIOLATION || 2A3550 HANDGUN VIOLATION || 2C3550 HANDGUN VIOLATION || 30233 HANDGUN VIOLATION I| 35250 HANDGUN VIOLATION | | 43550 HANDGUN VIOLATION (S\&S) I | 35250 HANDGUN VIOLATION / CDS || 11285 HANDGUN VIOLATION / CDS || 15212 HANDGUN VIOLATION/CDS | | 10692 HANDGUN VIOLATION/CDS | | 30233 HANDGUN VIOLATION/CDS || 10175 HANDGUN VIOLATION/CDS | | 10493 HANDGUN VIOLATION/CDS I | 10692 HANDGUN VIOLATION/CDS || 11106 HANDGUN VIOLATION/CDS || 11285 HANDGUN VIOLATION/CDS || 15212 HANDGUN VIOLATION/STOLEN AUTO | | 20480 HANDGUN VIOLATIONB | | 11119 HANDGUN VIOLATIONS || 10692 HANDGUN VIOLATIONS || 11106 HANDGUN VIOLATIONS || 11609 HANDGUN VIOLATON || 11609 HANDGUN VIOLTAION || 11106 HANDGUN VIOLTAION || 11609 HANDGUN VIOLTION || 11609 HANDGUN VOILATION || 10692 HANDGUN VOILATION || 11106 HANDGUN/CDS || 10175 HANDGUN/CDS || 10493 HANDGUN/CDS || 11106 HANDGUN/CDS || 11111 HANDGUN/CDS || 11454 HANDGUN/CDS VIOLATION || 20696 HANDGUN/NARCOTICS | | 30233 HANDGUUN VIOLATION | | 11285 HANDUN VIOLATION | | 10175 HANFGUN VIOLATION || 10692 HANGUN VIOLATION || 10692 HANGUN VIOLATION I| 11609 HANGUN VIOLATION || 15212

Hgv II

HGV II

HGV || 10175

HGV || 10233

HGV || 10439

HGV || 10487

HGV || 10493 
HGV || 10521

HGV || 10573

HGV || 10642

HGV || 10690

HGV || 10692

HGV || 10881

HGV || 10908

HGV | 11106

HGV || 11111

HGV | 11118

HGV || 11119

HGV || 11130

HGV || 11285

HGV || 11338

HGV || 11415

HGV || 11425

HGV || 11454

HGV || 11455

HGV || 11564

HGV || 11609

HGV || 11610

HGV || 11612

HGV || 11635

HGV || 12801

HGV || 15202

HGV || 15212

HGV || 15285

HGV || 15299

HGV || 1A1118

HGV I| 1A1119

HGV || 1A5212

HGV || 1C1118

HGV II 1C1119

HGV || 20105

HGV || 20233

HGV || 20239

HGV II 20480

HGV || 20696

HGV || 22030

HGV || 22210

HGV | | 22220

HGV || 23000

HGV || 23030

HGV II 23550

HGV || 25212

HGV || 2A0696

HGV | | 2A3550

HGV || 30233

HGV || 34025

HGV || 35250

HGV II 43550

HGV II 53550

HGV (S\&S) || 10493

HGV / CDS || 10233

HGV / CDS I I 10493

HGV / CDS I | 10573

HGV / CDS I | 11119

HGV / CDS || 11609

HGV / CDS | | 15212

HGV / CDS || 53550 


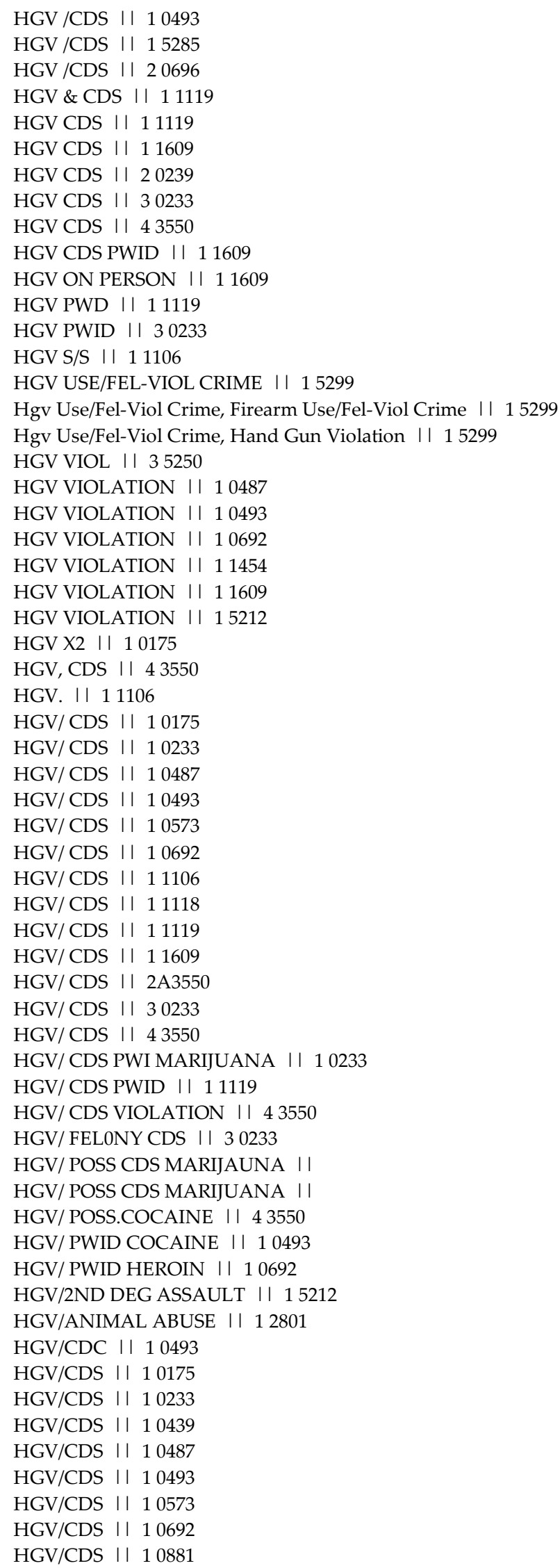




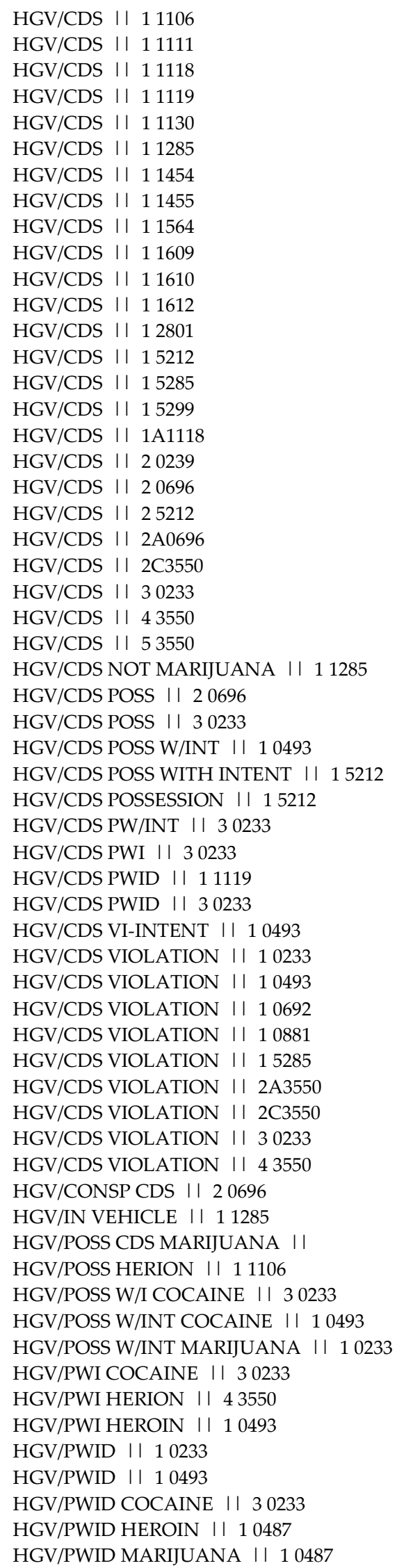


HHANDGUN VIOLATION || 10175

HNADGUN IN VEHICLE || 10175

HNADGUN VIOLATION || 11609

HUNDGUN VIOLATION || 10692

HUNDGUN VIOLATION || 11609

ILLEGAL POSS FIREARM || 11106

ILLEGAL POSSESSION OF FIREARM | | 11285

ILLEGAL POSSESSION REG FIREARM || 11609

KNOW ALTER FIREARM ID NUMBER || 11612

Know Alter Firearm Id Number, Firearms Violation I| 11612

Know Alter Firearm Id Number, Handgun Violation I| 11612

Know Alter Firearm Id Number, Hgv I I 11612

Know Alter Firearm Id Number, Hgv / Cds || 11612

Know Alter Firearm Id Number, Know Alter Firearm Id || 11612

Know Alter Firearm Id Number, Possession W/Int Herio I| 11612

LARCENY || 15212

LARCENY/HAND GUN VIOLATION || 15212

LOADED HANDGUN IN VEHICLE || 11454

LOADED HANDGUN ON PERSON I | 11455

LOADED HANDGUN ON PERSON I| 15212

LOITER/DEADLY WEAPON || 20050

LONG GUN VIOLATIION I I

Mal Destr Prop Value + \$500, Concealed Weapon II 34030

MCHNGUN:FAIL KEEP REGISTER | | 35200

P.W.I || 10692

POSS CDS W HANDGUN II 10493

POSS COCAINE /HGV II 15285

POSS DANGEROUS WEAPON || 15202

POSS DANGEROUS WEAPON II 20050

Poss Deadly Weapon II

POSS DEADLY WEAPON || 11415

POSS DEADLY WEAPON || 15200

POSS DEADLY WEAPON II 15202

POSS DEADLY WEAPON || 43550

POSS DEADLY WEAPON INT. I I 20050

POSS FIREARM || 11106

POSS FIREARM I| 11612

POSS HANDGUN $\mid$ |

POSS HANDGUN || 10692

POSS HANDGUN || 11106

POSS HANDGUN || 11285

POSS HANDGUN || 15212

POSS OF 12 GAUGE PUMP ACTION । ।

Poss Of A Deadly Weapon II

POSS OF A DEADLY WEAPON I| 15285

Poss Of Dang Weapon I।

Poss Of Deadly Weapon II

POSS OF DEADLY WEAPON I ।

POSS OF DEADLY WEAPON || 15202

POSS OF DEADLY WEAPON I| 35399

POSS OF FIREARM I| 11285

POSS OF FIREARM । I 11454

POSS OF FIREARM || 15212

POSS OF FIREARM MINOR I | 15285

POSS OF FIREARM/AMMO/MINOR I I 15285

Poss Of Firearm/Ammo/Minor, Firearms Violation || 15285

Poss Of Firearm/Ammo/Minor, Handgun Vio II 15285

Poss Of Firearm/Ammo/Minor, Handgun Violation || 15285

Poss Of Firearm/Ammo/Minor, Hgv I| 15285

Poss Of Firearm/Ammo/Minor, Poss Of Firearm Minor || 15285 
Poss Of Firearm/Ammo/Minor, Poss Of Firearm/Ammo/Min I| 15285

Poss Of Firearm/Ammo/Minor, Pwid Marijuana || 15285

Poss Of Firearm/Ammo/Minor, Reckless Endangerment || 15285

Poss Of Firearm/Ammo/Minor, S And S I| 15285

POSS OF FIREARMS I| 10692

POSS OF HANDGUN ।| 10692

POSS OF HANDGUN II 11106

POSS OF HANDGUN I| 11609

POSS OF HANDGUN || 15212

POSS OF HGV II 10493

POSS RIFLE SHOTGUN I| 11610

POSS W INT MARIJUANA || 15212

POSS W/ INTENT COCAINE/ HGV | | 10881

POSS W/I COCAINE || 11609

POSS W/I HANDGUN II 11609

POSS W/INT /HGV || 30233

POSS W/INT. COCAINE || 11609

POSS W/INT/HGV II 10493

POSS W/INTENT CDS I| 10692

POSS W/INTENT HANDGUN || 11119

POSS W/INTENT TO DIST COCAINE | | 11609

POSS: DEADLY WEAPON I| 15202

POSS. CDS/ DEADLY WEAPON || 15202

POSS. DEADLY WEAPON || 15202

POSS. FIREARM || 11610

POSS. FIREARM || 12801

POSS. HANDGUN || 10175

POSS. HANDGUN || 15212

POSS. OF A DANGEROUS WEAPON I | 15202

POSS. OF A LETHAL WEAPON I| 15202

POSS. OF DEADLY WEAPON || 15202

POSS. OF HANDGUN II 10175

POSS/ GUN I| 15212

POSS/HANDGUN/ WEAR AND CARRY I | 15285

POSS/REC WEAPON WHILE CONF/DET || 21055

POSSEION OF A DEADLY WEAPON | | 10521

Possesion Of Deadly Weapon II

POSSESION OF DEADLY WEAPON I I 11415

POSSESSION DEADLY WEAPON || 11415

POSSESSION DEADLY WEAPON || 15202

POSSESSION DEADLY WEAPON I| 22220

POSSESSION FIREARM | | 11454

POSSESSION HANDGUN || 10233

POSSESSION HERION II 11609

POSSESSION ILLEGAL HANDGUN WEA || 15212

POSSESSION OF A BB HANDGUN । ।

POSSESSION OF A DEADLY WEAPON || 15202

POSSESSION OF A FIREARM || 11609

POSSESSION OF A FIREARM WITH F || 11610

POSSESSION OF CONCEALED WEAPON I ।

POSSESSION OF DANGEROUS WEAPON I | 15202

POSSESSION OF DEADLY WEAPON I| 11415

POSSESSION OF FIREARM I| 10439

POSSESSION OF FIREARM | | 10692

POSSESSION OF FIREARM | | 11610

POSSESSION OF HANDGUN || 10175

POSSESSION OF HANDGUN || 11609

POSSESSION OF HANDGUN I| 30233

POSSESSION OF HEROIN II 10439

POSSESSION OF MARIJUANA || 10692 


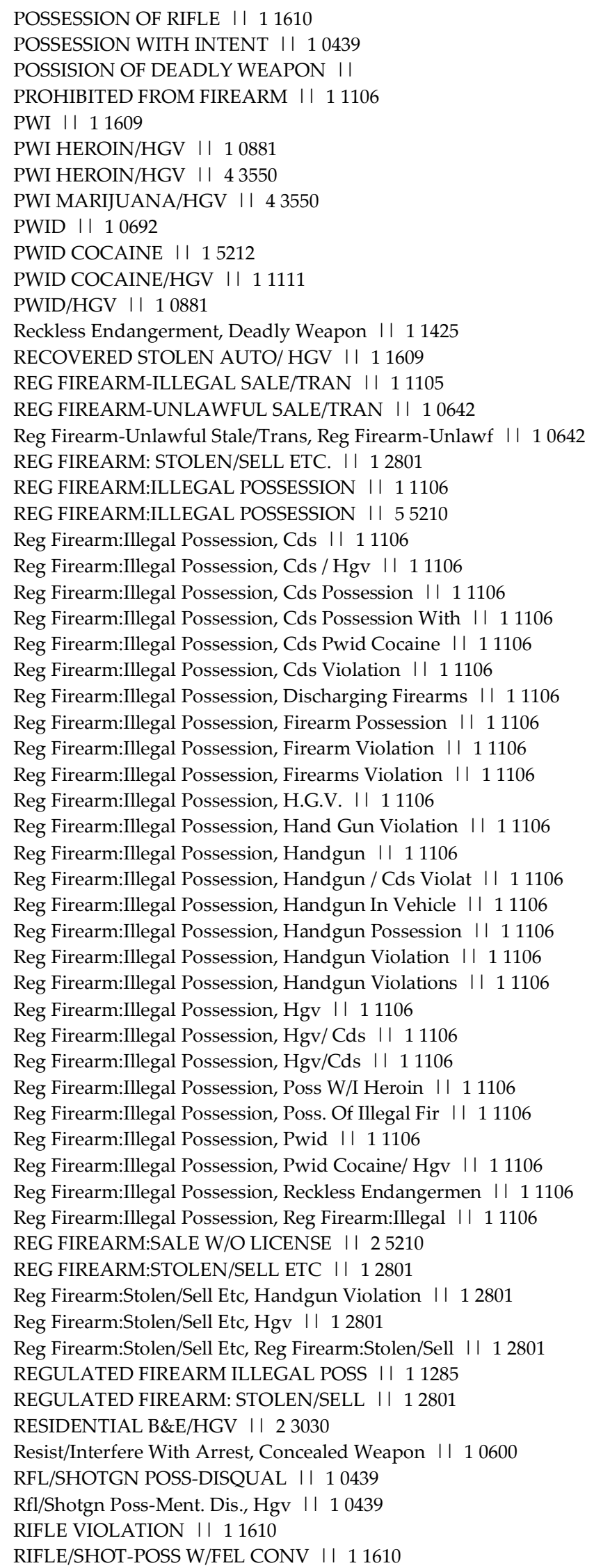


Rifle/Shot-Poss W/Fel Conv, Assault I| 11610

Rifle/Shot-Poss W/Fel Conv, Cds I| 11610

Rifle/Shot-Poss W/Fel Conv, Cds Violation I| 11610

Rifle/Shot-Poss W/Fel Conv, Dist Of Cocaine II 11610

Rifle/Shot-Poss W/Fel Conv, Poss. Firearm Drug Traf I| 11610

Rifle/Shot-Poss W/Fel Conv, Rifle/Shot-Poss W/Fel Co || 11610

Rifle/Shot-Poss W/Fel Conv, Theft II 11610

RIFLE/SHOTGUN UNREGISTERED I I

RIFLE/SHOTGUN:UNREGISTERED || 25212

Rifle/Shotgun:Unregistered, Handgun Violation I| 25212

Rifle/Shotgun:Unregistered, Hgv || 25212

Rifle/Shotgun:Unregistered, Rifle/Shotgun:Unregiste || 25212

Rifle/Shotgun:Unregistered, Rifle/Shotgun:Unregister || 25212

S.S. WARRANT/HGV II 11609

S\&S WARRANT/HGV II 11609

SHOTGUN VIOLATION || 10573

SPRING ASSISTED KNIFE । |

SPRING ASSISTED KNIFE || 10600

SPRING LOADED KNIFE I | 15202

SS HGV || 11609

STOLEN AUTO || 10175

STOLEN AUTO || 10692

STOLEN AUTO || 11609

STOLEN AUTO || 15212

STOLEN AUTO/ HAND GUN I| 11609

STOLEN AUTO/HGV || 11106

STOLEN HANDGUN || 10692

SWITCH BLADE I।

SWITCH BLADE KNIFE I |

SWITCH BLADE KNIFE || 15202

SWITCHBLADE KNIFE । |

THEFT || 10692

Theft: Less \$1,000 Value, Handgun Violation I | 10621

THREAT WITH DEADLY WEAPON II 11415

TRAFFIC/HGV || 11609

TRANSPORTING HANDGUN IN VEHICL I I 10175

Trespass: Private Property, Concealed Deadly Weapon I| 22220

Trespass: Private Property, Deadly Weapon I| 22220

Trespass: Private Property, Poss Of Deadly Weapon I| 22220

TRESPASS/ DEADLY WEAPON || 22220

TRESSPASSING || 15212

WEAPONS VIOLATION II

WEAPONS VIOLATION I| 35250

\section{Common Assault}

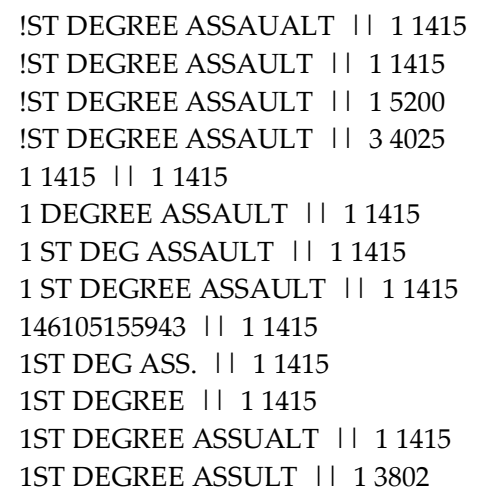


1ST DEGREE BURGLARY || 11415

1ST DEGREE SEX OFFENSE || 11415

2 DEGREE ASSAULT || 11415

2 DEGREE ASSULT I। 11415

2 ND DEGREE ASSAULT | | 11415

2N DEGREE ASSAULT | | 11415

2N DG ASSAULT || 11415

2ND SEGREE ASSAULT | | 11415

2ND AGG. ASSULT || 11415

2ND ASSAULT || 11415

2ND ASSAULT (DOMESTIC) | | 11415

2ND ASSAULT DOMESTIC || 11415

2ND ASSAULT/COMMON ASSAULT || 11415

2ND ASSUALT | | 11415

2ND COMMON ASSAULT I| 11415

2ND DEDREE ASSAULT । | 11415

2ND DEFREE ASSAULT || 11415

2ND DEG AGREE | | 11415

2ND DEG ASAULT | | 11415

2ND DEG ASLT || 11415

2ND DEG ASLT । | 20050

2ND DEG ASSAQULT । | 11415

2ND DEG ASSAULLT || 11415

2ND DEG ASSAULT | | 10505

2ND DEG ASSAULT || 11415

2ND DEG ASSAULT || 11425

2ND DEG ASSAULT || 20254

2ND DEG ASSAULT || 22220

2ND DEG ASSAULT । | 23030

2ND DEG ASSAULT || 35399

2ND DEG ASSAULT DOMESTIC || 11415

2ND DEG ASSAULT ON LAW ENFORCE I। 11415

2ND DEG ASSUALT । I 11415

2ND DEG ASSULT | | 11415

2ND DEG. ASSAULT || 11415

2ND DEG. ASSAULT || 34025

2ND DEG.ASSAULT || 11415

2ND DEGREE ASSAULT || 11415

2ND DEGREE || 11415

2ND DEGREE ASSAULT | | 11415

2ND DEGREE ASAULT || 11415

2ND DEGREE ASSAILT || 11415

2ND DEGREE ASSAULT । ।

2ND DEGREE ASSAULT | | 10173

2ND DEGREE ASSAULT | | 10521

2ND DEGREE ASSAULT | | 10621

2ND DEGREE ASSAULT | | 11415

2ND DEGREE ASSAULT | | 13802

2ND DEGREE ASSAULT || 14803

2ND DEGREE ASSAULT || 15200

2ND DEGREE ASSAULT । | 20050

2ND DEGREE ASSAULT | | 20105

2ND DEGREE ASSAULT | | 20254

2ND DEGREE ASSAULT | | 20480

2ND DEGREE ASSAULT । I 20700

2ND DEGREE ASSAULT | | 22220

2ND DEGREE ASSAULT || 23000

2ND DEGREE ASSAULT | | 23030

2ND DEGREE ASSAULT | | 34025

2ND DEGREE ASSAULT || 35399 
2ND DEGREE ASSAULT || 3A1005

2ND DEGREE ASSAULT । | 43550

2ND DEGREE ASSAULT (DOMESTIC) । | 11415

2ND DEGREE ASSAULT / COMMON AS I| 11415

2ND DEGREE ASSAULT 1_1415 || 11415

2ND DEGREE ASSAULT DOMESTIC । | 11415

2ND DEGREE ASSAULT(DOMESTIC) | | 11415

2ND DEGREE ASSAULT/4TH DEGREE | | 11415

2ND DEGREE ASSAULT/CHILD ABUS || 11415

2ND DEGREE ASSAULT/ DOMESTIC || 11415

2ND DEGREE ASSAULT/BURGLARY | | 11415

2ND DEGREE ASSAULT/DISORDERLY || 11415

2ND DEGREE ASSAUT | | 11415

2ND DEGREE ASSUALT | | 11415

2ND DEGREE ASSUAULT | | 11415

2ND DEGREE ASSULT || 11415

2ND DEGREE COMMON ASSAULT | | 11415

2ND DEGREE DEGREE | | 11415

2ND DEGREE DOMESTIC ASSALT | | 11415

2ND DEGREE DOMESTIC ASSAULT || 11415

2ND DEGRES ASSAULT I 11415

2ND DEHREE ASSAULT || 11415

2ND DERGREE ASSAULT || 20700

2ND DG ASSAULT DOMESTIC | | 11415

2ND DG. ASSAULT || 11415

2ND DGREE ASSAULT | | 11415

2ND DRGREE ASSAULT || 11415

2ND. DEG. ASSAULT || 11415

2ND. DEGREE ASSAULT | | 11415

2NE DEGREE ASSAULT | | 11415

2NS DEG ASSAULT || 11415

4TH BURGLARY || 20254

4TH DEG B\&E I| 11415

4TH DEG SEX OFFENSE | | 11415

4TH DEGREE ASSAULT || 11130

4TH DEGREE BURGLARY | | 11415

4TH DEGREE BURGLARY I | 20254

4TH DEGREE SEX OFFENSE | | 11415

4TH DEGREE SEXUAL ASSAULT || 11415

AAG ASSAULT | | 35399

ABDUCTION || 11415

AFFRAY || 11415

Affray, Assault I| 35399

Affray, Assault / Affray II 35399

Affray, Common Assault II 35399

AFFRAY/COMMON ASSAULT || 11415

AFRRAY | 11415

AGG AS;T I| 11415

AGG ASS. BY POINTING || 11415

AGG ASS/ B\&E || 11415

AGG ASSUALT || 11415

AGG ASSUALT BY CUTTING (DOSMES I। 11415

AGG ASSUALT BY THREAT | | 11106

AGG ASSULT | | 11415

AGG ASSULT || 14200

AGG ASSUSLY | | 11415

AGG. | I 11415

AGG. ASS BY THREAT || 11415

AGG. ASSUALT | 11415

AGG. ASSUALT CONSPIRACY || 11415 
AGG. ASSULT || 11415

AGG.ASSULT || 11415

AGG/ASSULT BY THREAT | | 11415

AGGRAAVATED | 11415

AGGRAVATED || 11415

AGGRAVATED ASSUALT || 11415

AGGRAVATED ASSUALT | | 23000

AGGRAVATED ASSULT || 11415

AGGRAVATTED || 11415

AGGRAVTED ASSUALT | | 11415

AGGRIVATED ASSULT || 11415

AGR ASSAULT I| 11415

AGRAVATED ASSAULT || 11415

AGRRAVATED ASSAULT || 11415

AGRRIVATED ASSAULT || 11415

Alc. Bev./Intox:Endanger, Assault I| 14200

Alc. Bev./Intox:Endanger, Assault 2Nd Degree I| 14200

Alc. Bev./Intox:Endanger, Assault By Threat I| 14200

Alc. Bev./Intox:Endanger, Assualt || 14200

Alc. Bev./Intox:Endanger, Common Assault I| 14200

Alc. Bev./Intox:Endanger, Second Degree Assault I| 14200

ARMED PERSON I| 11415

ASAAULT 2ND DEGREE || 11415

ASAULT || 11415

ASAULT SEC DEGREE | | 11415

ASLT || 11415

ASLT 2ND || 11415

ASLT 2ND DEG || 11415

ASLT 2ND DEGREE || 11415

ASLT SEC DEG I।

ASS. ON POLICE II 11415

ASSAILT || 11415

ASSASULT SECOND DEGREE I I 11415

ASSASULT- 2ND DEGREE || 11415

ASSAUALT 2ND DEGREE || 11415

ASSAUKT I| 23030

ASSAULLT-SECOND DEGREE | | 11415

Assault II

ASSAULT II

ASSAULT || 10042

ASSAULT || 10043

ASSAULT || 10173

ASSAULT || 10334

ASSAULT || 10340

ASSAULT || 10356

ASSAULT || 10521

ASSAULT || 10600

ASSAULT || 10621

ASSAULT || 10881

ASSAULT || 11093

ASSAULT || 11116

ASSAULT || 11119

ASSAULT || 11130

ASSAULT || 11137

ASSAULT || 11285

ASSAULT || 11313

ASSAULT || 11338

ASSAULT || 11415

ASSAULT I| 11416

ASSAULT || 11614 


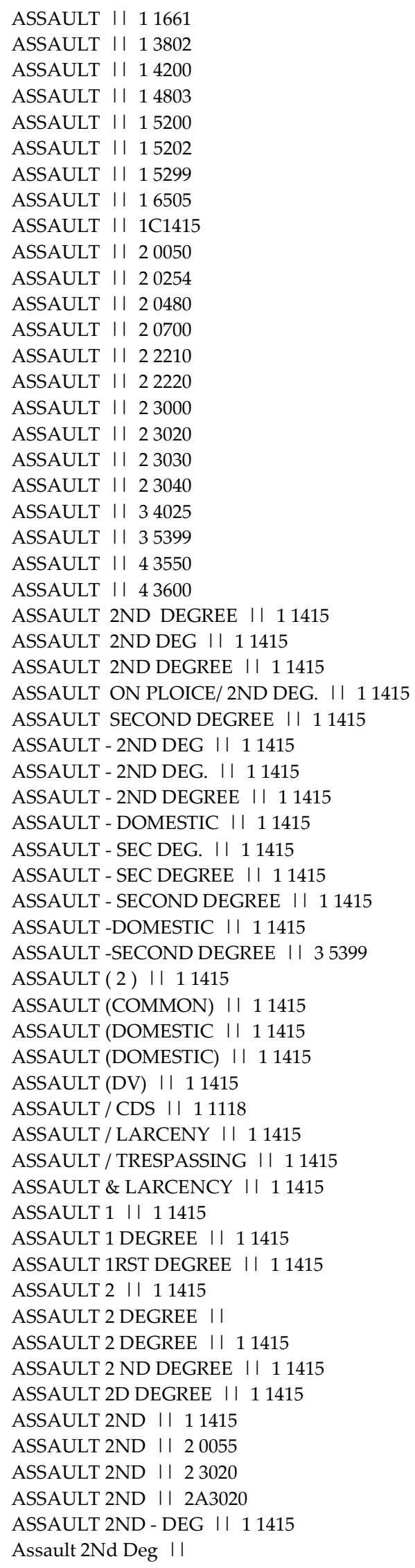


ASSAULT 2ND DEG । |

ASSAULT 2ND DEG || 10521

ASSAULT 2ND DEG || 10600

ASSAULT 2ND DEG | | 10621

ASSAULT 2ND DEG || 11415

ASSAULT 2ND DEG || 20254

ASSAULT 2ND DEG | | 22220

ASSAULT 2ND DEG. I| 11415

ASSAULT 2ND DEGREE || 11137

ASSAULT 2ND DEGREE || 11415

ASSAULT 2ND DEGREE || 13802

ASSAULT 2ND DEGREE || 14200

ASSAULT 2ND DEGREE || 20050

ASSAULT 2ND DEGREE || 20055

ASSAULT 2ND DEGREE I| 20254

ASSAULT 2ND DEGREE || 23030

ASSAULT 2ND DEGREE/ DOMESTIC । | 11415

ASSAULT 2ND DEGREE/ UNARMED RO | | 20700

ASSAULT 2ND DG | | 11415

ASSAULT 2ND DGE || 11415

ASSAULT 2ND DGR || 11415

ASSAULT 2ND DGREE | | 11415

ASSAULT 2ND DOMESTIC || 11415

ASSAULT 2ND. DEG. II 11415

ASSAULT 2ND. DEGREE || 11415

ASSAULT AND BATTERY || 11415

ASSAULT ASSAULT || 11415

ASSAULT BY POINTING || 11338

ASSAULT BY POINTING || 11415

ASSAULT BY THREAT | | 11415

ASSAULT BY THREAT | | 16505

ASSAULT BY THREAT । 1 C1415

ASSAULT BY THREAT I | 20050

ASSAULT BY THREAT I | 20055

ASSAULT BY THREAT || 20105

ASSAULT BY THREAT I | 22220

ASSAULT COMM || 11415

ASSAULT COMMON || 11415

ASSAULT DEGREE || 11415

ASSAULT DOMESTIC I| 11415

ASSAULT DOMESTIC VIOLENCE || 11415

ASSAULT DV || 11415

ASSAULT II || 11415

ASSAULT II DEGREE | | 11415

ASSAULT IN THE 2ND DEGREE | | 11415

ASSAULT ON DOC EMPLOYEE ETC. I I 21399

ASSAULT ON OFFICER II 11415

ASSAULT ON OFFICER 2ND DEGREE I। 11416

ASSAULT ON PLOICE || 11415

ASSAULT PISTOL ROSTER VIO || 35250

ASSAULT SEC DEG I ।

ASSAULT SEC DEG I| 11415

ASSAULT SEC DEG-LAW ENFORCMENT I। 11416

Assault Sec Deg-Law Enforcment, Assault Sec Deg-Law II 11416

Assault Sec Deg-Law Enforcment, Assault Sec Deg-Le O II 11416

Assault Sec Deg-Law Enforcment, Cds II 11416

Assault Sec Deg-Law Enforcment, Cds Violation I| 11416

Assault Sec Deg-Law Enforcment, Disorderly Conduct II 11416

ASSAULT SEC DEG-LE OFF/P\&P AG | | 11416

ASSAULT SEC DEG-LE/P\&P/FIRE/EM || 11416 
ASSAULT SEC DEG. I। 11415

ASSAULT SEC DEGREE || 10621

ASSAULT SEC DEGREE || 11415

ASSAULT SEC DEGREE I। 11416

ASSAULT SEC DEGREE. II

ASSAULT SEC-DEGREE || 11415

ASSAULT SEC. DEGREE | | 11415

ASSAULT SECOND | | 11415

ASSAULT SECOND DEGREE || 11415

ASSAULT SECOND DEGREE || 10043

ASSAULT SECOND DEGREE I | 11415

ASSAULT SECOND DEGREE | | 11661

ASSAULT SECOND DEGREE || 13802

ASSAULT SECOND DEGREE || 15200

ASSAULT SECOND DEGREE || 23020

ASSAULT SECOND DEGREE || 23030

ASSAULT SECOND DEGREE | | 43550

ASSAULT SECOND DEGRESS | | 11415

ASSAULT SECOND DGEREE I | 11415

ASSAULT WPNS DLR VIOLATION I | 10475

ASSAULT WPNS SALE-TRANSFER I | 10474

ASSAULT- 2ND DEGREE I I 11415

ASSAULT-SECOND DEGREE || 11415

ASSAULT-2ND DEG । ।

ASSAULT-2ND DEGREE | | 11415

ASSAULT-DOMESTIC || 11415

ASSAULT-SEC DEG । ।

ASSAULT-SEC DEGREE । ।

ASSAULT-SEC DEGREE | | 11415

ASSAULT-SECOND DEGREE | | 11415

ASSAULT-SECOND DGREE | | 11415

ASSAULT, 2ND DEGREE || 11415

Assault, Assault || 11313

ASSAULT, CDS POSS. I I 11415

ASSAULT, SECOND DEGREE || 11415

ASSAULT: SECOND DEGREE I| 11415

ASSAULT/ HOME INVASION || 11415

ASSAULT/SECOND DEGREE || 11415

ASSAULT/COMMON DOMESTIC || 10042

ASSAULT/DEST OF PROPERTY | | 11415

ASSAULT/DESTRUCTION OF PROPERT I। 11415

ASSAULT/DOMESTIC || 11415

ASSAULT/DUI || 11415

ASSAULT/HINDERING | 11415

ASSAULT/LARCENY || 11415

ASSAULT/SECOND DEGREE || 11415

ASSAULT/THEFT I| 10621

ASSAULT/THEFT I| 11415

ASSAUNT 2ND DEGREE || 11415

ASSAUT I| 11415

ASSAUT 2ND-DV I I 11415

ASSAUT/ HOME INVASION | | 11415

ASSAUTL 2ND DEGREE | | 11415

ASSLT 2ND/DOC EMPLOYEE ETC. I I 11440

Asslt 2Nd/Doc Employee Etc., Asslt 2Nd/Doc Employee I। 11440

Asslt-Sec Degree, 11415 Assault Sec I| 11415

Asslt-Sec Degree, 1 Deg Assault I| 11415

Asslt-Sec Degree, 1 Degree Assault | | 11415

Asslt-Sec Degree, 2 Nd Degree Assault | | 11415

Asslt-Sec Degree, 2Nd Deg Assault I| 11415 
Asslt-Sec Degree, 2Nd Degree Assault | | 11415

Asslt-Sec Degree, 2Nd Agree Assault || 11415

Asslt-Sec Degree, 2Nd Assault || 11415

Asslt-Sec Degree, 2Nd Assault Deg I 11415

Asslt-Sec Degree, 2Nd Deg Aslt | | 11415

Asslt-Sec Degree, 2Nd Deg Assault I | 11415

Asslt-Sec Degree, 2Nd Deg. Assault I| 11415

Asslt-Sec Degree, 2Nd Deg. Assualt I| 11415

Asslt-Sec Degree, 2Nd Deg.Assault | 11415

Asslt-Sec Degree, 2Nd Degree II 11415

Asslt-Sec Degree, 2Nd Degree Assasult I| 11415

Asslt-Sec Degree, 2Nd Degree Assault || 11415

Asslt-Sec Degree, 2Nd Degree Assault (Domestic) || 11415

Asslt-Sec Degree, 2Nd Degree Assult || 11415

Asslt-Sec Degree, 2Nd Degree Common Assault I| 11415

Asslt-Sec Degree, 2Nd Dgree Assault || 11415

Asslt-Sec Degree, 2Nd Dgreee Assault || 11415

Asslt-Sec Degree, 2Nd. Deg. Assault || 11415

Asslt-Sec Degree, Affarry I| 11415

Asslt-Sec Degree, Affray II 11415

Asslt-Sec Degree, Asault || 11415

Asslt-Sec Degree, Aslt I| 11415

Asslt-Sec Degree, Aslt 2Nd Degree || 11415

Asslt-Sec Degree, Assasult I| 11415

Asslt-Sec Degree, Assault I| 11415

Asslt-Sec Degree, Assault - 2Nd Degree II 11415

Asslt-Sec Degree, Assault / Affray II 11415

Asslt-Sec Degree, Assault 1 Degree || 11415

Asslt-Sec Degree, Assault 2 || 11415

Asslt-Sec Degree, Assault 2 Degree || 11415

Asslt-Sec Degree, Assault 2Nd || 11415

Asslt-Sec Degree, Assault 2Nd Degree II 11415

Asslt-Sec Degree, Assault 2Nd Deg I I 11415

Asslt-Sec Degree, Assault 2Nd Deg - Domestic Vio II 11415

Asslt-Sec Degree, Assault 2Nd Deg. I I 11415

Asslt-Sec Degree, Assault 2Nd Degre I| 11415

Asslt-Sec Degree, Assault 2Nd Degree || 11415

Asslt-Sec Degree, Assault By Pointing At I| 11415

Asslt-Sec Degree, Assault By Theat || 11415

Asslt-Sec Degree, Assault By Threat I| 11415

Asslt-Sec Degree, Assault By Threat/Domestic I| 11415

Asslt-Sec Degree, Assault Commom I| 11415

Asslt-Sec Degree, Assault Common II 11415

Asslt-Sec Degree, Assault In The Second Degree I| 11415

Asslt-Sec Degree, Assault Sec Degree I| 11415

Asslt-Sec Degree, Assault Sec. Deg I| 11415

Asslt-Sec Degree, Assault Sec. Degree I| 11415

Asslt-Sec Degree, Assault Second I| 11415

Asslt-Sec Degree, Assault Second Deg. I I 11415

Asslt-Sec Degree, Assault Second Degree || 11415

Asslt-Sec Degree, Assault Seg Degree II 11415

Asslt-Sec Degree, Assault With A Dangerous Weapo II 11415

Asslt-Sec Degree, Assault- Sec Degree II 11415

Asslt-Sec Degree, Assault- Second Degree I| 11415

Asslt-Sec Degree, Assault-Sec Degree || 11415

Asslt-Sec Degree, Assault, 2'Nd Degree || 11415

Asslt-Sec Degree, Assault, Second Degree II 11415

Asslt-Sec Degree, Assault/ Disorderly | | 11415

Asslt-Sec Degree, Assault/Cds II 11415

Asslt-Sec Degree, Assualt I| 11415 
Asslt-Sec Degree, Assualt 2Nd || 11415

Asslt-Sec Degree, Assualt 2Nd Degree || 11415

Asslt-Sec Degree, Assualt By Threat || 11415

Asslt-Sec Degree, Assualt On Officer II 11415

Asslt-Sec Degree, Assualt Second Degree II 11415

Asslt-Sec Degree, Assult 2Nd Degree | | 11415

Asslt-Sec Degree, Attempt B And E || 11415

Asslt-Sec Degree, Attempted Assault || 11415

Asslt-Sec Degree, Attempted B\&E I I 11415

Asslt-Sec Degree, Attempted Larceny I | 11415

Asslt-Sec Degree, B \& E I| 11415

Asslt-Sec Degree, B\&E I| 11415

Asslt-Sec Degree, Bb Gun Violation I| 11415

Asslt-Sec Degree, Breaking And Entering II 11415

Asslt-Sec Degree, Burglary II 11415

Asslt-Sec Degree, Burglary 3Rd Degree II 11415

Asslt-Sec Degree, Cds I| 11415

Asslt-Sec Degree, Cds Marijuana II 11415

Asslt-Sec Degree, Cds Poss Intent || 11415

Asslt-Sec Degree, Cds Poss Mari I| 11415

Asslt-Sec Degree, Cds Viol II 11415

Asslt-Sec Degree, Cds Violation II 11415

Asslt-Sec Degree, Child Abuse || 11415

Asslt-Sec Degree, Child Abuse Physical || 11415

Asslt-Sec Degree, Cmmmon Assault II 11415

Asslt-Sec Degree, Com Assault || 11415

Asslt-Sec Degree, Com. Assault I| 11415

Asslt-Sec Degree, Com/Assault || 11415

Asslt-Sec Degree, Comjmon Assault II 11415

Asslt-Sec Degree, Comm Assault || 11415

Asslt-Sec Degree, Comman Assault I| 11415

Asslt-Sec Degree, Commen Assault I I 11415

Asslt-Sec Degree, Commmon Assault II 11415

Asslt-Sec Degree, Commom Assault I| 11415

Asslt-Sec Degree, Common II 11415

Asslt-Sec Degree, Common Asault II 11415

Asslt-Sec Degree, Common Aslt || 11415

Asslt-Sec Degree, Common Assaualt II 11415

Asslt-Sec Degree, Common Assault || 11415

Asslt-Sec Degree, Common Assault (Domestic Viole II 11415

Asslt-Sec Degree, Common Assault (Domestic) I| 11415

Asslt-Sec Degree, Common Assault 2Nd Deg II 11415

Asslt-Sec Degree, Common Assault 2Nd Deg Assault I| 11415

Asslt-Sec Degree, Common Assault 2Nd Degree I| 11415

Asslt-Sec Degree, Common Assault Domestic II 11415

Asslt-Sec Degree, Common Assault On A Minor II 11415

Asslt-Sec Degree, Common Assault/ Second Degree II 11415

Asslt-Sec Degree, Common Assaut I I 11415

Asslt-Sec Degree, Common Asslt || 11415

Asslt-Sec Degree, Common Assualt || 11415

Asslt-Sec Degree, Common Assult || 11415

Asslt-Sec Degree, Commonassault || 11415

Asslt-Sec Degree, Commond Assault | | 11415

Asslt-Sec Degree, Commoun Assault | | 11415

Asslt-Sec Degree, Commount Assault || 11415

Asslt-Sec Degree, Cutting || 11415

Asslt-Sec Degree, Dest Property II 11415

Asslt-Sec Degree, Destruction Of Property I| 11415

Asslt-Sec Degree, Diorderly/ Resisting/Ftc || 11415

Asslt-Sec Degree, Disorderly I| 11415 
Asslt-Sec Degree, Disorderly Conduct II 11415

Asslt-Sec Degree, Disorderly Conduct || 11415

Asslt-Sec Degree, Dom Assault || 11415

Asslt-Sec Degree, Dom. Assault I| 11415

Asslt-Sec Degree, Domestic || 11415

Asslt-Sec Degree, Domestic 2Nd Degree I| 11415

Asslt-Sec Degree, Domestic Assault I I 11415

Asslt-Sec Degree, Domestic Assault - 2 Nd Degree II 11415

Asslt-Sec Degree, Domestic Assault Common II 11415

Asslt-Sec Degree, Domestic Common Assault I| 11415

Asslt-Sec Degree, Domestic Common Assualt || 11415

Asslt-Sec Degree, Domestic Violence II 11415

Asslt-Sec Degree, Drunk \& Disorderly || 11415

Asslt-Sec Degree, Dv. Common Assault I| 11415

Asslt-Sec Degree, Fail To Obey | | 11415

Asslt-Sec Degree, False Imprisonment I| 11415

Asslt-Sec Degree, Fourth Degree Sex Offense I| 11415

Asslt-Sec Degree, Frist Degree Assault I| 11415

Asslt-Sec Degree, Fto II 11415

Asslt-Sec Degree, Hindering II 11415

Asslt-Sec Degree, Hindering Investigation I| 11415

Asslt-Sec Degree, Home Invasion I I 11415

Asslt-Sec Degree, Ist Deg Assault || 11415

Asslt-Sec Degree, Ist Degree Assault || 11415

Asslt-Sec Degree, Larceny I| 11415

Asslt-Sec Degree, Lst Degree Assault || 11415

Asslt-Sec Degree, Mal Dest Of Prop I| 11415

Asslt-Sec Degree, Mal Destruction Of Property || 11415

Asslt-Sec Degree, Malacious Destruction Of Prope I| 11415

Asslt-Sec Degree, Maliic Destruction I| 11415

Asslt-Sec Degree, Mdop - 500 || 11415

Asslt-Sec Degree, Physical Child Abuse I| 11415

Asslt-Sec Degree, Pos Cds II 11415

Asslt-Sec Degree, Poss Cds || 11415

Asslt-Sec Degree, Poss Cocaine || 11415

Asslt-Sec Degree, Poss Marijuana I| 11415

Asslt-Sec Degree, Poss Of Cocaine || 11415

Asslt-Sec Degree, Poss W/Int Marijuana II 11415

Asslt-Sec Degree, Pwi Cocaine I| 11415

Asslt-Sec Degree, Resisting Arrest I 11415

Asslt-Sec Degree, Revoked \& Suspended || 11415

Asslt-Sec Degree, Sce Degree Assault I| 11415

Asslt-Sec Degree, Sec Deg Assault I I 11415

Asslt-Sec Degree, Sec Degree Assault I| 11415

Asslt-Sec Degree, Sec. Degree Assault || 11415

Asslt-Sec Degree, Second Deg Aslt I| 11415

Asslt-Sec Degree, Second Deg Assault || 11415

Asslt-Sec Degree, Second Degg Assault I| 11415

Asslt-Sec Degree, Second Degree Assault II 11415

Asslt-Sec Degree, Second Degree Asssault || 11415

Asslt-Sec Degree, Second Degree Assualt I| 11415

Asslt-Sec Degree, Second Degree Assult || 11415

Asslt-Sec Degree, Second Degrre Assault || 11415

Asslt-Sec Degree, Second Drgree Assault || 11415

Asslt-Sec Degree, Second-Degree Assault || 11415

Asslt-Sec Degree, Sex Offense I I 11415

Asslt-Sec Degree, Stolen Auto II 11415

Asslt-Sec Degree, Theft I I 11415

Asslt-Sec Degree, Theft /Assault 2Nd Deg II 11415

Asslt-Sec Degree, Theft Less Than \$500 I| 11415 
Asslt-Sec Degree, Theft Under \$1000 | | 11415

Asslt-Sec Degree, Theft Under 100.00 || 11415

Asslt-Sec Degree, Theft: Less Than \$100.00 || 11415

Asslt-Sec Degree, Trespass I| 11415

Asslt-Sec Degree, Trespassing || 11415

Asslt-Sec Degree, Trespassing/Disorderly I| 11415

Asslt-Sec Degree, Violation Of Exparte II 11415

Asslt-Sec Degree, Violation Peace Order I| 11415

ASSSAULT I| 11415

ASSSAULT SEC DEGREE I | 11415

ASSUALT I| 11415

ASSUALT 1ST DEGREE | | 11415

ASSUALT 2ND || 13802

Assualt 2Nd Degree II

ASSUALT 2ND DEGREE | | 11415

ASSUALT FIRST DEGREE || 11415

ASSUALT ON POLICE || 10600

ASSUALT ON POLICE II 11415

ASSUALT ON POLICE OFFICER/RESI || 11415

ASSUALT SEC DEGREE || 11415

ASSUALT-SEC || 11415

ASSUALT/ LARCENY | 11415

ASSULT || 11415

ASSULT I| 34025

ASSULT 2ND DEGREE || 11415

ASSULT COMMON || 11415

ASSULT ON POLICE || 11416

ASSULT ON POLICE || 20050

ATT B\&E || 11415

ATT ESCAPE || 11415

ATT-ASLT SEC DEG-LE OFC/P\&P AG I | 1 A1416

ATT-ASSAULT-SEC DEGREE || 1A1415

Att-Asslt-Sec Degree, Att-Assault-Sec Degree II 1A1415

Att-Burglary-Fourth Degree, 2Nd Degree Assault II 2A3030

Att-Burglary-Fourth Degree, Second Degree Assault I| 2A3030

Att-Escape - Second Degree, Assault I| 2A1020

Att-Kidnapping, Assault I| 3A1005

ATT-VIOLATE EXPARTE/PROT ORDER || 2A0254

ATT. 2ND DEGREE ASSAULT || 11415

ATT. ASSAULT || 11415

ATTEMPT ASSAULT || 11415

ATTEMPT B \& E || 11415

ATTEMPT B AND E I। 11415

ATTEMPT BURGLARY | | 11415

ATTEMPT STOLEN AUTO || 11415

ATTEMPTED 2ND DEGREE ASSAULT || 11415

ATTEMPTED ABDUCTION || 11415

ATTEMPTED B+E || 11415

ATTP. ASSAULT || 11415

AUTO THEFT || 20254

B \& E || 11415

B \& E || 20254

B \&E || 11415

B\&E || 11415

B\&E ATT । 11415

B\&E RESIDENTIAL | | 11415

B+E || 11415

BB GUN VIOLATION I| 11415

BREAK AND ENTERING । | 11415

BREAKING AND ENTERING || 11415 


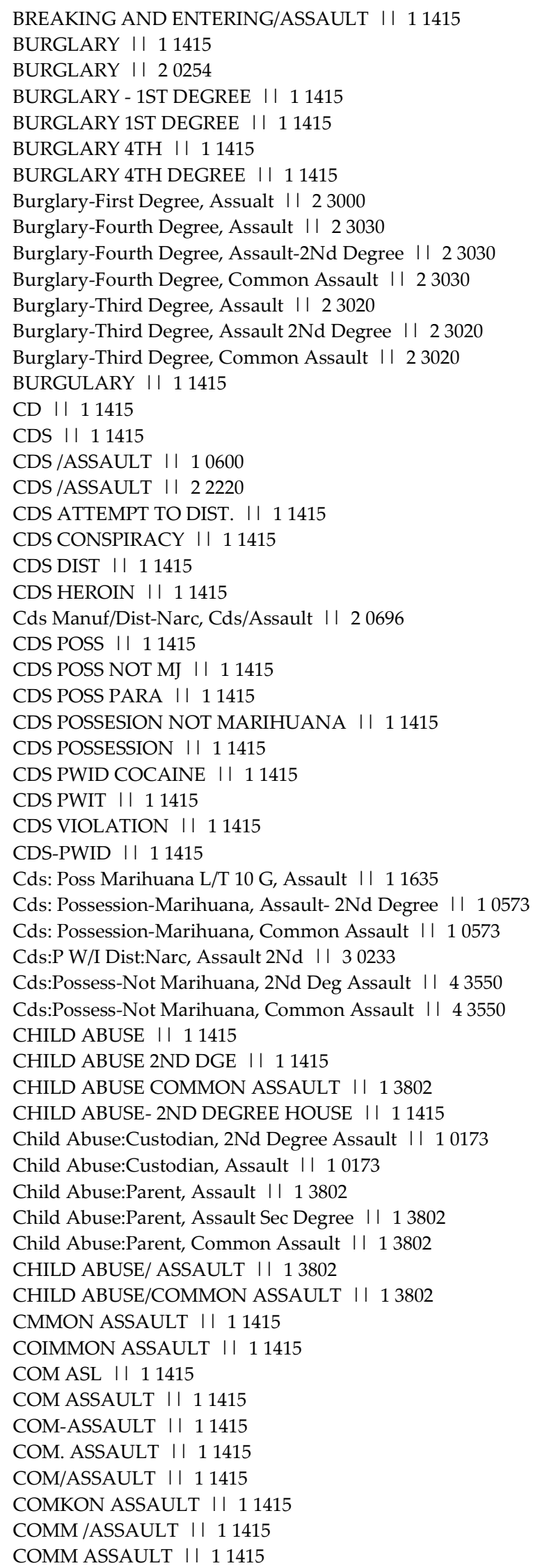




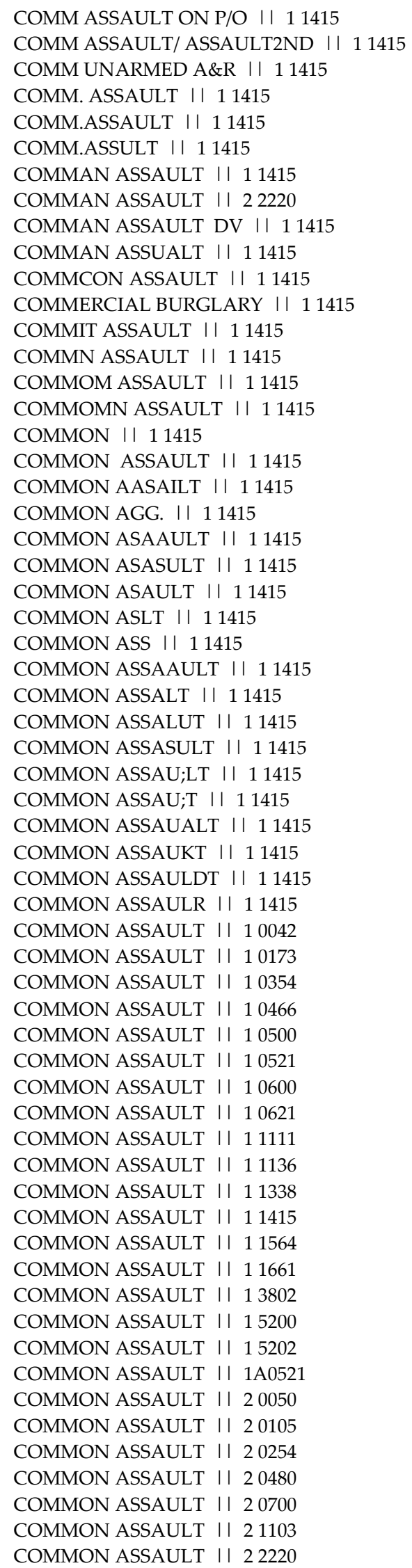


COMMON ASSAULT || 23000 COMMON ASSAULT || 23020 COMMON ASSAULT || 23030 COMMON ASSAULT || 2A3030 COMMON ASSAULT I| 34025 COMMON ASSAULT || 34030 COMMON ASSAULT || 35399 COMMON ASSAULT || 3A1005 COMMON ASSAULT || 43600 COMMON ASSAULT (DOMESTIC) I I 11415 COMMON ASSAULT ( DV ) | | 11415

COMMON ASSAULT (DOMESTIC) I। 11415 COMMON ASSAULT (DV RELATED) || 11415 COMMON ASSAULT (DV) | | 11415 COMMON ASSAULT 2ND || 11415 COMMON ASSAULT 2ND DEGREE || 11415 COMMON ASSAULT BY THREAT || 11415 COMMON ASSAULT DOMESTIC || 11415 COMMON ASSAULT DOMESTIC RELATE || 11415 COMMON ASSAULT DV RELATED || 11415 COMMON ASSAULT- DOMESTIC || 11415 COMMON ASSAULT-DOMESTIC || 11415 COMMON ASSAULT, DOMESTIC || 11415 COMMON ASSAULT(DOMESTIC) || 11415 COMMON ASSAULT/ DOMESTIC ASSAU || 11415 COMMON ASSAULT/2ND DEG || 11415 COMMON ASSAULT/CDS VIOLATION || 11415 COMMON ASSAULT/DOMESTIC || 11415 COMMON ASSAULTH || 10233 COMMON ASSAULTH || 11415 COMMON ASSAUOLT || 11415 COMMON ASSAUT I| 11415 COMMON ASSAUT-DOMESTIC || 11415 COMMON ASSAYLT || 11415 COMMON ASSLT (D.V.) || 11415 COMMON ASSSAULT || 11415 COMMON ASSUALT || 11415 COMMON ASSUALT || 13802 COMMON ASSUALT 2ND DEGREE || 11415 COMMON ASSUALT, DOMESTIC || 11415 COMMON ASSUALT/AFFRAY || 11415

COMMON ASSULT I| 11415 COMMON ASSULT || 30233 COMMON ASSULT (DV) I। 11415 COMMON DOMESTIC ASSAULT || 11415 COMMON-ASSAULT || 11415 COMMON. ASSAULT || 11415 COMMONASSAULT || 11415 COMMONN ASSAULT || 11415 COMMONON ASSAULT || 11415 COMMONT ASSAULT || 11415 COMON ASSAULT I| 11415 CON-ASSAULT-SEC DEGREE || 1C1415 CON-ASSAULT-SEC DEGRREE | |

Con-Asslt-Sec Degree, Con-Assault-Sec Degree II 1 C1415 CON-VIOLATE EXPARTE/PROT ORDER I | 2C0254

COOMMON ASSAULT || 11415 COOMON ASSAULT || 11415 CR 3203 || 11415

DERSTRUCTION OF PROPERTY || 11415 
DESORDELY || 11415

DEST OF PROP || 11415

DEST OF PROPERTY I। 11415

DEST. PROPERTY || 11415

DESTEVATION OF PROPERTY || 11415

DESTRUCTION OF PROP || 11415

DESTRUCTION OF PROPERTY || 11415

DESTRUCTION OF PROPERTY || 20254

DESTRUCTION PF PROPERTY || 11415

DESTRUCTION/ DOMESTIC I| 11415

DESTRUTION OF PROP || 11415

DESTSTRUCTION OF PROPERTY || 11415

DETSTRUCTION OF PROPERTY || 11415

DIRT BIKE VIOLATION || 11415

DIRTBIKE VIOLATION || 11415

Dis.Erly Conduct, 2Nd Deg Assault || 20050

Dis.Erly Conduct, 2Nd Degree Assault || 20050

Dis.Erly Conduct, Assault || 20050

Dis.Erly Conduct, Assault By Threat | 20050

Dis.Erly Conduct, Assault Law Enforcement I| 20050

Dis.Erly Conduct, Assault Second Degree I| 20050

Dis.Erly Conduct, Common Assault I| 20050

DISODERLY CONDUCT || 11415

DISORDERELY CONDUCT | | 11415

DISORDERLY || 11415

DISORDERLY / ASSAULT || 11415

DISORDERLY \& ASSAULT | | 11415

DISORDERLY CONDUCT || 11415

DISORDERLY CONDUCT/COMMON ASS |। 11415

DISORDERLY CONDUCTED | | 11415

DISORDERLY INTOX || 11415

DISORDERLY INTOXICATION || 11415

DISORDERLY/ASSAULT I| 11415

DOM ASSAULT || 11415

DOM. ASSAULT || 11415

DOMESTIC || 11415

DOMESTIC ASSAULT || 11415

DOMESTIC COMMON ASSAULT || 11415

DOMESTIC 2ND DEGREE ASSAULT || 11415

DOMESTIC 2ND DEGREE ASUALT | | 11415

DOMESTIC AGG ASSUALT || 11415

DOMESTIC ASLT || 11415

DOMESTIC ASSAULT I| 10621

DOMESTIC ASSAULT I| 11415

DOMESTIC ASSAULT || 15200

DOMESTIC ASSAULT || 23030

DOMESTIC ASSAULT (COMMON) | | 11415

DOMESTIC ASSAULT 2ND I | 11415

DOMESTIC ASSAULT 2ND DEGREE || 11415

DOMESTIC ASSAULT SEC DEGREE || 11415

DOMESTIC ASSUALT । 11415

DOMESTIC ASSUALT (2ND DEGREE) | | 11415

DOMESTIC ASSULT || 11415

DOMESTIC COMMON || 11415

DOMESTIC COMMON ASSAULT I | 11415

DOMESTIC COMMON ASSAULT || 14200

DOMESTIC COMMON ASSAULT || 31005

DOMESTIC RELATED COMMON ASSAUL | | 11415

DOMESTIC VIOLATION II 11415

DOMESTIC VIOLENCE || 11415 
DOMESTIC VIOLENCE ASSAULT || 11415

DOMESTIC VIOLENCE/COMMON ASSA || 11415

DOMESTIC/COMMON ASSAULT || 11415

DOMESTICVIOLENCE || 11415

DOMESTIV ASSAULT || 11415

DOMMESTIC ASSAULT || 11415

DOMOSTIC || 11415

DRIVING ON SUSPENDED LICENSE | | 11415

DRUNK AND DISORDERLY || 11415

DRUNKEN DISORDERLY || 11415

DSIORDERLY CONDUCT I| 11415

DV ASSAULT I| 11415

DV COMMON ASSAULT || 11415

DWI II 11415

EX PARTE VIOLATION I| 20254

EXPARTA VIOLATION || 20254

Fail Obey Renble/Lawfl, 2Nd Degree Assault I| 20055

Fail Obey Renble/Lawfl, Assault I| 20055

Fail Obey Renble/Lawfl, Assault 2Nd Degree II 20055

Fail Obey Renble/Lawfl, Assault Sec Deg | | 20055

Fail Obey Renble/Lawfl, Assault Second Degree I| 20055

Fail Obey Renble/Lawfl, Second Degree Assualt II 20055

FAIL TO OBEY || 11415

FAILURE TO OBEY || 11415

FAILURE TO OBEY POLICE OFFICER I| 20254

FALSE IMPRISIONMENT || 11415

FALSE IMPRISONMENT || 11415

False Imprisonment, Common Assault || 10042

FIRE ARMS VIOLATION | 11415

FLEEING AND ELUDING || 11415

FLEEING ON FOOT । | 11415

FTO || 11415

FTO/DISORDERLY I| 11415

FTO/DISORDERLY || 11415

GAMBLING || 11415

HARASSMENT I| 11415

HINDERING || 11415

HINDERING INVESTIGATION || 11415

HINDERSING | | 11415

HOME INVASION || 11415

HOME INVASION / ASSAULT AND RO | | 11338

HOME INVASION / ASSAULT AND RO || 1 C1338

ILL POSS AMMUNITION || 11415

INTERFERING WITH ARREST I I 11415

INTOXICATED PUBLIC DISTURBANCE || 11415

IST DEGREE ASSAULT || 11415

LARCENCY FROM AUTO || 11415

LARCENY | | 11415

LARCENY || 20254

LARCENY ATTEMPT || 11415

LARCENY FROM AUTO || 11415

LARCERNY || 11415

LEAVING THE SCENE OF ACCIDENT । | 11415

Mal Dest Prop/Valu - \$500, 2Nd Degree Assault II 34025

Mal Dest Prop/Valu - \$500, Assault I I 34025

Mal Dest Prop/Valu - \$500, Common Assault II 34025

Mal Dest Prop/Valu - \$500, Second Degree Assault I| 34025

Mal Destr Prop Value + \$500, Assault I I 34030

Mal Destr Prop Value + \$500, Common Assault I I 34030

MAL DESTRUCTION OF PROPERTY | | 11415 
MAL DESTRUCTION PROPERTY || 11415

MAL. DESTRUCTION OF PROP || 11415

MALICIOUS DESTRUCTION OF PROP || 11415

MALICIOUS DESTRUCTION OF PROPE I | 11415

MUTUAL AFFRAY || 11415

Obstructing \& Hindering, 2Nd Assualt || 10043

OBSTRUCTING AND HINDERING | | 11415

OPEN CONTAINER || 11415

OPEN CONTAINER FTO || 11415

PEACE ORDER VIOLATION II 20254

Peace Order: Fail To Comply, Common Assault II 20105

PHYSICAL CHILD ABUSE || 11415

POSS HEROIN || 11415

POSS MARIJUANA I | 11415

POSS WITH INTENT HEROIN I | 11415

POSS. OF COCAINE || 11415

POSSESSION CONTRABAND || 11415

POSSESSION OF BB GUN I| 11415

POSSESSION OF COCAINE || 11415

PROTECTION ORDER VIOLATED || 20254

PROTECTION ORDER VIOLATION I I 20254

PROTECTIVE ORDER II 20254

PROTECTIVE ORDER VIOLATION || 20254

PWI CDS || 11415

Reckless Endangerment, Assault || 11425

Reckless Endangerment, Domestic Assault II 11425

RESIDENTIAL B/E || 11415

RESIDENTIAL B\&E || 11415

RESIDENTIAL BREAKING AND ENTER | | 11415

RESIDENTIAL BURGLARY || 11415

RESIST ARREST || 11415

Resist/Interfere With Arrest, 2Nd Deg Assault I| 10600

Resist/Interfere With Arrest, 2Nd Degree Assault II 10600

Resist/Interfere With Arrest, Assault I| 10600

Resist/Interfere With Arrest, Assault 2Nd Deg || 10600

Resist/Interfere With Arrest, Common Assault II 10600

Resist/Interfere With Arrest, Common Assault I| 10600

RESISTING ARREST || 11415

ROGUE VAGABOND || 11415

SEC ASSAULT || 11415

SEC DEG ASLT | | 11415

SEC DEG ASSAULT || 11415

SEC DEGEE ASSAULT I I 11415

SEC DEGREE II 11415

SEC DEGREE ASSAULT I।

SEC DEGREE ASSAULT || 11415

SEC DEGREE ASSUALT । | 11415

SEC- DEGREE ASSAULT | | 11415

SEC-DEG ASSAULT || 11415

SEC-DEGREE ASSAULT || 11415

SEC. DEG. ASSAULT I| 11415

SEC. DEGREE ASSAULT I | 11415

SEC. DERGREE ASSAULT | | 11415

SECOND ASSAULT || 11415

SECOND DEG ASLT I| 11415

SECOND DEG ASSAULT | | 11415

SECOND DEG. ASSAULT || 11415

SECOND DEGREE ASAULLT I | 11415

SECOND DEGREE ASLT I | 11415

SECOND DEGREE ASSAULT । | 
SECOND DEGREE ASSAULT || 10173 SECOND DEGREE ASSAULT || 10600 SECOND DEGREE ASSAULT || 11106 SECOND DEGREE ASSAULT || 11415 SECOND DEGREE ASSAULT || 15200 SECOND DEGREE ASSAULT || 20700 SECOND DEGREE ASSAULT | | 23020 SECOND DEGREE ASSAULT || 23030 SECOND DEGREE ASSAULT | | 35399 SECOND DEGREE ASSAULT/ DESTRUC । | 11415 SECOND DEGREE ASSUALT | | 11415 SECOND DEGRRE ASSAULT || 11415 SECOND DGREE ASSAULT | | 11415 SECOND-DEGREE ASSAULT | | 11415 SECONDARY ASSAULT || 11415 SENCOND DEGREE ASSAULT || 11415 SEOND DEGREE ASSAULT || 11415 SERVICE OF PROTECTIVE ORDER || 20254

SEX OFFENSE II 11415

SEX OFFENSE 4TH DEGREE || 11415

SEXUAL ASSAULT || 43600

SIMPLE ASSAULT || 11415

STALKING || 11415

STOLEN AUTO || 11415

STOLEN VEHICLE || 11415

THEFT || 11415

THEFT || 20254

Theft Less Than \$100.00, 2Nd Degree Assault || 10521

Theft Less Than \$100.00, Assault I| 10521

THEFT U/\$1000 || 11415

THEFT UNDER \$100 || 11415

THEFT UNDER \$1000 || 11415

THEFT UNDER \$1000.00 || 11415

THEFT UNDER 100.00 || 11415

Theft: Less \$1,000 Value, Assault Sec Degree || 10621

Theft: Less \$1,000 Value, Common Assault I| 10621

Theft: Less \$1,000 Value, Common Assault 2Nd Degree I| 10621

THEFT/ ASSAULT || 10521

THEFT/ASSAULT || 10521

THEFT/ASSAULT || 11415

THEFT/LARCENY || 11415

THERFT I| 11415

TREPASSING || 11415

TREPASSING/ HOLDING AGAINST WI I| 11415

TRESPASS I| 11415

Trespass-Posted Property, Common Assault II 22210

Trespass: Private Property, Assault 2Nd || 22220

Trespass: Private Property, Assault 2Nd Degree I| 22220

TRESPASSING || 11415

TRESPASSING/ LARCERY | | 11415

TRESSPASSING I| 11415

UNARMED ASSAULT II 11415

UNARMED COMMERCIAL PROPERTY || 11415

UNAUTHORIZED USE || 11415

VILATION OF PROTECTIVE ORDER I | 20254

VILOATION OF PROTECTIVE ORDER | | 20254

VIO OF PROTECTION ORDER | | 20254

VIOALTION OF PROT ORDER I | 11415

VIOL OF PEACE ORDER I| 20254

VIOL OF PROTECTIVE ORDER || 20254 
VIOL PROTECT ORDER || 20254

VIOL PROTECTION ORDER I | 20254

VIOL PROTECTIVE ORDER || 20254

VIOL. EX. PARTE/ASSAULT || 11415

VIOL. OF PROTECTIVE ORDER || 20254

VIOL. OF TEMP. PROTECTIVE ORDE || 20254

VIOL. PROTECTIVE ORDER I| 20254

VIOLATE EX PARTE || 20254

VIOLATE EXPARTE || 20254

VIOLATE EXPARTE/PROT ORDER || 20254

Violate Exparte/Prot Order, Burglary I| 20254

Violate Exparte/Prot Order, Common Assault II 20254

Violate Exparte/Prot Order, Destruction Of Property I| 20254

Violate Exparte/Prot Order, Ex Parte II 20254

Violate Exparte/Prot Order, Fto I| 20254

Violate Exparte/Prot Order, No Front Plate || 20254

Violate Exparte/Prot Order, Protctive Order Violatio I| 20254

Violate Exparte/Prot Order, Second Degree Assault I| 20254

Violate Exparte/Prot Order, Viol. Prot. Order II 20254

Violate Exparte/Prot Order, Viol.Protective Order II 20254

Violate Exparte/Prot Order, Violate Exparte II 20254

Violate Exparte/Prot Order, Violate Exparte/Prot Or II 20254

Violate Exparte/Prot Order, Violate Exparte/Prot Ord II 20254

Violate Exparte/Prot Order, Violate Protection Orde II 20254

Violate Exparte/Prot Order, Violation Ex Parte II 20254

Violate Exparte/Prot Order, Violation Of A Protectiv II 20254

Violate Exparte/Prot Order, Violation Of An Ex-Part I| 20254

Violate Exparte/Prot Order, Violation Of Court Orde || 20254

Violate Exparte/Prot Order, Violation Of Court Order I| 20254

Violate Exparte/Prot Order, Violation Of Ex Parte II 20254

Violate Exparte/Prot Order, Violation Of Ex- Parte I| 20254

Violate Exparte/Prot Order, Violation Of Ex-Parte I| 20254

Violate Exparte/Prot Order, Violation Of Exparte II 20254

Violate Exparte/Prot Order, Violation Of Expate II 20254

Violate Exparte/Prot Order, Violation Of Final Prote I| 20254

Violate Exparte/Prot Order, Violation Of Order Of Pr I| 20254

Violate Exparte/Prot Order, Violation Of Peace Order I| 20254

Violate Exparte/Prot Order, Violation Of Probation I| 20254

Violate Exparte/Prot Order, Violation Of Prot Order I| 20254

Violate Exparte/Prot Order, Violation Of Protection II 20254

Violate Exparte/Prot Order, Violation Of Protective II 20254

Violate Exparte/Prot Order, Violation Of Temporary P || 20254

Violate Exparte/Prot Order, Violation Protective Or || 20254

Violate Exparte/Prot Order, Violtaion Of Protection I| 20254

VIOLATE OF EXPARTE || 11415

VIOLATE PROT ORDER || 20254

VIOLATE PROTECTION ORDER | | 20254

VIOLATE PROTECTIVE ORDER | | 20254

VIOLATED PROTECTIVE ORDER || 20254

VIOLATING PROTECTIVE ORDER || 20254

VIOLATION || 20254

VIOLATION EX PARTE || 20254

VIOLATION EX-PARTE || 20254

VIOLATION EX-PARTE || 20254

VIOLATION EXPARTE || 20254

VIOLATION EXPARTE ORDER || 20254

VIOLATION FINAL PROTECTIVE ORD || 20254

VIOLATION O FEXPARTE || 20254

VIOLATION OF A EXPARTE/PROTECT I| 20254

VIOLATION OF A PEACE ORDER I। 20254 
VIOLATION OF A PIECE ORDER || 20254

VIOLATION OF A PROTECTION ORDE || 20254 VIOLATION OF A PROTECTIVE ODER | | 20254

VIOLATION OF A PROTECTIVE ORDE || 20254

VIOLATION OF A TEMPORARY PROTE || 20254

VIOLATION OF AN EX-PARTE || 20254

VIOLATION OF AN EXPARTE || 20254

VIOLATION OF AN EXPARTE ORDER || 20254

VIOLATION OF COURT DOCUMENT || 11415

VIOLATION OF COURT ORDER I I 20254

VIOLATION OF EX PARTE II 20254

VIOLATION OF EX-PARTE || 20254

VIOLATION OF EX-PARTE || 20254

VIOLATION OF EX-PERTE || 20254

VIOLATION OF EXPARTE || 20254

VIOLATION OF EXPARTIE || 20254

VIOLATION OF FINAL PROTECTIVE || 20254

VIOLATION OF PEACE ORDER | | 11415

VIOLATION OF PEACE ORDER I | 20254

VIOLATION OF PROT ORDER || 20254

VIOLATION OF PROT. ORDER I I 20254

VIOLATION OF PROTECIVE ORDER I | 20254

VIOLATION OF PROTECTION I| 20254

VIOLATION OF PROTECTION ORDER ।| 20254

VIOLATION OF PROTECTION ORER I | 20254

VIOLATION OF PROTECTIVE I| 20254

VIOLATION OF PROTECTIVE COURT || 20254

VIOLATION OF PROTECTIVE ODER || 20254

VIOLATION OF PROTECTIVE ORDER || 11415

VIOLATION OF PROTECTIVE ORDER || 20254

VIOLATION OF PROTECTIVE ORDER/ | | 20254

VIOLATION PEACE ORDER II 20254

VIOLATION PEOTECTION ORDER I| 20254

VIOLATION PROTECTION ORDER || 20254

VIOLATION PROTECTIVE ORDER || 20254

VIOLATON OF EXPARTE II 20254

VIOLATON OF PROTECTION ORDER I| 20254

VIOLLATION OF PEACE ORDER || 20254

VOLIATION OF PEACE ORDER || 11415

VOP || 20254

VOPO || 20254

WITNESS INTIMIDATION || 11415

ZND DEGREE ASSAULT | | 11415

\section{Sex Offense (Subsequently Combined with Rape)}

1-1130 || 11130

1ST DEG B+E || 11130

1ST DEG SEX OFFENSE | | 21102

1ST DEG. SEX OFFENSE || 21102

1ST DEGREE || 11130

1ST DEGREE B \& E | | 11130

1ST DEGREE B\&E || 11130

1ST DEGREE BURGLARY || 11130

1ST DEGREE SEX OFFENSE || 21102

1ST DEGREE SEX OFFENSE | । 31005

2 DEG B \& E || 11130

2,3,4 DEGREE SEX OFFENSE I I 23600

2ND DEG BURGLARY || 11130 
2ND DEG SEX OFFENSE || 21103

2ND DEG SEX OFFENSE || 23600

2ND DEGREE B\&E | | 11130

2ND DEGREE BURGLARY | | 11130

2ND DEGREE SEX OFFENSE || 10322

2ND DEGREE SEX OFFENSE || 21102

2ND DEGREE SEX OFFENSE || 21110

2ND DEGREE SEX OFFENSE || 23600

2ND DEGREE SEXUAL CHILD ABUSE || 10173

3RD DEG BURGLARY | | 11130

3RD DEG SEX OFFENSE I | 33600

3RD DEG. SEX OFFENSE || 33600

3RD DEGREE BURGLARY || 11130

3RD DEGREE SEX OFFENSE || 10922

3RD DEGREE SEX OFFENSE I I 23600

3RD DEGREE SEX OFFENSE | | 33600

3RD DEGREE SEX OFFENSE I। 3A3600

4 DEGREE BURGLARY || 11130

4 TH DEGREE B+E || 11130

4 THE DEGREE BURGLARY || 11130

4T DEGREE BURGLARY | | 11130

4T H DEGREE B\&E | | 11130

4TH B \& E || 11130

4TH B\&E || 11130

4TH B+E || 11130

4TH BREAKING AND ENTERING | | 11130

4TH BURGLARY || 11130

4TH DEG B AND E || 11130

4TH DEG B/E || 11130

4TH DEG B\&E || 11130

4TH DEG B+E || 11130

4TH DEG BE || 11130

4TH DEG BREAKING AND ENTERING | | 11130

4TH DEG BURG I | 11130

4TH DEG BURGLARY || 11130

4TH DEG RES B\&E || 11130

4TH DEG SEX OFFENSE I I 10922

4TH DEG SEX OFFENSE || 43600

4TH DEG. B \& E || 11130

4TH DEG. B\&E || 11130

4TH DEG. BREAKING AND ENTERING | | 11130

4TH DEG. BURGLARY || 11130

4TH DEG. SEX OFFENSE I | 35715

4TH DEGREE BURGLARY || 11130

4TH DEGREE SEX OFFENSE || 43600

4TH DEGREE B \& E || 11130

4TH DEGREE B AND E || 11130

4TH DEGREE B-E || 11130

4TH DEGREE B/E || 11130

4TH DEGREE B\&E || 11130

4TH DEGREE B+E || 11130

4TH DEGREE B7E || 11130

4TH DEGREE BE || 11130

4TH DEGREE BREAKING \& ENTERING || 11130

4TH DEGREE BREAKING AND ENTERI । | 11130

4TH DEGREE BUGLARY | | 11130

4TH DEGREE BUR. I I 11130

4TH DEGREE BURG || 11130

4TH DEGREE BURGLARY || 11130

4TH DEGREE BURGLARY || 1A1130 
4TH DEGREE BURGLRY || 11130

4TH DEGREE BURGULARY || 11130

4TH DEGREE BURGURALY || 11130

4TH DEGREE RESIDENTIAL B \& E || 11130

4TH DEGREE RESIDENTIAL B\&E | | 11130

4TH DEGREE SEX OFFENSE | | 10922

4TH DEGREE SEX OFFENSE || 13605

4TH DEGREE SEX OFFENSE I। 33600

4TH DEGREE SEX OFFENSE | | 43600

4TH DEGREE SEX OFFENSE I| 63600

4TH DERGEE BURGLARY | | 11130

4TH DERGREE B\&E || 11130

4TH DG B\&E || 11130

4TH DG BURGLARY || 11130

4TH SEX OFFENCE II 43600

4TH. DEGREE SEX OFFENSE || 43600

ATT 1ST DEG SEX OFFENSE || 21125

ATT 2ND DEG SEX OFFENSE I | 21115

ATT 4TH DEG BURGLARY || 1A1130

ATT B\&E OF A GARAGE || 1A1130

ATT BURGLARY || 1A1130

ATT COMMERCIAL B AND E II 1A1130

ATT-BURGLARY || 1A1130

ATT-BURGLARY-4TH DEG-STORE || 1A1130

Att-Burglary-4Th Deg-Store, Att B \& E || 1A1130

Att-Burglary-4Th Deg-Store, Att. B+E || 1A1130

Att-Burglary-4Th Deg-Store, Attempt B \& E || 1 A1130

Att-Burglary-4Th Deg-Store, Attempt B And E || 1A1130

Att-Burglary-4Th Deg-Store, Attempt B/E || 1A1130

Att-Burglary-4Th Deg-Store, Attempt B\&E || 1A1130

Att-Burglary-4Th Deg-Store, Attempt Breaking And Ent II 1A1130

Att-Burglary-4Th Deg-Store, Attempt Burglary II 1A1130

Att-Burglary-4Th Deg-Store, Attempted Residential B I| 1A1130

Att-Burglary-4Th Deg-Store, Destruction Of Property II 1A1130

Att-Burglary-4Th Deg-Store, Theft -500.00 || 1A1130

Att-Burglary-4Th Deg-Store, Trespassing I| 1A1130

Att-Fail Reg Offender, Att-Fail Reg Offender/Flse I I I 1A1477

Att-Fail Reg Offender, Att-Fail Reg Offender/Flse In I I 1A1477

ATT-FAIL REG OFFENDER/FLSE INF || 1A1477

ATT-SEX OFF 4TH DEG-SEX CONT I| 4A3600

ATT-SEX OFF REG-FAIL NOTIFY/IN I | 2A1134

ATT-SEX OFFENSE SECOND DEGREE I| 2A3600

Att-Sex Offense Third Degree, 3Rd Degree Sex Offens II 3A3600

Att-Sex Offense Third Degree, Att-Sex Offense Third I| 3A3600

ATT. B \& E || 1A1130

ATT. B\&E || 1A1130

ATT. BREAKING \& ENTERING || 1 A1130

ATT. BURGLARY || 1A1130

ATT.BURGLARY || 1A1130

ATTEMPT 4TH DEGREE BURGLARY || 1 A 1130

ATTEMPT B \& E || 1 A 1130

ATTEMPT B + E || 11130

ATTEMPT B\&E || 11130

ATTEMPT B\&E || 1 A1130

ATTEMPT B\&E || 1C1130

ATTEMPT BREAKING \& ENTERING || 1A1130

ATTEMPT BREAKING AND ENTERING || 1 A1130

ATTEMPT BURGLARY | | 1A1130

ATTEMPT COMM B\&E I| 1A1130

ATTEMPT SEXUAL CHILD ABUSE I। 21115 
ATTEMPTED B \& E || 1A1130

ATTEMPTED B\&E 4TH DEGREE || 1A1130

ATTEMPTED BREAKING AND ENTERIN || 1A1130

ATTEMPTED BURGLARY || 1A1130

ATTEMPTED COMMERCIAL B\&E || 1A1130

ATTEMTED BURGLARY || 1A1130

B \& E - 4 DEGREE || 11130

B \& E || 11130

B \& E || 1A1130

B \& E (4TH DEG) ।| 11130

B \& E 4TH DEGREE || 11130

B \& E COMMERCIAL || 11130

B \& E OF A VACANT || 11130

B \&E RESIDENTIAL || 11130

B + E || 11130

B AND E || 11130

B AND E 4TH || 11130

B AND E 4TH DEG । | 11130

B/E || 11130

B/E 4TH || 11130

B/E 4TH DEGREE I। 11130

B\& E || 1A1130

B\&E || 11130

B\&E || 1A1130

B\&E (VACANT) || 11130

B\&E 2ND DEGREE || 11130

B\&E 3RD || 11130

B\&E 4 TH DEGREE || 11130

B\&E 4TH || 11130

B\&E 4TH DEG || 11130

B\&E 4TH DEGREE | | 11130

B\&E COMMERCIAL | 11130

B\&E DWELLING || 11130

B\&E OF VACANT || 11130

B\&E RESIDENT || 11130

B\&E RESIDENTIAL || 11130

B\&E VACANT I| 11130

B\&E4TH || 11130

B+E || 11130

$\mathrm{B}+\mathrm{E}$ 2ND DEGREE || 11130

B+E 4TH I| 11130

B+E 4TH DEGREE I। 11130

B+E SHED || 11130

B+E VACANT I| 11130

BREAK \& ENTER || 11130

BREAK AND ENTER || 11130

BREAKING \& ENTERING || 11130

BREAKING AND ENTEREING || 11130

BREAKING AND ENTERING || 11130

BREAKING AND ENTERING 4TH || 11130

BREAKING AND ENTERING 4TH DEGR | | 11130

BREAKING AND ENTRY | | 11130

BREAKING ENTERING | 1 A1130

BUGLARY || 11130

BURGALRY || 11130

BURGARY || 11130

BURGLARY || 11130

BURGLARY || 1A1130

BURGLARY - 4TH DEG I। 11130

BURGLARY - 4TH DEGREE || 11130 


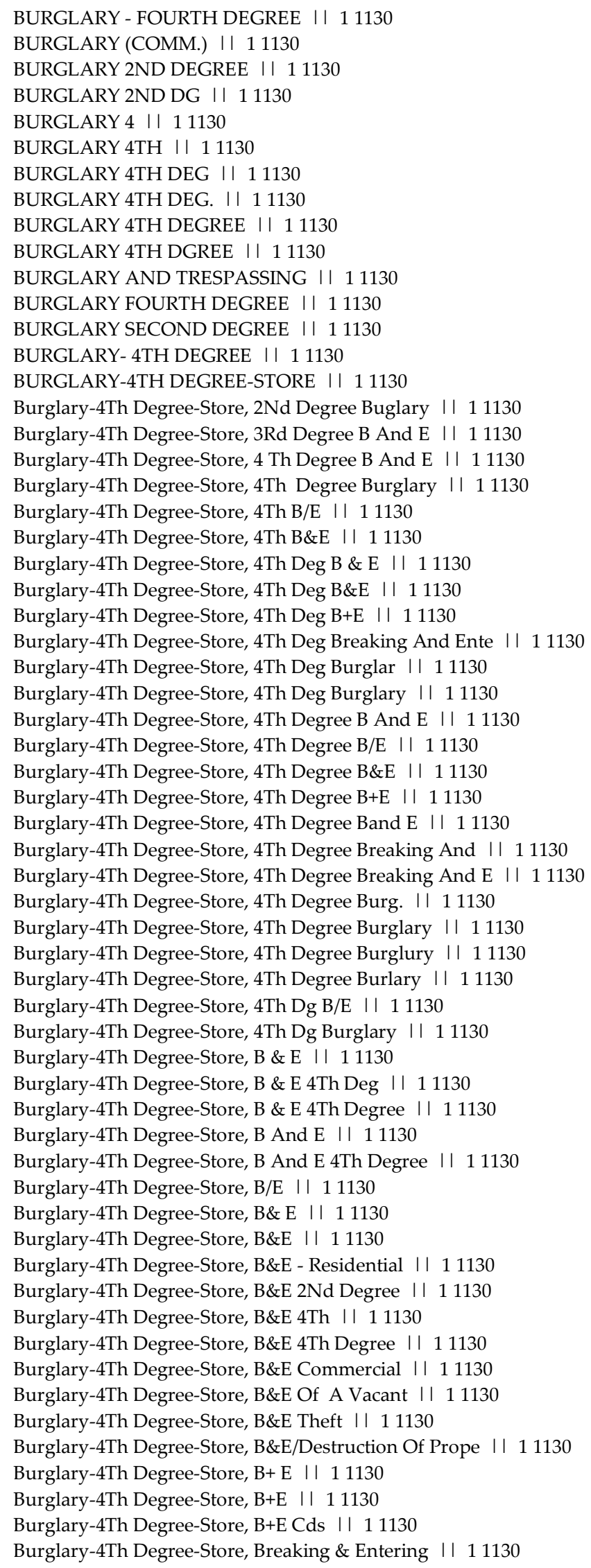


Burglary-4Th Degree-Store, Breaking And Entering I| 11130

Burglary-4Th Degree-Store, Breaking And Entering 4Th || 11130

Burglary-4Th Degree-Store, Burglary | | 11130

Burglary-4Th Degree-Store, Burglary 2Nd || 11130

Burglary-4Th Degree-Store, Burglary 2Nd Deg || 11130

Burglary-4Th Degree-Store, Burglary 4Th || 11130

Burglary-4Th Degree-Store, Burglary 4Th Deg I| 11130

Burglary-4Th Degree-Store, Burglary 4Th Degree I| 11130

Burglary-4Th Degree-Store, Burglary In The Fourth De || 11130

Burglary-4Th Degree-Store, Burglary-4Th Degree II 11130

Burglary-4Th Degree-Store, Burglary-4Th Degree-Stor I| 11130

Burglary-4Th Degree-Store, Burglary-4Th Degree-Store I| 11130

Burglary-4Th Degree-Store, Burglary4Th Degree I| 11130

Burglary-4Th Degree-Store, Cds I| 11130

Burglary-4Th Degree-Store, Cds Poos W/ Intent I| 11130

Burglary-4Th Degree-Store, Cds Poss. Cocaine I| 11130

Burglary-4Th Degree-Store, Cds Violation I| 11130

Burglary-4Th Degree-Store, Cds Violation/4Th B\&E || 11130

Burglary-4Th Degree-Store, Comm: B\& E || 11130

Burglary-4Th Degree-Store, Comm. B\&E || 11130

Burglary-4Th Degree-Store, Commercial B\&E I| 11130

Burglary-4Th Degree-Store, Commercial Breaking And II 11130

Burglary-4Th Degree-Store, Commercial Bulgary I| 11130

Burglary-4Th Degree-Store, Commercial Burglary || 11130

Burglary-4Th Degree-Store, Commerical Burglary II 11130

Burglary-4Th Degree-Store, False Infomation I| 11130

Burglary-4Th Degree-Store, Fourth Degree B\&E || 11130

Burglary-4Th Degree-Store, Fourth Degree Buglary || 11130

Burglary-4Th Degree-Store, Fourth Degree Burglary I| 11130

Burglary-4Th Degree-Store, Larceny || 11130

Burglary-4Th Degree-Store, Possession Cds || 11130

Burglary-4Th Degree-Store, Residential B And E I| 11130

Burglary-4Th Degree-Store, Residential B\&E || 11130

Burglary-4Th Degree-Store, Residential B\&E 4Th Degr I| 11130

Burglary-4Th Degree-Store, Residential Burglary || 11130

Burglary-4Th Degree-Store, Trepassing II 11130

Burglary-4Th Degree-Store, Tresp/Be/Cds I| 11130

Burglary-4Th Degree-Store, Trespass/4Th B \& E || 11130

Burglary-4Th Degree-Store, Trespassing || 11130

Burglary-4Th Degree-Store, Trespassing Private Prope I| 11130

Burglary-4Th Degree-Store, Tresspassing || 11130

BURGLARY-FOURTH DEGREE || 11130

BURGLAY 4TH II 11130

BURGLERY 4TH DEGREE I| 11130

BURGLURY 4TH DEGREE || 11130

BURLGARY 4TH || 11130

CDS || 11130

CDS POSS I| 11130

CDS POSS MARIJUANA I| 11130

CDS POSSESSION NOT MARIJUANA || 11130

CDS PWID || 11130

CDS VIOLATION || 11130

CDS: CONSPIRACY || 11130

CDS/4TH DEG BURGLARY || 11130

CHILD NEGLECT । | 11130

CHILD PORN PERMIT SEX SUBJ | | 10298

COMERCIAL B\&E || 11130

COMERCIAL BURGLARY || 11130

COMM B\&E || 11130

COMM BURG || 11130 


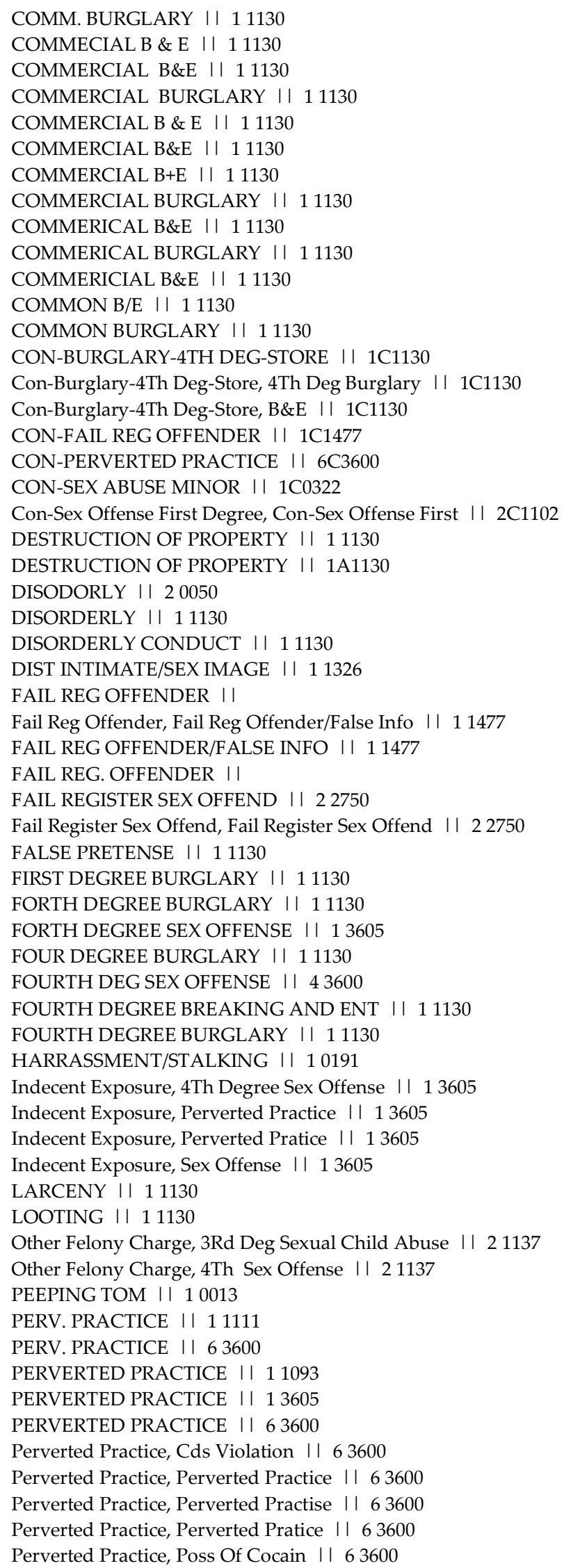


Perverted Practice, Preverted Practice I| 63600

Perverted Practice, Prostitution || 63600

Perverted Practice, Sex Offense || 63600

PERVERTED PRATICE || 63600

PERVERTED SEX ACT ।| 63600

PERVETED PRACTICE I।

PLACE INTIMATE/SEX IMAGE INTER || 11326

POSS HEROIN II 11130

POSS WITH INT MARIJUANA || 11130

POSSESSION NOT MARIJUANA || 11130

POSSESSION STOLEN VEHICLE I I 11130

PROSTITUTION/PERVERTED PRACTIC || 63600

PROSTITUTION/PERVERTED SEX PRA || 63600

RECREATION B\&E || 11130

RES. BURGLARY || 11130

RESIDENTAL BREAKING AND ENTERI || 11130

RESIDENTAL BREAKING ENTERING || 11130

RESIDENTAL BURG || 11130

RESIDENTAL BURGLARY || 11130

RESIDENTIAL B \& E || 11130

RESIDENTIAL B AND E || 11130

RESIDENTIAL B\&E || 11130

RESIDENTIAL B+E || 11130

RESIDENTIAL BURGLARY || 11130

RESIDENTIONAL BREAKING AND ENT || 11130

ROGUE \& VAGABOND || 1A1130

SEX ABUSE MINOR || 10322

SEX ABUSE MINOR || 21137

Sex Abuse Minor, Sex Abuse Minor I| 10322

Sex Abuse Minor, Sex Abuse Of A Minor I| 10322

Sex Abuse Minor, Sexual Child Abuse I| 10322

SEX ABUSE MINOR: HOUSE/FAM || 10922

SEX ABUSE MNR/CONT COURSE COND I| 21136

SEX ABUSE OF A MINOR || 21103

SEX ABUSE OF A MINOR || 33600

SEX OFF -FAIL NOTIFY/INCLD I ।

SEX OFF 1ST DEG I।

SEX OFF 4TH DEG ।| 43600

SEX OFF 4TH DEG-SEX ACT 14/15Y || 11623

SEX OFF 4TH DEG-SEX CONTACT || 43600

SEX OFF 4TH DEG-VAG INT 14/15 || 11624

Sex Off 4Th Sex Act/Un Age, Sex Off 4Th Sex Act/Un A I| 10301

SEX OFF 4TH- PERS POS AUTH I| 11625

SEX OFF REG-CHANGE ADDRESS I| 21134

Sex Off Reg-Change Address, Sex Off Reg-Change Addr II 21134

Sex Off Reg-Change Address, Sex Off Reg-Change Addre || 21134

Sex Off Reg-Change Address, Sex Off Reg-Fail Notify || 21134

Sex Off Reg-Change Address, Sex Off Reg-Fail Notify/ I I 21134

SEX OFF REG-FAIL ।

SEX OFF REG-FAIL NOTIFY । ।

SEX OFF REG-FAIL NOTIFY/INCLD । ।

SEX OFF REG-FAIL NOTIFY/INCLD || 21134

SEX OFF REG-FAIL NOTIFY/INCLUD । |

SEX OFFENCE || 13605

SEX OFFENCE II 23600

SEX OFFENSE || 10173

SEX OFFENSE || 10922

SEX OFFENSE || 11604

SEX OFFENSE || 13605

SEX OFFENSE || 16525 
SEX OFFENSE || 20050

SEX OFFENSE || 22210

SEX OFFENSE || 23600

SEX OFFENSE || 33600

SEX OFFENSE || 43600

SEX OFFENSE || 4A3600

SEX OFFENSE || 63600

SEX OFFENSE 3 RD DEGREE || 33600

SEX OFFENSE 3RD || 33600

SEX OFFENSE 3RD DEGREE || 10922

SEX OFFENSE 4TH DEGREE I | 43600

SEX OFFENSE FIRST DEGREE || 21102

Sex Offense First Degree, 1St Degree Sex Offense || 21102

Sex Offense First Degree, Sex Offense II 21102

Sex Offense First Degree, Sex Offense First Degree I| 21102

SEX OFFENSE FOURTH DEGREE || 43600

Sex Offense Fourth Degree, 4Th Deg Sex Offense II 43600

Sex Offense Fourth Degree, 4Th Degree Sex Offence II 43600

Sex Offense Fourth Degree, 4Th Degree Sex Offense II 43600

Sex Offense Fourth Degree, Sex Off 4Th Deg II 43600

Sex Offense Fourth Degree, Sex Off 4Th Deg-Sex Cont II 43600

Sex Offense Fourth Degree, Sex Offense II 43600

Sex Offense Fourth Degree, Sex Offense Fourth Degree II 43600

SEX OFFENSE OF MINOR I| 10322

SEX OFFENSE SECOND DEGREE I| 23600

Sex Offense Second Degree, Sex Offense II 23600

Sex Offense Second Degree, Sex Offense Second Degree II 23600

Sex Offense Second Degree, Sexual Child Abuse I| 23600

SEX OFFENSE THIRD DEGREE I| 33600

Sex Offense Third Degree, Sex Offense Third Degree II 33600

Sex Offense Third Degree, Sexual Child Abuse II 33600

SEX OFFESE || 33600

SEXUAL CHILD ABUSE I| 10322

SEXUAL CHILD ABUSE || 21103

SEXUAL CHILD ABUSE || 21137

SEXUAL CHILD ABUSE OF MINOR I| 10322

SEXUAL CHILD ABUSE OF MINOR II 21136

SEXUAL OFFENSE 4TH DEGREE || 43600

SEXUAL PHYSICAL CHILD ABUSE || 10322

SEXUAL SOLICITATION OF MINOR || 11604

SEXUAL SOLICITATION OF A MINOR || 11604

Sexual Solicitation Of A Minor, Sexual Solicitation || 11604

SODOMY I| 53600

Sodomy-Generally, Sodomy-Generally I| 53600

SOLICITING CUSTOMERS || 10665

STALKING || 15406

STALKING || 16525

Stalking, Stalking I| 16525

STOLEN AUTO || 11130

THEFT || 11130

THEIFT || 11130

THIRD DEGREE B\&E || 11130

THIRD DEGREE BURGLARY || 11130

TREPASSING || 11130

TRESPASS I| 11130

TRESPASS- PEEPING TOM || 22280

Trespass- Peeping Tom, Trespass- Peeping Tom I| 22280

TRESPASS-PEEPING TOM || 13611

Trespass: Private Property, 4Th Dg Sex Offense II 22220

TRESPASSING || 11130 
TRESPASSING || $1 \mathrm{~A} 1130$

TRESSPASSING || 11130

UNSUPERVISED CHILDREN || 11614

URGLARY || 11130

\section{Domestic}

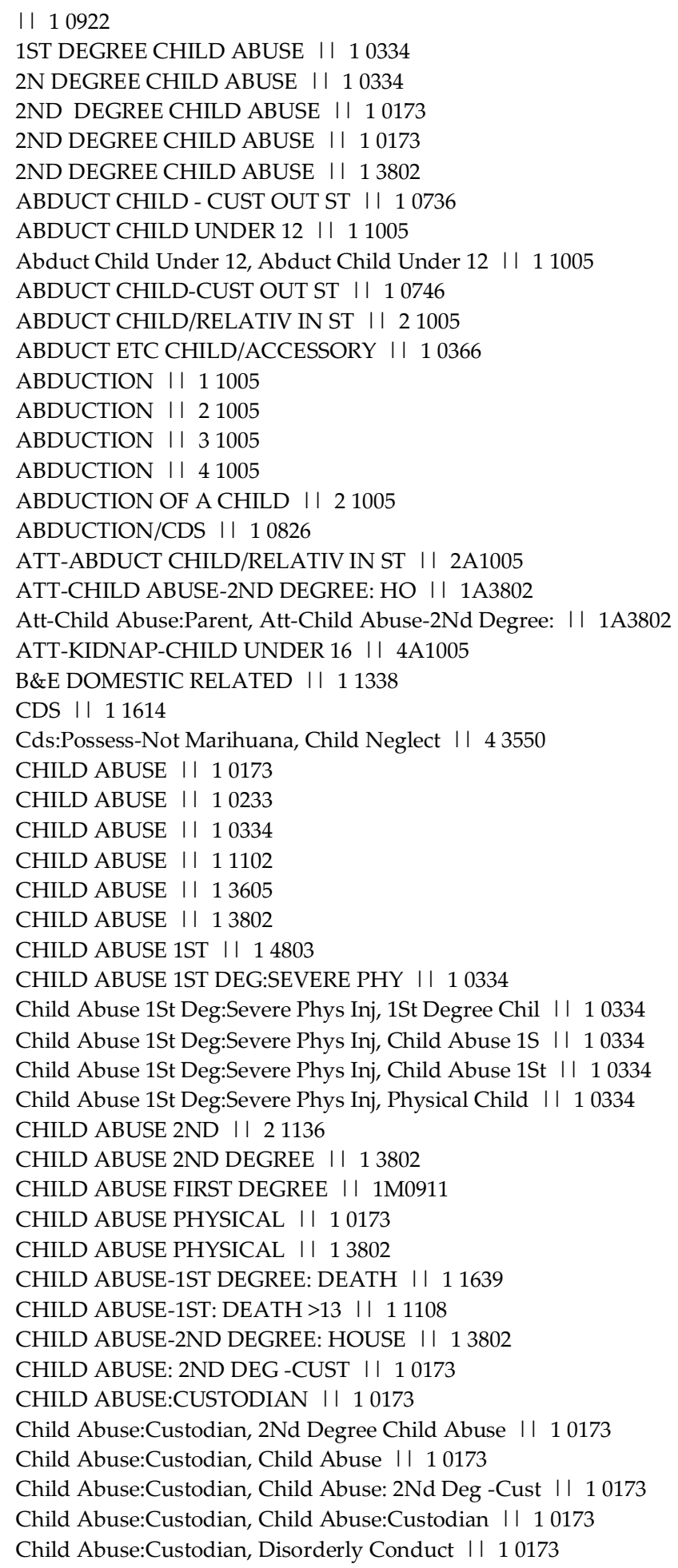


Child Abuse:Custodian, Physical Child Abuse II 10173

Child Abuse:Parent, 2Cd Degree Child Abuse By Pare I| 13802

Child Abuse:Parent, Child Abuse I| 13802

Child Abuse:Parent, Child Abuse-2Nd Degree: House I| 13802

Child Abuse:Parent, Fel. Child Abuse || 13802

Child Abuse:Parent, Phys Child Abuse I| 13802

Child Abuse:Parent, Physical Child Abuse I| 13802

CHILD ABUSED PYSICAL || 13802

CHILD ENDANGERMENT || 11425

CHILD ENDANGERMENT $\|$ | 11614

CHILD ENDANGERMENT || 14200

CHILD NEGLECT | | 10005

CHILD NEGLECT || 10043

CHILD NEGLECT । | 10600

CHILD NEGLECT । | 11425

CHILD NEGLECT || 11614

CHILD NEGLECT RESULT DEATH | | 11109

CHILD NEGLET || 10005

CHILD PORN COMPUTERS || 10315

CHILD PORN PROMOTE/DISTRB || 10300

Child Porn Promote/Distrb, Child Porn Promote/Distrb || 10300

Child Porn Solicit Subject, Child Porn Solicit Subje II 23799

COMM NUIS/ADMINISTER CDS | | 10238

CON-CHILD ABUSE-2ND DEGREE: HO I। 1 C3802

CONFINE UNATTENDED CHILD | | 10005

Confine Unattended Child, Child Neglect II 10005

Confine Unattended Child, Confine Unattended Child II 10005

Confine Unattended Child, Unattended Child II 10005

CONFINED UNATTENDED CHILD | | 10005

CONTRIBUTE TO COND OF CHLD | | 20238

Contribute To Cond Of Chld, Shoplifting || 20238

DESERTION-MINOR CHILD || 43899

DEST. OF PROPERTY (DOMESTIC) I। 34025

DET CHILD CUSTODIAN OUT ST । | 10737

DETAIN CHILD/RELATVE IN ST | | 10364

DOMESTIC B\&E || 23030

DOMESTIC DEST OF PROP I | 34025

DOMESTIC DESTRUCTION || 34025

DOMESTIC DESTRUCTION OF PROPER I| 34025

DOMESTIC LARCENY | | 10521

DOMESTIC LARCENY | | 10621

DOMESTIC LARCENY | | 11137

DOMESTIC VIOLENCE || 23030

DRIVING WHILE IMPAIRED BY DRUG | | 11614

DRUNKED DISORDERLY DOMESTIC || 14200

Fail Obey Renble/Lawfl, Child Neglect || 20055

FTO DISORDERLY DOMESTIC || 20050

GAMBLING || 20238

KIDNAP-CHILD UNDER 16 || 41005

Kidnap-Child Under 16, Kidnap-Child Under 16 || 41005

LARCENY /DOMESTIC || 10521

NEGLECT OF MINOR | | 11614

Neglect Of Minor, Child Neglect I I 11614

Neglect Of Minor, Neglect Of Minor I I 11614

NEGLIGENT/CHILD ABUSE | | 10005

NONSUPPORT - MINOR CHILD | | 33899

PARENTAL ABDOCTION || 11005

PARENTAL ABDUCTION || 11005

PARENTAL ABDUCTION I| 41005

PARTENAL ABDUCTION || 21005 
Peace Order: Fail To Comply, Violation Of Exparte O II 20105

PHYS CHILD ABUSE || 10173

PHYSICA CHILD ABUSE || 10334

PHYSICAL CHILD ABUSE || 10173

PHYSICAL CHILD ABUSE || 10334

PHYSICAL CHILD ABUSE || 13802

PHYSICAL CHILD ABUSED | | 13802

POSSESS CHILD PORNOGRAPHY || 11756

Possess Child Pornography, Possess Child Pornograph || 11756

RECKLESS ENDANGERMENT I| 20238

SCHOOL: FAIL SEND CHILD || 10928

SCHOOL:FAIL SEND CHILD || 10047

School:Fail Send Child, School:Fail Send Child || 10047

SECOND DEGREE CHILD ABUSE I I 10173

SOL-CHILD ABUSE-2ND DEGREE: HO || $1 S 3802$

Trespass: Private Property, Violation Of Exparte II 22220

UNATENDED CHILD || 10005

UNATTENDED CHILD || 10005

UNATTENDED CHILD || 20238

UNATTENED CHILD NEGLECT || 10005

UNNATENDED CHILD || 10005

VIOL EX-PARTE I| 20105

Violate Exparte II

VIOLATE EXPARTE I।

VIOLATE EXPARTE ORDER/PROT ORD I।

VIOLATE EXPARTE/PROT ORDER II 20354

VIOLATE EXPARTE/PROT ORDER-SUB I| 20354

Violate Exparte/Prot Order, Violate Exparte/Prot Or || 20354

Violate Exparte/Prot Order, Violate Exparte/Prot Ord II 20354

Violate Exparte/Prot Order, Violation Protective Ord I| 20354

VIOLATION EX PARTE || 20105

Violation Exparte/Protec Orde II

VIOLATION OF EX PARTE || 20105

Violation Of Ex-Parte II

VIOLATION OF EX-PARTE || 34025

VIOLATION OF EXPARTE I

VIOLATION OF EXPARTE || 20105

VUL ADULT ABUSE/CUSTODIAN | | 10466

Vul Adult Abuse/Custodian, Vul Adult Abuse/Custodia || 10466

Vul Adult Abuse/Custodian, Vul Adult Abuse/Custodian II 10466

\section{Non-Deadly Weapon}

II 11285

AIR PISTOL VIOLATION ||

AIR SOFT PISTOL I I

AIRGUN VIOLATION II

AMMO VIOLATION || 11285

AMMUNITION VIOLATION || 11285

B.B. Gun Violation II

B.B. GUN VIOLATION I |

B.B.GUN VIOLATION ।

BB GUN VIOLATION ।

Bb Gun II

BB GUN I |

BB GUN || 43550

BB GUN VIOLATION । |

Bb Gun Poss II

BB GUN POSSESSION ।| 10690 


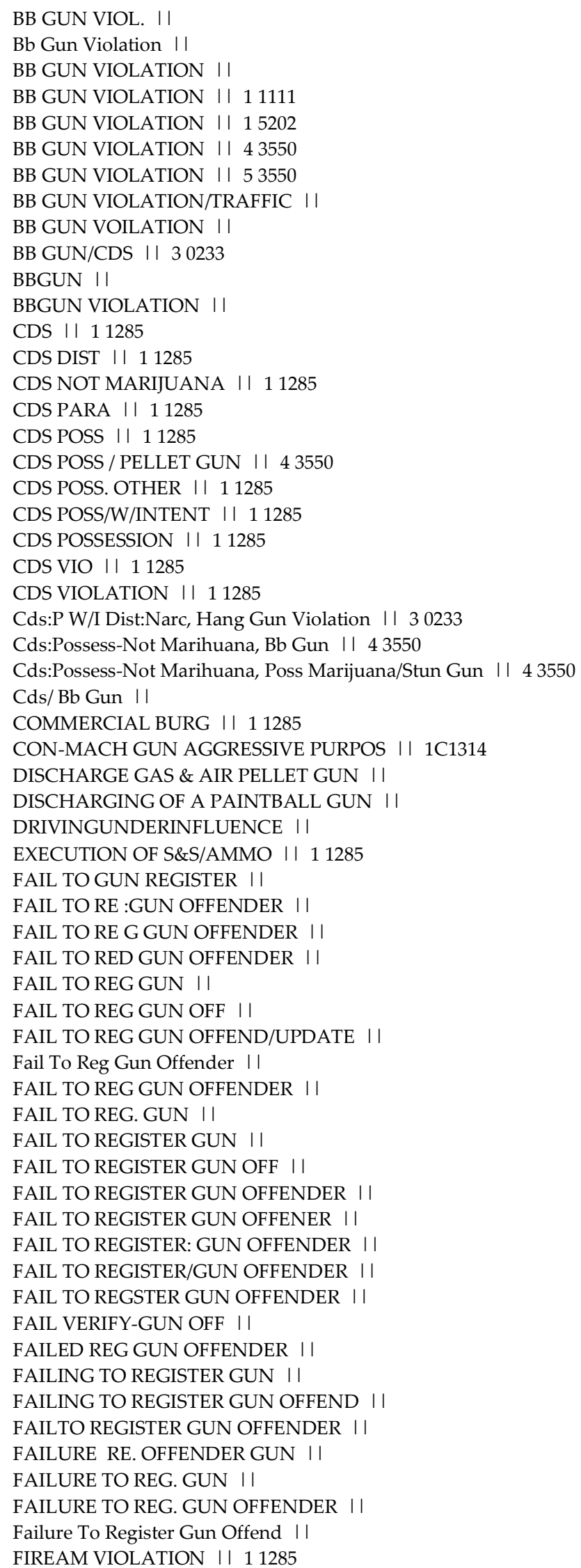




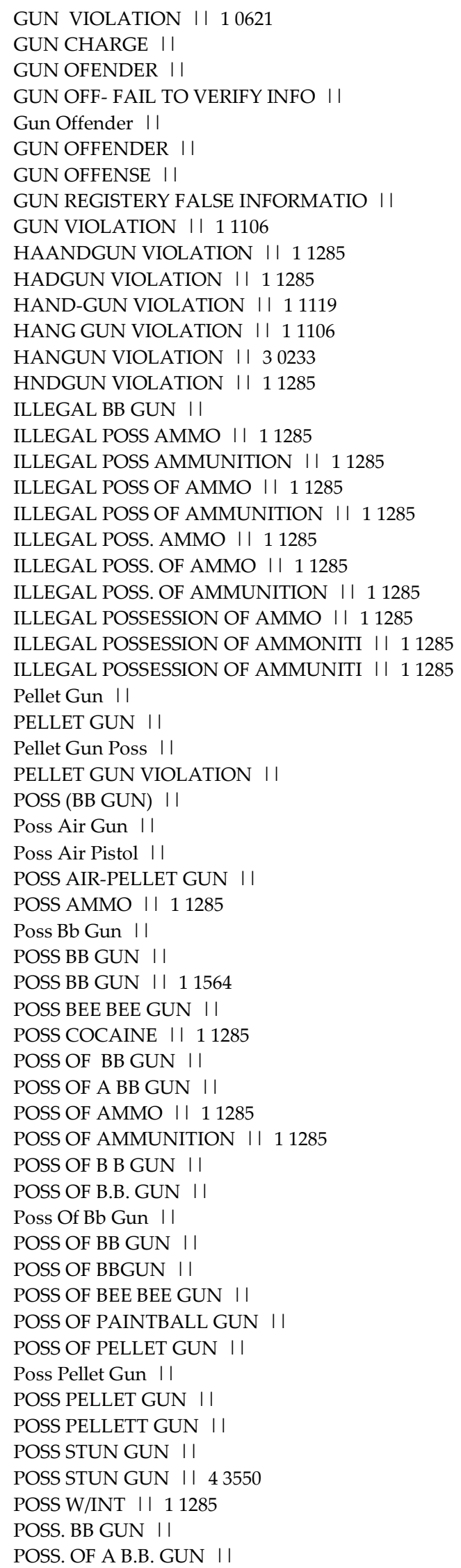




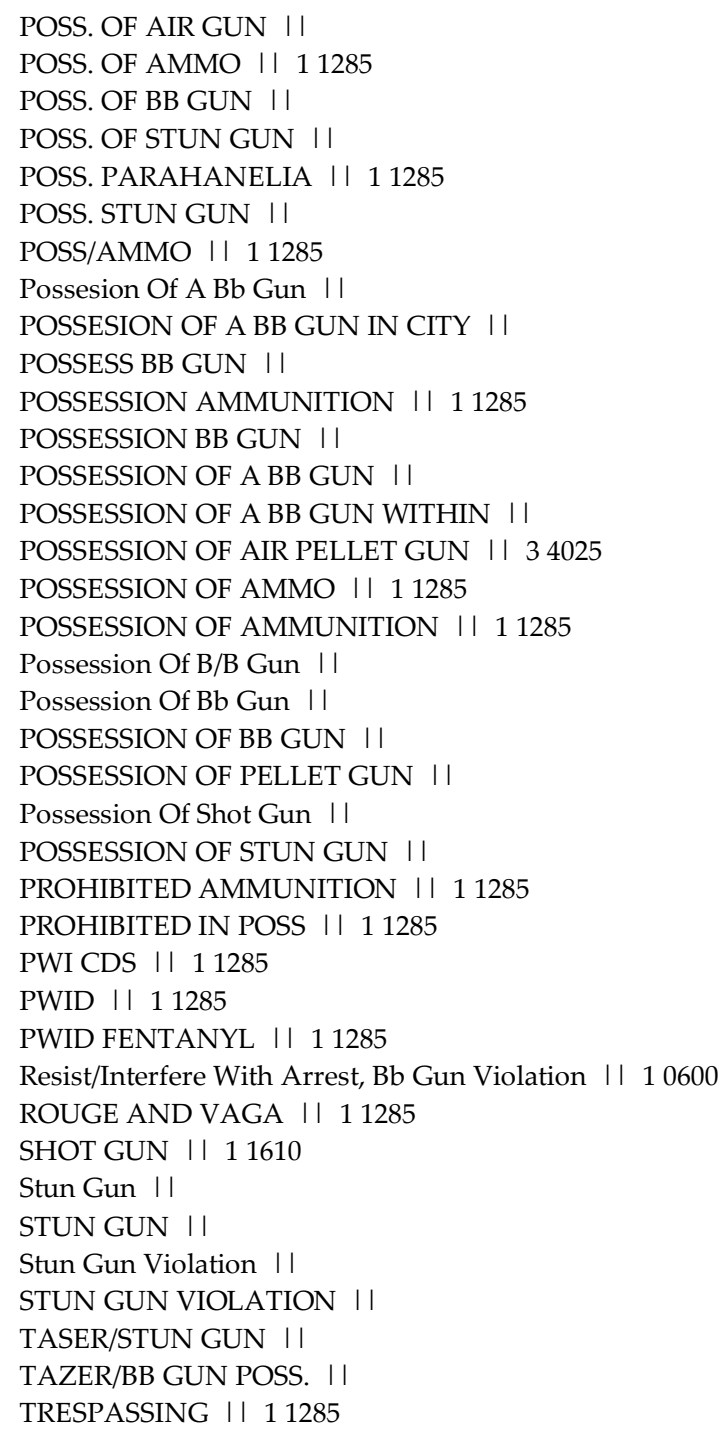

\section{Burglary}

1 DEG B\&E || 23000

1 ST DEGREE BURGLARY | | 34025

1ST BURGLARY | | 23040

1ST DEG BURGLARY || 23000

1ST DEG BURGLARY || 23030

1ST DEG BURGLARY || 53550

1ST DEG. BURGLARY || 23030

1ST DEG.BURGLARY || 23000

1ST DEGREE B \& E | | 23040

1ST DEGREE B\&E | | 23000

1ST DEGREE BREAKING AND ENTERI || 23000

1ST DEGREE BURGLARY || 11136

1ST DEGREE BURGLARY || 23000

1ST DEGREE BURGLARY || 23010

1ST DEGREE BURGLARY || 23030

1ST DEGREE BURGLARY || 23040

1ST DEGREE BURGLARY || 34025

1ST DEGREE BURGLARY (C) | | 23010 
1ST DEGREE COMMERCIAL BURGLARY || 23040

2 DEG B AND E || 23020

2ND DEG BURGLARY | 10622

2ND DEG BURGLARY | 20480

2ND DEG. BURGLARY || 10622

2ND DEGREE B\&E || 23000

2ND DEGREE B\&E || 23010

2ND DEGREE BURGLARY || 10600

2ND DEGREE BURGLARY || 23010

2ND DEGREE BURGLARY || 23030

2ND DEGREE BURGLARY | | 34030

3RD DEGREE BURGLARY || 23020

3RD DEGREE BURGLARY || 23030

3RD DEGREE BURLARY I | 23030

4 DEGREE BURGLARY | | 23030

4TH B \& E (ATTEMPT) | | 34025

4TH B\&E || 23030

4TH BURGLARY || 23030

4TH DEG B \& E || 1A0521

4TH DEG B\&E || 23030

4TH DEG B\&E || 23040

4TH DEG B+E I| 22220

4TH DEG B+E I| 23010

4TH DEG BURGLARY | 10621

4TH DEG BURGLARY || 22220

4TH DEG BURGLARY | | 23030

4TH DEG BURGLARY || 30233

4TH DEG BURGLARY | | 34025

4TH DEG BURGLARY/CDS । 43550

4TH DEG. B\&E || 22210

4TH DEG. BURGLARY || 23030

4TH DEG. RESIDENTIAL BURGLARY | | 23040

4TH DEGREE B \& E I| 23030

4TH DEGREE B \& E || 23030

4TH DEGREE B AND E || 23030

4TH DEGREE B/E I | 23030

4TH DEGREE B/E I। 34025

4TH DEGREE B\&E || 10621

4TH DEGREE B\&E || 11111

4TH DEGREE B\&E || 22220

4TH DEGREE B\&E || 23030

4TH DEGREE B\&E || 23040

4TH DEGREE B\&E || 23050

4TH DEGREE B\&E I | 34025

4TH DEGREE B\&E || 53550

4TH DEGREE B+E || 23030

4TH DEGREE B+E || 43550

4TH DEGREE B+E || 53550

4TH DEGREE BREAKING \& ENTERING || 23030

4TH DEGREE BREAKING AND ENTERI । । 43550

4TH DEGREE BURGLARY || 11111

4TH DEGREE BURGLARY | | 11119

4TH DEGREE BURGLARY | | 22210

4TH DEGREE BURGLARY || 22220

4TH DEGREE BURGLARY | | 23000

4TH DEGREE BURGLARY | | 23010

4TH DEGREE BURGLARY || 23030

4TH DEGREE BURGLARY || 23040

4TH DEGREE BURGLARY || 23090

4TH DEGREE BURGLARY || 2A3030 
4TH DEGREE BURGLARY || 2A3040

4TH DEGREE BURGLARY || 34025

4TH DEGREE BURGLARY || 34030

4TH DEGREE BURGLARY || 43550

4TH DEGREE BURGLARY || 53550

4TH DEGREE BURGULARY || 23030

4TH DEGREE BURLARY I| 23030

4TH DEGREE, B\&E I। 43550

4TH DGREE BURGLARY || 11137

4TH. DEGREE RESIDENTIAL BURGLA | । 34025

Alc. Bev./Intox:Endanger, 4Th Degree Burglary II 14200

ARMED HOME INVASION || 11338

ATREMPT BURGLARY || 2A3000

Att - Theft: Less $\$ 1,000$ Value, Commercial Burglary I| 1A0621

Att - Theft: Less \$1,000 Value, First Degree Burglar I| 1A0621

ATT B \& E || 23030

ATT B \& E || 2A3030

ATT B \& E I| 34025

ATT B \& E RESIDENTIAL || 2A3030

ATT B\&E || 10621

ATT B\&E || 11111

ATT B\&E || 1A2200

ATT B\&E || 23030

ATT B\&E || 2A3030

ATT B+E || 2A3030

ATT BURGLARY || 10621

ATT BURGLARY || 16525

ATT COMM B\&E || 2A3010

ATT-BURGLARY- FIRST DEGREE I ।

Att- Theft: \$1K To Under \$10K, Burglary I I 1 A0622

ATT-BURGLARY || 5A1313

ATT-BURGLARY-4TH DEG-DWELL || 2A3030

ATT-BURGLARY-4TH DEG I।

ATT-BURGLARY-4TH DEGREE THEFT || 2A3040

ATT-BURGLARY-FIRST DEGREE || 2A3000

Att-Burglary-First Degree, Att-Burglary-First Degree II 2A3000

Att-Burglary-First Degree, Cds Possession Marijuana I| 2A3000

Att-Burglary-Fourth Degree, 4Th Degree B\&E I| 2A3030

Att-Burglary-Fourth Degree, Att B\&E || 2A3030

Att-Burglary-Fourth Degree, Att B\&E (Resident) II 2A3030

Att-Burglary-Fourth Degree, Att-Burglary- 4Th Deg-D I| 2A3030

Att-Burglary-Fourth Degree, Att-Burglary-4Th Deg-Dw I| 2A3030

Att-Burglary-Fourth Degree, Att. B\&E[Residence] I| 2A3030

Att-Burglary-Fourth Degree, Attemp B\& E || 2A3030

Att-Burglary-Fourth Degree, Attempt 4Th Degree Burgl I| 2A3030

Att-Burglary-Fourth Degree, Attempt B \& E || 2A3030

Att-Burglary-Fourth Degree, Attempt B\&E || 2A3030

Att-Burglary-Fourth Degree, Attempt B+E I| 2A3030

Att-Burglary-Fourth Degree, Attempt Breaking And Ent I| 2A3030

Att-Burglary-Fourth Degree, Attempt Burglary || 2A3030

Att-Burglary-Fourth Degree, Attempted B\&E I| 2A3030

Att-Burglary-Fourth Degree, Attempted Breaking And E I| 2A3030

Att-Burglary-Fourth Degree, B \& E || 2A3030

Att-Burglary-Fourth Degree, B/E || 2A3030

Att-Burglary-Fourth Degree, B\& E Attempt I| 2A3030

Att-Burglary-Fourth Degree, B\&E || 2A3030

Att-Burglary-Fourth Degree, Burglary II 2A3030

Att-Burglary-Fourth Degree, Cds I| 2A3030

Att-Burglary-Fourth Degree, Destruction Of Prop II 2A3030

Att-Burglary-Fourth Degree, Destruction Of Property II 2A3030 
Att-Burglary-Fourth Degree, Malicious Destruction II 2A3030

Att-Burglary-Fourth Degree, Trespassing || 2A3030

ATT-BURGLARY-THIRD DEGREE || 2A3020

Att-Burglary-Third Degree, Att-Burglary-Third Degree II 2A3020

Att-Burglary-Third Degree, Burglary || 2A3020

Att-Burglary-Third Degree, Property Destruction II 2A3020

ATT-BURGLARY/2ND DEGREE/GENERA || 2A3010

Att-Burglary/2Nd Degree/General, Attempt B And E I| 2A3010

ATT-HOME INVASION || 1A1338

ATT. 1ST DEG BURGLARY || 2A3030

ATT. B\&E || 23030

ATT. B\&E || 2A3030

ATT. B+E || 34025

ATT. BURGLARY || 23040

ATT. BURGLARY || 2A3030

ATTEMP B \& E || 2A3030

ATTEMPT 4TH DEG B\&E || 2A3030

ATTEMPT B \& E || 2A3030

ATTEMPT B \& E I| 34025

ATTEMPT B\&E || 10600

ATTEMPT B\&E || 20050

ATTEMPT B\&E || 22210

ATTEMPT B\&E || 22220

ATTEMPT B\&E || 23030

ATTEMPT B\&E || 23050

ATTEMPT B\&E || 2A3000

ATTEMPT B\&E || 2A3030

ATTEMPT B\&E || 34025

ATTEMPT B\&E || 34030

ATTEMPT B\&E || 42399

ATTEMPT B\&E (RESIDENTIAL) || 2A3030

ATTEMPT B+E || 2A3030

ATTEMPT BREAKING AND ENTERING || 22220

ATTEMPT BREAKING AND ENTERING || 2A3030

ATTEMPT BREAKING AND ENTERING || 34025

ATTEMPT BULGLARY || 2A3030

ATTEMPT BURGLARY | | 22220

ATTEMPT BURGLARY || 23030

ATTEMPT BURGLARY || 2A3000

ATTEMPT BURGLARY || 2A3020

ATTEMPT BURGLARY || 2A3030

ATTEMPT BURGLARY || 34025

ATTEMPT BURGLAY || 2A3030

ATTEMPT COMMERCIAL B\&E | | 23045

ATTEMPT RESIDENTIAL BURGLARY || 2A3030

ATTEMPT T B AND E TRASPASS || 22210

ATTEMPTED 4TH DEGREE B\&E || 23030

ATTEMPTED B \& E || 34025

ATTEMPTED B\&E || 2A3000

ATTEMPTED B\&E || 2A3030

ATTEMPTED B\&E | | 34025

ATTEMPTED BREAKING AND ENTERIN || 2A3030

ATTEMPTED BURGLARY || 22220

ATTEMPTED BURGLARY || 23030

ATTEMPTED BURGLARY | | 2A3030

ATTEMPTED BURGLARY || 43550

ATTEMPTED BURGLARY 4TH DEGREE || 2A3030

ATTEMPY B\&E || 2A3030

ATTEMT B \& E || 2A3030

ATTEMTPED BURGLARY || 22210 
B \& E || 10521

B \& E || 10621

B \& E || 10622

B \& E || 11338

B \& E || 14200

B \& E || 1A0521

B \& E || 22210

B \& E || 22220

B \& E || 23000

B \& E || 23010

B \& E || 23020

B \& E || 23030

B \& E || 23040

B \& E || 2A3030

B \& E || 34025

B \& E || 43550

B \& E (ATT) || 2A3030

B \& E BURGLARY || 11136

$\begin{array}{llll}B & \& & E\end{array}$ BURGLARY || 2C3020

B \& E OF VACANT | | 34025

B \& E RESIDENTIAL | | 23030

B AND E || 10621

B AND E || 10622

B AND E || 11111

B AND E || 23000

B AND E || 23010

B AND E || 23020

B AND E || 23030

B AND E I| 23040

B AND E I| 34025

B/E || 10621

B/E || 23030

B/E || 2A3030

B\&E || 10600

B\&E || 10621

B\&E || 10622

B\&E || 10623

B\&E || 11111

B\&E || 11137

B\&E || 15202

B\&E || 1A0521

B\&E || 1A0621

B\&E || 22210

B\&E || 22220

B\&E || 23000

B\&E || 23010

B\&E || 23020

B\&E || 23030

B\&E || 23040

B\&E || 2A3030

B\&E || 34025

B\&E || 34030

B\&E || 43550

B\&E || 53550

B\&E 1ST DEGREE || 11106

B\&E 4Th I I

B\&E ATTEMPT || 2A3030

B\&E COMMERICAL || 23010

B\&E DWELLING || 12202

B\&E RESIDENCE || 23040 
B\&E RESIDENTAL || 23010

B\&E RESIDENTIAL || 23000

B\&E RESIDENTIAL || 23030

B\&E/CDS POSS || 11635

B\&E/TRESPASSING || 34025

$\mathrm{B}+\mathrm{E}$ || 22210

$\mathrm{B}+\mathrm{E} \quad|| 23000$

$\mathrm{B}+\mathrm{E} \quad$ || 23010

$\mathrm{B}+\mathrm{E} \quad$ || 23020

B+E || 23030

B+E || 23040

B+E || 34025

$\mathrm{B}+\mathrm{E}$ || 34030

B+E (RESIDENTIAL) || 23040

B+E (RESIDENTIAL) | | 34025

B+E 4TH DEGREE || 22210

B+E RESIDENTIAL | | 23030

BREAK AND ENTER | | 10621

BREAKING \& ENTER || 23030

BREAKING \& ENTERING || 10621

BREAKING \& ENTERING || 22220

BREAKING \& ENTERING || 23000

BREAKING \& ENTERING || 23010

BREAKING \& ENTERING || 23030

BREAKING \& ENTERING || 23040

BREAKING \& ENTERING || 34025

BREAKING \& ENTERING 4TH DEG || 23030

BREAKING \& ENTRY || 34025

BREAKING AND ENTERIMG | | 23000

BREAKING AND ENTERING | | 10621

BREAKING AND ENTERING | | 11338

BREAKING AND ENTERING I | 22210

BREAKING AND ENTERING | | 22220

BREAKING AND ENTERING || 23000

BREAKING AND ENTERING | | 23010

BREAKING AND ENTERING | | 23030

BREAKING AND ENTERING I | 23040

BREAKING AND ENTERING || 2A3030

BREAKING AND ENTERING || 2A3550

BREAKING AND ENTERING || 34025

BREAKING AND ENTERING || 43600

BREAKING AND ENTERING || 63600

BREAKING AND ENTERING (ATTEMPT || 2A3030

BREAKING AND ENTERING 2ND DEGR | | 23040

BREAKING AND ENTERING 4TH DEGR || 10622

BREAKING AND ENTERY || 23030

BREAKING AND ENTRY | | 23030

BREAKING ENTERING | | 23030

BREAKING IN AND ENTERING || 23010

BUGLARY || 11338

BUGLARY || 23030

BURG 3RD DEG । ।

BURG 4TH DEG I ।

BURG TOOLS-MOT. VEH/POSS I| 23080

Burg Tools-Mot. Veh/Poss, Att Stolen Auto II 23080

Burg Tools-Mot. Veh/Poss, Poss Criminal Tool I| 23080

Burg Tools-Mot. Veh/Poss, Rogue And Vagabond I| 23080

Burg Tools-Mot. Veh/Poss, Theft U/\$1000.00 || 23080

BURGALRY || 2A3030

BURGALRY 4TH DEGREE || 23030 


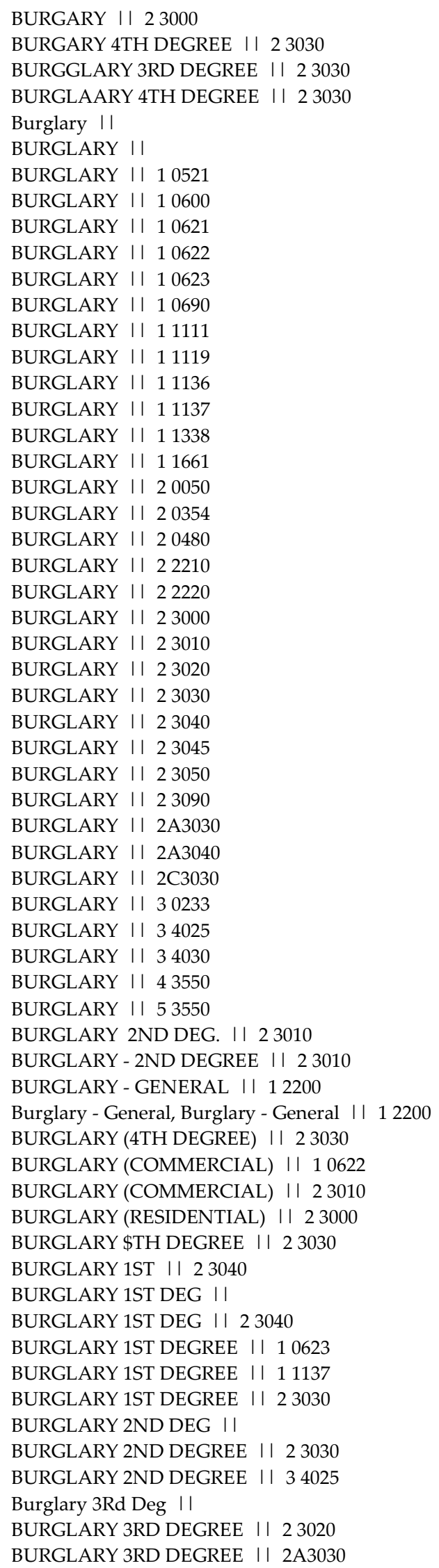




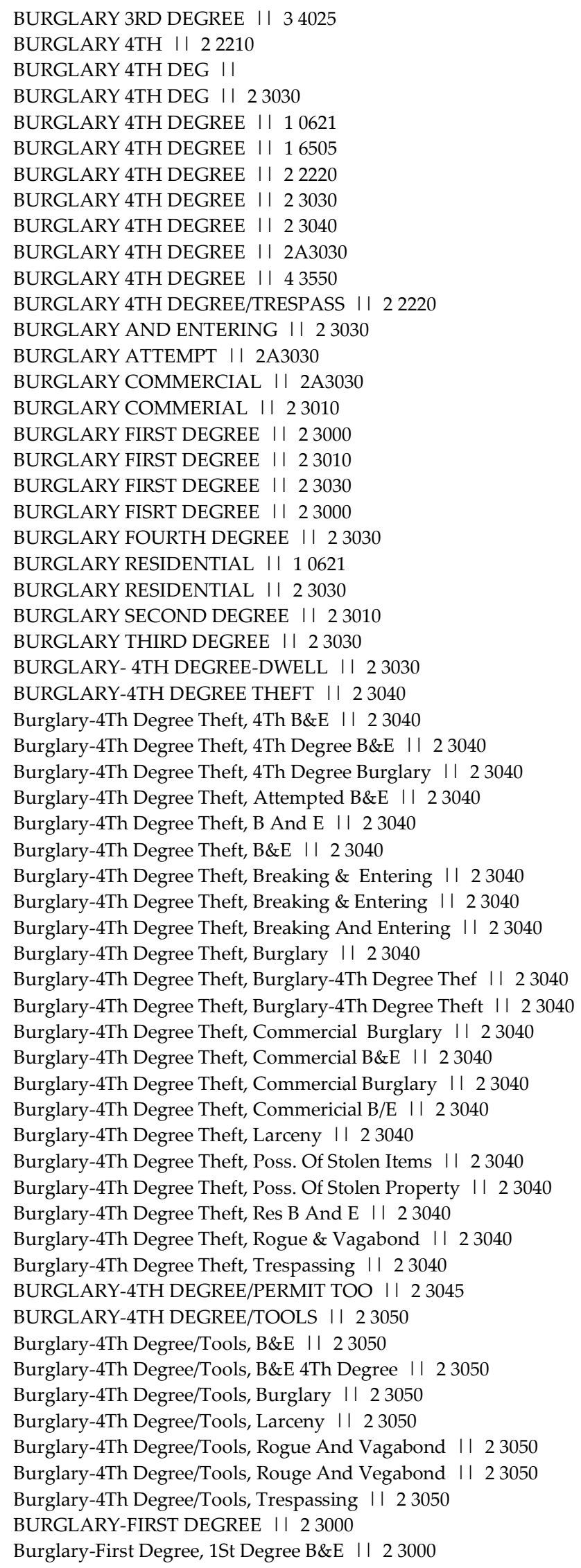


Burglary-First Degree, 1St Degree Breaking And Ente || 23000

Burglary-First Degree, 1St Degree Burglary || 23000

Burglary-First Degree, 4Th Degree Burglary || 23000

Burglary-First Degree, B/R || 23000

Burglary-First Degree, B\&E || 23000

Burglary-First Degree, B+E || 23000

Burglary-First Degree, Braking And Entering | | 23000

Burglary-First Degree, Breaking \& Entering || 23000

Burglary-First Degree, Burglary || 23000

Burglary-First Degree, Burglary 1St Deg || 23000

Burglary-First Degree, Burglary First Degree I| 23000

Burglary-First Degree, Burglary-First Degree I| 23000

Burglary-First Degree, Home Invasion || 23000

Burglary-First Degree, Residential B\&E || 23000

BURGLARY-FOURTH DEGREE I | 23030

Burglary-Fourth Degree, 1St Degree B\&E || 23030

Burglary-Fourth Degree, 3Rd Degree B\&E || 23030

Burglary-Fourth Degree, 3Rd Degree Buglary || 23030

Burglary-Fourth Degree, 4Th B/E | | 23030

Burglary-Fourth Degree, 4Th B\&E || 23030

Burglary-Fourth Degree, 4Th Deg B\&E || 23030

Burglary-Fourth Degree, 4Th Deg B+E || 23030

Burglary-Fourth Degree, 4Th Deg Burglary || 23030

Burglary-Fourth Degree, 4Th Deg. Burglary I| 23030

Burglary-Fourth Degree, 4Th Degree || 23030

Burglary-Fourth Degree, 4Th Degree B/E || 23030

Burglary-Fourth Degree, 4Th Degree B\&E || 23030

Burglary-Fourth Degree, 4Th Degree Burglary || 23030

Burglary-Fourth Degree, 4Th Dgree Burglary I| 23030

Burglary-Fourth Degree, B \& E || 23030

Burglary-Fourth Degree, B \& E || 23030

Burglary-Fourth Degree, B \& E 1St Degree I| 23030

Burglary-Fourth Degree, B \& E 2Nd Degree I| 23030

Burglary-Fourth Degree, B And E I| 23030

Burglary-Fourth Degree, B/E || 23030

Burglary-Fourth Degree, B/E Dwelling || 23030

Burglary-Fourth Degree, B\&E || 23030

Burglary-Fourth Degree, B\&E 4Th Degree II 23030

Burglary-Fourth Degree, B\&E- Second Degree || 23030

Burglary-Fourth Degree, B+E || 23030

Burglary-Fourth Degree, Breaking \& Entering I| 23030

Burglary-Fourth Degree, Breaking And Entering I| 23030

Burglary-Fourth Degree, Burglary I| 23030

Burglary-Fourth Degree, Burglary 4Th || 23030

Burglary-Fourth Degree, Burglary 4Th Degree I| 23030

Burglary-Fourth Degree, Burglary 4Th Dg. I | 23030

Burglary-Fourth Degree, Burglary Dwelling || 23030

Burglary-Fourth Degree, Burglary- 4Th Degree-Dwell || 23030

Burglary-Fourth Degree, Burglary-Fourth Degree I| 23030

Burglary-Fourth Degree, Burglury | | 23030

Burglary-Fourth Degree, Burlary I| 23030

Burglary-Fourth Degree, Cds I| 23030

Burglary-Fourth Degree, Cds Violation I| 23030

Burglary-Fourth Degree, Cds Violation Poss Of II 23030

Burglary-Fourth Degree, Commercial B\&E I| 23030

Burglary-Fourth Degree, Commercial Burglary || 23030

Burglary-Fourth Degree, Destruction Of Property II 23030

Burglary-Fourth Degree, Destruction Property II 23030

Burglary-Fourth Degree, Dirt Bike Violation I| 23030

Burglary-Fourth Degree, Fourth Degree Breaking And E I| 23030 
Burglary-Fourth Degree, Larceny || 23030

Burglary-Fourth Degree, Mal Destruction Of Prop || 23030

Burglary-Fourth Degree, Poss Of Marijuana II 23030

Burglary-Fourth Degree, Res B\&E || 23030

Burglary-Fourth Degree, Res. B\&E || 23030

Burglary-Fourth Degree, Residental B\&E || 23030

Burglary-Fourth Degree, Residential B\&E || 23030

Burglary-Fourth Degree, Residential Burglary I| 23030

Burglary-Fourth Degree, Trespassing || 23030

Burglary-Fourth Degree, Tresspassing || 23030

Burglary-General-Con, Con-Burglary-General || 1C2200

BURGLARY-THIRD DEG । |

BURGLARY-THIRD DEGREE || 23020

Burglary-Third Degree, 1St Degree Burglary II 23020

Burglary-Third Degree, 4Th Degree Burglary || 23020

Burglary-Third Degree, B/E || 23020

Burglary-Third Degree, B\&E || 23020

Burglary-Third Degree, Burglary || 23020

Burglary-Third Degree, Burglary-Third Degree I| 23020

Burglary-Third Degree, Residental Burglary || 23020

Burglary-Third Degree, Residential B\&E || 23020

Burglary-Third Degree, Stolen Auto II 23020

BURGLARY/2ND DEGREE/GENERAL || 23010

Burglary/2Nd Degree/General, 2Nd Degree Burglary || 23010

Burglary/2Nd Degree/General, 4Th Degree Burglary I| 23010

Burglary/2Nd Degree/General, Att B \& E || 23010

Burglary/2Nd Degree/General, B And E || 23010

Burglary/2Nd Degree/General, B\&E 4 Degree || 23010

Burglary/2Nd Degree/General, Breaking \& Entering || 23010

Burglary/2Nd Degree/General, Breaking And Entering || 23010

Burglary/2Nd Degree/General, Burglary || 23010

Burglary/2Nd Degree/General, Burglary 3Rd Degree I| 23010

Burglary/2Nd Degree/General, Burglary/2Nd Degree/Ge I| 23010

Burglary/2Nd Degree/General, Burglary/2Nd Degree/Gen I| 23010

Burglary/2Nd Degree/General, Commercial B And E I| 23010

Burglary/2Nd Degree/General, Commercial B\&E I| 23010

Burglary/2Nd Degree/General, Commercial Burglary || 23010

Burglary/2Nd Degree/General, Larceny II 23010

Burglary/2Nd Degree/General, Tresspassing || 23010

BURGLARY/THEFT || 23010

BURGULARY || 23030

BURGULARY || 34025

BURGULAY || 23000

BURLARY FROM AUTO || 23090

CDS / B\&E || 30233

Cds: Poss Marihuana L/T 10 G, 1St Degree Burglary || 11635

Cds: Poss Marihuana L/T 10 G, 4Th Degree B\&E/Cds I| 11635

Cds: Poss Marihuana L/T 10 G, B \& E I| 11635

Cds: Possession-Marihuana, Attempt B\&E || 10573

Cds: Possession-Marihuana, B\&E || 10573

Cds: Possession-Marihuana, Burglary || 10573

Cds:Possess-Not Marihuana, 4Th Degree B\&E I| 43550

Cds:Possess-Not Marihuana, B \& E || 43550

CDS/ B\&E || 30233

CDS/B\&E I| 43550

CMMERCIAL BURGLARY || 10622

COMMB/E || 23010

COMM B\&E || 23010

COMM BURGLARY || 23010

COMM. B\&E || 10621 
COMM. B\&E || 23010

COMM. BURGLARY || 10622

COMM. BURGLARY || 23040

COMMERCIAL B \& E || 10521

COMMERCIAL B \& E || 10621

COMMERCIAL B \& E || 23010

COMMERCIAL B \& E || 34025

COMMERCIAL B \& E || 34030

COMMERCIAL B/E || 23010

COMMERCIAL B\&E || 10622

COMMERCIAL B\&E || 23010

COMMERCIAL B\&E || 23040

COMMERCIAL B\&E || 23045

COMMERCIAL B\&E || 34025

COMMERCIAL B\&E || 34030

COMMERCIAL B+E || 10621

COMMERCIAL B+E || 23010

COMMERCIAL BURGLARY || 10621

COMMERCIAL BURGLARY | | 10622

COMMERCIAL BURGLARY || 11137

COMMERCIAL BURGLARY | | 22210

COMMERCIAL BURGLARY || 22220

COMMERCIAL BURGLARY || 23010

COMMERCIAL BURGLARY | | 23030

COMMERCIAL BURGLARY | | 23040

COMMERCIAL BURGLARY || 43550

COMMERCILA BURGLARY || 23010

COMMERICAL B\&E || 23010

COMMERICAL B\&E || 23040

COMMERICAL BURGLARY || 23040

COMMON B\&E || 10521

CON-BURGLARY-4TH DEG-DWELL || 2C3030

Con-Burglary-4Th Degree Theft, Poss Of Marijuana II 2C3040

CON-BURGLARY-FIRST DEGREE || 2C3000

Con-Burglary-Fourth Degree, B \& E || 2C3030

CON-BURGLARY-THIRD DEGREE || 2C3020

CON-BURGLARY/2ND DEGREE/GENERA I| 2C3010

Con-Burglary/2Nd Degree/General, 4Th Degree Burglary II 2C3010

CON-HOME INVASION || 1 C1338

DOM. B\&E || 2A3030

False Statement To Officer, 4Th Deg. B\&E I। 14803

FIRST DEGREE BUGLARY || 23000

FIRST DEGREE BURGLARY || 11136

FIRST DEGREE BURGLARY | | 23000

FIRST DEGREE BURGLARY || 23010

FIRST DEGREE BURGLARY || 23030

FIRST DEGREE BURGULARY || 23000

FIRST DEGREE BURLARY | | 23000

FIRST DREGREE BURGLARY || 23000

FOUR DEGREE BURGLARY || 23030

FOURTH DEGREE BURGLARY || 23030

FOURTH DEGREE BURGLARY I | 34025

Gaming/Cards- Dice- Etc., B \& E || 13903

Gaming/Cards- Dice- Etc., B \& E 4Th Degree II 13903

Gaming/Cards- Dice- Etc., B\&E || 13903

HOME INVASION || 11338

IST DEGREE BURGLARY || 23010

KIDNAPPING || 11338

Mal Dest Prop/Valu - \$500, Attempt B \& E I I 34025

Mal Dest Prop/Valu - \$500, Attempt Burglary II 34025 
Mal Dest Prop/Valu - \$500, B \& E I| 34025

Mal Dest Prop/Valu - \$500, B\&E || 34025

Mal Dest Prop/Valu - \$500, B+E II 34025

Mal Dest Prop/Valu - \$500, Burglary II 34025

Mal Dest Prop/Valu - \$500, Possession Of Burglary To II 34025

Mal Destr Prop Value + \$500, Attemp B\&E II 34030

Mal Destr Prop Value + \$500, Attempt B\&E II 34030

Mal Destr Prop Value + \$500, Attempted Burglary II 34030

Mal Destr Prop Value + \$500, B\&E I| 34030

Mal Destr Prop Value + \$500, B+E I| 34030

Mal Destr Prop Value + \$500, Burglary II 34030

Mal Destr Prop Value + \$500, Commercial B\&E/ Destruc II 34030

MAL DESTRUCTION OF PROPERTY || 1A1338

Peace Order: Fail To Comply, Burglary || 20105

RES. BURLARY || 23030

RES/ B\&E || 23030

RESIDENTAL B\&E || 23030

RESIDENTIAL B \& E || 23040

RESIDENTIAL B\&E || 23030

RESIDENTIAL B \& E || 23010

RESIDENTIAL B \& E || 23030

RESIDENTIAL B AND E || 23000

RESIDENTIAL B AND E | | 23030

RESIDENTIAL B AND E || 23050

RESIDENTIAL B AND E I | 34025

RESIDENTIAL B\&E || 10600

RESIDENTIAL B\&E || 10622

RESIDENTIAL B\&E || 22220

RESIDENTIAL B\&E || 23000

RESIDENTIAL B\&E || 23010

RESIDENTIAL B\&E || 23030

RESIDENTIAL B\&E || 23040

RESIDENTIAL B\&E I| 34025

RESIDENTIAL B\&E || 43550

RESIDENTIAL B+E || 23000

RESIDENTIAL B+E || 23030

RESIDENTIAL BREAKING AND ENTER || 23000

RESIDENTIAL BREAKING AND ENTER || 23030

RESIDENTIAL BURG || 23030

RESIDENTIAL BURGLARY | | 10621

RESIDENTIAL BURGLARY || 10622

RESIDENTIAL BURGLARY || 10826

RESIDENTIAL BURGLARY || 11338

RESIDENTIAL BURGLARY | | 23000

RESIDENTIAL BURGLARY || 23030

SECOND DEGREE B\&E || 23010

SECOND DEGREE BURGLARY || 10621

STOLEN AUTO/B\&E I| 23000

Theft Less Than \$100.00, B \& E || 10521

Theft Less Than \$100.00, B\&E || 10521

Theft Less Than $\$ 100.00$, Commercial Burglary || 10521

Theft: \$1,000 To Under \$10,000, 1St Degree Burglary II 10622

Theft: $\$ 1,000$ To Under $\$ 10,000$, B\&E 4Th Degree I| 10622

Theft: $\$ 1,000$ To Under $\$ 10,000$, Burglary I| 10622

Theft: $\$ 1,000$ To Under $\$ 10,000$, Burglary 1St Degree II 10622

Theft: $\$ 1,000$ To Under $\$ 10,000$, Burglary Residental || 10622

Theft: \$1,000 To Under \$10,000, Residential B \& E I| 10622

Theft: \$1,000 To Under \$10,000, Residential Burglary || 10622

Theft: Less \$1,000 Value, 4Th Degree Burglary II 10621

Theft: Less \$1,000 Value, 4Th Degree Burgulary I| 10621 
Theft: Less \$1,000 Value, Burglary || 10621

Theft: Less \$1,000 Value, Burglary 4Th Degree | | 10621

Theft: Less $\$ 1,000$ Value, Burglary First Degree I| 10621

Theft: Less \$1,000 Value, First Degree Burglary I| 10621

Trespass-Posted Property, 4Th Degree Burglary || 22210

Trespass-Posted Property, Att B/E || 22210

Trespass-Posted Property, B\&E || 22210

Trespass-Posted Property, Burglary || 22210

Trespass-Posted Property, Burglary - 4Th Degree || 22210

Trespass-Posted Property, Burglary 4Th Deg || 22210

Trespass-Posted Property, Burglary 4Th Degree I| 22210

Trespass: Private Property, 4Th Degree Burglary II 22220

Trespass: Private Property, B\&E || 22220

Trespass: Private Property, Burglary || 22220

TRESPASSING /4TH DEGREE BURGLA I | 2A0696

TRESPASSING/ BURGLARY || 23030

TRESPASSING/4TH DEGREE B+E I| 22210

VACANT B\&E || 22210

\section{Larceny}

Alc. Bev./Intox:Endanger, Larceny || 14200

ANAUTH USE || 22411

ATOU THEFT I| 20480

ATT - THEFT: LESS \$1,000 VALUE I । 1A0621

Att - Theft: Less \$1,000 Value, Atempted Theft II 1A0621

Att - Theft: Less \$1,000 Value, Att - Theft: Less \$1 I| 1A0621

Att - Theft: Less $\$ 1,000$ Value, Attempt Theft II 1A0621

Att - Theft: Less \$1,000 Value, Attempted Theft II 1A0621

Att - Theft: Less $\$ 1,000$ Value, Larceny II 1A0621

Att - Theft: Less \$1,000 Value, Theft || 1A0621

Att - Theft: Less \$1,000 Value, Uttering I| 1A0621

ATT -THEFT:\$10K TO UNDER \$100K I | 1A0623

Att -Theft:\$10K To Under \$100K, Trespassing || 1A0623

ATT LARCENY || 10622

ATT LARCENY || 1A0621

ATT LARCENY || 23090

ATT LARCENY I| 34025

ATT LARCENY FROM AUTO || 23090

ATT STOLEN AUTO । I 11119

ATT STOLEN AUTO || 2A0480

ATT STOLEN AUTO || 34030

ATT THEFT || 10521

ATT THEFT I| 34025

ATT- THEFT: \$1K TO UNDER \$10K || 1A0622

Att- Theft: \$1K To Under \$10K, Attempt Auto Theft II 1 A0622

Att- Theft: \$1K To Under \$10K, Attempt Stolen Auto II 1A0622

Att- Theft: \$1K To Under \$10K, Check Fraud II 1 A0622

Att- Theft: \$1K To Under \$10K, False Pretense I| $1 \mathrm{~A} 0622$

Att- Theft: $\$ 1 \mathrm{~K}$ To Under $\$ 10 \mathrm{~K}$, Stolen Auto II 1A0622

Att-Cds Manuf/Dist., Theft I I 2A3550

ATT-MOTOR VEH/UNLAWFUL TAKING I| 2A0480

Att-Motor Veh/Unlawful Taking, Att Stolen Auto I| 2A0480

ATT-ROGUE AND VAGABOND || 2A3090

ATT-THEFT LESS THAN \$100.00 | | 1A0521

Att-Theft Less Than $\$ 100.00$, Att Theft I| 1A0521

Att-Theft Less Than \$100.00, Att-Theft Less Than \$10 || 1A0521

Att-Theft Less Than \$100.00, Attempt Theft II 1A0521

Att-Theft Less Than $\$ 100.00$, Larceny | | 1 A0521 
Att-Theft Less Than $\$ 100.00$, Larceny From Auto II 1A0521 Att-Theft Less Than $\$ 100.00$, Larceny Shoplifting | | 1A0521 Att-Theft Less Than $\$ 100.00$, Theft II 1A0521

ATT-THEFT: \$1,500 TO UND \$25K I| 1A1136

ATT-THEFT: \$100 TO UND \$1,500 || 1A1137

ATT. LARCENY || 1A0521

ATT. LARCENY || 23090

ATT. LARCENY FROM AUTO || 23090

ATT. LARCENY FROM AUTO || 34025

ATT. LARCONY I| 10521

ATT. THEFT || 10621

ATT. THEFT || 1A0521

ATT. THEFT || 23090

ATTEMPT AUTO THEFT || 10623

ATTEMPT AUTO THEFT I | 34025

ATTEMPT GRAND LARCENY | | 34030

ATTEMPT LARCENCY FROM AUTO || 23090

ATTEMPT LARCENY || 10521

ATTEMPT LARCENY || 10622

ATTEMPT LARCENY || 11137

ATTEMPT LARCENY || 1A0521

ATTEMPT LARCENY | | 1A0621

ATTEMPT LARCENY | | 1A1136

ATTEMPT LARCENY || 1A1137

ATTEMPT LARCENY || 22220

ATTEMPT LARCENY || 23090

ATTEMPT LARCENY I 53550

ATTEMPT LARCENY FROM AUTO || 1A0621

ATTEMPT LARCENY FROM AUTO | | 23090

ATTEMPT LARCENY FROM AUTO I | 34025

ATTEMPT LARCENY OF AUTO | | 23090

ATTEMPT STOLEN AUTO I| 10622

ATTEMPT STOLEN AUTO | | 1A0622

ATTEMPT STOLEN AUTO I I 20480

ATTEMPT STOLEN AUTO || 23090

ATTEMPT STOLEN AUTO | | 2A0480

ATTEMPT STOLEN AUTO I | 34030

ATTEMPT THEFT । | 1A0521

ATTEMPT THEFT || 1A0621

ATTEMPT THEFT । $1 \mathrm{~A} 1137$

ATTEMPT THEFT || 23090

ATTEMPT THEFT \$1K-\$10K । | 1A0622

ATTEMPT THEFT FROM AUTO || 23090

ATTEMPTED LARCENY | | 10621

ATTEMPTED LARCENY | 1 A0621

ATTEMPTED LARCENY || 23090

ATTEMPTED LARCENY FROM AUTO || 23090

ATTEMPTED STOLEN AUTO || 1A0622

ATTEMPTED STOLEN AUTO || 23090

ATTEMPTED STOLEN AUTO || 2A2411

ATTEMPTED THEFT I I 1A0521

ATTEMPTED THEFT । | 1C1137

ATTEMPTED THEFT । 23090

ATTEMPTED THEFT I। 2A0480

ATTEMPTED THEIFT || 1A0623

ATTEMPTRD LARCENY || 23090

AUTO THEFT I।

AUTO THEFT || 10521

AUTO THEFT || 10621

AUTO THEFT || 10622 


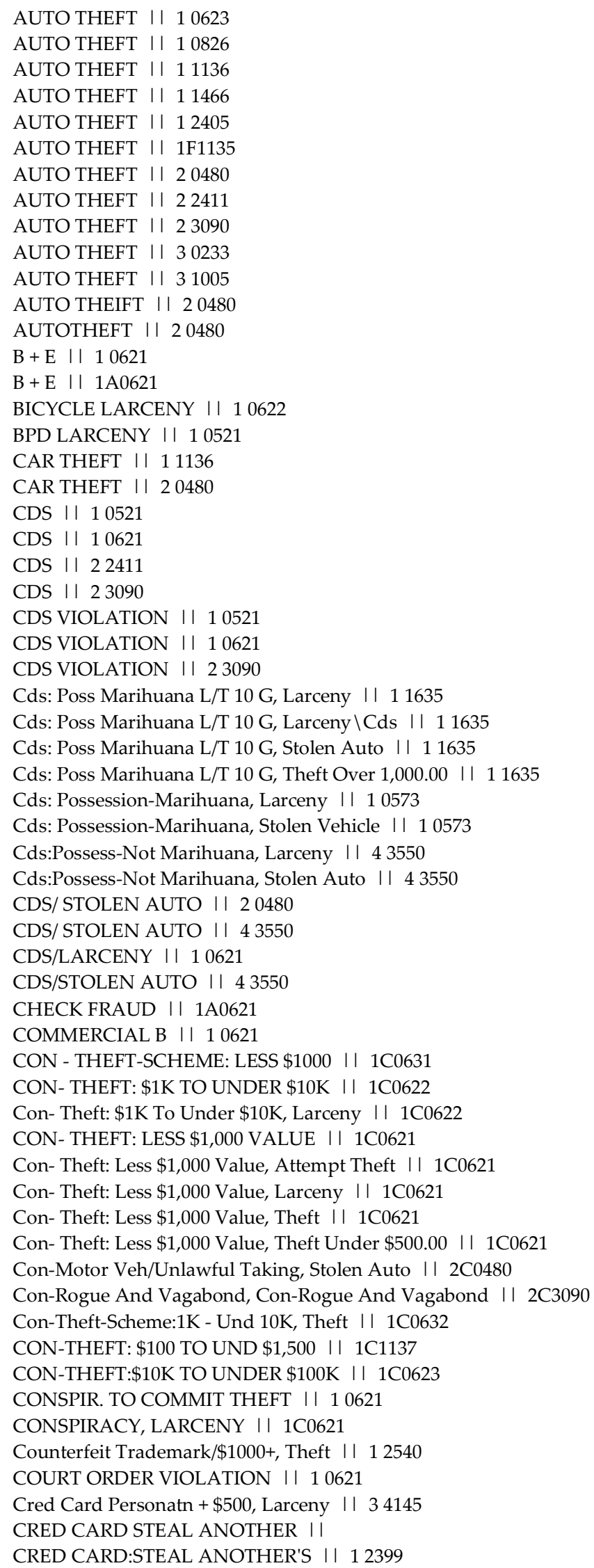


Cred Card:Steal Another'S, Cred Card:Steal Another'S I| 12399

Cred Card:Steal Another'S, Credit Card Fraud || 12399

Cred Card:Steal Another'S, Theft || 12399

Cred Crd Receiv Unlawfully, Theft || 12803

Currency:Manufacture/Counterfeit, Theft || 10067

DEST. OF PROPERTY || 23090

DEST. OF. PROP/LARCENY I | 34025

DESTRUCTION OF PROPERTY || 10521

DESTRUCTION OF PROPERTY || 10621

DESTRUCTION OF PROPERTY || 23090

DESTRUCTION OFF PROPERTY || 23090

DESTRUCTION PROPERTY || 23090

DESTUCTION OF PROPERTY | | 23090

DIRT BIKE VIOLATION || 10621

DIRT BIKE VIOLATION || 22411

DIRTBIKE VIOLATION/STOLEN AUTO । ।

DIRTBIKE VIOLATION/STOLEN AUTO || 10623

Dis.Erly Conduct, Larceny | | 20050

DISORDELY || 23090

DISORDERLY || 10621

DISORDERLY CONDUCT || 10521

DISORDERLY CONDUCT || 10621

DISPLAY REG. ISSUE TO ANOTHER | | 10521

DRIVING ON SUSPENDED LIC | | 22411

DRIVING STOLEN VECHILE | | 20480

DRIVING STOLEN VEHICLE || 11136

DRIVING UNINSURED VEH | | 10521

DRIVING WITHOUT LICENSE | | 10521

DRIVING WITHOUT LICENSE | | 22411

Electr Co:Tamper W/Meter, Larceny | | 10215

Fail Obey Renble/Lawfl, Larceny || 20055

FAIL TO OBEY || 23090

FAIL TO PAY TAXI || 10521

FALSE PRETENSE || 10621

FALSE PRETENSE || 10521

FALSE PRETENSE || 10621

FALSE PRETENSE || 1A0621

False Statement To Officer, Larceny || 14803

False Statement To Officer, Theft I| 14803

FALSE STATEMENT TO POLICE OFFI | | 10521

False Stmt To Peace Officer, Theft Under $\$ 1000.00$ || 10690

FELONY LARCENY || 11136

FELONY THEFT || 11136

FELONY THEFT OVER 1,000.00 | | 11137

Forgery-Priv Documents, Att Larceny I| 12502

Forgery-Priv Documents, Theft || 12502

FRAUD || 10521

FRAUD PER ID INFOTHEFT \$500+ | | 11301

Fraud Per Id Infotheft \$500+, Theft Over \$1000 || 11301

FRAUD-PER IDENT INFO THEFT || 11200

FRAUD-PER IDENT INFO THEFT I| 20071

FRAUD-PER IDENT INFO THEFT \$50 || 11200

Fraud-Per Ident Info Theft, Credit Card Fraud | | 11200

Fraud-Per Ident Info Theft, Fraud-Per Ident Info The I| 11200

Fraud-Per Ident Info Theft, Identity Theft I| 11200

FRAUD-PER IDENT INFO THEFT\$500 || 11201

GRAND LARCENY || 10622

GRAND LARCENY | | 10633

GRAND THEFT I I 10622

GRAND THEFT AUTO || 10623 
GRAND THEFT AUTO || 20480

GRAND THEFT AUTO || 23090

Grocery Carts-Removal, Larceny || 12411

HINDERING || 10621

ID THEFT/ FALSE STATEMENT || 11432

IDENITY THEFT I ।

IDENTITY THEFT । I 10196

IDENTITY THEFT || 11160

IDENTITY THEFT || 1A0622

LAARCENY || 10521

LACENY || 10621

LACRENY || 10521

LANCENY FROM AUTO || 23090

LAR/TRESPASSING || 10621

LARCANY || 10521

LARCEBY FROM AUTO || 23090

LARCENCY || 10521

LARCENCY || 10621

LARCENCY || 11137

LARCENCY || 15202

LARCENCY FROM AUTO || 10621

LARCENCY LESS THAN \$100 || 10521

LARCENEY || 10621

Larceny II

LARCENY || 10191

LARCENY || 10356

LARCENY || 10521

LARCENY || 10573

LARCENY | | 10600

LARCENY || 10621

LARCENY || 10622

LARCENY | | 10623

LARCENY || 10631

LARCENY || 10632

LARCENY | | 10642

LARCENY || 10690

LARCENY | 11111

LARCENY || 11135

LARCENY || 11136

LARCENY || 11137

LARCENY || 11160

LARCENY || 11286

LARCENY || 11416

LARCENY I| 12399

LARCENY || 15200

LARCENY || 15202

LARCENY || 16505

LARCENY || 1A0521

LARCENY || 1A0621

LARCENY || 1A1136

LARCENY || 1F0621

LARCENY || 20050

LARCENY || 20480

LARCENY || 20700

LARCENY || 22210

LARCENY || 22220

LARCENY || 22411

LARCENY || 23030

LARCENY | | 23040

LARCENY || 23090 


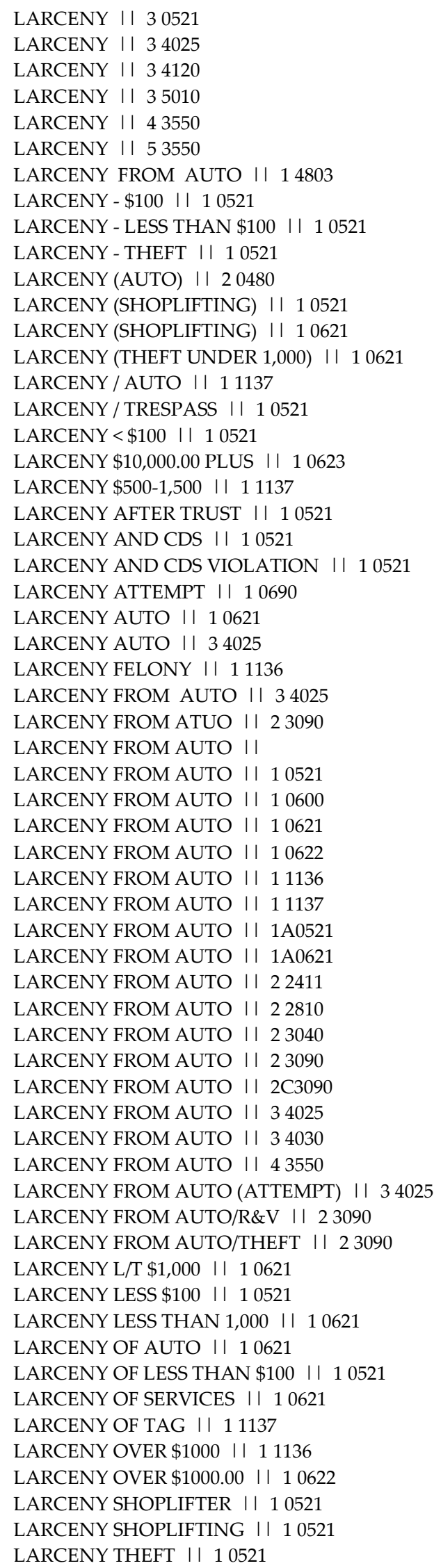




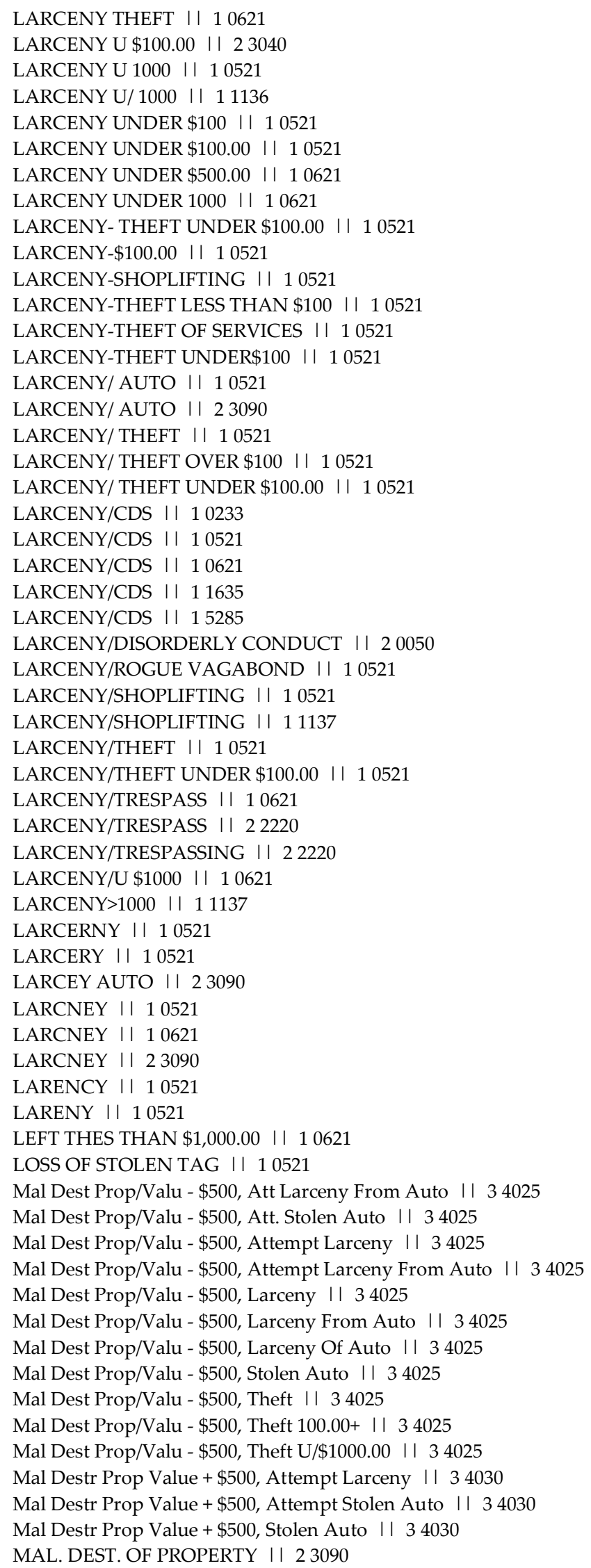


MOTER VEHICLE TAKING || 20480

MOTOE VEHICLE/ UNLAWFUL TAKING || 20480

MOTOR VECHICLE THEFT || 10623

MOTOR VECHILE THEFT || 10622

MOTOR VEH UNLAWFUL TAKING ।

MOTOR VEH/UNLAWFUL TAKING I| 20480

Motor Veh/Unlawful Taking, Auto Theft I I 20480

Motor Veh/Unlawful Taking, Car Theft I I 20480

Motor Veh/Unlawful Taking, Cds Poss II 20480

Motor Veh/Unlawful Taking, Cds Violatiion II 20480

Motor Veh/Unlawful Taking, Cds Violation II 20480

Motor Veh/Unlawful Taking, Destruction Of Property II 20480

Motor Veh/Unlawful Taking, Dirt Bike Violation II 20480

Motor Veh/Unlawful Taking, Dirtbike Violation II 20480

Motor Veh/Unlawful Taking, Motor Veh/Unlawful Takin II 20480

Motor Veh/Unlawful Taking, Motor Veh/Unlawful Taking I | 20480

Motor Veh/Unlawful Taking, Motor Vehcile Theft II 20480

Motor Veh/Unlawful Taking, Motor Vehicle Theft II 20480

Motor Veh/Unlawful Taking, Poss. Of Stolen Vehicle II 20480

Motor Veh/Unlawful Taking, Stloen Auto II 20480

Motor Veh/Unlawful Taking, Stolen Auto I| 20480

Motor Veh/Unlawful Taking, Stolen Auto II 20480

Motor Veh/Unlawful Taking, Stolen Auto/Unauthorize U II 20480

Motor Veh/Unlawful Taking, Stolen Auton II 20480

Motor Veh/Unlawful Taking, Stolen Motor Scooter II 20480

Motor Veh/Unlawful Taking, Stolen Vechicle II 20480

Motor Veh/Unlawful Taking, Theft II 20480

Motor Veh/Unlawful Taking, Theft \$10,000 - \$100,000 || 20480

Motor Veh/Unlawful Taking, Theft \$10,000-\$100,000 II 20480

Motor Veh/Unlawful Taking, Theft Over \$1,000 || 20480

Motor Veh/Unlawful Taking, Theft Over 1000.00 || 20480

Motor Veh/Unlawful Taking, Unathurized Use I| 20480

Motor Veh/Unlawful Taking, Unauthorize Used II 20480

Motor Veh/Unlawful Taking, Unauthorized Use II 20480

Motor Veh/Unlawful Taking, Unauthorized Use Of Auto I| 20480

Motor Veh/Unlawful Taking, Vehicle Theft II 20480

MOTOR VEHICLE THEFT I I 10622

MOTOR VEHICLE THEFT || 10623

MOTOR VEHICLE THEFT । | 11136

MOTOR VEHICLE THEFT I| 20480

MOTOR VEHICLE THEFT I| 22411

MOTOR VEHICLE UNLAWFUL TAKING || 20480

MOTOR VEHICLE/UNLAWFUL TAKING I।

MOTORVEH/UNLAWFULTAKING I I

MV THEFT II 20480

Obstructing \& Hindering, Larceny || 10043

OPEN CONTAINER II 10621

OPERATING A STOLEN VEHICLE | | 34025

OPERATING STOLEN VEHICLE I | 20480

OPERATIONS STOLEN VEHICLE || 20480

Other Non Felony Charge, Fraud-Per Ident Info Theft I I 20071

Other Non Felony Charge, Fraud-Per Ident Info Theft I I 20073

Other Non Felony Charge, Identity Theft I| 20073

Other Non Felony Charge, Theft I| 20071

POSESSION OF STOLEN TAGS I| 10521

POSS - STOLEN AUTO || 10623

POSS CRIMINAL TOOLS || 1A0621

POSS OF A STOLEN AUTO || 10622

POSS OF COUNTERFEIT MONEY I | 1A0521

POSS OF STOLEN AUTO || 11466 
POSS OF STOLEN AUTO || 20480 POSS OF STOLEN PLATES || 10521 POSS OF STOLEN PROP || 10621 POSS OF STOLEN PROPERTY | | 10521 POSS OF STOLEN PROPERTY | | 10621 POSS OF STOLEN PROPERTY | | 20055 POSS OF STOLEN PROPERTY I | 20480 POSS OF STOLEN PROPERTY || 22810 POSS OF STOLEN VEHICLE | | 20480 POSS OF STOLEN VEHICLE I| 22411 POSS STOLEN AUTO || 10622 POSS STOLEN AUTO || 11136 POSS STOLEN AUTO || 11466 POSS STOLEN AUTO || 11467 POSS STOLEN AUTO || 20480 POSS STOLEN LIC PLATE | | 10621 POSS STOLEN PROP || 10521 POSS STOLEN PROP || 10621 POSS STOLEN PROPERTY | | 10521 POSS STOLEN PROPERTY || 10621 POSS STOLEN PROPERTY I| 20480 POSS STOLEN TAG || 10521 POSS STOLEN TAGS || 22810 POSS STOLEN VEHICLE || 10622 POSS STOLEN VEHICLE | | 20480 POSS. OF STOLEN AUTO || 20480 POSS. OF STOLEN PROPERTY | | 10621 POSS. OF STOLEN TAG || 10521 POSS. OF STOLEN VEHICLE || 11136 POSS. OF STOLEN VEHICLE I I 20480 POSS. STOLEN AUTO || 10622 POSS. STOLEN AUTO || 20480 POSS. STOLEN BIKE || 10621 POSS. STOLEN PROPERTY || 10521 POSS. STOLEN PROPERTY || 11137 POSS. STOLEN TAG || 10521 POSS. STOLEN TAG || 11137 POSS. STOLEN TAGS || 10621 POSS. STOLEN VEHICLE I| 20480 POSS.STOLEN PROPERTY || 2R2803 POSSESION OF STOLEN AUTO || 10623 POSSESION OF STOLEN AUTO || 20480 POSSESION OF STOLEN PROPERTY I | 10521 POSSESION OF STOLEN PROPERTY I | 11137 POSSESION OF STOLEN TAGS || 10521 POSSESION OF STOLEN VEHICLE || 10623 POSSESION STOLEN AUTO || 10623 POSSESION STOLEN AUTO || 20480 POSSESS STOLEN AUTO || 20480

Possess/Issue Forged Currency, Attempted Larceny || 10494 Possess/Use False Govt Id Doc, Identity Theft II 11432 Possess/Use False Govt Id Doc, Idnetity Theft II 11432 POSSESSION I| 10621 POSSESSION OF STOLEN PRPERTY || 10621 POSSESSION OF A STOLEN AUTO || 20480 POSSESSION OF HEROIN II 22411

POSSESSION OF STOLEN AUTO | | 10622 POSSESSION OF STOLEN AUTO | | 11136 POSSESSION OF STOLEN AUTO | | 20480 POSSESSION OF STOLEN AUTO | | 22411 
POSSESSION OF STOLEN CELL PHON || 10621 POSSESSION OF STOLEN GOODS || 10621 POSSESSION OF STOLEN GOODS | | 11137 POSSESSION OF STOLEN PROP || 10521 POSSESSION OF STOLEN PROPERTY | | 10521 POSSESSION OF STOLEN PROPERTY | | 10621 POSSESSION OF STOLEN PROPERTY I | 10826 POSSESSION OF STOLEN PROPERTY || 11136 POSSESSION OF STOLEN PROPERTY || 11137 POSSESSION OF STOLEN PROPERTY || 20480 POSSESSION OF STOLEN PROPERTY | | 22810 POSSESSION OF STOLEN SCOOTER || 22411 POSSESSION OF STOLEN TAG || 22810 POSSESSION OF STOLEN VECHICLE I। 20480 POSSESSION OF STOLEN VEHICLE || 11136 POSSESSION OF STOLEN VEHICLE || 20480 POSSESSION OF STOLEN VEHICLE || 22411 POSSESSION STOLEN AUTO || 20480 POSSESSION STOLEN PROPERTY | | 10621 POSSESSION STOLEN PROPERTY I | 10622 POSSESSION STOLEN PROPERTY | | 11137 PURCHASE STOLEN AUTO PARTS | | 10622 PURSE SNATCH || 10521 RECEIVING STOLEN PROPERTY | | 11137 RECKLESS ENDANGERMENT | | 10521 RECOVEED STOLEN AUTO || 20480 RECOVERD STOLEN AUTO | | 20480 RECOVERED STOLEN I| 20480 RECOVERED STOLEN AUTO I । RECOVERED STOLEN AUTO | | 20480 RECOVERED STOLEN AUTO || 22411 RECOVERED STOLEN AUTO I| 34030 RECOVERED STOLEN PROPERTY || 10521 RECOVERED STOLEN PROPERTY || 11137 RECOVERED STOLEN PROPERTY || 30521 RECOVERED STOLEN TAG | | 11119 RECOVERED STOLEN TAG || 11137 RECOVERED STOLEN TAGS || 10521 RECOVERED STOLEN TAGS || 10621 RECOVERED/STOLENAUTO || 10622 REOVERED STOLEN AUTO | | 20480 RESISISTING ARREST । | 10521

Resist/Interfere With Arrest, Larceny | | 10600 ROGAN VAGABOND || 23090 ROGUE \& VAGABOND || 10521 ROGUE \& VAGABOND || 10621 ROGUE \& VAGABOND || 23090 ROGUE \& VAGABOND | | 2A3090 ROGUE \& VAGABONDS || 10621 ROGUE \& VAGOBOND || 23090 ROGUE A VAGABOND || 23090 ROGUE AND VAGABAND || 10521 ROGUE AND VAGABAND | | 23090 ROGUE AND VAGABON || 23090 ROGUE AND VAGABOND || 10521 ROGUE AND VAGABOND | | 10621 ROGUE AND VAGABOND || 1A0521 ROGUE AND VAGABOND || 1A0621 ROGUE AND VAGABOND | | 23090

Rogue And Vagabond, Att Larceny II 23090 
Rogue And Vagabond, Att Larceny From Auto II 23090 Rogue And Vagabond, Att. Larceny From Auto || 23090 Rogue And Vagabond, Attempt Auto Theft I| 23090

Rogue And Vagabond, Attempt Larceny From Auto II 23090

Rogue And Vagabond, Attempt Stolen Auto II 23090

Rogue And Vagabond, Attempt Theft From Auto II 23090

Rogue And Vagabond, Attempted Auto Theft II 23090

Rogue And Vagabond, Attepted Stolen Auto II 23090

Rogue And Vagabond, Destruction Of Prop || 23090

Rogue And Vagabond, Destruction Of Property II 23090

Rogue And Vagabond, Larcency From Auto I| 23090

Rogue And Vagabond, Larceny I| 23090

Rogue And Vagabond, Larceny From Auto II 23090

Rogue And Vagabond, Poss Of Stolen Property II 23090

Rogue And Vagabond, Ro;Gue\&Vagabond I| 23090

Rogue And Vagabond, Rogue \& Vaga Bond I| 23090

Rogue And Vagabond, Rogue \& Vagabon I | 23090

Rogue And Vagabond, Rogue \& Vagabond II 23090

Rogue And Vagabond, Rogue And Vagabond II 23090

Rogue And Vagabond, Rogue And Vegabond I| 23090

Rogue And Vagabond, Rogurt Vagabond I| 23090

Rogue And Vagabond, Rouge \& Vagabond I I 23090

Rogue And Vagabond, Rouge And Vagabond II 23090

Rogue And Vagabond, Rouge And Vogabond I I 23090

Rogue And Vagabond, Rougue And Vagabond II 23090

Rogue And Vagabond, Theft I| 23090

Rogue And Vagabond, Theft From Auto I| 23090

Rogue And Vagabond, Theft Over \$300 || 23090

Rogue And Vagabond, Theft Over 1000.00 || 23090

ROGUE AND VAGABOUND || 23090

ROGUE AND VAGENBOND || 23090

ROGUE AND VEGABON II 23090

ROGUE AND VEGABOND | | 23090

ROGUE VAGABOND || 23090

ROGUE\& VAGABOND || 1A0621

ROGUE\& VAGABOND || 23090

ROGUE\&VAGABOND || 23090

ROUGE \& VAGABOND || 23090

ROUGE + VAGABOND || 23090

ROUGE AND VAGABON I| 23090

ROUGE AND VAGABOND || 23090

ROUGE AND VAGABOND || 2A3090

ROUGE AND VAGABOUND || 23090

ROUGE VAGABOND || 23090

ROUGE VAGABOND || 2A3090

ROUGE VAGAVON || 23090

ROUGUE VAGABOND || 23090

SELL W/O LIC II 1A0521

SELL W/O LICENSE | | 1A0521

SELL/POSSES STOLEN MANF NO. I I 22810

SELLING W/OUT A LICENSE | | 10521

SELLING WITHOUT A LICENSE | | 10521

SHOP LIFTING I| 10521

SHOP LIFTING || 10621

SHOPLFTING || 10521

SHOPLIFITING || 10621

SHOPLIFT || 10521

SHOPLIFTING || 10521

SHOPLIFTING || 10621

SHOPLIFTING || 1F0621 
SHOPLIFTING UNDER \$100 || 10521

SHOPLIFTING UNDER \$100.00 || 10521

STOELN AUTO || 20480

STOLEN I| 20480

STOLEN AUTO || 10622

STOLEN AUTO || 11136

STOLEN AUTO || 20480

STOLEN ATO || 20480

STOLEN ATUO || 34025

STOLEN AUTIO II 20480

STOLEN AUTO I।

STOLEN AUTO || 10521

STOLEN AUTO || 10573

STOLEN AUTO II 10600

STOLEN AUTO || 10621

STOLEN AUTO || 10622

STOLEN AUTO || 10623

STOLEN AUTO || 10825

STOLEN AUTO || 10826

STOLEN AUTO || 11106

STOLEN AUTO || 11111

STOLEN AUTO || 11119

STOLEN AUTO || 11135

STOLEN AUTO || 11136

STOLEN AUTO || 11137

STOLEN AUTO || 11455

STOLEN AUTO || 11466

STOLEN AUTO || 11564

STOLEN AUTO || 11635

STOLEN AUTO || 15285

STOLEN AUTO || 20480

STOLEN AUTO || 22411

STOLEN AUTO || 23030

STOLEN AUTO || 23090

STOLEN AUTO || 30233

STOLEN AUTO I| 34025

STOLEN AUTO || 43550

STOLEN AUTO || 53550

STOLEN AUTO (BIKE) || 20480

STOLEN AUTO / CDS I | 30233

STOLEN AUTO / RECOVERED | | 22411

STOLEN AUTO TAGS | | 11137

STOLEN AUTO THEFT || 20480

STOLEN AUTO/ FALSE STATEMENT I | 11137

STOLEN AUTO/ V.V || 20480

STOLEN AUTO/CDS || 11111

STOLEN AUTO/CDS I| 20480

STOLEN AUTO/THEFT || 20480

STOLEN AUTTO || 20480

STOLEN BIKE || 10622

STOLEN CAR I| 10623

STOLEN CAR I| 20480

STOLEN CAR I| 34030

STOLEN DIRTBIKE || 20480

STOLEN LICENSE PLATE I ।

STOLEN LICENSE PLATE | | 10521

STOLEN LICENSE PLATE || 10621

STOLEN LICENSE PLATE || 22810

STOLEN LICENSE PLATE I | 34030

STOLEN MOTORCYCLE || 20480 
STOLEN PLATE (POSSESSION) | | 10621

STOLEN PROPERTY || 10621

STOLEN PROPERTY/THEFT I | 10621

STOLEN REAR TAG POSSESSION I I 10521

STOLEN RECOVERD TAGS || 10621

STOLEN TAG || 10521

STOLEN TAG || 10621

STOLEN TAG || 11119

STOLEN TAG || 11137

STOLEN TAG || 11564

STOLEN TAG || 22810

STOLEN TAG || 43550

STOLEN TAG LARCENY | | 10621

STOLEN TAGS || 10233

STOLEN TAGS || 10521

STOLEN TAGS || 10621

STOLEN TAGS || 11137

STOLEN TAGS || 11564

STOLEN TAGS || 22810

STOLEN TAGS/CDS || 11564

STOLEN THEFT GREATER 100 || 10521

STOLEN VECHILE I | 10622

STOLEN VEHC I| 20480

Stolen Vehicle II

STOLEN VEHICLE I |

STOLEN VEHICLE I| 10622

STOLEN VEHICLE || 10690

STOLEN VEHICLE || 11136

STOLEN VEHICLE || 20480

STOLEN VEHICLE I| 22411

STOLEN/UNATHORIZED USE || 20480

STOLENAUTO || 20480

TAKING VEH. W/O OWNER'S CONSEN I।

THEDT UNDER 1000.00 | | 10621

THEF LESS THAN \$100 || 10621

THEFR/LORCENY || 10521

Theft II

THEFT I।

THEFT || 10217

THEFT I| 10521

THEFT || 10621

THEFT I| 10622

THEFT I| 10623

THEFT I| 10631

THEFT I| 10632

THEFT || 10633

THEFT I| 10660

THEFT I| 10690

THEFT || 11111

THEFT || 11135

THEFT || 11136

THEFT || 11137

THEFT I| 11160

THEFT । 12399

THEFT I| 12513

THEFT || 1A0521

THEFT || 1A0621

THEFT I| 1A0622

THEFT I| 1A1137

THEFT || 1F1137 
THEFT || 20050

THEFT ।| 20480

THEFT II 20700

THEFT I| 22210

THEFT || 22220

THEFT || 22411

THEFT I| 22810

THEFT I| 23030

THEFT || 23040

THEFT || 23080

THEFT I| 23090

THEFT I | 30233

THEFT || 34025

THEFT II 34030

THEFT I| 43550

THEFT UNDER 100 | | 10521

THEFT - \$500 || 10521

THEFT - ATTEMPT || 10030

Theft - Attempt, Theft - Attempt I| 10030

THEFT - LESS I| 10521

THEFT -\$500.00 || 10621

THEFT -U- 100.00 || 10521

THEFT (ATT) I| 1A0521

THEFT (LARCENY FROM AUTO) | | 10621

THEFT (SHOPLIFTING) I । 10521

THEFT (SHOPLIFTING) I। 10621

THEFT +1000 || 10622

THEFT $<$ । I 10621

THEFT $<\$ 100$ | | 10521

THEFT $<\$ 100.00$ || 10521

THEFT $<\$ 1000$ || 10621

THEFT $<1 \mathrm{~K}$ || 10521

THEFT $<\$ 1,000$ | | 11137

THEFT $<\$ 100$ || 10521

THEFT $<\$ 100.00$ || 10521

THEFT < \$100/TRESPASSING || 10521

THEFT $<\$ 1000$ || 10621

THEFT $<\$ 500$ || 10621

THEFT $<100$ || 10521

THEFT $>\$ 1000 \quad$ | | 10622

THEFT $>1000$ || 10621

THEFT $\$ 1,000-\$ 10,000$ || 10632

THEFT \$1,000.00 || 10622

THEFT $\$ 10,000$ TO UNDER $\$ 100,00$ I ।

THEFT $\$ 100$ - \$1,000 || 10621

THEFT \$100 LESS || 10521

THEFT $\$ 100-\$ 1,000$ || 10621

THEFT \$100-\$1000 || 10621

THEFT \$100-1499 || 11137

THEFT \$1000 (STOLEN AUTO) | | 20480

THEFT 1000.00 PLUS I| 23040

THEFT 1500-2500 || 11136

THEFT ABOVE 1000 || 10623

THEFT AUTO || 11136

THEFT AUTO || 11466

THEFT AUTO I| 20480

THEFT FROM AUTO || 10521

THEFT FROM AUTO ।| 10621

THEFT FROM AUTO I। 23090

THEFT GREATER THAN \$1000 || 10622 
THEFT GREATER THAN \$500 || 12399

THEFT GREATER THAN 1000 | | 10622

THEFT L/T | | 10621

THEFT L/T \$1,000 || 1 C0621

THEFT L/T \$100 || 10521

THEFT L/T \$100 || 10621

THEFT L/T $\$ 1000$ | | 22220

THEFT L/T \$300 || 10521

THEFT L/T \$300 || 10621

THEFT L/T \$500.00 || 10521

THEFT L/T 500 || 11137

THEFT LESS || 10521

Theft Less $\$ 1,000$ । ।

THEFT LESS \$100 | | 10521

THEFT LESS \$100 || 10621

THEFT LESS $\$ 100.00$ । ।

THEFT LESS $\$ 100.00$ || 10521

THEFT LESS $\$ 100.00$ | | 10621

THEFT LESS \$1000 || 10521

THEFT LESS \$1000 || 10621

THEFT LESS $\$ 500$ | | 10521

THEFT LESS $\$ 500$ | | 10621

THEFT LESS 1,000 || 10621

THEFT LESS 1,000.00 || 10621

THEFT LESS 100. I I 10521

THEFT LESS THAN || 10521

THEFT LESS THAN \$ 100.00 | | 10521

THEFT LESS THAN $\$ 1,000$ | | 10521

THEFT LESS THAN $\$ 1,000$ | | 10621

THEFT LESS THAN \$1,000.00 || 30233

Theft Less Than $\$ 100$ I।

THEFT LESS THAN \$100 | | 10521

THEFT LESS THAN \$100 || 10621

THEFT LESS THAN \$100 || 21020

THEFT LESS THAN $\$ 100$ || 22220

THEFT LESS THAN $\$ 100.00$ । ।

THEFT LESS THAN \$100.00 | | 10521

THEFT LESS THAN \$100.00 || 10621

THEFT LESS THAN \$100.00 | | 1F0521

THEFT LESS THAN \$100.00 | | 22220

Theft Less Than \$100.00, Att Theft I| 10521

Theft Less Than \$100.00, Attempt Commercial Armed R I| 10521

Theft Less Than \$100.00, Attempt Larceny | | 10521

Theft Less Than \$100.00, Breaking And Entering II 10521

Theft Less Than $\$ 100.00$, Cds I I 10521

Theft Less Than \$100.00, Cds Poss || 10521

Theft Less Than \$100.00, Cds Poss Not Marijuana II 10521

Theft Less Than \$100.00, Cds Poss. Heron I| 10521

Theft Less Than \$100.00, Cds Possession || 10521

Theft Less Than \$100.00, Cds Violation I| 10521

Theft Less Than \$100.00, Disorderly I| 10521

Theft Less Than \$100.00, Disorderly Conduct I| 10521

Theft Less Than \$100.00, Dist Heroin I | 10521

Theft Less Than \$100.00, Dist Heroin Consp II 10521

Theft Less Than \$100.00, Fail To Pay II 10521

Theft Less Than \$100.00, False Pretense I| 10521

Theft Less Than \$100.00, Forge Document I | 10521

Theft Less Than \$100.00, Fraud I I 10521

Theft Less Than \$100.00, Laceny I| 10521

Theft Less Than \$100.00, Lacerny || 10521 
Theft Less Than \$100.00, Lanceny || 10521

Theft Less Than \$100.00, Lancery || 10521

Theft Less Than \$100.00, Larceny I| 10521

Theft Less Than $\$ 100.00$, Larceny 100.00 || 10521

Theft Less Than \$100.00, Larceny After Trust I I 10521

Theft Less Than \$100.00, Larceny Auto Theft I | 10521

Theft Less Than \$100.00, Larceny From Auto I | 10521

Theft Less Than \$100.00, Larceny Shoplifting II 10521

Theft Less Than \$100.00, Larceny Under \$100.00 || 10521

Theft Less Than \$100.00, Larceny Under \$1000.00 I | 10521

Theft Less Than \$100.00, Larceny Under 1,000,00 || 10521

Theft Less Than \$100.00, Larceny Under 100 || 10521

Theft Less Than \$100.00, Larceny/Shoplifting || 10521

Theft Less Than \$100.00, Larcney II 10521

Theft Less Than \$100.00, Larency || 10521

Theft Less Than \$100.00, Poss Stolen Prop I I 10521

Theft Less Than \$100.00, Poss Stolen Property II 10521

Theft Less Than \$100.00, Poss W/Int Cds I I 10521

Theft Less Than \$100.00, Possession Of Stolen Proper II 10521

Theft Less Than \$100.00, Possession Stolen Tags I| 10521

Theft Less Than $\$ 100.00$, Shop Lifting I| 10521

Theft Less Than $\$ 100.00$, Shoplifting II 10521

Theft Less Than $\$ 100.00$, Shoplifting/ Theft I| 10521

Theft Less Than \$100.00, Shopligting II 10521

Theft Less Than $\$ 100.00$, Stolen Placard I I 10521

Theft Less Than \$100.00, Stolen Tag I| 10521

Theft Less Than $\$ 100.00$, Stolen Tags I | 10521

Theft Less Than \$100.00, Stolen Tags/Larceny || 10521

Theft Less Than $\$ 100.00$, Theft I| 10521

Theft Less Than $\$ 100.00$, Theft Of Service II 10521

Theft Less Than $\$ 100.00$, Theft -100 || 10521

Theft Less Than \$100.00, Theft (-\$1000.00) || 10521

Theft Less Than \$100.00, Theft (1000.00) I| 10521

Theft Less Than \$100.00, Theft < I 10521

Theft Less Than \$100.00, Theft L/T \$1,000 || 10521

Theft Less Than $\$ 100.00$, Theft Les Than \$100 || 10521

Theft Less Than $\$ 100.00$, Theft Less $\$ 100$ || 10521

Theft Less Than $\$ 100.00$, Theft Less $\$ 100$ Value I| 10521

Theft Less Than $\$ 100.00$, Theft Less $\$ 100$. I I 10521

Theft Less Than \$100.00, Theft Less \$100.00 || 10521

Theft Less Than $\$ 100.00$, Theft Less Than $\$ 1,000$ | | 10521

Theft Less Than $\$ 100.00$, Theft Less Than \$100 || 10521

Theft Less Than \$100.00, Theft Less Than \$100 10521 || 10521

Theft Less Than $\$ 100.00$, Theft Less Than $\$ 100.00$ || 10521

Theft Less Than $\$ 100.00$, Theft Less Than $\$ 100.00$ II 1 F0521

Theft Less Than $\$ 100.00$, Theft Less Than $\$ 100.00 /$ La || 10521

Theft Less Than \$100.00, Theft Less Than \$500 II 10521

Theft Less Than \$100.00, Theft Less Than 1,000.00 || 10521

Theft Less Than \$100.00, Theft Less Than 100 || 10521

Theft Less Than \$100.00, Theft Less Than 500.00 || 10521

Theft Less Than $\$ 100.00$, Theft Less Than $\$ 500.00$ || 10521

Theft Less Than \$100.00, Theft Less Then 100 || 10521

Theft Less Than $\$ 100.00$, Theft Of Service II 10521

Theft Less Than $\$ 100.00$, Theft Of Services I| 10521

Theft Less Than \$100.00, Theft Over \$100 | | 10521

Theft Less Than \$100.00, Theft U /300 || 10521

Theft Less Than \$100.00, Theft U/ \$100 || 10521

Theft Less Than $\$ 100.00$, Theft U/\$100.00 || 10521

Theft Less Than $\$ 100.00$, Theft U/\$500 || 10521

Theft Less Than \$100.00, Theft Under || 10521 
Theft Less Than $\$ 100.00$, Theft Under $\$ 1,000.00$ || 10521 Theft Less Than $\$ 100.00$, Theft Under $\$ 100$ || 10521 Theft Less Than \$100.00, Theft Under \$100. I | 10521 Theft Less Than \$100.00, Theft Under \$100.00 || 10521 Theft Less Than \$100.00, Theft Under \$1000 || 10521 Theft Less Than \$100.00, Theft Under \$1000.00 || 10521 Theft Less Than $\$ 100.00$, Theft Under $\$ 500$ | | 10521 Theft Less Than $\$ 100.00$, Theft Under \$500.00 । I 10521 Theft Less Than \$100.00, Theft Under 1,000.00 || 10521 Theft Less Than \$100.00, Theft Under 100 || 10521 Theft Less Than \$100.00, Theft Under 100.00 || 10521 Theft Less Than \$100.00, Theft Under 500 || 10521 Theft Less Than \$100.00, Theft Under\$100 || 10521 Theft Less Than \$100.00, Theft Under\$100.00 I| 10521 Theft Less Than \$100.00, Theft Under\$1000 || 10521 Theft Less Than \$100.00, Theft Under1,000.00 || 10521 Theft Less Than \$100.00, Theft Undr \$100 || 10521 Theft Less Than $\$ 100.00$, Theft- Less $\$ 100$ | | 10521 Theft Less Than $\$ 100.00$, Theft: Less Than \$100 || 10521

Theft Less Than \$100.00, Theft: Poss Of Stolen Prope || 10521 Theft Less Than \$100.00, Theft/Trespassing I| 10521 Theft Less Than $\$ 100.00$, Theftless 100.00 II 10521 Theft Less Than \$100.00, Thief Of Service I| 10521 THEFT LESS THAN \$100/ TRESPASS || 10521 THEFT LESS THAN $\$ 1000$ || 10521 THEFT LESS THAN \$1000 || 10573 THEFT LESS THAN $\$ 1000$ || 10621 THEFT LESS THAN \$1000 || 11137 THEFT LESS THAN $\$ 1000$ | | 22220 THEFT LESS THAN $\$ 1000$ | | 34025

THEFT LESS THAN $\$ 1000,00$ | | 10621 THEFT LESS THAN \$1000.00 || 10521 THEFT LESS THAN $\$ 1000.00$ | | 10621 THEFT LESS THAN \$500 || 10521 THEFT LESS THAN $\$ 500$ | | 34120 THEFT LESS THAN $\$ 500.00$ | | 10621 THEFT LESS THAN 1,000 || 10521 THEFT LESS THAN 1,000 || 10621 THEFT LESS THAN 1,000.00 | | 10521 THEFT LESS THAN 100 | $\mid 10521$ THEFT LESS THAN 100.00 । । THEFT LESS THAN 100.00 | | 10521 THEFT LESS THAN 1000 || 10521 THEFT LESS THAN 1000.00 | | 10521 THEFT LESS THAN 1000.00 || 23090 THEFT LESS THAN 150.00 | | 11137 THEFT LESS THAN 1500 | | 11137 THEFT LESS THAN 500 | | 10521 THEFT LESS THAN 500 | | 10621 THEFT LESS THAN A 100.. | | 10521 THEFT LESS THAT $\$ 1,000$ | । 10621 THEFT LESS THE \$ 100 || 10521 THEFT LESS THEN $\$ 100$ | | 10521 THEFT LESS THEN $\$ 100.00$ | । 10621 THEFT LESS THEN 500 । । 10621 THEFT LOSS THAN 100.00 || 10521 THEFT MORE THAN \$1,000.00 || 10622 THEFT MORE THAN 500 || 11137 THEFT OF LICENSE PLATE | | 10621 THEFT OF LICENSE PLATES | | 10521 
THEFT OF MOTOR VEHICLE || 20480

THEFT OF PROPERTY || 23040

THEFT OF SERVICE I| 10521

THEFT OF SERVICE || 10621

THEFT OF SERVICE I| 11137

THEFT OF SERVICES I। 10521

THEFT OF SERVICES II 11137

THEFT OF SERVICES GREATER THAN I | 11136

THEFT OF TAGS II 10621

THEFT OF VEHICLE || 10623

THEFT OF VEHICLE TAG | | 10521

THEFT OVER $\$ 1,000$ || 10621

THEFT OVER $\$ 1,000$ || 10622

THEFT OVER $\$ 1,000$ || 11136

THEFT OVER \$1,000.00 || 10621

THEFT OVER $\$ 1,500.00$ || 11136

THEFT OVER $\$ 10,000$ || 20480

THEFT OVER \$100 | | 10621

THEFT OVER \$100 || 11137

THEFT OVER \$100.00 UNDER \$1,50 | | 11137

THEFT OVER $\$ 1000$ | । 10621

THEFT OVER \$1000 |। 10622

THEFT OVER \$1000 || 10623

THEFT OVER \$1000 || 11136

THEFT OVER $\$ 1000$ । | 20480

THEFT OVER \$1000- \$10,000 || 10622

THEFT OVER $\$ 1000.00$ | | 10621

THEFT OVER $\$ 1000.00$ || 10622

THEFT OVER \$1500 || 11137

THEFT OVER $\$ 1500$ I I 1 A1136

THEFT OVER \$1500-25000 || 11136

THEFT OVER \$500 । I 10621

THEFT OVER $\$ 500$ । I 10622

THEFT OVER $\$ 500$ । I 10631

THEFT OVER \$500 || 11137

THEFT OVER $\$ 500.00$ I। 10215

THEFT OVER $\$ 500.00$ I | 10622

THEFT OVER 1,000 || 10622

THEFT OVER 1,000 || 11136

THEFT OVER 1,000 || 11137

THEFT OVER $1,000.00$ । | 10622

THEFT OVER 10,000 || 20480

THEFT OVER 10,000 || 22411

THEFT OVER 100 | | 10621

THEFT OVER 100 || 11137

THEFT OVER 1000 || 10622

THEFT OVER 1000 || 23090

THEFT OVER 10000 | | 20480

THEFT OVER 1500 || 11136

THEFT OVER 1500 । | 11160

THEFT OVER 300.00 || 10621

THEFT OVER 500. I। 11137

THEFT OVER 500\$ I I 11137

THEFT OVER10,000 || 1A0623

THEFT SCHEME \$1,000-\$10,000 | । 10632

THEFT SCHEME: $\$ 1,500$ TO < \$25K | | 11160

THEFT SCHEME: \$100,000 PLUS | | 11303

THEFT SCHEME: $\$ 100$ TO $<\$ 1,500$ | | 11161

THEFT STOLEN AUTO | | 20480

THEFT U \$100 || 10521 
THEFT U 1000 || 11137

THEFT U/ 1,000 || 1C0621

THEFT U/\$100 || 10521

THEFT U/\$100 || 34025

THEFT U/\$100.00 । ।

THEFT U/\$100.00 || 10521

THEFT U/\$100.00 || 10631

THEFT U/\$1000 || 10621

THEFT U/\$1000.00 || 10621

THEFT U/100 | | 10521

THEFT U/100.00 || 10521

THEFT U/1000 || 10621

THEFT U/1000.00 || 10621

THEFT U/1000.00 || 34025

THEFT UN \$100 || 10521

THEFT UNDER || 10521

THEFT UNDER || 10621

THEFT UNDER | | 11137

THEFT UNDER \$ 100 || 10521

THEFT UNDER \$ 100.00 || 10521

THEFT UNDER \$1000,00 | | 10621

THEFT UNDER $\$ 1,000$ | | 10521

THEFT UNDER \$1,000 || 10621

THEFT UNDER $\$ 1,000$ || 23090

THEFT UNDER $\$ 1,000.00$ | । 10043

THEFT UNDER \$1,000.00 | | 10521

THEFT UNDER $\$ 1,000.00$ | | 10621

THEFT UNDER $\$ 1,000.00$ | | 22210

THEFT UNDER $\$ 1,000.00$ | | 22810

THEFT UNDER \$1.000 || 10621

THEFT UNDER $\$ 10,000$ । ।

THEFT UNDER \$100 | | 10521

THEFT UNDER \$100 | | 10621

THEFT UNDER \$100 || 20050

THEFT UNDER \$100 || 23090

THEFT UNDER \$100 | | 43550

THEFT UNDER \$100. I I 10521

THEFT UNDER $\$ 100.00$ || 10521

THEFT UNDER \$100.00 || 10621

THEFT UNDER \$100.00 || 11137

THEFT UNDER $\$ 100.00$ | | 1 A0521

THEFT UNDER \$1000 || 10521

THEFT UNDER \$1000 || 10621

THEFT UNDER \$1000 || 10631

THEFT UNDER \$1000 || 11137

THEFT UNDER \$1000 || 1A0521

THEFT UNDER $\$ 1000.00$ | | 10521

THEFT UNDER $\$ 1000.00$ | | 10621

THEFT UNDER $\$ 1000.00$ | | 10622

THEFT UNDER \$1000.00 || 11137

THEFT UNDER $\$ 1000.00$ | । 20480

THEFT UNDER \$1100 || 10521

THEFT UNDER $\$ 1500$ | | 23090

THEFT UNDER $\$ 1500.00$ । । 10521

THEFT UNDER \$300 | | 10621

THEFT UNDER \$300.00 | | 10521

THEFT UNDER \$500 || 10521

THEFT UNDER \$500 | | 10621

THEFT UNDER \$500 || 10690

THEFT UNDER \$500 || 11137 
THEFT UNDER $\$ 500.00$ || 10521 THEFT UNDER $\$ 500.00$ || 10621 THEFT UNDER $\$ 500.00$ | | 11137 THEFT UNDER $\$ 500.00$ || 43550 THEFT UNDER \$500/ LARCENY | | 10521 THEFT UNDER 1,000 || 10521 THEFT UNDER 1,000 || 10621 THEFT UNDER 1,000 || 10622 THEFT UNDER 1,000 || 22210 THEFT UNDER 1,000.00 || 10521 THEFT UNDER $1,000.00$ | | 10621 THEFT UNDER 1,000.00 || 23090 THEFT UNDER 100 || 10521 THEFT UNDER 100 || 10621 THEFT UNDER 100.00 || 10521 THEFT UNDER 100.00 || 10621 THEFT UNDER 1000 || 10521 THEFT UNDER 1000 | | 10621 THEFT UNDER 1000 || 11137 THEFT UNDER 1000.00 || 10521 THEFT UNDER 1000.00 || 10621 THEFT UNDER 1100.00 || 10521 THEFT UNDER 1500 || 11137 THEFT UNDER 1500.00 || 10521 THEFT UNDER 500 || 10521 THEFT UNDER 500 || 10621 THEFT UNDER 500 || 22220 THEFT UNDER 500 | | 35010 THEFT UNDER 500.00 | | 10521 THEFT UNDER 500.00 | | 35010 THEFT UNDER/10,000 || 10632 THEFT UNDER $\$ 100$ || 10521 THEFT UNDER $\$ 100.00$ | | 10521 THEFT UNDER $\$ 1000$ || 10521 THEFT UNDER $\$ 500.00$ || 10521 THEFT UNDER100.00 || 10521 THEFT- CDS || 10521

THEFT-SCHEME I।

THEFT-SCHEME: LESS \$100 || 11631 THEFT-SCHEME: LESS \$1000 || 10631

Theft-Scheme: Less \$1000, Theft-Scheme: Less \$1000 || 10631 THEFT-SCHEME:\$300 PLUS || 10282

Theft-Scheme:\$300 Plus, Theft-Scheme:\$300 Plus || 10282 THEFT-SCHEME:\$LESS \$300 || 10283 THEFT-SCHEME:10K TO UND 100K || 10633

Theft-Scheme:10K To Und 100K, Larceny | | 10633

Theft-Scheme:10K To Und 100K, Theft-Scheme:10K To Un I। 10633 THEFT-SCHEME:1K TO UNDER 10K | | 10632

THEFT-SCHEME:1K TO UNDER 10K || 1F0632

Theft-Scheme:1K To Under 10K, False Pretense || 10632

Theft-Scheme:1K To Under 10K, Larceny I | 10632

Theft-Scheme:1K To Under 10K, Theft I| 10632

Theft-Scheme:1K To Under 10K, Theft-Scheme:1K To Un || 10632

Theft-Scheme:1K To Under 10K, Theft-Scheme:1K To Und I। 10632 THEFT-SHOPLIFTING || 10621

THEFT: \$1,000 TO UNDER \$10,000 || 10622

THEFT: $\$ 1,000$ TO UNDER $\$ 10,000$ | | 1 F0622

Theft: $\$ 1,000$ To Under $\$ 10,000$, Auto Theft I| 10622

Theft: \$1,000 To Under \$10,000, Breaking And Enteri I| 10622

Theft: \$1,000 To Under \$10,000, Credit Card Fraud || 10622 
Theft: \$1,000 To Under \$10,000, Dirt Bike Violation II 10622 Theft: $\$ 1,000$ To Under $\$ 10,000$, Larceny || 10622

Theft: \$1,000 To Under \$10,000, Larceny Att Theft I | 10622

Theft: \$1,000 To Under \$10,000, Larceny From Auto I| 10622

Theft: \$1,000 To Under \$10,000, Larceny Over 1,000 || 10622

Theft: $\$ 1,000$ To Under $\$ 10,000$, Larency I| 10622

Theft: $\$ 1,000$ To Under \$10,000, Larency From Auto II 10622

Theft: $\$ 1,000$ To Under $\$ 10,000$, Motor Vehicle Theft II 10622

Theft: $\$ 1,000$ To Under $\$ 10,000$, Poss Of Stolen Prope II 10622 Theft: \$1,000 To Under \$10,000, Poss Stolen Auto II 10622

Theft: \$1,000 To Under \$10,000, Poss Stolen Property II 10622 Theft: $\$ 1,000$ To Under $\$ 10,000$, Possesion Of Stolen I| 10622

Theft: \$1,000 To Under \$10,000, Possession Of Stole || 10622

Theft: \$1,000 To Under \$10,000, Recovered Stolen Aut || 10622

Theft: $\$ 1,000$ To Under $\$ 10,000$, Stolen Auto II 10622

Theft: $\$ 1,000$ To Under $\$ 10,000$, Stolen Scooter II 10622

Theft: \$1,000 To Under \$10,000, Theft I| 10622

Theft: $\$ 1,000$ To Under $\$ 10,000$, Theft Greater Than \$ I 10622

Theft: $\$ 1,000$ To Under $\$ 10,000$, Theft Of Services II 1 F0622

Theft: $\$ 1,000$ To Under $\$ 10,000$, Theft Over $\$ 1000$ || 10622

Theft: $\$ 1,000$ To Under $\$ 10,000$, Theft Over $\$ 1000.00$ || 10622

Theft: $\$ 1,000$ To Under $\$ 10,000$, Theft Over $\$ 500$ II 10622

Theft: $\$ 1,000$ To Under $\$ 10,000$, Theft Under 10,000 || 10622

Theft: \$1,000 To Under $\$ 10,000$, Theft: $\$ 1,000$ To Un I| 10622

Theft: $\$ 1,000$ To Under $\$ 10,000$, Theft: $\$ 1,000$ To Un I| 1 F0622

Theft: $\$ 1,000$ To Under $\$ 10,000$, Theft: $\$ 1,000$ To Und II 10622

Theft: \$1,000 To Under \$10,000, Theft:\$500 Plus Valu || 10622

Theft: $\$ 1,000$ To Under $\$ 10,000$, Theft $>\$ 1000$ || 10622

Theft: \$1,000 To Under $\$ 10,000$, Unauthorized Use I| 10622

THEFT: \$1,500 TO UNDER \$25,000 || 11136

THEFT: $\$ 1,500$ TO UNDER $\$ 25,000$ || 1 F1136

THEFT: \$100 TO UNDER \$1,500 || 11137

THEFT: $\$ 100$ TO UNDER $\$ 1,500$ || 1 F1137

THEFT: \$100 TO UNDER \$1500 । |

THEFT: $\$ 100,000$ PLUS || 10624

THEFT: \$25,000 TO UND \$100,000 || 11135

THEFT: \$300 PLUS VALUE I| 32400

THEFT: \$500 PLUS VALUE I | 30521

THEFT: \$500 PLUS VALUE || 35020

Theft: \$500 Plus Value, Theft: \$500 Plus Value I। 30521

THEFT: LESS \$1,000 VALUE | | 10621

THEFT: LESS \$1,000 VALUE I | 1F0621

Theft: Less \$1,000 Value, Att. Larceny I| 10621

Theft: Less \$1,000 Value, Att. Theft II 10621

Theft: Less \$1,000 Value, B\& E / Cds Violation I | 10621

Theft: Less \$1,000 Value, Breaking \& Entering || 10621

Theft: Less \$1,000 Value, Breaking And Entering || 10621

Theft: Less \$1,000 Value, Cds || 10621

Theft: Less \$1,000 Value, Cds Violation || 10621

Theft: Less \$1,000 Value, Destruction Of Property || 10621

Theft: Less \$1,000 Value, Domestic Theft I| 10621

Theft: Less \$1,000 Value, False Pretense I| 10621

Theft: Less \$1,000 Value, Larceney | | 10621

Theft: Less \$1,000 Value, Larceny | | 10621

Theft: Less \$1,000 Value, Larceny Auto I| 10621

Theft: Less \$1,000 Value, Larceny From Auto II 10621

Theft: Less \$1,000 Value, Larceny Less Than $\$ 500$ || 10621

Theft: Less \$1,000 Value, Larceny Less Than 1,000 || 10621

Theft: Less \$1,000 Value, Larceny Shoplifting || 10621

Theft: Less \$1,000 Value, Larency || 10621 
Theft: Less \$1,000 Value, Motor Vehicle Theft. I I 10621

Theft: Less \$1,000 Value, Poss Of A Dangerous Weapo II 10621

Theft: Less \$1,000 Value, Poss Of Stolen Property II 10621

Theft: Less \$1,000 Value, Poss. Stolen Property II 10621

Theft: Less \$1,000 Value, Possess Stolen Property || 10621

Theft: Less \$1,000 Value, Possession Of Stolen Prope I| 10621

Theft: Less \$1,000 Value, Rec. Stol. Goods I| 10621

Theft: Less \$1,000 Value, Rogue \& Vagabond I| 10621

Theft: Less $\$ 1,000$ Value, Shoplifting I| 10621

Theft: Less \$1,000 Value, Shoplifting / Larceny < I 10621

Theft: Less $\$ 1,000$ Value, Stolen Auto I| 10621

Theft: Less \$1,000 Value, Stolen Tag || 10621

Theft: Less \$1,000 Value, Stolen Tag/Theft I| 10621

Theft: Less $\$ 1,000$ Value, Stolen Tags I| 10621

Theft: Less \$1,000 Value, Stolen Vehicle II 10621

Theft: Less \$1,000 Value, Theft [ \$1000.00] || 10621

Theft: Less $\$ 1,000$ Value, Theft II 10621

Theft: Less $\$ 1,000$ Value, Theft Less $\$ 1,000$ । I 10621

Theft: Less $\$ 1,000$ Value, Theft Less Than $\$ 500$ || 10621

Theft: Less $\$ 1,000$ Value, Theft: Less $\$ 1,000$ Value || 10621

Theft: Less \$1,000 Value, Theft +100 || 10621

Theft: Less $\$ 1,000$ Value, Theft $>\$ 500.00$ || 10621

Theft: Less \$1,000 Value, Theft From Auto || 10621

Theft: Less \$1,000 Value, Theft L/T \$500.00 || 10621

Theft: Less \$1,000 Value, Theft Less Than $\$ 500.00$ || 10621

Theft: Less $\$ 1,000$ Value, Theft Less $\$ 1000$ | | 10621

Theft: Less \$1,000 Value, Theft Less Than \$1000 || 10621

Theft: Less $\$ 1,000$ Value, Theft Less Than $\$ 1000.00$ || 10621

Theft: Less \$1,000 Value, Theft Less Than $\$ 500$ । I 10621

Theft: Less \$1,000 Value, Theft Less Than $\$ 500.00$ || 10621

Theft: Less \$1,000 Value, Theft Less Than 1000 | I 10621

Theft: Less \$1,000 Value, Theft Less Than 1000.00 || 10621

Theft: Less \$1,000 Value, Theft Less Than 500.00 || 10621

Theft: Less $\$ 1,000$ Value, Theft Less Then 100. I I 10621

Theft: Less \$1,000 Value, Theft Of Services II 10621

Theft: Less \$1,000 Value, Theft Over \$100 || 10621

Theft: Less $\$ 1,000$ Value, Theft Over $\$ 100.00$ || 10621

Theft: Less \$1,000 Value, Theft Over \$1000.00 || 10621

Theft: Less \$1,000 Value, Theft Over \$500.00 || 10621

Theft: Less \$1,000 Value, Theft Over 500 || 10621

Theft: Less $\$ 1,000$ Value, Theft Over $\$ 100.00$ || 10621

Theft: Less \$1,000 Value, Theft Over500.00 || 10621

Theft: Less \$1,000 Value, Theft U/\$1000.00 || 10621

Theft: Less \$1,000 Value, Theft Under \$1000 || 10621

Theft: Less \$1,000 Value, Theft Under \$1,000 | | 10621

Theft: Less $\$ 1,000$ Value, Theft Under $\$ 1,000.00$ || 10621

Theft: Less \$1,000 Value, Theft Under \$1000 || 10621

Theft: Less $\$ 1,000$ Value, Theft Under $\$ 1000.00$ | | 10621

Theft: Less $\$ 1,000$ Value, Theft Under $\$ 300.00$ | | 10621

Theft: Less $\$ 1,000$ Value, Theft Under $\$ 500$ | | 10621

Theft: Less $\$ 1,000$ Value, Theft Under $\$ 500.00$ | | 10621

Theft: Less \$1,000 Value, Theft Under 1,000 || 10621

Theft: Less $\$ 1,000$ Value, Theft Under 1,000.00 || 10621

Theft: Less \$1,000 Value, Theft Under 1000.00 | I 10621

Theft: Less \$1,000 Value, Theft Under 500 || 10621

Theft: Less \$1,000 Value, Theft-Shoplifting II 10621

Theft: Less \$1,000 Value, Theft: Less \$1,000 Value || 10621

Theft: Less $\$ 1,000$ Value, Theft: Less $\$ 1,000$ Value I| 1F0621

Theft: Less \$1,000 Value, Trespassing || 10621

Theft: Less \$1,000 Value, Tresspassing || 10621 
THEFT: LESS THAN \$1,000.00 || 10521

THEFT: LESS THAN \$100 || 10621

THEFT: LESS THAN 500 || 10621

THEFT: OVER \$1500.00 || 11136

THEFT:\$10,000 TO UND \$100,000 || 10623

Theft:\$10,000 To Und \$100,000, Larceny I| 10623

Theft:\$10,000 To Und \$100,000, Motor Vehicle Theft II 10623

Theft:\$10,000 To Und \$100,000, Poss Stolen Auto II 10623

Theft:\$10,000 To Und \$100,000, Stoeln Auto || 10623

Theft:\$10,000 To Und \$100,000, Stolen Auto II 10623

Theft:\$10,000 To Und \$100,000, Theft Auto II 10623

Theft: $\$ 10,000$ To Und $\$ 100,000$, Theft Over $\$ 10,000$ || 10623

Theft:\$10,000 To Und \$100,000, Theft Over \$10000 || 10623

Theft:\$10,000 To Und $\$ 100,000$, Theft:\$10,000 To Und II 10623

Theft:\$10,000 To Und \$100,000, Unauthorized Use I| 10623

THEFT:\$10,000 TO UNDER \$100,00 || 1F0623

THEFT:LESS $\$ 1,000$ । ।

THEFT:LESS \$300 VALUE | | 32399

THEFT:LESS \$500 VALUE || 35010

Theft:Less $\$ 500$ Value, Theft:Less $\$ 500$ Value I। 35010

THEFT/ FALSE STATEMENT || 10521

THEFT/ FELONY II 11136

THEFT/ SHOPLIFTING || 10521

THEFT/ TRESPASS || 22220

THEFT/COUNTERFIET CURRENCY || 10621

THEFT/LARCENY || 10521

THEFT/SHOPLIFTING || 10521

THEFT/STOLEN TAGS || 10521

THEFT/TRESPASS || 22220

THEFT/TRESPASSING || 10621

THEFTOVER \$100 || 11137

THEIFT UNDER \$1000 || 10521

THFET UNDER \$500 || 10621

TRESOASSING || 10521

Trespass-Posted Property, Larceny || 22210

Trespass: Private Property, Attempt Larceny I| 22220

Trespass: Private Property, Larceny || 22220

Trespass: Private Property, Larceny Shoplifting I| 22220

Trespass: Private Property, Theft I| 22220

Trespass: Private Property, Theft Under I| 22220

Trespass: Private Property, Theft Under \$100 || 22220

TRESPASS/ATTEMPT LARCENY || 22210

TRESPASS/LARCENCY || 10521

TRESPASSING || 10521

TRESPASSING || 10621

TRESPASSING || 1A0521

TRESPASSING || 23090

TRESPASSING THEFT || 22220

TRESPASSING/ROGUE AND VAGABOND || 23090

TRESS PASSING || 10521

TRESSPASS/THEFT || 22220

TRESSPASSING || 10521

TRESSPASSING || 10621

TRESSPASSING/THEFT | | 10521

U/U LIVESTOCK MV ETC I| 22411

U/U Livestock Mv Etc, Auto Theft I| 22411

U/U Livestock Mv Etc, Larceny || 22411

U/U Livestock Mv Etc, Motor Vehicle Theft I| 22411

U/U Livestock Mv Etc, Possession Stolen Veh I| 22411

U/U Livestock Mv Etc, Stolen Auto || 22411 
U/U Livestock Mv Etc, Stolen Vehicle/Cds || 22411

U/U Livestock Mv Etc, Theft || 22411

U/U Livestock Mv Etc, U/U Livestock Mv Etc || 22411

U/U Livestock Mv Etc, Unathorize Use II 22411

U/U Livestock Mv Etc, Unauth Removal Of Prop || 22411

U/U Livestock Mv Etc, Unautherized Used I| 22411

U/U Livestock Mv Etc, Unauthorize Use Of Motor Vehci || 22411

U/U Livestock Mv Etc, Unauthorize Vehicle II 22411

U/U Livestock Mv Etc, Unauthorized Use I| 22411

U/U Livestock Mv Etc, Unauthorized Use Of A Vehicle I| 22411

U/U Livestock Mv Etc, Unlawful Taking Of Vehicle II 22411

UNATH USE I| 22411

UNATHERIZED USE || 22411

UNATHORIZED USE || 22411

UNAUTH REMOVAL OF PROP || 22411

UNAUTH. USE || 22411

UNAUTHORIZE USE I| 22411

UNAUTHORIZED || 22411

UNAUTHORIZED DISP. \& USE OF RE || 10621

UNAUTHORIZED USE || 10521

UNAUTHORIZED USE || 22411

UNAUTHORIZED USE AUTO || 22411

UNAUTHORIZED USE OF VEHICLE || 22411

UNAUTHORIZED USE STOLEN AUTO || 11466

UNAUTHORIZIED USE || 22411

UNLAWFUL TAKING || 20480

Uttering False Document, Theft || 12513

VEH UNLAWFUL TAKING | |

VEHICLE LARCENY | | 20480

VEHICLE THEFT I| 10622

VEHICLE THEFT || 10825

VEHICLE THEFT II 20480

\title{
Drug Distribution (Subsequently Combined with Drug Possession)
}

\author{
11111 || 1A1118 \\ 11118 || $1 \mathrm{~A} 1118$ \\ 20696 || 30233 \\ 30233 || 2C0696 \\ 43550 I| 43550 \\ ATT CDS DIST 11 \\ ATT CDS DIST || 2A0696 \\ ATT CDS DIST I| 2A3550 \\ ATT CDS DIST I| 30233 \\ ATT CDS DIST NARC || 30233 \\ ATT CDS DIST-NARC । । \\ ATT CDS DIST. II 11119 \\ ATT CDS DISTRIBUTION || 2A3550 \\ ATT CDS DISTRIBUTION || 43550 \\ ATT DIS || 2A3550 \\ ATT DIS I| 30233 \\ ATT DIS MARIJUANA I| 23550 \\ ATT DIS OF HEROIN || 2A0696 \\ ATT DIST || 11111 \\ ATT DIST || 11119 \\ ATT DIST || 1A1118 \\ ATT DIST || 20696 \\ ATT DIST || 23550 \\ ATT DIST || 2A0696
}


ATT DIST || 30233

ATT DIST (HEROIN) । । 43550

ATT DIST ALPRAZOLAN || 2A3550

ATT DIST CDS । ।

ATT DIST CDS || 10573

ATT DIST CDS || 2A0696

ATT DIST CDS || 2A3550

ATT DIST CDS || 30233

ATT DIST CDS || 43550

ATT DIST COCAINE || 2A0696

ATT DIST COCAINE || 2C0696

ATT DIST COCAINE || 30233

ATT DIST COCAINE || 43550

ATT DIST HEROIN || 11118

ATT DIST HEROIN । 20480

ATT DIST HEROIN || 2A0696

ATT DIST HEROIN || 2A3550

ATT DIST HEROIN | | 30233

ATT DIST MARIJUANA I| 2A3550

ATT DIST NARC || 30233

ATT DIST OF COCAINE || 2A0696

ATT DIST OF COCAINE | | 30233

ATT DIST OF COCAINE || 43550

ATT DIST OF HERION || 10573

ATT DIST OF HEROIN I। 2A0696

ATT DIST OF HEROIN I I 30233

ATT DIST PWID || 2A3550

ATT DIST TO OFFICER || 30233

ATT DIST. I। 2A3550

ATT DIST. II 30233

ATT DIST. CDS I| 2A0696

ATT DISTRIBUTION ।| 2A3550

ATT DISTRIBUTION CDS I| 2A0696

ATT DISTRIBUTION CDS || 30233

ATT DISTRIBUTION MARIJUANA || 2A3550

ATT DISTRIBUTION/HEROIN || 1A1118

ATT POSS I| 43550

ATT TO DIST CDS || 2A3550

ATT TO DIST. HEROIN | | 30233

ATT TO DISTIBUTE CDS || 30233

ATT TO DISTRIBUTE I| 2A3550

ATT TO DISTRIBUTE HEROIN || 2A0696

Att- Cds: Poss Marihna L/T 10 G, Cds II 1A1635

ATT-CDS DIST NARC I।

ATT-CDS DIST-NARC I ।

ATT-CDS DIST-NARC || 1A1118

ATT-CDS DIST-NARC || 2A0696

ATT-CDS DIST/DISPENSE-LG AMT । । 1A0880

ATT-CDS DISTRIBUTE - OTHER || 2A3550

Att-Cds Manuf/Dist-Narc, 13550 || 2A0696

Att-Cds Manuf/Dist-Narc, Att Cds Dist II 2A0696

Att-Cds Manuf/Dist-Narc, Att Dist Cocaine II 2A0696

Att-Cds Manuf/Dist-Narc, Att Distributio Oxycodone II 2A0696

Att-Cds Manuf/Dist-Narc, Att Distribution Cocaine II 2A0696

Att-Cds Manuf/Dist-Narc, Att To Dist II 2A0696

Att-Cds Manuf/Dist-Narc, Att-Cds Dist-Narc II 2A0696

Att-Cds Manuf/Dist-Narc, Att. Dist Cocaine II 2A0696

Att-Cds Manuf/Dist-Narc, Attempt Dist Heroin II 2A0696

Att-Cds Manuf/Dist-Narc, C.D.S. Violation II 2A0696

Att-Cds Manuf/Dist-Narc, Cds I| 2A0696 
Att-Cds Manuf/Dist-Narc, Cds Violation I| 2A0696

Att-Cds Manuf/Dist-Narc, Cds - Distribution II 2A0696

Att-Cds Manuf/Dist-Narc, Cds Att Distribution II 2A0696

Att-Cds Manuf/Dist-Narc, Cds Cocaine II 2A0696

Att-Cds Manuf/Dist-Narc, Cds Consp. II 2A0696

Att-Cds Manuf/Dist-Narc, Cds Consp. To Dist. II 2A0696

Att-Cds Manuf/Dist-Narc, Cds Conspiracy II 2A0696

Att-Cds Manuf/Dist-Narc, Cds Dist II 2A0696

Att-Cds Manuf/Dist-Narc, Cds Dist Cocaine II 2A0696

Att-Cds Manuf/Dist-Narc, Cds Dist Crack Cocaine II 2A0696

Att-Cds Manuf/Dist-Narc, Cds Dist Heroin II 2A0696

Att-Cds Manuf/Dist-Narc, Cds Dist Heroin/ Pwi II 2A0696

Att-Cds Manuf/Dist-Narc, Cds Dist Not Marijuana I| 2A0696

Att-Cds Manuf/Dist-Narc, Cds Dist. II 2A0696

Att-Cds Manuf/Dist-Narc, Cds Dist. Cocaine I| 2A0696

Att-Cds Manuf/Dist-Narc, Cds Dist. Heroin II 2A0696

Att-Cds Manuf/Dist-Narc, Cds Distirbution II 2A0696

Att-Cds Manuf/Dist-Narc, Cds Distrbution II 2A0696

Att-Cds Manuf/Dist-Narc, Cds Distribute II 2A0696

Att-Cds Manuf/Dist-Narc, Cds Distribution I| 2A0696

Att-Cds Manuf/Dist-Narc, Cds Distribution Cocaine II 2A0696

Att-Cds Manuf/Dist-Narc, Cds Distribution Heroin II 2A0696

Att-Cds Manuf/Dist-Narc, Cds Heroin II 2A0696

Att-Cds Manuf/Dist-Narc, Cds Poss I| 2A0696

Att-Cds Manuf/Dist-Narc, Cds Poss Cocaine II 2A0696

Att-Cds Manuf/Dist-Narc, Cds Poss Distribution II 2A0696

Att-Cds Manuf/Dist-Narc, Cds Poss Heroin II 2A0696

Att-Cds Manuf/Dist-Narc, Cds Poss W Int I| 2A0696

Att-Cds Manuf/Dist-Narc, Cds Poss W/I I| 2A0696

Att-Cds Manuf/Dist-Narc, Cds Poss W/I Dist Cocaine II 2A0696

Att-Cds Manuf/Dist-Narc, Cds Poss W/Int I I 2A0696

Att-Cds Manuf/Dist-Narc, Cds Poss W/Intent I| 2A0696

Att-Cds Manuf/Dist-Narc, Cds Poss. II 2A0696

Att-Cds Manuf/Dist-Narc, Cds Poss. With Intent II 2A0696

Att-Cds Manuf/Dist-Narc, Cds Poss/Dist || 2A0696

Att-Cds Manuf/Dist-Narc, Cds Possession II 2A0696

Att-Cds Manuf/Dist-Narc, Cds Possession Cocaine II 2A0696

Att-Cds Manuf/Dist-Narc, Cds Possession Heroin I| 2A0696

Att-Cds Manuf/Dist-Narc, Cds Possession With Intent I| 2A0696

Att-Cds Manuf/Dist-Narc, Cds Possession-Not Marijua II 2A0696

Att-Cds Manuf/Dist-Narc, Cds Pwi II 2A0696

Att-Cds Manuf/Dist-Narc, Cds Pwid II 2A0696

Att-Cds Manuf/Dist-Narc, Cds Pwid Cocaine II 2A0696

Att-Cds Manuf/Dist-Narc, Cds Pwid Heroin II 2A0696

Att-Cds Manuf/Dist-Narc, Cds Viiolation II 2A0696

Att-Cds Manuf/Dist-Narc, Cds Viol I| 2A0696

Att-Cds Manuf/Dist-Narc, Cds Viol. II 2A0696

Att-Cds Manuf/Dist-Narc, Cds Violaation II 2A0696

Att-Cds Manuf/Dist-Narc, Cds Violation II 2A0696

Att-Cds Manuf/Dist-Narc, Cds Violation Ditriburion II 2A0696

Att-Cds Manuf/Dist-Narc, Cds Violation Poss. Heroin II 2A0696

Att-Cds Manuf/Dist-Narc, Cds Violation- Cocaine II 2A0696

Att-Cds Manuf/Dist-Narc, Cds W/Int Cocaine II 2A0696

Att-Cds Manuf/Dist-Narc, Cds-Distribution II 2A0696

Att-Cds Manuf/Dist-Narc, Cds: Distribution II 2A0696

Att-Cds Manuf/Dist-Narc, Cds: Poss W/Int II 2A0696

Att-Cds Manuf/Dist-Narc, Cds/ Dist || 2A0696

Att-Cds Manuf/Dist-Narc, Cds/Distribution II 2A0696

Att-Cds Manuf/Dist-Narc, Cocaine Distribution II 2A0696

Att-Cds Manuf/Dist-Narc, Cons Dist.Heroin II 2A0696 
Att-Cds Manuf/Dist-Narc, Cons. To Dist Heroin I| 2A0696

Att-Cds Manuf/Dist-Narc, Consp To Dist Heroin II 2A0696

Att-Cds Manuf/Dist-Narc, Consp To Dist. Heroin II 2A0696

Att-Cds Manuf/Dist-Narc, Consp. To Dist. Heroin II 2A0696

Att-Cds Manuf/Dist-Narc, Consp.To Sell Narc. II 2A0696

Att-Cds Manuf/Dist-Narc, Conspiracy II 2A0696

Att-Cds Manuf/Dist-Narc, Conspiracy Cds II 2A0696

Att-Cds Manuf/Dist-Narc, Conspiracy To Dist Heroin II 2A0696

Att-Cds Manuf/Dist-Narc, Conspiracy To Dist. II 2A0696

Att-Cds Manuf/Dist-Narc, Conspirary To Dist Cds II 2A0696

Att-Cds Manuf/Dist-Narc, Conspircy II 2A0696

Att-Cds Manuf/Dist-Narc, Dis Cds I| 2A0696

Att-Cds Manuf/Dist-Narc, Disrtibution Heroin I| 2A0696

Att-Cds Manuf/Dist-Narc, Dist - Marijuana II 2A0696

Att-Cds Manuf/Dist-Narc, Dist Cds II 2A0696

Att-Cds Manuf/Dist-Narc, Dist Cds Heroin II 2A0696

Att-Cds Manuf/Dist-Narc, Dist Cocain II 2A0696

Att-Cds Manuf/Dist-Narc, Dist Cocaine II 2A0696

Att-Cds Manuf/Dist-Narc, Dist Cocaine/Percocet II 2A0696

Att-Cds Manuf/Dist-Narc, Dist Herion II 2A0696

Att-Cds Manuf/Dist-Narc, Dist Heroin II 2A0696

Att-Cds Manuf/Dist-Narc, Dist Of Cds II 2A0696

Att-Cds Manuf/Dist-Narc, Dist Of Cocaine II 2A0696

Att-Cds Manuf/Dist-Narc, Dist Of Heroin I| 2A0696

Att-Cds Manuf/Dist-Narc, Dist Of Oxycodone II 2A0696

Att-Cds Manuf/Dist-Narc, Dist. Cds II 2A0696

Att-Cds Manuf/Dist-Narc, Dist. Cds Heroin II 2A0696

Att-Cds Manuf/Dist-Narc, Dist. Cocaine || 2A0696

Att-Cds Manuf/Dist-Narc, Dist. Heroin II 2A0696

Att-Cds Manuf/Dist-Narc, Dist. Of Cocaine II 2A0696

Att-Cds Manuf/Dist-Narc, Dist. Of Heroin II 2A0696

Att-Cds Manuf/Dist-Narc, Distibution Heroin II 2A0696

Att-Cds Manuf/Dist-Narc, Distrib. Heroin II 2A0696

Att-Cds Manuf/Dist-Narc, Distribution II 2A0696

Att-Cds Manuf/Dist-Narc, Distribution Cds I| 2A0696

Att-Cds Manuf/Dist-Narc, Distribution Cocaine II 2A0696

Att-Cds Manuf/Dist-Narc, Distribution Heroin II 2A0696

Att-Cds Manuf/Dist-Narc, Distribution Of Cds II 2A0696

Att-Cds Manuf/Dist-Narc, Distribution Of Cocaine I| 2A0696

Att-Cds Manuf/Dist-Narc, Distribution Of Herion II 2A0696

Att-Cds Manuf/Dist-Narc, Distribution Of Heroin II 2A0696

Att-Cds Manuf/Dist-Narc, Distribution Of Narcotics I| 2A0696

Att-Cds Manuf/Dist-Narc, Heroin Cds Violation II 2A0696

Att-Cds Manuf/Dist-Narc, Narcotics II 2A0696

Att-Cds Manuf/Dist-Narc, P.W.I Heroin II 2A0696

Att-Cds Manuf/Dist-Narc, Poss Heroin I| 2A0696

Att-Cds Manuf/Dist-Narc, Poss Cds II 2A0696

Att-Cds Manuf/Dist-Narc, Poss Cds-Not Marijuana II 2A0696

Att-Cds Manuf/Dist-Narc, Poss Cocaine I| 2A0696

Att-Cds Manuf/Dist-Narc, Poss Heroin I| 2A0696

Att-Cds Manuf/Dist-Narc, Poss Of Acetaminophen II 2A0696

Att-Cds Manuf/Dist-Narc, Poss Of Cocaine II 2A0696

Att-Cds Manuf/Dist-Narc, Poss Of Oxycodone II 2A0696

Att-Cds Manuf/Dist-Narc, Poss Oxycodone II 2A0696

Att-Cds Manuf/Dist-Narc, Poss Suboxone II 2A0696

Att-Cds Manuf/Dist-Narc, Poss W/ Cocaine II 2A0696

Att-Cds Manuf/Dist-Narc, Poss W/ Int Dist Heroin I| 2A0696

Att-Cds Manuf/Dist-Narc, Poss W/Cds II 2A0696

Att-Cds Manuf/Dist-Narc, Poss W/I Dist II 2A0696

Att-Cds Manuf/Dist-Narc, Poss W/I Heroin I| 2A0696 
Att-Cds Manuf/Dist-Narc, Poss W/Int || 2A0696

Att-Cds Manuf/Dist-Narc, Poss W/Int Dist Heroin I I 2A0696

Att-Cds Manuf/Dist-Narc, Poss W/Int Distribute II 2A0696

Att-Cds Manuf/Dist-Narc, Poss W/Int Heroin II 2A0696

Att-Cds Manuf/Dist-Narc, Poss W/Intent II 2A0696

Att-Cds Manuf/Dist-Narc, Poss W/Intent Cocaine || 2A0696

Att-Cds Manuf/Dist-Narc, Poss W/Intent Heroin II 2A0696

Att-Cds Manuf/Dist-Narc, Poss W/Intent To Dist II 2A0696

Att-Cds Manuf/Dist-Narc, Poss W/Itn Cocaine || 2A0696

Att-Cds Manuf/Dist-Narc, Poss With Int Heroin II 2A0696

Att-Cds Manuf/Dist-Narc, Poss With Intent II 2A0696

Att-Cds Manuf/Dist-Narc, Poss With The Intent I| 2A0696

Att-Cds Manuf/Dist-Narc, Poss. Cds II 2A0696

Att-Cds Manuf/Dist-Narc, Poss. Cocaine II 2A0696

Att-Cds Manuf/Dist-Narc, Poss. Heroin II 2A0696

Att-Cds Manuf/Dist-Narc, Poss. W./Int. Heroin II 2A0696

Att-Cds Manuf/Dist-Narc, Poss. W/ Int. II 2A0696

Att-Cds Manuf/Dist-Narc, Poss. W/I Heroin II 2A0696

Att-Cds Manuf/Dist-Narc, Poss. With Intent To Distri II 2A0696

Att-Cds Manuf/Dist-Narc, Poss./W/Intent I| 2A0696

Att-Cds Manuf/Dist-Narc, Poss.Cds II 2A0696

Att-Cds Manuf/Dist-Narc, Possession Heroin II 2A0696

Att-Cds Manuf/Dist-Narc, Possession Of Cds I| 2A0696

Att-Cds Manuf/Dist-Narc, Possession Of Cocaine II 2A0696

Att-Cds Manuf/Dist-Narc, Possession W Intent II 2A0696

Att-Cds Manuf/Dist-Narc, Possession W/ Intent II 2A0696

Att-Cds Manuf/Dist-Narc, Possession W/I Cocaine II 2A0696

Att-Cds Manuf/Dist-Narc, Possession W/Intent II 2A0696

Att-Cds Manuf/Dist-Narc, Possession With Intent I| 2A0696

Att-Cds Manuf/Dist-Narc, Possession With Intent To II 2A0696

Att-Cds Manuf/Dist-Narc, Possw/Int Dist Herion II 2A0696

Att-Cds Manuf/Dist-Narc, Pwi II 2A0696

Att-Cds Manuf/Dist-Narc, Pwi Cds II 2A0696

Att-Cds Manuf/Dist-Narc, Pwi Cds Cocaine II 2A0696

Att-Cds Manuf/Dist-Narc, Pwi Cocaine I| 2A0696

Att-Cds Manuf/Dist-Narc, Pwi Herion II 2A0696

Att-Cds Manuf/Dist-Narc, Pwi Heroin II 2A0696

Att-Cds Manuf/Dist-Narc, Pwi- Heroin II 2A0696

Att-Cds Manuf/Dist-Narc, Pwi-Cocaine II 2A0696

Att-Cds Manuf/Dist-Narc, Pwi/ Cds II 2A0696

Att-Cds Manuf/Dist-Narc, Pwid II 2A0696

Att-Cds Manuf/Dist-Narc, Pwid Cds II 2A0696

Att-Cds Manuf/Dist-Narc, Pwid Cocaine I| 2A0696

Att-Cds Manuf/Dist-Narc, Pwid Heroin II 2A0696

Att-Cds Manuf/Dist-Narc, Pwid-Cocaine || 2A0696

Att-Cds Manuf/Dist., 13550 || 2A3550

Att-Cds Manuf/Dist., Att Dist Marijuana II 2A3550

Att-Cds Manuf/Dist., Att. Dist. Marijuana II 2A3550

Att-Cds Manuf/Dist., Cds || 2A3550

Att-Cds Manuf/Dist., Cds - Distribution II 2A3550

Att-Cds Manuf/Dist., Cds Dist I I 2A3550

Att-Cds Manuf/Dist., Cds Dist Marijuana I| 2A3550

Att-Cds Manuf/Dist., Cds Dist Pwid Poss I| 2A3550

Att-Cds Manuf/Dist., Cds Dist. I| 2A3550

Att-Cds Manuf/Dist., Cds Distribution II 2A3550

Att-Cds Manuf/Dist., Cds Distribution/Poss I| 2A3550

Att-Cds Manuf/Dist., Cds Distru || 2A3550

Att-Cds Manuf/Dist., Cds Marijuana II 2A3550

Att-Cds Manuf/Dist., Cds Not Marijuana II 2A3550

Att-Cds Manuf/Dist., Cds Poss II 2A3550 
Att-Cds Manuf/Dist., Cds Poss Marijuana II 2A3550

Att-Cds Manuf/Dist., Cds Poss W Int I| 2A3550

Att-Cds Manuf/Dist., Cds Poss W/ Int I| 2A3550

Att-Cds Manuf/Dist., Cds Poss W/Intent I| 2A3550

Att-Cds Manuf/Dist., Cds Poss W/Intent I| 2A3550

Att-Cds Manuf/Dist., Cds Possesion Marijuana II 2A3550

Att-Cds Manuf/Dist., Cds Possession II 2A3550

Att-Cds Manuf/Dist., Cds Possession Marijuana II 2A3550

Att-Cds Manuf/Dist., Cds Possession With Intent II 2A3550

Att-Cds Manuf/Dist., Cds Pwid II 2A3550

Att-Cds Manuf/Dist., Cds Violation II 2A3550

Att-Cds Manuf/Dist., Cds- Dist I I 2A3550

Att-Cds Manuf/Dist., Cds-Distribution II 2A3550

Att-Cds Manuf/Dist., Cds: Dist. Marijuana II 2A3550

Att-Cds Manuf/Dist., Cons To Dist Marijuana II 2A3550

Att-Cds Manuf/Dist., Conspiracy To Distribute Cds I| 2A3550

Att-Cds Manuf/Dist., Csd Violation II 2A3550

Att-Cds Manuf/Dist., Dist Cds II 2A3550

Att-Cds Manuf/Dist., Dist Marijuana II 2A3550

Att-Cds Manuf/Dist., Dist. Marijuana I| 2A3550

Att-Cds Manuf/Dist., Dist. Of Cds II 2A3550

Att-Cds Manuf/Dist., Dist. Of Marijauna II 2A3550

Att-Cds Manuf/Dist., Dist. Of Marijuana II 2A3550

Att-Cds Manuf/Dist., Distribution II 2A3550

Att-Cds Manuf/Dist., Distribution Marihuana II 2A3550

Att-Cds Manuf/Dist., Distribution Marijuana II 2A3550

Att-Cds Manuf/Dist., Distribution Of Cocaine II 2A3550

Att-Cds Manuf/Dist., Distribution Of Marijuana II 2A3550

Att-Cds Manuf/Dist., Distrubution I | 2A3550

Att-Cds Manuf/Dist., Distrubution Marijuana II 2A3550

Att-Cds Manuf/Dist., Narcotic Violation II 2A3550

Att-Cds Manuf/Dist., Narcotics II 2A3550

Att-Cds Manuf/Dist., P/W/I Cocaine II 2A3550

Att-Cds Manuf/Dist., Poss Cds II 2A3550

Att-Cds Manuf/Dist., Poss Cocaine || 2A3550

Att-Cds Manuf/Dist., Poss Marijuana II 2A3550

Att-Cds Manuf/Dist., Poss Of Marijuana II 2A3550

Att-Cds Manuf/Dist., Poss Of Marujuana I| 2A3550

Att-Cds Manuf/Dist., Poss W/Int Dist Marijuana II 2A3550

Att-Cds Manuf/Dist., Poss W/Int Marijuana II 2A3550

Att-Cds Manuf/Dist., Poss W/Intent Marijuana II 2A3550

Att-Cds Manuf/Dist., Poss. Marijuana II 2A3550

Att-Cds Manuf/Dist., Poss. W/I Marijuana II 2A3550

Att-Cds Manuf/Dist., Possessiion/Marijuana II 2A3550

Att-Cds Manuf/Dist., Possession Marijuana II 2A3550

Att-Cds Manuf/Dist., Possession W/Intent I| 2A3550

Att-Cds Manuf/Dist., Possession With Intent II 2A3550

Att-Cds Manuf/Dist., Possession/Intent-Marijuana II 2A3550

Att-Cds Manuf/Dist., Pwi Marijuana || 2A3550

Att-Cds Manuf/Dist., Pwi-Marijuana II 2A3550

Att-Cds Manuf/Dist., Pwid Cds II 2A3550

Att-Cds Manuf/Dist., Pwid Marijuana II 2A3550

Att-Cds Manuf/Dist., Pwid Pecocet I| 2A3550

ATT-CDS-DIST I।

ATT-CDS-DISTRIBUTE | । 1A3550

ATT-CDS: POSS W/I DIST: NARC || 1A1119

Att-Cds: Possession - Marihuana, Cds II 1A0573

Att-Cds:Obtain By Fraud, Att-Cds:Obtain By Fraud || 1A0248

ATT-CDS:P W/I DIST:NARC || 3A0233

ATT-CDS:POSS W/INTENT DIST: NA || 3A0233 
Att-Cds:Possess-Not Marihuana, Att Cds Poss II 4A3550

Att-Cds:Possess-Not Marihuana, Att Dist Cocaine I| 4A3550

Att-Cds:Possess-Not Marihuana, Att To Poss Cds II 4A3550

Att-Cds:Possess-Not Marihuana, Att-Cds:Possess-Not M I| 4A3550

Att-Cds:Possess-Not Marihuana, Attemp To Possess Co I| 4A3550

Att-Cds:Possess-Not Marihuana, Attempt Poss Of Cds I| 4A3550

Att-Cds:Possess-Not Marihuana, Attempt Poss. Heroin II 4A3550

Att-Cds:Possess-Not Marihuana, Cds II 4A3550

Att-Cds:Possess-Not Marihuana, Cds Poss I| 4A3550

Att-Cds:Possess-Not Marihuana, Cds Poss - Att II 4A3550

Att-Cds:Possess-Not Marihuana, Cds Poss Heroin II 4A3550

Att-Cds:Possess-Not Marihuana, Cds Pwid II 4A3550

Att-Cds:Possess-Not Marihuana, Cds Violation II 4A3550

Att-Cds:Possess-Not Marihuana, Conspiracy II 4A3550

Att-Cds:Possess-Not Marihuana, Passing False Prescr I| 4A3550

Att-Cds:Possess-Not Marihuana, Poss Cocaine II 4A3550

Att-Cds:Possess-Not Marihuana, Poss Of Heroin I | 4A3550

ATT-CONTRABAND DELIVERY I| 2A1080

Att-Contraband Delivery, Con To Deliver Contraband II 2A1080

ATT-DIST HEROIN || 2A0696

ATT-POSS W/INT TO DIST । । 1A0233

ATT. CDS DISTRBUTION || 1A1118

ATT. CDS DISTRIBUTION । | 2A3550

ATT. DIST CDS || 11119

ATT. DIST CDS || 30233

ATT. DIST COCAINE I| 2A0696

ATT. DIST COCAINE || 30233

ATT. DIST COCAINE || 43550

ATT. DIST HEROIN ।| 43550

ATT. DIST OF HEROIN I| 43550

ATT. DIST OF LIQUID CODEINE || 43550

ATT. DIST. II 30233

ATT. DIST. II 43550

ATT. DIST. CDS I| 43550

ATT. DISTR. COCAINE || 43550

ATT. DISTR. HEROIN I| 20696

ATT. DISTRIBUTE HEROIN I| 2A0696

ATT. DISTRIBUTE MARIJUANA || 2A3550

ATT. DISTRIBUTION || 11111

ATT. DISTRIBUTION || 2A0696

ATT. DISTRIBUTION || 2A3550

ATT. DISTRIBUTION CDS || 10573

ATT. DISTRIBUTION CDS || 11111

ATT. DISTRIBUTION CDS | । 1A1118

ATT. DISTRIBUTION CDS । । 1C1118

ATT. DISTRIBUTION CDS || 2A0696

ATT. DISTRIBUTION CDS || 2A3550

ATT. DISTRIBUTION OF COCAINE || 2A0696

ATT. DISTRIBUTION SUBOXONE || 43550

ATT. DISTRUBUTION । 11111

ATT. POSS.OF HEROIN । 4 A3550

ATT.DIST.OF HEROIN || 2A0696

ATTEMPT DIST || 1A1118

ATTEMPT DIST CDS I। 1 C1118

ATTEMPT DIST HERION I| 30233

ATTEMPT DIST MARJ I| 10233

ATTEMPT DIST.COCAINE || 2A0696

ATTEMPT DISTRIBUTE COCAINE || 1A1118

ATTEMPT DISTRIBUTION । ।

ATTEMPT DISTRIBUTION || 1A1118 
ATTEMPT DISTRIBUTION || 23550

ATTEMPT DISTRIBUTION || 2A0696

ATTEMPT DISTRIBUTION || 2A3550

ATTEMPT DISTRIBUTION CDS || 11111

ATTEMPT DISTRIBUTION CDS || 1A1118

ATTEMPT DISTRUBUTION HERION || 30233

ATTEMPT TO DIST || 2A3550

ATTEMPT TO DIST CDS I। 43550

ATTEMPT TO DIST MARIJUANA || 2A3550

ATTEMPT TO DIST. CDS I I 2C0696

ATTEMPT TO DISTRIBUTE || 2A3550

ATTEMPT TO DISTRIBUTE CDS || 10043

ATTEMPT TO DISTRIBUTE HEROIN || 2A0696

ATTEMPT TO DISTRIBUTE MARIJUAN || 2A3550

Attempt-Cds Possess - Lg Amt, Attempt-Cds Possess - II 1A0881

ATTEMPTED DIST. OF HERION || 43550

ATTEMPTED DISTRIBUTION || 11111

ATTEMPTED DISTRIBUTION CDS | | 2A0696

B\& E || 10233

BALTIMORE || 2A3550

C.D.S || 30233

C.D.S || 43550

C.D.S. I| 43550

C.D.S. VIOLATION || 43550

CBD DIST MARIJUANA I| 2A3550

CCDS POSS II 43550

CD DIST || 2A3550

CD POSS || 30233

CD PWID || 11119

CD VIOALTION I| 2A3550

CD VIOLATION || 43550

CD-PAI || 2A3550

CDA || 10233

CDA || 43550

CDA VIOLATION || 43550

CDC COCAINE I| 2A0696

CDC DIST || 11118

CDDS VIOLATION || 43550

CDFS COCAINE || 2A0696

CDS || 10010

CDS || 10233

CDS || 10239

CDS || 10255

CDS I| 10493

CDS || 10881

CDS || 10908

CDS || 11118

CDS || 11119

CDS || 15409

CDS || 1A0010

CDS || 1A0233

CDS I| 1A0660

CDS || 1A0908

CDS || 1A1118

CDS || 1A1119

CDS I| 1A3550

CDS || 1C0233

CDS || 1C1118

CDS || 1C1119

CDS || 20239 


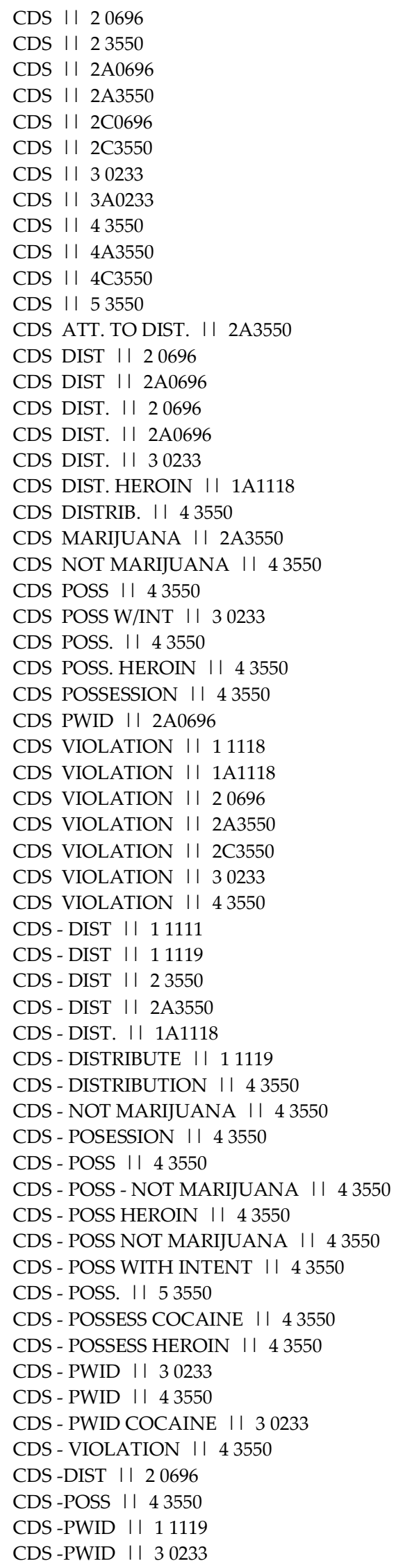


CDS : DISTRIBUTION HEROIN || 2A0696

CDS : HEROIN I| 43550

CDS : POSS COCAINE II 43550

CDS : POSSESS COCAINE I| 43550

CDS : POSSESS HEROIN । | 43550

CDS : PWID HEROIN I| 30233

CDS :ATT DIST(COCAINE) | | 2A0696

CDS (PWID) I| 10573

CDS [PSS. II 2A3550

CDS / POSS OF HEROIN II 30233

CDS / PWI I| 10573

CDS /DIST || 20696

CDS/PWI || 30233

CDS ADMIN EQUIP POSS/DIST || 10255

Cds Admin Equip Poss/Dist, Cds II 10255

Cds Admin Equip Poss/Dist, Cds Paraphernalia || 10255

Cds Admin Equip Poss/Dist, Cds Possession I| 10255

Cds Admin Equip Poss/Dist, Cds Violation I| 10255

Cds Admin Equip Poss/Dist, Paraphernalia || 10255

CDS AH DIST || 2A3550

CDS ATT /DIST. II 30233

CDS ATT DIS II 2A0696

CDS ATT DIS || 2A3550

CDS ATT DIST || 10233

CDS ATT DIST || 10573

CDS ATT DIST ।| 10879

CDS ATT DIST || 11111

CDS ATT DIST || 11119

CDS ATT DIST । | 1A1118

CDS ATT DIST ।| 1C1118

CDS ATT DIST || 2A0696

CDS ATT DIST || 2A3550

CDS ATT DIST || 30233

CDS ATT DIST । I 43550

CDS ATT DIST (COCAINE) || 2A0696

CDS ATT DIST COCAINE || 11111

CDS ATT DIST COCAINE || 43550

CDS ATT DIST/ HEROIN । 11119

CDS ATT DISTRIBUTE || 2A0696

CDS ATT DISTRIBUTE I| 2C0696

CDS ATT TO DIST || 2A3550

CDS ATT TO DIST । | 43550

CDS ATT TO DIST. I। 2A3550

CDS ATT. DIST । I 2A3550

CDS ATT. DIST || 30233

CDS ATT. DIST || 53550

CDS ATT. DIST. I। 11119

CDS ATT. DIST. II 1A1118

CDS ATT. DIST. | | 30233

CDS ATT. DIST. I। 43550

CDS ATT. DIST. COCAINE || 1A1118

CDS ATT. DISTRIBUTION || 11111

CDS ATT. DISTRIBUTION || 1A1118

CDS ATT. DISTRIBUTION || 2A0696

CDS ATT. DISTRIBUTION | | 30233

CDS ATT. TO DIST ।| 30233

CDS ATTEMPT DIST || 2A0696

CDS ATTEMPT DIST || 2A3550

CDS ATTEMPT DIST ।| 30233

CDS ATTEMPT DISTRIBUTE || 2A3550 
CDS ATTEMPT DISTRIBUTION || 11119 CDS ATTEMPT DISTRIBUTION || 11564 CDS ATTEMPT DISTRIBUTION || $1 \mathrm{~A} 1118$ CDS ATTEMPT DISTRIBUTION || 43550 CDS ATTEMPT TO DIST । | 2A3550

CDS ATTEMPT TO DISTRIBUTE || 30233 CDS ATTEMPTED DIST । | 30233

CDS ATTEMPTED DISTRIBUTION || 10573

CDS ATTMPT DISTRIBUTE || 2A0696

CDS CIOLATION II 20696

CDS COCAINE || 11118

CDS COCAINE || 11119

CDS COCAINE || 1A1118

CDS COCAINE || 1C1118

CDS COCAINE || 20696

CDS COCAINE || 2A0696

CDS COCAINE || 30233

CDS COCAINE || 43550

CDS COCAINE I| 53550

CDS COCAINE DIST || 11118

CDS COCAINE DIST || 1A1118

CDS COCAINE DIST I| 2A0696

CDS COCAINE DIST. I I 43550

CDS COMSPIRACY || 30233

CDS CON I| 2C0696

CDS CON DIST I| 20696

CDS CON DIST || 2A0696

CDS CON DIST COCAINE || 1A1118

CDS CONS I| 2A0696

CDS CONS DIST || 11118

CDS CONS DIST || 11119

CDS CONS TO DIST I। 2A3550

CDS CONS. DISTRIBUTE || 15409

CDS CONSP || 1C1118

CDS CONSP || 20696

CDS CONSP I| 2C0696

CDS CONSP DIST || $1 \mathrm{C} 1118$

CDS CONSP TO DIST । | 2C0696

CDS CONSP. II 2A0696

CDS CONSP. II 4A3550

CDS CONSP. TO BUY ।| 43550

CDS CONSPIR TO DIST || 2A0696

CDS CONSPIRACY || 10908

CDS CONSPIRACY || 11118

CDS CONSPIRACY || $1 \mathrm{C} 1118$

CDS CONSPIRACY || 20696

CDS CONSPIRACY I| 23550

CDS CONSPIRACY || 2A3550

CDS CONSPIRACY || 2C0696

CDS CONSPIRACY || 2C3550

CDS CONSPIRACY || 30233

CDS CONSPIRACY TO DISTRIBUTE || 2A0696

CDS CONSPIRACY TO DISTRIBUTE || 2C0696

CDS CONSPIRACY TO POSSESS | | 4A3550

CDS CONSPIRE II 2C0696

CDS CONSPIRE TO DIST || 2A3550

CDS CONSPIRE TO DISTRABUTE || 2A3550

CDS DIS || 1A1118

CDS DIS || 1C1118

CDS DIS || 20696 


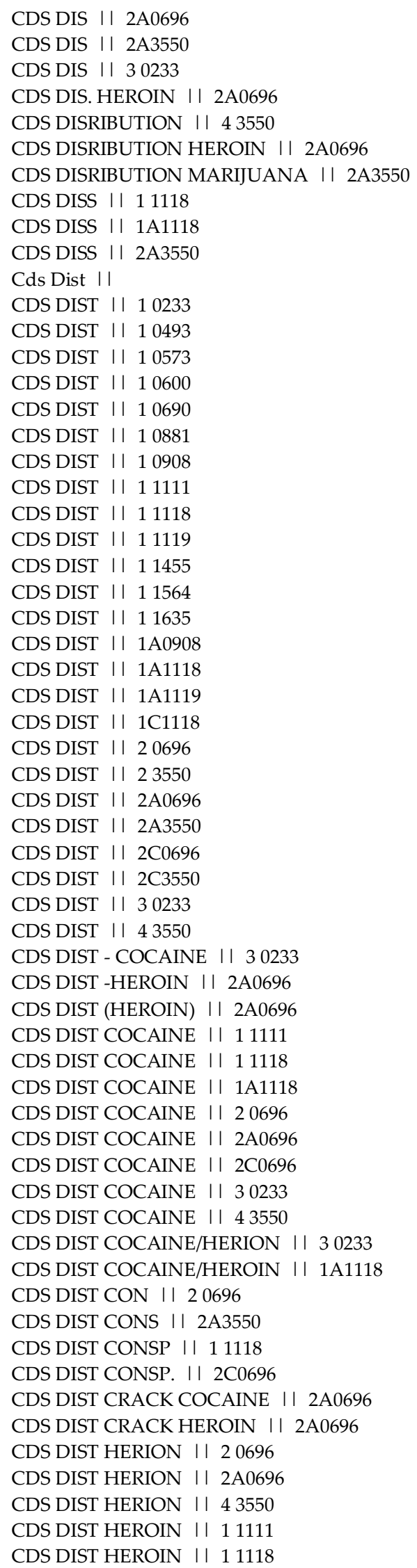


CDS DIST HEROIN । | 11119

CDS DIST HEROIN || 1A1118

CDS DIST HEROIN I I 1C1111

CDS DIST HEROIN || 1C1118

CDS DIST HEROIN । 20696

CDS DIST HEROIN || 2A0696

CDS DIST HEROIN । | 2C0696

CDS DIST HEROIN || 30233

CDS DIST HEROIN || 43550

CDS DIST HEROIN \& COCAINE || 2A0696

CDS DIST MARIJ I। 2A3550

CDS DIST MARIJUANA | | 11332

CDS DIST MARIJUANA || 11635

CDS DIST MARIJUANA || 23550

CDS DIST MARIJUANA I I 2A3550

Cds Dist Narc I |

CDS DIST NARCO | 1 A1118

CDS DIST NARCOTICS || 1A1118

CDS DIST NARCOTICS || 23550

CDS DIST NOT MARIJUANA || 2A0696

CDS DIST OF COCAINE || 30233

CDS DIST OF HEROIN II 20696

CDS DIST OF HEROIN I। 2A0696

CDS DIST- NOT MARIJUANA || 2A0696

CDS DIST-HEROIN I| 2A0696

CDS DIST-NARC । ।

CDS DIST-NARC || 11118

CDS DIST-NARC || 20696

CDS DIST-PWID || 43550

CDS DIST: COCAINE || 11118

CDS DIST: COCAINE || 1A1118

CDS DIST: HEROIN || 20696

CDS DIST:SCHOOL PROP/BUS I। 10483

CDS DIST. I I

CDS DIST. I। 10233

CDS DIST. II 11111

CDS DIST. II 11118

CDS DIST. II 11119

CDS DIST. I। 11564

CDS DIST. I। 11635

CDS DIST. II 1A0908

CDS DIST. I I 1A1118

CDS DIST. I। 20696

CDS DIST. I I 23550

CDS DIST. II 2A0696

CDS DIST. II 2A3550

CDS DIST. II 30233

CDS DIST. I। 43550

CDS DIST. COCAINE || 11118

CDS DIST. COCAINE || 11119

CDS DIST. COCAINE || 1A1118

CDS DIST. COCAINE I| 20696

CDS DIST. COCAINE || 2A0696

CDS DIST. COCAINE || 2C0696

CDS DIST. COCAINE/HEROIN || 11118

CDS DIST. COCAINE/HEROIN || 11119

CDS DIST. CONS || 20696

CDS DIST. HERION I| 2A0696

CDS DIST. HERION || 43550

CDS DIST. HEROIN || 11111 
CDS DIST. HEROIN || 11119 CDS DIST. HEROIN || 1A1118 CDS DIST. HEROIN I| 20696 CDS DIST. HEROIN II 2A0696 CDS DIST. HEROIN ।| 43550 CDS DIST. MARIJUANA || 23550 CDS DIST. MARIJUANA I| 2A3550 CDS DIST. MARIJUANA/COCAINE || 11111

CDS DIST. OF COCAINE || 2A0696 CDS DIST. OXYCODONE || 2A0696 CDS DIST.HEROIN || 11118

CDS DIST.OF MARIJUANA || 11119

CDS DIST/ HEROIN || 1A1118

CDS DIST/COCAINE || 11118

CDS DIST/COCAINE || 1A1118

CDS DIST/PWID || 11118

CDS DISTBUTION || 11118

CDS DISTIBUTION || 20696

CDS DISTIBUTION || 2A3550

Cds Distr I |

CDS DISTR I| 2A0696

CDS DISTR I I 30233

CDS DISTR. I। 2A0696

CDS DISTR. I। 2A3550

CDS DISTR. MARIJUANA || 2A3550

CDS DISTRABUTION || 1A1118

CDS DISTRABUTION || 2A3550

CDS DISTRBUTION || 11118

CDS DISTRBUTION I| 20696

CDS DISTRBUTION । | 43550

CDS DISTRIB || 23550

CDS DISTRIB COCAINE || 1A1118

CDS DISTRIB. II 2A0696

CDS DISTRIBATION || 1A1118

CDS DISTRIBUTE ।

CDS DISTRIBUTE - OTHER | | 23550

Cds Distribution I I

CDS DISTRIBUTION ।

CDS DISTRIBUTION || 10010 CDS DISTRIBUTION || 10233 CDS DISTRIBUTION || 10573

CDS DISTRIBUTION ।| 10600 CDS DISTRIBUTION || 10696 CDS DISTRIBUTION || 10908 CDS DISTRIBUTION || 11111 CDS DISTRIBUTION || 11118 CDS DISTRIBUTION || 11119 CDS DISTRIBUTION । | 11564 CDS DISTRIBUTION || 1A0010 CDS DISTRIBUTION || 1A0908 CDS DISTRIBUTION || 1A1118 CDS DISTRIBUTION ॥ 1C1118 CDS DISTRIBUTION || 20696 CDS DISTRIBUTION || 23550 CDS DISTRIBUTION || 2A0696 CDS DISTRIBUTION || 2A3550 CDS DISTRIBUTION || 2C0696 CDS DISTRIBUTION || 2C3550 CDS DISTRIBUTION || 30233 CDS DISTRIBUTION || 43550 
CDS DISTRIBUTION - HEROIN || 2C0696 CDS DISTRIBUTION -COCAINE || 2A0696 CDS DISTRIBUTION ( MARIJUANA) | | 2A3550 CDS DISTRIBUTION (CONSPIRACY) || 2C0696 CDS DISTRIBUTION COCAINE || 11111 CDS DISTRIBUTION COCAINE || 1A1118 CDS DISTRIBUTION COCAINE || 2A0696 CDS DISTRIBUTION COCAINE || 2C0696 CDS DISTRIBUTION COCAINE || 30233 CDS DISTRIBUTION HEROIN || 2A0696 CDS DISTRIBUTION HEROIN | | 30233 CDS DISTRIBUTION HEROIN || 43550 CDS DISTRIBUTION MARIJUANA || 23550 CDS DISTRIBUTION MARIJUANA || 2A3550 CDS DISTRIBUTION NARCOTICS || 1A1118 CDS DISTRIBUTION OF COCAINE || 11119 CDS DISTRIBUTION OF COCAINE || 2A0696 CDS DISTRIBUTION OF HEROIN || 2A0696 CDS DISTRIBUTION OF HEROIN || 43550 CDS DISTRIBUTION SUSP. HEROIN || 2A0696 CDS DISTRIBUTION VIOLATION || 2A0696 CDS DISTRIBUTION- MARIJUANA | | 23550 CDS DISTRIBUTION, NOT MARIJUAN | | 20696 CDS DISTRIBUTION; NOT MARIJUAN || 20696 CDS DISTRIBUTION/COCAINE || 1A1118

CDS DISTRIBUTION/HEROIN || 1A1118

CDS DISTRIBUTION/POSSESSION || 2A0696 CDS DISTRIBUTON || 30233 CDS DISTRO HEROIN || 2A0696 CDS DISTRUBITION || 20696

CDS DISTRUBTION || 20696 CDS DISTRUBTION || 2A0696 CDS DISTRUBTION I| 2A3550

CDS DISTRUBUTION || 2A0696

CDS DISTRUBUTION || 2A3550 CDS DISTRUBUTION MARIJUANA | | 10233 CDS DITRIBUTION | 11118

CDS DSIT || 2A0696

CDS HERIN PWID || 43550

CDS HERION || 11119

CDS HERION || 1A1118

CDS HERION I| 30233

CDS HERION I| 43550

CDS HEROIN I | 10881

CDS HEROIN || 11118

CDS HEROIN || 11119

CDS HEROIN I| 20696

CDS HEROIN || 2C0696

CDS HEROIN I| 30233

CDS HEROIN ।| 43550

CDS HEROIN DIST || 2A0696

CDS HEROIN POSS. II 43550

CDS HEROIN POSSESSION || 43550

CDS HYDROMORPHONE I| 43550

CDS INSIDE I| 43550

CDS INT TO DISTRIBUTE || 30233

CDS INTENT || 43550

CDS INTENT DIST. / POSS. I I 2A0696

CDS INTENT DISTRIBUTE | | 2A3550

CDS INTENT TO DISTRIBUTE || 11118 
CDS IOLATION || 11119

CDS LARGE AMOUNT/ PWI | | 10881

CDS MANUF/DIST-NARC || 20696

Cds Manuf/Dist-Narc, Cds I| 20696

Cds Manuf/Dist-Narc, Cds - Violation II 20696

Cds Manuf/Dist-Narc, Cds Conspiracy Cocaine || 20696

Cds Manuf/Dist-Narc, Cds Conspire Dist I| 20696

Cds Manuf/Dist-Narc, Cds Disribution II 20696

Cds Manuf/Dist-Narc, Cds Dist || 20696

Cds Manuf/Dist-Narc, Cds Dist Cocaine II 20696

Cds Manuf/Dist-Narc, Cds Dist Heroin II 20696

Cds Manuf/Dist-Narc, Cds Dist Narcotics I| 20696

Cds Manuf/Dist-Narc, Cds Dist-Narc II 20696

Cds Manuf/Dist-Narc, Cds Dist. I I 20696

Cds Manuf/Dist-Narc, Cds Distrabution II 20696

Cds Manuf/Dist-Narc, Cds Distribution I| 20696

Cds Manuf/Dist-Narc, Cds Distrubution II 20696

Cds Manuf/Dist-Narc, Cds Distrubution Cocaine II 20696

Cds Manuf/Dist-Narc, Cds Not Marijuana II 20696

Cds Manuf/Dist-Narc, Cds Poss I| 20696

Cds Manuf/Dist-Narc, Cds Poss Heroin I| 20696

Cds Manuf/Dist-Narc, Cds Poss W/ Int To Distribute II 20696

Cds Manuf/Dist-Narc, Cds Poss W/Intent I| 20696

Cds Manuf/Dist-Narc, Cds Poss With Intent || 20696

Cds Manuf/Dist-Narc, Cds Poss With Intent Heroin II 20696

Cds Manuf/Dist-Narc, Cds Poss With Intent To Distru || 20696

Cds Manuf/Dist-Narc, Cds Poss. Cocaine II 20696

Cds Manuf/Dist-Narc, Cds Poss... I| 20696

Cds Manuf/Dist-Narc, Cds Poss/ Intent Heroin || 20696

Cds Manuf/Dist-Narc, Cds Possesion I| 20696

Cds Manuf/Dist-Narc, Cds Possesion With Intent || 20696

Cds Manuf/Dist-Narc, Cds Possession Heroin II 20696

Cds Manuf/Dist-Narc, Cds Pwi II 20696

Cds Manuf/Dist-Narc, Cds Pwid II 20696

Cds Manuf/Dist-Narc, Cds Pwid Heroin I| 20696

Cds Manuf/Dist-Narc, Cds Viol. I I 20696

Cds Manuf/Dist-Narc, Cds Violation I I 20696

Cds Manuf/Dist-Narc, Cds Violaton Dist I| 20696

Cds Manuf/Dist-Narc, Cds- Dist Cocaine || 20696

Cds Manuf/Dist-Narc, Cds-Distribution II 20696

Cds Manuf/Dist-Narc, Cds/ Tresspassing II 20696

Cds Manuf/Dist-Narc, Cds/Trespassing I| 20696

Cds Manuf/Dist-Narc, Cocaine II 20696

Cds Manuf/Dist-Narc, Cocaine Distribution II 20696

Cds Manuf/Dist-Narc, Cons To Dist Heroin || 20696

Cds Manuf/Dist-Narc, Cons. Dist I| 20696

Cds Manuf/Dist-Narc, Cons. To Dist Heroin II 20696

Cds Manuf/Dist-Narc, Consp Cocaine II 20696

Cds Manuf/Dist-Narc, Consp Dist Heroin I| 20696

Cds Manuf/Dist-Narc, Consp Heroin II 20696

Cds Manuf/Dist-Narc, Consp To Distribute Cocaine I| 20696

Cds Manuf/Dist-Narc, Consp. Cocaine I| 20696

Cds Manuf/Dist-Narc, Consp. Dist. Cocaine II 20696

Cds Manuf/Dist-Narc, Conspi Dist Heroin I I 20696

Cds Manuf/Dist-Narc, Conspiracy I| 20696

Cds Manuf/Dist-Narc, Conspiracy Cocaine II 20696

Cds Manuf/Dist-Narc, Conspiracy To Distribute Heroi || 20696

Cds Manuf/Dist-Narc, Conspiracy To Distrubute Herio II 20696

Cds Manuf/Dist-Narc, Conspire Distribute Heroin II 20696

Cds Manuf/Dist-Narc, Destribution Of Heroin II 20696 
Cds Manuf/Dist-Narc, Dis. Heroin (Consp) I| 20696

Cds Manuf/Dist-Narc, Dist Cds || 20696

Cds Manuf/Dist-Narc, Dist Cocaine I| 20696

Cds Manuf/Dist-Narc, Dist Cocsine I| 20696

Cds Manuf/Dist-Narc, Dist Consp To Cocaine || 20696

Cds Manuf/Dist-Narc, Dist Herion I I 20696

Cds Manuf/Dist-Narc, Dist Heroin I I 20696

Cds Manuf/Dist-Narc, Dist Of Cds || 20696

Cds Manuf/Dist-Narc, Dist Of Cocaine || 20696

Cds Manuf/Dist-Narc, Dist Of Heroin II 20696

Cds Manuf/Dist-Narc, Dist. Cocaine I| 20696

Cds Manuf/Dist-Narc, Dist. Heroin I| 20696

Cds Manuf/Dist-Narc, Dist. -Cocaine || 20696

Cds Manuf/Dist-Narc, Dist. Cds || 20696

Cds Manuf/Dist-Narc, Dist. Cocaine II 20696

Cds Manuf/Dist-Narc, Dist. Heroin II 20696

Cds Manuf/Dist-Narc, Dist. Of Cds I| 20696

Cds Manuf/Dist-Narc, Dist. Of Cocaine I| 20696

Cds Manuf/Dist-Narc, Dist. Of Heroin II 20696

Cds Manuf/Dist-Narc, Dist.Cocaine || 20696

Cds Manuf/Dist-Narc, Distherion II 20696

Cds Manuf/Dist-Narc, Distibution Heroin I| 20696

Cds Manuf/Dist-Narc, Distribute Cds II 20696

Cds Manuf/Dist-Narc, Distributiion-Cocaine || 20696

Cds Manuf/Dist-Narc, Distribution I| 20696

Cds Manuf/Dist-Narc, Distribution Cocaine II 20696

Cds Manuf/Dist-Narc, Distribution Heroin I| 20696

Cds Manuf/Dist-Narc, Distribution Cds I| 20696

Cds Manuf/Dist-Narc, Distribution Cocain I| 20696

Cds Manuf/Dist-Narc, Distribution Cocaine I| 20696

Cds Manuf/Dist-Narc, Distribution Herion II 20696

Cds Manuf/Dist-Narc, Distribution Heroin II 20696

Cds Manuf/Dist-Narc, Distribution Heroin (Consp) I I 20696

Cds Manuf/Dist-Narc, Distribution Of Cocaine II 20696

Cds Manuf/Dist-Narc, Distribution Of Heroin I| 20696

Cds Manuf/Dist-Narc, Distribution- Heroin II 20696

Cds Manuf/Dist-Narc, Distribution-Cocaine II 20696

Cds Manuf/Dist-Narc, Distribution-Heroin I| 20696

Cds Manuf/Dist-Narc, Distribution/Cocaine I| 20696

Cds Manuf/Dist-Narc, Distrubution Cds II 20696

Cds Manuf/Dist-Narc, Distrubution Cocaine II 20696

Cds Manuf/Dist-Narc, Distrubution Herion || 20696

Cds Manuf/Dist-Narc, Ditribution Cocaine || 20696

Cds Manuf/Dist-Narc, Heroin Distribution I| 20696

Cds Manuf/Dist-Narc, Narcotics II 20696

Cds Manuf/Dist-Narc, P.W.I.D. II 20696

Cds Manuf/Dist-Narc, Pos Of Cds I| 20696

Cds Manuf/Dist-Narc, Poss Cds II 20696

Cds Manuf/Dist-Narc, Poss Cds-Not Marijuana || 20696

Cds Manuf/Dist-Narc, Poss Heroin I| 20696

Cds Manuf/Dist-Narc, Poss W/ Heroin II 20696

Cds Manuf/Dist-Narc, Poss W/I Cocaine II 20696

Cds Manuf/Dist-Narc, Poss W/Int II 20696

Cds Manuf/Dist-Narc, Poss W/Int Cocaine II 20696

Cds Manuf/Dist-Narc, Poss W/Int Cocanie II 20696

Cds Manuf/Dist-Narc, Poss W/Int Herion/Cocaine || 20696

Cds Manuf/Dist-Narc, Poss W/Int Heroin I| 20696

Cds Manuf/Dist-Narc, Poss W/Intent Cocaine II 20696

Cds Manuf/Dist-Narc, Poss W/Intent Heroin II 20696

Cds Manuf/Dist-Narc, Poss W/Intent Heroin \& Cocaine I| 20696 
Cds Manuf/Dist-Narc, Poss With Intent || 20696

Cds Manuf/Dist-Narc, Poss With Intent Cocaine || 20696

Cds Manuf/Dist-Narc, Poss With Intent Heroin I I 20696

Cds Manuf/Dist-Narc, Poss With Intet || 20696

Cds Manuf/Dist-Narc, Possession Heroin II 20696

Cds Manuf/Dist-Narc, Possession Of Cocaine II 20696

Cds Manuf/Dist-Narc, Possession W/I Heroin I | 20696

Cds Manuf/Dist-Narc, Possession W/Int Dist II 20696

Cds Manuf/Dist-Narc, Possession With Intent To Dist || 20696

Cds Manuf/Dist-Narc, Pwi Cds II 20696

Cds Manuf/Dist-Narc, Pwi Cocaine II 20696

Cds Manuf/Dist-Narc, Pwi Heroin I| 20696

Cds Manuf/Dist-Narc, Pwi/Cocaine || 20696

Cds Manuf/Dist-Narc, Pwid II 20696

Cds Manuf/Dist-Narc, Pwid Cocaine II 20696

Cds Manuf/Dist-Narc, Pwid Heroin I| 20696

Cds Manuf/Dist-Narc, Tresspass || 20696

Cds Manuf/Dist., Cds II 23550

Cds Manuf/Dist., Cds Conspiracy II 23550

Cds Manuf/Dist., Cds Dist Marijuana I| 23550

Cds Manuf/Dist., Cds Distirbution I| 23550

Cds Manuf/Dist., Cds Distribute - Other II 23550

Cds Manuf/Dist., Cds Distribution I I 23550

Cds Manuf/Dist., Cds Marijuana I | 23550

Cds Manuf/Dist., Cds Poss I| 23550

Cds Manuf/Dist., Cds Poss Marijuana I| 23550

Cds Manuf/Dist., Cds Poss W Intent Marijuana | | 23550

Cds Manuf/Dist., Cds Poss W/Int || 23550

Cds Manuf/Dist., Cds Poss. I 23550

Cds Manuf/Dist., Cds Possession I| 23550

Cds Manuf/Dist., Cds Violation II 23550

Cds Manuf/Dist., Cds/Marijuana I| 23550

Cds Manuf/Dist., Cdsviolation II 23550

Cds Manuf/Dist., Con Cds Dist I I 23550

Cds Manuf/Dist., Cons. To Dist Marijuana || 23550

Cds Manuf/Dist., Consp Dist Of Marijuana II 23550

Cds Manuf/Dist., Consp. Distribution I| 23550

Cds Manuf/Dist., Conspi Distribute || 23550

Cds Manuf/Dist., Conspiracy Dist. Marijuana || 23550

Cds Manuf/Dist., Cospire To Distribute Cds || 23550

Cds Manuf/Dist., Css II 23550

Cds Manuf/Dist., Dist || 23550

Cds Manuf/Dist., Dist Marijuana || 23550

Cds Manuf/Dist., Dist. Cds II 23550

Cds Manuf/Dist., Dist. Marijuana II 23550

Cds Manuf/Dist., Distribution | | 23550

Cds Manuf/Dist., Distribution Cds I| 23550

Cds Manuf/Dist., Distribution Maijuana I| 23550

Cds Manuf/Dist., Distribution Marijauana I| 23550

Cds Manuf/Dist., Distribution Marijuana I| 23550

Cds Manuf/Dist., Distribution Marjuana || 23550

Cds Manuf/Dist., Distribution Of Marijuana II 23550

Cds Manuf/Dist., Distribution-Marijana I| 23550

Cds Manuf/Dist., Distribution-Marijuana || 23550

Cds Manuf/Dist., Distrubution Marijuana I| 23550

Cds Manuf/Dist., Marijuana II 23550

Cds Manuf/Dist., Marijuana Distribution I| 23550

Cds Manuf/Dist., Marijuana Possesion I I 23550

Cds Manuf/Dist., Poss Cocaine II 23550

Cds Manuf/Dist., Poss Marijuana I| 23550 
Cds Manuf/Dist., Poss W/ Int Cds || 23550

Cds Manuf/Dist., Poss. Cds Marijuana I| 23550

Cds Manuf/Dist., Poss.Cds II 23550

Cds Manuf/Dist., Poss.Marijauana I| 23550

Cds Manuf/Dist., Pwid Marijuana II 23550

CDS MANUFACTORING || 30233

Cds Manufacture-Lg Amount, Cds Manufacture-Lg Amount II 10879

CDS MANUFACTURING | | 10881

CDS MARIJAUA || 2A3550

CDS MARIJUANA || 10233

CDS MARIJUANA I| 2A3550

CDS MARIJUANA || 30233

CDS MARIJUANA || 43550

CDS MARIJUANA DIST I| 10573

CDS MARIJUANA DIST || 2A3550

CDS MARIJUANA DIST. I I 2A3550

CDS MARIJUANA POSS W/ INTENT । | 11635

CDS MDMA I| 43550

CDS METHYLENEDIOXYMETHAMPHETAM || 10233

CDS MJ PWID || 10233

CDS MPOSS W/INTENT || 20696

CDS NARC PWID || 11119

CDS NARC PWID || 11111

CDS NOT MARIJUANA || 11119

CDS NOT MARIJUANA || 23550

CDS NOT MARIJUANA || 2A0696

CDS NOT MARIJUANA I | 30233

CDS NOT MARIJUANA I| 43550

CDS NOT MARIJUANA DISTRIBUTION || 2A0696

CDS NOT MARIJUANA POSS I 43550

CDS NOT MARIJUANA PWID || 2A0696

CDS NOT MARIJUANA PWID || 43550

CDS NOT MARIJUNA HEROIN | | 43550

CDS NOT MARJ. II 43550

CDS NOT MARJIAUNA || 43550

CDS OXYCODONE I| 43550

CDS P || 10233

CDS P W/INT || 11119

CDS P W/INT I| 30233

CDS P W/INT DIST ।| 10690

CDS P.W.I.D || 11119

CDS P/W/I || 10573

CDS P/W/I || 30233

Cds Pack Materl Poss/Distr, Cds Violation I| 10256

Cds Pack Materl Poss/Distr, Poss Marijuana II 10256

Cds Pack Materl Poss/Distr, Pwi/ Heroin I| 10256

CDS PARAPHANALIA I| 53550

CDS PARAPHANLIA || 53550

CDS PARAPHENALIA || 53550

CDS PARAPHENILIA || 53550

CDS PARAPHERNAILIA || 53550

CDS PARAPHERNALIA || 53550

CDS PARPH. II 53550

CDS PARPHANELIA I| 53550

CDS POCESSION I| 11119

CDS POOS W/I || 30233

CDS POS || 2A0696

CDS POS I| 43550

CDS POS I| 53550

CDS POSEESION || 43550 
CDS POSESS HEROIN । । 43550

CDS POSESS PERCOCET । | 43550

CDS POSESSION I| 2A0696

CDS POSESSION I| 43550

CDS POSESSION W/INT । | 11564

CDS POSESSION W/INTENT || 11610

CDS POSS || 10233

CDS POSS || 10255

CDS POSS || 10493

CDS POSS || 10881

CDS POSS I| 10908

CDS POSS || 11118

CDS POSS || 11119

CDS POSS || 1A1118

CDS POSS I| 1C1118

CDS POSS || 20696

CDS POSS I| 23550

CDS POSS I| 2A0696

CDS POSS I| 2A3550

CDS POSS || 2C0696

CDS POSS I| 30233

CDS POSS ।| 43550

CDS POSS II 53550

CDS POSS - NOT MAR ।| 43550

CDS POSS - NOT MARIJUANA I | 43550

CDS POSS (COCAINE) I। 43550

CDS POSS (CON) HEROIN II 30233

CDS POSS (HEROIN) I। 43550

CDS POSS (NOT MARIJUANA | | 43550

CDS POSS AND DIST । । 10573

CDS POSS COC W/I || 30233

CDS POSS COCAIN ।| 43550

CDS POSS COCAINE || 1A1118

CDS POSS COCAINE I| 23550

CDS POSS COCAINE || 2A0696

CDS POSS COCAINE I| 30233

CDS POSS COCAINE || 43550

CDS POSS COCAINE || 53550

CDS POSS COCAINE / HEROIN || 11119

CDS POSS COCAINE / HEROIN I | 43550

CDS POSS COCAINE \& HEROIN || 43550

CDS POSS COCAINE HEROIN I I 20696

CDS POSS COCAINE HEROIN I | 43550

CDS POSS COCAINE PARA II 53550

CDS POSS COCAINE WITH INTENT । | 30233

CDS POSS COCAINE/HEROIN || 43550

CDS POSS COCAINE/MARIJUANA I| 43550

CDS POSS COCIANE I| 43550

CDS POSS CRACK CAOINE || 43550

CDS POSS CRACK COCAINE || 43550

CDS POSS CRACK COCAINE/HEROIN I| 43550

CDS POSS CRACK/ HEROIN I I 30233

CDS POSS DIST I I 10487

CDS POSS DIST || 2A3550

CDS POSS FOR DISTRIBUTION | | 10272

Cds Poss For Distribution, Cds Poss For Distribution I| 10272

CDS POSS HERION || 2A0696

CDS POSS HERION I| 43550

CDS POSS HERION/COCAINE I | 43550

CDS POSS HEROIN II 10908 
CDS POSS HEROIN । | 11119

CDS POSS HEROIN । | 1A1118

CDS POSS HEROIN ।| 20696

CDS POSS HEROIN II 2A0696

CDS POSS HEROIN । | 30233

CDS POSS HEROIN ।| 43550

CDS POSS HEROIN I। 53550

CDS POSS HEROIN \& COCAINE I| 43550

CDS POSS HEROIN/COCAINE || 11119

CDS POSS HEROIN/COCAINE I | 30233

CDS POSS HEROIN/COCAINE I | 43550

CDS POSS I.T.D. I 30233

CDS POSS INT || 30233

CDS POSS INTENT || 30233

CDS POSS INTENT || 43550

CDS POSS INTENT DIST. | | 11111

CDS POSS LG AMT । | 10233

CDS POSS MARI I I 43550

CDS POSS MARIJUANA I| 10233

CDS POSS MARIJUANA || 23550

CDS POSS MARIJUANA I| 2A3550

CDS POSS MARIJUANA II 43550

CDS POSS MARIJUANA W INTENT ｜| 43550

CDS POSS MORPHINE || 43550

CDS POSS NARC I| 10908

CDS POSS NOT MAR I| 43550

CDS POSS NOT MARI । | 43550

CDS POSS NOT MARIHUANA || 43550

CDS POSS NOT MARIJ. I I 43550

CDS POSS NOT MARIJUANA || 30233

CDS POSS NOT MARIJUANA || 43550

CDS POSS NOT MARIJUNA I | 43550

CDS POSS NOT MJ । I 43550

CDS POSS OF COCAINE I| 2A0696

CDS POSS OF COCAINE ।| 30233

CDS POSS OF COCAINE ।| 43550

CDS POSS OF COCAINE AND HEROIN । | 43550

CDS POSS OF COCAINE/HEROIN || 11119

CDS POSS OF HEROIN || 30233

CDS POSS OF HEROIN । | 43550

CDS POSS OF MARIJUANA I | 43550

CDS POSS OTHER || 43550

CDS POSS OXY || 43550

CDS POSS PARAP || 53550

CDS POSS PARAPHERNALIA I I 53550

CDS POSS PWID || 10573

CDS POSS PWID || 11119

CDS POSS PWID || 20696

CDS POSS PWID || 30233

CDS POSS SUBOXONE || 43550

CDS POSS TO DISTRIBUTE | | 43550

CDS POSS TRAMADOL । 43550

CDS POSS W INT || 30233

CDS POSS W INT I| 43550

CDS POSS W INTENT II 11119

CDS POSS W INTENT I| 2A3550

CDS POSS W INTENT || 30233

CDS POSS W INTENT || 43550

CDS POSS W INTENT CRACK CAOINE I। 43550

CDS POSS W INTENT HEROIN || 43550 
CDS POSS W. INT || 43550

CDS POSS W.INTENT || 11119

CDS POSS W/ INT || 10493

CDS POSS W/ INT I| 10573

CDS POSS W/ INT । 10908

CDS POSS W/ INT || 11111

CDS POSS W/ INT || 11118

CDS POSS W/ INT I| 11119

CDS POSS W/ INT || 1A1118

CDS POSS W/ INT II 2A0696

CDS POSS W/ INT I| 2A3550

CDS POSS W/ INT || 30233

CDS POSS W/ INT || 43550

CDS POSS W/ INT । | 53550

CDS POSS W/ INT-HEROIN I I 30233

CDS POSS W/ INT: HEROIN || 30233

CDS POSS W/ INT: HEROIN I I 43550

CDS POSS W/ INTENT । I 10233

CDS POSS W/ INTENT I I 11119

CDS POSS W/ INTENT ।| 23550

CDS POSS W/ INTENT I| 2A0696

CDS POSS W/ INTENT I I 30233

CDS POSS W/ INTENT । 43550

CDS POSS W/ INTENT HEROIN । । 43550

CDS POSS W/ INTENT NOT MARIJ I । 43550

CDS POSS W/ INTENT TO DIST. I I 11119

CDS POSS W/ INTENT \ | 11119

CDS POSS W/I ।| 10493

CDS POSS W/I । | 11119

CDS POSS W/I II 43550

CDS POSS W/I COCAINE || 30233

CDS POSS W/I HEROIN II 11119

CDS POSS W/I HEROIN I I 30233

CDS POSS W/INENT TO DISTRIBUTE । । $1 \mathrm{~A} 1118$

CDS POSS W/INT || 10233

CDS POSS W/INT I| 10493

CDS POSS W/INT I। 10573

CDS POSS W/INT ।| 11111

CDS POSS W/INT ।| 11119

CDS POSS W/INT ।| 20696

CDS POSS W/INT || 2A0696

CDS POSS W/INT ।| 2A3550

CDS POSS W/INT I| 2C0696

CDS POSS W/INT II 30233

CDS POSS W/INT I| 43550

CDS POSS W/INT COCAINE || 11111

CDS POSS W/INT HEROIN I 30233

CDS POSS W/INT HEROIN I I 43550

CDS POSS W/INT MARIJUANA || 10573

CDS POSS W/INT NOT MARIJUA | | 43550

CDS POSS W/INT TO DIST । ।

CDS POSS W/INT TO DIST । 10233

CDS POSS W/INT TO DIST I। 23550

CDS POSS W/INT TO DIST || 30233

Cds Poss W/Int To Dist, Att. Cds Distribution II 10233

Cds Poss W/Int To Dist, Cds || 10233

Cds Poss W/Int To Dist, Cds Consp. To Distribute || 10233

Cds Poss W/Int To Dist, Cds Dist I | 10233

Cds Poss W/Int To Dist, Cds Dist Marijuana I| 10233

Cds Poss W/Int To Dist, Cds Distribution I| 10233 
Cds Poss W/Int To Dist, Cds Distrubution || 10233

Cds Poss W/Int To Dist, Cds Distubution || 10233

Cds Poss W/Int To Dist, Cds Marijuana I| 10233

Cds Poss W/Int To Dist, Cds Para || 10233

Cds Poss W/Int To Dist, Cds Poss || 10233

Cds Poss W/Int To Dist, Cds Poss Heroin I| 10233

Cds Poss W/Int To Dist, Cds Poss Marijuana II 10233

Cds Poss W/Int To Dist, Cds Poss W/ Int II 10233

Cds Poss W/Int To Dist, Cds Poss W/I || 10233

Cds Poss W/Int To Dist, Cds Poss W/Int I| 10233

Cds Poss W/Int To Dist, Cds Poss W/Int To Dist I | 10233

Cds Poss W/Int To Dist, Cds Poss-Not Mar. I I 10233

Cds Poss W/Int To Dist, Cds Poss. I I 10233

Cds Poss W/Int To Dist, Cds Possession || 10233

Cds Poss W/Int To Dist, Cds Possession With Intent I| 10233

Cds Poss W/Int To Dist, Cds Possession With Intent T || 10233

Cds Poss W/Int To Dist, Cds Pwi || 10233

Cds Poss W/Int To Dist, Cds Pwid I I 10233

Cds Poss W/Int To Dist, Cds Pwid Marijuana I| 10233

Cds Poss W/Int To Dist, Cds Pwid/Marijuana || 10233

Cds Poss W/Int To Dist, Cds Viol II 10233

Cds Poss W/Int To Dist, Cds Violation I I 10233

Cds Poss W/Int To Dist, Cds-Pwi Marijuana || 10233

Cds Poss W/Int To Dist, Cds-Pwid || 10233

Cds Poss W/Int To Dist, Conspiracy Marijuana I| 10233

Cds Poss W/Int To Dist, Dist Cds I I 10233

Cds Poss W/Int To Dist, Dist Marijuana || 10233

Cds Poss W/Int To Dist, Dist Of Mara || 10233

Cds Poss W/Int To Dist, Dist. Cds || 10233

Cds Poss W/Int To Dist, Dist. Marijuana || 10233

Cds Poss W/Int To Dist, Distirubtion || 10233

Cds Poss W/Int To Dist, Distribution I| 10233

Cds Poss W/Int To Dist, Distribution Marijuana || 10233

Cds Poss W/Int To Dist, Distribution Of Marihuana || 10233

Cds Poss W/Int To Dist, Distribution Of Marijauna || 10233

Cds Poss W/Int To Dist, Distribution Of Marijuana I| 10233

Cds Poss W/Int To Dist, Distribution- Marijuana II 10233

Cds Poss W/Int To Dist, Distribution-Marijuana || 10233

Cds Poss W/Int To Dist, Distrubution Of Marijuana || 10233

Cds Poss W/Int To Dist, Marijajuana II 10233

Cds Poss W/Int To Dist, Marijuana Dist. I I 10233

Cds Poss W/Int To Dist, Marijuana Distribution || 10233

Cds Poss W/Int To Dist, Marijuana Pwid II 10233

Cds Poss W/Int To Dist, P/W/I Marijuana II 10233

Cds Poss W/Int To Dist, Poss Cds || 10233

Cds Poss W/Int To Dist, Poss Int. Marijuana || 10233

Cds Poss W/Int To Dist, Poss Marijuana I| 10233

Cds Poss W/Int To Dist, Poss W/ Int Marijuana || 10233

Cds Poss W/Int To Dist, Poss W/ Intent || 10233

Cds Poss W/Int To Dist, Poss W/ Mar I| 10233

Cds Poss W/Int To Dist, Poss W/I Heroin || 10233

Cds Poss W/Int To Dist, Poss W/I Marijauna II 10233

Cds Poss W/Int To Dist, Poss W/I Marijuana II 10233

Cds Poss W/Int To Dist, Poss W/Int Dist Cds | 10233

Cds Poss W/Int To Dist, Poss W/Int Marijuana II 10233

Cds Poss W/Int To Dist, Poss W/Intent || 10233

Cds Poss W/Int To Dist, Poss W/Intent Marijuana || 10233

Cds Poss W/Int To Dist, Poss Wint Cocaine I| 10233

Cds Poss W/Int To Dist, Poss With Int Marijuana II 10233

Cds Poss W/Int To Dist, Poss With Intent Marij || 10233 
Cds Poss W/Int To Dist, Poss.Marijunna || 10233

Cds Poss W/Int To Dist, Poss.W/Int. Dist Marijuana || 10233

Cds Poss W/Int To Dist, Possession W/ Int To Dist | | 10233

Cds Poss W/Int To Dist, Possession W/I Marijuana II 10233

Cds Poss W/Int To Dist, Possession W/Int Marijuana || 10233

Cds Poss W/Int To Dist, Pwi Cocaine || 10233

Cds Poss W/Int To Dist, Pwi Marijuana I | 10233

Cds Poss W/Int To Dist, Pwid Heroin I| 10233

Cds Poss W/Int To Dist, Pwid Marijuana || 10233

Cds Poss W/Int To Dist, Pwid/Marijuana II 10233

CDS POSS W/INT. COCAINE I| 2A0696

CDS POSS W/INTENT । | 10573

CDS POSS W/INTENT ॥ 11111

CDS POSS W/INTENT । 11118

CDS POSS W/INTENT । 11119

CDS POSS W/INTENT । | 30233

CDS POSS W/INTENT I। 43550

CDS POSS W/INTENT - COCAINE || 43550

CDS POSS W/INTENT COCAINE || 2A0696

CDS POSS W/INTENT COCAINE || 30233

CDS POSS W/INTENT HEROIN I| 30233

CDS POSS W/INTENT MARIJUANA | | 10233

CDS POSS W/INTENT MARIJUANA | | 10573

CDS POSS W/INTENT TO DIST । | 11111

CDS POSS W/INTENT TO DIST || 11119

CDS POSS W/INTENT TO DIST । | 43550

CDS POSS W/INTENT TO DISTRBUTE || 11119

CDS POSS W/INTENT TO DISTRIBD । ।

CDS POSS W/INTENT TO DISTRIBUT । | 10487

CDS POSS W/INTENT TO DISTRIBUT । | 10573

CDS POSS W/INTENT TO DISTRIBUT । | 11111

CDS POSS W/INTENT TO DISTRIBUT । | 11119

CDS POSS W/INTENT TO DISTRIBUT ।| 20696

CDS POSS W/INTENT TO DISTRIBUT । | 30233

CDS POSS W/INTENT TO DISTRIBUT । | 43550

CDS POSS WI I I 30233

CDS POSS WITH INETNT || 30233

CDS POSS WITH INT || 20696

CDS POSS WITH INT || 30233

CDS POSS WITH INT I| 43550

CDS POSS WITH INT COCAINE || 43550

CDS POSS WITH INTENT I I 10573

CDS POSS WITH INTENT II 11111

CDS POSS WITH INTENT || 11119

CDS POSS WITH INTENT || 11564

CDS POSS WITH INTENT | | 11635

CDS POSS WITH INTENT || 2A0696

CDS POSS WITH INTENT | | 30233

CDS POSS WITH INTENT | | 43550

CDS POSS WITH INTENT HEROIN || 43550

CDS POSS WITH INTENT TO DISTRI | | 10573

CDS POSS WITH INTENT. I I 11111

CDS POSS- COCAINE I| 43550

CDS POSS- HEROIN || 43550

CDS POSS- NOT MARIJUANA I | 43550

CDS POSS- NOT MARJUANA || 43550

CDS POSS-COCAINE || 43550

CDS POSS-HERION । | 43550

CDS POSS-HEROIN । | 43550

CDS POSS-MARIJUANA || 43550 
CDS POSS-NOT MARIJ || 43550

CDS POSS-NOT MARIJUANA || 43550

CDS POSS, DIST II 2A0696

CDS POSS; NOT MARIJUANA I I 43550

CDS POSS: DIST. I I 30233

CDS POSS: HEROIN || 43550

CDS POSS: NOT MARIJUANA || 11119

CDS POSS: NOT MARIJUANA || 43550

CDS POSS: OTHER ।| 43550

CDS POSS: PWID I| 43550

CDS POSS: W INTENT । | 43550

CDS POSS:HEROIN || 43550

CDS POSS:NOT MARIJUANA || 43550

CDS POSS. II 10233

CDS POSS. II 11119

CDS POSS. II 1A1118

CDS POSS. II 20696

CDS POSS. II 2A0696

CDS POSS. II 30233

CDS POSS. II 43550

CDS POSS. II 53550

CDS POSS. COCAINE || 11118

CDS POSS. COCAINE || 1A1118

CDS POSS. COCAINE || 30233

CDS POSS. COCAINE I| 43550

CDS POSS. COCAINE/ HEROIN || 43550

CDS POSS. HERION I| 43550

CDS POSS. HEROIN || 2C0696

CDS POSS. HEROIN I| 30233

CDS POSS. HEROIN I| 43550

CDS POSS. INT. DISTRIBUTE || 30233

CDS POSS. INTENT । I 30233

CDS POSS. MARIJUANA I| 2A3550

CDS POSS. NOT MAR I| 43550

CDS POSS. NOT MARIJUANA || 43550

CDS POSS. OF COCAINE I। 43550

CDS POSS. OTHER || 43550

CDS POSS. PARAPHERNALIA || 53550

CDS POSS. PERCOCET || 43550

CDS POSS. SUBOXONE || 43550

CDS POSS. W INTENT TO DISTRIBU । | 43550

CDS POSS. W/ INT ।| 11111

CDS POSS. W/ INT ।| 11119

CDS POSS. W/ INTENT I| 10233

CDS POSS. W/ INTENT । 10881

CDS POSS. W/ INTENT ।| 11635

CDS POSS. W/INT ।| 11119

CDS POSS. W/INT I| 30233

CDS POSS. W/INTENT ।| 30233

CDS POSS. WIT INTENT || 43550

CDS POSS. WITH INTENT | | 10233

CDS POSS. WITH INTENT | | 10573

CDS POSS. WITH INTENT || 30233

CDS POSS. WITH INTENT I| 43550

CDS POSS.-NOT MARIJUANA || 43550

CDS POSS.. I। 43550

CDS POSS.COCAINE || 43550

CDS POSS.HEROIN || 43550

CDS POSS.PWID || 11119

CDS POSS.W/INTENT ॥ 30233 


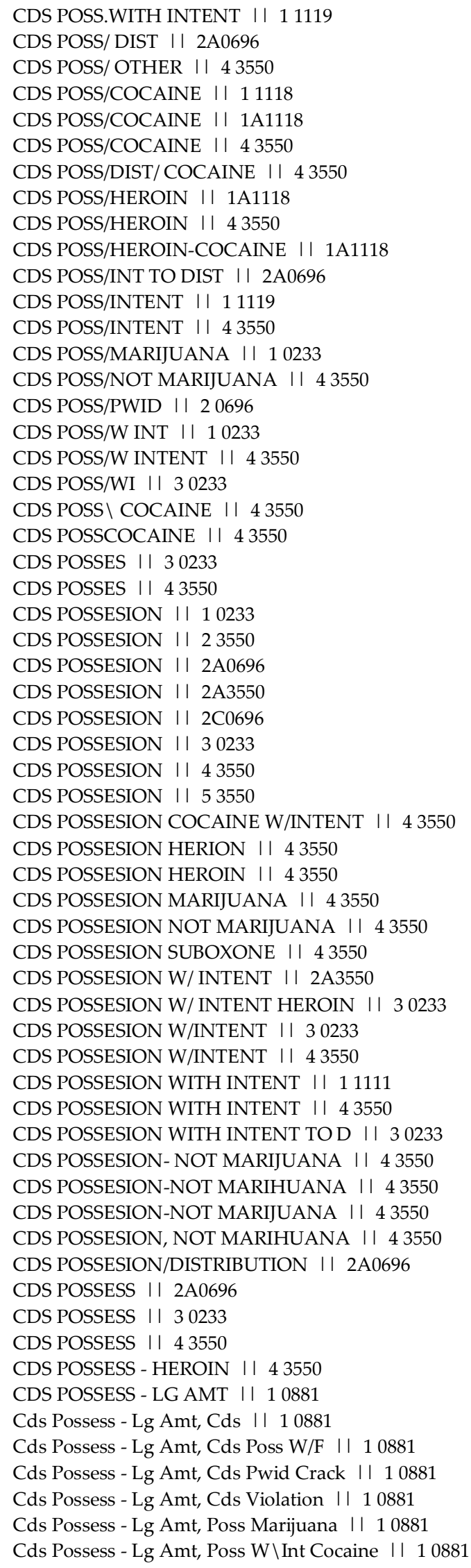


Cds Possess - Lg Amt, Poss With Intent To Dist I| 10881 Cds Possess - Lg Amt, Poss. With Int. Heroin I। 10881 Cds Possess - Lg Amt, Poss. With Intent Heroin I| 10881 Cds Possess - Lg Amt, Pwi Heroin II 10881

Cds Possess - Lg Amt, Pwid Cocaine || 10881

Cds Possess - Lg Amt, Pwid Heroin I| 10881

CDS POSSESS COCAINE || 43550

CDS POSSESS HEROIN || 43550

CDS POSSESS MARI || 10233

CDS POSSESS MARIJUANA II 2A3550

CDS POSSESS W/ INTENT TO DISTR । I 11119

CDS POSSESS-HEROIN II 43550

CDS POSSESSION || 10233

CDS POSSESSION || 10255

CDS POSSESSION || 10881

CDS POSSESSION || 11118

CDS POSSESSION || 11119

CDS POSSESSION || 1A0233

CDS POSSESSION || 1A1118

CDS POSSESSION || 20696

CDS POSSESSION || 23550

CDS POSSESSION I| 2A0696

CDS POSSESSION || 2A3550

CDS POSSESSION || 2C0696

CDS POSSESSION || 2C3550

CDS POSSESSION || 30233

CDS POSSESSION ।| 43550

CDS POSSESSION || 4A3550

CDS POSSESSION ।| 53550

CDS POSSESSION HEROIN || 43550

CDS POSSESSION - HEROIN I । 43550

CDS POSSESSION - NOT MAR || 43550

CDS POSSESSION COCAINE | | 11119

CDS POSSESSION COCAINE || 20696

CDS POSSESSION COCAINE || 30233

CDS POSSESSION COCAINE I| 43550

CDS POSSESSION COCAINE || 53550

CDS POSSESSION COCAINE \& HEROI || 43550

CDS POSSESSION COCAINE/MARIJUA || 43550

CDS POSSESSION DIST || 2A0696

CDS POSSESSION DIST | | 30233

CDS POSSESSION HEROIN || 1A1118

CDS POSSESSION HEROIN | | 30233

CDS POSSESSION HEROIN I| 43550

CDS POSSESSION HEROIN I | 53550

CDS POSSESSION MARIJUANA I | 43550

CDS POSSESSION NOT MAR I| 43550

CDS POSSESSION NOT MARIJ | | 43550

CDS POSSESSION NOT MARIJUANA | | 43550

CDS POSSESSION NOT MJ || 43550

CDS POSSESSION OF COCAINE || 2A0696

CDS POSSESSION OF COCAINE || 43550

CDS POSSESSION OF HEROIN | | 11119

CDS POSSESSION OF HEROIN || 2A0696

CDS POSSESSION OF HEROIN I I 30233

CDS POSSESSION OF HEROIN I | 43550

CDS POSSESSION OF HEROIN W/ IN I| 30233

CDS POSSESSION PARAPHERNALIA || 53550

CDS POSSESSION SUBOXONE || 43550

CDS POSSESSION W/ INT || 11119 
CDS POSSESSION W/ INT | | 30233

CDS POSSESSION W/ INTENT | | 10573

CDS POSSESSION W/ INTENT | | 43550

CDS POSSESSION W/ INTENT TO DI I I 43550

CDS POSSESSION W/I || 43550

CDS POSSESSION W/INT I| 30233

CDS POSSESSION W/INT I| 43550

CDS POSSESSION W/INTENT | | 30233

CDS POSSESSION W/INTENT | | 43550

CDS POSSESSION W/INTENT TO DIS || 11111

CDS POSSESSION W/INTENT TO DIS I । 43550

CDS POSSESSION WITH INTENT | | 10233

CDS POSSESSION WITH INTENT || 10573

CDS POSSESSION WITH INTENT || 10881

CDS POSSESSION WITH INTENT I | 11111

CDS POSSESSION WITH INTENT | | 11119

CDS POSSESSION WITH INTENT || 11564

CDS POSSESSION WITH INTENT || 20696

CDS POSSESSION WITH INTENT || 30233

CDS POSSESSION WITH INTENT || 43550

Cds Possession With Intent To II

CDS POSSESSION WITH INTENT TO I। 10573

CDS POSSESSION WITH INTENT TO । | 11119

CDS POSSESSION WITH INTENT TO || 2A0696

CDS POSSESSION WITH INTENT TO || 30233

CDS POSSESSION WITH INTENT TO I | 43550

CDS POSSESSION WITH THE INTENT । । 11119

CDS POSSESSION WITH THE INTENT || 2A0696

CDS POSSESSION WITH THE INTENT ।| 30233

CDS POSSESSION WITH THE INTENT I| 43550

CDS POSSESSION- COCAINE || 30233

CDS POSSESSION- NOT MARIJUANA || 43550

CDS POSSESSION-HEROIN I| 43550

CDS POSSESSION, NOT MARIJUANA I| 43550

CDS POSSESSION; NOT MARIJUANA || 30233

CDS POSSESSION; NOT MARIJUANA || 43550

CDS POSSESSION; NOT MARIJUANA || 53550

CDS POSSESSION: HEROIN || 43550

CDS POSSESSION: MARIJUANA || 53550

CDS POSSESSION/CONSPIRACY | | 43550

CDS POSSESSION/W INTENT | | 30233

CDS POSSESSION/COCAINE || 11119

CDS POSSESSION/DISTRIBUTION | 30233

CDS POSSESSION/HEROIN || 11119

CDS POSSESSION/INT DIST || 30233

CDS POSSESSON || 43550

CDS POSSESSSION || 11119

CDS POSSESSSION ।| 2A0696

CDS POSSESTION W/INT || 11119

CDS POSSISSION ।| 43550

CDS POSSS W/INTENT I| 43550

CDS POSSSION || 43550

CDS POSSSSION I| 43550

CDS PSSSESSION ॥ 43550

CDS PW/INT I| 30233

CDS PWD/DISTR || 20696

CDS PWI || 10233

CDS PWI ।| 10484

CDS PWI ।| 10573

CDS PWI || 10881 


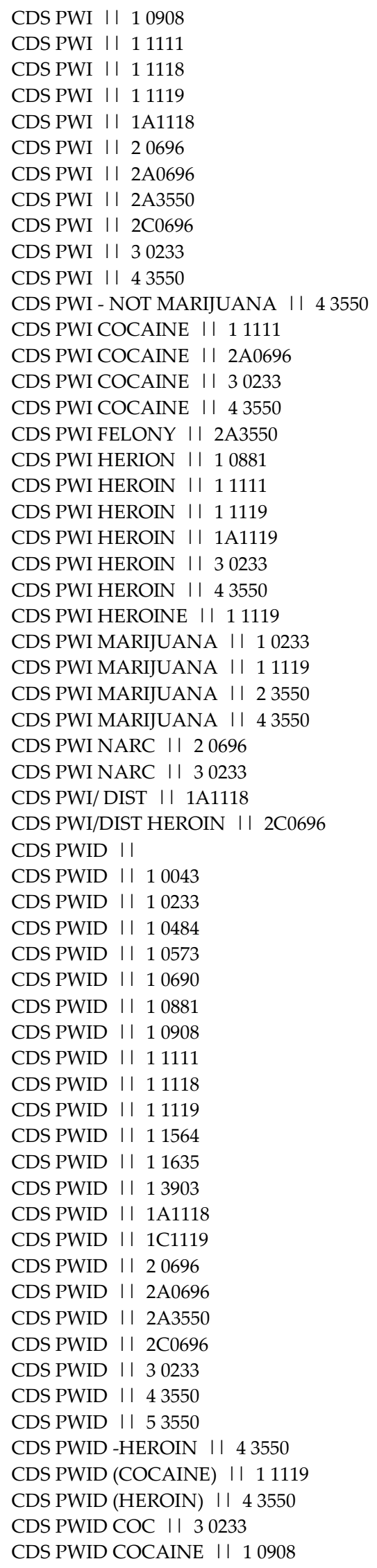


CDS PWID COCAINE || 11111 CDS PWID COCAINE || 11118 CDS PWID COCAINE || 11119 CDS PWID COCAINE || 11454 CDS PWID COCAINE || 1A1118 CDS PWID COCAINE || 2A0696 CDS PWID COCAINE || 30233 CDS PWID COCAINE || 43550 CDS PWID FENTANYL | | 10908 CDS PWID HERION || 11119 CDS PWID HERION I | 30233 CDS PWID HERION/COCAINE | | 30233 CDS PWID HEROIN || 11111 CDS PWID HEROIN || 11119 CDS PWID HEROIN || 2A0696 CDS PWID HEROIN || 30233 CDS PWID HEROIN । | 43550 CDS PWID HEROIN COCAINE | | 11111 CDS PWID HEROIN/COCAINE | 10881 CDS PWID HEROIN/FENTANYL MIX || 11119 CDS PWID HEROIN/MARIJUANA || 30233 CDS PWID MARFIJUANA I | 10573 CDS PWID MARIJUAN || 43550 CDS PWID MARIJUANA || 10233 CDS PWID MARIJUANA || 10573 CDS PWID MARIJUANA || 11564 CDS PWID MARIJUANA || 11635 CDS PWID NARC || 10493 CDS PWID NARC I| 10881 CDS PWID NARC | | 10908 CDS PWID NARC | 11111 CDS PWID NARC । 11119 CDS PWID NARC || 1A1118 CDS PWID NARC || 2A0696 CDS PWID NARC || 43550 CDS PWID NARCOTICS । 10908 CDS PWID NARCOTICS || 11118 CDS PWID NOT MARIJUANA || 11111 CDS PWID NOT MARIJUANA | | 11119 CDS PWID NOT MARIJUANA I | 30233 CDS PWID NOT MARIJUANA || 43550 CDS PWID OTHER | | 11119 CDS PWID- HEROIN । 43550 CDS PWID- HEROIN/COCAINE | | 30233 CDS PWID-COCAINE I| 43550

CDS PWID-DIST. I। 43550

CDS PWID-HEROIN || 43550

CDS PWID-MARIJUANA || 10573

CDS PWID/COCAINE || 11118

CDS PWID/HEROIN || 1A1118

CDS PWID/HEROIN/COCAINE | | 11119

CDS PWIP || 11119

CDS PWIT || 10233

CDS PWIT I| 43550

CDS S\&S || 11119

CDS SYNTHETHIC MARIJUANA | | 43550

CDS VACATION || 2A3550

CDS VIOLATION I| 43550

CDS VIIOLATION I| 2A0696

CDS VIJOLATION || 43550 
CDS VILATION || 11119

CDS VILATION || 2A3550

CDS VILATION || 43550

CDS VILLATION || 43550

CDS VILOATION I| 43550

CDS VILOTION I| 43550

CDS VIO I| 43550

CDS VIOALTION || 10233

CDS VIOALTION || 2A0696 CDS VIOALTION I| 2A3550 CDS VIOALTION I। 30233

CDS VIOALTION || 43550

CDS VIOATION || 11119

CDS VIOATION || 20696

CDS VIOATION I| 43550

CDS VIOL || 10233

CDS VIOL || 10908

CDS VIOL || 11118

CDS VIOL || 1A1118

CDS VIOL || 23550

CDS VIOL I| 2A3550

CDS VIOL | | 30233

CDS VIOL || 43550

CDS VIOL - POSSES CDS NOT MARI । | 43550

CDS VIOL COMP TO DIST. HEROIN । | 15409

CDS VIOL PWID || 30233

CDS VIOL. II 11119

CDS VIOL. II 20696

CDS VIOL. I I 2A0696

CDS VIOL. II 2C0696

CDS VIOL. I I 30233

CDS VIOL. II 43550

CDS VIOLAION || 23550

CDS VIOLAION || 2A3550

CDS VIOLAION || 43550

CDS VIOLAITION || 1A1118

CDS VIOLAITION || 43550

CDS VIOLAITON || 43550

CDS VIOLALTION || 43550

CDS VIOLATIION || 2A0696

CDS VIOLATIION II 43550

CDS VIOLATIN II 43550

CDS VIOLATIO II 43550

CDS VIOLATIOIN | | 10255

CDS VIOLATIOIN || 43550

CDS VIOLATIOM || 11118

CDS VIOLATIOM || 43550

CDS VIOLATION || 10010

CDS VIOLATION || 10233

CDS VIOLATION || 10246

CDS VIOLATION I| 10255

CDS VIOLATION I। 10493

CDS VIOLATION I। 10696

CDS VIOLATION I। 10881

CDS VIOLATION I| 10908

CDS VIOLATION ॥ 11118

CDS VIOLATION ॥ 11119

CDS VIOLATION || 1A0010

CDS VIOLATION I I 1A0233

CDS VIOLATION || 1A0696 
CDS VIOLATION || 1A0908 CDS VIOLATION || 1A1118 CDS VIOLATION || 1A1119 CDS VIOLATION || 1 C1118 CDS VIOLATION || 1C1119 CDS VIOLATION || 20696 CDS VIOLATION I| 23550 CDS VIOLATION I| 2A0696 CDS VIOLATION || 2A3550 CDS VIOLATION I| 2C0230 CDS VIOLATION II 2C0696 CDS VIOLATION || 2C3550 CDS VIOLATION || 30233 CDS VIOLATION || 3A0233 CDS VIOLATION || 3C0233 CDS VIOLATION I| 43550 CDS VIOLATION || 4A3550 CDS VIOLATION || 4C3550 CDS VIOLATION II 53550 CDS VIOLATION || 63550 CDS VIOLATION - DIST I। 2A0696 CDS VIOLATION - HEROIN I I 43550

CDS VIOLATION - POSSESSION || 43550 CDS VIOLATION (HEROIN) | | 43550

CDS VIOLATION (NOT MARIJUANA) | | 30233 CDS VIOLATION (NOT MARIJUANA) I | 43550 CDS VIOLATION (POSS. OF HEROIN || 43550 CDS VIOLATION (POSS.) || 43550 CDS VIOLATION (PWI) || 43550 CDS VIOLATION (PWID) | | 43550 CDS VIOLATION DIST । 11118 CDS VIOLATION DIST । I 20696 CDS VIOLATION DIST । | 23550 CDS VIOLATION DIST || 2A3550 CDS VIOLATION DIST. I I 43550 CDS VIOLATION DIST. MARIJUANA || 2A3550 CDS VIOLATION DISTRIBUTION || 2A3550

CDS VIOLATION HEROIN || 43550 CDS VIOLATION HEROIN POSSESSIO || 43550 CDS VIOLATION HEROIN PWID | | 30233 CDS VIOLATION PHARMACY W/O LIC I | 30233 CDS VIOLATION POSS II 43550 CDS VIOLATION POSS OXYCOTINE || 2A3550 CDS VIOLATION POSS W/INT I| 30233

CDS VIOLATION POSS. HEROIN | | 10881 CDS VIOLATION POSS.HEROIN || 43550 CDS VIOLATION PWI || 2A3550 CDS VIOLATION PWID || 10573 CDS VIOLATION PWID || 11111 CDS VIOLATION PWID || 11118 CDS VIOLATION PWID || 11119 CDS VIOLATION PWID || 11564 CDS VIOLATION PWID || 43550 CDS VIOLATION PWID HEROIN || 11111 CDS VIOLATION PWID HEROIN I। 11119 CDS VIOLATION S\&S I| 43550

CDS VIOLATION-DIST । | 43550 CDS VIOLATION-DIST I | 2A3550 CDS VIOLATION-DIST-HEROIN || 2A0696 CDS VIOLATION-DIST. ATTEMPT । | 43550 
CDS VIOLATION-HEROIN PWID || 43550

CDS VIOLATION-POSS || 43550

CDS VIOLATION-POSS HEROIN || 43550

CDS VIOLATION-POSS. HEROIN || 43550

CDS VIOLATION; ATT DIST I। 2A0696

CDS VIOLATION; DISTRIBUTION || 2A0696

CDS VIOLATION; NOT MARIJUANA I| 20696

CDS VIOLATION; NOT MARIJUANA || 30233

CDS VIOLATION; NOT MARIJUANA | | 43550

CDS VIOLATION; POSS II 43550

CDS VIOLATION; POSSESSION I| 43550

CDS VIOLATION; PWID || 30233

CDS VIOLATION(PWI COCAINE) | | 30233

CDS VIOLATION/ DISTRIBUTION || 2A0696

CDS VIOLATION/DISTRIBUTION || 43550

CDS VIOLATION/ INTENT | | 43550

CDS VIOLATION/ NOT MARIJUANA I | 43550

CDS VIOLATION/ POSS W/INT COCA I I 43550

CDS VIOLATION/ POSSESSION I 43550

CDS VIOLATION/S\&S | | 10881

CDS VIOLATION/CONSPIRACY || 2C0696

CDS VIOLATION/DISTRIBUTION || 30233

CDS VIOLATION/POSS I| 43550

CDS VIOLATION/PWI । 10573

CDS VIOLATION/TRESPASS | | 43550

CDS VIOLATIONS || 11119

CDS VIOLATIONS I| 2A0696

CDS VIOLATIONS || 2A3550

CDS VIOLATIONS || 30233

CDS VIOLATIONS || 43550

CDS VIOLATIONS || 53550

CDS VIOLATOIN || 1A1118

CDS VIOLATOIN || 2A3550

CDS VIOLATON || 10493

CDS VIOLATON || 2A0696

CDS VIOLATON I| 30233

CDS VIOLATON I। 43550

CDS VIOLATOPM ।| 11119

CDS VIOLATOR I| 30233

CDS VIOLATTION I| 2A3550

CDS VIOLOATION || 30233

CDS VIOLOATION I| 43550

CDS VIOLTAION I| 43550

CDS VIOLTATION । 43550

CDS VIOLTION || 11119

CDS VIOLTION ।| 43550

CDS VIOOATION || 43550

CDS VOI I। 43550

CDS VOI./POSS. NOT MARIJAUNA | | 43550

CDS VOILATION II 20696

CDS VOILATION I| 43550

CDS VOILATION POSS COCAINE | | 11118

CDS VOLATION II 2A0696

CDS VOLATION I| 30233

CDS VVIOLATION || 11119

CDS W/ INT I I 30233

CDS W/ INT TO DIST || 11119

CDS W/ INT TO DIST । | 30233

CDS W/ INTENT I | 10573

CDS W/ INTENT । | 11119 
CDS W/ INTENT || 11635 CDS W/ INTENT । I 20696 CDS W/ INTENT || 30233 CDS W/ INTENT II 43550 CDS W/MARIJUANA I| 43550 CDS W/I I| 2A0696 CDS W/INT | | 11119 CDS W/INT I| 43550

CDS W/INT TO DISTRIBUTION || 2A0696 CDS W/INTENT I| 10233 CDS W/INTENT I| 10573 CDS W/INTENT || 23550 CDS W/INTENT ।| 30233 CDS W/INTENT । | 43550 CDS WITH INTENT I ।

CDS WITH INTENT | | 10573 CDS WITH INTENT || 11111 CDS WITH INTENT || 11564 CDS WITH INTENT I | 30233

CDS WITH INTENT TO DISTRIBUTE || 2A0696 CDS WITH INTENT TO DISTRIBUTE || 43550 CDS WITH INTENT TO SELL I I 2A0696 CDS_DIST I| 43550

CDS- COCAINE || 43550

CDS- CONSP. TO DIST । | 20696

CDS- DIST I| 2A3550

CDS- DIST. COCAINE || 2A0696

CDS- DISTRIBUTE || 30233

CDS- DISTRIBUTION || 20696

CDS- DISTRIBUTION ।| 2A0696

CDS- HEROIN || 43550

CDS- PARAPHENELIA || 10255

CDS- POSS II 43550

CDS- POSS COCAINE/HEROIN I| 30233

CDS- POSS ECSTASY || 43550

CDS- POSS OF HERION I| 43550

CDS- POSS OF HEROIN || 2A0696

CDS- POSS OF HEROIN । | 43550

CDS- POSS W/ INTENT । । 10573

CDS- POSS W/ INTENT | | 30233

CDS- POSS. HERION || 43550

CDS- POSS. HEROIN I| 43550

CDS- POSSESS || 43550

CDS- POSSESSION I| 30233

CDS- POSSESSION I| 43550

CDS- POSSESSION OF HEROIN | | 43550

CDS- PWID II 43550

CDS- VIOLATION I। 43550

CDS-CONSPIPIRACY || 2A0696

CDS-CONSPIRACY || 20696

CDS-DIS || 2A3550

CDS-DIST || 11118

CDS-DIST || 20696

CDS-DIST || 23550

CDS-DIST || 2A0696

CDS-DIST || 2A3550

CDS-DIST || 2C0696

CDS-DIST || 30233

CDS-DIST COCAINE || 2A0696

CDS-DIST MARIJUANA || 2A3550 
CDS-DIST NARC ।

CDS-DIST OF HEROIN । | 43550

CDS-DIST-NARC I।

CDS-DIST. HEROIN I| 2C0696

CDS-DIST. NARCOTICS || 20696

CDS-DIST. OF HEROIN । | 20696

Cds-Dist., Cds Violation I| 13550

Cds-Dist., Cds-Distribute || 13550

CDS-DISTR- COCAINE || 2A0696

CDS-DISTRIBUTION || 11118

CDS-DISTRIBUTION || 1A1118

CDS-DISTRIBUTION || 2A0696

CDS-DISTRIBUTION || 2A3550

CDS-DISTRIBUTION || 2C0696

CDS-DISTRIBUTION || 43550

CDS-HEROIN || 43550

CDS-MARIJUANA-DIST. I I 2A3550

CDS-NOT MARIJUANA I | 43550

CDS-POSS I| 11119

CDS-POSS I| 20696

CDS-POSS II 43550

CDS-POSS I| 53550

CDS-POSS COCAINE ।| 1A1118

CDS-POSS HERION । 43550

CDS-POSS HEROIN II 43550

CDS-POSS OF HEROIN I। 43550

Cds-Poss W/I Del Confinement, Cds || 21097

Cds-Poss W/I Del Confinement, Cds Violation I I 21097

CDS-POSS W/INTENT I ।

CDS-POSS W/INTENT DISTRIBUTION । | 43550

CDS-POSS. BUPRENORPHINE || 43550

CDS-POSS. COCAINE II 43550

CDS-POSS. HEROIN II 43550

CDS-POSS. W/INTENT COCAINE || 20696

CDS-POSSESION || 43550

CDS-POSSESION HERION I। 43550

CDS-POSSESS I| 43550

CDS-POSSESSION || 43550

CDS-POSSESSION HEROIN || 30233

CDS-POSSESSOIN || 43550

CDS-PWI I| 10233

CDS-PWI I| 2A0696

CDS-PWI I| 30233

CDS-PWID || 10233

CDS-PWID || 10573

CDS-PWID || 11111

CDS-PWID || 11119

CDS-PWID || 20696

CDS-PWID || 23550

CDS-PWID || 2A0696

CDS-PWID || 30233

CDS-PWID || 43550

CDS-PWID (COCAINE) | | 11119

CDS-PWID HEROIN || 2C0696

CDS-PWID HEROIN I| 30233

CDS-PWID MARJUANA || 43550

CDS-PWID-HEROIN || 43550

CDS-PWID-MARIJUANA | | 10573

CDS-VIOLATION || 10233

CDS-VIOLATION || 11119 
CDS-VIOLATION || 30233

CDS: ATT DIST (COCAINE) || 2A0696

CDS: ATTEMPT DISTRO || 1A1118

CDS: DIST (HEROIN) I| 2A0696

CDS: DIST-HEROIN || 30233

CDS: DIST-MARIJUANA || 10233

CDS: DIST-PCP/LSD || 10696

CDS: DISTRIBUTION || 11111

CDS: DISTRIBUTION || 20696

CDS: DISTRIBUTION || 23550

CDS: DISTRIBUTION || 2A0696

CDS: DISTRIBUTION || 30233

CDS: DISTRO || 10881

CDS: DISTRO || 11111

CDS: DISTRO I| 11118

CDS: DISTRO || 1A1118

CDS: DISTRO || 1C1118

CDS: DISTRO || 2A0696

CDS: PARA.(NOT MARIJ) I| 53550

CDS: PARAPHERNALIA || 53550

CDS: POSESS COCAINE I| 43550

CDS: POSS II 43550

CDS: POSS (COCAINE) I| 43550

CDS: POSS COCAINE || 43550

CDS: POSS COCAINE/MARIJUANA || 43550

CDS: POSS HEROIN / COCAINE I | 43550

CDS: POSS INTENT || 11119

Cds: Poss Marihuana L/T 10 G, Att Dist Heroin || 11635

Cds: Poss Marihuana L/T 10 G, Cds Dist I | 11635

Cds: Poss Marihuana L/T 10 G, Cds Distribution I| 11635

Cds: Poss Marihuana L/T 10 G, Cds Poss W/Int | | 11635

Cds: Poss Marihuana L/T 10 G, Cds Poss With Intent || 11635

Cds: Poss Marihuana L/T 10 G, Cds Pwid II 11635

Cds: Poss Marihuana L/T 10 G, Distribution Marijuan II 11635

Cds: Poss Marihuana L/T 10 G, P/W/I Marijuana II 11635

Cds: Poss Marihuana L/T 10 G, Poss W/I Cds II 11635

Cds: Poss Marihuana L/T 10 G, Poss W/I Marijuana II 11635

Cds: Poss Marihuana L/T 10 G, Poss W/Int Marijuana || 11635

Cds: Poss Marihuana L/T 10 G, Poss With Intent Marij I| 11635

Cds: Poss Marihuana L/T 10 G, Poss/W/Intent I | 11635

Cds: Poss Marihuana L/T 10 G, Pwid Cocaine II 11635

Cds: Poss Marihuana L/T 10 G, Pwid Heroin I| 11635

Cds: Poss Marihuana L/T 10 G, Pwid Marijuana I| 11635

CDS: POSS OF COCAINE II 43550

CDS: POSS PARAPHERNALIA || 53550

CDS: POSS W/ INT I| 30233

CDS: POSS W/I DIST: NARC || 11119

CDS: POSS W/I DIST:NARC I |

CDS: POSS W/INT || 10573

CDS: POSS W/INTENT DIST: NARC । ।

CDS: POSS-HEROIN II 43550

CDS: POSS-W/INT. I। 10233

CDS: POSS. COCAINE || 30233

CDS: POSS. COCAINE || 43550

CDS: POSS. W/ INTENT I। 43550

CDS: POSS. W/INT. II 10233

CDS: POSSESION HERION || 43550

CDS: POSSESION-NOT MARIJUANA | | 43550

CDS: POSSESS COCAINE I| 43550

CDS: POSSESSION || 30233 
CDS: POSSESSION || 43550

CDS: POSSESSION NOT MARIJUANA || 2A0696

CDS: POSSESSION NOT MARIJUANA || 43550

CDS: POSSESSION WITH INTENT NO || 30233

CDS: POSSESSION- NOT MARIJUANA || 11118

Cds: Possession-Marihuana, Att Distribution Marijuan I| 10573

Cds: Possession-Marihuana, Att. Cds Distribution I| 10573

Cds: Possession-Marihuana, Attempt Dist I| 10573

Cds: Possession-Marihuana, Cds Dist I | 10573

Cds: Possession-Marihuana, Cds Distribution II 10573

Cds: Possession-Marihuana, Cds Poss W/I Dist Marijua II 10573

Cds: Possession-Marihuana, Cds Poss W/I Marijuana || 10573

Cds: Possession-Marihuana, Cds Poss W/Int || 10573

Cds: Possession-Marihuana, Cds Poss W/Int Dist II 10573

Cds: Possession-Marihuana, Cds Poss W/Int Marijuana I| 10573

Cds: Possession-Marihuana, Cds Poss W/Intent || 10573

Cds: Possession-Marihuana, Cds Poss With Intent || 10573

Cds: Possession-Marihuana, Cds Possession W/Int || 10573

Cds: Possession-Marihuana, Cds Pwid II 10573

Cds: Possession-Marihuana, Cds Pwid Heroin II 10573

Cds: Possession-Marihuana, Cds Pwid Mairjuana || 10573

Cds: Possession-Marihuana, Cds Pwid Marijuana I| 10573

Cds: Possession-Marihuana, Cds W/Int || 10573

Cds: Possession-Marihuana, Cds W/Int Marijuana || 10573

Cds: Possession-Marihuana, Cds With Intent To Distri || 10573

Cds: Possession-Marihuana, Cds-Pwid II 10573

Cds: Possession-Marihuana, Conspiracy To Dist || 10573

Cds: Possession-Marihuana, Dist Marijuana || 10573

Cds: Possession-Marihuana, Dist Of Marijuana II 10573

Cds: Possession-Marihuana, Dist W/Int Heroin I| 10573

Cds: Possession-Marihuana, Dist. Marijuana || 10573

Cds: Possession-Marihuana, Dist.Heroin II 10573

Cds: Possession-Marihuana, Distribution Marijuana I| 10573

Cds: Possession-Marihuana, Distribution Of Marijuana I| 10573

Cds: Possession-Marihuana, Marijuana Distribution || 10573

Cds: Possession-Marihuana, Marijuana Pwid II 10573

Cds: Possession-Marihuana, Poss Cds W/Intent I| 10573

Cds: Possession-Marihuana, Poss W/I - Marijuana || 10573

Cds: Possession-Marihuana, Poss W/I Marijuana || 10573

Cds: Possession-Marihuana, Poss W/Int || 10573

Cds: Possession-Marihuana, Poss W/Int Dis Cocaine I| 10573

Cds: Possession-Marihuana, Poss W/Int Marijauna || 10573

Cds: Possession-Marihuana, Poss W/Int Marijuana || 10573

Cds: Possession-Marihuana, Poss W/Int Mariuana I| 10573

Cds: Possession-Marihuana, Poss W/Int. Dist. I| 10573

Cds: Possession-Marihuana, Poss W/Intent Marijuana || 10573

Cds: Possession-Marihuana, Poss W/Intent Marj I I 10573

Cds: Possession-Marihuana, Poss With Intent Marijuan I| 10573

Cds: Possession-Marihuana, Poss. Cds W/Intent || 10573

Cds: Possession-Marihuana, Poss. W/I Cocaine || 10573

Cds: Possession-Marihuana, Poss. W/I Marijuana || 10573

Cds: Possession-Marihuana, Poss. W/Int Marijuana II 10573

Cds: Possession-Marihuana, Poss. W/Int. Marijuana || 10573

Cds: Possession-Marihuana, Poss. With Intent To Dist || 10573

Cds: Possession-Marihuana, Possesion W/Int Marijuana II 10573

Cds: Possession-Marihuana, Possession W/Int Marijuan I| 10573

Cds: Possession-Marihuana, Possession W/Intent Marij || 10573

Cds: Possession-Marihuana, Possession With Intent || 10573

Cds: Possession-Marihuana, Pwid II 10573

Cds: Possession-Marihuana, Pwid Cds II 10573 
Cds: Possession-Marihuana, Pwid Cocaine || 10573

Cds: Possession-Marihuana, Pwid Marijajuana || 10573

Cds: Possession-Marihuana, Pwid Marijuana I| 10573

Cds: Possession-Marihuana, Pwid Mj I| 10573

Cds: Possession-Marihuana, Pwid Of Marijuana || 10573

CDS: PWI COCAINE / HEROIN | | 30233

CDS: PWID || 10881

CDS: PWID || 11111

CDS: PWID || 11118

CDS: PWID || 11119

CDS: PWID || 1A1118

CDS: PWID || 30233

CDS: PWID (COCAINE) || 30233

CDS: PWID (COCAINE/HEROIN) | | 43550

CDS: PWID COCAINE || 11119

CDS:COM NUIS: DIST. NARC || 20330

CDS:DIST || 1A1118

CDS:DIST (COCAINE) || 2A0696

CDS:DIST-NARC I |

CDS:IMPORT INTO STATE || 33550

Cds:P W/I Dist: Schl Bus/Prop, Cds Violation I। 10484

CDS:P W/I DIST:NARC I 30233

Cds:P W/I Dist:Narc, B And E/ Cds Violation II 30233

Cds:P W/I Dist:Narc, Cds I| 30233

Cds:P W/I Dist:Narc, Cds Poss W/I || 30233

Cds:P W/I Dist:Narc, Cds Poss. I I 30233

Cds:P W/I Dist:Narc, Cds Pwid || 30233

Cds:P W/I Dist:Narc, Cds Cocaine || 30233

Cds:P W/I Dist:Narc, Cds Cocaine Pwid | | 30233

Cds:P W/I Dist:Narc, Cds Dist I I 30233

Cds:P W/I Dist:Narc, Cds Dist And Possession I| 30233

Cds:P W/I Dist:Narc, Cds Dist Herion || 30233

Cds:P W/I Dist:Narc, Cds Dist Heroin I | 30233

Cds:P W/I Dist:Narc, Cds Dist Marijuana I| 30233

Cds:P W/I Dist:Narc, Cds Dist/Heroin I I 30233

Cds:P W/I Dist:Narc, Cds Distribution I | 30233

Cds:P W/I Dist:Narc, Cds Distrubution Cocaine I| 30233

Cds:P W/I Dist:Narc, Cds Herion || 30233

Cds:P W/I Dist:Narc, Cds Intent To Distribute | | 30233

Cds:P W/I Dist:Narc, Cds Manufacturing/Distribution I| 30233

Cds:P W/I Dist:Narc, Cds Narc Pwi I I 30233

Cds:P W/I Dist:Narc, Cds Not Maj || 30233

Cds:P W/I Dist:Narc, Cds Not Marijuana || 30233

Cds:P W/I Dist:Narc, Cds P.W.I.D. I I 30233

Cds:P W/I Dist:Narc, Cds P/W/I II 30233

Cds:P W/I Dist:Narc, Cds Pcss W/ Intent || 30233

Cds:P W/I Dist:Narc, Cds Poss I | 30233

Cds:P W/I Dist:Narc, Cds Poss Cocaine I| 30233

Cds:P W/I Dist:Narc, Cds Poss Cocaine/Heroin I| 30233

Cds:P W/I Dist:Narc, Cds Poss Heroin I | 30233

Cds:P W/I Dist:Narc, Cds Poss Heroin/Cocaine I| 30233

Cds:P W/I Dist:Narc, Cds Poss Marijuana || 30233

Cds:P W/I Dist:Narc, Cds Poss W Intent I | 30233

Cds:P W/I Dist:Narc, Cds Poss W Intent Heroin I I 30233

Cds:P W/I Dist:Narc, Cds Poss W/ In I 30233

Cds:P W/I Dist:Narc, Cds Poss W/ Int I| 30233

Cds:P W/I Dist:Narc, Cds Poss W/ Intent || 30233

Cds:P W/I Dist:Narc, Cds Poss W/I I | 30233

Cds:P W/I Dist:Narc, Cds Poss W/I Cocaine I | 30233

Cds:P W/I Dist:Narc, Cds Poss W/I/Dist I | 30233 
Cds:P W/I Dist:Narc, Cds Poss W/Int || 30233

Cds:P W/I Dist:Narc, Cds Poss W/Int Cocaine I| 30233

Cds:P W/I Dist:Narc, Cds Poss W/Intent I | 30233

Cds:P W/I Dist:Narc, Cds Poss W/Intent -Cocaine I| 30233

Cds:P W/I Dist:Narc, Cds Poss With Int || 30233

Cds:P W/I Dist:Narc, Cds Poss With Intent I| 30233

Cds:P W/I Dist:Narc, Cds Poss With Intent Dist I| 30233

Cds:P W/I Dist:Narc, Cds Poss With Intent Heroin I| 30233

Cds:P W/I Dist:Narc, Cds Poss.- Dist. || 30233

Cds:P W/I Dist:Narc, Cds Poss/ Int I| 30233

Cds:P W/I Dist:Narc, Cds Possesion I | 30233

Cds:P W/I Dist:Narc, Cds Possession || 30233

Cds:P W/I Dist:Narc, Cds Possession Cocaine || 30233

Cds:P W/I Dist:Narc, Cds Possession Heroin || 30233

Cds:P W/I Dist:Narc, Cds Possession W/ Intent I| 30233

Cds:P W/I Dist:Narc, Cds Possession W/ Intent Cocai || 30233

Cds:P W/I Dist:Narc, Cds Possession W/Intent I| 30233

Cds:P W/I Dist:Narc, Cds Possession With Intent I| 30233

Cds:P W/I Dist:Narc, Cds Possession With Intent Her || 30233

Cds:P W/I Dist:Narc, Cds Possession With Intent To || 30233

Cds:P W/I Dist:Narc, Cds Pwi I I 30233

Cds:P W/I Dist:Narc, Cds Pwi Cocaine I| 30233

Cds:P W/I Dist:Narc, Cds Pwi Heroin || 30233

Cds:P W/I Dist:Narc, Cds Pwi/ Disorderly || 30233

Cds:P W/I Dist:Narc, Cds Pwid || 30233

Cds:P W/I Dist:Narc, Cds Pwid Cocaine I| 30233

Cds:P W/I Dist:Narc, Cds Pwid Crack Cocaine || 30233

Cds:P W/I Dist:Narc, Cds Pwid Dist || 30233

Cds:P W/I Dist:Narc, Cds Pwid Heroin I | 30233

Cds:P W/I Dist:Narc, Cds Pwid Poss I| 30233

Cds:P W/I Dist:Narc, Cds Violation I 30233

Cds:P W/I Dist:Narc, Cds Violation Dist Heroin || 30233

Cds:P W/I Dist:Narc, Cds Violation Poss W/Int Cocai I| 30233

Cds:P W/I Dist:Narc, Cds Violation W/ Int II 30233

Cds:P W/I Dist:Narc, Cds Violations || 30233

Cds:P W/I Dist:Narc, Cds Violatoin I I 30233

Cds:P W/I Dist:Narc, Cds Violtion I I 30233

Cds:P W/I Dist:Narc, Cds W/ Intent I| 30233

Cds:P W/I Dist:Narc, Cds Wit I | 30233

Cds:P W/I Dist:Narc, Cds With Intent To Distribute I| 30233

Cds:P W/I Dist:Narc, Cds- Pwid I I 30233

Cds:P W/I Dist:Narc, Cds-Distribution || 30233

Cds:P W/I Dist:Narc, Cds-Possess W/ Intent || 30233

Cds:P W/I Dist:Narc, Cds-Possession-Cocaine II 30233

Cds:P W/I Dist:Narc, Cds-Pwid II 30233

Cds:P W/I Dist:Narc, Cds: Poss W/ Int Distr || 30233

Cds:P W/I Dist:Narc, Cds: Poss. I 30233

Cds:P W/I Dist:Narc, Cds:P W/I Dist:Narc II 30233

Cds:P W/I Dist:Narc, Cds:Poss Heroin || 30233

Cds:P W/I Dist:Narc, Cds:Poss W/Intent Dist: Narc || 30233

Cds:P W/I Dist:Narc, Cdsw Pwi Cocaine II 30233

Cds:P W/I Dist:Narc, Cdsw/Intent I| 30233

Cds:P W/I Dist:Narc, Cocaine Distribution I| 30233

Cds:P W/I Dist:Narc, Cocaine Pwid I| 30233

Cds:P W/I Dist:Narc, Con Poss W/I Cds II 30233

Cds:P W/I Dist:Narc, Consp Cds I I 30233

Cds:P W/I Dist:Narc, Consp To Dist I 30233

Cds:P W/I Dist:Narc, Consp. Dist Cds || 30233

Cds:P W/I Dist:Narc, Consp. Dist Cocaine I| 30233

Cds:P W/I Dist:Narc, Consp. Dist. Heroin I| 30233 
Cds:P W/I Dist:Narc, Consp.Dist.Herion I 30233

Cds:P W/I Dist:Narc, Conspi Distribution Marijuana || 30233

Cds:P W/I Dist:Narc, Conspiracy | | 30233

Cds:P W/I Dist:Narc, Conspiracy Distribute Cocaine || 30233

Cds:P W/I Dist:Narc, Conspiracy To Distribute I| 30233

Cds:P W/I Dist:Narc, Conspiracy To Distribute Cocai I| 30233

Cds:P W/I Dist:Narc, Dist (Consp) Heroin | | 30233

Cds:P W/I Dist:Narc, Dist Cds I| 30233

Cds:P W/I Dist:Narc, Dist Cocaine || 30233

Cds:P W/I Dist:Narc, Dist Heroin || 30233

Cds:P W/I Dist:Narc, Dist Marijuana II 30233

Cds:P W/I Dist:Narc, Dist Of Cocaine || 30233

Cds:P W/I Dist:Narc, Dist Of Heroin || 30233

Cds:P W/I Dist:Narc, Dist. Cocaine I| 30233

Cds:P W/I Dist:Narc, Dist. Herion || 30233

Cds:P W/I Dist:Narc, Dist. Heroin || 30233

Cds:P W/I Dist:Narc, Dist.- Heroin I I 30233

Cds:P W/I Dist:Narc, Distibution Cocaine I| 30233

Cds:P W/I Dist:Narc, Distribution I 30233

Cds:P W/I Dist:Narc, Distribution Heroin I| 30233

Cds:P W/I Dist:Narc, Distribution Of Heroin I I 30233

Cds:P W/I Dist:Narc, Distribution Cds I | 30233

Cds:P W/I Dist:Narc, Distribution Cocaine || 30233

Cds:P W/I Dist:Narc, Distribution Heroin || 30233

Cds:P W/I Dist:Narc, Distribution Of Cocaine I| 30233

Cds:P W/I Dist:Narc, Distribution Of Heroin I| 30233

Cds:P W/I Dist:Narc, Heroin Distribution I | 30233

Cds:P W/I Dist:Narc, Heroin Poss W/ Intent || 30233

Cds:P W/I Dist:Narc, Narcotics I | 30233

Cds:P W/I Dist:Narc, P.W.I.D. I 30233

Cds:P W/I Dist:Narc, P/W/I Cocaine || 30233

Cds:P W/I Dist:Narc, P/W/I Heroin I| 30233

Cds:P W/I Dist:Narc, Poss II 30233

Cds:P W/I Dist:Narc, Poss Cds I| 30233

Cds:P W/I Dist:Narc, Poss Cocaine || 30233

Cds:P W/I Dist:Narc, Poss Cocaine W Int I | 30233

Cds:P W/I Dist:Narc, Poss Heroin I| 30233

Cds:P W/I Dist:Narc, Poss Intent Marijuana || 30233

Cds:P W/I Dist:Narc, Poss Mdma || 30233

Cds:P W/I Dist:Narc, Poss Of Cds || 30233

Cds:P W/I Dist:Narc, Poss Of Cocaine || 30233

Cds:P W/I Dist:Narc, Poss Of Heroin I | 30233

Cds:P W/I Dist:Narc, Poss Oxycodone I| 30233

Cds:P W/I Dist:Narc, Poss W Int Cds I| 30233

Cds:P W/I Dist:Narc, Poss W. Int || 30233

Cds:P W/I Dist:Narc, Poss W/ I | 30233

Cds:P W/I Dist:Narc, Poss W/ Cds || 30233

Cds:P W/I Dist:Narc, Poss W/ Cocaine II 30233

Cds:P W/I Dist:Narc, Poss W/ Heroin I | 30233

Cds:P W/I Dist:Narc, Poss W/ Int || 30233

Cds:P W/I Dist:Narc, Poss W/ Int Cocaine I| 30233

Cds:P W/I Dist:Narc, Poss W/ Intent I | 30233

Cds:P W/I Dist:Narc, Poss W/ Intent Dist I | 30233

Cds:P W/I Dist:Narc, Poss W/Cds I| 30233

Cds:P W/I Dist:Narc, Poss W/Cocaine I| 30233

Cds:P W/I Dist:Narc, Poss W/I Heroin I| 30233

Cds:P W/I Dist:Narc, Poss W/I Cocain || 30233

Cds:P W/I Dist:Narc, Poss W/I Cocaine || 30233

Cds:P W/I Dist:Narc, Poss W/I Dist Cocaine II 30233

Cds:P W/I Dist:Narc, Poss W/I Heroin I| 30233 
Cds:P W/I Dist:Narc, Poss W/I Heron || 30233

Cds:P W/I Dist:Narc, Poss W/I Marijuana || 30233

Cds:P W/I Dist:Narc, Poss W/I To Distribute I 30233

Cds:P W/I Dist:Narc, Poss W/Int Not Marijuana II 30233

Cds:P W/I Dist:Narc, Poss W/Int Cocaine I| 30233

Cds:P W/I Dist:Narc, Poss W/Int Dist I I 30233

Cds:P W/I Dist:Narc, Poss W/Int Dist Cocaine I | 30233

Cds:P W/I Dist:Narc, Poss W/Int Heroin I | 30233

Cds:P W/I Dist:Narc, Poss W/Int Heroin Cocaine || 30233

Cds:P W/I Dist:Narc, Poss W/Int Marijuana II 30233

Cds:P W/I Dist:Narc, Poss W/Intent | 30233

Cds:P W/I Dist:Narc, Poss W/Intent Cocaine || 30233

Cds:P W/I Dist:Narc, Poss W/Intent Herion I I 30233

Cds:P W/I Dist:Narc, Poss W/Intent Heroin I I 30233

Cds:P W/I Dist:Narc, Poss W/Intent Marijuana I| 30233

Cds:P W/I Dist:Narc, Poss W/Intent To Distribute Cd || 30233

Cds:P W/I Dist:Narc, Poss With Intent Cocaine || 30233

Cds:P W/I Dist:Narc, Poss With Intent Heroin I | 30233

Cds:P W/I Dist:Narc, Poss With The Intent I| 30233

Cds:P W/I Dist:Narc, Poss. I I 30233

Cds:P W/I Dist:Narc, Poss. Cocaine W/Int | | 30233

Cds:P W/I Dist:Narc, Poss. Cocaine W/Int Dist I I 30233

Cds:P W/I Dist:Narc, Poss. Cocaine With Intent I | 30233

Cds:P W/I Dist:Narc, Poss. Heroin || 30233

Cds:P W/I Dist:Narc, Poss. Of Cds || 30233

Cds:P W/I Dist:Narc, Poss. W.I Heroin I | 30233

Cds:P W/I Dist:Narc, Poss. W/ Intent To Dist. I I 30233

Cds:P W/I Dist:Narc, Poss. W/I Heroin I| 30233

Cds:P W/I Dist:Narc, Poss. W/Int | | 30233

Cds:P W/I Dist:Narc, Poss. W/Int Cocaine I| 30233

Cds:P W/I Dist:Narc, Poss. W/Int. Cocaine || 30233

Cds:P W/I Dist:Narc, Poss./W/Int.Dist.Cocaine || 30233

Cds:P W/I Dist:Narc, Poss.W/Int Dist Heroin I| 30233

Cds:P W/I Dist:Narc, Poss.W/Int.Dist.Cocaine || 30233

Cds:P W/I Dist:Narc, Poss/ Dist Cds II 30233

Cds:P W/I Dist:Narc, Poss/W/Intent Heroin I | 30233

Cds:P W/I Dist:Narc, Possesion W/ Intent | | 30233

Cds:P W/I Dist:Narc, Possesion W/Intent Cds || 30233

Cds:P W/I Dist:Narc, Possess With Intent To Distrib || 30233

Cds:P W/I Dist:Narc, Possession Cds || 30233

Cds:P W/I Dist:Narc, Possession Cocaine || 30233

Cds:P W/I Dist:Narc, Possession Cocaine/Herion I| 30233

Cds:P W/I Dist:Narc, Possession Heroin I| 30233

Cds:P W/I Dist:Narc, Possession Marijuana II 30233

Cds:P W/I Dist:Narc, Possession Not Marijuana || 30233

Cds:P W/I Dist:Narc, Possession Of Cocaine II 30233

Cds:P W/I Dist:Narc, Possession Of Heroin I| 30233

Cds:P W/I Dist:Narc, Possession W Intent I| 30233

Cds:P W/I Dist:Narc, Possession W/ Int Cocaine I| 30233

Cds:P W/I Dist:Narc, Possession W/I Cocaine I| 30233

Cds:P W/I Dist:Narc, Possession W/I Distr Cds || 30233

Cds:P W/I Dist:Narc, Possession W/I Heroin I I 30233

Cds:P W/I Dist:Narc, Possession With Intent I | 30233

Cds:P W/I Dist:Narc, Possession With Intent Cds I| 30233

Cds:P W/I Dist:Narc, Possession With Intent Heroin I | 30233

Cds:P W/I Dist:Narc, Possession With Intent To Dist I| 30233

Cds:P W/I Dist:Narc, Possession/Intent-Cocaine || 30233

Cds:P W/I Dist:Narc, Possw/ Intent Heroin I 30233

Cds:P W/I Dist:Narc, Pwi || 30233

Cds:P W/I Dist:Narc, Pwi Cds || 30233 
Cds:P W/I Dist:Narc, Pwi Cds Heroin || 30233

Cds:P W/I Dist:Narc, Pwi Cocaine I| 30233

Cds:P W/I Dist:Narc, Pwi Cocaine \& Marijuana || 30233

Cds:P W/I Dist:Narc, Pwi Herion I I 30233

Cds:P W/I Dist:Narc, Pwi Heroin || 30233

Cds:P W/I Dist:Narc, Pwi Marijuana || 30233

Cds:P W/I Dist:Narc, Pwi Marijuana/ Herion I| 30233

Cds:P W/I Dist:Narc, Pwi. Cds I| 30233

Cds:P W/I Dist:Narc, Pwi/Cds || 30233

Cds:P W/I Dist:Narc, Pwi/Herion/ Cocaine I| 30233

Cds:P W/I Dist:Narc, Pwid I 30233

Cds:P W/I Dist:Narc, Pwid Cds I| 30233

Cds:P W/I Dist:Narc, Pwid Cocaine || 30233

Cds:P W/I Dist:Narc, Pwid Herion || 30233

Cds:P W/I Dist:Narc, Pwid Heroin I 30233

Cds:P W/I Dist:Narc, Pwid-Cocaine || 30233

Cds:P W/I Dist:Narc, Pwid-Heroin I| 30233

Cds:P W/I Dist:Narc, Pwit || 30233

Cds:P W/I Dist:Narc, Rogue And Vagabond I | 30233

CDS:POS W/I DIST:PCP/LSD || 20233

Cds:Pos W/I Dist:Pcp/Lsd, Cds || 20233

Cds:Pos W/I Dist:Pcp/Lsd, Cds Violation II 20233

Cds:Pos W/I Dist:Pcp/Lsd, Cds:Pos W/I Dist:Pcp/Lsd I| 20233

Cds:Pos W/I Dist:Pcp/Lsd, Poss Cds I| 20233

CDS:POSS I| 30233

CDS:POSS (COCAINE) I| 43550

CDS:POSS COCAINE || 43550

CDS:POSS HEROIN || 43550

Cds:Poss Para, Cds I| 53550

Cds:Poss Para, Cds - Violation I| 53550

Cds:Poss Para, Cds .Poss I| 53550

Cds:Poss Para, Cds Cocaine II 53550

Cds:Poss Para, Cds Dist. II 53550

Cds:Poss Para, Cds Not Marijuana II 53550

Cds:Poss Para, Cds Par I| 53550

Cds:Poss Para, Cds Para II 53550

Cds:Poss Para, Cds Paraphenalia/Possession I| 53550

Cds:Poss Para, Cds Paraphenelia I| 53550

Cds:Poss Para, Cds Paraphenilia || 53550

Cds:Poss Para, Cds Paraphernalia I| 53550

Cds:Poss Para, Cds Pern. II 53550

Cds:Poss Para, Cds Pos Cocaine || 53550

Cds:Poss Para, Cds Poss II 53550

Cds:Poss Para, Cds Poss Cocaine I| 53550

Cds:Poss Para, Cds Poss Heroin || 53550

Cds:Poss Para, Cds Poss Not Marij I 53550

Cds:Poss Para, Cds Poss Paraphernalia || 53550

Cds:Poss Para, Cds Poss W/Intent Herion I| 53550

Cds:Poss Para, Cds Poss. I| 53550

Cds:Poss Para, Cds Possesion || 53550

Cds:Poss Para, Cds Possession || 53550

Cds:Poss Para, Cds Possession Marijuana I| 53550

Cds:Poss Para, Cds Pwid II 53550

Cds:Poss Para, Cds Pwid Heroin II 53550

Cds:Poss Para, Cds Viol I| 53550

Cds:Poss Para, Cds Violation II 53550

Cds:Poss Para, Cds Violation Poss Marijuana || 53550

Cds:Poss Para, Cds Violation Poss Of Marij II 53550

Cds:Poss Para, Cds Violaton II 53550

Cds:Poss Para, Cds Voilation || 53550 
Cds:Poss Para, Cds- Posession || 53550

Cds:Poss Para, Cds-Paraphernalia || 53550

Cds:Poss Para, Cds-Pwid I| 53550

Cds:Poss Para, Cds: Poss II 53550

Cds:Poss Para, Cds:Poss Paraphernalia || 53550

Cds:Poss Para, Dist. Cds || 53550

Cds:Poss Para, Distribution I| 53550

Cds:Poss Para, Drving On Suspended License I| 53550

Cds:Poss Para, Paraphilla || 53550

Cds:Poss Para, Poss Cds II 53550

Cds:Poss Para, Poss Cds Paraphernalia I| 53550

Cds:Poss Para, Poss Cocaine I| 53550

Cds:Poss Para, Poss Heroin || 53550

Cds:Poss Para, Poss Marijuana I| 53550

Cds:Poss Para, Poss Of Cocaine I| 53550

Cds:Poss Para, Poss Of Heroin || 53550

Cds:Poss Para, Poss Of Marijuana I| 53550

Cds:Poss Para, Poss Of Parapher. I 53550

Cds:Poss Para, Poss Of Paraphernalia || 53550

Cds:Poss Para, Poss Paraphernalia || 53550

Cds:Poss Para, Poss Suboxone I 53550

Cds:Poss Para, Poss. Cds II 53550

Cds:Poss Para, Poss. Cocaine I| 53550

Cds:Poss Para, Poss. Marijuana || 53550

Cds:Poss Para, Poss. Of Marijuana I| 53550

Cds:Poss Para, Poss. W/Int Dist. Marihuana I| 53550

Cds:Poss Para, Poss. With Int Marijuana I| 53550

Cds:Poss Para, Possesion Of Heroin I| 53550

Cds:Poss Para, Possession Cocaine || 53550

Cds:Poss Para, Possession Marijuana || 53550

Cds:Poss Para, Possession Not Marijuana || 53550

Cds:Poss Para, Possession Of Heroin II 53550

Cds:Poss Para, Possession Of Para || 53550

Cds:Poss Para, Possession Of Paraphernalia I| 53550

Cds:Poss Para, Possession Paraphenilia || 53550

Cds:Poss Para, Pwi Marijuana II 53550

Cds:Poss Para, Pwid Cocaine || 53550

Cds:Poss Para, Tresspassing || 53550

CDS:POSS PARAPHERNALIA || 53550

CDS:POSS W/INTENT DIST: NARC I 30233

CDS:POSS. II 43550

Cds:Possess-Not Marihuana, 4Th Degree B\&G || 43550

Cds:Possess-Not Marihuana, Alcohol Violation I| 43550

Cds:Possess-Not Marihuana, Att Dist Of Cocaine II 43550

Cds:Possess-Not Marihuana, Att Dist. Heroin I| 43550

Cds:Possess-Not Marihuana, Att Distribution Cocaine I| 43550

Cds:Possess-Not Marihuana, Att. Cds-Dist. I | 43550

Cds:Possess-Not Marihuana, Att. Dist Cocaine II 43550

Cds:Possess-Not Marihuana, Att. Dist Of Heroin I| 43550

Cds:Possess-Not Marihuana, B And E I| 43550

Cds:Possess-Not Marihuana, C.D.S. Possession I| 43550

Cds:Possess-Not Marihuana, C.D.S. Possession Not Ma I| 43550

Cds:Possess-Not Marihuana, C.D.S. Violation II 43550

Cds:Possess-Not Marihuana, Ccds Violation I| 43550

Cds:Possess-Not Marihuana, Cd Violation II 43550

Cds:Possess-Not Marihuana, Cds II 43550

Cds:Possess-Not Marihuana, Cds Distribution I| 43550

Cds:Possess-Not Marihuana, Cds Poss II 43550

Cds:Possess-Not Marihuana, Cds Poss With Intent I| 43550

Cds:Possess-Not Marihuana, Cds Poss. I| 43550 
Cds:Possess-Not Marihuana, Cds Possession I| 43550

Cds:Possess-Not Marihuana, Cds Pwid I| 43550

Cds:Possess-Not Marihuana, Cds Viol. II 43550

Cds:Possess-Not Marihuana, Cds Violation II 43550

Cds:Possess-Not Marihuana, Cds - Int Dist I| 43550

Cds:Possess-Not Marihuana, Cds - Poss II 43550

Cds:Possess-Not Marihuana, Cds - Poss Cocaine I| 43550

Cds:Possess-Not Marihuana, Cds - Poss Heroin I| 43550

Cds:Possess-Not Marihuana, Cds - Poss Not Marihuana I| 43550

Cds:Possess-Not Marihuana, Cds - Possession II 43550

Cds:Possess-Not Marihuana, Cds -Poss Herion II 43550

Cds:Possess-Not Marihuana, Cds /Poss I| 43550

Cds:Possess-Not Marihuana, Cds \& Marijuana I| 43550

Cds:Possess-Not Marihuana, Cds Alprazolam I 43550

Cds:Possess-Not Marihuana, Cds Att. Dist. I I 43550

Cds:Possess-Not Marihuana, Cds Attempt Dist I| 43550

Cds:Possess-Not Marihuana, Cds Attempt To Distribute || 43550

Cds:Possess-Not Marihuana, Cds Cocaine I| 43550

Cds:Possess-Not Marihuana, Cds Consp. To Distribute I| 43550

Cds:Possess-Not Marihuana, Cds Cviolation II 43550

Cds:Possess-Not Marihuana, Cds Dist I | 43550

Cds:Possess-Not Marihuana, Cds Dist Cocaine II 43550

Cds:Possess-Not Marihuana, Cds Dist Heroin I| 43550

Cds:Possess-Not Marihuana, Cds Dist. I | 43550

Cds:Possess-Not Marihuana, Cds Dist. Cocaine I| 43550

Cds:Possess-Not Marihuana, Cds Dist/Pwid/Poss II 43550

Cds:Possess-Not Marihuana, Cds Distr I| 43550

Cds:Possess-Not Marihuana, Cds Distribution I | 43550

Cds:Possess-Not Marihuana, Cds Distrubition I | 43550

Cds:Possess-Not Marihuana, Cds Herion I| 43550

Cds:Possess-Not Marihuana, Cds Heroin I| 43550

Cds:Possess-Not Marihuana, Cds Methadone I| 43550

Cds:Possess-Not Marihuana, Cds Narcotics I| 43550

Cds:Possess-Not Marihuana, Cds Not Marijuana II 43550

Cds:Possess-Not Marihuana, Cds Oxycodone II 43550

Cds:Possess-Not Marihuana, Cds Paraphanalia II 43550

Cds:Possess-Not Marihuana, Cds Paraphenila || 43550

Cds:Possess-Not Marihuana, Cds Pos I| 43550

Cds:Possess-Not Marihuana, Cds Posession II 43550

Cds:Possess-Not Marihuana, Cds Poss I| 43550

Cds:Possess-Not Marihuana, Cds Poss - Cocaine II 43550

Cds:Possess-Not Marihuana, Cds Poss - Not Marijuana I| 43550

Cds:Possess-Not Marihuana, Cds Poss ( Not Marijuana I| 43550

Cds:Possess-Not Marihuana, Cds Poss (Heroin) I। 43550

Cds:Possess-Not Marihuana, Cds Poss (Not Marijuana) II 43550

Cds:Possess-Not Marihuana, Cds Poss /W Intent I| 43550

Cds:Possess-Not Marihuana, Cds Poss Clonazepam I| 43550

Cds:Possess-Not Marihuana, Cds Poss Cocaina II 43550

Cds:Possess-Not Marihuana, Cds Poss Cocaine I| 43550

Cds:Possess-Not Marihuana, Cds Poss Cocanie I| 43550

Cds:Possess-Not Marihuana, Cds Poss Cociane II 43550

Cds:Possess-Not Marihuana, Cds Poss Cocsine I| 43550

Cds:Possess-Not Marihuana, Cds Poss Crack Cocaine An || 43550

Cds:Possess-Not Marihuana, Cds Poss Herion I| 43550

Cds:Possess-Not Marihuana, Cds Poss Heroin II 43550

Cds:Possess-Not Marihuana, Cds Poss Heroin/Cocaine I| 43550

Cds:Possess-Not Marihuana, Cds Poss Heron I| 43550

Cds:Possess-Not Marihuana, Cds Poss Mari I| 43550

Cds:Possess-Not Marihuana, Cds Poss Marijuana II 43550

Cds:Possess-Not Marihuana, Cds Poss Mdma || 43550 
Cds:Possess-Not Marihuana, Cds Poss No Marijuana || 43550

Cds:Possess-Not Marihuana, Cds Poss Not Maijuana I| 43550

Cds:Possess-Not Marihuana, Cds Poss Not Marihuana I| 43550

Cds:Possess-Not Marihuana, Cds Poss Not Marij II 43550

Cds:Possess-Not Marihuana, Cds Poss Not Marijuana I| 43550

Cds:Possess-Not Marihuana, Cds Poss Of Heroin II 43550

Cds:Possess-Not Marihuana, Cds Poss Of Oxycodene I| 43550

Cds:Possess-Not Marihuana, Cds Poss Other I| 43550

Cds:Possess-Not Marihuana, Cds Poss Oxycodone I| 43550

Cds:Possess-Not Marihuana, Cds Poss Oxycontin II 43550

Cds:Possess-Not Marihuana, Cds Poss Percocet I| 43550

Cds:Possess-Not Marihuana, Cds Poss Suboxine || 43550

Cds:Possess-Not Marihuana, Cds Poss Subxone I| 43550

Cds:Possess-Not Marihuana, Cds Poss Violation I| 43550

Cds:Possess-Not Marihuana, Cds Poss W Intent || 43550

Cds:Possess-Not Marihuana, Cds Poss W/ Int I| 43550

Cds:Possess-Not Marihuana, Cds Poss W/Intent I| 43550

Cds:Possess-Not Marihuana, Cds Poss W/I I| 43550

Cds:Possess-Not Marihuana, Cds Poss W/I Cocaine II 43550

Cds:Possess-Not Marihuana, Cds Poss W/I Cocine || 43550

Cds:Possess-Not Marihuana, Cds Poss W/I Dist Cocaine I| 43550

Cds:Possess-Not Marihuana, Cds Poss W/I Marijuana I| 43550

Cds:Possess-Not Marihuana, Cds Poss W/Int || 43550

Cds:Possess-Not Marihuana, Cds Poss W/Int Heroin I| 43550

Cds:Possess-Not Marihuana, Cds Poss W/Int- Heroin II 43550

Cds:Possess-Not Marihuana, Cds Poss W/Intent II 43550

Cds:Possess-Not Marihuana, Cds Poss W/Intent Cds Po || 43550

Cds:Possess-Not Marihuana, Cds Poss W/Intent Cocaine || 43550

Cds:Possess-Not Marihuana, Cds Poss W/Intent To Dist || 43550

Cds:Possess-Not Marihuana, Cds Poss With Intent II 43550

Cds:Possess-Not Marihuana, Cds Poss With Intent Not || 43550

Cds:Possess-Not Marihuana, Cds Poss- Cocaine II 43550

Cds:Possess-Not Marihuana, Cds Poss- Not Marijuana II 43550

Cds:Possess-Not Marihuana, Cds Poss-Synthetic Marij I| 43550

Cds:Possess-Not Marihuana, Cds Poss-Xanax I| 43550

Cds:Possess-Not Marihuana, Cds Poss: Marijuana / Coc II 43550

Cds:Possess-Not Marihuana, Cds Poss: Marijuna / Coca I| 43550

Cds:Possess-Not Marihuana, Cds Poss: Not Marijuana I| 43550

Cds:Possess-Not Marihuana, Cds Poss:Heroin I 43550

Cds:Possess-Not Marihuana, Cds Poss. II 43550

Cds:Possess-Not Marihuana, Cds Poss. Cocaine I| 43550

Cds:Possess-Not Marihuana, Cds Poss. Herion II 43550

Cds:Possess-Not Marihuana, Cds Poss. Heroin II 43550

Cds:Possess-Not Marihuana, Cds Poss. Horoin II 43550

Cds:Possess-Not Marihuana, Cds Poss. Not Marijuana || 43550

Cds:Possess-Not Marihuana, Cds Poss. Other || 43550

Cds:Possess-Not Marihuana, Cds Poss. W/ Intent I| 43550

Cds:Possess-Not Marihuana, Cds Poss. W/Intent (Cocai II 43550

Cds:Possess-Not Marihuana, Cds Poss... I| 43550

Cds:Possess-Not Marihuana, Cds Poss/ Trespassing || 43550

Cds:Possess-Not Marihuana, Cds Poss/Poss W/Intent I| 43550

Cds:Possess-Not Marihuana, Cds Poss> I| 43550

Cds:Possess-Not Marihuana, Cds Possecion II 43550

Cds:Possess-Not Marihuana, Cds Posses II 43550

Cds:Possess-Not Marihuana, Cds Posses Heroin II 43550

Cds:Possess-Not Marihuana, Cds Possesion II 43550

Cds:Possess-Not Marihuana, Cds Possesion Heroin || 43550

Cds:Possess-Not Marihuana, Cds Possesion Of Heroin II 43550

Cds:Possess-Not Marihuana, Cds Possesion With Inten I| 43550

Cds:Possess-Not Marihuana, Cds Possesion With Intent || 43550 
Cds:Possess-Not Marihuana, Cds Possess I| 43550

Cds:Possess-Not Marihuana, Cds Possession I| 43550

Cds:Possess-Not Marihuana, Cds Possession - Heroin I| 43550

Cds:Possess-Not Marihuana, Cds Possession - Not Mari I| 43550

Cds:Possess-Not Marihuana, Cds Possession - Other II 43550

Cds:Possess-Not Marihuana, Cds Possession -Not Mari I| 43550

Cds:Possess-Not Marihuana, Cds Possession Cocaine I| 43550

Cds:Possess-Not Marihuana, Cds Possession Herion I| 43550

Cds:Possess-Not Marihuana, Cds Possession Heroin I| 43550

Cds:Possess-Not Marihuana, Cds Possession Marihuana II 43550

Cds:Possess-Not Marihuana, Cds Possession Marijuana I| 43550

Cds:Possess-Not Marihuana, Cds Possession Not Mariju || 43550

Cds:Possess-Not Marihuana, Cds Possession Of Cocain I| 43550

Cds:Possess-Not Marihuana, Cds Possession Of Cocaine || 43550

Cds:Possess-Not Marihuana, Cds Possession Of Heroin I| 43550

Cds:Possess-Not Marihuana, Cds Possession W/ Intent I| 43550

Cds:Possess-Not Marihuana, Cds Possession W/ Intent- I| 43550

Cds:Possess-Not Marihuana, Cds Possession W/Int I| 43550

Cds:Possess-Not Marihuana, Cds Possession W/Int Hero II 43550

Cds:Possess-Not Marihuana, Cds Possession With Int || 43550

Cds:Possess-Not Marihuana, Cds Possession With Inten I| 43550

Cds:Possess-Not Marihuana, Cds Possession- Heroin I| 43550

Cds:Possess-Not Marihuana, Cds Possession- Methadone || 43550

Cds:Possess-Not Marihuana, Cds Possession-Cocaine I| 43550

Cds:Possess-Not Marihuana, Cds Possession-Heroin I| 43550

Cds:Possess-Not Marihuana, Cds Possession-Not Mariju I| 43550

Cds:Possess-Not Marihuana, Cds Possession-Oxycodone I| 43550

Cds:Possess-Not Marihuana, Cds Possession-Xanax I| 43550

Cds:Possess-Not Marihuana, Cds Possession(Cocaine) I| 43550

Cds:Possess-Not Marihuana, Cds Possession/Distribut I| 43550

Cds:Possess-Not Marihuana, Cds Possesssion II 43550

Cds:Possess-Not Marihuana, Cds Pwi || 43550

Cds:Possess-Not Marihuana, Cds Pwi Cocaine II 43550

Cds:Possess-Not Marihuana, Cds Pwi Cociane II 43550

Cds:Possess-Not Marihuana, Cds Pwid || 43550

Cds:Possess-Not Marihuana, Cds Pwid - Heroin I| 43550

Cds:Possess-Not Marihuana, Cds Pwid Cocaine I| 43550

Cds:Possess-Not Marihuana, Cds Pwid Heroin I| 43550

Cds:Possess-Not Marihuana, Cds Suboxne || 43550

Cds:Possess-Not Marihuana, Cds Suboxone II 43550

Cds:Possess-Not Marihuana, Cds Vi0Lation II 43550

Cds:Possess-Not Marihuana, Cds Viiolation II 43550

Cds:Possess-Not Marihuana, Cds Viloation II 43550

Cds:Possess-Not Marihuana, Cds Vilolation II 43550

Cds:Possess-Not Marihuana, Cds Vio I| 43550

Cds:Possess-Not Marihuana, Cds Vioaltion I| 43550

Cds:Possess-Not Marihuana, Cds Vioation II 43550

Cds:Possess-Not Marihuana, Cds Viol I| 43550

Cds:Possess-Not Marihuana, Cds Viol. I I 43550

Cds:Possess-Not Marihuana, Cds Violatin I | 43550

Cds:Possess-Not Marihuana, Cds Violation I I 43550

Cds:Possess-Not Marihuana, Cds Violation (Heroin) II 43550

Cds:Possess-Not Marihuana, Cds Violation (Not Marij I| 43550

Cds:Possess-Not Marihuana, Cds Violation (Poss Cocai I| 43550

Cds:Possess-Not Marihuana, Cds Violation (Poss) I | 43550

Cds:Possess-Not Marihuana, Cds Violation Cocaine I| 43550

Cds:Possess-Not Marihuana, Cds Violation Poss I| 43550

Cds:Possess-Not Marihuana, Cds Violation Poss Cocai II 43550

Cds:Possess-Not Marihuana, Cds Violation Poss Heroi I| 43550

Cds:Possess-Not Marihuana, Cds Violation Poss W/Int || 43550 
Cds:Possess-Not Marihuana, Cds Violation Poss. Coca || 43550

Cds:Possess-Not Marihuana, Cds Violation Possession I| 43550

Cds:Possess-Not Marihuana, Cds Violation Pwid II 43550

Cds:Possess-Not Marihuana, Cds Violation-Not Mariju I| 43550

Cds:Possess-Not Marihuana, Cds Violation/ Poss II 43550

Cds:Possess-Not Marihuana, Cds Violation/Dist Heroin I| 43550

Cds:Possess-Not Marihuana, Cds Violation/Heroin II 43550

Cds:Possess-Not Marihuana, Cds Violation/Poss W/Int || 43550

Cds:Possess-Not Marihuana, Cds Violations I| 43550

Cds:Possess-Not Marihuana, Cds Violaton II 43550

Cds:Possess-Not Marihuana, Cds Violtion II 43550

Cds:Possess-Not Marihuana, Cds Volation II 43550

Cds:Possess-Not Marihuana, Cds Voliation I I 43550

Cds:Possess-Not Marihuana, Cds W/ Int To Distribute II 43550

Cds:Possess-Not Marihuana, Cds W/Int I I 43550

Cds:Possess-Not Marihuana, Cds W/Intent || 43550

Cds:Possess-Not Marihuana, Cds With Intent To Distri || 43550

Cds:Possess-Not Marihuana, Cds Xanax II 43550

Cds:Possess-Not Marihuana, Cds- Dist I| 43550

Cds:Possess-Not Marihuana, Cds- Distribution I| 43550

Cds:Possess-Not Marihuana, Cds- Poss Cocaine I| 43550

Cds:Possess-Not Marihuana, Cds- Poss Of Cocaine I| 43550

Cds:Possess-Not Marihuana, Cds- Possess I| 43550

Cds:Possess-Not Marihuana, Cds- Possession I| 43550

Cds:Possess-Not Marihuana, Cds- Possession Not Mari I| 43550

Cds:Possess-Not Marihuana, Cds-Cocaine II 43550

Cds:Possess-Not Marihuana, Cds-Poss II 43550

Cds:Possess-Not Marihuana, Cds-Poss Cocaine I| 43550

Cds:Possess-Not Marihuana, Cds-Poss Heroin II 43550

Cds:Possess-Not Marihuana, Cds-Poss Not Marijuana II 43550

Cds:Possess-Not Marihuana, Cds-Possess I| 43550

Cds:Possess-Not Marihuana, Cds-Pwi II 43550

Cds:Possess-Not Marihuana, Cds-Pwi Cocaine II 43550

Cds:Possess-Not Marihuana, Cds-Pwid II 43550

Cds:Possess-Not Marihuana, Cds-Violation II 43550

Cds:Possess-Not Marihuana, Cds: Poss II 43550

Cds:Possess-Not Marihuana, Cds: Poss Not Marijuana II 43550

Cds:Possess-Not Marihuana, Cds: Poss-Cocaine I| 43550

Cds:Possess-Not Marihuana, Cds: Poss-Heroin I| 43550

Cds:Possess-Not Marihuana, Cds: Possesion II 43550

Cds:Possess-Not Marihuana, Cds: Possession I I 43550

Cds:Possess-Not Marihuana, Cds: Possession Not Mari | | 43550

Cds:Possess-Not Marihuana, Cds: Possession- Not Mari I| 43550

Cds:Possess-Not Marihuana, Cds: Pwid II 43550

Cds:Possess-Not Marihuana, Cds:Poss Cocaine II 43550

Cds:Possess-Not Marihuana, Cds:Poss Heroin I| 43550

Cds:Possess-Not Marihuana, Cds:Poss Marijuana || 43550

Cds:Possess-Not Marihuana, Cds:Poss W/Intent I| 43550

Cds:Possess-Not Marihuana, Cds:Possess-Not Marihuan I| 43550

Cds:Possess-Not Marihuana, Cds:Possess-Not Marihuana || 43550

Cds:Possess-Not Marihuana, Cds:Possess-Not Marijuan || 43550

Cds:Possess-Not Marihuana, Cds. Poss II 43550

Cds:Possess-Not Marihuana, Cds. Violation II 43550

Cds:Possess-Not Marihuana, Cds.Poss II 43550

Cds:Possess-Not Marihuana, Cds/Disorderly I | 43550

Cds:Possess-Not Marihuana, Cdsviolation II 43550

Cds:Possess-Not Marihuana, Cdsw Poss I| 43550

Cds:Possess-Not Marihuana, Cocaine Distribution I| 43550

Cds:Possess-Not Marihuana, Cocaine Poss II 43550

Cds:Possess-Not Marihuana, Cocaine Poss W/Intent || 43550 
Cds:Possess-Not Marihuana, Cocaine Possession I| 43550

Cds:Possess-Not Marihuana, Cocaine Pwid I| 43550

Cds:Possess-Not Marihuana, Con-Cds Poss Heroin I| 43550

Cds:Possess-Not Marihuana, Consp Distribution Of Her I| 43550

Cds:Possess-Not Marihuana, Consp To Dist. Heroin I| 43550

Cds:Possess-Not Marihuana, Conspiracy Herion II 43550

Cds:Possess-Not Marihuana, Cs Violation I | 43550

Cds:Possess-Not Marihuana, Csd Dist. Heroin II 43550

Cds:Possess-Not Marihuana, Csd Violation I| 43550

Cds:Possess-Not Marihuana, Dcs Violation II 43550

Cds:Possess-Not Marihuana, Destruction Of Property I| 43550

Cds:Possess-Not Marihuana, Disorderly II 43550

Cds:Possess-Not Marihuana, Dist Cds || 43550

Cds:Possess-Not Marihuana, Dist Cds/Heroin I| 43550

Cds:Possess-Not Marihuana, Dist Cocaine || 43550

Cds:Possess-Not Marihuana, Dist Cocanie I| 43550

Cds:Possess-Not Marihuana, Dist Heroin I| 43550

Cds:Possess-Not Marihuana, Dist Of Cds I| 43550

Cds:Possess-Not Marihuana, Dist Of Cocaine II 43550

Cds:Possess-Not Marihuana, Dist Of Heroin I| 43550

Cds:Possess-Not Marihuana, Dist. Cds II 43550

Cds:Possess-Not Marihuana, Dist. Cocaine I| 43550

Cds:Possess-Not Marihuana, Dist. Heroin I| 43550

Cds:Possess-Not Marihuana, Dist. Of Cocaine I| 43550

Cds:Possess-Not Marihuana, Dist. Of Heroin II 43550

Cds:Possess-Not Marihuana, Dist.Cocaine I| 43550

Cds:Possess-Not Marihuana, Distibution Heroin I| 43550

Cds:Possess-Not Marihuana, Distributiion Heroin I| 43550

Cds:Possess-Not Marihuana, Distribution I| 43550

Cds:Possess-Not Marihuana, Distribution Cds II 43550

Cds:Possess-Not Marihuana, Distribution Cocaine || 43550

Cds:Possess-Not Marihuana, Distribution Heroin I| 43550

Cds:Possess-Not Marihuana, Distribution Of Cocaine I| 43550

Cds:Possess-Not Marihuana, Distribution Of Herion I| 43550

Cds:Possess-Not Marihuana, Distribution Of Heroin I| 43550

Cds:Possess-Not Marihuana, Distribution-Cocaine I| 43550

Cds:Possess-Not Marihuana, Distrubution Herion I| 43550

Cds:Possess-Not Marihuana, Dui || 43550

Cds:Possess-Not Marihuana, Dui Of Alcohol || 43550

Cds:Possess-Not Marihuana, Dwi Heroin I| 43550

Cds:Possess-Not Marihuana, Felony Pwid I | 43550

Cds:Possess-Not Marihuana, Gambling II 43550

Cds:Possess-Not Marihuana, H.G.V I | 43550

Cds:Possess-Not Marihuana, Heroin Distribution II 43550

Cds:Possess-Not Marihuana, Heroin Poss II 43550

Cds:Possess-Not Marihuana, Heroin Poss W/ Intent || 43550

Cds:Possess-Not Marihuana, Heroin Possession II 43550

Cds:Possess-Not Marihuana, Heroin Pwid II 43550

Cds:Possess-Not Marihuana, Loitering I| 43550

Cds:Possess-Not Marihuana, Nacotics || 43550

Cds:Possess-Not Marihuana, Narcotic Violation II 43550

Cds:Possess-Not Marihuana, Narcotics II 43550

Cds:Possess-Not Marihuana, Operator W/O Seatbelt I| 43550

Cds:Possess-Not Marihuana, P.W.I.D. C.D.S. Violation I| 43550

Cds:Possess-Not Marihuana, P/W Int Heroin II 43550

Cds:Possess-Not Marihuana, P/W/I Cocaine || 43550

Cds:Possess-Not Marihuana, P/W/I Heroin I| 43550

Cds:Possess-Not Marihuana, Plvid Heroin II 43550

Cds:Possess-Not Marihuana, Popss Not Marijuan- Herio II 43550

Cds:Possess-Not Marihuana, Pos Of Heroin I| 43550 
Cds:Possess-Not Marihuana, Pos. Cds || 43550

Cds:Possess-Not Marihuana, Posession Of Heroin I| 43550

Cds:Possess-Not Marihuana, Poss II 43550

Cds:Possess-Not Marihuana, Poss Cds II 43550

Cds:Possess-Not Marihuana, Poss Not Marijuana I| 43550

Cds:Possess-Not Marihuana, Poss - Cocaine II 43550

Cds:Possess-Not Marihuana, Poss - Heroin I | 43550

Cds:Possess-Not Marihuana, Poss -Cocaine || 43550

Cds:Possess-Not Marihuana, Poss Alprazolam || 43550

Cds:Possess-Not Marihuana, Poss Cds II 43550

Cds:Possess-Not Marihuana, Poss Cds (Cocaine) I | 43550

Cds:Possess-Not Marihuana, Poss Cds (Heroin) I| 43550

Cds:Possess-Not Marihuana, Poss Cds Cocaine || 43550

Cds:Possess-Not Marihuana, Poss Cds Heroin I| 43550

Cds:Possess-Not Marihuana, Poss Cds W/Intent || 43550

Cds:Possess-Not Marihuana, Poss Cds-Not Marijuana I| 43550

Cds:Possess-Not Marihuana, Poss Clonazepam I| 43550

Cds:Possess-Not Marihuana, Poss Clonzapan I| 43550

Cds:Possess-Not Marihuana, Poss Coc II 43550

Cds:Possess-Not Marihuana, Poss Cocain I| 43550

Cds:Possess-Not Marihuana, Poss Cocaine I| 43550

Cds:Possess-Not Marihuana, Poss Codine I| 43550

Cds:Possess-Not Marihuana, Poss Ecstacy || 43550

Cds:Possess-Not Marihuana, Poss Ecstasy || 43550

Cds:Possess-Not Marihuana, Poss Herion II 43550

Cds:Possess-Not Marihuana, Poss Heroin II 43550

Cds:Possess-Not Marihuana, Poss Heroin Cds II 43550

Cds:Possess-Not Marihuana, Poss Heroin W/ Int || 43550

Cds:Possess-Not Marihuana, Poss Heroin/Cocaine II 43550

Cds:Possess-Not Marihuana, Poss Heroine II 43550

Cds:Possess-Not Marihuana, Poss Mari Cds || 43550

Cds:Possess-Not Marihuana, Poss Marij I | 43550

Cds:Possess-Not Marihuana, Poss Marijuana I 43550

Cds:Possess-Not Marihuana, Poss Mdma || 43550

Cds:Possess-Not Marihuana, Poss Not Marijuana I| 43550

Cds:Possess-Not Marihuana, Poss Not Marj I| 43550

Cds:Possess-Not Marihuana, Poss Not Mj | | 43550

Cds:Possess-Not Marihuana, Poss Of Buprenorphine I| 43550

Cds:Possess-Not Marihuana, Poss Of Cds || 43550

Cds:Possess-Not Marihuana, Poss Of Cds Dist I | 43550

Cds:Possess-Not Marihuana, Poss Of Cds- Suspected Co I| 43550

Cds:Possess-Not Marihuana, Poss Of Cocain II 43550

Cds:Possess-Not Marihuana, Poss Of Cocaine I| 43550

Cds:Possess-Not Marihuana, Poss Of Cocaine/Marijuana II 43550

Cds:Possess-Not Marihuana, Poss Of Coccaine II 43550

Cds:Possess-Not Marihuana, Poss Of Excasty || 43550

Cds:Possess-Not Marihuana, Poss Of Herion II 43550

Cds:Possess-Not Marihuana, Poss Of Heroin I| 43550

Cds:Possess-Not Marihuana, Poss Of Heroin \& Cocaine I| 43550

Cds:Possess-Not Marihuana, Poss Of Marijuana || 43550

Cds:Possess-Not Marihuana, Poss Of Methadone I| 43550

Cds:Possess-Not Marihuana, Poss Of Oxicodone II 43550

Cds:Possess-Not Marihuana, Poss Of Percocet I| 43550

Cds:Possess-Not Marihuana, Poss Oxcodone II 43550

Cds:Possess-Not Marihuana, Poss Oxycodone I | 43550

Cds:Possess-Not Marihuana, Poss Percocet II 43550

Cds:Possess-Not Marihuana, Poss Pf Heroin I| 43550

Cds:Possess-Not Marihuana, Poss Suboxine || 43550

Cds:Possess-Not Marihuana, Poss Suboxone I| 43550

Cds:Possess-Not Marihuana, Poss W Int Heroin || 43550 
Cds:Possess-Not Marihuana, Poss W Intent || 43550

Cds:Possess-Not Marihuana, Poss W. Intent I| 43550

Cds:Possess-Not Marihuana, Poss W/ Cocaine II 43550

Cds:Possess-Not Marihuana, Poss W/ Heroin II 43550

Cds:Possess-Not Marihuana, Poss W/ I Cocaine II 43550

Cds:Possess-Not Marihuana, Poss W/ Int || 43550

Cds:Possess-Not Marihuana, Poss W/ Int Cocaine I| 43550

Cds:Possess-Not Marihuana, Poss W/ Int Heroin I| 43550

Cds:Possess-Not Marihuana, Poss W/ Int Oxycodone I| 43550

Cds:Possess-Not Marihuana, Poss W/ Intent I | 43550

Cds:Possess-Not Marihuana, Poss W/ Intent Cocaine I| 43550

Cds:Possess-Not Marihuana, Poss W/ Intent Heroin || 43550

Cds:Possess-Not Marihuana, Poss W/ Mdma || 43550

Cds:Possess-Not Marihuana, Poss W/Cocaine II 43550

Cds:Possess-Not Marihuana, Poss W/I I| 43550

Cds:Possess-Not Marihuana, Poss W/I Cds || 43550

Cds:Possess-Not Marihuana, Poss W/I Cds: Not Marijua II 43550

Cds:Possess-Not Marihuana, Poss W/I Dist Cocaine II 43550

Cds:Possess-Not Marihuana, Poss W/I Distr Cds II 43550

Cds:Possess-Not Marihuana, Poss W/I Heroin II 43550

Cds:Possess-Not Marihuana, Poss W/I To Dist Heroin II 43550

Cds:Possess-Not Marihuana, Poss W/I To Distribute I| 43550

Cds:Possess-Not Marihuana, Poss W/Int I| 43550

Cds:Possess-Not Marihuana, Poss W/Int Cds I| 43550

Cds:Possess-Not Marihuana, Poss W/Int Cocaine I| 43550

Cds:Possess-Not Marihuana, Poss W/Int Dist Cocaine II 43550

Cds:Possess-Not Marihuana, Poss W/Int Herion II 43550

Cds:Possess-Not Marihuana, Poss W/Int Heroin I| 43550

Cds:Possess-Not Marihuana, Poss W/Intent I | 43550

Cds:Possess-Not Marihuana, Poss W/Intent Cocaine I| 43550

Cds:Possess-Not Marihuana, Poss W/Intent Herion I| 43550

Cds:Possess-Not Marihuana, Poss W/Intent Heroin || 43550

Cds:Possess-Not Marihuana, Poss With Int Cocaine II 43550

Cds:Possess-Not Marihuana, Poss With Int Dist Cocain I| 43550

Cds:Possess-Not Marihuana, Poss With Int Heroin I| 43550

Cds:Possess-Not Marihuana, Poss With Intent Cocaine II 43550

Cds:Possess-Not Marihuana, Poss With Intent Heroin II 43550

Cds:Possess-Not Marihuana, Poss With Intent Marijuan I| 43550

Cds:Possess-Not Marihuana, Poss With Intent To Distr || 43550

Cds:Possess-Not Marihuana, Poss With The Intent I| 43550

Cds:Possess-Not Marihuana, Poss Xanax I| 43550

Cds:Possess-Not Marihuana, Poss-Cds II 43550

Cds:Possess-Not Marihuana, Poss-Cocaine II 43550

Cds:Possess-Not Marihuana, Poss-Herion I | 43550

Cds:Possess-Not Marihuana, Poss-Not Marijuana/Cds II 43550

Cds:Possess-Not Marihuana, Poss. I | 43550

Cds:Possess-Not Marihuana, Poss. Of Cocaine I| 43550

Cds:Possess-Not Marihuana, Poss. / W Intent Cds I| 43550

Cds:Possess-Not Marihuana, Poss. Cds I| 43550

Cds:Possess-Not Marihuana, Poss. Cds Not Marijuana I| 43550

Cds:Possess-Not Marihuana, Poss. Cocain I | 43550

Cds:Possess-Not Marihuana, Poss. Cocaine I| 43550

Cds:Possess-Not Marihuana, Poss. Herion I| 43550

Cds:Possess-Not Marihuana, Poss. Heroin I | 43550

Cds:Possess-Not Marihuana, Poss. Hreoin I | 43550

Cds:Possess-Not Marihuana, Poss. Marijuana II 43550

Cds:Possess-Not Marihuana, Poss. Not Marijuana || 43550

Cds:Possess-Not Marihuana, Poss. Of Cds I| 43550

Cds:Possess-Not Marihuana, Poss. Of Cocaine II 43550

Cds:Possess-Not Marihuana, Poss. Of Cocaine \& Heroin I| 43550 
Cds:Possess-Not Marihuana, Poss. Of Heroin || 43550

Cds:Possess-Not Marihuana, Poss. Of Marijuana || 43550

Cds:Possess-Not Marihuana, Poss. Of Methylenedioxyme I| 43550

Cds:Possess-Not Marihuana, Poss. Oxcodone I| 43550

Cds:Possess-Not Marihuana, Poss. Oxicodone I| 43550

Cds:Possess-Not Marihuana, Poss. Sched. 2 Narcotic I| 43550

Cds:Possess-Not Marihuana, Poss. W./Int Cocaine I| 43550

Cds:Possess-Not Marihuana, Poss. W./Int Heroin II 43550

Cds:Possess-Not Marihuana, Poss. W./Int. Cocaine I| 43550

Cds:Possess-Not Marihuana, Poss. W/I Cocaine II 43550

Cds:Possess-Not Marihuana, Poss. W/I Heroin I | 43550

Cds:Possess-Not Marihuana, Poss. W/Int Cocaine I| 43550

Cds:Possess-Not Marihuana, Poss. W/Int Heroin || 43550

Cds:Possess-Not Marihuana, Poss. W/Intent I| 43550

Cds:Possess-Not Marihuana, Poss. W/Intent Heroin II 43550

Cds:Possess-Not Marihuana, Poss. W/Intent Cocaine || 43550

Cds:Possess-Not Marihuana, Poss. With Intent Cocain I| 43550

Cds:Possess-Not Marihuana, Poss. With Intent Heroin I| 43550

Cds:Possess-Not Marihuana, Poss./W. Int. Heroin II 43550

Cds:Possess-Not Marihuana, Poss.Cds I| 43550

Cds:Possess-Not Marihuana, Poss.Cds Not Marijauna || 43550

Cds:Possess-Not Marihuana, Poss.Cocaine II 43550

Cds:Possess-Not Marihuana, Poss.Herion I| 43550

Cds:Possess-Not Marihuana, Poss.Heroin I| 43550

Cds:Possess-Not Marihuana, Poss.Marijuana II 43550

Cds:Possess-Not Marihuana, Poss.Not Marijuana I| 43550

Cds:Possess-Not Marihuana, Poss.Of Heroin I| 43550

Cds:Possess-Not Marihuana, Poss.W./Int Heroin I| 43550

Cds:Possess-Not Marihuana, Poss.W/ Int. Cocaine I| 43550

Cds:Possess-Not Marihuana, Poss.W/Int. Heroin II 43550

Cds:Possess-Not Marihuana, Poss.W/Int.Dist Cocaine I| 43550

Cds:Possess-Not Marihuana, Poss/W/Int. Cds II 43550

Cds:Possess-Not Marihuana, Posseion Of Heroin II 43550

Cds:Possess-Not Marihuana, Possesion I| 43550

Cds:Possess-Not Marihuana, Possesion Cocaine || 43550

Cds:Possess-Not Marihuana, Possesion Heroin II 43550

Cds:Possess-Not Marihuana, Possesion Of Cds II 43550

Cds:Possess-Not Marihuana, Possesion Of Cocaine I| 43550

Cds:Possess-Not Marihuana, Possesion With Intent Her || 43550

Cds:Possess-Not Marihuana, Possess/Cocaine II 43550

Cds:Possess-Not Marihuana, Possessession Of Cds II 43550

Cds:Possess-Not Marihuana, Possession II 43550

Cds:Possess-Not Marihuana, Possession Herion I| 43550

Cds:Possess-Not Marihuana, Possession Of Heroin I| 43550

Cds:Possess-Not Marihuana, Possession - Cocaine II 43550

Cds:Possess-Not Marihuana, Possession Cds || 43550

Cds:Possess-Not Marihuana, Possession Cocain I| 43550

Cds:Possess-Not Marihuana, Possession Cocaine I| 43550

Cds:Possess-Not Marihuana, Possession Cocaine/Heroin I| 43550

Cds:Possess-Not Marihuana, Possession Herion || 43550

Cds:Possess-Not Marihuana, Possession Heroin II 43550

Cds:Possess-Not Marihuana, Possession Heroin With In I| 43550

Cds:Possess-Not Marihuana, Possession Heroin/ Mariju || 43550

Cds:Possess-Not Marihuana, Possession Marijuana I| 43550

Cds:Possess-Not Marihuana, Possession Not Marijuana II 43550

Cds:Possess-Not Marihuana, Possession Of Cds || 43550

Cds:Possess-Not Marihuana, Possession Of Cocaine || 43550

Cds:Possess-Not Marihuana, Possession Of Cocaine / H I| 43550

Cds:Possess-Not Marihuana, Possession Of Cocane II 43550

Cds:Possess-Not Marihuana, Possession Of Herion I| 43550 
Cds:Possess-Not Marihuana, Possession Of Heroin || 43550

Cds:Possess-Not Marihuana, Possession Of Marihuana I| 43550

Cds:Possess-Not Marihuana, Possession Of Marijuana I| 43550

Cds:Possess-Not Marihuana, Possession Of Oxycodone I| 43550

Cds:Possess-Not Marihuana, Possession Oxycodone I| 43550

Cds:Possess-Not Marihuana, Possession Oxycontin I| 43550

Cds:Possess-Not Marihuana, Possession W Int I | 43550

Cds:Possess-Not Marihuana, Possession W/I Cocaine I| 43550

Cds:Possess-Not Marihuana, Possession W/I Distr Cds || 43550

Cds:Possess-Not Marihuana, Possession W/I Heroin II 43550

Cds:Possess-Not Marihuana, Possession W/I Marijuana II 43550

Cds:Possess-Not Marihuana, Possession W/Int Cocaine I| 43550

Cds:Possess-Not Marihuana, Possession W/Intent || 43550

Cds:Possess-Not Marihuana, Possession With Intent || 43550

Cds:Possess-Not Marihuana, Possession With Intent C || 43550

Cds:Possess-Not Marihuana, Possession With Intent He || 43550

Cds:Possess-Not Marihuana, Possession With Intent Ma I| 43550

Cds:Possess-Not Marihuana, Possession With Intent To || 43550

Cds:Possess-Not Marihuana, Possession With Intent-Co || 43550

Cds:Possess-Not Marihuana, Possession- Not Marijuan I| 43550

Cds:Possess-Not Marihuana, Possession-Cocaine II 43550

Cds:Possess-Not Marihuana, Possession-Marijuana || 43550

Cds:Possess-Not Marihuana, Possession/Heroin II 43550

Cds:Possess-Not Marihuana, Possessionof Cocaine I| 43550

Cds:Possess-Not Marihuana, Possof Heroin I| 43550

Cds:Possess-Not Marihuana, Prostitution I| 43550

Cds:Possess-Not Marihuana, Pwi || 43550

Cds:Possess-Not Marihuana, Pwi Dist. Heroin || 43550

Cds:Possess-Not Marihuana, Pwi - Heroin I| 43550

Cds:Possess-Not Marihuana, Pwi -Cocaine I| 43550

Cds:Possess-Not Marihuana, Pwi Cds || 43550

Cds:Possess-Not Marihuana, Pwi Cds Cocaine II 43550

Cds:Possess-Not Marihuana, Pwi Cocaine I| 43550

Cds:Possess-Not Marihuana, Pwi Cocaine/Marijuana II 43550

Cds:Possess-Not Marihuana, Pwi Dist. Heroin I | 43550

Cds:Possess-Not Marihuana, Pwi Herion I| 43550

Cds:Possess-Not Marihuana, Pwi Heroin I| 43550

Cds:Possess-Not Marihuana, Pwi Heroin/Cocaine/Mariju || 43550

Cds:Possess-Not Marihuana, Pwi Heron I| 43550

Cds:Possess-Not Marihuana, Pwi Marijuana II 43550

Cds:Possess-Not Marihuana, Pwi Not Marijuana I| 43550

Cds:Possess-Not Marihuana, Pwi Oxycodone \& Cocaine || 43550

Cds:Possess-Not Marihuana, Pwi To Distribute II 43550

Cds:Possess-Not Marihuana, Pwi- Cocaine I| 43550

Cds:Possess-Not Marihuana, Pwi-Cocaine II 43550

Cds:Possess-Not Marihuana, Pwi-Cocaine/Marijuan || 43550

Cds:Possess-Not Marihuana, Pwi-Heroin II 43550

Cds:Possess-Not Marihuana, Pwi-Marijuana II 43550

Cds:Possess-Not Marihuana, Pwi-Marijuana-Cocaine I| 43550

Cds:Possess-Not Marihuana, Pwi//Herion I| 43550

Cds:Possess-Not Marihuana, Pwid II 43550

Cds:Possess-Not Marihuana, Pwid Cds II 43550

Cds:Possess-Not Marihuana, Pwid Cocaine I| 43550

Cds:Possess-Not Marihuana, Pwid Cocaine/Marijuana II 43550

Cds:Possess-Not Marihuana, Pwid Cociane II 43550

Cds:Possess-Not Marihuana, Pwid Herion I| 43550

Cds:Possess-Not Marihuana, Pwid Heroin I| 43550

Cds:Possess-Not Marihuana, Pwid Hydrocodone I| 43550

Cds:Possess-Not Marihuana, Pwid Marijuana II 43550

Cds:Possess-Not Marihuana, Pwid Of Cds I| 43550 
Cds:Possess-Not Marihuana, Pwid Of Cocaine || 43550

Cds:Possess-Not Marihuana, Pwid Of Cocaine/Heroin I| 43550

Cds:Possess-Not Marihuana, Pwid Of Heroin II 43550

Cds:Possess-Not Marihuana, Pwid Oxycodone II 43550

Cds:Possess-Not Marihuana, Pwid Vicodin I| 43550

Cds:Possess-Not Marihuana, Pwid Xanax I| 43550

Cds:Possess-Not Marihuana, Pwu Cocaine I| 43550

Cds:Possess-Not Marihuana, Rogues And Vagabond II 43550

Cds:Possess-Not Marihuana, S\&S Warrant/Cds Poss II 43550

Cds:Possess-Not Marihuana, Simple Posession Of A Nar I| 43550

Cds:Possess-Not Marihuana, Traffic/Cds II 43550

Cds:Possess-Not Marihuana, Trespassing I| 43550

Cds:Possess-Not Marihuana, Unauthorized Use || 43550

Cds:Possess-Not Marihuana, Violation Cds I| 43550

CDS:POSSESS-NOT MARIJUANA I I 43550

CDS:PWID || 11111

CDS:PWID || 30233

CDS:PWID || 43550

CDS:PWID COCAINE || 10233

CDS:PWID COCAINE || 30233

CDS:PWID COCAINE || 43550

CDS:VIOLATION POSSESSION HEROI II 43550

CDS. II 2A0696

CDS. DIST || 11119

CDS. DIST I| 2A0696

CDS. DIST HEROIN I | 30233

CDS. DIST. II 2A3550

CDS. DISTRIBUTION || 11118

CDS. DISTRIBUTION || 2A0696

CDS. INT. DIST. I। 43550

CDS. POSS || 10233

CDS. POSS || 43550

CDS. POSS. COCAINE || 43550

CDS. VIOLATION I| 2A3550

CDS.DIST || 20696

CDS.DIST. II 1A1119

CDS.POSS || 10233

CDS.POSS || 43550

CDS.VIOLATION || 43550

CDS/ COCAINE I| 43550

CDS/ DIST I| 20696

CDS/ DIST COCAINE || 20696

Cds/ Pharmacy Without License II

CDS/ PWID || 10573

CDS/ PWID || 11118

CDS/ PWID || 11119

CDS/ATT DISTRIBUTION || 11111

CDS/ATT DISTRIBUTION || 2A0696

CDS/COCAINE || 20696

CDS/COCAINE || 30233

CDS/COCAINE || 43550

CDS/COCAINE POSS || 43550

CDS/CONSP || 23550

CDS/DIST || 11111

CDS/DIST I| 2A0696

CDS/DIST || 2A3550

CDS/DIST HEROIN || $1 \mathrm{~A} 1118$

CDS/DIST. II 2A3550

CDS/DISTRIBUTION || 20696

CDS/DISTRIBUTION || 23550 
CDS/DISTRIBUTION || 2C0696 CDS/DISTRIBUTION COCAINE || 1A1118 CDS/FALSE STATEMENT II 43550

CDS/GAMBLING || 11119

CDS/GAMBLING || 43550

CDS/HEROIN || 2A0696

CDS/HEROIN || 30233

CDS/HEROIN || 43550

CDS/POSS || 11119

CDS/POSS II 30233

CDS/POSS II 43550

CDS/PRESC:ILLEGAL MANUFAC । 10010

CDS/PWI || 11111

CDS/PWI I| 11564

CDS/PWI I| 20696

CDS/PWI I| 30233

CDS/PWI || 53550

CDS/PWI COCAINE || 30233

CDS/PWID || 10233

CDS/PWID || 10573

CDS/PWID || 11111

CDS/PWID || 11118

CDS/PWID || 11119

CDS/PWID || 11564

CDS/PWID || 1 C1118

CDS/PWID || 30233

CDS/PWID || 43550

CDS/PWID CCAINE || 11119

CDS/PWID COCAINE || 11119

CDS/PWID COCAINE || 30233

CDS/PWID/HEROIN || 11119

CDS/SEARCH \& SEIZURE || 11119

CDS/SSW II 11119

CDS/TRAFFIC II 43550

CDS/VIOLATION I| 30233

CDS/W/INTENT TO DIST. I I 30233

CDSDISTRIBUTION || 2C0696

CDSDS VIOLATION || 43550

CDSPWID || 11119

CDSS VIOLATION | 43550

CDSVIOLATION || 10233

CDSVIOLATION I| 30233

CDSVIOLATION II 43550

CDV VIOLATION I| 43550

CED VIOLATION I | 43550

COCAINE || 43550

COCAINE DIST || 30233

COCAINE DISTRIBUTION || 11118

COCAINE POSSESSION I| 43550

COCAINE PWID || 30233

CON CDS I| 2C3550

CON DIST HEROIN I| 2C0696

CON POSS W/INT DIST COCAINE || 30233

CON PWI COCAINE || 30233

CON TO DIST I| 2C0696

CON TO DIST CDS I। 2A0696

CON-CDS DIST-NARC || 2C0696

CON-CDS DISTRIBUTE - OTHER I | 2C3550

Con-Cds Manuf/Dist-Narc, Cds II 2C0696

Con-Cds Manuf/Dist-Narc, Cds Conspiracy I| 2C0696 
Con-Cds Manuf/Dist-Narc, Cds Dist II 2C0696

Con-Cds Manuf/Dist-Narc, Cds Dist Heroin II 2C0696

Con-Cds Manuf/Dist-Narc, Cds Dist. I I 2C0696

Con-Cds Manuf/Dist-Narc, Cds Distrbution II 2C0696

Con-Cds Manuf/Dist-Narc, Cds Distribution I I 2C0696

Con-Cds Manuf/Dist-Narc, Cds Distrubution II 2C0696

Con-Cds Manuf/Dist-Narc, Cds Poss With Intent Cocain II 2C0696

Con-Cds Manuf/Dist-Narc, Cds Pwi Cocaine I| 2C0696

Con-Cds Manuf/Dist-Narc, Cds Violation II 2C0696

Con-Cds Manuf/Dist-Narc, Cds Violation-Dist II 2C0696

Con-Cds Manuf/Dist-Narc, Cds- Pwid II 2C0696

Con-Cds Manuf/Dist-Narc, Cds/Trespassing I| 2C0696

Con-Cds Manuf/Dist-Narc, Con To Dist II 2C0696

Con-Cds Manuf/Dist-Narc, Con-Cds Dist-Narc II 2C0696

Con-Cds Manuf/Dist-Narc, Con. To Dist. II 2C0696

Con-Cds Manuf/Dist-Narc, Cons To Dist. Heroin II 2C0696

Con-Cds Manuf/Dist-Narc, Cons To Distrib I| 2C0696

Con-Cds Manuf/Dist-Narc, Consp Dist Heroin II 2C0696

Con-Cds Manuf/Dist-Narc, Consp To Dist Heroin II 2C0696

Con-Cds Manuf/Dist-Narc, Consp- Dist Heroin II 2C0696

Con-Cds Manuf/Dist-Narc, Consp, Dist Heroin II 2C0696

Con-Cds Manuf/Dist-Narc, Conspi-Distribution Heroin II 2C0696

Con-Cds Manuf/Dist-Narc, Conspiracy II 2C0696

Con-Cds Manuf/Dist-Narc, Conspiracy Cds Dist II 2C0696

Con-Cds Manuf/Dist-Narc, Conspiracy Distribute Coca II 2C0696

Con-Cds Manuf/Dist-Narc, Conspiracy To Dist II 2C0696

Con-Cds Manuf/Dist-Narc, Conspiracy To Distribute II 2C0696

Con-Cds Manuf/Dist-Narc, Conspircy II 2C0696

Con-Cds Manuf/Dist-Narc, Dist Heroin I| 2C0696

Con-Cds Manuf/Dist-Narc, Dist Of Cocaine I| 2C0696

Con-Cds Manuf/Dist-Narc, Dist Of Heroin I| 2C0696

Con-Cds Manuf/Dist-Narc, Distribution Cocaine II 2C0696

Con-Cds Manuf/Dist-Narc, Distribution Heroin II 2C0696

Con-Cds Manuf/Dist-Narc, Distribution Of Cds II 2C0696

Con-Cds Manuf/Dist-Narc, Distribution Of Heroin I| 2C0696

Con-Cds Manuf/Dist-Narc, Heroin Distribution II 2C0696

Con-Cds Manuf/Dist-Narc, P[Oss W/Int Cocaine II 2C0696

Con-Cds Manuf/Dist-Narc, Poss Herion I I 2C0696

Con-Cds Manuf/Dist-Narc, Poss Heroin I| 2C0696

Con-Cds Manuf/Dist-Narc, Poss W/ Cds II 2C0696

Con-Cds Manuf/Dist-Narc, Poss W/ Heroin II 2C0696

Con-Cds Manuf/Dist-Narc, Poss W/In To Distribute I| 2C0696

Con-Cds Manuf/Dist-Narc, Possession W/I Heroin I| 2C0696

Con-Cds Manuf/Dist-Narc, Possession W/Intent II 2C0696

Con-Cds Manuf/Dist-Narc, Possession With Intent II 2C0696

Con-Cds Manuf/Dist-Narc, Pwi Marijuana II 2C0696

Con-Cds Manuf/Dist., Cds II 2C3550

Con-Cds Manuf/Dist., Cds Con II 2C3550

Con-Cds Manuf/Dist., Cds Distribution I| 2C3550

Con-Cds Manuf/Dist., Cds Poss I| 2C3550

Con-Cds Manuf/Dist., Cds Violation II 2C3550

Con-Cds Manuf/Dist., Con To Dist Cds II 2C3550

Con-Cds Manuf/Dist., Con-Cds Distribute - Other I| 2C3550

Con-Cds Manuf/Dist., Conspiracy To Intent To Poss II 2C3550

Con-Cds Manuf/Dist., Dist Cds II 2C3550

Con-Cds Manuf/Dist., Distribution I| 2C3550

Con-Cds Manuf/Dist., Distribution Marijuana II 2C3550

Con-Cds Manuf/Dist., Distribution Of Marijuana I| 2C3550

Con-Cds Manuf/Dist., Heroin Distribution II 2C3550

Con-Cds Manuf/Dist., Poss Marijuana II 2C3550 
Con-Cds Manuf/Dist., Poss/Wit Int || 2C3550

Con-Cds Manuf/Dist., Possession With Intent I| 2C3550

CON-CDS-DISTRIBUTE I I 1C3550

CON-CDS: POSS W/I DIST: NARC । $1 \mathrm{C} 1119$

Con-Cds:P W/I Dist:Narc, Cds II 3C0233

Con-Cds:P W/I Dist:Narc, Cds Poss W/Int || 3C0233

Con-Cds:P W/I Dist:Narc, Cds Possession I I 3C0233

Con-Cds:P W/I Dist:Narc, Cds Pwi I I 3C0233

Con-Cds:P W/I Dist:Narc, Cds Violation I| 3C0233

Con-Cds:P W/I Dist:Narc, Cds/With Intent || 3C0233

Con-Cds:P W/I Dist:Narc, Con-Cds:Poss W/Intent Dist: I| 3C0233

Con-Cds:P W/I Dist:Narc, Pwi Cds I| 3C0233

CON-CDS:POSS W/INTENT DIST: NA || 3C0233

Con-Cds:Possess-Not Marihuana, Cd Violation II 4C3550

Con-Cds:Possess-Not Marihuana, Cds II 4C3550

Con-Cds:Possess-Not Marihuana, Cds Not Marijuana I| 4C3550

Con-Cds:Possess-Not Marihuana, Cds Poss II 4C3550

Con-Cds:Possess-Not Marihuana, Con, Cds II 4C3550

Con-Cds:Possess-Not Marihuana, Consp. To Posses II 4C3550

Con-Cds:Possess-Not Marihuana, Conspiracy II 4C3550

Con-Cds:Possess-Not Marihuana, Narcotics II 4C3550

CON-CONTRABAND DELIVERY || 2C1080

Con-Contraband Delivery, Con To Deliver Contraband II 2C1080

CON-CONTRABAND POSS W/I DEL || 2C1085

CON-DIST/PWD FENT/HEROIN MIX || 1C0908

CON-POSS W/INT TO DIST I I 1 C0233

CON. POSS. CDS I| 4C3550

CON. TO DIST HEROIN || 2A0696

CON. TO DISTRIBUTE || 2C0696

CONPIRACY TO POSS II 2C0696

CONPIRECY DIST. HEROIN || 15409

CONS CDS DIST II 30233

CONS DIST I| 2A0696

CONS POSS W/INT DIST I | 43550

CONS PWI CDS || 53550

CONS TO DIST CRACK COCAINE I| 2A0696

CONS TO DIST OXYCODONE I I 30233

CONS TO DIST. COCAINE || 2A0696

CONS TO DISTR HEROIN | | 2C0696

CONS. DIST CDS II 11119

CONSIP TO DIST HEROIN || 2C0696

CONSP CDS I| 20696

CONSP DIST CDS || 1C1111

CONSP DIST CDS || 2A0696

CONSP DIST CDS || 2C0696

CONSP DIST CDS । । 30233

CONSP DIST HEROIN I| 30233

CONSP TO DIS HEROIN I I 2C0696

CONSP TO DIST । 1 C1118

CONSP TO DIST । | 2C0696

CONSP TO DIST HERION | | 2C0696

CONSP TO DIST HEROIN | | 20696

CONSP TO DIST HEROIN || 2A0696

CONSP TO DIST HEROIN I | 2C0696

CONSP TO DISTRIBUTE COCAINE || 2A0696

CONSP. II 20696

CONSP. CDS I| 2C0696

CONSP. DIST. COCAINE || 2A0696

CONSP. DIST. HEROIN I| 2C0696

CONSP. TO DIST I| 2A0696 
CONSP. TO DIST CDS || 20696

CONSP. TO DIST HEROIN | | 2C0696

CONSP. TO DIST NARCOTICS I| 20696

CONSP. TO DIST. II 2C0696

CONSP. TO DIST. COCAINE || 20696

CONSP. TO DIST. HEROIN || 11111

CONSP. TO DIST. HEROIN I| 20696

CONSP. TO DISTR. HEROIN || 2A0696

CONSP. TO DISTR. HEROIN || 2C0696

CONSP. TO DISTR. HEROIN II 30233

CONSP. TO DISTRIBUTE HEROIN || 20696

CONSP/ PWID COCAINE || 43550

CONSP/DIST OF HEROIN | | 30233

CONSPI TO DIST || 2C0696

CONSPIRACY || 11118

CONSPIRACY || 2A0696

CONSPIRACY || 2A3550

CONSPIRACY || 2C0696

CONSPIRACY I| 43550

CONSPIRACY CDS || $1 \mathrm{C} 1118$

CONSPIRACY CDS DISTRIBUTION || 11118

CONSPIRACY CDS DISTRIBUTION || 2C0696

CONSPIRACY CDS MANUFACTURING || 43550

CONSPIRACY DIST ।| 10573

CONSPIRACY DIST COCAINE || 20696

CONSPIRACY DIST. CDS I| 1C1118

CONSPIRACY DISTRIBUTE CDS || 2A0696

CONSPIRACY DISTRIBUTE COCAINE || 2C0696

CONSPIRACY POSS W/I COCAINE || 2C0696

CONSPIRACY POSSW/I COCAINE I| 2C0696

CONSPIRACY TO DIS. CDS || 2A0696

CONSPIRACY TO DIST || 20696

CONSPIRACY TO DIST I| 30233

CONSPIRACY TO DIST COCAINE || 20696

CONSPIRACY TO DIST MJ || 2C3550

CONSPIRACY TO DIST-COCAINE || 2A0696

CONSPIRACY TO DIST. CDS || 30233

CONSPIRACY TO DIST. COCAINE || 2C0696

CONSPIRACY TO DISTRIBUTE || 20696

CONSPIRACY TO DISTRIBUTE || 30233

CONSPIRACY TO DISTRIBUTE CDS || 20696

CONSPIRACY TO DISTRIBUTE CDS || 2A0696

CONSPIRACY TO DISTRIBUTE CDS || 2C0696

CONSPIRACY TO DISTRIBUTE HEROI I। 20696

CONSPIRACY TO DISTRIBUTE HEROI I| 2A0696

CONSPIRACY TO DISTRIBUTE HEROI || 2C0696

CONSPIRACY TO POSSESS I| 20696

CONSPIRACY TO SELL HERION I 43550

CONSPIRACY; CDS DISTRIBUTION || 20696

CONSPIRE DISTRIBUTE HEROIN || 30233

CONSPIRE PWID COCAINE I | 30233

CONSPIRE TO DISTRIBUTE HEROIN || 2C0696

CONSPIRE TO POSS HEROIN I 43550

CONSPRIRACY DIST CDS NOT MARIJ I। 4A3550

CONSPRIRACY TO DIST I I 20696

CONTRABAND || 11835

CONTRABAND || 21080

CONTRABAND || 43550

CONTRABAND DELIVERY || 21080

CONTRABAND IN JAIL | | 21080 
CONTRABAND POSS || 11835

Contraband-Conceal In Jail, Contraband-Conceal In J || 14903

CPS PWID I| 30233

CS VIOLATION || 10233

CS: POSSESS-NOT MARIJUANA || 43550

CSD DIST || 2A0696

CSD POSS II 43550

CSD POSS MARIJUANA I| 10233

CSD VIOLATION I| 43550

CSDS VIOLATION I| 2A0696

CSDS VIOLATION II 43550

CURFEW VIOLATION II 30233

CURRENCY:MANUFACTURE/COUNTERFE || 10067

Currency:Manufacture/Counterfeit, Counterfeit Curren I| 10067

Currency:Manufacture/Counterfeit, Currency:Manufactu || 10067

Currency:Manufacture/Counterfeit, Forged Currency || 10067

CWID COCAINE || 11119

CXDS VIOLATION II 30233

DEST OF PROPERTY || 10233

DIRT BIKE I| 43550

DIS CDS/PWIT || 1A1118

DIS OF MARIJUANA I| 2A3550

DIS. COCAINE || 2A0696

DISORDERLY CONDUCT || 43550

DISORDERLY CONDUCT //CDS VIOLA I | 43550

DISP HEROIN || 43550

DISRIBUTION || 23550

DISRTIBUTION || 2A0696

DISST HEROIN || 2A0696

DIST || 11118

DIST || 1A1118

DIST || 20696

DIST I| 2A0696

DIST || 2A3550

DIST || 30233

DIST I| 53550

DIST COCAINE || 20696

DIST OF HEROIN || 2A0696

DIST OF HEROIN I| 30233

DIST OF MARIJUANA II 2A3550

DIST . HEROIN || 43550

DIST CDS || 10233

DIST CDS || 10573

DIST CDS | | 11111

DIST CDS || 11118

DIST CDS || 11119

DIST CDS || 1A1118

DIST CDS I| 20696

DIST CDS || 2A0696

DIST CDS || 2A3550

DIST CDS || 2C0696

DIST CDS || 30233

DIST CDS I| 43550

DIST CDS / PWIT । 1A1118

DIST COCAINE || 11111

DIST COCAINE || 11118

DIST COCAINE || 1A1118

DIST COCAINE || 20696

DIST COCAINE I| 2A0696

DIST COCAINE || 30233 
DIST COCAINE || 43550

DIST COCAINE/HEROIN || 2A0696

DIST COCAINE/MARIJUANA || 20696

DIST COKE HEROIN II 20696

DIST HERION । | 43550

DIST HEROIN || 11118

DIST HEROIN || 11119

DIST HEROIN || 1A1118

DIST HEROIN || 20696

DIST HEROIN I | 2A0696

DIST HEROIN || 30233

DIST HEROIN || 43550

DIST MARIJAUNA || 10573

DIST MARIJAUNA || 2A3550

DIST MARIJUANA | | 10233

DIST MARIJUANA || 10493

DIST MARIJUANA || 23550

DIST MARIJUANA I| 2A0696

DIST MARIJUANA || 2A3550

DIST MARIJUANA || 30233

DIST MARJIUANA || 10573

DIST MDMA I| 10696

DIST MJ I| 10233

DIST MJ || 23550

DIST MJ || 2A3550

DIST MJ || 2C3550

DIST NARC ।

DIST OF MARIJUANA || 23550

DIST OF CACAINE || 11118

DIST OF CDS || 10573

DIST OF CDS || 11111

DIST OF CDS I। 20696

DIST OF CDS || 2A0696

DIST OF CDS || 30233

DIST OF CDS || 43550

DIST OF CO || 2C0696

DIST OF COC I| 2A0696

DIST OF COCAINE || 11119

DIST OF COCAINE || 1 A1118

DIST OF COCAINE || 20696

DIST OF COCAINE || 23550

DIST OF COCAINE || 2A0696

DIST OF COCAINE I| 2C0696

DIST OF COCAINE I| 30233

DIST OF COCAINE || 43550

DIST OF COCAINE/HEROIN || 2A0696

DIST OF HEORIN || 43550

DIST OF HERION । | 2A0696

DIST OF HEROIN || 11111

DIST OF HEROIN । | 11118

DIST OF HEROIN I| 11119

DIST OF HEROIN । | 20696

DIST OF HEROIN । I 2A0696

DIST OF HEROIN || 2C0696

DIST OF HEROIN I I 30233

DIST OF HEROIN । | 43550

DIST OF HEROIN/COCAINE || 43550

DIST OF MARIJUANA || 10233

DIST OF MARIJUANA || 10573

DIST OF MARIJUANA || 23550 
DIST OF MARIJUANA || 2A0696 DIST OF MARIJUANA || 2A3550 DIST OF MARIJUANA | | 2C3550 DIST OF MARIJUANA || 43550 DIST OF MARIJUNA || 2A3550 DIST OF METHADONE | | 1A1118 DIST OF NARCOTICS || 10233 DIST OF OXYCODONE I| 20696 DIST OF OXYCODONE || 2A0696 DIST OF OXYCODONE I| 43550 DIST OF PRESCRIBED MED. I।

DIST OF PROPERTY I | 34030

DIST OXYCODONE || 2A0696 DIST W/I || 2A0696 DIST-CDS || 11118

DIST-CDS || 20696

DIST-CDS || 23550

DIST-CDS || 30233

DIST-COCAINE || 2A0696

DIST,OF MARIJUNNA || 2A3550

DIST. II 2A3550

DIST. CDS || 20696

DIST. CDS I।

DIST. CDS || 10233

DIST. CDS || 10573

DIST. CDS || 11111

DIST. CDS || $1 \mathrm{~A} 1118$

DIST.CDS || 20696

DIST. CDS || 2A0696

DIST. CDS || 2A3550

DIST.CDS || 43550

DIST. COCAINE || 11118

DIST. COCAINE || 20696

DIST. COCAINE I| 2A0696

DIST. COCAINE || 43550

DIST. HERION || 11111

DIST. HERION || 20696

DIST. HERION || 2A0696

DIST. HERION || 30233

DIST. HERION || 43550

DIST. HERION/COCAINE | | 2A0696

DIST. HEROIN || $1 \mathrm{C} 1118$

DIST. HEROIN || 20696

DIST. HEROIN I| 2A0696

DIST. HEROIN || 2C0696

DIST. HEROIN || 30233

DIST. HEROIN || 43550

DIST. HEROIN/ CDS VIOLATION I I 20696

DIST. HEROIN/COCAINE || 20696

DIST. HEROINE || 2C0696

DIST. MARIJUANA || 10573

DIST. MARIJUANA I| 2A3550

DIST. OF ALPRAZOLAM || 2A3550

DIST. OF CDS || 11111

DIST. OF CDS II 20696

DIST. OF CDS || 2A0696

DIST. OF CDS || 43550

DIST. OF COCAINE || 1A1118

DIST. OF COCAINE || 20696

DIST. OF COCAINE || 2A0696 
DIST. OF COCAINE || 30233

DIST. OF COCAINE || 43550

DIST. OF HEROIN || 10573

DIST. OF HEROIN || 11118

DIST. OF HEROIN || 11119

DIST. OF HEROIN || 20696

DIST. OF HEROIN | | 2A0696

DIST. OF HEROIN || 2A3550

DIST. OF HEROIN || 30233

DIST. OF HEROIN I| 43550

DIST. OF MARIJUANA | 10573

DIST. OF MARIJUANA I 23550

DIST. OF MARIJUANA || 2A0696

DIST. OF MARIJUANA I| 2A3550

DIST. OF MDMA II 20696

DIST. OF NARCOTICS || 10233

DIST. OF PROP. II 34025

DIST. OF PROPERTY | | 34030

DIST. OF SUBOXONE || 2A3550

DIST. PROPERTY || 34025

DIST. SUBOXONE || 2A3550

DIST.CDS I| 11119

DIST.COCAINE || 1A1118

DIST.COCAINE || 2A0696

DIST.COCAINE || 30233

DIST.HEROIN || 20696

DIST.HEROIN || 2A0696

DIST.OF MARIJUANA || 2A3550

DIST/MARIJUANA || 2C3550

DIST/CDS || 11111

DIST/PWID FENTNYL/HEROIN MIX | | 10908

DISTIBUTION || 20696

DISTIBUTION || 2A3550

DISTIBUTION COCAINE || 2C0696

DISTIBUTION OF BUPRENORPHIN || 2A3550

DISTIBUTION OF HERION | | 23550

DISTR I| 2A0696

DISTR HEROIN || 11111

DISTR HEROIN || 20696

DISTR HEROIN I| 2A0696

DISTR OF HEROIN || 2A0696

DISTR. COCAINE || 11111

DISTR. COCAINE || 11118

DISTR. COCAINE || 1A1118

DISTR. COCAINE || 20696

DISTR. HEROIN || 20696

DISTR. HEROIN I| 2A0696

DISTR. HEROIN || 2C0696

DISTR. HEROIN || 30233

DISTR. HEROIN || 43550

DISTR. MARIJUANA || 10573

DISTR. MARIJUANA || 2A3550

DISTR.COCAINE || 11118

DISTRBUTION || 10233

DISTRIB HEROIN I| 2A0696

DISTRIBUITION OF COCAINE || 20696

DISTRIBUTE CDS || 2A0696

DISTRIBUTION || 10233

DISTRIBUTION || 10573

DISTRIBUTION || 11111 
DISTRIBUTION || 11118 DISTRIBUTION || 11119 DISTRIBUTION || 15202 DISTRIBUTION || 1A1118 DISTRIBUTION || 20696 DISTRIBUTION || 23550 DISTRIBUTION || 2A0696 DISTRIBUTION || 2A3550 DISTRIBUTION || 2C0696 DISTRIBUTION || 2C3550 DISTRIBUTION || 30233 DISTRIBUTION || 43550 DISTRIBUTION CDS || 20696 DISTRIBUTION -HEROIN || 30233 DISTRIBUTION ALPRAZOLAM || 2A3550 DISTRIBUTION CDS । | DISTRIBUTION CDS || 10233 DISTRIBUTION CDS || 11111 DISTRIBUTION CDS || 1A1118 DISTRIBUTION CDS || 20696 DISTRIBUTION CDS || 2A0696 DISTRIBUTION CDS || 2A3550 DISTRIBUTION CDS || 30233 DISTRIBUTION CDS || 43550 DISTRIBUTION CDS MARIJUANA || 2A3550 DISTRIBUTION CLONAZEPAM || 2A3550

DISTRIBUTION COCAINE || 11111 DISTRIBUTION COCAINE || 11118 DISTRIBUTION COCAINE || 11119 DISTRIBUTION COCAINE || 1A1118 DISTRIBUTION COCAINE || 20696 DISTRIBUTION COCAINE || 2A0696 DISTRIBUTION COCAINE || 30233 DISTRIBUTION COCAINE || 43550 DISTRIBUTION COCAINE/HEROIN || 11119 DISTRIBUTION HERION I| 1A1118 DISTRIBUTION HERION || 2A0696 DISTRIBUTION HEROIN || 1A1118 DISTRIBUTION HEROIN || 20696 DISTRIBUTION HEROIN || 2A0696 DISTRIBUTION HEROIN || 2C0696 DISTRIBUTION HEROIN || 30233 DISTRIBUTION HEROIN I । 43550 DISTRIBUTION MARIHUANA || 2A3550 DISTRIBUTION MARIJUANA || 11635 DISTRIBUTION MARIJUANA || 23550 DISTRIBUTION MARIJUANA || 2A3550 DISTRIBUTION OF CDS | । 1A1118 DISTRIBUTION OF CDS || 20696 DISTRIBUTION OF CDS || 2A0696 DISTRIBUTION OF CDS I। 2A3550 DISTRIBUTION OF CDS || 30233 DISTRIBUTION OF CDS || 43550 DISTRIBUTION OF CLONAZEPAM || 23550 DISTRIBUTION OF COCAINE || 11111 DISTRIBUTION OF COCAINE || 11118 DISTRIBUTION OF COCAINE || 11119 DISTRIBUTION OF COCAINE || 1A1118 DISTRIBUTION OF COCAINE || 20696 DISTRIBUTION OF COCAINE || 2A0696 
DISTRIBUTION OF COCAINE || 30233 DISTRIBUTION OF COCAINE || 43550 DISTRIBUTION OF CONTRABAND || 21085 DISTRIBUTION OF HERION || 2A0696 DISTRIBUTION OF HEROIN | | 11119 DISTRIBUTION OF HEROIN || 20696 DISTRIBUTION OF HEROIN | | 2A0696 DISTRIBUTION OF HEROIN | | 30233 DISTRIBUTION OF HEROIN || 43550 DISTRIBUTION OF MARIJUANA || 10494 DISTRIBUTION OF MARIJUANA || 10690 DISTRIBUTION OF MARIJUANA || 11635 DISTRIBUTION OF MARIJUANA || 23550 DISTRIBUTION OF MARIJUANA || 2A3550 DISTRIBUTION OXYCODONE || 2A0696 DISTRIBUTION-COCAINE || 20696 DISTRIBUTION-COCAINE || 2A0696 DISTRUBUTION CDS || 20696 DISTRUBUTION HERION | | 2A0696 DISTRUBUTION OF COCAINE || 1A1118 DISTRUBUTION OF MARIJUANA || 2A3550 DISTRUCTION OF PROPERTY | | 14200 DISTRUCTION OF PROPERTY | | 34025 DISTRUCTION OF PROPERTY | | 34030 DISTRUCTION PROPERTY | | 34025 DISTTRIBUTION MARIHUANA | | 2A3550 DOST HEROIN I| 2A0696 DRUG PARAPHERNALIA || 43550 DRUG PARAPHERNALIA || 53550 DRVING WITHOUT LICENSE | | 10233

DSIT. OF COCAINE || 20696

DUI || 43550

DUI || 53550

DUI/CDS POSS || 53550

DWI HEROIN || 43550

EDS POSS I| 10233

EXECUTION OF S.S/CDS || 43550

EXECUTION OF SEARCH \& SEIZURE || 30233

EXECUTION SEARCH+SEIZURE || 30233

FAILURE TO OBEY I I 43550

FAKE CDS: POSS W/I DIST || 10246

False Presc With Intent Dist, False Pretense || 10728

FALSE PRETENSE || 43550

FALSE STATEMENT I| 53550

False Stmt To Peace Officer, Pwid Heroin I| 10690

False Stmt To Peace Officer, Pwid Marijuana || 10690

FELONY CDS I| 11119

FRAUD || 30233

GAMBLING || 30233

GAMBLING || 43550

GAMBLING/CDS || 43550

HARASS FOL. ANOTH W/INT TO || 15406

Harass Fol. Anoth W/Int To, Harass Fol. Anoth W/Int I| 15406

HERION || 43550

HERION POSSESSION W/ INTENT I I 1A1118

HEROIN II 43550

HEROIN COCAINE POSS || 43550

HEROIN DIST I| 2A0696

HEROIN DISTRIBUTION II 30233

HEROIN POSSESSION I| 43550 
HEROIN PWID || 30233

HEROIN/PILLS || 43550

HEROINE POSS. II 30233

HGC/CDS II 10493

HINDERING || 2A0696

Hire Etc Minor: Dist Cds, Cds Violation I| 15409

Hire Etc Minor: Dist Cds, Consp. To. Dist. Cocaine II 15409

HIT AND RUN | | 30233

ID MISUSE || 43550

ILLEG. AMMO POSSEISION || 43550

ILLEGAL AMMO POSS I| 43550

ILLEGAL NIGHT CLUB || 43550

ILLEGAL PHARMACY । |

ILLEGAL PHARMACY || 15202

Import Minor: Drug Felony, Import Minor: Drug Felony | I 10489

INT DIST || 30233

INTENT TO DISTRIBUTE || 10573

INTENT TO DISTRIBUTE | | 11564

INTENT TO DISTRIBUTE | | 43550

LOTTERING || 43550

MANUFACTURE CDS - NARCOTIC || 10661

MARIJUANA +10GRAMS || 2A3550

MARIJUANA DISTRIB || 11564

MARIJUANA PWID || 10233

NARC POSS W/INTENT- LG AMT I | 20231

Narc Poss W/Intent- Lg Amt, Cds Poss W/F I| 20231

Narc Poss W/Intent- Lg Amt, Cds Poss W/I I| 20231

Narc Poss W/Intent- Lg Amt, Cds Poss W/Int I | 20231

Narc Poss W/Intent- Lg Amt, Cds Violation I | 20231

Narc Poss W/Intent- Lg Amt, Poss W/I Cocaine II 20231

Narc Poss W/Intent- Lg Amt, Poss W/I Heroin II 20231

Narc Poss W/Intent- Lg Amt, Possession With Intent H || 20231

Narc Poss W/Intent- Lg Amt, Possession With Intent- I| 20231

Narc Poss W/Intent- Lg Amt, Possession With Intent-H I| 20231

NARCORTICS I| 43550

NARCOTIC VIOLATION I I 23550

NARCOTIC VIOLATION II 30233

NARCOTICS || 11119

NARCOTICS || 30233

NARCOTICS II 43550

NARCOTICS VIOLATION | | 30233

NARCOTICS VIOLATION II 43550

OPEN CONTAINER I| 1A1118

OPEN CONTAINER I। 43550

Operating Pharmacy W/O License I I

OPR. PHARM. W/OUT LICENSE । |

P W/INT COCAINE II 11119

P-W-I- HEROIN || 30233

P.W.I HEROIN I| 43550

P.W.I. DIST.HEROIN || 43550

P.W.I.D. II 30233

P.WIT. COCAINE || 43550

P.WIT. DIS. COCAINE || 30233

P.WIT. HEROIN I| 2A0696

P.WIT.HEROIN II 30233

P/W.INT HEROIN || 30233

P/W.INT HEROIN/COCAINE || 20696

P/W.INT. MARIJUANA I| 2A3550

P/W.INT.COCAINE I| 2A0696

P/W.INT.HEROIN/COCAINE || 30233 
P/W.INTCOCAINE/HERLOIN || 2A0696

P/W/I COCAINE || 30233

P/W/I HEROIN I| 2A0696

P/W/I HEROIN II 30233

P/W/I HEROIN I| 43550

P/W/I HEROIN/ COCAINE I| 10881

P/W/I MARIJUANA I 10233

P/W/I MARIJUANA II 10573

P/W/I MARIJUANA || 11119

PARA II 53550

PARAPHERNALIA || 53550

PARAPHERNALIA POSSESSION I| 53550

PEWID COCIANE || 43550

PHARMACY W/O LIC I |

PHARMACY W/O LIC. I I

PHARMACY W/O LIC. I I 11111

PHARMACY W/O LICENSE I |

PHARMACY W/O LICENSE I | 2A0696

PHARMACY W/O LICENSE I I 2A3550

PHARMACY WITHOUT LICENSE । |

PHARMENCY WITHOUT LICENSE I I

PILLS/CDS I| 43550

POESSION W/INT OF COCAINE || 2A0696

POO. HEROIN I| 43550

POPSS HEROIN I| 43550

POS HEROIN II 43550

POS MARIJUANA I| 43550

POS W/ INT MARIJUANA | | 10573

POS: HEROIN I| 43550

POS. OF COCAINE II 43550

POS.W.INT. HEROIN/COCAINE || 2A0696

POSESSION I| 43550

POSESSION CDS I| 43550

POSESSION OF COCAINE I| 2A0696

POSESSION OF COCAINE || 43550

POSESSION OF HEROIN II 43550

POSESSION WITH INTENT I I 11119

POSESSION WITH INTENT MARIJUAN || 10233

POSS || 1A0660

POSS I| 30233

POSS I| 43550

POSS COCAINE || 43550

POSS COCAINE I| 53550

POSS HEROIN I| 30233

POSS HEROIN II 43550

POSS MARIJUANA || 10233

POSS - HEROIN II 43550

POSS -CDS II 43550

POSS /INT HEROIN || 30233

POSS ALPRAZOLAM || 43550

POSS CAOCAINE || 43550

POSS CDS || 10233

POSS CDS || 10881

POSS CDS || 11119

POSS CDS I| 1A1118

POSS CDS || 20696

POSS CDS || 2A0696

POSS CDS I| 2A3550

POSS CDS || 30233

POSS CDS || 43550 


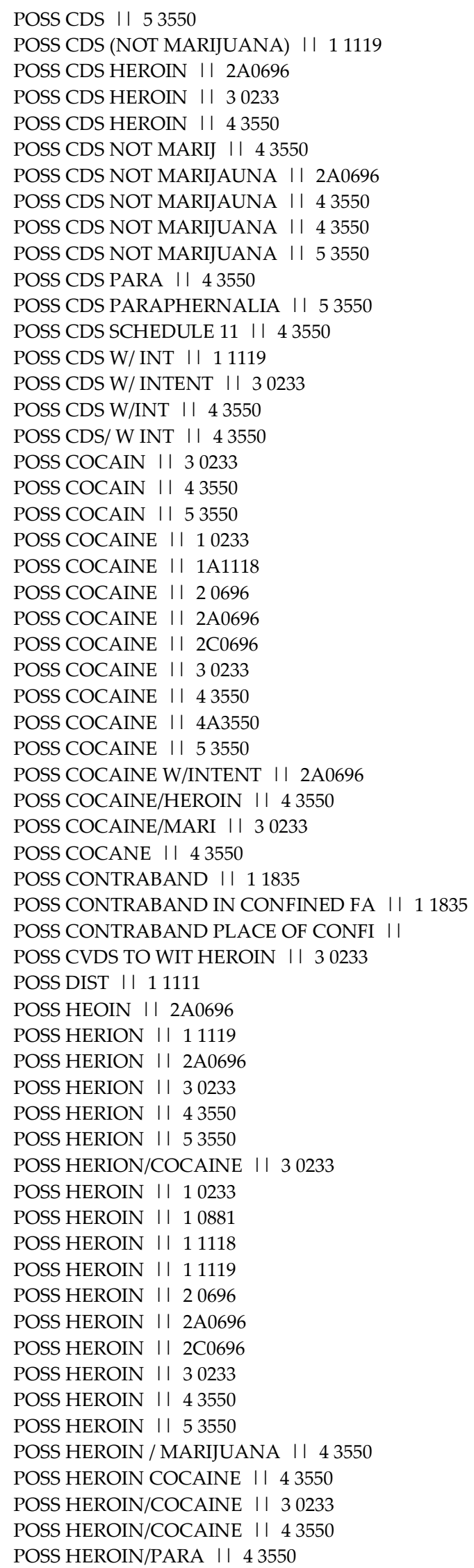


POSS HEROIN/PWID HEROIN | | 11119

POSS HEROINE I| 43550

POSS HERROIN || 43550

POSS INT CDS I| 30233

POSS INT COCAINE || 11119

POSS INT CONSPIRACY DISTRIBUTI || 30233

POSS INTENT I | 43550

POSS INTENT COCAINE || 43550

POSS INTENT COCANE || 43550

POSS INTENT DIST. HEROIN || 10881

POSS K2 I I 43550

POSS MARIJUAN PWID । | 10573

POSS MARIJUANA || 10233

POSS MARIJUANA II 23550

POSS MARIJUANA I| 2A3550

POSS MARIJUANA || 30233

POSS MARIJUANA || 43550

POSS MARIJUANA II 53550

POSS MARIJUANA W I DIST I I 10573

POSS MARIJUANA W INT DIST || 10573

POSS MARIJUANA W/INT || 10233

POSS MARIJUANA W/INT I I 10573

POSS MARIJUANA W/INTENT TO DIS || 10573

POSS MARIJUANA/HEROIN || 2A0696

POSS MARJ II 2A3550

POSS MDMA I| 43550

POSS MJ W/ INT I| 10573

POSS MJ/COCAINE PWID || 10881

POSS MOLLY I| 43550

POSS NOT MAJ I | 43550

POSS NOT MARI I | 43550

POSS NOT MARIJUANA || 43550

POSS O COCAINE I| 2A0696

POSS OF CDS I| 10233

POSS OF CDS || $1 \mathrm{~A} 1118$

POSS OF CDS I। 2A0696

POSS OF CDS II 30233

POSS OF CDS I| 43550

POSS OF CDS I| 53550

POSS OF CDS (PRESCRIPTION) | | 43550

POSS OF CO I| 43550

POSS OF CO || 53550

POSS OF COC ।| 43550

POSS OF COCAINE I| 20696

POSS OF COCAINE I| 2A0696

POSS OF COCAINE || 30233

POSS OF COCAINE || 43550

POSS OF COCAINE I| 53550

POSS OF COCAINE WITH INT || 30233

POSS OF COCAINE/HEROIN || 43550

POSS OF CONTRABAND I | 11835

POSS OF FIREWORKS W/ INTENT I I 1A0029

POSS OF HEOIN/COCAINE | | 43550

POSS OF HERIN II 30233

POSS OF HERION II 43550

POSS OF HEROIN I I 10233

POSS OF HEROIN । | 11119

POSS OF HEROIN I| 2A0696

POSS OF HEROIN I I 30233

POSS OF HEROIN । | 43550 
POSS OF HEROIN । | 53550

POSS OF HEROIN CDS I| 43550

POSS OF HEROIN W/ INT I I 30233

POSS OF HEROIN W/ INTENT I | 30233

POSS OF HEROIN W/INTENT || 30233

POSS OF HEROIN W/INTENT I । 43550

POSS OF HEROIN/COCAINE | | 43550

POSS OF HEROINE II 43550

POSS OF MARIJANA || 10233

POSS OF MARIJUAN/ COCAINE I | 43550

POSS OF MARIJUANA I| 10233

POSS OF MARIJUANA I| 23550

POSS OF MARIJUANA || 2A3550

POSS OF MARIJUANA | | 30233

POSS OF MARIJUANA II 43550

POSS OF MARIJUANA W/ INTENT || 10233

POSS OF OXY || 43550

POSS OF OXYCODONE I| 2A0696

POSS OF OXYCODONE || 30233

POSS OF OXYCODONE || 43550

POSS OF OXYCONTIN II 43550

POSS OF PARAPHERNALIA I| 53550

POSS OF PARAPHERNELIA I| 53550

POSS OF PERCOCET II 43550

POSS OF PERSCRIPTION DRUGS I। 43550

POSS OF SUBOXONE II 43550

POSS OF SUS HERION I| 43550

POSS OF TRAMADOL HYDROCHLORIDE ।| 2A3550

POSS OF XANAX II 43550

POSS OXY || 43550

POSS OXYCODINE I| 43550

POSS OXYCODONE I| 43550

POSS PARAPHERNALIA || 30233

POSS PARAPHERNALIA || 53550

POSS PECOCET || 43550

POSS PERCOCET || 43550

POSS PRESCRIPTION DRUGS I| 43550

POSS PROMETHAZINE || 43550

POSS SUBOXONE || 2A0696

POSS SUBOXONE II 43550

POSS W /INT MARIJUANA || 10233

POSS W HERION I | 43550

POSS W HEROIN II 2A0696

POSS W HEROIN I I 30233

POSS W INT COCAINE || 2A0696

POSS W INT COCAINE || 30233

POSS W INT COCAINE || 43550

POSS W INT DIST I | 43550

POSS W INT HEROIN | | 43550

POSS W INT HEROIN \& COCAINE || 30233

POSS W INT HEROIN COCAINE I | 43550

POSS W INTENT I| 10233

POSS W INTENT | | 30233

POSS W INTENT || 43550

POSS W INTENT COCAINE I I 43550

POSS W. INT HEROIN I I 30233

POSS W. INTENT HEROIN | | 30233

POSS W./INT COCAINE || 43550

POSS W./INT HEROIN I I 43550

POSS W/ I I 30233 
POSS W/ CDS II 30233

POSS W/CDS I। 43550

POSS W/ COCAINE I| 2A0696

POSS W/ COCAINE || 30233

POSS W/ COCAINE I| 43550

POSS W/ HEROIN I| 30233

POSS W/ HEROIN I | 43550

POSS W/ I COCAINE I| 30233

POSS W/ INT || 10233

POSS W/ INT || 10881

POSS W/ INT I| 1A0233

POSS W/ INT I| 2A0696

POSS W/ INT || 30233

POSS W/ INT I | 43550

POSS W/ INT CDS I। 43550

POSS W/ INT COCAINE | | 10493

POSS W/ INT COCAINE | | 11111

POSS W/ INT COCAINE I I 20696

POSS W/ INT COCAINE I | 30233

POSS W/ INT COCAINE | | 43550

POSS W/ INT CRACK COCAINE || 30233

POSS W/ INT DIST COCAINE I | 43550

POSS W/ INT DIST HERION | | 30233

POSS W/ INT DIST HEROIN | | 30233

POSS W/ INT HEROIN I I 20696

POSS W/ INT HEROIN II 2A0696

POSS W/ INT HEROIN I| 30233

POSS W/ INT HEROIN I | 43550

POSS W/ INT MARIJUANA | | 10233

POSS W/ INT MARIJUANA | | 10573

POSS W/ INT MARIJUANA || 11635

POSS W/ INT MARIJUANA I I 23550

POSS W/ INT MARIJUANA I I 43550

POSS W/ INT MOLLY II 43550

POSS W/ INT PERKS || 43550

POSS W/ INT TO DIST । I 10881

POSS W/ INT. DIS HEROIN | | 43550

POSS W/ INTENT | | 10233

POSS W/ INTENT । | 10573

POSS W/ INTENT I। 11564

POSS W/ INTENT I| 2A0696

POSS W/ INTENT I | 30233

POSS W/ INTENT || 43550

POSS W/ INTENT -COCAINE | | 11118

POSS W/ INTENT CDS I I 30233

POSS W/ INTENT CDS । । 43550

POSS W/ INTENT COCAINE | | 11119

POSS W/ INTENT COCAINE | | 30233

POSS W/ INTENT DISTRIBUTE MARI | | 10573

POSS W/ INTENT HEROIN | | 11119

POSS W/ INTENT HEROIN I | 11610

POSS W/ INTENT HEROIN I I 2A0696

POSS W/ INTENT HEROIN I I 30233

POSS W/ INTENT HEROIN | | 43550

POSS W/ INTENT HEROIN AND COCA I | 20696

POSS W/ INTENT MARIJUANA | | 10573

POSS W/ INTENT MJ || 2A3550

POSS W/ INTENT TO DIST I I 11118

POSS W/ INTENT TO DIST HERION I I 30233

POSS W/ INTENT TO DIST HEROIN || 43550 
POSS W/ INTENT TO DIST MARIJUA | | 10233 POSS W/ INTENT TO DISTRIBUTE | | 11119 POSS W/ MARIJUANA I I 10233

POSS W/ MARIJUANA I | 2A3550

POSS W/COCAIE II 43550

POSS W/COCAINE || 43550

POSS W/HERION I| 43550

POSS W/I II 43550

POSS W/I CDS ।| 10233

POSS W/I CDS || 30233

POSS W/I COC II 43550

POSS W/I COCAINE || 10493

POSS W/I COCAINE || 11111

POSS W/I COCAINE || 11119

POSS W/I COCAINE || 20696

POSS W/I COCAINE || 2C0696

POSS W/I COCAINE || 30233

POSS W/I COCAINE I| 43550

POSS W/I DIST COC I | 10881

POSS W/I DIST COCAINE || 30233

POSS W/I DIST CRACK COCAINE || 30233

POSS W/I DIST NARC MARIJUANA I | 10573

POSS W/I HEOIN I I 20696

POSS W/I HERION । | 43550

POSS W/I HEROIN । | 10233

POSS W/I HEROIN II 10660

POSS W/I HEROIN । | 10881

POSS W/I HEROIN । | 11111

POSS W/I HEROIN । | 11119

POSS W/I HEROIN I I 20696

POSS W/I HEROIN I I 2A0696

POSS W/I HEROIN II 30233

POSS W/I HEROIN II 43550

POSS W/I MAR I I 10573

POSS W/I MARIJUANA | | 10233

POSS W/I MARIJUANA I I 10493

POSS W/I MARIJUANA I I 10573

POSS W/I MARIJUANA I| 30233

POSS W/I MARIJUANA I | 43550

POSS W/I MARJUANA | | 10233

POSS W/I MARUJUANA || 10233

POSS W/I MARUJUANA I I 10573

POSS W/I TO DISTR I। 2A0696

POSS W/I TO DISTR I I 30233

POSS W/IND I| 30233

POSS W/INENT TO DISTRIBUTE | | 11111

POSS W/INT || 10233

POSS W/INT || 10487

POSS W/INT || 10573

POSS W/INT || 10881

POSS W/INT I| 11119

POSS W/INT I| 11564

POSS W/INT I| 11635

POSS W/INT I| 2A0696

POSS W/INT I| 30233

POSS W/INT I| 43550

POSS W/INT CDS || 2A0696

POSS W/INT CDS | | 30233

POSS W/INT CO I I 30233

POSS W/INT COCAINE || 10881 
POSS W/INT COCAINE || 11111 POSS W/INT COCAINE || 2A0696 POSS W/INT COCAINE || 30233 POSS W/INT COCAINE || 43550 POSS W/INT COCAINE/ HEROIN || 20696 POSS W/INT COCAINE/ HEROIN || 30233 POSS W/INT DIST । I 10573

POSS W/INT DIST । | 30233

POSS W/INT DIST । | 43550

POSS W/INT DIST COCAINE || 20696 POSS W/INT DIST COCAINE I | 30233 POSS W/INT DIST ECSTASY | | 30233 POSS W/INT DIST HEROIN || 2A0696 POSS W/INT DIST HEROIN | | 30233 POSS W/INT DIST MET. I I 30233 POSS W/INT HERION I| 2A0696 POSS W/INT HEROIN I I 10233 POSS W/INT HEROIN । | 10881 POSS W/INT HEROIN । | 11118 POSS W/INT HEROIN ।| 1 C1118 POSS W/INT HEROIN II 20696 POSS W/INT HEROIN I| 2A0696 POSS W/INT HEROIN I। 2C0696 POSS W/INT HEROIN || 30233 POSS W/INT HEROIN I | 43550 POSS W/INT MARI I I 10573 POSS W/INT MARIJUANA । । POSS W/INT MARIJUANA || 10233 POSS W/INT MARIJUANA | | 10573 POSS W/INT MARIJUANA I| 2A3550 POSS W/INT MARIJUANA | | 30233 POSS W/INT MARIJUANA | | 43550 POSS W/INT MJ I| 10573

POSS W/INT TO DIST । ।

POSS W/INT TO DIST || 11111

POSS W/INT. II 11119

POSS W/INT. II 2A0696

POSS W/INT. II 43550

POSS W/INT. MARIJUANA || 10233

POSS W/INT.COCAINE | | 43550

POSS W/INTE | | 10573

POSS W/INTENT || 10233

POSS W/INTENT || 10573

POSS W/INTENT I I 2A0696

POSS W/INTENT || 30233

POSS W/INTENT || 43550

POSS W/INTENT CDS | | 30233

POSS W/INTENT CDS I। 43550

POSS W/INTENT COCAINE || 11111 POSS W/INTENT COCAINE || 20696 POSS W/INTENT COCAINE | | 30233 POSS W/INTENT COCAINE | | 43550 POSS W/INTENT DIST । I 30233 POSS W/INTENT DIST COCAINE || 30233 POSS W/INTENT HEROIN | | 10690 POSS W/INTENT HEROIN I। 10881 POSS W/INTENT HEROIN || 11111 POSS W/INTENT HEROIN I I 11119 POSS W/INTENT HEROIN I| 2A0696 POSS W/INTENT HEROIN || 30233 
POSS W/INTENT HEROIN | | 43550

POSS W/INTENT MARIJUANA || 10233

POSS W/INTENT MARIJUANA | | 10573

POSS W/INTENT MARIJUANA | | 43550

POSS W/INTENT NOT MARIJUANA | | 43550

POSS W/INTENT PERCOCET | | 10493

POSS W/INTENT TO DIST | | 10881

POSS W/INTENT TO DIST । I 11111

POSS W/INTENT TO DIST | | 11119

POSS W/INTENT TO DIST COCAINE || 11119

POSS W/INTENT TO DIST COCAINE I| 30233

POSS W/INTENT TO DIST HEROIN । | 43550

POSS W/INTENT TO DISTRIBUTE | | 11119

POSS W/INTENT TO DISTRIBUTE | । 1A1118

POSS W/INTENT TO DISTRIBUTE CD || 11119

POSS W/INTENT TO DISTRIBUTE CO || 30233

POSS W/NT || 30233

POSS WID I | 43550

POSS WID COCAINE I| 30233

POSS WID HERION I| 2A0696

POSS WITH COCAINE II 30233

POSS WITH INT I | 30233

POSS WITH INT COCAINE || 11119

POSS WITH INT COCAINE || 30233

POSS WITH INT COCAINE || 43550

POSS WITH INT HEROIN I I 30233

POSS WITH INT MARIJUANA | | 30233

POSS WITH INT TO DIST HEROIN | | 30233

POSS WITH INT TO DISTRIBUTE HE | | 30233

POSS WITH INT. CDS || 43550

POSS WITH INTENT || 10493

POSS WITH INTENT || 10573

POSS WITH INTENT II 10881

POSS WITH INTENT I। 11119

POSS WITH INTENT || 20696

POSS WITH INTENT || 2A0696

POSS WITH INTENT I| 30233

POSS WITH INTENT । । 43550

POSS WITH INTENT CDS || 10493

POSS WITH INTENT CDS || 2A0696

POSS WITH INTENT CDS I | 30233

POSS WITH INTENT COCAINE || 11111

POSS WITH INTENT COCAINE || 11119

POSS WITH INTENT COCAINE I | 30233

POSS WITH INTENT COCAINE || 43550

POSS WITH INTENT CRACK COCANE || 43550

POSS WITH INTENT DIST. MARIJUA I| 2A3550

POSS WITH INTENT HERION | | 10573

POSS WITH INTENT HEROIN || 11119

POSS WITH INTENT HEROIN || 30233

POSS WITH INTENT HEROIN I | 43550

POSS WITH INTENT MARIJUANA || 10233

POSS WITH INTENT MARIJUANA || 10573

POSS WITH INTENT MARJUANA I I 10573

POSS WITH INTENT TO DISTRIBUTE || 10573

POSS WITH INTENT TO DISTRIBUTE || 11119

POSS WITH INTENT TO DISTRIBUTE || 30233

POSS WITH INTNENT II 10233

POSS WITH THE INTENT I| 30233

POSS WITH THE INTENT TO DIST || 10573 
POSS WITH THE INTENT TO DIST || 30233

POSS XANAX || 43550

POSS-CDS || 11119

POSS-CDS COCAINE I| 43550

POSS-COCAINE || 43550

POSS-HEROIN || 43550

POSS, W/ INTENT HEROIN I I 20696

POSS: HEROIN I| 43550

POSS: MARIJUANA || 10881

POSS: WITH INTENT TO DISTRIBUT I I 10573

POSS. II 43550

POSS. CDS || 10233

POSS. CDS I| 2A0696

POSS. CDS I| 2A3550

POSS. CDS I| 30233

POSS. CDS || 43550

POSS. CDS VIOLATION || 43550

POSS. CDS-HEROIN I| 43550

POSS. COCAINE I| 20696

POSS. COCAINE || 2A0696

POSS. COCAINE || 30233

POSS. COCAINE I| 43550

POSS. COCAINE/HEROIN || 43550

POSS. COCAINE/HEROIN || 43550

POSS. HERION II 2A0696

POSS. HERION || 43550

POSS. HERION \& COCAINE || 43550

POSS. HEROIN || 20696

POSS. HEROIN I| 2A0696

POSS. HEROIN || 30233

POSS. HEROIN || 43550

POSS. HEROIN || 53550

POSS. HEROIN/ COCAINE I | 30233

POSS. HEROIN/COCAINE || 43550

POSS. HEROIN/COCAINE || 30233

POSS. HEROINE I| 30233

POSS. INT COCAINE I| 30233

POSS. INT HEROIN || 10881

POSS. INT HEROIN || 30233

POSS. MARIJAUNA II 43550

POSS. MARIJAUNA W/INTENT || 10233

POSS. MARIJUANA || 2A3550

POSS. MARIJUANA || 43550

POSS. OF CDS I| 43550

POSS. OF ALPRAZOLOM || 43550

POSS. OF CDS || 2A0696

POSS. OF CDS I| 43550

POSS. OF COCAINE I| 20696

POSS. OF COCAINE || 2A0696

POSS. OF COCAINE || 30233

POSS. OF COCAINE II 43550

POSS. OF CONTRABAND || 11835

POSS. OF ECSTASY || 43550

POSS. OF HERION || 43550

POSS. OF HEROIN II 2A0696

POSS. OF HEROIN I| 30233

POSS. OF HEROIN ।| 43550

POSS. OF OXYCODONE I| 43550

POSS. OXYCODONE II 43550

POSS. OXYCONTIN || 30233 
POSS. PERCOCETS || 43550

POSS. W. INTENT TO DISTRIBUTE || 43550

POSS. W./INT I I 1 C1118

POSS. W./INT COCAINE || 43550

POSS. W./INT. COCAINE || 30233

POSS. W./INT. COCAINE I। 43550

POSS. W.INT HEROIN II 43550

POSS. W/ INT COCAINE | | 30233

POSS. W/ INT COCAINE || 43550

POSS. W/ INT HEROIN I I 30233

POSS. W/ INT. DIST COCAINE I I 30233

POSS. W/ INT.HEROIN I | 30233

POSS. W/ INTENT / DISTRIB. I I 43550

POSS. W/ INTENT । | 10573

POSS. W/ INTENT । | 30233

POSS. W/ INTENT COCAINE || 30233

POSS. W/ INTENT HEROIN | | 43550

POSS. W/I I| 10573

POSS. W/I I| 2A3550

POSS. W/I COCAINE || 30233

POSS. W/I COCOAINE || 30233

POSS. W/I HEROIN II 11118

POSS. W/I MARIJUANA || 10573

POSS. W/I MARIJUANA || 2A3550

POSS. W/I/DIST. HEROIN I| 30233

POSS. W/INT I| 2A0696

POSS. W/INT I| 30233

POSS. W/INT I| 43550

POSS. W/INT CDS I| 11111

POSS. W/INT COCAINE || 30233

POSS. W/INT COCAINE || 43550

POSS. W/INT COCAINE, HEROIN | | 11119

POSS. W/INT HEROIN II 11111

POSS. W/INT HEROIN । I 11118

POSS. W/INT HEROIN || 2A0696

POSS. W/INT HEROIN I I 30233

POSS. W/INT HEROIN/COCAINE || 30233

POSS. W/INT MOLLIE || 11111

POSS. W/INT. I। 20696

POSS. W/INT. II 2A0696

POSS. W/INT. I I 30233

POSS. W/INT. CDS || 11119

POSS. W/INT. COCAINE I| 1A1118

POSS. W/INT. COCAINE II 2C0696

POSS. W/INT. HEROIN II 20696

POSS. W/INT. MARI || 2A3550

Poss. W/Int. Marijuana I।

POSS. W/INTENT I| 11111

POSS. W/INTENT CDS | | 10573

POSS. W/INTENT COCAINE || 10881

POSS. WINT COCAINE I| 2C0696

POSS. WITH INT I| 11119

POSS. WITH INT COCAINE || 2A0696

POSS. WITH INT COCAINE || 43550

POSS. WITH INT TO DISTR | | 43550

POSS. WITH INT. COCAINE || 43550

POSS. WITH INT. ECSTASY || 43550

POSS. WITH INT. HEROIN || 30233

POSS. WITH INTENT I| 30233

POSS. WITH INTENT CDS | | 30233 
POSS. WITH INTENT COCAINE | | 30233

POSS. WITH INTENT HEROIN || 43550

POSS. WITH INTENT MARIJUANA I| 2A3550

POSS. WITH INTENT TO DISTRIBUT || 2A0696

POSS.CDS I| 43550

POSS.CDS/HEROIN I| 30233

POSS.COCAINE || 43550

POSS.COCAINE || 53550

POSS.COCAINE/HEROIN || 43550

POSS.HERION I| 43550

POSS.HEROIN II 43550

POSS.OF COCAINE I| 43550

POSS.PARAPHERNALIA || 53550

POSS.SUBOXONE I| 43550

POSS.W.INT HEROIN || 30233

POSS.W.INT. COCAINE || 2A0696

POSS.W.INT. COCAINE || 30233

POSS.W.INT. COCAINE/HEROIN || 43550

POSS.W.INT. HEROIN || 10881

POSS.W.INT. HEROIN/COCAINE || 30233

POSS.W.INT.COCAINE || 2A0696

POSS.W.INT.COCAINE || 30233

POSS.W.INT.COCAINE || 43550

POSS.W.INT.COCAINE/HEROIN || 30233

POSS.W.INT.HEROIN/COCAINE || 30233

POSS.W.INT.MARIJUANA | | 2A3550

POSS.W.INT/HEROIN/COCAINE || 43550

POSS.W/ INTENT II 30233

POSS.W/IDIST. I I 30233

POSS.W/INT DIST.COCAINE || 2A0696

POSS.W/INT DIST.HASHISH || 10573

POSS.W/INT DIST/HEROIN || 30233

POSS.W/INT HEROIN II 2C0696

POSS.W/INT HEROIN I। 43550

POSS.W/INT. COCAINE || 1A1118

POSS.W/INT. DIST.HEROIN I| 20696

POSS.W/INT. DIST.HEROIN || 30233

POSS.W/INT. HEROIN | | 30233

POSS.W/INT.COCAINE || 2A0696

POSS.W/INT.DIST HASHISH || 10573

POSS.W/INT.DIST HEROIN || 30233

POSS.W/INT.DIST. HEROIN I| 43550

POSS.W/INT.DIST.HEROIN || 10233

POSS.W/INT.DIST.HEROIN || 30233

POSS.W/INT.DIST.HEROIN || 43550

POSS.W/INT.DIST/HEROIN || 30233

POSS.W/INT/DIST.HEROIN || 43550

POSS.W/INTENT I| 20696

POSS.W/INTENT || 2A3550

POSS.WINT. HEROIN || 30233

POSS.WINT.DIST HEROIN | | 30233

POSS.WINT.DIST.COCAINE | | 30233

POSS/HEROIN II 30233

POSS/ W INT DIST. I I 43550

POSS/ W/ INTENT COCAINE | I 11119

POSS/ W/I COCAINE II 43550

POSS/CDS VIOLATION I| 43550

POSS/COC II 43550

POSS/COCAINE II 43550

POSS/DIST || 43550 
POSS/HEROIN || 30233

POSS/HEROIN I| 43550

POSS/INT MARIJUANA I| 10233

POSS/PWID || 10573

POSS/W/INT MARIJUANA || 2A3550

POSS/WINT INT || 30233

POSSES CDS II 43550

POSSES W. INTENT TO DISTRIBUTR ॥ 10233

POSSES. CDS || 43550

POSSESION COCAINE || 43550

POSSESION HERION I| 43550

POSSESION HEROIN || 43550

POSSESION OF CDS || 2A0696

POSSESION OF CDS || 43550

POSSESION OF CDS NON MARIJUANA | | 43550

POSSESION OF CDS NOT MARIJUANA | | 43550

POSSESION OF CDS NOT MARIJUNA I | 43550

POSSESION OF COCAINE || 43550

POSSESION OF COCAINE W/ INTENT I| 30233

POSSESION OF HERION || 43550

POSSESION W INTENT TO DISTR. I । 10573

POSSESION W/ INTENT I I 43550

POSSESION W/ INTENT HEROIN | | 30233

POSSESION W/I DIST MARIJUANA || 10233

POSSESION W/I HEROIN I| 43550

POSSESION W/INTENT | | 43550

POSSESION W/INTENT CDS || 43550

POSSESION W/INTENT ECSTACY || 2A0696

POSSESION WITH INTENT I I 30233

POSSESION WITH INTENT COCAINE I| 43550

POSSESS CDS ॥ 43550

POSSESS CDS SCHED II || 43550

POSSESS CDS W/I DISTR I। 20696

POSSESSION I| 11118

POSSESSION || 30233

POSSESSION I| 43550

POSSESSION WITH INTENT || 10573

POSSESSION ALPRAZOLAM || 1A1118

POSSESSION ALPRAZOLAM || 43550

POSSESSION APLRAZOLAM || 43550

POSSESSION CDS I| 2C0696

POSSESSION CDS || 30233

POSSESSION CDS || 43550

POSSESSION CLONAZEPAM || 43550

POSSESSION COCAINE || 2A0696

POSSESSION COCAINE || 2C0696

POSSESSION COCAINE || 30233

POSSESSION COCAINE || 43550

POSSESSION CODEINE || 43550

POSSESSION CON I| 43550

POSSESSION HERION I| 43550

POSSESSION HEROIN I I 20696

POSSESSION HEROIN II 2A0696

POSSESSION HEROIN I I 30233

POSSESSION HEROIN II 43550

POSSESSION HEROIN || 53550

POSSESSION MARIJUANA || 23550

POSSESSION MARIJUANA | | 43550

POSSESSION MARIJUANA WITH INTE I | 23550

POSSESSION MDMA || 43550 
POSSESSION METHADONE || 43550 POSSESSION METHYLENEDIOXYMETHA || 43550 POSSESSION OF HEROIN I I 30233

POSSESSION OF CDS ।| 10908 POSSESSION OF CDS || 2A0696 POSSESSION OF CDS || 30233 POSSESSION OF CDS I। 43550 POSSESSION OF CDS (NOT MARIJUA || 43550 POSSESSION OF CDS NOT MARIJUAN I | 43550 POSSESSION OF CDS WITH THE INT I | 11119 POSSESSION OF COAINE || 43550 POSSESSION OF COCAINE || 20696 POSSESSION OF COCAINE || 2C0696 POSSESSION OF COCAINE || 30233 POSSESSION OF COCAINE || 43550 POSSESSION OF COCAINE || 53550 POSSESSION OF COCAINE W/INT || 43550 POSSESSION OF CONTRABAND || 11835 POSSESSION OF CONTROLLED SUBST || 43550 POSSESSION OF DRUG PARAPHERNAL || 53550 POSSESSION OF ECSTASY I | 43550 POSSESSION OF HERION II 11118 POSSESSION OF HERION | | 30233 POSSESSION OF HERION || 43550 POSSESSION OF HEROIN I I 11119 POSSESSION OF HEROIN || 2A0696 POSSESSION OF HEROIN I 30233 POSSESSION OF HEROIN || 43550 POSSESSION OF HEROIN I | 53550 POSSESSION OF MARIJUANA || 10233 POSSESSION OF MARIJUANA I| 2A3550 POSSESSION OF MARIJUANA I| 30233 POSSESSION OF MARIJUANA || 43550 POSSESSION OF METHYLENEDIOXYME II 43550 POSSESSION OF OXYCODONE || 43550 POSSESSION OF PARAPHERNALIA I| 53550 POSSESSION OF SCHEDULE I NARCO | | 43550 POSSESSION OF SUBOXONE || 43550 POSSESSION OF SUBOXONE | | 4A3550 POSSESSION OXYCODONE || 43550 POSSESSION OXYCONTIN || 43550 POSSESSION PARAPHERNALIA || 53550 POSSESSION W INTENT I| 20696 POSSESSION W/ INT TO DISTRIBUT | | 10573 POSSESSION W/ INTENT I I 2A0696 POSSESSION W/ INTENT || 30233 POSSESSION W/ INTENT TO DIST. I I 10573 POSSESSION W/I ALPRAZOLAM || 10233 POSSESSION W/I ALPRAZOLAM || 23550 POSSESSION W/I CDS | | 10233 POSSESSION W/I CDS || 11118 POSSESSION W/ICDS || 23550 POSSESSION W/I CDS I| 2A3550 POSSESSION W/I CDS HEROIN | | 30233 POSSESSION W/I COCAINE || 30233 POSSESSION W/I COCAINE || 43550 POSSESSION W/I DIST. COCAINE || 11119 POSSESSION W/I DISTRIBUTION || 10233 POSSESSION W/I DISTRIBUTION || 30233 POSSESSION W/I HEROIN I। 20696 
POSSESSION W/I HEROIN || 2A0696 POSSESSION W/I HEROIN || 30233 POSSESSION W/I HEROIN || 43550 POSSESSION W/I HEROIN/COCAINE || 30233 POSSESSION W/I INTENT TO DISTR || 11119 POSSESSION W/I MARIJUANA | | 10573 POSSESSION W/I TO DIST. I I 10233

Possession W/I To Distribute R I | POSSESSION W/INT || 11119 POSSESSION W/INT I| 43550 POSSESSION W/INT DISTRUBUTE | | 10573 POSSESSION W/INT HERION || 10493 POSSESSION W/INT MARIJUANA | | 10573 POSSESSION W/INT/DIST COCAINE || 43550 POSSESSION W/INTENT HEROIN | | 33550 POSSESSION W/INTENT TO POSSESS || 10573 POSSESSION WITH INT. TO DISTRI । | 11119 POSSESSION WITH INTENT || 10233 POSSESSION WITH INTENT || 10493 POSSESSION WITH INTENT || 10573 POSSESSION WITH INTENT | | 10908 POSSESSION WITH INTENT I | 11118 POSSESSION WITH INTENT || 11119 POSSESSION WITH INTENT || 11564 POSSESSION WITH INTENT || 23550 POSSESSION WITH INTENT || 2A0696 POSSESSION WITH INTENT || 2A3550 POSSESSION WITH INTENT || 30233 POSSESSION WITH INTENT || 43550 POSSESSION WITH INTENT AND DIS | । 30233 POSSESSION WITH INTENT CDS || 11564 POSSESSION WITH INTENT COCAINE || 10233 POSSESSION WITH INTENT COCAINE || 30233 POSSESSION WITH INTENT COCAINE || 43550 POSSESSION WITH INTENT CRACK C || 2A0696 POSSESSION WITH INTENT DISTRIB || 30233 POSSESSION WITH INTENT HEROIN | | 43550 POSSESSION WITH INTENT MARIJUA | | 10233 POSSESSION WITH INTENT MARIJUA | | 10573 POSSESSION WITH INTENT MARIJUA | | 2A3550 POSSESSION WITH INTENT TO DIST | | 10573 POSSESSION WITH INTENT TO DIST | | 10880 POSSESSION WITH INTENT TO DIST I | 11119 POSSESSION WITH INTENT TO DIST I | 20696 POSSESSION WITH INTENT TO DIST I I 21085 POSSESSION WITH INTENT TO DIST || 23550 POSSESSION WITH INTENT TO DIST || 30233 POSSESSION WITH INTENT TO DIST I | 43550 POSSESSION XANAX || 43550 POSSESSION-COCAINE || 43550 POSSESSION-HEROIN I| 43550 POSSESSION-NARCOTICS I| 43550 POSSESSION, NOT MARIJUANA || 30233 POSSESSION/COCAINE I| 43550 POSSESSION/DISTRIBUTION || 2A0696 POSSESSION/HEROIN I| 43550 POSSESSION/INTENT HEROIN || 11119 POSSESSION/INTENT-COCAINE || 20696 POSSESSION/INTENT-COCAINE || 43550 POSSESSION/INTENT-HEROIN || 30233 
POSSESSION/W INTENT HEROIN || 30233

POSSESSIONO OF HEROIN || 43550

POSSSESSION W/I HEROIN || 43550

POSSSSION I| 43550

POSSW/I HEROIN I | 30233

POSSW/INT MARIJUANA | | 10573

POSSW/INT.DIST.HEROIN || 30233

POSSW/INTENT MARIJUANA | | 30233

PRACTICING PHARMACEUTICALS I।

PRES ILL POS W/IN DIST I| 10264

PRESC OBTN CONCEAL/FALSE INFO | | 10266

PRESC:ILLEGAL MANUFAC || 10010

PROHIBTIED PERSON IN POSSESION || 11118

PROSTITUTION || 10233

PROSTITUTION II 43550

PROSTITUTION || 53550

PSID HEROIN || 11119

PSSESSION WITH INTENT TO DISTR | | 30233

PW INT COCAINE II 43550

PW.INT. HEROIN || 2C0696

PWI | | 10233

PWI || 10573

PWI || 10881

PWI || 20696

PWI I| 23550

PWI || 2A3550

PWI || 30233

PWI || 43550

PWI COCAINE || 30233

PWI COCAINE/HEROIN || 30233

PWI CDS || 10233

PWI CDS || 10573

PWI CDS I| 11111

PWI CDS I| 11119

PWI CDS || 2A0696

PWI CDS I| 30233

PWI CDS II 43550

PWI CDS I| 53550

PWI CDS - NOT MARIJUANA || 1C1118

PWI CDS OXYCODONE I | 43550

PWI CLONIDINE || 43550

PWI COC I| 43550

PWI COCAIN/HEROIN | | 30233

PWI COCAINE I| 10233

PWI COCAINE || 10573

PWI COCAINE || 10881

PWI COCAINE || 11111

PWI COCAINE II 11118

PWI COCAINE || 11119

PWI COCAINE || 20696

PWI COCAINE I| 2A0696

PWI COCAINE || 2C0696

PWI COCAINE I| 30233

PWI COCAINE I। 43550

PWI COCAINE HEROIN I| 2A0696

PWI COCAINE/HEROIN I I 30233

PWI COCAINE/HEROIN । | 43550

PWI CRACK I| 43550

PWI DIST COCAINE || 30233

PWI DIST HERION । | 43550 
PWI DIST HEROIN || 30233

PWI HEOIN || 30233

PWI HERION || 10881

PWI HERION || 2A0696

PWI HERION || 30233

PWI HERION || 43550

PWI HEROIN I | 10881

PWI HEROIN || 11111

PWI HEROIN || 11119

PWI HEROIN II 20696

PWI HEROIN || 2A0696

PWI HEROIN I| 2C0696

PWI HEROIN || 30233

PWI HEROIN || 43550

PWI HEROIN | | 53550

PWI HEROIN AND COCAINE || 30233

PWI HEROIN/ COCAINE || 30233

PWI HEROIN/COCAINE || 2A0696

PWI HEROIN/COCAINE I | 30233

PWI HEROIN/COCAINE | | 43550

PWI MAIJUANA || 10573

PWI MARIHUANA || 10233

PWI MARIJUANA || 10233

PWI MARIJUANA || 10573

PWI MARIJUANA || 11564

PWI MARIJUANA | I 11635

PWI MARIJUANA || 23550

PWI MARIJUANA || 2A3550

PWI MARIJUANA || 43550

PWI MARIJUANA I| 53550

PWI MARIJUANA/COCAINE || 30233

PWI MARIJUNA || 10573

PWI MARIJUNA || 11564

PWI MARJUANA | | 10573

PWI MJ || 10233

PWI MJ || 10573

PWI MJ I| 2A3550

PWI NARC || 10881

PWI NOT MARIJUANA | | 1A1118

PWI OXYCODONE || 30233

PWI OXYCODONE I| 43550

PWI PERCOCET । | 43550

PWI XANAX || 43550

PWI XANEX I| 10233

PWI-COCAINE || 43550

PWI-HERION/COCAINE || 43550

PWI-HEROIN || 43550

PWI-MARIJUANA || 10233

PWI-MARIJUANA || 10573

PWI-MARIJUANA || 11111

PWI-XANEX II 43550

PWI/ CDS || 2A0696

PWI/ HEROIN I| 2A0696

PWI/ HEROIN || 43550

PWI/HEROIN I। 43550

PWID | |

PWID || 10233

PWID || 10493

PWID || 10573

PWID || 10881 


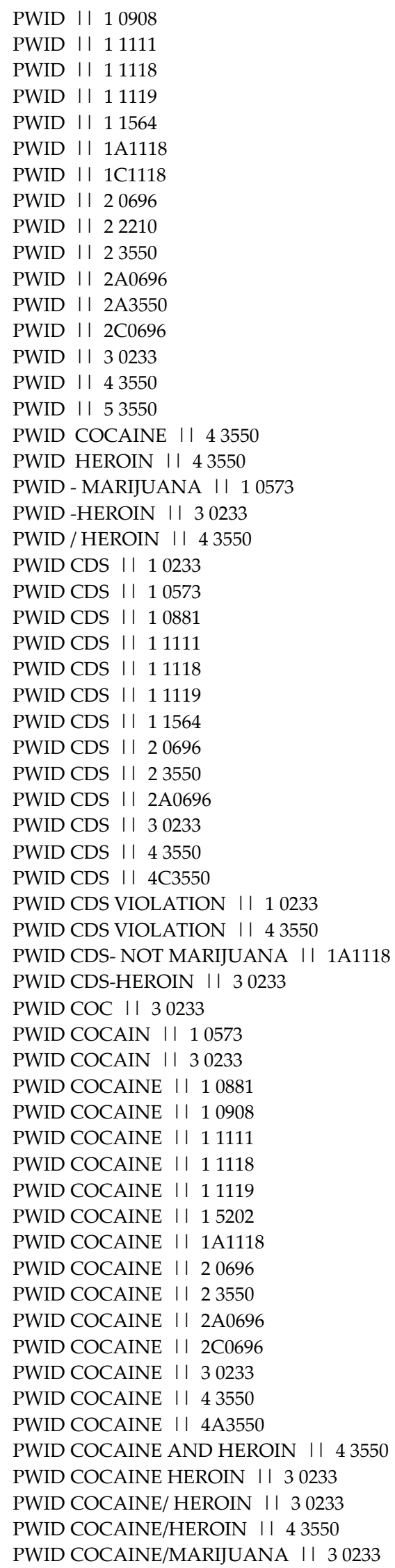


PWID COCAINE\&HEROIN || 30233

PWID COCIANE/HEROIN || 11119

PWID COCOAINE || 43550

PWID ECSTASY || 30233

PWID HERION || 10493

PWID HERION || 11106

PWID HERION || 11119

PWID HERION || 20696

PWID HERION || 2A0696

PWID HERION II 30233

PWID HERION I | 43550

PWID HERION/COCAINE || 43550

PWID HEROIN || 10233

PWID HEROIN || 10493

PWID HEROIN || 10573

PWID HEROIN || 10881

PWID HEROIN । | 10908

PWID HEROIN I| 11111

PWID HEROIN || 11118

PWID HEROIN || 11119

PWID HEROIN I| 1A1118

PWID HEROIN I| 20696

PWID HEROIN || 23550

PWID HEROIN || 2A0696

PWID HEROIN I I 30233

PWID HEROIN I | 43550

PWID HEROIN I| 53550

PWID HEROIN / COCAINE | | 30233

PWID HEROIN AND COCAINE || 30233

PWID HEROIN/ COCAINE || 11119

PWID HEROIN/ FENTENYL || 10881

PWID HEROIN/COCAINE || 30233

PWID HEROIN/OXYCODONE I| 30233

PWID HEROINE/COCAINE I। 43550

PWID MAR || 10233

PWID MARIHUANA | | 23550

PWID MARIJAUNA | | 10573

PWID MARIJAUNA || 11564

PWID MARIJUANA || 10233

PWID MARIJUANA || 10573

PWID MARIJUANA | 11119

PWID MARIJUANA || 11564

PWID MARIJUANA || 11635

PWID MARIJUANA | | 14801

PWID MARIJUANA II 23550

PWID MARIJUANA || 2A0696

PWID MARIJUANA || 2A3550

PWID MARIJUANA I | 30233

PWID MARIJUANA || 43550

PWID MARIJUANNA || 10573

PWID MJ I | 10573

PWID MOLLY || 2A0696

PWID MOLLY/ MJ || 30233

PWID MOT MARIJUANA | 11119

PWID NOT MARIJUANA | | 11118

PWID OF CDS || 30233

PWID OXY || 43550

PWID OXYCODINE || 20696

PWID OXYCODON || 43550

PWID OXYCODONE || 30233 
PWID OXYCODONE || 43550

PWID POSS HERION I| 30233

PWID SUBOXNE || 43550

PWID SUBOXONE || 2A3550

PWID TRAMADOL ।| 23550

PWID XANAX || 43550

PWID- HERION I। 43550

PWID- HEROIN I| 20696

PWID- HEROIN || 23030

PWID- HEROIN I| 2A0696

PWID- HEROIN I| 30233

PWID- NOT MARIJUANA || 30233

PWID-CDS || 11564

PWID-CDS || 30233

PWID-COCAINE || 2A0696

PWID-COCAINE I| 30233

PWID-COCAINE || 43550

PWID-HERION I। 43550

PWID-HEROIN || 30233

PWID-HEROIN || 43550

PWID-MARIJUANA || 10233

PWID-MARIJUANA || 10573

PWID-NARCOTICS || 10233

PWID-NARCOTICS || 2A0696

PWID-NARCOTICS || 43550

PWID. MARIJUANA II 11564

PWID/COCAINE || 2A0696

PWID/COCAINE || 30233

PWID/COCAINE-HEROIN || 11119

PWID/HEROIN || 11119

PWID/HEROIN || 30233

PWID/MAR || 10573

PWID/TRAFFIC II 11111

PWIDCOCAINE/MARIJUANA || 30233

PWIN HEROIN || 10881

PWIND MARIJUANA || 11635

PWINT COCAINE | | 30233

PWINT. COCAINE || 2C0696

PWINT.HEROIN || 43550

PWIT COCAINE II 20696

PWIT CDS || 11118

PWIT CDS MARIJUANA || 2A3550

PWIT CDS NOT MARIJAUANA | | 11119

PWIT CDS NOT MARIJUANA I| 10600

PWIT CDS NOT MARIJUANA || 11111

PWIT CDS NOT MARIJUANA || 11119

PWIT CDS NOT MARIJUANA | IA1118

PWIT CDS NOT MARIJUANA I | 2A0696

PWIT. COCAINE || 20696

PWIT. HEROIN || 2A0696

PWIT.COCAINE I| 20696

PWIT $>$ HEOIN || 43550

PWIW HEROIN I। 43550

PWRB HEROINE || 11118

RESIST ARREST I। 43550

SEARCH \& SEIZURE/CDS || 43550

SEARCH+SEIZURE CDS || 10493

SGV || 30233

SUSPENDED LICENSE | | 43550

TRAFFIC I| 43550 
TRAFFIC PWID || 11119

TRESPASS / CDS POSS I। 43550

Trespass-Posted Property, Cds Dist Heroin I| 22210

TRESPASS/CDS PWID I। 43550

TRESPASS/CDS VIOLATION I| 43550

TRESPASSING || 11119

TRESPASSING || 30233

TRESPASSING || 43550

TRESPASSING || 53550

TRESSPASS I| 53550

TRESSPASSING || 43550

UNATHORIZED VEH REG PLATE W/IN । ।

UNLICENSED PHARMACY | |

UNLICENSED PHARMACY || 2A0696

VDS POSS W/ INT || 43550

VDS VIOLATION I| 43550

VIOLATION CDS || 30233

VIOLATION CDS I| 43550

VIOLATION OF CDS I| 43550

WITNESS INTIMIDATION ｜～ 23550

\section{Court Non-Compliance}

2ND ESCAPE || 2A1020

A Order Of This Court II

Alc. Bev./Intox:Endanger, Violation Of Probation I| 14200

An Order Of Court II

An Order Of The Court II

An Order Of This Court II

ATT VERBAL EXTORT/THRT INJURY | | 1A1704

ATT-ESCAPE - FIRST DEGREE || 2A1010

ATT-ESCAPE - SECOND DEGREE || 2A1020

ATT-FAILURE TO APPEAR || 1A0077

Att-Failure To Appear, Att-Failure To Appear || 1 A0077

ATT-INDUCE/INHIBIT TESTIMONY || 1A0354

ATTEMPT ESCAPE || 21020

ATTEMPT ESCAPE || 21020

ATTEMPT-FTA/MISD DEF/WITNESS || 1A1475

ATTEMPTING TO ESCAPE || 21020

CON-FAILURE TO APPEAR I | 1 C0077

Con-Failure To Appear, Con-Failure To Appear I। $1 \mathrm{C} 0077$

CON-RETALIATE-WITNESS || 1C0356

Condition Of Probation II

Conditions Of Probation II

Contempt II

CONTEMPT ।।

CONTEMPT II 10087

DRIVING UNINSURED VEHICLE(FTA) । ।

DRIVING W/O LICENSE (FTA) I ।

DRIVING W/O LICENSE(FTA) I ।

ESCAPE II 10600

ESCAPE || 15505

ESCAPE || 2A1020

ESCAPE - 2ND DEG-COND OF RELS I। 10766

Escape - 2Nd Deg-Cond Of Rels, Escape - 2Nd Deg-Con I| 10766

Escape - 2Nd Deg-Cond Of Rels, Escape - 2Nd Deg-Cond I| 10766 ESCAPE - FIRST DEGREE I। 21010

Escape - First Degree, Escape - First Degree I| 21010

Escape - First Degree, Escape - First Degree I| 21015 
ESCAPE - SECOND DEGREE || 21020

ESCAPE - SECOND DEGREE || 21025

ESCAPE - SECOND DEGREE || 21030

Escape - Second Degree, Cds Violation II 21020

Escape - Second Degree, Disorderly II 21020

Escape - Second Degree, Escape - Second Degree I| 21020

Escape - Second Degree, Escape - Second Degree I| 21025

Escape - Second Degree, Resisting Arrest I| 21020

Escape - Second Degree, Trespassing I| 21020

ESCAPE FROM CUSTODY || 21020

ESCAPE- ATTEMPT I| 21020

Escape, Escape II 15505

ESCAPE/FALSE STATEMENT | | 2A1020

FAIL APPEAR-CITATION II 11476

FAIL TO APPEAR I |

FAIL TO FINE FINE । |

FAIL TO OBEY COURT ORDER I।

FAIL TO PAY FINE I I

Fail To Pay Fine II

FAIL TO PAY FINE । |

FAILE TO PAY FINE I।

Failing To Appear II

FAILING TO APPEAR I ।

FAILING TO OBEY COURT ORDER । |

FAILING TO OBEY ORDER OF COURT I ।

Failing To Pay Fine II

FAILTO PAY FINE I।

FAILURE TO APPEAR । ।

Failure To Appear II

FAILURE TO APPEAR I ।

FAILURE TO APPEAR | | 10077

FAILURE TO APPEAR || 20055

FAILURE TO APPEAR I| 690000

FAILURE TO APPEAR OR PAY FINE I I

Failure To Appear, Failure To Appear || 10077

Failure To Appear, Failure To Appear I| 690000

FAILURE TO APPEAR. I I

FAILURE TO APPEAR/PAY FINE I।

Failure To Apy Fine I।

FAILURE TO OBEY AN COURT ORDER I।

Failure To Obey Court Order II

FAILURE TO OBEY COURT ORDER I ।

FAILURE TO OBEY ORDER OF COURT I ।

Failure To Pay Fine II

FAILURE TO PAY FINE I।

Follow Condition Of Probation I।

Fta II

FTA $\mid 1$

FTA || 20050

FTA || 20055

FTA OR PAY FINE । ।

FTA SUBPOENA - SEC CORR SVC II 10656

FTA- SUSP LICENSE I ।

FTA/FELONY DEFENDANT | | 11474

FTA/FELONY DEFENDANT I| 15001

Fta/Felony Defendant, Fta/Felony Defendant II 11474

FTA/MISD DEF/WITNESS || 11475

Fta/Misd Def/Witness, Fta/Misd Def/Witness II 11475

FTA/MISDEMEANOR DEFENDANT I I 12474

Fta/Misdemeanor Defendant, Fta/Misdemeanor Defendan II 12474 
Fta/Misdemeanor Defendant, Fta/Misdemeanor Defendant || 12474

FUG FROM ALABAMA । |

FUG. FRM. GEORGIA । ।

FUGIIVE FROM PA I I

FUGIIVE FROM TEXAS I।

FUGITICE FROM PENNSYLVANIA । ।

FUGITITVE FROM JUSTICE - NEW J I ।

FUGITIV FROM PENNSYLVANIA | |

Fugitive ||

FUGITIVE I|

FUGITIVE FOR PENNSYLVANIA I।

FUGITIVE FROM JUSTICE - TEXAS I।

FUGITIVE FROM JUSTICE DELEWAR । ।

FUGITIVE FROM TEXAS । ।

FUGITIVE - VIRGINIA I |

FUGITIVE (NEW JERSEY) । |

FUGITIVE FOR ALABAMA । |

Fugitive For Delaware I I

FUGITIVE FOR NEW JERSEY | |

FUGITIVE FOR PA. I।

FUGITIVE FOR PENN I।

FUGITIVE FOR PENNSLYVANIA I |

Fugitive For Pennsylvania II

FUGITIVE FOR PENNSYLVANIA | |

FUGITIVE FOR VA । ।

FUGITIVE FOR VIRGINIA I।

FUGITIVE FORM JUSTICE I ।

FUGITIVE FORM PENNSYLVANIA । |

FUGITIVE FORM PENNSYVANIA । ।

FUGITIVE FR PA II

FUGITIVE FR DELAWARE । ।

FUGITIVE FR MICHIGAN । ।

Fugitive Fr Pa I।

FUGITIVE FR PA । ।

FUGITIVE FR PENNSYLANIA । |

Fugitive Fr Pennsylvania II

FUGITIVE FR PENNSYLVANIA । ।

FUGITIVE FR VA । ।

FUGITIVE FR VIRGINIA । ।

FUGITIVE FR VIRIGINIA । |

FUGITIVE FRM KENTUCKY । |

FUGITIVE FRO PA । ।

FUGITIVE FRO VIRGINIA । ।

FUGITIVE FRO WEST VA I।

FUGITIVE FROM GEORGIA I।

FUGITIVE FROM JUSTICE NYC । |

FUGITIVE FROM VA I।

Fugitive From Alabama I I

FUGITIVE FROM ALABAMA | |

FUGITIVE FROM ARIZONA । ।

Fugitive From Arkansas I I

FUGITIVE FROM BUCKS COUNTY PA I |

FUGITIVE FROM CA I।

Fugitive From California I।

FUGITIVE FROM CALIFORNIA । |

FUGITIVE FROM CAMDEN CO. SHERI I।

FUGITIVE FROM CHESTER CITY PA । ।

FUGITIVE FROM CHESTER CO PA I ।

FUGITIVE FROM CHESTERFIELD, VA I।

Fugitive From Colorado I I 
FUGITIVE FROM COLORADO । |

FUGITIVE FROM COMMONWEALTH PA I।

FUGITIVE FROM CONNECTICUT । |

FUGITIVE FROM CONNETICUT । ।

Fugitive From Dallas Tx I।

Fugitive From Delaware II

FUGITIVE FROM DELAWARE । |

FUGITIVE FROM DELAWARE COUNTY, I ।

FUGITIVE FROM DELEWARE । |

FUGITIVE FROM FAIRFAX,VA. I I

FUGITIVE FROM FL I ।

FUGITIVE FROM FLORDIA । |

Fugitive From Florida I I

FUGITIVE FROM FLORIDA । ।

FUGITIVE FROM GA I ।

Fugitive From Georgia II

FUGITIVE FROM GEORGIA । ।

FUGITIVE FROM GREENSVILLE. CO. I ।

Fugitive From Illinois I I

FUGITIVE FROM ILLINOIS | |

FUGITIVE FROM IN । ।

Fugitive From Indiana I |

FUGITIVE FROM INDIANA ||

FUGITIVE FROM JUSITCE - VIRGIN । ।

FUGITIVE FROM JUSTCE - PENNSYL I ।

FUGITIVE FROM JUSTIC I ।

Fugitive From Justice II

FUGITIVE FROM JUSTICE । ।

FUGITIVE FROM JUSTICE - ALABAM । ।

FUGITIVE FROM JUSTICE - ARIZON I।

FUGITIVE FROM JUSTICE - CALIFO । ।

FUGITIVE FROM JUSTICE - DELAWA I।

FUGITIVE FROM JUSTICE - DELWAR I I

FUGITIVE FROM JUSTICE - FLORID I ।

FUGITIVE FROM JUSTICE - GEORGI । ।

FUGITIVE FROM JUSTICE - INDIAN । ।

FUGITIVE FROM JUSTICE - KANSAS I ।

FUGITIVE FROM JUSTICE - KENTUC । । FUGITIVE FROM JUSTICE - LOUISA । ।

FUGITIVE FROM JUSTICE - MASSAC I । FUGITIVE FROM JUSTICE - MISSIS I ।

FUGITIVE FROM JUSTICE - MISSOU I । FUGITIVE FROM JUSTICE - NEW JE I । FUGITIVE FROM JUSTICE - NEW YO I। FUGITIVE FROM JUSTICE - NORTH I ।

FUGITIVE FROM JUSTICE - OHIO । ।

FUGITIVE FROM JUSTICE - OREGON I ।

FUGITIVE FROM JUSTICE - PA I ।

FUGITIVE FROM JUSTICE - PENNSL I । FUGITIVE FROM JUSTICE - PENNSY I । FUGITIVE FROM JUSTICE - SOUTH I। FUGITIVE FROM JUSTICE - TENNES I। FUGITIVE FROM JUSTICE - TEXAS I। FUGITIVE FROM JUSTICE - VIRGIN । । FUGITIVE FROM JUSTICE - WEST V I I FUGITIVE FROM JUSTICE -ARKANSA I । FUGITIVE FROM JUSTICE -NEW YOR I । FUGITIVE FROM JUSTICE -PENNSYL I I FUGITIVE FROM JUSTICE : PENNSY I ।

FUGITIVE FROM JUSTICE DELAWARE I। 
FUGITIVE FROM JUSTICE FLORIDA | । FUGITIVE FROM JUSTICE FROM PEN । । FUGITIVE FROM JUSTICE GEORGIA । । FUGITIVE FROM JUSTICE LOUISANN I । FUGITIVE FROM JUSTICE MISSOURI । । FUGITIVE FROM JUSTICE NEW YORK I । FUGITIVE FROM JUSTICE NJ I । FUGITIVE FROM JUSTICE NORTH CA I । FUGITIVE FROM JUSTICE PA । | FUGITIVE FROM JUSTICE PA. I। FUGITIVE FROM JUSTICE PENNSYLV I । FUGITIVE FROM JUSTICE PENNYLVA I। FUGITIVE FROM JUSTICE TEXAS । । FUGITIVE FROM JUSTICE VA । । FUGITIVE FROM JUSTICE VIRGIINI I । FUGITIVE FROM JUSTICE VIRGINIA । । FUGITIVE FROM JUSTICE- DELAWAR । । FUGITIVE FROM JUSTICE- FLORIDA I । Fugitive From Justice- Georgia I I FUGITIVE FROM JUSTICE- NEW YOR I । FUGITIVE FROM JUSTICE- NORTH C I । FUGITIVE FROM JUSTICE- PENNSYL I I FUGITIVE FROM JUSTICE- TEXAS । । FUGITIVE FROM JUSTICE- VA I। FUGITIVE FROM JUSTICE- VIRGINI । । FUGITIVE FROM JUSTICE-DELAWARE I । FUGITIVE FROM JUSTICE-FLORDIA । । FUGITIVE FROM JUSTICE-GEORGIA । । FUGITIVE FROM JUSTICE-NEW JERS । । FUGITIVE FROM JUSTICE-NEW MEXI I । FUGITIVE FROM JUSTICE-PA I । FUGITIVE FROM JUSTICE-PENNSYLV I । Fugitive From Justice-Virginia I I FUGITIVE FROM JUSTICE-VIRGINIA । । Fugitive From Justice-West Vir | | FUGITIVE FROM JUSTICE-WEST VIR I । FUGITIVE FROM JUSTICE, PENNSYL I । Fugitive From Justice,,Virgini I I FUGITIVE FROM JUSTICE: DE । । FUGITIVE FROM JUSTICE: DELAWAR । । FUGITIVE FROM JUSTICE: FLORIDA । | FUGITIVE FROM JUSTICE: IDAHO । | FUGITIVE FROM JUSTICE: NEW JER I । FUGITIVE FROM JUSTICE: NORTH C I । FUGITIVE FROM JUSTICE: PENNSYL । । FUGITIVE FROM JUSTICE: VA । । FUGITIVE FROM JUSTICE: VIRGINI । । FUGITIVE FROM JUSTICE:VIRGINIA । । FUGITIVE FROM JUSTICE..PA | । FUGITIVE FROM JUSTICE//PENNSYL । । FUGITIVE FROM JUSTICE/VIRGINIA । । FUGITIVE FROM JUTICE - PENNSYL । । Fugitive From Kansas I I FUGITIVE FROM KANSAS । । Fugitive From Kentucky I । FUGITIVE FROM KENTUCKY I |

FUGITIVE FROM LA । |

Fugitive From Loisiana I।

Fugitive From Louisiana II

FUGITIVE FROM LOUISIANA | | 


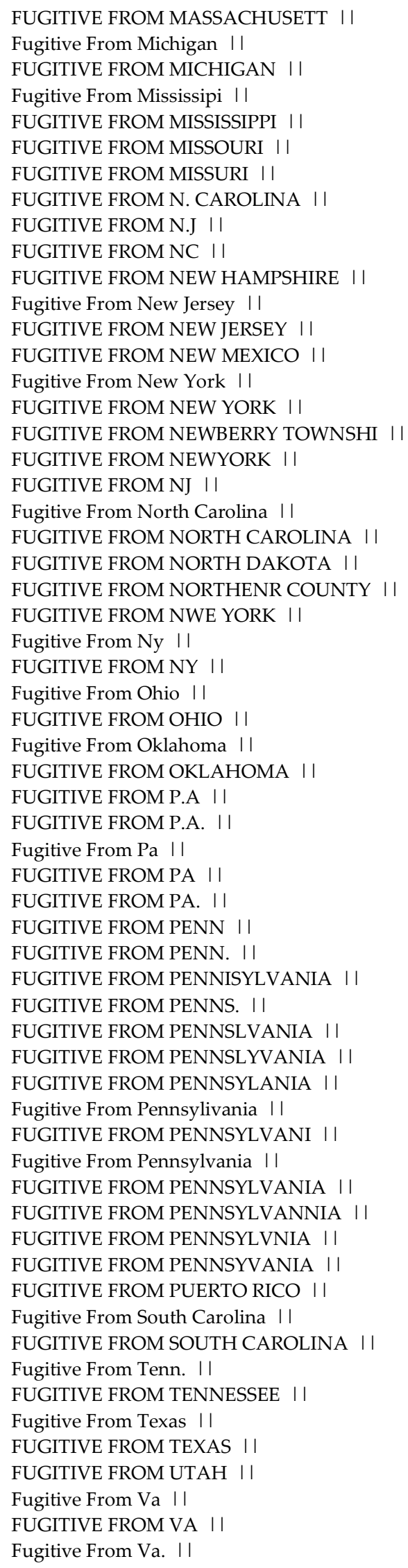


FUGITIVE FROM VA. I।

Fugitive From Virgina I।

FUGITIVE FROM VIRGINA । |

Fugitive From Virginia I I

FUGITIVE FROM VIRGINIA । |

FUGITIVE FROM VIRIGINA | |

FUGITIVE FROM VIRINIA | |

FUGITIVE FROM W.V । I

FUGITIVE FROM W.V. I।

FUGITIVE FROM WASHINGTON D.C. I ।

FUGITIVE FROM WASHINGTON DC I ।

Fugitive From West Viaginia I I

Fugitive From West Virginia । |

FUGITIVE FROM WEST VIRGINIA I।

FUGITIVE FROM WISCONSIN I I

FUGITIVE FROM YORK COUNTY SHER I ।

FUGITIVE FROM YORK COUTNY SHER I ।

Fugitive From York, Pa I I

FUGITIVE FROM- DELAWARE । ।

FUGITIVE FROMVIRGINIA । |

Fugitive In Virginia I I

FUGITIVE NORTH CAROLINA I |

Fugitive Of Delaware II

FUGITIVE OF PENNSYLVANIA | |

FUGITIVE OKLAHOMA I।

Fugitive Pa I|

FUGITIVE PA । |

Fugitive Pennsylvania | |

FUGITIVE PENNSYLVANIA । |

FUGITIVE ROM JUSTICE- KANSAS । ।

FUGITIVE SOUTH CAROLINA । |

FUGITIVE TENNESSEE । |

Fugitive Virgina II

FUGITIVE VIRGINIA । ।

Fugitive W Vir I I

FUGITIVE WARRANT I।

Fugitive- Pennsylvania II

Fugitive-Delaware II

FUGITIVE/ARIZONA । ।

FUGITIVE/VIRGINIA ।

FUGITIVI FROM VA I I

FUGITIVIE FROM CONNECTICUT । ।

FUGITIVR FROM JUSTICE VA I ।

Fugitivr From West Virginia I I

Fugittive From Florida II

FUGITVE FROM JUSTICE GEORGIA । ।

FUGITVE FROM JUSTICE VIRGINIA I ।

Fugitve From Kentucky I I

FUGITVE FROM PENNSYLVANIA | |

FUGITVE FROM VIRGINIA । |

Fugive From Illinois I |

FUGIVITIVE FROM PA I |

FUGIVITVE FROM PA I ।

FUGIVTIVE FROM VIRGINIA । ।

Fugtiive From Ohio II

FUGTIVE FROM JUSTICE - GEORGIA I ।

FUGTIVE FROM JUSTICE PA । ।

FUGTIVE FROM JUSTICE- P.A. I I

FUGUTIVE FROM GEORGIA I ।

Fugutive From Pennsylvania | | 
FUGUTIVE FROM PENNSYLVANIA । ।

Fugutive From Va I।

FUGUTIVE PA । |

Fuigtive From Pa II

Fuitive From Pa II

FUOITIVE I|

GRAND JURY IND I।

HARBOR FELON/FUGITIVE || 10232

Harbor Felon/Fugitive, Harbor Felon/Fugitive || 10232

Harbor Felon/Fugitive, Hindering/Harboring II 10232

HARBORING A FUGITIVE I| 10043

INDUCE INHIBIT TEST-FELONY OFF || 10355

INDUCE/INHIBIT TESTIMONY || 10354

Induce/Inhibit Testimony, Induce/Inhibit Testimony || 10354

INTENT TO INJURE W/ DEADLY WEA I | 15200

INTIMIDATE/INFL JUROR । |

INTIMIDATE/INFLU JUROR-FELONY | | 10359

INTIMIDATE/INFLUENCE JUROR I | 10358

INTIMIDATE/INFLUENCE JUROR | | 11571

INTIMIDATE/INFLUENCE JUROR-MIS || 10358

Intimidate/Influence Juror, Intimidate/Influence Ju || 10358

Intimidate/Influence Juror, Intimidate/Influence Jur || 10358

MACE/CHEM DEVICE-INT/INJURE | | 25200

Mal Dest Prop/Valu - \$500, Witness Intimidation I| 34025

Material Witness II

ORDER OF THE COURT I।

PAY FINE II

Peace Order: Fail To Comply, Violation Of Court Ord I| 20105

Peace Order: Fail To Comply, Violation Of Court Orde II 20105

Peace Order: Fail To Comply, Violation Of Probation I| 20105

PENNSYLVANIA FUGITIVE I |

PERJURY/AFFID REQ BY LAW || 10307

POSS CONTBND-PLACE OF CONFINEM | | 11835

RETALIATE - WITNESS || 11567

Retaliate Witness II

RETALIATE-WITNESS || 10356

RETALIATE-WITNESS MISDEMEANOR I | 10356

RETALIATE-WITNESS-FELONY OFF || 10357

Retaliate-Witness, Retaliate-Witness || 10356

SOLICIT/ INTIMIDUTE WITNESS || 11565

THREAT-SEND/DELIVER-INJURY || 11707

THREAT/ST OFFICIAL/TO INJURE || 10477

Trespass: Private Property, Fta || 22220

VERBAL EXTORTION/THRT INJURY I | 11704

VIOLATION COURT ORDER II 20105

VIOLATION OF A COURT ORDER || 11465

VIOLATION OF COURT ORDER I| 10043

Violation Of Probation II

Violation Of Probation, Violation Of Probation I| 10088

Violation Of Probation, Violation Of Probation I| 999999

VIRGINIA FUGITIVE । |

Vop II

VOP I

VOP || 20105

VOP/DESTRUCTION OF PROPERTY I| 34025

VUL ADULT ABUSE PHYSICAL INJUR || 21138

WITNESS INTIMIDATION || 10355

WITNESS INTIMIDATION || 10356

WITNESS INTIMIDATION || 10357

WITNESS INTIMIDATION || 11565 
WITNESS INTIMIDATION || 16525

\section{Property Destruction}

4TH DEGREE B\& E || 34025

Alc. Bev./Intox:Endanger, Dest Of Property II 14200

Alc. Bev./Intox:Endanger, Destruction Of Property || 14200

Alc. Bev./Intox:Endanger, Malicious Dest Of Prop II 14200

ANIMAL CRUELTY || 34025

ATT-DESTRUCTION-ELECTION EQUIP || 2A0383

ATT-MAL DEST PROP VALUE $+\$ 1,00$ | | 3A4030

ATT-MAL DEST PROP/VALU - \$1,00 || 3A4025

ATT-MAL DEST PROP/VALU - \$500 || 3A4025

Att-Mal Dest Prop/Valu - \$500, Att-Mal Dest Prop/Val I| 3A4025

ATT.DEST.OF PROPERTY || 3A4025

B /E || 34025

CDS || 34025

CDS VIOLATION II 34025

CON-MAL DEST PROP VALUE +\$1,00 || 3C4030

Con-Mal Dest Prop/Valu - \$500, Destruction Of Prope II 3C4025

Con-Mal Dest Prop/Valu - \$500, Mal Dest $<\$ 1000$ II 3C4025

CONSPIRACY DESTRUCT OF EVIDENC | | 11480

DANAGE PROPERTY || 34025

DESRTRUCTION OF PROPERTY || 34025

Dest Device Man/Pos/Dist, Possession Of Explosives I| 10720

DEST OF PROP || 2A3030

DEST OF PROP || 34025

DEST OF PROP || 34030

DEST OF PROP ATTEMPT । | 20055

DEST OF PROPERRTY || 34030

DEST OF PROPERTS || 34025

Dest Of Property II

DEST OF PROPERTY || 20050

DEST OF PROPERTY || 22220

DEST OF PROPERTY || 23030

DEST OF PROPERTY || 2A3030

DEST OF PROPERTY I। 34025

DEST OF PROPERTY || 34030

DEST OF PROPORTY I 34025

DEST PROPERTY | | 31314

DEST, OF PROPERTY || 34025

DEST, OF PROPERTY | | 34030

DEST. OF PROP. II 20050

DEST. OF PROP. II 34025

DEST. OF PROP. II 34030

DEST. OF PROPERTY I। 11466

DEST. OF PROPERTY || 16505

DEST. OF PROPERTY || 34025

DEST. OF PROPERTY || 34030

DEST. PROPERTY || 34025

DEST. PROPERTY | | 34030

DEST. PROPERTY LESS THAN 1000 | | 34025

DESTRICTION OF PROPERTY || 34025

DESTRUCCTION OF PROPERTY || 34025

DESTRUCITON OF PROPERTY | | 34025

DESTRUCT OF PROP || 14200

DESTRUCTION || 34025

DESTRUCTION OF PROPERTY | | 34025

DESTRUCTION OF PROPRTEY || 34025 
DESTRUCTION OF || 34025

DESTRUCTION OF PROPERTY || 34030

DESTRUCTION OF PEROPERTY | | 34025

DESTRUCTION OF PROERTY || 34025

DESTRUCTION OF PROP || 34025

DESTRUCTION OF PROP || 34030

Destruction Of Property I I

DESTRUCTION OF PROPERTY | | 10191

DESTRUCTION OF PROPERTY || 10600

DESTRUCTION OF PROPERTY | | 10622 DESTRUCTION OF PROPERTY || 11111 DESTRUCTION OF PROPERTY || 11137 DESTRUCTION OF PROPERTY | | 11425 DESTRUCTION OF PROPERTY || 14200 DESTRUCTION OF PROPERTY || 14803 DESTRUCTION OF PROPERTY || 16503 DESTRUCTION OF PROPERTY || 1A0622 DESTRUCTION OF PROPERTY | | 20050 DESTRUCTION OF PROPERTY || 20065 DESTRUCTION OF PROPERTY || 22210 DESTRUCTION OF PROPERTY | | 22220 DESTRUCTION OF PROPERTY || 23000 DESTRUCTION OF PROPERTY || 23030 DESTRUCTION OF PROPERTY || 23050 DESTRUCTION OF PROPERTY || 23080 DESTRUCTION OF PROPERTY || 2A3030 DESTRUCTION OF PROPERTY | | 34025 DESTRUCTION OF PROPERTY I | 34030 DESTRUCTION OF PROPERTY/DISOR I | 34025 DESTRUCTION OF PROPERY I | 34030 DESTRUCTION OF PROPETY || 34030 DESTRUCTION OF PROPOERTY I I 34030 DESTRUCTION OR PROPERTY I | 34025 DESTRUCTION PROPERTY || 22220 DESTRUCTION PROPERTY || 34025 DESTRUCTION PROPERTY | | 34030 DESTRUCTIVE DEVICE | | 10720

DESTRUCTOIN OF PROPERTY | | 34025 DESTRUCTTION OF PROPERTY || 34025 DESTRUCTUION OF PROEPRTY || 22210 DESTRUTION OF PROPERTY | | 14200 DESTRUTION OF PROPERTY | | 34025 DESTRUTION PROPERTY I 34025 DESTRUTION PROPERTY I 34030 DESTUCTION OF PROPERTY || 13802 DESTUCTION OF PROPERTY || 23030 DESTUCTION OF PROPERTY I | 34025 DESTUCTION OF PROPERTY | | 34030 DET. OF PROP || 34025

DIS. OF PROPERTY || 34025

Dis.Erly Conduct, Destruction Of Property II 20050

Dis.Erly Conduct, Mal Destruction Of Property I| 20050

DISORDERLY I| 34025

DISORDERLY CONDUCT || 34025

DOMESITC/DEST.PROP || 34025

Extortion:Value \$500. Plus, Dest Of Property I| 35060

False Statement To Officer, Dest.Prop. Police I| 14803

False Stmt To Peace Officer, Destruction Of Property || 10690

GRAFFITI I| 34025

HINDERING || 34025 
MAL DEST MISSILES AT VEH. | | 12903

Mal Dest Missiles At Veh., Disorderly Conduct I| 12903

MAL DEST OF PROP I। 34025

MAL DEST OF PROPERTY I 34025

MAL DEST OF PROPERTY I 34030

MAL DEST PROP । ।

MAL DEST PROP 1,000 । ।

MAL DEST PROP/1000 । ।

MAL DEST PROP/VALU - \$1,000 || 34025

MAL DEST PROP/VALU - \$300 || 12900

Mal Dest Prop/Valu - \$300, Mal Dest Prop/Valu - \$30 I| 12900

MAL DEST PROP/VALU - \$500 | | 34025

Mal Dest Prop/Valu - \$500, Atemp. B And E II 34025

Mal Dest Prop/Valu - \$500, Attemp Breaking And Enter II 34025

Mal Dest Prop/Valu - \$500, B E II 34025

Mal Dest Prop/Valu - \$500, Breaking And Entering I| 34025

Mal Dest Prop/Valu - \$500, Desruction Of Property II 34025

Mal Dest Prop/Valu - \$500, Dest Of Property II 34025

Mal Dest Prop/Valu - \$500, Dest Property II 34025

Mal Dest Prop/Valu - \$500, Dest. Of Prop II 34025

Mal Dest Prop/Valu - \$500, Dest. Of Property II 34025

Mal Dest Prop/Valu - \$500, Dest. Of. Property II 34025

Mal Dest Prop/Valu - \$500, Dest. Prop I| 34025

Mal Dest Prop/Valu - \$500, Destruc Of Prop II 34025

Mal Dest Prop/Valu - \$500, Destruction II 34025

Mal Dest Prop/Valu - \$500, Destruction Of Prooperty II 34025

Mal Dest Prop/Valu - \$500, Destruction Of Prop II 34025

Mal Dest Prop/Valu - \$500, Destruction Of Property || 34025

Mal Dest Prop/Valu - \$500, Destruction Pof Property II 34025

Mal Dest Prop/Valu - \$500, Destruction Property II 34025

Mal Dest Prop/Valu - \$500, Destuction Of Property II 34025

Mal Dest Prop/Valu - \$500, Disorderly II 34025

Mal Dest Prop/Valu - \$500, Disorderly Conduct II 34025

Mal Dest Prop/Valu - \$500, Dist Of Property II 34025

Mal Dest Prop/Valu - \$500, Distruction Of Property II 34025

Mal Dest Prop/Valu - \$500, Domestic Violence II 34025

Mal Dest Prop/Valu - \$500, Larcency From Auto II 34025

Mal Dest Prop/Valu - \$500, Larcety From Auto I I 34025

Mal Dest Prop/Valu - \$500, Mal Dest Of Property II 34025

Mal Dest Prop/Valu - \$500, Mal Dest Prop/Valu - \$1, I I 34025

Mal Dest Prop/Valu - \$500, Mal Dest Prop/Valu - \$50 I| 34025

Mal Dest Prop/Valu - \$500, Mal Dest Prop/Valu - \$500 I| 34025

Mal Dest Prop/Valu - \$500, Mal Destruction II 34025

Mal Dest Prop/Valu - \$500, Mal Destruction Of Prope II 34025

Mal Dest Prop/Valu - \$500, Mal. Destruction Of Prop II 34025

Mal Dest Prop/Valu - \$500, Mal.Dest.Of Property II 34025

Mal Dest Prop/Valu - \$500, Maldestruction Of Propert I I 34025

Mal Dest Prop/Valu - \$500, Malicious Dest Of Propert I I 34025

Mal Dest Prop/Valu - \$500, Malicious Destrouctions II 34025

Mal Dest Prop/Valu - \$500, Malicious Destruction Of I| 34025

Mal Dest Prop/Valu - \$500, Malicious Destruction/Dis II 34025

Mal Dest Prop/Valu - \$500, Mals. Dest Of Property II 34025

Mal Dest Prop/Valu - \$500, Mdop -\$500 II 34025

Mal Dest Prop/Valu - \$500, Poss Cds II 34025

Mal Dest Prop/Valu - \$500, Rogue Vagabond I I 34025

Mal Dest Prop/Valu - \$500, Trespassing II 34025

Mal Dest Prop/Valu - \$500, Violation Of Final Protec I| 34025

MAL DEST PROP/VALU $<\$ 1,000$ | | 34025

MAL DEST PROPERTY I I

MAL DEST PROPERTY || 34025 
MAL DEST PROPERTY || 34030

MAL DESTR I।

MAL DESTR OF PROP || 34025

MAL DESTR OF PROPERTY | | 34025

MAL DESTR PROP VALUE ।

MAL DESTR PROP VALUE $\$ 1,000+$ | | 34030

MAL DESTR PROP VALUE + $\$ 1,000$ | | 34030

MAL DESTR PROP VALUE + \$300 || 10431

Mal Destr Prop Value + \$300, Mal Destr Prop Value + II 10431

MAL DESTR PROP VALUE + \$500 I। 34030

Mal Destr Prop Value + \$500, Assautl/ Dest. Prop II 34030

Mal Destr Prop Value + \$500, Desctruction Of Propert II 34030

Mal Destr Prop Value + \$500, Dest Of Property I| 34030

Mal Destr Prop Value + \$500, Dest. Of Prop II 34030

Mal Destr Prop Value + \$500, Dest. Of Property II 34030

Mal Destr Prop Value + \$500, Dest. Of Property Over II 34030

Mal Destr Prop Value + \$500, Dest. Property II 34030

Mal Destr Prop Value + \$500, Destruct Of Prop II 34030

Mal Destr Prop Value + \$500, Destruction II 34030

Mal Destr Prop Value + \$500, Destruction Od Propert I| 34030

Mal Destr Prop Value + \$500, Destruction Of Prop I I 34030

Mal Destr Prop Value + \$500, Destruction Of Propert II 34030

Mal Destr Prop Value + \$500, Destruction Of Property II 34030

Mal Destr Prop Value + \$500, Destruction Pf Property || 34030

Mal Destr Prop Value + \$500, Detsruction Of Property II 34030

Mal Destr Prop Value + \$500, Distruction Of Property II 34030

Mal Destr Prop Value + \$500, Failure To Obey $\mid$ | 34030

Mal Destr Prop Value + \$500, Mal Des Of Prop II 34030

Mal Destr Prop Value + \$500, Mal Dest. Private Prope I| 34030

Mal Destr Prop Value + \$500, Mal Destr Prop Value + II 34030

Mal Destr Prop Value + \$500, Mal Destruction II 34030

Mal Destr Prop Value + \$500, Mal Destruction Of Prop II 34030

Mal Destr Prop Value + \$500, Mal Distruct Of Prop I | 34030

Mal Destr Prop Value + \$500, Mal. Destr Of Property I I 34030

Mal Destr Prop Value + \$500, Mal. Destruction Of Pro || 34030

Mal Destr Prop Value + \$500, Malacious Destruction II 34030

Mal Destr Prop Value + \$500, Malic Detruction Of Pro I I 34030

Mal Destr Prop Value + \$500, Malicious Dest. Of Prop II 34030

Mal Destr Prop Value + \$500, Malicious Destruction II 34030

Mal Destr Prop Value + \$500, Malicious Destruction O II 34030

Mal Destr Prop Value + \$500, Malicious Destructuion II 34030

Mal Destr Prop Value + \$500, Malicious Destrution Of II 34030

Mal Destr Prop Value + \$500, Malious Destruction Of I | 34030

Mal Destr Prop Value + \$500, Reckless Endangerment II 34030

MAL DESTR PROP VALUE $+1,000$ । ।

MAL DESTR PROP/VALUE -\$1,000.0 || 34025

MAL DESTRUCTION II 34025

MAL DESTRUCTION OF PROP || 34025

MAL DESTRUCTION OF PROP || 34030

MAL DESTRUCTION OF PROPERTY || 22210

MAL DESTRUCTION OF PROPERTY || 34025

MAL DESTRUCTION OF PROPERTY || 34030

MAL DESTRUCTION PROP || 20050

MAL. DEST OF PROPERTY | 34025

MAL. DEST. II 34025

MAL. DEST. OF PROP. I I 34030

MAL. DEST. OF PROPERTY || 34025

MAL. DEST. PROPERTY | | 34025

MAL. DESTRUCTION I I

MAL. DESTRUCTION || 34025 
MAL. DESTRUCTION OF PROP. I I 34025

MAL. DESTRUCTION OF PROPERTY || 34025

MAL. DESTRUCTION PROPERTY | | 34030

MAL.DESTRUCTION OF PROPERTY || 34025

MAL/DESTRUCTION OF PROPERTY I | 34025

MALCIOUS DESTRUCTION OF PROPER I I 34025

MALDEST II

MALDESTRUCTION OF PROPERTY || 34025

MALIC DESTRUCTION | | 34025

MALICE DESTRUCTION || 34025

MALICIOUS DESTRUCTION || 34030

MALICIOUS DAMAGE TO PROPERTY || 34025

MALICIOUS DEST OF PROP || 34030

MALICIOUS DEST OF PROPERTY I| 34025

MALICIOUS DEST. I I 34025

MALICIOUS DEST. OF PROPERTY || 34025

MALICIOUS DEST. OF PROPERTY I 34030

MALICIOUS DESTRCUTION OF PROPE I। 34025

MALICIOUS DESTRUCTION || 11137

MALICIOUS DESTRUCTION || 20700

MALICIOUS DESTRUCTION I | 34025

MALICIOUS DESTRUCTION I| 34030

MALICIOUS DESTRUCTION || 34035

MALICIOUS DESTRUCTION OF PROPE || 11661

MALICIOUS DESTRUCTION OF PROPE I I 20055

MALICIOUS DESTRUCTION OF PROPE || 34025

MALICIOUS DESTRUCTION OF PROPE I| 34030

MALICIOUS DESTRUCTION OF PROPE || 3A4025

MALICOUS DESTRUCTION | | 34025

MALICOUS DESTRUCTION I I 34030

MALICOUS DESTRUCTION OF PROPER || 20055

MALIOUS DESTRUCTION || 34025

MALIOUS DESTRUCTION PROPERTY I| 23030

MALIS DESTRUTION II 34025

MDOP -500 || 34025

MILICIOUS DESTRUCTION OF PROPE I | 34025

PROP II 34025

Reckless Endangerment, Destruction Of Property || 11425

Reckless Endangerment, Malicious Destruction Of Prop || 11425

Resist/Interfere With Arrest, Destruction Of Prop II 10600

Resist/Interfere With Arrest, Destruction Of Propert I| 10600

ROGUE \& VAGABOND || 34025

ROGUE AND VAGABOND | | 34025

THREAT II 34025

Trespass: Private Property, Destruction Of Property I| 22220

TRESPASSING || 34025

TRESSPASSING || 34025

VANDALISM || 34025

VANDALIZING PROPERTY || 34025

\section{Forgery, Fraud, and Extortion}

|| 11228

II 11262

ATT-BD CK/PASS/NSF/UND \$1000 || 1A1235

ATT-CDS:OBTAIN BY FRAUD || 1A0248

Att-Fraud Conversn/Lease Goods, Att-Fraud Conversn/L I| 2A2399

ATT-UTTERING FALSE DOCUMENT | | 1A2513

ATTEMPT CDS BY FRAUD । । 


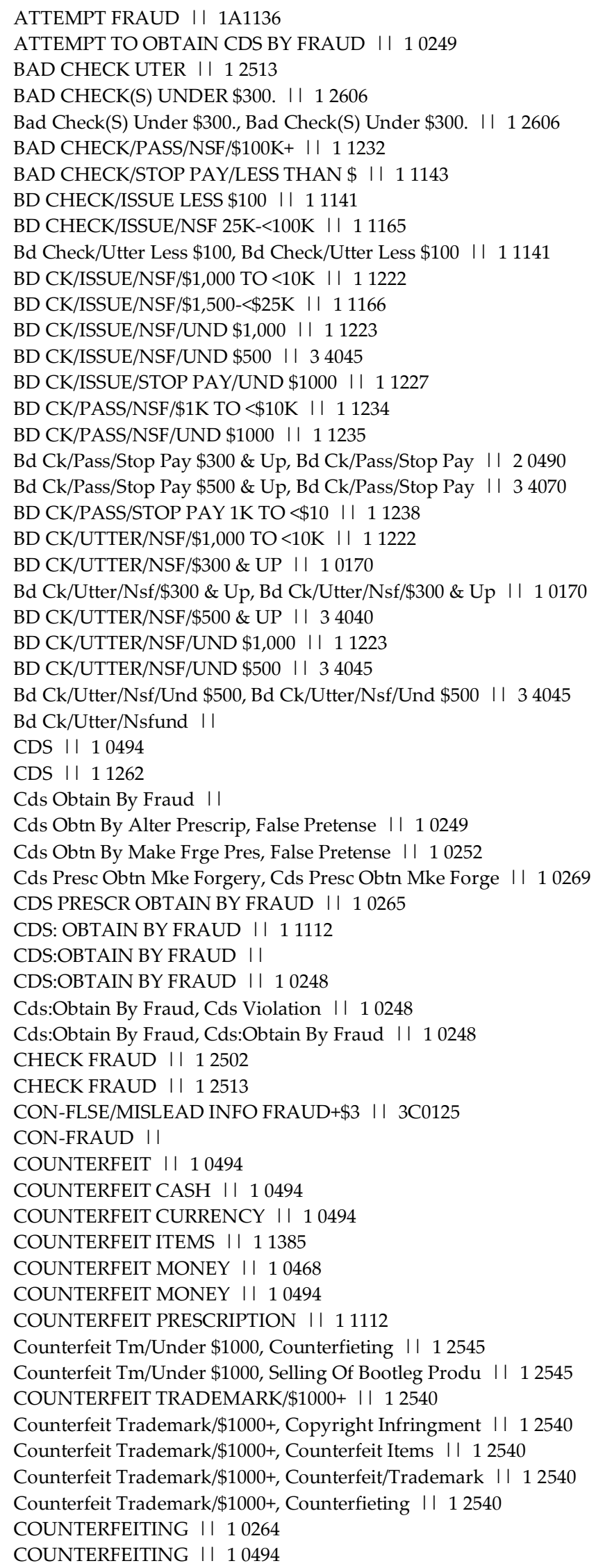




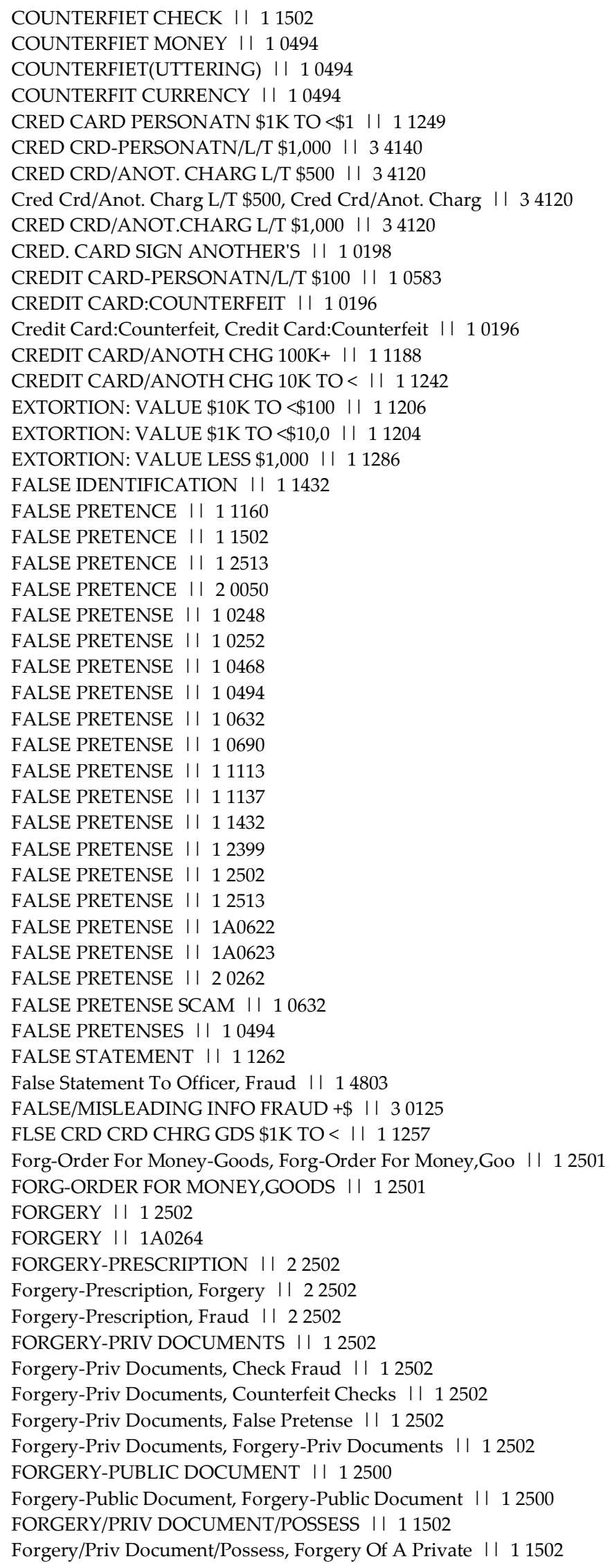


Forgery/Priv Document/Possess, Forgery/Priv Documen II 11502

Forgery/Priv Document/Possess, Forgery/Priv Document || 11502

Fraud II

FRAUD I |

FRAUD || 11111

FRAUD || 11137

FRAUD || 20480

FRAUD || 22820

FRAUD - PRESCRIPTION DRUG || 10026

FRAUD CONVERSN/LEASE GOODS I| 22399

Fraud Conversn/Lease Goods, Fraud Conversn/Lease Goo I| 22399

FRAUD ID INFO THFT $\$ 100-<\$ 1500 \quad|| 11369$

FRAUD ID INFO THFT $\$ 100-<1500 \quad|| 11362$

FRAUD ID INFO THFT \$1500-<25K || 11361

FRAUD ID INFO THFT \$1K $-<\$ 10 \mathrm{~K}$ | | 11260

FRAUD ID INFO THFT \$25K-<\$100K || 11360

FRAUD IDENT INFO THFT \$10K-<\$1 || 11259

FRAUD IDENT INFO THFT $\$ 1 \mathrm{~K}-<\$ 1 \quad$ | | 11260

FRAUD IDENT INFO THFT UND \$1,0 || 11261

FRAUD IDENT INFO THFT UND \$1,0 || 11266

Fraud Per Id Infotheft \$500+, Fraud Per Id Infotheft || 11301

Fraud-Per Id Infotheft \$500+, Fraud-Per Id Infothef I| 11300

FRAUD-PER IDENT AVOID PAY \$100 || 11267

FRAUD-PER IDENT AVOID PROS || 11203

FRAUD-PER IDENT AVOID PROS || 20082

Fraud-Per Ident Avoid Pros, Fraud-Per Ident Avoid Pr I| 11203

FRAUD-PER. IDENT. AVOID PROS || 11364

FRAUD-PER.IDENT.AVOID PROS || 11262

Fraud-Welfare, Fraud-Welfare || 72607

FRAUDULENT CHECK I| 1A0622

FRAUDULENT IDENTIFICATION || 11432

IDENTITY FRAUD - INDUCE ANOTH || 11280

ISSUE COUNTERFEIT ORDER || 22510

ISSUE FALSE DOCUMENT || 12513

ISSUE FALSE DOCUMENTS । |

OBTAIN DRUG BY FRAUD || 10180

Obtain Goods- Money-Forgery, Obtain Goods, Money-Fo II 22520

Obtain Goods- Money-Forgery, Obtain Goods, Money-For I| 22520

OBTAIN GOODS, MONEY-FORGERY || 22520

Other Non Felony Charge, Fraud-Per Ident Avoid Pros I| 20082

PASS FORGED PUBLIC DOC. I I 12504

POSS COUNTERFIT MONEY I | 10494

POSS OF CONTERFEIT CURRENCY I I 10494

POSS OF COUNTERFEIT II 10494

POSS OF COUNTERFEIT MONEY || 10494

POSS OF FAKE US CURRENCY || 10494

POSS OF FALSE DOCUMENTS || 11432

POSS OF MARIJUANA II 11262

POSS/ISSUE FORGED CURRENCY I ।

POSSESS FORGED PRESCRIP || 22530

POSSESS/ ISSUE FORGED CURRENCE I | 10494

POSSESS/ISSUE FORGED CURRENCY | | 10494

Possess/Issue Forged Currency, False Pretense II 10494

Possess/Issue Forged Currency, Poss Of Counterfeit I| 10494

Possess/Issue Forged Currency, Possess/Issue Forg I| 10494

Possess/Issue Forged Currency, Uttering | | 10494

POSSESSING COUNTERFEIT US CURR | | 10494

POSSESSION OF COUNTERFEIT CURR I | 10494

POSSESSION OF COUNTERFEIT US C I | 10468

POSSESSION OF COUNTERFIT CURRA । । 10494 


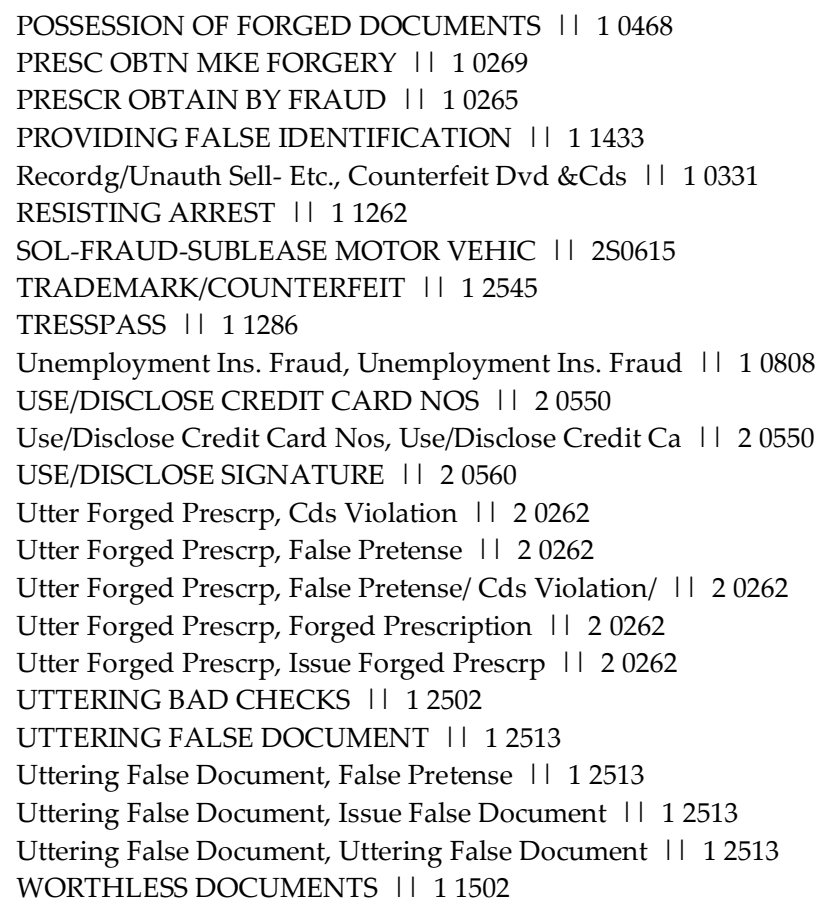

\section{Police Non-Compliance}

Alc. Bev./Intox:Endanger, Disorderly Fto || 14200

Alc. Bev./Intox:Endanger, Failure To Obey II 14200

ATT BY DRIVER TO ELUDE । ।

ATT BY DRIVER TO ELUDE POLICE । |

ATT BY DRIVER TO ELUDE UNIFORM । ।

ATT ELDUDE POLICE I।

ATT ELUDE POLICE I।

Att Elude Police Fail To Stop | |

ATT TO ELUDE POLICE I।

ATT TO ELUDE POLICE BY FAILING । |

ATT TO ELUDE UNIFORMED POLICE । | ATT. TO ELUDE UNIFORM POLICE I | ATTEMP BY DRIVING TO ELUDE POL I। Attemp To Elude Police By Fail I I

ATTEMPT BY DRIVER TO ELUDE UN । । ATTEMPT BY DRIVE TO ELUDE UNIF । | ATTEMPT BY DRIVER TO ELUDE । । Attempt By Driver To Elude Pol I I ATTEMPT BY DRIVER TO ELUDE POL I । Attempt By Driver To Elude Uni I I ATTEMPT BY DRIVER TO ELUDE UNI । । ATTEMPT BY DRIVER TO RLUDE POL I। ATTEMPT BY DRIVER, FLEEING AND I। Attempt By Driving To Elude Po I | ATTEMPT TO ELLUDE POLICE I । ATTEMPT TO ELUCE POLICE I। ATTEMPT TO ELUDE I। ATTEMPT TO ELUDE OFFICER I । ATTEMPT TO ELUDE POLICE । | ATTEMPT TO ELUDE POLICE AND FA । । ATTEMPT TO ELUDE POLICE BY FLE I । Attempt To Elude Police Office I I 
ATTEMPT TO ELUDE UNIFORM OFFIC । | ATTEMPT TO ELUDE UNIFORMED POL । । ATTEMPT TO ELUDING UNIFORM POL । । Attempt. To Elude II

Attempted To Elude Police II

ATTEMPTING TO ELUDE POLICE । ।

BENCH FTO I।

CDS || 10043

CDS \& RESISTING ARREST || 10600

CDS VIOLATION || 10043

Cds: Poss Marihuana L/T 10 G, Hit And Run II 11635

Cds: Possession-Marihuana, Cds/Resisting Arrest || 10573

CONSPIRACY || 10043

DETAIN ONLY BALT. CO POLICE । ।

Dis.Erly Conduct, Disorderly / Fto || 20050

Dis.Erly Conduct, Disorderly/Fto || 20050

Dis.Erly Conduct, Disorderly/Fto || 20050

Dis.Erly Conduct, Disordlery/Fto | | 20050

Dis.Erly Conduct, Fail To Obey | 20050

Dis.Erly Conduct, Failure To Obey || 20050

Dis.Erly Conduct, Failure To Obey A Lawful Order || 20050

Dis.Erly Conduct, Failure To Obey/Disorderly Co || 20050

Dis.Erly Conduct, Fto II 20050

Dis.Erly Conduct, Fto / Disorderly || 20050

Dis.Erly Conduct, Fto Disorderly || 20050

Dis.Erly Conduct, Fto Disorderly / Hindering | 20050

Dis.Erly Conduct, Fto/Disorderly || 20050

Dis.Erly Conduct, Resisting Arrest I| 20050

DISODERLY FTO || 20055

DISORDERLY || 10043

DISORDERLY || 20060

DISORDERLY / FTO || 20050

DISORDERLY / FTO । I 20055

DISORDERLY / HINDERING || 10043

DISORDERLY /FTO || 10395

DISORDERLY CONDUCT | | 10043

DISORDERLY CONDUCT । | 20060

DISORDERLY CONDUCT/FTO । 20050

DISORDERLY CONDUCT/FTO || 20055

DISORDERLY PUB DISOBEY POL | | 10395

Disorderly/ Fto I I

DISORDERLY/FTO || 10395

DISORDERLY/FTO || 20050

DISORDERLY/FTO || 20055

Disorderly/Fto I I

DISORDERLY/FTO || 10395

DISORDERLY/FTO || 20050

DISORDERLY/FTO I। 20055

DISORDERLY/FTO || 22220

DISTURB PEACE || 20060

Disturb Peace - Loud Noise, Failure To Obey || 20065

Disturb Peace - Loud Noise, Fto I| 20065

DISTURB THE PEACE || 20060

DRIVEN TO FLUDE POLICE I।

DRIVER ATTEMPT TO ELUDE POLICE I ।

DRIVER ELUDE POLICE । |

Driver Elude Unif. Police I |

DRIVING UNINSURED VEHICLE | | 10043

Elude From Police II

Elude Police || 
ELUDE POLICE । |

ELUDE POLICE BY FAILING TO STO । ।

ELUDE POLICE BY FLEEING ON FOO । ।

ELUDE POLICE BY VEHICLE । ।

ELUDE UNIFORMED POLICE BY FLEE । । ELUDE UNIFORMED POLICE OFFICER । । ELUDING । |

Eluding And Fleeing II

Eluding Police II

ELUDING POLICE । ।

ELUDING POLICE BY FAILING TO S । ।

Eluding Uniform Police I I

ELUDING UNIFORMED POLICE OFFIC । ।

ELUDING UNIFORMED POLICE ON FO । ।

FAIL OBEY RENBLE/LAWFL | | 20055

Fail Obey Renble/Lawfl, Cds Possession - Other I| 20055

Fail Obey Renble/Lawfl, Dirt Bike Violation I | 20055

Fail Obey Renble/Lawfl, Dirtbike Violation I I 20055

Fail Obey Renble/Lawfl, Disorderly | 20055

Fail Obey Renble/Lawfl, Disorderly /Fto || 20055

Fail Obey Renble/Lawfl, Disorderly Conduct II 20055

Fail Obey Renble/Lawfl, Disorderly Conduct / Fto I I 20055

Fail Obey Renble/Lawfl, Disorderly Conduct Fto I| 20055

Fail Obey Renble/Lawfl, Disorderly Conducted I| 20055

Fail Obey Renble/Lawfl, Disorderly Fail To Obey || 20055

Fail Obey Renble/Lawfl, Disorderly Fto I| 20055

Fail Obey Renble/Lawfl, Disorderly/Fto || 20055

Fail Obey Renble/Lawfl, Disorerly Conduct | | 20055

Fail Obey Renble/Lawfl, Drunk And Disorderly I 20055

Fail Obey Renble/Lawfl, Dsiorderly Conduct I| 20055

Fail Obey Renble/Lawfl, F.T.O || 20055

Fail Obey Renble/Lawfl, Fail Obey Renble/Lawfl || 20055

Fail Obey Renble/Lawfl, Fail To Obey I I 20055

Fail Obey Renble/Lawfl, Failure To Obey II 20055

Fail Obey Renble/Lawfl, Failure To Obey A Lawful Ord || 20055

Fail Obey Renble/Lawfl, Failure To Obey A Lawfull Or I| 20055

Fail Obey Renble/Lawfl, Fto II 20055

Fail Obey Renble/Lawfl, Fto / Disorderly || 20055

Fail Obey Renble/Lawfl, Fto / Disorderly / Resisting || 20055

Fail Obey Renble/Lawfl, Fto Disorderly I 20055

Fail Obey Renble/Lawfl, Fto/Disorderly | 20055

Fail Obey Renble/Lawfl, Fto/Disorderly Conduct II 20055

Fail Obey Renble/Lawfl, Hindering I| 20055

Fail Obey Renble/Lawfl, Loitering I| 20055

Fail Obey Renble/Lawfl, Loitering/Fto II 20055

Fail Obey Renble/Lawfl, Lotering I I 20055

Fail Obey Renble/Lawfl, Open Container I| 20055

Fail Obey Renble/Lawfl, Panhandling || 20055

Fail Obey Renble/Lawfl, Resisting Arrest || 20055

Fail Obey Renble/Lawfl, Traffic Violation | | 20055

Fail Obey Renble/Lawfl, Trespassing | | 20055

Fail Obey Renble/Lawfl, Tresspassing | | 20055

Fail To Obey II

FAIL TO OBEY I ।

FAIL TO OBEY || 10043

FAIL TO OBEY || 14899

FAIL TO OBEY || 15406

FAIL TO OBEY || 20050

FAIL TO OBEY || 20055

FAIL TO OBEY || 22220 
Fail To Obey Abatement Notice, Fail To Obey Abateme I| 10682 FAIL TO OBEY ORDER OF THE COUR I।

Fail To Obey, Fail To Obey I I 14899

FAIL TO OBEY/HINDERING || 20050

FAIL TO REMAIN AT SCENE ACCIDE I।

FAIL TO REMAIN AT SCENE OF ACC I।

FAIL TO REMAINAT SCENE OF ACCI I ।

Failing To Obey II

FAILING TO OBEY | |

Failing To Obey An Order I।

FAILING TO OBEY AN ORDER OF TH I ।

Failing To Obey Traffic Device I I

FAILTURE TO OBEY || 22220

FAILUIRE TO OBEY । I

Failure To Obey II

FAILURE TO OBEY I I

FAILURE TO OBEY || 10043

FAILURE TO OBEY | | 10592

FAILURE TO OBEY || 10600

FAILURE TO OBEY || 10690

FAILURE TO OBEY | I 12545

FAILURE TO OBEY I | 13605

FAILURE TO OBEY || 14200

FAILURE TO OBEY || 20050

FAILURE TO OBEY | | 20055

FAILURE TO OBEY || 22220

FAILURE TO OBEY || 2A3030

FAILURE TO OBEY A LAWFUL ORDER | | 20050

FAILURE TO OBEY AN ORDER I ।

FAILURE TO OBEY AN ORDER OF CO II

FAILURE TO OBEY AN ORDER OF TH I ।

FAILURE TO OBEY LAWFUL ORDER || 20055

FAILURE TO OBEY LAWFUL ORDER/T I | 20055

FAILURE TO OBEY ORDER I I

FAILURE TO OBEY ORDER OF THE C I ।

FAILURE TO OBEY TRAFFIC DEVICE । ।

FAILURE TO OBEY TRAFFIC DEVICE I| 20480

FAILURE TO OBEY/LITTERING || 10640

FAILURE TO OBEY/TRESPASS || 22220

Failure To Remain /Return To T । |

FAILURE TO REMAIN AT ACCIDENT I ।

FAILURE TO REMAIN AT SCENE I।

FAILURE TO REMAIN AT SCENE OF I।

FAILURE TO REMAIN AT SEEN OF A I।

FAILURE TO REMAIN AT THE SCENE I।

Failure To Remain On Scene II

FAILURE TO REMAIN OR RETURN TO । ।

FAILURE TO RETURN \& REMAIN AT I |

FAILURE TO RETURN TO \& REMAIN । ।

FAILURE TO RETURN/REMAIN AT SC । |

FAILURE TO STAY ON SCENE I I

FAILURE TO STAY ON SCENE OF AC I I

FAILURE TO STAY ON SENE OF ACC I।

FALILURE TO OBEY I |

FALIURE TO OBEY | | 20050

FALSE INFO TO POLICE | । 14803

FALSE STATEMENT || 10043

FALSE STATEMENT TO OFFICER I I 10690

FALSE STATEMENT TO OFFICER I | 14803

False Statement To Officer, Cds Violation I| 14803 
False Statement To Officer, Disorderly II 14803

False Statement To Officer, Disorderly Conduct || 14803

False Statement To Officer, False Report I I 14803

False Statement To Officer, False Report To A Polic I| 14803

False Statement To Officer, False Report To Police II 14803

False Statement To Officer, False Statement I| 14803

False Statement To Officer, False Statement To Offi I| 14803

False Statement To Officer, False Statement To Offic I| 14803

False Statement To Officer, False Statment | | 14803

False Statement To Officer, Hindering II 14803

False Statement To Officer, Hit And Run II 14803

False Statement To Officer, Open Container In Vehicl || 14803

FALSE STATEMENT TO POLICE || 10690

FALSE STATEMENT TO POLICE || 11364

FALSE STATEMENT TO POLICE I I 11433

FALSE STMT TO PEACE OFFICER | | 10690

False Stmt To Peace Officer, Cds || 10690

False Stmt To Peace Officer, Cds Violation II 10690

False Stmt To Peace Officer, Conspiracy To Distribut I| 10690

False Stmt To Peace Officer, Disorderly Conduct I | 10690

False Stmt To Peace Officer, Disorderly Drinking II 10690

False Stmt To Peace Officer, False Statement II 10690

False Stmt To Peace Officer, False Statement Of Poli I| 10690

False Stmt To Peace Officer, False Stmt To Peace Off || 10690

False Stmt To Peace Officer, Flase Statment After Ar || 10690

False Stmt To Peace Officer, Hindering/False Stateme I| 10690

False Stmt To Peace Officer, Littering | | 10690

False Stmt To Peace Officer, Open Container I| 10690

False Stmt To Peace Officer, Poss Withintent I| 10690

False Stmt To Peace Officer, Trespassing II 10690

Feiling To Obey II

Flee \& Eluding | |

FLEE AND ELUDE I।

FLEE AND ELUDING I।

FLEE FROM ACCIDENT SCENE | |

FLEEDING AND ELUDING । ।

FLEEING || 20480

FLEEING / ALLUDING । ।

FLEEING \& ELUDING । ।

FLEEING \& ELUDING ON FOOT । ।

FLEEING \& ELUDING POLICE । |

Fleeing And Alluding I I

FLEEING AND ALUDING । ।

Fleeing And Elude I I

Fleeing And Eluding II

FLEEING AND ELUDING । |

FLEEING AND ELUDING | | 10043

FLEEING AND ELUDING || 10600

FLEEING AND ELUDING || 20050

FLEEING AND ELUDING POLICE । ।

FLEEING AND ELUDINGJE34752 । ।

FLEEING ON FOOT । ।

FLEEING THE SCENE OF AN ACCIDE । ।

FLEEING THE SEEN OF AN ACCIDEN । ।

FLEEING/ ELUDING I ।

FLEEING/ELUDING । |

FLEEING/ELUDING ON FOOT । ।

FLEEING/ELUDING VEHICLE । ।

FLSE STMNT TO OFFICER CAUSE I। 10207

Flse Stmnt To Officer Cause, Flse Stmnt To Officer C || 10207 


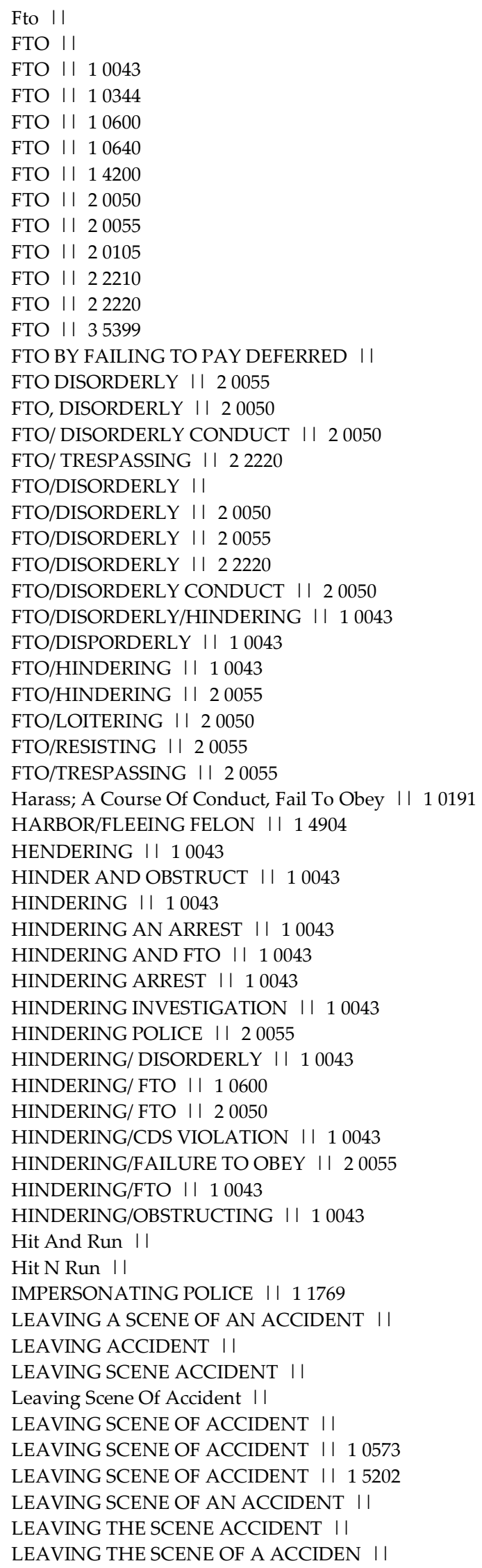


LEAVING THE SCENE OF ACCIDENT । |

LEAVING THE SCENE OF AN ACCIDE । |

Leavingthesceneofaccident I I

Litter/Dump: Under 100 Lbs, Fail To Obey || 10640

LITTERING || 10043

LOITERING/FTO || 22210

OBSTRUCT AND HINDERING || 10043

OBSTRUCT/ HINDERING | | 10043

OBSTRUCT/HINDER || 10043

OBSTRUCTING || 10043

OBSTRUCTING \& HINDERING || 10043

Obstructing \& Hindering, Cds || 10043

Obstructing \& Hindering, Cds Violation || 10043

Obstructing \& Hindering, Disorderly || 10043

Obstructing \& Hindering, Disorderly Conduct || 10043

Obstructing \& Hindering, Drunken Disorderly || 10043

Obstructing \& Hindering, Fail Obey Lawful Order || 10043

Obstructing \& Hindering, Fail To Obey || 10043

Obstructing \& Hindering, Failure To Obey || 10043

Obstructing \& Hindering, Failure To Obey/Disorderly || 10043

Obstructing \& Hindering, False Statement || 10043

Obstructing \& Hindering, Fto || 10043

Obstructing \& Hindering, Fto/ Disorderly || 10043

Obstructing \& Hindering, Fto/Disorderly || 10043

Obstructing \& Hindering, Harboring || 10043

Obstructing \& Hindering, Hendering || 10043

Obstructing \& Hindering, Hindereing || 10043

Obstructing \& Hindering, Hindering || 10043

Obstructing \& Hindering, Hindering Investigation || 10043

Obstructing \& Hindering, Hindering/Fto || 10043

Obstructing \& Hindering, Hindering/Obstructing || 10043

Obstructing \& Hindering, Hindering/Obstruction || 10043

Obstructing \& Hindering, Interfere With Arrest || 10043

Obstructing \& Hindering, Obstructing \& Hindering || 10043

Obstructing \& Hindering, Obstructing And Hindering || 10043

Obstructing \& Hindering, Obstruction || 10043

Obstructing \& Hindering, Resist Arrest || 10043

OBSTRUCTING AND HINDERING | | 10043

OBSTRUCTION || 10043

Personate Police Officer, Imped Police || 11769

Personate Police Officer, Impersonating Police Offi || 11769

POLICE IMPERSONATION || 11769

POSS MARIJUANA || 10043

PUBLIC INTOXICATION || 10043

Reckless Endangerment, Fto || 11425

RESIST ARREST || 10600

RESIST ARREST FAIL TO OBEY || 10600

RESIST/INTERFERE WITH ARREST || 10600

Resist/Interfere With Arrest, Affray || 10600

Resist/Interfere With Arrest, Attempt Breaking \& En || 10600

Resist/Interfere With Arrest, Cds I| 10600

Resist/Interfere With Arrest, Cds Poss I| 10600

Resist/Interfere With Arrest, Cds Possession || 10600

Resist/Interfere With Arrest, Cds Violation || 10600

Resist/Interfere With Arrest, Con Poss / Fto || 10600

Resist/Interfere With Arrest, Defecating In Public || 10600

Resist/Interfere With Arrest, Disorderely || 10600

Resist/Interfere With Arrest, Disorderly || 10600

Resist/Interfere With Arrest, Disorderly Conduct I| 10600

Resist/Interfere With Arrest, Driving On Revolked || 10600 
Resist/Interfere With Arrest, Failure To Obey II 10600

Resist/Interfere With Arrest, Fleeing And Eluding || 10600

Resist/Interfere With Arrest, Fto II 10600

Resist/Interfere With Arrest, Hindering I| 10600

Resist/Interfere With Arrest, Loitering || 10600

Resist/Interfere With Arrest, Open Container I| 10600

Resist/Interfere With Arrest, Poss Cocaine II 10600

Resist/Interfere With Arrest, Poss Of Marijuana II 10600

Resist/Interfere With Arrest, Public Urination II 10600

Resist/Interfere With Arrest, Refusing To Comply Law II 10600

Resist/Interfere With Arrest, Resist/Interfere With I| 10600

Resist/Interfere With Arrest, Resisting Arrest II 10600

Resist/Interfere With Arrest, Resisting Arrest I| 10600

RESISTING || 10600

RESISTING || 20050

RESISTING ARREST । ।

RESISTING ARREST । 10600

RESISTING ARREST I| 14801

RESISTING ARREST || 15202

RESISTING ARREST || 20050

Resisting Arrest, Failure To Obey || 14801

Resisting Arrest, Poss Cocaine II 14801

Resisting Arrest, Resist Arrest I| 14801

RESISTING ARREST//OPEN CONTAIN || 10600

RESISTING ARRESTING || 10600

School: Disturb Operation, Fto II 10070

Trespass-Posted Property, Failure To Obey || 22210

Trespass-Posted Property, Fto || 22210

Trespass-Posted Property, Resisting Arrest I| 22210

Trespass-Posted Property, Tresspassing/Fto I| 22210

Trespass: Private Property, Fail To Obey || 22220

Trespass: Private Property, Failure To Obey || 22220

Trespass: Private Property, Fto I| 22220

TRESPASSING || 20060

TRESPASSING/ FAIL TO OBEY || 22220

TRESPASSING/FTO || 22220

\section{Driving Violation}

(DRIVING, ATT.TO DRIVE) VEH. W I I

(DRIVING, ATTEMPTING TO DRIVE I।

(DRIVING, ATTEMPTING TO DRIVE) । ।

ATT-UNAUTH REMOVAL OF PROP || 2A2411

ATTACHING UNAUTHORIZED TAGS I ।

Attempt To Drive Motor Veh W/O II

Attempt To Drive Under Influen I |

ATTEMPT TO DRIVE W/O REQ LICEN । ।

ATTEMPTED DRIVING I।

ATTEMPTING DRIVING MOTOR VEH.W I ।

ATTEMPTING TO DRIVE A MOTOR VE । ।

ATTEMPTING TO DRIVE MOTOR VEH- II

Cds: Poss Marihuana L/T 10 G, Driving On Suspended L I | 11635

Cds: Poss Marihuana L/T 10 G, Driving W/ O/ License I| 11635

Cds: Possession-Marihuana, Driving On Suspended I| 10573

Cds: Possession-Marihuana, Driving On Suspended Lic I| 10573

CDS/SUSPENDED DRIVER || 10573

CON-UNAUTH REMOVAL OF PROP || 2C2411

Consuming Alcohol In Vehicle I I

D U I | | 
D.U.I, I|

D.U.I. II

DERIVING W/O LICENSE । ।

DID RIDE \& DRIVE DIRT BIKE | |

Dis.Erly Conduct, Dui || 20050

DISPLAY EXP. REG. PLATES ।

DISPLAY EXPIRED PLATED । ।

DISPLAY REG PLATE ISSUED TO AN । ।

Displaying Expired License Pla | |

Diving W/Out License I |

Drining Without License II

DRIV O/O LIC I ।

DRIV ON REVOKED LIC \& PRIV । ।

DRIV ON SISP LIC I।

DRIV ON SUPS I।

DRIV ON SUS LIC ।

Driv On Susp II

DRIV ON SUSP LIC \& PRIV I ।

Driv On Susp Lic II

DRIV ON SUSP LIC I ।

Driv On Susp Lic \& Priv I I

DRIV ON SUSP LIC \& PRIV । |

DRIV ON SUSP/CDS || 43550

DRIV ON SUSPENDED LIC । ।

DRIV OS SUSP LIC I ।

DRIV RENTED MOTOR VEH IN VIOL I ।

Driv Turnin Veh W/O Give Appro I I

Driv Und Infl Alcohol । ।

Driv Under Inf Of Alcohol | |

Driv Under Infl Alcohol I।

DRIV UNDER INFL ALCOHOL । |

DRIV UNDER INFL OF ALCH I ।

Driv Under Infl Of Alcohol I ।

DRIV UNDER INFL OF ALCOHOL I ।

DRIV UNDER INFLUENCE OF ALCOHO । ।

DRIV W OUT LIC I I

Driv W/O Headlight Or Fog Ligh I ।

Driv W/O Lic I|

DRIV W/O LIC I |

Driv W/O License I |

DRIV W/O LICENSE I I

DRIV. ON SUSP LIC I।

DRIV. ON SUSP. II

DRIV. ON SUSP. LIC. I I

DRIV. W/O LIC I।

DRIV. W/O LIC. I।

DRIV. WITHOUT LIC. I।

Drive On Suspended II

Drive On Suspended License I |

DRIVE SUSPENDED । ।

Drive Uninsured Vehicle II

DRIVE W O LICENSE I ।

Drive W/O License II

DRIVE W/O LICENSE I।

Drive W/Out License II

DRIVE W/OUT LICENSE I ।

DRIVE WHILE IMPAIRED । ।

DRIVE WITH OUT LICENSE I ।

DRIVE WITHOUT LICENSE I I

DRIVEND ON SUSP । । 


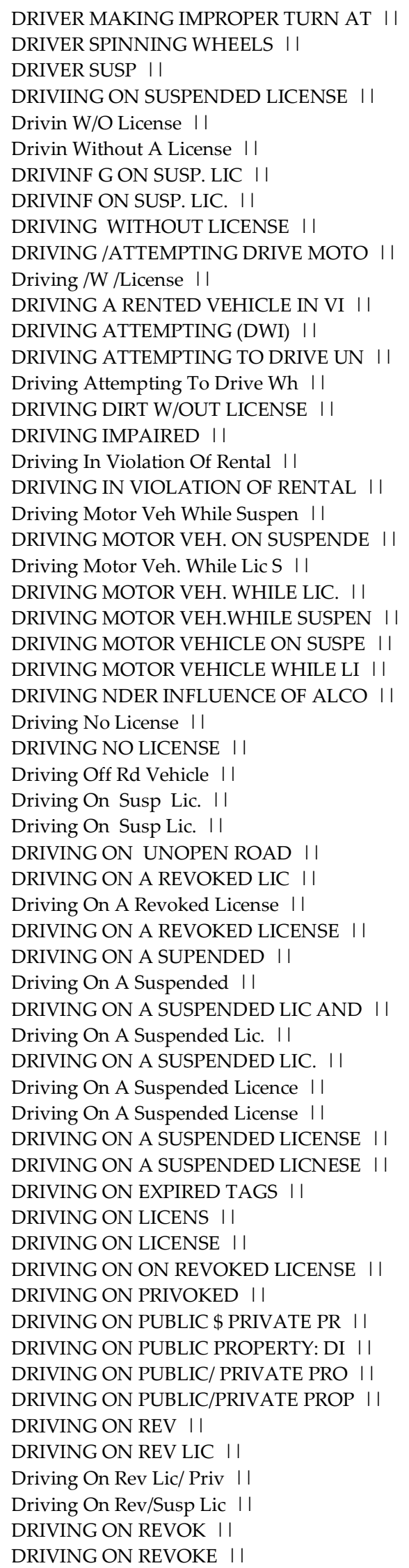




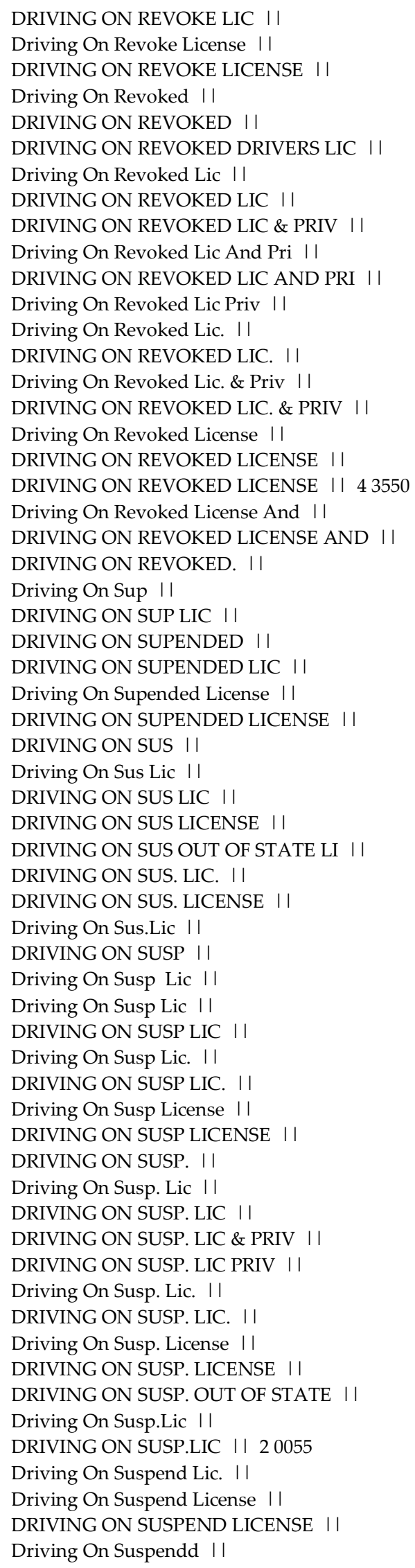


Driving On Suspende License I I DRIVING ON SUSPENDE LICENSE । ।

Driving On Suspended I I

DRIVING ON SUSPENDED | ।

DRIVING ON SUSPENDED || 10690

DRIVING ON SUSPENDED AND REVOK I ।

Driving On Suspended Lic I |

DRIVING ON SUSPENDED LIC I ।

Driving On Suspended Lic / Pri I |

Driving On Suspended Lic \& Pri I I

DRIVING ON SUSPENDED LIC \& PRI । ।

Driving On Suspended Lic And P I I

DRIVING ON SUSPENDED LIC AND P । ।

Driving On Suspended Lic Priv I I

DRIVING ON SUSPENDED LIC PRIV । ।

Driving On Suspended Lic. I I

DRIVING ON SUSPENDED LIC. I ।

Driving On Suspended Lic. \& Pr I |

DRIVING ON SUSPENDED LIC. \& PR । ।

DRIVING ON SUSPENDED LIC. \& PR ।| 43550

Driving On Suspended Lic. And II

DRIVING ON SUSPENDED LIC. AND I।

Driving On Suspended Licence I I

DRIVING ON SUSPENDED LICENCE । ।

Driving On Suspended License I I

DRIVING ON SUSPENDED LICENSE I ।

DRIVING ON SUSPENDED LICENSE | | 10573

Driving On Suspended License A I |

DRIVING ON SUSPENDED LICENSE A । ।

DRIVING ON SUSPENDED LICENSE F I ।

DRIVING ON SUSPENDED LICENSES । ।

DRIVING ON SUSPENDED LICNSE I ।

Driving On Suspended Lics II

Driving On Suspended Licsense II

DRIVING ON SUSPENDED OUT । |

Driving On Suspended Out Of St I I

DRIVING ON SUSPENDED OUT OF ST I ।

Driving On Suspended Outstate II

Driving On Suspended Revoked I |

DRIVING ON SUSPENDED1 । ।

DRIVING ON SUSPENDEDLIC | |

Driving On Suspendedn License I |

DRIVING ON SUSPENED । ।

DRIVING ON SUSPENED LIC I ।

DRIVING ON SUSPENED LIC. I।

Driving On Suspened Lic. \& Pri I।

DRIVING ON SUSPENED LICENCE I ।

DRIVING ON SUSPENED LICENSE I ।

DRIVING ON SUSPENED OUT OF STA । ।

Driving On Suspenede License I |

DRIVING ON SUSPENEDE LICENSE । ।

Driving On Susupended License II

DRIVING ONSUSPEND LIC । |

DRIVING ONSUSPENDED । ।

Driving Rental Veh Viol Of Agr I I

DRIVING REVOCKED I ।

DRIVING REVOKED । ।

DRIVING REVOKED LIC I ।

DRIVING SCOOTER WITH OUT LICEN I ।

DRIVING SUSOENDED । । 


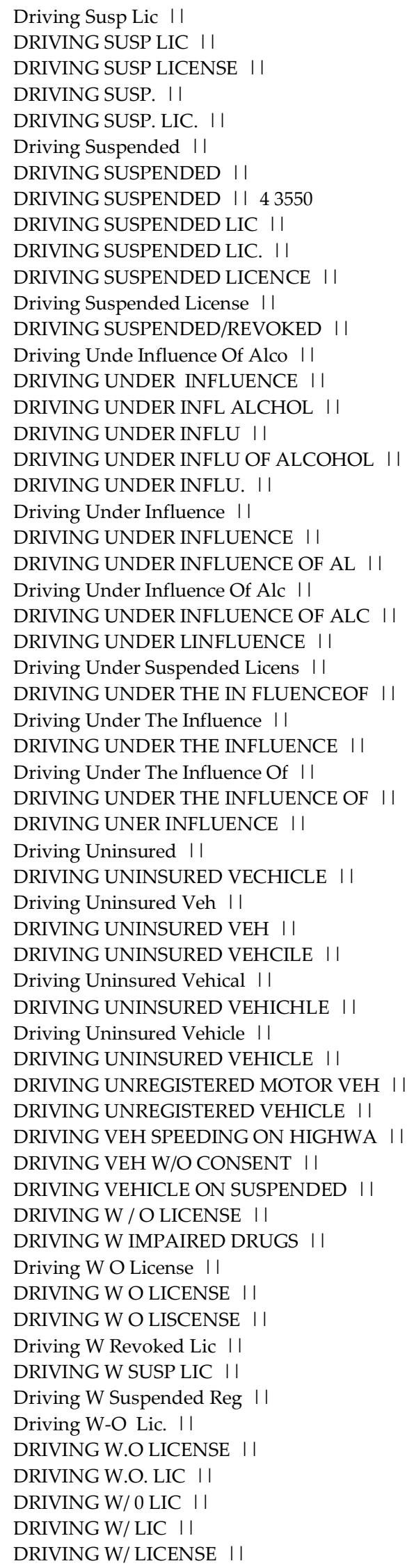




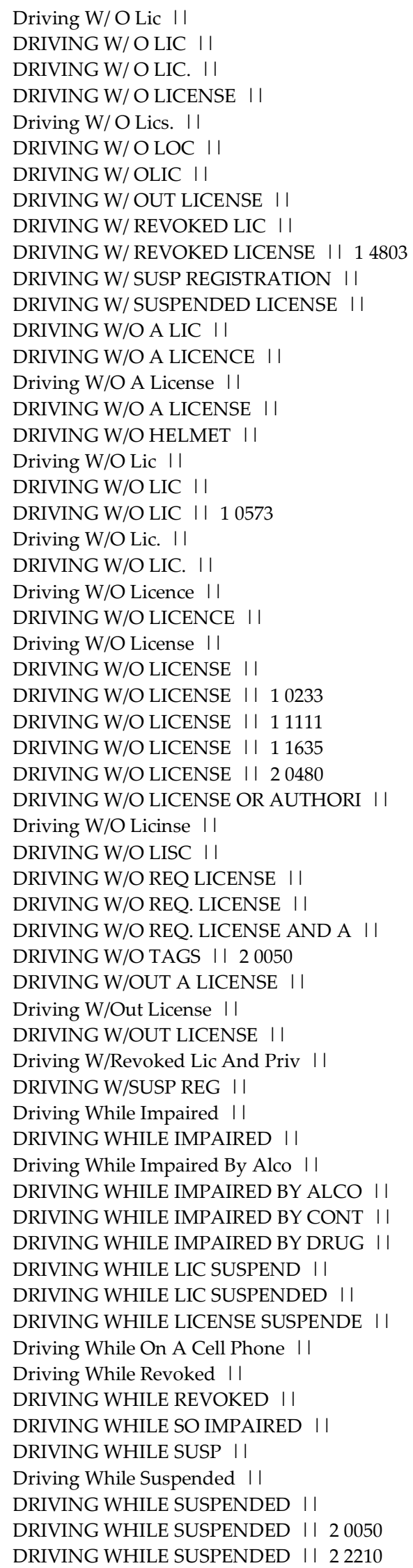


DRIVING WHILE SUSPENDENT । DRIVING WHILE UNDER THE । | DRIVING WI/O LICENSE I । DRIVING WIHOUT LICENSE I । DRIVING WIHTOUT LICENSE । । Driving Wiitout A License I | Driving With A License I | DRIVING WITH NO LIC ।

Driving With Out A License I | DRIVING WITH OUT A LICENSE I। DRIVING WITH OUT LIC I I DRIVING WITH OUT LICENCE । । Driving With Out License I | DRIVING WITH OUT LICENSE । । DRIVING WITH OUT LICENSE | | 10573 DRIVING WITH OUT LISC । । DRIVING WITH REVOKED LIC. \& PR । । DRIVING WITH REVOKED LICENSE । ।

Driving With Susp Licence I I DRIVING WITH SUSP. LIC । । Driving With Suspended Lic I I DRIVING WITH SUSPENDED LIC । ।

Driving With Suspended License II DRIVING WITH SUSPENDED LICENSE । । Driving Witho9Ut License II

Driving Withou Lic II

DRIVING WITHOUT । ।

Driving Without License I। DRIVING WITHOUT A LICENSE । ।

Driving Without A Lic II

DRIVING WITHOUT A LIC । । DRIVING WITHOUT A LICENCE I । Driving Without A License I I DRIVING WITHOUT A LICENSE I । DRIVING WITHOUT A LICENSE || 10600 DRIVING WITHOUT A LICENSE | | 43550 DRIVING WITHOUT A LICESNE I ।

Driving Without A Lics I। DRIVING WITHOUT A LISCENSE | | DRIVING WITHOUT AN LICENSE । । DRIVING WITHOUT CONSENT I । DRIVING WITHOUT DRIVING LICENS । । Driving Without Headlights II

Driving Without Lic I I

DRIVING WITHOUT LIC I ।

Driving Without Lic. II DRIVING WITHOUT LIC. I I

Driving Without Licence II

DRIVING WITHOUT LICENCE I ।

Driving Without License II DRIVING WITHOUT LICENSE I | DRIVING WITHOUT LICENSE | | 10690 DRIVING WITHOUT LICENSE | | 15202 DRIVING WITHOUT LICENSE | | 20050 DRIVING WITHOUT LICENSE I | 20480 DRIVING WITHOUT LICENSE | | 43550

Driving Without Lics II DRIVING WITHOUT LISCENCE I | DRIVING WITHOUT LIVENSE I । DRIVING WITHOUT OWNERS CONSENT । । 
DRIVING WITHOUT REQUIRED LIC. I । DRIVING WITHOUT REQUIRED LICEN I । DRIVING WITOUT LICENSE । ।

DRIVING WO A LICENSE I ।

DRIVING WO LIC I ।

DRIVING WO LICENSE I ।

DRIVING WO LICENSE | | 10573

DRIVING WO LISC I ।

DRIVING WRONG DIRECTION ON A O । । DRIVING WRONG DIRECTION ON ONE I । DRIVING WTHOUT A LICENSE I I

DRIVING/ ATT DRIVE W/O REQ LIC I I DRIVING/ATTEMPING DRIVE MOTOR । । Driving/Attempt Drive Motor Ve I I DRIVING/ATTEMPTING DRIVE MOTO । ।

Driving/Attempting Drive Motor I I DRIVING/ATTEMPTING DRIVE MOTOR । । DRIVING/ATTEMPTING ON HWY W/0 I। DRIVING/ATTEMPTING TO DRIVE MO I । DRIVING/ATTEMPTING TO DRIVE VE । । DRIVING/W OUT LIC ।

DRIVINGG ON SUSP LIC I ।

DRIVINGON SUSPENDED LIC । ।

Drivingonrevoked |।

Drivingonrevokedlic II

DRIVINGONREVOKEDLIC I |

DRIVINGONREVOKRD । |

Drivingonsuspendedlic I |

DRIVINGONSUSPENDEDLIC । ।

Drivingw/Oalic I I

Drivingwithoutalic I|

DRIVINGWITHOUTALIC I |

Drivinig Without A License I |

DRIVINING WITHOUT LICENSE I |

DRIVINNG W/O LICENSE । ।

DRIVIVINGWITHOUTALIC I |

DRIVIVNG ON SUSPENDED । ।

DRIVN W/IMPAIRED । I

DRIVNG SUSPENDED । ।

DRIVNG W OUT LIC । ।

DRIVNG W/O LIC I ।

DRIVNG WHILE SUSPENED । ।

DRIVONG W/O LICENSE । ।

Drv Suspended License I |

DRVING W/O LICENSE I ।

DRVING W\O LICENSE । ।

DRVING WITH OUT LICENSE । ।

Drving Without A License II

Drving Without License II

DRVING WITHOUT LICENSE । ।

DRVING WITHOUT LICENSEI । ।

DRVING WITHOUT LICENSES I ।

Dui II

DUI I।

DUI || 11111

DUI || 20050

DUI - ALCOHOL । |

DUI ALCOHOL I |

DUI ALOCHOL I |

DUI DRIVE WHILE SUSPENDED OR R । । 
Dui Of Alcohol | |

DUI OF ALCOHOL । |

DUI OF ALCOHOL PER SE I |

DUI/ DRIVING W/OUT LIC । ।

Dui/Dwi II

DUI/DWI ।

Dwi II

DWI ।

DWI || 10600

DWI || 11111

DWI/DUI । |

Fail Equip Vehicle Rear Stop L I ।

FAIL OF DRIVER TO STOP AFTER A I ।

Fail To Attach Front Plate I I

FAIL TO ATTACH FRONT PLATE I |

FAIL TO ATTACH LIC PLATES AT F | |

FAIL TO ATTACH PLATES । |

Fail To Attach Plates At Fron I I

FAIL TO ATTACH PLATES AT FRONT I।

Fail To Display Head Laghts I |

Fail To Display Lic. On Demand I I

Fail To Display License On Dem I |

FAIL TO DISPLAY LICENSE ON DEM I ।

FAIL TO DRIVE RIGHT OF CENTER । ।

Fail To Stop II

FAIL TO STOP I I

Fail To Stop After Accident I।

FAIL TO STOP AFTER ACCIDENT । ।

FAIL TO STOP AFTER ACCIDENT AT I ।

FAIL TO STOP AFTER UNATTENDED I ।

Fail To Stop After Veh Unatten I I

FAIL TO STOP AT ACCIDENT INVOL I ।

FAIL TO STOP AT STEADY CIRCULA I।

FAIL TO STOP AT STOP SIGN I।

FAIL TO STOP VEHICLE AT ACCIDE । ।

Fail Veh Driver In Acc To Rpt I |

FAILED TO STOP AT ACCIDENT SCE I।

FAILING TO STOP । ।

FAILING TO STOP VEHICLE AT SCE । ।

FAILURE TO STOP AFTER VEHICLE I।

FAILURE DRIVE RIGHT CENTER I ।

FAILURE DRIVER TO STOP AFTER U । । FAILURE OF DRIVER TO REPORT AC I I FAILURE OF DRIVER TO STOP AFTE I।

FAILURE OF VEH TO STOP AFTER A II

FAILURE OF VEH TO STOP AFTER U । ।

Failure Of Veh-Driver To Stop II

FAILURE OF VEH. DRIVER TO STOP I।

FAILURE OF VEH.DRIVER TO STOP । ।

FAILURE TO ATTACH FRONT PLATE । ।

FAILURE TO ATTACH PLATE AT FRO I।

Failure To Attatch Plates To F I|

FAILURE TO DRIVE RIGHT CENTER I।

FAILURE TO IMMEDIATELY STOP VE । ।

Failure To Stop I I

FAILURE TO STOP । ।

FAILURE TO STOP ACCIDENT | |

Failure To Stop After Acc Inv I I

Failure To Stop After Accident I I

FAILURE TO STOP AFTER ACCIDENT I। 
FAILURE TO STOP AFTER ACCIDENT || 11111

FAILURE TO STOP AFTER ACCUDENT I।

FAILURE TO STOP AFTER ACIDENT । |

FAILURE TO STOP AFTER AN ACCID । ।

FAILURE TO STOP AFTER UNATTENE । ।

FAILURE TO STOP AT A ACCIDENT । ।

Failure To Stop At A Stop Sign II

FAILURE TO STOP AT ACCIDENT I I

FAILURE TO STOP AT ACCIDENT SC । ।

FAILURE TO STOP AT AN ACCIDENT I।

FAILURE TO STOP AT RED CIRCULA I |

Failure To Stop At Scene II

FAILURE TO STOP AT SCENE OF AC । ।

FAILURE TO STOP AT STOP SIGN I |

FAILURE TO STOP AT THE ACCIDEN I।

FAILURE TO STOP AT THE SCENE । ।

FAILURE TO STOP AT THE SCENE O । ।

FAILURE TO STOP VECHICLE AT AC I ।

FAILURE TO STOP VEH AT SCENE O I।

FAILURE TO STOP VEH SCENE OF A I।

FAILURE TO STOP VEH. AT SCENE I ।

FAILURE TO STOP VEHICLE AT SCE I।

Failure To Stop/ Bodily Injury I I

FALSIFIED VEH REG PLATE । |

FISHING W/O LICENSE I ।

HOMICIDE-MV/VESSEL-IMPAIR ALC || 10693

INTENTIONAL MOTOR VECHICLE ACC । ।

INTENTIONAL MOTOR VEHICLE ACCI । ।

KNOWINGLY DRIVING UNINSURED VE । |

License Required Hawkers, Aggresive Pan Handling I| 10855

LICENSE REVOKED । I

LICENSE/TRADER'S: VIOLATION || 10071

Motor Bike ( Dirt Bike Violati I I

MOTOR VEHICLE OFFENSE I।

MOTORBIKES I।

NEGLIGENT DRIVING ।

No Front Md. License Plate I |

Open Container In Vehicle I |

OPEN CONTAINER IN VEHICLE । ।

Operating A Motor Scooter With II

Operating Motor Scooter Withou II

OPERATING SCOOTER W/O LICENSE I।

OPERATING UNREG MOTOR VEH II

OPERATING UNREGISTERED MOTOR I I

Operating Unregistered Motor V I I

OPERATING UNREGISTERED MOTOR V । ।

OPERATING UNREGISTERED VEHICLE I ।

PEDDLING WITHOUT LICENSE I ।

Person Driving Driving Motor V I I

PERSON DRIVING MOTOR VECH ON ( I |

PERSON DRIVING MOTOR VEH ON HW I।

PERSON DRIVING MOTOR VEH ON SU I ।

PERSON DRIVING MOTOR VEH- ON H I ।

PERSON DRIVING MOTOR VEH-ON HI I।

PERSON DRIVING MOTOR VEH. ON ( I I

PERSON DRIVING MOTOR VEH. ON H I I

PERSON DRIVING MOTOR VEH. ON R । । PERSON DRIVING MOTOR VEH. ON(H I। PERSON DRIVING ON MOTOR VEH. O I I PERSON DRIVING ON REVOKED LICE । । 
PERSON DRIVING VEH O N SUSPEND । ।

Person Drving Motor Veh On Hwy II

POSS COCAINE/ UNAUTHORIZED USE I| 20480

Possessing Suspended License I |

Reckles Driving II

RECKLESS DRIVING । ।

RECKLESS DRIVING || 10600

RECKLESS DRIVING || 11111

RECLESS DRIVING ।

RECLKES DRIVING ।

RESTAURANT-LICENSE FAILURE || 10036

REVOKE LICENSE I I

Revoked License I|

REVOKED LICENSE । ।

RIDE/DRIVE DIRTBIKE I |

Seat Belt Violation I |

SELL W/O LICENSE । ।

Sell Without License I |

SELLING W/O LICENSE I ।

SELLING W/O LICENSE I । 12545

SELLING W/O LICENSE | | 20050

SELLING WITHOUT A LICENSE | | 10045

Selling Without License II

SELLING WITHOUT LICENSE । ।

SELLING WITHOUT LICENSE || 10855

Speed Greater Than II

Speed Greater Than Reasonable II

SUSP. LICENSE । |

Suspend. Lic II

Suspended II

SUSPENDED DRIVER ||

Suspended Lic II

Suspended License II

SUSPENDED LICENSE | |

SUSPENDED LICENSE OUT OF STATE । ।

SUSPENDED LICENSE OUT STATE I ।

SUSPENDED OUT OF STATE LICENSE I।

Suspendedlicense II

Suspened License I|

TRAFFIC VIOLATION DUI I I

UNATHORIZE USE OF VEHICLE || 20480

UNATHORIZED USE (VEHICLE) | | 20480

UNAUTH REMOVAL OF MV I। 11466

UNAUTH REMOVAL OF PROP I ।

UNAUTH REMOVAL OF PROP | | 11467

UNAUTH USE MOTOR VEHICLE || 20480

UNAUTH. USE | | 20480

UNAUTHORISED USE || 11466

UNAUTHORIZE USE || 10622

UNAUTHORIZE USE || 10623

UNAUTHORIZE USE I| 11466

UNAUTHORIZE USE || 20480

UNAUTHORIZED DISPLAY AND USE O । ।

Unauthorized Motor Vehicle I I

UNAUTHORIZED USE I।

UNAUTHORIZED USE I| 10622

UNAUTHORIZED USE || 10623

UNAUTHORIZED USE || 11136

UNAUTHORIZED USE I | 11326

UNAUTHORIZED USE || 11466 


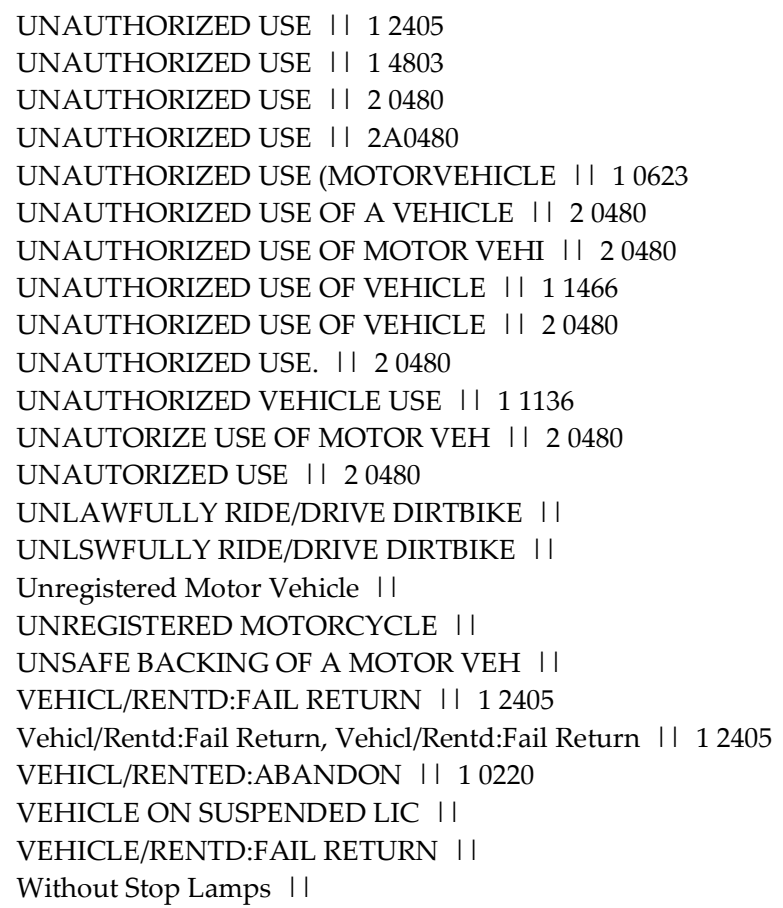

\section{Gambling (Subsequently Demoted to the Category "Ungrouped")}

CDS / GAMBLING || 13903

Cds: Poss Marihuana L/T 10 G, Cds Possession / Gambl || 11635

Cds: Possession-Marihuana, Gambling II 10573

DICE/GAMBLING || 13903

GAMBELING || 13903

GAMBILING || 13903

GAMBING || 13903

GAMBLIND || 13903

Gambling II

GAMBLING | |

GAMBLING || 10573

GAMBLING || 11111

GAMBLING || 13903

GAMBLING || 15202

GAMBLING || 15285

GAMBLING || 22210

GAMBLING (DICE) | | 13903

GAMBLING / DICE || 13903

GAMBLING /DICE || 13903

GAMBLING 13903 || 13903

GAMBLING DICE || 13903

GAMBLING OUTSIDE || 13903

GAMBLING/CDS || 13903

GAMBLING/TRESPASSING || 13903

GAMBLINGS || 13903

GAMBLNG || 13903

GAMING || 13903

Gaming-Keep Table/Place, Gaming-Keep Table/Place I| 13921

Gaming/Cards- Dice- Etc., Cds II 13903

Gaming/Cards- Dice- Etc., Cds Violation I| 13903

Gaming/Cards- Dice- Etc., Gambling I| 13903

Gaming/Cards- Dice- Etc., Gaming || 13903 
Gaming/Cards- Dice- Etc., Gaming/Cards, Dice, Etc. I| 13903 GAMING/CARDS, DICE, ETC. I| 13903

GAMLING || 13903

ILLEGAL GAMBLING || 13903

\section{Trespass}

4TH DEG B \&E ।| 22210

ALCOHOL VIOLATION || 22210

ATT-TRESPASS-POSTED PROPERTY I I 1A5707

CDS || 22210

CDS NOT MARIJUANA || 22210

CDS POSS I| 22210

CDS POSSESSION || 22210

CDS VIOLATION || 22210

Cds: Poss Marihuana L/T 10 G, Cds \& Tresspassing || 11635

Dis.Erly Conduct, Disorderly/Trespassiing || 20050

Dis.Erly Conduct, Trespassing I| 20050

Dis.Erly Conduct, Trespassing/Disorderly || 20050

Dis.Erly Conduct, Tresspass/ Private Property II 20050

Dis.Erly Conduct, Tresspassing || 20050

DISORDERLY || 22210

DISORDERLY CONDUCT I | 22210

DISORDERLY/TRESPASS || 20050

DISORDERLY/TRESPASS || 10344

DISORDERLY/TRESPASS || 20050

FALSE STATEMENT I| 22210

FALSE STATEMENT TO PEACE OFC I| 22210

Harass; A Course Of Conduct, Trespassing I| 10191

INDECENT EXPOSURE || 22210

LITTERING || 22210

LOITERING || 22210

LOTTERING || 22210

NO TRESPASSING || 22210

OPEN CONTAINER I| 22210

POSSESION W/ INT. CDS || 22210

ROGUE AND VAGABOND || 22210

SCHOOL: TRESPASS ON GROUND || 75707

School: Trespass On Ground, Cds I| 75707

School: Trespass On Ground, Trespassing || 75707

School: Trespass On Ground, Trespassing School Prope I| 75707

TREAPASSING || 22210

TREPASS I| 22220

TREPASS PUB AGNCY DUR HRS I| 10344

TREPASSING || 22210

TREPASSING || 22220

TREPASSING || 2A3030

TRESOASSING || 22210

TRESPASING || 22220

TRESPASS II

TRESPASS || 10344

TRESPASS || 10600

TRESPASS || 14803

TRESPASS || 20050

TRESPASS || 20055

TRESPASS || 20480

TRESPASS || 22210

TRESPASS || 22220 
TRESPASS || 23030

TRESPASS || 35399

TRESPASS || 35707

TRESPASS - POSTED || 22210

TRESPASS ON PRIVATE PROPERTY || 22220

TRESPASS POSTED || 22210

TRESPASS POSTED PROPERTY || 22210

TRESPASS POSTED PROPERTY || 22220

TRESPASS PUB AGNCY DUR HRS || 10344

Trespass Pub Agncy Dur Hrs, Disorderly Conduct I| 10344

Trespass Pub Agncy Dur Hrs, Hindering II 10344

Trespass Pub Agncy Dur Hrs, Trepass Pub Agncy Dur H I| 10344

Trespass Pub Agncy Dur Hrs, Trespass Pub Agncy Dur H I। 10344

Trespass Pub Agncy Dur Hrs, Trespassing I| 10344

Trespass Pub Agncy Dur Hrs, Tresspassing II 10344

TRESPASS PUBLIC AGENCY || 35707

TRESPASS-POSTED || 22210

TRESPASS-POSTED PROPERTY || 15707

TRESPASS-POSTED PROPERTY || 22210

Trespass-Posted Property, Breaking In Entering || 22210

Trespass-Posted Property, Buglary || 22210

Trespass-Posted Property, Cds I| 22210

Trespass-Posted Property, Cds Paraphenalia || 22210

Trespass-Posted Property, Cds Poss || 22210

Trespass-Posted Property, Cds Possession || 22210

Trespass-Posted Property, Cds Violation I| 22210

Trespass-Posted Property, Cds/ Trespassing || 22210

Trespass-Posted Property, Disorderly || 22210

Trespass-Posted Property, Disorderly Conduct || 22210

Trespass-Posted Property, Dist Of Cocaine I| 22210

Trespass-Posted Property, Loitering || 22210

Trespass-Posted Property, Open Container I| 22210

Trespass-Posted Property, Open Containers II 22210

Trespass-Posted Property, Poss Cds I| 22210

Trespass-Posted Property, Poss Cocaine I| 22210

Trespass-Posted Property, Poss Heroin || 22210

Trespass-Posted Property, Poss Marijuana || 22210

Trespass-Posted Property, Poss Of Heroin I| 22210

Trespass-Posted Property, Poss. Cocaine || 22210

Trespass-Posted Property, Poss. Heroin || 22210

Trespass-Posted Property, Poss.Herion I| 22210

Trespass-Posted Property, Possession Cds Heroine || 22210

Trespass-Posted Property, Possession Of Heroin || 22210

Trespass-Posted Property, Tesspassing I| 22210

Trespass-Posted Property, Trepass I| 22210

Trespass-Posted Property, Trepassing || 22210

Trespass-Posted Property, Trespass I| 22210

Trespass-Posted Property, Trespass - Post Property I| 22210

Trespass-Posted Property, Trespass - Posted Property II 22210

Trespass-Posted Property, Trespass-Posted Property || 15707

Trespass-Posted Property, Trespass-Posted Property || 22210

Trespass-Posted Property, Trespassing I| 15707

Trespass-Posted Property, Trespassing || 22210

Trespass-Posted Property, Trespassing - Posted I| 22210

Trespass-Posted Property, Trespassing - Posted Prope II 22210

Trespass-Posted Property, Trespassing Posted I| 22210

Trespass-Posted Property, Trespassing- Posted || 22210

Trespass-Posted Property, Trespassing/Open Containe I| 22210

Trespass-Posted Property, Trespassinng || 22210

Trespass-Posted Property, Trespassng || 22210 
Trespass-Posted Property, Trespsassing || 22210

Trespass-Posted Property, Tresspass || 22210

Trespass-Posted Property, Tresspassing I| 22210

Trespass-Posted Property, Tresspassing City Porperty I| 22210

Trespass-Posted Property, Violation Of Park Rule I| 22210

TRESPASS: PRIVATE PROPERTY || 22220

Trespass: Private Property, Breaking And Entering || 22220

Trespass: Private Property, Cds Violation II 22220

Trespass: Private Property, Disorderly I| 22220

Trespass: Private Property, Disorderly Conduct II 22220

Trespass: Private Property, Drunk And Disorderly || 22220

Trespass: Private Property, Harrasment I| 22220

Trespass: Private Property, Loitering || 22220

Trespass: Private Property, Loitering/Tresspassing || 22220

Trespass: Private Property, Shoplifting I| 22220

Trespass: Private Property, Trespasing || 22220

Trespass: Private Property, Trespass I| 22220

Trespass: Private Property, Trespass: Private Prope I| 22220

Trespass: Private Property, Trespass: Private Proper I| 22220

Trespass: Private Property, Trespassing I| 22220

Trespass: Private Property, Trespassing Warned II 22220

Trespass: Private Property, Trespassing/Disorderly || 22220

Trespass: Private Property, Tresspasing I| 22220

Trespass: Private Property, Tresspass II 22220

Trespass: Private Property, Tresspassing I| 22220

TRESPASS:PRIVATE PROPERTY | | 25707

TRESPASSIMG || 22220

TRESPASSIN || 22220

Trespassing I।

TRESPASSING । ।

TRESPASSING || 10191

TRESPASSING || 10344

TRESPASSING || 10573

TRESPASSING || 10600

TRESPASSING || 10604

TRESPASSING || 11111

TRESPASSING || 11137

TRESPASSING || 13605

TRESPASSING || 14200

TRESPASSING || 14803

TRESPASSING || 15202

TRESPASSING || 15707

TRESPASSING || 16505

TRESPASSING || $1 \mathrm{~A} 6503$

TRESPASSING || 20050

TRESPASSING || 20055

TRESPASSING || 20105

TRESPASSING || 22210

TRESPASSING || 22220

TRESPASSING || 23030

TRESPASSING || 23040

TRESPASSING || 2A2220

TRESPASSING || 2A3030

TRESPASSING || 35399

TRESPASSING || 35707

TRESPASSING || 75707

TRESPASSING - PRIVATE PROPERTY || 22220

TRESPASSING POSTED || 22210

TRESPASSING PRIVATE PROPERTY || 22220

TRESPASSING-POSTED || 22210 
TRESPASSING: PRIVATE PROPERTY || 22220

TRESPASSING/CDS || 22220

TRESPASSING/CDS VIOLATION | | 15202

TRESPASSING/CDS VIOLATION I I 22210

TRESPASSING/DISORDELY || 22220

TRESPASSING/DISORDERLY || 20050

TRESPASSING/DISORDERLY | | 22220

Trespassing/Loitering I |

TRESPASSISNG || 22220

TRESPPASING || 22220

TRESSPASING || 22210

TRESSPASING || 22220

TRESSPASS ।

TRESSPASS || 22210

TRESSPASS II 22220

TRESSPASS || 23030

Tresspassing II

TRESSPASSING । ।

TRESSPASSING || 10344

TRESSPASSING || 10600

TRESSPASSING || 11137

TRESSPASSING || 1A1137

TRESSPASSING || 20045

TRESSPASSING || 20050

TRESSPASSING || 20055

TRESSPASSING I| 22210

TRESSPASSING || 22220

TRESSPASSING || 23030

TRESSPASSING || 2A3030

TRESSPASSING || 35707

TRESSPASSING I| 75707

TRESSPASSING/DISORDERLY || 22220

URINATING IN PUBLIC/TRESPASSIN || 22220

VANDALISM || 22210

WANTON TRESPASS || 22220

\section{Disorderly Conduct}

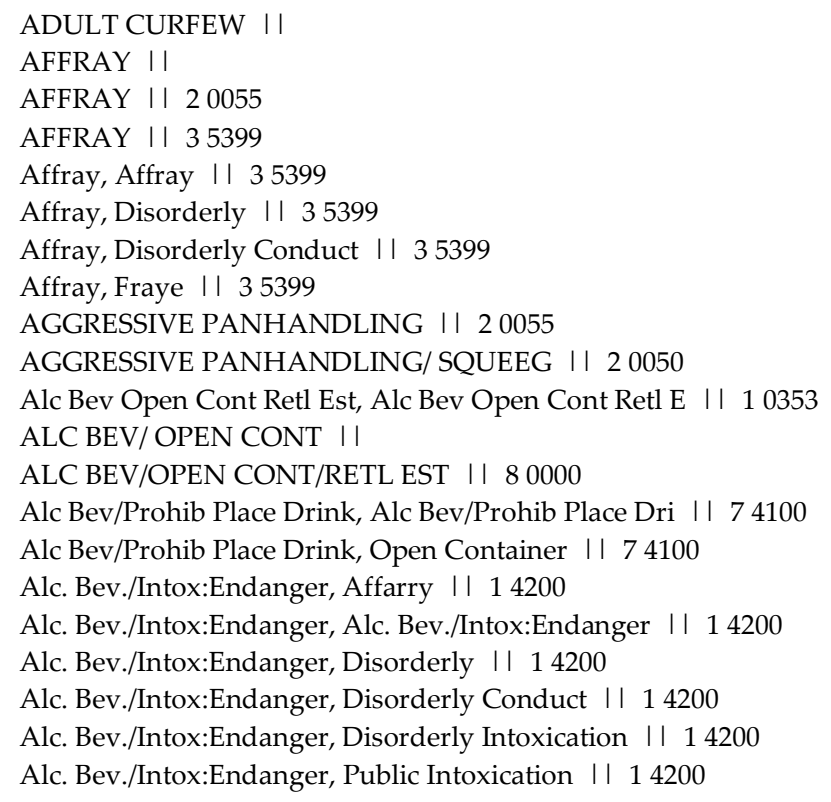


Alc. Bev./Intox:Endanger, Rouge \& Vagabond/ I I 14200

ALCOHOL INTOX ENDANGER || 14200

Alcohol Violation I I

ALCOHOL VIOLATION I।

Att-Harass; A Course Of Conduct, Att-Harass; A Cour II 1A0191

ATT-PEACE ORDER FAIL TO COMPL । ।

CAUSE LITTER/DUMP: L/T 100 LB || 10333

Cause Litter/Dump: L/T 100 Lbs, Littering || 10333

CDS || 10640

CDS || 20050

CDS I| 20055

CDS MARIJUANA || 20050

CDS POSS || 10640

CDS POSS || 20050

CDS POSSESSION OF PARAPHERNALI || 10640

CDS VIOLATION || 20050

CDS VIOLATION || 20055

Cds: Poss Marihuana L/T 10 G, Disorderly Conduct I | 11635

Cds: Poss Marihuana L/T 10 G, Disorderly/Cds I | 11635

Cds: Poss Marihuana L/T 10 G, Open Container I| 11635

Cds: Possession-Marihuana, Disorderly || 10573

Cds: Possession-Marihuana, Disorderly/Cds || 10573

Cds:Possess-Not Marihuana, Cds Disturbution II 43550

CDS/FALSE STATEMENT I 10640

CDS/INDECENT EXPOSURE || 22220

CITY CURFEW VIOLATION II

CIVIL DISOBEDIENCE || 20055

Con-Harass; A Course Of Conduct, Con-Harass; A Cours I| 1C0191 CONDUCTING BUSINESS OF DEALER ।

CONSPIRACY TO POSS COCAINE || 20050

CONSPIRACY TO POSS COCOAINE I| 20050

CONSUME ALC BEV PUBLIC I| 11663

CONSUME ALCOHOL IN PUBLIC I ।

Consuming Alcohol II

Consuming Alcohol On Public St I |

CURFEW II

CURFEW VIOATION II

CURFEW VIOL II

CURFEW VIOL. II

CURFEW VIOLATION I |

CURFEW VIOLATION II 10690

CURFEW VIOLATION- EXECUTIVE OR I।

CURFEW VIOLATIONS I I

CURFEW VIOLATON II

DEFECATION IN PUBLIC I ।

DIRSORDERLY CONDUCT || 20050

DIRT BIKE VIOLATION || 20050

DIS CON I| 20050

DIS CONDUCT ।| 20050

DIS INTOX || 20050

DIS ORDERLY CONDCUT । | 20050

Dis.Erly Conduct, Affray || 20050

Dis.Erly Conduct, Aggressive Panhandling I| 20050

Dis.Erly Conduct, Bicycle Violation I| 20050

Dis.Erly Conduct, Cds I| 20050

Dis.Erly Conduct, Cds Poss I| 20050

Dis.Erly Conduct, Cds Violation I| 20050

Dis.Erly Conduct, Cursing In Park || 20050

Dis.Erly Conduct, Disoderly || 20050

Dis.Erly Conduct, Disoderly Intoxication I| 20050 
Dis.Erly Conduct, Disordely || 20050

Dis.Erly Conduct, Disordely Conduct || 20050

Dis.Erly Conduct, Disorderly || 20050

Dis.Erly Conduct, Disorderly Conduct I| 20050

Dis.Erly Conduct, Disorderly Conducted I| 20050

Dis.Erly Conduct, Disorderly Condut I| 20050

Dis.Erly Conduct, Disorderly Conudct I| 20050

Dis.Erly Conduct, Disorderly House I| 20050

Dis.Erly Conduct, Disorderly Intox || 20050

Dis.Erly Conduct, Disorderly Intoxicated || 20050

Dis.Erly Conduct, Disorderly/Loitering | | 20050

Dis.Erly Conduct, Disorderlyconduct | | 20050

Dis.Erly Conduct, Disordery Conduct || 20050

Dis.Erly Conduct, Disorederly Conduct I| 20050

Dis.Erly Conduct, Dissorderly || 20050

Dis.Erly Conduct, Dissorderly Conduct || 20050

Dis.Erly Conduct, Drunk Disorderly || 20050

Dis.Erly Conduct, Drunken Disorderly || 20050

Dis.Erly Conduct, F.T.O. I I 20050

Dis.Erly Conduct, Fail To Sign Citation I| 20050

Dis.Erly Conduct, Hindering I| 20050

Dis.Erly Conduct, Indecent Exposure || 20050

Dis.Erly Conduct, Loitering I| 20050

Dis.Erly Conduct, Open Container I| 20050

Dis.Erly Conduct, Resitan An Arrest I| 20050

Dis.Erly Conduct, Solicitation I| 20050

Dis.Erly Conduct, Trepassing || 20050

DISCRDERLY CONDUCT || 20050

DISODERLY || 20050

Disoderly Conduct I।

DISODERLY CONDUCT || 20050

DISOERDLY CONDUCT ।| 13605

DISOERDLY CONDUCT । 20050

DISORDELY || 20050

DISORDELY CONDUCT ।| 14200

DISORDELY CONDUCT । 14803

DISORDELY CONDUCT ।| 20050

DISORDELY CONDUCT ।| 20055

DISORDERELY CONDUCT । | 20050

DISORDERLEY | 20050

DISORDERLEY CONDUCT । | 20050

DISORDERLEY CONDUCT । | 20065

Disorderly II

DISORDERLY I ।

DISORDERLY || 10016

DISORDERLY || 10344

DISORDERLY || 10395

DISORDERLY || 10592

DISORDERLY || 10600

DISORDERLY || 10622

DISORDERLY | | 10640

DISORDERLY || 10690

DISORDERLY || 11661

DISORDERLY || 13605

DISORDERLY || 14200

DISORDERLY || 14803

DISORDERLY || 20045

DISORDERLY || 20050

DISORDERLY || 20055

DISORDERLY || 20105 
DISORDERLY || 22220

DISORDERLY || 23010

DISORDERLY || 23040

DISORDERLY || 31314

DISORDERLY || 34030

DISORDERLY || 35311

DISORDERLY || 35399

DISORDERLY || 55311

DISORDERLY CONDUCT || 20055

DISORDERLY - INTOXICATED || 22220

DISORDERLY CODUCT I| 20050

Disorderly Conduct I |

DISORDERLY CONDUCT । |

DISORDERLY CONDUCT । | 10344

DISORDERLY CONDUCT || 10573

DISORDERLY CONDUCT || 10600

DISORDERLY CONDUCT | | 10640

DISORDERLY CONDUCT I I 10690

DISORDERLY CONDUCT । | 11425

DISORDERLY CONDUCT || 11661

DISORDERLY CONDUCT I| 12903

DISORDERLY CONDUCT I| 13605

DISORDERLY CONDUCT || 14200

DISORDERLY CONDUCT । | 20045

DISORDERLY CONDUCT I| 20050

DISORDERLY CONDUCT || 20055

DISORDERLY CONDUCT | | 20065

DISORDERLY CONDUCT | | 22003

DISORDERLY CONDUCT । 22220

DISORDERLY CONDUCT || 23010

DISORDERLY CONDUCT || 2A3030

DISORDERLY CONDUCT I I 35399

DISORDERLY CONDUCT PUBLIC INTO । ।

DISORDERLY CONDUCT/LOITERING । ।

DISORDERLY CONDUST || 20050

DISORDERLY CONUCT I| 20050

DISORDERLY DRINKING | | 20050

DISORDERLY HOUSEHOLD || 20065

DISORDERLY HOUSEHOLD || 55311

DISORDERLY INTOX । |

DISORDERLY INTOX || 20050

DISORDERLY INTOX || 20065

DISORDERLY INTOX || 22220

DISORDERLY INTOXICATED | | 20050

DISORDERLY INTOXICATION || 10349

DISORDERLY INTOXICATION || 14200

DISORDERLY INTOXICATION | | 20050

DISORDERLY INTOXICATION || 22220

DISORDERLY ORDER || 10600

DISORDERLY PUBLIC INTOXICATION || 20055

DISORDERLY-INTOXICATED || 14200

DISORDERLY/ CURFEW I ।

DISORDERLY/INTOXICATION || 14200

DISORDERLY/OPEN CONTAINER | | 20050

DISORDERLYCONDUCT I| 20050

DISORDERY I| 10640

DISORDERY || 20050

DISORDERY CONDUCT I| 20050

DISORDERYL CONDUCT । I 20050

DISORDLERY || 20055 


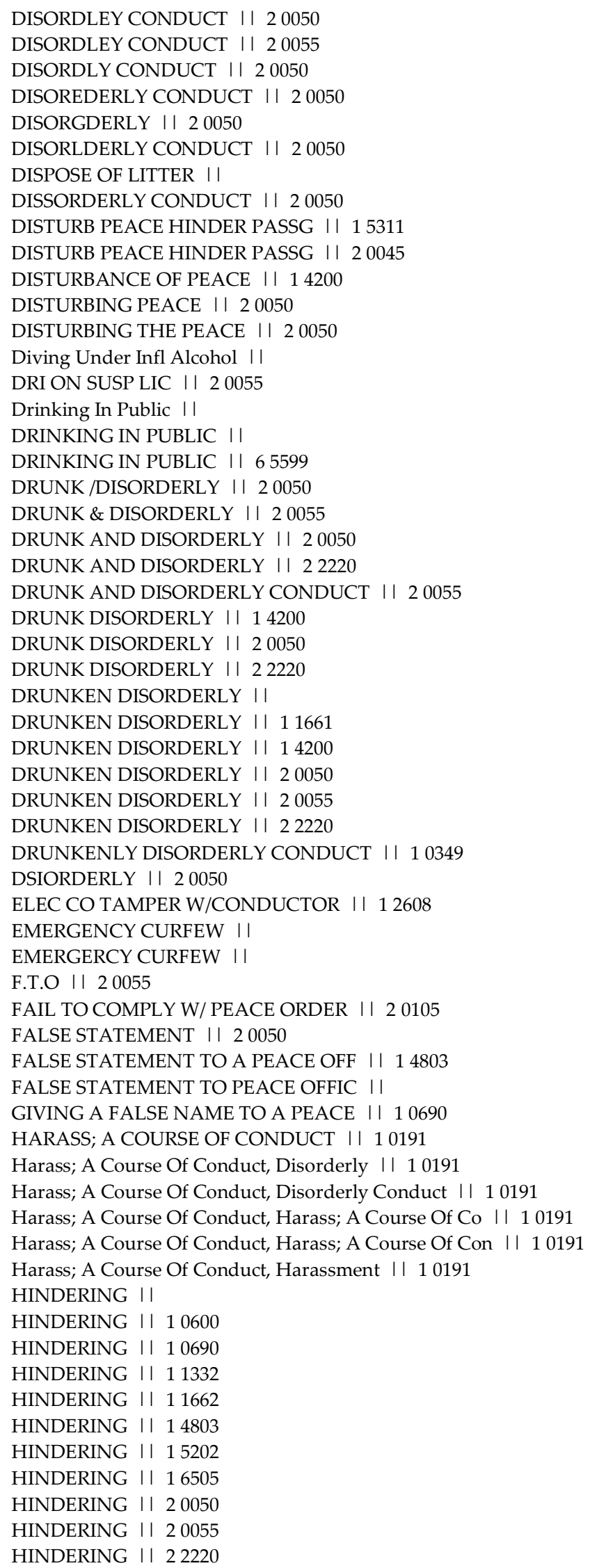


HINDERING WITH ARREST | | 10600

HINDERING/ DISORDERLY || 20050

HINDERING/DISORDERLY | | 20050

ILLEGAL DUMPING | | 10643

ILLEGAL DUMPING || 10645

ILLEGAL POSS OF AMMO | | 20055

INDECENT EXPOSE CORRECTION EMP || 21133

Indecent Exposure II

INDECENT EXPOSURE । ।

INDECENT EXPOSURE || 10600

INDECENT EXPOSURE || 13605

INDECENT EXPOSURE || 43600

INDECENT EXPOSURE || 63600

Indecent Exposure, Cds || 13605

Indecent Exposure, Cds Violation I| 13605

Indecent Exposure, Disorderly Coduct Indecent Exp || 13605

Indecent Exposure, Disorderly Conduct || 13605

Indecent Exposure, Ind. Exposure || 13605

Indecent Exposure, Indecent Exposure || 13605

Indecent Exposure, Indecent Exsposure || 13605

Indecent Exposure, Indicent Exposure || 13605

Indecent Exposure, Lewd Act || 13605

Indecent Exposure, Loitering || 13605

Indecent Exposure, Urinating In Public || 13605

INDECENT WXPOSURE || 63600

INDENCT EXPOSURE || 13605

INDICENT EXPOSURE || 13605

INTOX. DISORDERLY || 14200

LAUNCHING A MISSILE I | 20050

LIOTERING I।

LITTER/DUMP: OVER 500 LBS | | 10645

LITTER/DUMP: UNDER 100 LBS || 10640

Litter/Dump: Under 100 Lbs, Cds Poss II 10640

Litter/Dump: Under 100 Lbs, Cds Poss W Intent Mariju || 10640

Litter/Dump: Under 100 Lbs, Cds Violation II 10640

Litter/Dump: Under 100 Lbs, Drinking In Public I| 10640

Litter/Dump: Under 100 Lbs, Littering || 10640

Litter/Dump: Under 100 Lbs, Poss Not Marijuana || 10640

Littering ||

LITTERING || 10600

LITTERING || 10640

LITTERING \& DISORDERLY || 10640

LITTERING/ DISORDERLY CONDUCT | | 22220

LOITER/DISORDERLY I ।

LOITERING || 20050

LOITERING || 20055

LOITERING/DISORDERLY CONDUCT I ।

LOTTERING || 10640

NIGHT TIME CURFEW । ।

NIGHTIME CURFEW । ।

OBLIGATORY CURFEW I।

OBSTRUCT JUSTICE I| 25006

Obstruct Justice, Obstruct Justice | | 25006

OBSTRUCT/HINDER I| 10600

OBSTRUCTING I| 10600

OBSTRUCTION || 10600

OBSTRUCTION || 20050

Open Container II

Open Cantainer II

Open Comtainer II 


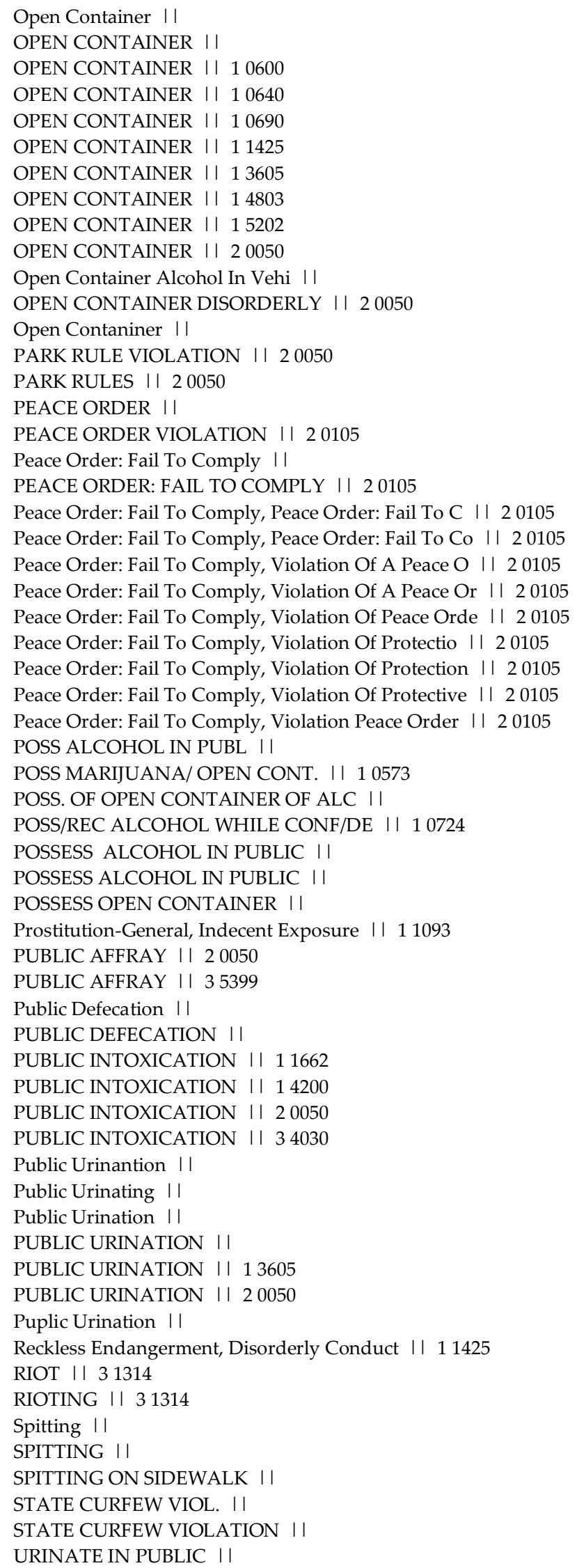




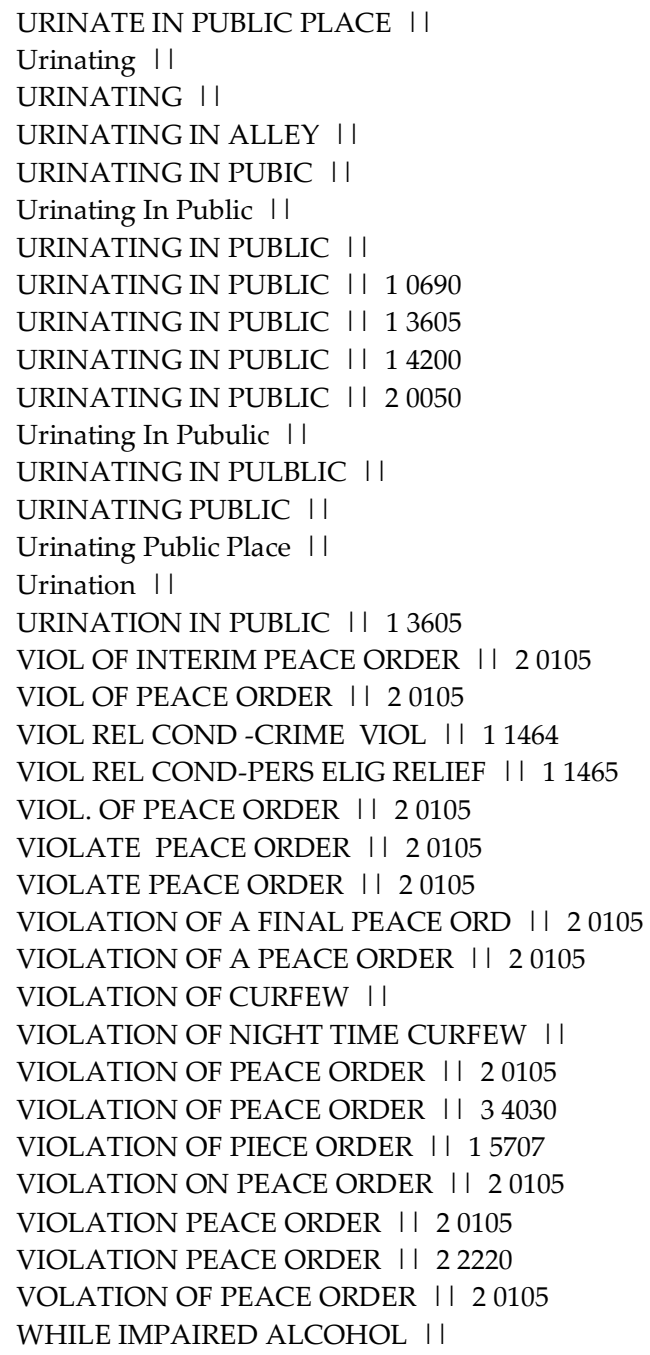

\section{Drug Possession (Subsequently Combined with Drug Distribution)}

1-0573 || 10573

ALTERED PRESCRIPTION I। 10249

Animal Cruel Fail: Provide, Cds Violation || 10503

ATT-CDS-SELL SUBSTITUTE DRUG | | 1A0018

C.D.S. Possession II

C.D.S. POSSESSION TO WIT MARIJ | | 10573

CD POSS NOT MARIJUANA । |

Cd Posses Not Marijuana II

CDS POSS. II 11111

Cds $\mid 1$

CDS II

CDS || 10005

CDS || 10021

CDS || 10271

CDS || 10356

CDS || 10487

CDS || 10488

CDS || 10573

CDS || 10600

CDS || 10622 


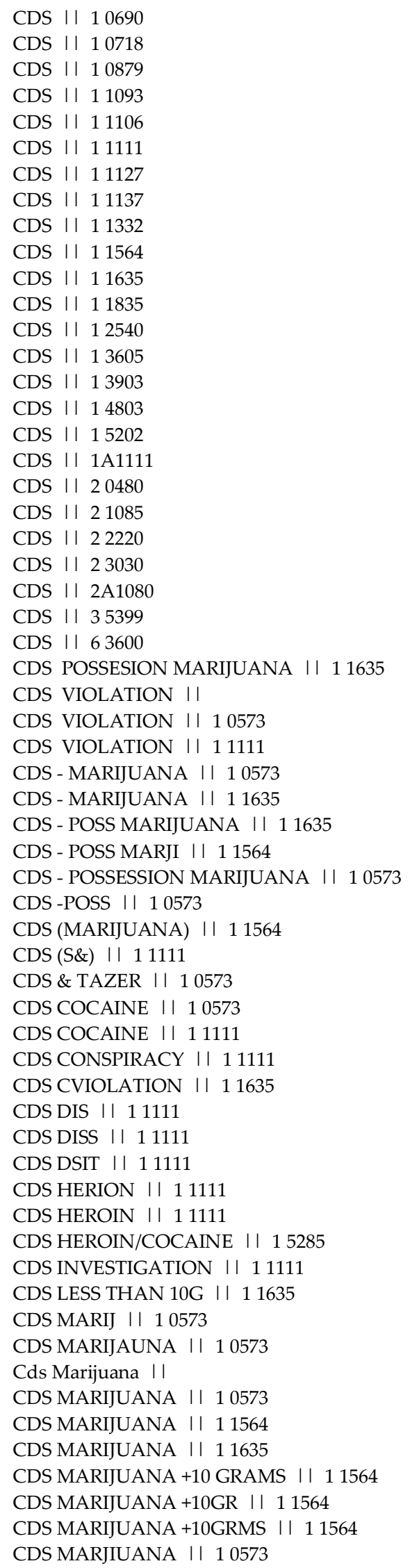




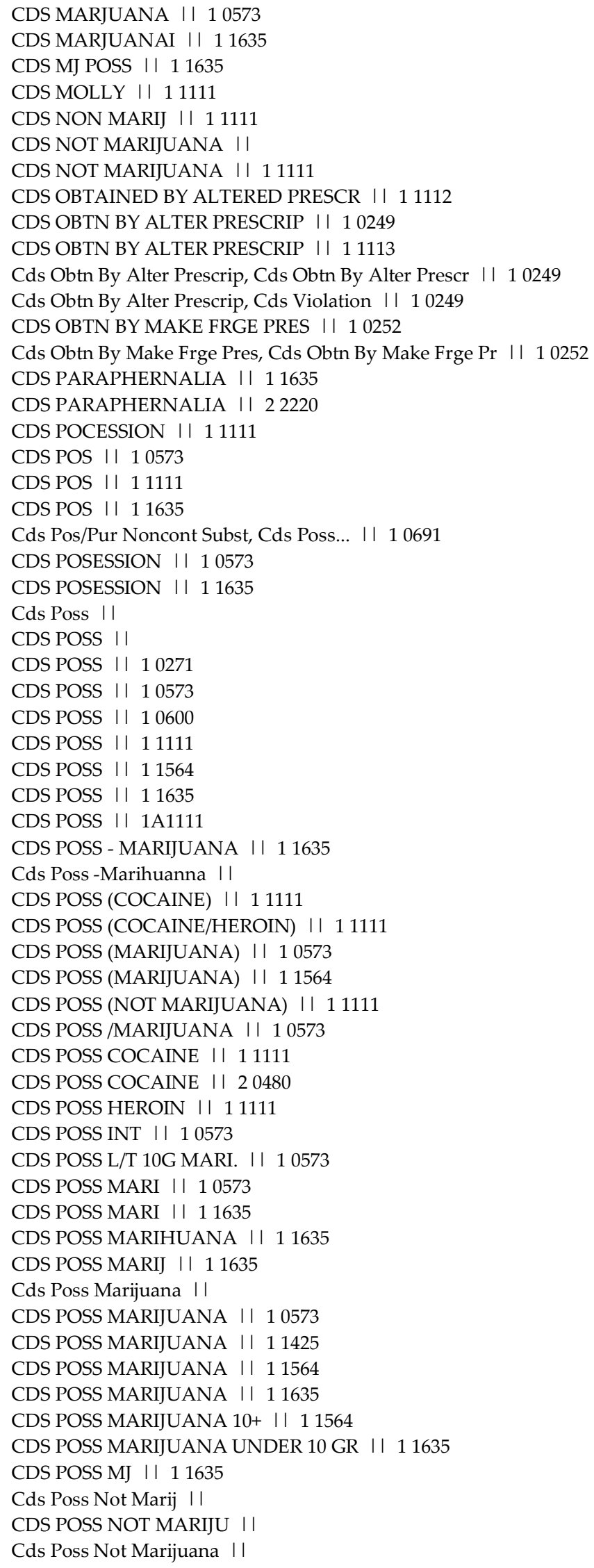


CDS POSS NOT MARIJUANA I । CDS POSS NOT MARIJUANA || 10573 CDS POSS NOT MARIJUANA || 11111 CDS POSS OF COCAINE || 11111 CDS POSS OF HERION । | 11111 CDS POSS OF MARIJ । 11635 CDS POSS OF MARIJUANA I | 10573 CDS POSS OF MARIJUANA || 11635 CDS POSS OTHER || 11111 CDS POSS OXYCODONE I I 10573 CDS POSS PARAPHERNELIA I| 11111 CDS POSS VIOLATION || 10573

CDS POSS W INT || 11111

CDS POSS WITH INT || 10573

Cds Poss-Marijuana II

CDS POSS, NOT MARIJUANA || 11111 CDS POSS: MARIJUANA || 10573

CDS POSS: MARIJUANA I | 11635 CDS POSS: NOT MARIJUANA || 11111 Cds Poss. II

CDS POSS. II 10573

CDS POSS. I। 11111

CDS POSS. II 11564

CDS POSS. I। 15202

CDS POSS. II 22220

CDS POSS. COAINE || 11111

CDS POSS. COCAINE || 11111

CDS POSS. CRYSTAL METH । 11111

CDS POSS. HERION || 11111

CDS POSS. HEROIN ।| 11111

CDS POSS. HEROIN/ COCAINE | | 11111 CDS POSS. MARIHUANA || 11635

Cds Poss. Marijuana II

CDS POSS. MARIJUANA | | 10573

CDS POSS. MARIJUANA || 11564

CDS POSS. MARIJUANA || 11635

CDS POSS. MAURIJUANA || 11635

CDS POSS. MJ || 11635

CDS POSS. OTHER I| 11111

CDS POSS.MARIJUANA || 11635

CDS POSS(MARIJUANA) | | 10573

CDS POSS/COCAINE || 11111

CDS POSS/HEROIN || 11111

CDS POSS/HEROIN,COCAINE || 11111

CDS POSSE || 10573

CDS POSSE MARIJUANA । ।

Cds Posses II

CDS POSSES || 11111

CDS POSSES INTENT ।| 10573

Cds Possesion 11

CDS POSSESION । ।

CDS POSSESION || 10573

CDS POSSESION I| 11111

CDS POSSESION || 11635

CDS POSSESION COCAINE I| 11635

CDS POSSESION MARIJUANA || 11635

CDS POSSESION OF MARIJUANA | | 11635

CDS POSSESION WITH THE INTENT || 10573

CDS POSSESION-NOT MARIJUANA | | 11111

CDS POSSESS || 10573 


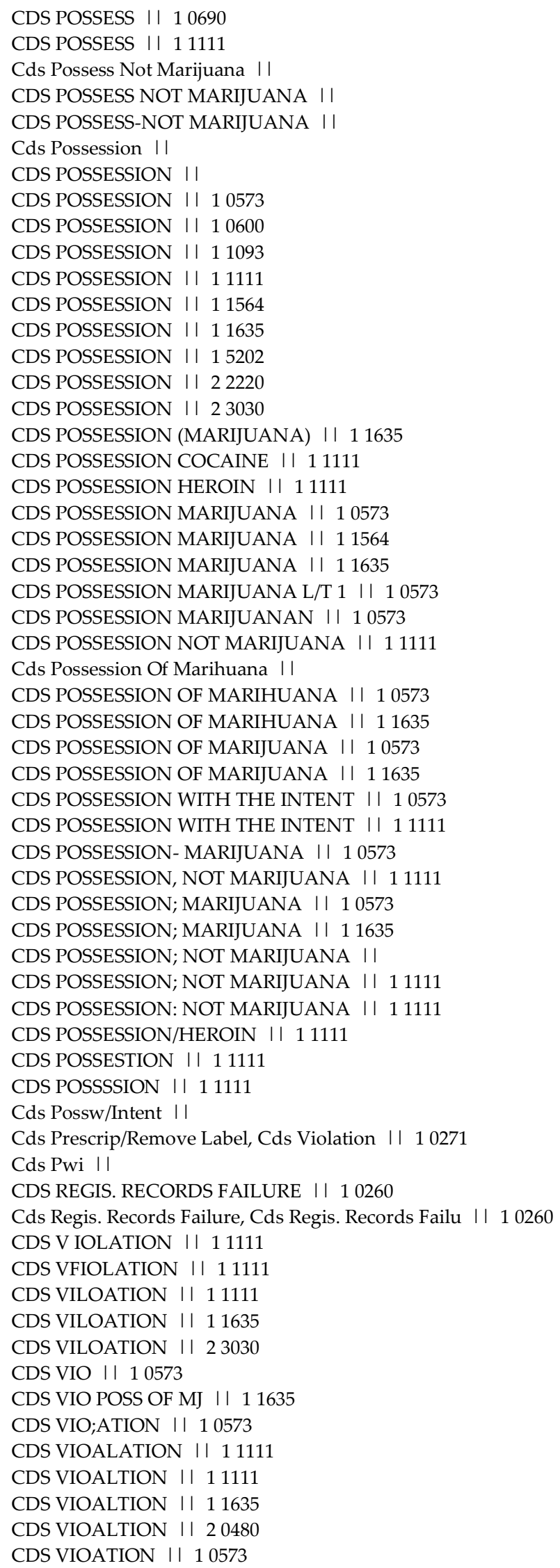


CDS VIOATION || 11111

CDS VIOL || 10573

CDS VIOL || 11564

CDS VIOL I| 15202

CDS VIOL-POSS. MARIJUANA | 11635

CDS VIOL. II 11111

CDS VIOL. II 11635

CDS VIOL. POSS. MARIJ. I I 11635

CDS VIOLAION || 11111

CDS VIOLAION || 11564

CDS VIOLATIN || 10573

CDS VIOLATIN || 11111

CDS VIOLATIOIN || 11635

CDS VIOLATIOM || 10573

Cds Violation I I

CDS VIOLATION $\mid$ |

CDS VIOLATION || 10249

CDS VIOLATION || 10256

CDS VIOLATION || 10271

CDS VIOLATION || 10487

CDS VIOLATION I| 10573

CDS VIOLATION I। 10600

CDS VIOLATION I| 10622

CDS VIOLATION || 10623

CDS VIOLATION I| 10690

CDS VIOLATION || 11106

CDS VIOLATION || 11111

CDS VIOLATION || 11113

CDS VIOLATION || 11136

CDS VIOLATION || 11137

CDS VIOLATION || 11364

CDS VIOLATION ।| 11454

CDS VIOLATION I| 11455

CDS VIOLATION || 11564

CDS VIOLATION || 11567

CDS VIOLATION I| 11610

CDS VIOLATION || 11635

CDS VIOLATION ॥ 11835

CDS VIOLATION || 13903

CDS VIOLATION || 14803

CDS VIOLATION || 15202

CDS VIOLATION || 15285

CDS VIOLATION || 1A0826

CDS VIOLATION I| 1A1111

CDS VIOLATION || 1A1137

CDS VIOLATION || 1C1111

CDS VIOLATION I| 20262

CDS VIOLATION II 20480

CDS VIOLATION $\| 21020$

CDS VIOLATION || 21085

CDS VIOLATION || 22220

CDS VIOLATION I| 23000

CDS VIOLATION I| 23030

CDS VIOLATION II 23040

CDS VIOLATION I| 2A3599

CDS VIOLATION I| 35250

CDS VIOLATION || 53555

CDS VIOLATION - MARIJUANA | | 10573

CDS VIOLATION - MARIJUANA POSS I। 11635

CDS VIOLATION INSIDE || 10573 
CDS VIOLATION MARIJUANA | | 11635 CDS VIOLATION MARIJUANA MORE T || 10573 CDS VIOLATION MARIJUANA POSS | | 11635 CDS VIOLATION POSS I| 11111 CDS VIOLATION POSS MARIJ | | 11635 CDS VIOLATION POSS MARIJU || 11635 CDS VIOLATION POSSESSION | 11111 CDS VIOLATION POSSESSION OF MA || 10573 CDS VIOLATION-POSS. MARIJUANA || 11635 CDS VIOLATION; NOT MARIJUANA || 11111 CDS VIOLATION; POSSESSION I| 10573 CDS VIOLATION;POSSESSION || 11635 CDS VIOLATION: POSSESSION OF M || 11564 CDS VIOLATION/ POSSESSION II 11111 CDS VIOLATION/ POSS MARIJUANA I| 11635 CDS VIOLATION/ POSS. OF MARIJU || 10573 CDS VIOLATION/TRAFFIC || 11111 CDS VIOLATION' I | 11111 CDS VIOLATIONS I। CDS VIOLATIONS || 11111 CDS VIOLATIONS || 11564 CDS VIOLATIONS || 11635 CDS VIOLATON || 10573 CDS VIOLATON || 11111 CDS VIOLATON || 11635 CDS VIOLATTION I| 11111 CDS VIOLATTION || 11635 CDS VIOLATUION || 10573 CDS VIOLLATION || 11564 CDS VIOLTAION || 10573

CDS VIOLTION || 11111 CDS VOILATION || 11111 CDS VOL I| 11635

CDS VOLATION || 11111

CDS VOLATION || 11635 CDS VVIOLATION || 11111 CDS- MARIJUANA II 10573 CDS- MARIJUANA || 11635 CDS- POSS || 10573 CDS- POSS || 11111 CDS- POSSESSION || 10573

Cds-Inhale Harm Substance, Cds I| 15599 CDS-MARIJUANA || 11635 CDS-NOT MARIJUANA || 10573 CDS-NOT MARIJUANA || 11111

CDS-POSS || 10573 CDS-POSS || 11111 CDS-POSS || 11451 CDS-POSS || 11635 CDS-POSS MARIJUANA | | 10573 CDS-POSS NOT MARIJUANA I । CDS-POSSESS || 10573 CDS-POSSESS || 11635 CDS-POSSESSION || 10573 CDS-POSSESSION || 11635

Cds-Unsolicit Mailing, Cds-Unsolicit Mailing I| 10025 CDS-VIOLATION || 11635

CDS, LARC, FALSE STMT | | 14803

Cds: Poss Marihuana L/T 10 G, C.D.S Violation I। 11635

Cds: Poss Marihuana L/T 10 G, C.D.S. Possession || 11635 
Cds: Poss Marihuana L/T 10 G, C.D.S. Violation || 11635

Cds: Poss Marihuana L/T 10 G, Cda Violation II 11635

Cds: Poss Marihuana L/T 10 G, Cdfs Poss Marijuana || 11635

Cds: Poss Marihuana L/T 10 G, Cds I I 11635

Cds: Poss Marihuana L/T 10 G, Cds Marijuana || 11635

Cds: Poss Marihuana L/T 10 G, Cds Violation I| 11635

Cds: Poss Marihuana L/T 10 G, Cds - Poss I| 11635

Cds: Poss Marihuana L/T 10 G, Cds - Poss Marijuana II 11635

Cds: Poss Marihuana L/T 10 G, Cds - Possession I| 11635

Cds: Poss Marihuana L/T 10 G, Cds :Poss Marijuana II 11635

Cds: Poss Marihuana L/T 10 G, Cds Cocaine II 11635

Cds: Poss Marihuana L/T 10 G, Cds Maraijuana II 11635

Cds: Poss Marihuana L/T 10 G, Cds Marijana || 11635

Cds: Poss Marihuana L/T 10 G, Cds Marijnana II 11635

Cds: Poss Marihuana L/T 10 G, Cds Marijuana II 11635

Cds: Poss Marihuana L/T 10 G, Cds Marijuana Less Tha || 11635

Cds: Poss Marihuana L/T 10 G, Cds Marjiana I| 11635

Cds: Poss Marihuana L/T 10 G, Cds Maurijauna I| 11635

Cds: Poss Marihuana L/T 10 G, Cds Pos Marijuana II 11635

Cds: Poss Marihuana L/T 10 G, Cds Posession I| 11635

Cds: Poss Marihuana L/T 10 G, Cds Poss I| 11635

Cds: Poss Marihuana L/T 10 G, Cds Poss - Mj Ltg | | 11635

Cds: Poss Marihuana L/T 10 G, Cds Poss (Marijuana) I। 11635

Cds: Poss Marihuana L/T 10 G, Cds Poss / Marijuana || 11635

Cds: Poss Marihuana L/T 10 G, Cds Poss Mari I I 11635

Cds: Poss Marihuana L/T 10 G, Cds Poss Marihuana II 11635

Cds: Poss Marihuana L/T 10 G, Cds Poss Marij II 11635

Cds: Poss Marihuana L/T 10 G, Cds Poss Marijuana || 11635

Cds: Poss Marihuana L/T 10 G, Cds Poss Marijuana <10 || 11635

Cds: Poss Marihuana L/T 10 G, Cds Poss Marj I I 11635

Cds: Poss Marihuana L/T 10 G, Cds Poss Marjuana I| 11635

Cds: Poss Marihuana L/T 10 G, Cds Poss Mj II 11635

Cds: Poss Marihuana L/T 10 G, Cds Poss Murijuan I| 11635

Cds: Poss Marihuana L/T 10 G, Cds Poss Of Marijuan I| 11635

Cds: Poss Marihuana L/T 10 G, Cds Poss Of Marihuana || 11635

Cds: Poss Marihuana L/T 10 G, Cds Poss Of Marijuana I| 11635

Cds: Poss Marihuana L/T 10 G, Cds Poss W Intent Mar I| 11635

Cds: Poss Marihuana L/T 10 G, Cds Poss W Intent Mari || 11635

Cds: Poss Marihuana L/T 10 G, Cds Poss- Marijuana I| 11635

Cds: Poss Marihuana L/T 10 G, Cds Poss-Marijuana || 11635

Cds: Poss Marihuana L/T 10 G, Cds Poss, I I 11635

Cds: Poss Marihuana L/T 10 G, Cds Poss, Marijuana || 11635

Cds: Poss Marihuana L/T 10 G, Cds Poss: Marijuana || 11635

Cds: Poss Marihuana L/T 10 G, Cds Poss. I | 11635

Cds: Poss Marihuana L/T 10 G, Cds Poss. Marijauna I| 11635

Cds: Poss Marihuana L/T 10 G, Cds Poss. Marijuana || 11635

Cds: Poss Marihuana L/T 10 G, Cds Poss... I I 11635

Cds: Poss Marihuana L/T 10 G, Cds Poss/ Intent II 11635

Cds: Poss Marihuana L/T 10 G, Cds Poss/Marijuana || 11635

Cds: Poss Marihuana L/T 10 G, Cds Possesion || 11635

Cds: Poss Marihuana L/T 10 G, Cds Possesion Marijuan I| 11635

Cds: Poss Marihuana L/T 10 G, Cds Possess I| 11635

Cds: Poss Marihuana L/T 10 G, Cds Possess Marijuana II 11635

Cds: Poss Marihuana L/T 10 G, Cds Possess-Marihuana || 11635

Cds: Poss Marihuana L/T 10 G, Cds Possession II 11635

Cds: Poss Marihuana L/T 10 G, Cds Possession - Marij II 11635

Cds: Poss Marihuana L/T 10 G, Cds Possession Marihu || 11635

Cds: Poss Marihuana L/T 10 G, Cds Possession Mariju I| 11635

Cds: Poss Marihuana L/T 10 G, Cds Possession Marijua I| 11635

Cds: Poss Marihuana L/T 10 G, Cds Possession Of Mar I| 11635 
Cds: Poss Marihuana L/T 10 G, Cds Possession Of Mari || 11635 Cds: Poss Marihuana L/T 10 G, Cds Possession W/ Int || 11635 Cds: Poss Marihuana L/T 10 G, Cds Possession W/ Inte || 11635 Cds: Poss Marihuana L/T 10 G, Cds Possession With In || 11635 Cds: Poss Marihuana L/T 10 G, Cds Possession- Mariju || 11635 Cds: Poss Marihuana L/T 10 G, Cds Possession-Marihua || 11635 Cds: Poss Marihuana L/T 10 G, Cds Possession-Marijua || 11635 Cds: Poss Marihuana L/T 10 G, Cds Possession/Mariju || 11635

Cds: Poss Marihuana L/T 10 G, Cds Possesson I| 11635

Cds: Poss Marihuana L/T 10 G, Cds Pss II 11635

Cds: Poss Marihuana L/T 10 G, Cds Pwi || 11635

Cds: Poss Marihuana L/T 10 G, Cds Pwi Marijuana || 11635

Cds: Poss Marihuana L/T 10 G, Cds Vilation I | 11635

Cds: Poss Marihuana L/T 10 G, Cds Viloation I I 11635

Cds: Poss Marihuana L/T 10 G, Cds Vio I | 11635

Cds: Poss Marihuana L/T 10 G, Cds Vioaltion I I 11635

Cds: Poss Marihuana L/T 10 G, Cds Viol I I 11635

Cds: Poss Marihuana L/T 10 G, Cds Viol. I I 11635

Cds: Poss Marihuana L/T 10 G, Cds Violatiom I | 11635

Cds: Poss Marihuana L/T 10 G, Cds Violation || 11635

Cds: Poss Marihuana L/T 10 G, Cds Violation Marijua I| 11635

Cds: Poss Marihuana L/T 10 G, Cds Violation Marijuan I| 11635

Cds: Poss Marihuana L/T 10 G, Cds Violation Poss. Of I| 11635

Cds: Poss Marihuana L/T 10 G, Cds Violation. I I 11635

Cds: Poss Marihuana L/T 10 G, Cds Violations II 11635

Cds: Poss Marihuana L/T 10 G, Cds Violaton I| 11635

Cds: Poss Marihuana L/T 10 G, Cds Violstion I I 11635

Cds: Poss Marihuana L/T 10 G, Cds Voilation II 11635

Cds: Poss Marihuana L/T 10 G, Cds Volation I| 11635

Cds: Poss Marihuana L/T 10 G, Cds- Poss Mj || 11635

Cds: Poss Marihuana L/T 10 G, Cds-Mar || 11635

Cds: Poss Marihuana L/T 10 G, Cds-Marijuana II 11635

Cds: Poss Marihuana L/T 10 G, Cds-Mj I| 11635

Cds: Poss Marihuana L/T 10 G, Cds-Poss || 11635

Cds: Poss Marihuana L/T 10 G, Cds-Poss-Marijuana || 11635

Cds: Poss Marihuana L/T 10 G, Cds-Possess II 11635

Cds: Poss Marihuana L/T 10 G, Cds-Possession II 11635

Cds: Poss Marihuana L/T 10 G, Cds; Possession- Mari || 11635

Cds: Poss Marihuana L/T 10 G, Cds: Poss Marihuana L | | 11635

Cds: Poss Marihuana L/T 10 G, Cds: Poss Marihuana L/ | | 11635

Cds: Poss Marihuana L/T 10 G, Cds: Poss Marijuana I| 11635

Cds: Poss Marihuana L/T 10 G, Cds: Poss Marijuana L || 11635

Cds: Poss Marihuana L/T 10 G, Cds: Poss-Marijuana I| 11635

Cds: Poss Marihuana L/T 10 G, Cds: Poss. I I 11635

Cds: Poss Marihuana L/T 10 G, Cds: Possession II 11635

Cds: Poss Marihuana L/T 10 G, Cds: Possession Mariju II 11635

Cds: Poss Marihuana L/T 10 G, Cds:Poss Marijuana II 11635

Cds: Poss Marihuana L/T 10 G, Cds. Violation II 11635

Cds: Poss Marihuana L/T 10 G, Cds/Possession I| 11635

Cds: Poss Marihuana L/T 10 G, Cdsa Marijuana || 11635

Cds: Poss Marihuana L/T 10 G, Cdsposs II 11635

Cds: Poss Marihuana L/T 10 G, Cdss Violation I I 11635

Cds: Poss Marihuana L/T 10 G, Cdv Violation I | 11635

Cds: Poss Marihuana L/T 10 G, Exceed Max Speed I | 11635

Cds: Poss Marihuana L/T 10 G, Marijuana II 11635

Cds: Poss Marihuana L/T 10 G, Marijuana Poss I | 11635

Cds: Poss Marihuana L/T 10 G, Narcotics || 11635

Cds: Poss Marihuana L/T 10 G, Pos Of Marijuana I I 11635

Cds: Poss Marihuana L/T 10 G, Posession Of Marajuana I। 11635

Cds: Poss Marihuana L/T 10 G, Posession Of Marijuan I | 11635 
Cds: Poss Marihuana L/T 10 G, Poss || 11635

Cds: Poss Marihuana L/T 10 G, Poss Of Marijuana || 11635

Cds: Poss Marihuana L/T 10 G, Poss Of Marijuana II 11635

Cds: Poss Marihuana L/T 10 G, Poss - Marijuana II 11635

Cds: Poss Marihuana L/T 10 G, Poss Cds || 11635

Cds: Poss Marihuana L/T 10 G, Poss Cds (Marijuana) || 11635

Cds: Poss Marihuana L/T 10 G, Poss Cds Marijuana I I 11635

Cds: Poss Marihuana L/T 10 G, Poss Cds-Marijuana I| 11635

Cds: Poss Marihuana L/T 10 G, Poss Cocaine II 11635

Cds: Poss Marihuana L/T 10 G, Poss Ession Of Marijua I| 11635

Cds: Poss Marihuana L/T 10 G, Poss Heroin II 11635

Cds: Poss Marihuana L/T 10 G, Poss Int Marij | | 11635

Cds: Poss Marihuana L/T 10 G, Poss Less 10G Marijua II 11635

Cds: Poss Marihuana L/T 10 G, Poss Mairjauna I | 11635

Cds: Poss Marihuana L/T 10 G, Poss Maj I | 11635

Cds: Poss Marihuana L/T 10 G, Poss Mari Cds || 11635

Cds: Poss Marihuana L/T 10 G, Poss Mariajuana || 11635

Cds: Poss Marihuana L/T 10 G, Poss Marihuana II 11635

Cds: Poss Marihuana L/T 10 G, Poss Marij I | 11635

Cds: Poss Marihuana L/T 10 G, Poss Marijuan I| 11635

Cds: Poss Marihuana L/T 10 G, Poss Marijuana || 11635

Cds: Poss Marihuana L/T 10 G, Poss Marijuana Less 10 || 11635

Cds: Poss Marihuana L/T 10 G, Poss Marijuanna I| 11635

Cds: Poss Marihuana L/T 10 G, Poss Marijuna II 11635

Cds: Poss Marihuana L/T 10 G, Poss Marijuuana I| 11635

Cds: Poss Marihuana L/T 10 G, Poss Marj I | 11635

Cds: Poss Marihuana L/T 10 G, Poss Merijuana I। 11635

Cds: Poss Marihuana L/T 10 G, Poss Mj || 11635

Cds: Poss Marihuana L/T 10 G, Poss Not Marijuana II 11635

Cds: Poss Marihuana L/T 10 G, Poss Of Cds || 11635

Cds: Poss Marihuana L/T 10 G, Poss Of Mara | | 11635

Cds: Poss Marihuana L/T 10 G, Poss Of Mariijuana II 11635

Cds: Poss Marihuana L/T 10 G, Poss Of Marijauan I| 11635

Cds: Poss Marihuana L/T 10 G, Poss Of Marijauna I| 11635

Cds: Poss Marihuana L/T 10 G, Poss Of Marijuana || 11635

Cds: Poss Marihuana L/T 10 G, Poss Of Marijuanna II 11635

Cds: Poss Marihuana L/T 10 G, Poss W Intent Marijuan I| 11635

Cds: Poss Marihuana L/T 10 G, Poss W/ Intent Cds I| 11635

Cds: Poss Marihuana L/T 10 G, Poss W/ Intent Marijua || 11635

Cds: Poss Marihuana L/T 10 G, Poss W/ Marijuana II 11635

Cds: Poss Marihuana L/T 10 G, Poss. Cds I| 11635

Cds: Poss Marihuana L/T 10 G, Poss. Marijauana || 11635

Cds: Poss Marihuana L/T 10 G, Poss. Marijuana || 11635

Cds: Poss Marihuana L/T 10 G, Poss. Maurijuana I| 11635

Cds: Poss Marihuana L/T 10 G, Poss. Of Cds II 11635

Cds: Poss Marihuana L/T 10 G, Poss. Of Heroin || 11635

Cds: Poss Marihuana L/T 10 G, Poss. Of Marijauana I| 11635

Cds: Poss Marihuana L/T 10 G, Poss. Of Marijuana || 11635

Cds: Poss Marihuana L/T 10 G, Poss.Cds II 11635

Cds: Poss Marihuana L/T 10 G, Poss.Marijuana | | 11635

Cds: Poss Marihuana L/T 10 G, Poss.Maurijauna I| 11635

Cds: Poss Marihuana L/T 10 G, Poss.Of Mariguana || 11635

Cds: Poss Marihuana L/T 10 G, Poss.Of Marijuana I| 11635

Cds: Poss Marihuana L/T 10 G, Poss/ Marijauana I| 11635

Cds: Poss Marihuana L/T 10 G, Poss/Mairjuana II 11635

Cds: Poss Marihuana L/T 10 G, Poss/Marijuana | | 11635

Cds: Poss Marihuana L/T 10 G, Possesion Of Marijuana || 11635

Cds: Poss Marihuana L/T 10 G, Possessiobn Of Marijua || 11635

Cds: Poss Marihuana L/T 10 G, Possession I I 11635

Cds: Poss Marihuana L/T 10 G, Possession Cds || 11635 
Cds: Poss Marihuana L/T 10 G, Possession Cocaine || 11635

Cds: Poss Marihuana L/T 10 G, Possession Marajuana || 11635

Cds: Poss Marihuana L/T 10 G, Possession Marijuana || 11635

Cds: Poss Marihuana L/T 10 G, Possession Marijuna I| 11635

Cds: Poss Marihuana L/T 10 G, Possession Mariquana II 11635

Cds: Poss Marihuana L/T 10 G, Possession Of Cds || 11635

Cds: Poss Marihuana L/T 10 G, Possession Of Marijua || 11635

Cds: Poss Marihuana L/T 10 G, Possession Of Marijuan I| 11635

Cds: Poss Marihuana L/T 10 G, Possession Of Possible I| 11635

Cds: Poss Marihuana L/T 10 G, Possession W Intent I | 11635

Cds: Poss Marihuana L/T 10 G, Possession/Marijuana II 11635

Cds: Poss Marihuana L/T 10 G, Posssession Of Marijua II 11635

Cds: Poss Marihuana L/T 10 G, Pwi - Marijuana || 11635

Cds: Poss Marihuana L/T 10 G, Pwi Cds || 11635

Cds: Poss Marihuana L/T 10 G, Pwi Marijuana II 11635

Cds: Poss Marihuana L/T 10 G, Pwi-Marijuana || 11635

CDS: POSS MARIJUANA $10 \mathrm{GM}+\mid$ | 11564

CDS: POSS MARIJUANA L/T 10 G I | 11635

CDS: POSS-MARIJUANA I| 10573

CDS: POSS-MOT MARJI I।

CDS: POSS. MARIJUANA I| 11635

CDS: POSSE MARIJUANA 10GM+ | | 10573

CDS: POSSESS-NOT MARIJUANA I| 11111

CDS: POSSESSION || 11111

CDS: POSSESSION MARIJUANA || 10573

CDS: POSSESSION MARIJUANA I | 11564

CDS: POSSESSION MARIJUANA | | 11635

Cds: Possession-Marihuana, Attempted First Degree As || 10573

Cds: Possession-Marihuana, C.D.S. Violation I| 10573

Cds: Possession-Marihuana, Cdfs Violation II 10573

Cds: Possession-Marihuana, Cds I| 10573

Cds: Possession-Marihuana, Cds Poss Marijuana W / | | 10573

Cds: Possession-Marihuana, Cds Violation II 10573

Cds: Possession-Marihuana, Cds - Poss Marijuana I| 10573

Cds: Possession-Marihuana, Cds - Pwi Marijuana || 10573

Cds: Possession-Marihuana, Cds Marijuana II 10573

Cds: Possession-Marihuana, Cds Marijuana Possession I| 10573

Cds: Possession-Marihuana, Cds Mariuana || 10573

Cds: Possession-Marihuana, Cds Paraphenlia || 10573

Cds: Possession-Marihuana, Cds Pos. II 10573

Cds: Possession-Marihuana, Cds Poss II 10573

Cds: Possession-Marihuana, Cds Poss - Marjiuana || 10573

Cds: Possession-Marihuana, Cds Poss Heroin II 10573

Cds: Possession-Marihuana, Cds Poss Marij II 10573

Cds: Possession-Marihuana, Cds Poss Marijuana II 10573

Cds: Possession-Marihuana, Cds Poss Marijuana W/ Int || 10573

Cds: Possession-Marihuana, Cds Poss Marijuna II 10573

Cds: Possession-Marihuana, Cds Poss Of Marijuana I| 10573

Cds: Possession-Marihuana, Cds Poss Oxycodone I| 10573

Cds: Possession-Marihuana, Cds Poss W Marij I I 10573

Cds: Possession-Marihuana, Cds Poss W/ Int I| 10573

Cds: Possession-Marihuana, Cds Poss W/ Intent I| 10573

Cds: Possession-Marihuana, Cds Poss W $\backslash$ Int | | 10573

Cds: Possession-Marihuana, Cds Poss-Not Marijuana || 10573

Cds: Possession-Marihuana, Cds Poss, Marijauna II 10573

Cds: Possession-Marihuana, Cds Poss: Maijuana II 10573

Cds: Possession-Marihuana, Cds Poss. I I 10573

Cds: Possession-Marihuana, Cds Poss. Marijuana || 10573

Cds: Possession-Marihuana, Cds Poss.Marijuana || 10573

Cds: Possession-Marihuana, Cds Poss.Marijuana L/T 10 || 10573 
Cds: Possession-Marihuana, Cds Poss/Marijuana || 10573

Cds: Possession-Marihuana, Cds Posses || 10573

Cds: Possession-Marihuana, Cds Possesion I| 10573

Cds: Possession-Marihuana, Cds Possesion Cocaine I| 10573

Cds: Possession-Marihuana, Cds Possess I| 10573

Cds: Possession-Marihuana, Cds Possession I| 10573

Cds: Possession-Marihuana, Cds Possession Marijuana I| 10573

Cds: Possession-Marihuana, Cds Possession Mj I| 10573

Cds: Possession-Marihuana, Cds Possession Not Mariju || 10573

Cds: Possession-Marihuana, Cds Possession Of Marijua II 10573

Cds: Possession-Marihuana, Cds Possession W Intent I| 10573

Cds: Possession-Marihuana, Cds Possession With Inten || 10573

Cds: Possession-Marihuana, Cds Possession- Marijuana || 10573

Cds: Possession-Marihuana, Cds Possw/Intent Marijuan I| 10573

Cds: Possession-Marihuana, Cds Pwi II 10573

Cds: Possession-Marihuana, Cds Pwi Marijuana || 10573

Cds: Possession-Marihuana, Cds Vioaltion II 10573

Cds: Possession-Marihuana, Cds Viol || 10573

Cds: Possession-Marihuana, Cds Viol. I | 10573

Cds: Possession-Marihuana, Cds Violaltion I| 10573

Cds: Possession-Marihuana, Cds Violation II 10573

Cds: Possession-Marihuana, Cds Violation - Poss Mar I| 10573

Cds: Possession-Marihuana, Cds Violation Marijuana II 10573

Cds: Possession-Marihuana, Cds Violation Poss Marij I| 10573

Cds: Possession-Marihuana, Cds Violation Poss Mariju I| 10573

Cds: Possession-Marihuana, Cds Violaton I| 10573

Cds: Possession-Marihuana, Cds- Marijuana || 10573

Cds: Possession-Marihuana, Cds- Poss W/ Intent || 10573

Cds: Possession-Marihuana, Cds-Poss I| 10573

Cds: Possession-Marihuana, Cds-Possession II 10573

Cds: Possession-Marihuana, Cds-Pwi || 10573

Cds: Possession-Marihuana, Cds; Marijuana II 10573

Cds: Possession-Marihuana, Cds: Poss Marijuana II 10573

Cds: Possession-Marihuana, Cds: Poss-Marihuana II 10573

Cds: Possession-Marihuana, Cds: Poss-Marijuana || 10573

Cds: Possession-Marihuana, Cds: Possession Of Mariju I| 10573

Cds: Possession-Marihuana, Cds: Possession-Marihuan I| 10573

Cds: Possession-Marihuana, Cds: Possession-Marihuana || 10573

Cds: Possession-Marihuana, Cds: Possession-Marijuan || 10573

Cds: Possession-Marihuana, Cds:Marijuana || 10573

Cds: Possession-Marihuana, Cds. Possession I| 10573

Cds: Possession-Marihuana, Cdsn Violation I| 10573

Cds: Possession-Marihuana, Cdsposs.Marijuana || 10573

Cds: Possession-Marihuana, Csd Violation II 10573

Cds: Possession-Marihuana, Exe S\& S/ Cds || 10573

Cds: Possession-Marihuana, Marijuana II 10573

Cds: Possession-Marihuana, Marijuana Poss W/ Intent I| 10573

Cds: Possession-Marihuana, Narcotic I| 10573

Cds: Possession-Marihuana, Narcotics || 10573

Cds: Possession-Marihuana, Poss || 10573

Cds: Possession-Marihuana, Poss - Marijuana II 10573

Cds: Possession-Marihuana, Poss Cds I| 10573

Cds: Possession-Marihuana, Poss Cds (Marijuana) | | 10573

Cds: Possession-Marihuana, Poss Cds Marih II 10573

Cds: Possession-Marihuana, Poss Cds Marijuana I| 10573

Cds: Possession-Marihuana, Poss Cds-Marijuana I| 10573

Cds: Possession-Marihuana, Poss Cocaine || 10573

Cds: Possession-Marihuana, Poss Herion I| 10573

Cds: Possession-Marihuana, Poss Heroin || 10573

Cds: Possession-Marihuana, Poss Marij || 10573 
Cds: Possession-Marihuana, Poss Marijuana II 10573

Cds: Possession-Marihuana, Poss Marijuana/Cocaine I| 10573

Cds: Possession-Marihuana, Poss Marijuanna II 10573

Cds: Possession-Marihuana, Poss Marijuna II 10573

Cds: Possession-Marihuana, Poss Not Marijuana || 10573

Cds: Possession-Marihuana, Poss Of Cds || 10573

Cds: Possession-Marihuana, Poss Of Cocaine II 10573

Cds: Possession-Marihuana, Poss Of Heroin I| 10573

Cds: Possession-Marihuana, Poss Of Marajuana || 10573

Cds: Possession-Marihuana, Poss Of Marijauna II 10573

Cds: Possession-Marihuana, Poss Of Marijuan || 10573

Cds: Possession-Marihuana, Poss Of Marijuana II 10573

Cds: Possession-Marihuana, Poss Of Marji I | 10573

Cds: Possession-Marihuana, Poss Percocet || 10573

Cds: Possession-Marihuana, Poss W Int Marijuana || 10573

Cds: Possession-Marihuana, Poss W/ Int Marijuana || 10573

Cds: Possession-Marihuana, Poss W/ Marijuana II 10573

Cds: Possession-Marihuana, Poss With Int Marijuana I| 10573

Cds: Possession-Marihuana, Poss: Marijuana I| 10573

Cds: Possession-Marihuana, Poss. Cds || 10573

Cds: Possession-Marihuana, Poss. Herion I| 10573

Cds: Possession-Marihuana, Poss. Marihuana II 10573

Cds: Possession-Marihuana, Poss. Marijauana || 10573

Cds: Possession-Marihuana, Poss. Marijuana || 10573

Cds: Possession-Marihuana, Poss. Of Marijuana I| 10573

Cds: Possession-Marihuana, Poss. Of Marjiuana || 10573

Cds: Possession-Marihuana, Poss.Cds I| 10573

Cds: Possession-Marihuana, Poss/Cds || 10573

Cds: Possession-Marihuana, Possesion W/ Intent || 10573

Cds: Possession-Marihuana, Possess Cds I| 10573

Cds: Possession-Marihuana, Possession I| 10573

Cds: Possession-Marihuana, Possession - Marijuana || 10573

Cds: Possession-Marihuana, Possession Cds || 10573

Cds: Possession-Marihuana, Possession Marijuana || 10573

Cds: Possession-Marihuana, Possession Of Cds || 10573

Cds: Possession-Marihuana, Possession Of Cocaine I| 10573

Cds: Possession-Marihuana, Possession Of Marijuana I| 10573

Cds: Possession-Marihuana, Possession Of Mariuana || 10573

Cds: Possession-Marihuana, Possession W/ Intent Mari | | 10573

Cds: Possession-Marihuana, Possession/Intent-Marijua || 10573

Cds: Possession-Marihuana, Possession/Marijuana || 10573

Cds: Possession-Marihuana, Pox Marijuana I| 10573

Cds: Possession-Marihuana, Pwd Marijuana II 10573

Cds: Possession-Marihuana, Pwi Marijuana II 10573

Cds: Possession-Marihuana, Pwi - Marijuana II 10573

Cds: Possession-Marihuana, Pwi Cds || 10573

Cds: Possession-Marihuana, Pwi Cocaine II 10573

Cds: Possession-Marihuana, Pwi Marijauna | | 10573

Cds: Possession-Marihuana, Pwi Marijuana II 10573

Cds: Possession-Marihuana, Pwi-Marijuana || 10573

CDS: POSSESSION-MARIJUANA || 10573

Cds:Admin - Not Marihuana, Cds:Admin - Not Marihuana I| 10247

Cds:Admin - Not Marihuana, Cds:Admin - Not Marijuan I| 10247

CDS:ADMIN - NOT MARIJUANA I| 10247

CDS:COUNTERFIT EQUIP:PCP/LSD | | 10523

CDS:POSS MARIJUANA || 10573

CDS:POSS MARIJUANA || 11635

Cds:Poss Para, Cds Violation I| 53555

CDS:POSS PARAPHERNALIA || 53555

CDS:POSSES-NOT MAR । 
CDS. POSS || 10573

CDS. POSS || 11111

CDS. POSS MARIJUANA || 10573

CDS/ POSS HEROIN || 11111

CDS/ POSSESSION || 10573

CDS/HEROIN || 11111

CDS/POS I| 11111

CDS/POS W INTENT । I 10573

CDS/POSS || 10573

CDS/POSS || 11111

CDS/POSS I| 11635

CDS/POSS COAINE || 11111

CDS/POSS COCAINE || 11111

CDS/TAZER II

CDSD VIOLATION I I

CDSPOSS MARIJUANA I| 11635

CDSVIOLATION || 10573

CDSVIOLATION || 11111

CDSVIOLATION || 11635

COCAINE || 11111

COCAINE POSS || 11111

CON- CDS: POSS MARIJNA L/T 10 | । 1C1635

Con-Cds Attempt- Conspiracy, Cds Violation II 2C3599

Con-Cds: Possession - Marihuana, Cds II 1C0573

Con-Cds: Possession - Marihuana, Con-Cds:Poss-Marihu II 1C0573

CON-CDS: POSSESSION - MARIJUAN I I 1C0573

CS VIOLATION || 10573

CSD, POSS. COCAINE || 11111

DRI ON SUSP LIC || 10573

FELONY CDS || 10488

FELONY CDS || 11564

Hire Etc Minor: Dist Cds II

HIT AND RUN/CDS I। 10573

MARIJUANA +10 GRAMS | | 11564

MARIJUANA LESS THAN 10 GRMS || 11635

MARIJUANA POSS I| 10573

MARIJUANA POSS || 11635

MARIJUANA POSSESSION || 10573

MARIJUANA POSSESSION WITH INTE || 10573

Other Non Felony Charge, Cds II 11449

Other Non Felony Charge, Cds I| 20082

P.W.I MARIJUANA || 11564

P.W.I.D. II 10573

POOS OF MARJ II 11635

POSS || 10573

POSS MARIJUANA || 10573

POSS OF MARIJUANA II 10573

POSS CDS II

POSS CDS || 10573

POSS CDS || 11106

POSS CDS || 11111

POSS CDS || 11564

POSS CDS || 11635

POSS CDS (NOT MARIJUANA) || 11111

POSS CDS ABOVE 10 GRAMS I I 10573

POSS CDS MARIJ L/T 10G I | 11635

POSS CDS MARIJUANA || 10573

POSS CDS MARIJUANA II 11635

POSS CDS NOT MARIJUANA || 11111

POSS CDS NOT MARJ. I I 11564 
POSS CDS-MARIJ I| 11635

Poss Cds-Marijuana I।

Poss Cocaine II

POSS COCAINE || 11111

POSS COCAINE || 14801

POSS HERION || 11111

POSS HEROIN I ।

POSS HEROIN I| 11111

POSS HEROIN || 11416

POSS HEROINE || 11111

POSS INT MARIJUANA I| 10487

POSS MARIJ I| 10573

POSS MARIJ || 11635

POSS MARIJ L/T 10G I | 11635

POSS MARIJANA I| 11635

POSS MARIJAUA || 11635

POSS MARIJAUANA || 10573

POSS MARIJAUNA || 10573

POSS MARIJAUNA | | 11635

Poss Marijuana II

POSS MARIJUANA I।

POSS MARIJUANA I I 10573

POSS MARIJUANA | | 11564

POSS MARIJUANA || 11635

POSS MARIJUANA +10 GRAMS || 11564

POSS MARIJUNA I I 10573

POSS MARIJUNA || 11635

POSS MARJ I| 10573

POSS MARJ I| 11635

POSS MARJIUANA I| 10573

POSS MARJUANA | | 10573

POSS MJ || 10573

POSS NOT MARIJUANA I | 11111

POSS NOT MARIJUANNA II 11111

POSS OF CDS || 10573

POSS OF CDS I| 11111

POSS OF CDS I| 11635

POSS OF CDS || 22220

POSS OF CDS NOT MARIJUANA || 11111

Poss Of Cocaine II

POSS OF COCAINE || 11111

POSS OF HERION || 11111

POSS OF HEROIN ।| 11111

POSS OF MARIJ. I I 10573

POSS OF MARIJANNA || 11635

Poss Of Marijauna II

POSS OF MARIJUAN I| 11635

Poss Of Marijuana II

POSS OF MARIJUANA II 10573

POSS OF MARIJUANA || 11564

POSS OF MARIJUANA I| 11635

POSS OF MARIJUNA I| 10573

POSS OF MARIJUNAN I| 11635

POSS OF MARJUAN I| 10573

POSS OF MARJUANA I I 10573

POSS OF MDMA I| 11111

POSS OF METHADONE || 11111

POSS OF OXYCODONE/XANAX || 11111

POSS W INT MARIJUANA I I 10573

POSS W INTENT I I 10573 
POSS W/ MARIJUANA I| 10573

POSS WITH MARIJUANA | | 10573

POSS. CDS I| 10573

POSS. CDS || 11111

POSS. CDS || 11635

POSS. CDS- MARIJUANA || 10573

POSS. CDS-MARIJUANA || 11635

POSS. COCAINE || 11111

POSS. HERION || 11111

POSS. HEROIN || 11111

POSS. MARIHUANA || 10573

POSS. MARIJAUANA || 11635

POSS. MARIJAUNA || 10573

POSS. MARIJUANA || 10573

POSS. MARIJUANA || 11564

POSS. MARIJUANA || 11635

POSS. MARJ. II 11635

POSS. OF CDS || 10573

POSS. OF COCAINE || 11111

POSS. OF HERION । | 11111

POSS. OF HEROIN || 11111

POSS. OF MARIJAUANA || 10573

Poss. Of Marijuana I I

POSS. OF MARIJUANA || 10573

POSS. OF MARIJUANA II 11635

POSS. W./INT. MARIJUANA I| 10573

POSS. WITH INT COCAINE || 11111

POSS. WITH INT MARIJUANA || 10573

POSS./WINT. II 10573

POSS.CDS I| 10573

POSS.CDS || 11635

POSS.MARIH || 10573

POSS.MARIHUANA || 10573

POSS.MARIJUANA || 10573

POSS.MARIJUANA I| 11564

POSS.MARIJUANA || 11635

POSS.OF CDS I| 11111

POSS.OF MARIJUANA || 11564

POSS/CDS I| 11111

POSS/REC CDS WHILE CONF/DET I I 10718

POSS/W INTENT MARIJUANA || 10573

POSSESION CDS I।

POSSESION CDS || 11111

POSSESION CDS || 11635

Possesion Marijuana II

POSSESION MARIJUANA || 11635

POSSESION OF CDS II 10573

POSSESION OF CDS || 11111

POSSESION OF MARIJUANA || 10573

POSSESION OF SCHEDULE I NARCOT || 11111

POSSESS CDS I| 10573

POSSESS CDS I| 11564

POSSESS MARIJUANA || 10573

Possess/Use False Govt Id Doc, Cds || 11432

POSSESSION II 10573

POSSESSION OF MARIJUANA || 10573

POSSESSION CDS || 10573

POSSESSION CDS || 11111

POSSESSION CDS || 11564

POSSESSION CDS || 11635 


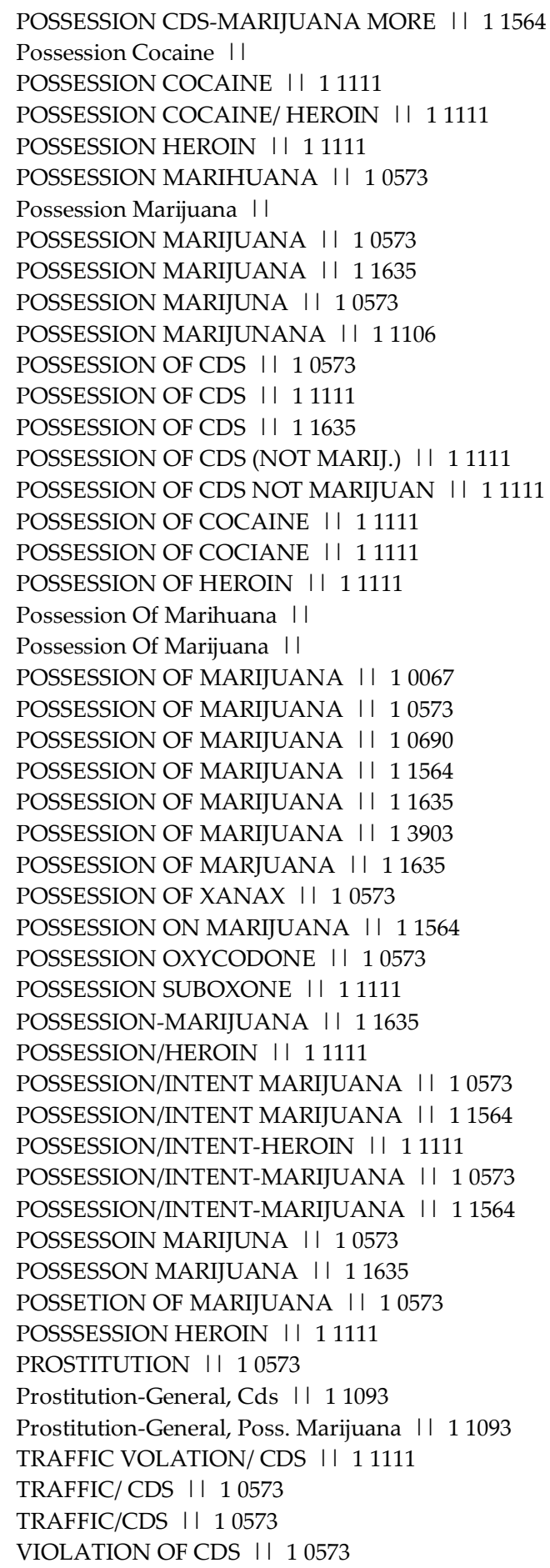

\section{Loitering and Vagabond}

AGG PANHANDLING I| 22220

Aggressive Pandhandling I | AGGRESSIVE PANDHANDLING । |

Aggressive Panhandeling II AGGRESSIVE PANHANDELING | । Aggressive Panhandling II 
AGGRESSIVE PANHANDLING | |

AGRESSIVE PANHANDLING | |

AGRESSIVE PANHANDLING || 22220

Loitering II

LOITERING ।

LOITERING || 10600

LOITERING || 10690

LOITERING || 15202

LOITERING || 22220

LOITERING WITHIN 50 FEET । |

Pan Handling II

PAN HANDLING | |

Panhandling | |

PANHANDLING । |

ROGUE \& VAGABOND | | 10622

ROGUE \& VAGABOND || 16300

ROGUE \& VAGABOND || 23050

ROGUE \& VAGABOND || 23080

ROGUE AND VAGABOND || 10271

ROGUE AND VAGABOND || 10600

ROGUE AND VAGABOND | | 11137

ROGUE VAGABOND || 31314

ROUGE AND VAGABOND || 11137

ROUGE AND VAGABOND || 1A1466

ROUGE AND VAGABOND | | 23050

\section{Bike and Dirtbike (Subsequently Combined with Disorderly Conduct)}

Biccle Violation I

BICYCLE ON SIDEWALK । |

Bicycle Violation II

BICYCLE VIOLATION I ।

Bike Violation I |

BIKE VIOLATION । ।

Diat Bike Violation I I

DID POSS DIRTBIKE ON PUBLIC ST । ।

DIKE BIKE VIOLATION ।

DIRK BIKE VIOLATION I I

Dirt Bike I |

DIRT BIKE I।

DIRT BIKE || 10600

DIRT BIKE IN CITY । ।

Dirt Bike Vio II

DIRT BIKE VIO । ।

Dirt Bike Vio. I I

DIRT BIKE VIOL I |

DIRT BIKE VIOL. I I

DIRT BIKE VIOLARION । ।

Dirt Bike Violation I |

DIRT BIKE VIOLATION I ।

DIRT BIKE VIOLATION | | 10622

DIRT BIKE VIOLATION || 20480

DIRT BIKE VIOLATON । ।

DIRT BKE I।

Dirt Violation I।

DIRT VIOLATION I।

DIRT- BIKE-VIOLATION । ।

Dirtbike I |

DIRTBIKE । । 


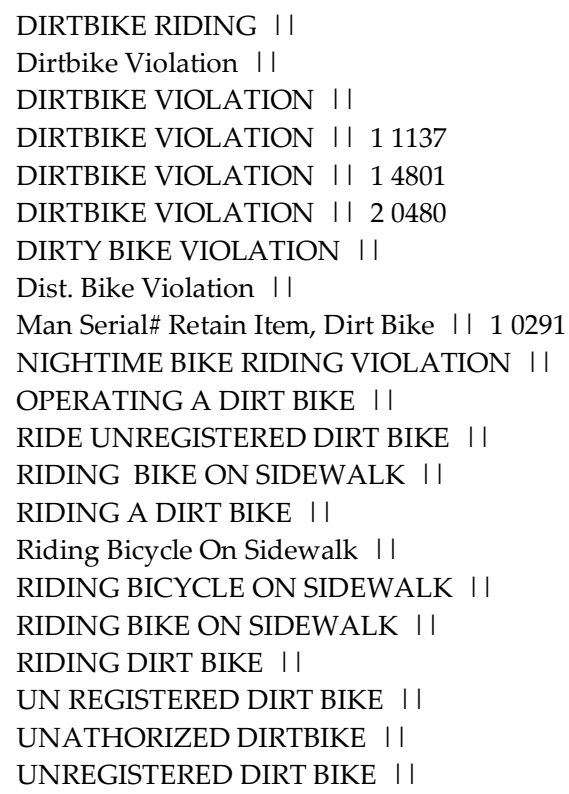

\section{Prostitution}

ATT-PROSTITUTION-GENERAL || 1A1093 HUMAN TRAFFIC || 11093

Human Traffic-Benefit Financ, Prostitution I| 10758 HUMAN TRAFFICKING || 11093

PROSITITUTION || 11093

PROSITUTION || 11093

PROST || 11093

PROST. II 11093

PROSTITION || 11093

PROSTITITION || 11093

PROSTITITUTION || 11093

PROSTITTION || 11093

PROSTITTUTION || 11093

PROSTITUION || 11093

PROSTITUITION । ।

PROSTITUITION || 11093

PROSTITUITON || 11093

Prostitution II

PROSTITUTION I ।

PROSTITUTION || 10600

PROSTITUTION || 10752

PROSTITUTION || 10758

PROSTITUTION || 11093

PROSTITUTION || 11635

PROSTITUTION || 13605

PROSTITUTION || 20480

PROSTITUTION || 20980

PROSTITUTION || 63600

PROSTITUTION-GENERAL || 11093

Prostitution-General, Prositution I| 11093

Prostitution-General, Prost || 11093

Prostitution-General, Prostitiution I| 11093

Prostitution-General, Prostittution I| 11093

Prostitution-General, Prostituion || 11093

Prostitution-General, Prostitutiion || 11093 
Prostitution-General, Prostitutiojn || 11093

Prostitution-General, Prostitution I| 11093

Prostitution-General, Prostitution-General || 11093

Prostitution-General, Prostituton I| 11093

Prostitution-General, Prostitutuion I| 11093

Prostitution-General, Prostiution I| 11093

Prostitution-General, Prostution || 11093

Prostitution-General, Prostutition I| 11093

Prostitution-General, Solicit Prostitution || 11093

PROSTITUTIUON || 11093

PROSTITUTTION || 11093

PROSTITUTUION || 11093

PROSTITUTUION/HUMAN TRAFFIC || 11093

PROSTITUTUTION || 11093

PROSTIUTION || 11093

PROSTTUTION || 11093

PROSTUTION || 11093

PROSTUTITION || 11093

PROSTUTUTION || 11093

PROTITUTION || 11093

SOLICITATION OF PROSITUTION || 11093

SOLICITATION OF PROSTITUTION || 11093

SOLICITATION OF PROTITUTION || 11093

TRAFFICKING || 11093

\section{Only Detained Temporarily}

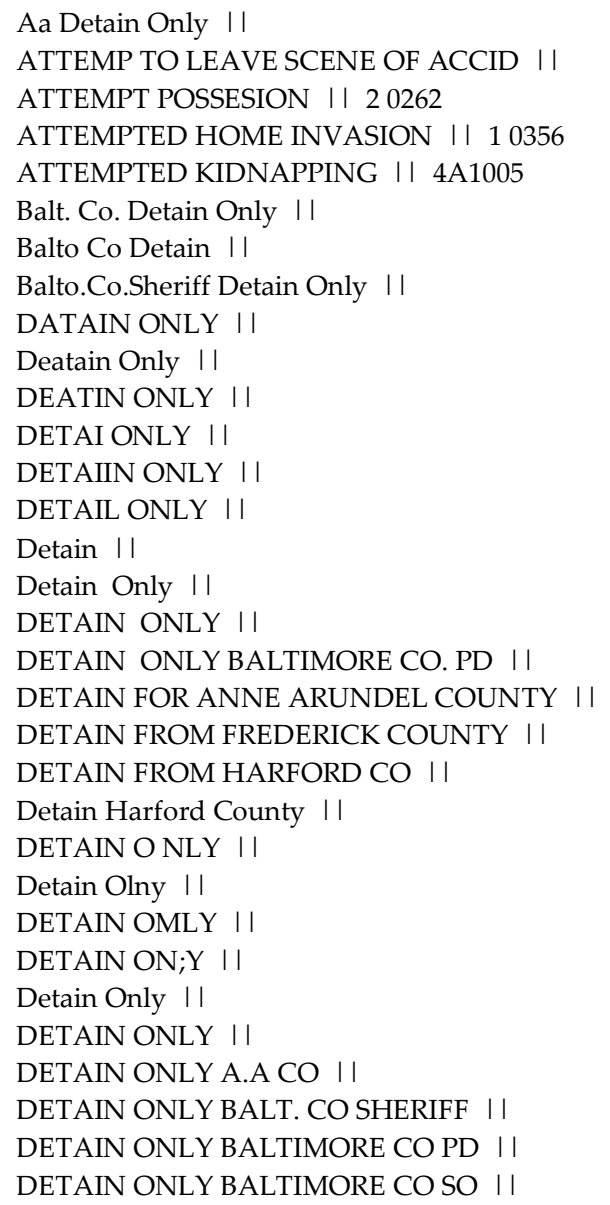


DETAIN ONLY BALTIMORE CO. I। DETAIN ONLY BALTIMORE COUNTY I। Detain Only Baltimore County P I | DETAIN ONLY BALTIMORE COUNTY P I | DETAIN ONLY BALTO CO PD । । DETAIN ONLY BALTO CO. I I DETAIN ONLY BALTO CO. SHERIFFS । । DETAIN ONLY BALTO. CO । | DETAIN ONLY CARROLL CO. I। DETAIN ONLY CECIL COUNTY I | DETAIN ONLY FOR AA COUNTY I | DETAIN ONLY FOR AA CO SHERIFF । | DETAIN ONLY FOR AA CO. I। DETAIN ONLY FOR AA COUNTY | | DETAIN ONLY FOR ANN ARUNDEL CO । । DETAIN ONLY FOR ANNE ARUNDEL C । । DETAIN ONLY FOR BALT CO. I I DETAIN ONLY FOR BALT. COSO । | DETAIN ONLY FOR BALT. CO. I । DETAIN ONLY FOR BALTIMORE CO. I। DETAIN ONLY FOR BALTIMORE COUN । । DETAIN ONLY FOR BALTIOMRE COUN I। DETAIN ONLY FOR BALTO COUNTY | | DETAIN ONLY FOR CAROLINE CO. I । DETAIN ONLY FOR CARROLL CO. I I DETAIN ONLY FOR CARROLL COUNTY I । DETAIN ONLY FOR CHARLES CO.SH I। DETAIN ONLY FOR HARFORD CO.SO । । DETAIN ONLY FOR HARFORD COUNTY I । DETAIN ONLY FOR PRINCE GEORGE I। DETAIN ONLY FOR QUEEN ANNE'S C I । DETAIN ONLY FOR SOMERSET COUNT II DETAIN ONLY FOR WASHINGTON COU I। DETAIN ONLY FOR WICOMICO CO. II DETAIN ONLY FREDERICK CO । । DETAIN ONLY FROM CHARLES CO. I I DETAIN ONLY HARFORD CO I I DETAIN ONLY HARFORD CO. SHERIF । | DETAIN ONLY HARFORD COUNTY I | DETAIN ONLY HARFORD COUNTY SHE । । DETAIN ONLY HOWARD | | DETAIN ONLY HOWARD COUNTY I । DETAIN ONLY ICE I। DETAIN ONLY PRINCE GEORGE COUN I । DETAIN ONLY WASHINGTON I। DETAIN ONLY WICOMICO SO । । Detain Only-Caroline County Sh I | Detain Only. II

DETAIN ONLY/AWOL || DETAIN ONLYFOR CONNECTICUT । | DETAIN ONYY | |

Detain. Only I |

Detaine Only II DETAINE ONLY | | DETAINED ONLY । | Detainer Only I I DETAINER ONLY | |

Detainonly II DETAINONLY FOR BALTO CO | |

DETAN ONLY I । 


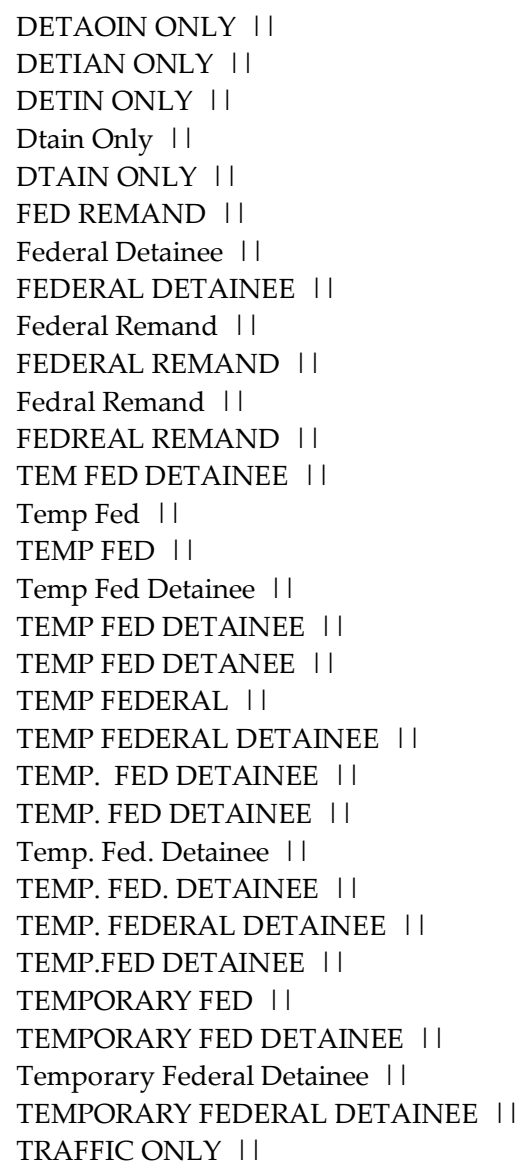

\section{Unknown}

Unknown Charge II

\section{Ungrouped (Subsequently Incorporates Gambling)}

... DID VIOLATE A PROTECTIVE O । | 20105

II 10892

II 10990

0Sc31736 । ।

0Sc86845 । I

0SD65435-40 । ।

11111 || 11111

11635 || 11635

$16303 \mathrm{C} \mathrm{II}$

$196010(3) \quad||$

20480 || 20480

2-3010 || 23010

21 902A1 । ।

4TH DEG B\& E || 23030

A FRAY || 35399

A/R I| 20700

ABUSE OF ANIMALS I I

ABUSE/NEGLECT REPORTING || 10734

ACCESS AFTER STATUTE | | 11480

Accessory After Fact, Accessory After Fact I| 10056

ACCESSORY AFTER THE FACT || 11332 


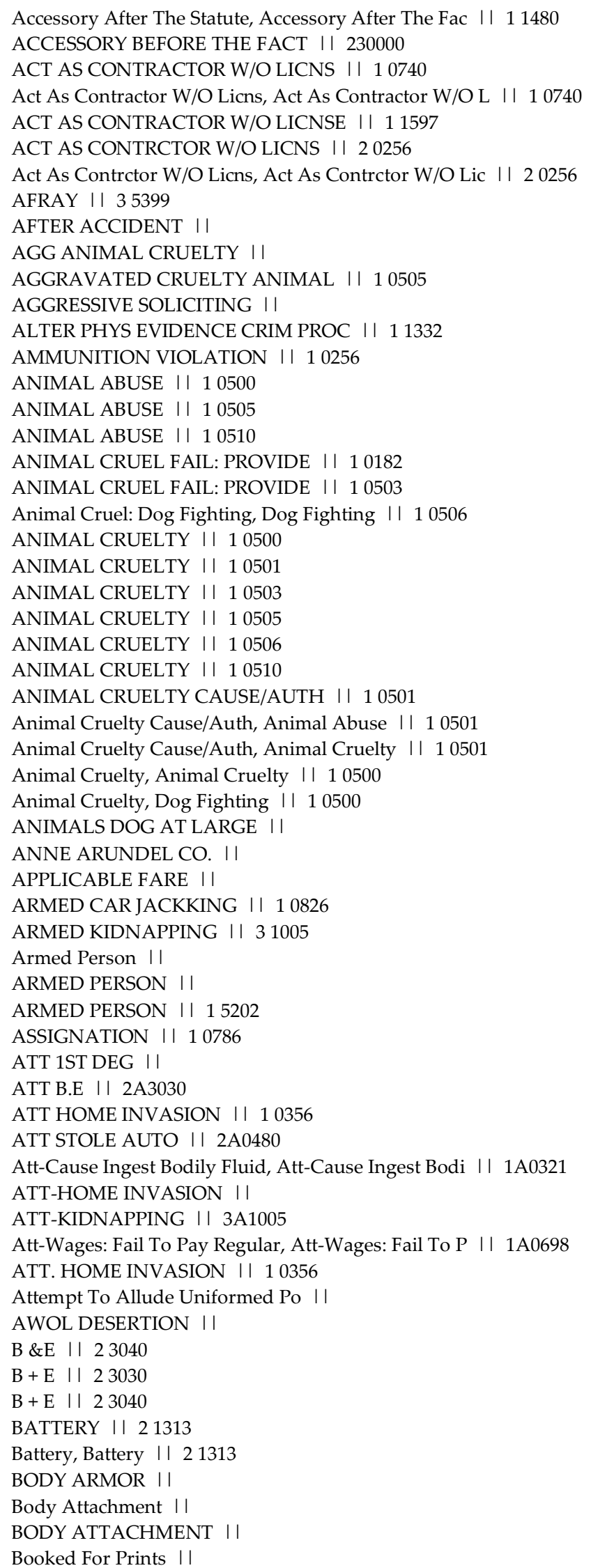


BRIBERY || 15101

C.D.S. POSSESSION || 63600

CD || 11111

CD'S MARIHUANA || 11564

CD'S POSSESSION || 11564

CDW || 15202

CED || 22220

CERFEW VIOLATION II

CHID ABUSE PHYSICAL | 13802

CITY ORD. TRAFFIC I।

City Ordinance Violation II

CODE ZONING I |

COMMIT ।

Commitment II

COMMITMENT I।

Community Service II

COMPUTERS ILLEGAL ACCESS ||

Computers Illegal Access, Computers Illegal Access I| 12804

CON TO COMMIT 1ST DEGREE ATT M I | 1C0990

CON-FAILURE FOREIGN CORP REGIS || 1 C0002

CON-KIDNAPPING || 3C1005

CON-RECKLESS ENDANGERMENT I| 1C1425

CONCEALED DEADLY WEASPON || 15202

CONCEALED DEADLY WEPON || 15202

CONSP. TO COMMIT 2ND DEGREE MU I| 11480

CONSPIRACY || 17399

CONSPIRACY || 22820

Consuming ||

CONSUMING I।

CONSUMING FOOD ON TRANSIT I।

Cs Violation II

Currency:Make Image, Currency:Make Image I| 10069

DEADLY CONCEALED WEAPOM I| 15202

Def In Public II

DEF ON REVOKED LIC । ।

Defendant Released In Error I |

DEFERED PAYMENT I।

Deferred Payment II

DEFERRED PAYMENT । ।

DEFERRED PYMT I।

DISCAHRGING I |

DISCHARGING FIREWORKS || 10016

DISCHARGING IN CITY LIMITS || 11106

DISODERLY || 10600

DISODERLY || 13903

DISODERLY || 14801

DISORDELY || 23040

DISORDELY || 35715

DISOREDRLY || 22220

DISORERLY || 10855

Display Govt Id Of Another, False Statement To Peac I| 11433

DOG AT LARGE I ।

DOGFIGHTING || 10505

DRI ON REVOKED LIC I ।

DRI ON SUSP LIC I।

DRI ON SUSP REVOKE । ।

DRI W/O LIC । |

DRINGON ON SUSPENDED | ।

DRUG CONSPIRACY | | 10880

DRV ON SUSP LIC I | 


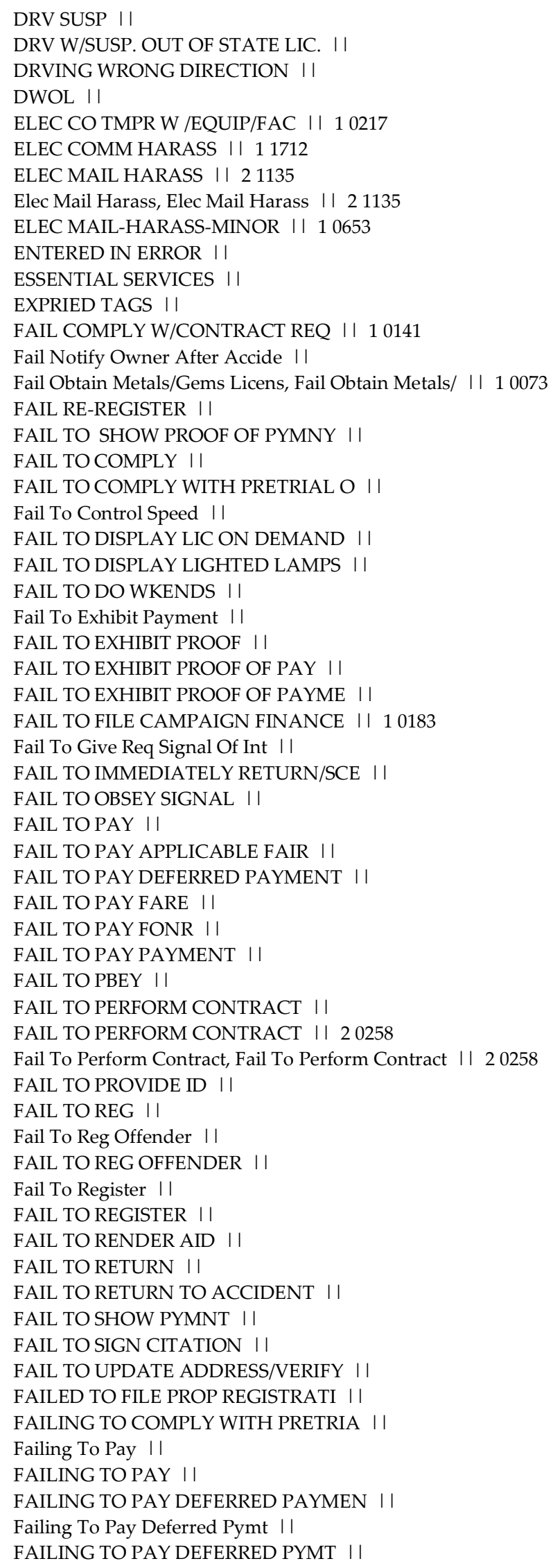


FAILING TO PAY PYMT ||

Failing To Register II

FAILING TO REGISTER । ।

FAILING TO REPORT I।

FAILING TO VERIFY | |

FAILING TO VERIFY INFO । ।

FAILING TO VERIFY INFOMATION I ।

Failing To Verify Information I |

FAILING TO VERIFY INFORMATION । ।

FAILURE TO COMPLY I I

FAILURE FOREIGN CORP REGIS I | 10002

FAILURE OBTAIN CIGARETTE LICEN || 10064

FAILURE TO COMPLETE WEEKDENDS । ।

Failure To Comply II

FAILURE TO DISPLAY LIGHTED LAM । ।

Failure To Exhibit Proof Of Pa II

FAILURE TO EXHIBIT PROOF OF PA । ।

Failure To Exhivit Proof Of Pa I I

FAILURE TO EXIBIT PROOF OF PAY I।

FAILURE TO GIVE INSURANCE INFO । ।

FAILURE TO IMMEDIATEL RETURN A I।

FAILURE TO IMMEDIATELY RETURN I।

Failure To Notify Owner Of Dam I I

FAILURE TO PARK 12IN OF RT CUR । ।

Failure To Pay II

FAILURE TO PAY | |

FAILURE TO PAY DEFERRED PAYME । ।

FAILURE TO PAY A DEFERRED PAYM । ।

Failure To Pay Applicable Fare II

FAILURE TO PAY APPLICABLE FARE I।

FAILURE TO PAY DEFERRED PAYMEN । ।

FAILURE TO PAY DEFERRED PMT. II

FAILURE TO PAY DEFFERED PAYMEN I।

FAILURE TO PAY DEFFERRED PAYME I।

Failure To Pay Fare I।

FAILURE TO PAY FARE I ।

FAILURE TO PAY IN REQUIRED MAN I I

FAILURE TO PAY WAGES || 210000

Failure To Pay Wages, Failure To Pay Wages || 210000

FAILURE TO REG । ।

FAILURE TO REG OFFENDER । ।

FAILURE TO REGISTER । ।

Failure To Return \& Ramain On I|

FAILURE TO RETURN SCENE OF ACC I ।

FAILURE TO RETURN TO A TRAFFFI I ।

FAILURE TO RETURN TO ACCIDENT । |

FAILURE TO SIGN CITATION I |

FAILURE TO SIGN TICKETS I|

FAILURE TO VERIFY । ।

FAILURE TO VERIFY IFNORMATION । ।

FAILURE TO VERIFY INFORMATION । |

FAILURE TOE PAY FARE IN REG. M I |

FAILURE TOP PAY FARE II

FALESSTATEMENT || 14803

FALIURE TO RETURN SCENE OF ACC I ।

FALSE ACCIDENT REPORT || 14803

FALSE ALARM || 15308

FALSE ALARM/AMBULANC CAUSE || 10208

FALSE ALARM/FIRE:CAUSE || 15308

False Alarm/Fire:Cause, False Alarm Of Fire || 15308 
FALSE CRIME REPORT || 14803

FALSE IMPRISONMENT | | 10042

FALSE IMPRISONMENT | | 31005

False Imprisonment, False Imprisonment | | 10042

FALSE IMPROSNMENT || 10042

FALSE OR MISLEADING APPLICATIO । ।

FALSE PRESCRIPTION || 11112

FALSE REPORT || 10207

FALSE REPORT || 14803

FALSE REPORTING I| 14803

False Statement I I

FALSE STATEMENT I।

FALSE STATEMENT || 10690

FALSE STATEMENT || 11364

FALSE STATEMENT I | 11432

FALSE STATEMENT || 11433

FALSE STATEMENT I| 14803

FALSE STATEMENT I| 23050

FALSE STATEMENT BEFORE ARREST || 14803

FALSE STATEMENT CAUSING AN INV || 14803

FALSE STATEMNT || 13903

FALSE STMTS-AUDIOLOGISTS | | 11521

FALSE/MISLEADING INFO \$300+ || 20125

False/Misleading Info \$300+, False/Misleading Info F || 30125

FALSIFYING IMFORMATION I ।

FALSIFYING/ATT TO FALSIFY W/FR । ।

FEDERAL REMAN I।

FELON IN POSSESSION || 11612

FELON IN POSSESSION OF AMMUNIT | | 11111

Figitive From Virginia I |

FILING FALSE REPORT | | 14803

Fireworks:Sale W/O Permit, Illegal Dist. Of Fire Wor || 10029

Fireworks:Sale W/O Permit, Illegal Dist. Of Firework I| 10029

Fireworks:Sale W/O Permit, Illegal Fireworks II 10029

Fireworks:Sale W/O Permit, Illegal Sale Of Fireworks || 10029

FIUGITIVE FROM VIRGINIA I |

FLASE REPORT I| 14803

FLASE STATEMENT || 14803

FOEGE PRESCRIPTIONS || 20262

FREY I| 35399

FROM NORTH CAROLINA I ।

From Pennsylvania II

FUCITIVE FROM JUSTICE: PENNSYL । ।

FUTIGIVE FROM NEW YORK I I

FUVITIVE FROM PA I।

GRAFFITI VANDALISM || 34030

GRAFFITI/VANDALISM | | 34030

GTA || 11466

GTA || 20480

H.G.V. || 11106

H.G.V. II 15285

Hacking II

HACKING I|

HACKING I| 10600

HARASSMENT II

HARASSMENT || 22220

HARBOR/ABDUCTED CHLD UN 12 || 10163

HARRASSMENT II

HARRASSMENT I| 10191

HAZARDOUS SUBST-DISPOSAL | | 10012 
HG || 11106

HGU || 11106

HIDE CHLD/ABD BY REL IN ST | | 10365

HIT \& RUN | |

HIT \& RUN || 21020

HIT AND RUN । ।

HIT AND RUN | | 10600

HITANDRUN I |

HOME INVASION | | 10500

HOME INVASION || 23000

HOME INVASION || 23030

Hr61390 I|

HUMAN TRAFFIC || 11080

HUMAN TRAFFIC-BENEFIT FINANC || 10757

HUMAN TRAFFICKING । ।

HUMAN TRAFFICKING || 10758

HUMAN TRAFFICKING || 10786

HUMAN TRAFFICKING | | 11080

HUMAN TRAFFICKING || 11082

HUMAN TRAFFICKING - COMPENSATI || 11082

HUMAN TRAFFICKING-TAKE CAUSE || 11080

HUMAN TRAFFICKING-TAKE/CAUSE I। 10786

ID MISUSE || 11433

ILL POSS AMMO || 11564

ILLEGAL LOCKOUT I।

ILLEGAL LOOKOUT I ।

ILLEGAL POSS AMMO || 11111

ILLEGAL POSSESION OF AMMO I ।

ILLEGAL POSSESSION OF A FIREAR | | 11106

ILLEGAL POSSESSION OF AMMUNITI || 11111

ILLEGAL TRANSPORTATION OF HAND || 11425

ILLEGAL VIDEOTAPING MOVIE | | 10336

ILLEGALLY PARKED I ।

Impeding Traffic I I

INCEST || 13604

INDENCENT EXPOSURE || 13605

INMATE CAUSE CONT W/ BODY FLUI I | 10222

INTOXICATED ENDANGER || 11661

KIDNAPING || 31005

KIDNAPPING || 10042

KIDNAPPING || 10163

KIDNAPPING || 22220

KIDNAPPING || 23000

KIDNAPPING || 31005

KIDNAPPING || 41005

Kidnapping, Kidnapping | 31005

KNOWINGLY FAILING TO SIGN ACKN । ।

KNOWINGLY USING FALEFIED REG P । ।

Knowingly Using Falsified Vehi I I

KNOWINGLY USING FALSIFIED VEHI । ।

LARCANY || 10622

Left Scene Of Accident II

LEWD ACT || 13605

LIFE THRT INJ/VEH-BOAT/UNDER । |

LOOTING || 15202

LOOTING || 23010

LOOTING || 23040

Mace/Chem Device-Int/Injure, Mace/Chem Device-Int/In I| 25200

MAN SERIAL\# RETAIN ITEM | | 10291

Man Serial\#: Remov/Oblit, Man Serial\#: Remov/Oblit II 22800 
METHYLENEDIOXYMETHAMPHETAMINE || 11111

MJ 10+ || 11564

MUDER 1ST I| 10990

MUTUAL COMBAT I| 35399

NO FRONT TAG I।

NOT RESTRAINED । ।

Obscene Mat/Puclsh/Dist., Obscene Mat/Puclsh/Distrib || 10295

Obtn Prop Of Vul Adult-Ovr \$500, Obt Prop Of Vul Adu I। 10782

OPEN COINTAINER ।|

OPEN OCNTAINER I।

OPER A MTR SCTR ON HWY W.O REQ I I

OPER NOT REST I।

OPERATING A SCOOTER WITH A LIC ।

OPERATING AN UNREGISTERED MOTO । ।

OPERATING MC W/O REQUIRED HEAD I I

OPERATOR NOT RESTRAINED BY SEA I।

OPERATOR W/O SEAT BELT II

OPR PHAR W/OUT LIC II

Other II

Other Non Felony Charge, False Statement I| 20082

Paraphenalia II

PARK RULE VIOLATION I |

PARK VIOLATION I।

PED WALK ALONG ROAD WONG WAY T I।

PENNSYLVANIA ।

Permitting Unauthorized Person II

PHONE MISUSE || 10340

POSS || 10468

POSS || 11111

Poss Contbnd-Place Of Confinement, Poss Contbnd-Pla || 11835

Poss Contbnd-Place Of Confinement, Poss Contbnd-Plac || 11835

POSS MJ || 11635

POSS OF BODY ARMER I I 11449

POSS OF CONTRBAND । I

POSS OF LESS THAN .10 GRAMS MA || 11635

POSS OF MARI. I I 11635

POSS OF XANAX || 11111

POSS W INTENT || 15202

POSS WLESS THAN 10GRAM OF MARI || 11635

Poss. II

POSS. I| 11111

POSS. DANGEROUS WEAPN || 15202

POSS. MAR. II 11564

POSS. MARIHUANA I| 11635

POSS. OF A TASSER II

POSS. PF MAR. I| 15202

Poss. With Int. II

POSS/OFFER/FOR SALES OF TURTLE I ।

POSS/REC TELECOM DEV || 11330

POSS/REC TELECOMM DEVICE || 10723

Poss/Rec Telecomm Device, Poss/Rec Telecomm Device II 10723

POSS/TRAIN/ DOG FOR DOGFIGHT । | 10510

POSSESION MARIHUANA || 11635

POSSESS/USE FALSE GOV'T ID DOC | | 11432

POSSESSING FICTITIOUS LIC । ।

Possessing Susp Lic II

POSSESSING SUSPENDED LIC । ।

Possession II

POSSESSION I|

POSSESSION || 11111 


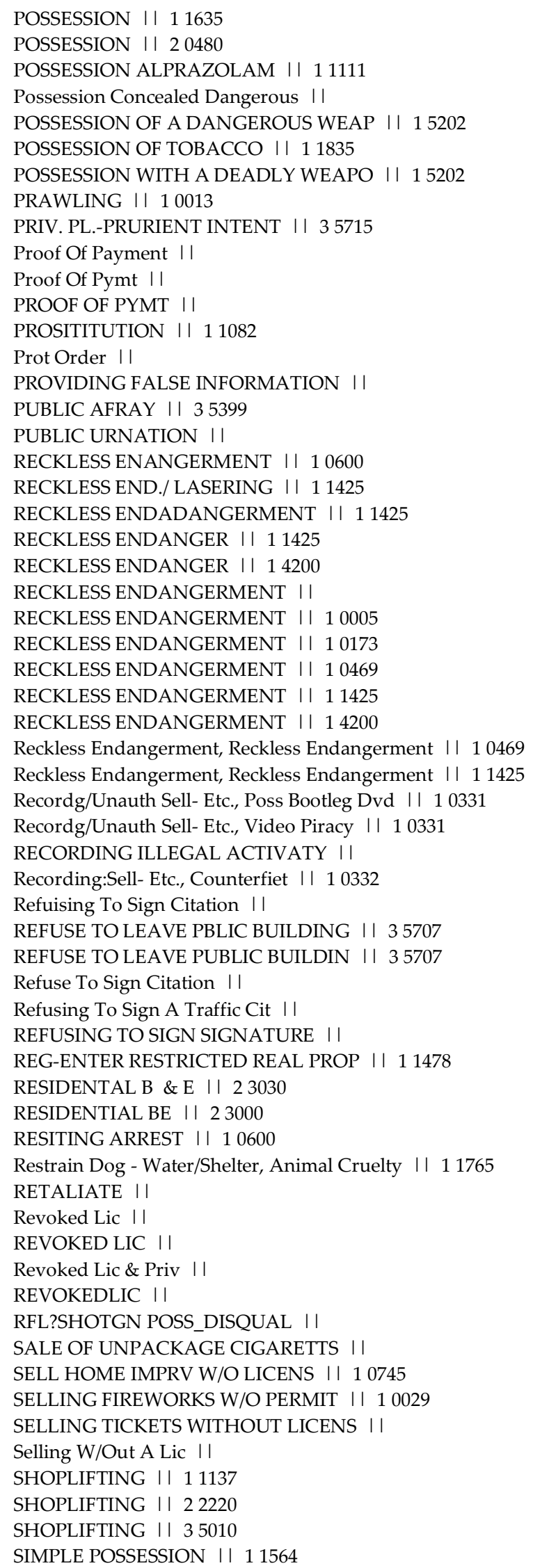


SMOKING ON TRANSIT । ।

Smoking/Carrying Tobacco । |

SOL-POSS/REC WEPON -CONF/DET || 2S1055

SOLICIT TO COMMIT CRIME I| 10034

SOLICITATION(COMAR) ।

SOLICITING ।

SOLICITING || 21125

SOLICITING GOODS । ।

Speedinng Grater II

SPINNING WHEELS I।

SRIVING W/OUT LIC I I

STLOEN AUTO || 20480

STOEN AUTO || 20480

STOLE ATUO II 20480

STOLE AUTO II 11136

STOLEM AUTO || 11466

STOLLEN AUTO || 20480

SUS LIC I।

SUSP.LIC I।

SUSPENDED LIC । ।

SUSPENDED LICENCE I ।

SUSPENDED OUT STATE LIC I ।

SUSPENDED REGISTRATION । ।

SUSPENDED/REVOKED । ।

SUSPENDEDLIC I |

Ta $16101 \mathrm{~A}$ । I

TA $16101 \mathrm{~A}$ । ।

Ta $16303 \mathrm{D}$ । ।

Ta 20105 A I।

TA-16-303-C I |

TEESPASSING || 22220

TEL MISUSE । ।

TEL MISUSE REPEAT PHONE CALLS I ।

TEL MISUSE: OBSCENE I | 10341

TEL MISUSE:REPEAT CALLS || 10340

Tel Misuse:Repeat Calls, Tel Misuse:Repeat Calls II 10340

Tel Misuse:Repeat Calls, Telephone Misuse II 10340

TEL MISUSE:SINGLE CALL || 15309

Tel Misuse:Single Call, Tel Misuse:Single Call || 15309

TELE MISUSE I।

Telephone Misuse II

TELEPHONE MISUSE | | 10340

TENNESSEE । |

TFAFFIC II

THIEF || 11137

THREAT OF MASS VIOLENCE | | 11335

THREATS / ST OFFICIAL/ TO INJU I I

Tobacco Violation II

TRADE MMARK INFRINGMENT || 12540

Traffic II

TRAFFIC I

Traffic Arrest II

TRAFFIC VIOLATION I I

TRANSFER HIV TO ANOTHER I | 47360

TRASPASING I| 11564

TREASPASSING || 22220

TRSPASSING || 22220

UNARMED A\&R I| 20700

UNATHORIZED USE I I 10622

UNATHORIZED USE || 20480 


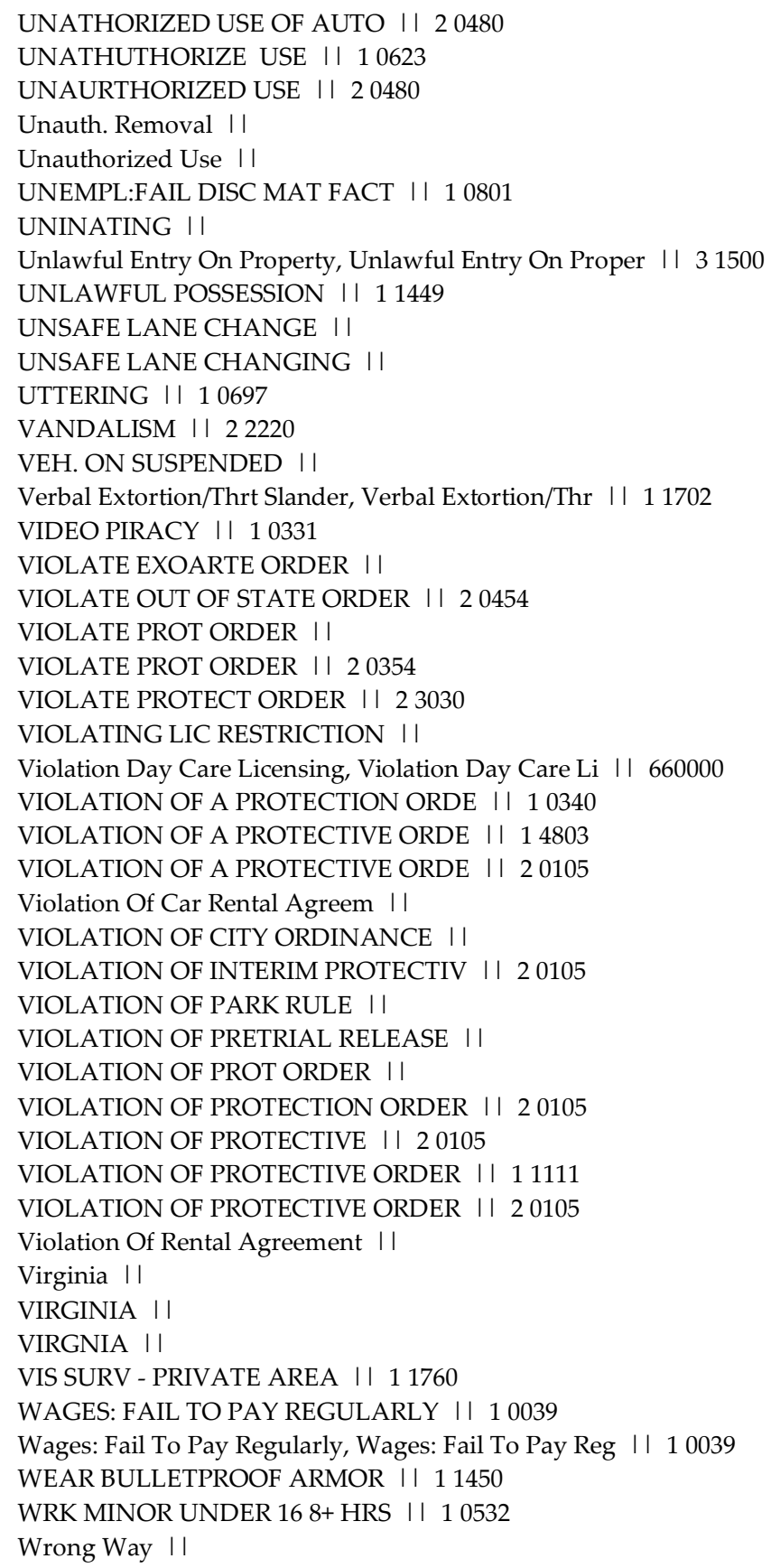




\section{Appendix 2}

\section{Comprehensive Analysis of BPD Crime Data}

\author{
Stephen L. Morgan \\ Johns Hopkins University
}

\author{
Rhiannon N. Miller \\ Johns Hopkins University
}

May 11, 2020

\section{Navigation of this Document}

In order to jump to results of interest, display the Table of Contents for this pdf file in the left pane of your pdf viewer. The top level of the Table of Contents contains labeled sections for each outcome, within which subsections are nested for each piece of the analysis. Clicking the items in the pane will take you to the relevant set of results.

\section{Introduction}

For this updated analysis of BPD's crime data, we offer comprehensive results that extend the results summarized in a Spring 2016 report and its Fall 2016 update:

- Morgan, Stephen L. and Joel A. Pally. 2016. "Ferguson, Gray, and Davis: An Analysis of Recorded Crime Incidents and Arrests in Baltimore City, March 2010 through December 2015." A Report Prepared for the 21st Century Cities Initiative at Johns Hopkins University. Baltimore, MD: Johns Hopkins University, March 15, 2016. (URL: https://osf.io/preprints/socarxiv/nshme/)

- Morgan, Stephen L. 2016. "Fall 2016 Update for Ferguson, Gray, and Davis: An Analysis of Recorded Crime Incidents and Arrests in Baltimore City, March 2010 through October 2016." Baltimore, MD: Johns Hopkins University, November 15, 2016. (URL: https://osf.io/preprints/socarxiv/6gdra/)

The results in the original report and its update were based on crime data through December 2015 and October 2016, respectively. In this final report, we offer models that incorporate crime data through Sunday, March 1, 2020.

The basic analysis strategy is the same as in the original report. We first model the prevailing cyclical crime trend from Monday, March 1st, 2010 through Sunday, August 10, 2014. Then, we use that estimated model to predict a counterfactual crime trend, as if the protest events and any changes in police practice from August 11, 2014 onward had not occurred. We then estimate period effects as average differences between observed and counterfactual values from August 11, 2014 onward in distinct time intervals.

Unlike the original report, with the availability of additional data it is reasonable to now divide the interval after Police Commissioner Anthony Batts was fired into (1) a transition period while Kevin Davis was the Interim Police Commissioner and (2) two additional periods after Kevin Davis was sworn in as Batts' permanent replacement and with a five-year contract as the city's 38th Police Commmissioner. For the analysis reported here, the transition period begins with the week of Monday, July 13, 2015 and ends on Sunday, October 18, 2015. The first period for Davis as commissioner encompasses the first full year of his term, from the week of Monday, October 19, 2015 (10/19/15 to 10/25/15) through the week of Monday, October 10, 2016 (10/10/16 to 10/16/16). The second period for Davis begins with the second full year of his term, from the week of Monday, October 17, 2016 (10/17/16 to 10/23/16) through his final week as commissioner, Monday, January 15, 2018 (1/15/18 to 1/21/18).

More than three full years have passed since 2016, and the city has had three new police commissioners since then, necessitating three new periods for a parallel structure of models. The fourth period for De 
Sousa as commissioner commences with the week of Monday, January 22, 2018 (1/22/18 to 1/28/18) and ends with the week of Monday, May 7, 2018 (5/7/18 to 5/13/18). The fifth period for Tuggle as commissioner commences with the week of Monday, May 14, 2018 (5/14/18 to 5/20/18) and ends with the week of Monday, February 4, 2019 (2/4/19 to 2/10/19). Finally, the sixth period for Harrison as commissioner commences with the week of Monday, February 11, 2019 (2/11/19 to 2/17/19) and ends with the week of February 24, 2020 (2/24/20 to 3/1/20), which is the final full week before Governor Hogan declared a state of emergency because of the COVID-19 pandemic. Thus, the final period is approximately the first full year of Harrison's term as commissioner.

\section{Descriptive Summary of the Data}

The types of crime incidents analyzed in subsequent sections are listed and summarized in Table 2.1 in the Data section, after which a breakdown of all types of crime incidents is provided by police district in Table 2.2. For this final report, we have moved the crime incident category of assaults by threat to the end of this appendix. At some point in the last three years, the BPD changed its categorization of incidents, backcoding some of the data on assaults by threat that we analyzed for the prior two reports. As shown below, under this new categorization assaults by threat declined abruptly to zero in the pre-Ferguson period and remained at zero through March 1, 2020. We have handled this change by allowing assaults by threat, which number only about 10 per week when categorized for the first portion of the time series, to continue to count as a category within the total crime incident count that we analyze. We then estimate models for assault by threat for completeness in order to show when the coding change occurred, but we do not include conclusions about assaults by treat in the main document of this report.

Two figures then display the distributions of the variables utilized for subsequent models: (1) variables for weather and hours of darkness; (2) variables that parameterize types of days per week, such as holidays, vacations, and other days without school in the Baltimore City Schools. For this final report, we introduce a new set of variables for types of days within each week, separating holidays and vacation days from other types of non-school days.

\section{Results for Each Outcome}

For each outcome, we first offer a table with coefficients from four estimated models:

- Model 1 is a naive model with nine estimated coefficients for the period indicator variables for the full time series;

- Model 2 is a "pretreatment" model, estimated only for data prior to August 11, 2014, that fits coefficients for time, weather, hours of darkness, and types of days;

- Model 3 is analogous to Model 1 but utilizes an outcome variable for the full time series that has been de-trended using coefficients for time, weather, hours of darkness, and types of days estimated by Model 2 using data from the "pretreatment" period only;

- Model 4 is an alternative to Model 3 that fits coefficients for weather, hours of darkness, and types of days simultaneously with coefficients for the six period indicator variables.

The period effects estimated for Model 3 are our preferred estimates, and the period effects estimated for Model 4 are plausible alternatives.

We then offer two figures that depict the fit of Model 2 as well as the variation that generates the period effects estimated for the preferred Model 3. For both figures, the black line is the predicted time series from Model 2 while the red line is an extrapolated cyclical trend, generated by forming predictions that utilize the coefficients from Model 2 and the observed values for weather, hours of darkness, and school days from August 11, 2014 onward.

For this final report, we only allow the time trend coefficient to structure the counterfactual red line through the first year of Davis' term as commissioner. Beginning with his second year, the contribution 
from the time trend coefficient is fixed for the rest of the time series. The cyclical pattern of the counterfactual red trend line continues to be generated by the other variables specified as predictors for Model 2.

The first figure of each pair overlays gray dots for the observed weekly total for each outcome, and the second figure substitutes a light blue line for the three-week moving average of the observed weekly totals. The differences between the gray dots (or the light blue line) and the red line is the source of variation that generates the period effects estimated for the preferred Model 3. Additional details of Model 3 are offered in the main document for this updated report.

Finally, for each outcome a diagnostic model is also fit to assess the extent to which the least squares estimation of Model 2 adequately represents the underlying time series up through August 10, 2014. For this alternative model, a poisson distribution is assumed for the outcome because it is a weekly count bounded by zero. In general, these alternative models support the decision to utilize the simpler Model 2 to estimate the underlying time series that structures the extrapolated counterfactual trend. In this final report, we continue to offer the $\mathrm{R}$ output for the estimated poisson regression so that it can be checked against the similar results in the original report. However, we no longer offer analogous figures to demonstrate the near equivalence of results based on Model 2 and its poisson regression alternative. Interested readers can consult the close similarity from the original report, which holds for this updated analysis as well. 


\section{Data}

Table 2.1: Descriptive Statistics for Weekly Reported Incidents of Victim-Based Crime, with Breakdown by Type of Crime

\begin{tabular}{lccccccc}
\hline \hline Statistic & Mean & St. Dev. & Min & Pctl(25) & Median & Pctl(75) & Max \\
\hline homicide & 5.3 & 2.7 & 0 & 3 & 5 & 7 & 17 \\
shooting & 10.4 & 5.5 & 0 & 6 & 9 & 14 & 33 \\
rape & 5.8 & 2.7 & 0 & 4 & 5 & 7 & 16 \\
robbery.carjacking & 6.2 & 4.7 & 0 & 3 & 5 & 8 & 30 \\
robbery.street & 60.0 & 17.7 & 24 & 46 & 57.5 & 71 & 128 \\
robbery.commercial & 14.1 & 6.2 & 2 & 10 & 13 & 18 & 44 \\
robbery.residence & 9.8 & 3.9 & 1 & 7 & 9 & 12 & 27 \\
aggravated.assault & 98.7 & 21.0 & 41 & 83 & 98.5 & 112.8 & 178 \\
common.assault & 159.7 & 26.4 & 78 & 141 & 159 & 178 & 241 \\
burglary & 140.5 & 31.6 & 66 & 120 & 141 & 158.8 & 403 \\
larceny.from.auto & 127.2 & 27.8 & 52 & 108 & 125 & 145 & 217 \\
larceny & 205.6 & 30.4 & 105 & 186 & 206 & 227.8 & 302 \\
auto.theft & 81.6 & 17.4 & 46 & 69 & 79 & 92 & 146 \\
arson & 4.6 & 3.1 & 0 & 2 & 4 & 6 & 33 \\
total & 934.4 & 110.8 & 539 & 859.5 & 949.5 & $1,021.8$ & 1,161 \\
\hline
\end{tabular}

Table 2.2: Descriptive Statistics for Weekly Reported Incidents of Victim-Based Crime, with Breakdown by District

\begin{tabular}{lccccccc}
\hline \hline Statistic & Mean & St. Dev. & Min & Pctl(25) & Median & Pctl(75) & Max \\
\hline northwestern & 93.0 & 16.0 & 51 & 83 & 92.5 & 103 & 181 \\
northern & 103.0 & 19.6 & 56 & 90 & 103 & 116 & 172 \\
northeastern & 142.8 & 22.3 & 79 & 130 & 144 & 156 & 207 \\
western & 75.4 & 14.9 & 43 & 65 & 75 & 84 & 220 \\
central & 111.3 & 22.5 & 56 & 95 & 111 & 127 & 193 \\
eastern & 80.0 & 14.4 & 41 & 70 & 79 & 90 & 119 \\
southwestern & 90.6 & 17.5 & 42 & 78 & 90 & 103 & 150 \\
southern & 108.0 & 21.6 & 50 & 94 & 108 & 123 & 226 \\
southeastern & 129.2 & 23.2 & 63 & 114 & 129 & 145 & 233 \\
total & 934.4 & 110.8 & 539 & 859.5 & 949.5 & $1,021.8$ & 1,161 \\
\hline
\end{tabular}


Weather and Darkness Variables for Subsequent Models

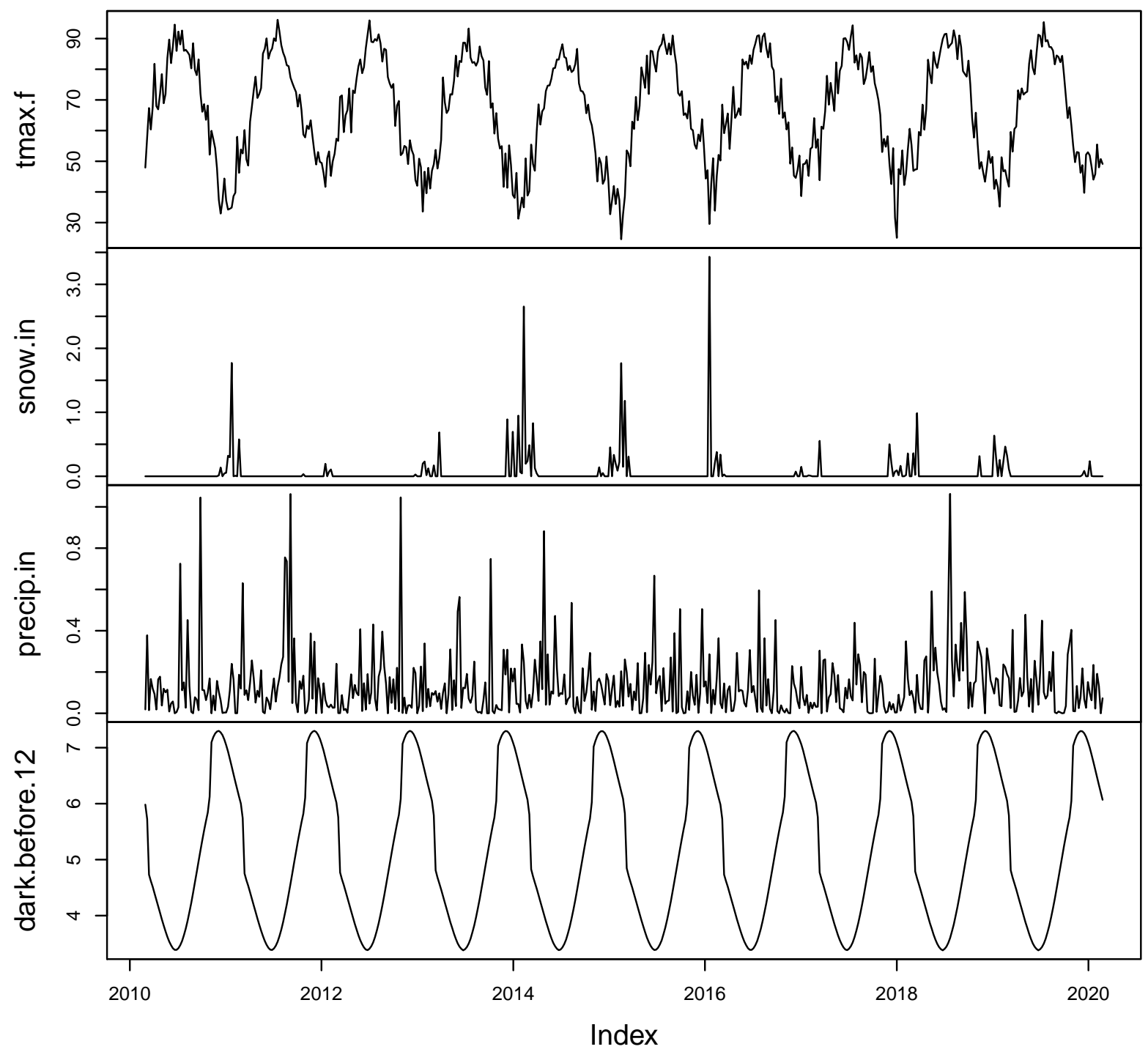

- tmax.f is the weekly average of the daily maximum temperature, measured in degrees Fahrenheit

- snow.in is the weekly average of total daily snowfall, measured in inches

- precip.in is the weekly average of total daily precipitation, measured in inches

- dark.before.12 is the weekly average of daily hours between sunset and midnight 
Types of Day Variables for Subsequent Models
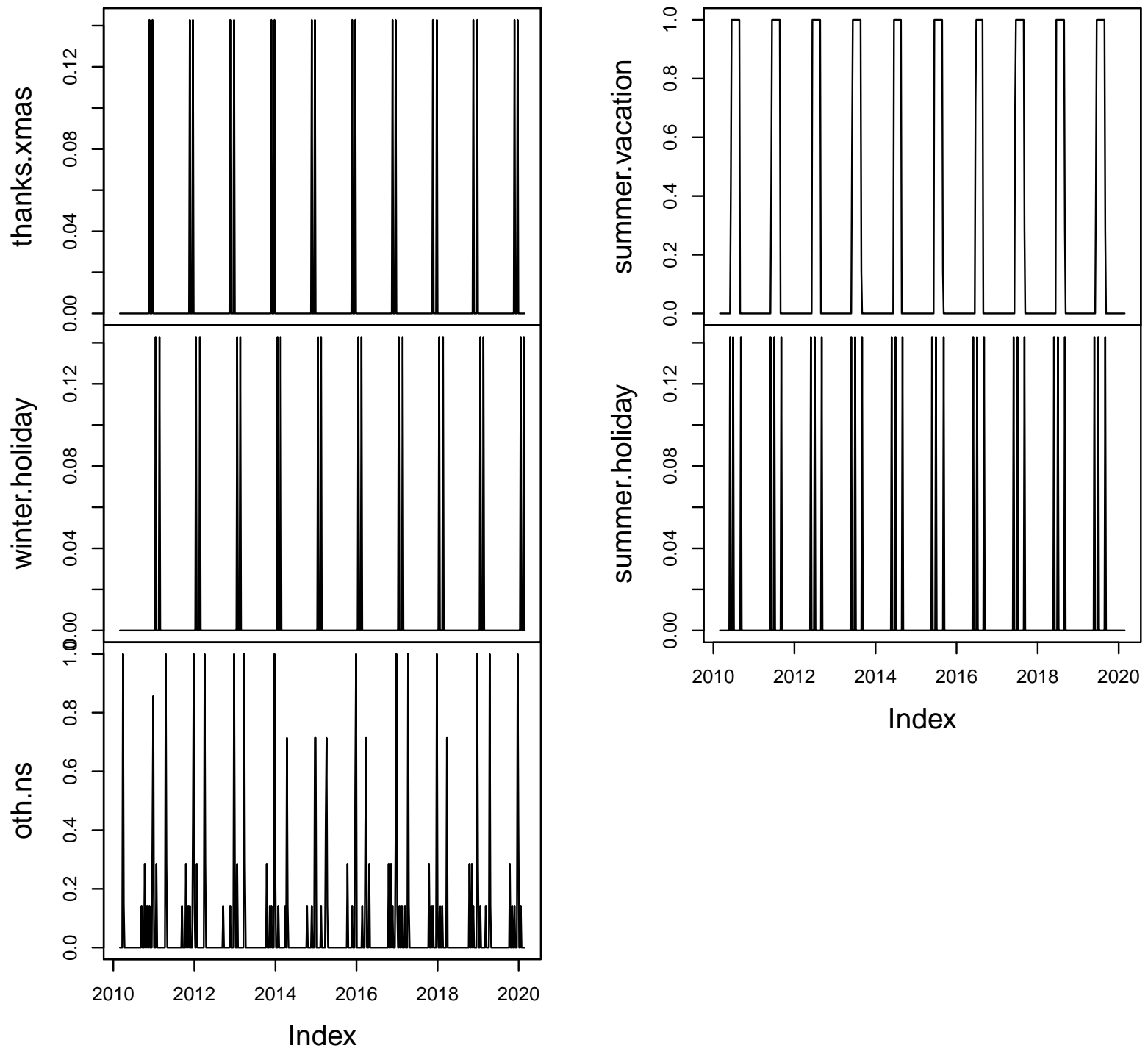

- thanks.xmas is the proportion of days of the week that are either Thanksgiving or Christmas

- winter.holiday is the proportion of days of the week that are a winter holiday other than Thanksgiving or Christmas

- other.ns is the proportion of days of the week that are other types of non-school days for the Baltimore City Schools (e.g., professional development days and winter vacation days)

- summer.vacation is the proportion of days of the week that are summer vacation for the Baltimore City Schools

- summer.holiday is the proportion of days of the week that are a summer holiday (e.g., 4th of July) 


\section{Results for total}

\subsection{Summary Values for total}

Table 3.1: Descriptives for Outcome Before Ferguson Protests Begin

\begin{tabular}{lcccccccc}
\hline \hline Statistic & $\mathrm{N}$ & Mean & St. Dev. & Min & Pctl(25) & Median & Pctl(75) & Max \\
\hline total & 232 & 945.272 & 98.921 & 612 & 875.5 & 960 & $1,023.2$ & 1,119 \\
\hline
\end{tabular}

Table 3.2: Descriptives for Outcome After Ferguson Protests Begin

\begin{tabular}{lcccccccr}
\hline \hline Statistic & $\mathrm{N}$ & Mean & St. Dev. & Min & Pctl(25) & Median & Pctl(75) & Max \\
\hline total & 290 & 925.724 & 118.939 & 539 & 851 & 943 & $1,014.8$ & 1,161 \\
\hline
\end{tabular}




\subsection{Four Models for total}

Table 3.3: Four Models that Differ on the Specification of Adjustment and Intervention Variables

\begin{tabular}{|c|c|c|c|c|}
\hline & \multicolumn{4}{|c|}{ Outcome: Count Per Week } \\
\hline & (1) & $(2)$ & (3) & $(4)$ \\
\hline Time (counter in weeks) & -0.35 & -0.19 & & -0.20 \\
\hline After Ferguson Protests Begin (week of $8 / 11 / 14$ onward) & -65.98 & & -37.35 & -34.85 \\
\hline After Gray Protests Begin (week of 4/20/15 onward) & 202.45 & & 110.13 & 107.27 \\
\hline Unrest and National Guard (4/27/15 - 5/3/2015) & 113.57 & & 126.39 & 139.87 \\
\hline After Batts Fired (week of 7/13/15 onward) & -11.78 & & -46.13 & -46.39 \\
\hline After Davis Confirmed (week of 10/19/15 onward) & -82.96 & & -24.64 & -20.69 \\
\hline After Davis First Year (week of 10/17/16 onward) & 99.87 & & 88.41 & 94.98 \\
\hline After De Sousa Begins (week of 1/19/18 onward) & -149.35 & & -114.06 & -104.99 \\
\hline After Tuggle Begins (week of 5/14/18 onward) & 140.41 & & 77.84 & 76.09 \\
\hline After Harrison Begins (week of 2/11/19 onward) & -79.34 & & -88.03 & -75.08 \\
\hline Average Maximum Temperature to 50 Degrees & & 4.49 & & 5.94 \\
\hline Plus Degrees in the 50s & & 5.35 & & 0.95 \\
\hline Plus Degrees in the 60s & & -2.89 & & 1.28 \\
\hline Plus Degrees in the 70s & & -4.90 & & -4.00 \\
\hline Plus Degrees Greater Than 80 & & -1.02 & & -2.35 \\
\hline Snowfall (inches) & & -65.58 & & -47.26 \\
\hline Precipitation (inches) & & -28.96 & & -23.24 \\
\hline Darkness Before Midnight (hours) & & 23.87 & & 26.38 \\
\hline Thanksgiving/Christmas (proportion of week) & & -32.28 & & -44.45 \\
\hline Winter Holiday (proportion of week) & & -211.39 & & -269.33 \\
\hline Other Out-of-School Days (proportion of week) & & -27.91 & & -54.92 \\
\hline Summer Vacation (proportion of week) & & 20.88 & & 1.81 \\
\hline Summer Holiday (proportion of week) & & -261.19 & & -222.81 \\
\hline Observations & 522 & 232 & 522 & 522 \\
\hline $\mathrm{R}^{2}$ & 0.23 & 0.70 & 0.30 & 0.71 \\
\hline
\end{tabular}




\subsection{Least Squares Updated Model (2) for total total}

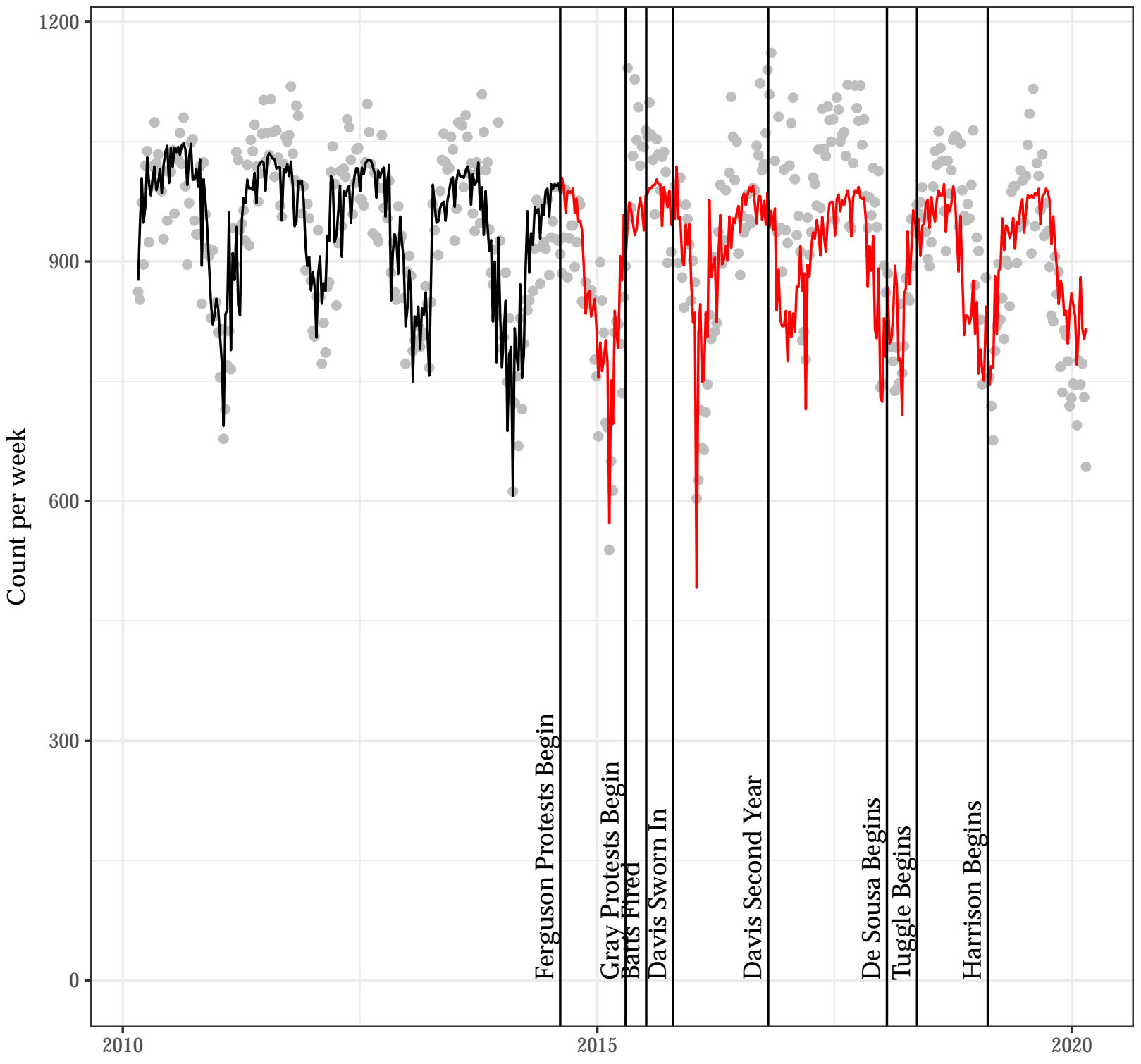




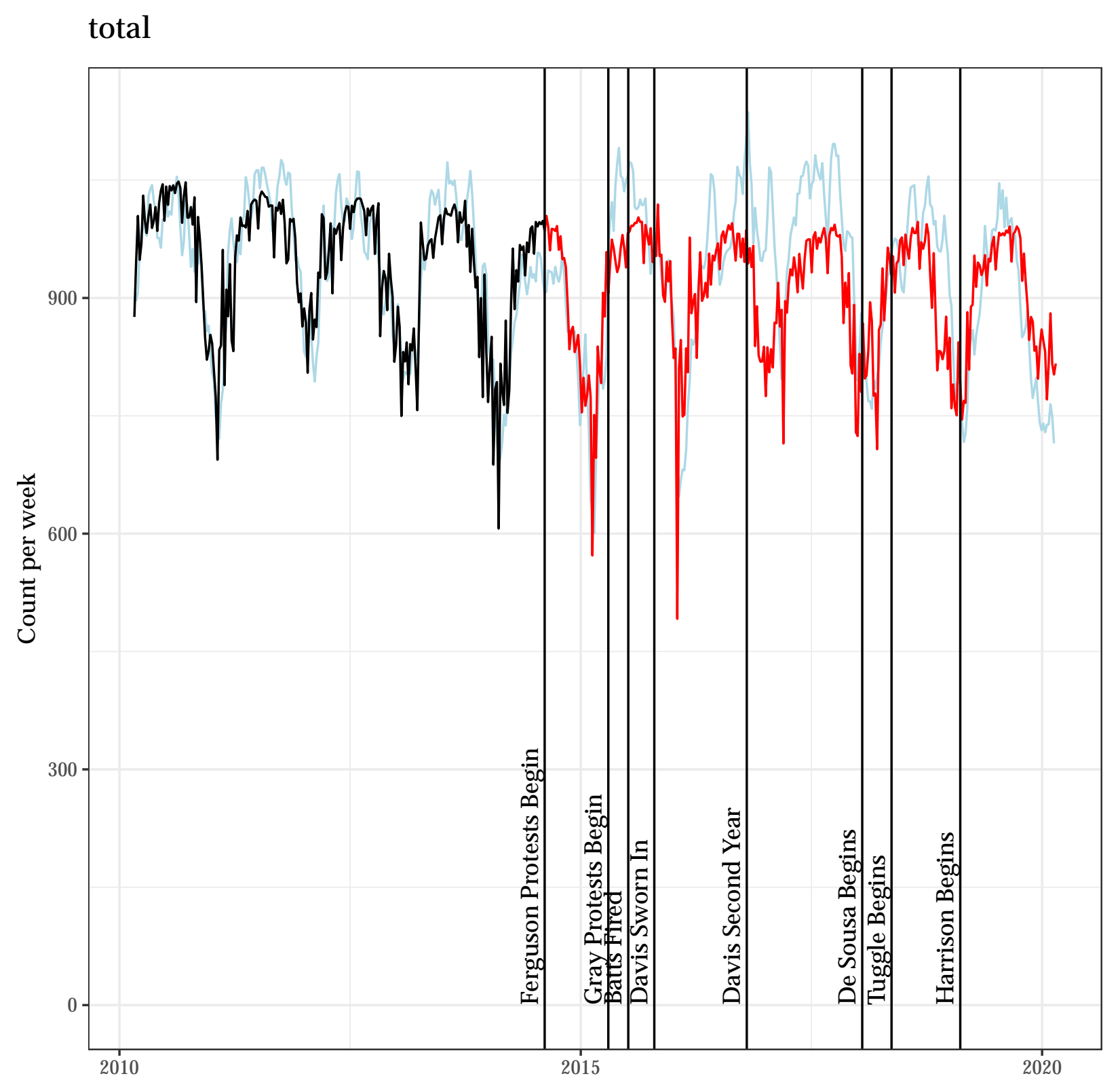


Autocorrelation Function for the Observed Outcome for Updated Model (2)

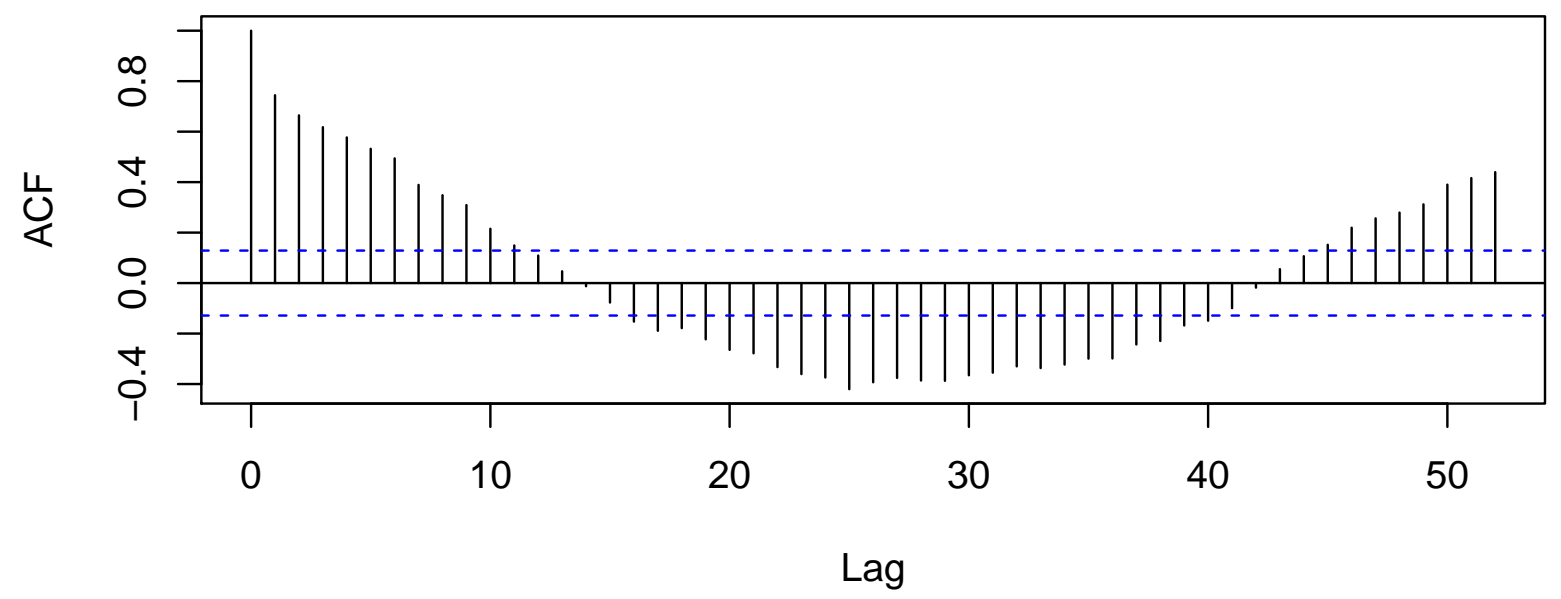

Autocorrelation Function for the Residuals from Updated Model (2)

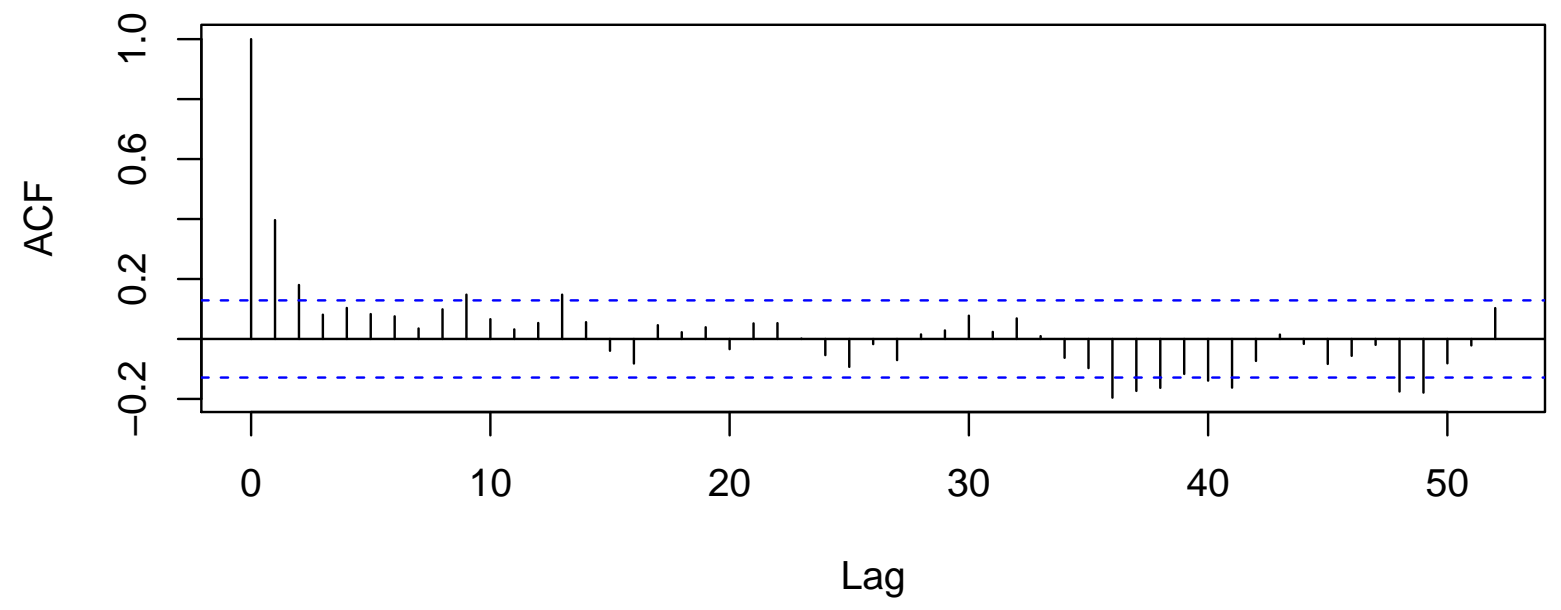




\subsection{A Poisson Regression Model as an Alternative to Model (2) for total}

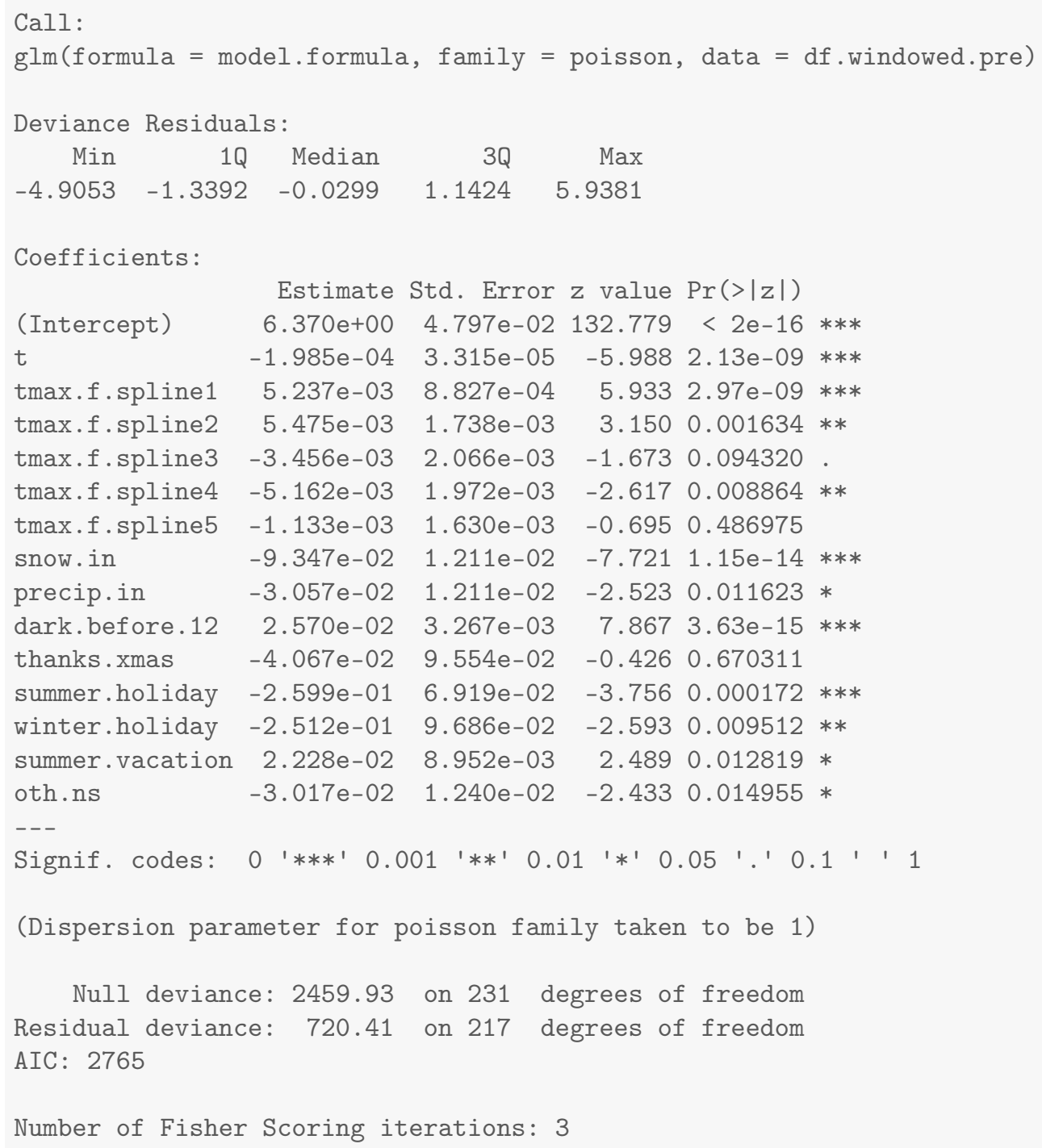




\section{Results for homicide}

\subsection{Summary Values for homicide}

Table 4.1: Descriptives for Outcome Before Ferguson Protests Begin

\begin{tabular}{lcccccccc}
\hline \hline Statistic & $\mathrm{N}$ & Mean & St. Dev. & Min & Pctl(25) & Median & Pctl(75) & Max \\
\hline homicide & 232 & 4.198 & 2.159 & 0 & 2.8 & 4 & 6 & 11 \\
\hline
\end{tabular}

Table 4.2: Descriptives for Outcome After Ferguson Protests Begin

\begin{tabular}{lcccccccc}
\hline \hline Statistic & $\mathrm{N}$ & Mean & St. Dev. & Min & Pctl(25) & Median & Pctl(75) & Max \\
\hline homicide & 290 & 6.183 & 2.846 & 0 & 4 & 6 & 8 & 17 \\
\hline
\end{tabular}




\subsection{Four Models for homicide}

Table 4.3: Four Models that Differ on the Specification of Adjustment and Intervention Variables

\begin{tabular}{|c|c|c|c|c|}
\hline & \multicolumn{4}{|c|}{ Outcome: Count Per Week } \\
\hline & $(1)$ & $(2)$ & (3) & $(4)$ \\
\hline Time (counter in weeks) & 0.0005 & 0.001 & & 0.001 \\
\hline After Ferguson Protests Begin (week of $8 / 11 / 14$ onward) & -0.29 & & -0.20 & -0.14 \\
\hline After Gray Protests Begin (week of 4/20/15 onward) & 4.65 & & 3.92 & 3.98 \\
\hline Unrest and National Guard $(4 / 27 / 15-5 / 3 / 2015)$ & 0.37 & & 1.00 & 0.76 \\
\hline After Batts Fired (week of 7/13/15 onward) & -1.14 & & -0.98 & -1.34 \\
\hline After Davis Confirmed (week of 10/19/15 onward) & -1.32 & & -0.99 & -0.82 \\
\hline After Davis First Year (week of 10/17/16 onward) & 0.25 & & 0.22 & 0.20 \\
\hline After De Sousa Begins (week of 1/19/18 onward) & -1.18 & & -0.77 & -0.76 \\
\hline After Tuggle Begins (week of 5/14/18 onward) & 0.75 & & 0.42 & 0.24 \\
\hline After Harrison Begins (week of 2/11/19 onward) & 0.63 & & 0.71 & 0.66 \\
\hline Average Maximum Temperature to 50 Degrees & & -0.07 & & -0.03 \\
\hline Plus Degrees in the $50 \mathrm{~s}$ & & 0.16 & & 0.09 \\
\hline Plus Degrees in the 60s & & -0.11 & & -0.01 \\
\hline Plus Degrees in the 70s & & 0.14 & & 0.01 \\
\hline Plus Degrees Greater Than 80 & & -0.15 & & -0.03 \\
\hline Snowfall (inches) & & -0.82 & & -0.59 \\
\hline Precipitation (inches) & & -1.29 & & -1.02 \\
\hline Darkness Before Midnight (hours) & & 0.04 & & 0.18 \\
\hline Thanksgiving/Christmas (proportion of week) & & -3.06 & & -6.90 \\
\hline Winter Holiday (proportion of week) & & -7.92 & & -3.86 \\
\hline Other Out-of-School Days (proportion of week) & & 0.61 & & 0.12 \\
\hline Summer Vacation (proportion of week) & & -0.29 & & 0.01 \\
\hline Summer Holiday (proportion of week) & & 9.71 & & 6.70 \\
\hline Observations & 522 & 232 & 522 & 522 \\
\hline $\mathrm{R}^{2}$ & 0.21 & 0.12 & 0.18 & 0.27 \\
\hline
\end{tabular}




\subsection{Least Squares Updated Model (2) for homicide}

homicide

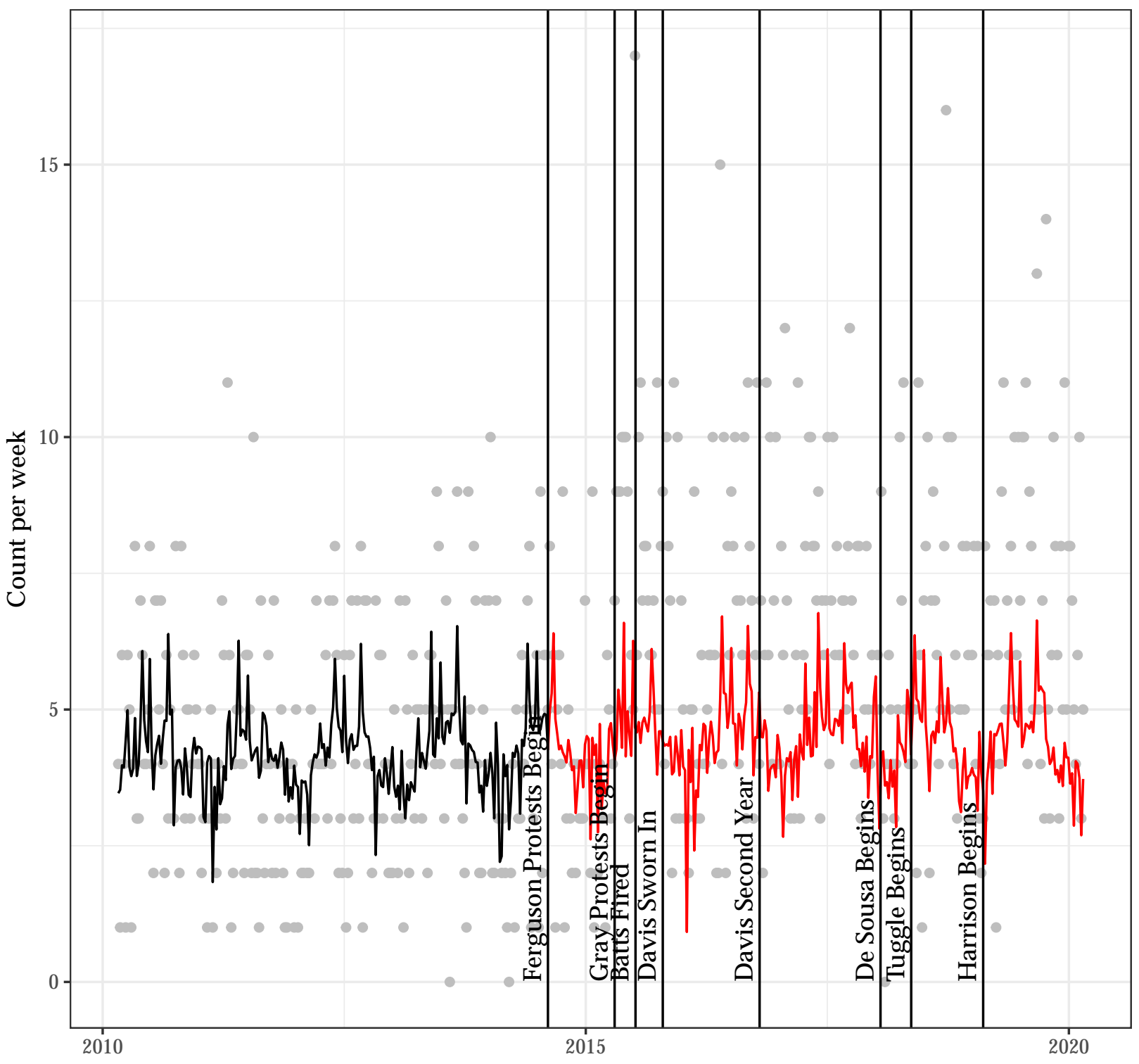




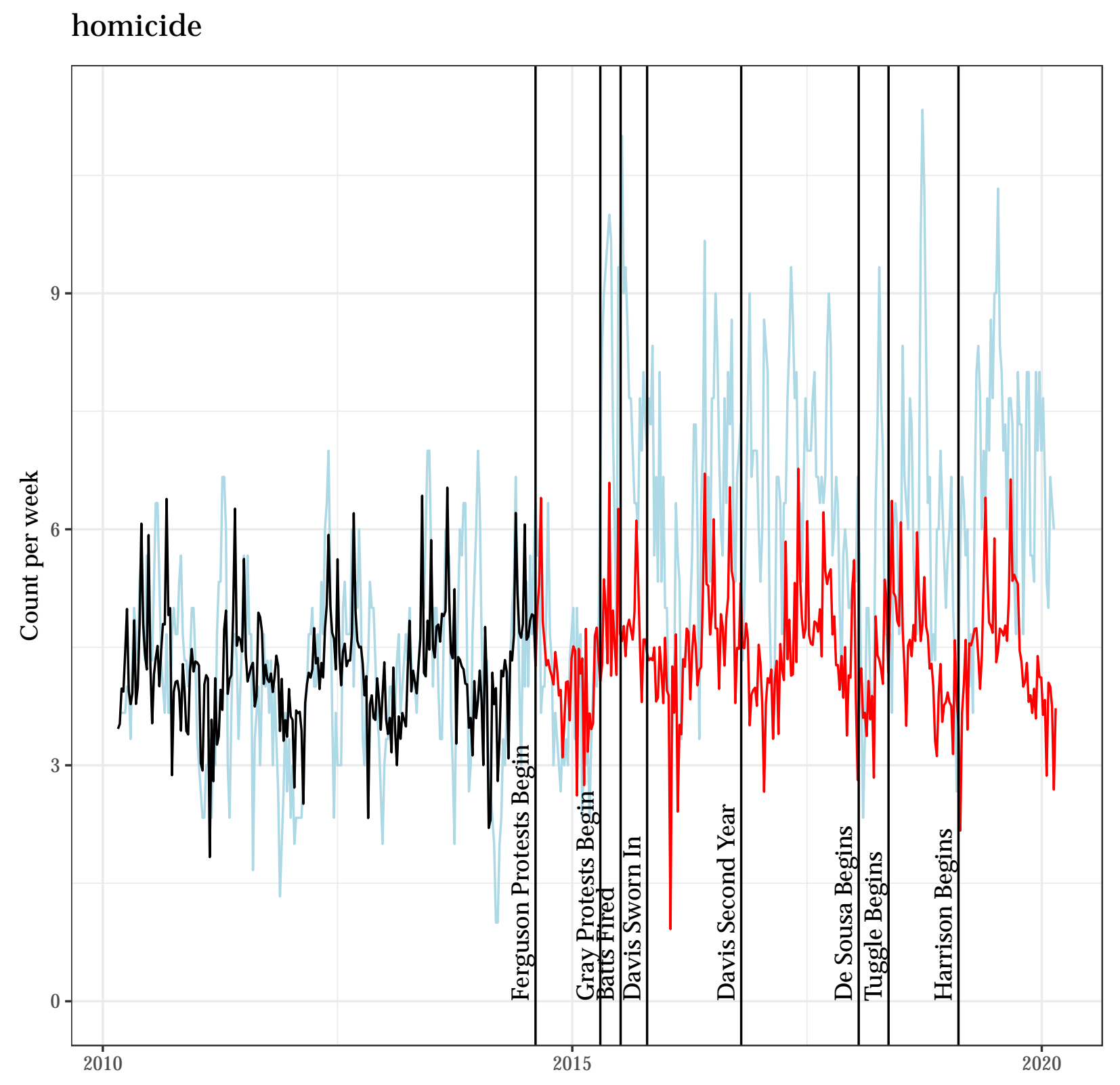


Autocorrelation Function for the Observed Outcome for Updated Model (2)

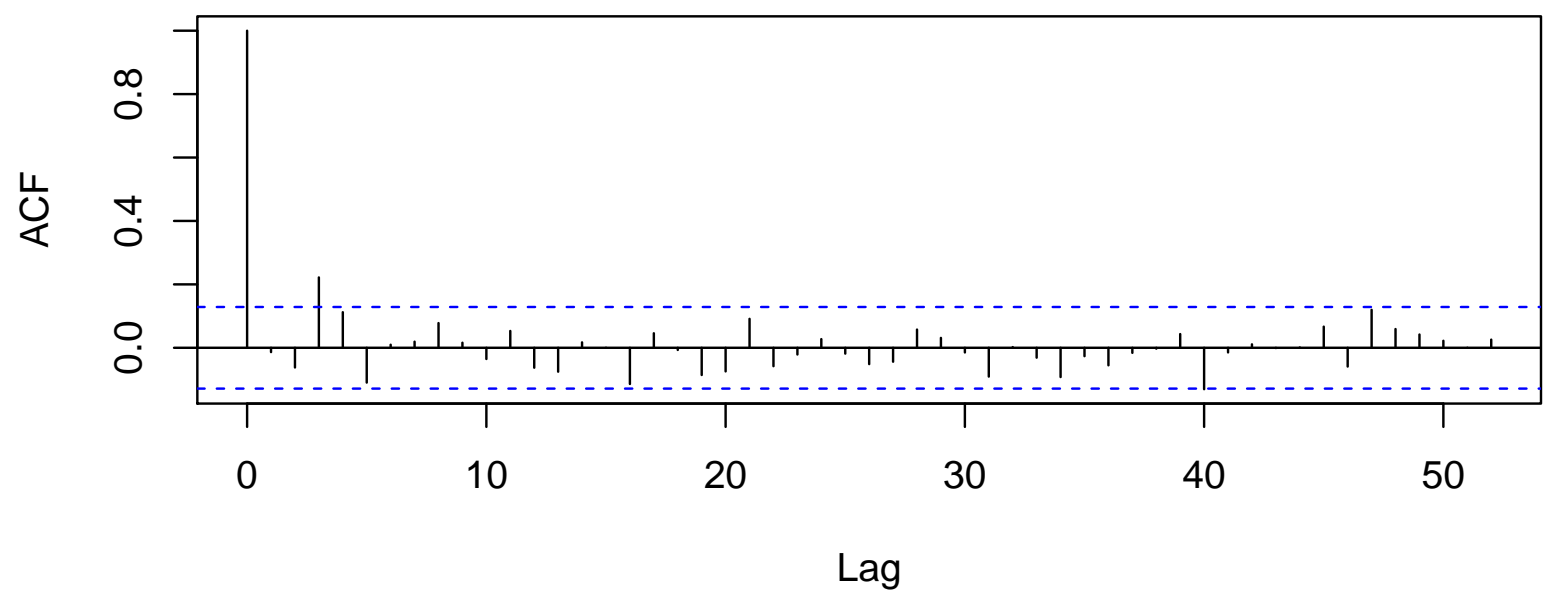

Autocorrelation Function for the Residuals from Updated Model (2)

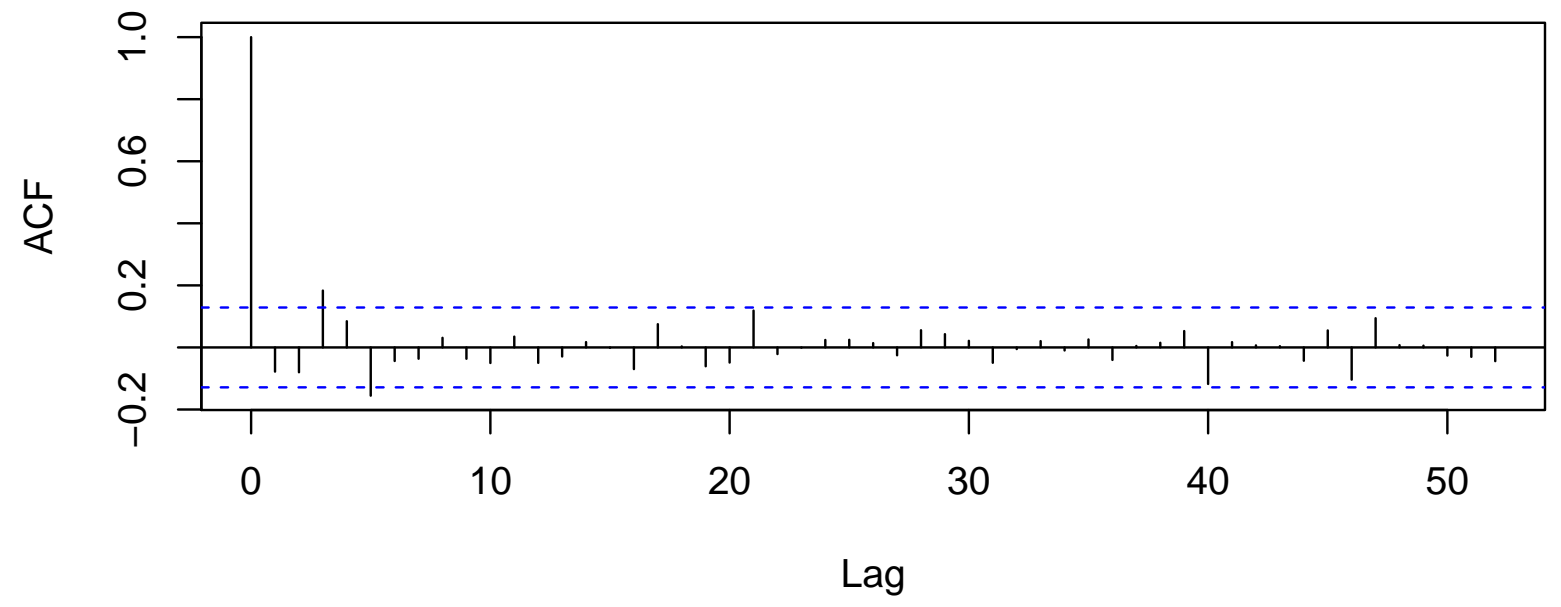




\subsection{A Poisson Regression Model as an Alternative to Model (2) for homicide}

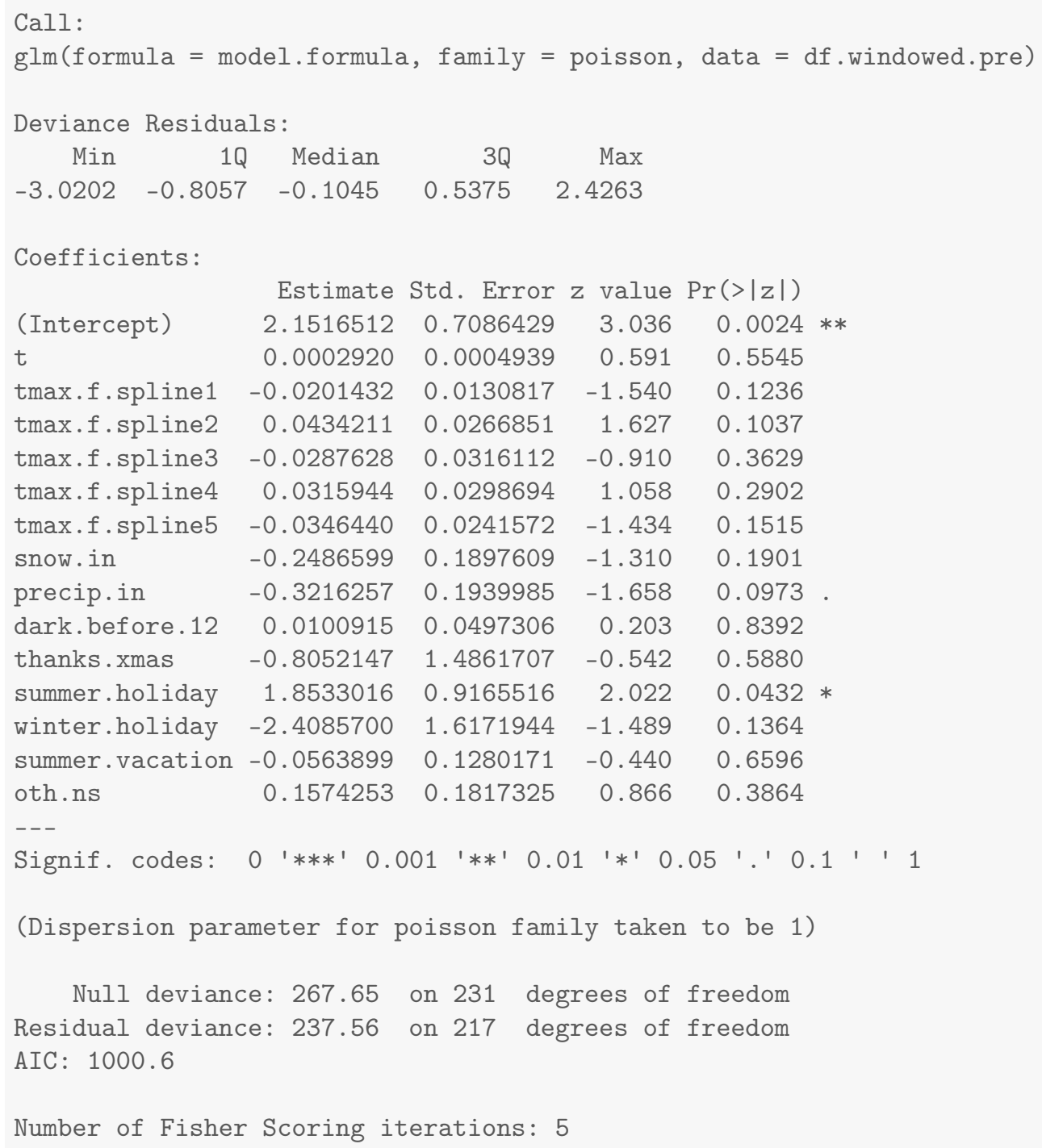




\section{Results for shooting}

\subsection{Summary Values for shooting}

Table 5.1: Descriptives for Outcome Before Ferguson Protests Begin

\begin{tabular}{lcccccccc}
\hline \hline Statistic & $\mathrm{N}$ & Mean & St. Dev. & Min & Pctl(25) & Median & Pctl(75) & Max \\
\hline shooting & 232 & 7.526 & 3.623 & 0 & 5 & 7 & 10 & 22 \\
\hline
\end{tabular}

Table 5.2: Descriptives for Outcome After Ferguson Protests Begin

\begin{tabular}{lcccccccc}
\hline \hline Statistic & $\mathrm{N}$ & Mean & St. Dev. & Min & Pctl(25) & Median & Pctl(75) & Max \\
\hline shooting & 290 & 12.703 & 5.704 & 1 & 8.2 & 12 & 16 & 33 \\
\hline
\end{tabular}




\subsection{Four Models for shooting}

Table 5.3: Four Models that Differ on the Specification of Adjustment and Intervention Variables

\begin{tabular}{|c|c|c|c|c|}
\hline & \multicolumn{4}{|c|}{ Outcome: Count Per Week } \\
\hline & $(1)$ & $(2)$ & $(3)$ & $(4)$ \\
\hline Time (counter in weeks) & -0.004 & -0.01 & & -0.003 \\
\hline After Ferguson Protests Begin (week of $8 / 11 / 14$ onward) & -0.13 & & 0.97 & 0.97 \\
\hline After Gray Protests Begin (week of 4/20/15 onward) & 12.15 & & 9.84 & 9.42 \\
\hline Unrest and National Guard (4/27/15 - 5/3/2015) & -4.93 & & -2.87 & -3.00 \\
\hline After Batts Fired (week of 7/13/15 onward) & -4.86 & & -4.40 & -5.01 \\
\hline After Davis Confirmed (week of $10 / 19 / 15$ onward) & -1.08 & & 0.21 & 0.71 \\
\hline After Davis First Year (week of 10/17/16 onward) & 0.29 & & -0.03 & 0.16 \\
\hline After De Sousa Begins (week of 1/19/18 onward) & -2.12 & & -0.94 & -0.77 \\
\hline After Tuggle Begins (week of 5/14/18 onward) & 3.60 & & 2.17 & 2.05 \\
\hline After Harrison Begins (week of 2/11/19 onward) & 0.48 & & 0.46 & 0.67 \\
\hline Average Maximum Temperature to 50 Degrees & & 0.05 & & 0.06 \\
\hline Plus Degrees in the $50 \mathrm{~s}$ & & -0.04 & & -0.03 \\
\hline Plus Degrees in the 60s & & 0.12 & & 0.12 \\
\hline Plus Degrees in the $70 \mathrm{~s}$ & & 0.13 & & 0.11 \\
\hline Plus Degrees Greater Than 80 & & -0.62 & & -0.44 \\
\hline Snowfall (inches) & & -0.74 & & -0.80 \\
\hline Precipitation (inches) & & -1.80 & & -2.38 \\
\hline Darkness Before Midnight (hours) & & 0.24 & & 0.16 \\
\hline Thanksgiving/Christmas (proportion of week) & & 9.55 & & 4.43 \\
\hline Winter Holiday (proportion of week) & & -4.16 & & -2.62 \\
\hline Other Out-of-School Days (proportion of week) & & 0.34 & & 0.03 \\
\hline Summer Vacation (proportion of week) & & 2.43 & & 1.94 \\
\hline Summer Holiday (proportion of week) & & 10.66 & & -0.76 \\
\hline Observations & 522 & 232 & 522 & 522 \\
\hline $\mathrm{R}^{2}$ & 0.34 & 0.26 & 0.45 & 0.47 \\
\hline
\end{tabular}




\subsection{Least Squares Updated Model (2) for shooting}

shooting

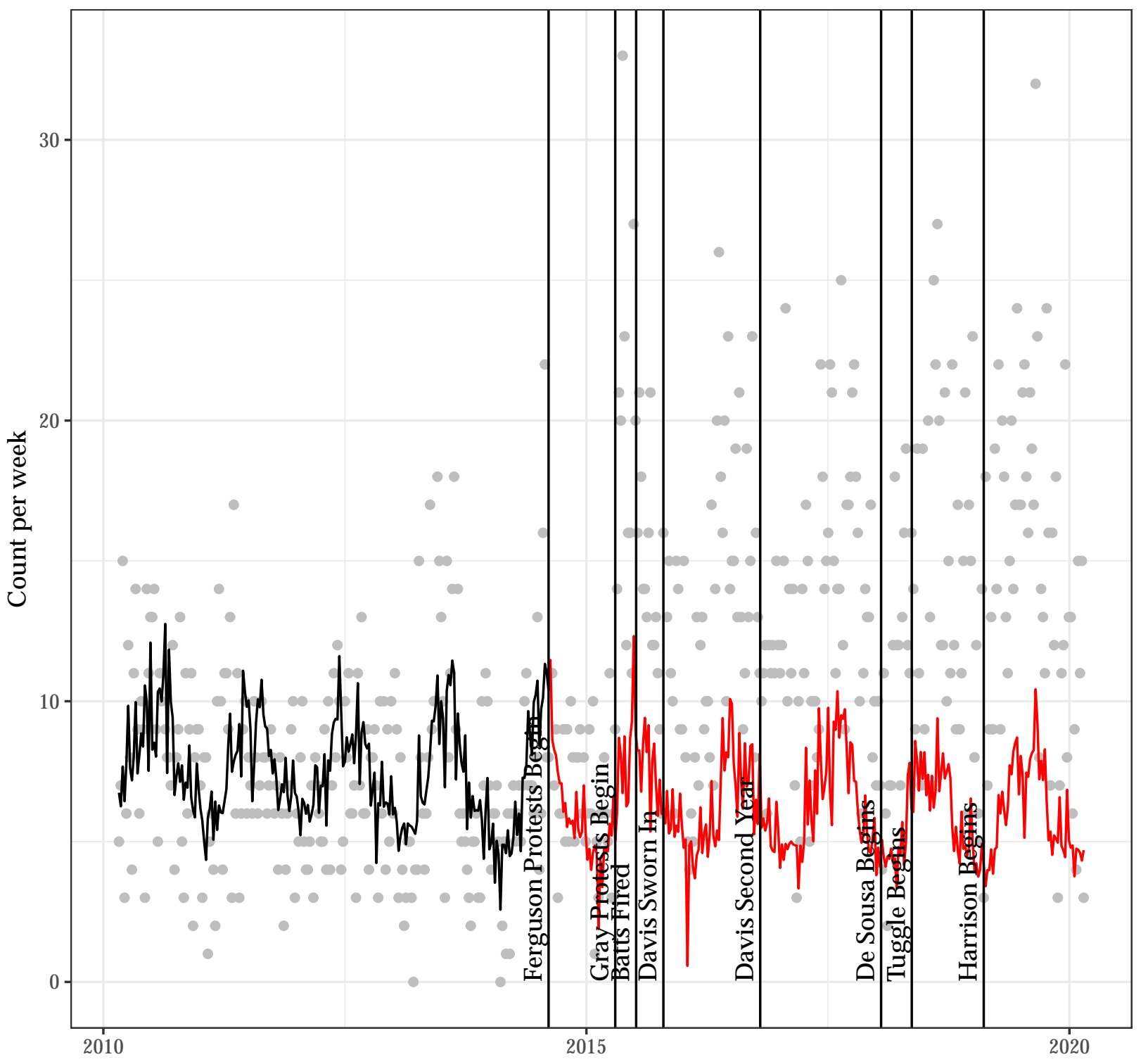




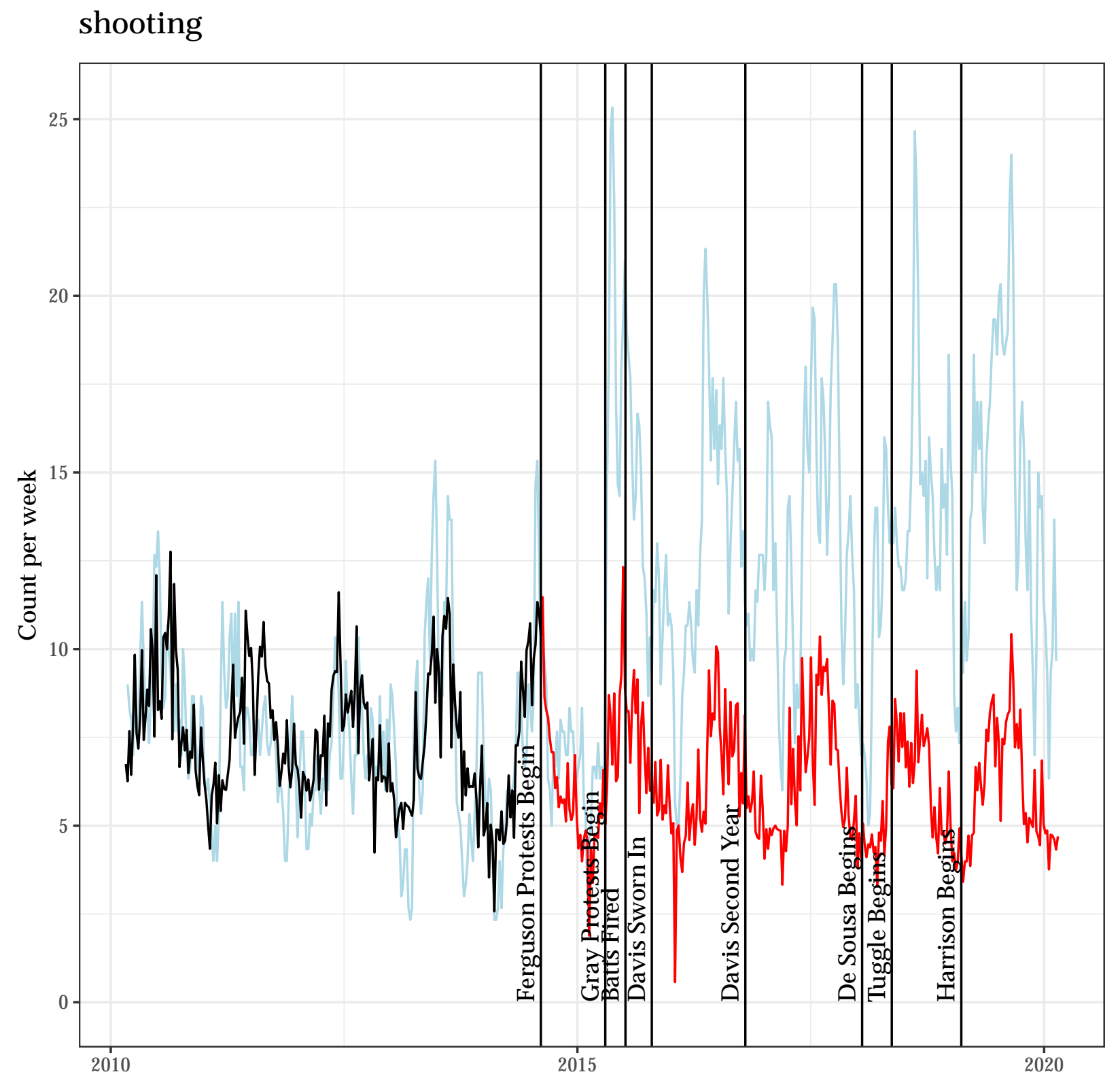


Autocorrelation Function for the Observed Outcome for Updated Model (2)

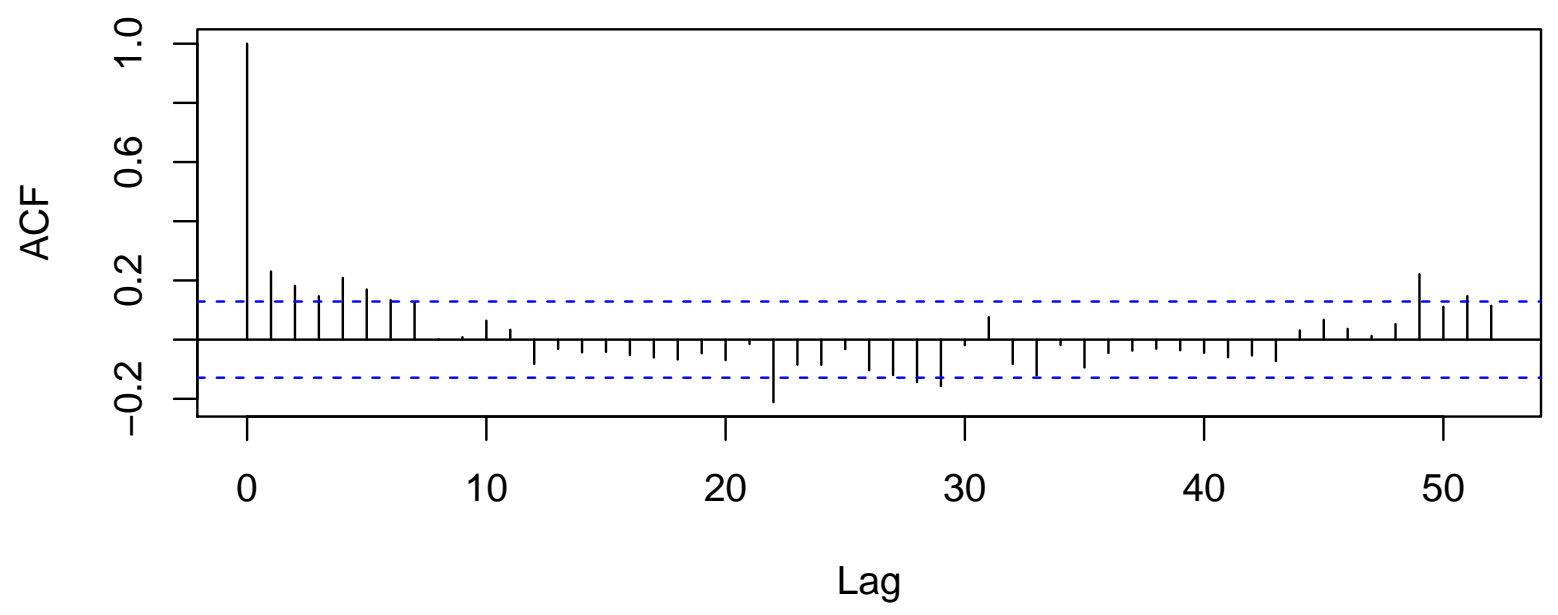

Autocorrelation Function for the Residuals from Updated Model (2)

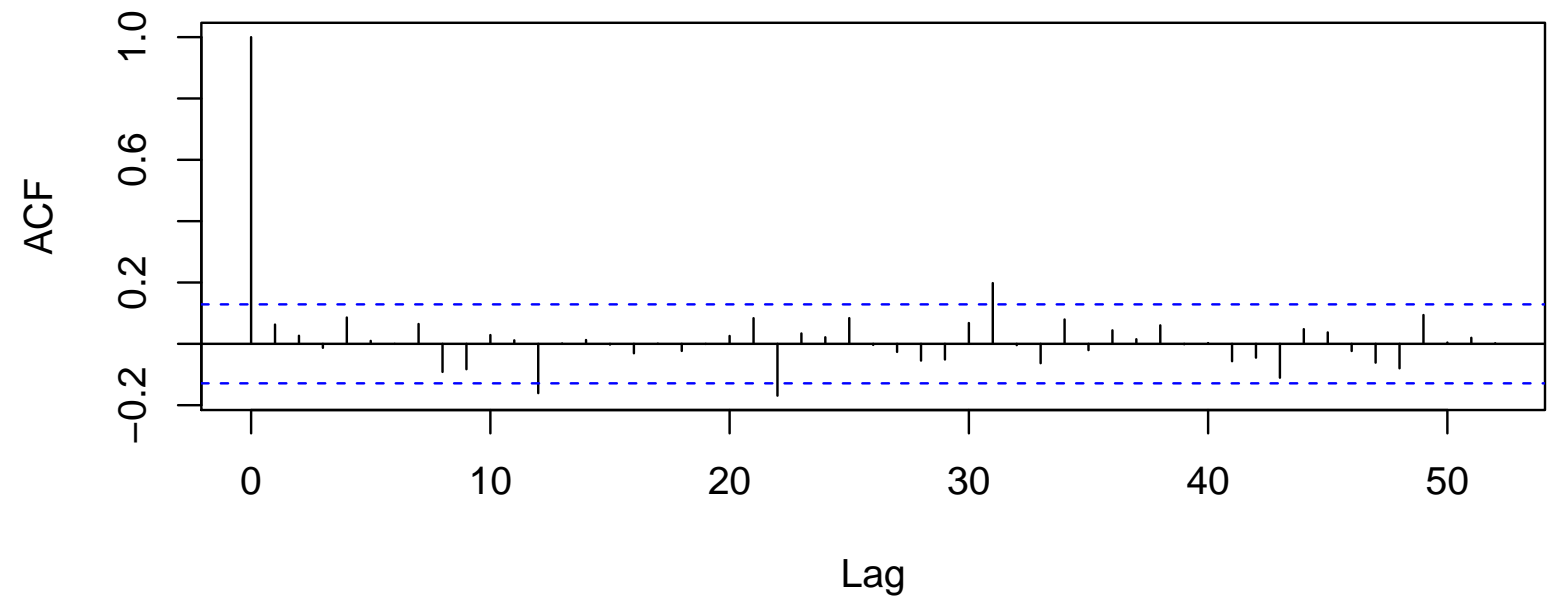




\subsection{A Poisson Regression Model as an Alternative to Model (2) for shooting}

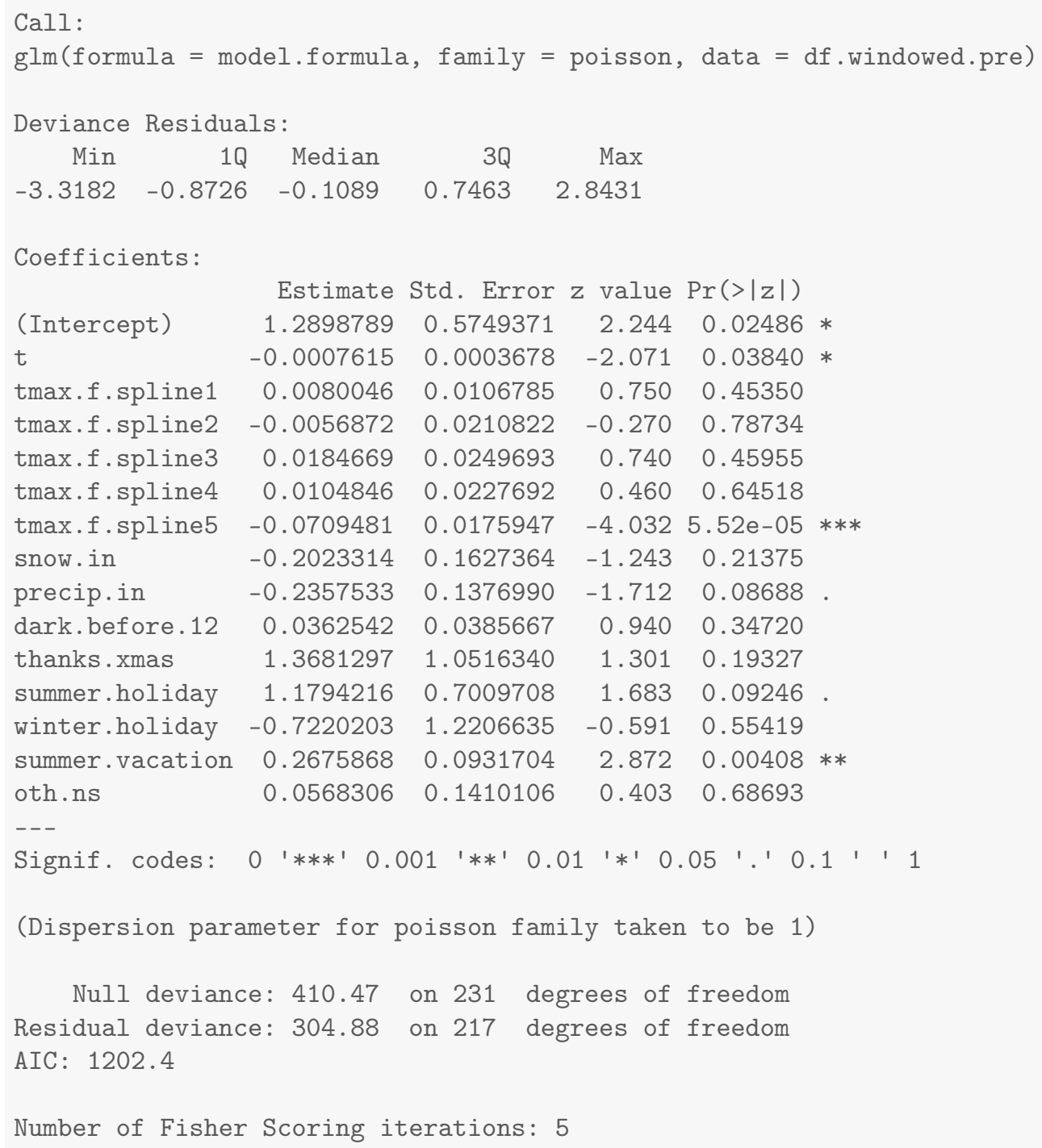




\section{Results for rape}

\subsection{Summary Values for rape}

Table 6.1: Descriptives for Outcome Before Ferguson Protests Begin

\begin{tabular}{lcccccccc}
\hline \hline Statistic & $\mathrm{N}$ & Mean & St. Dev. & Min & Pctl(25) & Median & Pctl(75) & Max \\
\hline rape & 232 & 5.647 & 2.530 & 0 & 4 & 5 & 7 & 16 \\
\hline
\end{tabular}

Table 6.2: Descriptives for Outcome After Ferguson Protests Begin

\begin{tabular}{lcccccccc}
\hline \hline Statistic & $\mathrm{N}$ & Mean & St. Dev. & Min & Pctl(25) & Median & Pctl(75) & Max \\
\hline rape & 290 & 5.838 & 2.855 & 0 & 4 & 6 & 8 & 14 \\
\hline
\end{tabular}




\subsection{Four Models for rape}

Table 6.3: Four Models that Differ on the Specification of Adjustment and Intervention Variables

\begin{tabular}{|c|c|c|c|c|}
\hline & \multicolumn{4}{|c|}{ Outcome: Count Per Week } \\
\hline & (1) & $(2)$ & (3) & (4) \\
\hline Time (counter in weeks) & -0.004 & -0.002 & & -0.003 \\
\hline After Ferguson Protests Begin (week of $8 / 11 / 14$ onward) & -0.55 & & -0.38 & -0.30 \\
\hline After Gray Protests Begin (week of 4/20/15 onward) & 2.20 & & 1.57 & 1.30 \\
\hline Unrest and National Guard $(4 / 27 / 15-5 / 3 / 2015)$ & 0.26 & & 0.22 & 0.18 \\
\hline After Batts Fired (week of 7/13/15 onward) & -1.68 & & -1.95 & -1.48 \\
\hline After Davis Confirmed (week of 10/19/15 onward) & 0.70 & & 1.11 & 1.09 \\
\hline After Davis First Year (week of 10/17/16 onward) & 1.39 & & 1.33 & 1.51 \\
\hline After De Sousa Begins (week of 1/19/18 onward) & 0.33 & & 0.22 & 0.21 \\
\hline After Tuggle Begins (week of 5/14/18 onward) & -0.45 & & -0.80 & -0.48 \\
\hline After Harrison Begins (week of 2/11/19 onward) & -1.12 & & -1.22 & -1.17 \\
\hline Average Maximum Temperature to 50 Degrees & & 0.05 & & -0.002 \\
\hline Plus Degrees in the 50s & & -0.05 & & -0.01 \\
\hline Plus Degrees in the 60s & & 0.04 & & 0.05 \\
\hline Plus Degrees in the 70s & & -0.07 & & -0.08 \\
\hline Plus Degrees Greater Than 80 & & 0.14 & & 0.06 \\
\hline Snowfall (inches) & & -0.63 & & -0.13 \\
\hline Precipitation (inches) & & 0.50 & & -0.14 \\
\hline Darkness Before Midnight (hours) & & -0.07 & & -0.34 \\
\hline Thanksgiving/Christmas (proportion of week) & & -4.95 & & -7.07 \\
\hline Winter Holiday (proportion of week) & & -3.38 & & -8.29 \\
\hline Other Out-of-School Days (proportion of week) & & 0.01 & & 0.06 \\
\hline Summer Vacation (proportion of week) & & -0.12 & & 0.01 \\
\hline Summer Holiday (proportion of week) & & -4.11 & & 0.47 \\
\hline Observations & 522 & 232 & 522 & 522 \\
\hline $\mathrm{R}^{2}$ & 0.06 & 0.06 & 0.07 & 0.12 \\
\hline
\end{tabular}




\subsection{Least Squares Updated Model (2) for rape}

rape

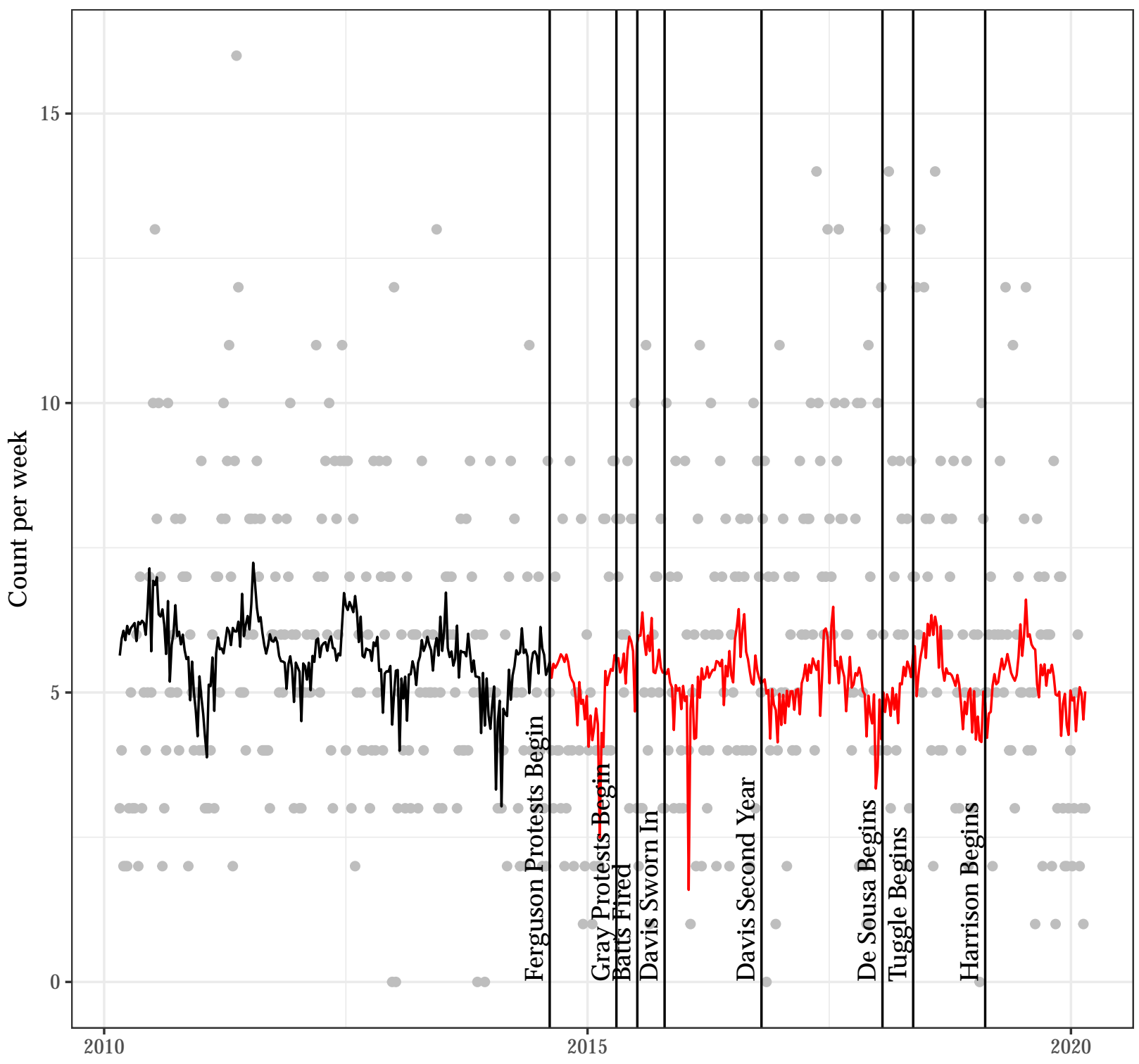




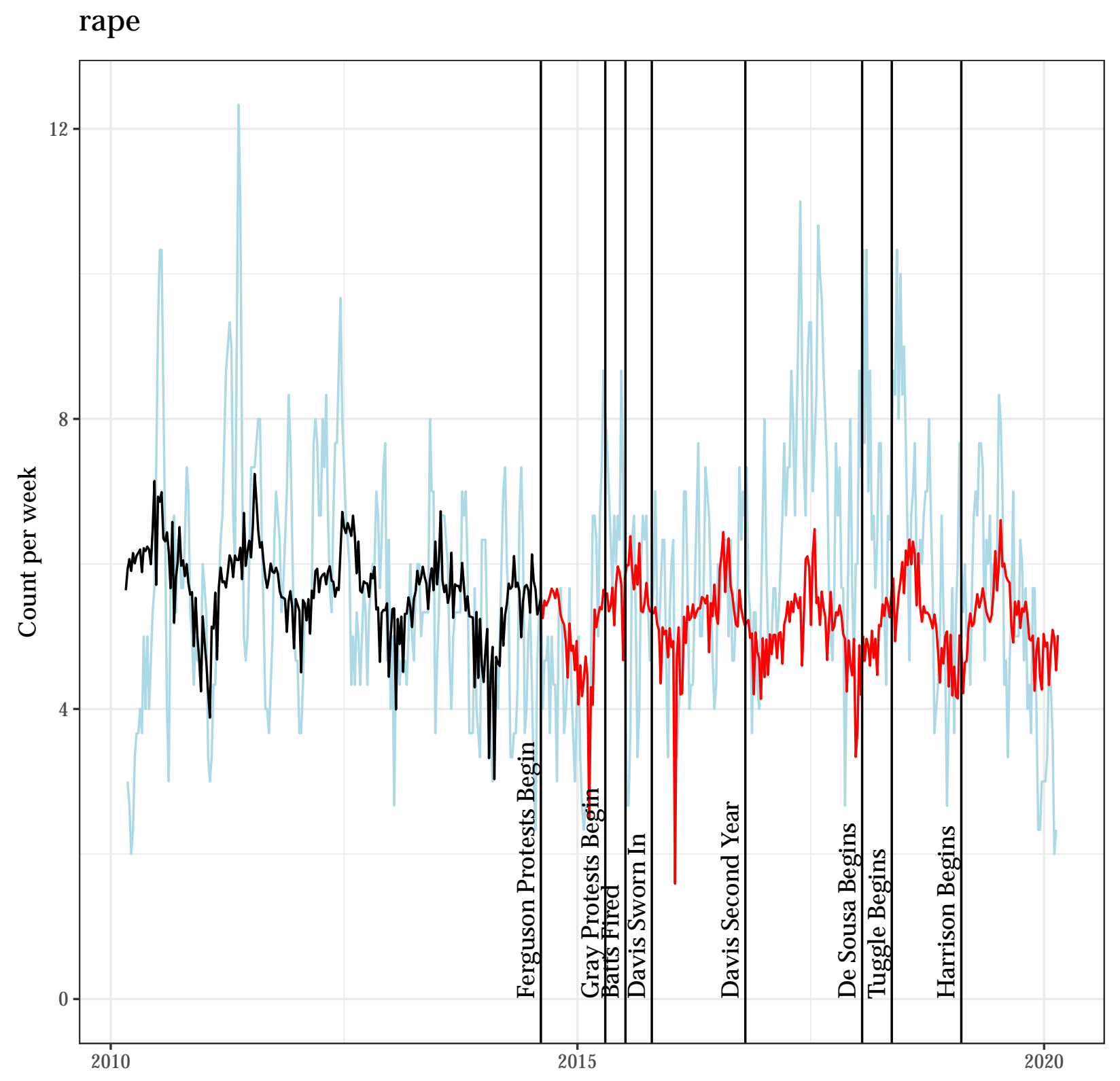


Autocorrelation Function for the Observed Outcome for Updated Model (2)

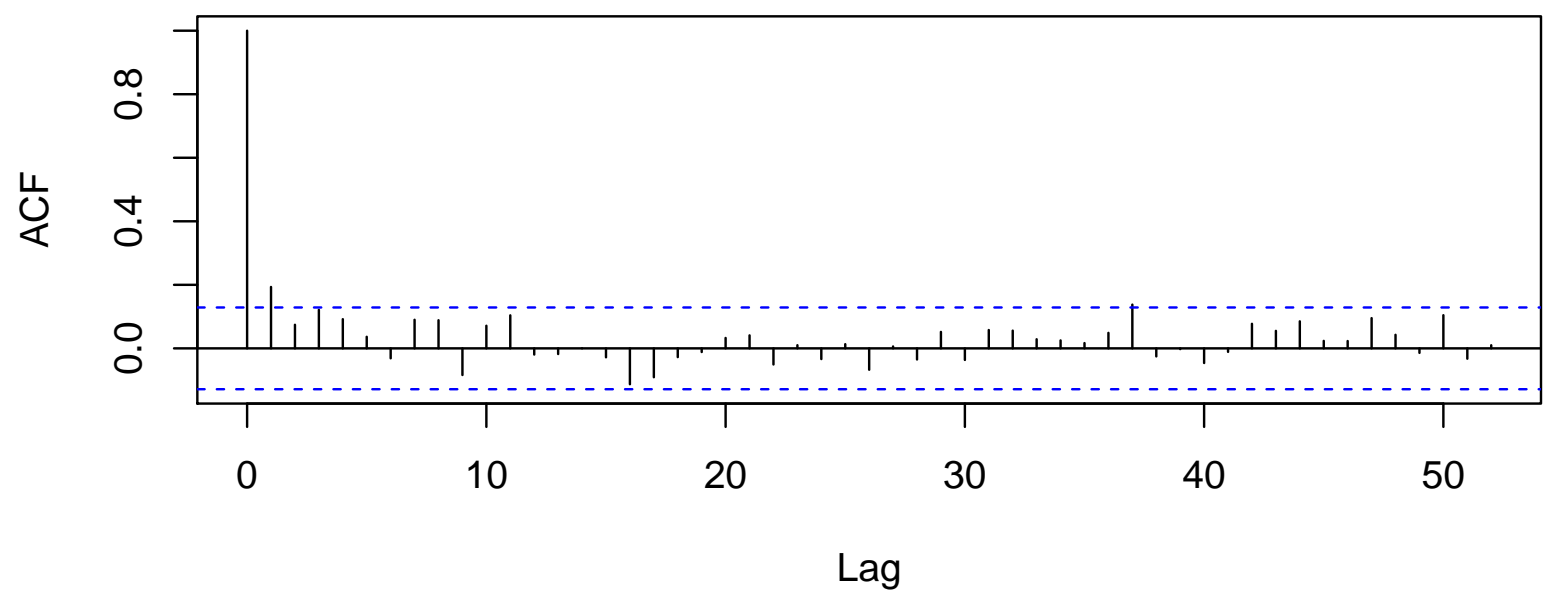

Autocorrelation Function for the Residuals from Updated Model (2)

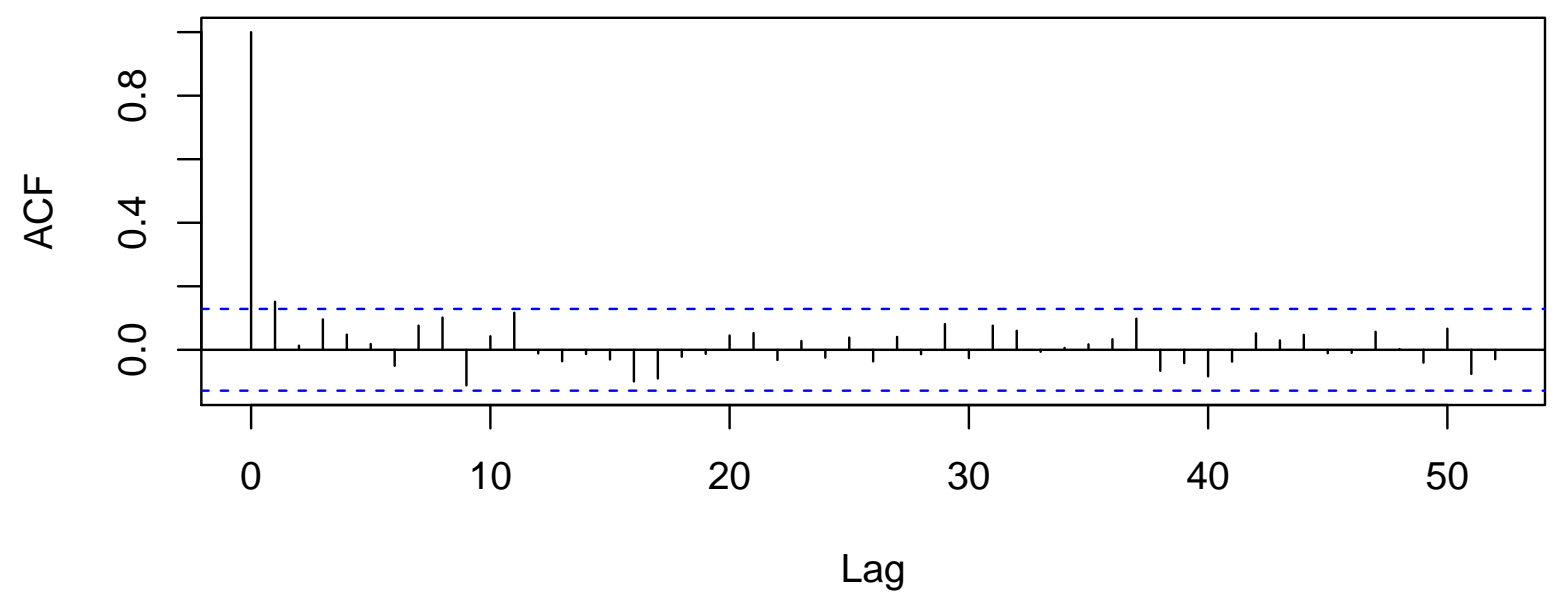




\subsection{A Poisson Regression Model as an Alternative to Model (2) for rape}

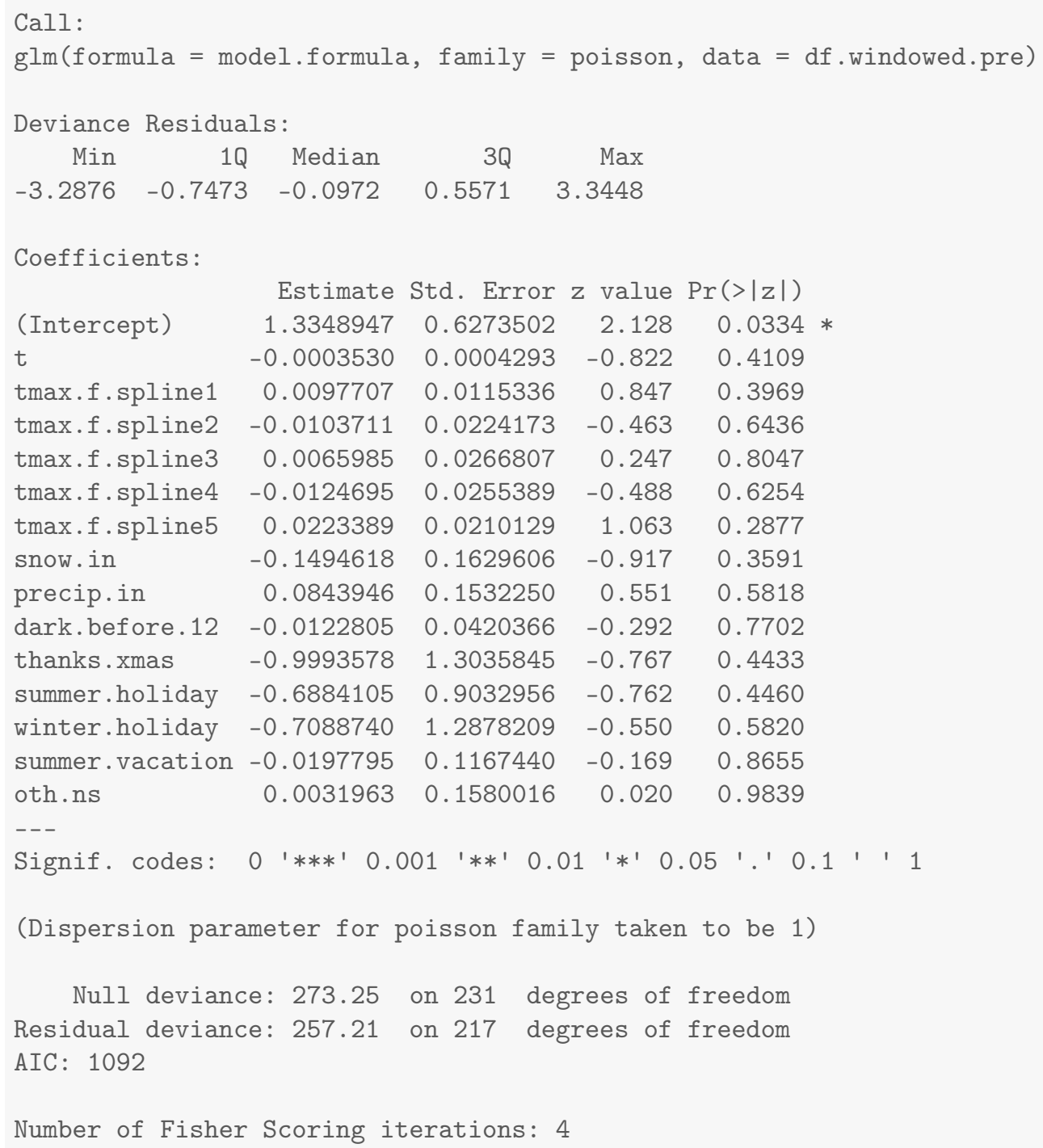




\section{Results for robbery.carjacking}

\subsection{Summary Values for robbery.carjacking}

Table 7.1: Descriptives for Outcome Before Ferguson Protests Begin

\begin{tabular}{lcccccccc}
\hline \hline Statistic & $\mathrm{N}$ & Mean & St. Dev. & Min & Pctl(25) & Median & Pctl(75) & Max \\
\hline robbery.carjacking & 232 & 3.155 & 2.071 & 0 & 2 & 3 & 4.2 & 11 \\
\hline
\end{tabular}

Table 7.2: Descriptives for Outcome After Ferguson Protests Begin

\begin{tabular}{lcccccccc}
\hline \hline Statistic & $\mathrm{N}$ & Mean & St. Dev. & Min & Pctl(25) & Median & Pctl(75) & Max \\
\hline robbery.carjacking & 290 & 8.638 & 4.736 & 0 & 5 & 8 & 11 & 30 \\
\hline
\end{tabular}




\subsection{Four Models for robbery.carjacking}

Table 7.3: Four Models that Differ on the Specification of Adjustment and Intervention Variables

\begin{tabular}{|c|c|c|c|c|}
\hline & \multicolumn{4}{|c|}{ Outcome: Count Per Week } \\
\hline & $(1)$ & $(2)$ & $(3)$ & $(4)$ \\
\hline Time (counter in weeks) & -0.004 & -0.01 & & -0.004 \\
\hline After Ferguson Protests Begin (week of $8 / 11 / 14$ onward) & 1.25 & & 1.72 & 1.46 \\
\hline After Gray Protests Begin (week of 4/20/15 onward) & 1.63 & & 1.14 & 1.49 \\
\hline Unrest and National Guard (4/27/15 - 5/3/2015) & 9.62 & & 9.83 & 10.06 \\
\hline After Batts Fired (week of 7/13/15 onward) & -0.24 & & -0.22 & -1.15 \\
\hline After Davis Confirmed (week of 10/19/15 onward) & 2.57 & & 3.12 & 3.36 \\
\hline After Davis First Year (week of 10/17/16 onward) & 3.63 & & 3.55 & 3.49 \\
\hline After De Sousa Begins (week of 1/19/18 onward) & -2.89 & & -2.61 & -1.98 \\
\hline After Tuggle Begins (week of 5/14/18 onward) & 1.82 & & 1.08 & 0.54 \\
\hline After Harrison Begins (week of 2/11/19 onward) & 1.92 & & 1.90 & 2.24 \\
\hline Average Maximum Temperature to 50 Degrees & & -0.03 & & 0.03 \\
\hline Plus Degrees in the $50 \mathrm{~s}$ & & 0.01 & & -0.08 \\
\hline Plus Degrees in the 60s & & 0.18 & & 0.23 \\
\hline Plus Degrees in the $70 \mathrm{~s}$ & & -0.18 & & -0.25 \\
\hline Plus Degrees Greater Than 80 & & -0.02 & & 0.15 \\
\hline Snowfall (inches) & & -0.03 & & -0.19 \\
\hline Precipitation (inches) & & 0.56 & & 0.76 \\
\hline Darkness Before Midnight (hours) & & 0.11 & & 0.57 \\
\hline Thanksgiving/Christmas (proportion of week) & & 3.98 & & 9.94 \\
\hline Winter Holiday (proportion of week) & & -4.43 & & 1.29 \\
\hline Other Out-of-School Days (proportion of week) & & -0.60 & & -0.64 \\
\hline Summer Vacation (proportion of week) & & 0.46 & & 1.66 \\
\hline Summer Holiday (proportion of week) & & 1.03 & & -0.25 \\
\hline Observations & 522 & 232 & 522 & 522 \\
\hline $\mathrm{R}^{2}$ & 0.52 & 0.12 & 0.61 & 0.55 \\
\hline
\end{tabular}




\subsection{Least Squares Updated Model (2) for robbery.carjacking}

robbery.carjacking

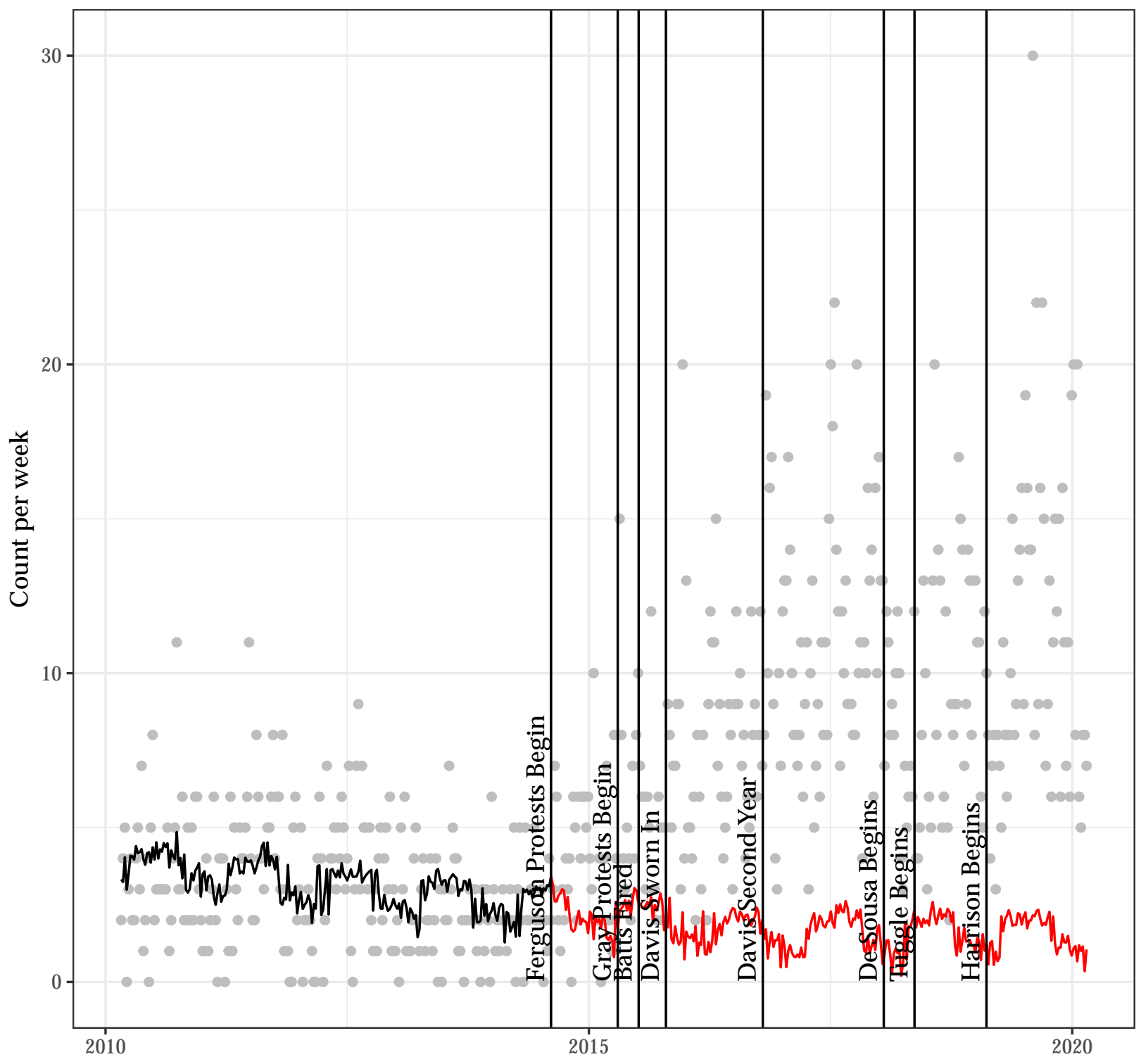




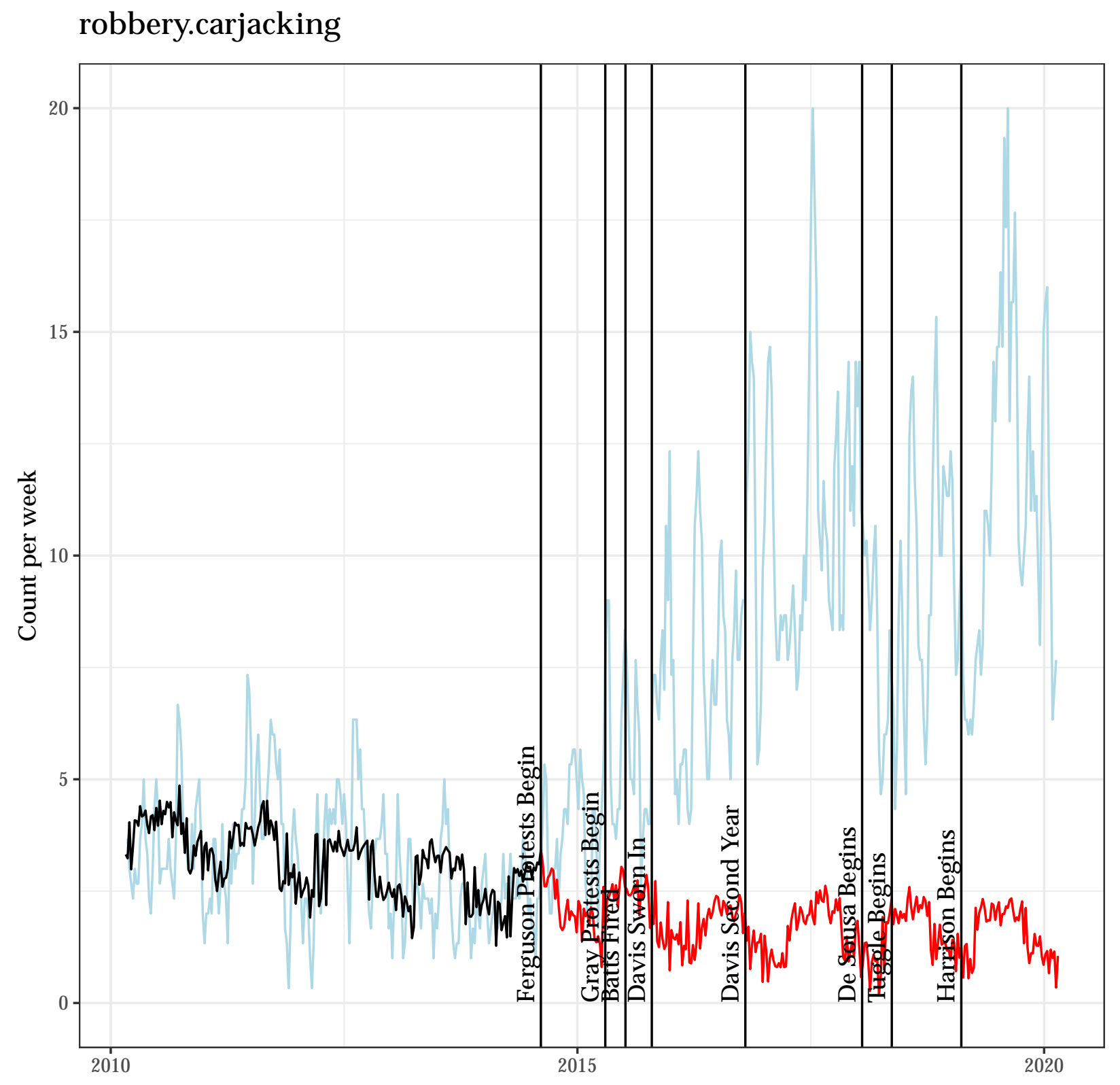


Autocorrelation Function for the Observed Outcome for Updated Model (2)

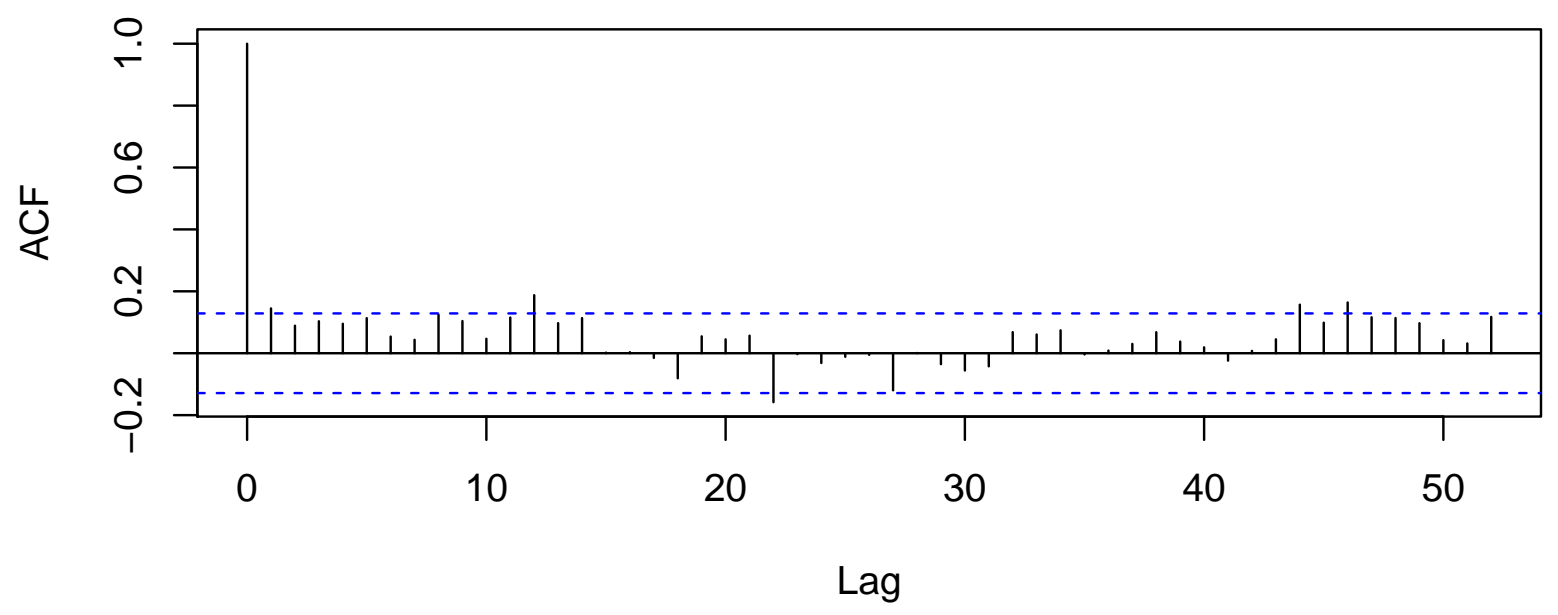

Autocorrelation Function for the Residuals from Updated Model (2)

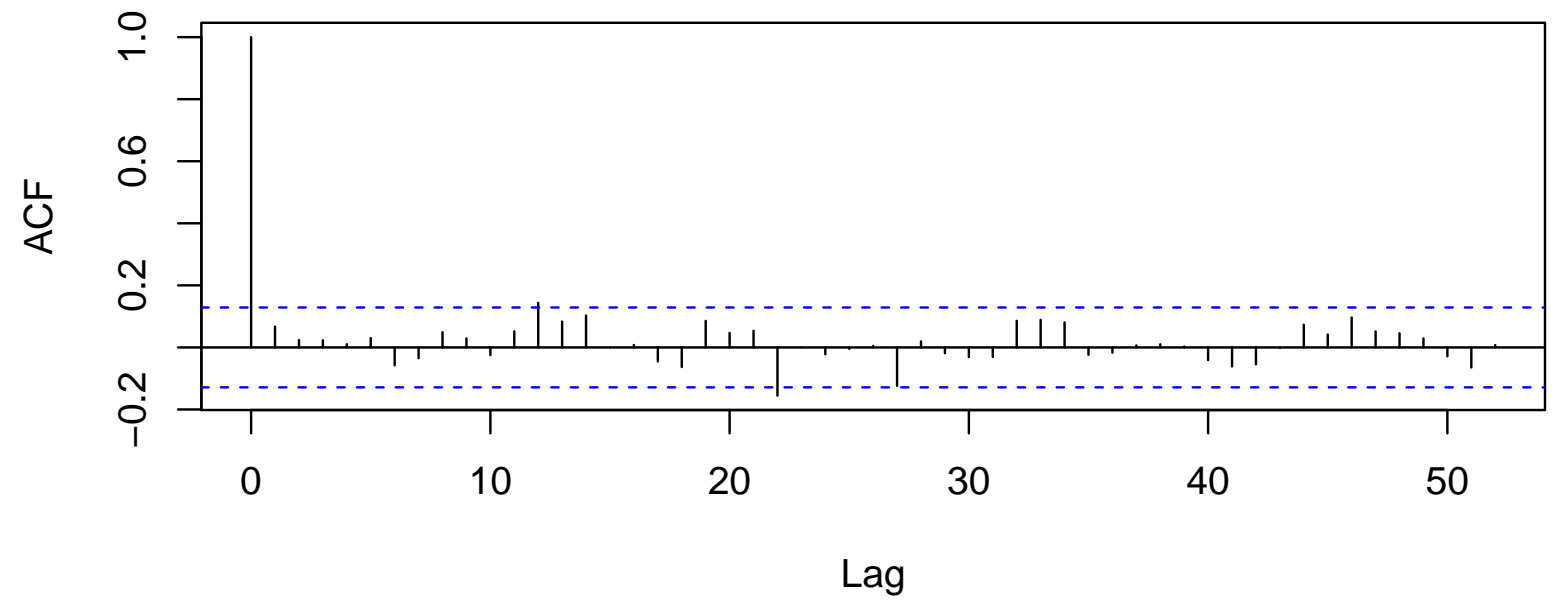




\subsection{A Poisson Regression Model as an Alternative to Model (2) for robbery.carjacking}

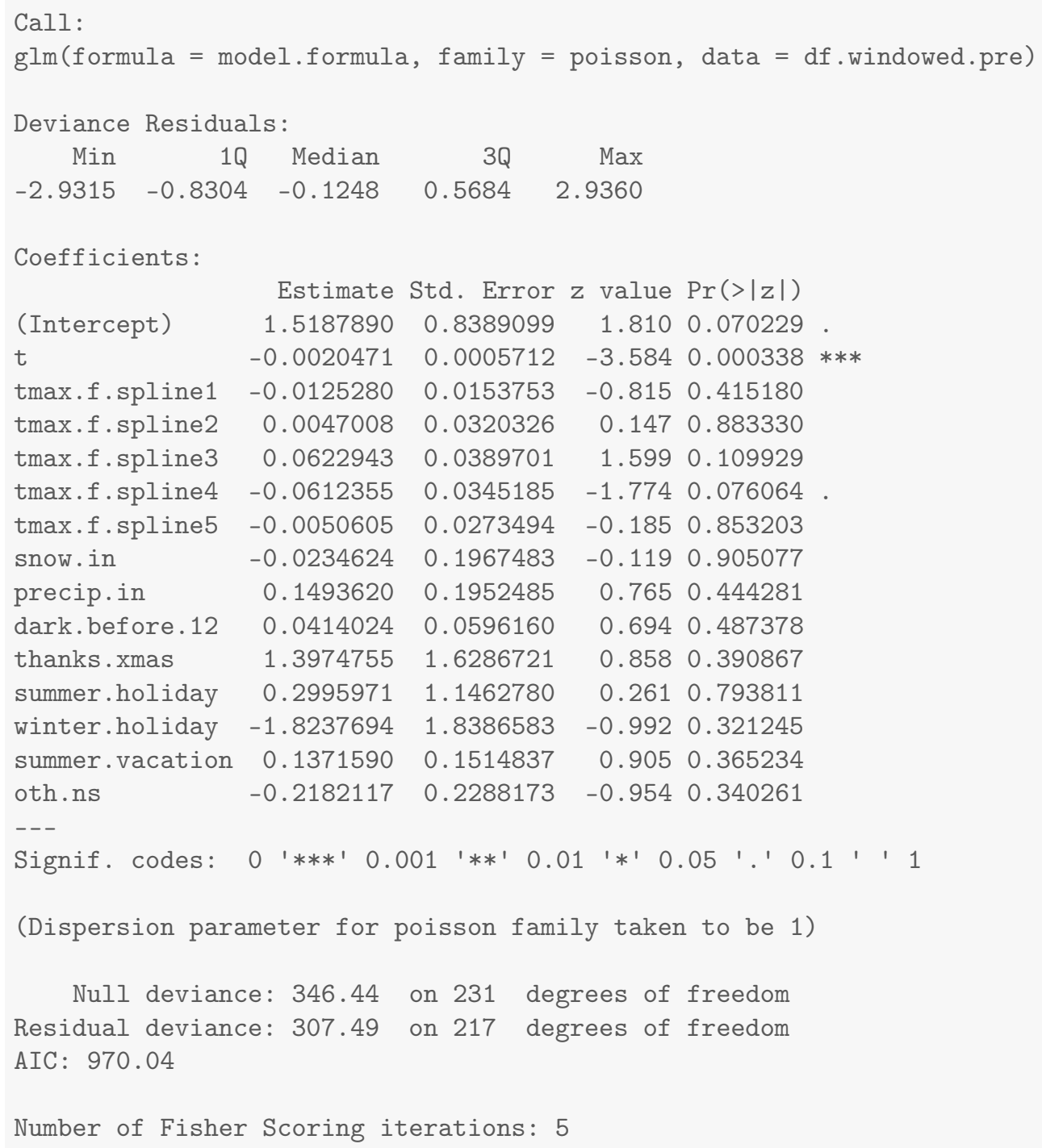




\section{Results for robbery.street}

\subsection{Summary Values for robbery.street}

Table 8.1: Descriptives for Outcome Before Ferguson Protests Begin

\begin{tabular}{lcccccccc}
\hline \hline Statistic & $\mathrm{N}$ & Mean & St. Dev. & Min & Pctl(25) & Median & Pctl(75) & Max \\
\hline robbery.street & 232 & 50.642 & 12.483 & 24 & 42 & 50 & 58.2 & 97 \\
\hline
\end{tabular}

Table 8.2: Descriptives for Outcome After Ferguson Protests Begin

\begin{tabular}{lcccccccc}
\hline \hline Statistic & N & Mean & St. Dev. & Min & Pctl(25) & Median & Pctl(75) & Max \\
\hline robbery.street & 290 & 67.490 & 17.742 & 24 & 55 & 68 & 79.8 & 128 \\
\hline
\end{tabular}




\subsection{Four Models for robbery.street}

Table 8.3: Four Models that Differ on the Specification of Adjustment and Intervention Variables

\begin{tabular}{|c|c|c|c|c|}
\hline & \multicolumn{4}{|c|}{ Outcome: Count Per Week } \\
\hline & (1) & (2) & (3) & $(4)$ \\
\hline Time (counter in weeks) & 0.01 & 0.02 & & 0.02 \\
\hline After Ferguson Protests Begin (week of $8 / 11 / 14$ onward) & -0.28 & & 0.20 & 1.36 \\
\hline After Gray Protests Begin (week of 4/20/15 onward) & 18.09 & & 17.02 & 13.93 \\
\hline Unrest and National Guard (4/27/15 - 5/3/2015) & -19.04 & & -16.19 & -14.59 \\
\hline After Batts Fired (week of 7/13/15 onward) & -2.22 & & -8.44 & -8.86 \\
\hline After Davis Confirmed (week of 10/19/15 onward) & 1.08 & & 5.05 & 6.59 \\
\hline After Davis First Year (week of 10/17/16 onward) & 7.41 & & 6.20 & 6.19 \\
\hline After De Sousa Begins (week of 1/19/18 onward) & -18.93 & & -11.56 & -12.50 \\
\hline After Tuggle Begins (week of 5/14/18 onward) & 12.62 & & 5.14 & 3.54 \\
\hline After Harrison Begins (week of 2/11/19 onward) & -9.26 & & -6.81 & -7.45 \\
\hline Average Maximum Temperature to 50 Degrees & & 0.49 & & 0.65 \\
\hline Plus Degrees in the 50s & & -0.07 & & -0.06 \\
\hline Plus Degrees in the 60s & & 0.63 & & 0.39 \\
\hline Plus Degrees in the 70s & & -0.80 & & -0.37 \\
\hline Plus Degrees Greater Than 80 & & -0.27 & & -0.22 \\
\hline Snowfall (inches) & & -5.98 & & -2.66 \\
\hline Precipitation (inches) & & 4.77 & & 3.51 \\
\hline Darkness Before Midnight (hours) & & 6.40 & & 6.26 \\
\hline Thanksgiving/Christmas (proportion of week) & & -8.54 & & -6.25 \\
\hline Winter Holiday (proportion of week) & & -31.03 & & -35.92 \\
\hline Other Out-of-School Days (proportion of week) & & 0.55 & & -4.67 \\
\hline Summer Vacation (proportion of week) & & 5.23 & & 0.40 \\
\hline Summer Holiday (proportion of week) & & -21.35 & & -24.11 \\
\hline Observations & 522 & 232 & 522 & 522 \\
\hline $\mathrm{R}^{2}$ & 0.34 & 0.23 & 0.25 & 0.49 \\
\hline
\end{tabular}


8.3 Least Squares Updated Model (2) for robbery.street

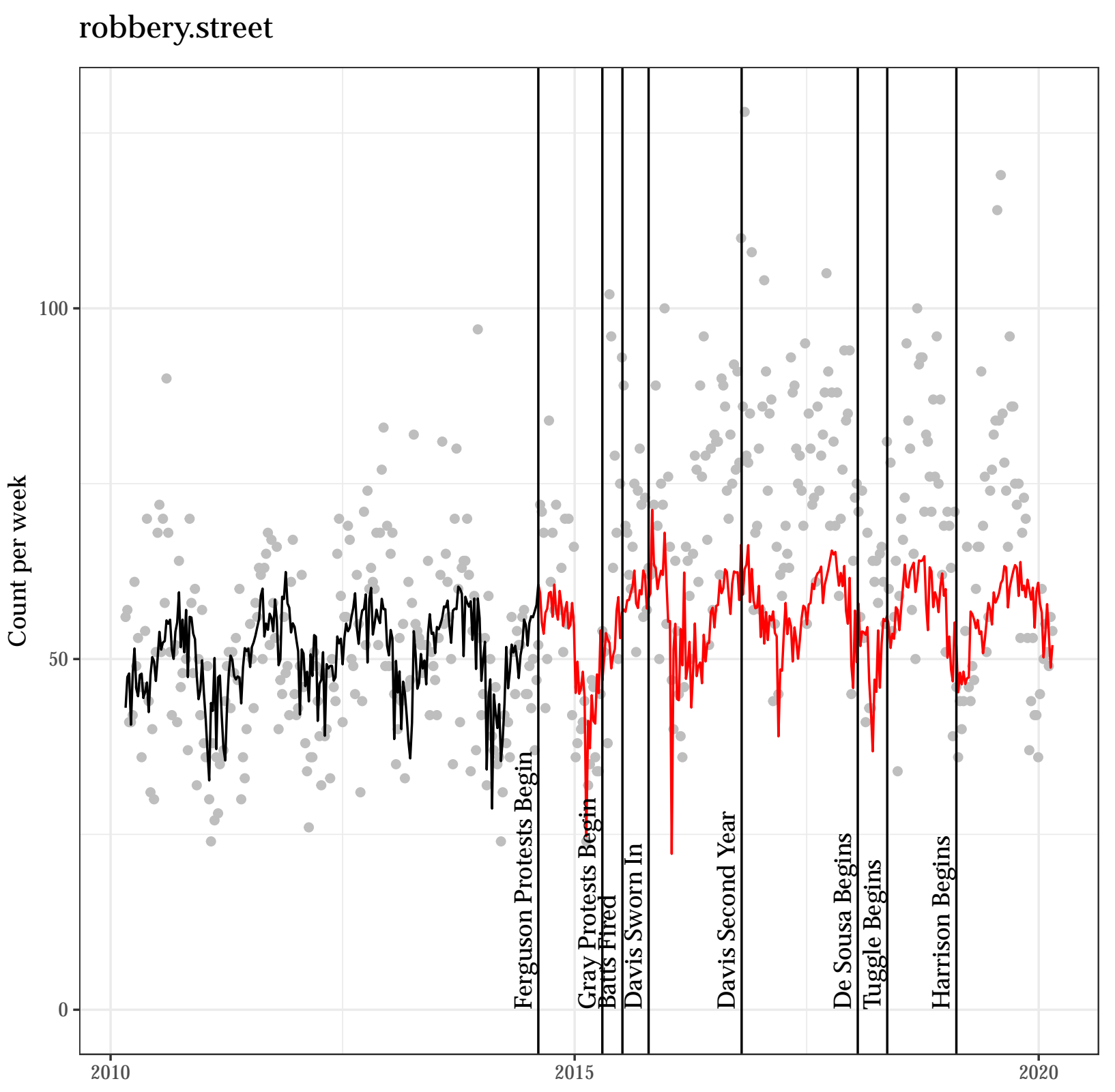




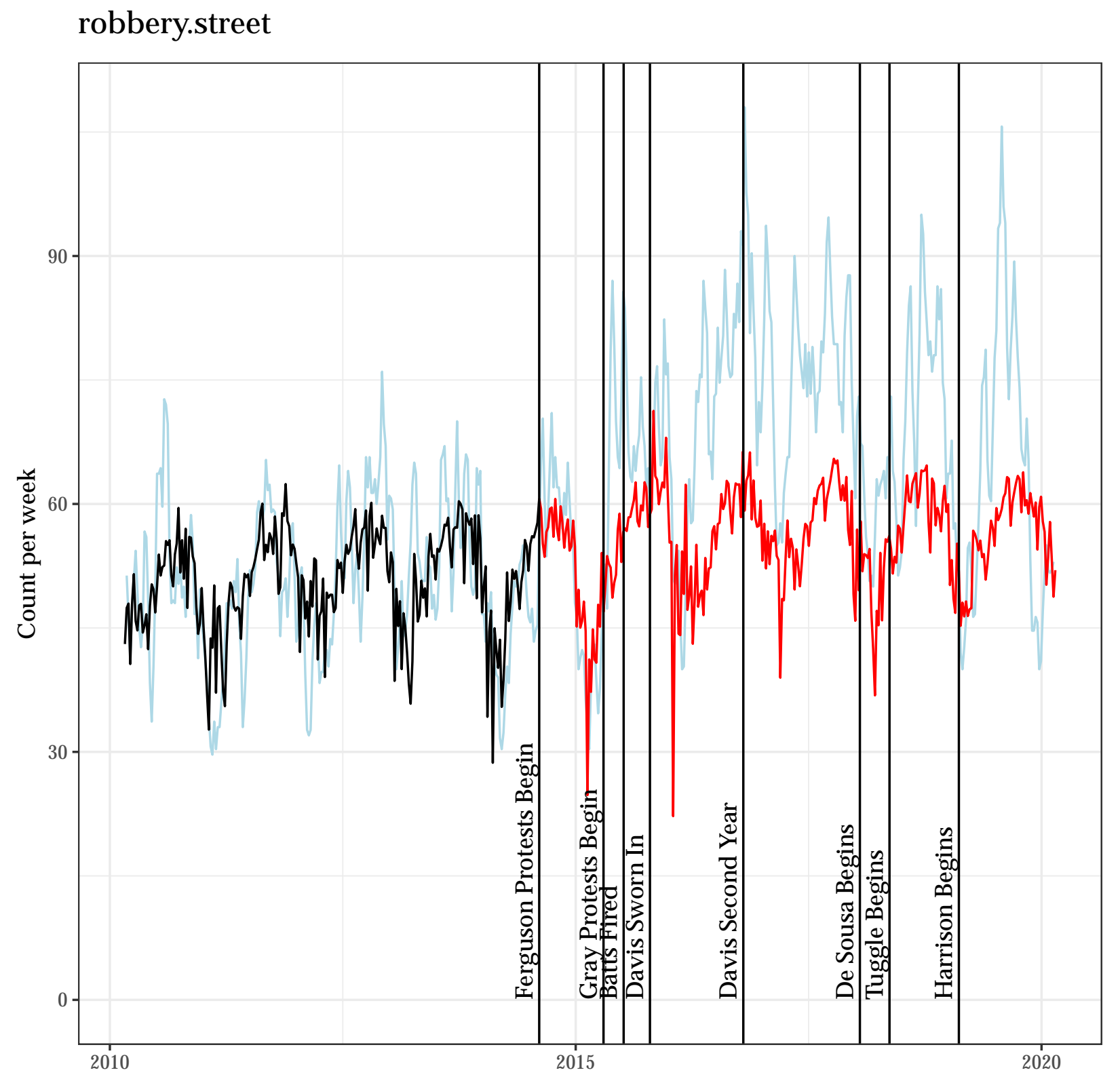


Autocorrelation Function for the Observed Outcome for Updated Model (2)

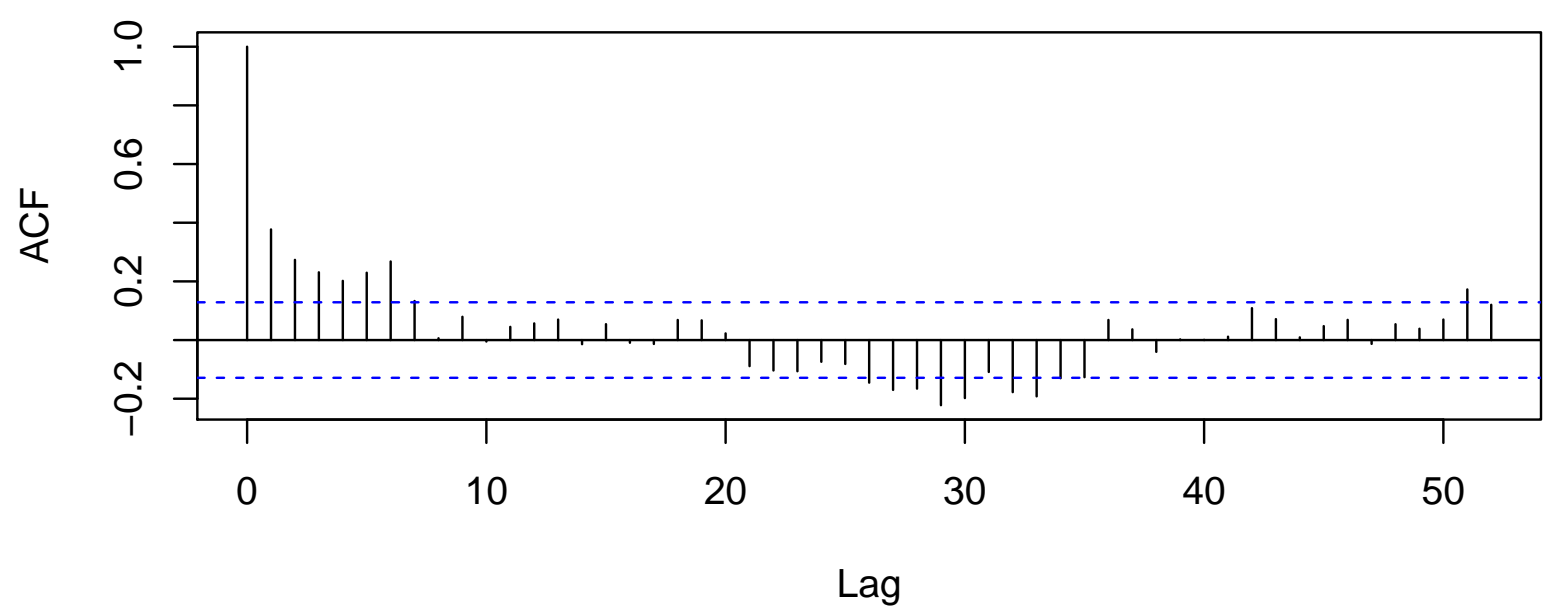

Autocorrelation Function for the Residuals from Updated Model (2)

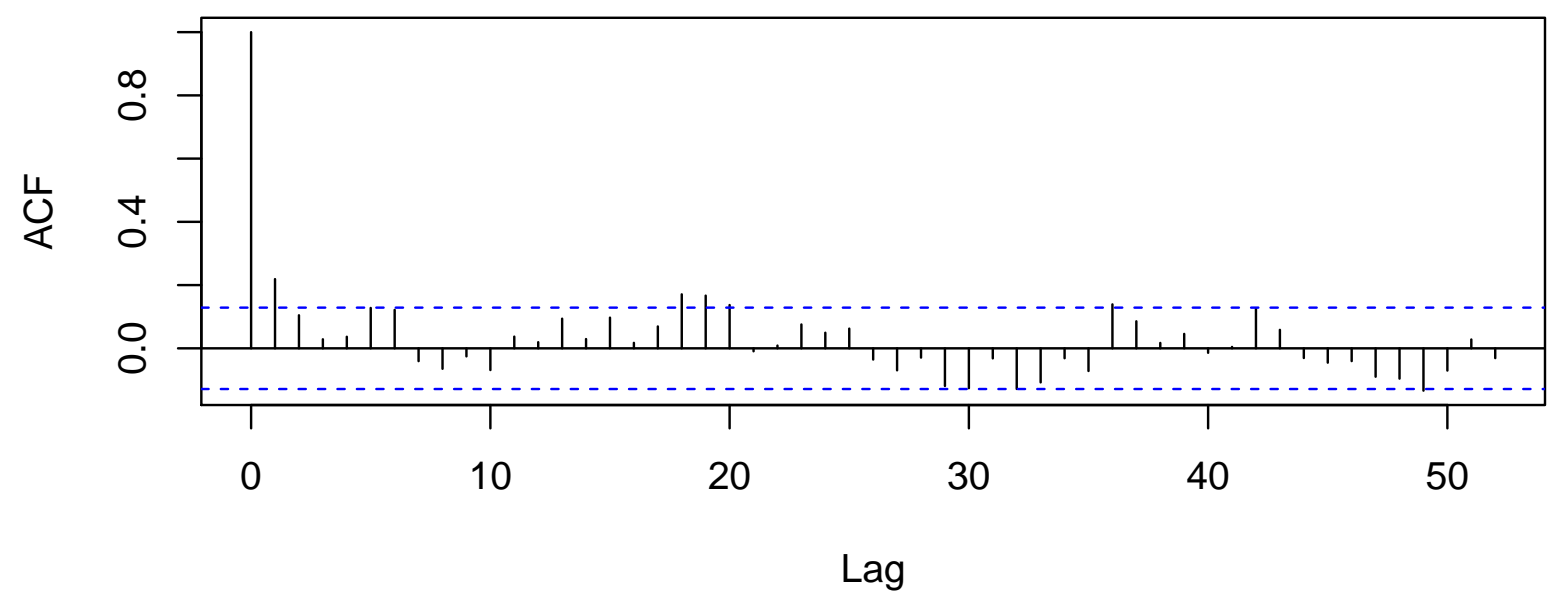




\subsection{A Poisson Regression Model as an Alternative to Model (2) for robbery.street}

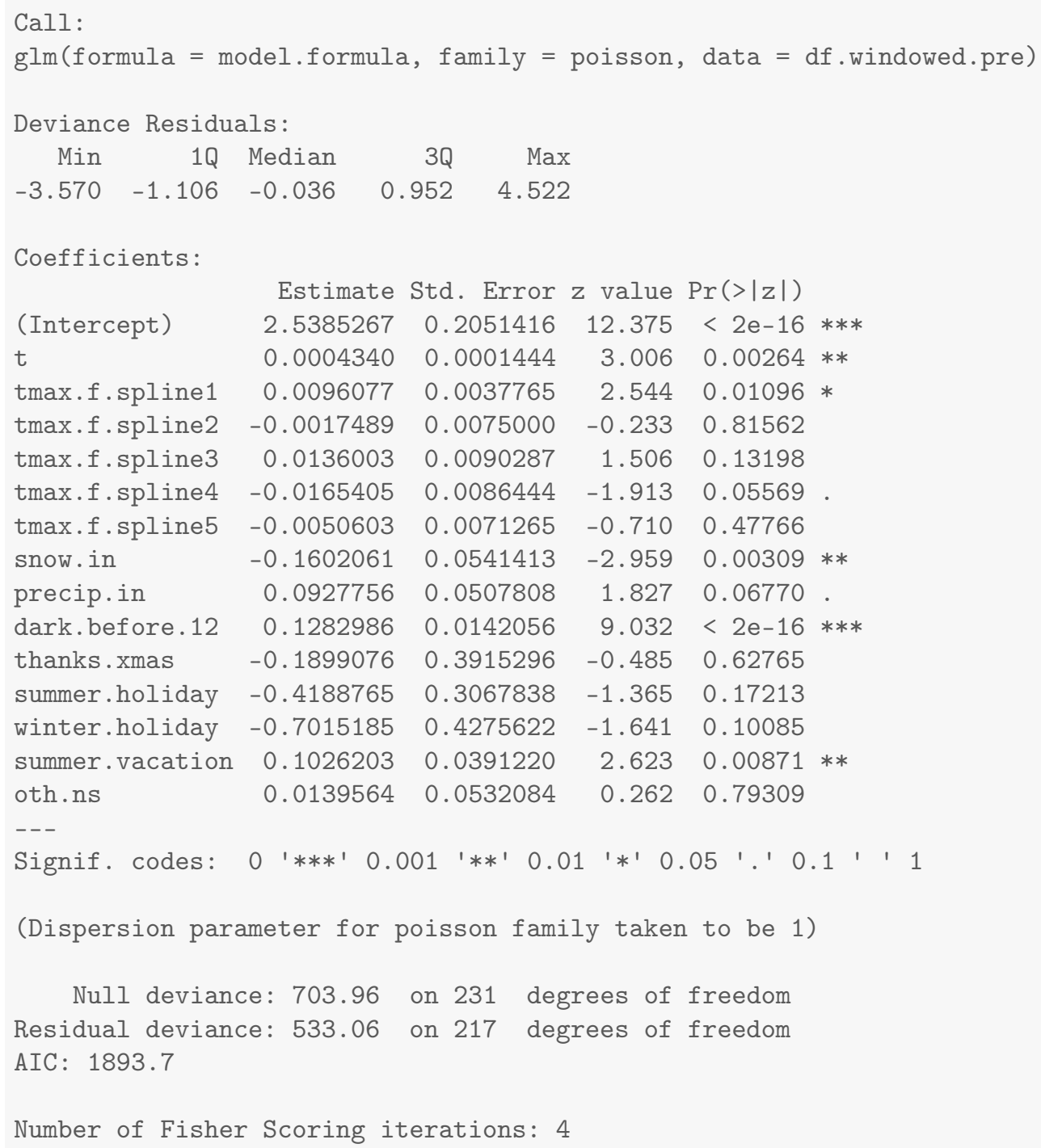




\section{Results for robbery.commercial}

\subsection{Summary Values for robbery.commercial}

Table 9.1: Descriptives for Outcome Before Ferguson Protests Begin

\begin{tabular}{lcccccccc}
\hline \hline Statistic & $\mathrm{N}$ & Mean & St. Dev. & Min & Pctl(25) & Median & Pctl(75) & Max \\
\hline robbery.commercial & 232 & 10.578 & 4.377 & 2 & 8 & 10 & 13 & 25 \\
\hline
\end{tabular}

Table 9.2: Descriptives for Outcome After Ferguson Protests Begin

\begin{tabular}{lcccccccc}
\hline \hline Statistic & $\mathrm{N}$ & Mean & St. Dev. & Min & Pctl(25) & Median & Pctl(75) & Max \\
\hline robbery.commercial & 290 & 16.938 & 6.062 & 4 & 12 & 16 & 21 & 44 \\
\hline
\end{tabular}




\subsection{Four Models for robbery.commercial}

Table 9.3: Four Models that Differ on the Specification of Adjustment and Intervention Variables

\begin{tabular}{|c|c|c|c|c|}
\hline & \multicolumn{4}{|c|}{ Outcome: Count Per Week } \\
\hline & (1) & $(2)$ & $(3)$ & $(4)$ \\
\hline Time (counter in weeks) & 0.003 & 0.005 & & 0.003 \\
\hline After Ferguson Protests Begin (week of $8 / 11 / 14$ onward) & 2.88 & & 2.20 & 2.69 \\
\hline After Gray Protests Begin (week of 4/20/15 onward) & 8.18 & & 9.75 & 9.35 \\
\hline Unrest and National Guard $(4 / 27 / 15-5 / 3 / 2015)$ & -1.17 & & -0.85 & -0.44 \\
\hline After Batts Fired (week of 7/13/15 onward) & -5.72 & & -7.30 & -6.80 \\
\hline After Davis Confirmed (week of 10/19/15 onward) & -0.69 & & -0.24 & -0.37 \\
\hline After Davis First Year (week of 10/17/16 onward) & 4.74 & & 4.36 & 4.30 \\
\hline After De Sousa Begins (week of 1/19/18 onward) & -7.25 & & -5.32 & -5.76 \\
\hline After Tuggle Begins (week of 5/14/18 onward) & 5.21 & & 3.29 & 3.57 \\
\hline After Harrison Begins (week of 2/11/19 onward) & -5.63 & & -4.86 & -5.11 \\
\hline Average Maximum Temperature to 50 Degrees & & -0.10 & & 0.04 \\
\hline Plus Degrees in the $50 \mathrm{~s}$ & & 0.09 & & -0.13 \\
\hline Plus Degrees in the 60s & & 0.42 & & 0.42 \\
\hline Plus Degrees in the $70 \mathrm{~s}$ & & -0.54 & & -0.38 \\
\hline Plus Degrees Greater Than 80 & & 0.10 & & -0.09 \\
\hline Snowfall (inches) & & -0.66 & & -0.42 \\
\hline Precipitation (inches) & & 2.77 & & 2.89 \\
\hline Darkness Before Midnight (hours) & & 1.80 & & 1.59 \\
\hline Thanksgiving/Christmas (proportion of week) & & -9.10 & & -8.01 \\
\hline Winter Holiday (proportion of week) & & -0.75 & & 5.91 \\
\hline Other Out-of-School Days (proportion of week) & & 1.96 & & 1.68 \\
\hline Summer Vacation (proportion of week) & & 2.20 & & 2.23 \\
\hline Summer Holiday (proportion of week) & & 4.73 & & 4.31 \\
\hline Observations & 522 & 232 & 522 & 522 \\
\hline $\mathrm{R}^{2}$ & 0.39 & 0.13 & 0.34 & 0.44 \\
\hline
\end{tabular}


9.3 Least Squares Updated Model (2) for robbery.commercial robbery.commercial

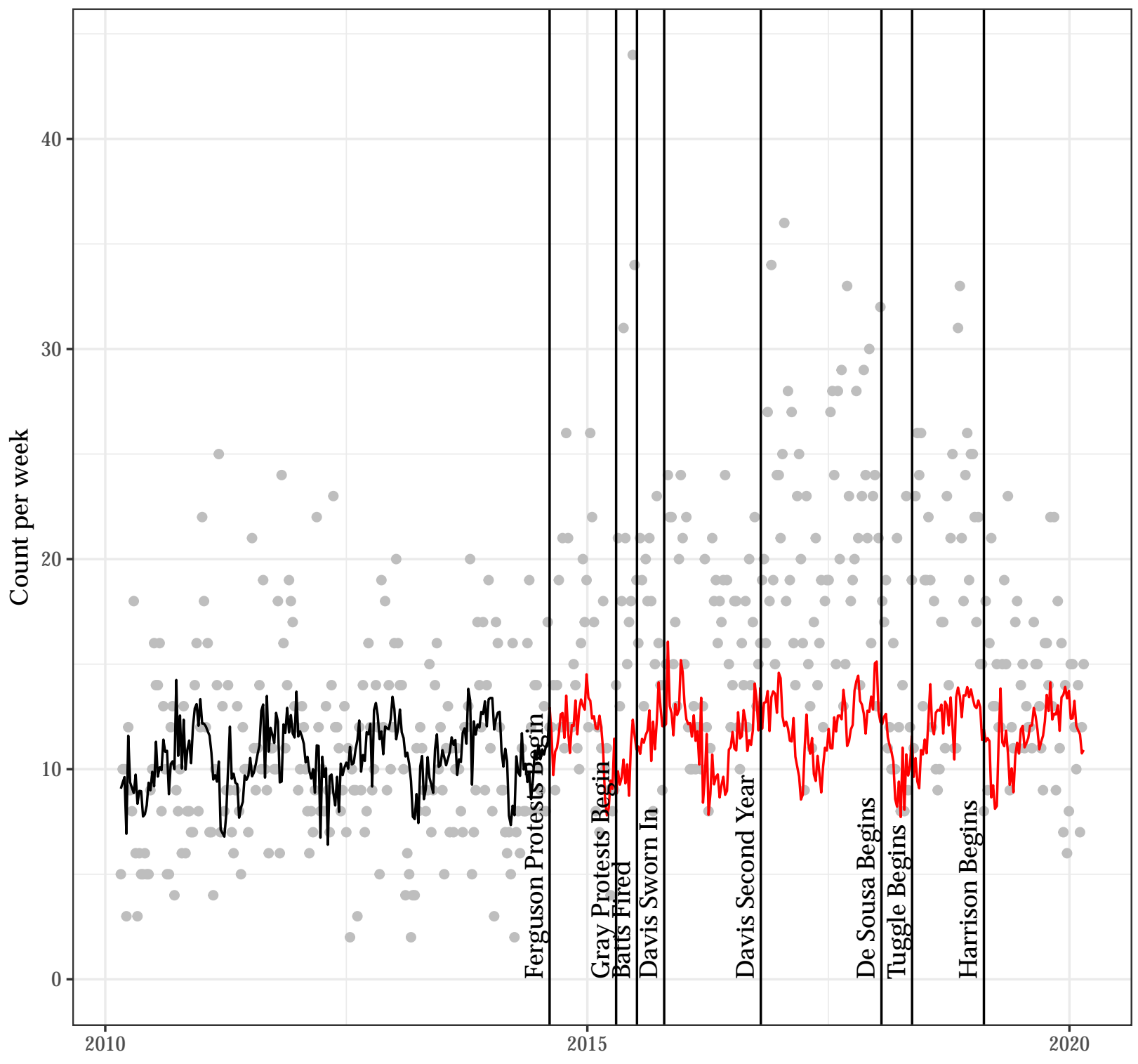




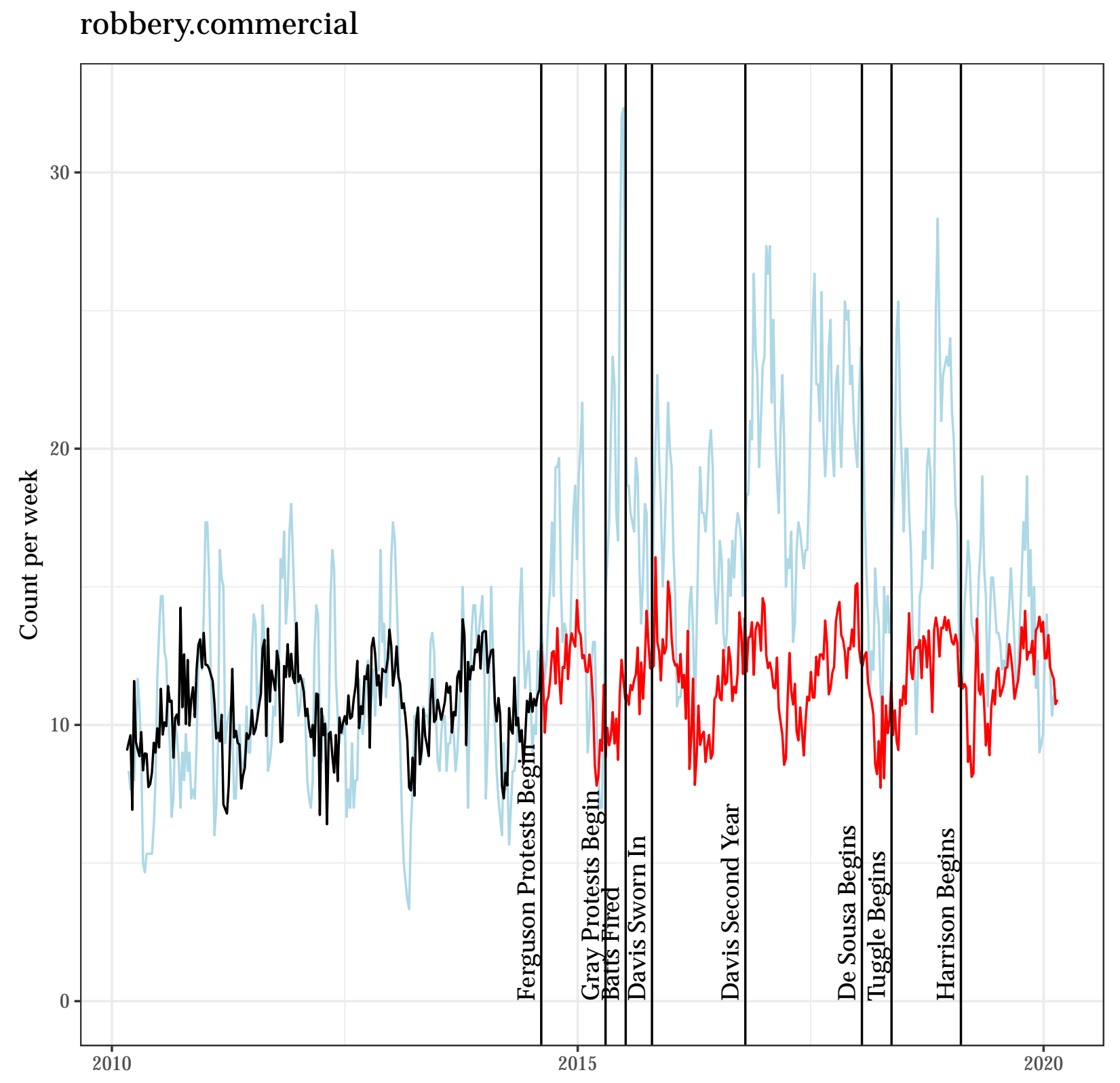


Autocorrelation Function for the Observed Outcome for Updated Model (2)

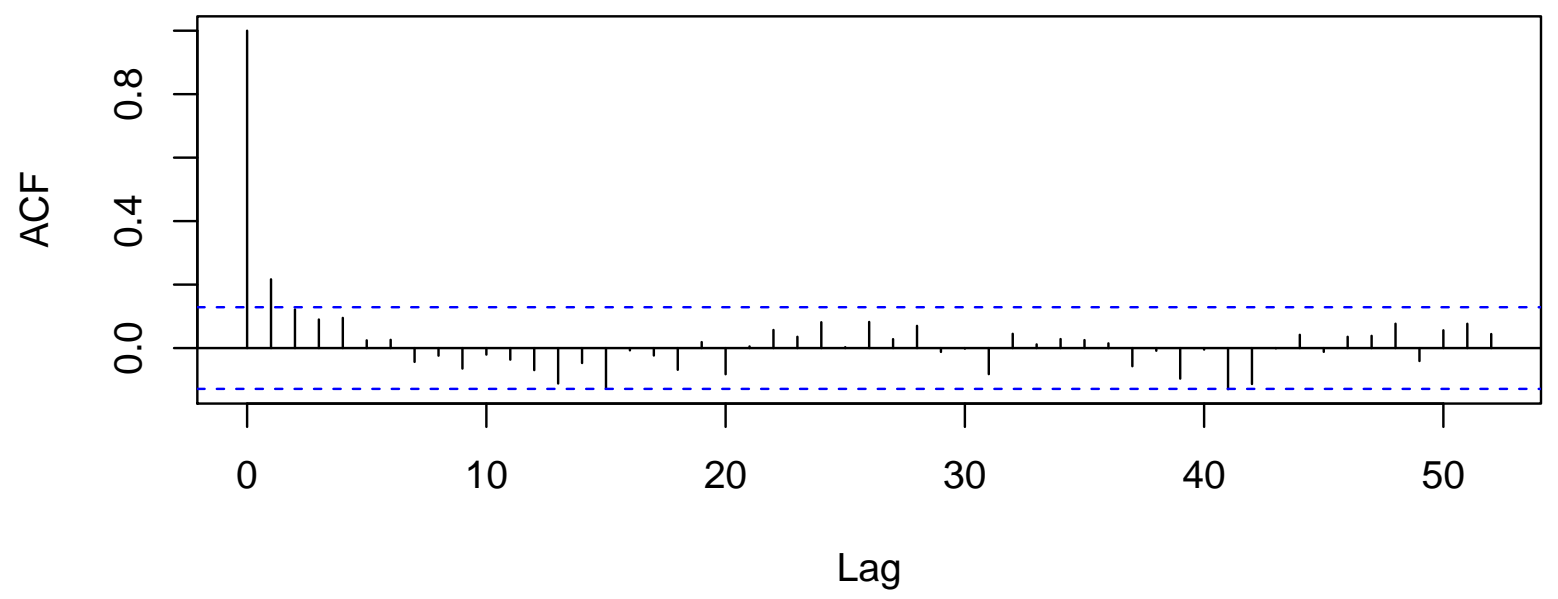

Autocorrelation Function for the Residuals from Updated Model (2)

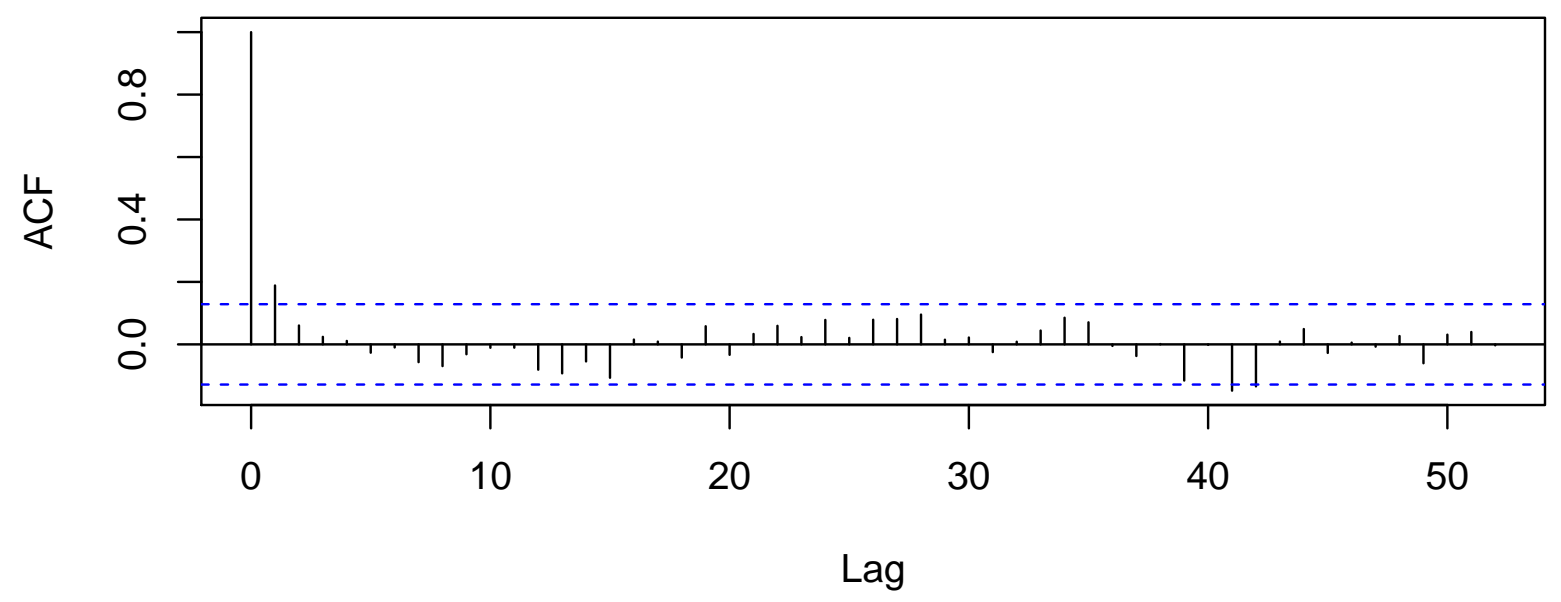




\subsection{A Poisson Regression Model as an Alternative to Model (2) for robbery.commercial}

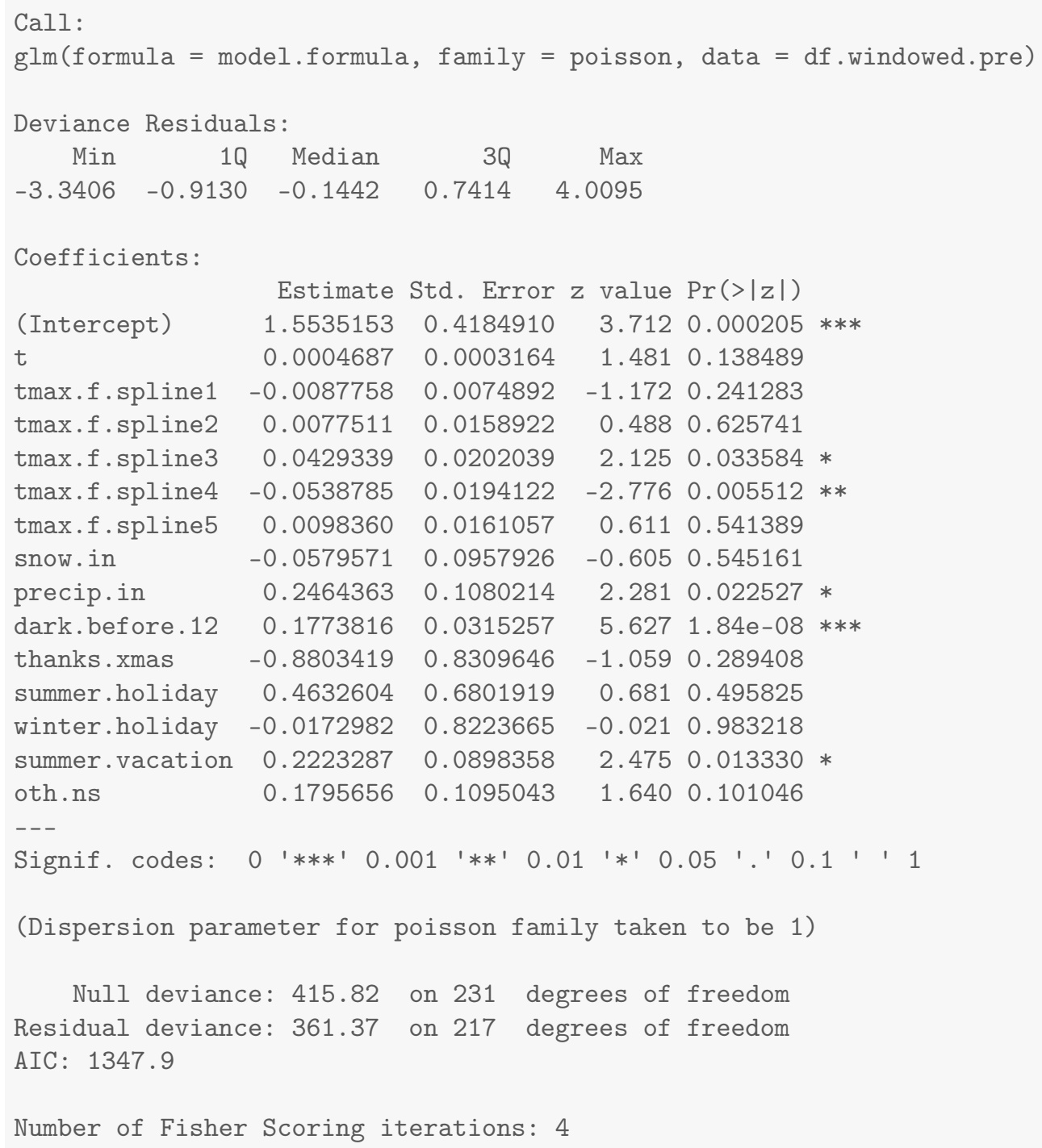




\section{Results for robbery.residence}

\subsection{Summary Values for robbery.residence}

Table 10.1: Descriptives for Outcome Before Ferguson Protests Begin

\begin{tabular}{lcccccccc}
\hline \hline Statistic & $\mathrm{N}$ & Mean & St. Dev. & Min & Pctl(25) & Median & Pctl(75) & Max \\
\hline robbery.residence & 232 & 10.280 & 4.052 & 1 & 8 & 10 & 13 & 27 \\
\hline
\end{tabular}

Table 10.2: Descriptives for Outcome After Ferguson Protests Begin

\begin{tabular}{lcccccccc}
\hline \hline Statistic & $\mathrm{N}$ & Mean & St. Dev. & Min & Pctl(25) & Median & Pctl(75) & Max \\
\hline robbery.residence & 290 & 9.500 & 3.764 & 1 & 7 & 9 & 12 & 27 \\
\hline
\end{tabular}




\subsection{Four Models for robbery.residence}

Table 10.3: Four Models that Differ on the Specification of Adjustment and Intervention Variables

\begin{tabular}{|c|c|c|c|c|}
\hline & \multicolumn{4}{|c|}{ Outcome: Count Per Week } \\
\hline & $(1)$ & $(2)$ & $(3)$ & $(4)$ \\
\hline Time (counter in weeks) & -0.004 & -0.01 & & -0.003 \\
\hline After Ferguson Protests Begin (week of $8 / 11 / 14$ onward) & -0.91 & & -1.00 & -0.91 \\
\hline After Gray Protests Begin (week of 4/20/15 onward) & 1.08 & & 2.13 & 1.42 \\
\hline Unrest and National Guard (4/27/15 - 5/3/2015) & 1.16 & & 0.93 & 1.17 \\
\hline After Batts Fired (week of $7 / 13 / 15$ onward) & -2.13 & & -2.85 & -3.28 \\
\hline After Davis Confirmed (week of 10/19/15 onward) & 1.87 & & 1.92 & 2.39 \\
\hline After Davis First Year (week of 10/17/16 onward) & 1.73 & & 1.64 & 1.60 \\
\hline After De Sousa Begins (week of 1/19/18 onward) & 0.35 & & 0.74 & 1.04 \\
\hline After Tuggle Begins (week of 5/14/18 onward) & -1.77 & & -2.21 & -2.59 \\
\hline After Harrison Begins (week of 2/11/19 onward) & -0.45 & & -0.60 & -0.35 \\
\hline Average Maximum Temperature to 50 Degrees & & -0.12 & & 0.05 \\
\hline Plus Degrees in the $50 \mathrm{~s}$ & & 0.27 & & -0.04 \\
\hline Plus Degrees in the 60s & & -0.11 & & 0.11 \\
\hline Plus Degrees in the $70 \mathrm{~s}$ & & -0.15 & & -0.17 \\
\hline Plus Degrees Greater Than 80 & & 0.17 & & 0.20 \\
\hline Snowfall (inches) & & 1.16 & & 0.56 \\
\hline Precipitation (inches) & & -1.19 & & -0.97 \\
\hline Darkness Before Midnight (hours) & & 0.63 & & 0.61 \\
\hline Thanksgiving/Christmas (proportion of week) & & -6.85 & & -0.16 \\
\hline Winter Holiday (proportion of week) & & 7.90 & & 2.01 \\
\hline Other Out-of-School Days (proportion of week) & & 0.77 & & 1.16 \\
\hline Summer Vacation (proportion of week) & & 1.73 & & 0.90 \\
\hline Summer Holiday (proportion of week) & & 15.11 & & 0.81 \\
\hline Observations & 522 & 232 & 522 & 522 \\
\hline $\mathrm{R}^{2}$ & 0.05 & 0.07 & 0.05 & 0.08 \\
\hline
\end{tabular}


10.3 Least Squares Updated Model (2) for robbery.residence robbery.residence

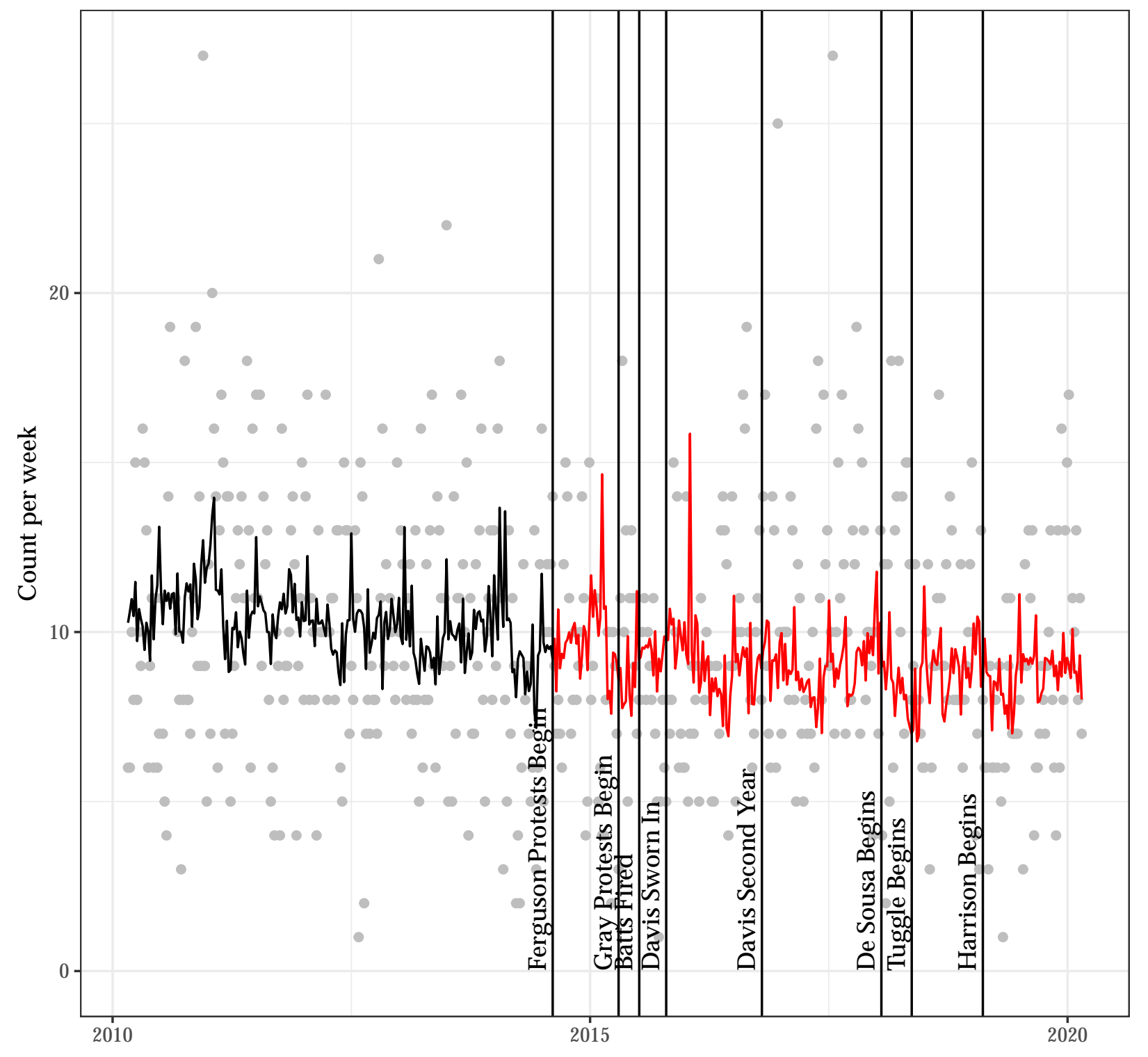




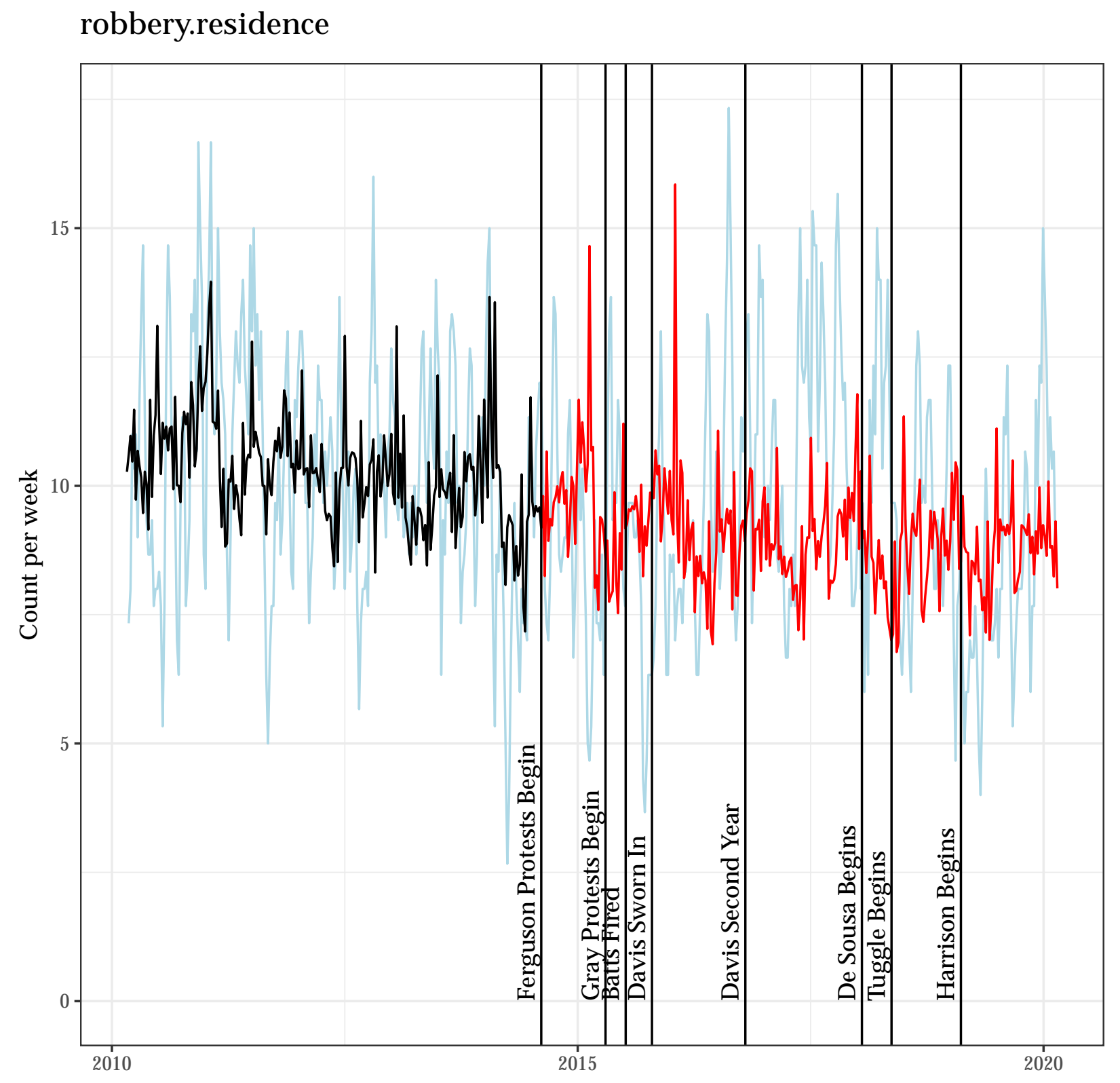


Autocorrelation Function for the Observed Outcome for Updated Model (2)

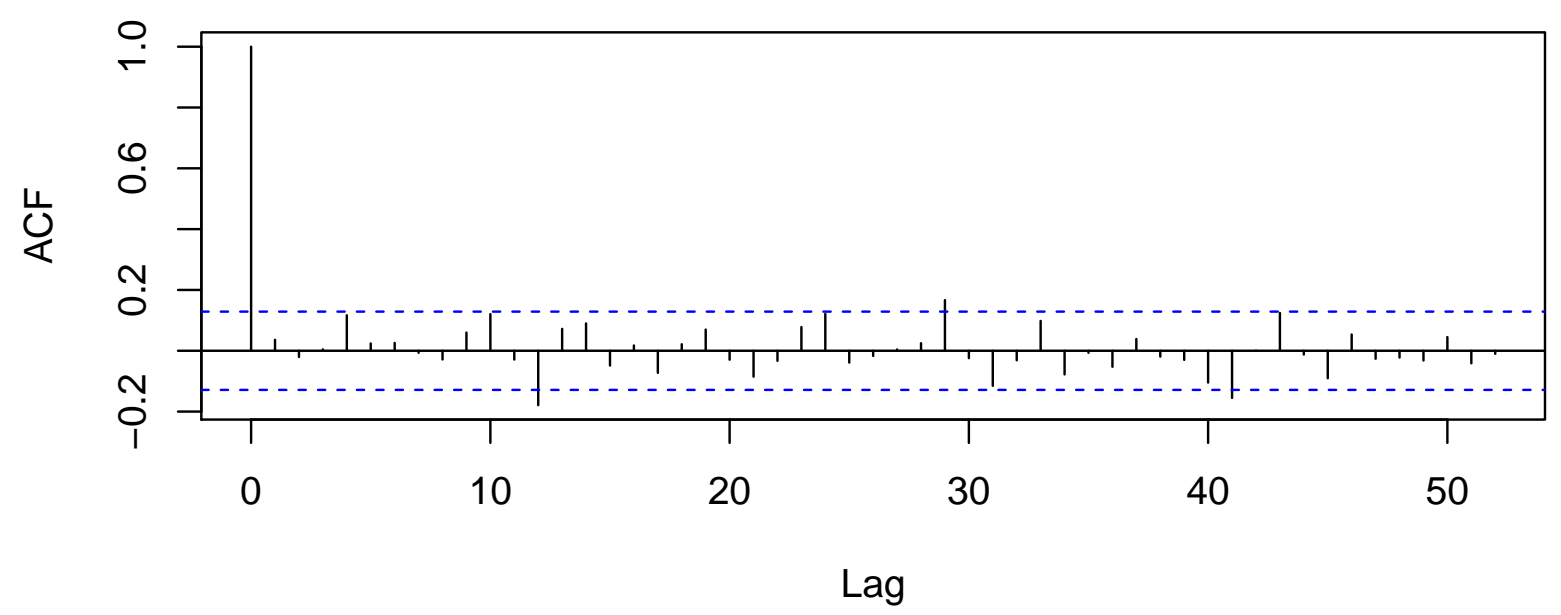

Autocorrelation Function for the Residuals from Updated Model (2)

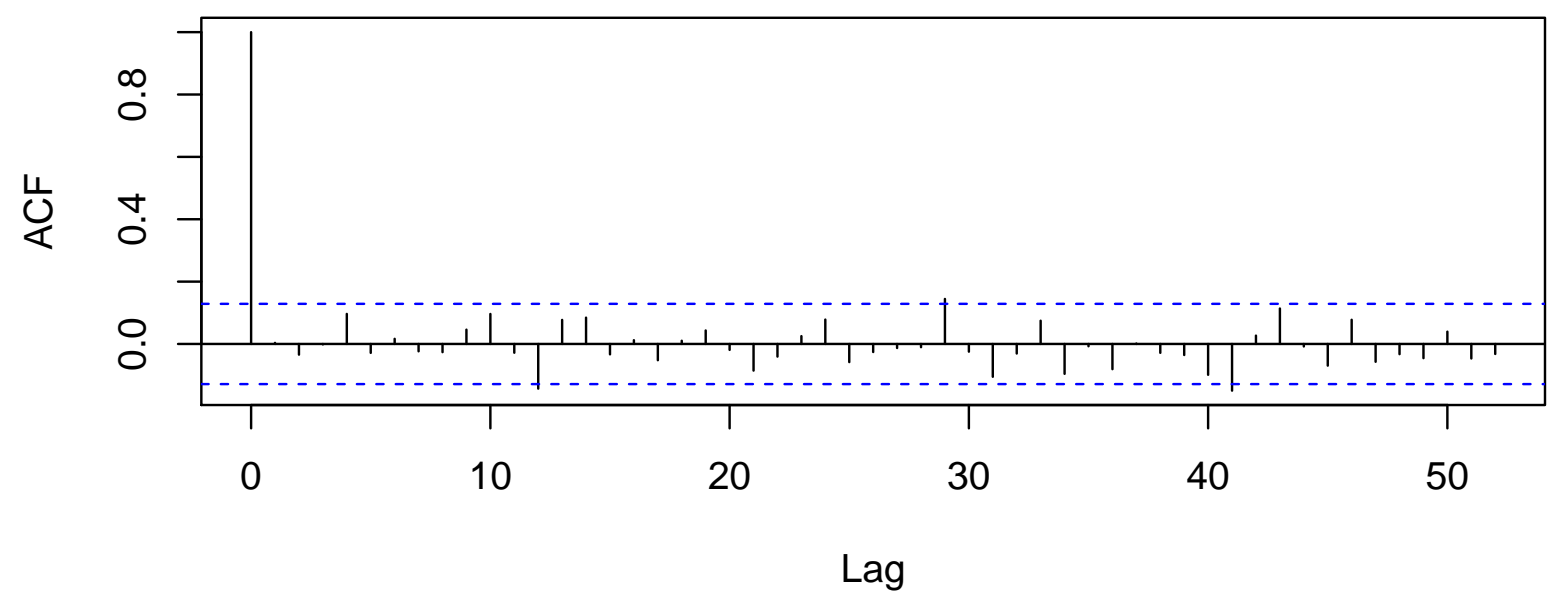




\subsection{A Poisson Regression Model as an Alternative to Model (2) for robbery.residence}

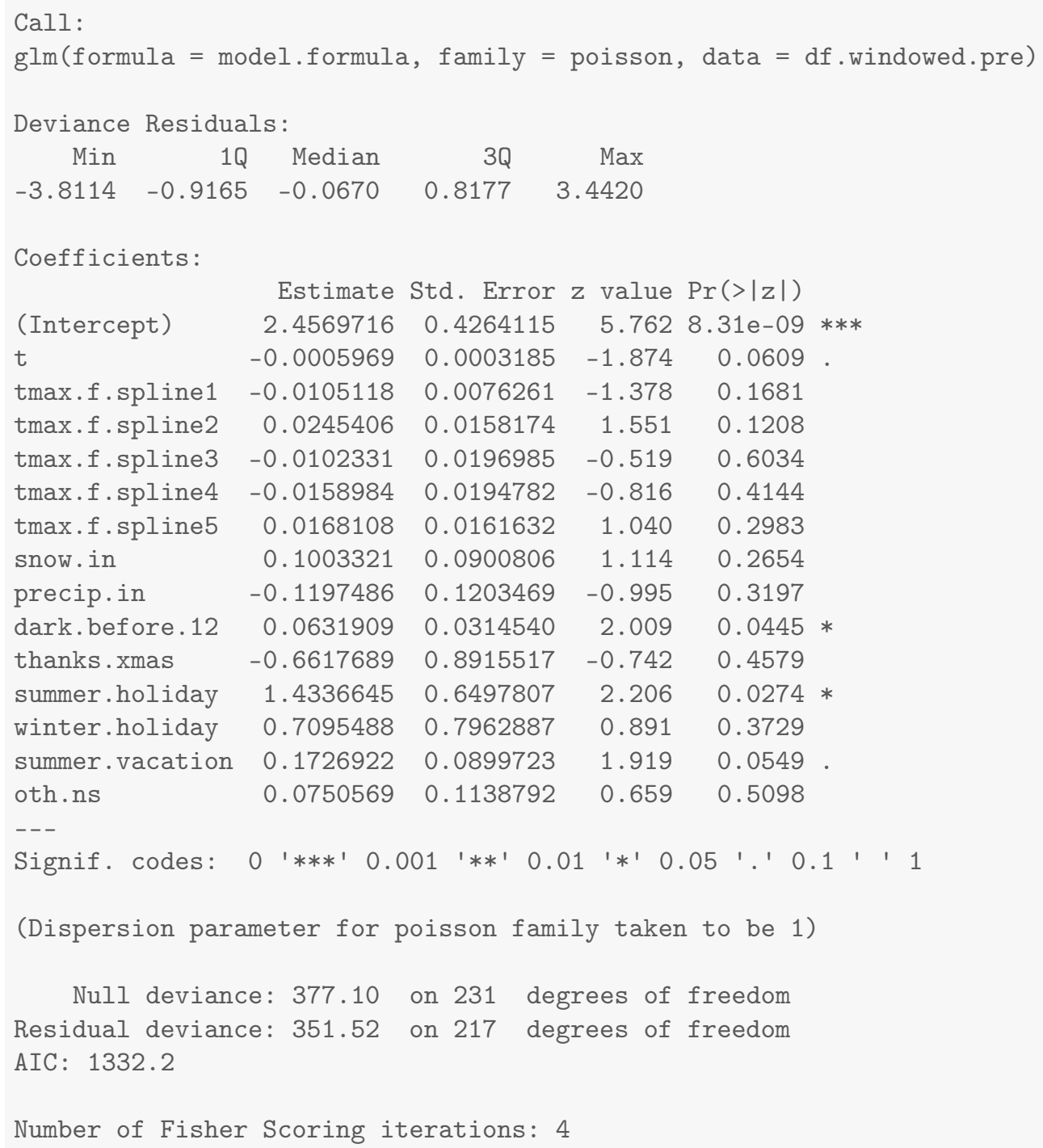




\section{Results for aggravated.assault}

\subsection{Summary Values for aggravated.assault}

Table 11.1: Descriptives for Outcome Before Ferguson Protests Begin

\begin{tabular}{lcccccccc}
\hline \hline Statistic & $\mathrm{N}$ & Mean & St. Dev. & Min & Pctl(25) & Median & Pctl(75) & Max \\
\hline aggravated.assault & 232 & 95.405 & 19.107 & 47 & 81 & 94.5 & 108 & 150 \\
\hline
\end{tabular}

Table 11.2: Descriptives for Outcome After Ferguson Protests Begin

\begin{tabular}{lcccccccc}
\hline \hline Statistic & $\mathrm{N}$ & Mean & St. Dev. & Min & Pctl(25) & Median & Pctl(75) & Max \\
\hline aggravated.assault & 290 & 101.314 & 22.076 & 41 & 84.2 & 102 & 117.8 & 178 \\
\hline
\end{tabular}




\subsection{Four Models for aggravated.assault}

Table 11.3: Four Models that Differ on the Specification of Adjustment and Intervention Variables

\begin{tabular}{|c|c|c|c|c|}
\hline & \multicolumn{4}{|c|}{ Outcome: Count Per Week } \\
\hline & $(1)$ & $(2)$ & $(3)$ & $(4)$ \\
\hline Time (counter in weeks) & -0.14 & -0.13 & & -0.12 \\
\hline After Ferguson Protests Begin (week of $8 / 11 / 14$ onward) & -1.43 & & 4.47 & 4.60 \\
\hline After Gray Protests Begin (week of $4 / 20 / 15$ onward) & 42.60 & & 25.13 & 23.22 \\
\hline Unrest and National Guard $(4 / 27 / 15-5 / 3 / 2015)$ & 46.68 & & 48.61 & 51.23 \\
\hline After Batts Fired (week of 7/13/15 onward) & -13.25 & & -9.54 & -10.77 \\
\hline After Davis Confirmed (week of 10/19/15 onward) & -0.17 & & 6.04 & 8.04 \\
\hline After Davis First Year (week of 10/17/16 onward) & 21.87 & & 19.43 & 22.62 \\
\hline After De Sousa Begins (week of $1 / 19 / 18$ onward) & -3.62 & & -9.93 & -2.68 \\
\hline After Tuggle Begins (week of 5/14/18 onward) & 14.65 & & 11.09 & 11.59 \\
\hline After Harrison Begins (week of 2/11/19 onward) & 2.90 & & -4.67 & 1.80 \\
\hline Average Maximum Temperature to 50 Degrees & & 0.34 & & 0.54 \\
\hline Plus Degrees in the $50 \mathrm{~s}$ & & 0.23 & & -0.31 \\
\hline Plus Degrees in the 60s & & -0.53 & & 0.20 \\
\hline Plus Degrees in the $70 \mathrm{~s}$ & & 0.44 & & 0.42 \\
\hline Plus Degrees Greater Than 80 & & -0.28 & & -0.75 \\
\hline Snowfall (inches) & & -3.35 & & -6.17 \\
\hline Precipitation (inches) & & -11.95 & & -12.13 \\
\hline Darkness Before Midnight (hours) & & -5.12 & & -4.11 \\
\hline Thanksgiving/Christmas (proportion of week) & & -51.45 & & -25.66 \\
\hline Winter Holiday (proportion of week) & & -4.89 & & -1.08 \\
\hline Other Out-of-School Days (proportion of week) & & 3.31 & & -0.18 \\
\hline Summer Vacation (proportion of week) & & -6.64 & & -5.12 \\
\hline Summer Holiday (proportion of week) & & 17.83 & & -3.90 \\
\hline Observations & 522 & 232 & 522 & 522 \\
\hline$\underline{\mathrm{R}^{2}}$ & 0.29 & 0.60 & 0.69 & 0.61 \\
\hline
\end{tabular}


11.3 Least Squares Updated Model (2) for aggravated.assault aggravated.assault

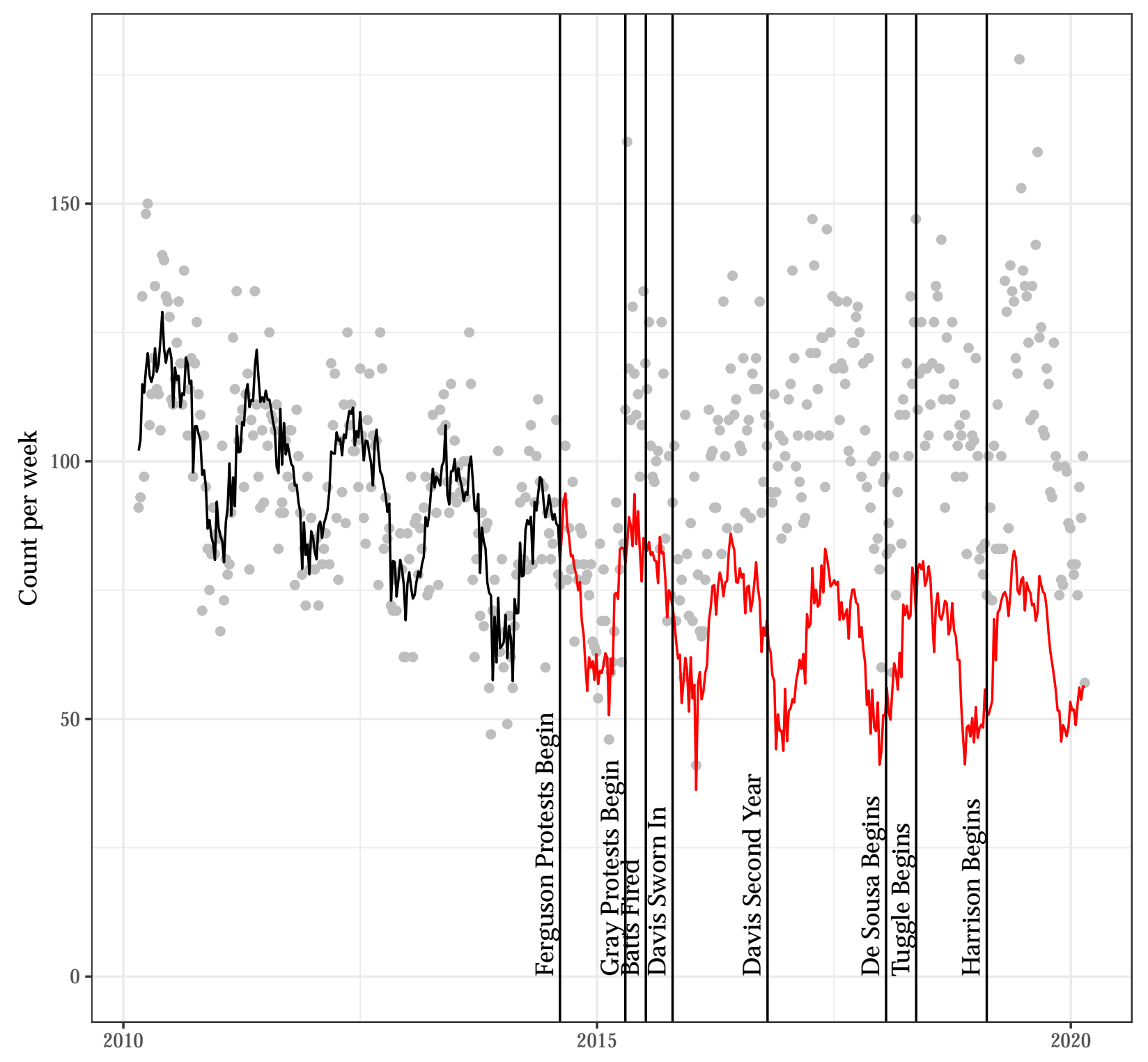




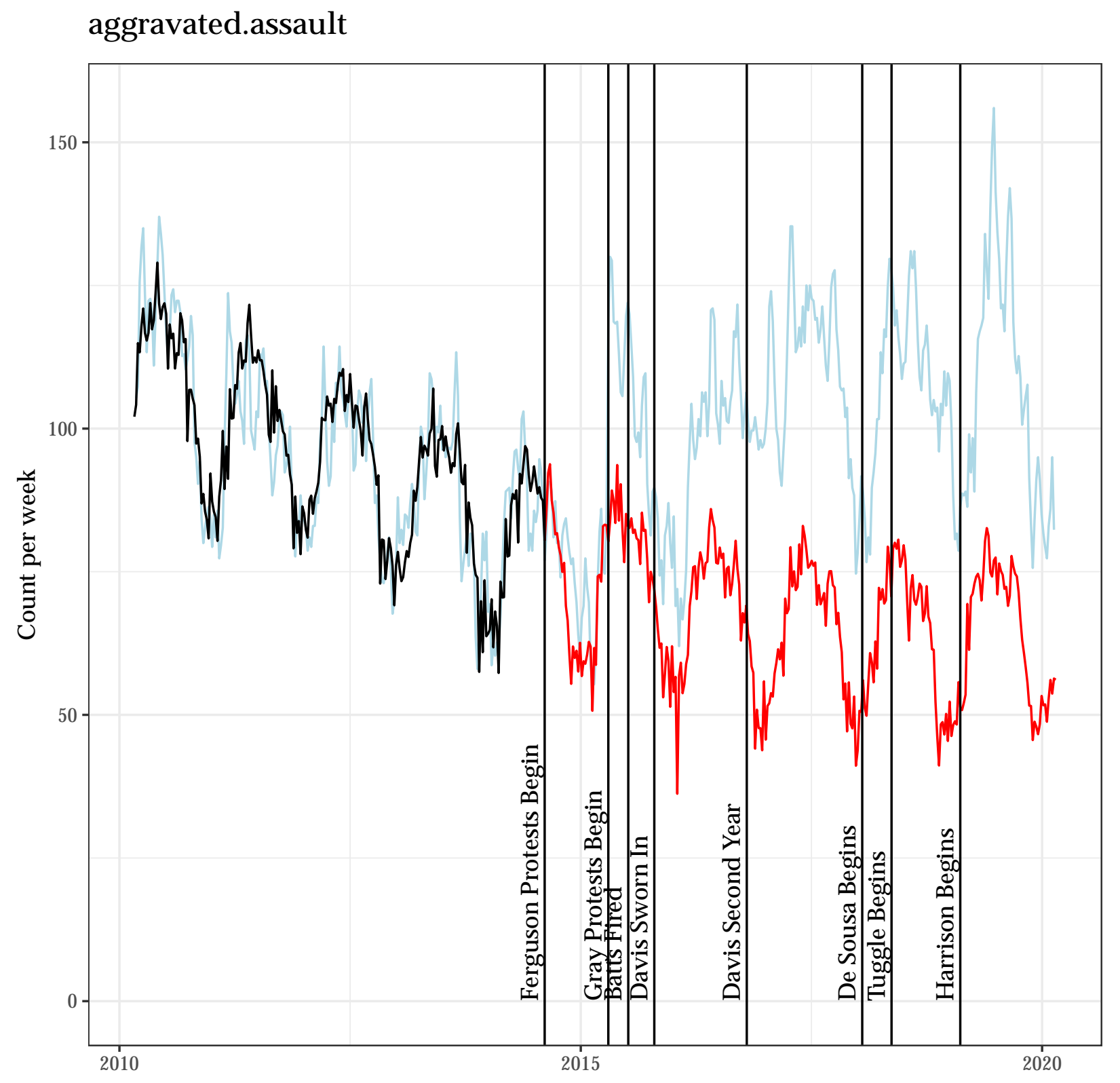


Autocorrelation Function for the Observed Outcome for Updated Model (2)

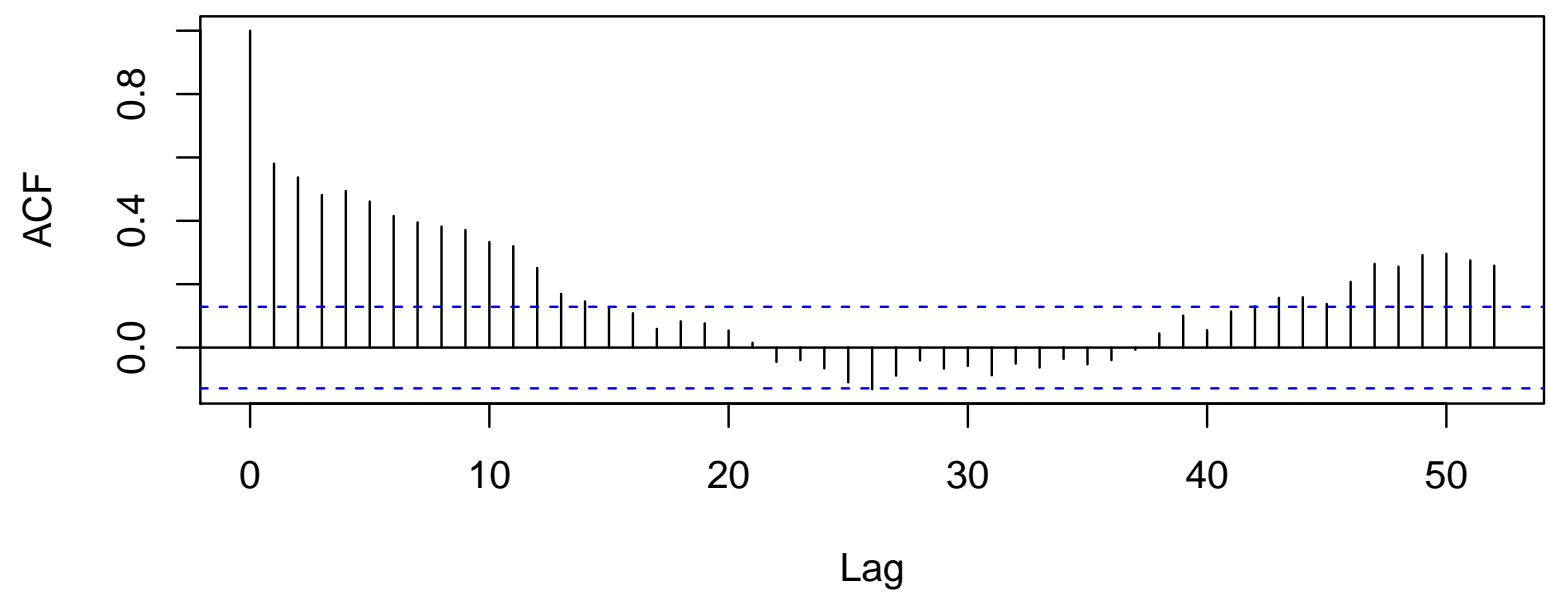

Autocorrelation Function for the Residuals from Updated Model (2)

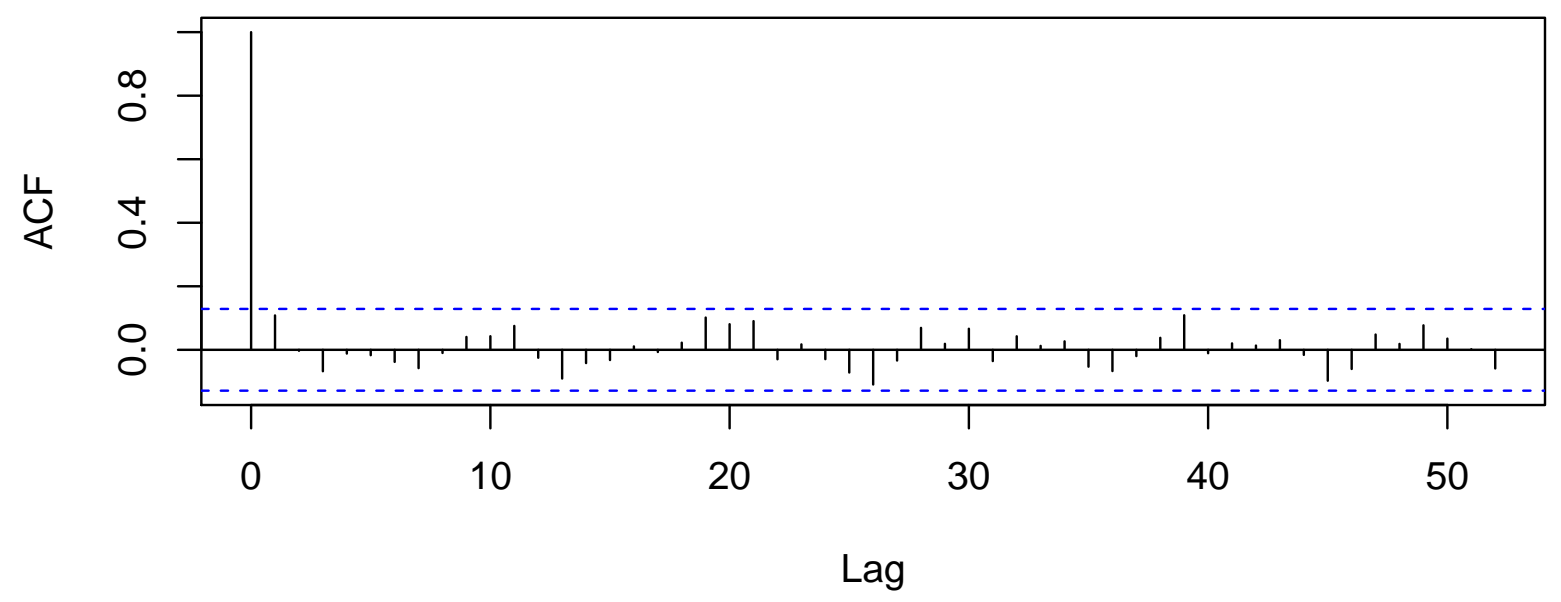




\subsection{A Poisson Regression Model as an Alternative to Model (2) for aggravated.assault}

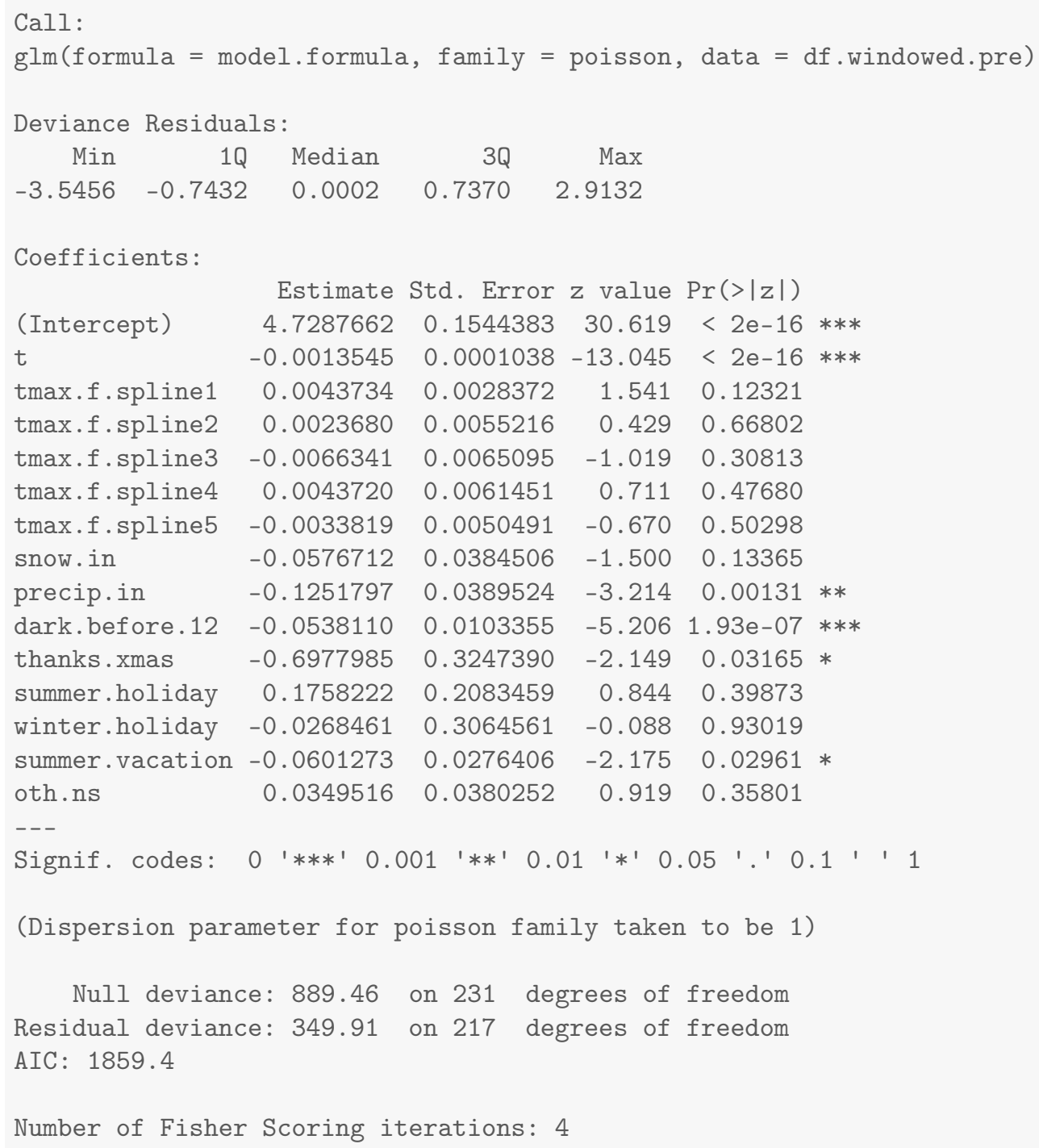




\section{Results for common.assault}

\subsection{Summary Values for common.assault}

Table 12.1: Descriptives for Outcome Before Ferguson Protests Begin

\begin{tabular}{lcccccccc}
\hline \hline Statistic & $\mathrm{N}$ & Mean & St. Dev. & Min & Pctl(25) & Median & Pctl(75) & Max \\
\hline common.assault & 232 & 170.216 & 25.481 & 100 & 153 & 170.5 & 184.2 & 241 \\
\hline
\end{tabular}

Table 12.2: Descriptives for Outcome After Ferguson Protests Begin

\begin{tabular}{lcccccccc}
\hline \hline Statistic & N & Mean & St. Dev. & Min & Pctl(25) & Median & Pctl(75) & Max \\
\hline common.assault & 290 & 151.328 & 24.091 & 78 & 135.2 & 151 & 167 & 215 \\
\hline
\end{tabular}




\subsection{Four Models for common.assault}

Table 12.3: Four Models that Differ on the Specification of Adjustment and Intervention Variables

\begin{tabular}{|c|c|c|c|c|}
\hline & \multicolumn{4}{|c|}{ Outcome: Count Per Week } \\
\hline & $(1)$ & (2) & (3) & $(4)$ \\
\hline Time (counter in weeks) & -0.13 & -0.10 & & -0.10 \\
\hline After Ferguson Protests Begin (week of 8/11/14 onward) & -23.02 & & -19.58 & -19.76 \\
\hline After Gray Protests Begin (week of 4/20/15 onward) & 21.27 & & 2.39 & 2.55 \\
\hline Unrest and National Guard $(4 / 27 / 15-5 / 3 / 2015)$ & -38.75 & & -46.82 & -44.96 \\
\hline After Batts Fired (week of 7/13/15 onward) & -8.03 & & -4.74 & -5.49 \\
\hline After Davis Confirmed (week of 10/19/15 onward) & 6.15 & & 10.17 & 11.34 \\
\hline After Davis First Year (week of 10/17/16 onward) & 33.62 & & 30.55 & 34.19 \\
\hline After De Sousa Begins (week of 1/19/18 onward) & -3.80 & & -10.50 & -5.66 \\
\hline After Tuggle Begins (week of 5/14/18 onward) & 10.46 & & 8.73 & 9.98 \\
\hline After Harrison Begins (week of 2/11/19 onward) & 1.96 & & -6.47 & -0.88 \\
\hline Average Maximum Temperature to 50 Degrees & & 1.04 & & 0.78 \\
\hline Plus Degrees in the $50 \mathrm{~s}$ & & -0.19 & & -0.10 \\
\hline Plus Degrees in the 60s & & 0.07 & & 0.31 \\
\hline Plus Degrees in the 70s & & -0.64 & & -0.73 \\
\hline Plus Degrees Greater Than 80 & & 0.56 & & 0.78 \\
\hline Snowfall (inches) & & -18.55 & & -12.60 \\
\hline Precipitation (inches) & & -9.83 & & -10.88 \\
\hline Darkness Before Midnight (hours) & & -3.46 & & -3.65 \\
\hline Thanksgiving/Christmas (proportion of week) & & -16.01 & & 4.39 \\
\hline Winter Holiday (proportion of week) & & -37.18 & & -66.01 \\
\hline Other Out-of-School Days (proportion of week) & & -12.54 & & -14.90 \\
\hline Summer Vacation (proportion of week) & & -32.32 & & -28.74 \\
\hline Summer Holiday (proportion of week) & & -45.94 & & -33.05 \\
\hline Observations & 522 & 232 & 522 & 522 \\
\hline $\mathrm{R}^{2}$ & 0.32 & 0.54 & 0.36 & 0.64 \\
\hline
\end{tabular}


12.3 Least Squares Updated Model (2) for common.assault common.assault

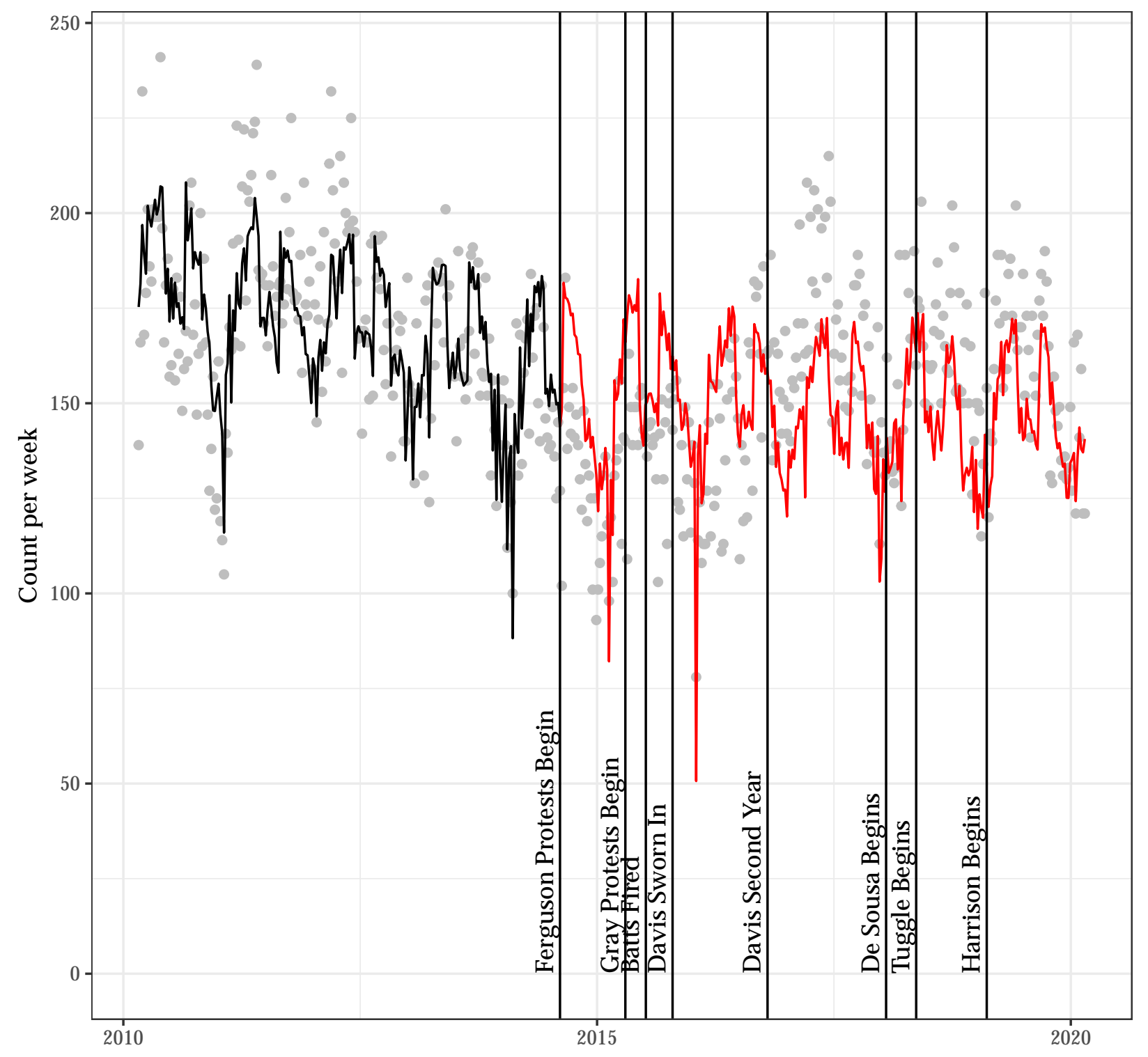




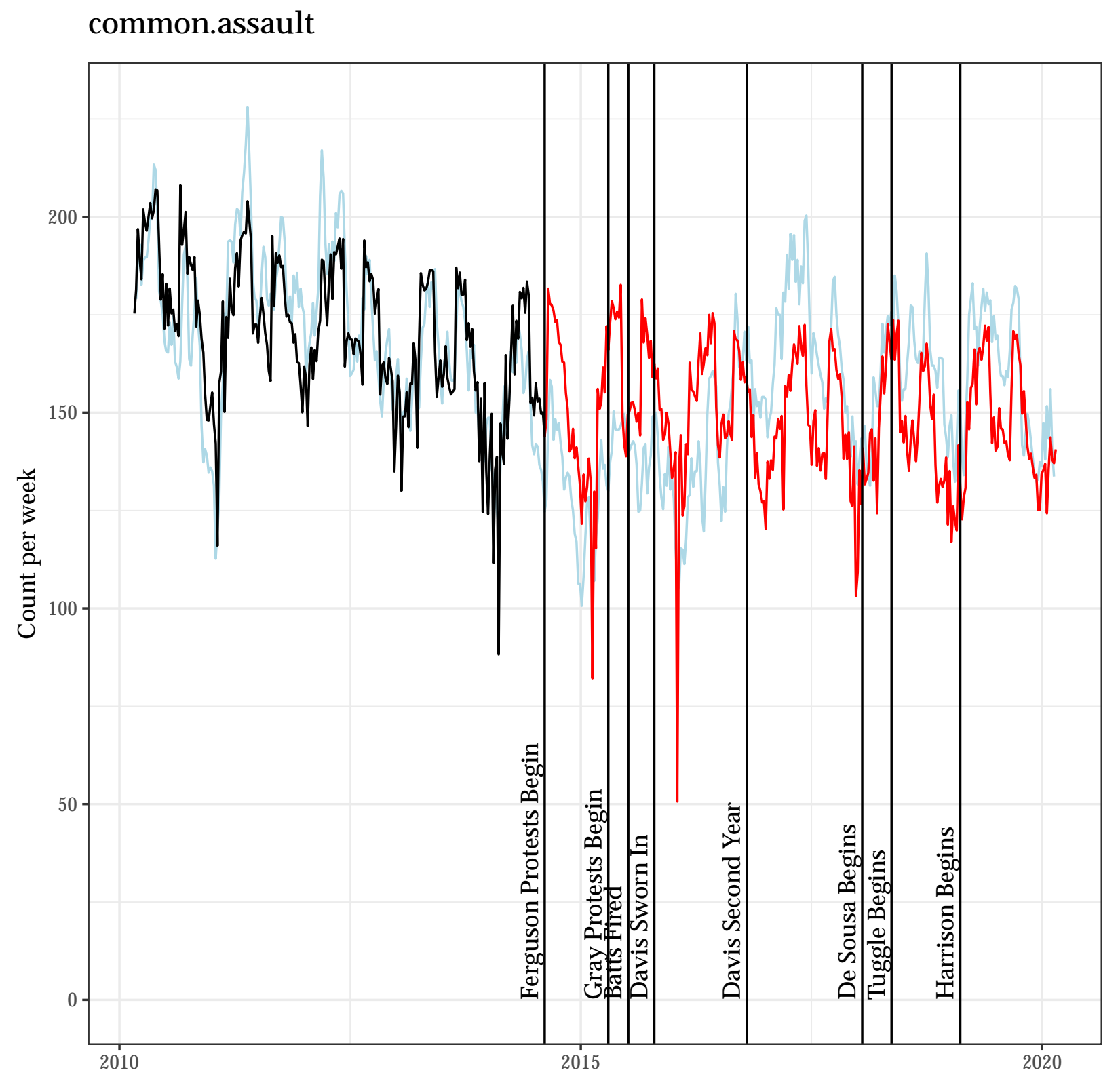


Autocorrelation Function for the Observed Outcome for Updated Model (2)

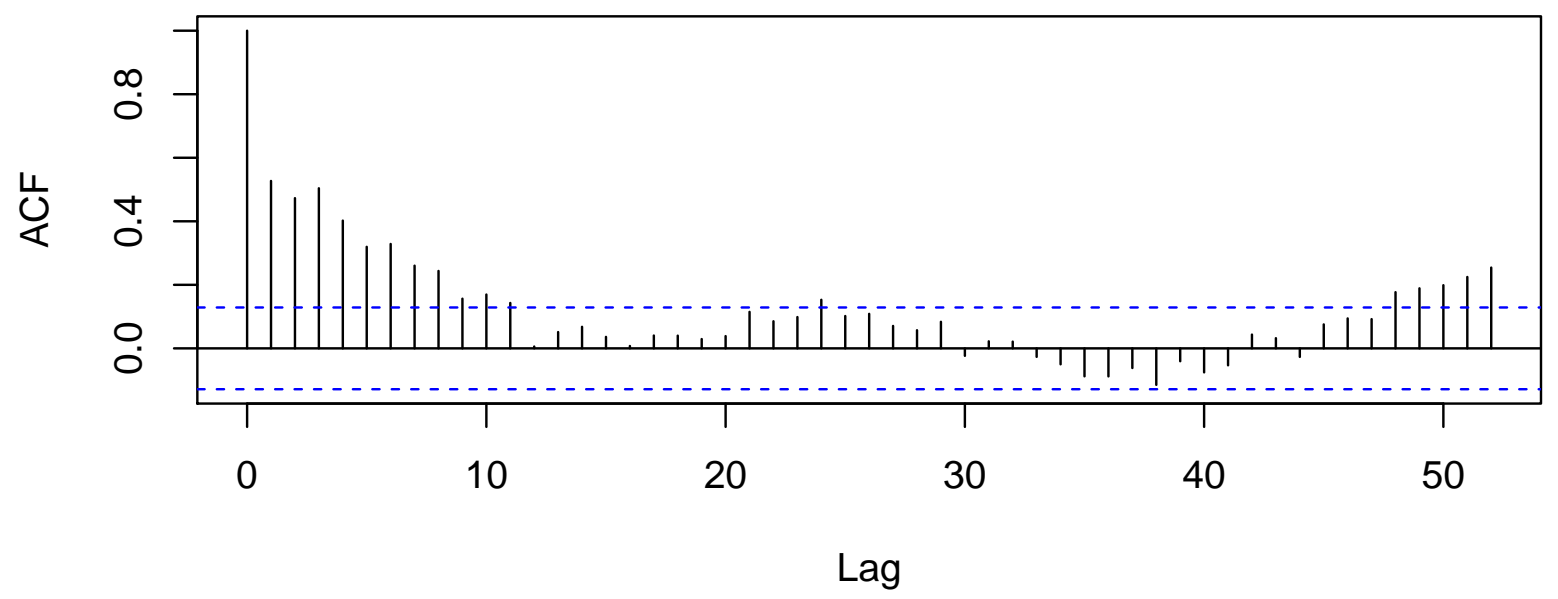

Autocorrelation Function for the Residuals from Updated Model (2)

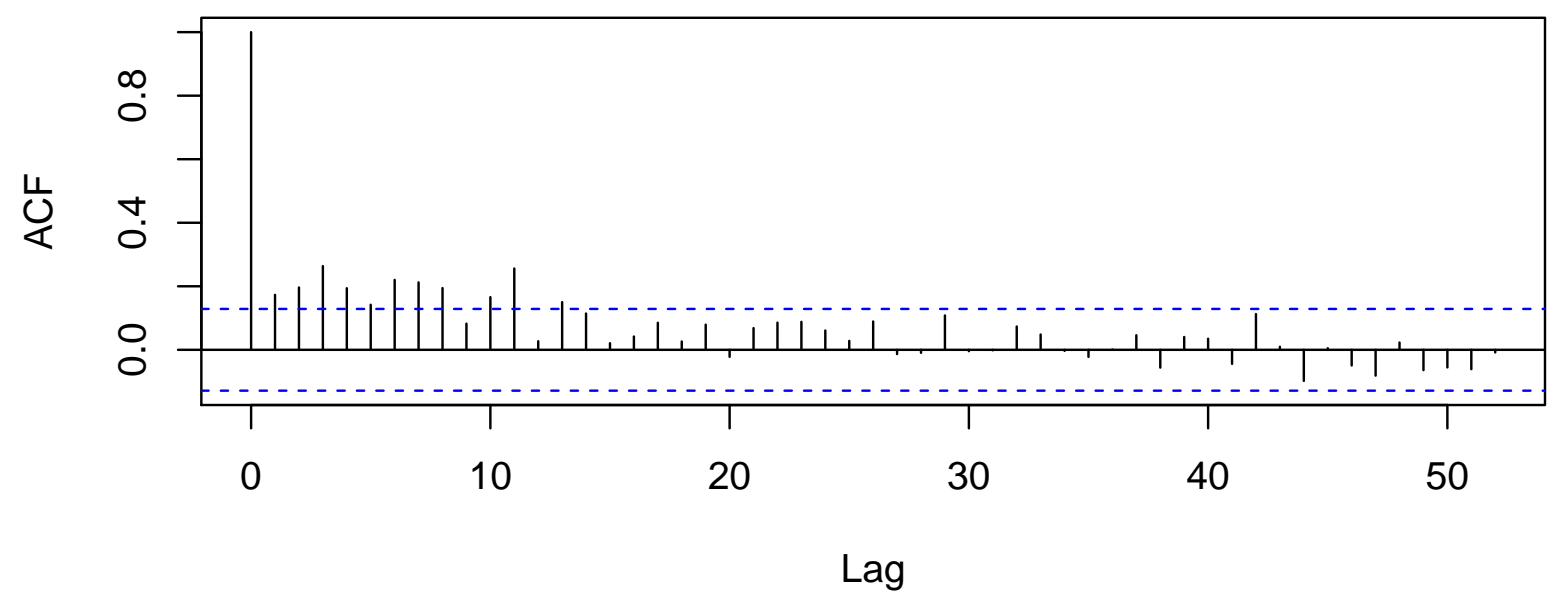




\subsection{A Poisson Regression Model as an Alternative to Model (2) for common.assault}

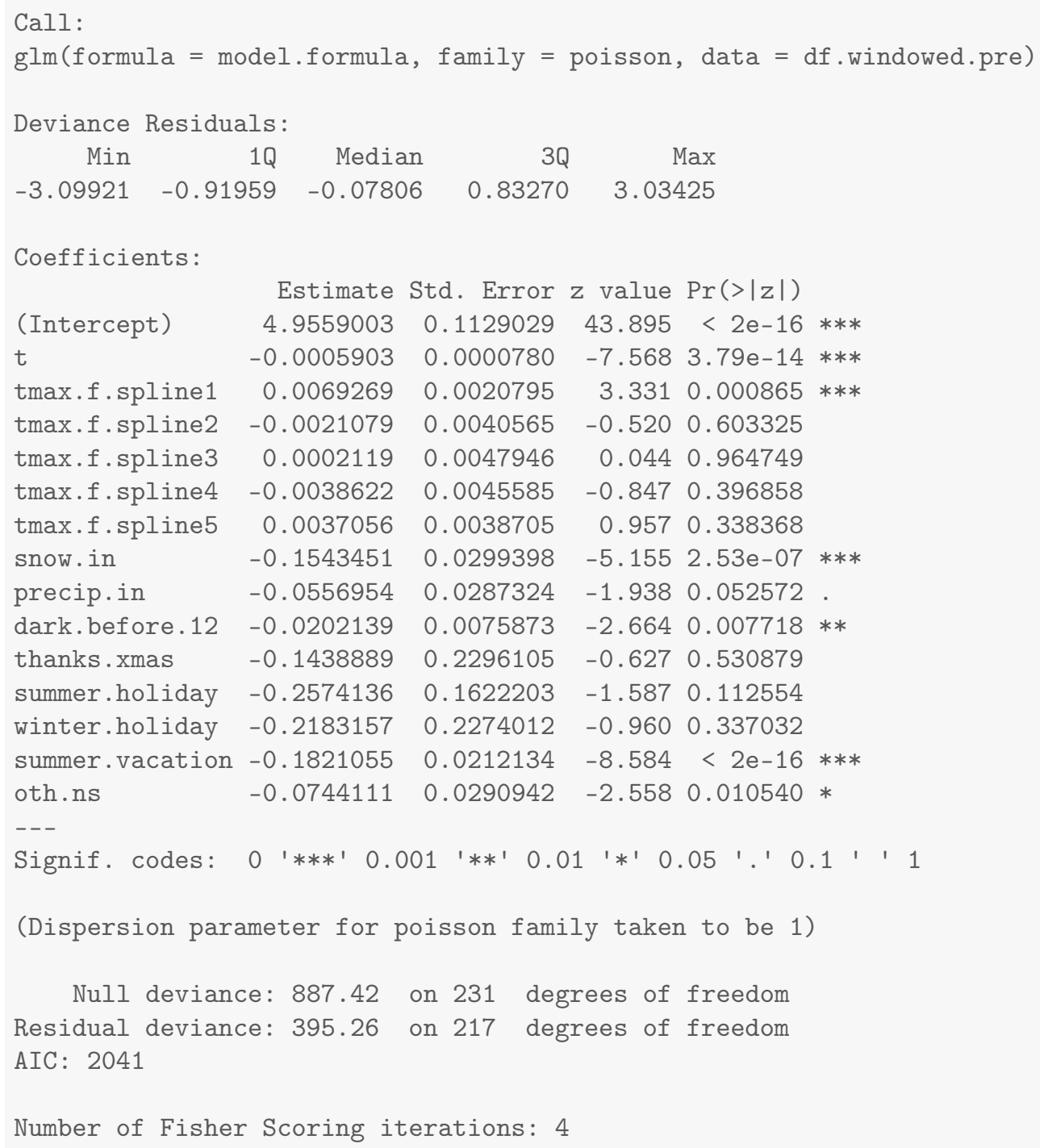




\section{Results for burglary}

\subsection{Summary Values for burglary}

Table 13.1: Descriptives for Outcome Before Ferguson Protests Begin

\begin{tabular}{lcccccccc}
\hline \hline Statistic & $\mathrm{N}$ & Mean & St. Dev. & Min & Pctl(25) & Median & Pctl(75) & Max \\
\hline burglary & 232 & 149.763 & 25.720 & 85 & 133 & 149 & 164 & 240 \\
\hline
\end{tabular}

Table 13.2: Descriptives for Outcome After Ferguson Protests Begin

\begin{tabular}{lcccccccc}
\hline \hline Statistic & $\mathrm{N}$ & Mean & St. Dev. & Min & Pctl(25) & Median & Pctl(75) & Max \\
\hline burglary & 290 & 133.093 & 33.876 & 66 & 108 & 132 & 153 & 403 \\
\hline
\end{tabular}




\subsection{Four Models for burglary}

Table 13.3: Four Models that Differ on the Specification of Adjustment and Intervention Variables

\begin{tabular}{|c|c|c|c|c|}
\hline & \multicolumn{4}{|c|}{ Outcome: Count Per Week } \\
\hline & (1) & (2) & (3) & $(4)$ \\
\hline Time (counter in weeks) & -0.12 & -0.10 & & -0.10 \\
\hline After Ferguson Protests Begin (week of $8 / 11 / 14$ onward) & -1.47 & & 0.46 & 1.49 \\
\hline After Gray Protests Begin (week of 4/20/15 onward) & 22.83 & & 18.45 & 15.44 \\
\hline Unrest and National Guard (4/27/15 - 5/3/2015) & 250.67 & & 260.33 & 260.70 \\
\hline After Batts Fired (week of 7/13/15 onward) & 11.24 & & -2.88 & 1.48 \\
\hline After Davis Confirmed (week of 10/19/15 onward) & -15.12 & & -6.04 & -6.99 \\
\hline After Davis First Year (week of 10/17/16 onward) & 18.98 & & 12.19 & 17.06 \\
\hline After De Sousa Begins (week of 1/19/18 onward) & -41.82 & & -29.12 & -29.69 \\
\hline After Tuggle Begins (week of 5/14/18 onward) & 17.49 & & -5.69 & 1.23 \\
\hline After Harrison Begins (week of 2/11/19 onward) & -15.43 & & -15.82 & -11.67 \\
\hline Average Maximum Temperature to 50 Degrees & & 0.96 & & 1.06 \\
\hline Plus Degrees in the 50s & & 1.03 & & 0.62 \\
\hline Plus Degrees in the 60s & & -0.17 & & -0.30 \\
\hline Plus Degrees in the 70s & & -1.08 & & -0.22 \\
\hline Plus Degrees Greater Than 80 & & -0.65 & & -1.16 \\
\hline Snowfall (inches) & & -8.39 & & -3.02 \\
\hline Precipitation (inches) & & 12.25 & & 12.50 \\
\hline Darkness Before Midnight (hours) & & 14.47 & & 11.67 \\
\hline Thanksgiving/Christmas (proportion of week) & & 161.76 & & 63.96 \\
\hline Winter Holiday (proportion of week) & & -10.48 & & -22.59 \\
\hline Other Out-of-School Days (proportion of week) & & 3.17 & & -9.19 \\
\hline Summer Vacation (proportion of week) & & 14.33 & & 5.03 \\
\hline Summer Holiday (proportion of week) & & -11.94 & & -4.74 \\
\hline Observations & 522 & 232 & 522 & 522 \\
\hline $\mathrm{R}^{2}$ & 0.48 & 0.44 & 0.45 & 0.63 \\
\hline
\end{tabular}


13.3 Least Squares Updated Model (2) for burglary burglary

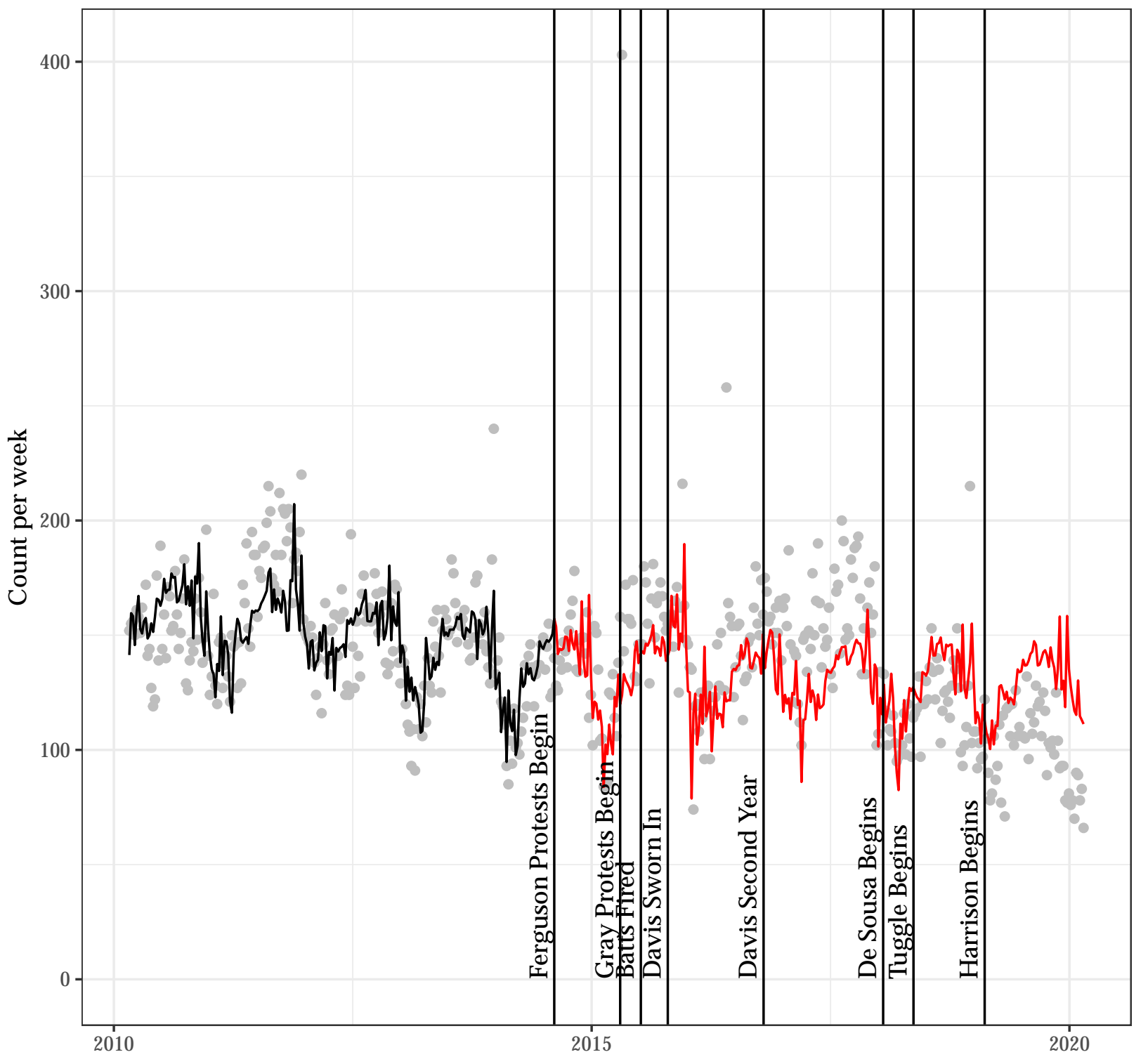




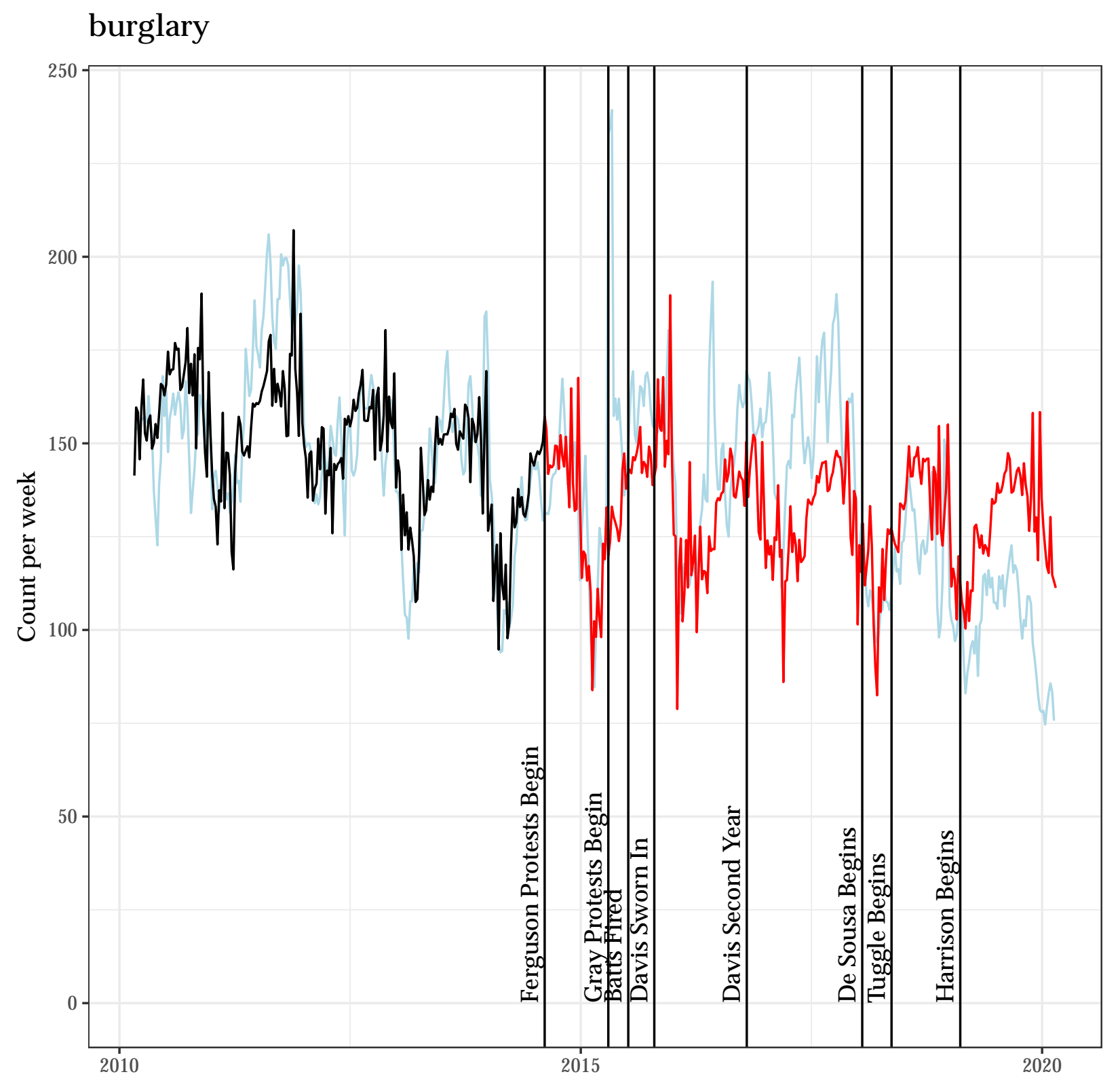


Autocorrelation Function for the Observed Outcome for Updated Model (2)

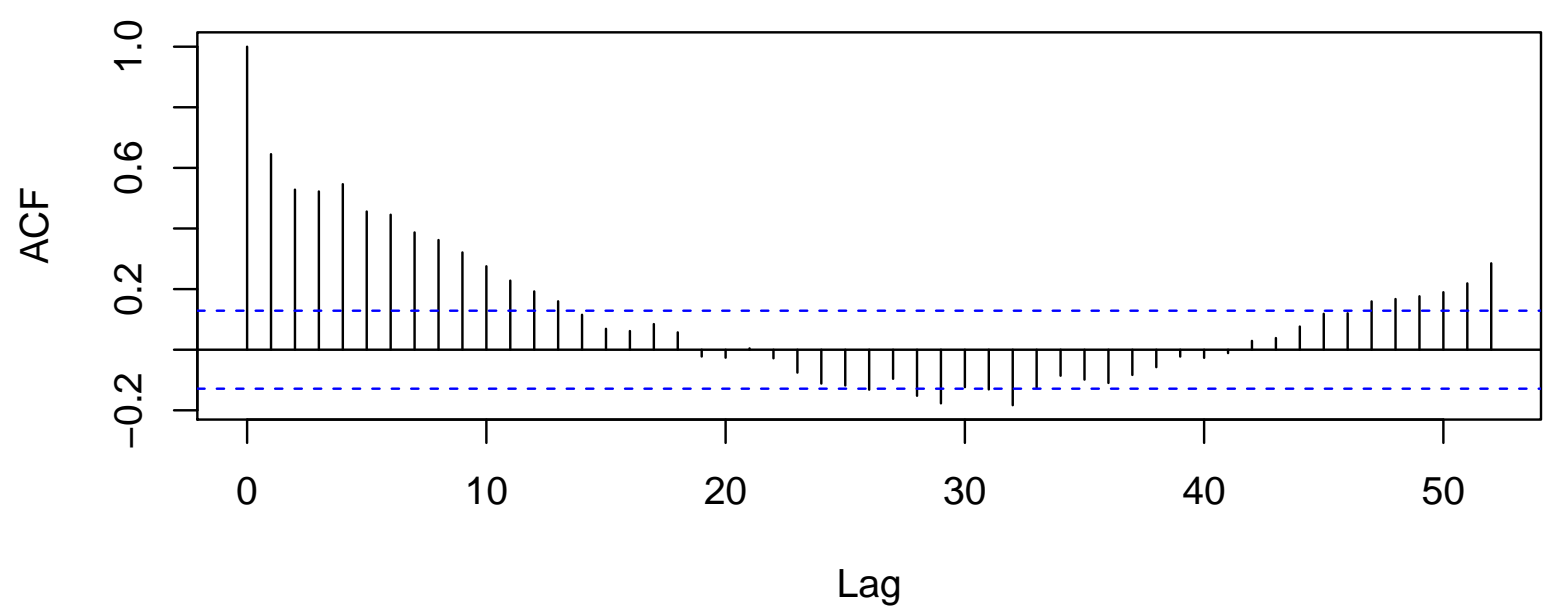

Autocorrelation Function for the Residuals from Updated Model (2)

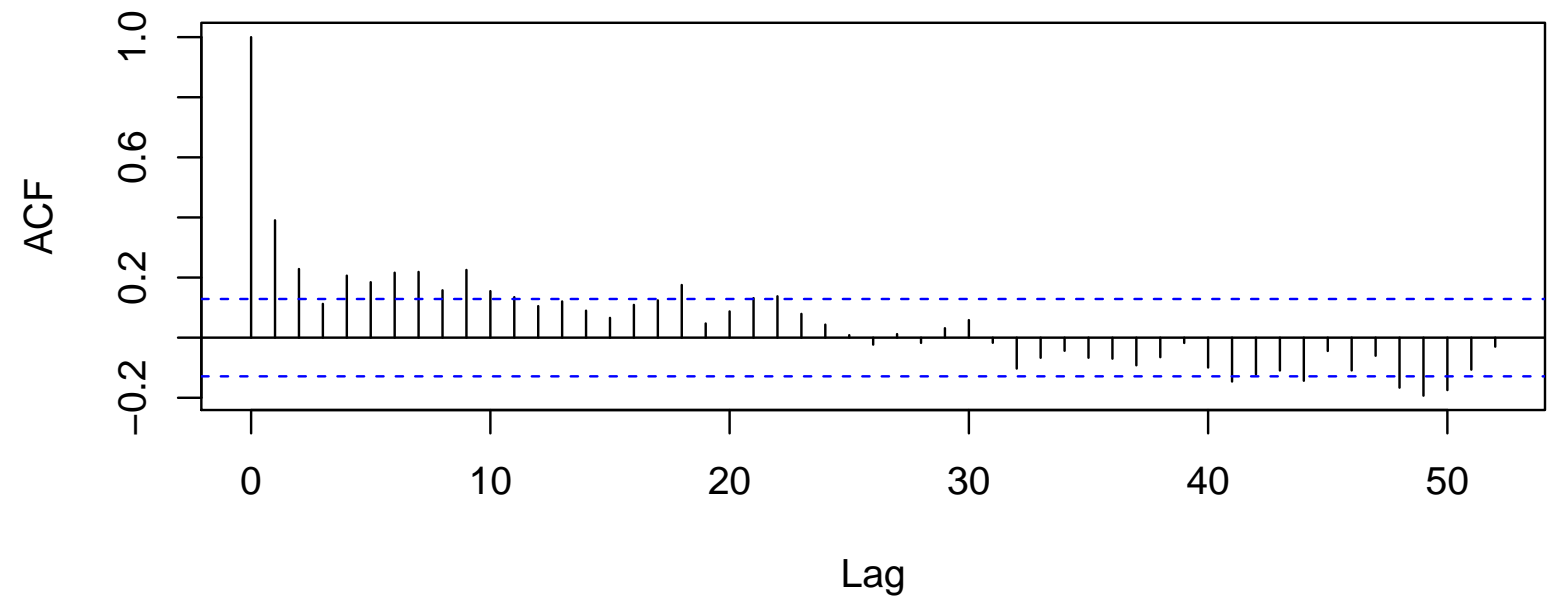




\subsection{A Poisson Regression Model as an Alternative to Model (2) for burglary}

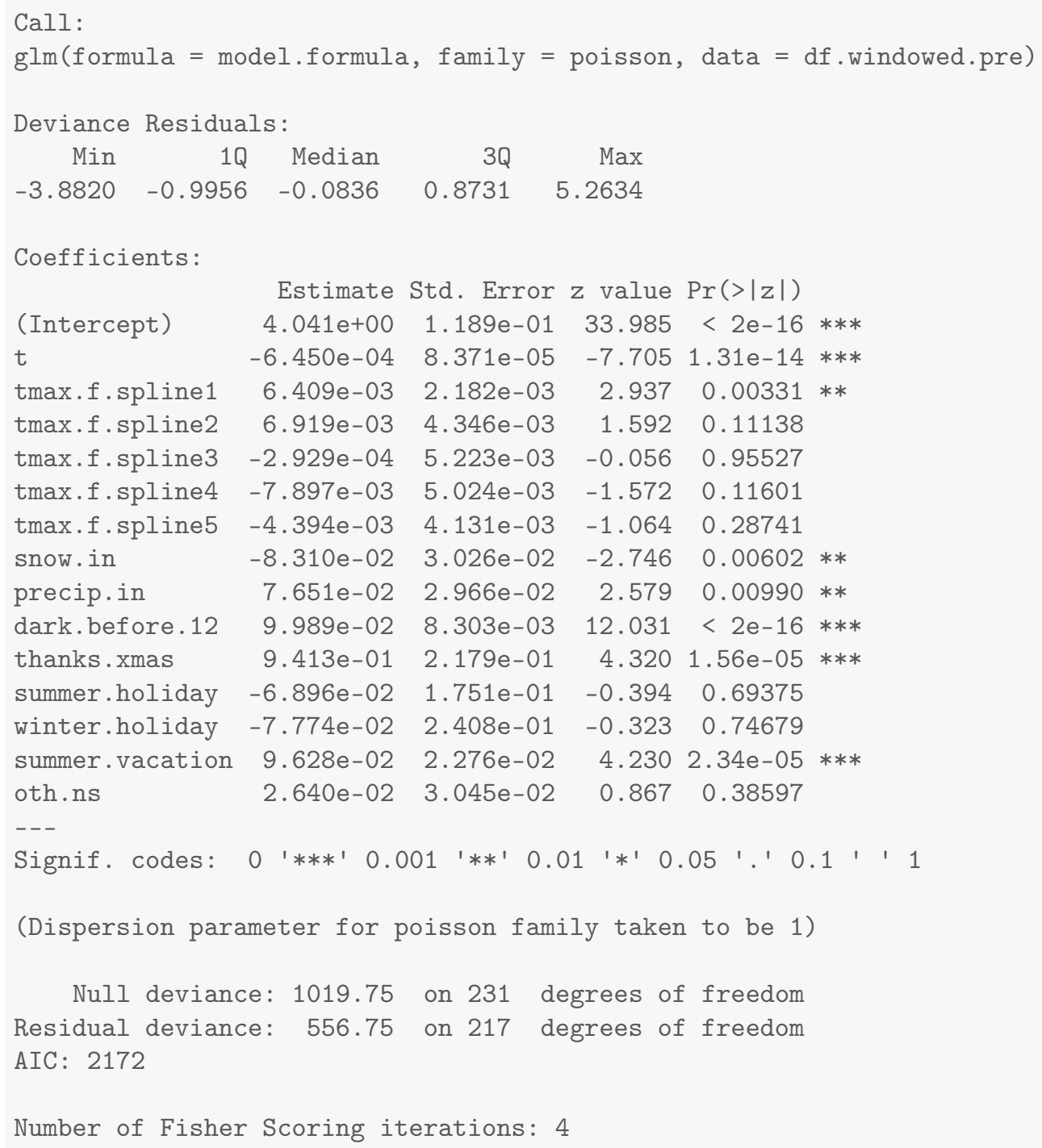




\section{Results for larceny.from.auto}

\subsection{Summary Values for larceny.from.auto}

Table 14.1: Descriptives for Outcome Before Ferguson Protests Begin

\begin{tabular}{lcccccccc}
\hline \hline Statistic & $\mathrm{N}$ & Mean & St. Dev. & Min & Pctl(25) & Median & Pctl(75) & Max \\
\hline larceny.from.auto & 232 & 133.720 & 26.546 & 75 & 115 & 131 & 152.2 & 207 \\
\hline
\end{tabular}

Table 14.2: Descriptives for Outcome After Ferguson Protests Begin

\begin{tabular}{lcccccccc}
\hline \hline Statistic & $\mathrm{N}$ & Mean & St. Dev. & Min & Pctl(25) & Median & Pctl(75) & Max \\
\hline larceny.from.auto & 290 & 122.066 & 27.813 & 52 & 102.2 & 122 & 141 & 217 \\
\hline
\end{tabular}




\subsection{Four Models for larceny.from.auto}

Table 14.3: Four Models that Differ on the Specification of Adjustment and Intervention Variables

\begin{tabular}{|c|c|c|c|c|}
\hline & \multicolumn{4}{|c|}{ Outcome: Count Per Week } \\
\hline & (1) & (2) & (3) & $(4)$ \\
\hline Time (counter in weeks) & -0.09 & -0.08 & & -0.07 \\
\hline After Ferguson Protests Begin (week of $8 / 11 / 14$ onward) & 2.56 & & 7.73 & 8.38 \\
\hline After Gray Protests Begin (week of 4/20/15 onward) & 32.47 & & 19.64 & 20.02 \\
\hline Unrest and National Guard (4/27/15 - 5/3/2015) & -72.46 & & -77.19 & -71.33 \\
\hline After Batts Fired (week of 7/13/15 onward) & 2.46 & & -2.92 & -5.70 \\
\hline After Davis Confirmed (week of 10/19/15 onward) & -28.93 & & -20.84 & -19.79 \\
\hline After Davis First Year (week of 10/17/16 onward) & 3.31 & & 1.63 & 2.50 \\
\hline After De Sousa Begins (week of 1/19/18 onward) & -15.41 & & -13.84 & -6.85 \\
\hline After Tuggle Begins (week of 5/14/18 onward) & 32.76 & & 26.49 & 23.44 \\
\hline After Harrison Begins (week of 2/11/19 onward) & -22.12 & & -26.98 & -22.16 \\
\hline Average Maximum Temperature to 50 Degrees & & 0.34 & & 1.54 \\
\hline Plus Degrees in the 50s & & 2.16 & & 0.23 \\
\hline Plus Degrees in the 60s & & -2.15 & & -0.63 \\
\hline Plus Degrees in the 70s & & -1.54 & & -1.25 \\
\hline Plus Degrees Greater Than 80 & & 0.84 & & -0.37 \\
\hline Snowfall (inches) & & -3.02 & & -1.20 \\
\hline Precipitation (inches) & & -8.09 & & -5.61 \\
\hline Darkness Before Midnight (hours) & & 1.22 & & 6.60 \\
\hline Thanksgiving/Christmas (proportion of week) & & 34.05 & & 26.89 \\
\hline Winter Holiday (proportion of week) & & -31.38 & & -11.26 \\
\hline Other Out-of-School Days (proportion of week) & & -8.49 & & -9.81 \\
\hline Summer Vacation (proportion of week) & & 23.66 & & 18.28 \\
\hline Summer Holiday (proportion of week) & & -84.02 & & -56.43 \\
\hline Observations & 522 & 232 & 522 & 522 \\
\hline $\mathrm{R}^{2}$ & 0.20 & 0.33 & 0.13 & 0.43 \\
\hline
\end{tabular}


14.3 Least Squares Updated Model (2) for larceny.from.auto larceny.from.auto

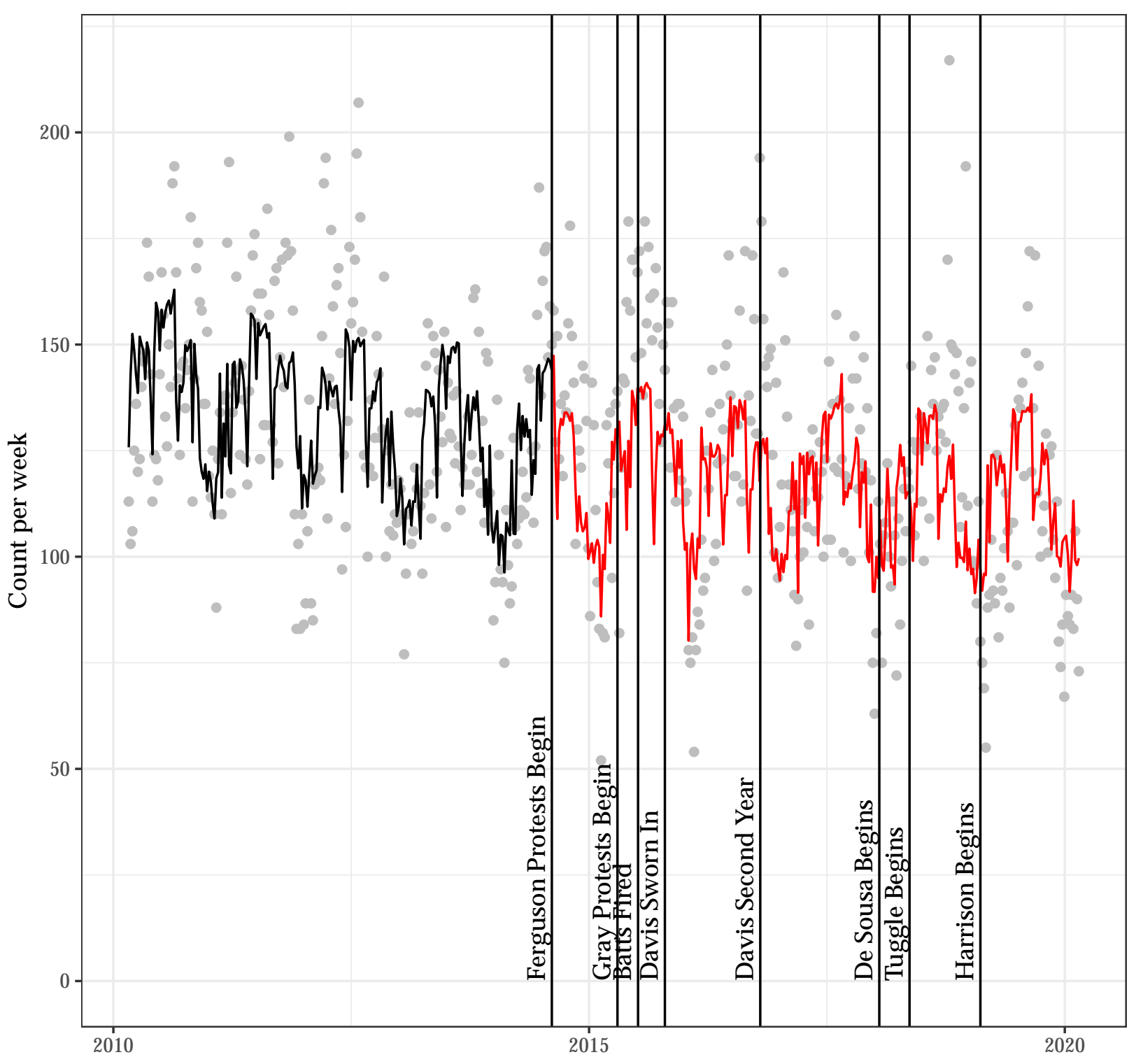




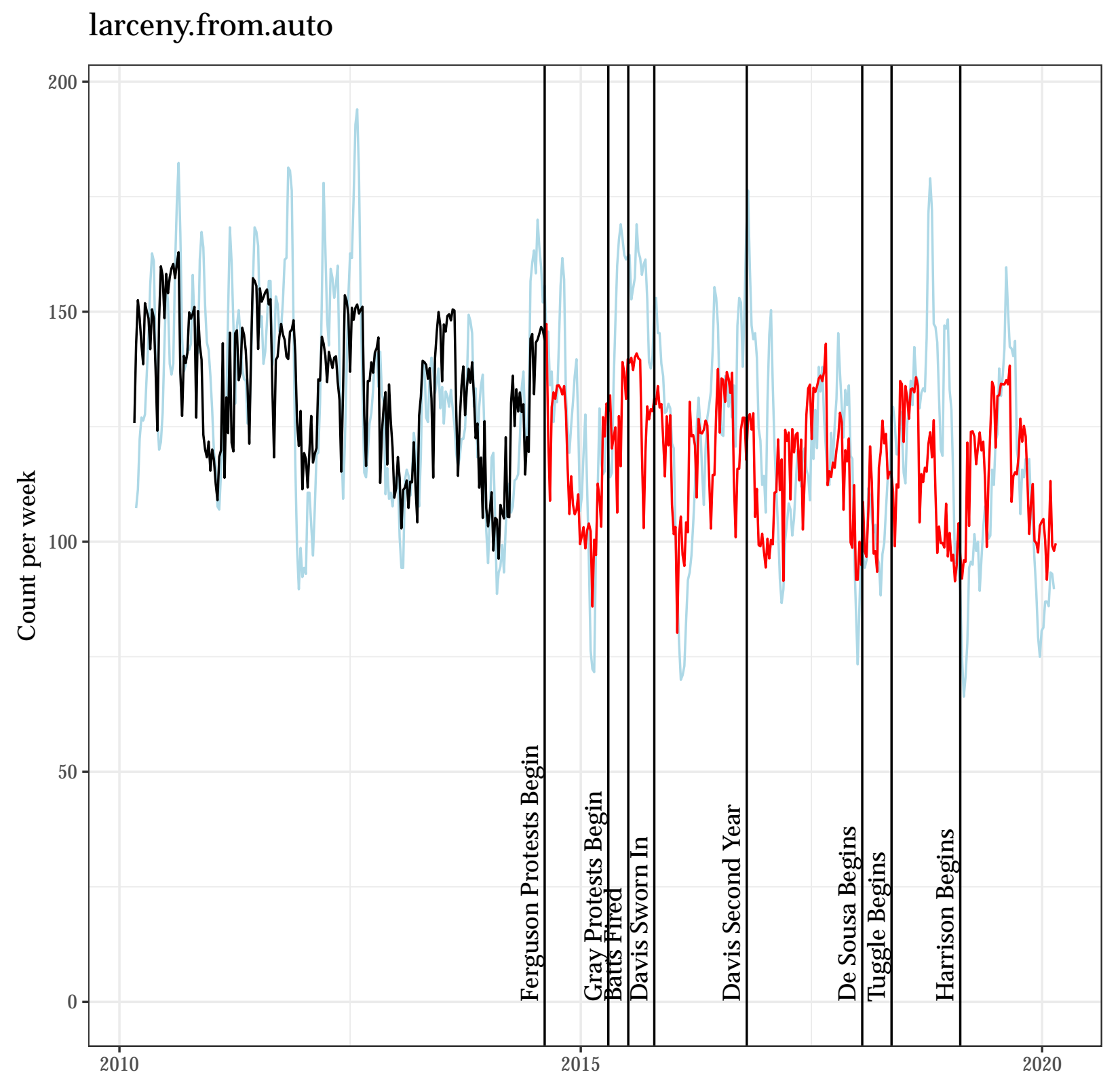


Autocorrelation Function for the Observed Outcome for Updated Model (2)

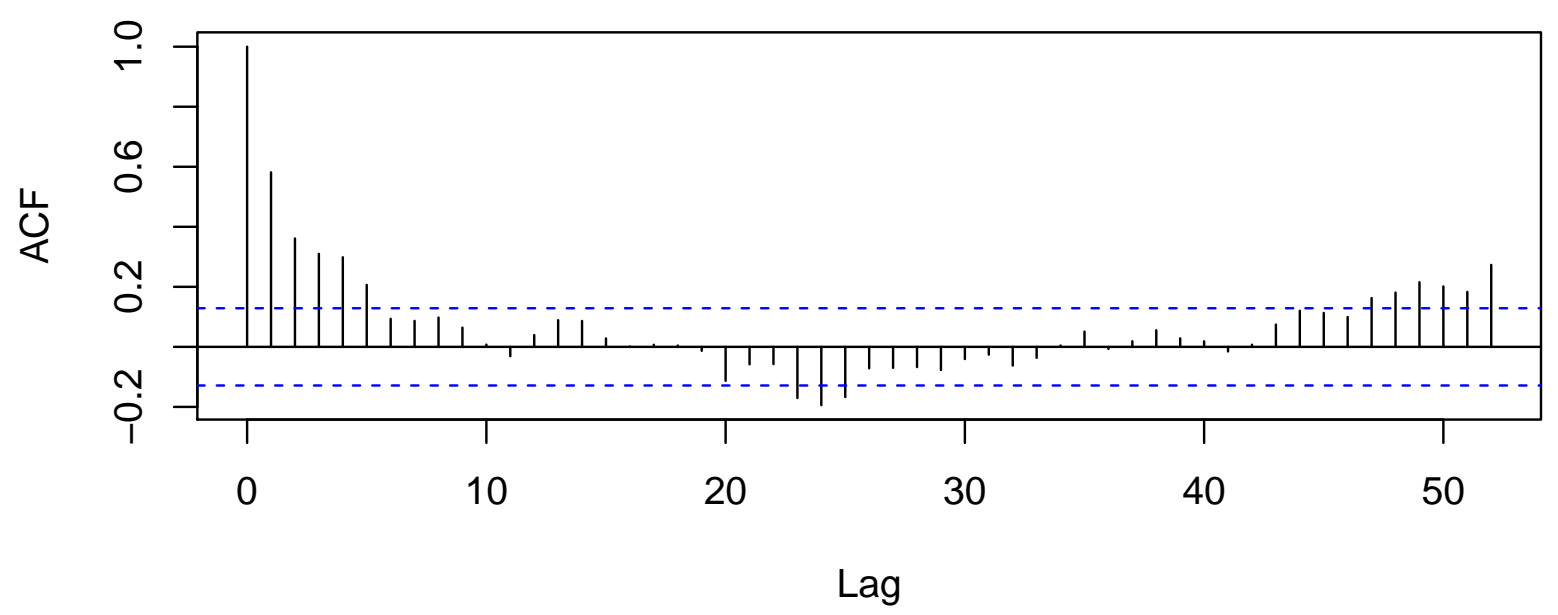

Autocorrelation Function for the Residuals from Updated Model (2)

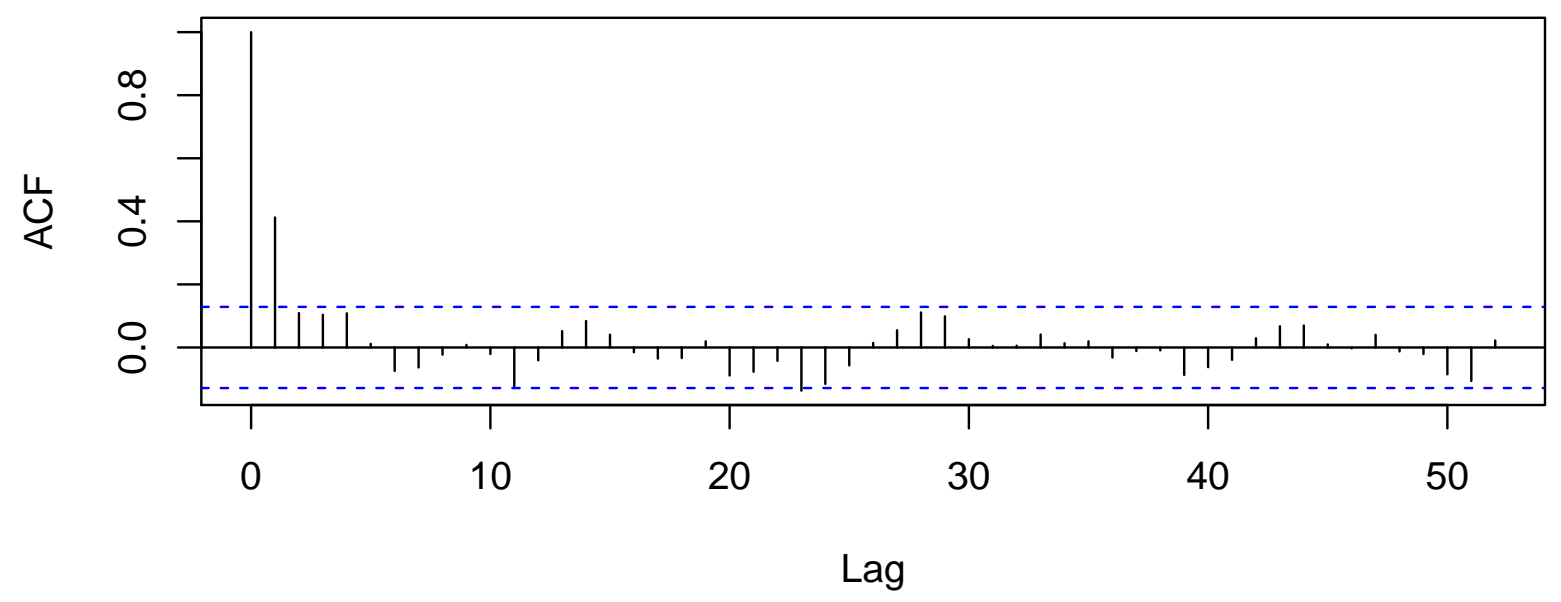




\subsection{A Poisson Regression Model as an Alternative to Model (2) for larceny.from.auto}

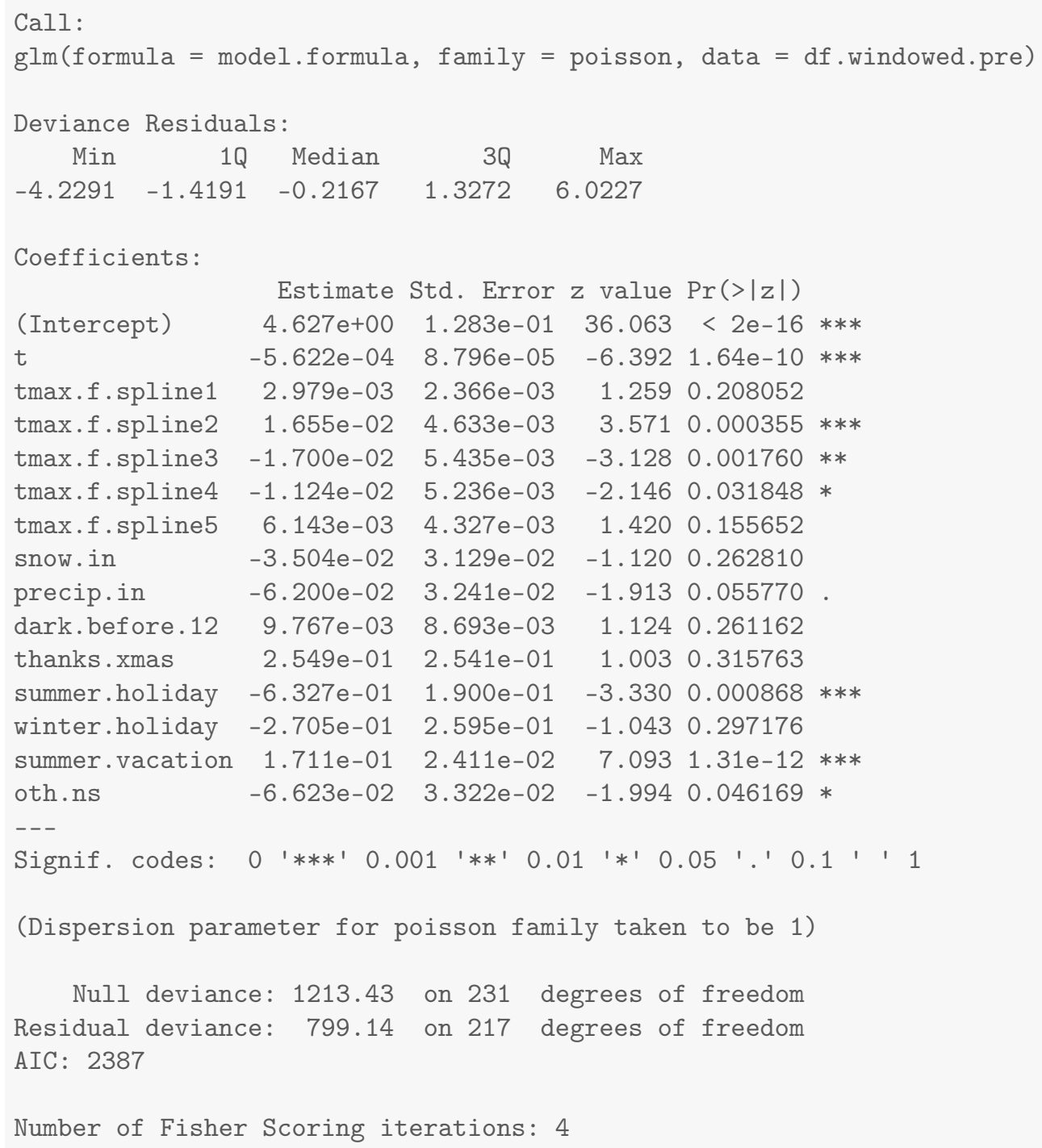




\section{Results for larceny}

\subsection{Summary Values for larceny}

Table 15.1: Descriptives for Outcome Before Ferguson Protests Begin

\begin{tabular}{lcccccccc}
\hline \hline Statistic & $\mathrm{N}$ & Mean & St. Dev. & Min & Pctl(25) & Median & Pctl(75) & Max \\
\hline larceny & 232 & 206.784 & 32.840 & 115 & 186 & 205 & 230.2 & 302 \\
\hline
\end{tabular}

Table 15.2: Descriptives for Outcome After Ferguson Protests Begin

\begin{tabular}{lcccccccc}
\hline \hline Statistic & $\mathrm{N}$ & Mean & St. Dev. & Min & Pctl(25) & Median & Pctl(75) & Max \\
\hline larceny & 290 & 204.655 & 28.312 & 105 & 186 & 206 & 224.8 & 277 \\
\hline
\end{tabular}




\subsection{Four Models for larceny}

Table 15.3: Four Models that Differ on the Specification of Adjustment and Intervention Variables

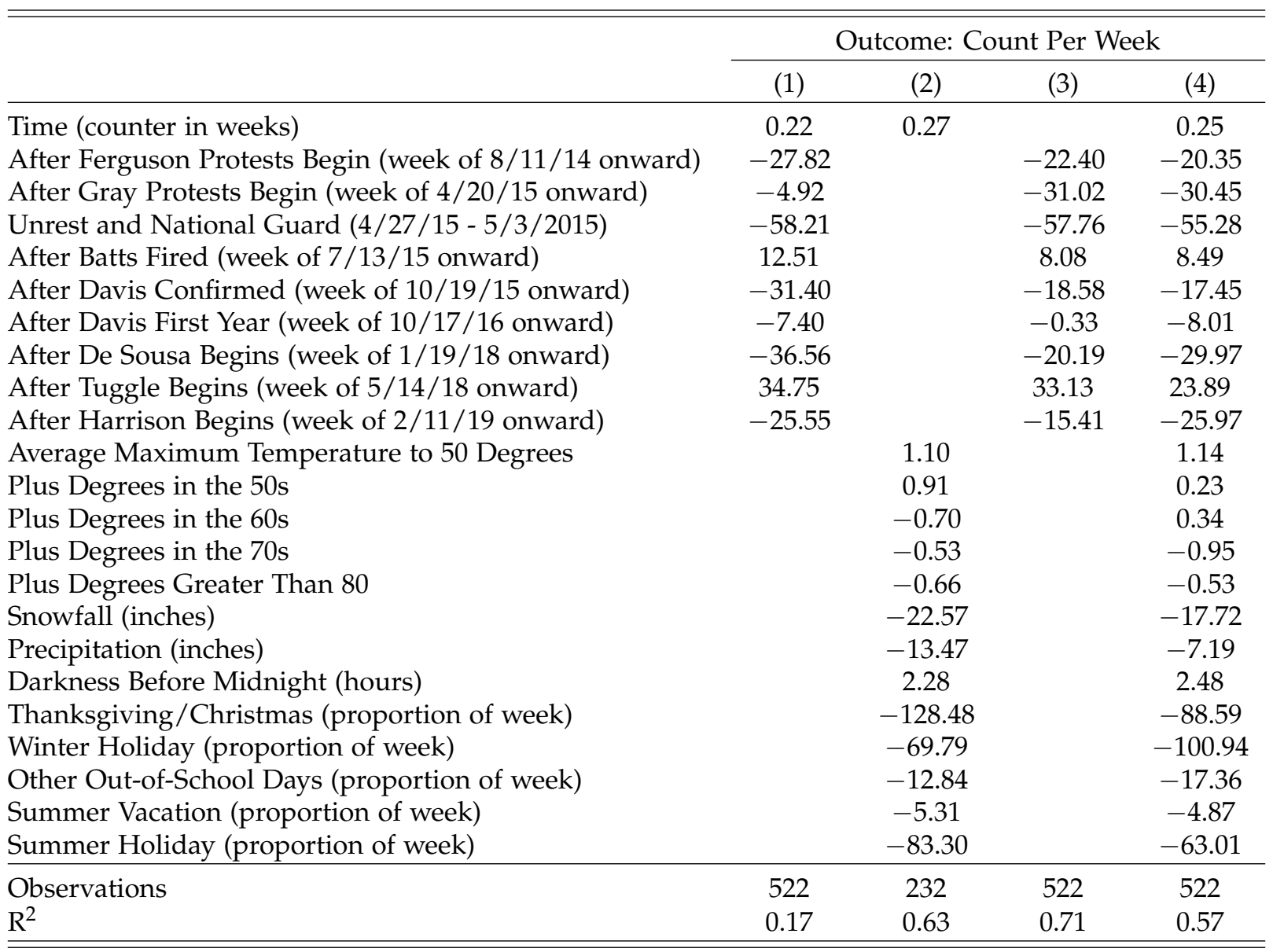


15.3 Least Squares Updated Model (2) for larceny larceny

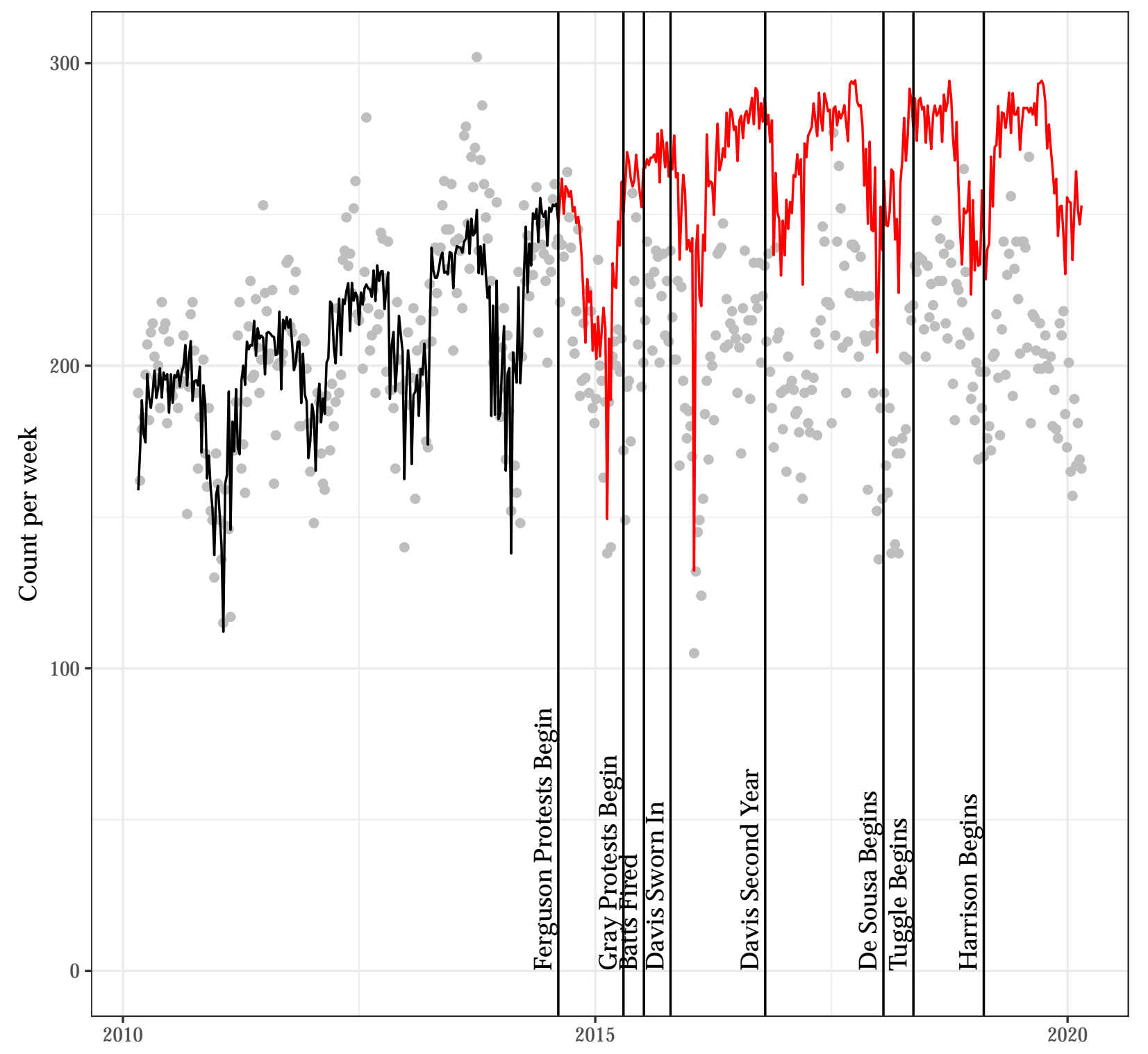




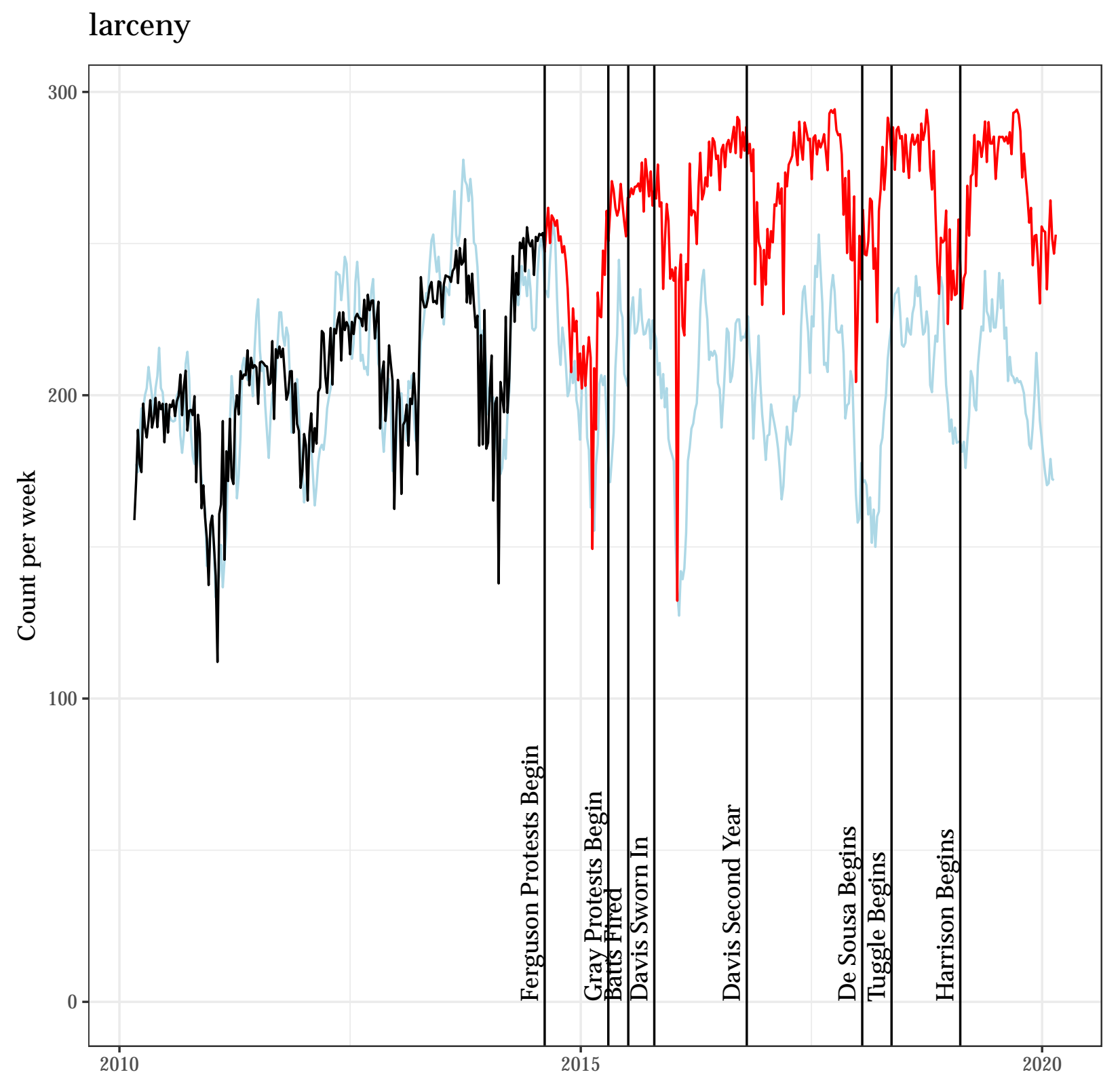


Autocorrelation Function for the Observed Outcome for Updated Model (2)

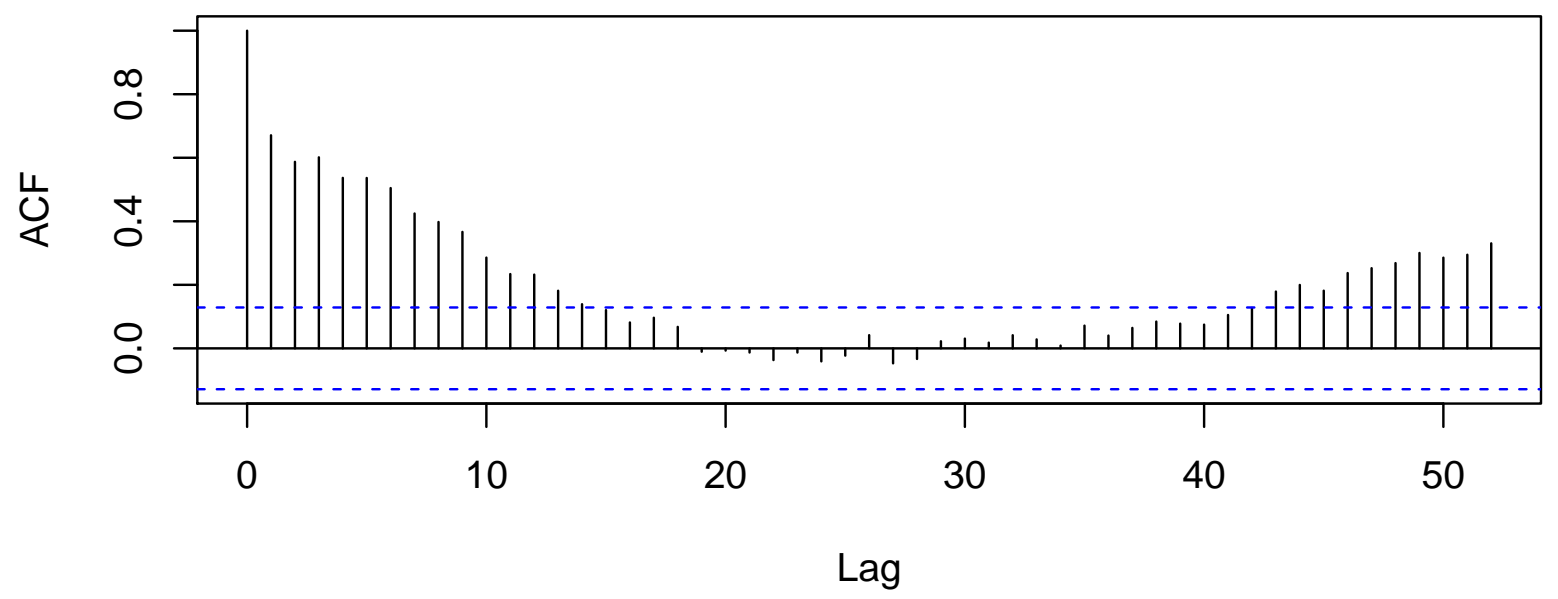

Autocorrelation Function for the Residuals from Updated Model (2)

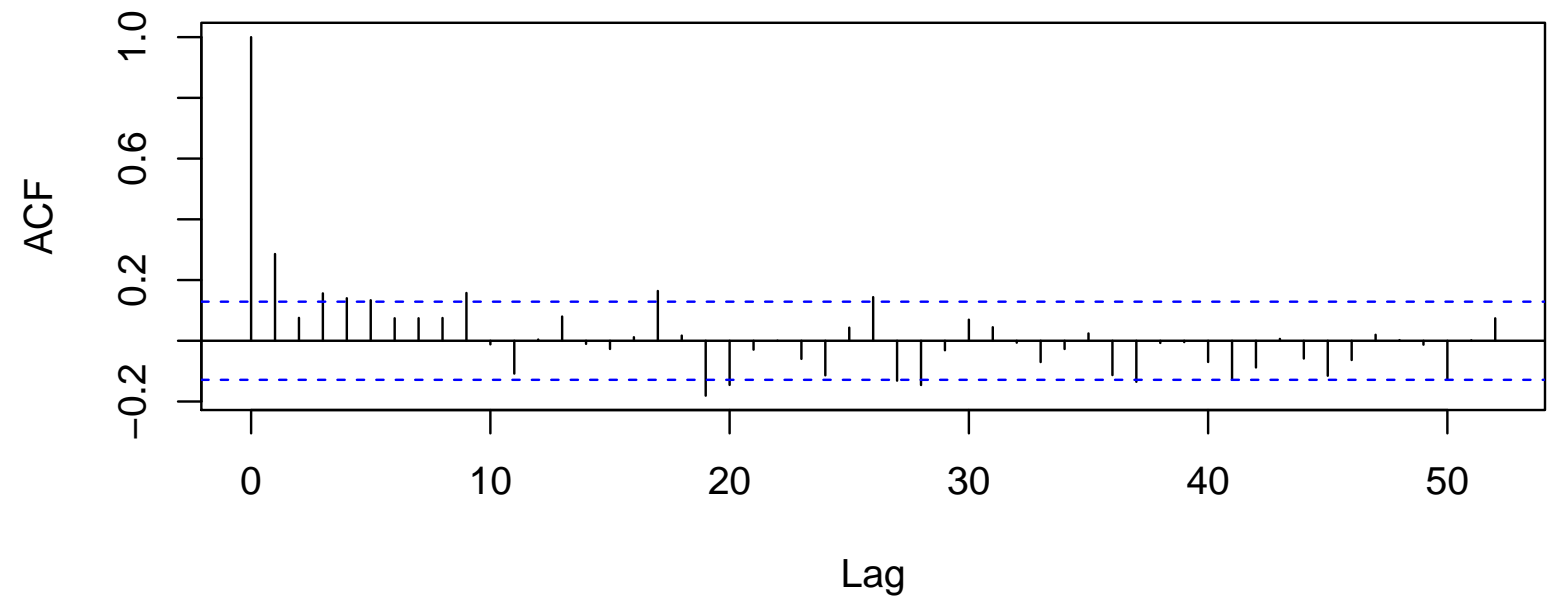




\subsection{A Poisson Regression Model as an Alternative to Model (2) for larceny}

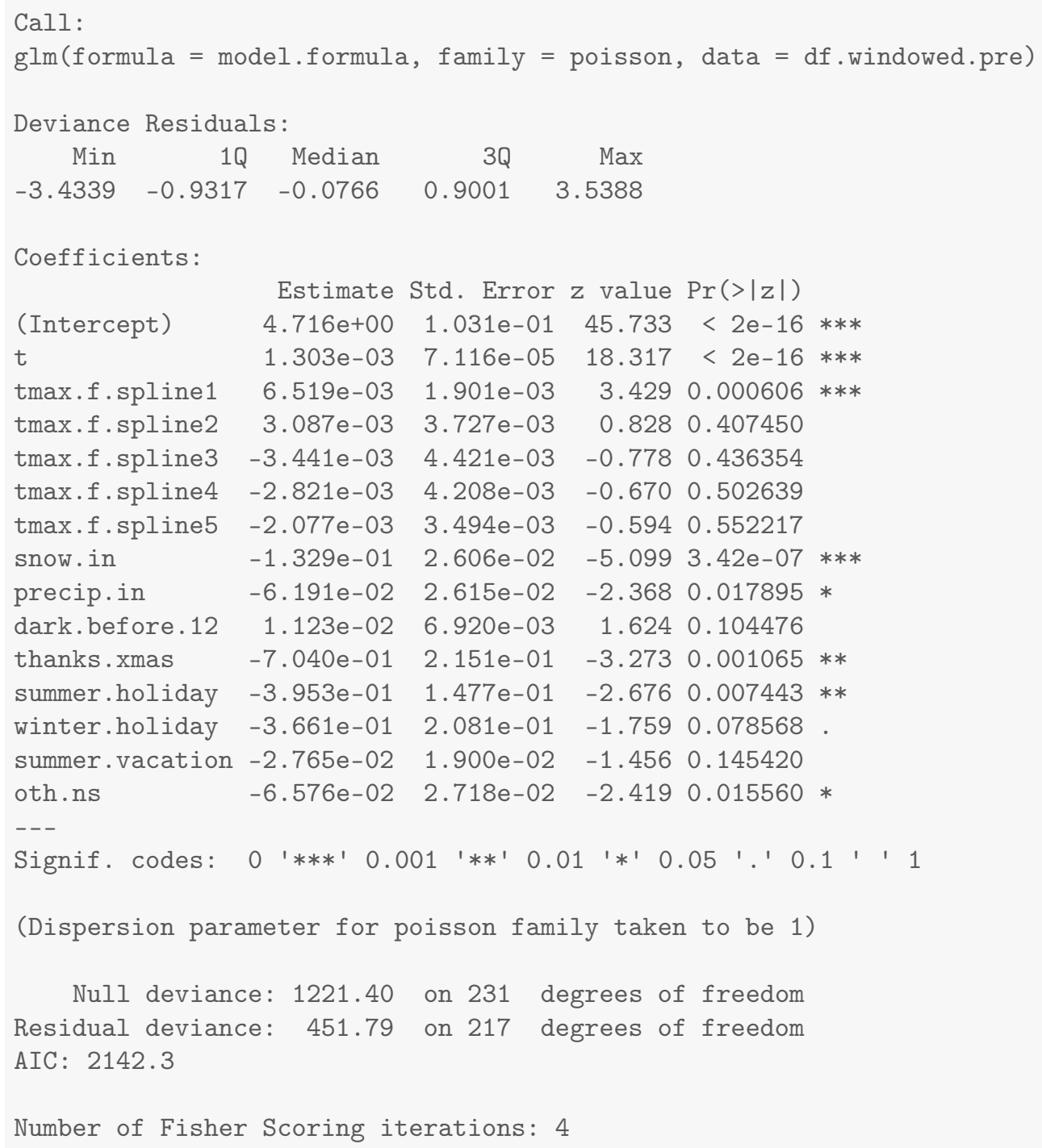




\section{Results for auto.theft}

\subsection{Summary Values for auto.theft}

Table 16.1: Descriptives for Outcome Before Ferguson Protests Begin

\begin{tabular}{lcccccccc}
\hline \hline Statistic & $\mathrm{N}$ & Mean & St. Dev. & Min & Pctl(25) & Median & Pctl(75) & Max \\
\hline auto.theft & 232 & 81.034 & 18.144 & 47 & 67.8 & 78 & 92 & 146 \\
\hline
\end{tabular}

Table 16.2: Descriptives for Outcome After Ferguson Protests Begin

\begin{tabular}{lcccccccc}
\hline \hline Statistic & $\mathrm{N}$ & Mean & St. Dev. & Min & Pctl(25) & Median & Pctl(75) & Max \\
\hline auto.theft & 290 & 81.976 & 16.775 & 46 & 70 & 80 & 92.8 & 145 \\
\hline
\end{tabular}




\subsection{Four Models for auto.theft}

Table 16.3: Four Models that Differ on the Specification of Adjustment and Intervention Variables

\begin{tabular}{|c|c|c|c|c|}
\hline & \multicolumn{4}{|c|}{ Outcome: Count Per Week } \\
\hline & $(1)$ & (2) & (3) & $(4)$ \\
\hline Time (counter in weeks) & -0.02 & -0.01 & & -0.01 \\
\hline After Ferguson Protests Begin (week of 8/11/14 onward) & -11.55 & & -7.70 & -9.69 \\
\hline After Gray Protests Begin (week of 4/20/15 onward) & 37.05 & & 28.91 & 33.83 \\
\hline Unrest and National Guard $(4 / 27 / 15-5 / 3 / 2015)$ & -27.37 & & -18.56 & -20.67 \\
\hline After Batts Fired (week of 7/13/15 onward) & 0.77 & & -8.44 & -7.17 \\
\hline After Davis Confirmed (week of 10/19/15 onward) & -17.68 & & -6.92 & -10.14 \\
\hline After Davis First Year (week of 10/17/16 onward) & 7.28 & & 6.71 & 6.56 \\
\hline After De Sousa Begins (week of 1/19/18 onward) & -16.70 & & -8.31 & -9.79 \\
\hline After Tuggle Begins (week of 5/14/18 onward) & 8.04 & & -4.69 & -1.71 \\
\hline After Harrison Begins (week of 2/11/19 onward) & -10.11 & & -7.52 & -7.63 \\
\hline Average Maximum Temperature to 50 Degrees & & -0.21 & & -0.23 \\
\hline Plus Degrees in the $50 \mathrm{~s}$ & & 1.58 & & 0.90 \\
\hline Plus Degrees in the 60s & & -0.81 & & -0.16 \\
\hline Plus Degrees in the 70s & & -0.11 & & -0.06 \\
\hline Plus Degrees Greater Than 80 & & 0.08 & & 0.18 \\
\hline Snowfall (inches) & & -0.70 & & -1.22 \\
\hline Precipitation (inches) & & 1.19 & & -0.27 \\
\hline Darkness Before Midnight (hours) & & 4.85 & & 4.40 \\
\hline Thanksgiving/Christmas (proportion of week) & & 0.52 & & -7.04 \\
\hline Winter Holiday (proportion of week) & & -27.37 & & -26.92 \\
\hline Other Out-of-School Days (proportion of week) & & -2.05 & & 0.62 \\
\hline Summer Vacation (proportion of week) & & 18.86 & & 11.36 \\
\hline Summer Holiday (proportion of week) & & -59.20 & & -43.43 \\
\hline Observations & 522 & 232 & 522 & 522 \\
\hline $\mathrm{R}^{2}$ & 0.23 & 0.42 & 0.19 & 0.40 \\
\hline
\end{tabular}


16.3 Least Squares Updated Model (2) for auto.theft auto.theft

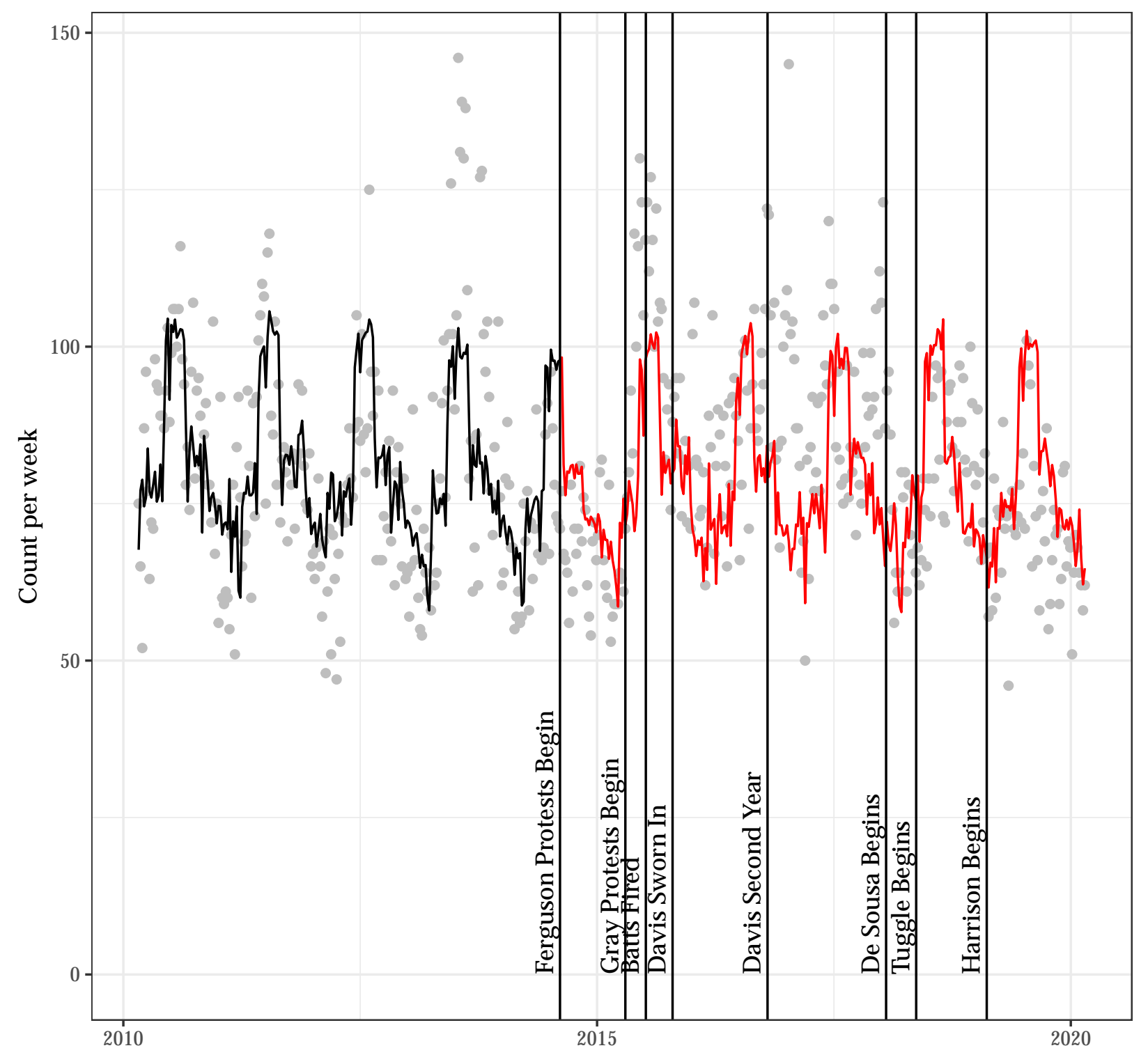




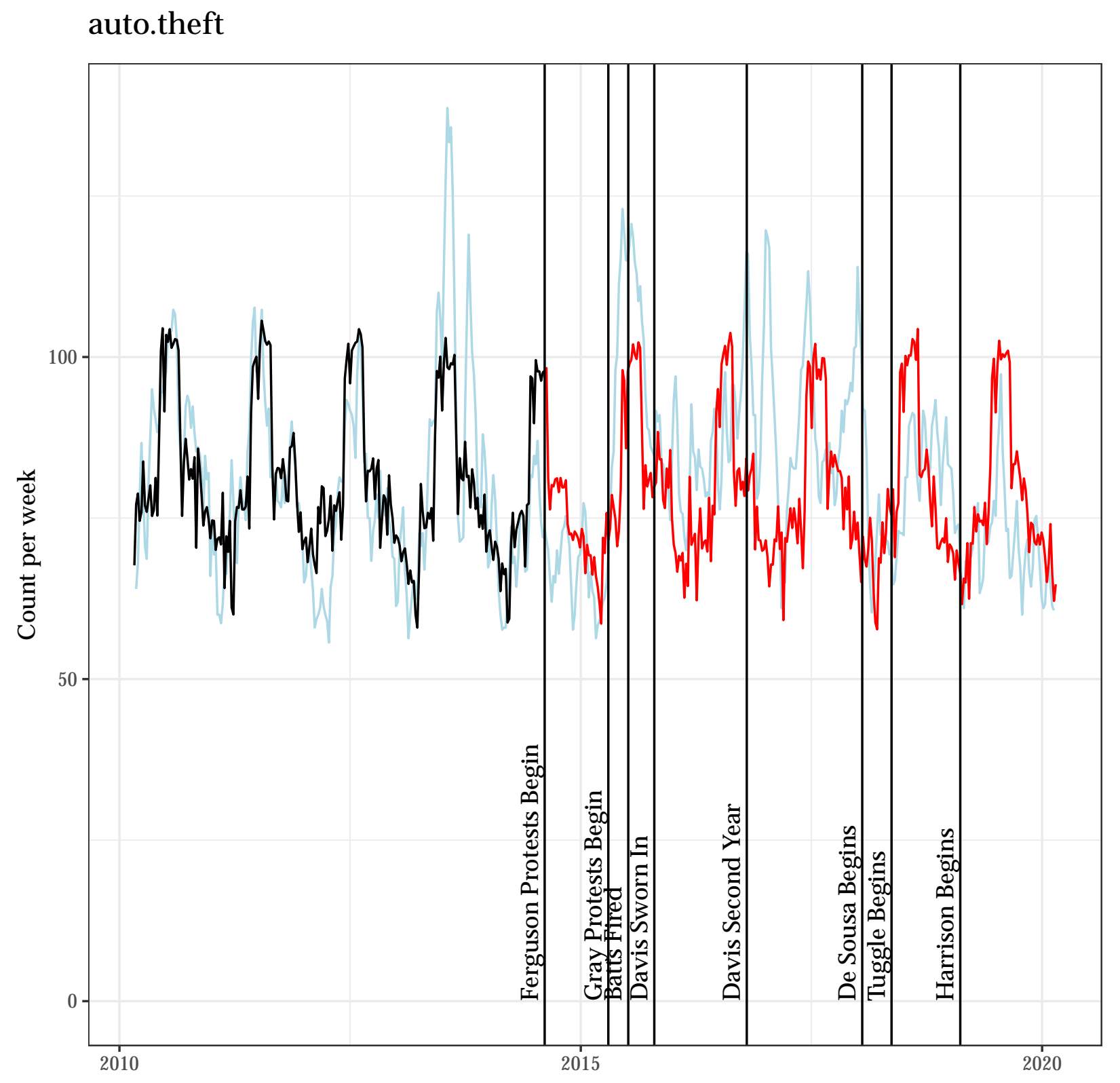


Autocorrelation Function for the Observed Outcome for Updated Model (2)

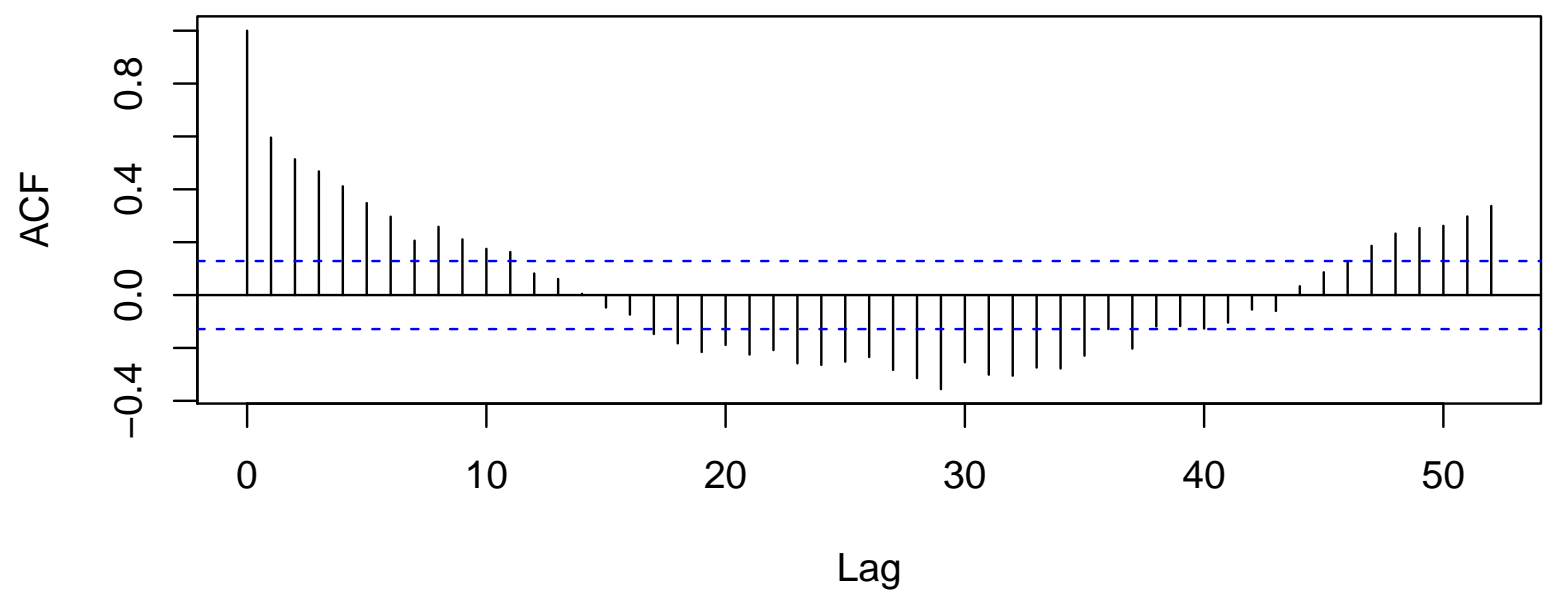

Autocorrelation Function for the Residuals from Updated Model (2)

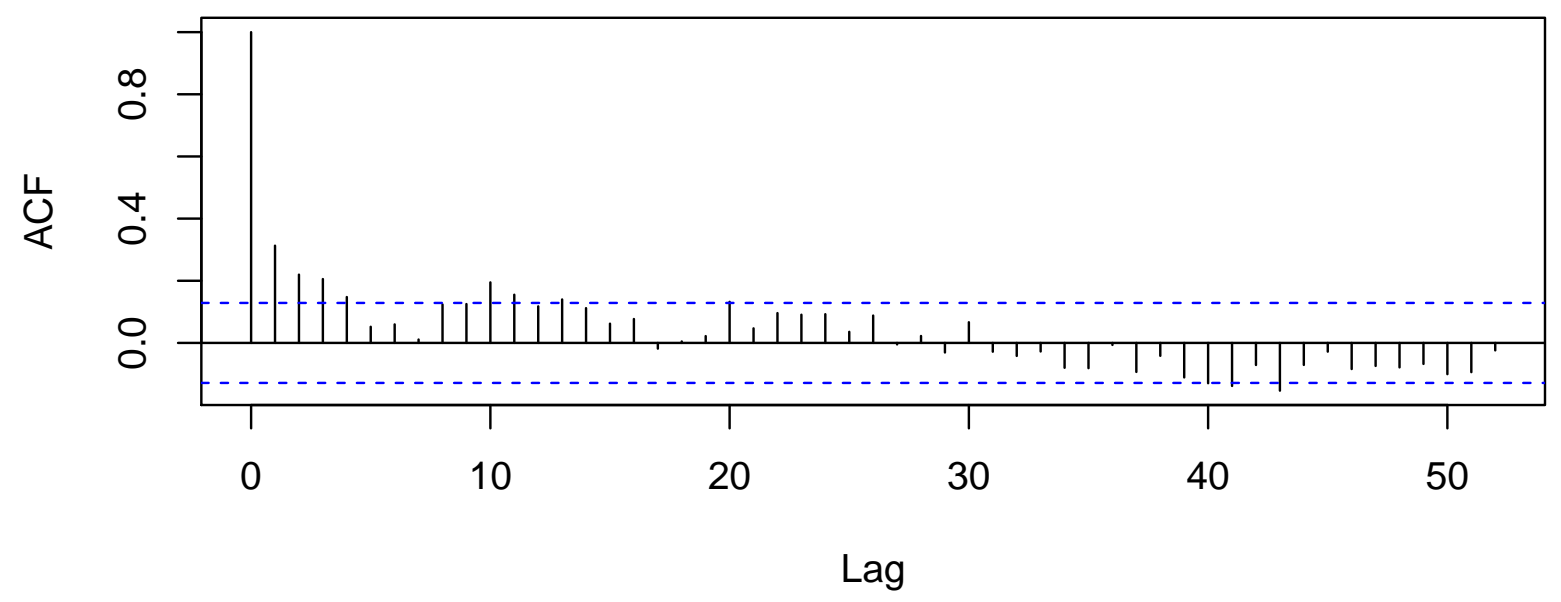




\subsection{A Poisson Regression Model as an Alternative to Model (2) for auto.theft}

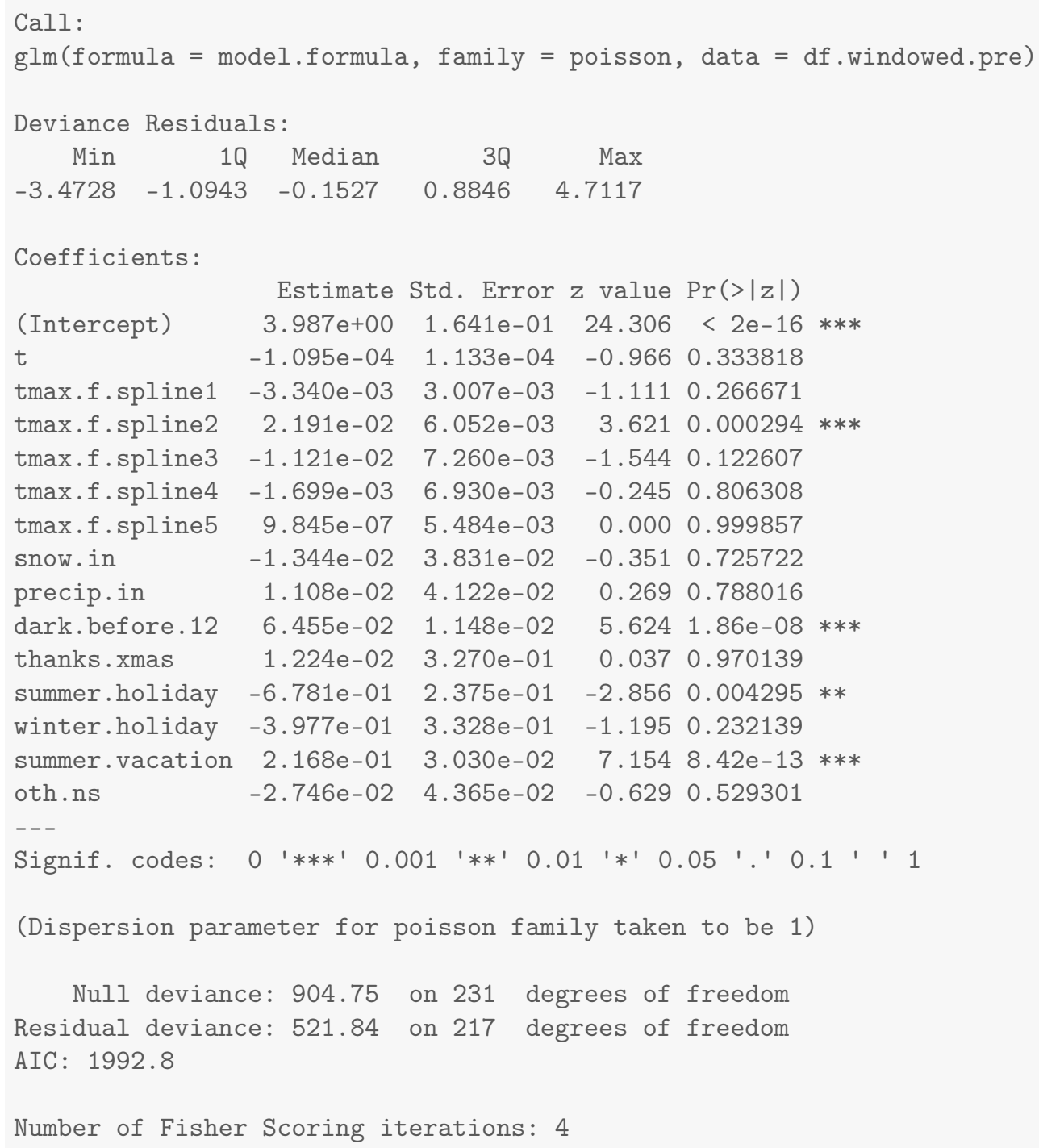




\section{Results for arson}

\subsection{Summary Values for arson}

Table 17.1: Descriptives for Outcome Before Ferguson Protests Begin

\begin{tabular}{lcccccccc}
\hline \hline Statistic & $\mathrm{N}$ & Mean & St. Dev. & Min & Pctl(25) & Median & Pctl(75) & Max \\
\hline arson & 232 & 5.289 & 2.914 & 0 & 3 & 5 & 7 & 15 \\
\hline
\end{tabular}

Table 17.2: Descriptives for Outcome After Ferguson Protests Begin

\begin{tabular}{lcccccccc}
\hline \hline Statistic & $\mathrm{N}$ & Mean & St. Dev. & Min & Pctl(25) & Median & Pctl(75) & Max \\
\hline arson & 290 & 4.003 & 3.155 & 0 & 2 & 4 & 5 & 33 \\
\hline
\end{tabular}




\subsection{Four Models for arson}

Table 17.3: Four Models that Differ on the Specification of Adjustment and Intervention Variables

\begin{tabular}{lcccc}
\hline \hline & \multicolumn{3}{c}{ Outcome: Count Per Week } \\
\cline { 2 - 5 } & $(1)$ & $(2)$ & $(3)$ & $(4)$ \\
\hline Time (counter in weeks) & -0.01 & -0.01 & & -0.01 \\
After Ferguson Protests Begin (week of $8 / 11 / 14$ onward) & 0.21 & & 0.77 & 0.61 \\
After Gray Protests Begin (week of $4 / 20 / 15$ onward) & 2.14 & & 0.99 & 1.08 \\
Unrest and National Guard (4/27/15 - 5/3/2015) & 26.95 & & 27.76 & 27.26 \\
After Batts Fired (week of 7/13/15 onward) & -0.01 & & 0.39 & 0.40 \\
After Davis Confirmed (week of 10/19/15 onward) & -0.32 & & -0.14 & -0.06 \\
After Davis First Year (week of 10/17/16 onward) & 0.30 & & -0.01 & 0.28 \\
After De Sousa Begins (week of 1/19/18 onward) & -1.46 & & -1.87 & -1.43 \\
After Tuggle Begins (week of 5/14/18 onward) & -0.66 & & -0.91 & -0.65 \\
After Harrison Begins (week of 2/11/19 onward) & 0.47 & & -0.08 & 0.38 \\
Average Maximum Temperature to 50 Degrees & & 0.14 & & 0.08 \\
Plus Degrees in the 50s & & -0.09 & & -0.10 \\
Plus Degrees in the 60s & & -0.13 & & 0.02 \\
Plus Degrees in the 70s & & -0.29 & & 0.09 \\
Plus Degrees Greater Than 80 & & -0.03 & & -0.21 \\
Snowfall (inches) & & -0.51 & & -0.65 \\
Precipitation (inches) & & -0.01 & & -0.17 \\
Darkness Before Midnight (hours) & & -0.37 & & 1.43 \\
Thanksgiving/Christmas (proportion of week) & & 5.98 & & -2.16 \\
Winter Holiday (proportion of week) & & -0.29 & & -0.98 \\
Other Out-of-School Days (proportion of week) & & -1.70 & & -0.09 \\
Summer Vacation (proportion of week) & & & & -1.66 \\
Summer Holiday (proportion of week) & 522 & 232 & 522 & 522 \\
\hline Observations & 0.35 & 0.14 & 0.31 & 0.40 \\
R & & & \\
\hline \hline
\end{tabular}




\subsection{Least Squares Updated Model (2) for arson}

arson

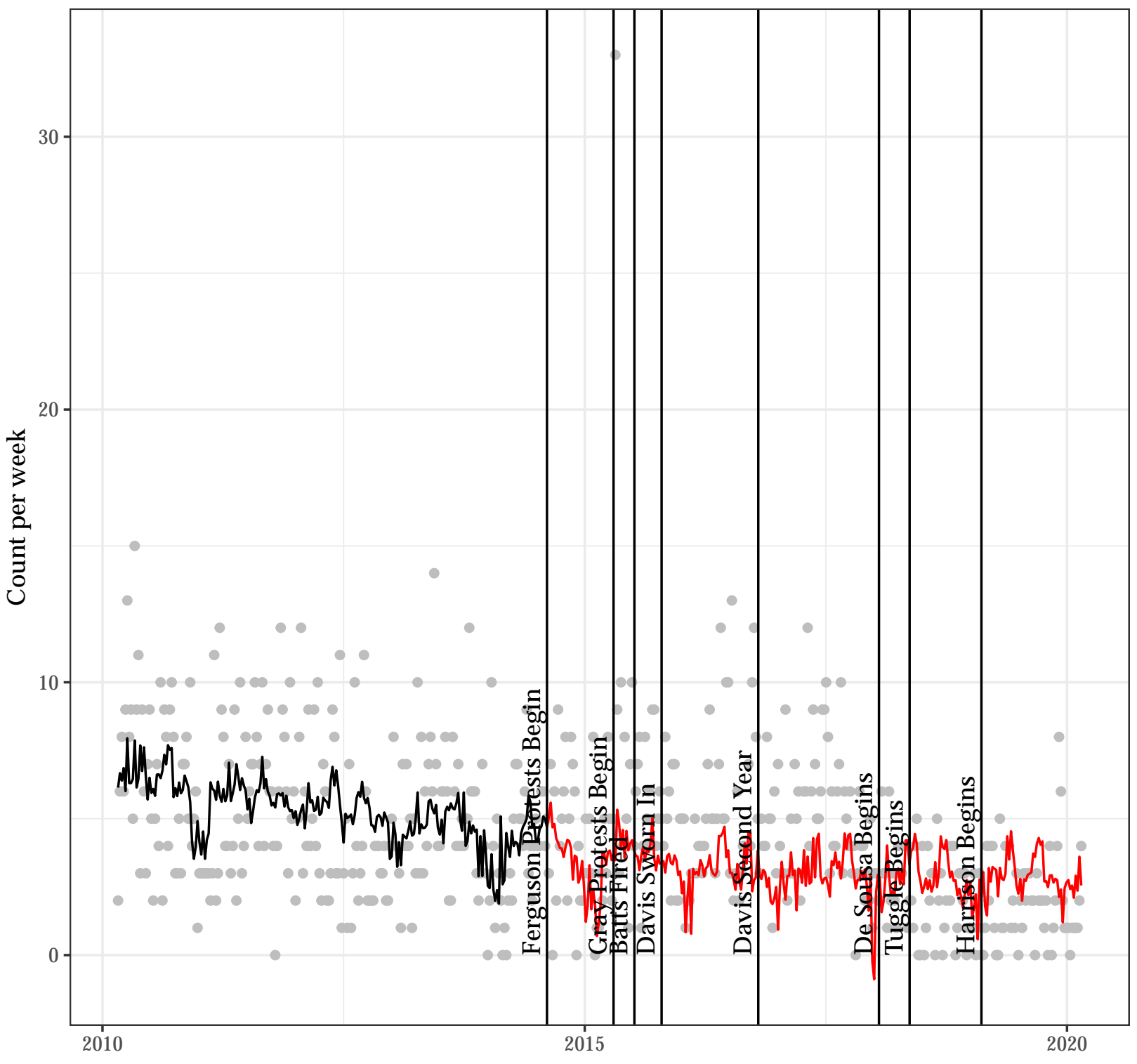




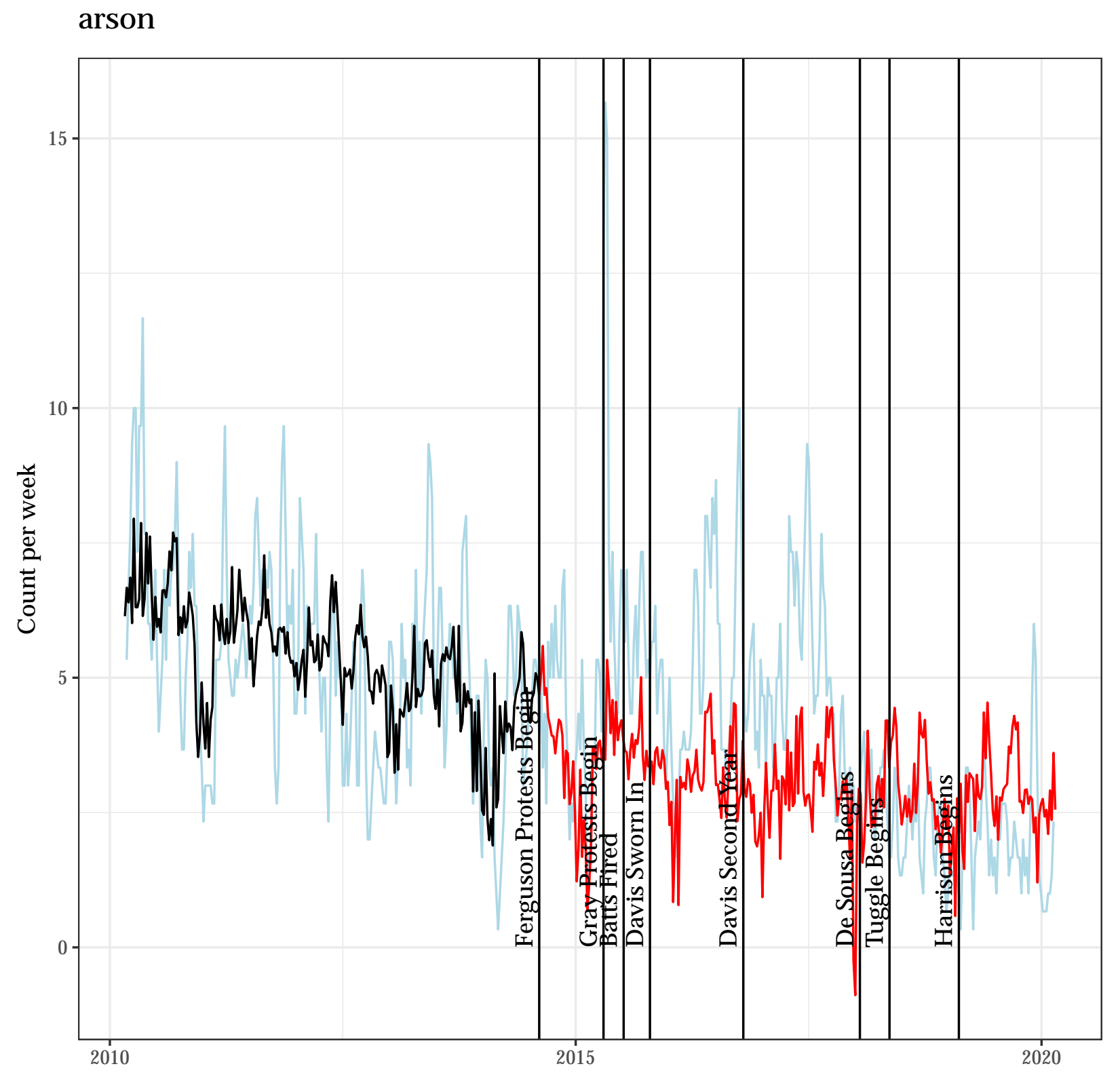


Autocorrelation Function for the Observed Outcome for Updated Model (2)

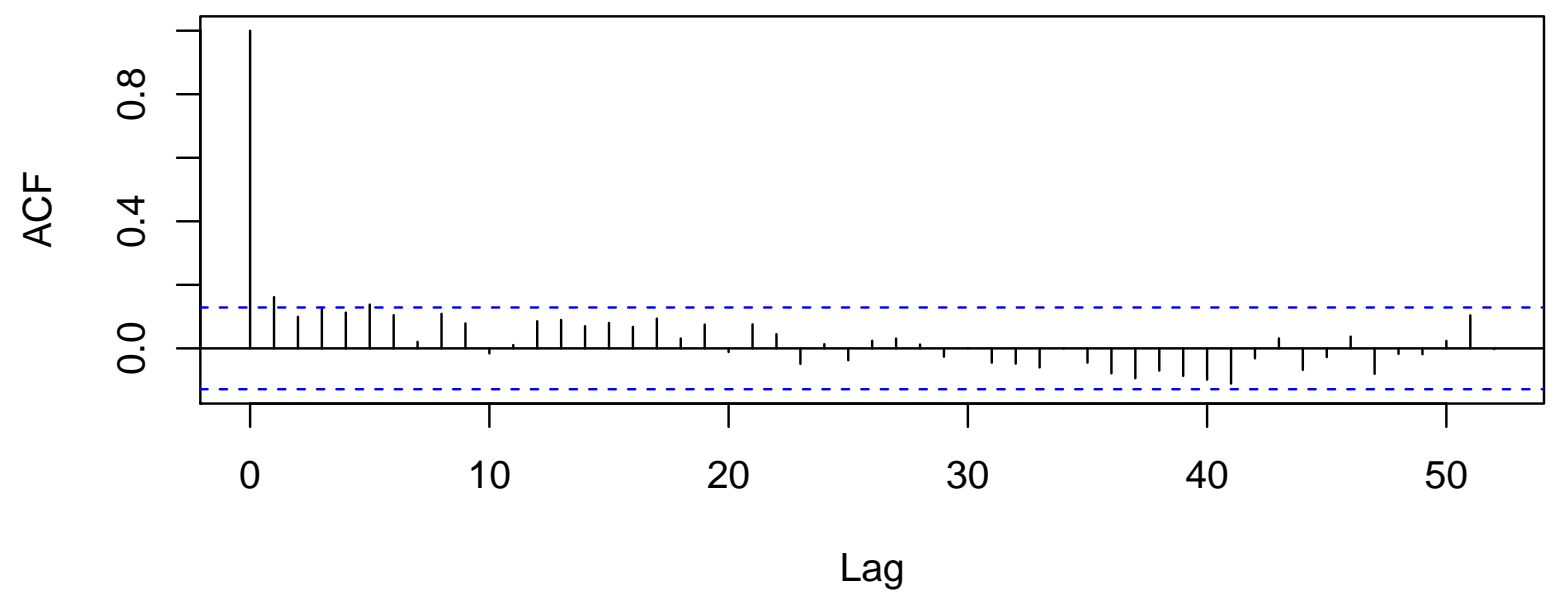

Autocorrelation Function for the Residuals from Updated Model (2)

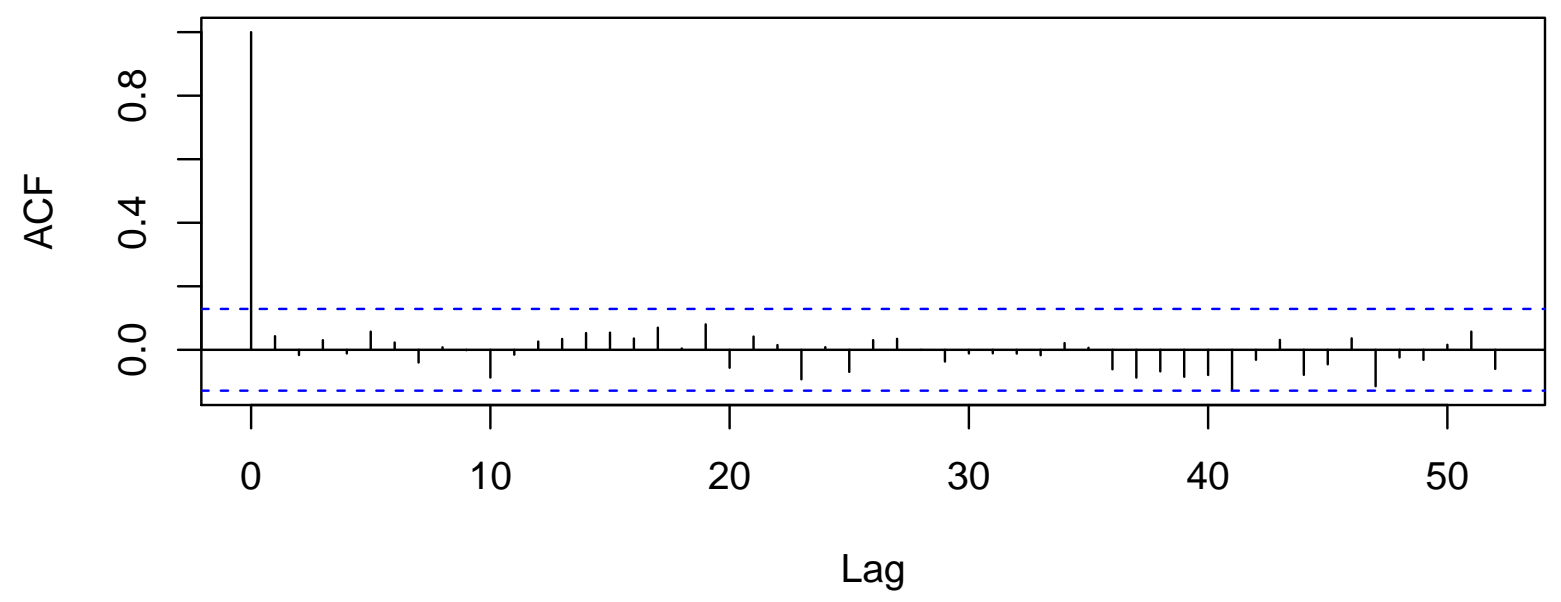




\subsection{A Poisson Regression Model as an Alternative to Model (2) for arson}

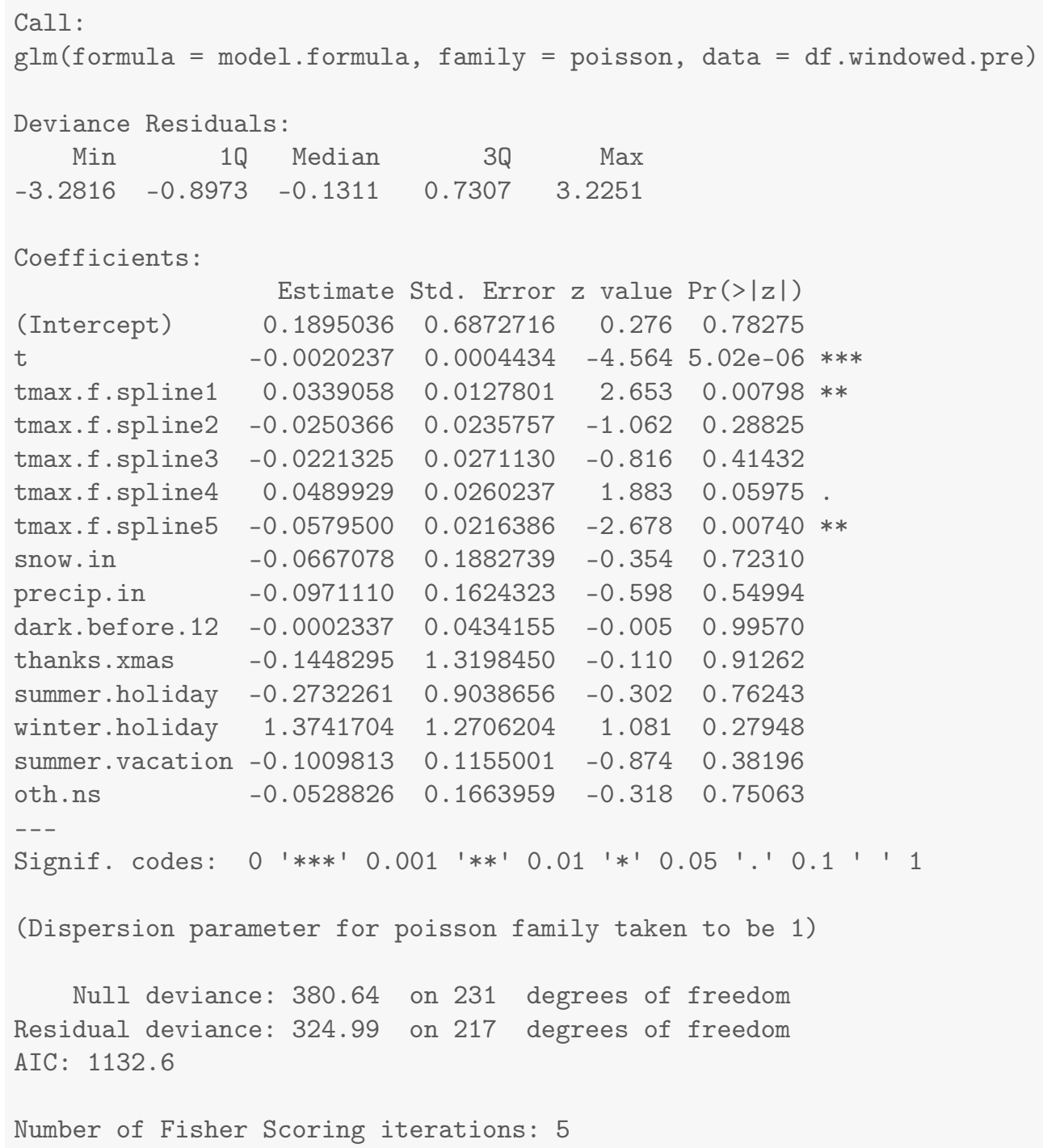




\section{Results for northwestern}

\subsection{Summary Values for northwestern}

Table 18.1: Descriptives for Outcome Before Ferguson Protests Begin

\begin{tabular}{lcccccccc}
\hline \hline Statistic & $\mathrm{N}$ & Mean & St. Dev. & Min & $\operatorname{Pctl}(25)$ & Median & $\operatorname{Pctl}(75)$ & Max \\
\hline northwestern & 232 & 95.052 & 15.257 & 56 & 85 & 94 & 105 & 151 \\
\hline
\end{tabular}

Table 18.2: Descriptives for Outcome After Ferguson Protests Begin

\begin{tabular}{lcccccccc}
\hline \hline Statistic & $\mathrm{N}$ & Mean & St. Dev. & Min & $\operatorname{Pctl}(25)$ & Median & $\operatorname{Pctl}(75)$ & Max \\
\hline northwestern & 290 & 91.317 & 16.424 & 51 & 81 & 91 & 100 & 181 \\
\hline
\end{tabular}




\subsection{Four Models for northwestern}

Table 18.3: Four Models that Differ on the Specification of Adjustment and Intervention Variables

\begin{tabular}{|c|c|c|c|c|}
\hline & \multicolumn{4}{|c|}{ Outcome: Count Per Week } \\
\hline & $(1)$ & (2) & (3) & $(4)$ \\
\hline Time (counter in weeks) & -0.01 & -0.001 & & 0.01 \\
\hline After Ferguson Protests Begin (week of 8/11/14 onward) & -10.32 & & -7.29 & -8.91 \\
\hline After Gray Protests Begin (week of 4/20/15 onward) & 16.35 & & 12.07 & 11.62 \\
\hline Unrest and National Guard $(4 / 27 / 15-5 / 3 / 2015)$ & 5.23 & & 5.65 & 6.80 \\
\hline After Batts Fired (week of 7/13/15 onward) & -7.19 & & -12.92 & -13.20 \\
\hline After Davis Confirmed (week of 10/19/15 onward) & -2.82 & & 1.39 & 2.16 \\
\hline After Davis First Year (week of 10/17/16 onward) & 13.09 & & 11.69 & 11.85 \\
\hline After De Sousa Begins (week of 1/19/18 onward) & -21.62 & & -15.61 & -15.63 \\
\hline After Tuggle Begins (week of 5/14/18 onward) & 9.73 & & 3.33 & 1.95 \\
\hline After Harrison Begins (week of 2/11/19 onward) & -1.05 & & -0.78 & -0.19 \\
\hline Average Maximum Temperature to 50 Degrees & & 1.32 & & 0.58 \\
\hline Plus Degrees in the $50 \mathrm{~s}$ & & -0.80 & & 0.28 \\
\hline Plus Degrees in the 60s & & 0.52 & & 0.20 \\
\hline Plus Degrees in the 70s & & -0.83 & & -0.74 \\
\hline Plus Degrees Greater Than 80 & & -0.17 & & 0.001 \\
\hline Snowfall (inches) & & -6.08 & & -5.32 \\
\hline Precipitation (inches) & & -1.74 & & 0.71 \\
\hline Darkness Before Midnight (hours) & & 6.01 & & 5.74 \\
\hline Thanksgiving/Christmas (proportion of week) & & 26.44 & & 1.08 \\
\hline Winter Holiday (proportion of week) & & 8.96 & & -8.68 \\
\hline Other Out-of-School Days (proportion of week) & & -6.18 & & -6.38 \\
\hline Summer Vacation (proportion of week) & & 1.98 & & -0.57 \\
\hline Summer Holiday (proportion of week) & & -15.53 & & -21.17 \\
\hline Observations & 522 & 232 & 522 & 522 \\
\hline $\mathrm{R}^{2}$ & 0.12 & 0.27 & 0.11 & 0.31 \\
\hline
\end{tabular}


18.3 Least Squares Updated Model (2) for northwestern northwestern

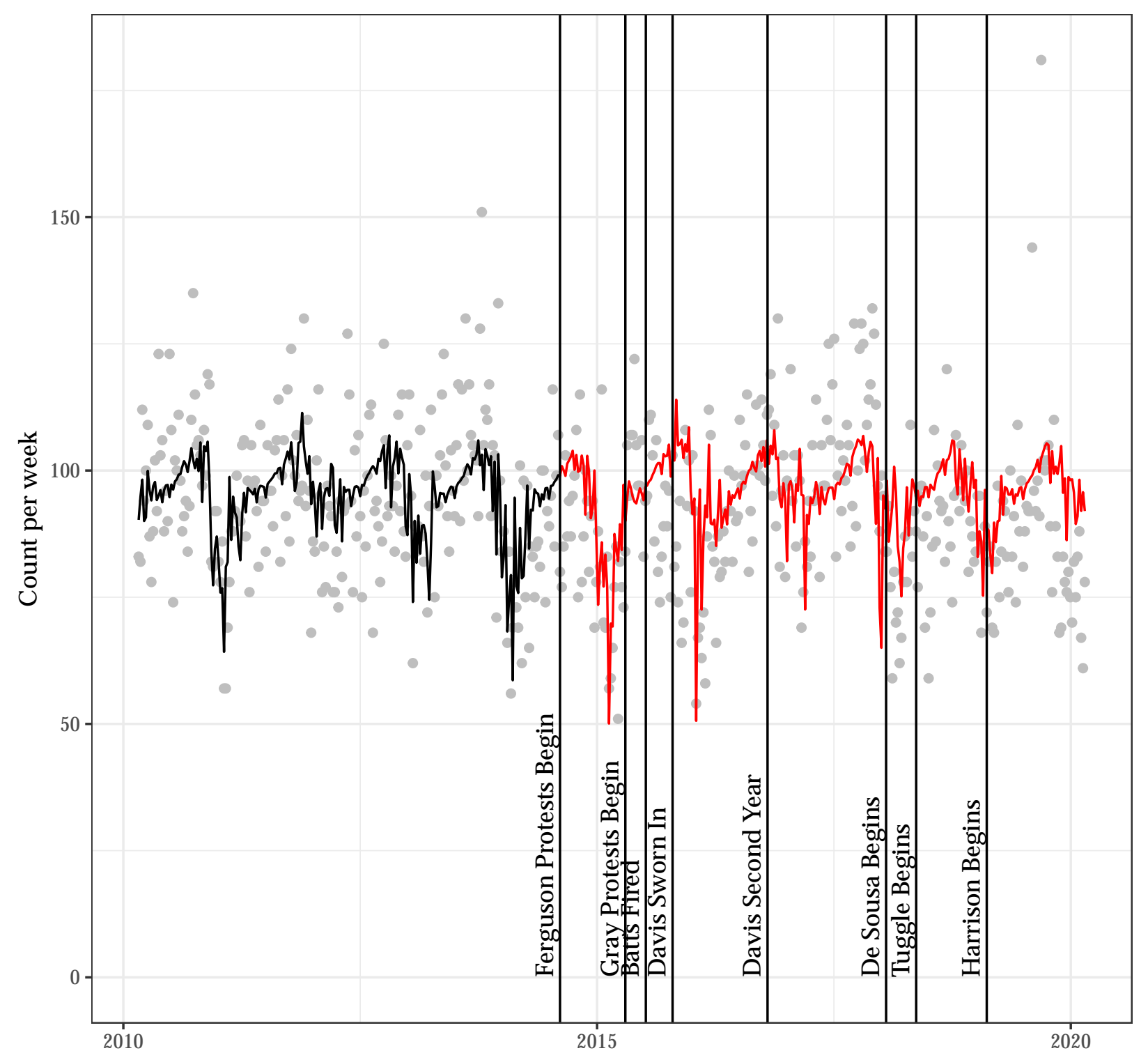




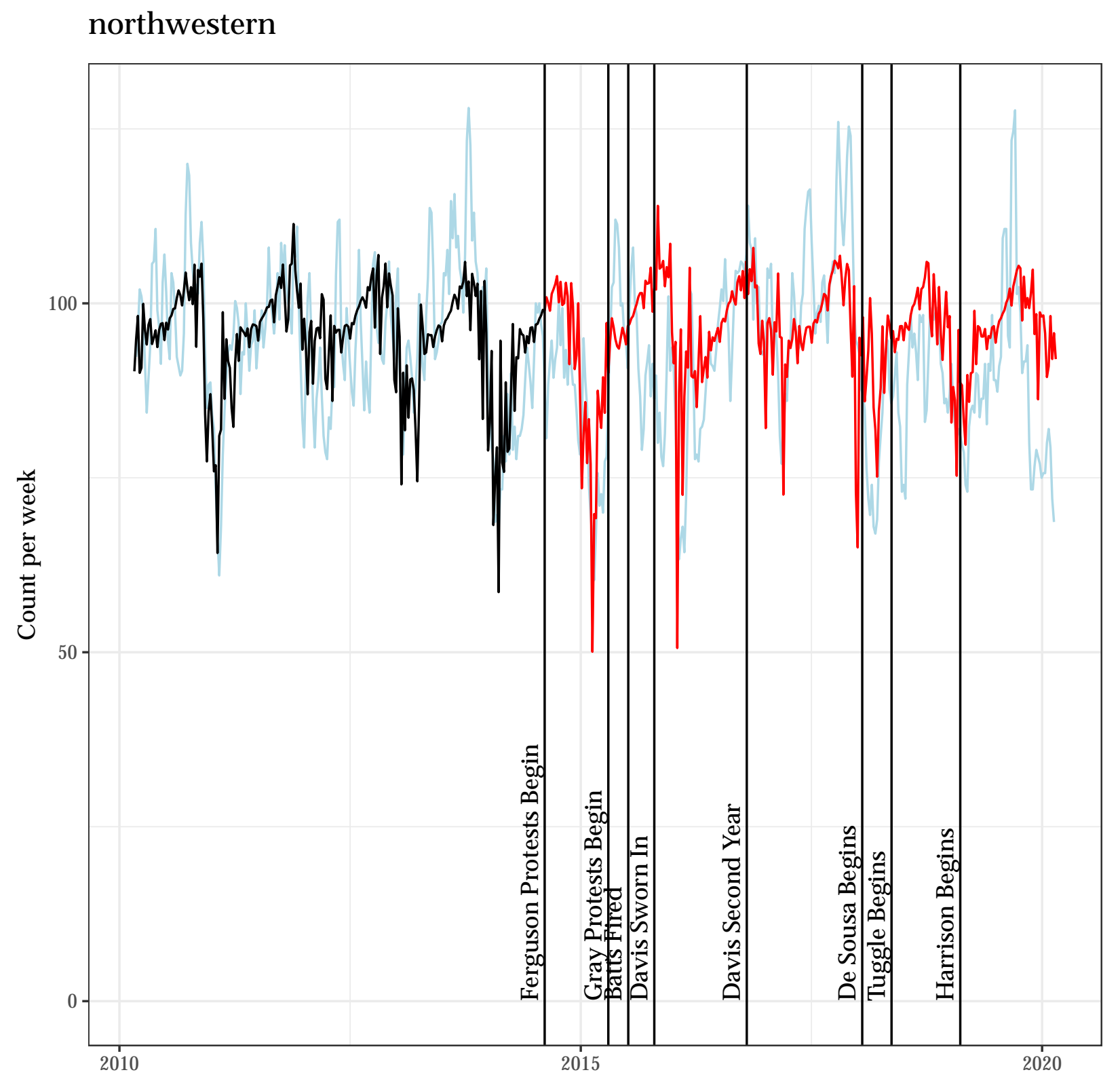


Autocorrelation Function for the Observed Outcome for Updated Model (2)

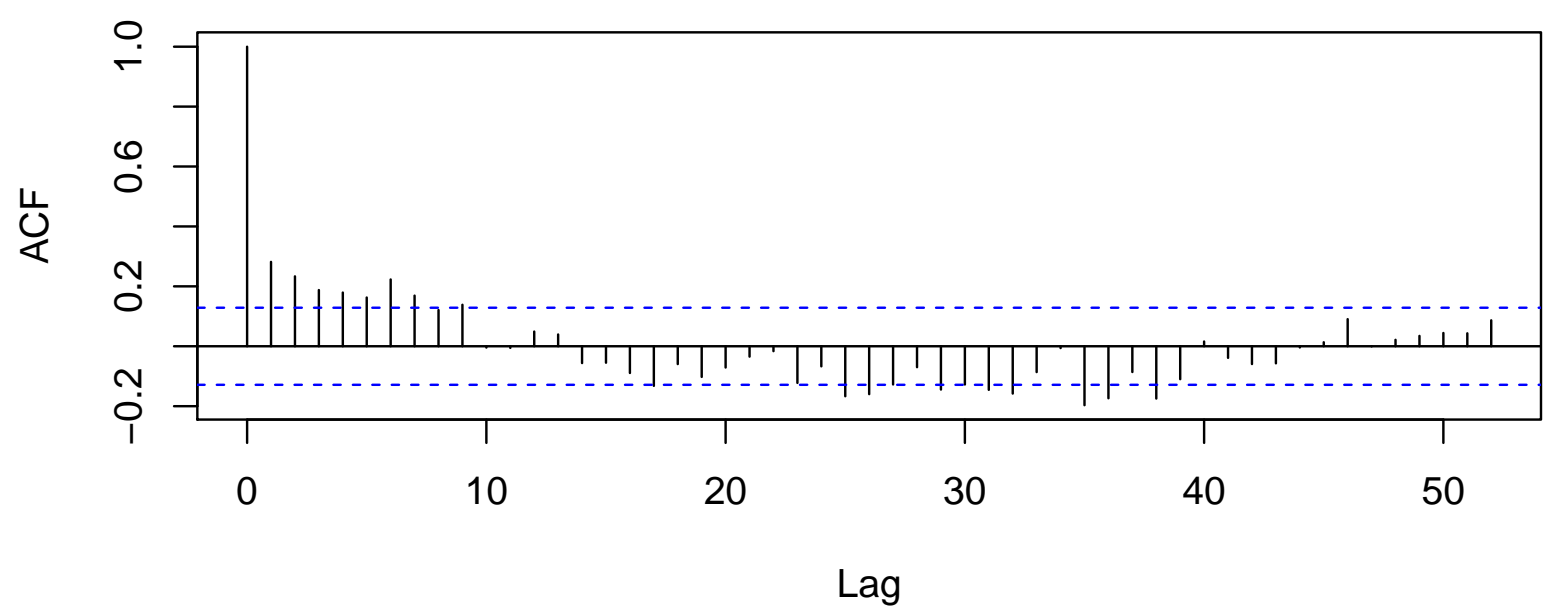

Autocorrelation Function for the Residuals from Updated Model (2)

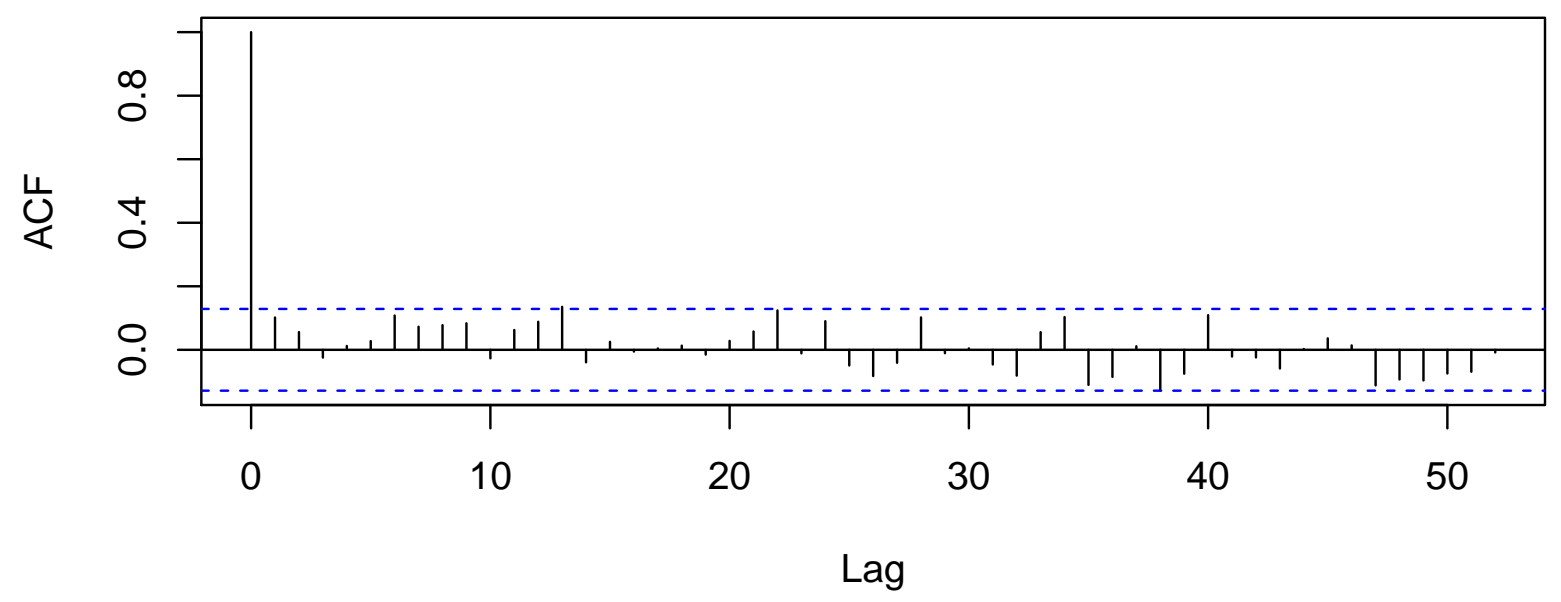




\subsection{A Poisson Regression Model as an Alternative to Model (2) for northwestern}

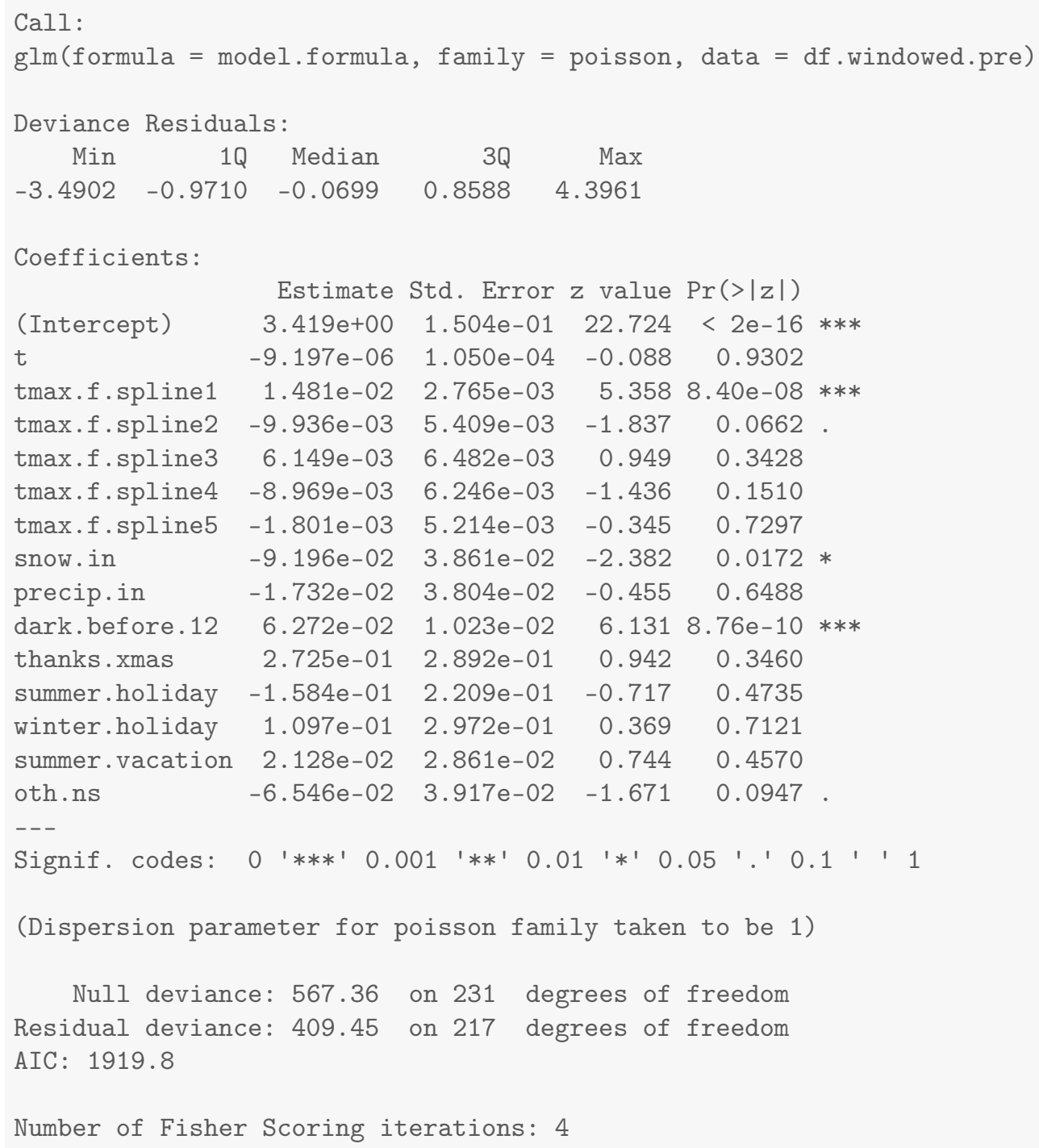




\section{Results for northern}

\subsection{Summary Values for northern}

Table 19.1: Descriptives for Outcome Before Ferguson Protests Begin

\begin{tabular}{lcccccccc}
\hline \hline Statistic & $\mathrm{N}$ & Mean & St. Dev. & Min & Pctl(25) & Median & Pctl(75) & Max \\
\hline northern & 232 & 104.272 & 17.723 & 56 & 93 & 104.5 & 116 & 149 \\
\hline
\end{tabular}

Table 19.2: Descriptives for Outcome After Ferguson Protests Begin

\begin{tabular}{lcccccccc}
\hline \hline Statistic & $\mathrm{N}$ & Mean & St. Dev. & Min & Pctl(25) & Median & Pctl(75) & Max \\
\hline northern & 290 & 102.014 & 21.026 & 58 & 87 & 102 & 115 & 172 \\
\hline
\end{tabular}




\subsection{Four Models for northern}

Table 19.3: Four Models that Differ on the Specification of Adjustment and Intervention Variables

\begin{tabular}{|c|c|c|c|c|}
\hline & \multicolumn{4}{|c|}{ Outcome: Count Per Week } \\
\hline & $(1)$ & (2) & (3) & $(4)$ \\
\hline Time (counter in weeks) & 0.01 & 0.03 & & 0.03 \\
\hline After Ferguson Protests Begin (week of 8/11/14 onward) & -5.83 & & -2.44 & -2.02 \\
\hline After Gray Protests Begin (week of $4 / 20 / 15$ onward) & 33.61 & & 20.50 & 22.29 \\
\hline Unrest and National Guard $(4 / 27 / 15-5 / 3 / 2015)$ & -17.03 & & -10.33 & -12.58 \\
\hline After Batts Fired (week of 7/13/15 onward) & -8.75 & & -11.90 & -13.29 \\
\hline After Davis Confirmed (week of 10/19/15 onward) & -23.48 & & -15.74 & -16.03 \\
\hline After Davis First Year (week of 10/17/16 onward) & 5.86 & & 6.29 & 5.24 \\
\hline After De Sousa Begins (week of 1/19/18 onward) & -25.15 & & -18.15 & -19.87 \\
\hline After Tuggle Begins (week of 5/14/18 onward) & 16.85 & & 9.63 & 8.93 \\
\hline After Harrison Begins (week of 2/11/19 onward) & -15.17 & & -12.73 & -14.39 \\
\hline Average Maximum Temperature to 50 Degrees & & 0.23 & & 0.75 \\
\hline Plus Degrees in the $50 \mathrm{~s}$ & & 1.17 & & 0.18 \\
\hline Plus Degrees in the 60s & & -1.02 & & -0.34 \\
\hline Plus Degrees in the 70s & & 0.99 & & 0.20 \\
\hline Plus Degrees Greater Than 80 & & -1.42 & & -0.47 \\
\hline Snowfall (inches) & & -6.07 & & -5.30 \\
\hline Precipitation (inches) & & -5.08 & & -3.79 \\
\hline Darkness Before Midnight (hours) & & 2.55 & & 3.14 \\
\hline Thanksgiving/Christmas (proportion of week) & & 8.41 & & 20.15 \\
\hline Winter Holiday (proportion of week) & & -67.06 & & -67.50 \\
\hline Other Out-of-School Days (proportion of week) & & 0.13 & & -6.63 \\
\hline Summer Vacation (proportion of week) & & -2.35 & & -0.22 \\
\hline Summer Holiday (proportion of week) & & -47.83 & & -37.86 \\
\hline Observations & 522 & 232 & 522 & 522 \\
\hline $\mathrm{R}^{2}$ & 0.20 & 0.36 & 0.27 & 0.44 \\
\hline
\end{tabular}


19.3 Least Squares Updated Model (2) for northern northern

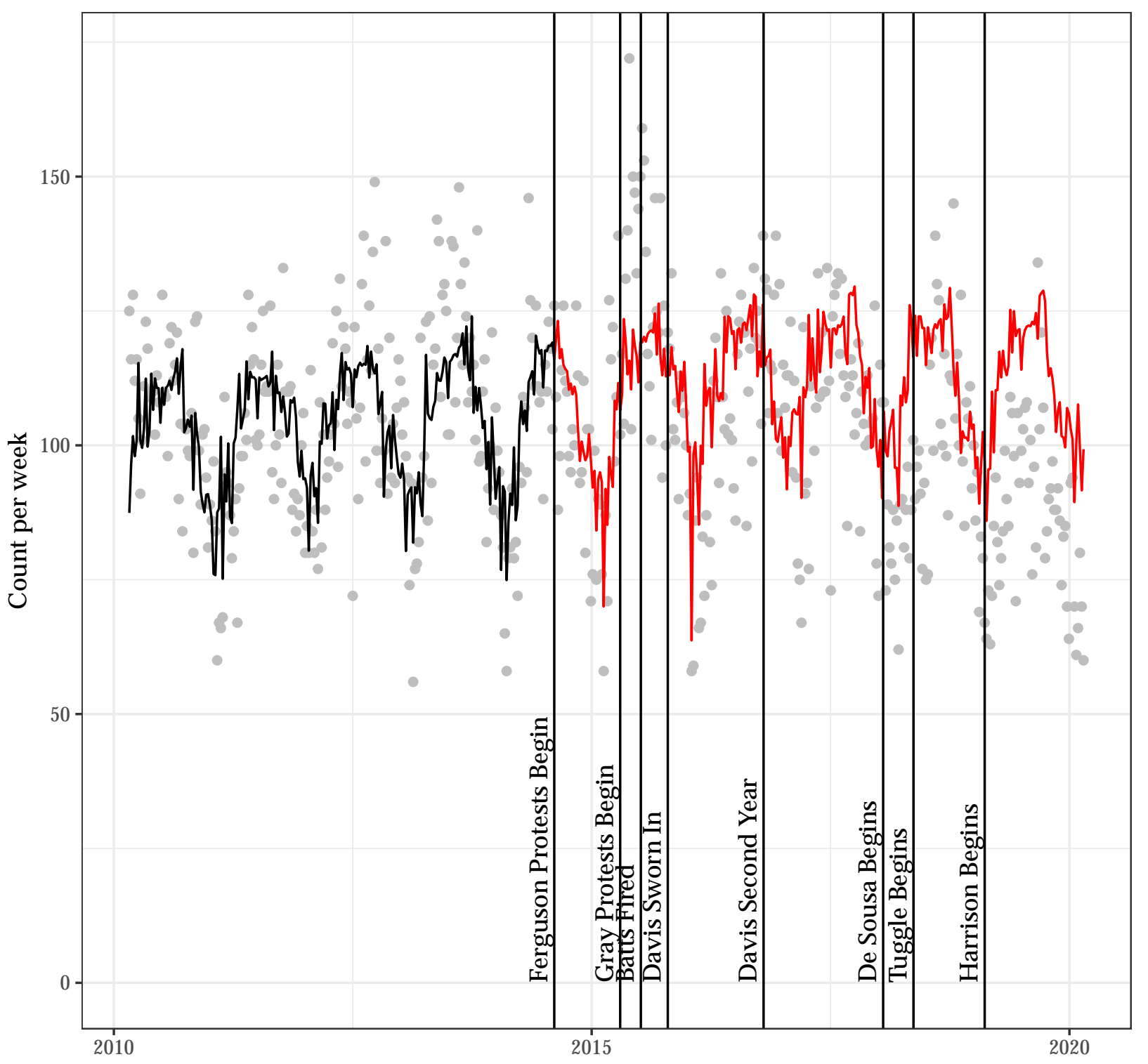




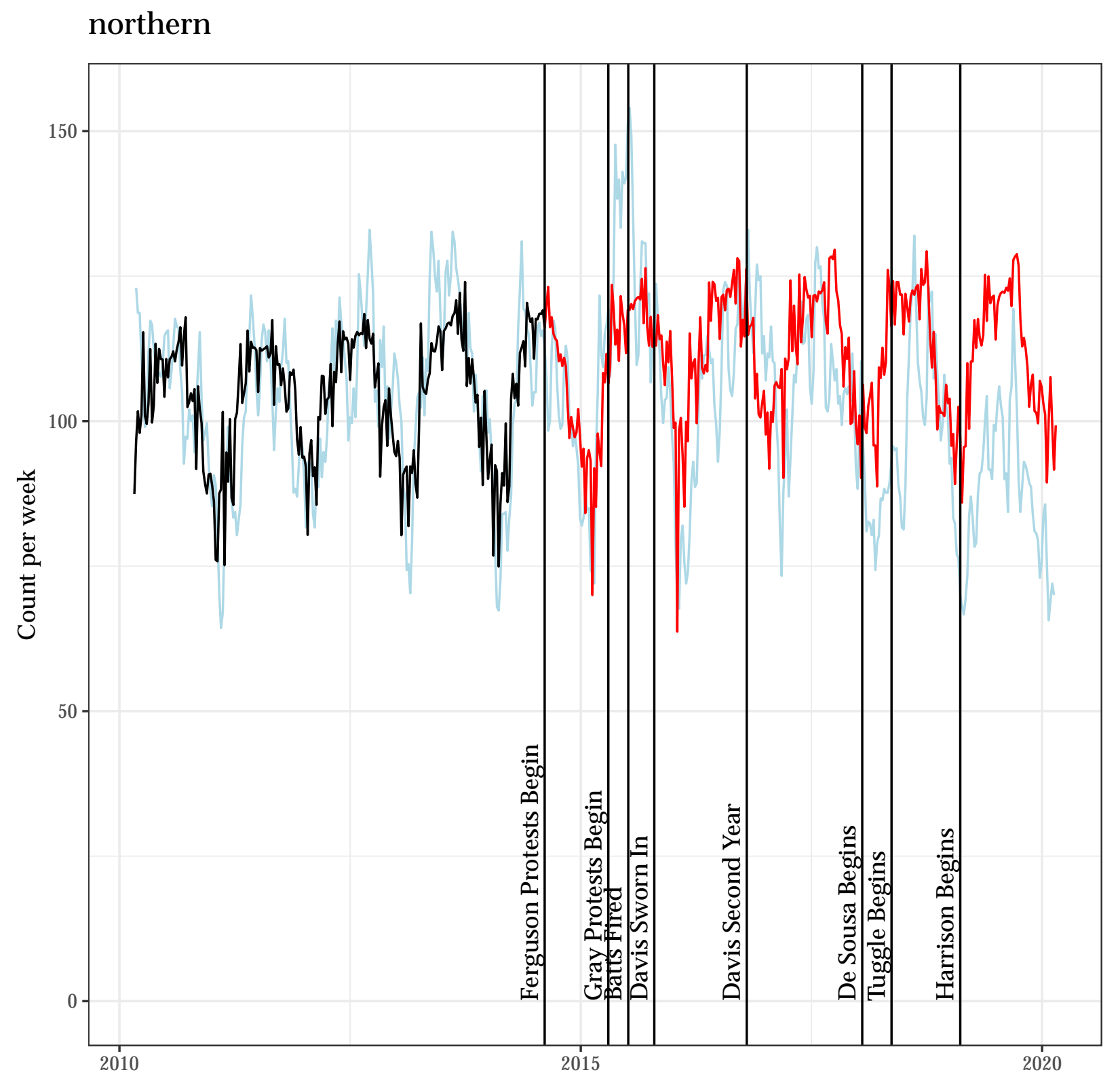


Autocorrelation Function for the Observed Outcome for Updated Model (2)

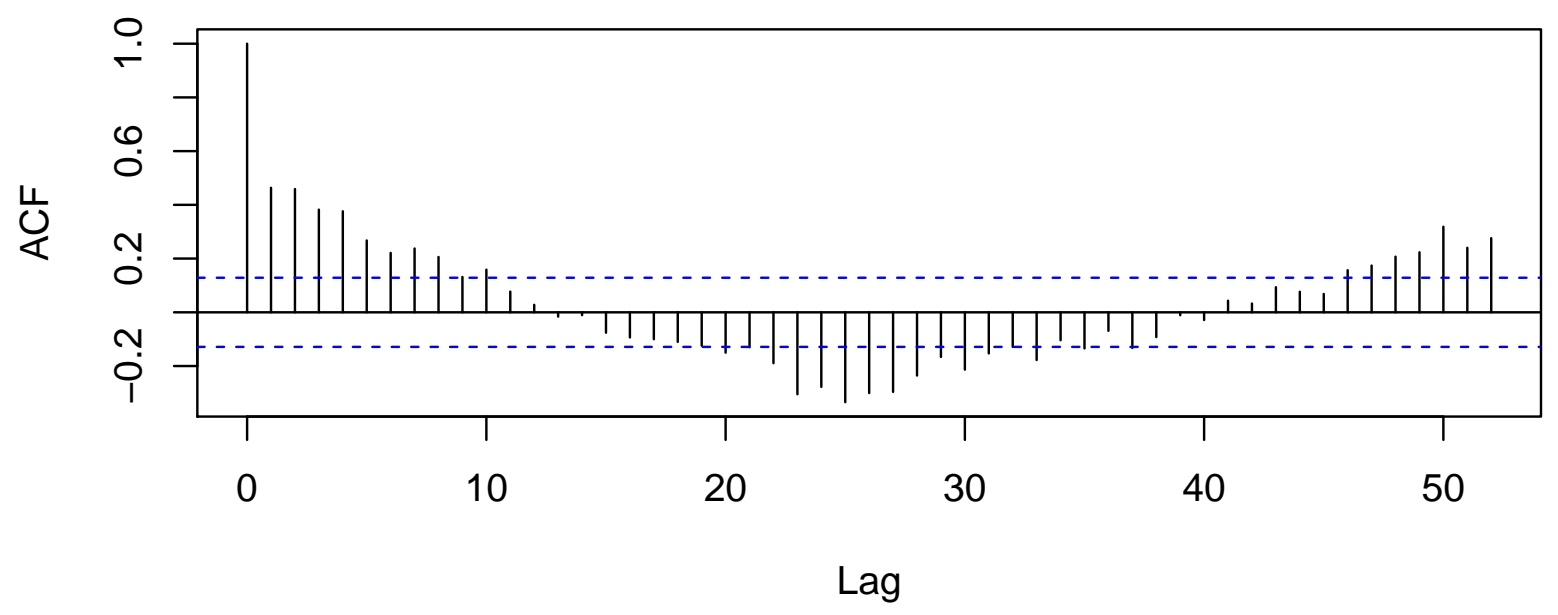

Autocorrelation Function for the Residuals from Updated Model (2)

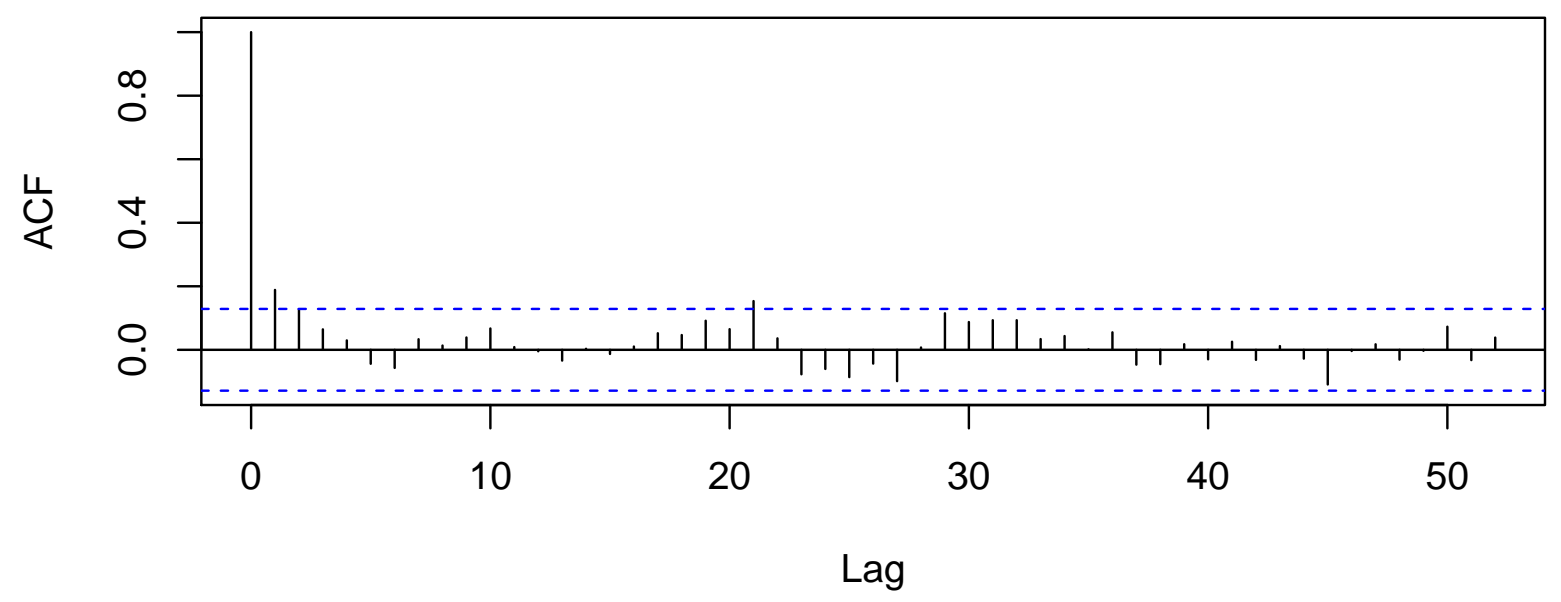




\subsection{A Poisson Regression Model as an Alternative to Model (2) for northern}

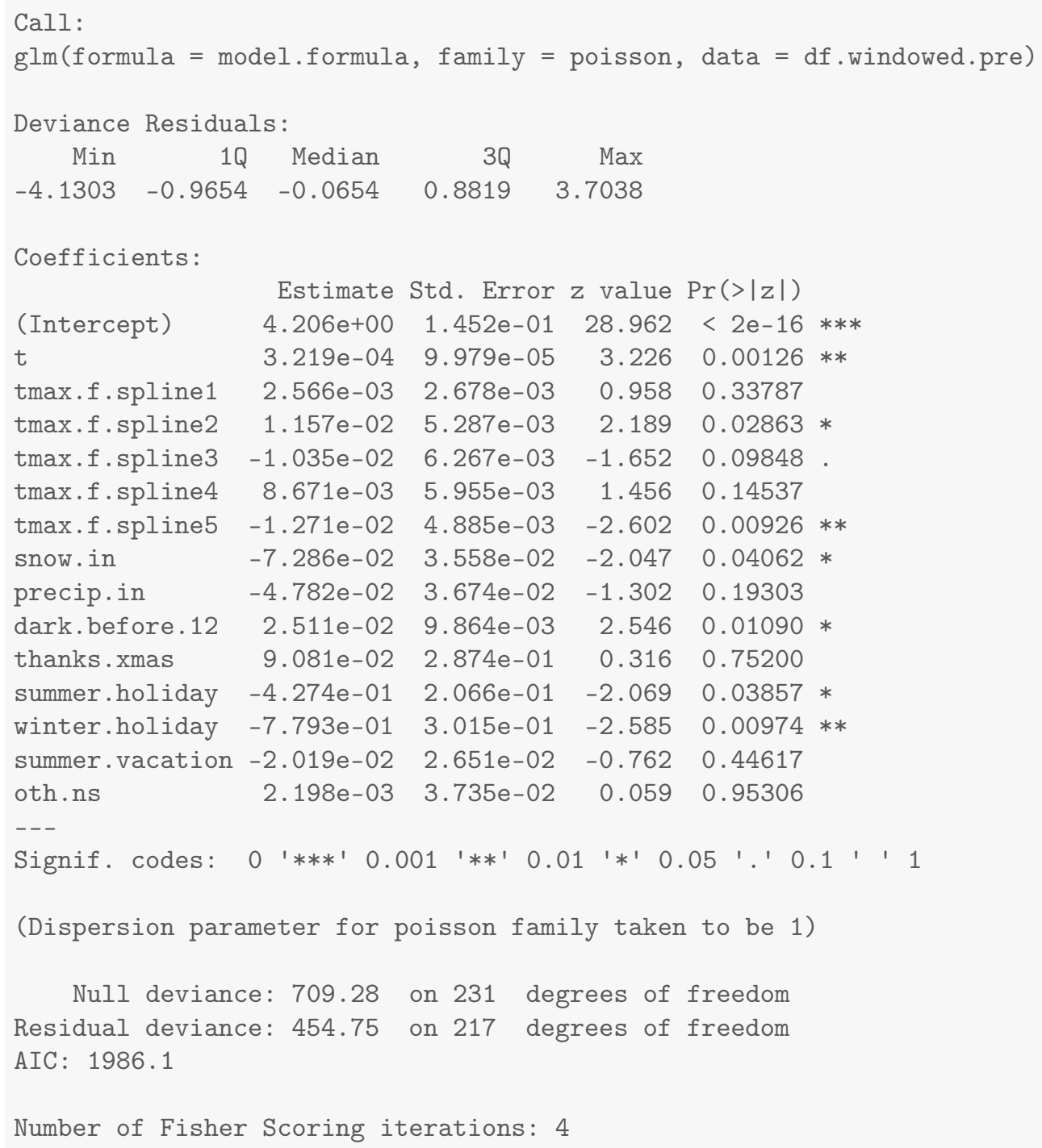




\section{Results for northeastern}

\subsection{Summary Values for northeastern}

Table 20.1: Descriptives for Outcome Before Ferguson Protests Begin

\begin{tabular}{lcccccccc}
\hline \hline Statistic & $\mathrm{N}$ & Mean & St. Dev. & Min & $\operatorname{Pctl}(25)$ & Median & $\operatorname{Pctl}(75)$ & Max \\
\hline northeastern & 232 & 149.030 & 20.718 & 84 & 134.8 & 148 & 162.2 & 207 \\
\hline
\end{tabular}

Table 20.2: Descriptives for Outcome After Ferguson Protests Begin

\begin{tabular}{lcccccccc}
\hline \hline Statistic & $\mathrm{N}$ & Mean & St. Dev. & Min & $\operatorname{Pctl}(25)$ & Median & $\operatorname{Pctl}(75)$ & Max \\
\hline northeastern & 290 & 137.790 & 22.366 & 79 & 126 & 138 & 152 & 206 \\
\hline
\end{tabular}




\subsection{Four Models for northeastern}

Table 20.3: Four Models that Differ on the Specification of Adjustment and Intervention Variables

\begin{tabular}{|c|c|c|c|c|}
\hline & \multicolumn{4}{|c|}{ Outcome: Count Per Week } \\
\hline & $(1)$ & (2) & (3) & $(4)$ \\
\hline Time (counter in weeks) & -0.04 & -0.02 & & -0.02 \\
\hline After Ferguson Protests Begin (week of 8/11/14 onward) & -4.88 & & -2.10 & -2.26 \\
\hline After Gray Protests Begin (week of 4/20/15 onward) & 20.28 & & 12.17 & 10.59 \\
\hline Unrest and National Guard $(4 / 27 / 15-5 / 3 / 2015)$ & 5.54 & & 6.69 & 9.89 \\
\hline After Batts Fired (week of 7/13/15 onward) & -3.71 & & -8.42 & -8.36 \\
\hline After Davis Confirmed (week of 10/19/15 onward) & -26.02 & & -21.01 & -19.49 \\
\hline After Davis First Year (week of 10/17/16 onward) & 28.13 & & 25.89 & 26.75 \\
\hline After De Sousa Begins (week of 1/19/18 onward) & -18.08 & & -13.37 & -12.83 \\
\hline After Tuggle Begins (week of 5/14/18 onward) & -1.07 & & -8.53 & -9.72 \\
\hline After Harrison Begins (week of 2/11/19 onward) & -1.64 & & -2.39 & -0.60 \\
\hline Average Maximum Temperature to 50 Degrees & & 0.60 & & 0.55 \\
\hline Plus Degrees in the $50 \mathrm{~s}$ & & 0.91 & & 0.42 \\
\hline Plus Degrees in the 60s & & -1.20 & & -0.43 \\
\hline Plus Degrees in the 70s & & -0.15 & & 0.12 \\
\hline Plus Degrees Greater Than 80 & & 0.04 & & -0.16 \\
\hline Snowfall (inches) & & -20.25 & & -15.55 \\
\hline Precipitation (inches) & & 3.10 & & 3.90 \\
\hline Darkness Before Midnight (hours) & & 4.37 & & 4.07 \\
\hline Thanksgiving/Christmas (proportion of week) & & 10.60 & & -4.25 \\
\hline Winter Holiday (proportion of week) & & -30.52 & & -51.56 \\
\hline Other Out-of-School Days (proportion of week) & & -0.13 & & -8.31 \\
\hline Summer Vacation (proportion of week) & & 1.87 & & -2.80 \\
\hline Summer Holiday (proportion of week) & & -17.98 & & -24.15 \\
\hline Observations & 522 & 232 & 522 & 522 \\
\hline $\mathrm{R}^{2}$ & 0.22 & 0.25 & 0.21 & 0.40 \\
\hline
\end{tabular}


20.3 Least Squares Updated Model (2) for northeastern northeastern

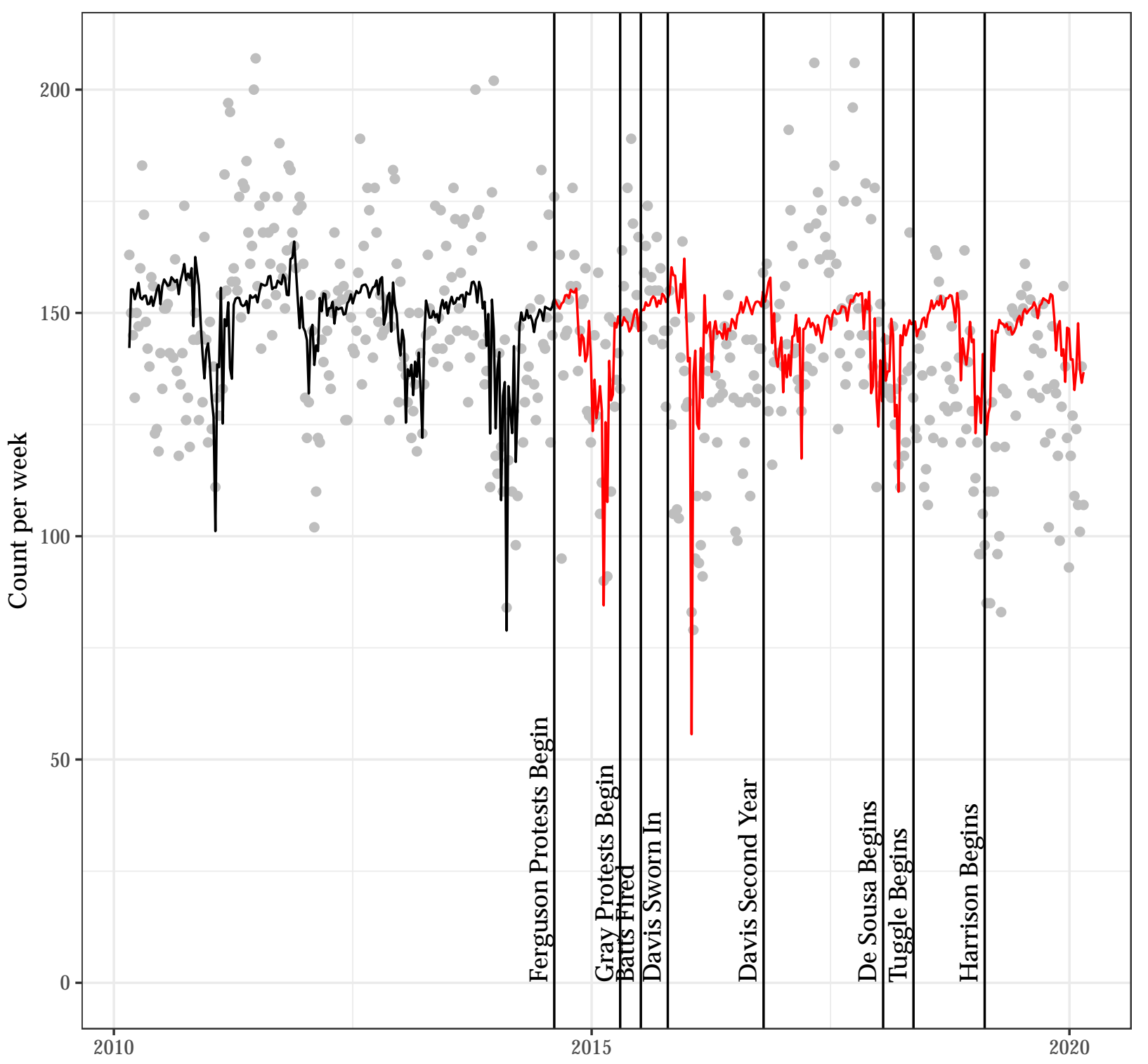




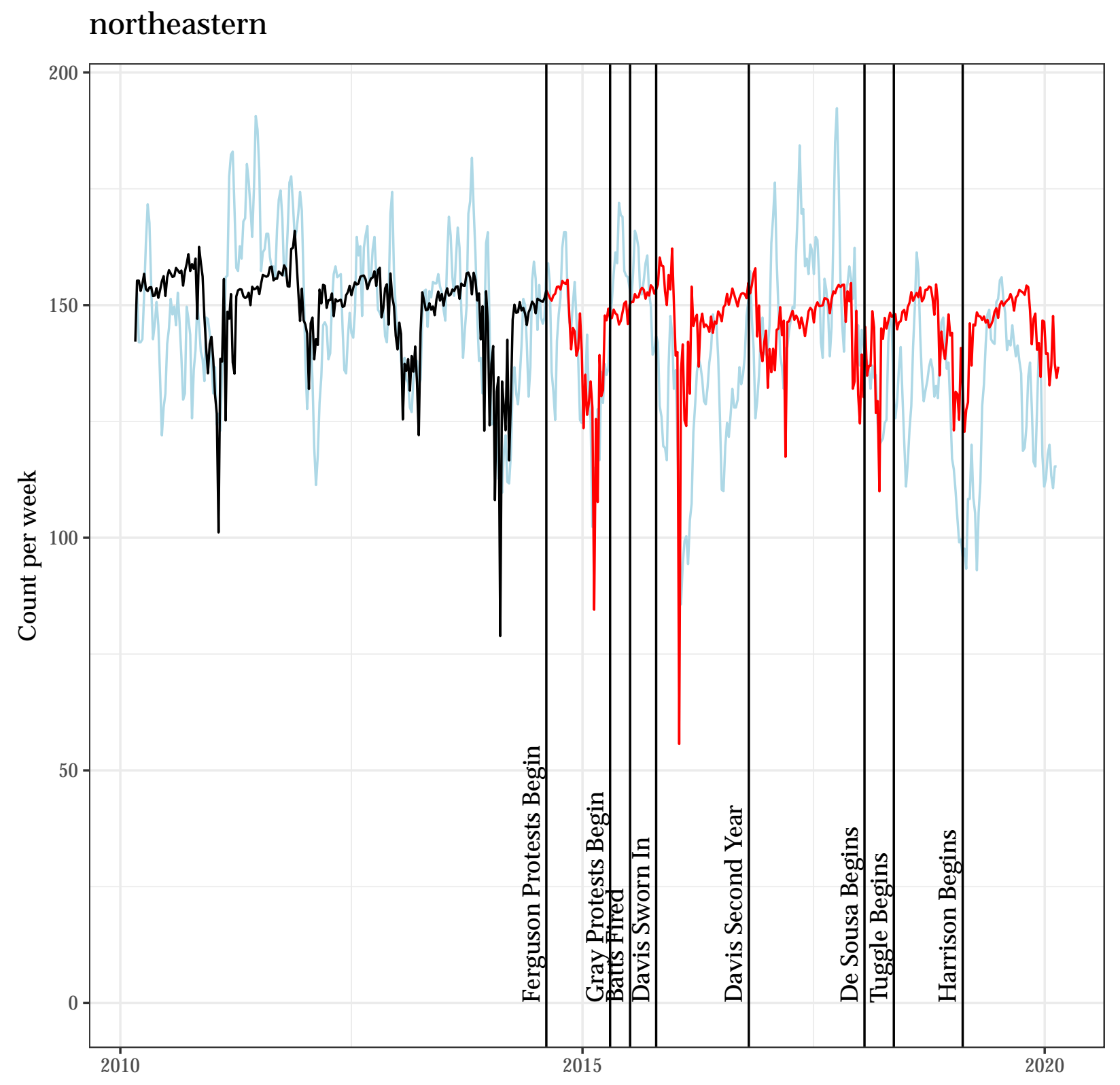


Autocorrelation Function for the Observed Outcome for Updated Model (2)

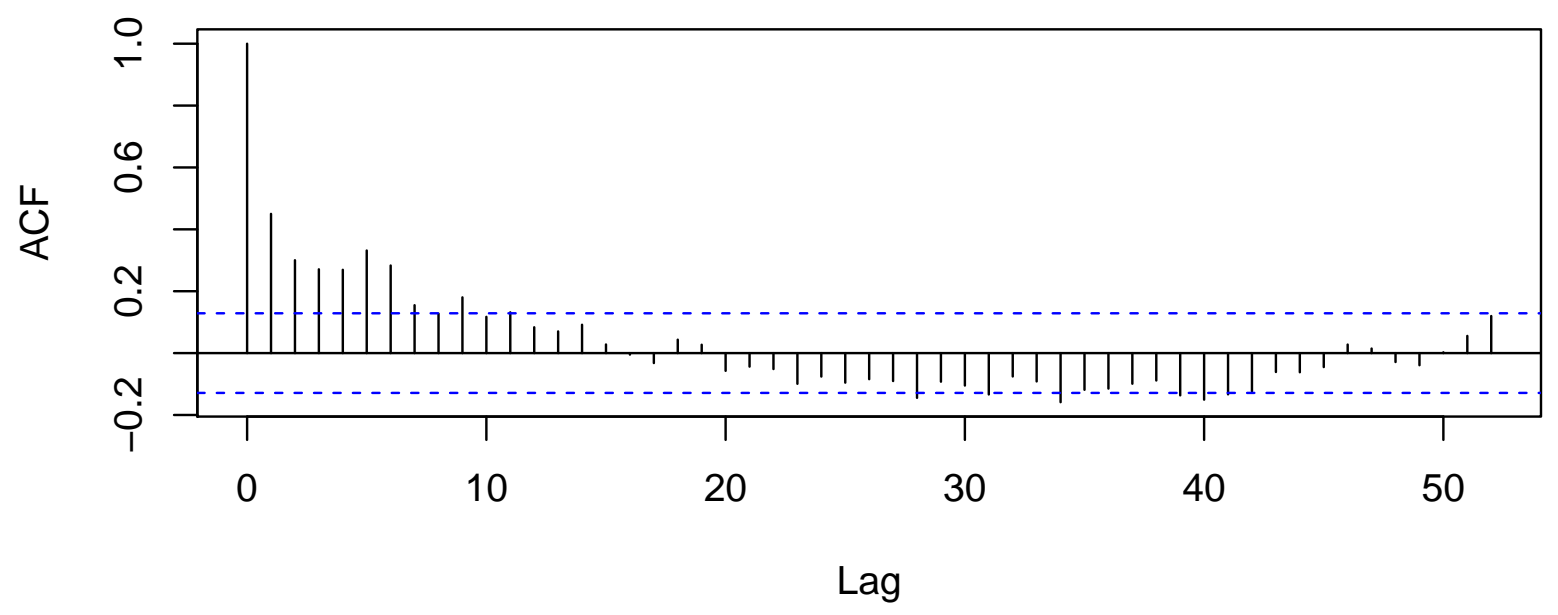

Autocorrelation Function for the Residuals from Updated Model (2)

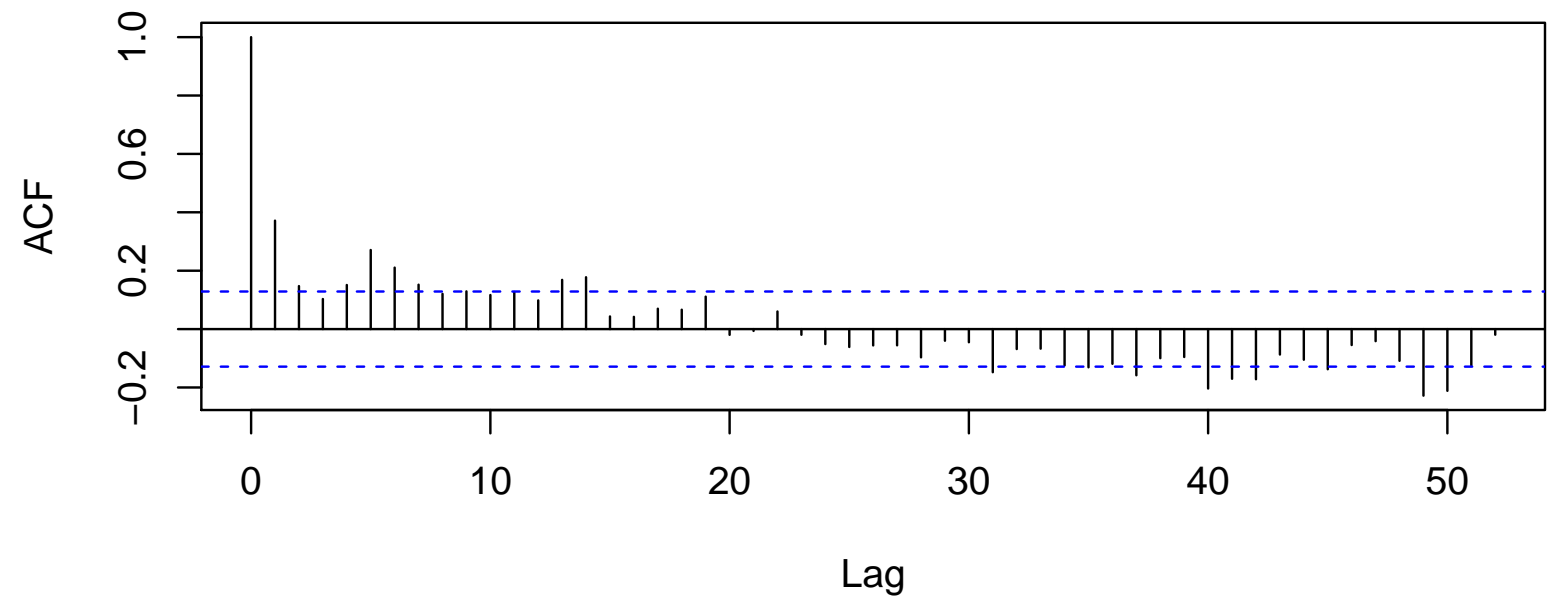




\subsection{A Poisson Regression Model as an Alternative to Model (2) for northeastern}

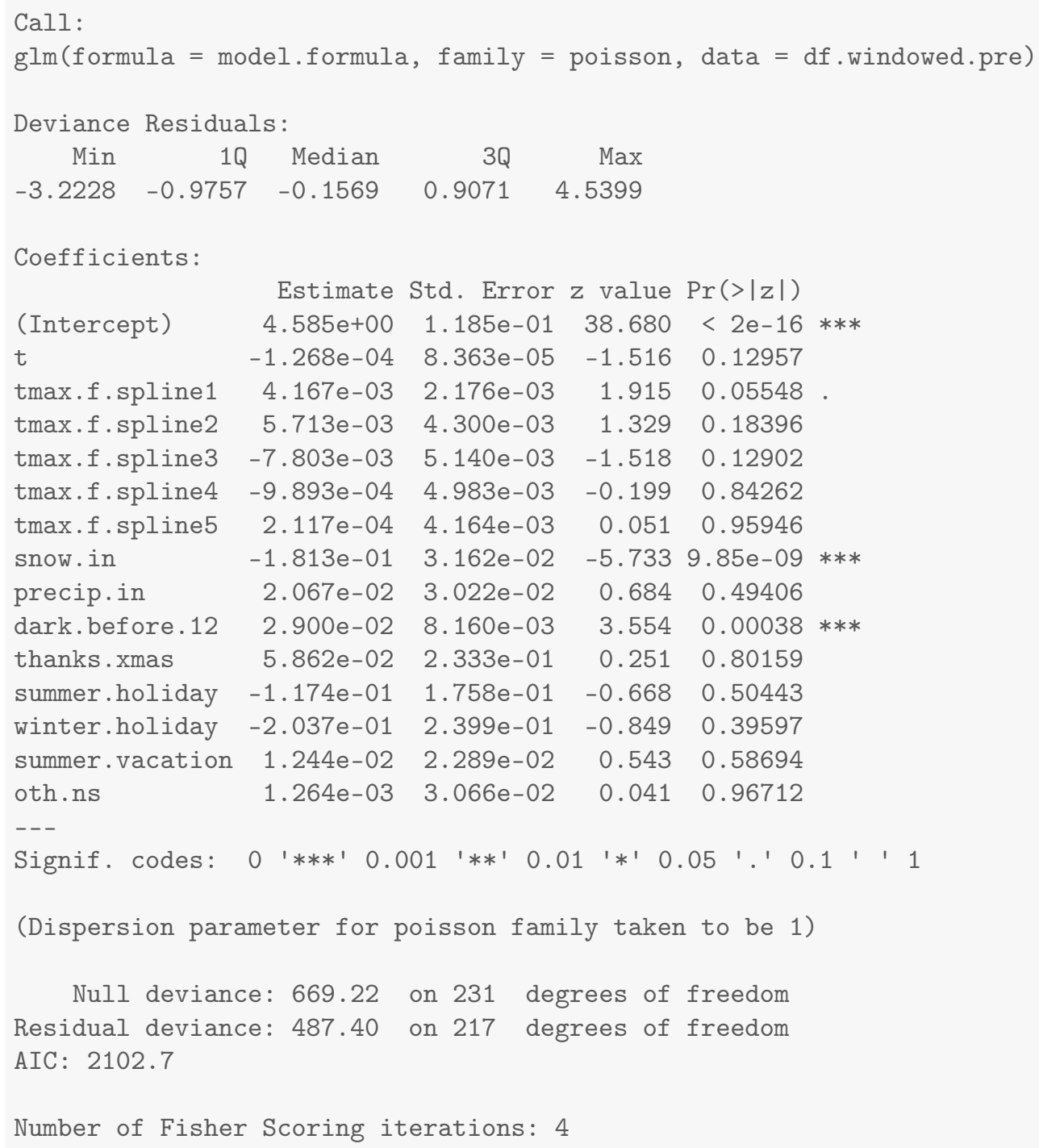




\section{Results for western}

\subsection{Summary Values for western}

Table 21.1: Descriptives for Outcome Before Ferguson Protests Begin

\begin{tabular}{lcccccccc}
\hline \hline Statistic & $\mathrm{N}$ & Mean & St. Dev. & Min & Pctl(25) & Median & Pctl(75) & Max \\
\hline western & 232 & 77.522 & 13.491 & 43 & 67.8 & 77 & 87 & 113 \\
\hline
\end{tabular}

Table 21.2: Descriptives for Outcome After Ferguson Protests Begin

\begin{tabular}{lcccccccc}
\hline \hline Statistic & $\mathrm{N}$ & Mean & St. Dev. & Min & Pctl(25) & Median & Pctl(75) & Max \\
\hline western & 290 & 73.652 & 15.766 & 43 & 64 & 74.5 & 82 & 220 \\
\hline
\end{tabular}




\subsection{Four Models for western}

Table 21.3: Four Models that Differ on the Specification of Adjustment and Intervention Variables

\begin{tabular}{lcccc}
\hline \hline & \multicolumn{3}{c}{ Outcome: Count Per Week } \\
\cline { 2 - 5 } & $(1)$ & $(2)$ & $(3)$ & $(4)$ \\
\hline Time (counter in weeks) & -0.05 & -0.04 & -0.04 \\
After Ferguson Protests Begin (week of 8/11/14 onward) & -5.26 & & -3.89 & -3.48 \\
After Gray Protests Begin (week of 4/20/15 onward) & 20.69 & & 13.51 & 13.58 \\
Unrest and National Guard (4/27/15 - 5/3/2015) & 135.02 & & 134.50 & 136.94 \\
After Batts Fired (week of 7/13/15 onward) & -7.79 & & -9.41 & -9.08 \\
After Davis Confirmed (week of 10/19/15 onward) & 3.33 & & 6.71 & 6.72 \\
After Davis First Year (week of 10/17/16 onward) & 0.55 & & -0.39 & 0.52 \\
After De Sousa Begins (week of 1/19/18 onward) & -6.02 & & -5.29 & -3.66 \\
After Tuggle Begins (week of 5/14/18 onward) & 10.46 & & 6.09 & 6.43 \\
After Harrison Begins (week of 2/11/19 onward) & -6.39 & & -8.10 & -5.95 \\
Average Maximum Temperature to 50 Degrees & & 0.05 & & 0.38 \\
Plus Degrees in the 50s & & 1.30 & & 0.36 \\
Plus Degrees in the 60s & & -0.96 & & -0.33 \\
Plus Degrees in the 70s & & -0.25 & & -0.03 \\
Plus Degrees Greater Than 80 & & 0.02 & & -0.24 \\
Snowfall (inches) & & -3.24 & & 1.33 \\
Precipitation (inches) & & 1.96 & & 1.69 \\
Darkness Before Midnight (hours) & & -32.69 & & 1.72 \\
Thanksgiving/Christmas (proportion of week) & & -65.85 & & -44.32 \\
Winter Holiday (proportion of week) & & -4.80 & & -3.67 \\
Other Out-of-School Days (proportion of week) & & -4.07 & & -3.87 \\
Summer Vacation (proportion of week) & -1.77 & & 0.29 \\
Summer Holiday (proportion of week) & 522 & 232 & 522 & 522 \\
Observations & 0.30 & 0.33 & 0.29 & 0.44 \\
\hline R & & & & \\
\hline \hline
\end{tabular}




\subsection{Least Squares Updated Model (2) for western}

western

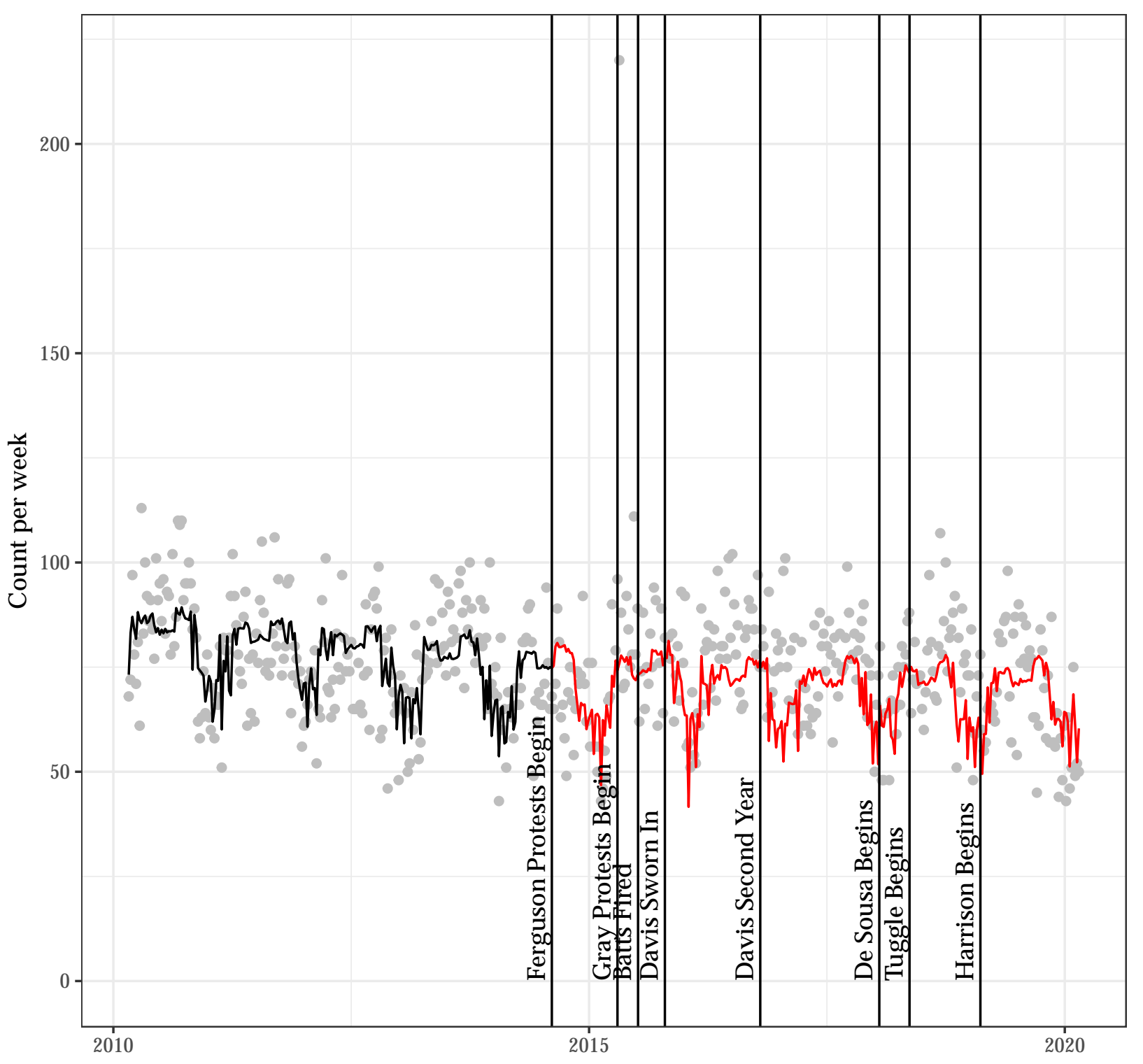




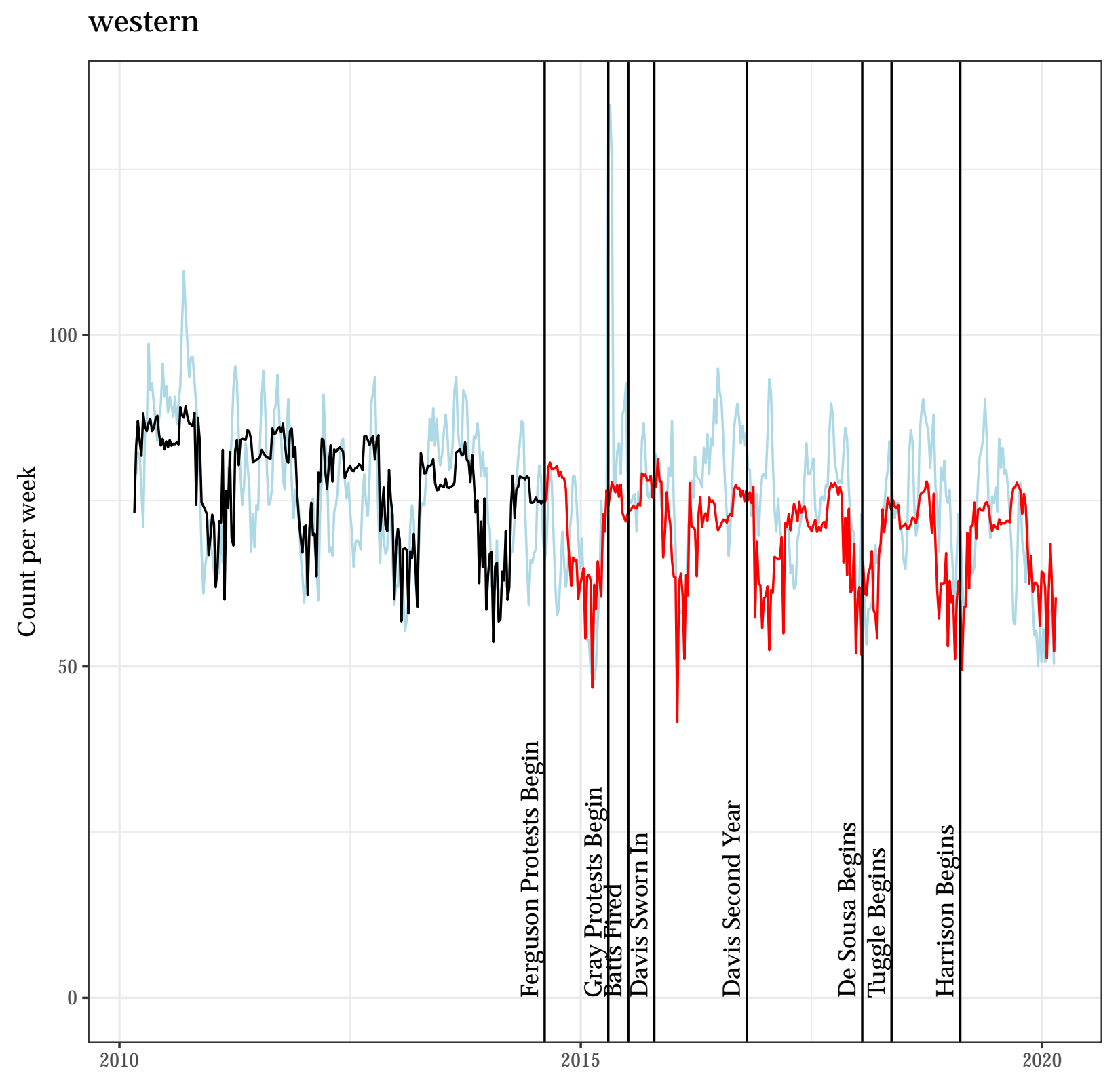


Autocorrelation Function for the Observed Outcome for Updated Model (2)

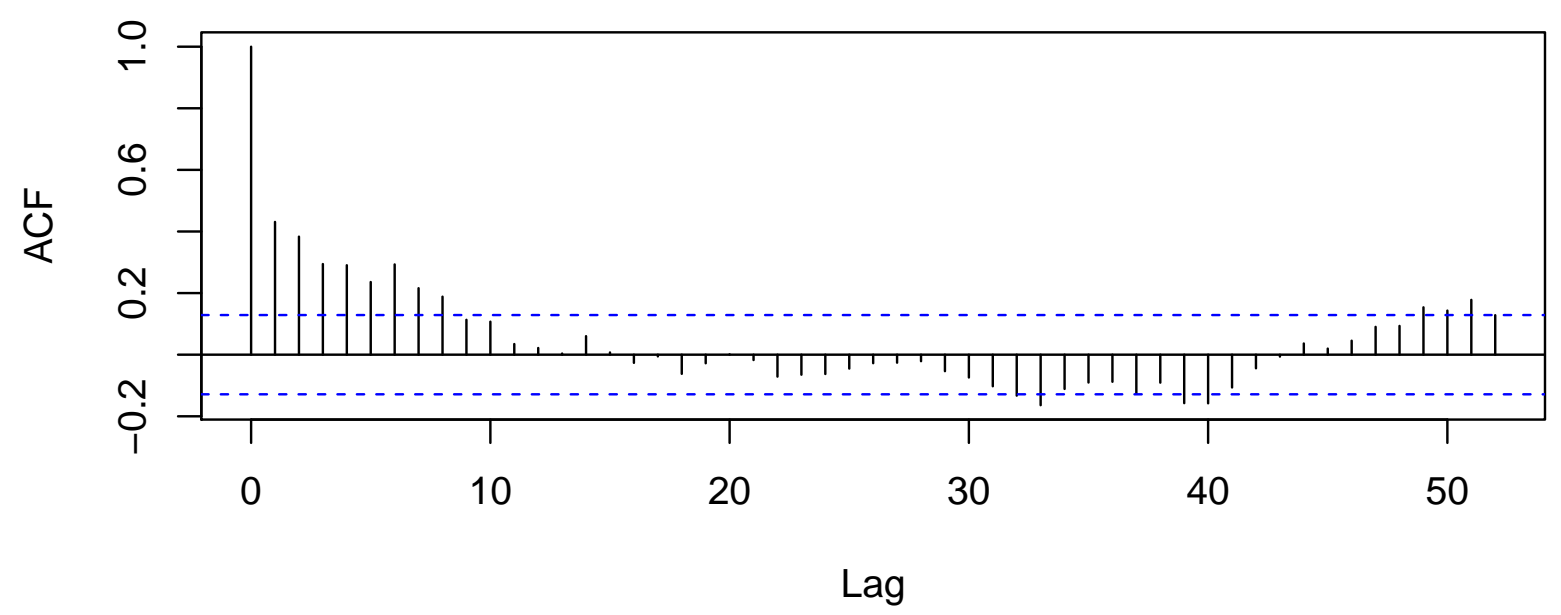

Autocorrelation Function for the Residuals from Updated Model (2)

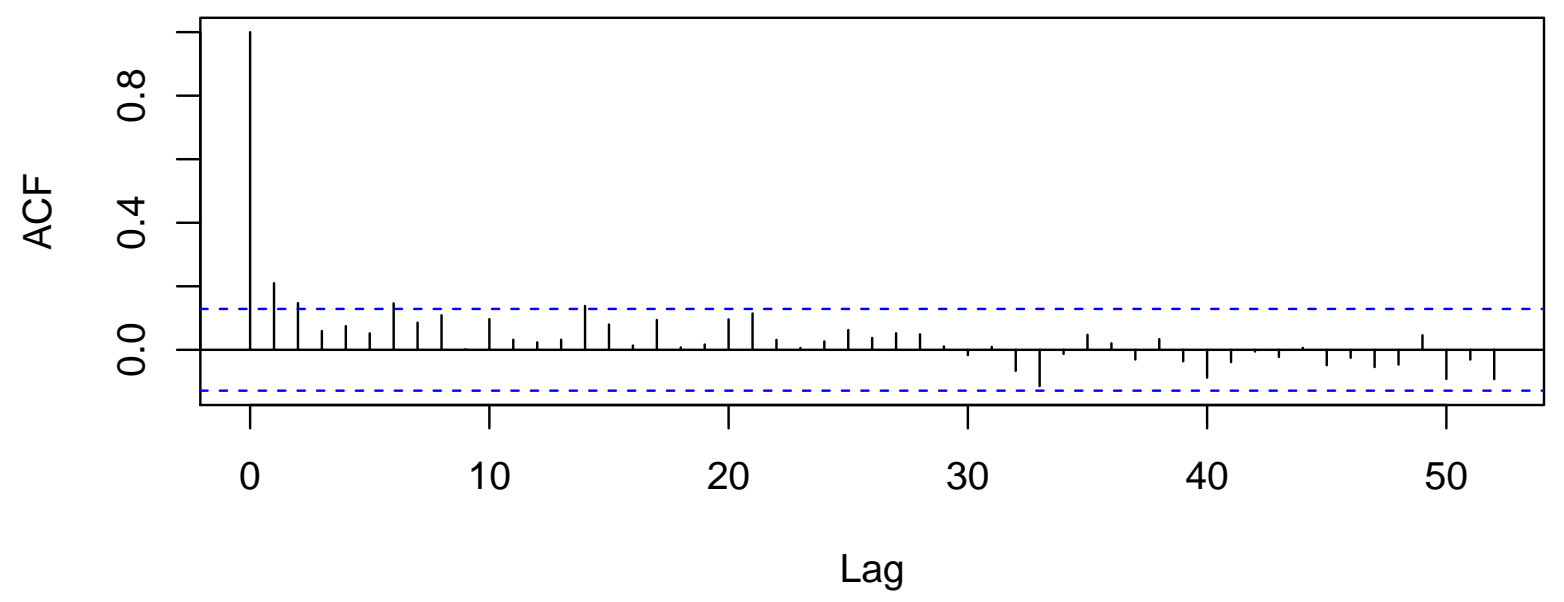




\subsection{A Poisson Regression Model as an Alternative to Model (2) for western}

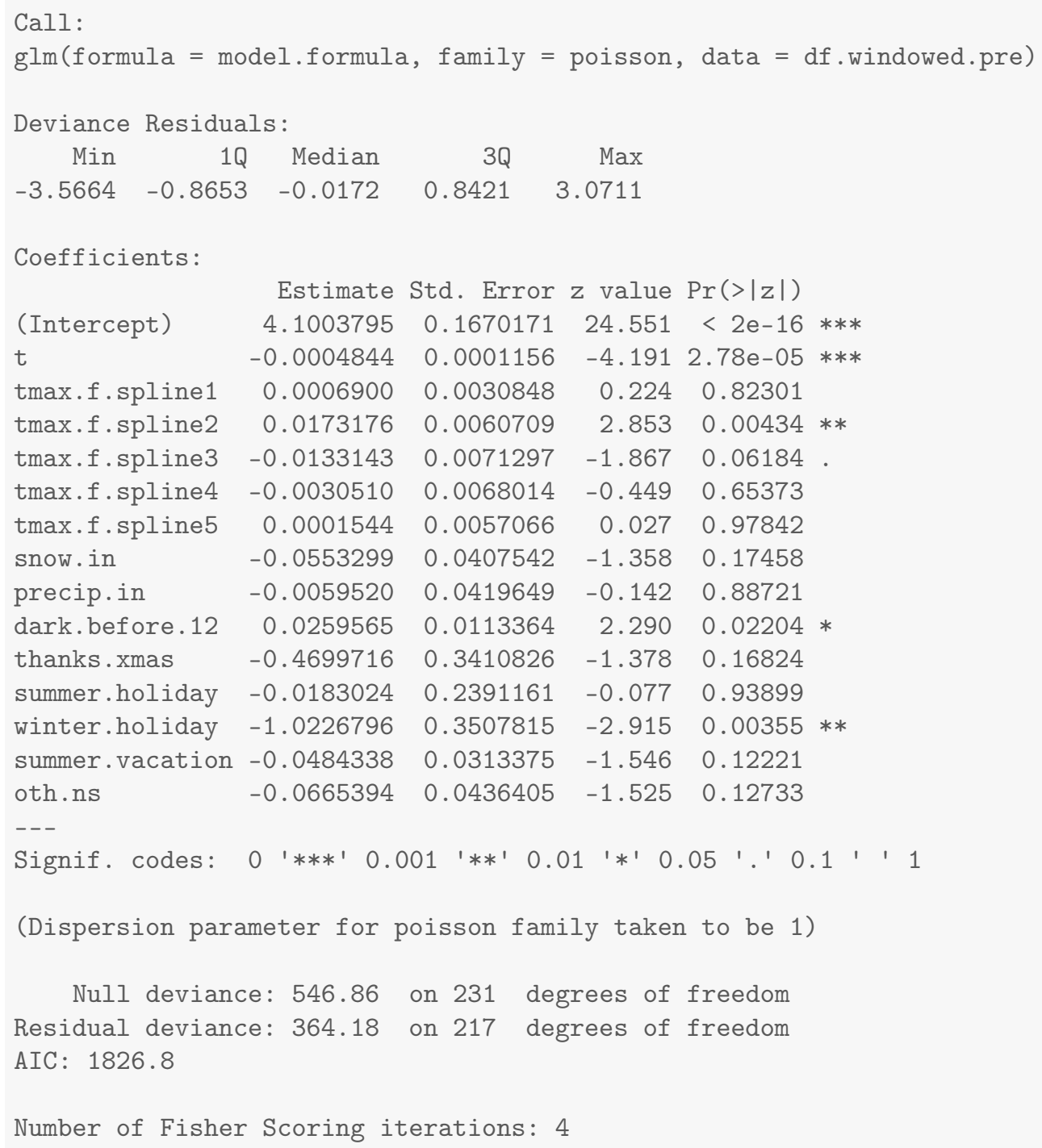




\section{Results for central}

\subsection{Summary Values for central}

Table 22.1: Descriptives for Outcome Before Ferguson Protests Begin

\begin{tabular}{lcccccccc}
\hline \hline Statistic & $\mathrm{N}$ & Mean & St. Dev. & Min & Pctl(25) & Median & Pctl(75) & Max \\
\hline central & 232 & 116.155 & 23.837 & 56 & 98 & 115 & 133 & 193 \\
\hline
\end{tabular}

Table 22.2: Descriptives for Outcome After Ferguson Protests Begin

\begin{tabular}{lcccccccc}
\hline \hline Statistic & $\mathrm{N}$ & Mean & St. Dev. & Min & Pctl(25) & Median & Pctl(75) & Max \\
\hline central & 290 & 107.403 & 20.527 & 61 & 94 & 107 & 121 & 184 \\
\hline
\end{tabular}




\subsection{Four Models for central}

Table 22.3: Four Models that Differ on the Specification of Adjustment and Intervention Variables

\begin{tabular}{|c|c|c|c|c|}
\hline & \multicolumn{4}{|c|}{ Outcome: Count Per Week } \\
\hline & $(1)$ & (2) & (3) & $(4)$ \\
\hline Time (counter in weeks) & -0.12 & -0.10 & & -0.10 \\
\hline After Ferguson Protests Begin (week of 8/11/14 onward) & -9.17 & & -2.16 & -2.80 \\
\hline After Gray Protests Begin (week of 4/20/15 onward) & 22.11 & & 2.45 & 3.80 \\
\hline Unrest and National Guard $(4 / 27 / 15-5 / 3 / 2015)$ & 6.70 & & 4.86 & 8.11 \\
\hline After Batts Fired (week of 7/13/15 onward) & 6.38 & & 2.28 & 2.62 \\
\hline After Davis Confirmed (week of 10/19/15 onward) & -2.60 & & 9.41 & 8.38 \\
\hline After Davis First Year (week of 10/17/16 onward) & 5.36 & & 2.89 & 5.68 \\
\hline After De Sousa Begins (week of 1/19/18 onward) & -10.53 & & -9.56 & -5.59 \\
\hline After Tuggle Begins (week of 5/14/18 onward) & 30.01 & & 21.13 & 23.04 \\
\hline After Harrison Begins (week of 2/11/19 onward) & -5.06 & & -10.86 & -5.67 \\
\hline Average Maximum Temperature to 50 Degrees & & 0.66 & & 0.85 \\
\hline Plus Degrees in the $50 \mathrm{~s}$ & & 0.63 & & 0.21 \\
\hline Plus Degrees in the 60s & & 0.24 & & 0.33 \\
\hline Plus Degrees in the 70s & & -1.92 & & -1.05 \\
\hline Plus Degrees Greater Than 80 & & 0.37 & & -0.44 \\
\hline Snowfall (inches) & & -5.15 & & -3.08 \\
\hline Precipitation (inches) & & -12.29 & & -9.34 \\
\hline Darkness Before Midnight (hours) & & -0.16 & & 1.16 \\
\hline Thanksgiving/Christmas (proportion of week) & & -71.39 & & -63.80 \\
\hline Winter Holiday (proportion of week) & & -26.90 & & -8.87 \\
\hline Other Out-of-School Days (proportion of week) & & -4.09 & & -8.36 \\
\hline Summer Vacation (proportion of week) & & 10.40 & & 4.49 \\
\hline Summer Holiday (proportion of week) & & -90.31 & & -85.40 \\
\hline Observations & 522 & 232 & 522 & 522 \\
\hline $\mathrm{R}^{2}$ & 0.17 & 0.55 & 0.24 & 0.54 \\
\hline
\end{tabular}




\subsection{Least Squares Updated Model (2) for central}

central

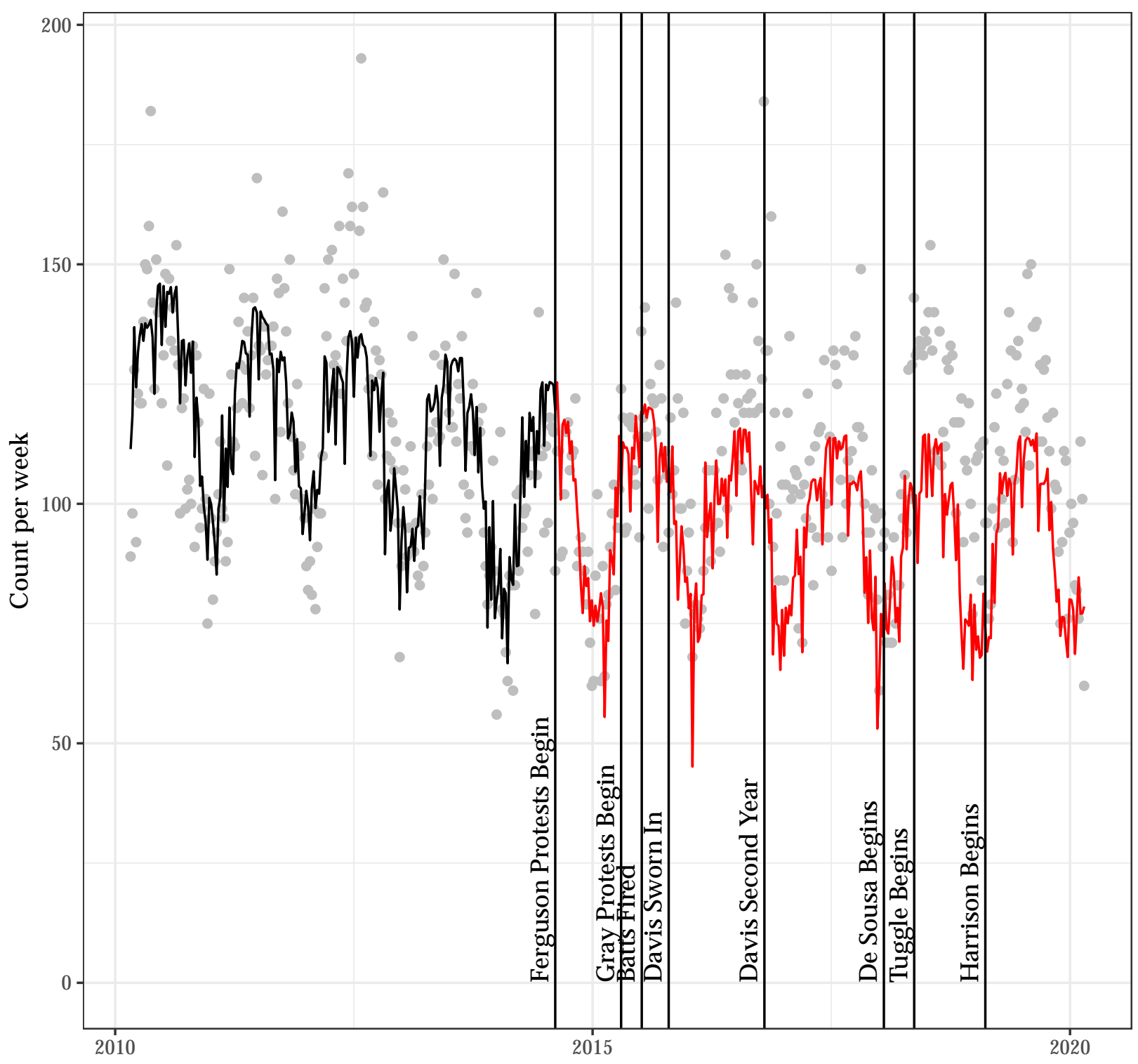




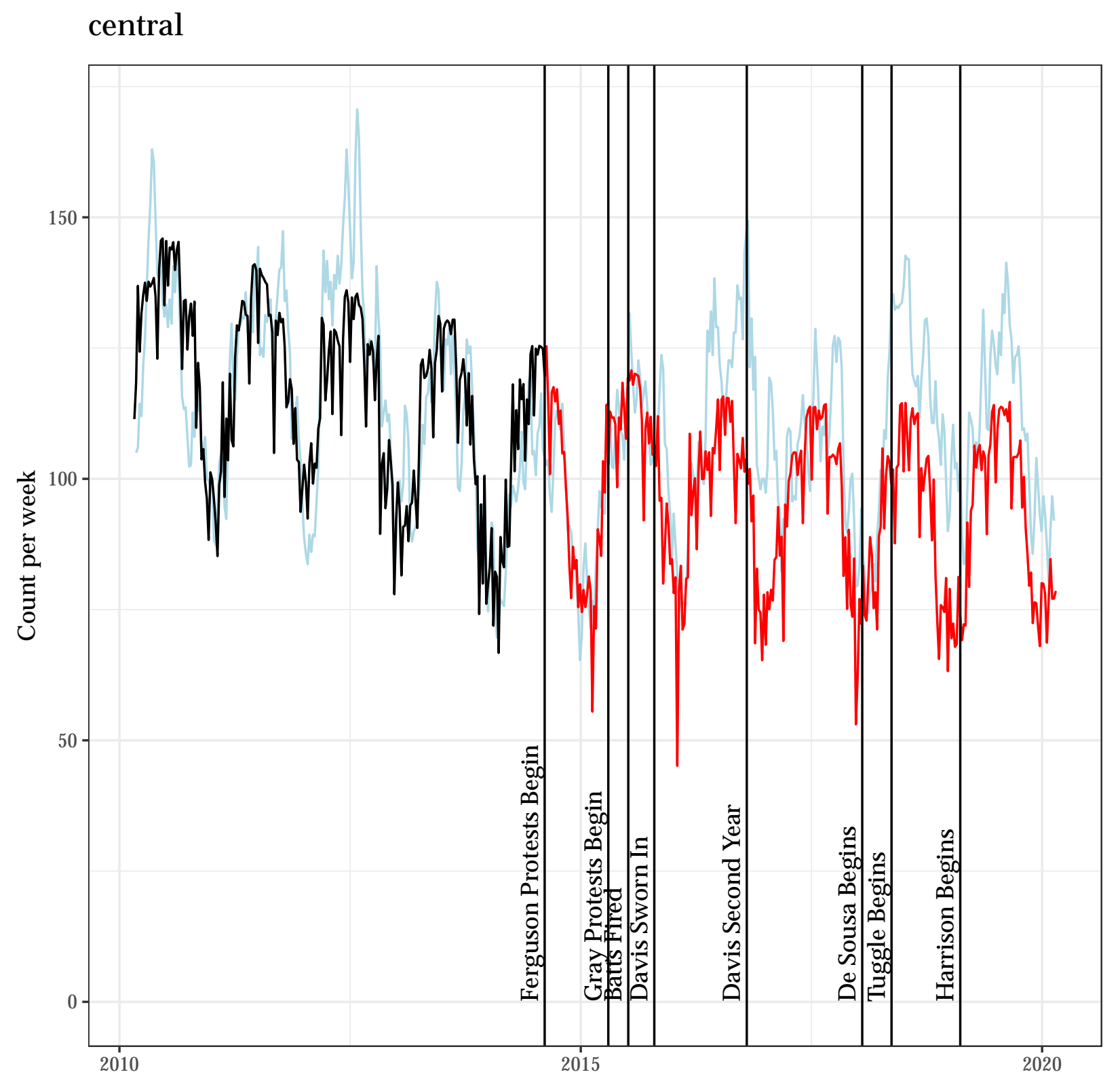


Autocorrelation Function for the Observed Outcome for Updated Model (2)

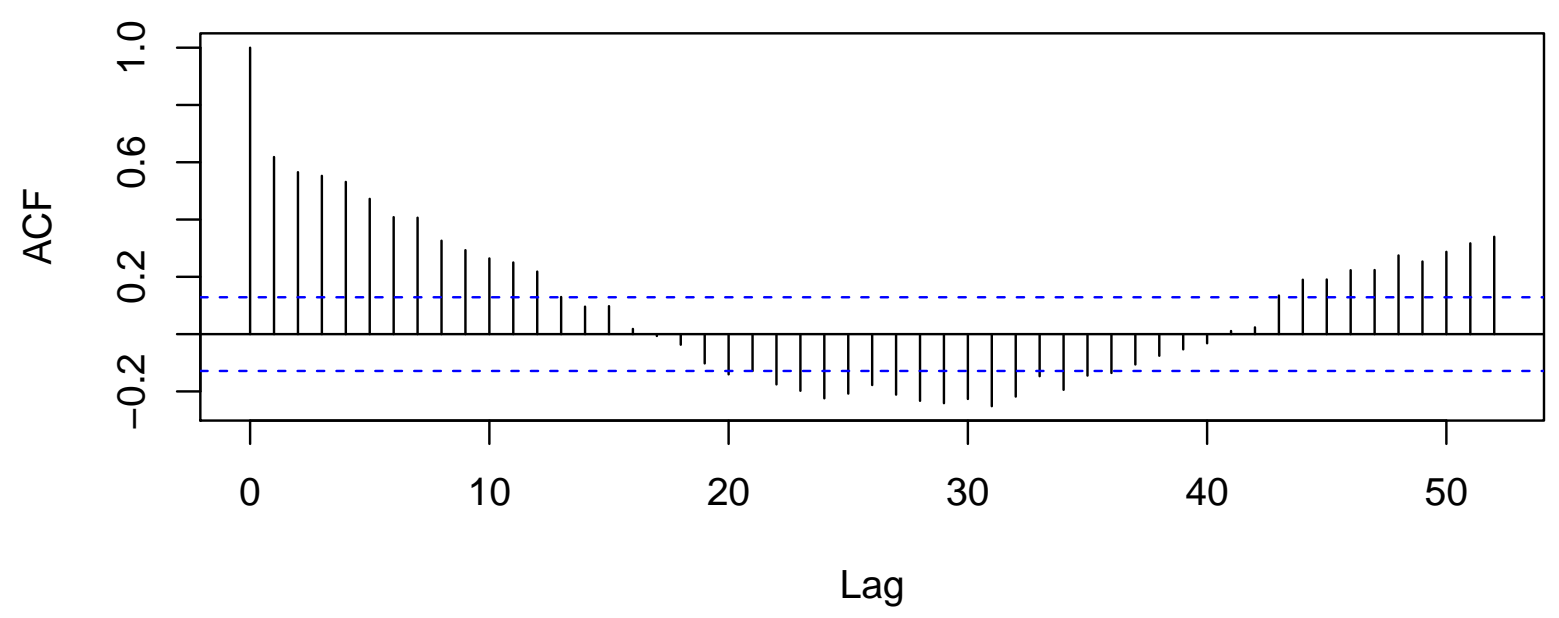

Autocorrelation Function for the Residuals from Updated Model (2)

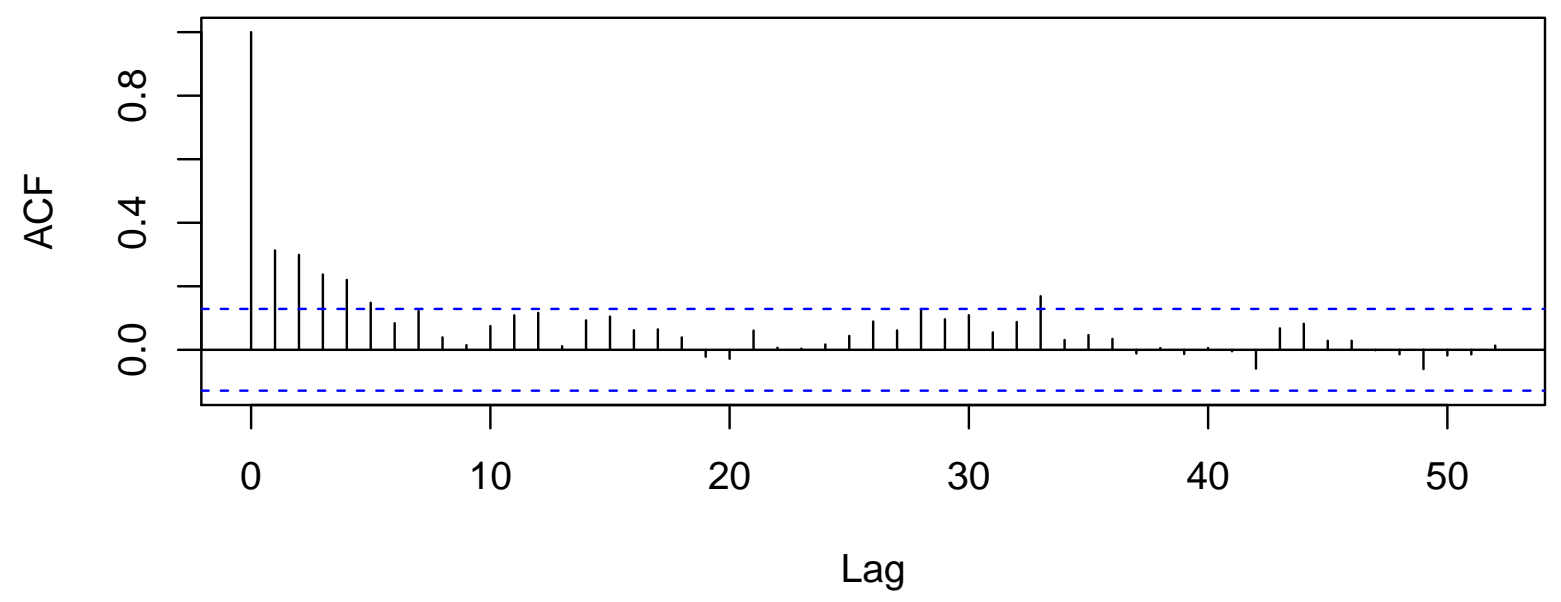




\subsection{A Poisson Regression Model as an Alternative to Model (2) for central}

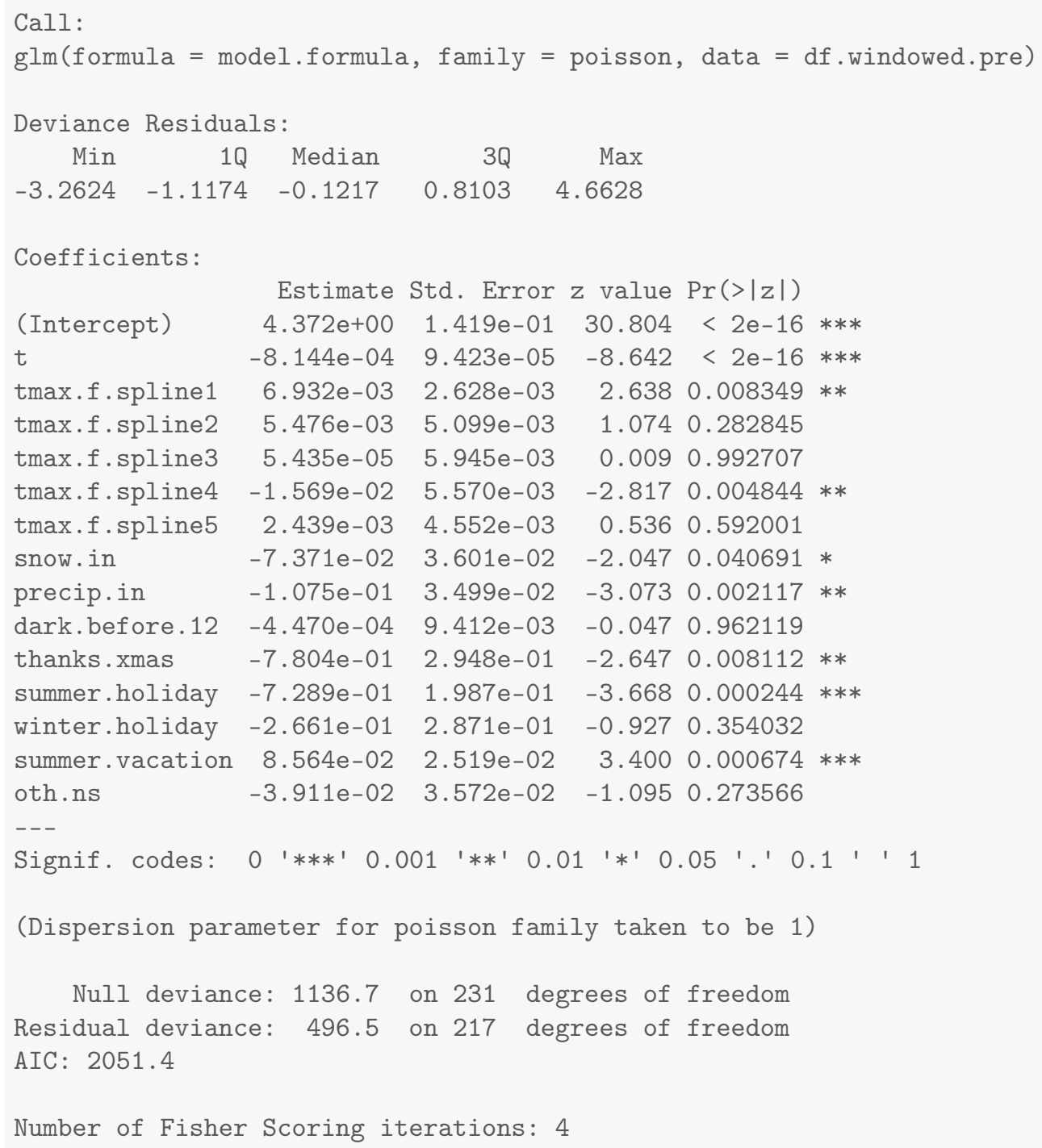




\section{Results for eastern}

\subsection{Summary Values for eastern}

Table 23.1: Descriptives for Outcome Before Ferguson Protests Begin

\begin{tabular}{lcccccccc}
\hline \hline Statistic & $\mathrm{N}$ & Mean & St. Dev. & Min & Pctl(25) & Median & Pctl(75) & Max \\
\hline eastern & 232 & 78.927 & 14.044 & 41 & 69 & 78 & 90 & 117 \\
\hline
\end{tabular}

Table 23.2: Descriptives for Outcome After Ferguson Protests Begin

\begin{tabular}{lcccccccc}
\hline \hline Statistic & $\mathrm{N}$ & Mean & St. Dev. & Min & Pctl(25) & Median & Pctl(75) & Max \\
\hline eastern & 290 & 80.793 & 14.629 & 44 & 72 & 81 & 90 & 119 \\
\hline
\end{tabular}




\subsection{Four Models for eastern}

Table 23.3: Four Models that Differ on the Specification of Adjustment and Intervention Variables

\begin{tabular}{|c|c|c|c|c|}
\hline & \multicolumn{4}{|c|}{ Outcome: Count Per Week } \\
\hline & $(1)$ & $(2)$ & $(3)$ & (4) \\
\hline Time (counter in weeks) & -0.02 & -0.01 & & -0.01 \\
\hline After Ferguson Protests Begin (week of $8 / 11 / 14$ onward) & -8.86 & & -6.05 & -6.14 \\
\hline After Gray Protests Begin (week of 4/20/15 onward) & 20.06 & & 10.48 & 10.97 \\
\hline Unrest and National Guard (4/27/15 - 5/3/2015) & 0.17 & & 1.92 & 1.51 \\
\hline After Batts Fired (week of 7/13/15 onward) & -7.10 & & -8.06 & -7.33 \\
\hline After Davis Confirmed (week of 10/19/15 onward) & 1.54 & & 6.29 & 5.85 \\
\hline After Davis First Year (week of 10/17/16 onward) & 10.34 & & 9.97 & 10.32 \\
\hline After De Sousa Begins (week of 1/19/18 onward) & -8.65 & & -7.67 & -7.79 \\
\hline After Tuggle Begins (week of 5/14/18 onward) & 3.86 & & 0.95 & 1.85 \\
\hline After Harrison Begins (week of 2/11/19 onward) & -4.15 & & -5.12 & -4.93 \\
\hline Average Maximum Temperature to 50 Degrees & & 0.18 & & 0.29 \\
\hline Plus Degrees in the $50 \mathrm{~s}$ & & 0.58 & & 0.04 \\
\hline Plus Degrees in the 60s & & -0.77 & & -0.15 \\
\hline Plus Degrees in the 70s & & 0.29 & & 0.09 \\
\hline Plus Degrees Greater Than 80 & & -0.05 & & -0.04 \\
\hline Snowfall (inches) & & -10.00 & & -7.61 \\
\hline Precipitation (inches) & & -3.40 & & -5.77 \\
\hline Darkness Before Midnight (hours) & & -0.72 & & -0.96 \\
\hline Thanksgiving/Christmas (proportion of week) & & -30.43 & & -38.85 \\
\hline Winter Holiday (proportion of week) & & 16.44 & & 16.45 \\
\hline Other Out-of-School Days (proportion of week) & & -1.09 & & -3.00 \\
\hline Summer Vacation (proportion of week) & & 0.51 & & -1.93 \\
\hline Summer Holiday (proportion of week) & & -25.74 & & -4.51 \\
\hline Observations & 522 & 232 & 522 & 522 \\
\hline $\mathrm{R}^{2}$ & 0.13 & 0.26 & 0.14 & 0.31 \\
\hline
\end{tabular}




\subsection{Least Squares Updated Model (2) for eastern}

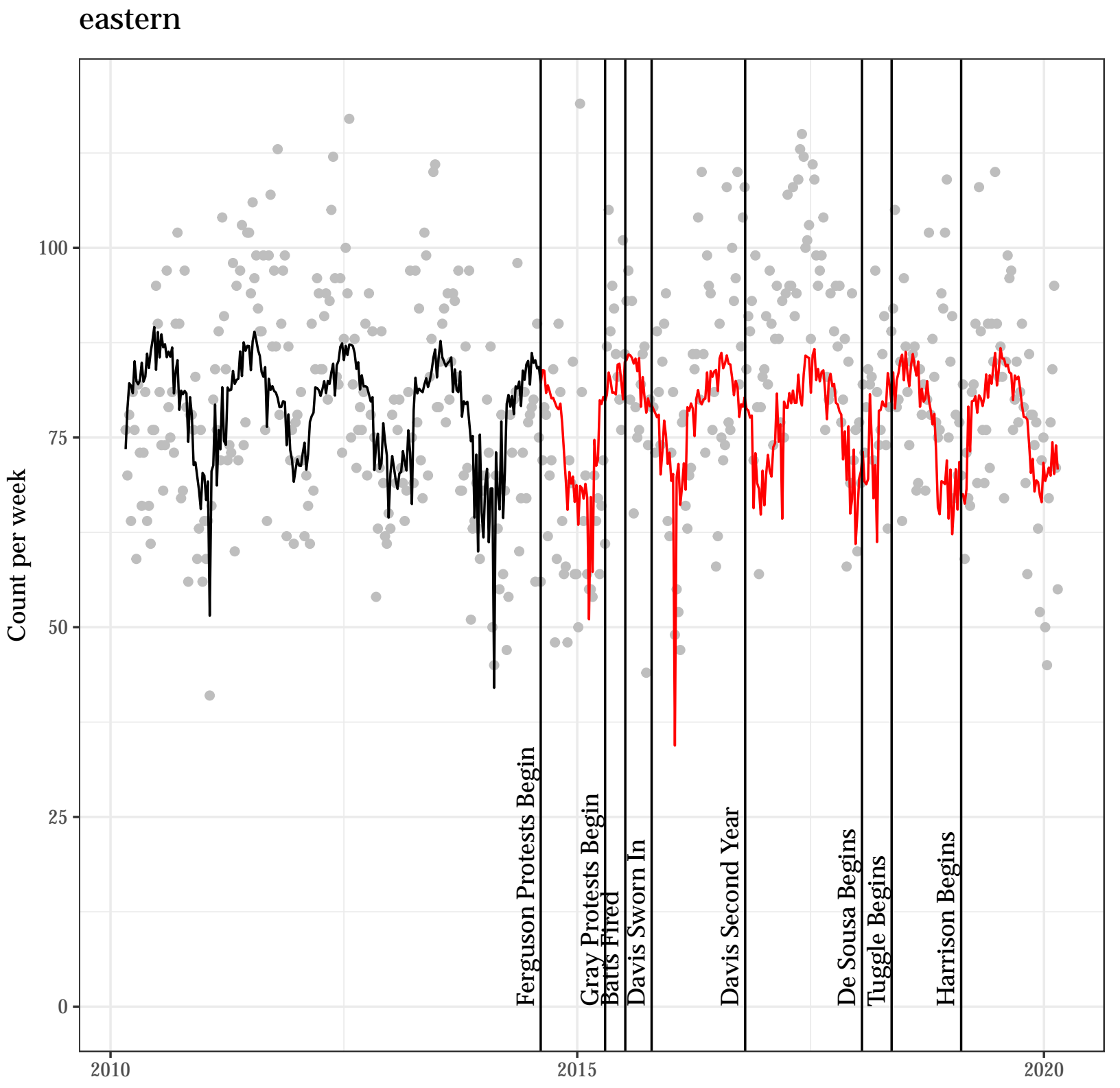




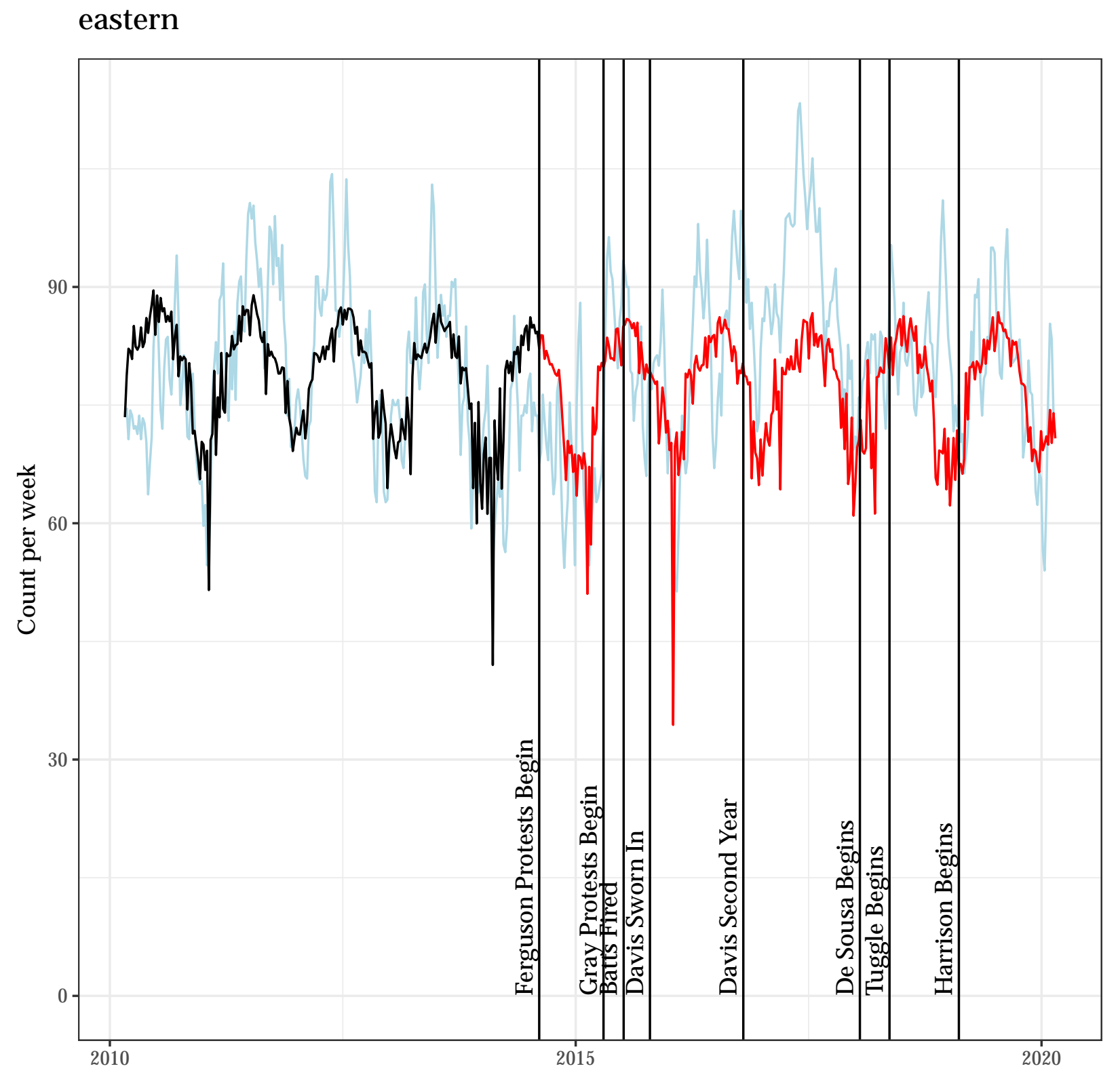


Autocorrelation Function for the Observed Outcome for Updated Model (2)

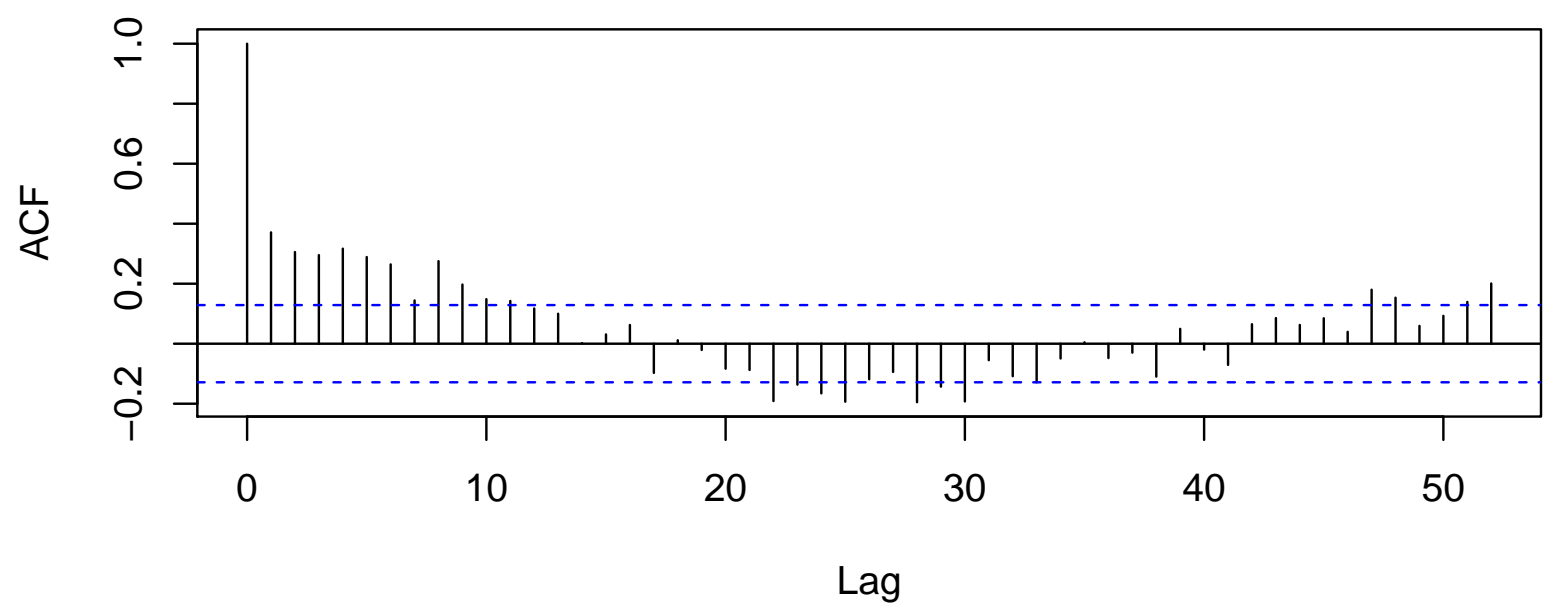

Autocorrelation Function for the Residuals from Updated Model (2)

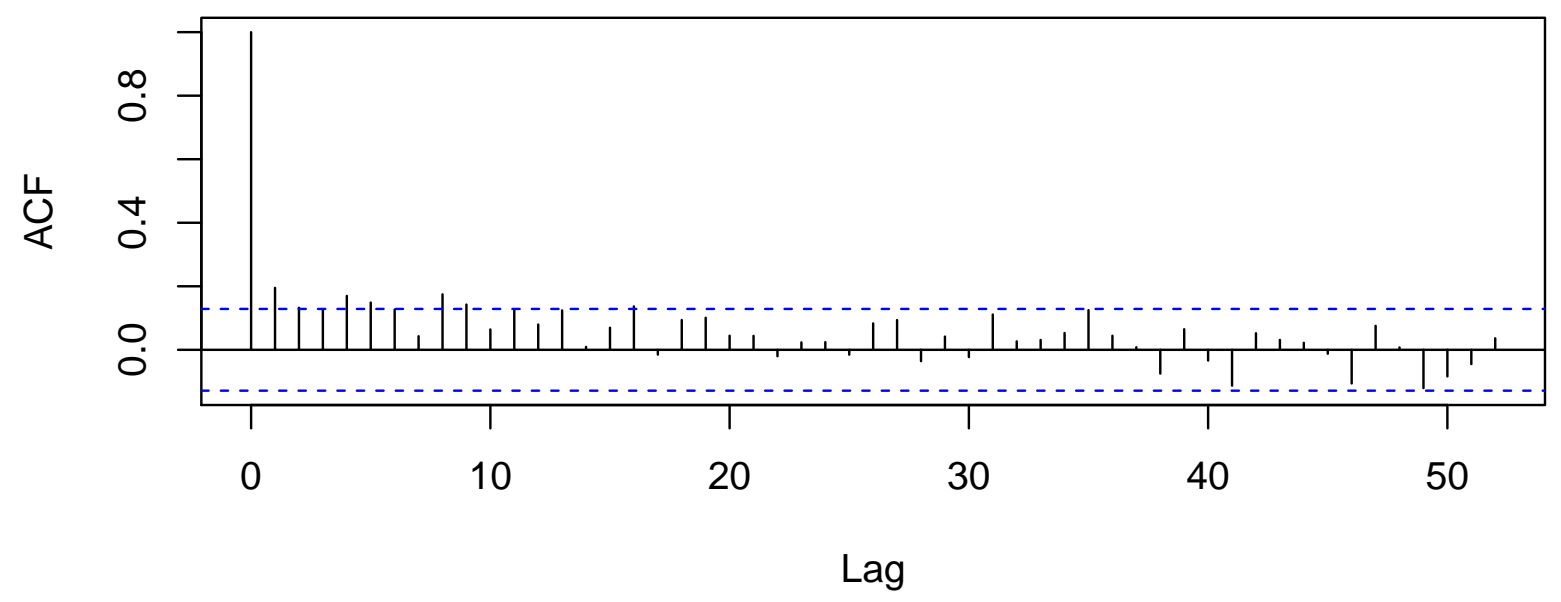




\subsection{A Poisson Regression Model as an Alternative to Model (2) for eastern}

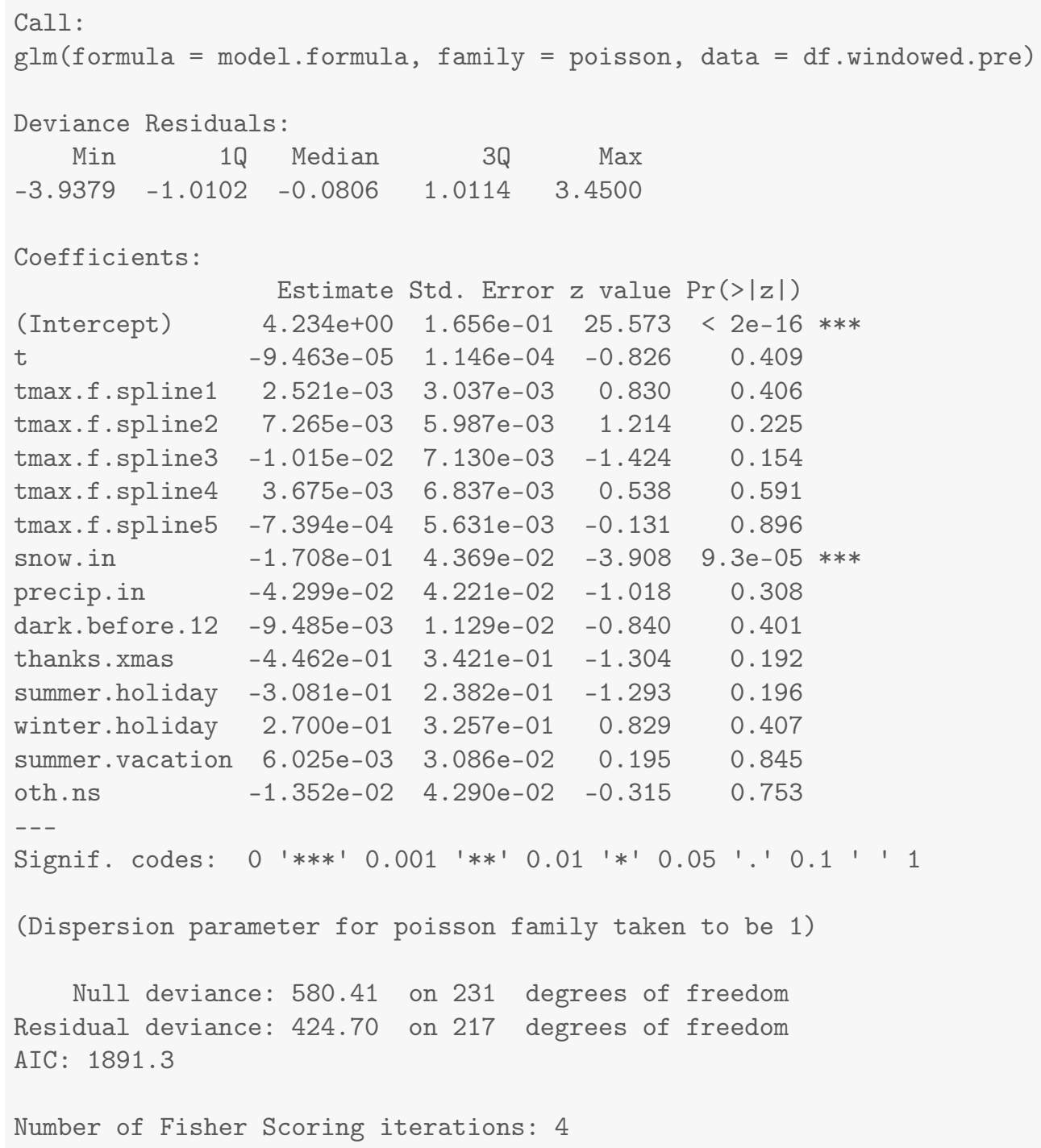




\section{Results for southwestern}

\subsection{Summary Values for southwestern}

Table 24.1: Descriptives for Outcome Before Ferguson Protests Begin

\begin{tabular}{lcccccccc}
\hline \hline Statistic & $\mathrm{N}$ & Mean & St. Dev. & Min & Pctl(25) & Median & Pctl(75) & Max \\
\hline southwestern & 232 & 83.599 & 13.437 & 52 & 75 & 83 & 92 & 122 \\
\hline
\end{tabular}

Table 24.2: Descriptives for Outcome After Ferguson Protests Begin

\begin{tabular}{lcccccccc}
\hline \hline Statistic & N & Mean & St. Dev. & Min & Pctl(25) & Median & Pctl(75) & Max \\
\hline southwestern & 290 & 96.131 & 18.369 & 42 & 84 & 97 & 109 & 150 \\
\hline
\end{tabular}




\subsection{Four Models for southwestern}

Table 24.3: Four Models that Differ on the Specification of Adjustment and Intervention Variables

\begin{tabular}{|c|c|c|c|c|}
\hline & \multicolumn{4}{|c|}{ Outcome: Count Per Week } \\
\hline & $(1)$ & $(2)$ & $(3)$ & $(4)$ \\
\hline Time (counter in weeks) & -0.01 & -0.003 & & -0.002 \\
\hline After Ferguson Protests Begin (week of 8/11/14 onward) & -4.17 & & -2.88 & -1.52 \\
\hline After Gray Protests Begin (week of 4/20/15 onward) & 13.92 & & 9.07 & 6.20 \\
\hline Unrest and National Guard (4/27/15 - 5/3/2015) & -3.07 & & -3.50 & -0.50 \\
\hline After Batts Fired (week of 7/13/15 onward) & 2.97 & & -0.65 & -1.00 \\
\hline After Davis Confirmed (week of 10/19/15 onward) & -2.53 & & 1.78 & 3.25 \\
\hline After Davis First Year (week of 10/17/16 onward) & 13.70 & & 12.58 & 13.12 \\
\hline After De Sousa Begins (week of 1/19/18 onward) & -12.61 & & -8.01 & -8.11 \\
\hline After Tuggle Begins (week of 5/14/18 onward) & 17.85 & & 12.38 & 11.55 \\
\hline After Harrison Begins (week of 2/11/19 onward) & -7.49 & & -7.41 & -6.90 \\
\hline Average Maximum Temperature to 50 Degrees & & 0.15 & & 0.54 \\
\hline Plus Degrees in the $50 \mathrm{~s}$ & & 0.56 & & 0.02 \\
\hline Plus Degrees in the 60s & & 0.11 & & 0.13 \\
\hline Plus Degrees in the 70s & & -0.91 & & -0.22 \\
\hline Plus Degrees Greater Than 80 & & 0.10 & & -0.18 \\
\hline Snowfall (inches) & & -8.01 & & -3.19 \\
\hline Precipitation (inches) & & -3.29 & & -4.44 \\
\hline Darkness Before Midnight (hours) & & 3.25 & & 2.75 \\
\hline Thanksgiving/Christmas (proportion of week) & & 9.67 & & 21.22 \\
\hline Winter Holiday (proportion of week) & & -57.34 & & -49.21 \\
\hline Other Out-of-School Days (proportion of week) & & -6.27 & & -6.63 \\
\hline Summer Vacation (proportion of week) & & 3.38 & & 0.27 \\
\hline Summer Holiday (proportion of week) & & -5.89 & & -25.03 \\
\hline Observations & 522 & 232 & 522 & 522 \\
\hline $\mathrm{R}^{2}$ & 0.28 & 0.22 & 0.32 & 0.44 \\
\hline
\end{tabular}




\subsection{Least Squares Updated Model (2) for southwestern}

southwestern

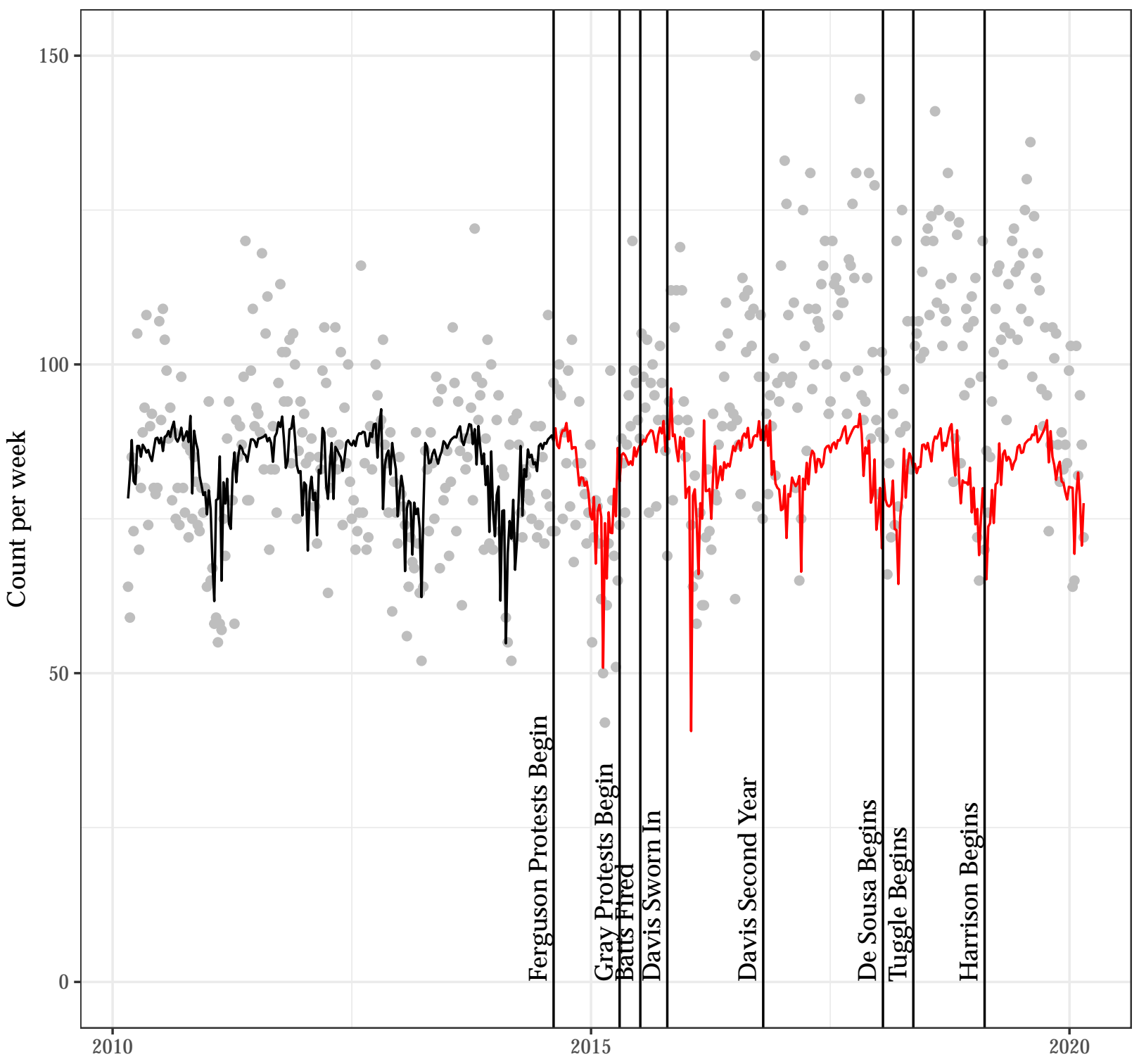




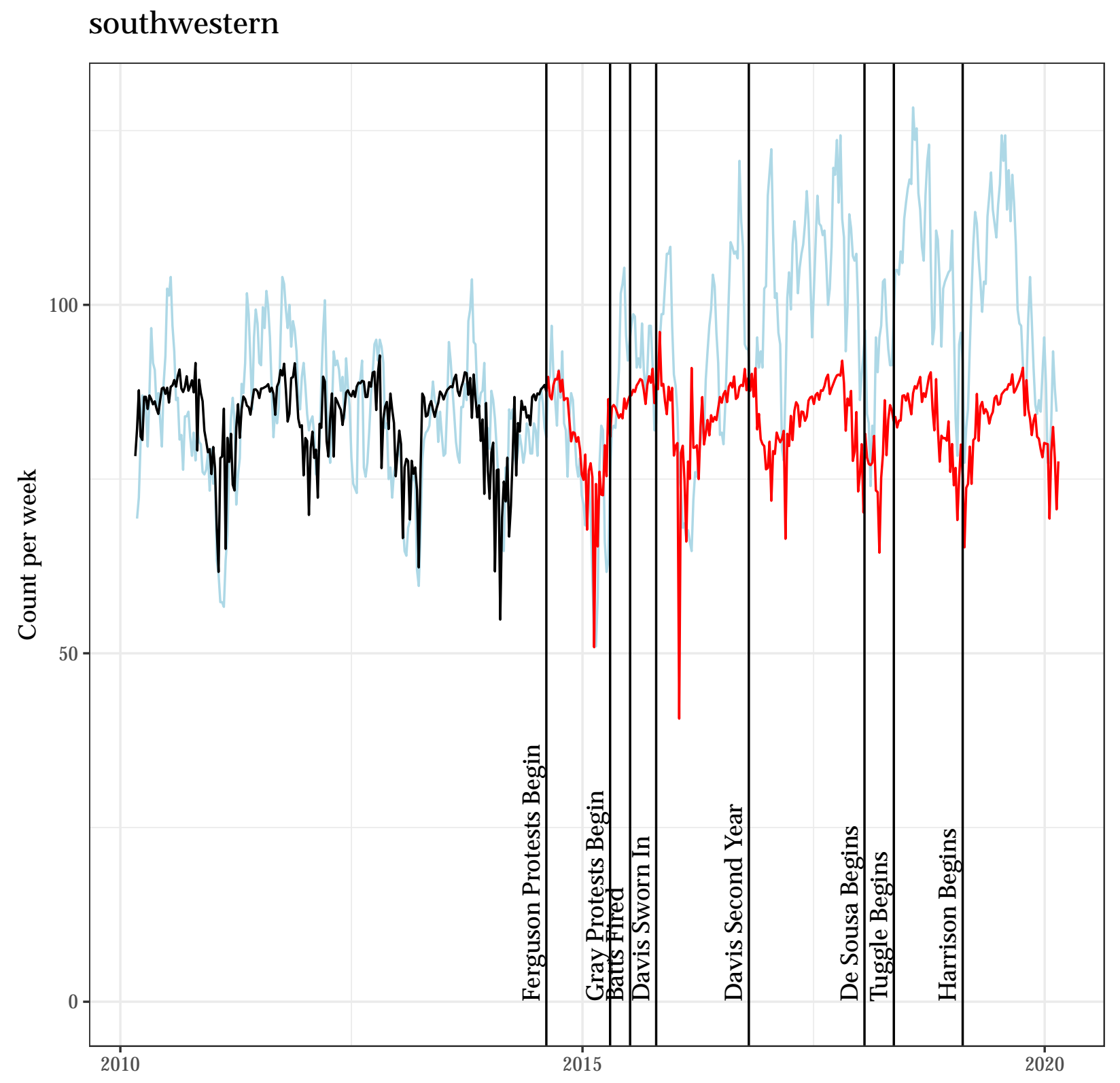


Autocorrelation Function for the Observed Outcome for Updated Model (2)

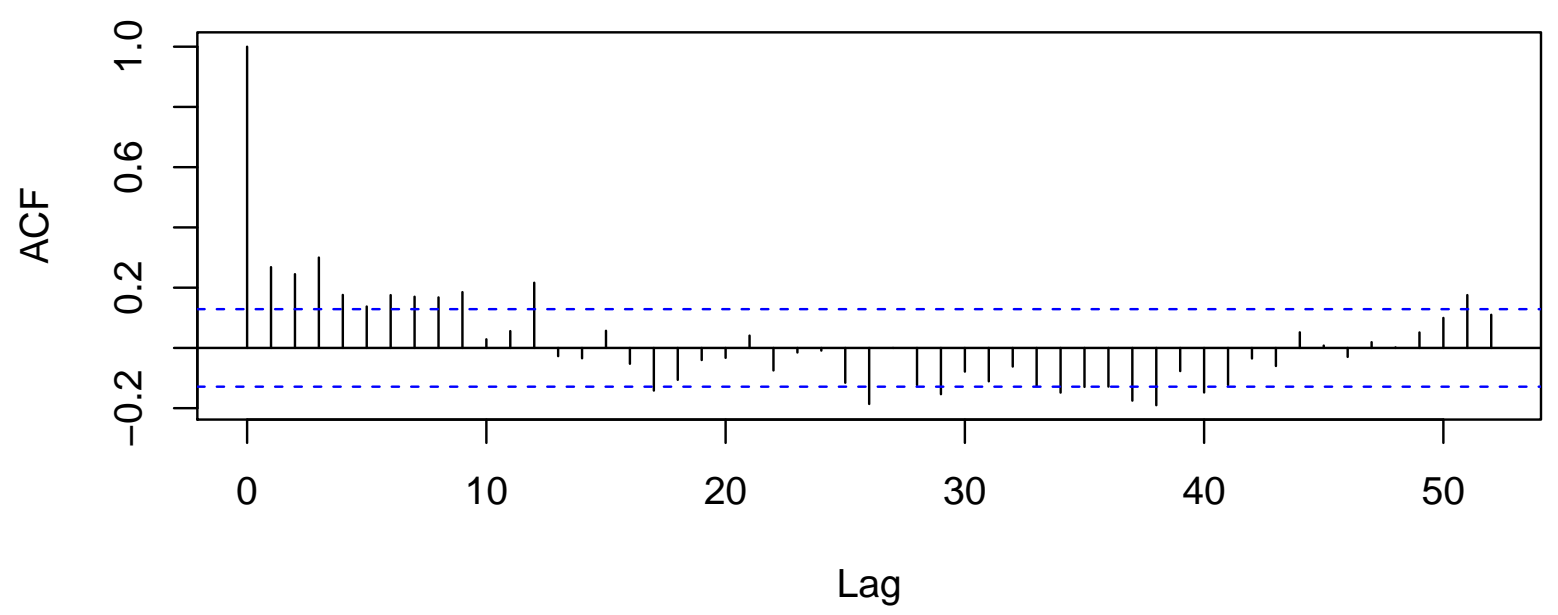

Autocorrelation Function for the Residuals from Updated Model (2)

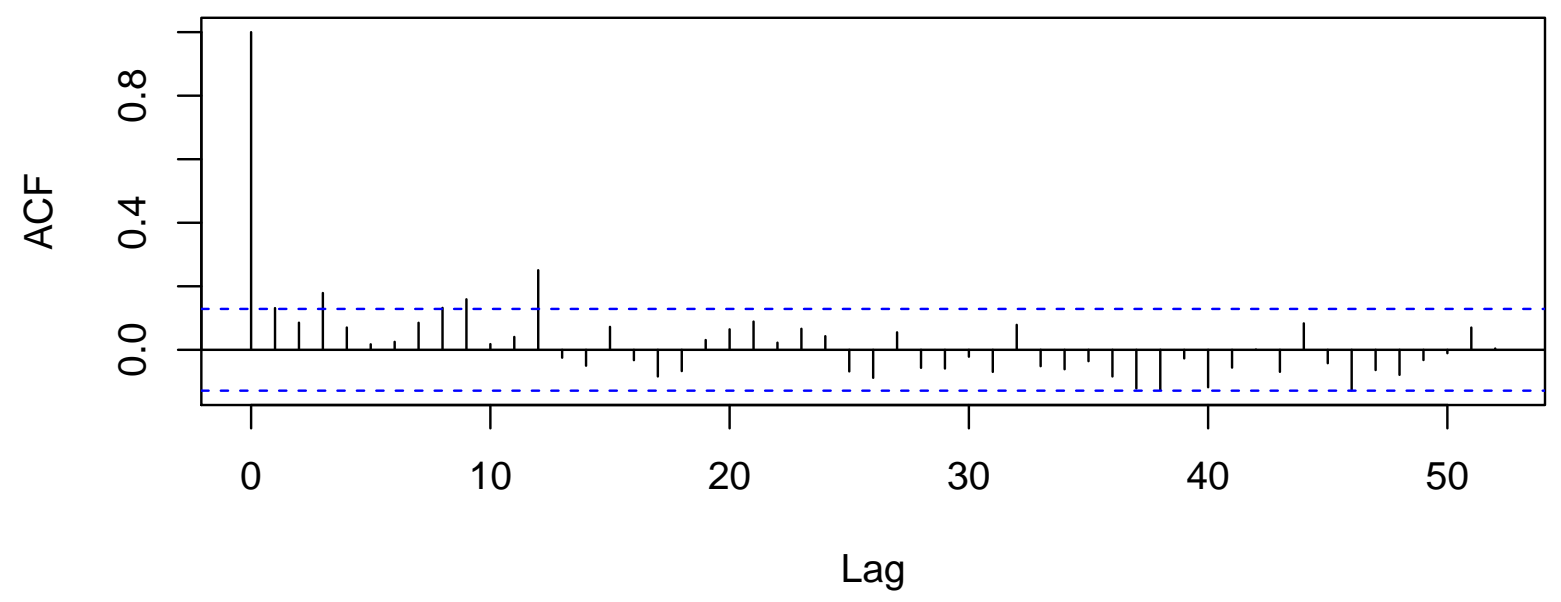




\subsection{A Poisson Regression Model as an Alternative to Model (2) for southwestern}

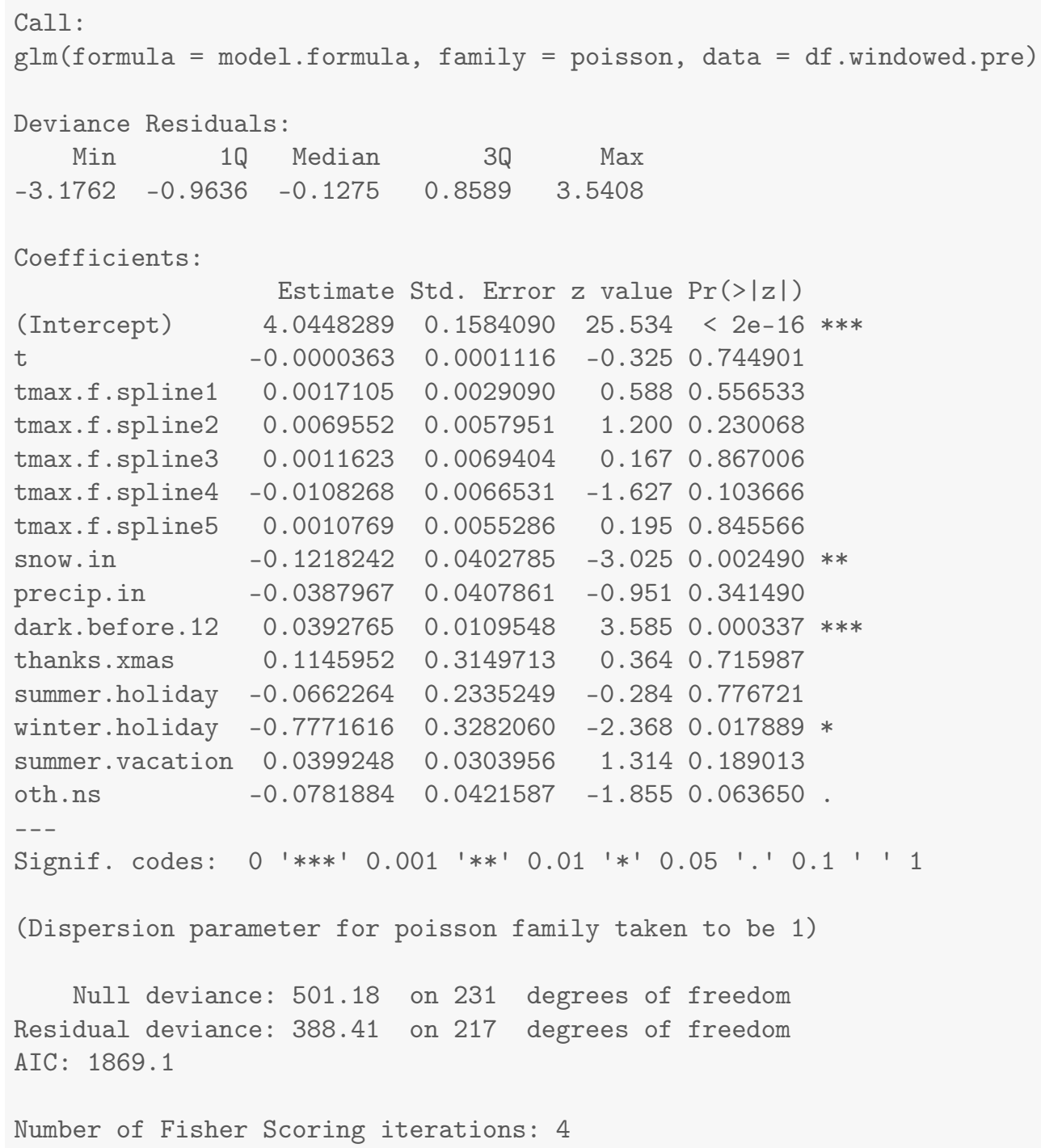




\section{Results for southern}

\subsection{Summary Values for southern}

Table 25.1: Descriptives for Outcome Before Ferguson Protests Begin

\begin{tabular}{lcccccccc}
\hline \hline Statistic & $\mathrm{N}$ & Mean & St. Dev. & Min & Pctl(25) & Median & Pctl(75) & Max \\
\hline southern & 232 & 113.078 & 20.885 & 57 & 98.8 & 112 & 127 & 185 \\
\hline
\end{tabular}

Table 25.2: Descriptives for Outcome After Ferguson Protests Begin

\begin{tabular}{lcccccccc}
\hline \hline Statistic & $\mathrm{N}$ & Mean & St. Dev. & Min & Pctl(25) & Median & Pctl(75) & Max \\
\hline southern & 290 & 103.872 & 21.332 & 50 & 88 & 104 & 118.8 & 226 \\
\hline
\end{tabular}




\subsection{Four Models for southern}

Table 25.3: Four Models that Differ on the Specification of Adjustment and Intervention Variables

\begin{tabular}{|c|c|c|c|c|}
\hline & \multicolumn{4}{|c|}{ Outcome: Count Per Week } \\
\hline & (1) & $(2)$ & (3) & $(4)$ \\
\hline Time (counter in weeks) & -0.15 & -0.14 & & -0.13 \\
\hline After Ferguson Protests Begin (week of $8 / 11 / 14$ onward) & -4.28 & & -0.09 & 0.80 \\
\hline After Gray Protests Begin (week of 4/20/15 onward) & 22.90 & & 10.26 & 7.56 \\
\hline Unrest and National Guard (4/27/15 - 5/3/2015) & -12.17 & & -11.94 & -7.67 \\
\hline After Batts Fired (week of 7/13/15 onward) & 5.46 & & 4.24 & 3.55 \\
\hline After Davis Confirmed (week of 10/19/15 onward) & 0.46 & & 6.81 & 9.31 \\
\hline After Davis First Year (week of 10/17/16 onward) & 15.34 & & 11.22 & 15.15 \\
\hline After De Sousa Begins (week of 1/19/18 onward) & -17.55 & & -18.50 & -11.92 \\
\hline After Tuggle Begins (week of 5/14/18 onward) & 25.27 & & 16.52 & 17.00 \\
\hline After Harrison Begins (week of 2/11/19 onward) & -12.74 & & -19.42 & -12.21 \\
\hline Average Maximum Temperature to 50 Degrees & & 0.40 & & 0.80 \\
\hline Plus Degrees in the $50 \mathrm{~s}$ & & 0.75 & & -0.49 \\
\hline Plus Degrees in the 60s & & -0.18 & & 1.16 \\
\hline Plus Degrees in the 70s & & -0.69 & & -0.94 \\
\hline Plus Degrees Greater Than 80 & & -0.37 & & -0.45 \\
\hline Snowfall (inches) & & -3.61 & & -2.77 \\
\hline Precipitation (inches) & & -8.79 & & -5.67 \\
\hline Darkness Before Midnight (hours) & & 1.10 & & 1.40 \\
\hline Thanksgiving/Christmas (proportion of week) & & 48.35 & & 21.61 \\
\hline Winter Holiday (proportion of week) & & -14.12 & & -30.37 \\
\hline Other Out-of-School Days (proportion of week) & & -5.76 & & -5.25 \\
\hline Summer Vacation (proportion of week) & & -2.33 & & -0.58 \\
\hline Summer Holiday (proportion of week) & & -1.55 & & 12.75 \\
\hline Observations & 522 & 232 & 522 & 522 \\
\hline $\mathrm{R}^{2}$ & 0.26 & 0.45 & 0.40 & 0.50 \\
\hline
\end{tabular}




\subsection{Least Squares Updated Model (2) for southern southern}

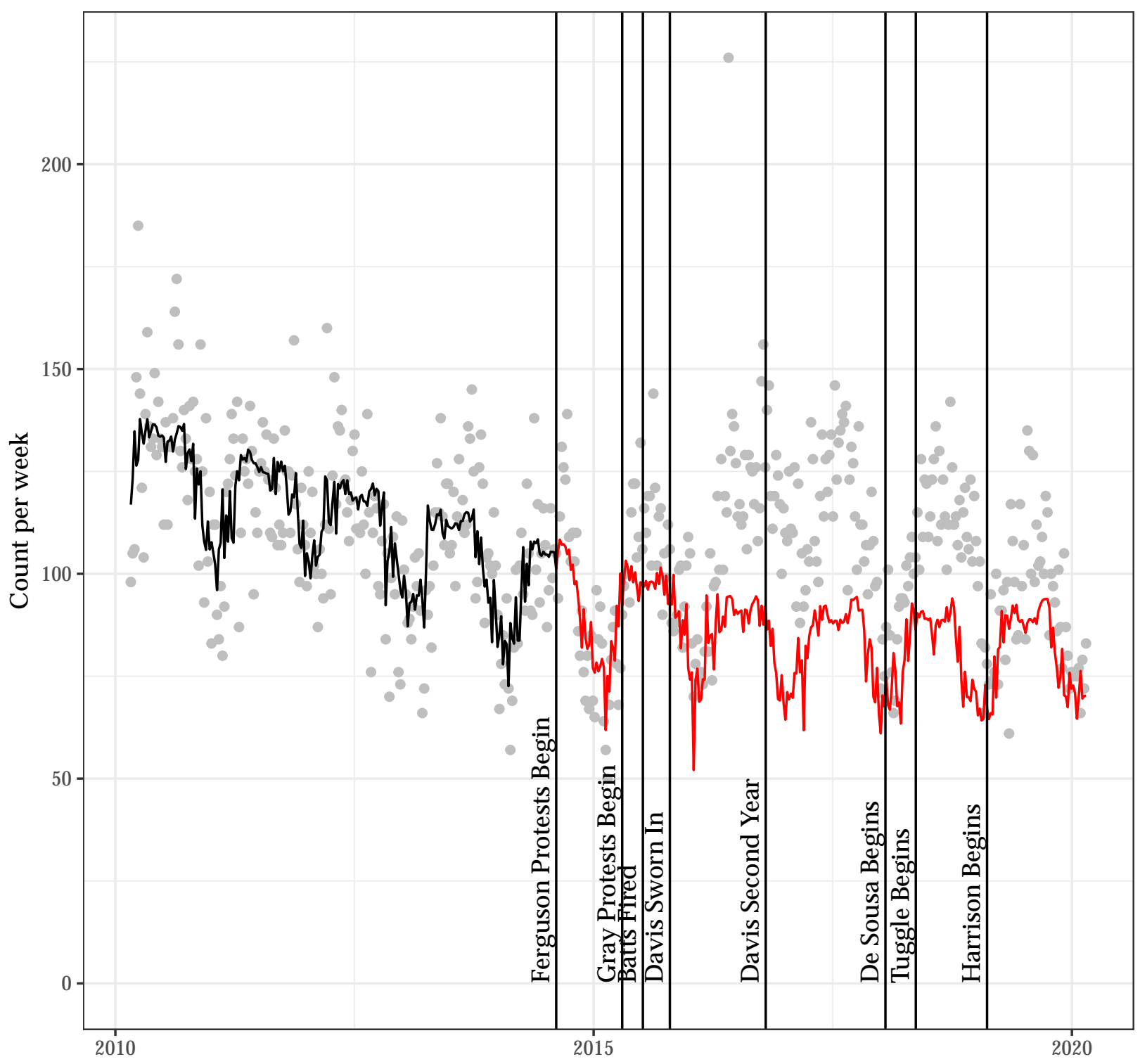




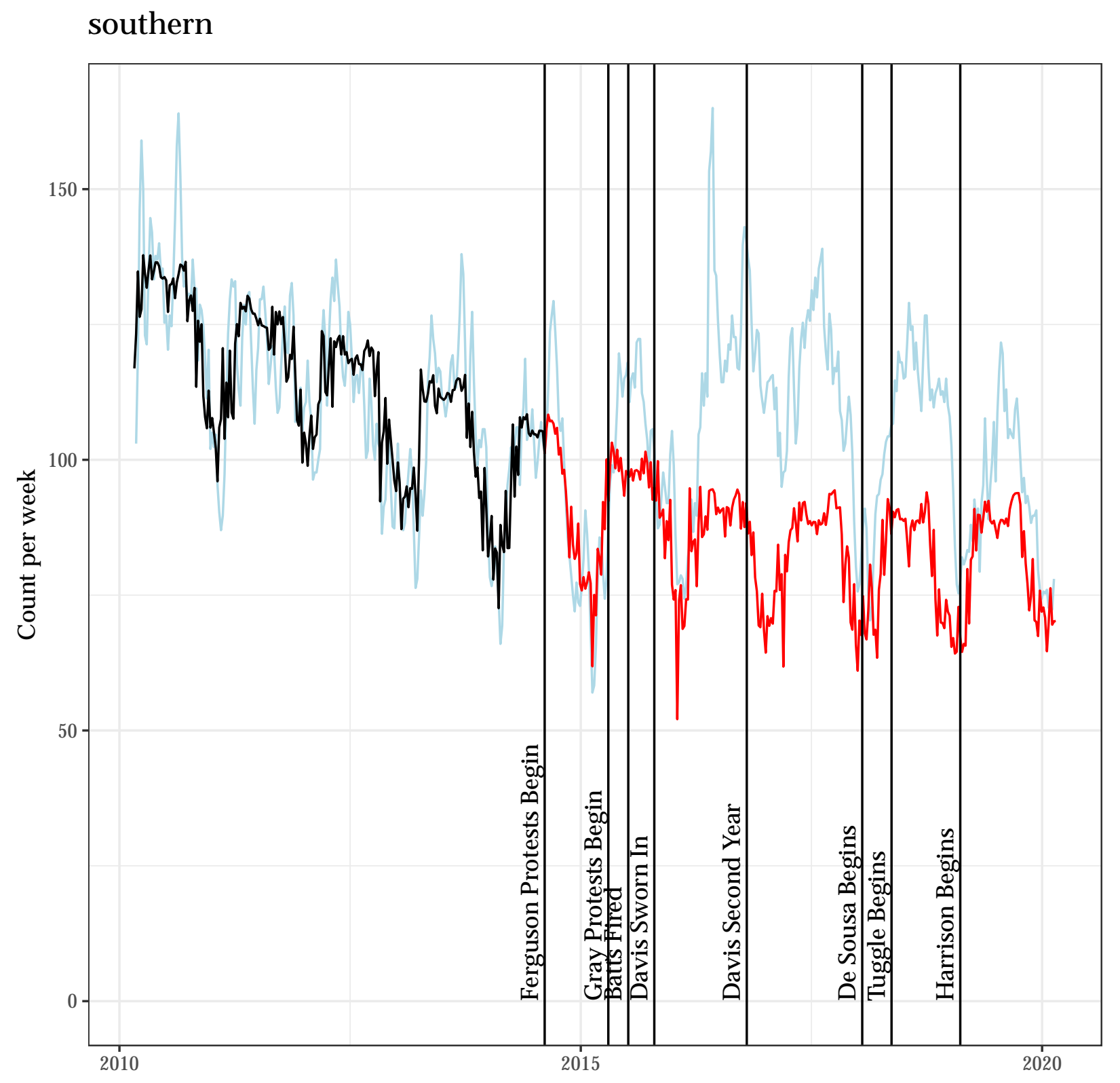


Autocorrelation Function for the Observed Outcome for Updated Model (2)

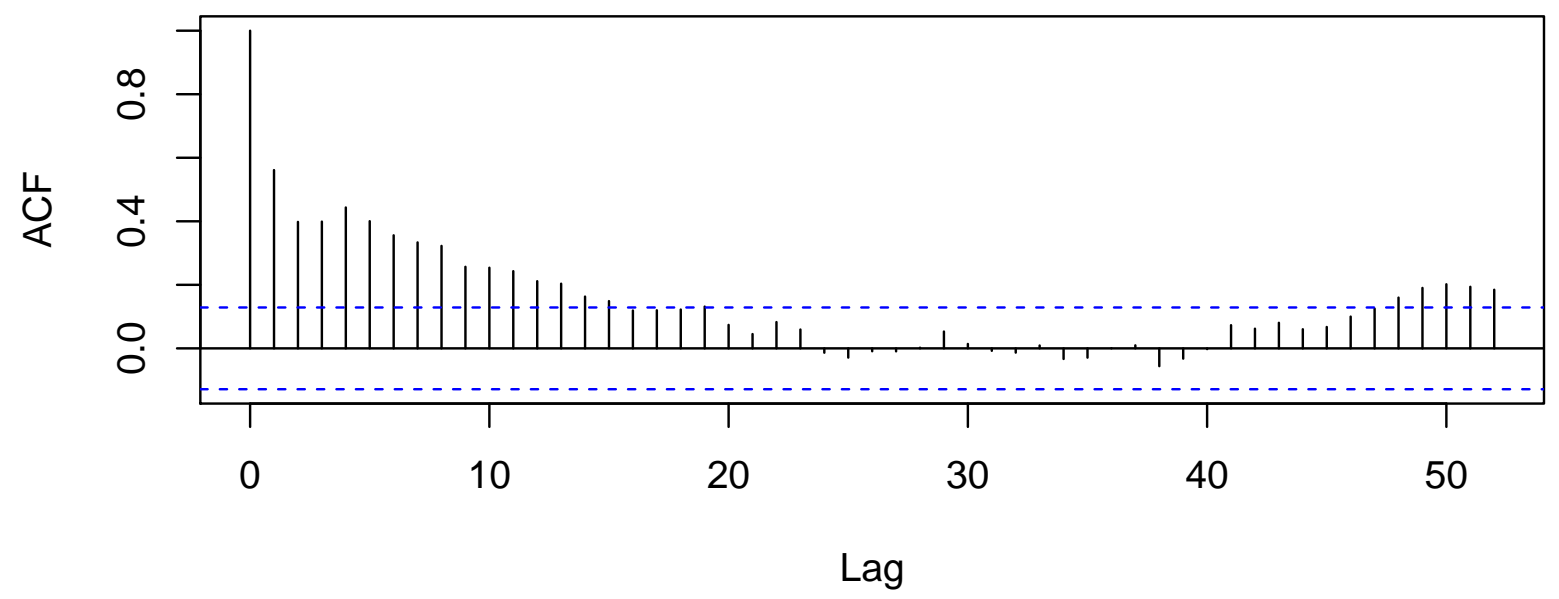

Autocorrelation Function for the Residuals from Updated Model (2)

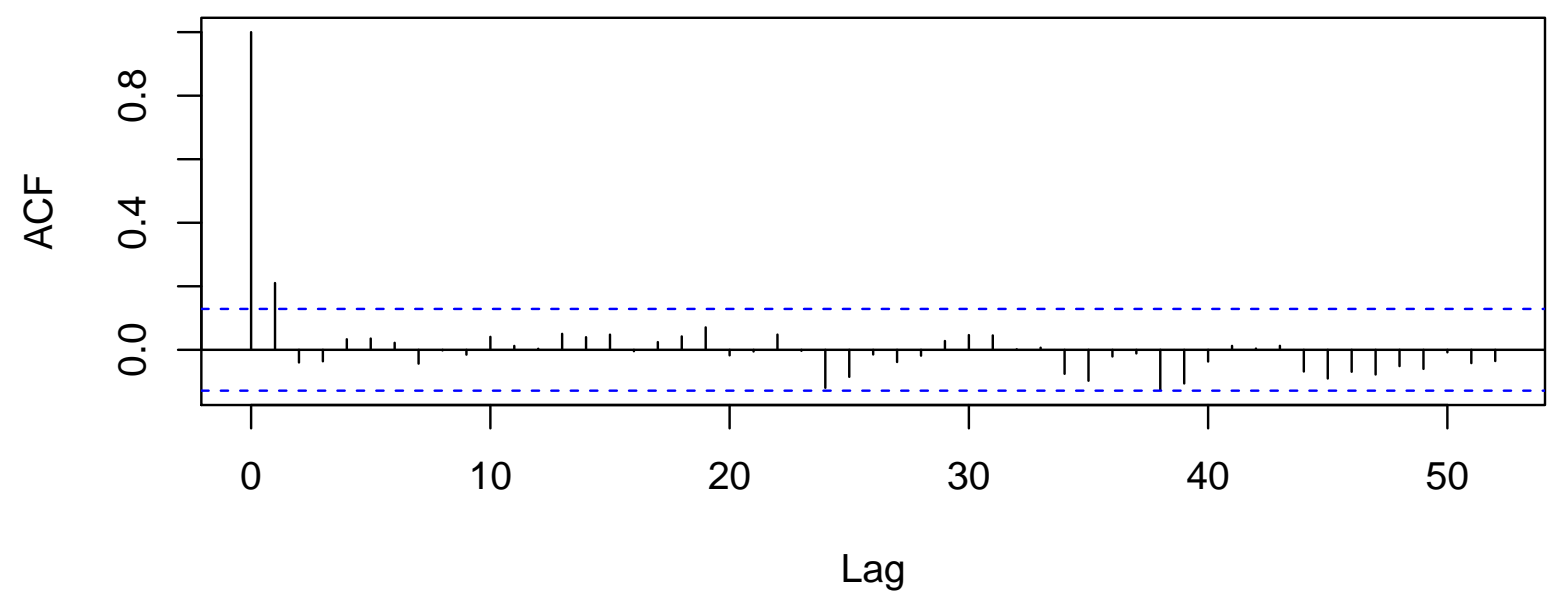




\subsection{A Poisson Regression Model as an Alternative to Model (2) for southern}

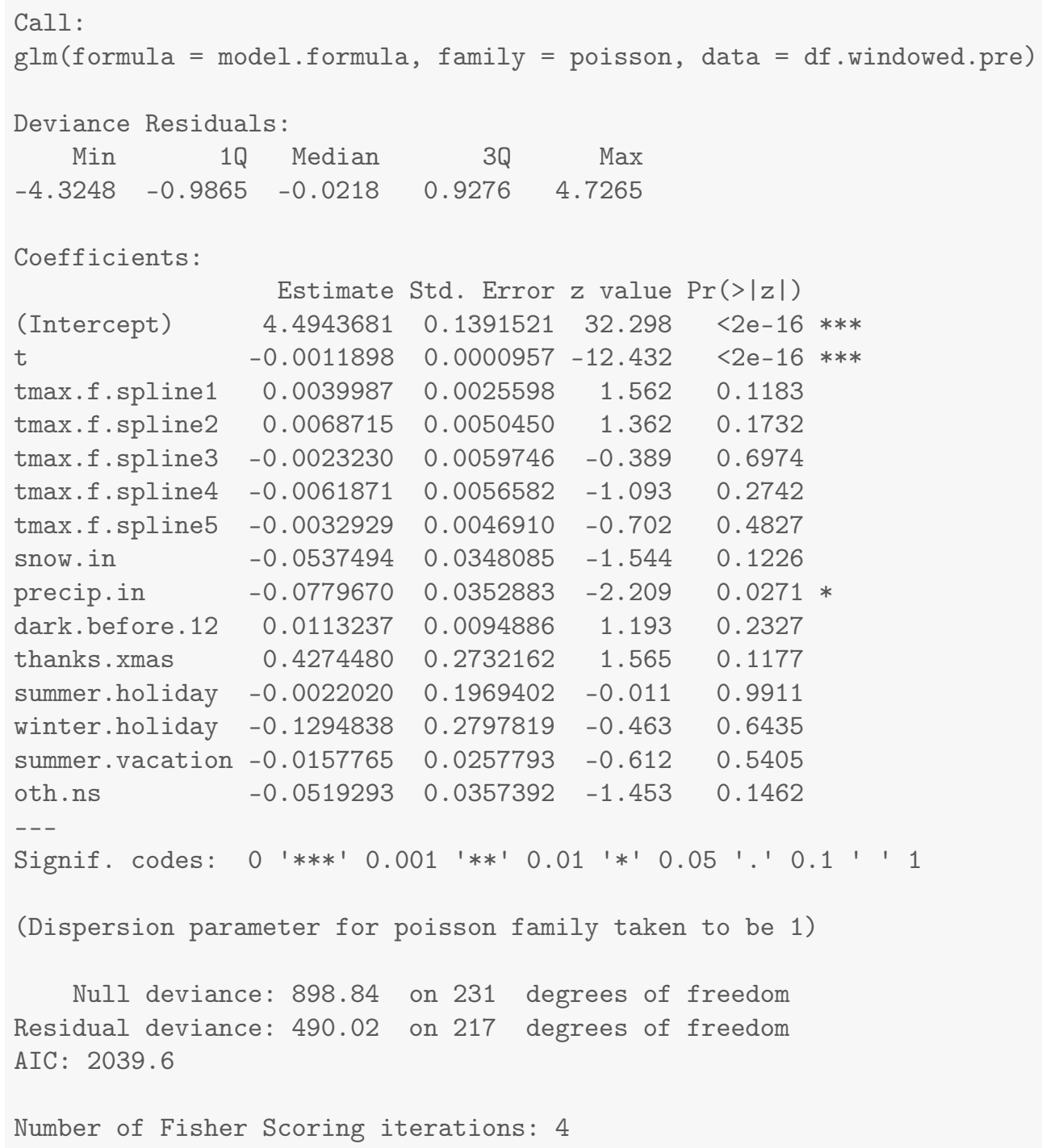




\section{Results for southeastern}

\subsection{Summary Values for southeastern}

Table 26.1: Descriptives for Outcome Before Ferguson Protests Begin

\begin{tabular}{lcccccccc}
\hline \hline Statistic & $\mathrm{N}$ & Mean & St. Dev. & Min & $\operatorname{Pctl}(25)$ & Median & $\operatorname{Pctl}(75)$ & Max \\
\hline southeastern & 232 & 126.487 & 20.270 & 79 & 113 & 126.5 & 142 & 181 \\
\hline
\end{tabular}

Table 26.2: Descriptives for Outcome After Ferguson Protests Begin

\begin{tabular}{lcccccccc}
\hline \hline Statistic & $\mathrm{N}$ & Mean & St. Dev. & Min & $\operatorname{Pctl}(25)$ & Median & $\operatorname{Pctl}(75)$ & Max \\
\hline southeastern & 290 & 131.390 & 25.125 & 63 & 117 & 129.5 & 148 & 233 \\
\hline
\end{tabular}




\subsection{Four Models for southeastern}

Table 26.3: Four Models that Differ on the Specification of Adjustment and Intervention Variables

\begin{tabular}{|c|c|c|c|c|}
\hline & \multicolumn{4}{|c|}{ Outcome: Count Per Week } \\
\hline & $(1)$ & (2) & (3) & $(4)$ \\
\hline Time (counter in weeks) & 0.02 & 0.06 & & 0.04 \\
\hline After Ferguson Protests Begin (week of 8/11/14 onward) & -15.56 & & -12.55 & -10.90 \\
\hline After Gray Protests Begin (week of $4 / 20 / 15$ onward) & 36.03 & & 23.32 & 24.23 \\
\hline Unrest and National Guard $(4 / 27 / 15-5 / 3 / 2015)$ & -5.70 & & -0.63 & -1.68 \\
\hline After Batts Fired (week of 7/13/15 onward) & 8.95 & & -1.15 & 0.68 \\
\hline After Davis Confirmed (week of 10/19/15 onward) & -29.36 & & -18.41 & -19.46 \\
\hline After Davis First Year (week of 10/17/16 onward) & 7.88 & & 8.05 & 6.72 \\
\hline After De Sousa Begins (week of 1/19/18 onward) & -28.02 & & -16.71 & -18.48 \\
\hline After Tuggle Begins (week of 5/14/18 onward) & 27.49 & & 15.33 & 15.14 \\
\hline After Harrison Begins (week of 2/11/19 onward) & -25.76 & & -21.91 & -24.38 \\
\hline Average Maximum Temperature to 50 Degrees & & 1.08 & & 1.24 \\
\hline Plus Degrees in the $50 \mathrm{~s}$ & & -0.06 & & -0.15 \\
\hline Plus Degrees in the 60s & & 0.73 & & 0.78 \\
\hline Plus Degrees in the 70s & & -1.75 & & -1.50 \\
\hline Plus Degrees Greater Than 80 & & 0.65 & & -0.33 \\
\hline Snowfall (inches) & & -2.66 & & -1.80 \\
\hline Precipitation (inches) & & 2.75 & & -0.15 \\
\hline Darkness Before Midnight (hours) & & 6.17 & & 7.44 \\
\hline Thanksgiving/Christmas (proportion of week) & & 0.94 & & -1.71 \\
\hline Winter Holiday (proportion of week) & & 30.03 & & -24.14 \\
\hline Other Out-of-School Days (proportion of week) & & 0.59 & & -6.36 \\
\hline Summer Vacation (proportion of week) & & 11.95 & & 6.84 \\
\hline Summer Holiday (proportion of week) & & -52.84 & & -36.22 \\
\hline Observations & 522 & 232 & 522 & 522 \\
\hline $\mathrm{R}^{2}$ & 0.20 & 0.41 & 0.20 & 0.46 \\
\hline
\end{tabular}




\subsection{Least Squares Updated Model (2) for southeastern}

southeastern

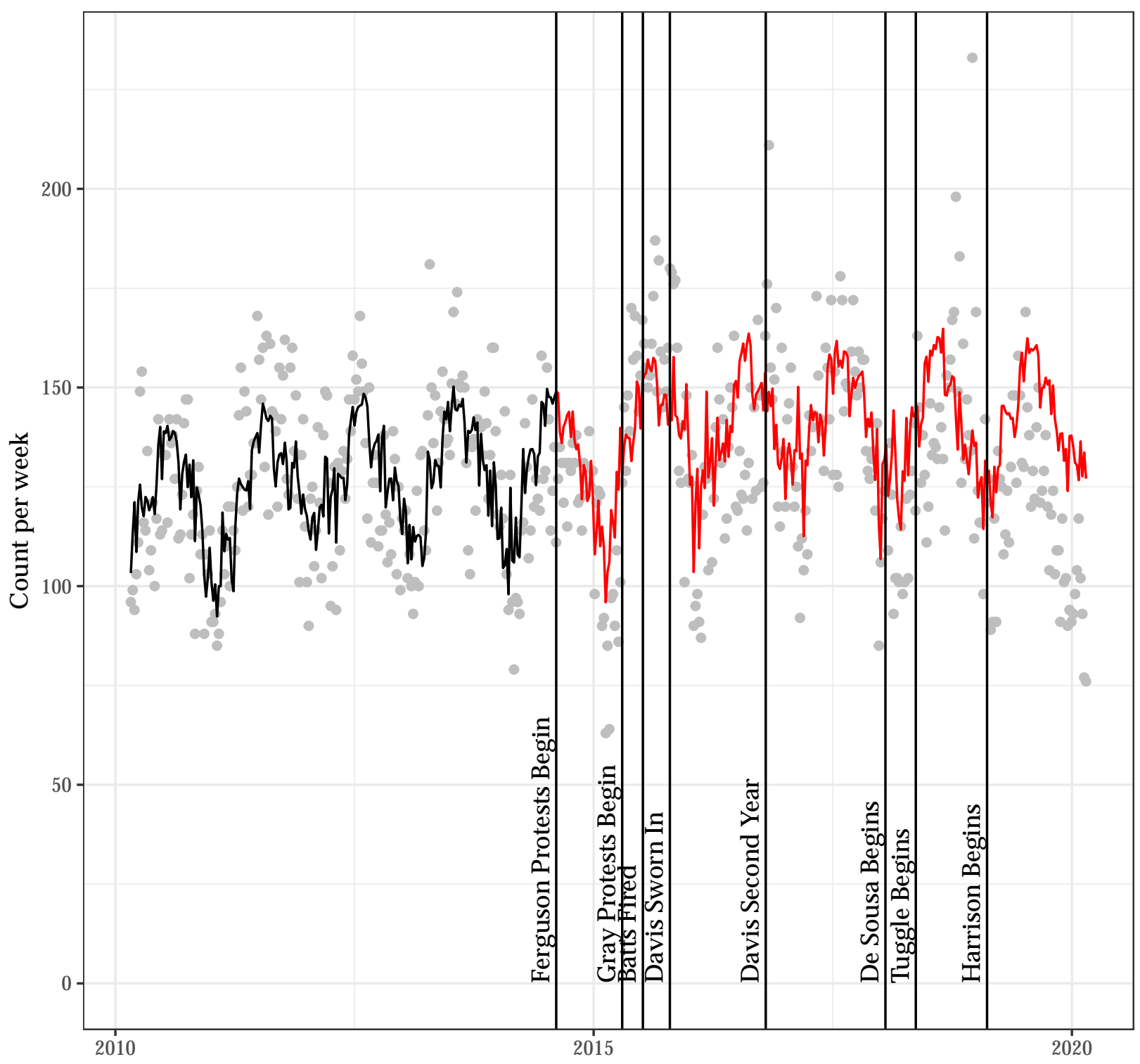




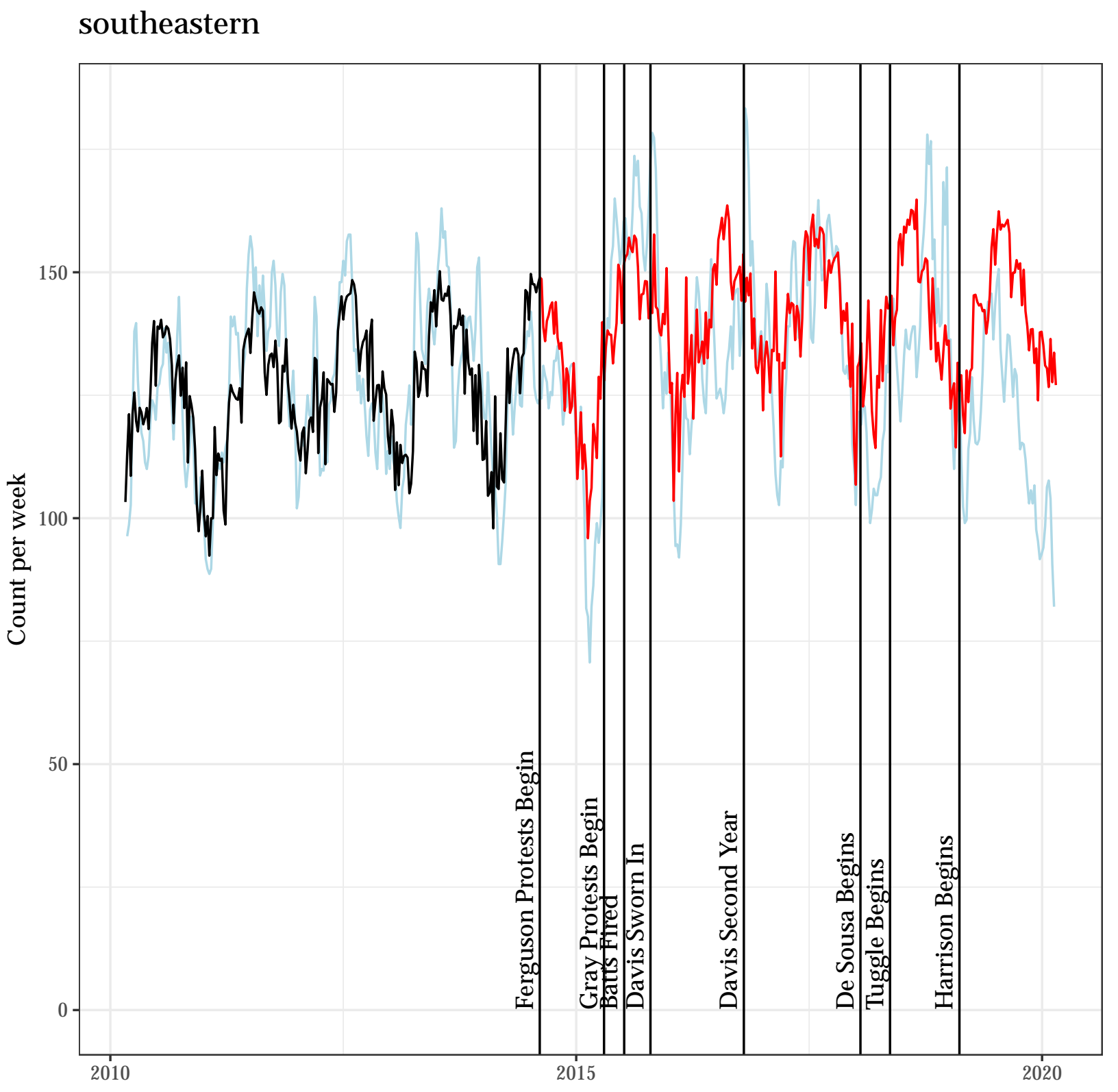


Autocorrelation Function for the Observed Outcome for Updated Model (2)

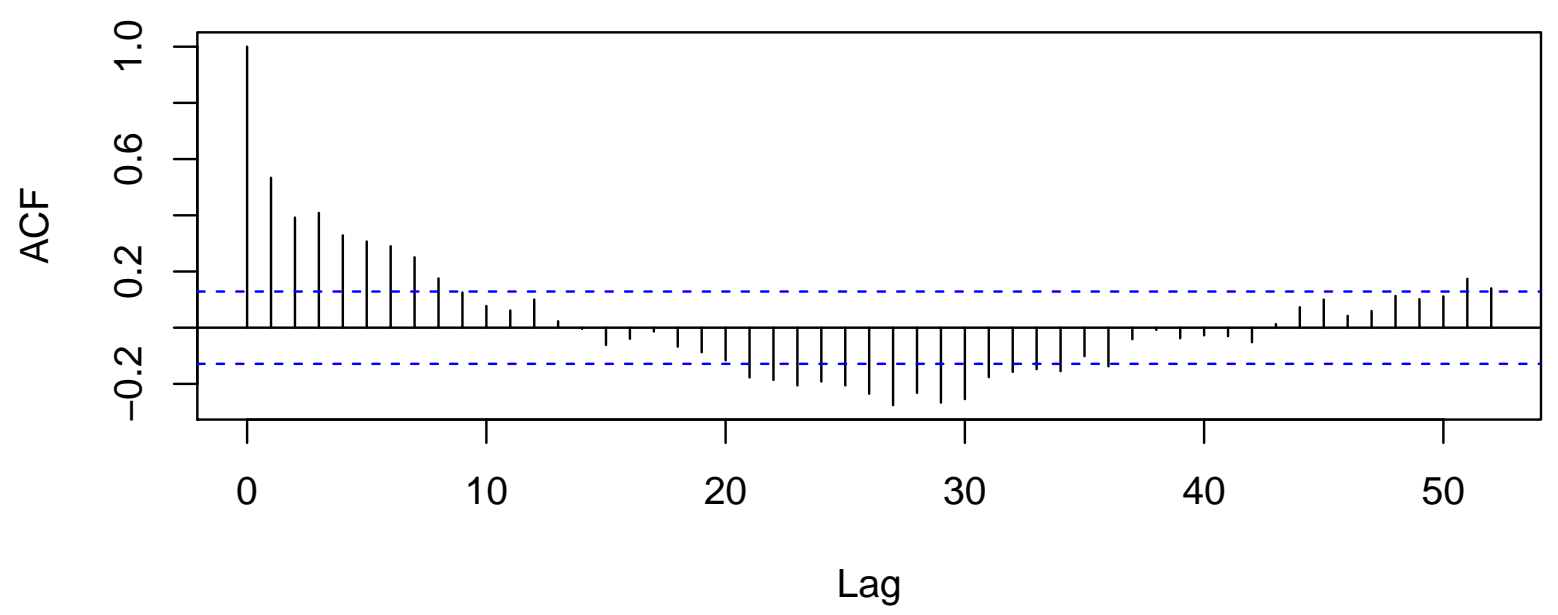

Autocorrelation Function for the Residuals from Updated Model (2)

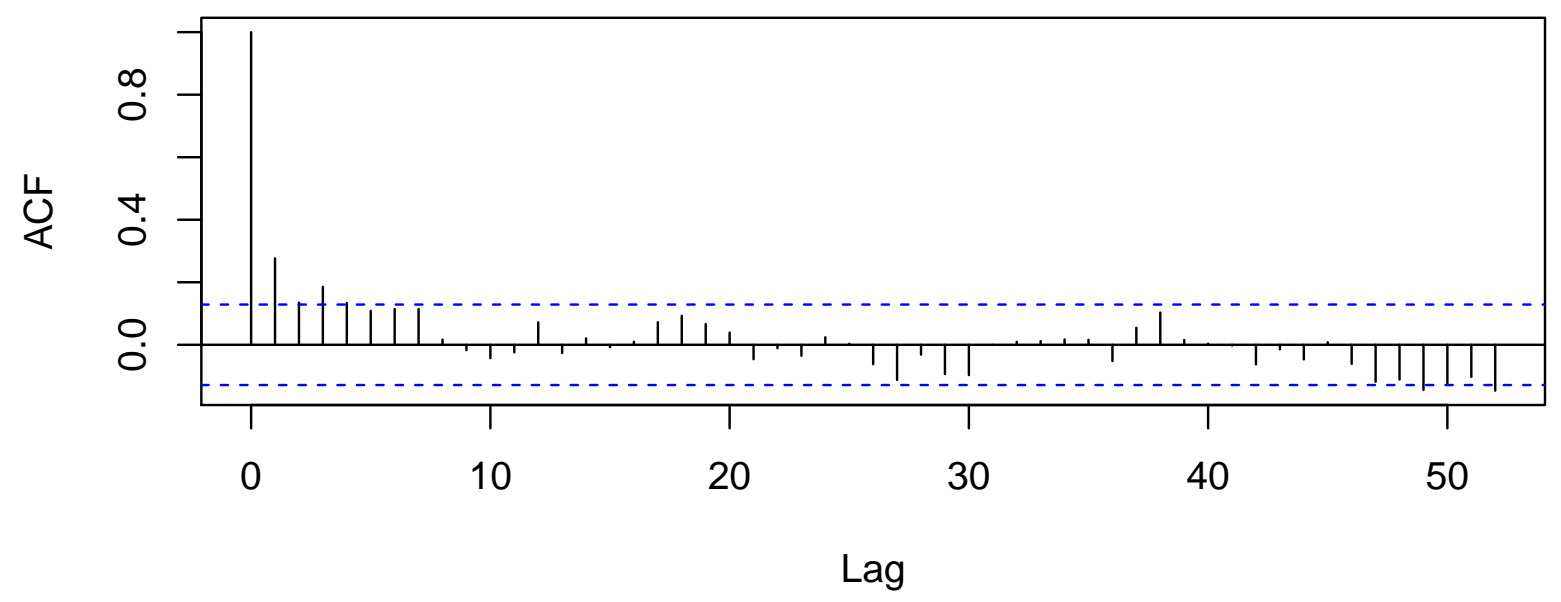




\subsection{A Poisson Regression Model as an Alternative to Model (2) for southeastern}

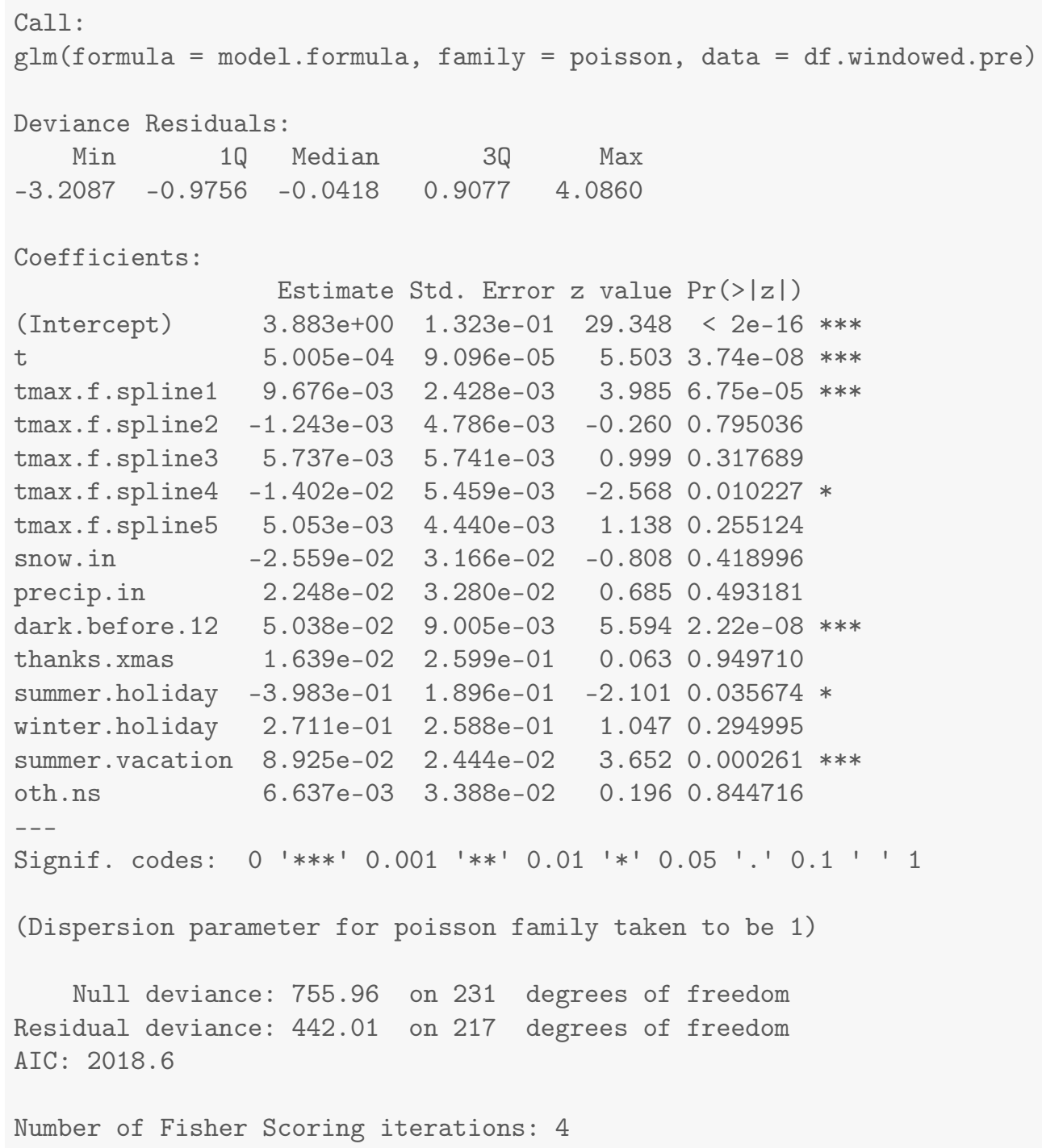




\section{Results for homicide.northwestern}

\subsection{Summary Values for homicide.northwestern}

Table 27.1: Descriptives for Outcome Before Ferguson Protests Begin

\begin{tabular}{lcccccccc}
\hline \hline Statistic & $\mathrm{N}$ & Mean & St. Dev. & Min & Pctl(25) & Median & Pctl(75) & Max \\
\hline homicide.northwestern & 232 & 0.539 & 0.749 & 0 & 0 & 0 & 1 & 4 \\
\hline
\end{tabular}

Table 27.2: Descriptives for Outcome After Ferguson Protests Begin

\begin{tabular}{lcccccccc}
\hline \hline Statistic & $\mathrm{N}$ & Mean & St. Dev. & Min & Pctl(25) & Median & Pctl(75) & Max \\
\hline homicide.northwestern & 290 & 0.790 & 0.889 & 0 & 0 & 1 & 1 & 4 \\
\hline
\end{tabular}




\subsection{Four Models for homicide.northwestern}

Table 27.3: Four Models that Differ on the Specification of Adjustment and Intervention Variables

\begin{tabular}{|c|c|c|c|c|}
\hline & \multicolumn{4}{|c|}{ Outcome: Count Per Week } \\
\hline & $(1)$ & $(2)$ & (3) & $(4)$ \\
\hline Time (counter in weeks) & 0.001 & 0.002 & & 0.001 \\
\hline After Ferguson Protests Begin (week of $8 / 11 / 14$ onward) & -0.04 & & -0.11 & -0.02 \\
\hline After Gray Protests Begin (week of $4 / 20 / 15$ onward) & 0.46 & & 0.39 & 0.36 \\
\hline Unrest and National Guard $(4 / 27 / 15-5 / 3 / 2015)$ & 1.91 & & 1.76 & 1.88 \\
\hline After Batts Fired (week of 7/13/15 onward) & -0.10 & & -0.23 & -0.22 \\
\hline After Davis Confirmed (week of 10/19/15 onward) & -0.34 & & -0.21 & -0.25 \\
\hline After Davis First Year (week of 10/17/16 onward) & 0.20 & & 0.20 & 0.21 \\
\hline After De Sousa Begins (week of 1/19/18 onward) & -0.41 & & -0.20 & -0.33 \\
\hline After Tuggle Begins (week of 5/14/18 onward) & 0.11 & & -0.04 & 0.001 \\
\hline After Harrison Begins (week of 2/11/19 onward) & 0.07 & & 0.14 & 0.07 \\
\hline Average Maximum Temperature to 50 Degrees & & -0.02 & & 0.001 \\
\hline Plus Degrees in the $50 \mathrm{~s}$ & & 0.02 & & 0.04 \\
\hline Plus Degrees in the $60 \mathrm{~s}$ & & 0.06 & & -0.03 \\
\hline Plus Degrees in the 70s & & -0.10 & & -0.02 \\
\hline Plus Degrees Greater Than 80 & & 0.05 & & 0.03 \\
\hline Snowfall (inches) & & -0.18 & & 0.09 \\
\hline Precipitation (inches) & & -0.08 & & -0.09 \\
\hline Darkness Before Midnight (hours) & & 0.09 & & 0.08 \\
\hline Thanksgiving/Christmas (proportion of week) & & -0.61 & & -0.12 \\
\hline Winter Holiday (proportion of week) & & -2.65 & & 0.03 \\
\hline Other Out-of-School Days (proportion of week) & & -0.25 & & -0.12 \\
\hline Summer Vacation (proportion of week) & & 0.11 & & 0.01 \\
\hline Summer Holiday (proportion of week) & & 0.53 & & 0.47 \\
\hline Observations & 522 & 232 & 522 & 522 \\
\hline $\mathrm{R}^{2}$ & 0.05 & 0.07 & 0.02 & 0.08 \\
\hline
\end{tabular}


27.3 Least Squares Updated Model (2) for homicide.northwestern homicide.northwestern

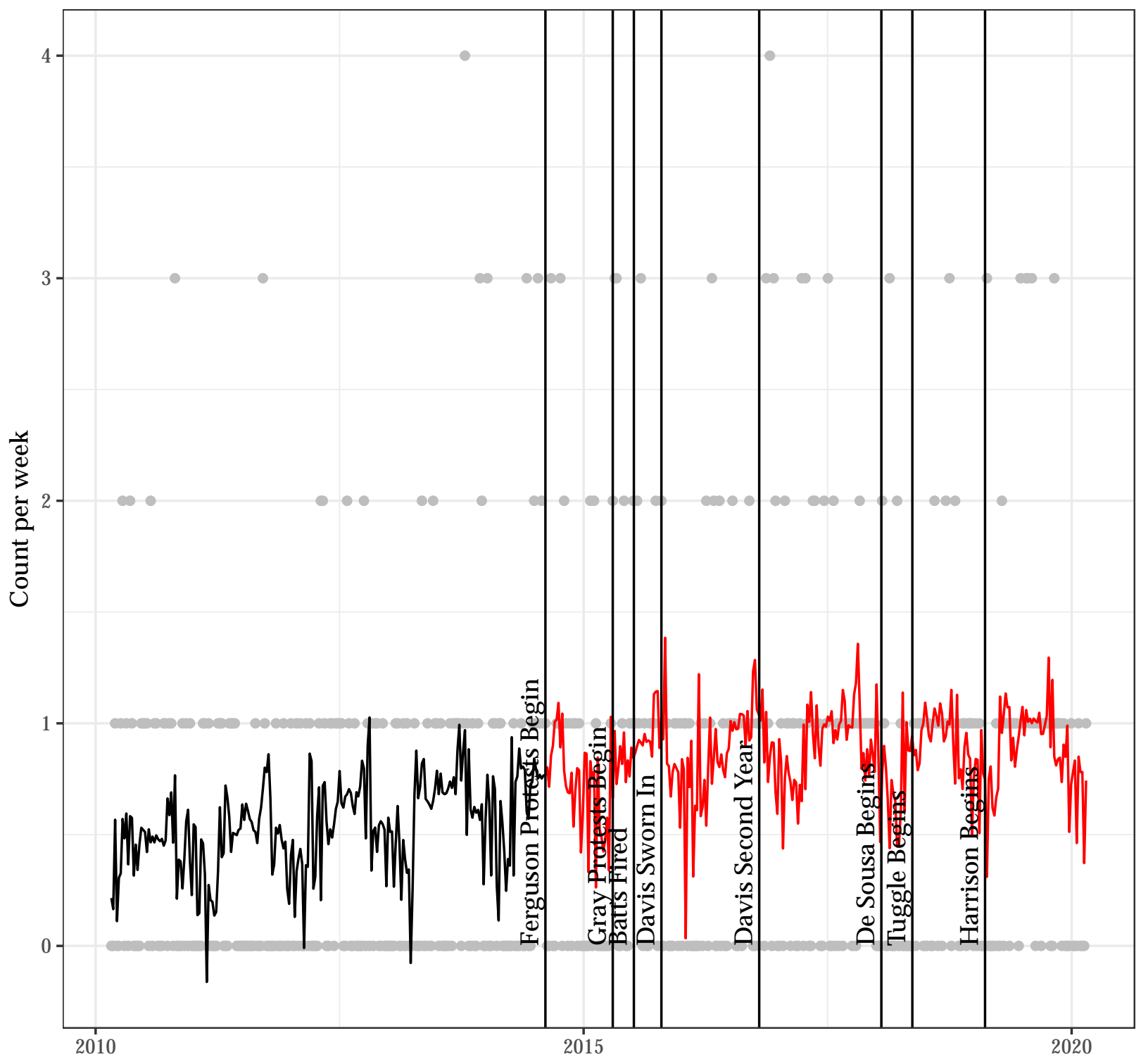




\section{homicide.northwestern}

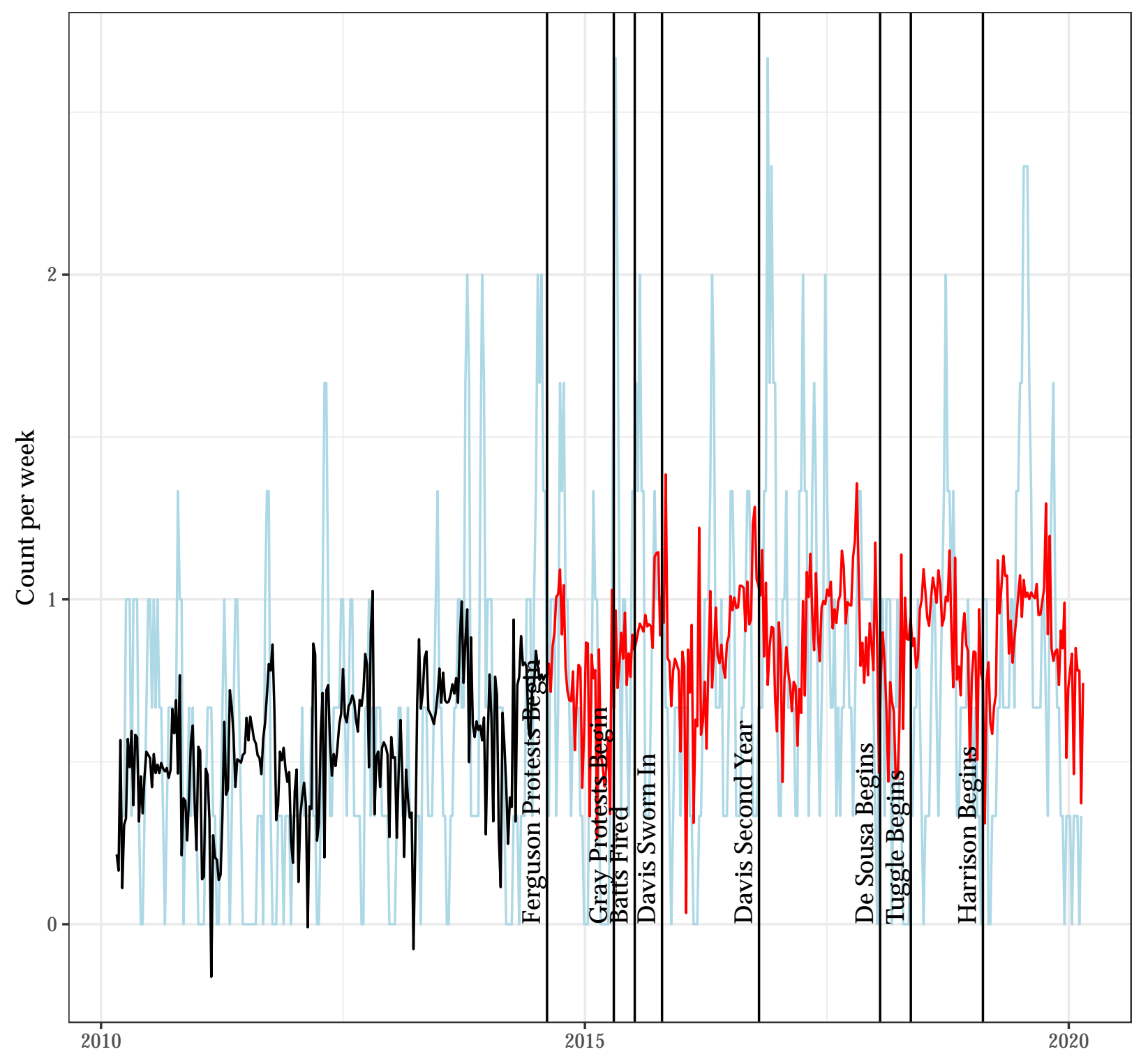


Autocorrelation Function for the Observed Outcome for Updated Model (2)

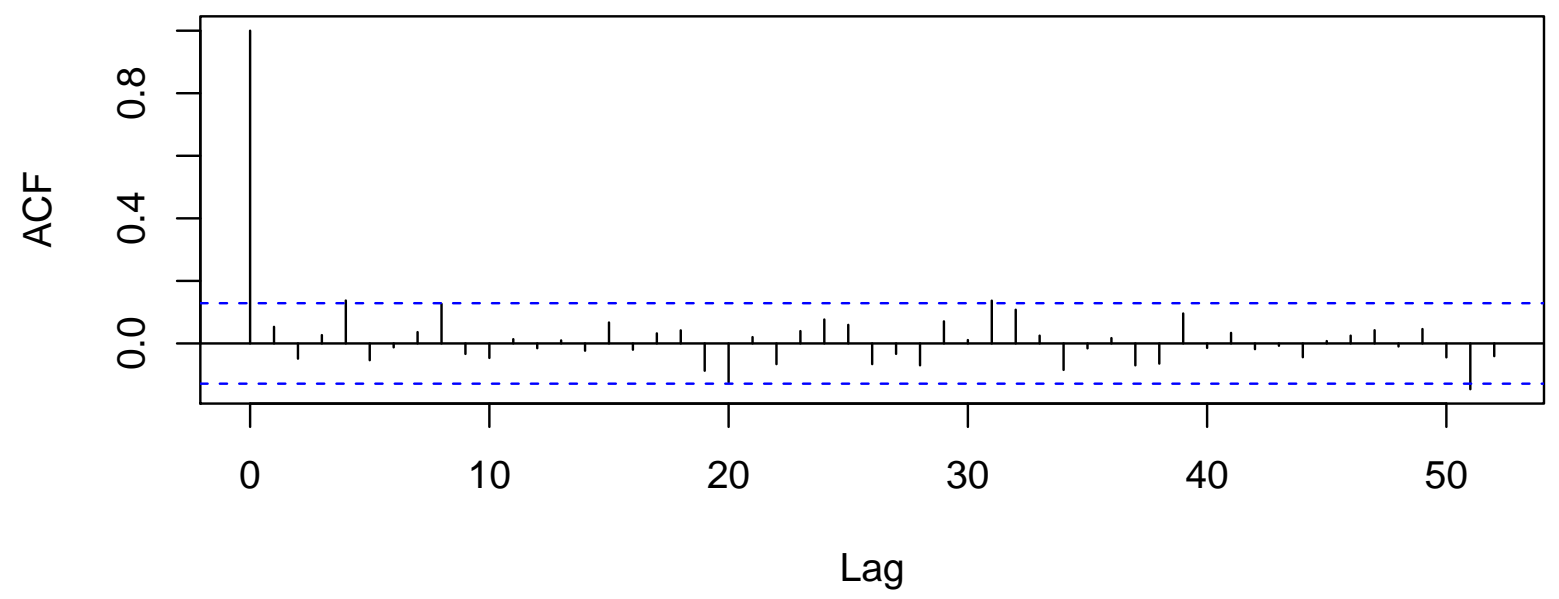

Autocorrelation Function for the Residuals from Updated Model (2)

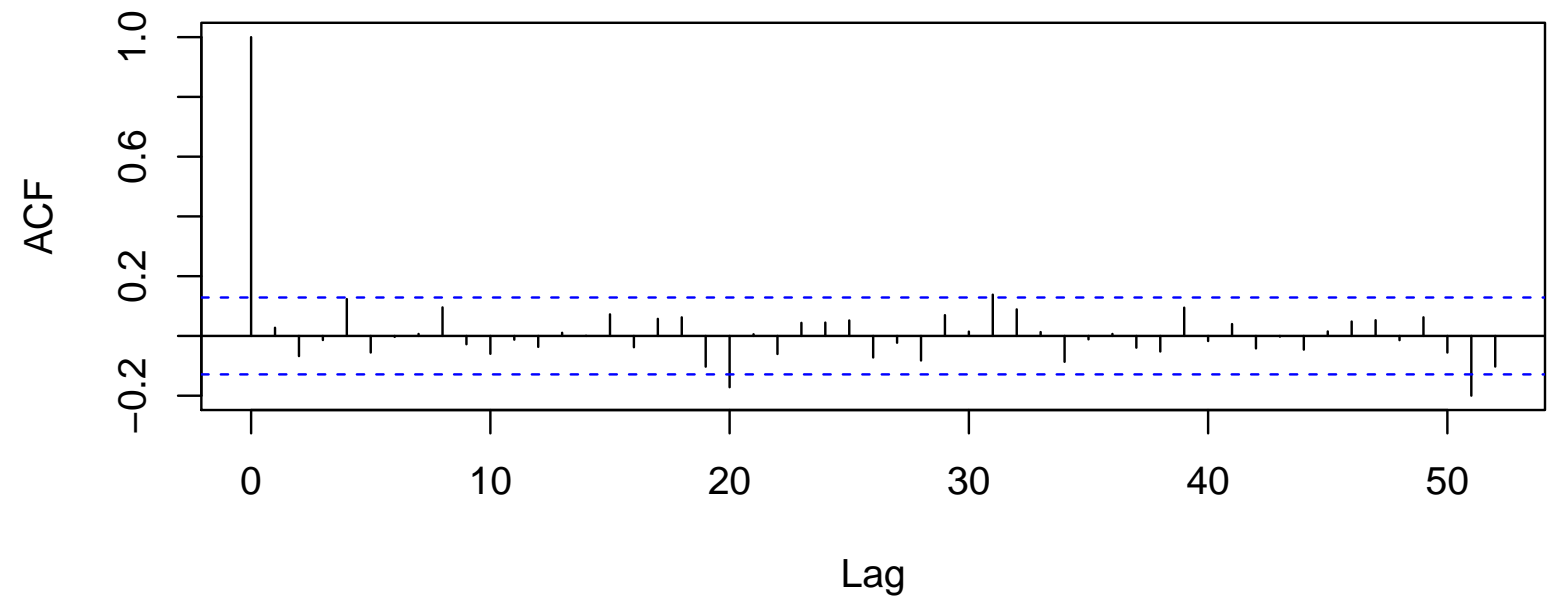




\subsection{A Poisson Regression Model as an Alternative to Model (2) for homicide.northwestern}

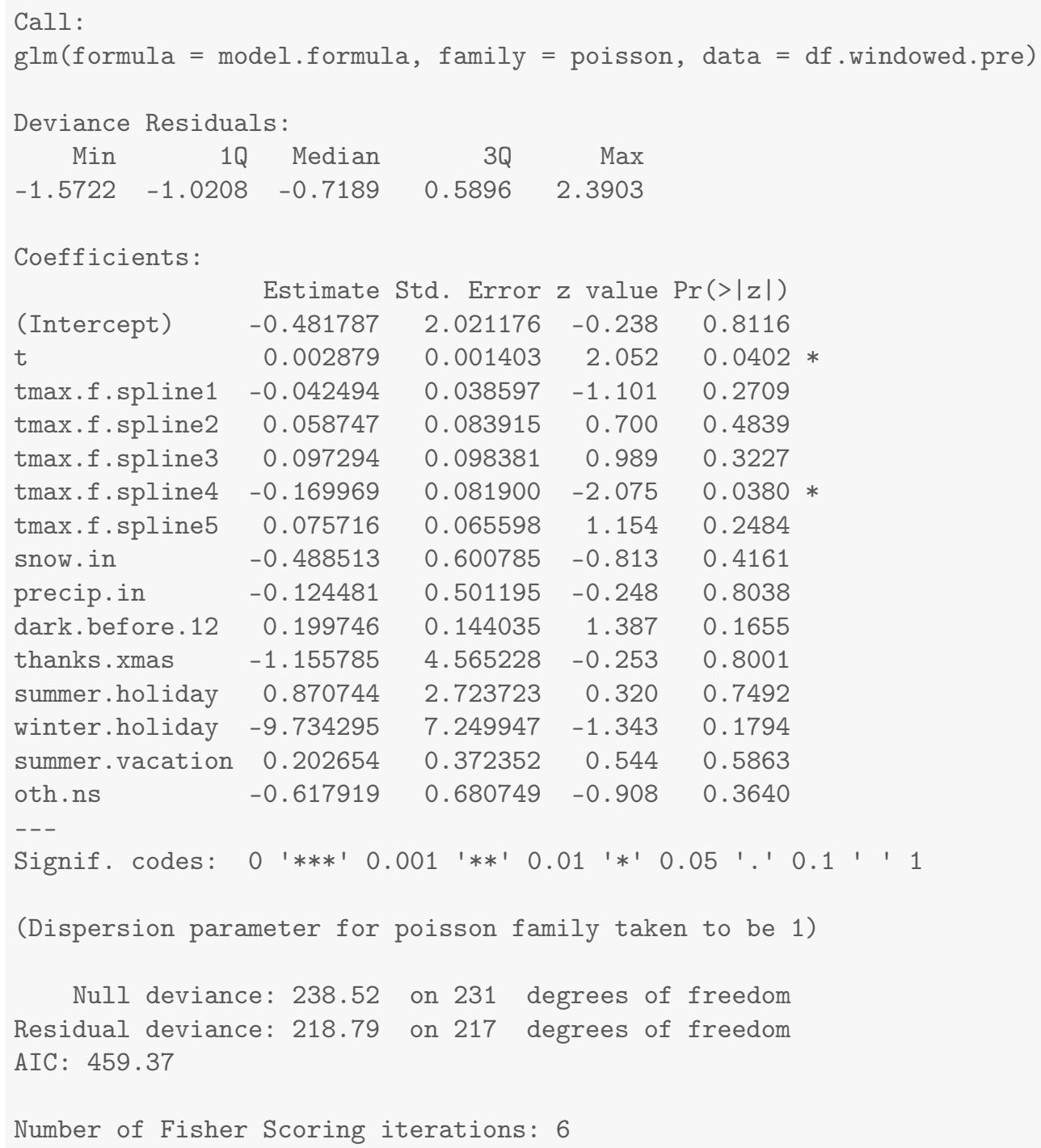




\section{Results for homicide.northern}

\subsection{Summary Values for homicide.northern}

Table 28.1: Descriptives for Outcome Before Ferguson Protests Begin

\begin{tabular}{lcccccccc}
\hline \hline Statistic & $\mathrm{N}$ & Mean & St. Dev. & Min & Pctl(25) & Median & Pctl(75) & Max \\
\hline homicide.northern & 232 & 0.345 & 0.612 & 0 & 0 & 0 & 1 & 3 \\
\hline
\end{tabular}

Table 28.2: Descriptives for Outcome After Ferguson Protests Begin

\begin{tabular}{lcccccccc}
\hline \hline Statistic & $\mathrm{N}$ & Mean & St. Dev. & Min & Pctl(25) & Median & Pctl(75) & Max \\
\hline homicide.northern & 290 & 0.421 & 0.683 & 0 & 0 & 0 & 1 & 3 \\
\hline
\end{tabular}




\subsection{Four Models for homicide.northern}

Table 28.3: Four Models that Differ on the Specification of Adjustment and Intervention Variables

\begin{tabular}{|c|c|c|c|c|}
\hline & \multicolumn{4}{|c|}{ Outcome: Count Per Week } \\
\hline & (1) & (2) & (3) & $(4)$ \\
\hline Time (counter in weeks) & 0.0003 & 0.0000 & & 0.0002 \\
\hline After Ferguson Protests Begin (week of 8/11/14 onward) & -0.08 & & -0.04 & -0.06 \\
\hline After Gray Protests Begin (week of 4/20/15 onward) & 0.23 & & 0.10 & 0.16 \\
\hline Unrest and National Guard (4/27/15 - 5/3/2015) & -0.54 & & -0.18 & -0.30 \\
\hline After Batts Fired (week of 7/13/15 onward) & 0.02 & & 0.22 & 0.08 \\
\hline After Davis Confirmed (week of 10/19/15 onward) & -0.29 & & -0.34 & -0.26 \\
\hline After Davis First Year (week of 10/17/16 onward) & 0.19 & & 0.21 & 0.19 \\
\hline After De Sousa Begins (week of 1/19/18 onward) & 0.05 & & -0.004 & 0.06 \\
\hline After Tuggle Begins (week of 5/14/18 onward) & -0.19 & & -0.15 & -0.25 \\
\hline After Harrison Begins (week of 2/11/19 onward) & 0.06 & & 0.11 & 0.11 \\
\hline Average Maximum Temperature to 50 Degrees & & -0.02 & & -0.01 \\
\hline Plus Degrees in the $50 \mathrm{~s}$ & & 0.04 & & 0.004 \\
\hline Plus Degrees in the $60 \mathrm{~s}$ & & -0.08 & & -0.01 \\
\hline Plus Degrees in the 70s & & 0.13 & & 0.05 \\
\hline Plus Degrees Greater Than 80 & & -0.10 & & -0.05 \\
\hline Snowfall (inches) & & -0.14 & & -0.09 \\
\hline Precipitation (inches) & & 0.18 & & 0.23 \\
\hline Darkness Before Midnight (hours) & & -0.06 & & -0.01 \\
\hline Thanksgiving/Christmas (proportion of week) & & 0.15 & & -0.99 \\
\hline Winter Holiday (proportion of week) & & -0.73 & & -1.43 \\
\hline Other Out-of-School Days (proportion of week) & & 0.13 & & 0.13 \\
\hline Summer Vacation (proportion of week) & & -0.33 & & -0.11 \\
\hline Summer Holiday (proportion of week) & & 0.13 & & -0.11 \\
\hline Observations & 522 & 232 & 522 & 522 \\
\hline $\mathrm{R}^{2}$ & 0.02 & 0.08 & 0.01 & 0.04 \\
\hline
\end{tabular}


28.3 Least Squares Updated Model (2) for homicide.northern homicide.northern

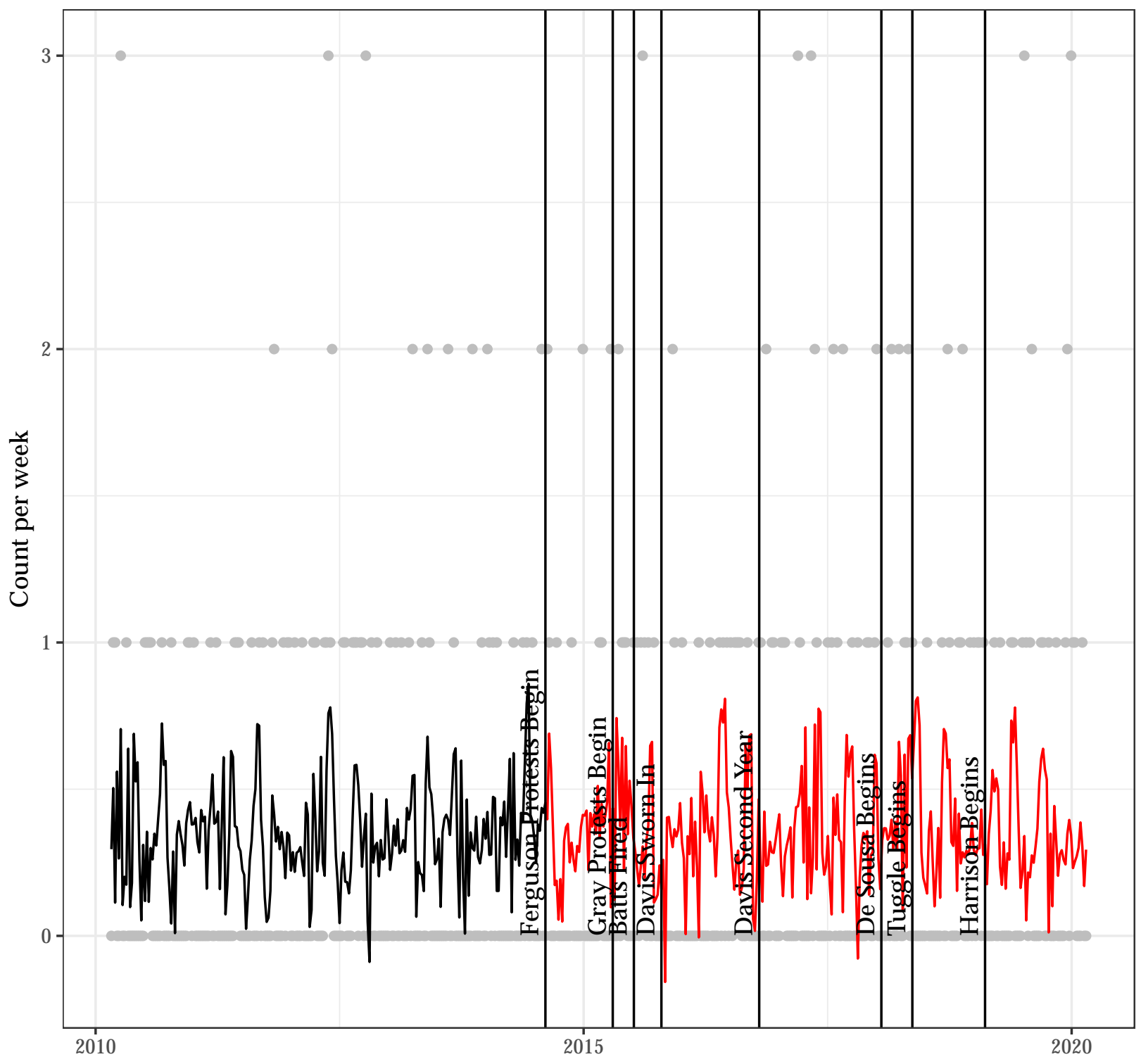




\section{homicide.northern}

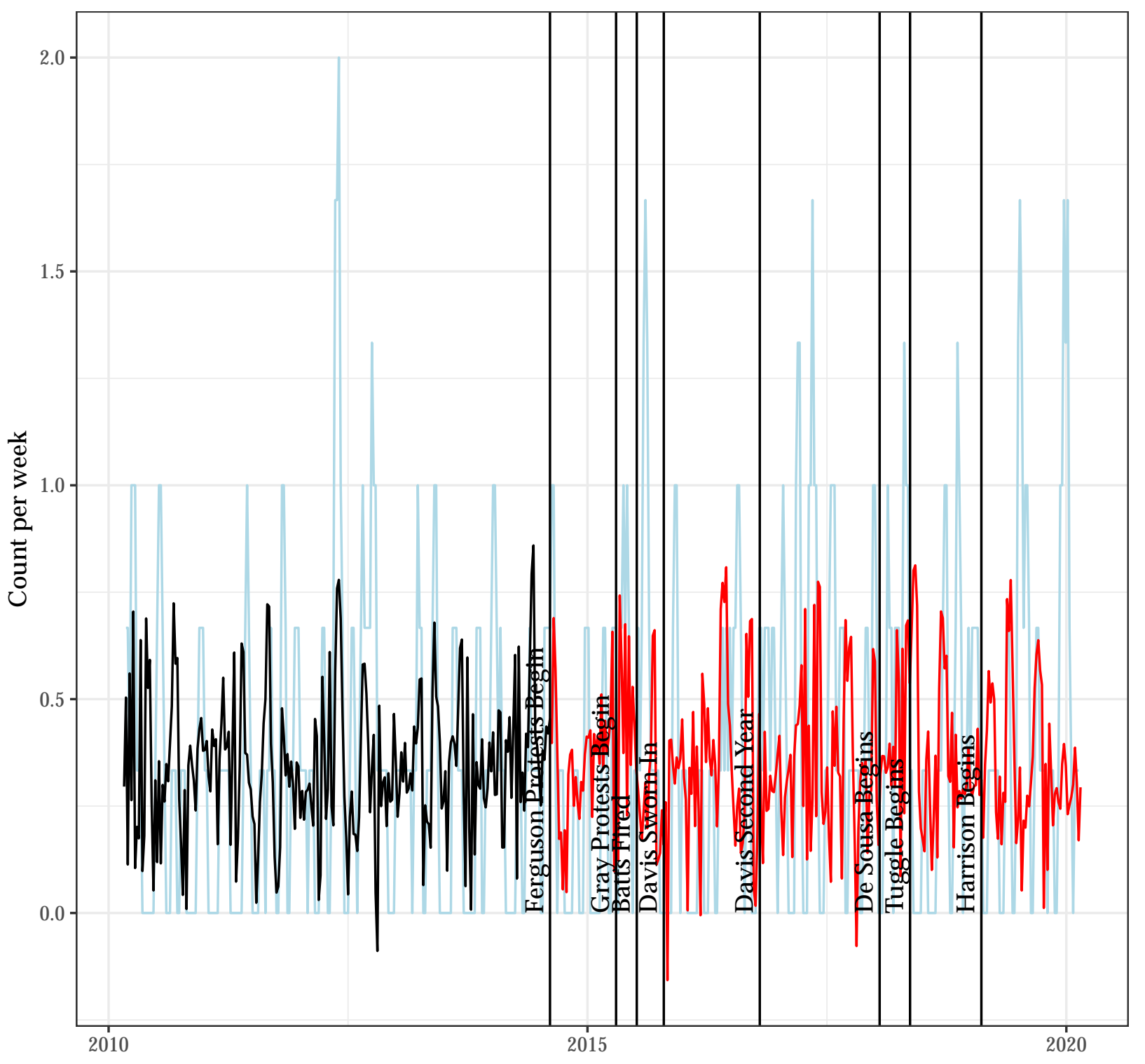


Autocorrelation Function for the Observed Outcome for Updated Model (2)

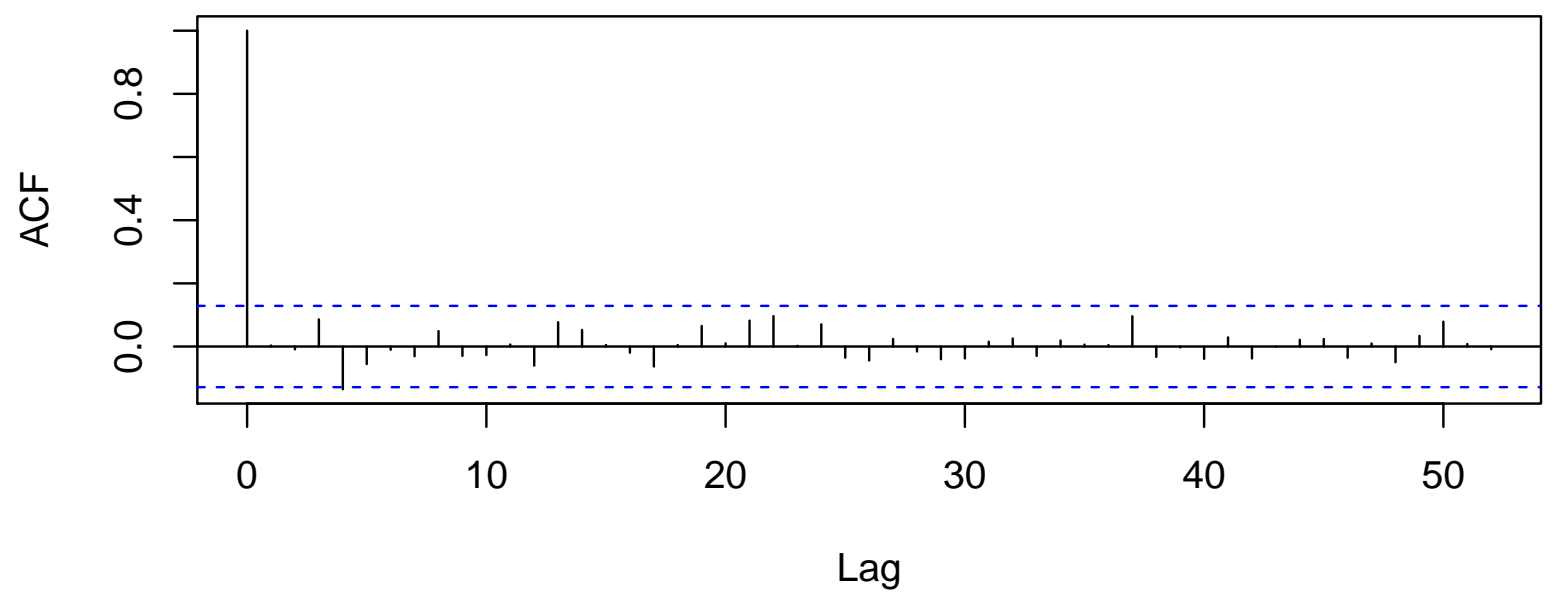

Autocorrelation Function for the Residuals from Updated Model (2)

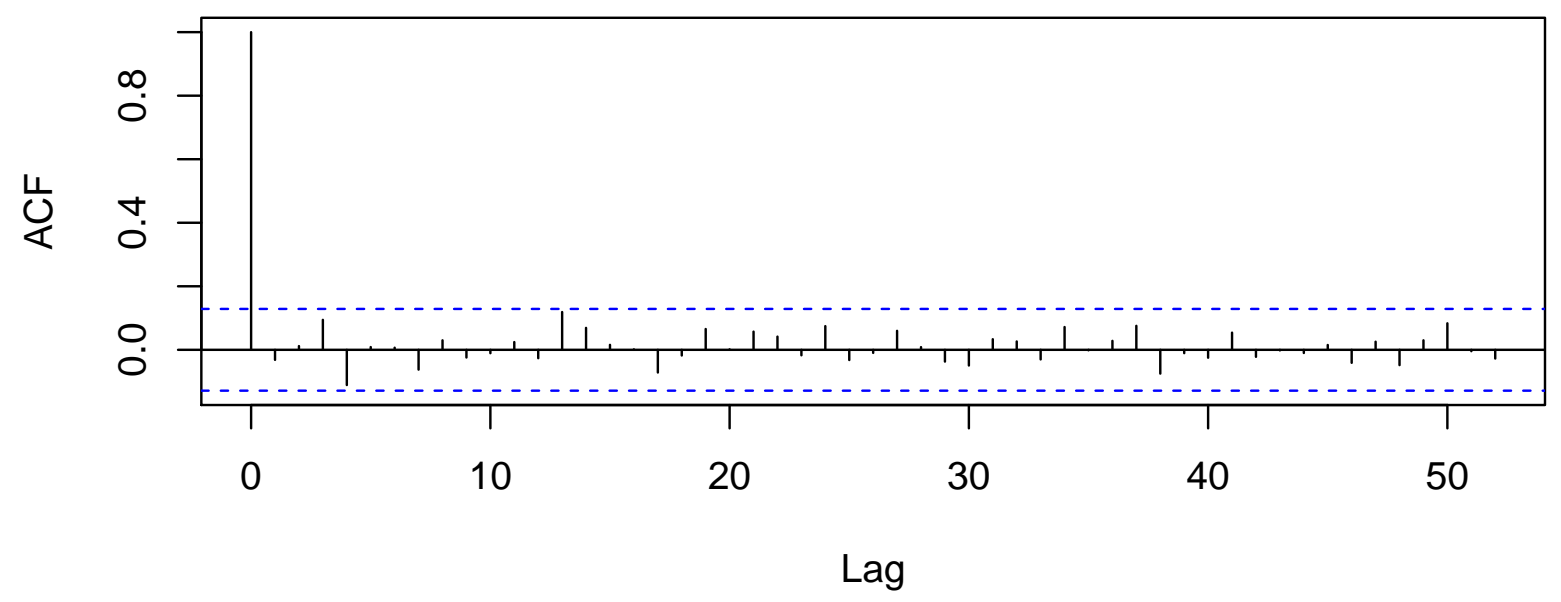




\subsection{A Poisson Regression Model as an Alternative to Model (2) for homicide.northern}

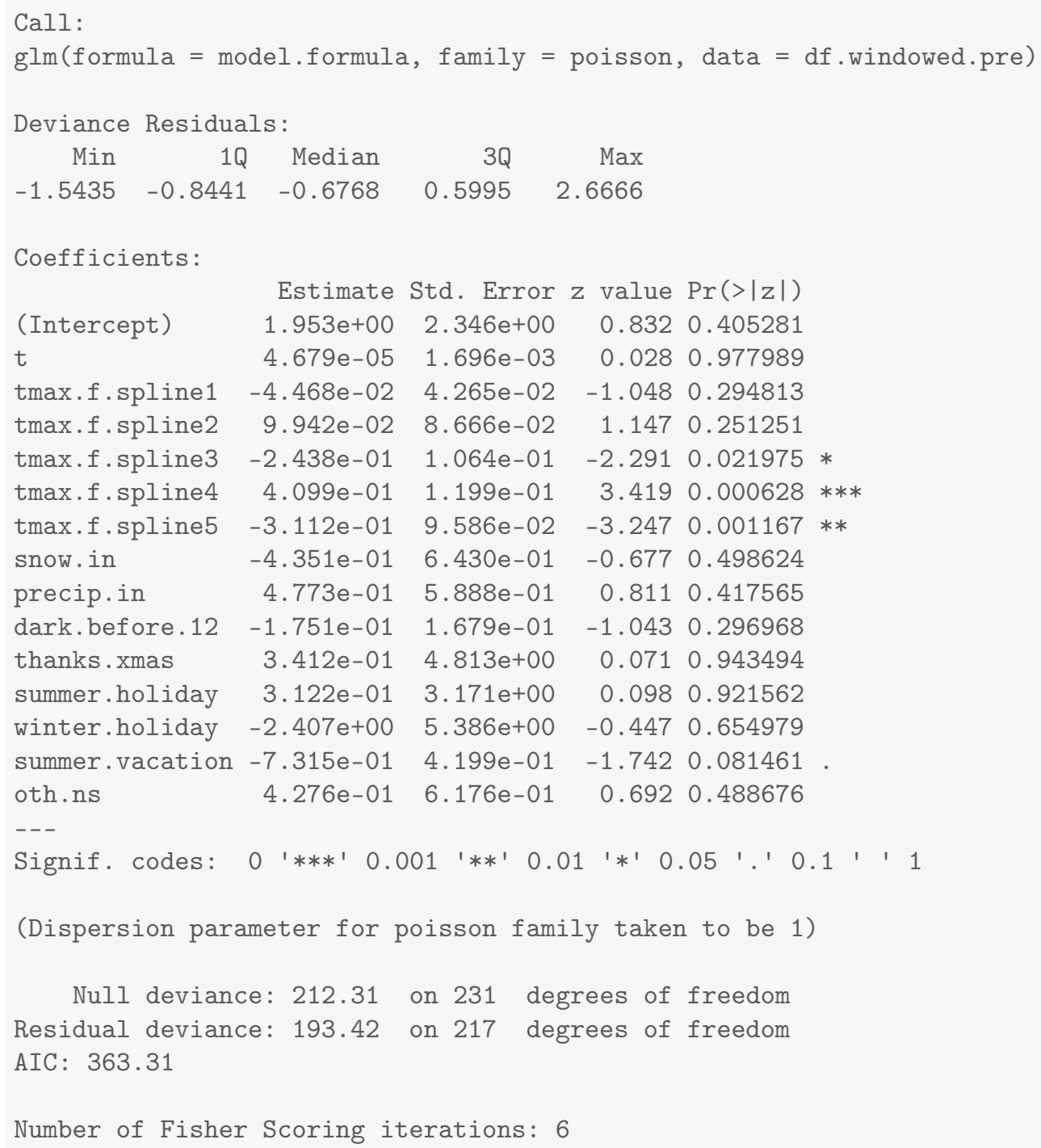




\section{Results for homicide.northeastern}

\subsection{Summary Values for homicide.northeastern}

Table 29.1: Descriptives for Outcome Before Ferguson Protests Begin

\begin{tabular}{lcccccccc}
\hline \hline Statistic & $\mathrm{N}$ & Mean & St. Dev. & Min & Pctl(25) & Median & Pctl(75) & Max \\
\hline homicide.northeastern & 232 & 0.625 & 0.849 & 0 & 0 & 0 & 1 & 5 \\
\hline
\end{tabular}

Table 29.2: Descriptives for Outcome After Ferguson Protests Begin

\begin{tabular}{lcccccccc}
\hline \hline Statistic & $\mathrm{N}$ & Mean & St. Dev. & Min & Pctl(25) & Median & Pctl(75) & Max \\
\hline homicide.northeastern & 290 & 0.817 & 0.972 & 0 & 0 & 1 & 1 & 5 \\
\hline
\end{tabular}




\subsection{Four Models for homicide.northeastern}

Table 29.3: Four Models that Differ on the Specification of Adjustment and Intervention Variables

\begin{tabular}{|c|c|c|c|c|}
\hline & \multicolumn{4}{|c|}{ Outcome: Count Per Week } \\
\hline & $(1)$ & $(2)$ & (3) & $(4)$ \\
\hline Time (counter in weeks) & -0.001 & -0.002 & & -0.002 \\
\hline After Ferguson Protests Begin (week of 8/11/14 onward) & 0.49 & & 0.50 & 0.52 \\
\hline After Gray Protests Begin (week of $4 / 20 / 15$ onward) & 0.39 & & 0.29 & 0.28 \\
\hline Unrest and National Guard $(4 / 27 / 15-5 / 3 / 2015)$ & -1.28 & & -1.09 & -1.11 \\
\hline After Batts Fired (week of 7/13/15 onward) & -0.25 & & -0.18 & -0.21 \\
\hline After Davis Confirmed (week of 10/19/15 onward) & -0.16 & & -0.14 & -0.09 \\
\hline After Davis First Year (week of 10/17/16 onward) & 0.04 & & 0.03 & 0.03 \\
\hline After De Sousa Begins (week of 1/19/18 onward) & -0.12 & & -0.20 & -0.04 \\
\hline After Tuggle Begins (week of 5/14/18 onward) & 0.20 & & 0.14 & 0.10 \\
\hline After Harrison Begins (week of 2/11/19 onward) & 0.24 & & 0.21 & 0.28 \\
\hline Average Maximum Temperature to 50 Degrees & & -0.03 & & -0.01 \\
\hline Plus Degrees in the $50 \mathrm{~s}$ & & 0.05 & & 0.01 \\
\hline Plus Degrees in the 60s & & -0.05 & & 0.01 \\
\hline Plus Degrees in the 70s & & 0.06 & & 0.01 \\
\hline Plus Degrees Greater Than 80 & & -0.03 & & -0.04 \\
\hline Snowfall (inches) & & 0.05 & & -0.14 \\
\hline Precipitation (inches) & & -0.10 & & -0.02 \\
\hline Darkness Before Midnight (hours) & & -0.03 & & 0.03 \\
\hline Thanksgiving/Christmas (proportion of week) & & -2.11 & & -1.96 \\
\hline Winter Holiday (proportion of week) & & -2.34 & & -0.76 \\
\hline Other Out-of-School Days (proportion of week) & & -0.06 & & -0.10 \\
\hline Summer Vacation (proportion of week) & & -0.29 & & 0.09 \\
\hline Summer Holiday (proportion of week) & & 2.18 & & 1.96 \\
\hline Observations & 522 & 232 & 522 & 522 \\
\hline $\mathrm{R}^{2}$ & 0.03 & 0.06 & 0.08 & 0.05 \\
\hline
\end{tabular}


29.3 Least Squares Updated Model (2) for homicide.northeastern homicide.northeastern

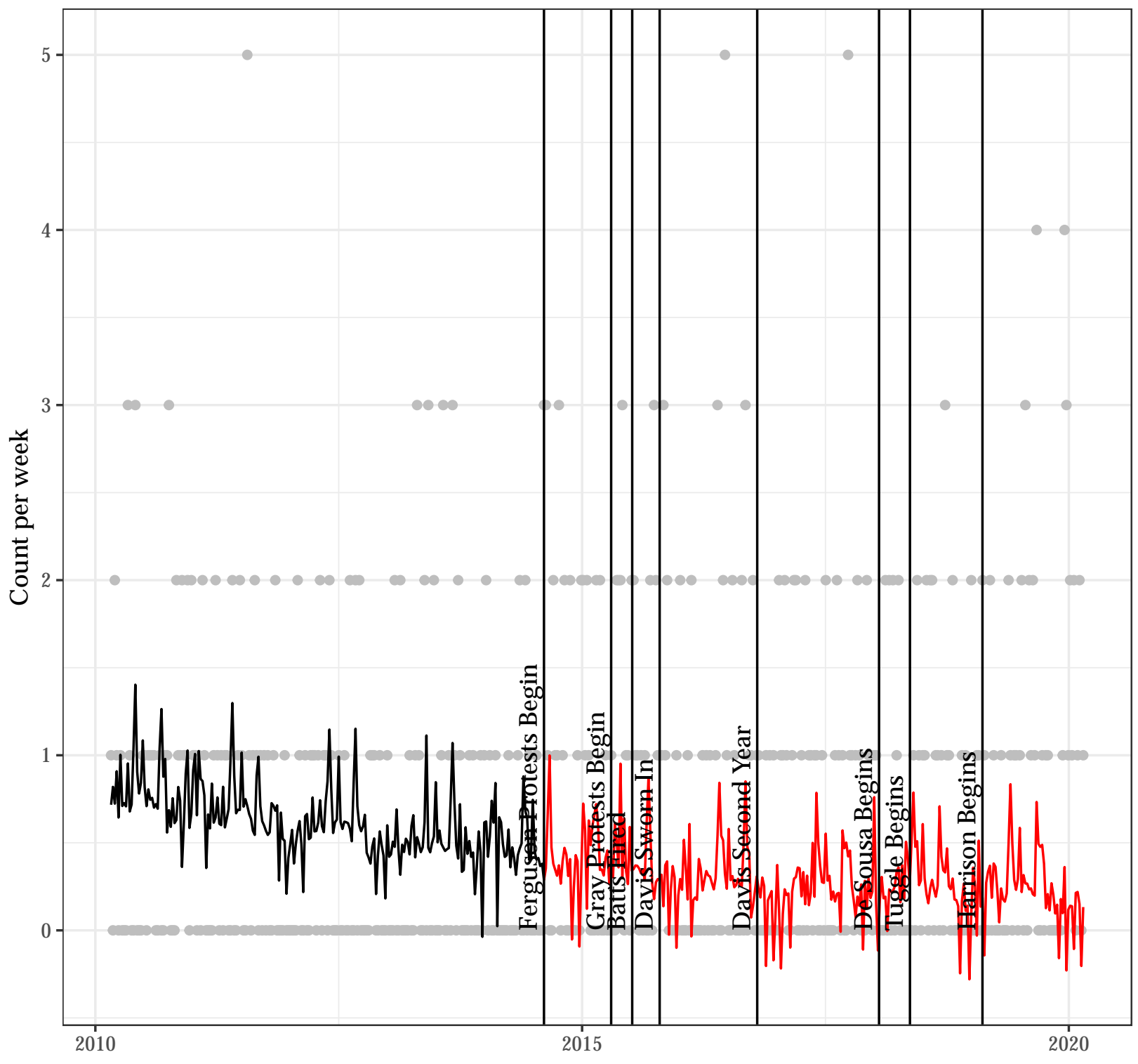




\section{homicide.northeastern}

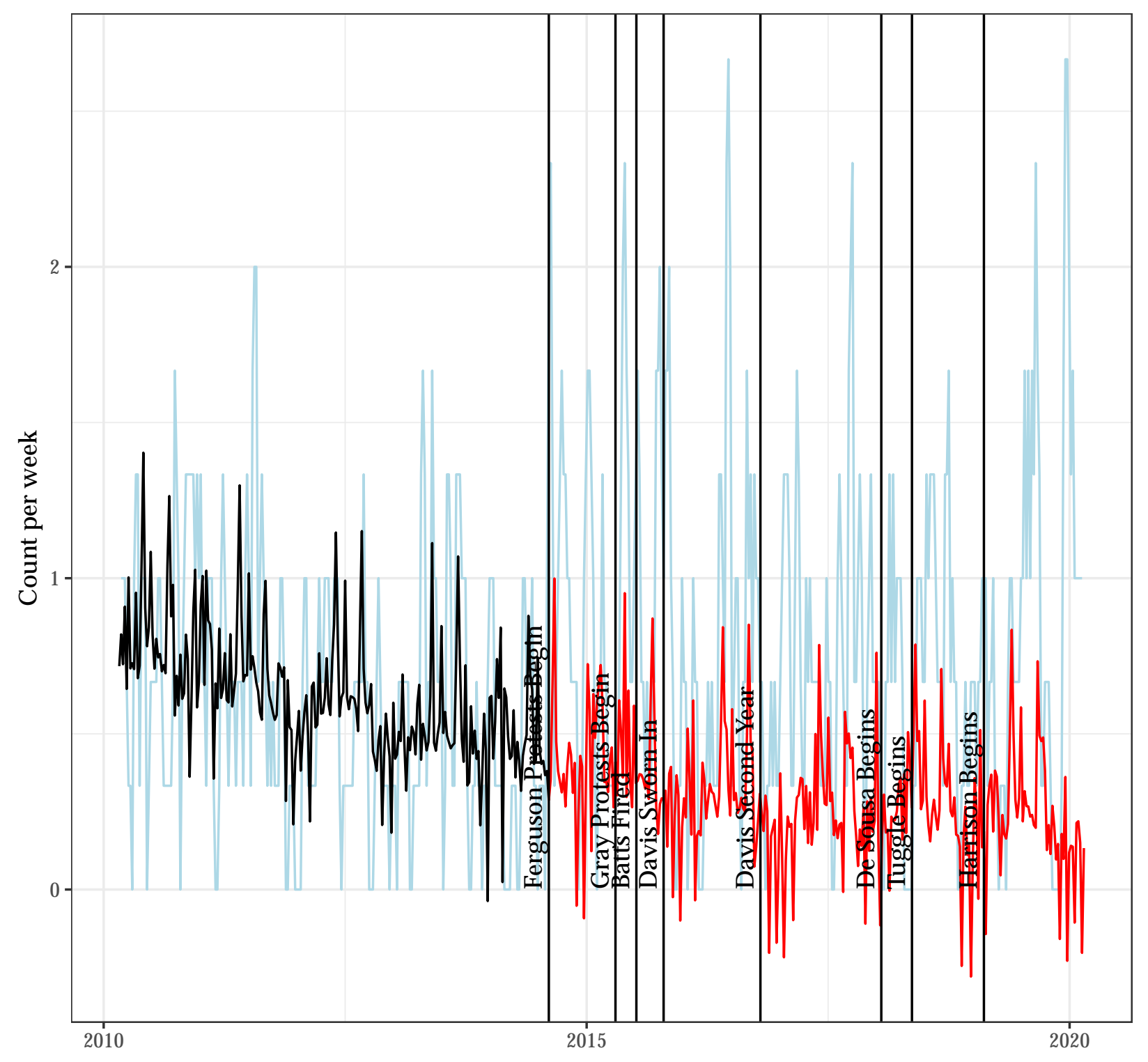


Autocorrelation Function for the Observed Outcome for Updated Model (2)

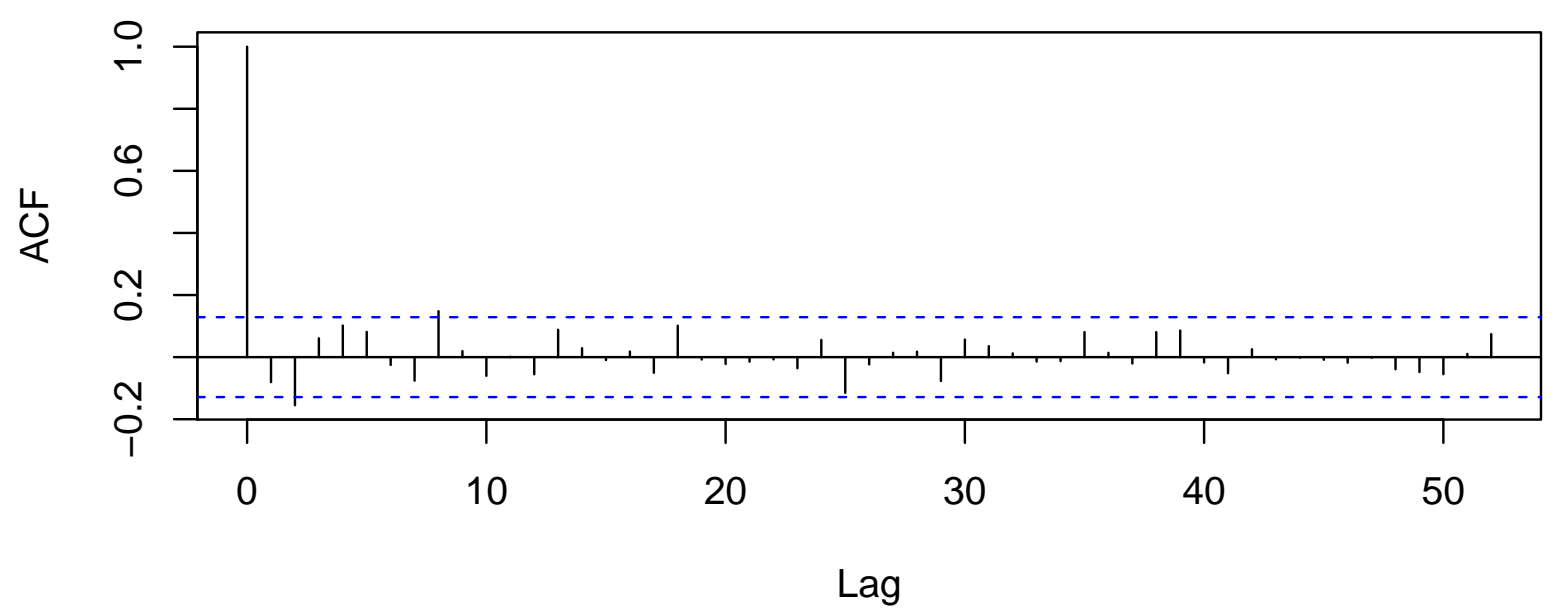

Autocorrelation Function for the Residuals from Updated Model (2)

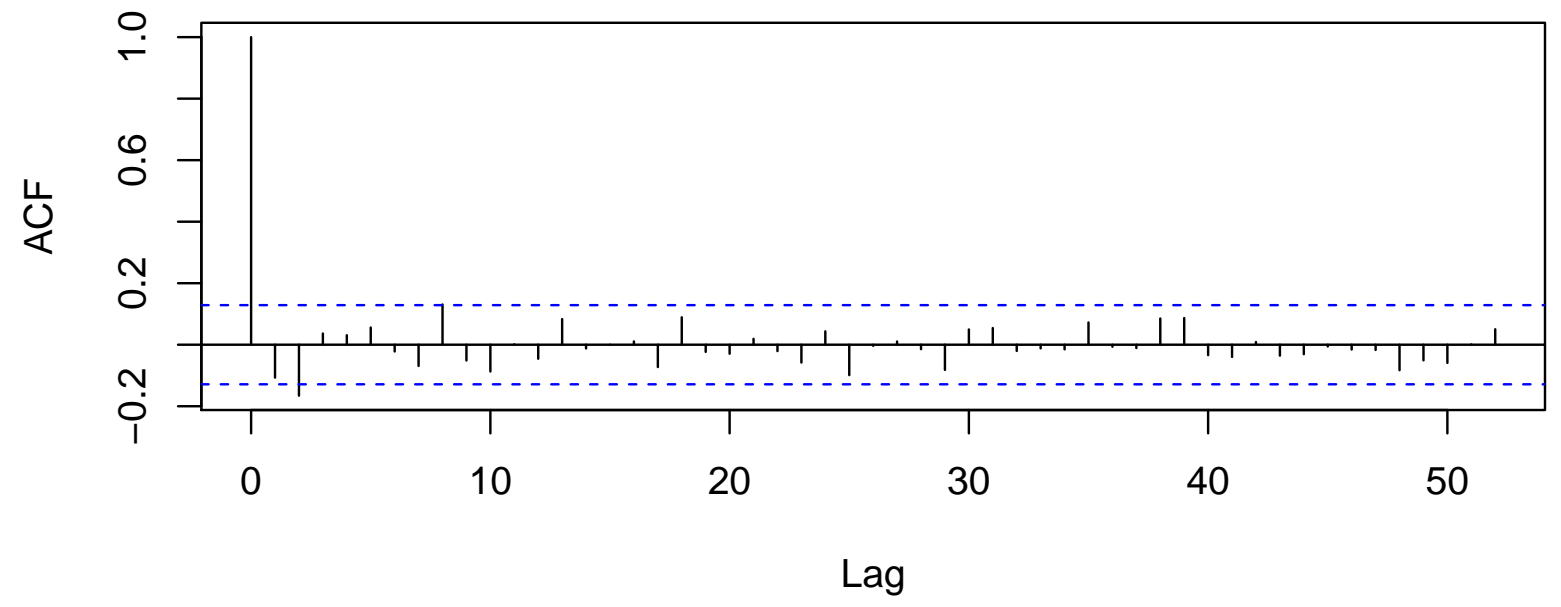




\subsection{A Poisson Regression Model as an Alternative to Model (2) for homicide.northeastern}

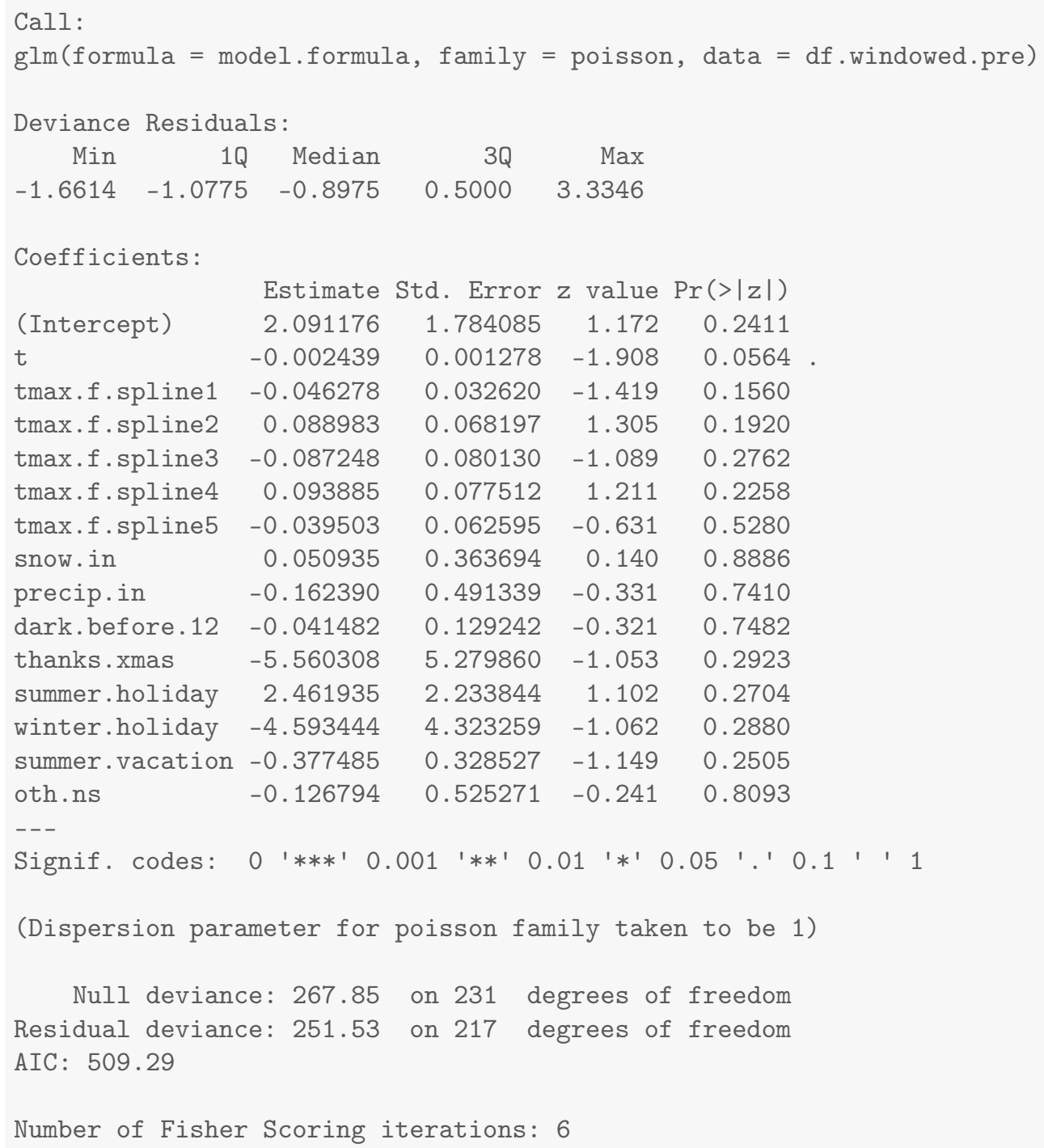




\section{Results for homicide.western}

\subsection{Summary Values for homicide.western}

Table 30.1: Descriptives for Outcome Before Ferguson Protests Begin

\begin{tabular}{lcccccccc}
\hline \hline Statistic & $\mathrm{N}$ & Mean & St. Dev. & Min & $\operatorname{Pctl}(25)$ & Median & Pctl(75) & Max \\
\hline homicide.western & 232 & 0.616 & 0.808 & 0 & 0 & 0 & 1 & 3 \\
\hline
\end{tabular}

Table 30.2: Descriptives for Outcome After Ferguson Protests Begin

\begin{tabular}{lcccccccc}
\hline \hline Statistic & $\mathrm{N}$ & Mean & St. Dev. & Min & Pctl(25) & Median & Pctl(75) & Max \\
\hline homicide.western & 290 & 1.021 & 1.097 & 0 & 0 & 1 & 1 & 5 \\
\hline
\end{tabular}




\subsection{Four Models for homicide.western}

Table 30.3: Four Models that Differ on the Specification of Adjustment and Intervention Variables

\begin{tabular}{lcccc}
\hline \hline & \multicolumn{3}{c}{ Outcome: Count Per Week } \\
\cline { 2 - 5 } & $(1)$ & $(2)$ & $(3)$ & $(4)$ \\
\hline Time (counter in weeks) & 0.0001 & 0.0003 & & 0.0003 \\
After Ferguson Protests Begin (week of 8/11/14 onward) & -0.16 & & -0.17 & -0.18 \\
After Gray Protests Begin (week of 4/20/15 onward) & 0.98 & & 1.00 & 0.99 \\
Unrest and National Guard $(4 / 27 / 15$ - 5/3/2015) & 0.55 & & 0.54 & 0.43 \\
After Batts Fired (week of 7/13/15 onward) & 0.12 & & 0.06 & 0.06 \\
After Davis Confirmed (week of 10/19/15 onward) & -0.38 & & -0.40 & -0.39 \\
After Davis First Year (week of 10/17/16 onward) & -0.26 & & -0.29 & -0.29 \\
After De Sousa Begins (week of 1/19/18 onward) & -0.07 & & -0.01 & -0.04 \\
After Tuggle Begins (week of 5/14/18 onward) & 0.05 & & 0.05 & 0.07 \\
After Harrison Begins (week of 2/11/19 onward) & 0.25 & & 0.23 & 0.20 \\
Average Maximum Temperature to 50 Degrees & & 0.01 & & 0.002 \\
Plus Degrees in the 50s & & 0.01 & & 0.01 \\
Plus Degrees in the 60s & & -0.02 & & -0.01 \\
Plus Degrees in the 70s & & 0.02 & & -0.01 \\
Plus Degrees Greater Than 80 & & -0.01 & & 0.02 \\
Snowfall (inches) & & -0.26 & & -0.21 \\
Precipitation (inches) & & 0.04 & & -0.31 \\
Darkness Before Midnight (hours) & & 0.89 & & -1.52 \\
Thanksgiving/Christmas (proportion of week) & & 2.80 & & 0.23 \\
Winter Holiday (proportion of week) & & 0.32 & & 0.19 \\
Other Out-of-School Days (proportion of week) & & -0.01 & & 0.03 \\
Summer Vacation (proportion of week) & 522 & 232 & 522 & 522 \\
Summer Holiday (proportion of week) & 0.08 & 0.03 & 0.07 & 0.10 \\
\hline Observations & & & &
\end{tabular}


30.3 Least Squares Updated Model (2) for homicide.western homicide.western

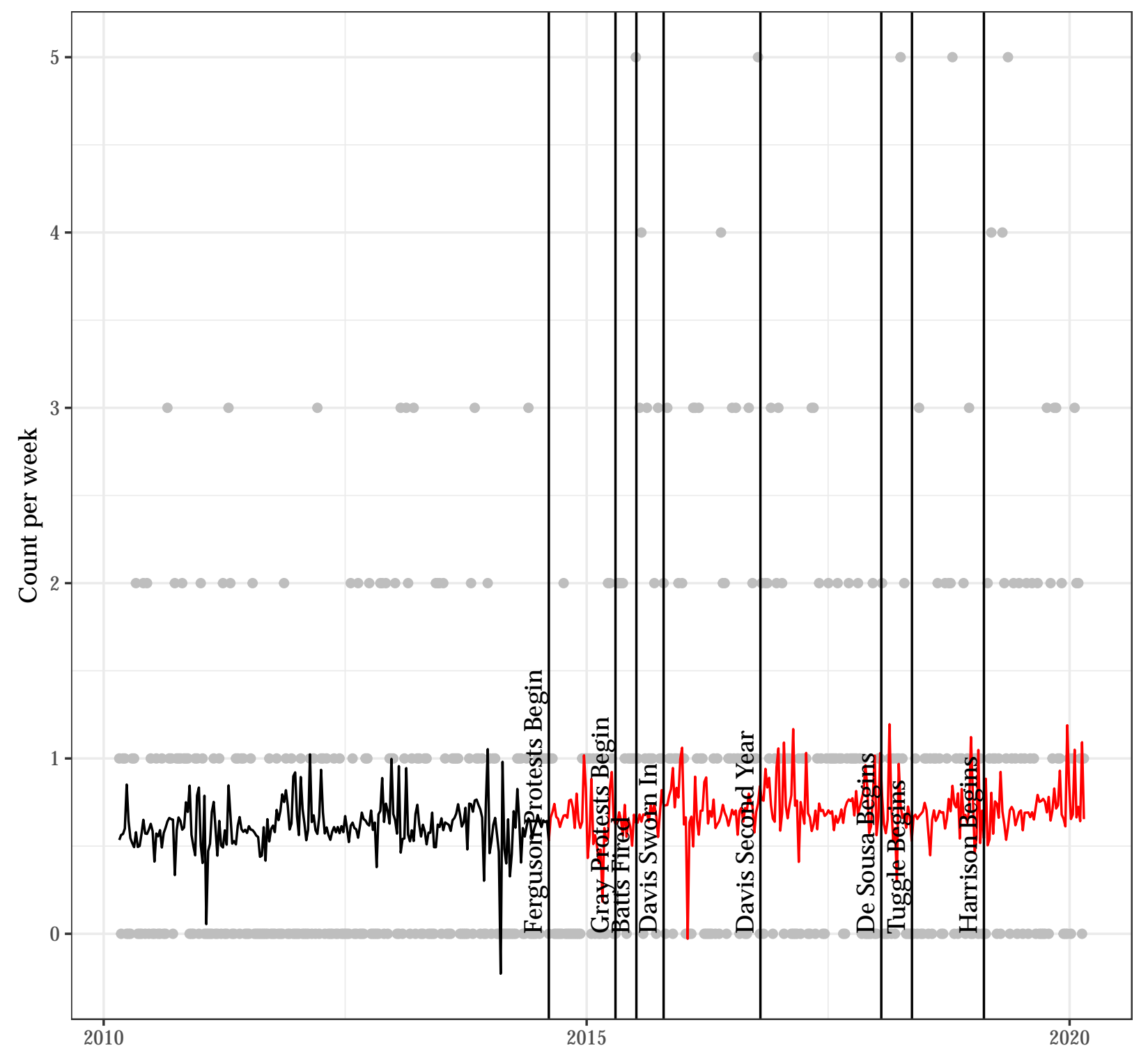




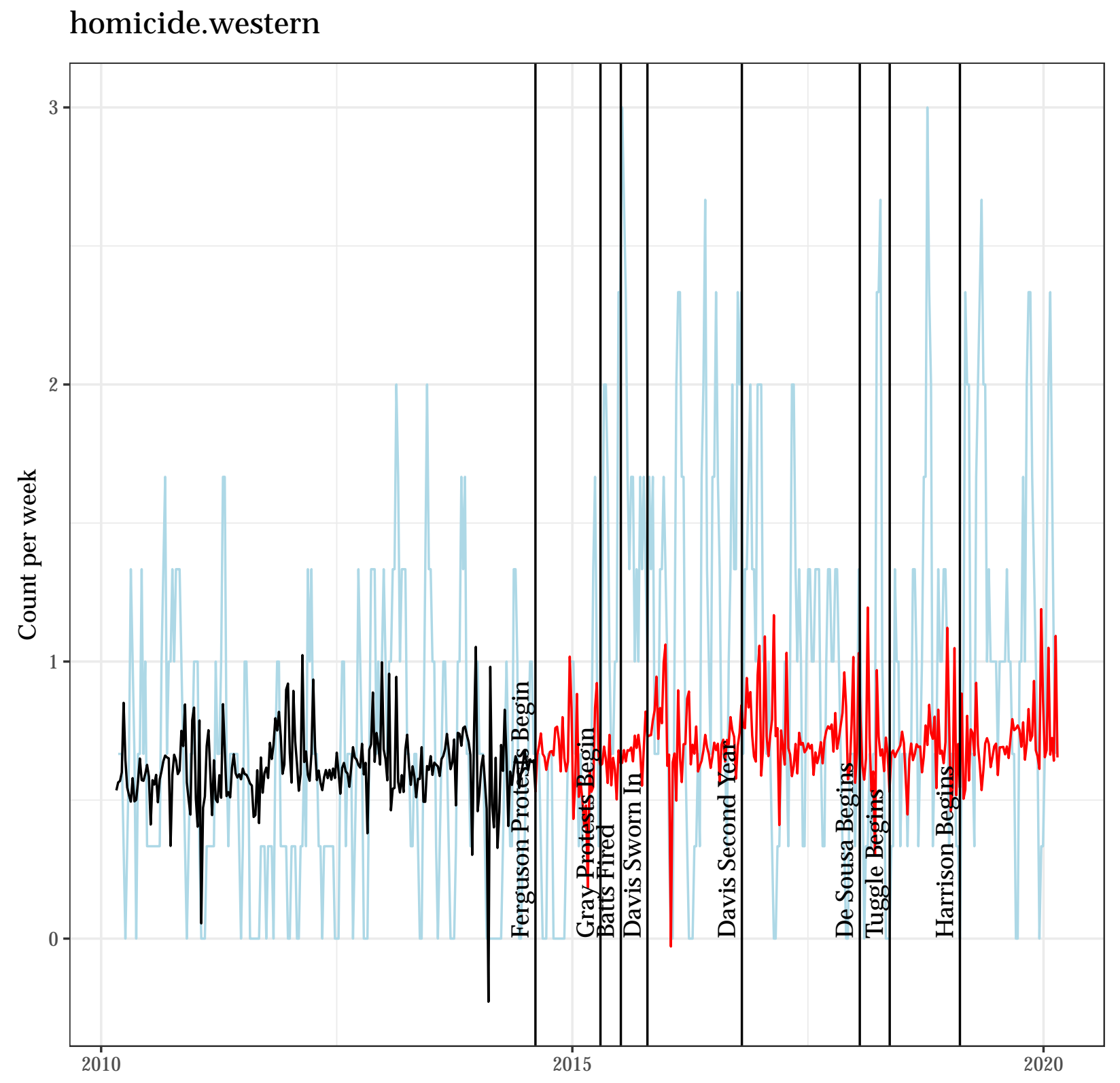


Autocorrelation Function for the Observed Outcome for Updated Model (2)

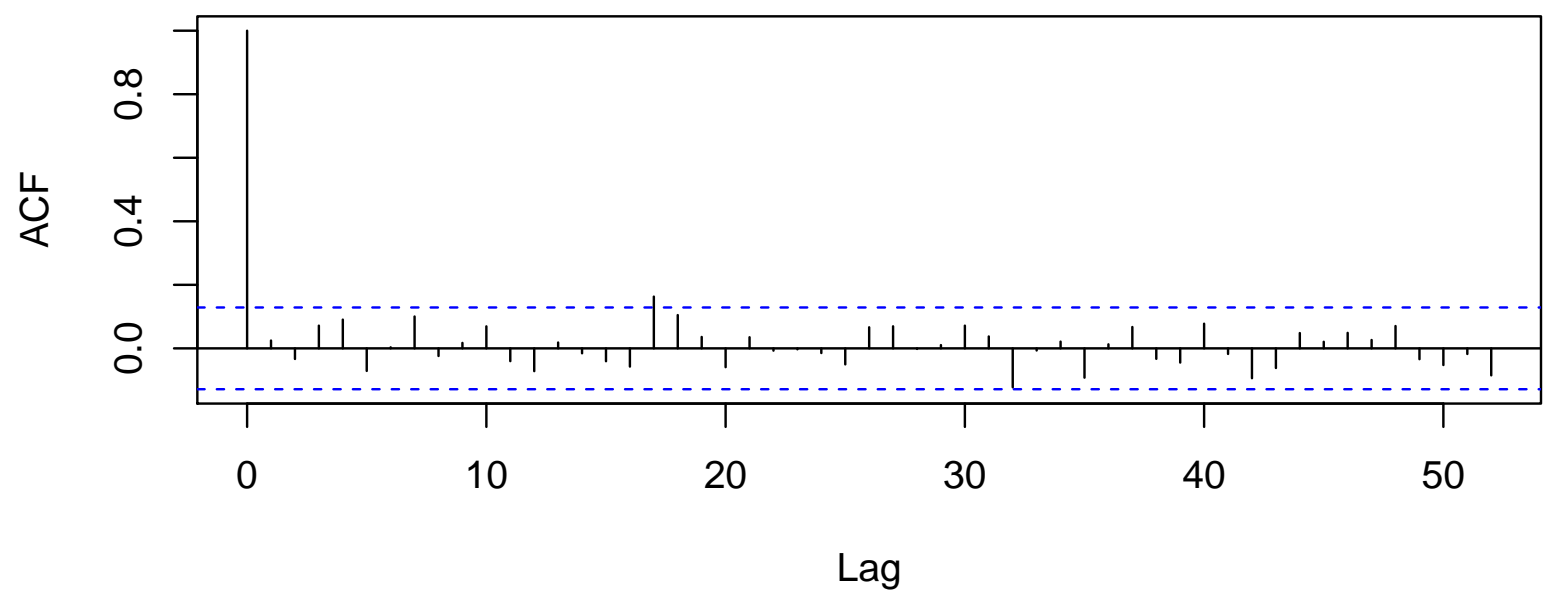

Autocorrelation Function for the Residuals from Updated Model (2)

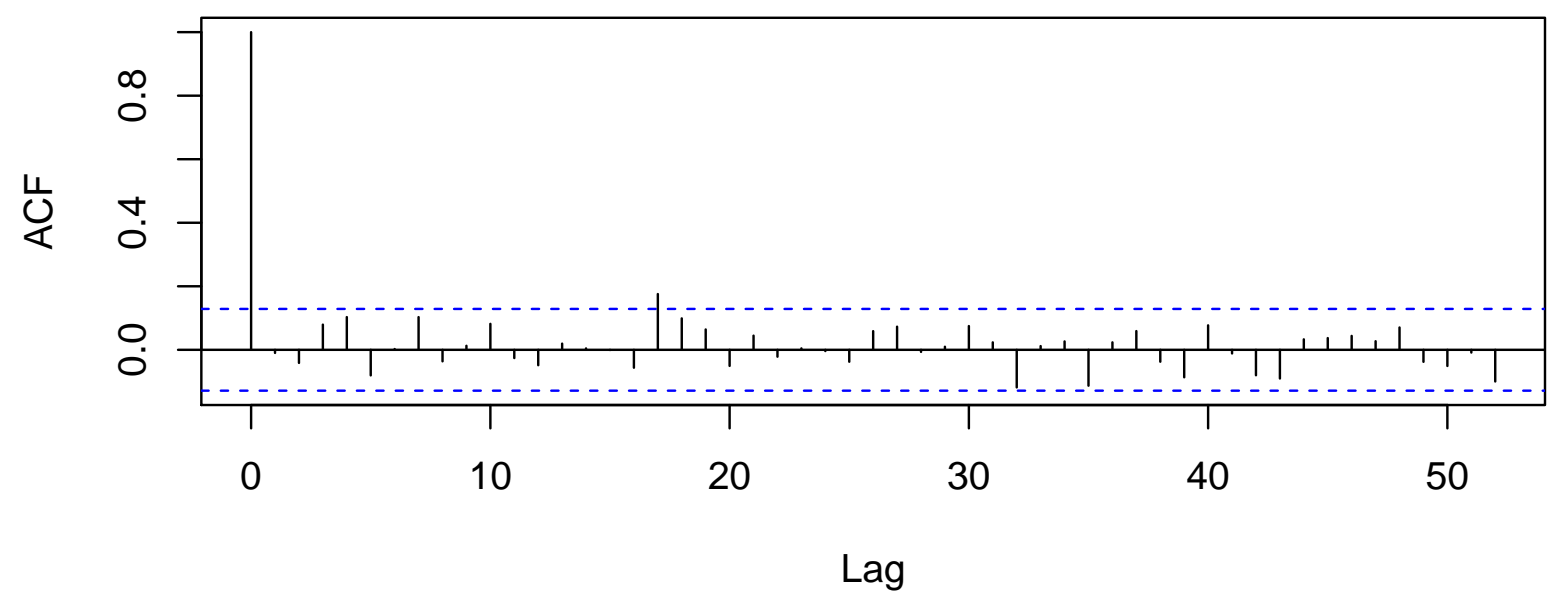




\subsection{A Poisson Regression Model as an Alternative to Model (2) for homicide.western}

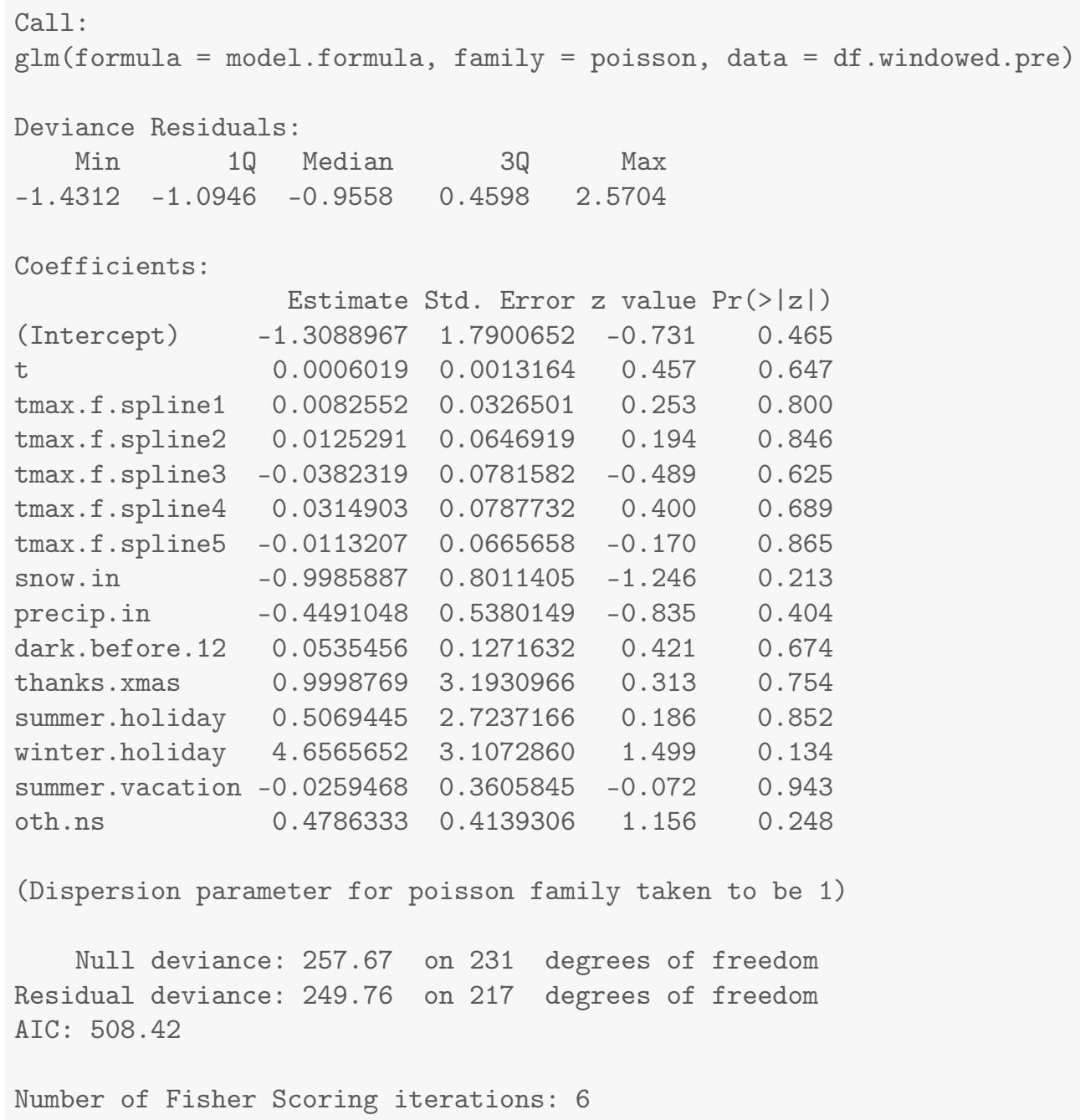




\section{Results for homicide.central}

\subsection{Summary Values for homicide.central}

Table 31.1: Descriptives for Outcome Before Ferguson Protests Begin

\begin{tabular}{lcccccccc}
\hline \hline Statistic & $\mathrm{N}$ & Mean & St. Dev. & Min & Pctl(25) & Median & Pctl(75) & Max \\
\hline homicide.central & 232 & 0.289 & 0.595 & 0 & 0 & 0 & 0 & 3 \\
\hline
\end{tabular}

Table 31.2: Descriptives for Outcome After Ferguson Protests Begin

\begin{tabular}{lcccccccc}
\hline \hline Statistic & $\mathrm{N}$ & Mean & St. Dev. & Min & Pctl(25) & Median & Pctl(75) & Max \\
\hline homicide.central & 290 & 0.372 & 0.616 & 0 & 0 & 0 & 1 & 3 \\
\hline
\end{tabular}




\subsection{Four Models for homicide.central}

Table 31.3: Four Models that Differ on the Specification of Adjustment and Intervention Variables

\begin{tabular}{lcccc}
\hline \hline & \multicolumn{3}{c}{ Outcome: Count Per Week } \\
\cline { 2 - 5 } & $(1)$ & $(2)$ & $(3)$ & $(4)$ \\
\hline Time (counter in weeks) & 0.001 & 0.001 & & 0.001 \\
After Ferguson Protests Begin (week of 8/11/14 onward) & -0.08 & & -0.06 & -0.06 \\
After Gray Protests Begin (week of 4/20/15 onward) & 0.07 & -0.04 & -0.01 \\
Unrest and National Guard (4/27/15 - 5/3/2015) & -0.36 & -0.47 & -0.43 \\
After Batts Fired (week of 7/13/15 onward) & 0.13 & & 0.17 & 0.13 \\
After Davis Confirmed (week of 10/19/15 onward) & -0.11 & & -0.11 & -0.07 \\
After Davis First Year (week of 10/17/16 onward) & 0.005 & & 0.07 & 0.02 \\
After De Sousa Begins (week of 1/19/18 onward) & -0.02 & & -0.04 & -0.01 \\
After Tuggle Begins (week of 5/14/18 onward) & -0.02 & & 0.07 & -0.02 \\
After Harrison Begins (week of 2/11/19 onward) & -0.22 & & -0.23 & -0.25 \\
Average Maximum Temperature to 50 Degrees & & -0.01 & & -0.001 \\
Plus Degrees in the 50s & & 0.04 & & 0.002 \\
Plus Degrees in the 60s & & -0.04 & & 0.01 \\
Plus Degrees in the 70s & & -0.02 & & -0.02 \\
Plus Degrees Greater Than 80 & & 0.04 & & 0.02 \\
Snowfall (inches) & & 0.005 & & -0.03 \\
Precipitation (inches) & & -0.21 & & -0.03 \\
Darkness Before Midnight (hours) & & -1.30 & & 0.46 \\
Thanksgiving/Christmas (proportion of week) & & -1.75 & & -0.34 \\
Winter Holiday (proportion of week) & & 0.04 & & -0.19 \\
Other Out-of-School Days (proportion of week) & & 0.11 & & -0.02 \\
Summer Vacation (proportion of week) & 3.32 & & 1.66 \\
Summer Holiday (proportion of week) & 522 & 232 & 522 & 522 \\
Observations & 0.02 & 0.09 & 0.01 & 0.05 \\
R $^{2}$ & & & &
\end{tabular}


31.3 Least Squares Updated Model (2) for homicide.central homicide.central

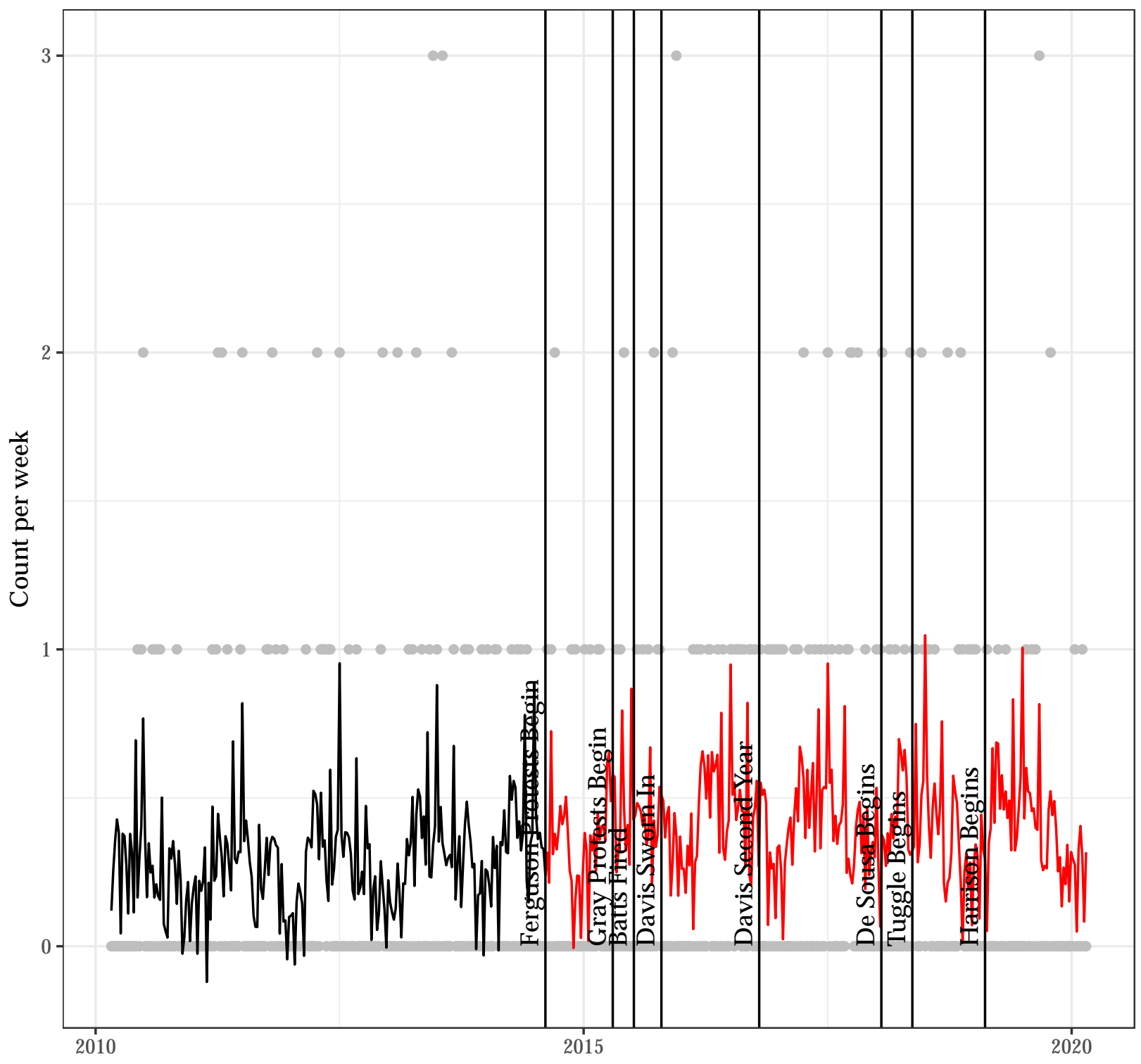




\section{homicide.central}

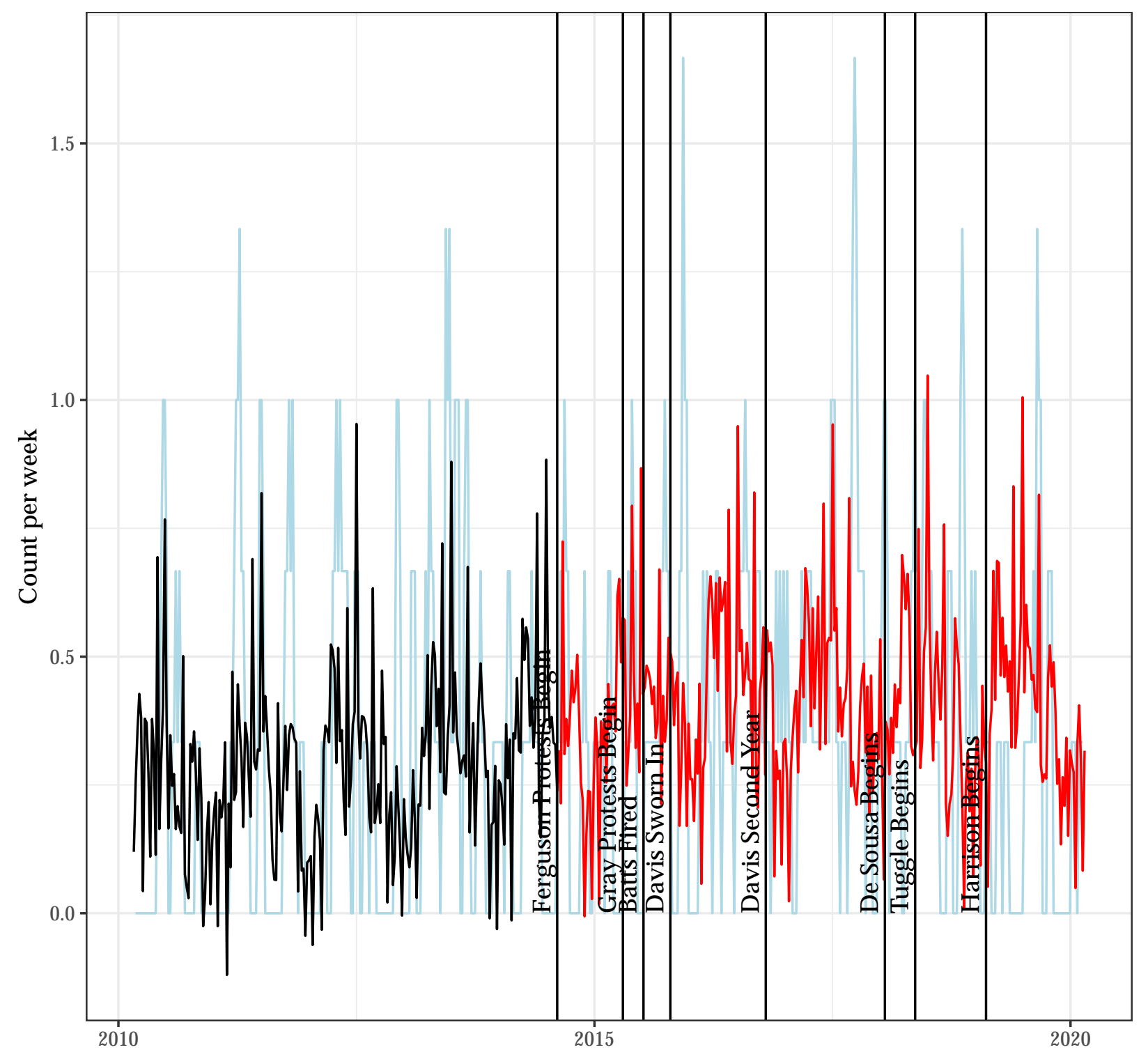


Autocorrelation Function for the Observed Outcome for Updated Model (2)

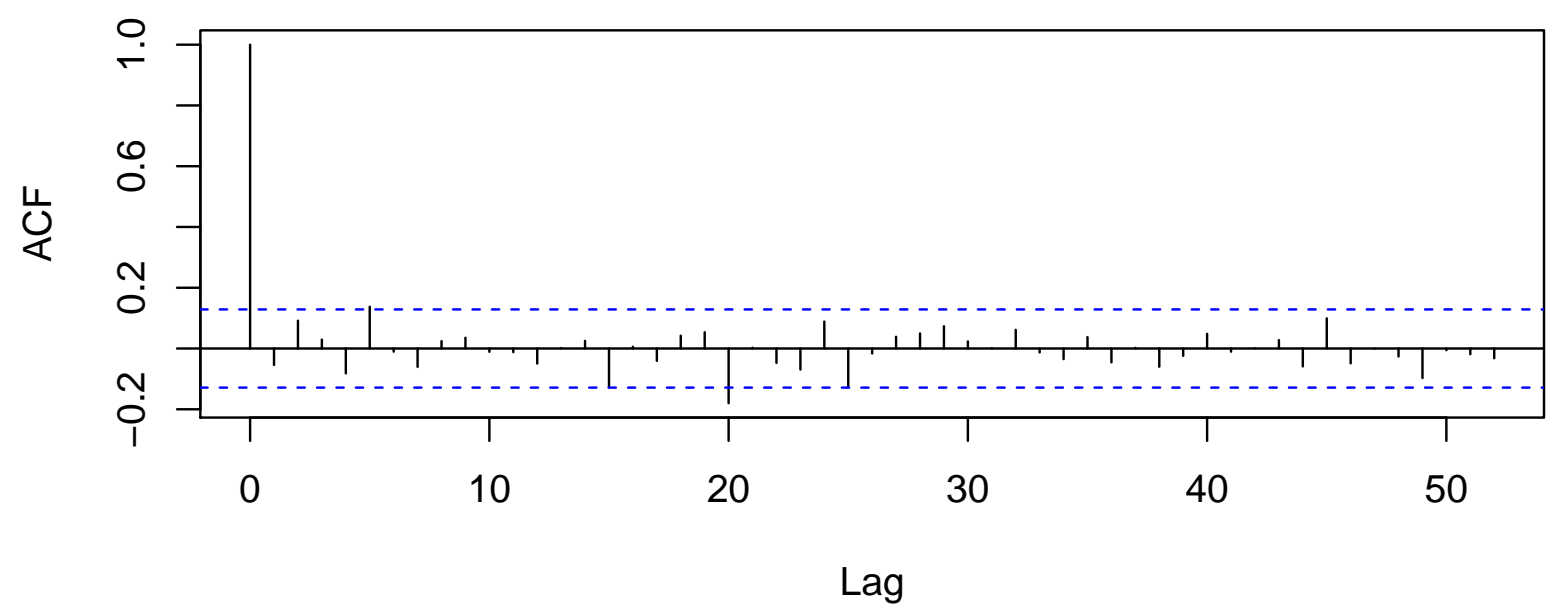

Autocorrelation Function for the Residuals from Updated Model (2)

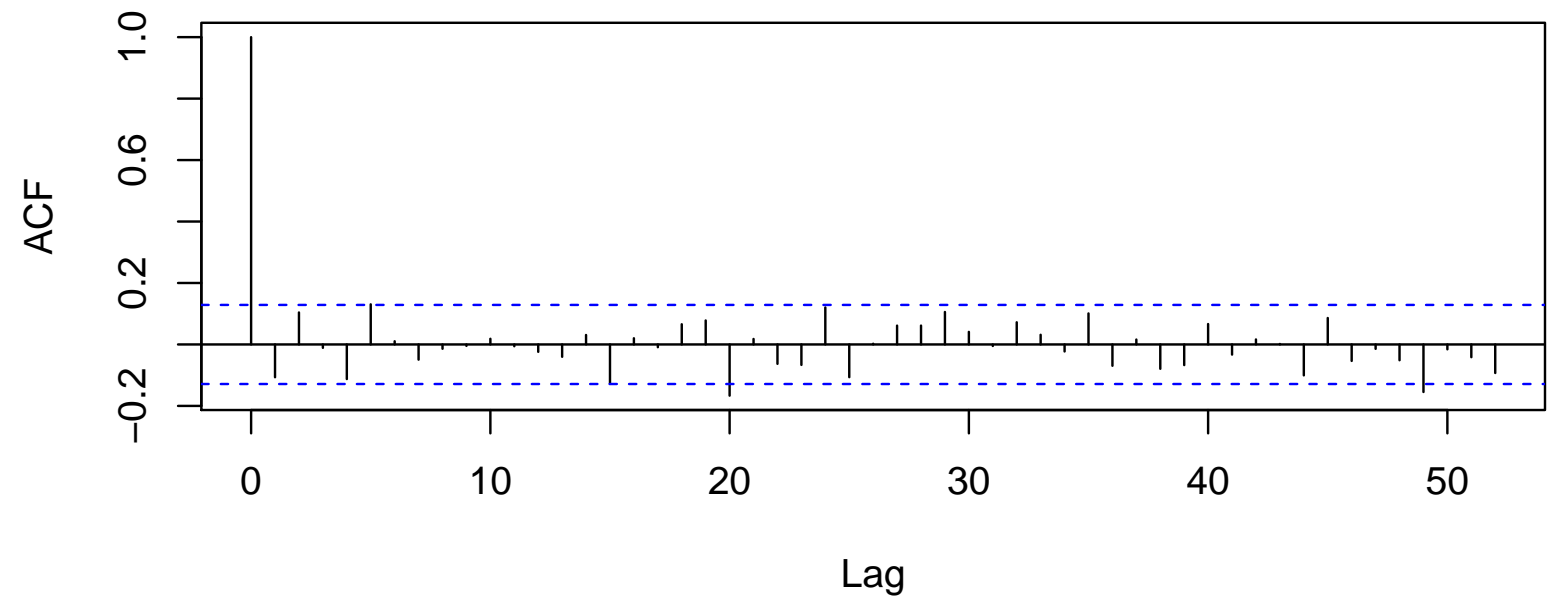




\subsection{A Poisson Regression Model as an Alternative to Model (2) for homicide.central}

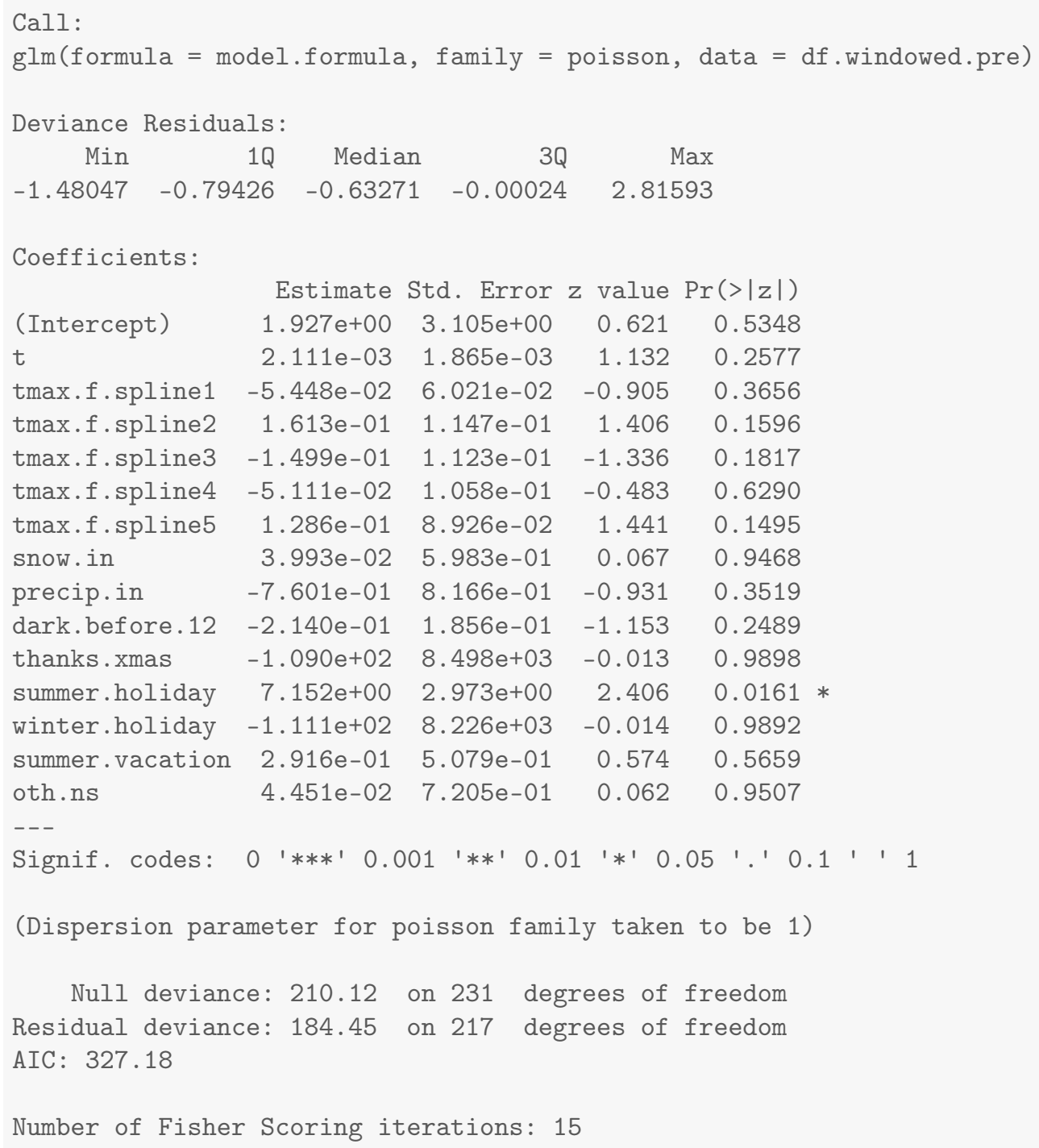




\section{Results for homicide.eastern}

\subsection{Summary Values for homicide.eastern}

Table 32.1: Descriptives for Outcome Before Ferguson Protests Begin

\begin{tabular}{lcccccccc}
\hline \hline Statistic & $\mathrm{N}$ & Mean & St. Dev. & Min & Pctl(25) & Median & Pctl(75) & Max \\
\hline homicide.eastern & 232 & 0.634 & 0.805 & 0 & 0 & 0 & 1 & 3 \\
\hline
\end{tabular}

Table 32.2: Descriptives for Outcome After Ferguson Protests Begin

\begin{tabular}{lcccccccc}
\hline \hline Statistic & $\mathrm{N}$ & Mean & St. Dev. & Min & $\operatorname{Pctl}(25)$ & Median & Pctl(75) & Max \\
\hline homicide.eastern & 290 & 0.903 & 1.038 & 0 & 0 & 1 & 1 & 5 \\
\hline
\end{tabular}




\subsection{Four Models for homicide.eastern}

Table 32.3: Four Models that Differ on the Specification of Adjustment and Intervention Variables

\begin{tabular}{|c|c|c|c|c|}
\hline & \multicolumn{4}{|c|}{ Outcome: Count Per Week } \\
\hline & (1) & $(2)$ & $(3)$ & $(4)$ \\
\hline Time (counter in weeks) & -0.001 & -0.0005 & & -0.0004 \\
\hline After Ferguson Protests Begin (week of 8/11/14 onward) & -0.17 & & -0.07 & -0.11 \\
\hline After Gray Protests Begin (week of 4/20/15 onward) & 0.99 & & 0.71 & 0.80 \\
\hline Unrest and National Guard $(4 / 27 / 15-5 / 3 / 2015)$ & -1.37 & & -1.27 & -1.23 \\
\hline After Batts Fired (week of $7 / 13 / 15$ onward) & -0.36 & & -0.29 & -0.36 \\
\hline After Davis Confirmed (week of 10/19/15 onward) & 0.08 & & 0.15 & 0.17 \\
\hline After Davis First Year (week of 10/17/16 onward) & -0.04 & & -0.05 & -0.06 \\
\hline After De Sousa Begins (week of 1/19/18 onward) & -0.28 & & -0.26 & -0.21 \\
\hline After Tuggle Begins (week of 5/14/18 onward) & 0.20 & & 0.18 & 0.14 \\
\hline After Harrison Begins (week of 2/11/19 onward) & 0.13 & & 0.08 & 0.12 \\
\hline Average Maximum Temperature to 50 Degrees & & 0.02 & & 0.02 \\
\hline Plus Degrees in the 50s & & -0.01 & & -0.02 \\
\hline Plus Degrees in the 60s & & -0.02 & & 0.02 \\
\hline Plus Degrees in the 70s & & 0.03 & & 0.02 \\
\hline Plus Degrees Greater Than 80 & & -0.04 & & -0.04 \\
\hline Snowfall (inches) & & 0.01 & & -0.04 \\
\hline Precipitation (inches) & & -0.41 & & -0.30 \\
\hline Darkness Before Midnight (hours) & & -0.03 & & 0.05 \\
\hline Thanksgiving/Christmas (proportion of week) & & -0.95 & & -4.37 \\
\hline Winter Holiday (proportion of week) & & -0.77 & & -0.08 \\
\hline Other Out-of-School Days (proportion of week) & & 0.20 & & 0.30 \\
\hline Summer Vacation (proportion of week) & & -0.02 & & -0.10 \\
\hline Summer Holiday (proportion of week) & & 2.41 & & 1.41 \\
\hline Observations & 522 & 232 & 522 & 522 \\
\hline $\mathrm{R}^{2}$ & 0.05 & 0.07 & 0.06 & 0.09 \\
\hline
\end{tabular}


32.3 Least Squares Updated Model (2) for homicide.eastern homicide.eastern

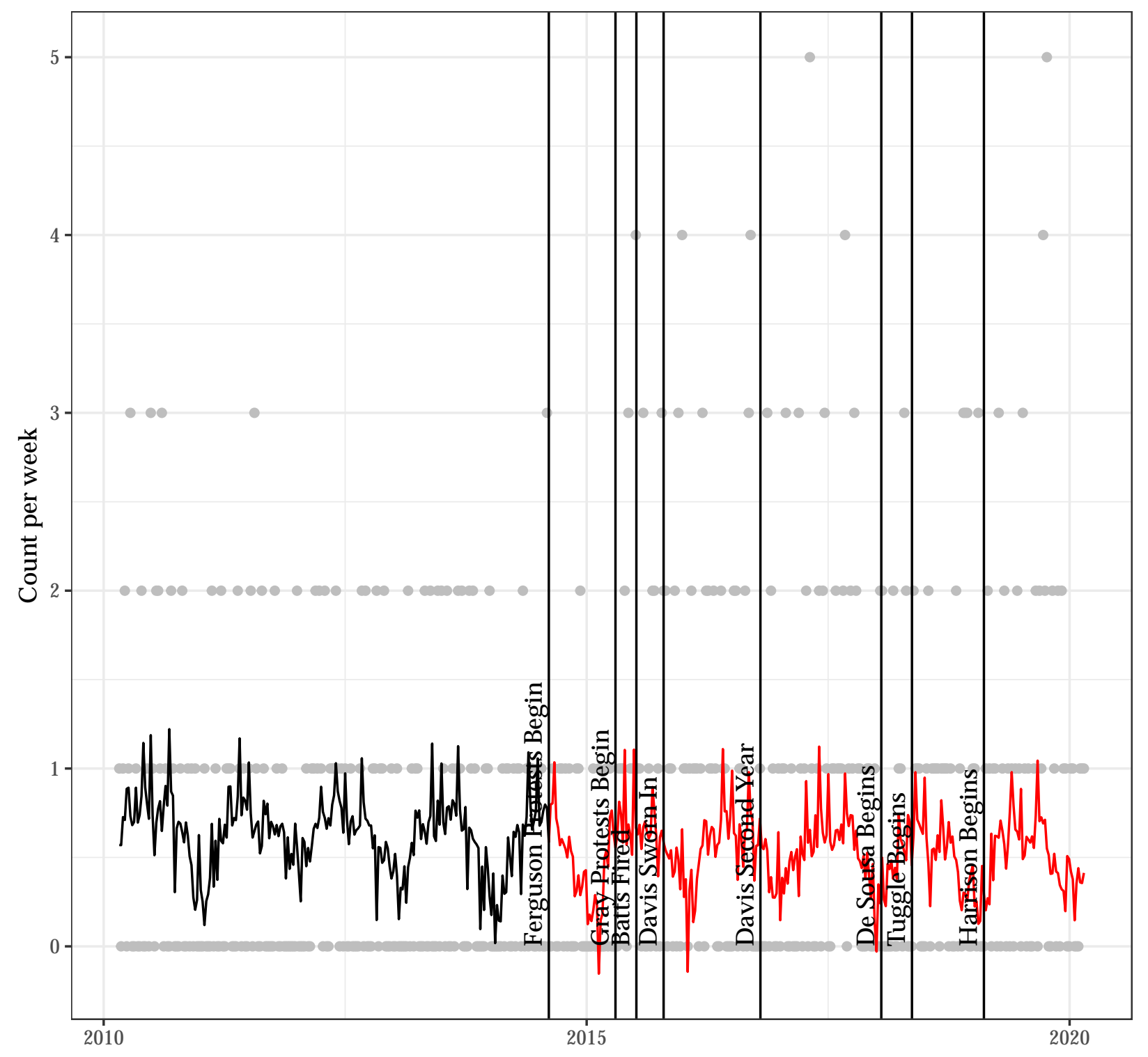




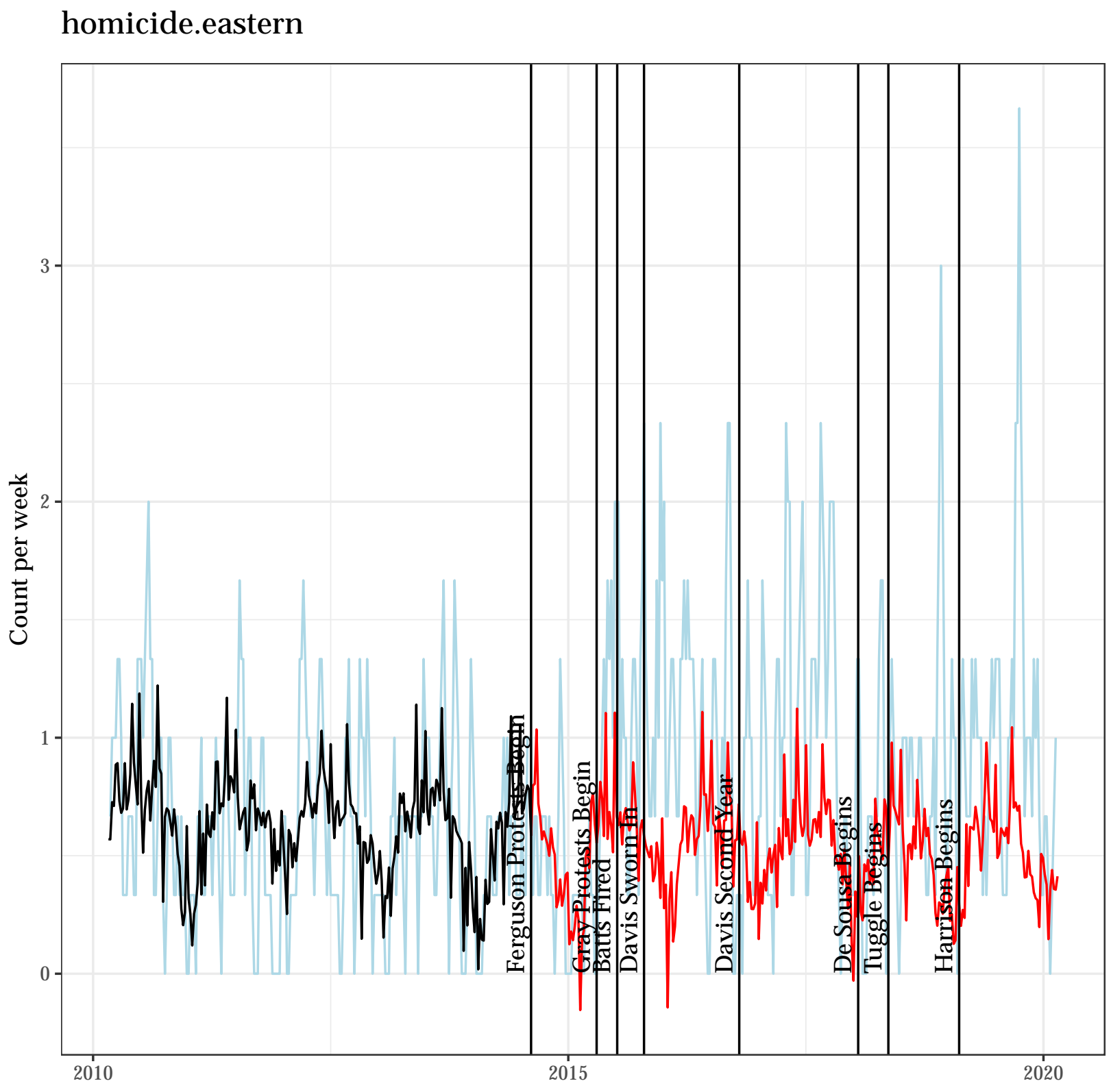


Autocorrelation Function for the Observed Outcome for Updated Model (2)

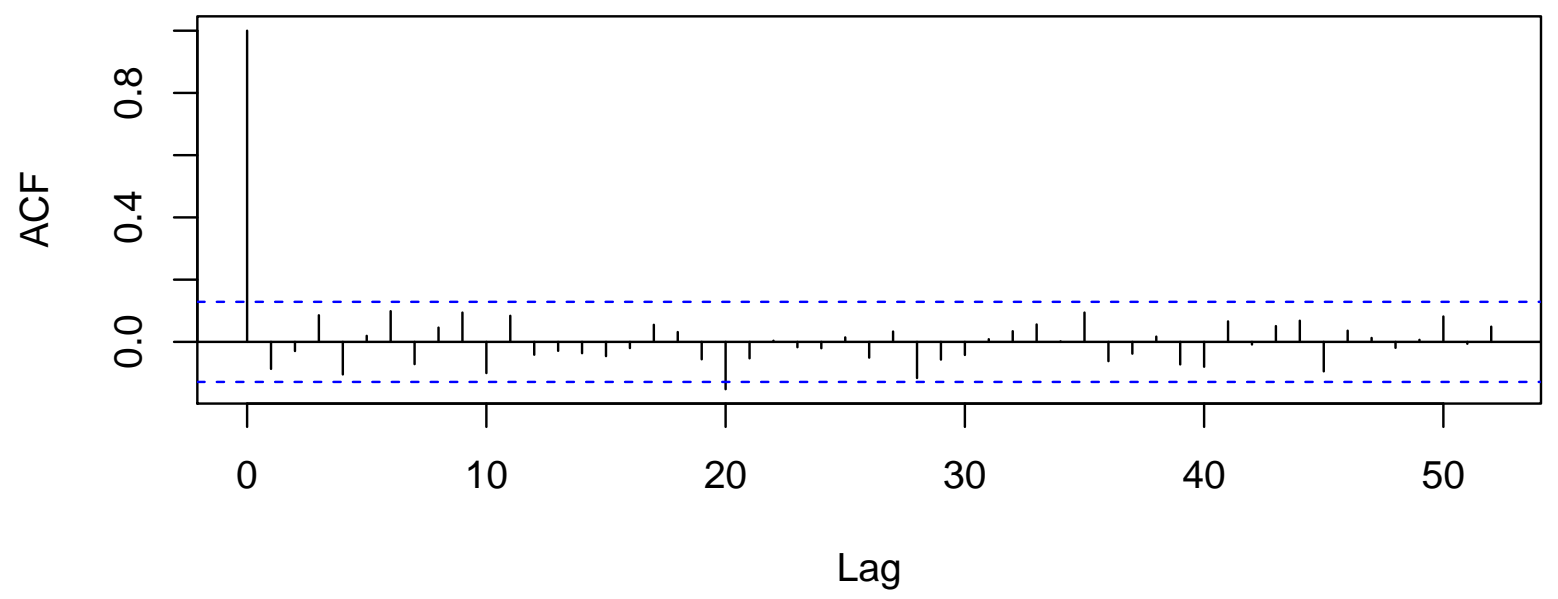

Autocorrelation Function for the Residuals from Updated Model (2)

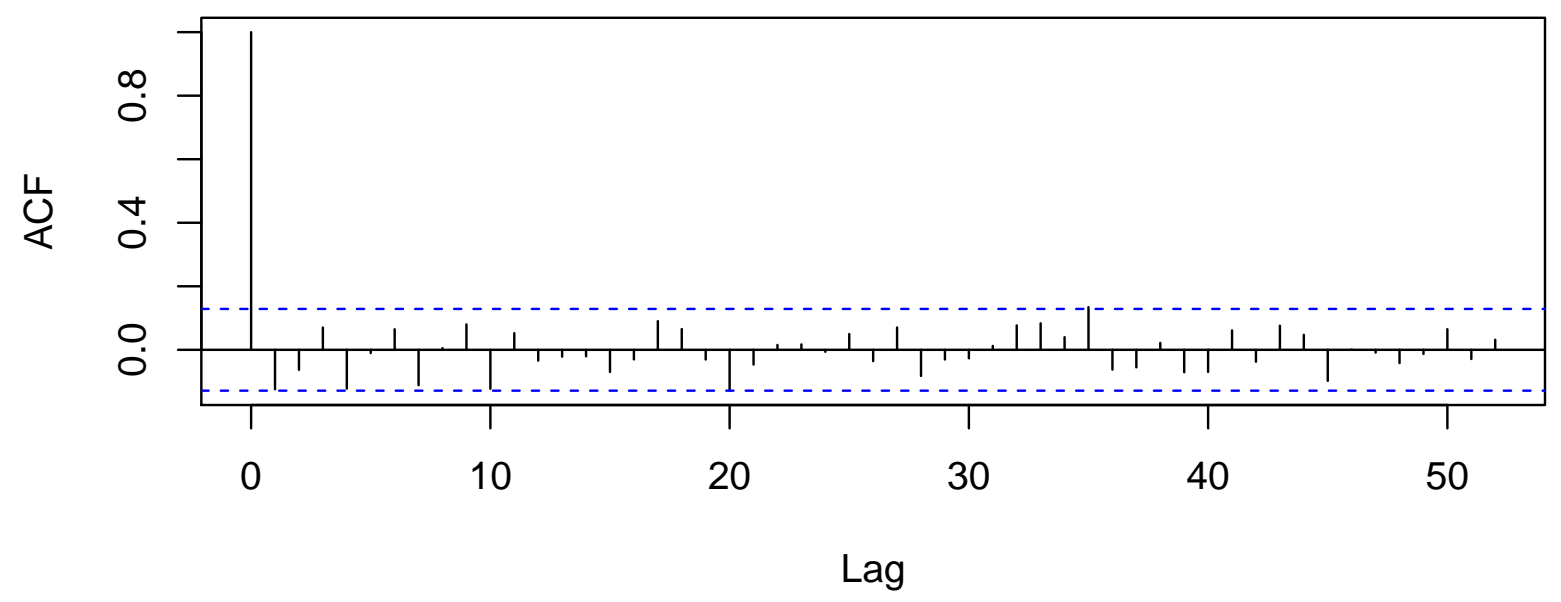




\subsection{A Poisson Regression Model as an Alternative to Model (2) for homicide.eastern}

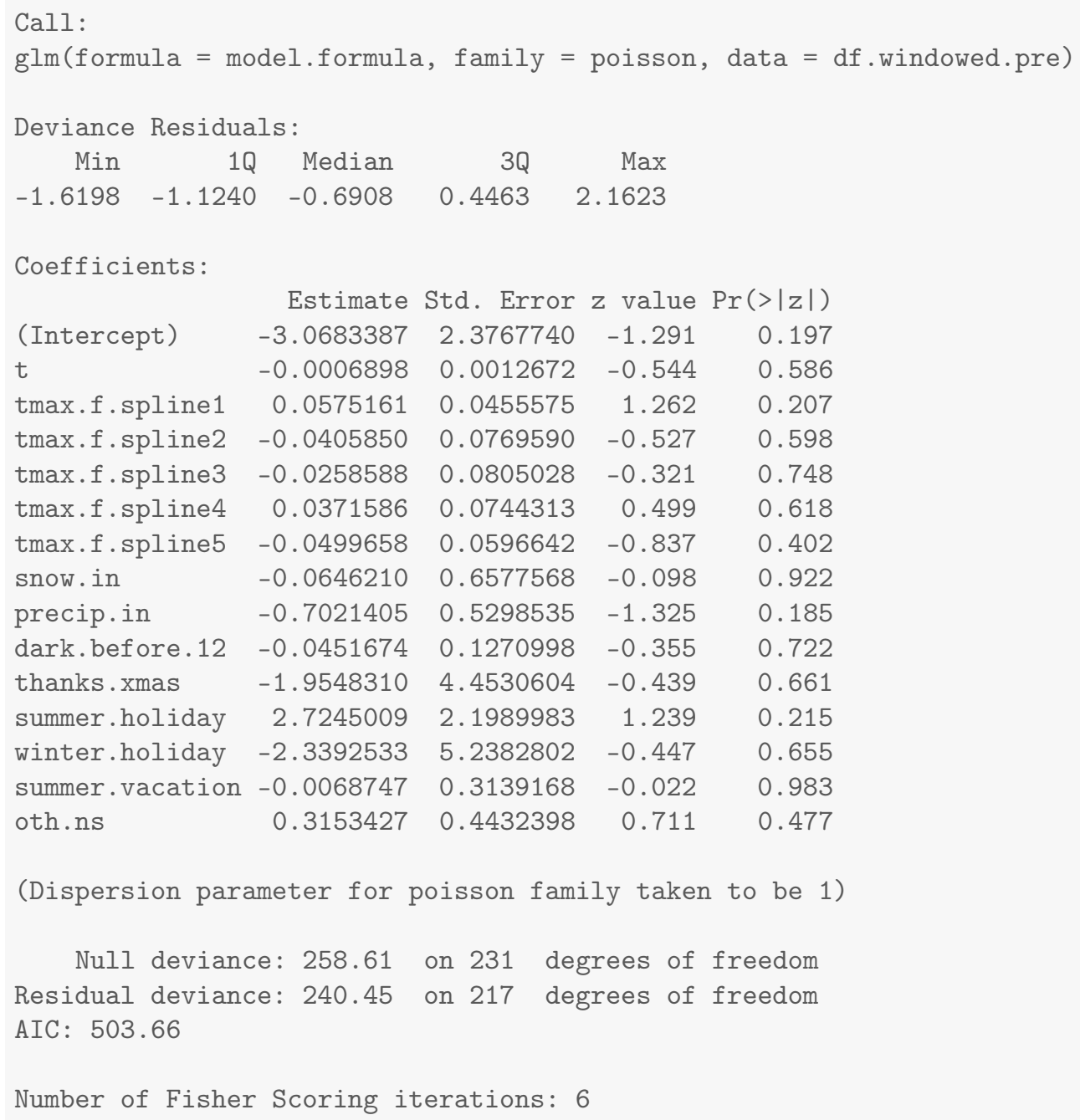




\section{Results for homicide.southwestern}

\subsection{Summary Values for homicide.southwestern}

Table 33.1: Descriptives for Outcome Before Ferguson Protests Begin

\begin{tabular}{lcccccccc}
\hline \hline Statistic & $\mathrm{N}$ & Mean & St. Dev. & Min & Pctl(25) & Median & Pctl(75) & Max \\
\hline homicide.southwestern & 232 & 0.526 & 0.720 & 0 & 0 & 0 & 1 & 4 \\
\hline
\end{tabular}

Table 33.2: Descriptives for Outcome After Ferguson Protests Begin

\begin{tabular}{lcccccccc}
\hline \hline Statistic & N & Mean & St. Dev. & Min & Pctl(25) & Median & Pctl(75) & Max \\
\hline homicide.southwestern & 290 & 0.855 & 0.934 & 0 & 0 & 1 & 1 & 4 \\
\hline
\end{tabular}




\subsection{Four Models for homicide.southwestern}

Table 33.3: Four Models that Differ on the Specification of Adjustment and Intervention Variables

\begin{tabular}{|c|c|c|c|c|}
\hline & \multicolumn{4}{|c|}{ Outcome: Count Per Week } \\
\hline & $(1)$ & $(2)$ & (3) & $(4)$ \\
\hline Time (counter in weeks) & 0.001 & 0.001 & & 0.001 \\
\hline After Ferguson Protests Begin (week of 8/11/14 onward) & -0.01 & & -0.02 & 0.02 \\
\hline After Gray Protests Begin (week of 4/20/15 onward) & 0.86 & & 0.83 & 0.75 \\
\hline Unrest and National Guard $(4 / 27 / 15-5 / 3 / 2015)$ & -0.45 & & -0.25 & -0.35 \\
\hline After Batts Fired (week of 7/13/15 onward) & -0.46 & & -0.57 & -0.59 \\
\hline After Davis Confirmed (week of 10/19/15 onward) & -0.29 & & -0.17 & -0.13 \\
\hline After Davis First Year (week of 10/17/16 onward) & 0.06 & & 0.01 & 0.05 \\
\hline After De Sousa Begins (week of 1/19/18 onward) & -0.28 & & -0.09 & -0.19 \\
\hline After Tuggle Begins (week of 5/14/18 onward) & 0.53 & & 0.34 & 0.34 \\
\hline After Harrison Begins (week of 2/11/19 onward) & -0.18 & & -0.10 & -0.13 \\
\hline Average Maximum Temperature to 50 Degrees & & -0.01 & & -0.01 \\
\hline Plus Degrees in the $50 \mathrm{~s}$ & & 0.01 & & 0.02 \\
\hline Plus Degrees in the $60 \mathrm{~s}$ & & 0.01 & & 0.002 \\
\hline Plus Degrees in the 70s & & 0.03 & & -0.01 \\
\hline Plus Degrees Greater Than 80 & & -0.04 & & 0.03 \\
\hline Snowfall (inches) & & -0.32 & & -0.17 \\
\hline Precipitation (inches) & & -0.09 & & 0.10 \\
\hline Darkness Before Midnight (hours) & & 0.08 & & 0.02 \\
\hline Thanksgiving/Christmas (proportion of week) & & 0.94 & & 1.05 \\
\hline Winter Holiday (proportion of week) & & -0.95 & & 0.11 \\
\hline Other Out-of-School Days (proportion of week) & & 0.09 & & -0.18 \\
\hline Summer Vacation (proportion of week) & & 0.06 & & 0.04 \\
\hline Summer Holiday (proportion of week) & & -1.58 & & -1.39 \\
\hline Observations & 522 & 232 & 522 & 522 \\
\hline $\mathrm{R}^{2}$ & 0.07 & 0.05 & 0.03 & 0.10 \\
\hline
\end{tabular}


33.3 Least Squares Updated Model (2) for homicide.southwestern homicide.southwestern

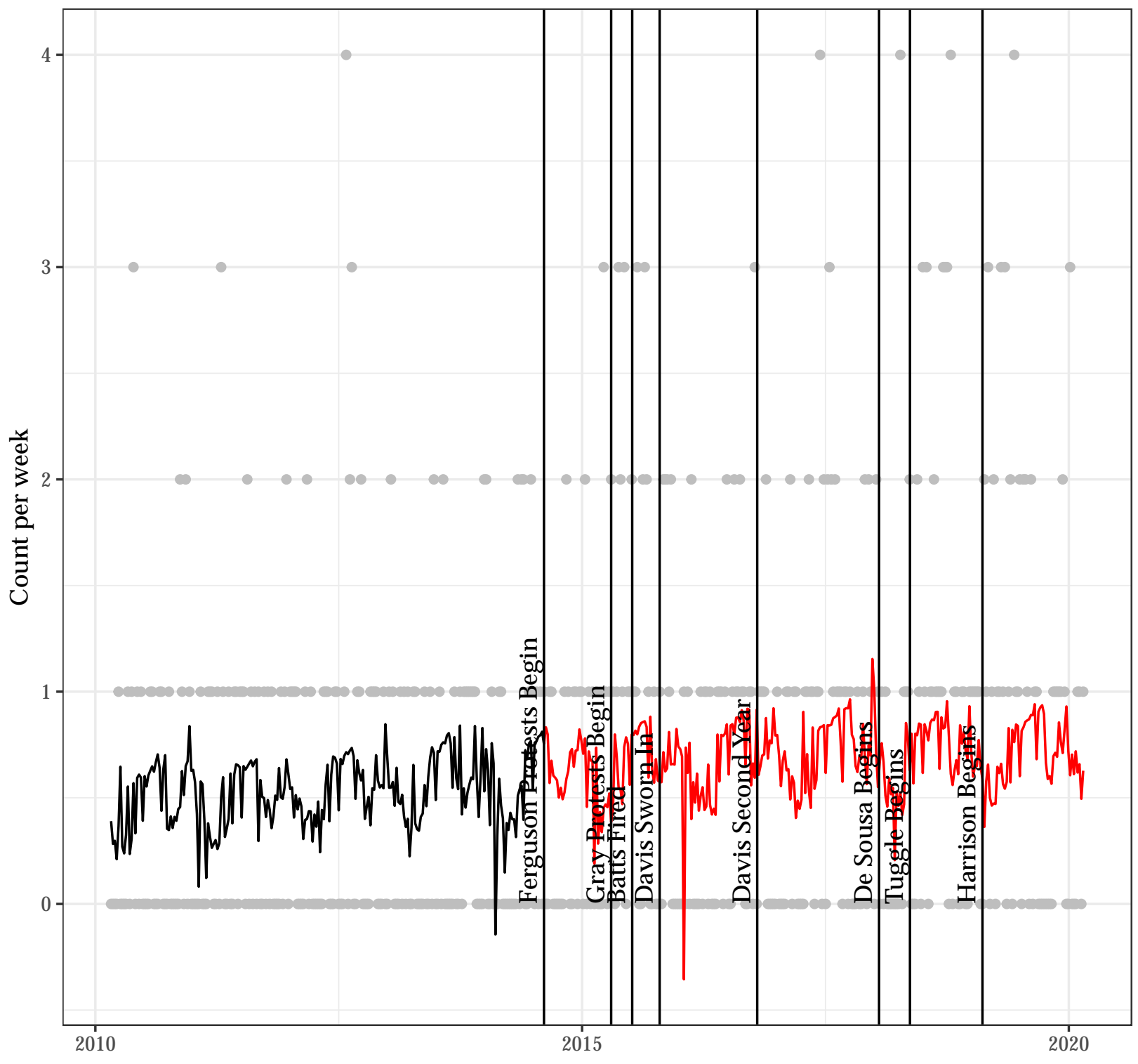




\section{homicide.southwestern}

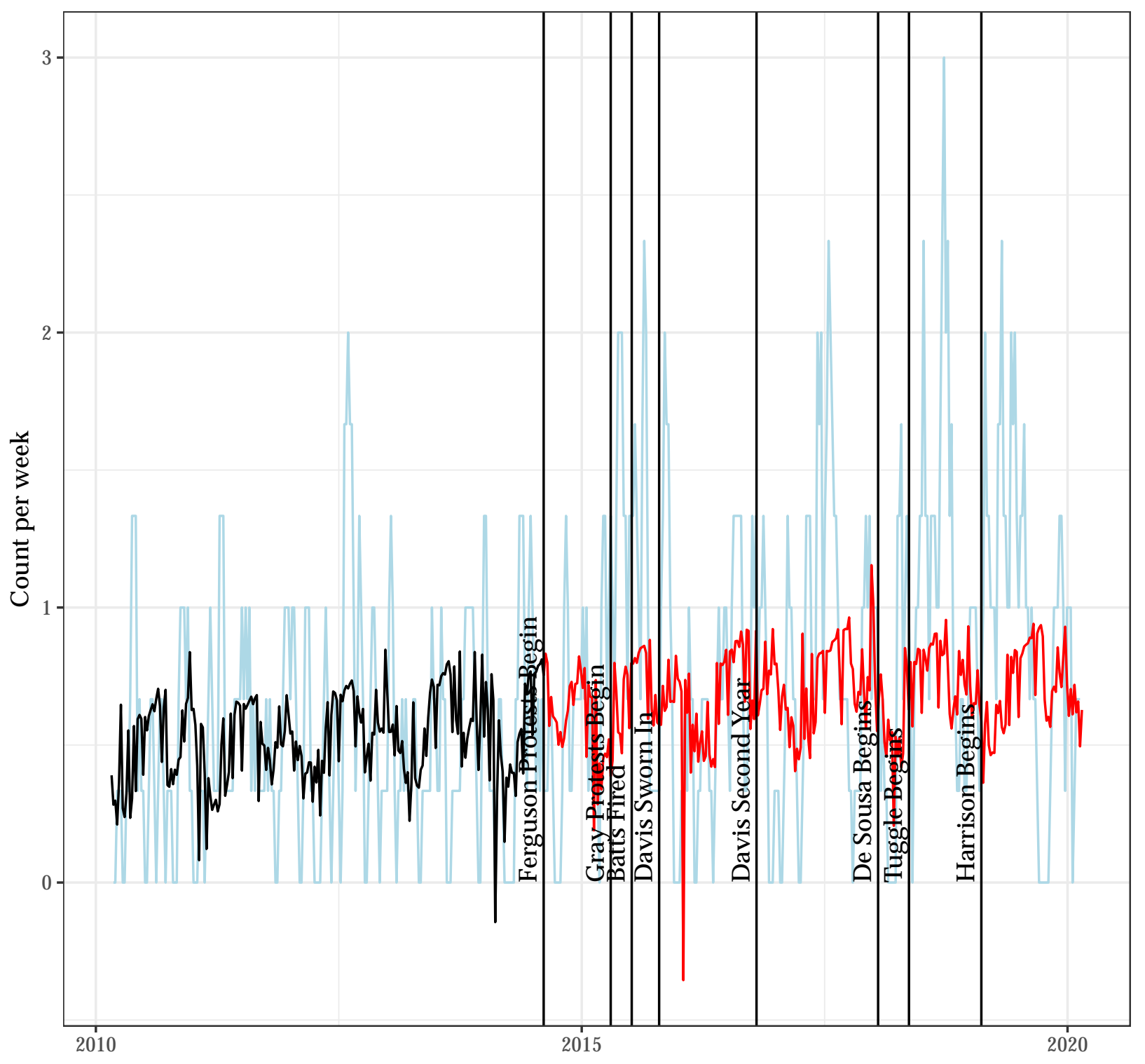


Autocorrelation Function for the Observed Outcome for Updated Model (2)

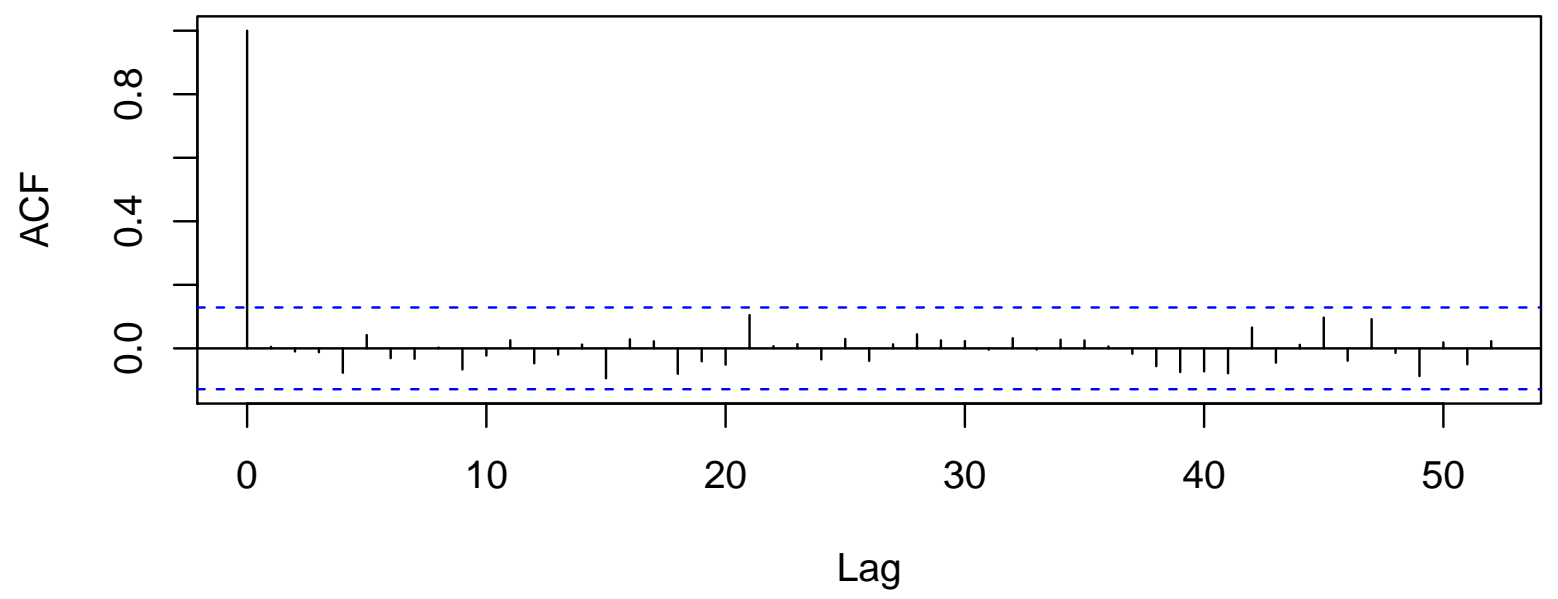

Autocorrelation Function for the Residuals from Updated Model (2)

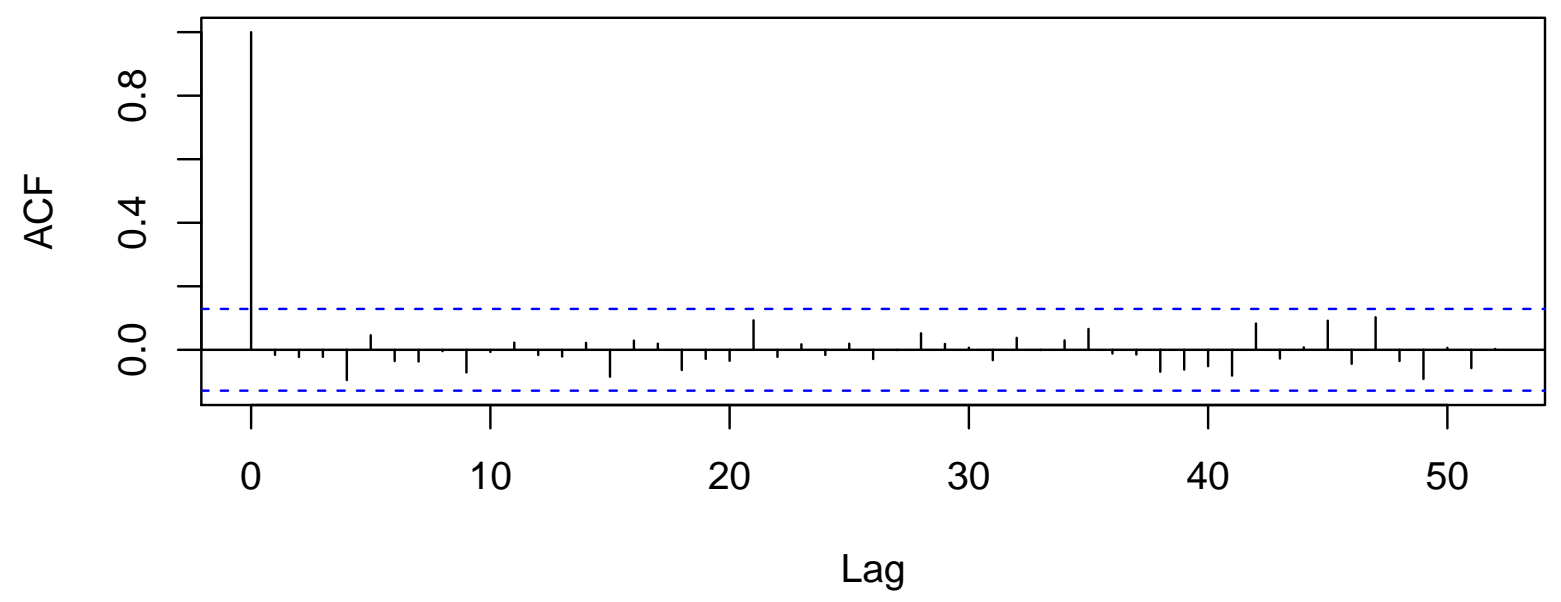




\subsection{A Poisson Regression Model as an Alternative to Model (2) for homicide.southwestern}

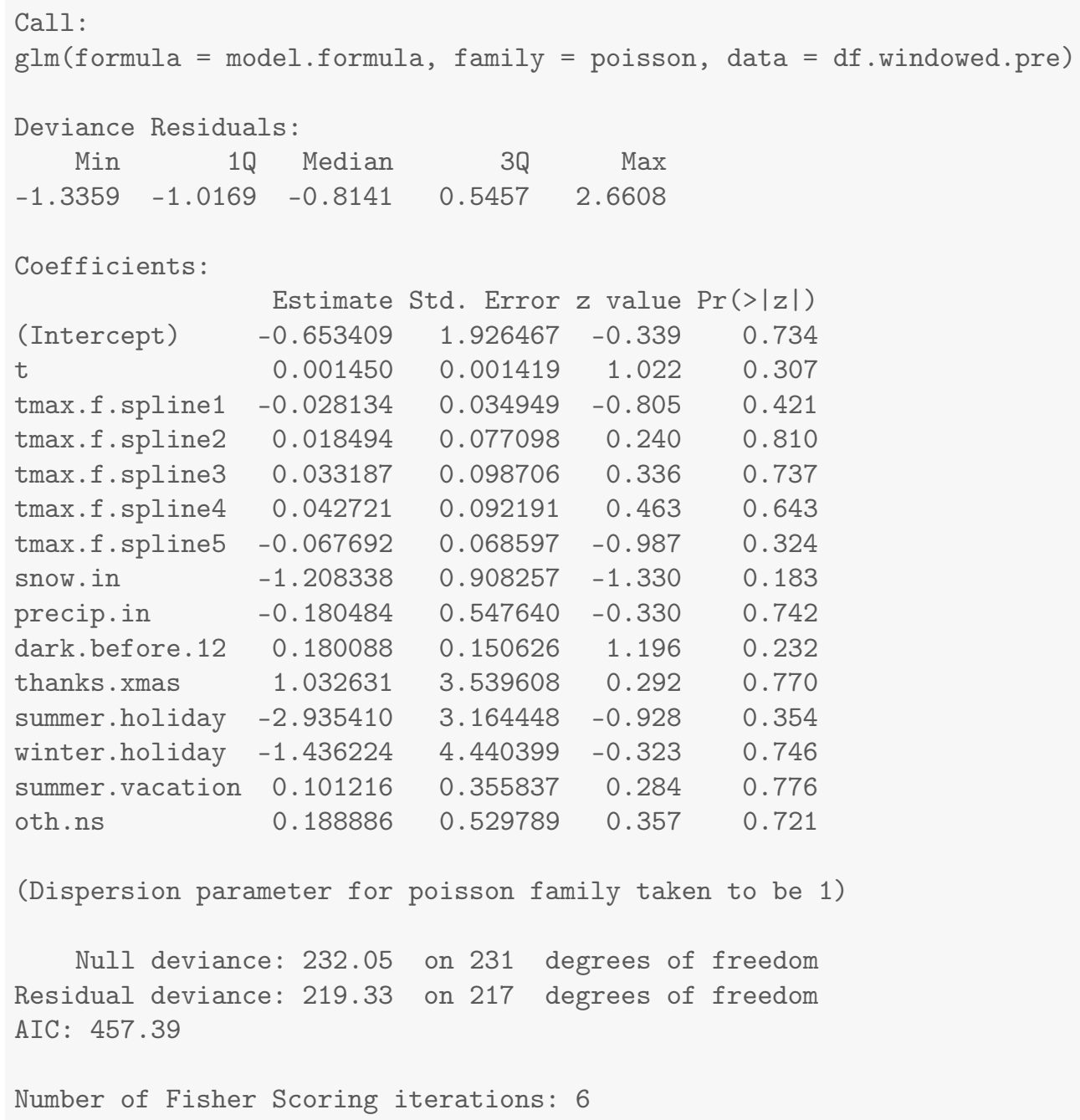




\section{Results for homicide.southern}

\subsection{Summary Values for homicide.southern}

Table 34.1: Descriptives for Outcome Before Ferguson Protests Begin

\begin{tabular}{lcccccccc}
\hline \hline Statistic & $\mathrm{N}$ & Mean & St. Dev. & Min & $\operatorname{Pctl}(25)$ & Median & Pctl(75) & Max \\
\hline homicide.southern & 232 & 0.379 & 0.598 & 0 & 0 & 0 & 1 & 3 \\
\hline
\end{tabular}

Table 34.2: Descriptives for Outcome After Ferguson Protests Begin

\begin{tabular}{lcccccccc}
\hline \hline Statistic & $\mathrm{N}$ & Mean & St. Dev. & Min & Pctl(25) & Median & Pctl(75) & Max \\
\hline homicide.southern & 290 & 0.586 & 0.777 & 0 & 0 & 0 & 1 & 4 \\
\hline
\end{tabular}




\subsection{Four Models for homicide.southern}

Table 34.3: Four Models that Differ on the Specification of Adjustment and Intervention Variables

\begin{tabular}{lcccc}
\hline \hline & \multicolumn{3}{c}{ Outcome: Count Per Week } \\
\cline { 2 - 5 } & $(1)$ & $(2)$ & $(3)$ & $(4)$ \\
\hline Time (counter in weeks) & 0.0001 & -0.0002 & & 0.0000 \\
After Ferguson Protests Begin (week of 8/11/14 onward) & -0.17 & & -0.14 & -0.17 \\
After Gray Protests Begin (week of 4/20/15 onward) & 0.59 & & 0.57 & 0.59 \\
Unrest and National Guard (4/27/15 - 5/3/2015) & 1.18 & & 1.20 & 1.14 \\
After Batts Fired (week of 7/13/15 onward) & -0.32 & & -0.25 & -0.31 \\
After Davis Confirmed (week of 10/19/15 onward) & 0.07 & & 0.07 & 0.07 \\
After Davis First Year (week of 10/17/16 onward) & 0.06 & & 0.04 & 0.05 \\
After De Sousa Begins (week of 1/19/18 onward) & 0.11 & & 0.14 & 0.14 \\
After Tuggle Begins (week of 5/14/18 onward) & -0.06 & & -0.05 & -0.05 \\
After Harrison Begins (week of 2/11/19 onward) & -0.10 & & -0.12 & -0.11 \\
Average Maximum Temperature to 50 Degrees & & 0.01 & & 0.003 \\
Plus Degrees in the 50s & & -0.03 & -0.01 \\
Plus Degrees in the 60s & & 0.04 & 0.01 \\
Plus Degrees in the 70s & & -0.01 & -0.04 \\
Plus Degrees Greater Than 80 & & 0.18 & & -0.01 \\
Snowfall (inches) & & -0.33 & & -0.09 \\
Precipitation (inches) & & -0.01 & & 0.001 \\
Darkness Before Midnight (hours) & & 1.09 & & 0.52 \\
Thanksgiving/Christmas (proportion of week) & & -1.27 & & -1.00 \\
Winter Holiday (proportion of week) & & 0.04 & & 0.17 \\
Other Out-of-School Days (proportion of week) & & 1.08 & & -0.01 \\
Summer Vacation (proportion of week) & 522 & 232 & 522 & 522 \\
Summer Holiday (proportion of week) & 0.05 & 0.03 & 0.07 & 0.06 \\
\hline Observations & & & & \\
$R^{2}$ & & & & \\
\hline \hline
\end{tabular}


34.3 Least Squares Updated Model (2) for homicide.southern homicide.southern

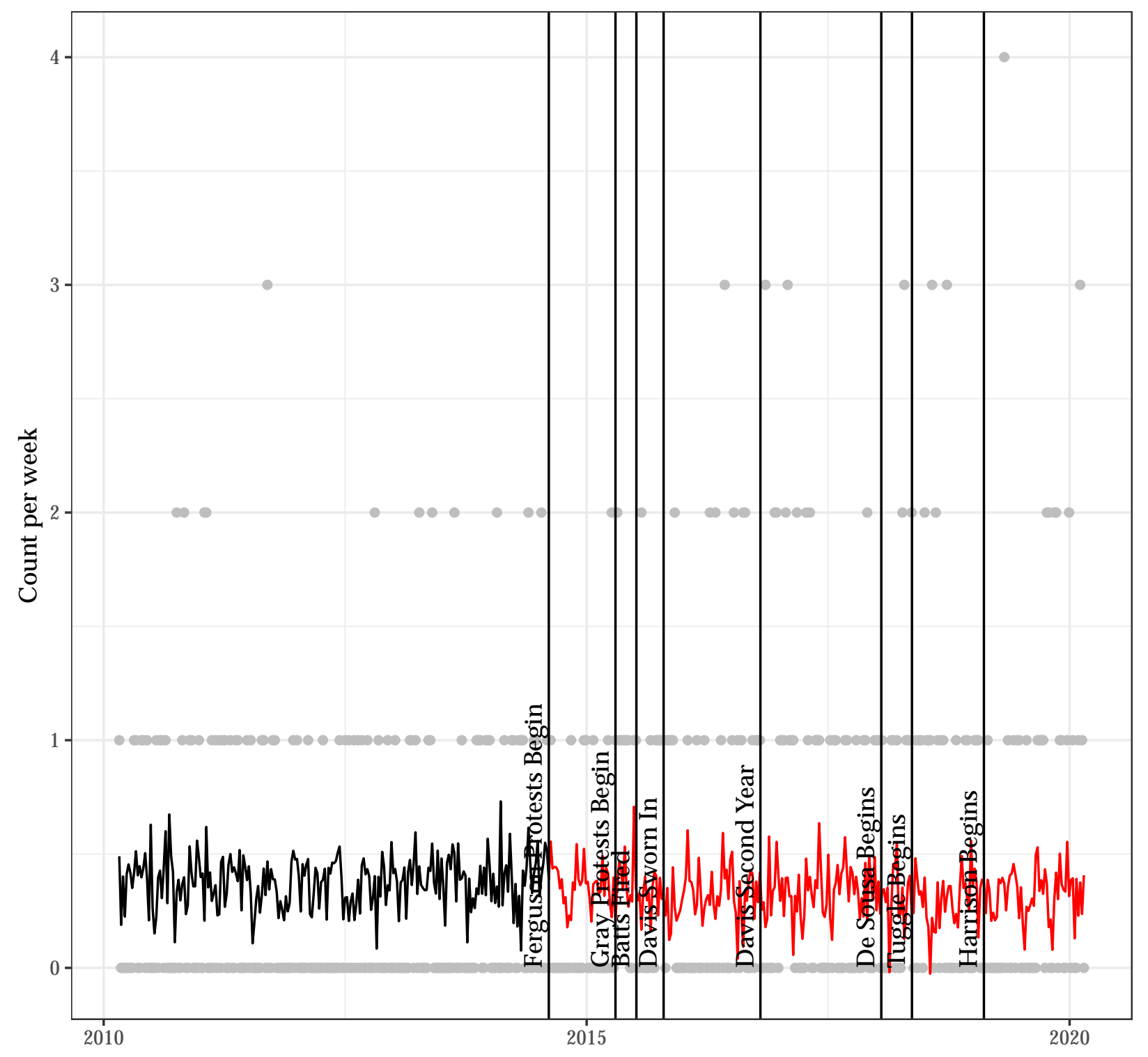




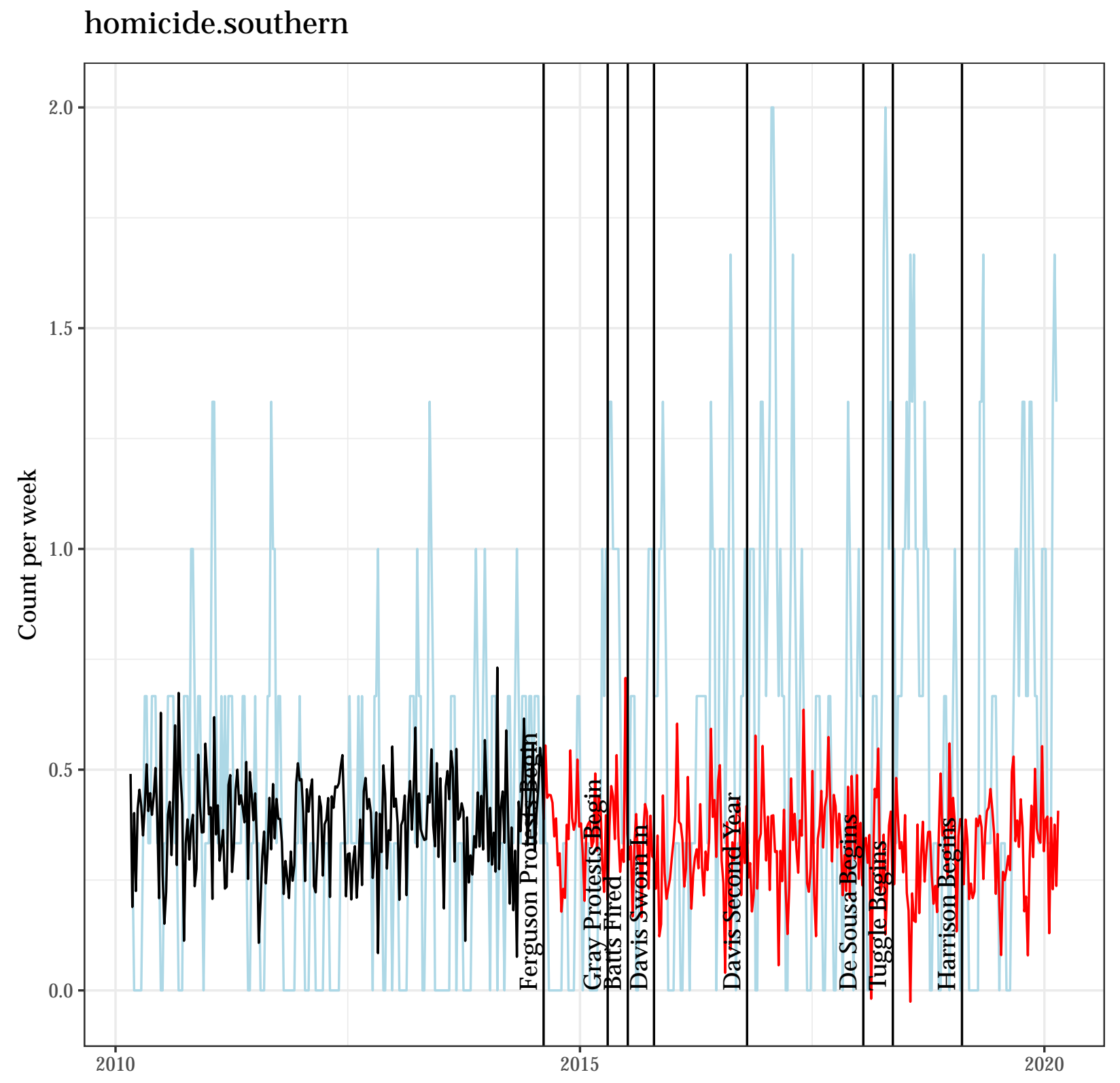


Autocorrelation Function for the Observed Outcome for Updated Model (2)

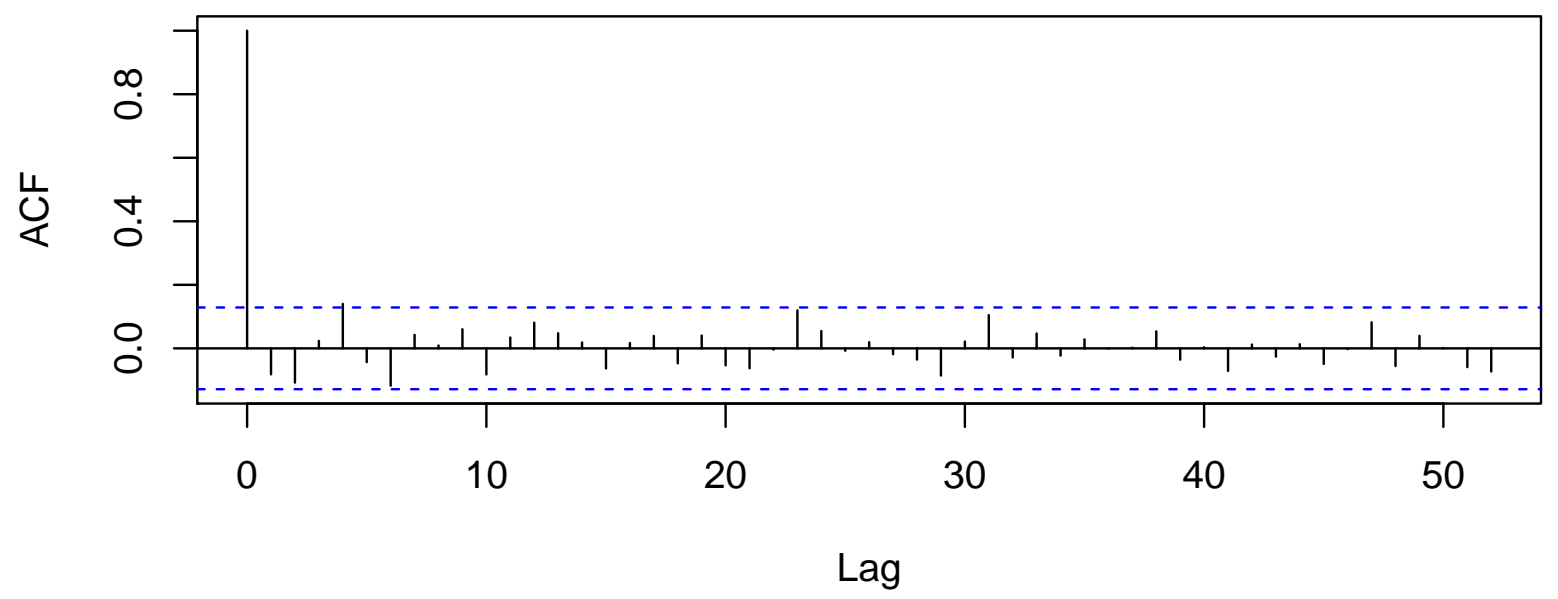

Autocorrelation Function for the Residuals from Updated Model (2)

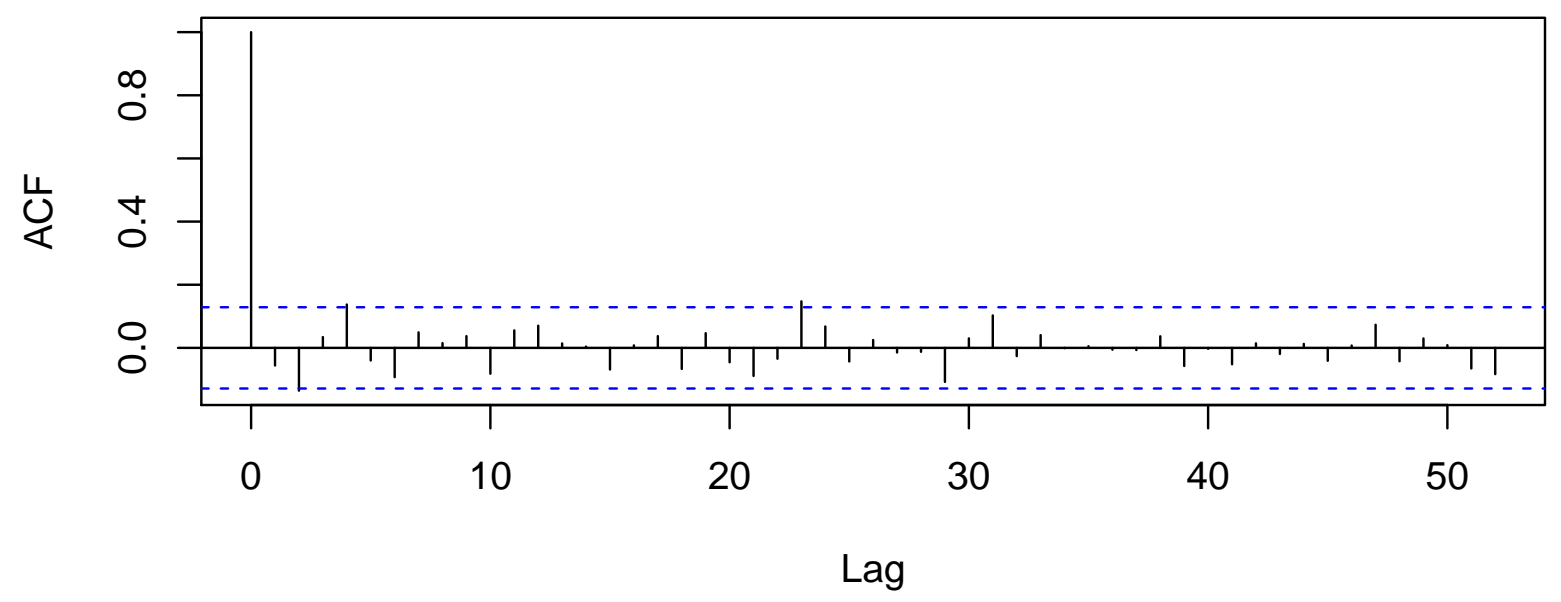




\subsection{A Poisson Regression Model as an Alternative to Model (2) for homicide.southern}

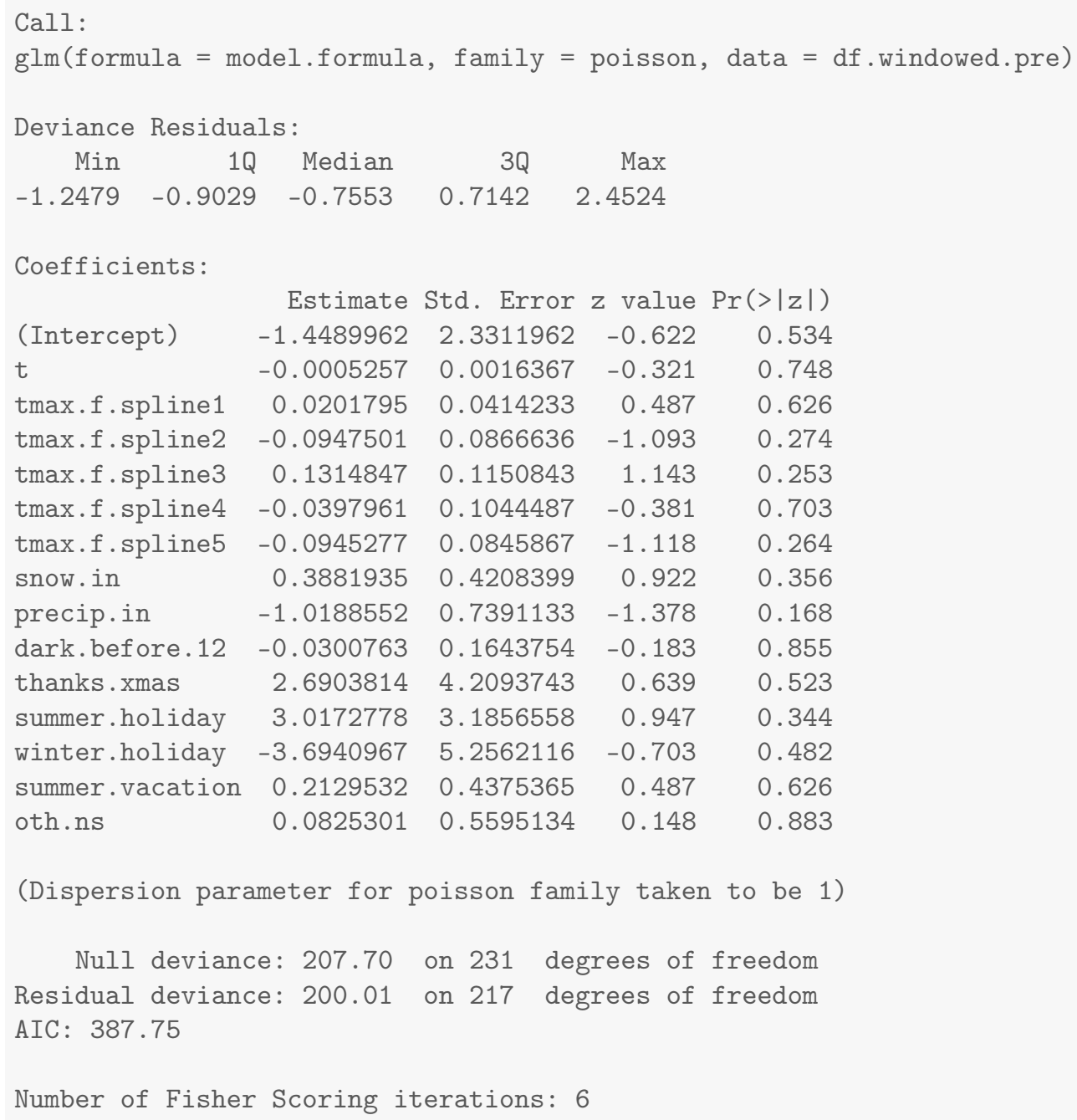




\section{Results for homicide.southeastern}

\subsection{Summary Values for homicide.southeastern}

Table 35.1: Descriptives for Outcome Before Ferguson Protests Begin

\begin{tabular}{lcccccccc}
\hline \hline Statistic & $\mathrm{N}$ & Mean & St. Dev. & Min & $\operatorname{Pctl}(25)$ & Median & Pctl(75) & Max \\
\hline homicide.southeastern & 232 & 0.246 & 0.505 & 0 & 0 & 0 & 0 & 2 \\
\hline
\end{tabular}

Table 35.2: Descriptives for Outcome After Ferguson Protests Begin

\begin{tabular}{lcccccccc}
\hline \hline Statistic & $\mathrm{N}$ & Mean & St. Dev. & Min & Pctl(25) & Median & Pctl(75) & Max \\
\hline homicide.southeastern & 290 & 0.417 & 0.601 & 0 & 0 & 0 & 1 & 3 \\
\hline
\end{tabular}




\subsection{Four Models for homicide.southeastern}

Table 35.3: Four Models that Differ on the Specification of Adjustment and Intervention Variables

\begin{tabular}{|c|c|c|c|c|}
\hline & \multicolumn{4}{|c|}{ Outcome: Count Per Week } \\
\hline & $(1)$ & $(2)$ & (3) & $(4)$ \\
\hline Time (counter in weeks) & 0.0001 & 0.0001 & & 0.0002 \\
\hline After Ferguson Protests Begin (week of 8/11/14 onward) & -0.07 & & -0.08 & -0.08 \\
\hline After Gray Protests Begin (week of $4 / 20 / 15$ onward) & 0.07 & & 0.06 & 0.05 \\
\hline Unrest and National Guard $(4 / 27 / 15-5 / 3 / 2015)$ & 0.73 & & 0.78 & 0.73 \\
\hline After Batts Fired (week of 7/13/15 onward) & 0.08 & & 0.07 & 0.08 \\
\hline After Davis Confirmed (week of 10/19/15 onward) & 0.10 & & 0.14 & 0.13 \\
\hline After Davis First Year (week of 10/17/16 onward) & 0.0001 & & 0.003 & -0.01 \\
\hline After De Sousa Begins (week of 1/19/18 onward) & -0.16 & & -0.10 & -0.13 \\
\hline After Tuggle Begins (week of 5/14/18 onward) & -0.06 & & -0.13 & -0.09 \\
\hline After Harrison Begins (week of 2/11/19 onward) & 0.37 & & 0.40 & 0.37 \\
\hline Average Maximum Temperature to 50 Degrees & & -0.02 & & -0.02 \\
\hline Plus Degrees in the $50 \mathrm{~s}$ & & 0.04 & & 0.03 \\
\hline Plus Degrees in the 60s & & -0.01 & & -0.01 \\
\hline Plus Degrees in the 70s & & -0.002 & & -0.001 \\
\hline Plus Degrees Greater Than 80 & & 0.001 & & 0.001 \\
\hline Snowfall (inches) & & -0.14 & & -0.15 \\
\hline Precipitation (inches) & & -0.001 & & -0.11 \\
\hline Darkness Before Midnight (hours) & & 0.02 & & 0.004 \\
\hline Thanksgiving/Christmas (proportion of week) & & -1.15 & & 0.04 \\
\hline Winter Holiday (proportion of week) & & -0.27 & & -0.63 \\
\hline Other Out-of-School Days (proportion of week) & & 0.11 & & -0.08 \\
\hline Summer Vacation (proportion of week) & & -0.01 & & 0.07 \\
\hline Summer Holiday (proportion of week) & & 1.14 & & 0.96 \\
\hline Observations & 522 & 232 & 522 & 522 \\
\hline $\mathrm{R}^{2}$ & 0.06 & 0.03 & 0.06 & 0.08 \\
\hline
\end{tabular}


35.3 Least Squares Updated Model (2) for homicide.southeastern homicide.southeastern

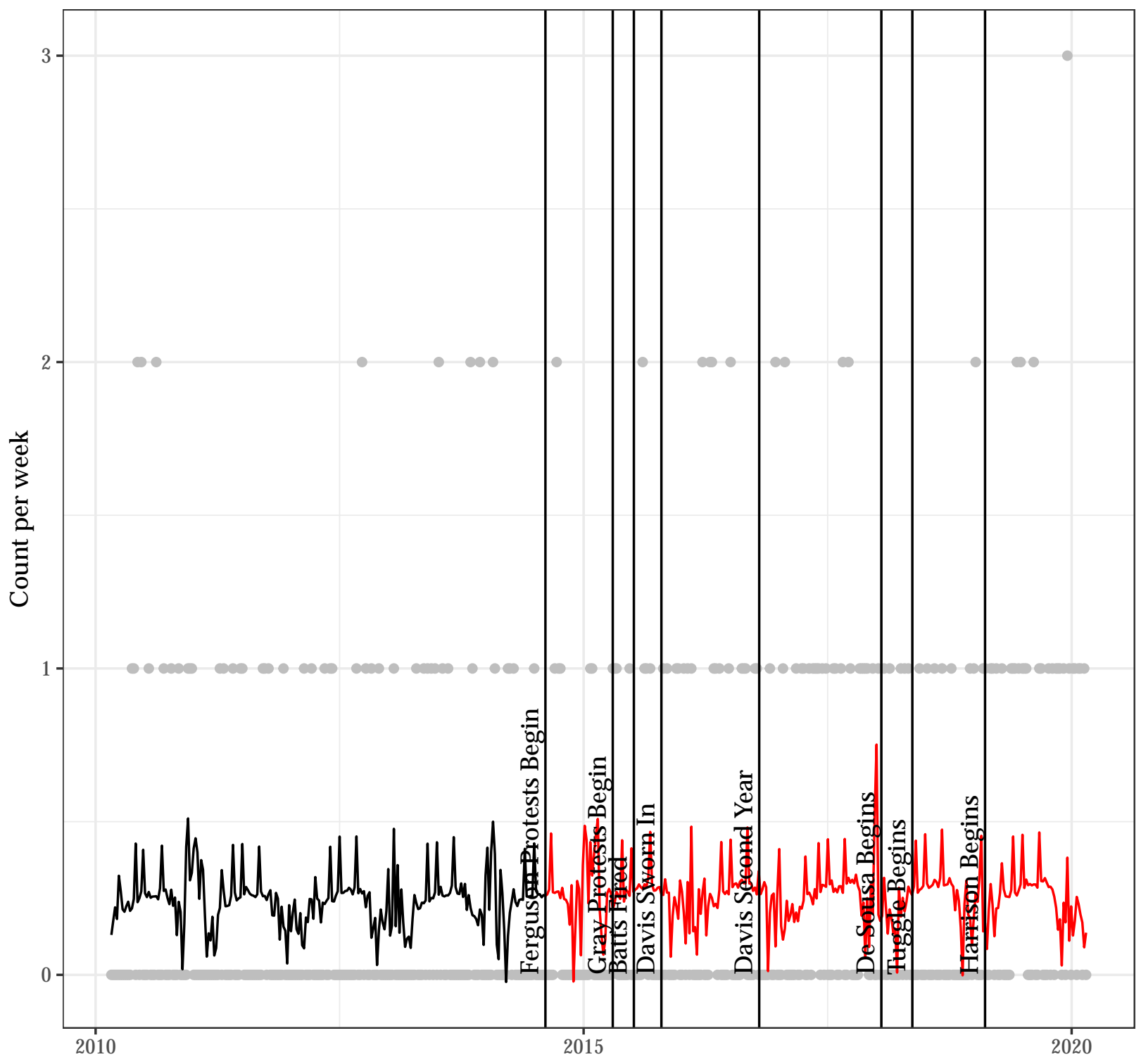




\section{homicide.southeastern}

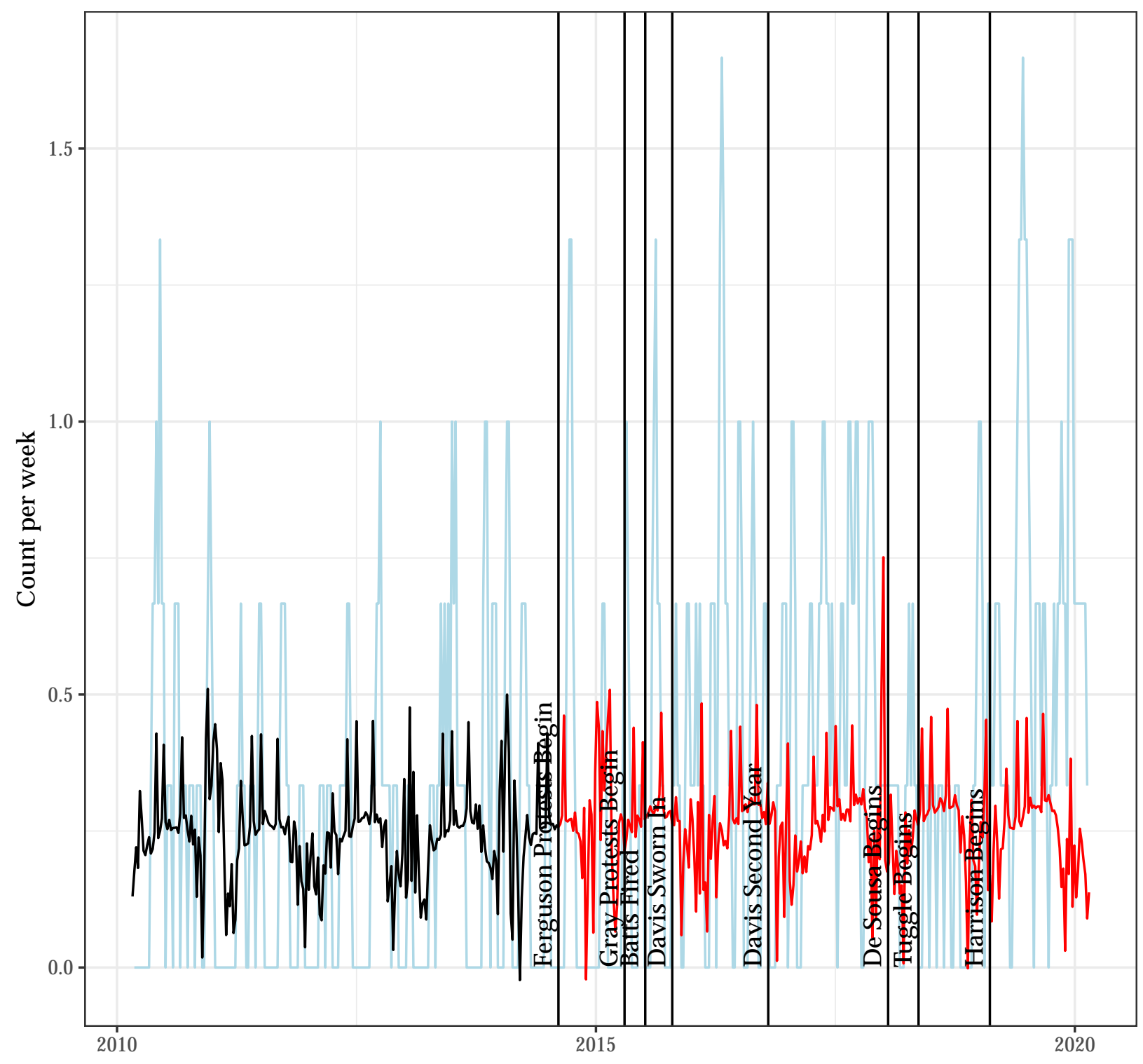


Autocorrelation Function for the Observed Outcome for Updated Model (2)

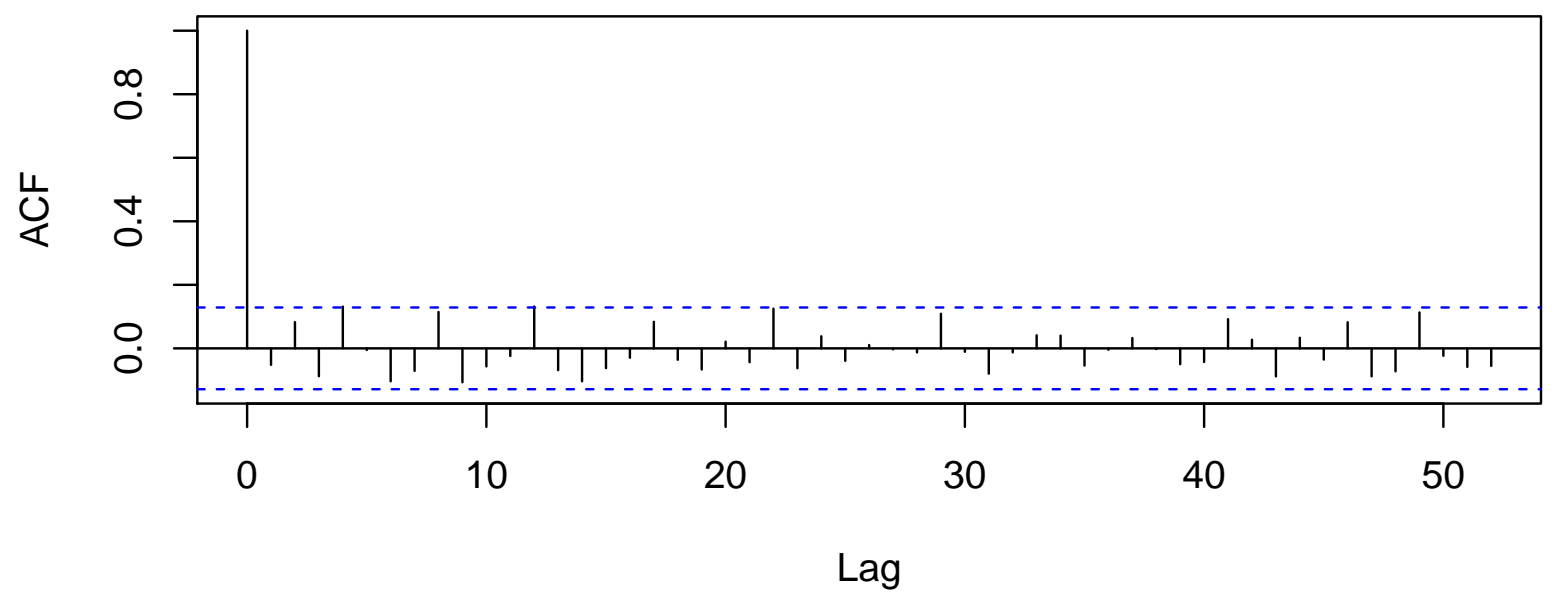

Autocorrelation Function for the Residuals from Updated Model (2)

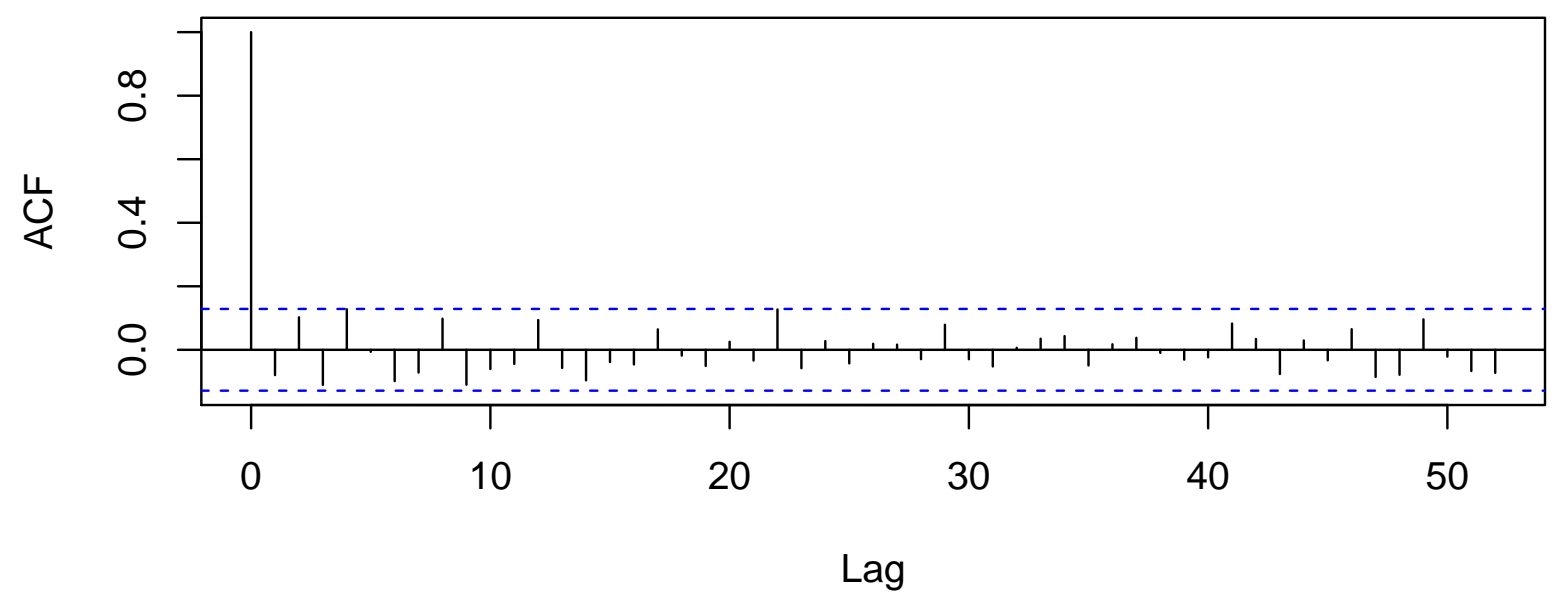




\subsection{A Poisson Regression Model as an Alternative to Model (2) for homicide.southeastern}

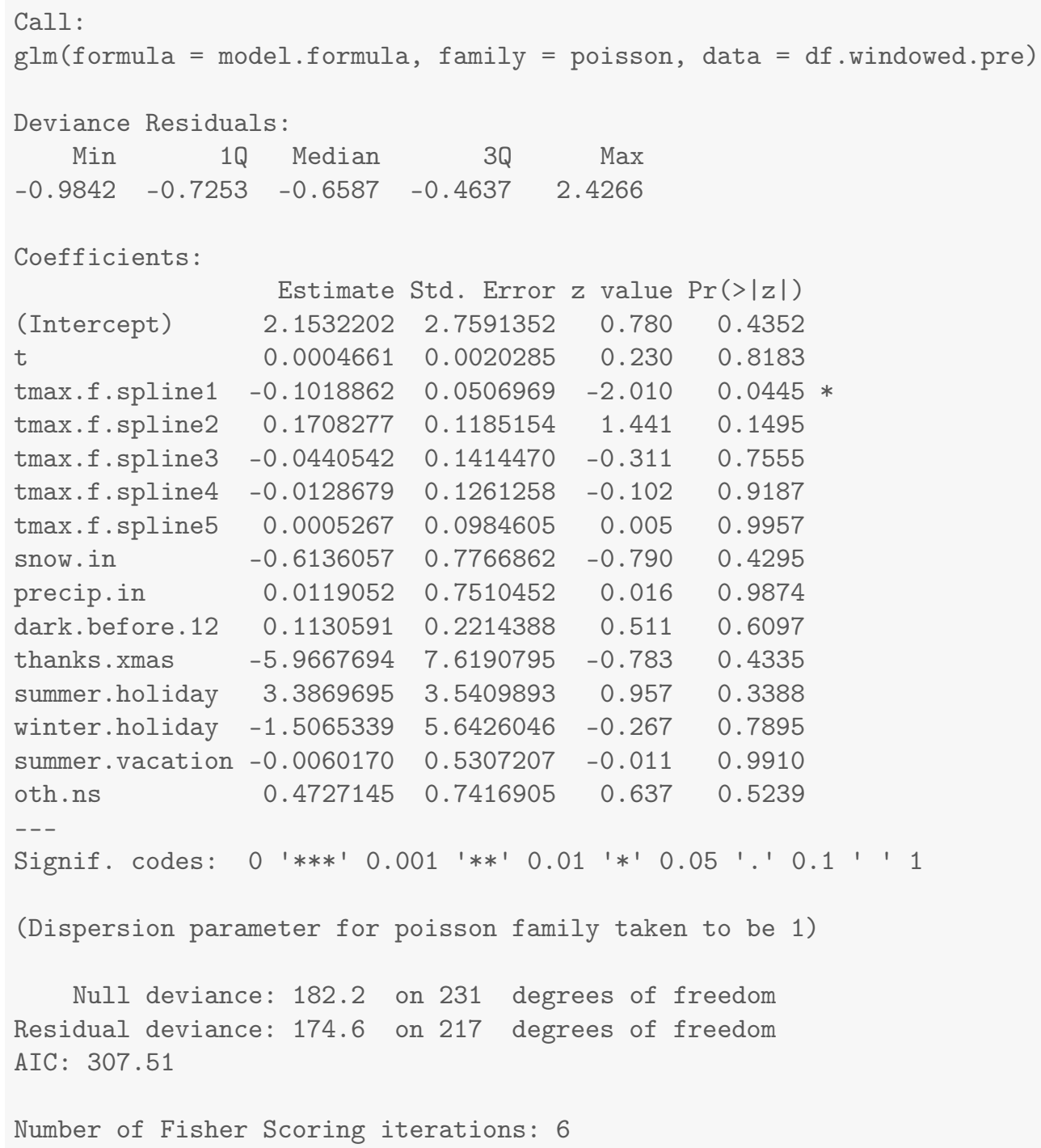




\section{Results for shooting.northwestern}

\subsection{Summary Values for shooting.northwestern}

Table 36.1: Descriptives for Outcome Before Ferguson Protests Begin

\begin{tabular}{lcccccccc}
\hline \hline Statistic & $\mathrm{N}$ & Mean & St. Dev. & Min & Pctl(25) & Median & Pctl(75) & Max \\
\hline shooting.northwestern & 232 & 0.806 & 1.101 & 0 & 0 & 0 & 1 & 6 \\
\hline
\end{tabular}

Table 36.2: Descriptives for Outcome After Ferguson Protests Begin

\begin{tabular}{lcccccccc}
\hline \hline Statistic & $\mathrm{N}$ & Mean & St. Dev. & Min & Pctl(25) & Median & Pctl(75) & Max \\
\hline shooting.northwestern & 290 & 1.438 & 1.615 & 0 & 0 & 1 & 2 & 10 \\
\hline
\end{tabular}




\subsection{Four Models for shooting.northwestern}

Table 36.3: Four Models that Differ on the Specification of Adjustment and Intervention Variables

\begin{tabular}{|c|c|c|c|c|}
\hline & \multicolumn{4}{|c|}{ Outcome: Count Per Week } \\
\hline & $(1)$ & $(2)$ & $(3)$ & $(4)$ \\
\hline Time (counter in weeks) & -0.001 & -0.001 & & -0.001 \\
\hline After Ferguson Protests Begin (week of $8 / 11 / 14$ onward) & 0.33 & & 0.50 & 0.43 \\
\hline After Gray Protests Begin (week of $4 / 20 / 15$ onward) & 0.30 & & 0.02 & 0.14 \\
\hline Unrest and National Guard $(4 / 27 / 15-5 / 3 / 2015)$ & 2.72 & & 3.01 & 2.82 \\
\hline After Batts Fired (week of 7/13/15 onward) & 0.24 & & 0.31 & 0.08 \\
\hline After Davis Confirmed (week of 10/19/15 onward) & -0.39 & & -0.24 & -0.21 \\
\hline After Davis First Year (week of 10/17/16 onward) & 0.68 & & 0.57 & 0.61 \\
\hline After De Sousa Begins (week of $1 / 19 / 18$ onward) & -0.72 & & -0.62 & -0.45 \\
\hline After Tuggle Begins (week of 5/14/18 onward) & 1.12 & & 0.98 & 0.90 \\
\hline After Harrison Begins (week of 2/11/19 onward) & -0.52 & & -0.59 & -0.52 \\
\hline Average Maximum Temperature to 50 Degrees & & 0.05 & & 0.03 \\
\hline Plus Degrees in the $50 \mathrm{~s}$ & & -0.10 & & -0.03 \\
\hline Plus Degrees in the 60s & & 0.08 & & 0.04 \\
\hline Plus Degrees in the $70 \mathrm{~s}$ & & 0.02 & & -0.02 \\
\hline Plus Degrees Greater Than 80 & & -0.14 & & -0.07 \\
\hline Snowfall (inches) & & -0.06 & & -0.08 \\
\hline Precipitation (inches) & & -0.24 & & -0.31 \\
\hline Darkness Before Midnight (hours) & & 0.06 & & 0.20 \\
\hline Thanksgiving/Christmas (proportion of week) & & -2.30 & & -0.12 \\
\hline Winter Holiday (proportion of week) & & -0.98 & & 0.57 \\
\hline Other Out-of-School Days (proportion of week) & & 0.23 & & 0.06 \\
\hline Summer Vacation (proportion of week) & & 0.41 & & 0.33 \\
\hline Summer Holiday (proportion of week) & & -2.03 & & -2.54 \\
\hline Observations & 522 & 232 & 522 & 522 \\
\hline $\mathrm{R}^{2}$ & 0.09 & 0.09 & 0.13 & 0.12 \\
\hline
\end{tabular}


36.3 Least Squares Updated Model (2) for shooting.northwestern shooting.northwestern

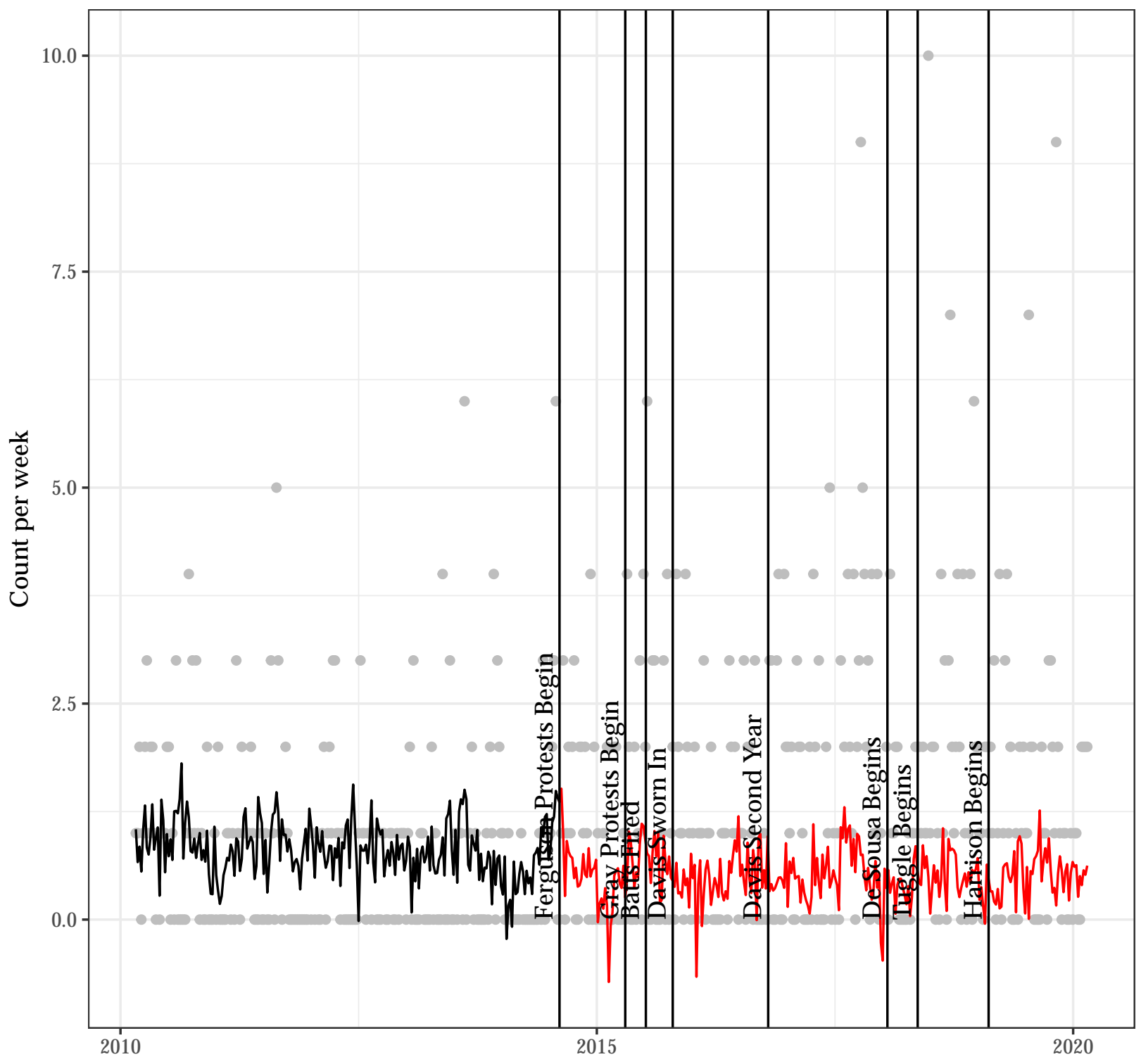


shooting.northwestern

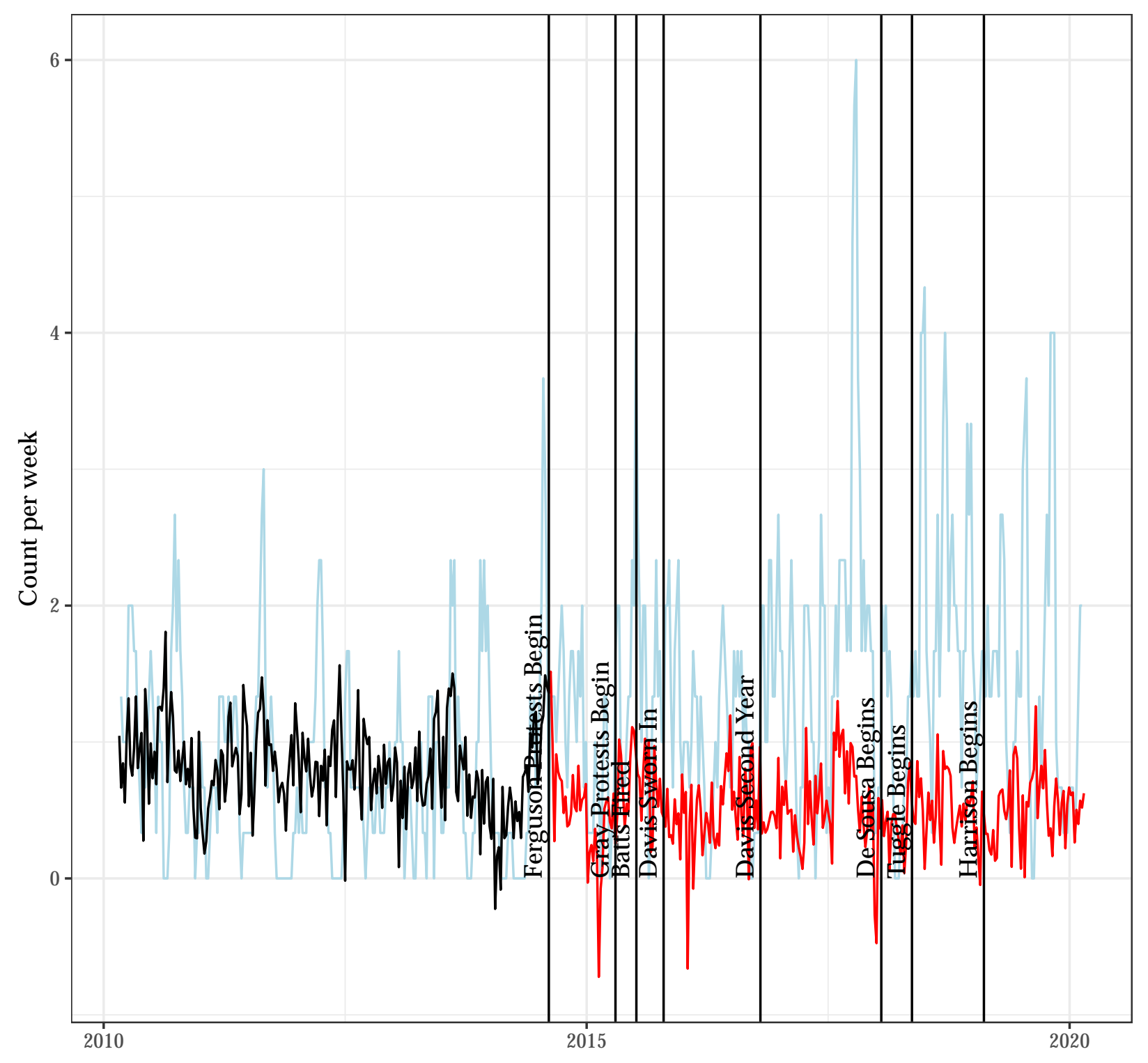


Autocorrelation Function for the Observed Outcome for Updated Model (2)

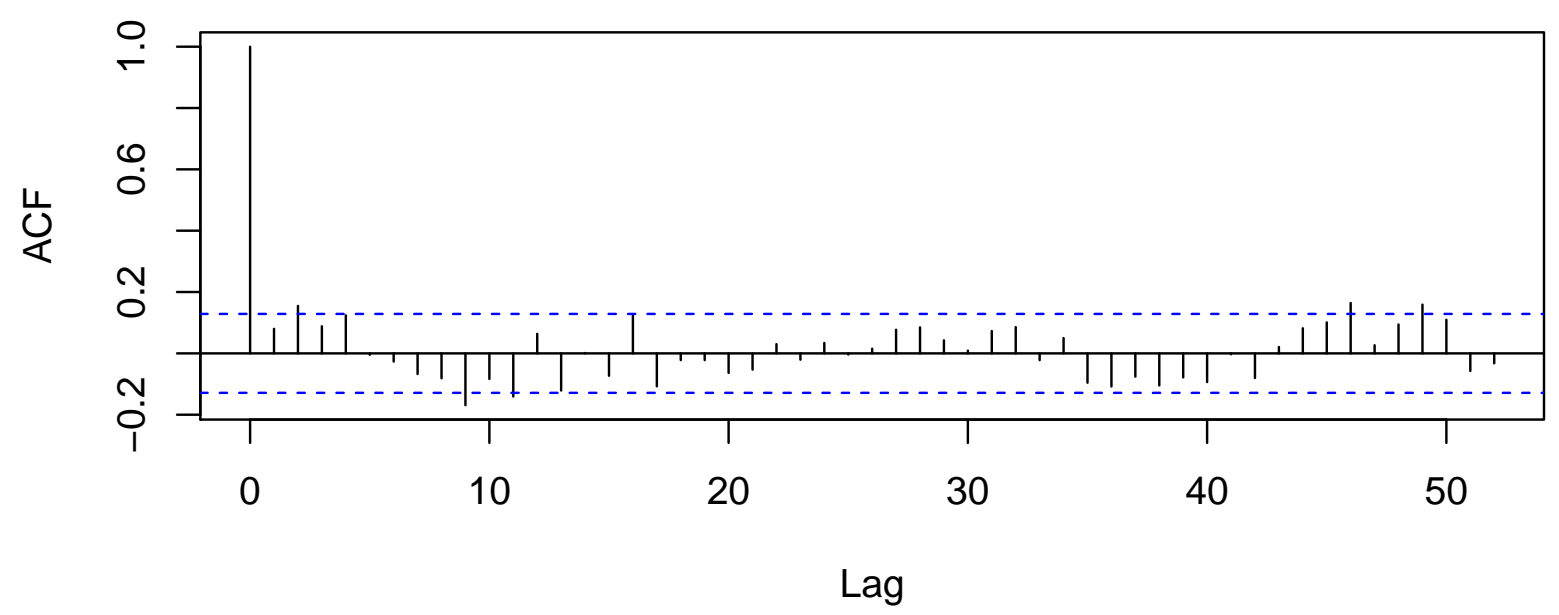

Autocorrelation Function for the Residuals from Updated Model (2)

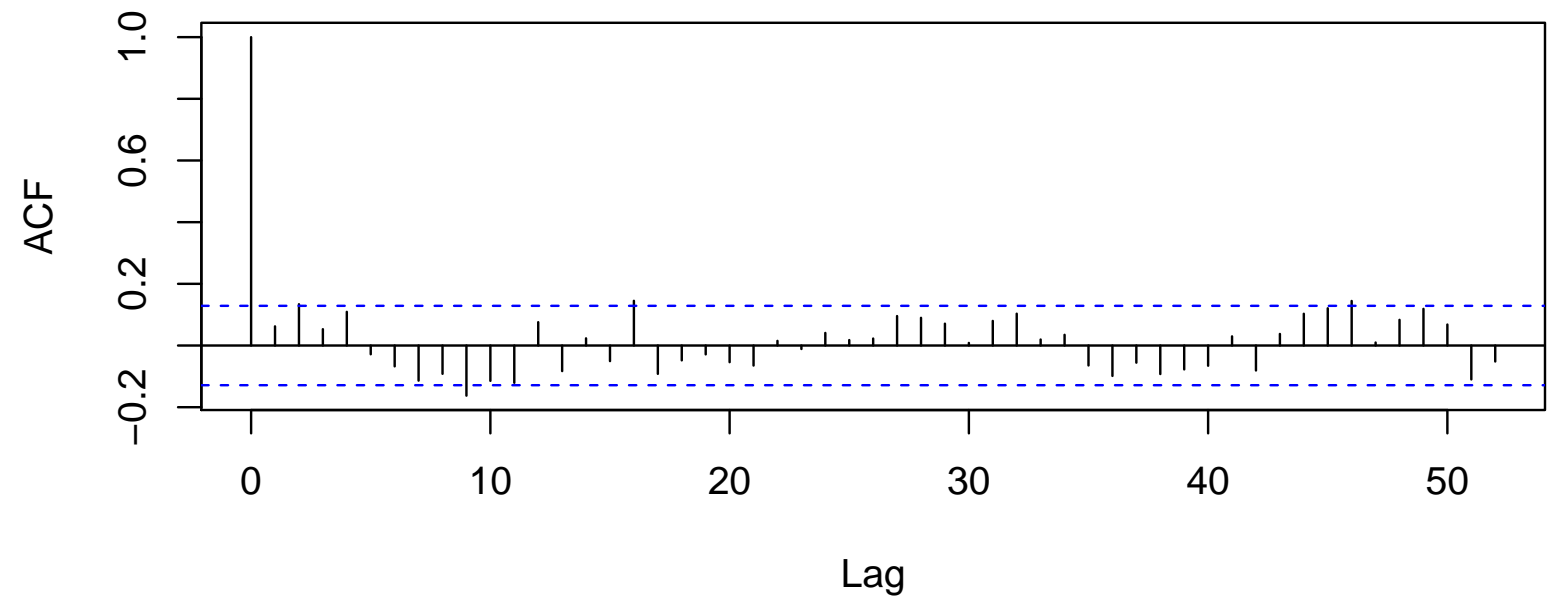




\subsection{A Poisson Regression Model as an Alternative to Model (2) for shooting.northwestern}

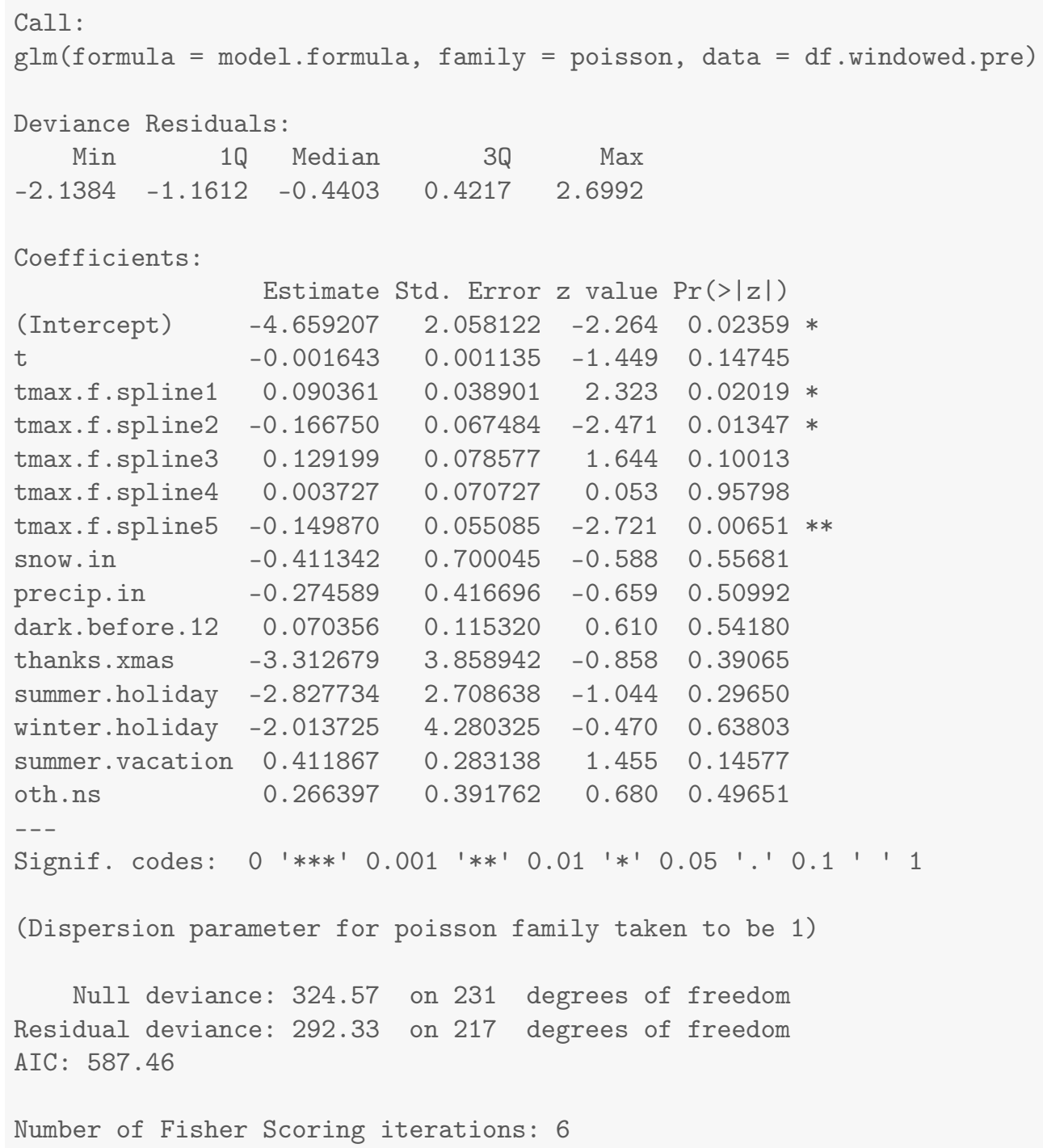




\section{Results for shooting.northern}

\subsection{Summary Values for shooting.northern}

Table 37.1: Descriptives for Outcome Before Ferguson Protests Begin

\begin{tabular}{lcccccccc}
\hline \hline Statistic & $\mathrm{N}$ & Mean & St. Dev. & Min & Pctl(25) & Median & Pctl(75) & Max \\
\hline shooting.northern & 232 & 0.517 & 0.800 & 0 & 0 & 0 & 1 & 4 \\
\hline
\end{tabular}

Table 37.2: Descriptives for Outcome After Ferguson Protests Begin

\begin{tabular}{lcccccccc}
\hline \hline Statistic & $\mathrm{N}$ & Mean & St. Dev. & Min & Pctl(25) & Median & Pctl(75) & Max \\
\hline shooting.northern & 290 & 0.862 & 1.162 & 0 & 0 & 1 & 1 & 8 \\
\hline
\end{tabular}




\subsection{Four Models for shooting.northern}

Table 37.3: Four Models that Differ on the Specification of Adjustment and Intervention Variables

\begin{tabular}{|c|c|c|c|c|}
\hline & \multicolumn{4}{|c|}{ Outcome: Count Per Week } \\
\hline & $(1)$ & $(2)$ & $(3)$ & (4) \\
\hline Time (counter in weeks) & -0.001 & -0.0003 & & -0.0004 \\
\hline After Ferguson Protests Begin (week of $8 / 11 / 14$ onward) & -0.02 & & 0.06 & 0.05 \\
\hline After Gray Protests Begin (week of 4/20/15 onward) & 0.23 & & 0.08 & -0.02 \\
\hline Unrest and National Guard $(4 / 27 / 15-5 / 3 / 2015)$ & -0.64 & & -0.47 & -0.43 \\
\hline After Batts Fired (week of 7/13/15 onward) & 1.09 & & 1.01 & 1.10 \\
\hline After Davis Confirmed (week of $10 / 19 / 15$ onward) & -0.75 & & -0.67 & -0.65 \\
\hline After Davis First Year (week of 10/17/16 onward) & -0.11 & & -0.13 & -0.09 \\
\hline After De Sousa Begins (week of 1/19/18 onward) & 0.10 & & 0.14 & 0.11 \\
\hline After Tuggle Begins (week of 5/14/18 onward) & 0.17 & & 0.02 & 0.06 \\
\hline After Harrison Begins (week of 2/11/19 onward) & -0.13 & & -0.11 & -0.09 \\
\hline Average Maximum Temperature to 50 Degrees & & 0.04 & & 0.005 \\
\hline Plus Degrees in the $50 \mathrm{~s}$ & & -0.04 & & 0.003 \\
\hline Plus Degrees in the 60s & & -0.01 & & -0.02 \\
\hline Plus Degrees in the 70s & & 0.05 & & 0.05 \\
\hline Plus Degrees Greater Than 80 & & -0.02 & & -0.01 \\
\hline Snowfall (inches) & & -0.09 & & -0.08 \\
\hline Precipitation (inches) & & 0.09 & & 0.10 \\
\hline Darkness Before Midnight (hours) & & 0.03 & & -0.05 \\
\hline Thanksgiving/Christmas (proportion of week) & & 5.57 & & 2.91 \\
\hline Winter Holiday (proportion of week) & & 1.26 & & 0.01 \\
\hline Other Out-of-School Days (proportion of week) & & -0.28 & & -0.37 \\
\hline Summer Vacation (proportion of week) & & -0.28 & & -0.21 \\
\hline Summer Holiday (proportion of week) & & -2.41 & & -0.69 \\
\hline Observations & 522 & 232 & 522 & 522 \\
\hline $\mathrm{R}^{2}$ & 0.06 & 0.08 & 0.06 & 0.09 \\
\hline
\end{tabular}




\subsection{Least Squares Updated Model (2) for shooting.northern shooting.northern}

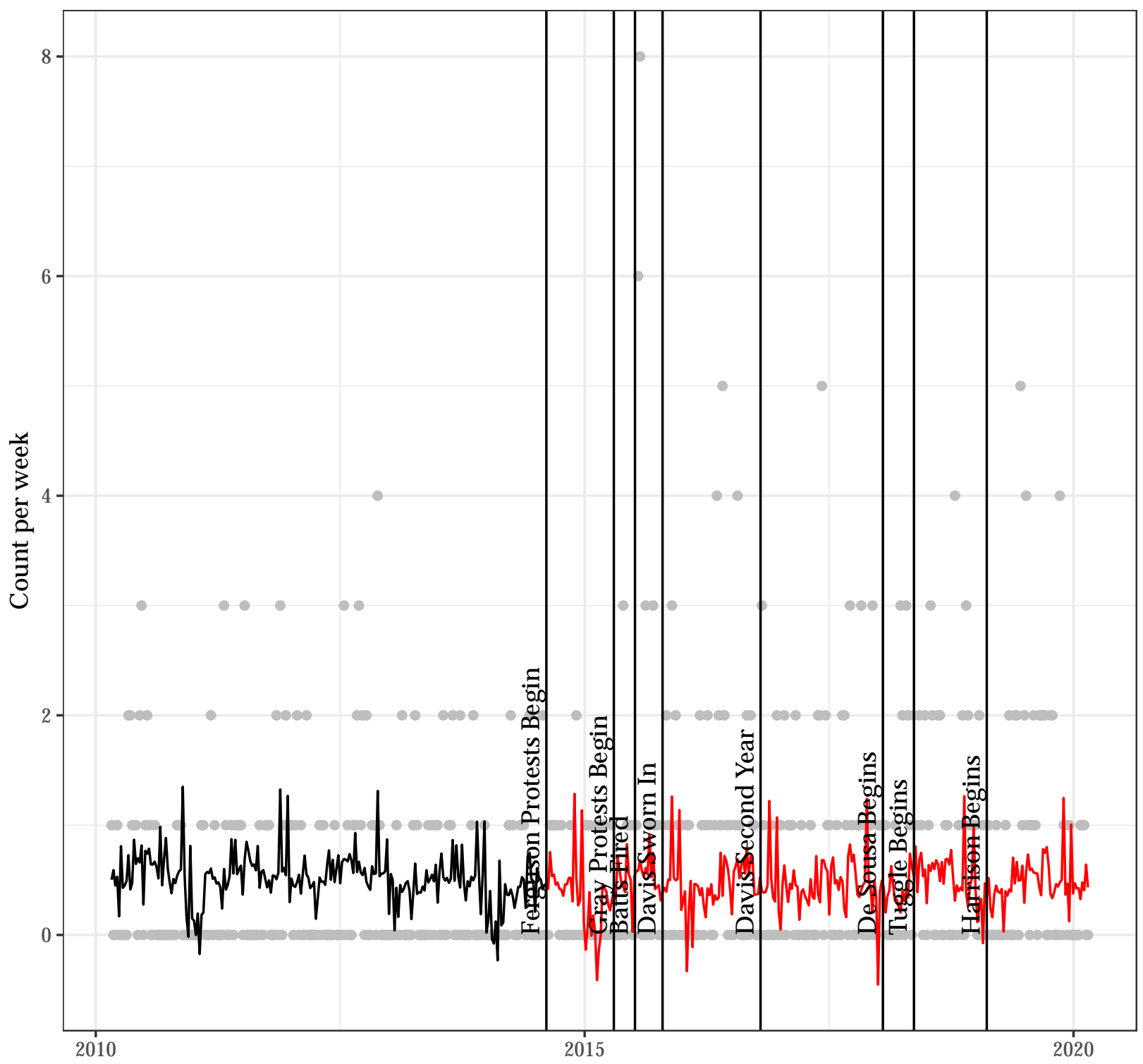




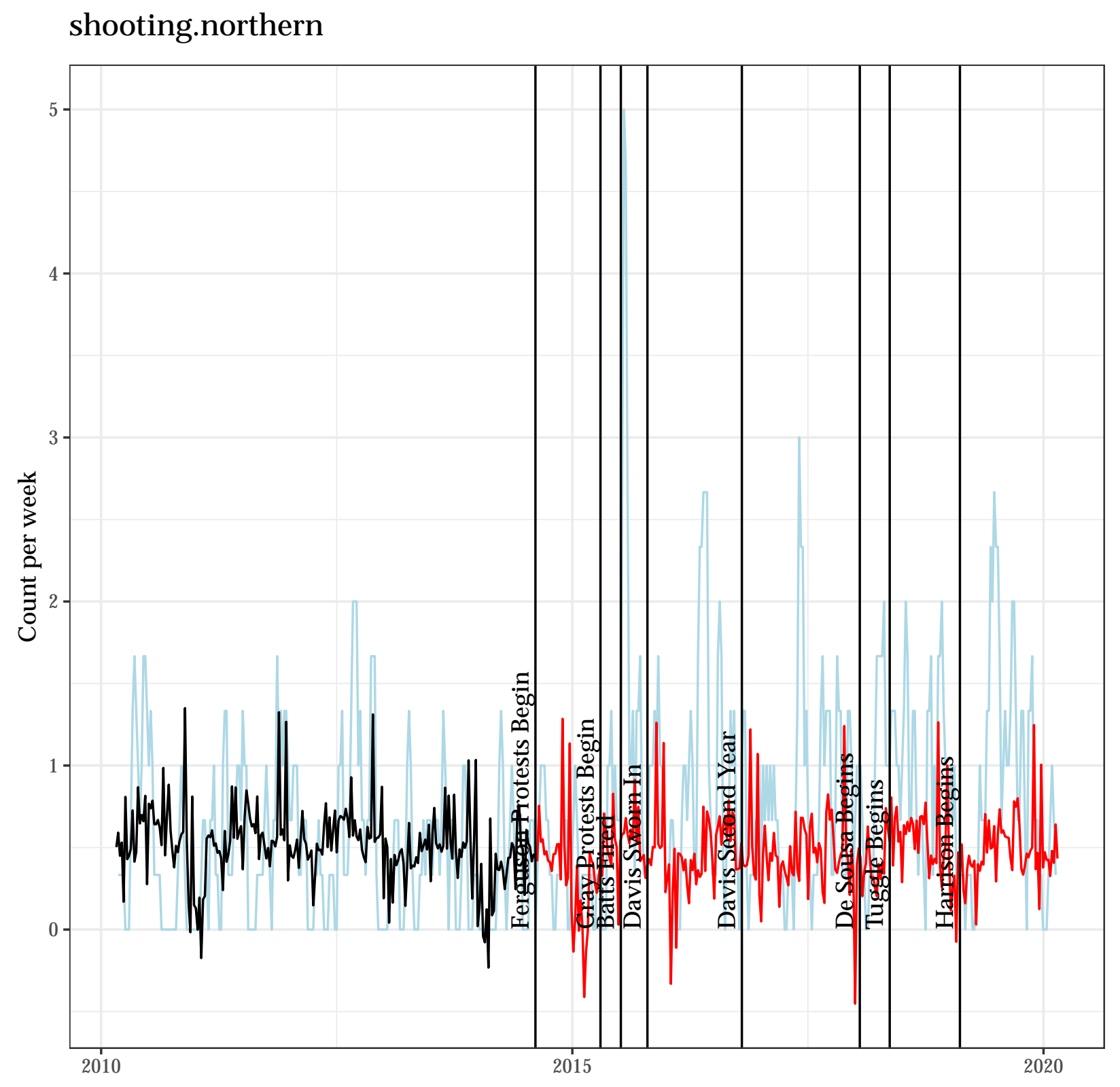


Autocorrelation Function for the Observed Outcome for Updated Model (2)

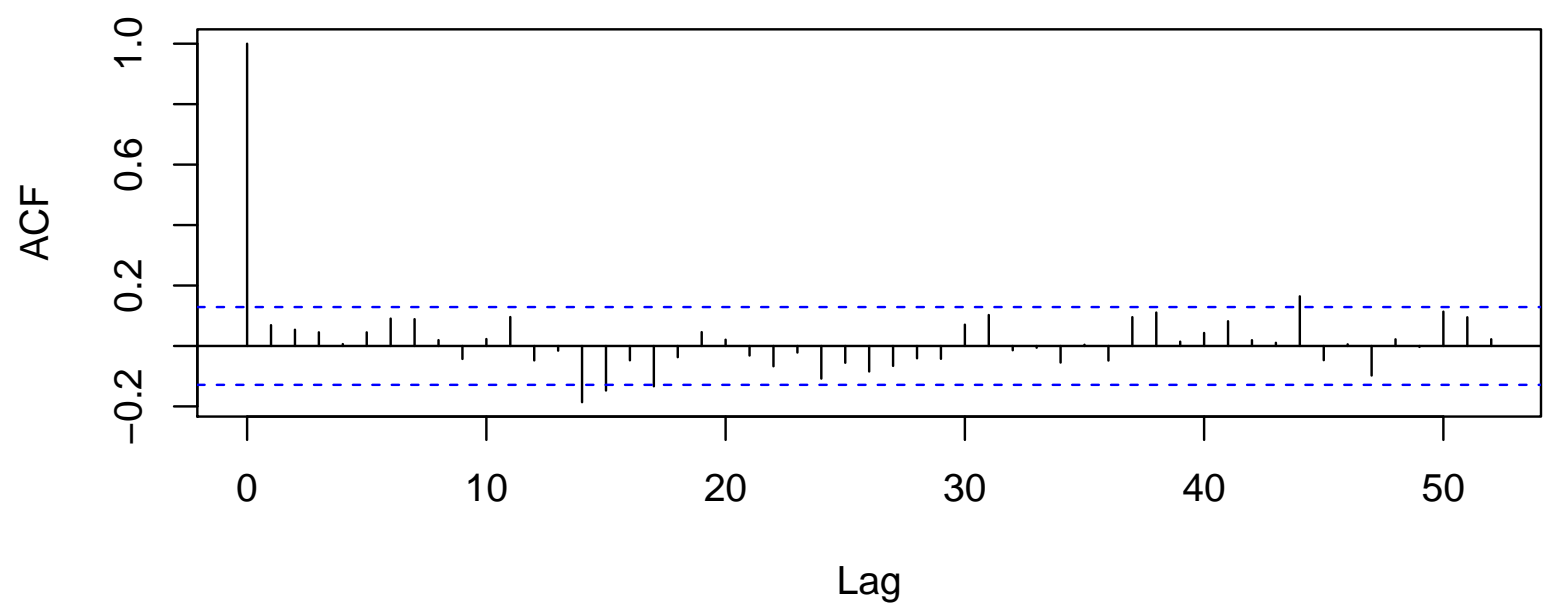

Autocorrelation Function for the Residuals from Updated Model (2)

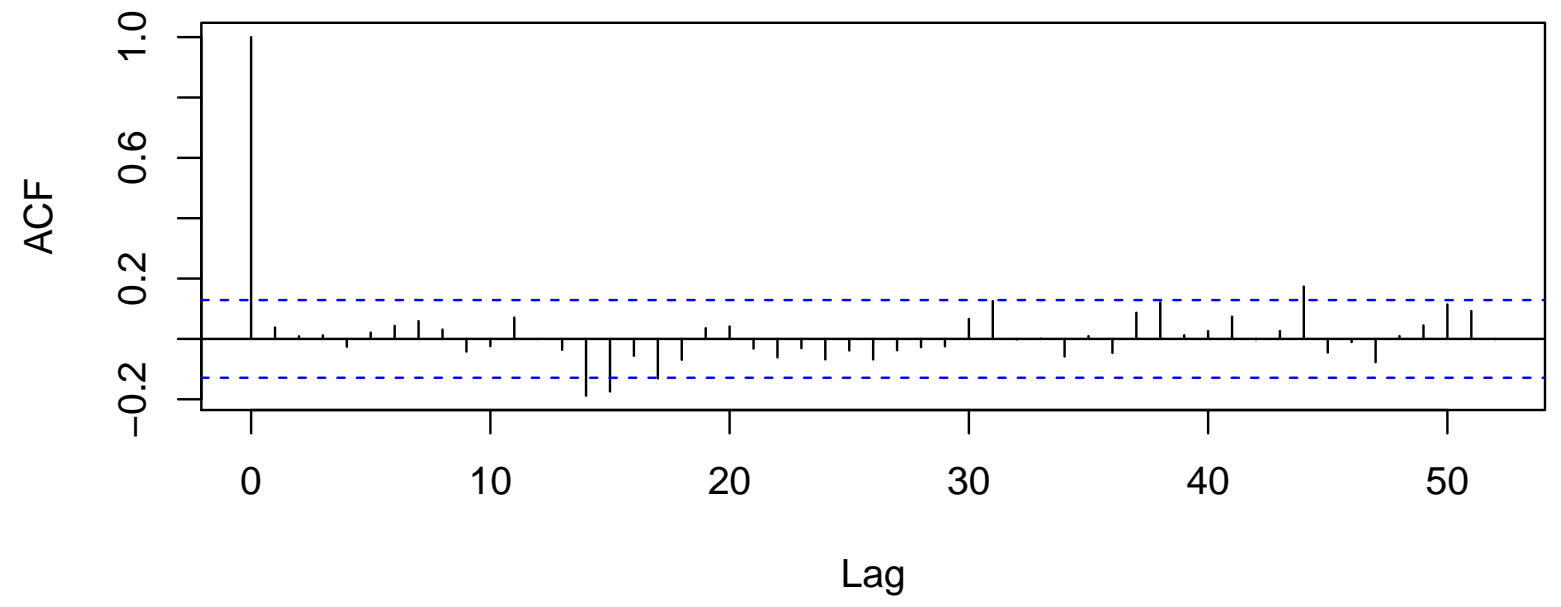




\subsection{A Poisson Regression Model as an Alternative to Model (2) for shooting.northern}

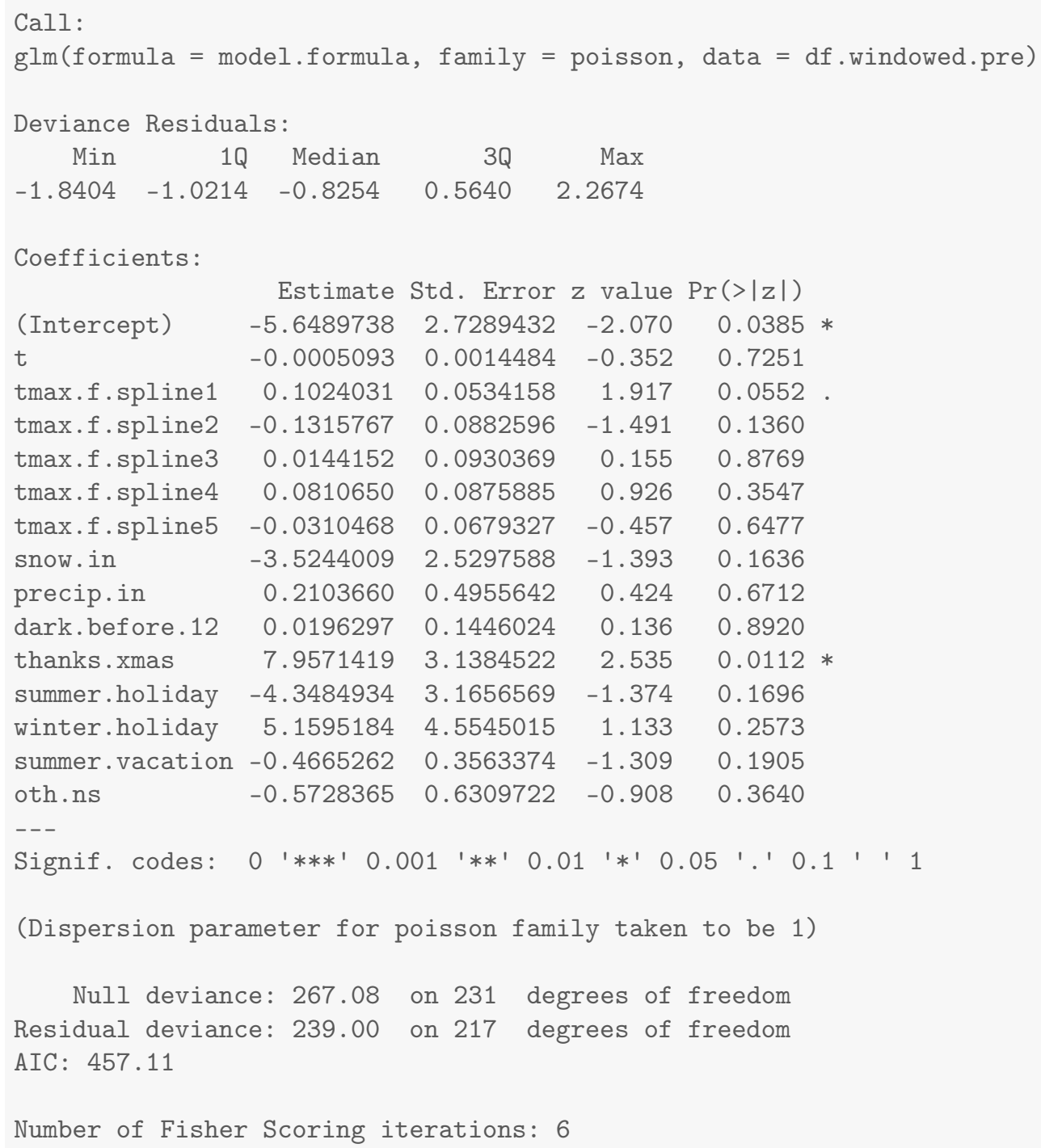




\section{Results for shooting.northeastern}

\subsection{Summary Values for shooting.northeastern}

Table 38.1: Descriptives for Outcome Before Ferguson Protests Begin

\begin{tabular}{lcccccccc}
\hline \hline Statistic & $\mathrm{N}$ & Mean & St. Dev. & Min & Pctl(25) & Median & Pctl(75) & Max \\
\hline shooting.northeastern & 232 & 0.970 & 1.145 & 0 & 0 & 1 & 1 & 6 \\
\hline
\end{tabular}

Table 38.2: Descriptives for Outcome After Ferguson Protests Begin

\begin{tabular}{lcccccccc}
\hline \hline Statistic & $\mathrm{N}$ & Mean & St. Dev. & Min & Pctl(25) & Median & Pctl(75) & Max \\
\hline shooting.northeastern & 290 & 1.414 & 1.498 & 0 & 0 & 1 & 2 & 7 \\
\hline
\end{tabular}




\subsection{Four Models for shooting.northeastern}

Table 38.3: Four Models that Differ on the Specification of Adjustment and Intervention Variables

\begin{tabular}{|c|c|c|c|c|}
\hline & \multicolumn{4}{|c|}{ Outcome: Count Per Week } \\
\hline & (1) & (2) & (3) & $(4)$ \\
\hline Time (counter in weeks) & -0.001 & -0.001 & & -0.001 \\
\hline After Ferguson Protests Begin (week of $8 / 11 / 14$ onward) & -0.09 & & 0.01 & 0.03 \\
\hline After Gray Protests Begin (week of 4/20/15 onward) & 1.03 & & 0.79 & 0.70 \\
\hline Unrest and National Guard (4/27/15 - 5/3/2015) & 1.18 & & 1.54 & 1.65 \\
\hline After Batts Fired (week of 7/13/15 onward) & -0.60 & & -0.39 & -0.51 \\
\hline After Davis Confirmed (week of 10/19/15 onward) & 0.23 & & 0.36 & 0.46 \\
\hline After Davis First Year (week of 10/17/16 onward) & 0.06 & & -0.02 & -0.01 \\
\hline After De Sousa Begins (week of 1/19/18 onward) & -0.18 & & -0.10 & 0.03 \\
\hline After Tuggle Begins (week of 5/14/18 onward) & 0.15 & & -0.004 & -0.08 \\
\hline After Harrison Begins (week of 2/11/19 onward) & 0.41 & & 0.42 & 0.46 \\
\hline Average Maximum Temperature to 50 Degrees & & -0.02 & & -0.03 \\
\hline Plus Degrees in the 50s & & -0.03 & & 0.01 \\
\hline Plus Degrees in the 60s & & 0.07 & & 0.02 \\
\hline Plus Degrees in the 70s & & 0.06 & & 0.08 \\
\hline Plus Degrees Greater Than 80 & & -0.13 & & -0.13 \\
\hline Snowfall (inches) & & -0.35 & & -0.59 \\
\hline Precipitation (inches) & & -0.20 & & -0.26 \\
\hline Darkness Before Midnight (hours) & & -0.13 & & -0.05 \\
\hline Thanksgiving/Christmas (proportion of week) & & 4.20 & & 1.30 \\
\hline Winter Holiday (proportion of week) & & -2.26 & & -0.89 \\
\hline Other Out-of-School Days (proportion of week) & & 0.49 & & 0.60 \\
\hline Summer Vacation (proportion of week) & & -0.20 & & 0.16 \\
\hline Summer Holiday (proportion of week) & & -2.69 & & -2.14 \\
\hline Observations & 522 & 232 & 522 & 522 \\
\hline $\mathrm{R}^{2}$ & 0.05 & 0.09 & 0.09 & 0.12 \\
\hline
\end{tabular}


38.3 Least Squares Updated Model (2) for shooting.northeastern shooting.northeastern

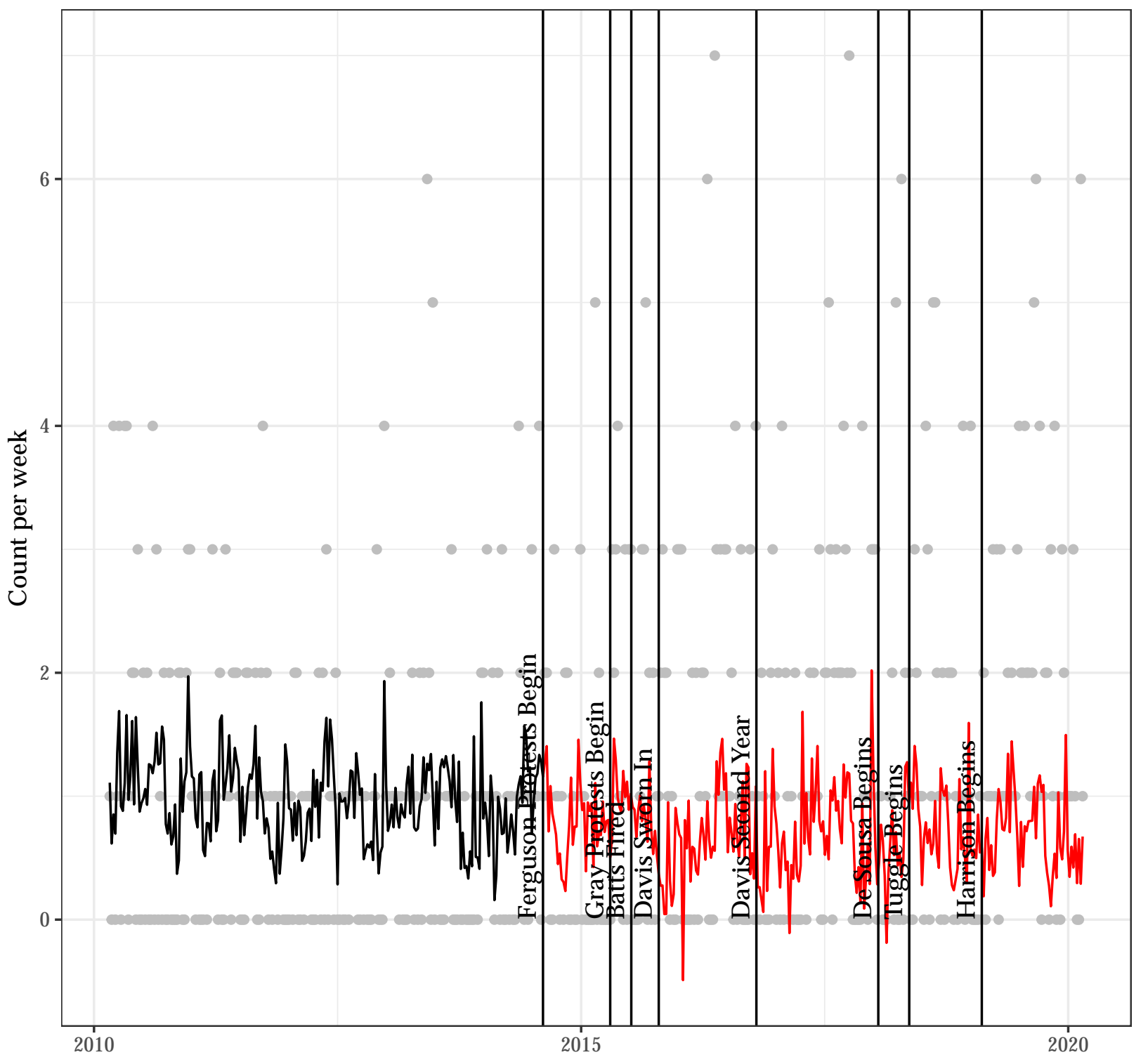




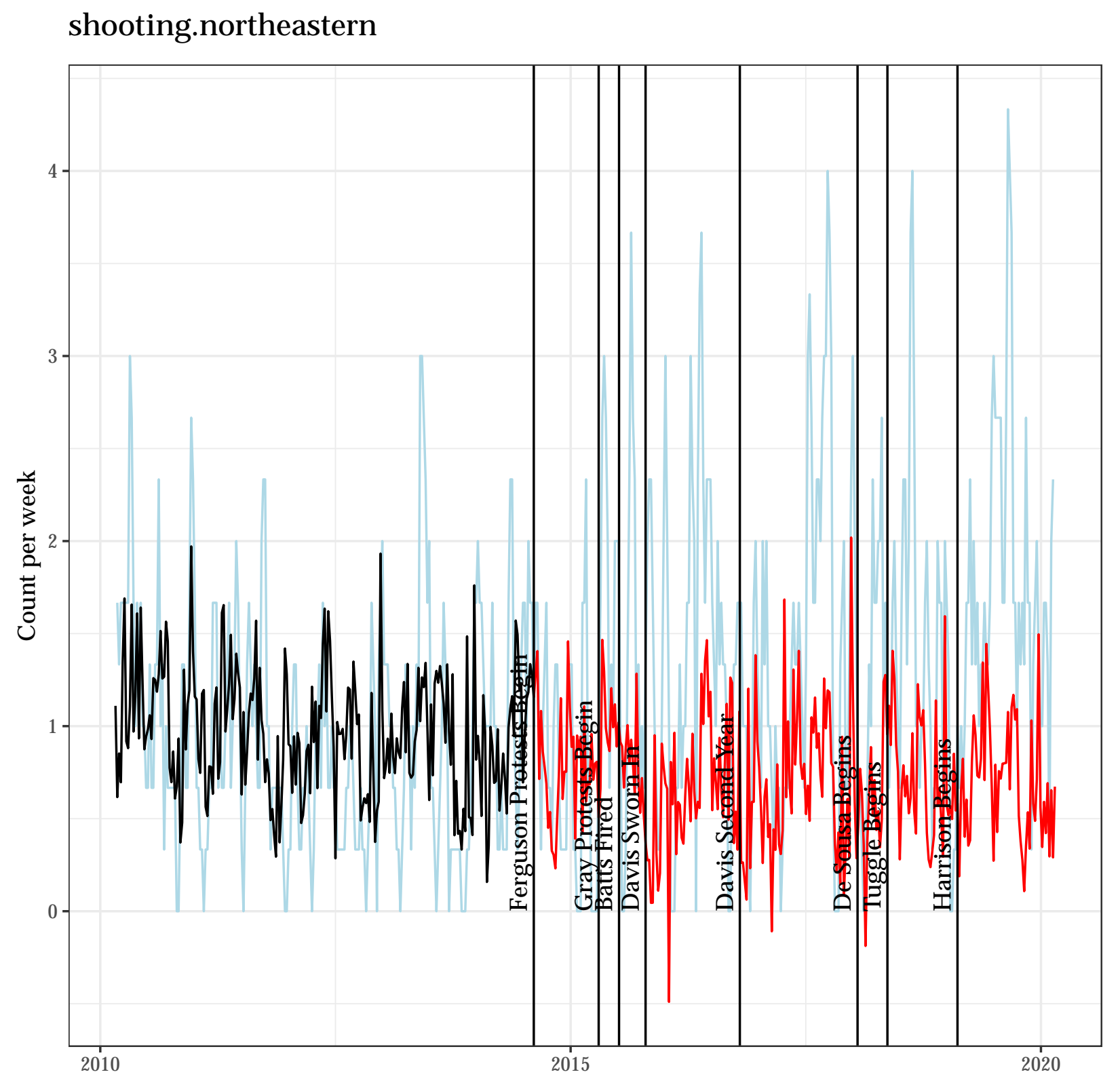


Autocorrelation Function for the Observed Outcome for Updated Model (2)

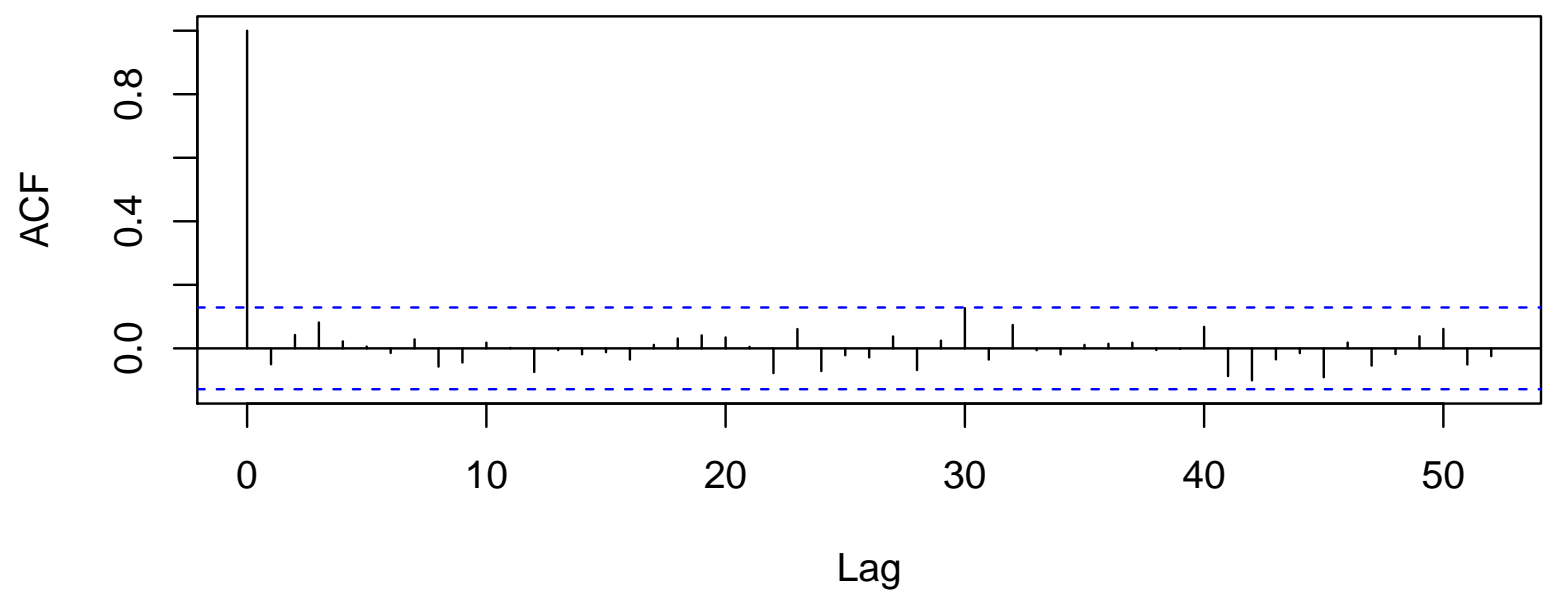

Autocorrelation Function for the Residuals from Updated Model (2)

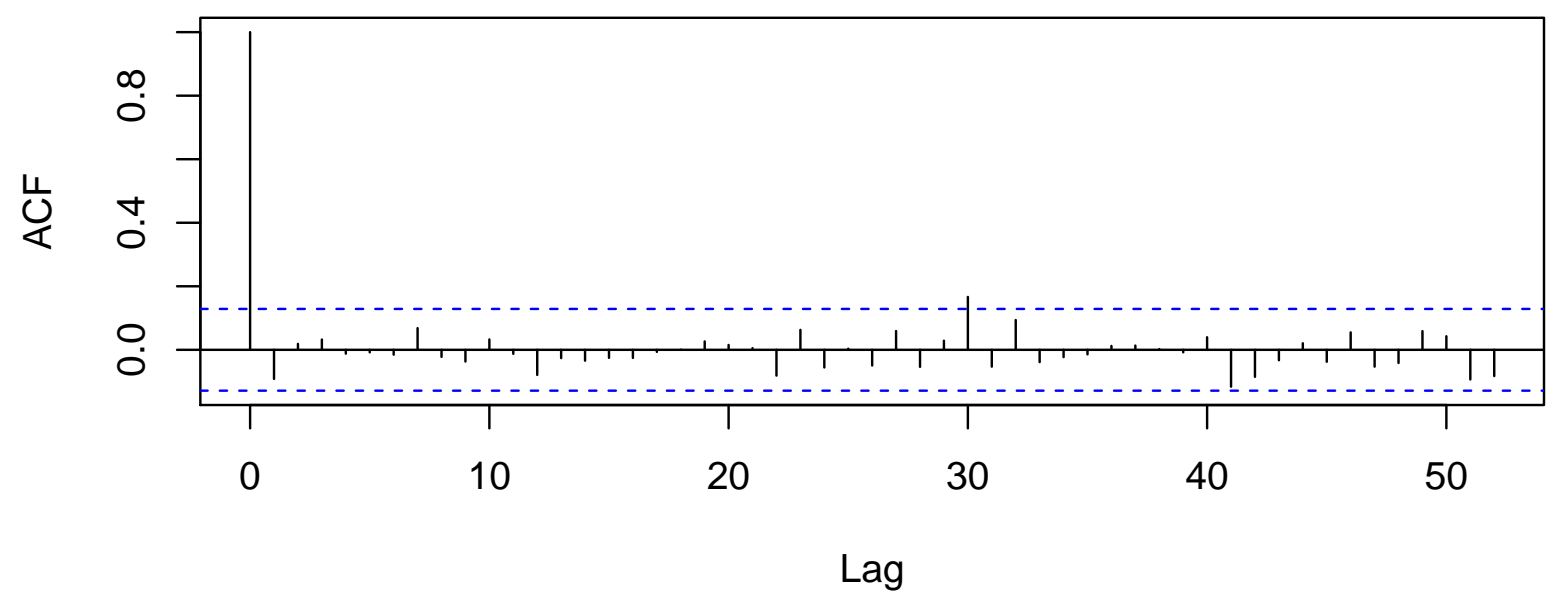




\subsection{A Poisson Regression Model as an Alternative to Model (2) for shooting.northeastern}

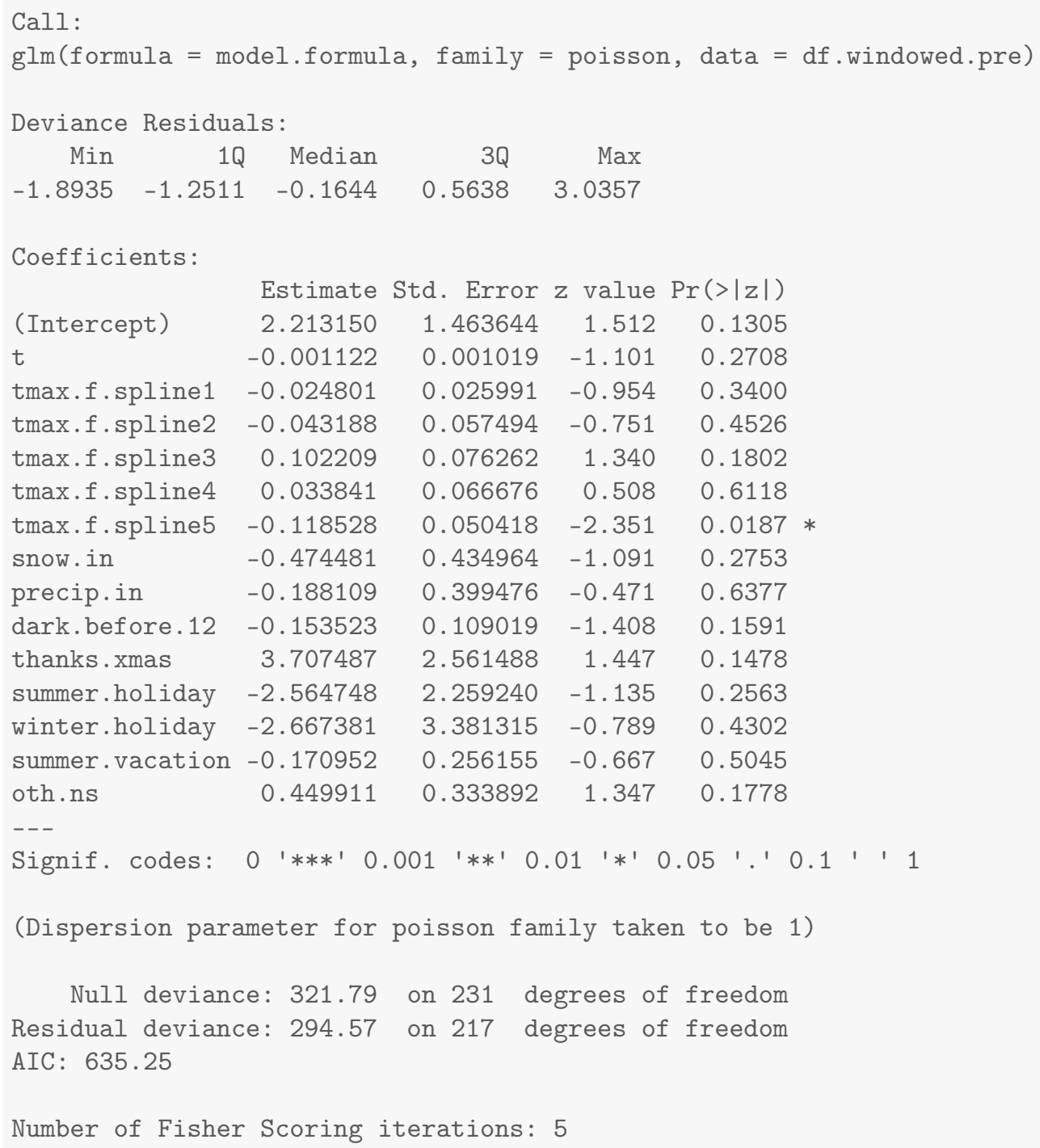




\section{Results for shooting.western}

\subsection{Summary Values for shooting.western}

Table 39.1: Descriptives for Outcome Before Ferguson Protests Begin

\begin{tabular}{lcccccccc}
\hline \hline Statistic & $\mathrm{N}$ & Mean & St. Dev. & Min & $\operatorname{Pctl}(25)$ & Median & Pctl(75) & Max \\
\hline shooting.western & 232 & 1.241 & 1.378 & 0 & 0 & 1 & 2 & 7 \\
\hline
\end{tabular}

Table 39.2: Descriptives for Outcome After Ferguson Protests Begin

\begin{tabular}{lcccccccc}
\hline \hline Statistic & $\mathrm{N}$ & Mean & St. Dev. & Min & $\operatorname{Pctl}(25)$ & Median & Pctl(75) & Max \\
\hline shooting.western & 290 & 2.134 & 1.864 & 0 & 1 & 2 & 3 & 10 \\
\hline
\end{tabular}




\subsection{Four Models for shooting.western}

Table 39.3: Four Models that Differ on the Specification of Adjustment and Intervention Variables

\begin{tabular}{|c|c|c|c|c|}
\hline & \multicolumn{4}{|c|}{ Outcome: Count Per Week } \\
\hline & $(1)$ & (2) & (3) & $(4)$ \\
\hline Time (counter in weeks) & -0.001 & -0.001 & & -0.001 \\
\hline After Ferguson Protests Begin (week of $8 / 11 / 14$ onward) & 0.28 & & 0.46 & 0.45 \\
\hline After Gray Protests Begin (week of $4 / 20 / 15$ onward) & 3.60 & & 3.06 & 3.15 \\
\hline Unrest and National Guard $(4 / 27 / 15-5 / 3 / 2015)$ & -3.00 & & -2.58 & -2.81 \\
\hline After Batts Fired (week of 7/13/15 onward) & -3.20 & & -2.87 & -2.95 \\
\hline After Davis Confirmed (week of 10/19/15 onward) & 0.45 & & 0.59 & 0.54 \\
\hline After Davis First Year (week of 10/17/16 onward) & 0.09 & & 0.08 & 0.14 \\
\hline After De Sousa Begins (week of 1/19/18 onward) & -0.41 & & -0.29 & -0.40 \\
\hline After Tuggle Begins (week of $5 / 14 / 18$ onward) & 0.18 & & 0.05 & 0.23 \\
\hline After Harrison Begins (week of 2/11/19 onward) & 0.17 & & 0.15 & 0.16 \\
\hline Average Maximum Temperature to 50 Degrees & & -0.03 & & 0.002 \\
\hline Plus Degrees in the $50 \mathrm{~s}$ & & 0.07 & & 0.02 \\
\hline Plus Degrees in the 60s & & -0.07 & & -0.05 \\
\hline Plus Degrees in the 70s & & 0.06 & & 0.05 \\
\hline Plus Degrees Greater Than 80 & & -0.14 & & -0.09 \\
\hline Snowfall (inches) & & -0.12 & & 0.18 \\
\hline Precipitation (inches) & & -0.31 & & -0.32 \\
\hline Darkness Before Midnight (hours) & & -0.10 & & -0.18 \\
\hline Thanksgiving/Christmas (proportion of week) & & 4.45 & & 4.37 \\
\hline Winter Holiday (proportion of week) & & -2.30 & & -2.49 \\
\hline Other Out-of-School Days (proportion of week) & & -0.24 & & 0.07 \\
\hline Summer Vacation (proportion of week) & & 0.72 & & 0.36 \\
\hline Summer Holiday (proportion of week) & & 5.46 & & 0.70 \\
\hline Observations & 522 & 232 & 522 & 522 \\
\hline $\mathrm{R}^{2}$ & 0.14 & 0.10 & 0.17 & 0.17 \\
\hline
\end{tabular}


39.3 Least Squares Updated Model (2) for shooting.western shooting.western

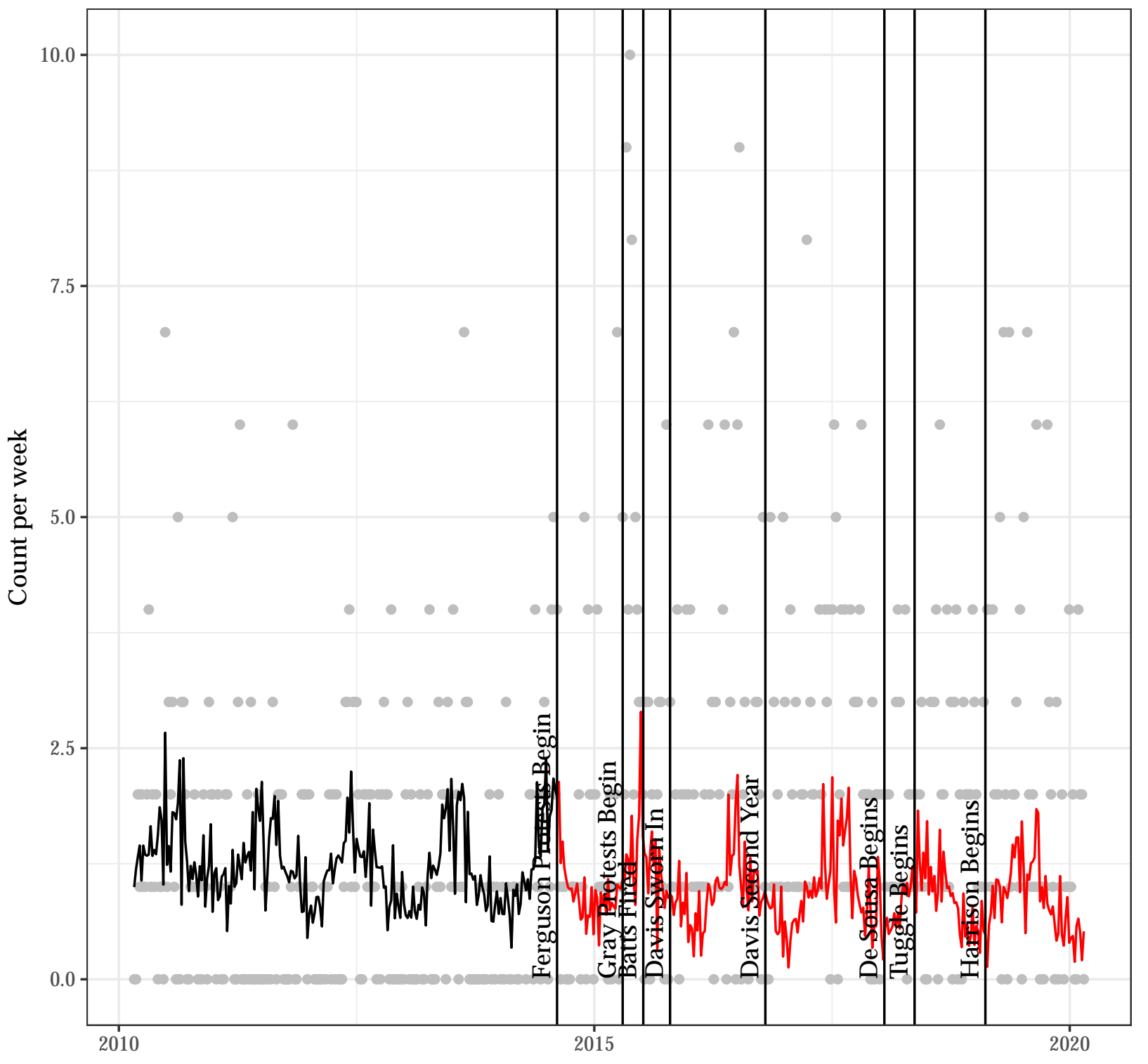




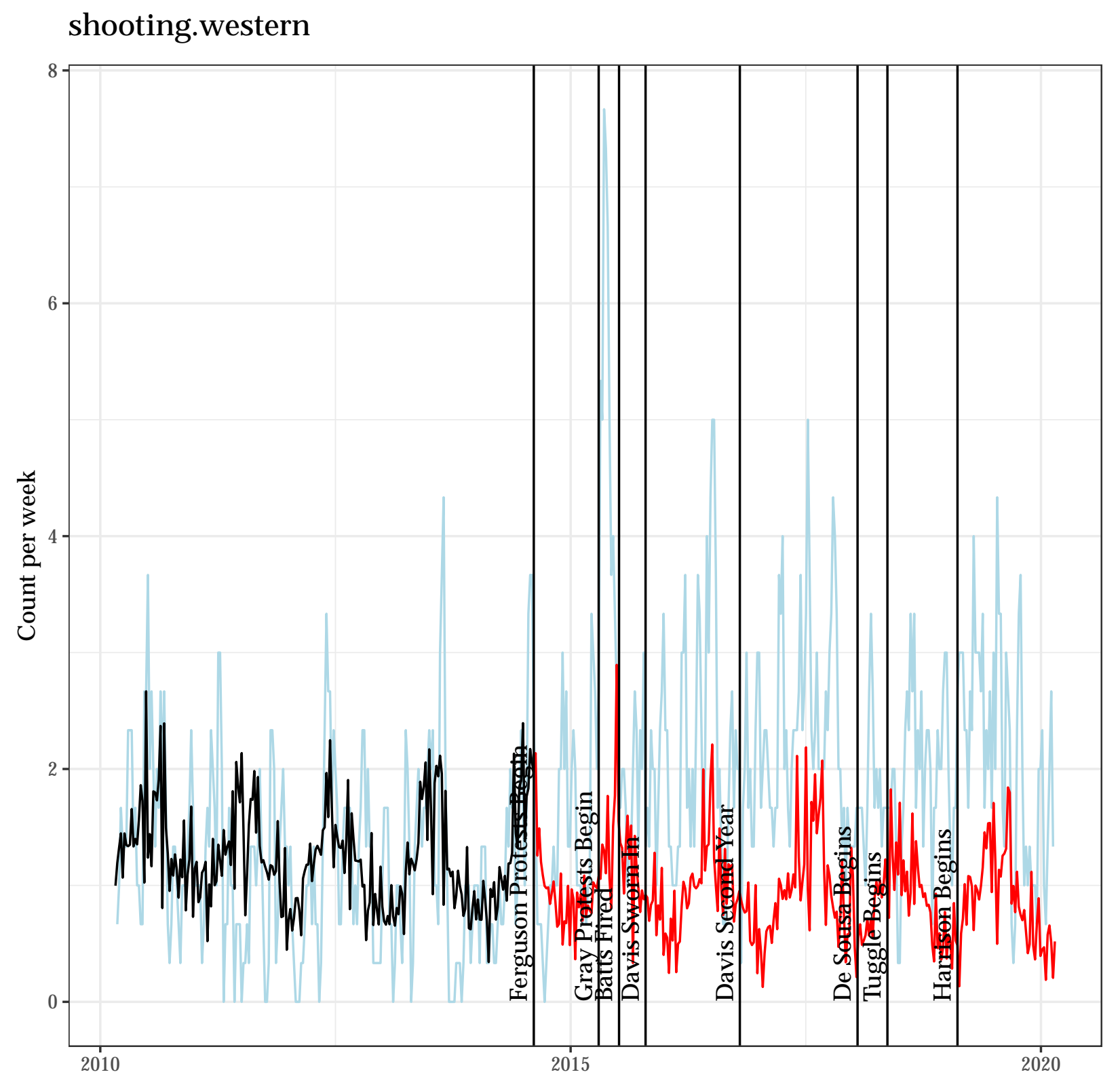


Autocorrelation Function for the Observed Outcome for Updated Model (2)

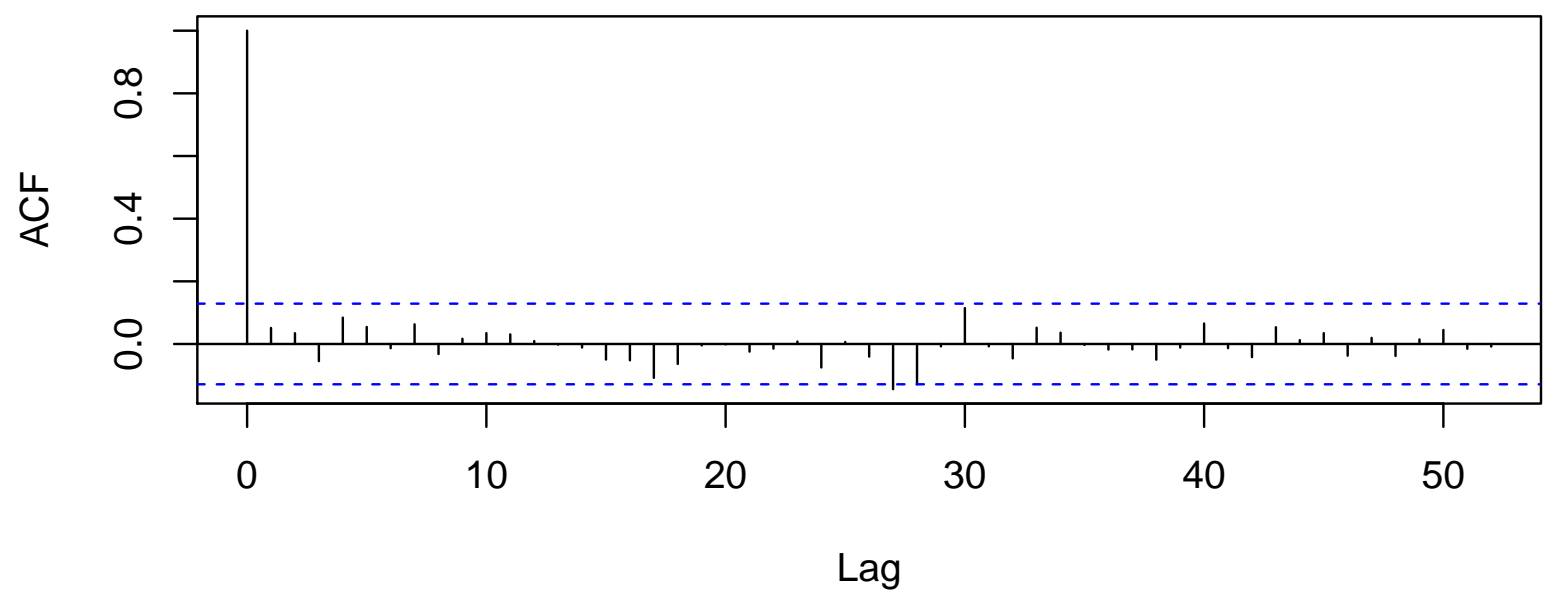

Autocorrelation Function for the Residuals from Updated Model (2)

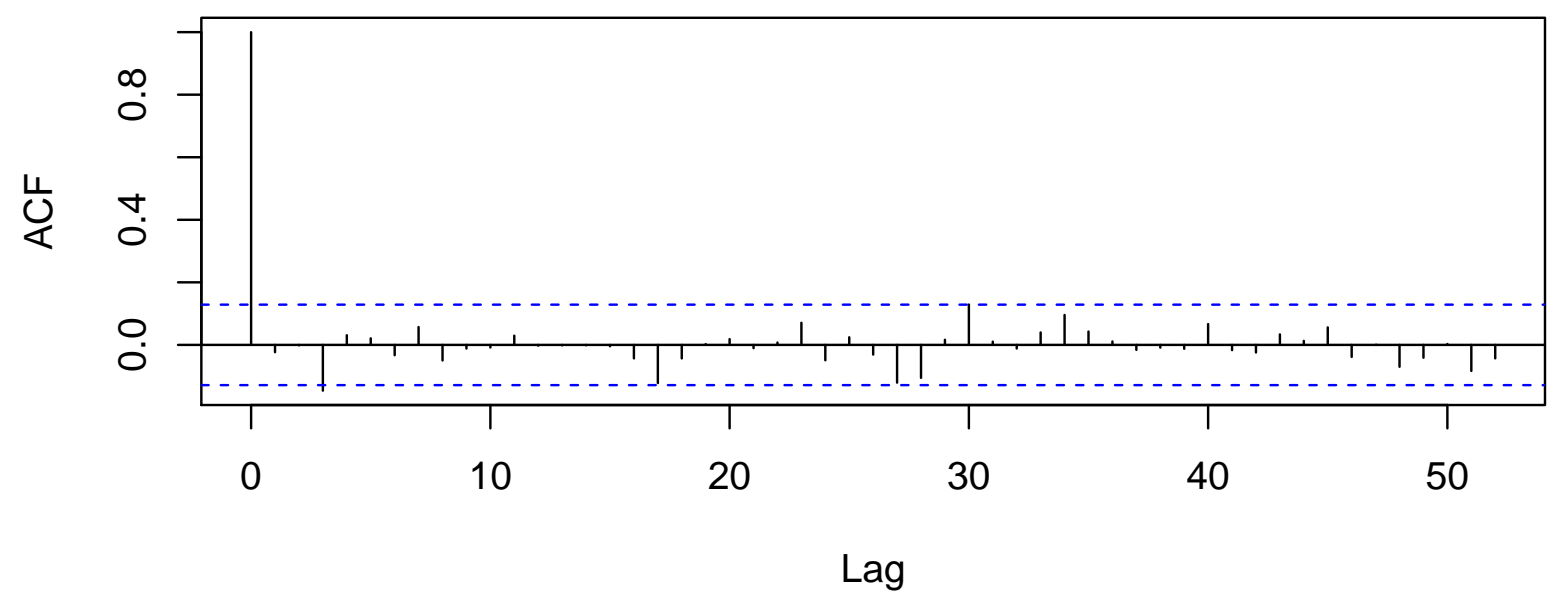




\subsection{A Poisson Regression Model as an Alternative to Model (2) for shooting.western}

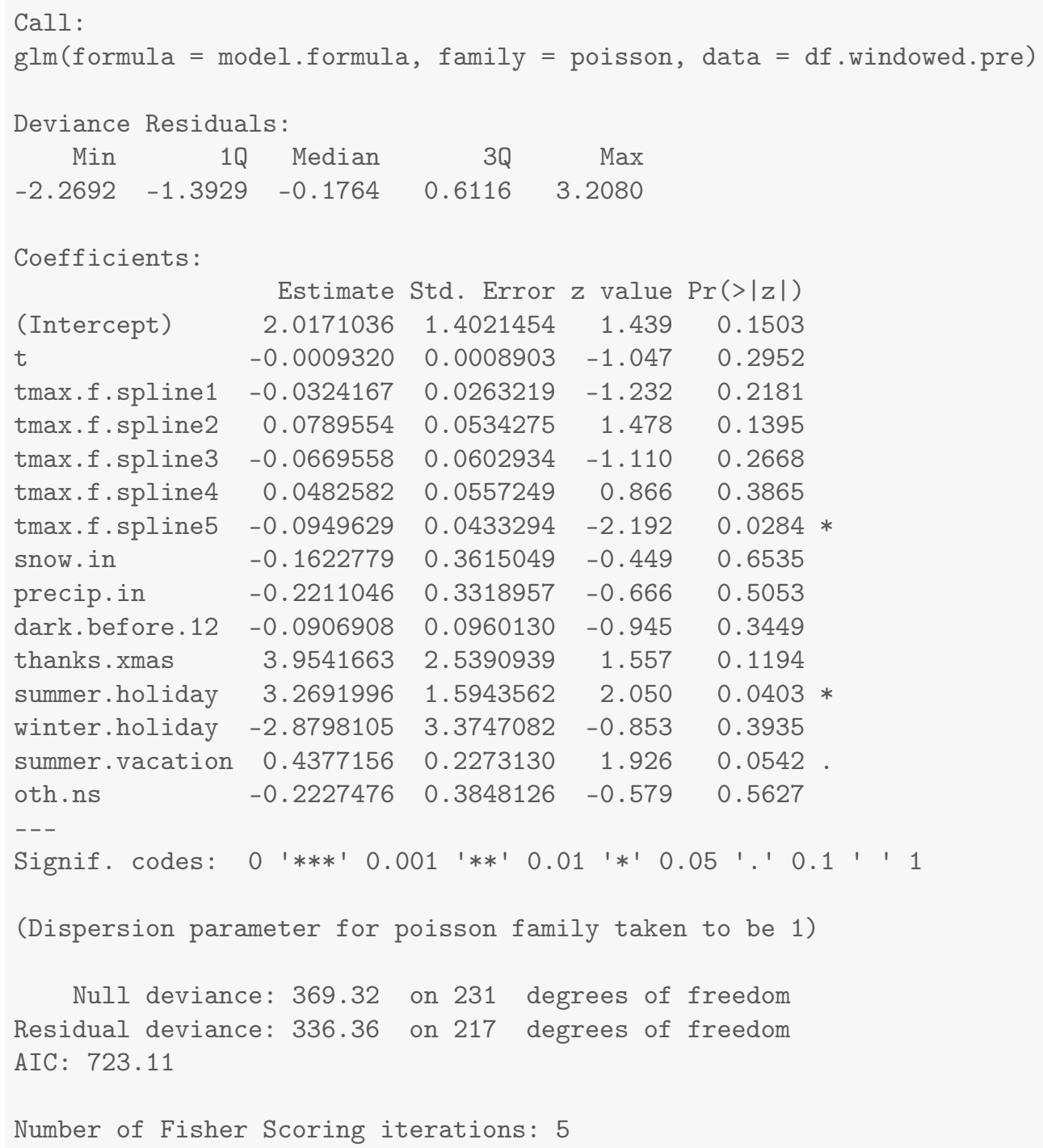




\section{Results for shooting.central}

\subsection{Summary Values for shooting.central}

Table 40.1: Descriptives for Outcome Before Ferguson Protests Begin

\begin{tabular}{lcccccccc}
\hline \hline Statistic & $\mathrm{N}$ & Mean & St. Dev. & Min & Pctl(25) & Median & Pctl(75) & Max \\
\hline shooting.central & 232 & 0.552 & 0.910 & 0 & 0 & 0 & 1 & 5 \\
\hline
\end{tabular}

Table 40.2: Descriptives for Outcome After Ferguson Protests Begin

\begin{tabular}{lcccccccc}
\hline \hline Statistic & $\mathrm{N}$ & Mean & St. Dev. & Min & $\operatorname{Pctl}(25)$ & Median & $\operatorname{Pctl}(75)$ & Max \\
\hline shooting.central & 290 & 0.914 & 1.309 & 0 & 0 & 0 & 1 & 10 \\
\hline
\end{tabular}




\subsection{Four Models for shooting.central}

Table 40.3: Four Models that Differ on the Specification of Adjustment and Intervention Variables

\begin{tabular}{lcccc}
\hline \hline & \multicolumn{3}{c}{ Outcome: Count Per Week } \\
\cline { 2 - 5 } & $(1)$ & $(2)$ & $(3)$ & $(4)$ \\
\hline Time (counter in weeks) & -0.001 & -0.0004 & -0.001 \\
After Ferguson Protests Begin (week of 8/11/14 onward) & -0.25 & & -0.26 & -0.16 \\
After Gray Protests Begin (week of 4/20/15 onward) & 0.83 & & 0.91 & 0.69 \\
Unrest and National Guard (4/27/15 - 5/3/2015) & -1.00 & & -0.97 & -0.83 \\
After Batts Fired (week of 7/13/15 onward) & -0.49 & & -0.86 & -0.72 \\
After Davis Confirmed (week of 10/19/15 onward) & 0.41 & & 0.57 & 0.67 \\
After Davis First Year (week of 10/17/16 onward) & 0.07 & & 0.06 & 0.06 \\
After De Sousa Begins (week of 1/19/18 onward) & -0.25 & & -0.08 & -0.04 \\
After Tuggle Begins (week of 5/14/18 onward) & 0.50 & & 0.42 & 0.22 \\
After Harrison Begins (week of 2/11/19 onward) & 0.41 & & 0.46 \\
Average Maximum Temperature to 50 Degrees & & 0.01 & 0.01 \\
Plus Degrees in the 50s & & 0.02 & -0.02 \\
Plus Degrees in the 60s & & -0.01 & & 0.05 \\
Plus Degrees in the 70s & & 0.10 & & -0.04 \\
Plus Degrees Greater Than 80 & & 0.43 & & 0.01 \\
Snowfall (inches) & & -0.01 & & -0.31 \\
Precipitation (inches) & & 0.25 & & 0.12 \\
Darkness Before Midnight (hours) & -3.72 & & -2.43 \\
Thanksgiving/Christmas (proportion of week) & & -0.15 & & 0.28 \\
Winter Holiday (proportion of week) & & -0.19 & & -0.04 \\
Other Out-of-School Days (proportion of week) & & 0.71 & & 0.30 \\
Summer Vacation (proportion of week) & 4.66 & & 0.65 \\
Summer Holiday (proportion of week) & & 232 & 522 & 522 \\
Observations & & & & 0.13 \\
$\mathrm{R}^{2}$ & & & \\
\hline \hline
\end{tabular}


40.3 Least Squares Updated Model (2) for shooting.central shooting.central

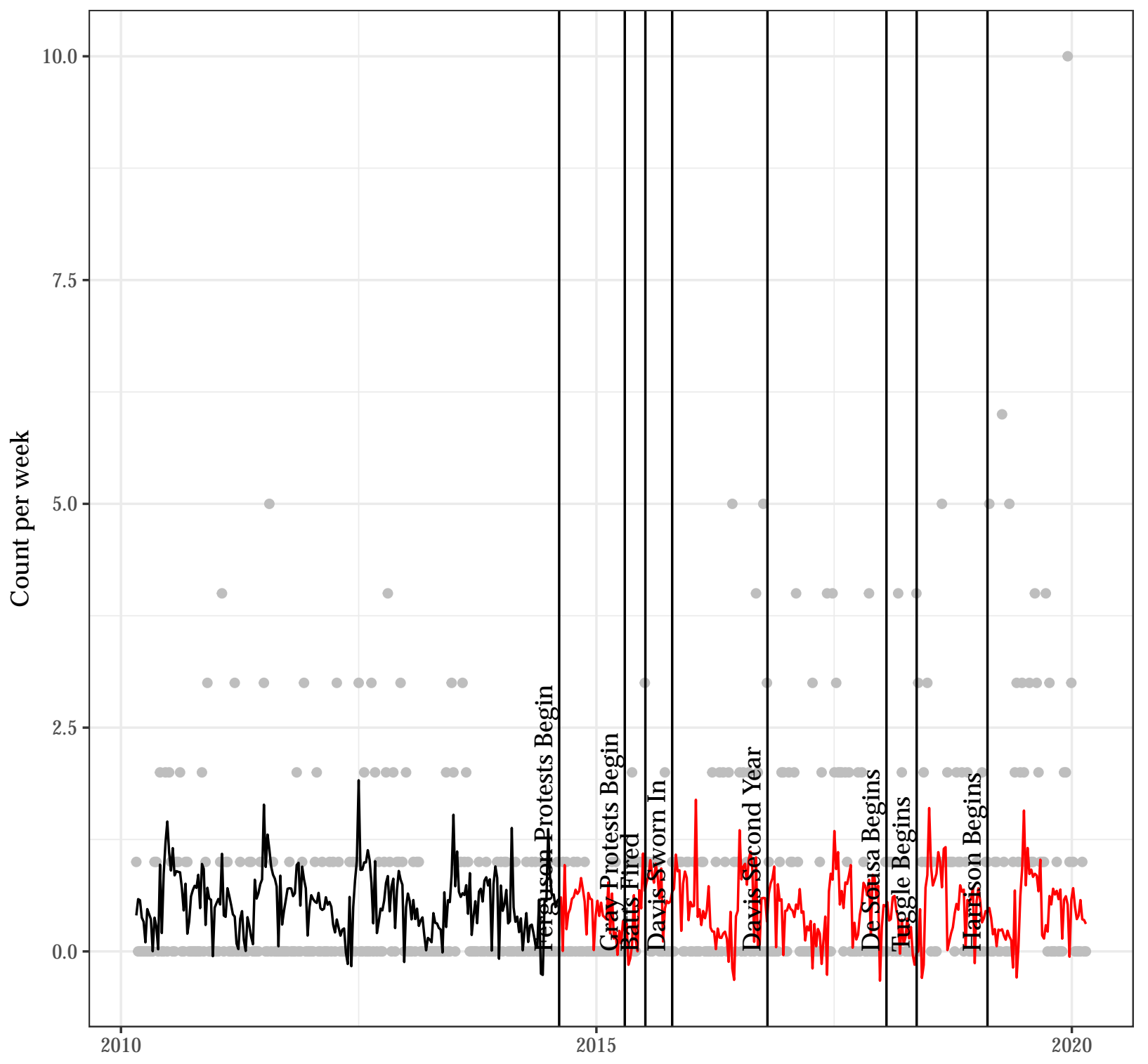




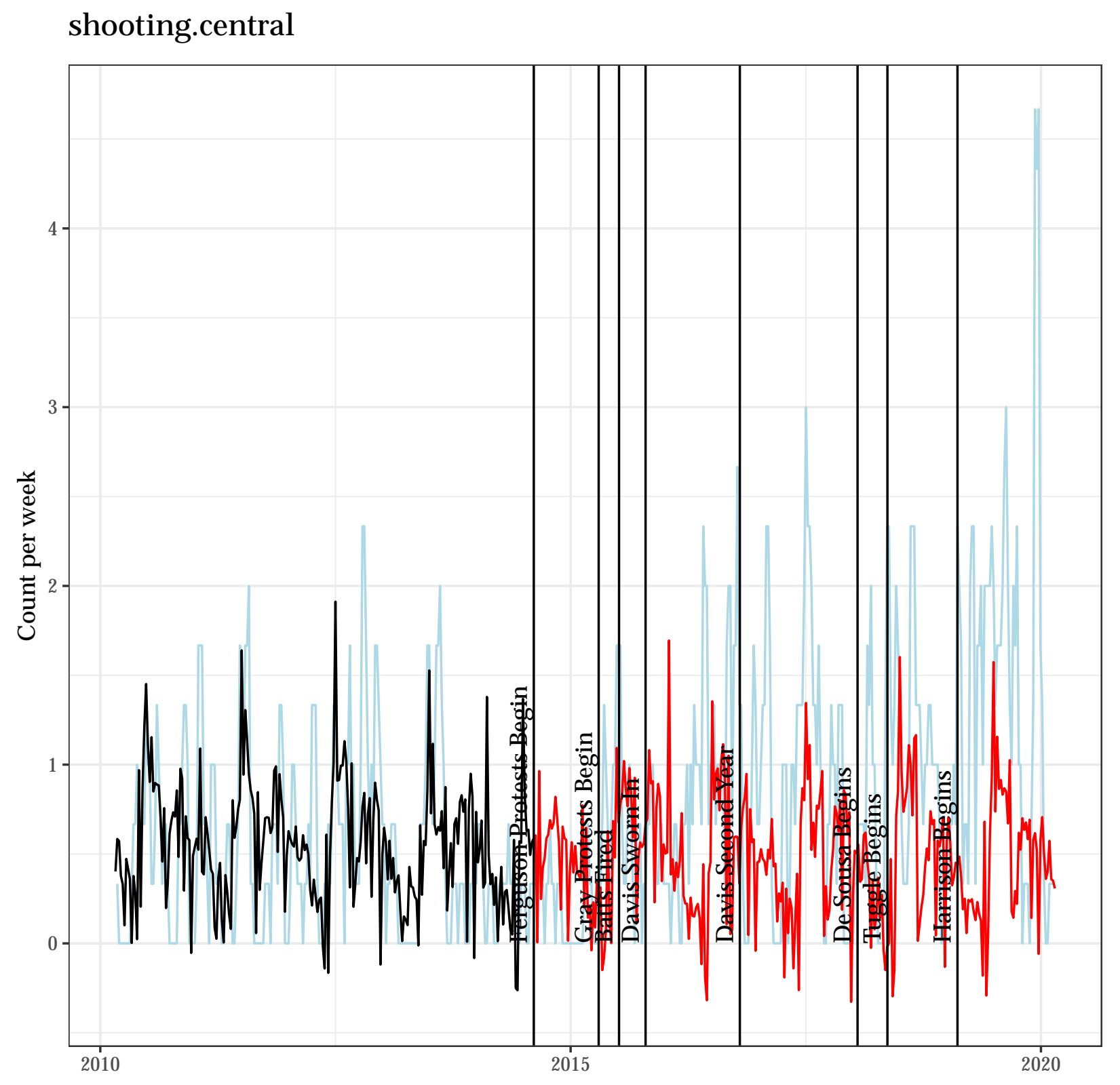


Autocorrelation Function for the Observed Outcome for Updated Model (2)

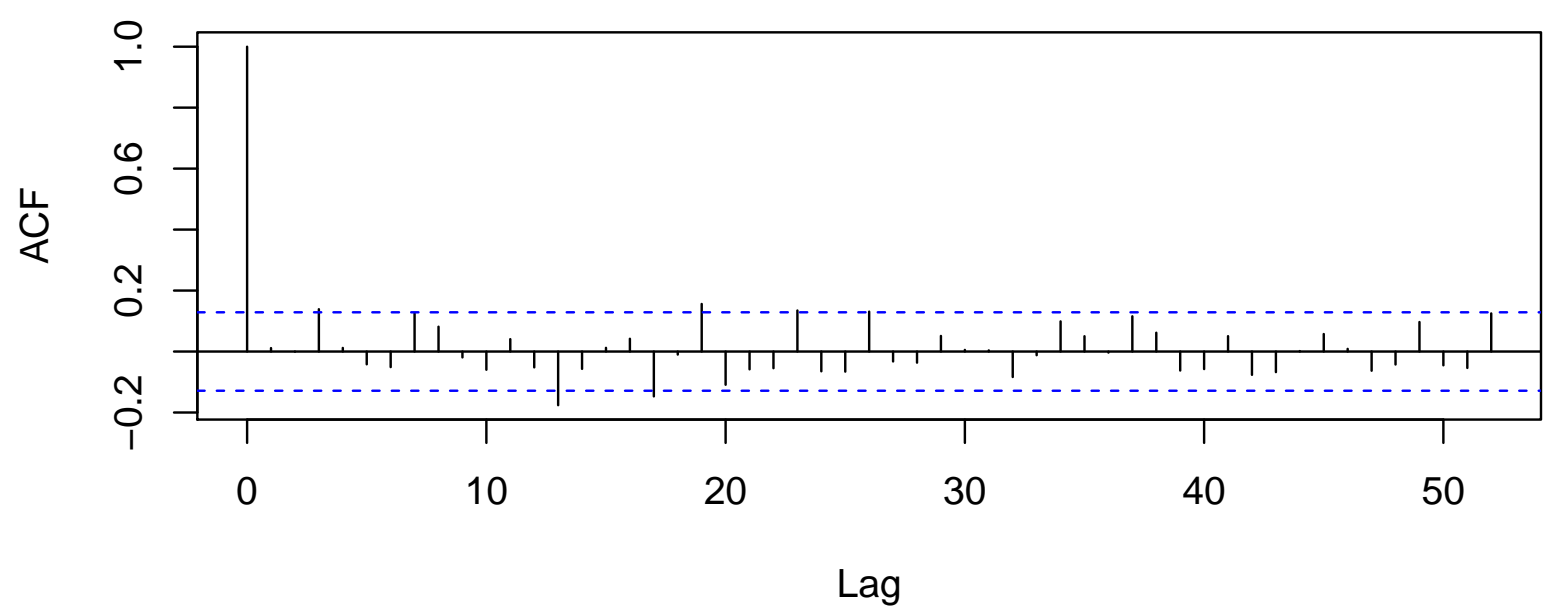

Autocorrelation Function for the Residuals from Updated Model (2)

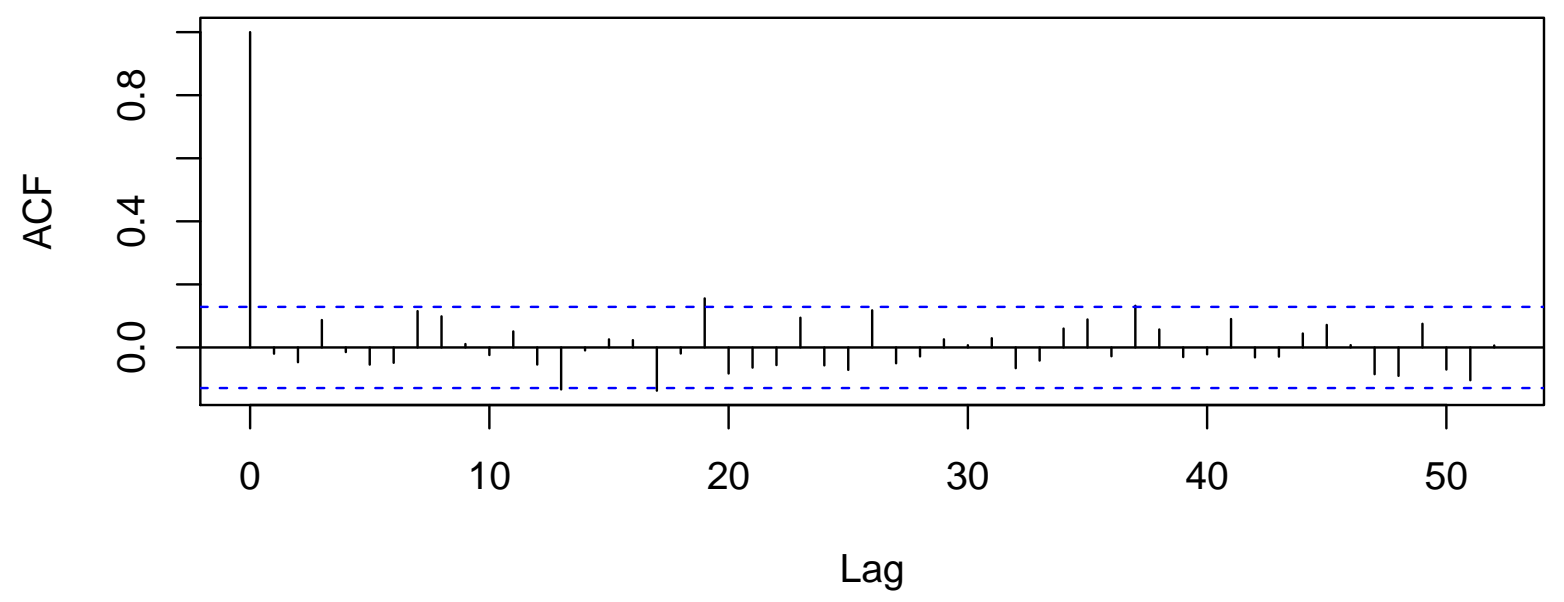




\subsection{A Poisson Regression Model as an Alternative to Model (2) for shooting.central}

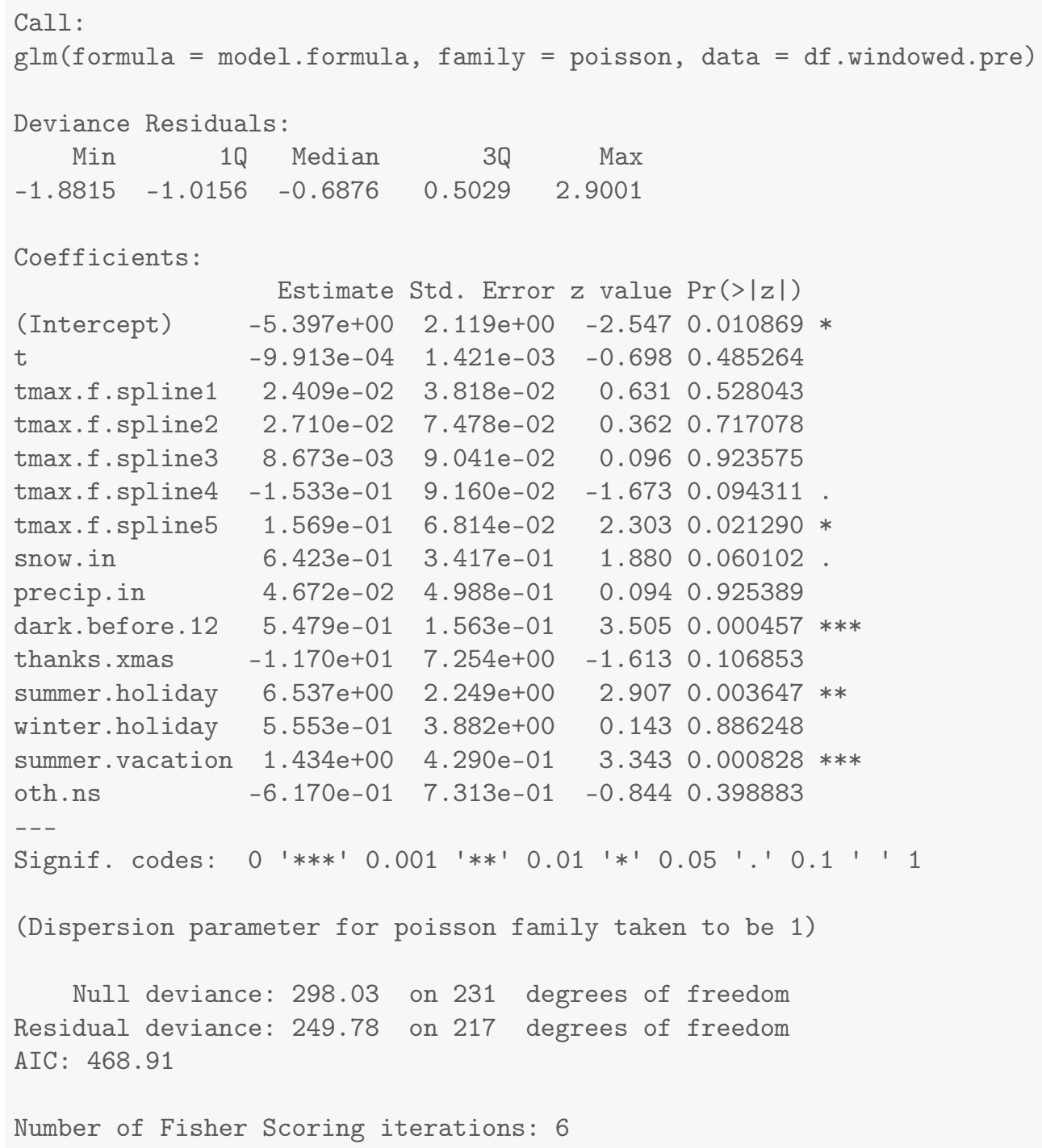




\section{Results for shooting.eastern}

\subsection{Summary Values for shooting.eastern}

Table 41.1: Descriptives for Outcome Before Ferguson Protests Begin

\begin{tabular}{lcccccccc}
\hline \hline Statistic & $\mathrm{N}$ & Mean & St. Dev. & Min & $\operatorname{Pctl}(25)$ & Median & $\operatorname{Pctl}(75)$ & Max \\
\hline shooting.eastern & 232 & 1.181 & 1.393 & 0 & 0 & 1 & 2 & 8 \\
\hline
\end{tabular}

Table 41.2: Descriptives for Outcome After Ferguson Protests Begin

\begin{tabular}{lcccccccc}
\hline \hline Statistic & $\mathrm{N}$ & Mean & St. Dev. & Min & Pctl(25) & Median & Pctl(75) & Max \\
\hline shooting.eastern & 290 & 1.914 & 1.934 & 0 & 0.2 & 1.5 & 3 & 11 \\
\hline
\end{tabular}




\subsection{Four Models for shooting.eastern}

Table 41.3: Four Models that Differ on the Specification of Adjustment and Intervention Variables

\begin{tabular}{|c|c|c|c|c|}
\hline & \multicolumn{4}{|c|}{ Outcome: Count Per Week } \\
\hline & $(1)$ & (2) & $(3)$ & (4) \\
\hline Time (counter in weeks) & -0.0005 & -0.001 & & -0.0004 \\
\hline After Ferguson Protests Begin (week of $8 / 11 / 14$ onward) & -0.26 & & 0.01 & -0.02 \\
\hline After Gray Protests Begin (week of 4/20/15 onward) & 3.70 & & 3.10 & 3.03 \\
\hline Unrest and National Guard $(4 / 27 / 15-5 / 3 / 2015)$ & -4.55 & & -3.98 & -4.24 \\
\hline After Batts Fired (week of $7 / 13 / 15$ onward) & -2.68 & & -2.31 & -2.53 \\
\hline After Davis Confirmed (week of $10 / 19 / 15$ onward) & 0.25 & & 0.43 & 0.54 \\
\hline After Davis First Year (week of 10/17/16 onward) & -0.51 & & -0.53 & -0.53 \\
\hline After De Sousa Begins (week of 1/19/18 onward) & -0.04 & & 0.14 & 0.22 \\
\hline After Tuggle Begins (week of 5/14/18 onward) & 0.64 & & 0.44 & 0.43 \\
\hline After Harrison Begins (week of 2/11/19 onward) & 0.24 & & 0.26 & 0.22 \\
\hline Average Maximum Temperature to 50 Degrees & & -0.01 & & 0.01 \\
\hline Plus Degrees in the $50 \mathrm{~s}$ & & 0.04 & & 0.02 \\
\hline Plus Degrees in the 60s & & -0.03 & & 0.01 \\
\hline Plus Degrees in the $70 \mathrm{~s}$ & & 0.07 & & -0.01 \\
\hline Plus Degrees Greater Than 80 & & -0.18 & & -0.11 \\
\hline Snowfall (inches) & & -0.27 & & -0.26 \\
\hline Precipitation (inches) & & -0.04 & & -0.53 \\
\hline Darkness Before Midnight (hours) & & -0.07 & & -0.01 \\
\hline Thanksgiving/Christmas (proportion of week) & & 2.78 & & 0.30 \\
\hline Winter Holiday (proportion of week) & & -1.60 & & 1.88 \\
\hline Other Out-of-School Days (proportion of week) & & 0.56 & & 0.16 \\
\hline Summer Vacation (proportion of week) & & 0.50 & & 0.59 \\
\hline Summer Holiday (proportion of week) & & 5.47 & & 4.13 \\
\hline Observations & 522 & 232 & 522 & 522 \\
\hline $\mathrm{R}^{2}$ & 0.13 & 0.11 & 0.16 & 0.20 \\
\hline
\end{tabular}




\subsection{Least Squares Updated Model (2) for shooting.eastern shooting.eastern}

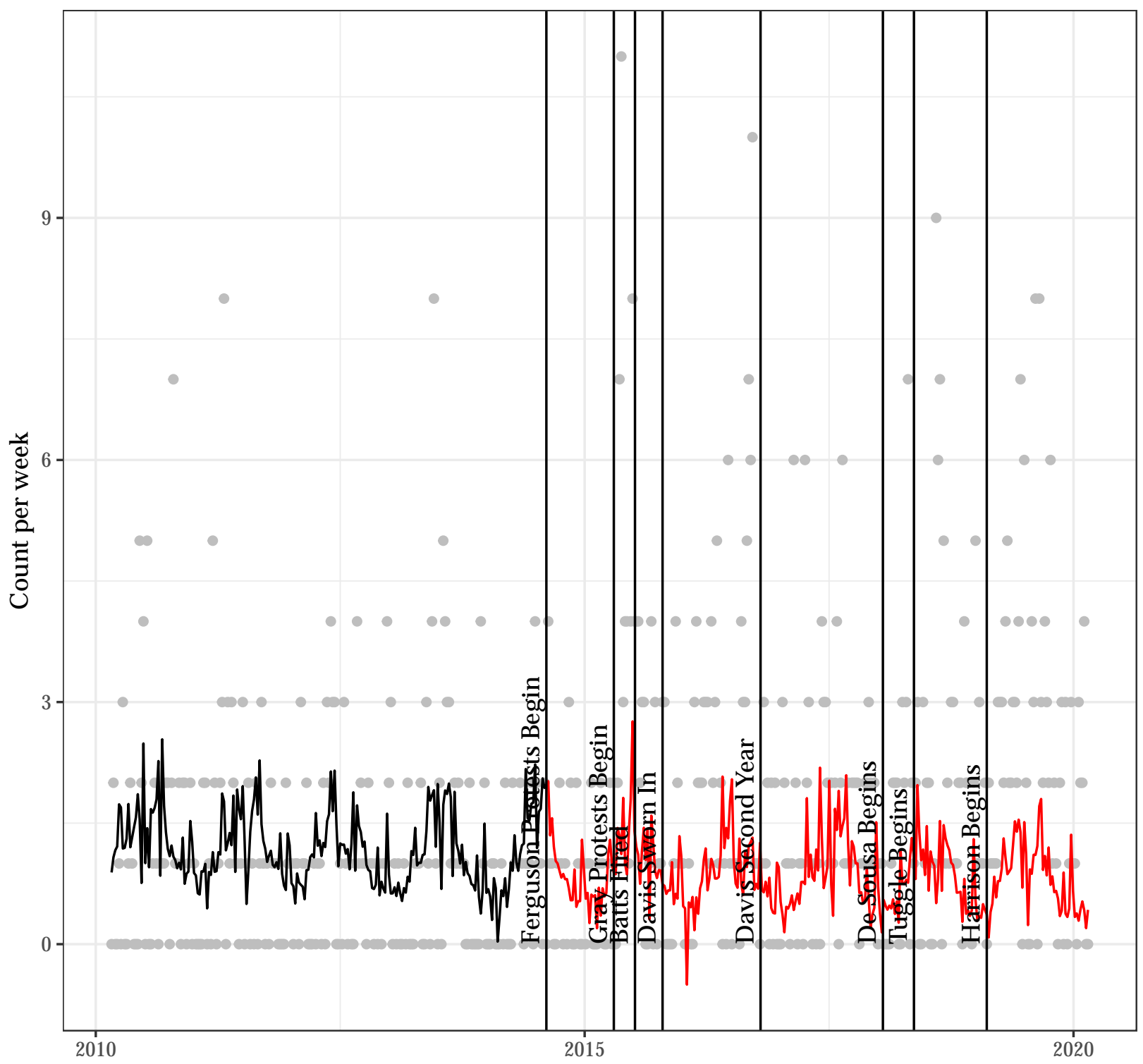




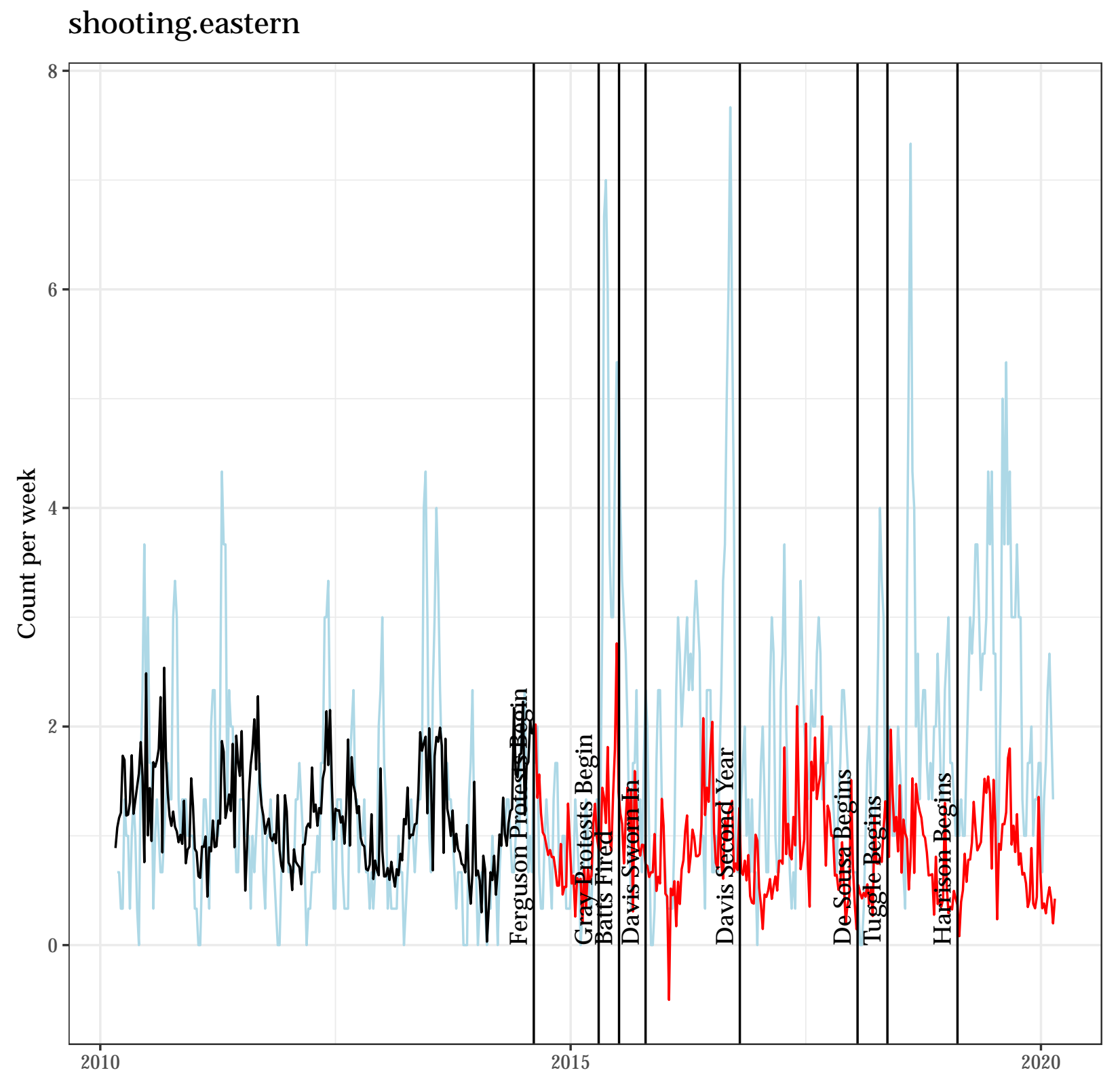


Autocorrelation Function for the Observed Outcome for Updated Model (2)

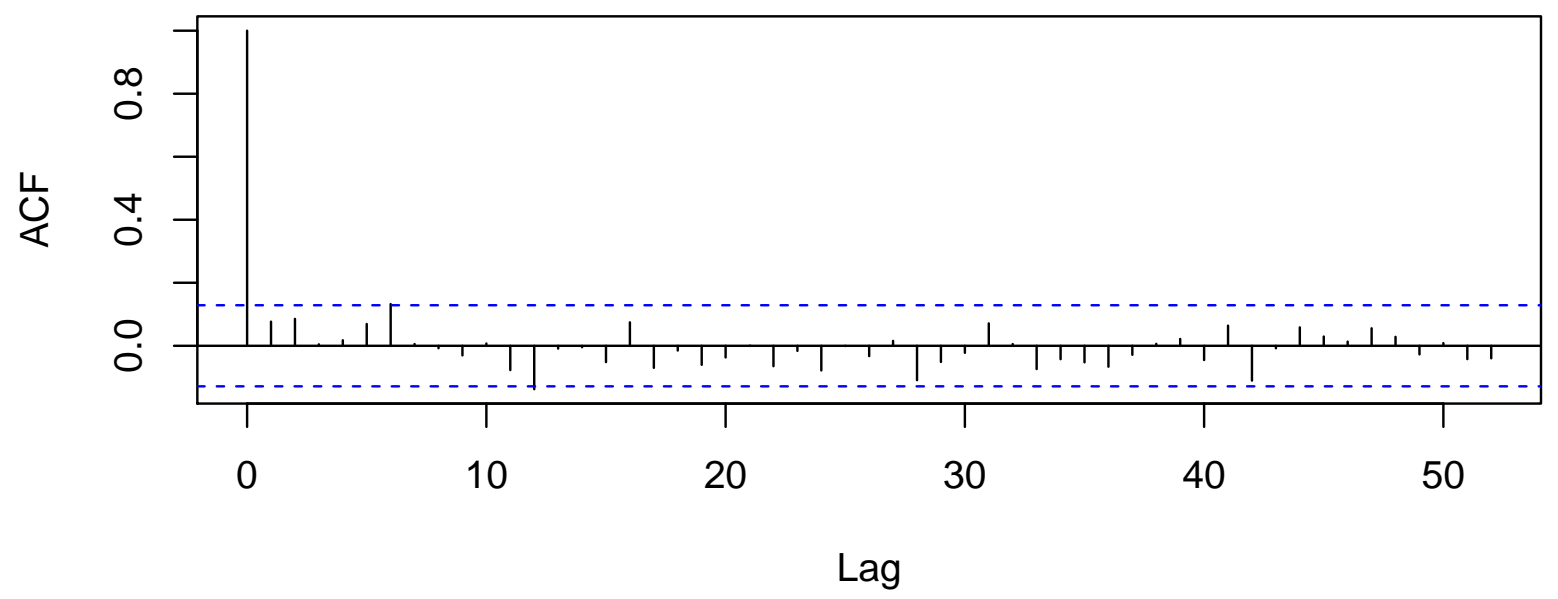

Autocorrelation Function for the Residuals from Updated Model (2)

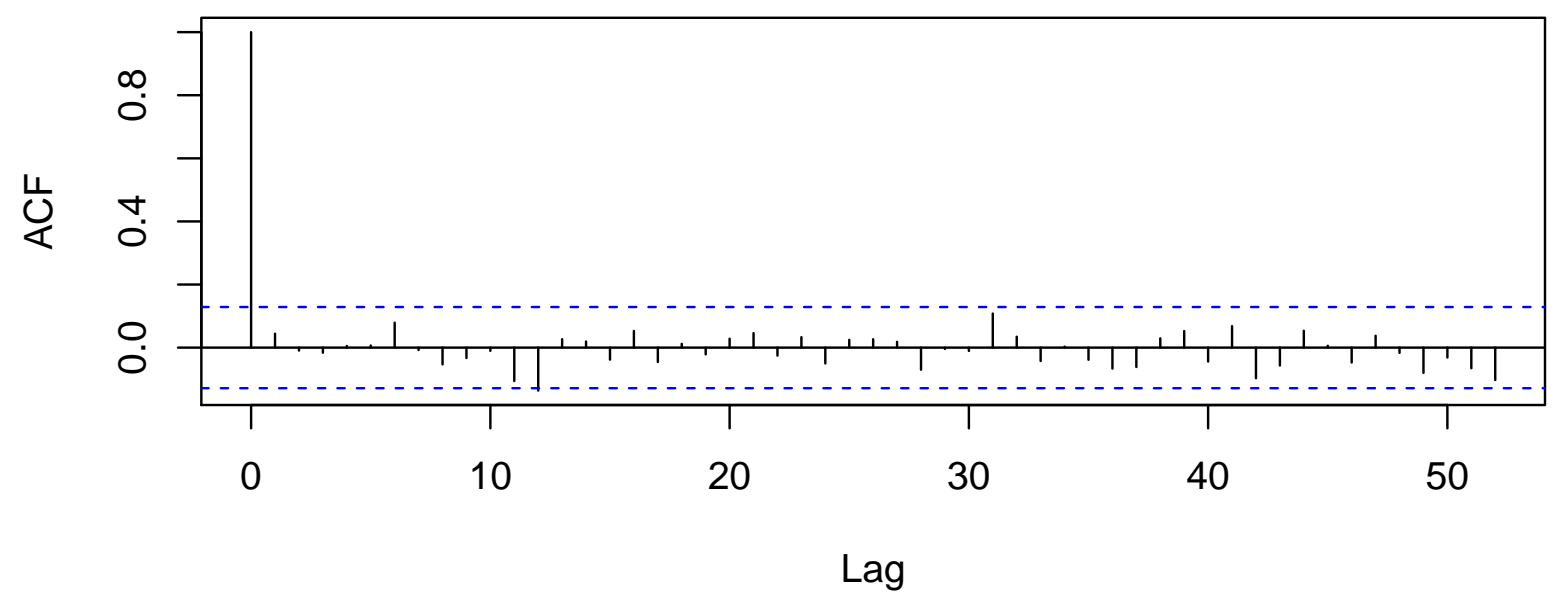




\subsection{A Poisson Regression Model as an Alternative to Model (2) for shooting.eastern}

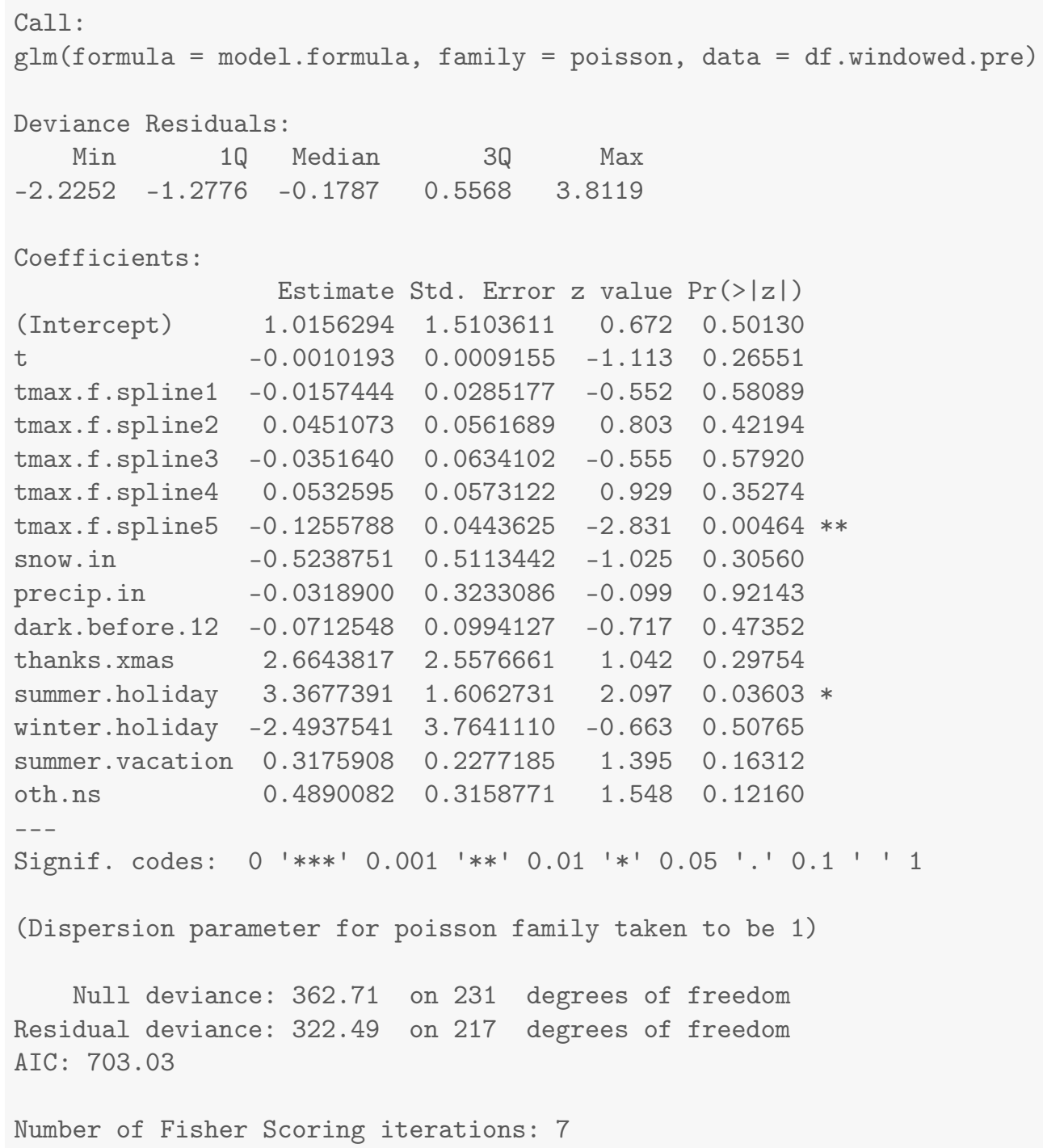




\section{Results for shooting.southwestern}

\subsection{Summary Values for shooting.southwestern}

Table 42.1: Descriptives for Outcome Before Ferguson Protests Begin

\begin{tabular}{lcccccccc}
\hline \hline Statistic & $\mathrm{N}$ & Mean & St. Dev. & Min & $\operatorname{Pctl}(25)$ & Median & Pctl(75) & Max \\
\hline shooting.southwestern & 232 & 0.948 & 1.123 & 0 & 0 & 1 & 1 & 6 \\
\hline
\end{tabular}

Table 42.2: Descriptives for Outcome After Ferguson Protests Begin

\begin{tabular}{lcccccccc}
\hline \hline Statistic & $\mathrm{N}$ & Mean & St. Dev. & Min & $\operatorname{Pctl}(25)$ & Median & Pctl(75) & Max \\
\hline shooting.southwestern & 290 & 1.752 & 1.680 & 0 & 1 & 1 & 2 & 8 \\
\hline
\end{tabular}




\subsection{Four Models for shooting.southwestern}

Table 42.3: Four Models that Differ on the Specification of Adjustment and Intervention Variables

\begin{tabular}{lcccc}
\hline \hline & \multicolumn{3}{c}{ Outcome: Count Per Week } \\
\cline { 2 - 5 } & $(1)$ & $(2)$ & $(3)$ & $(4)$ \\
\hline Time (counter in weeks) & 0.001 & 0.001 & & 0.001 \\
After Ferguson Protests Begin (week of 8/11/14 onward) & 0.06 & & 0.19 & 0.21 \\
After Gray Protests Begin (week of 4/20/15 onward) & 0.93 & & 0.66 & 0.53 \\
Unrest and National Guard (4/27/15 - 5/3/2015) & -0.09 & 0.26 & 0.28 \\
After Batts Fired (week of 7/13/15 onward) & 0.83 & 0.71 & 0.70 \\
After Davis Confirmed (week of 10/19/15 onward) & -0.98 & & -0.74 & -0.71 \\
After Davis First Year (week of 10/17/16 onward) & -0.40 & & -0.38 & -0.38 \\
After De Sousa Begins (week of 1/19/18 onward) & 0.14 & & 0.45 & 0.25 \\
After Tuggle Begins (week of 5/14/18 onward) & -0.02 & & -0.31 & -0.26 \\
After Harrison Begins (week of 2/11/19 onward) & -0.23 & & -0.10 & -0.18 \\
Average Maximum Temperature to 50 Degrees & & 0.003 & & 0.02 \\
Plus Degrees in the 50s & & 0.02 & & -0.01 \\
Plus Degrees in the 60s & & 0.01 & & -0.02 \\
Plus Degrees in the 70s & & -0.04 & & -0.07 \\
Plus Degrees Greater Than 80 & & 0.05 & & 0.27 \\
Snowfall (inches) & & -0.29 & & -0.37 \\
Precipitation (inches) & & 0.21 & & 0.05 \\
Darkness Before Midnight (hours) & & -2.60 & & -1.41 \\
Thanksgiving/Christmas (proportion of week) & & -1.57 & & -2.41 \\
Winter Holiday (proportion of week) & & -0.14 & & -0.05 \\
Other Out-of-School Days (proportion of week) & & 0.93 & & -0.18 \\
Summer Vacation (proportion of week) & 522 & 232 & 522 & 522 \\
Summer Holiday (proportion of week) & 0.10 & 0.09 & 0.07 & 0.15 \\
\hline Observations & & & & \\
$\mathrm{R}^{2}$ & & & & \\
\hline \hline
\end{tabular}


42.3 Least Squares Updated Model (2) for shooting.southwestern shooting.southwestern

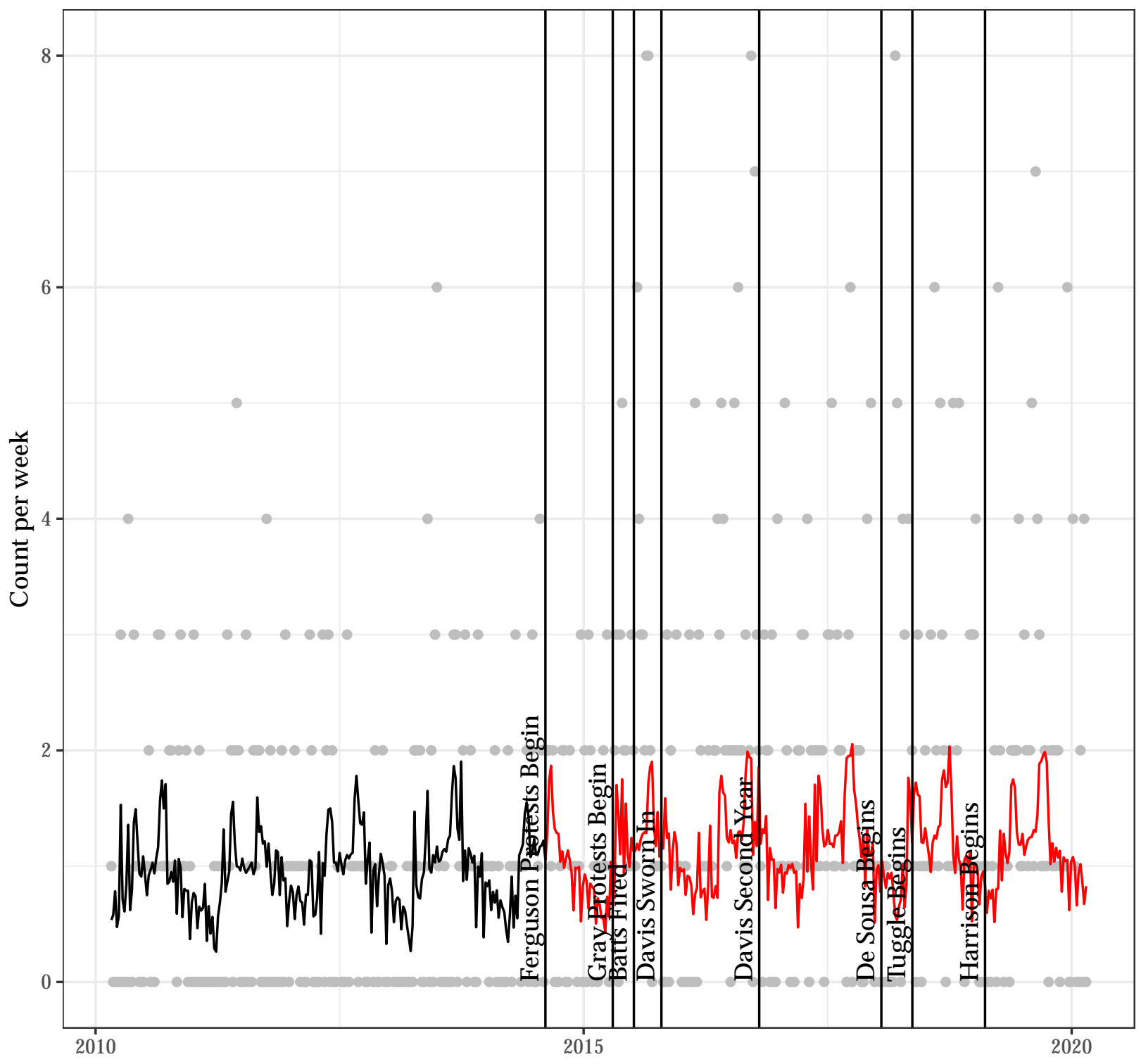




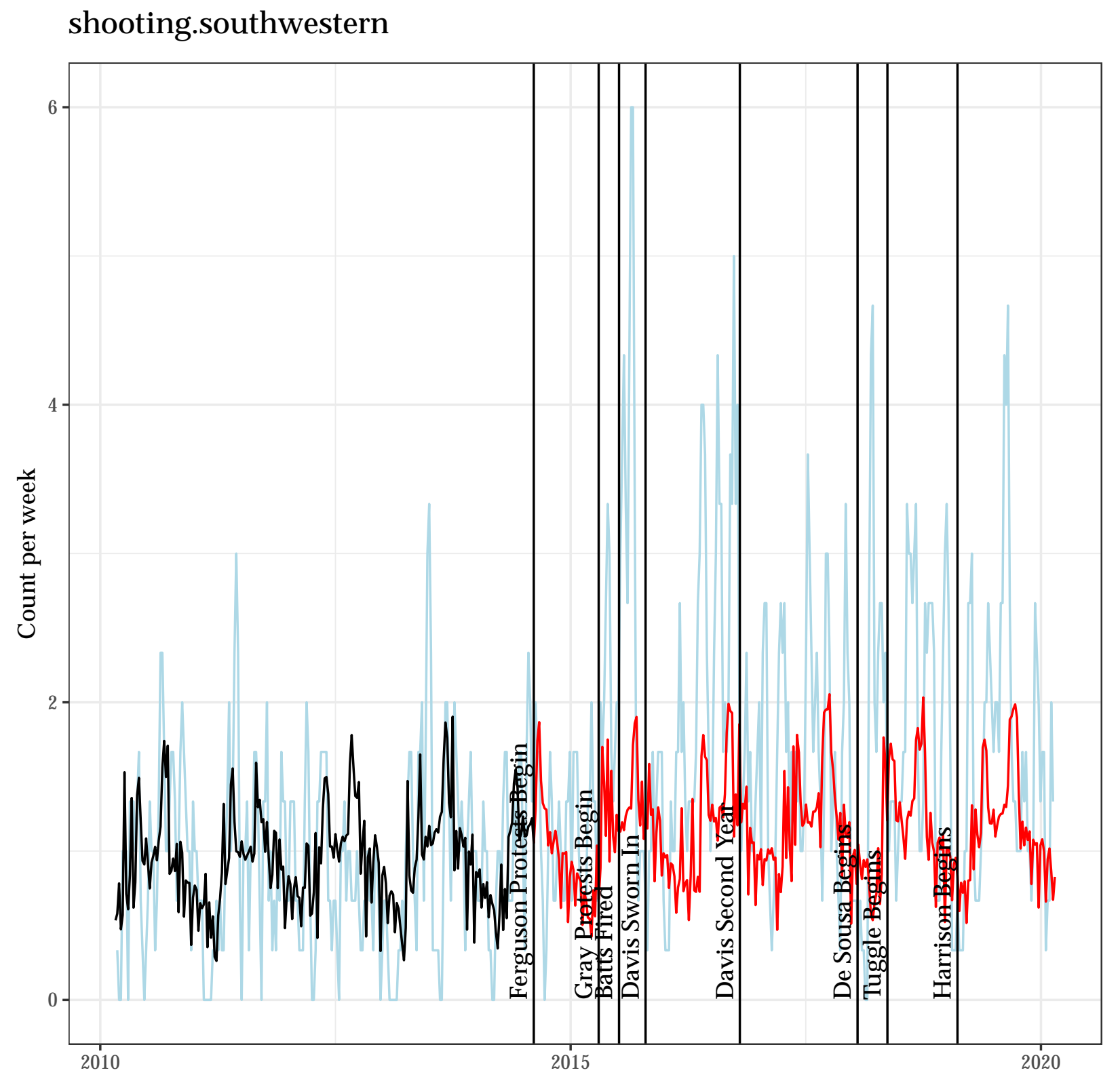


Autocorrelation Function for the Observed Outcome for Updated Model (2)

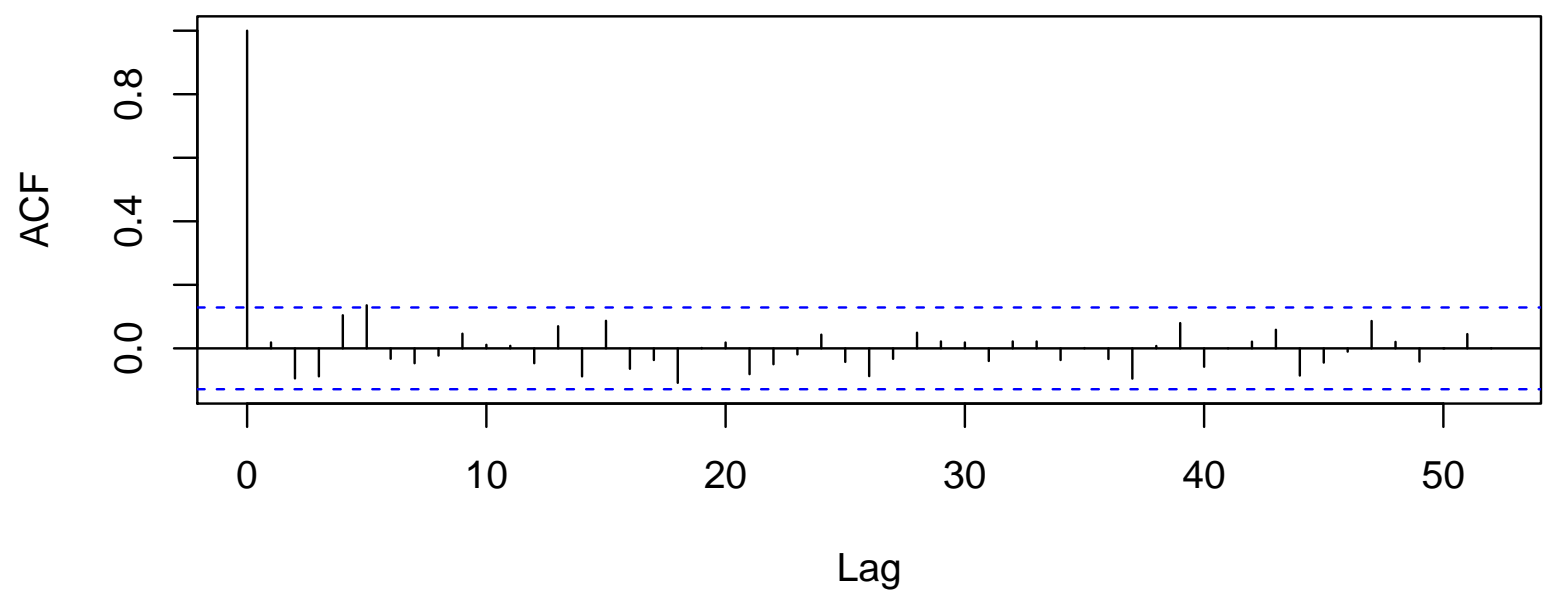

Autocorrelation Function for the Residuals from Updated Model (2)

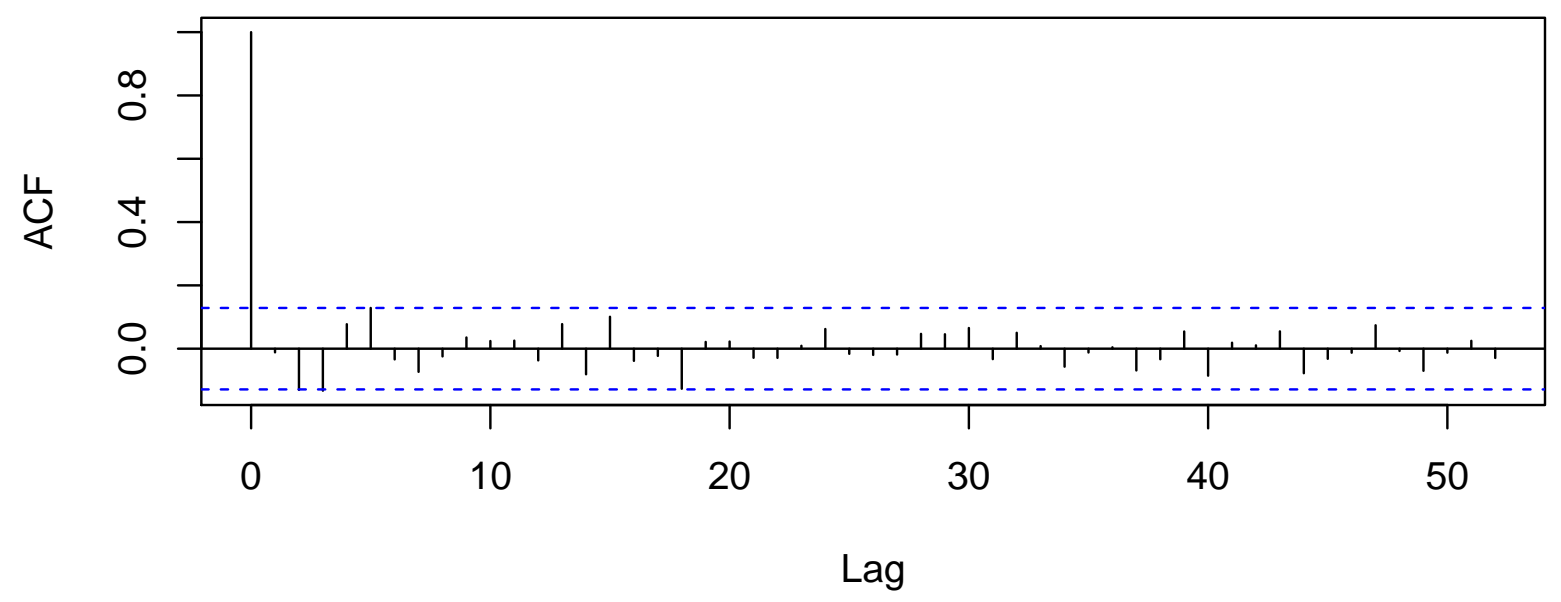




\subsection{A Poisson Regression Model as an Alternative to Model (2) for shooting.southwestern}

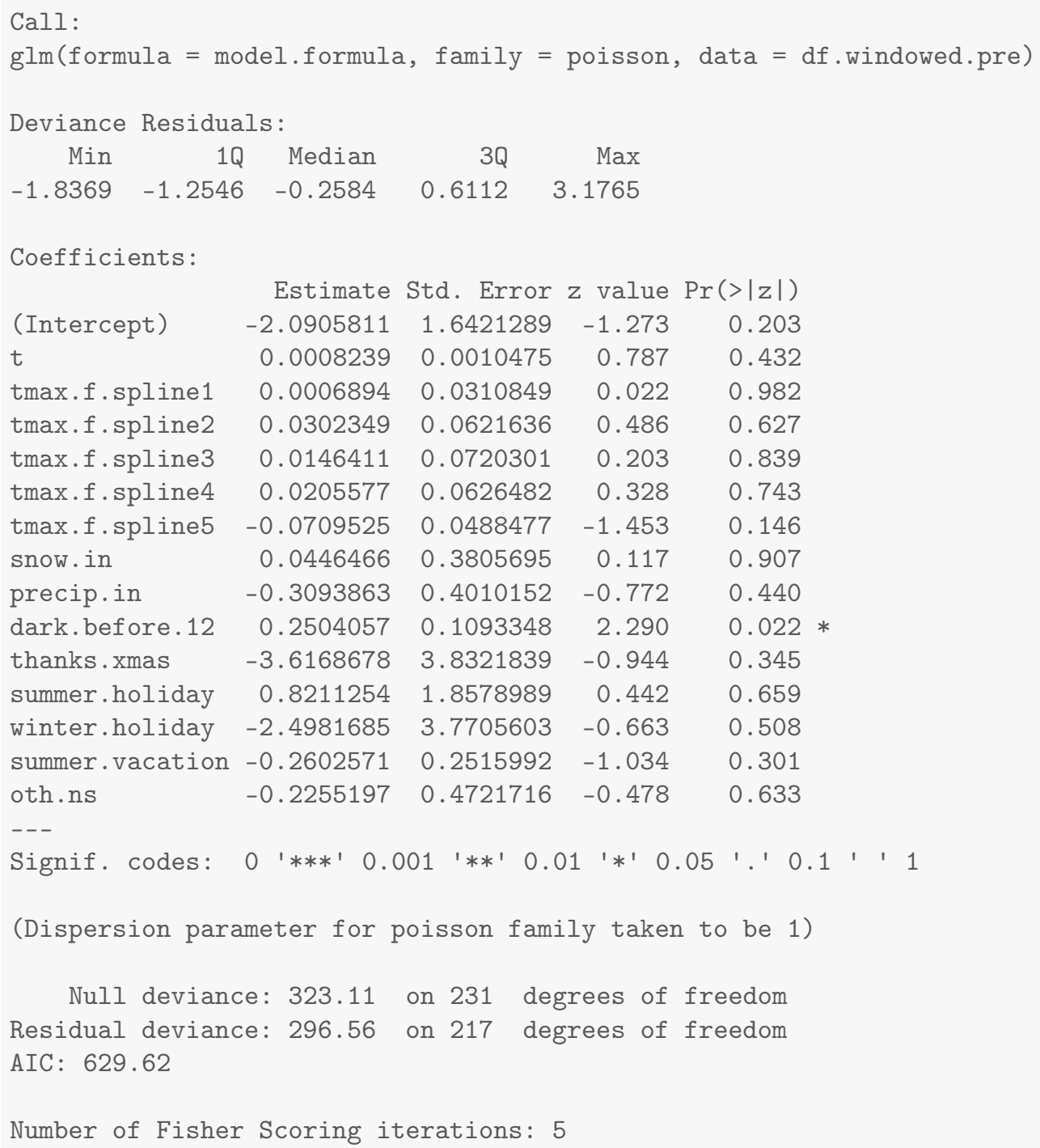




\section{Results for shooting.southern}

\subsection{Summary Values for shooting.southern}

Table 43.1: Descriptives for Outcome Before Ferguson Protests Begin

\begin{tabular}{lcccccccc}
\hline \hline Statistic & $\mathrm{N}$ & Mean & St. Dev. & Min & Pctl(25) & Median & Pctl(75) & Max \\
\hline shooting.southern & 232 & 0.776 & 1.058 & 0 & 0 & 0 & 1 & 5 \\
\hline
\end{tabular}

Table 43.2: Descriptives for Outcome After Ferguson Protests Begin

\begin{tabular}{lcccccccc}
\hline \hline Statistic & $\mathrm{N}$ & Mean & St. Dev. & Min & Pctl(25) & Median & Pctl(75) & Max \\
\hline shooting.southern & 290 & 1.462 & 1.520 & 0 & 0 & 1 & 2 & 8 \\
\hline
\end{tabular}




\subsection{Four Models for shooting.southern}

Table 43.3: Four Models that Differ on the Specification of Adjustment and Intervention Variables

\begin{tabular}{|c|c|c|c|c|}
\hline & \multicolumn{4}{|c|}{ Outcome: Count Per Week } \\
\hline & (1) & (2) & (3) & $(4)$ \\
\hline Time (counter in weeks) & -0.0004 & -0.0003 & & -0.0002 \\
\hline After Ferguson Protests Begin (week of $8 / 11 / 14$ onward) & -0.14 & & -0.04 & -0.03 \\
\hline After Gray Protests Begin (week of 4/20/15 onward) & 1.24 & & 0.97 & 0.96 \\
\hline Unrest and National Guard (4/27/15 - 5/3/2015) & -0.82 & & -0.94 & -0.80 \\
\hline After Batts Fired (week of 7/13/15 onward) & 0.04 & & 0.08 & -0.05 \\
\hline After Davis Confirmed (week of 10/19/15 onward) & -0.32 & & -0.19 & -0.08 \\
\hline After Davis First Year (week of 10/17/16 onward) & 0.46 & & 0.44 & 0.44 \\
\hline After De Sousa Begins (week of 1/19/18 onward) & -1.00 & & -0.92 & -0.86 \\
\hline After Tuggle Begins (week of 5/14/18 onward) & 0.51 & & 0.48 & 0.29 \\
\hline After Harrison Begins (week of 2/11/19 onward) & -0.02 & & -0.10 & -0.01 \\
\hline Average Maximum Temperature to 50 Degrees & & 0.001 & & 0.02 \\
\hline Plus Degrees in the 50s & & 0.01 & & -0.05 \\
\hline Plus Degrees in the 60s & & 0.03 & & 0.10 \\
\hline Plus Degrees in the 70s & & -0.07 & & -0.08 \\
\hline Plus Degrees Greater Than 80 & & 0.003 & & 0.03 \\
\hline Snowfall (inches) & & -0.25 & & -0.21 \\
\hline Precipitation (inches) & & -0.43 & & -0.09 \\
\hline Darkness Before Midnight (hours) & & -0.03 & & 0.04 \\
\hline Thanksgiving/Christmas (proportion of week) & & -0.49 & & -0.75 \\
\hline Winter Holiday (proportion of week) & & 3.80 & & 1.24 \\
\hline Other Out-of-School Days (proportion of week) & & -0.18 & & -0.45 \\
\hline Summer Vacation (proportion of week) & & 0.34 & & 0.06 \\
\hline Summer Holiday (proportion of week) & & 1.54 & & 0.11 \\
\hline Observations & 522 & 232 & 522 & 522 \\
\hline $\mathrm{R}^{2}$ & 0.11 & 0.06 & 0.13 & 0.15 \\
\hline
\end{tabular}


43.3 Least Squares Updated Model (2) for shooting.southern shooting.southern

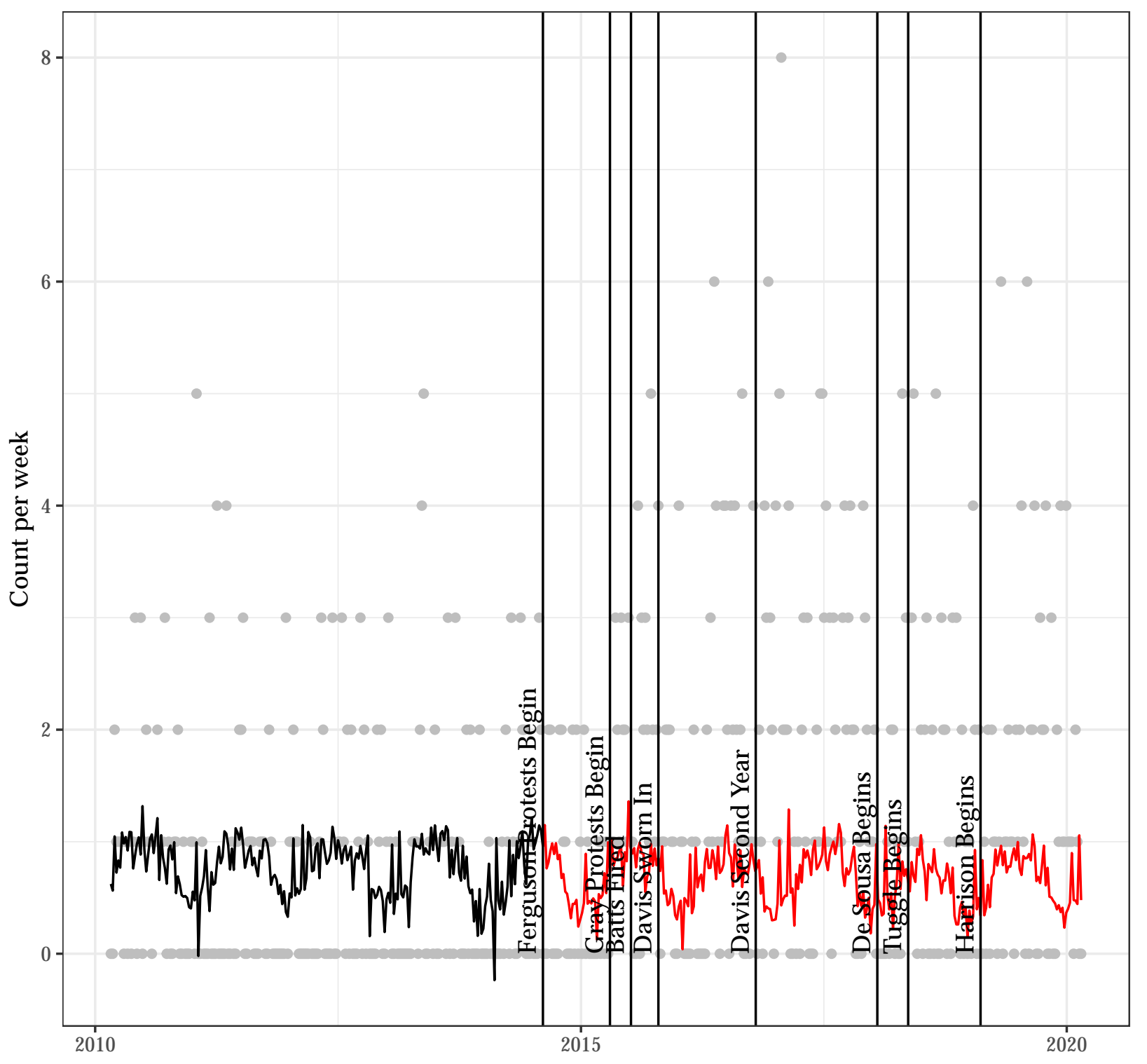




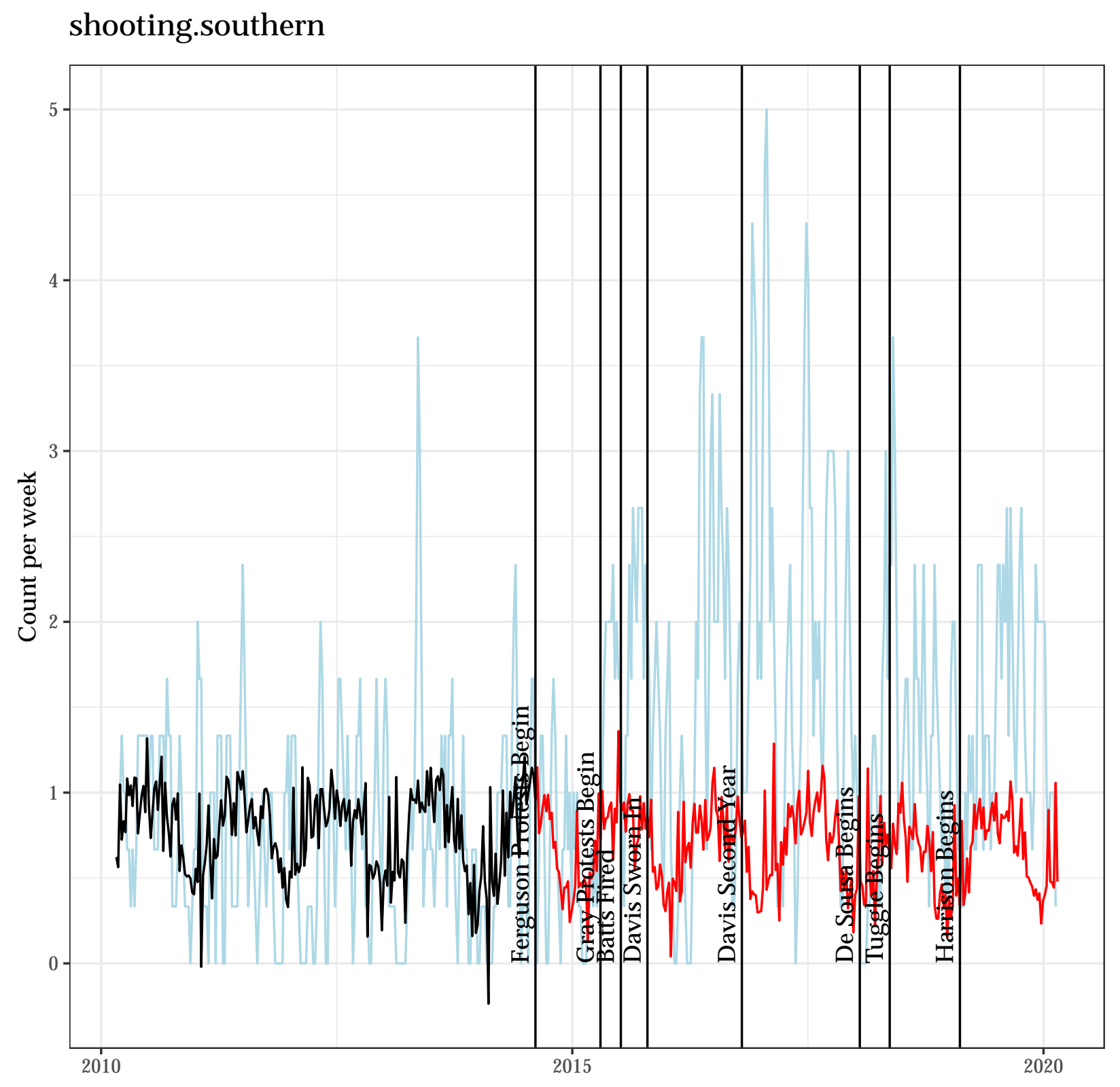


Autocorrelation Function for the Observed Outcome for Updated Model (2)

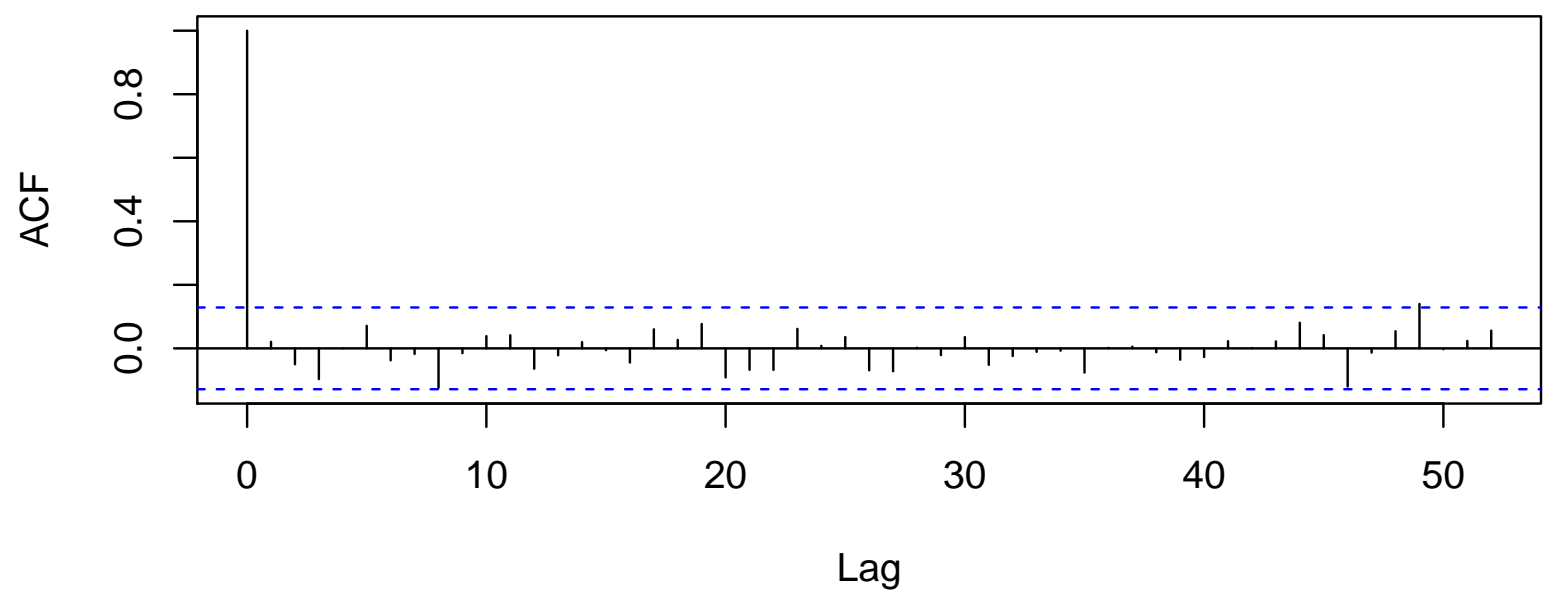

Autocorrelation Function for the Residuals from Updated Model (2)

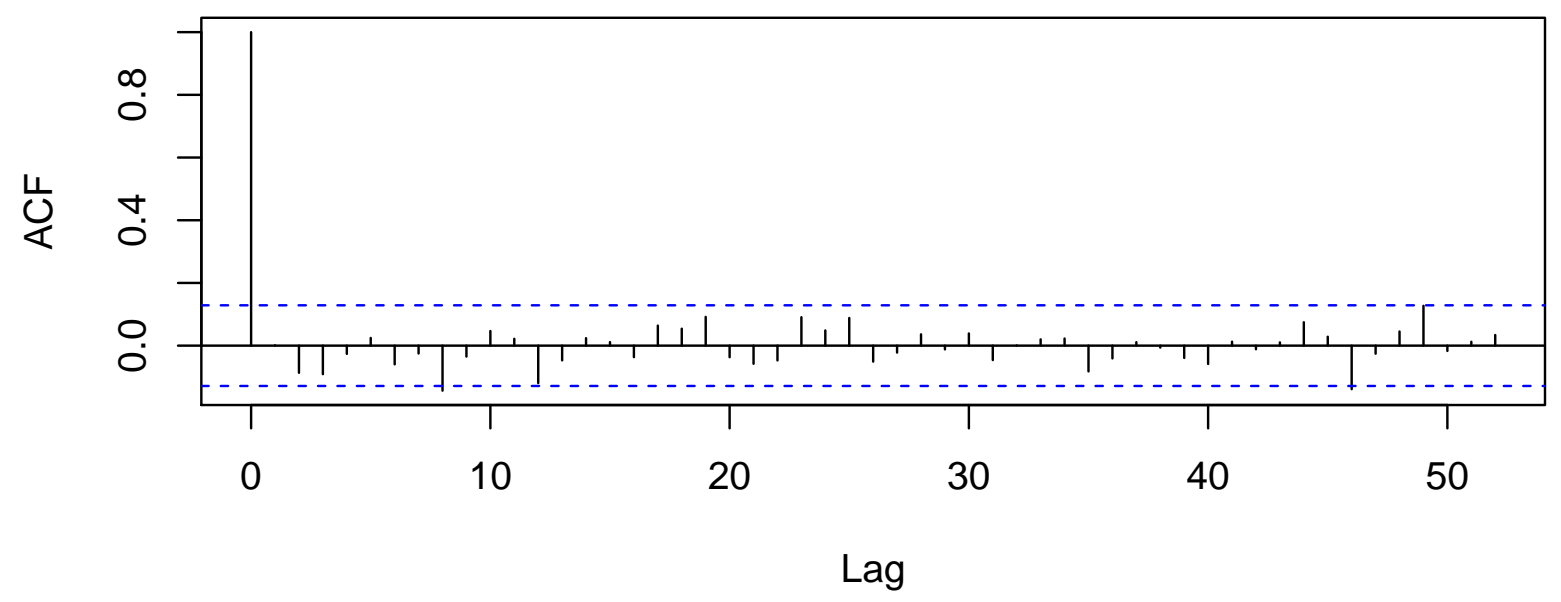




\subsection{A Poisson Regression Model as an Alternative to Model (2) for shooting.southern}

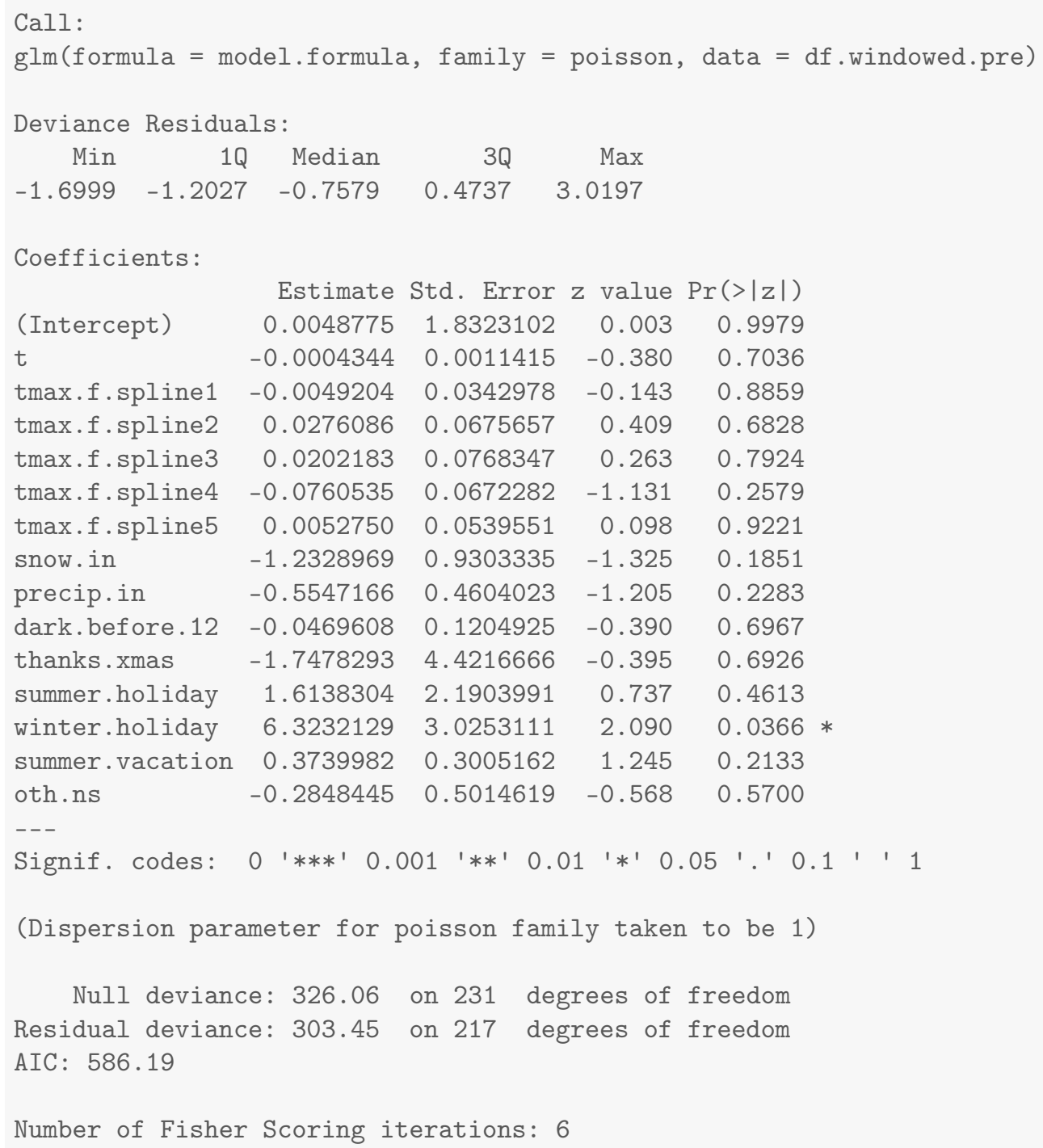




\section{Results for shooting.southeastern}

\subsection{Summary Values for shooting.southeastern}

Table 44.1: Descriptives for Outcome Before Ferguson Protests Begin

\begin{tabular}{lcccccccc}
\hline \hline Statistic & $\mathrm{N}$ & Mean & St. Dev. & Min & $\operatorname{Pctl}(25)$ & Median & Pctl(75) & Max \\
\hline shooting.southeastern & 232 & 0.534 & 0.872 & 0 & 0 & 0 & 1 & 5 \\
\hline
\end{tabular}

Table 44.2: Descriptives for Outcome After Ferguson Protests Begin

\begin{tabular}{lcccccccc}
\hline \hline Statistic & $\mathrm{N}$ & Mean & St. Dev. & Min & Pctl(25) & Median & Pctl(75) & Max \\
\hline shooting.southeastern & 290 & 0.814 & 1.081 & 0 & 0 & 0 & 1 & 6 \\
\hline
\end{tabular}




\subsection{Four Models for shooting.southeastern}

Table 44.3: Four Models that Differ on the Specification of Adjustment and Intervention Variables

\begin{tabular}{|c|c|c|c|c|}
\hline & \multicolumn{4}{|c|}{ Outcome: Count Per Week } \\
\hline & $(1)$ & $(2)$ & $(3)$ & (4) \\
\hline Time (counter in weeks) & -0.0004 & -0.001 & & -0.001 \\
\hline After Ferguson Protests Begin (week of $8 / 11 / 14$ onward) & -0.03 & & 0.03 & 0.02 \\
\hline After Gray Protests Begin (week of 4/20/15 onward) & 0.29 & & 0.24 & 0.23 \\
\hline Unrest and National Guard (4/27/15 - 5/3/2015) & 1.27 & & 1.25 & 1.36 \\
\hline After Batts Fired (week of 7/13/15 onward) & -0.08 & & -0.08 & -0.13 \\
\hline After Davis Confirmed (week of 10/19/15 onward) & 0.02 & & 0.09 & 0.14 \\
\hline After Davis First Year (week of 10/17/16 onward) & -0.05 & & -0.12 & -0.09 \\
\hline After De Sousa Begins (week of 1/19/18 onward) & 0.25 & & 0.35 & 0.38 \\
\hline After Tuggle Begins (week of 5/14/18 onward) & 0.35 & & 0.31 & 0.27 \\
\hline After Harrison Begins (week of 2/11/19 onward) & 0.16 & & 0.09 & 0.16 \\
\hline Average Maximum Temperature to 50 Degrees & & 0.01 & & -0.01 \\
\hline Plus Degrees in the 50s & & -0.03 & & 0.02 \\
\hline Plus Degrees in the 60s & & 0.04 & & -0.01 \\
\hline Plus Degrees in the $70 \mathrm{~s}$ & & -0.03 & & 0.01 \\
\hline Plus Degrees Greater Than 80 & & -0.04 & & -0.03 \\
\hline Snowfall (inches) & & -0.07 & & -0.22 \\
\hline Precipitation (inches) & & -0.38 & & -0.30 \\
\hline Darkness Before Midnight (hours) & & 0.02 & & 0.03 \\
\hline Thanksgiving/Christmas (proportion of week) & & 1.67 & & 0.25 \\
\hline Winter Holiday (proportion of week) & & -0.36 & & -0.81 \\
\hline Other Out-of-School Days (proportion of week) & & 0.10 & & 0.05 \\
\hline Summer Vacation (proportion of week) & & 0.64 & & 0.51 \\
\hline Summer Holiday (proportion of week) & & -0.26 & & -0.67 \\
\hline Observations & 522 & 232 & 522 & 522 \\
\hline $\mathrm{R}^{2}$ & 0.07 & 0.05 & 0.11 & 0.10 \\
\hline
\end{tabular}


44.3 Least Squares Updated Model (2) for shooting.southeastern shooting.southeastern

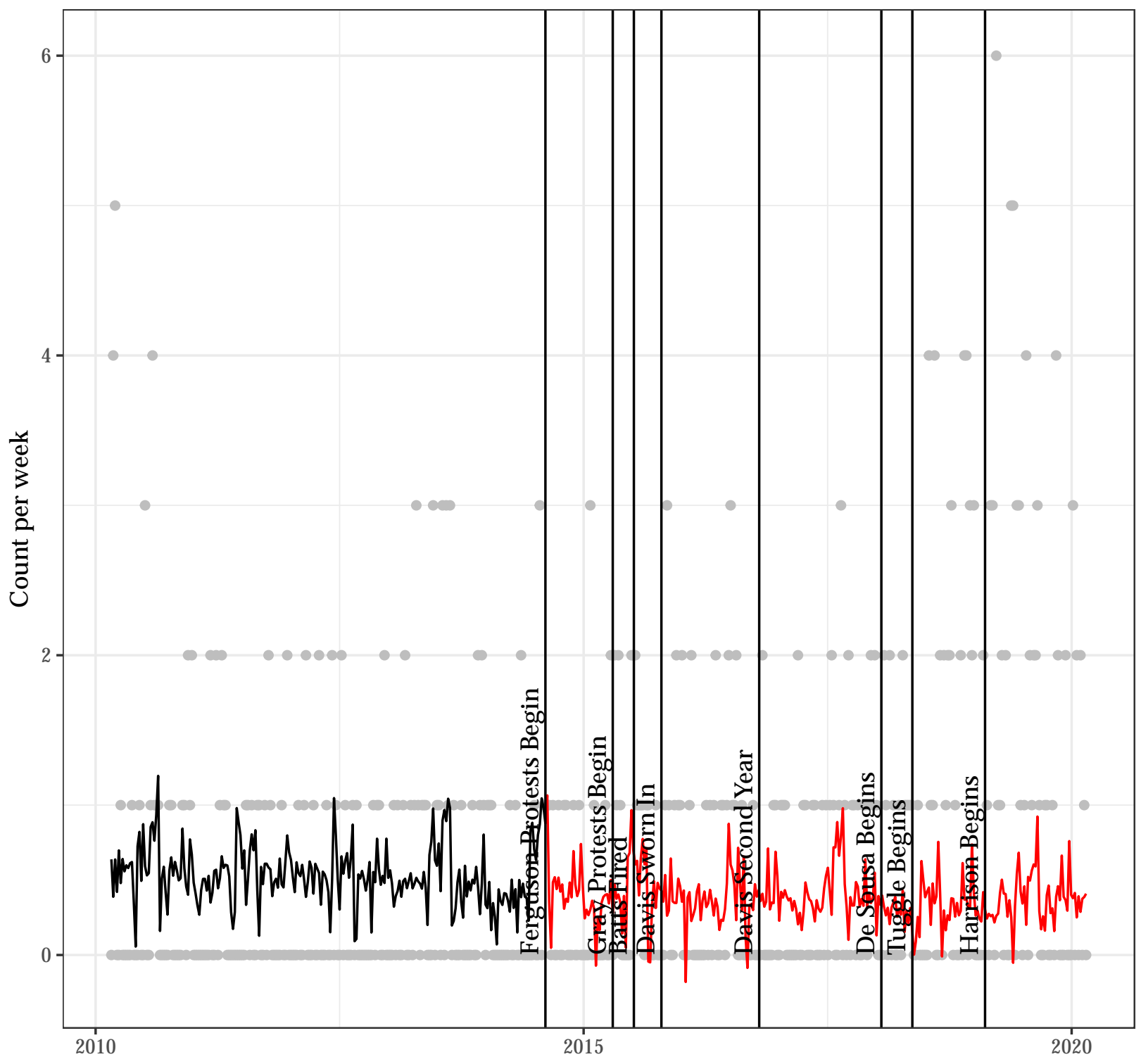




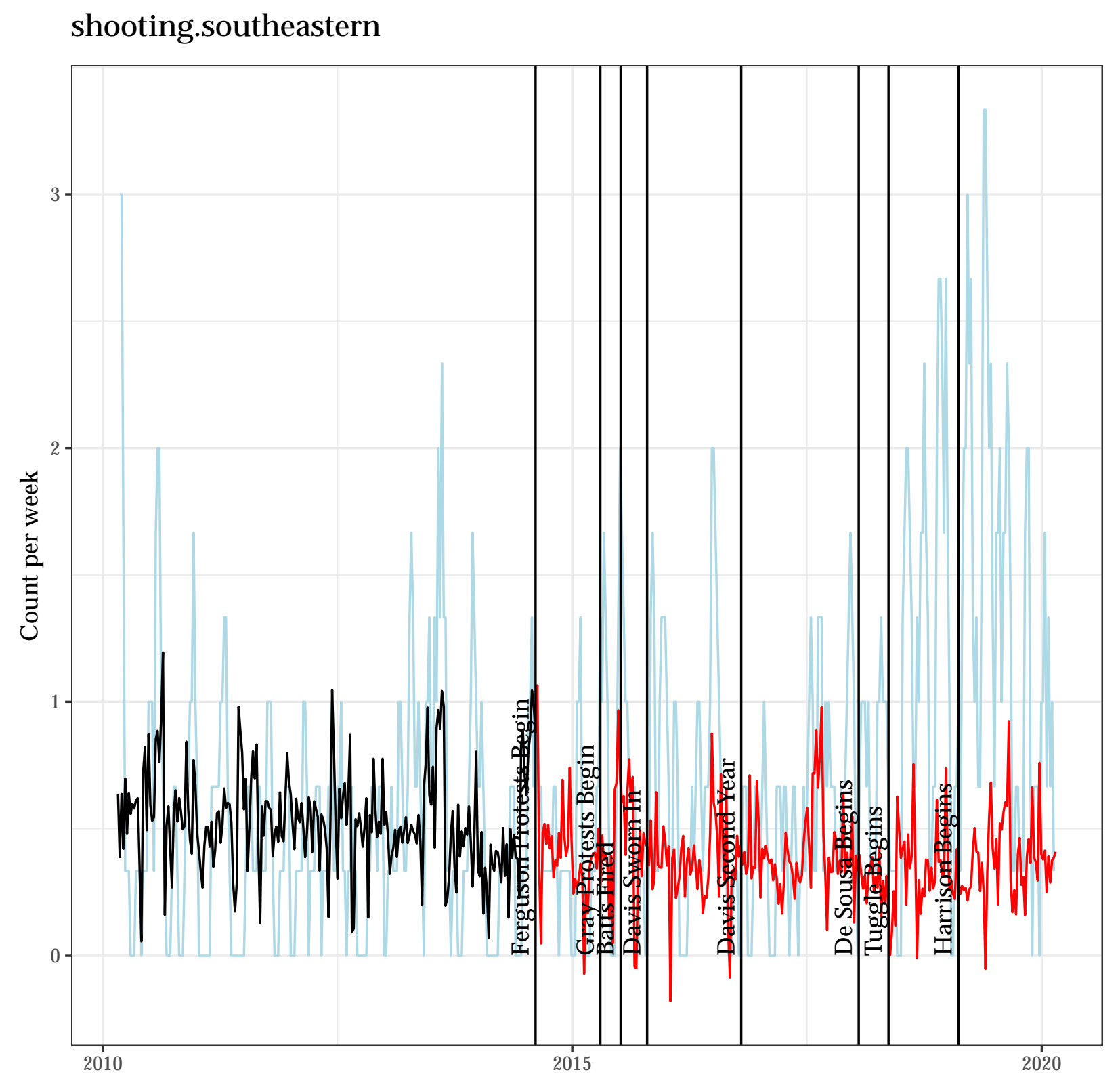


Autocorrelation Function for the Observed Outcome for Updated Model (2)

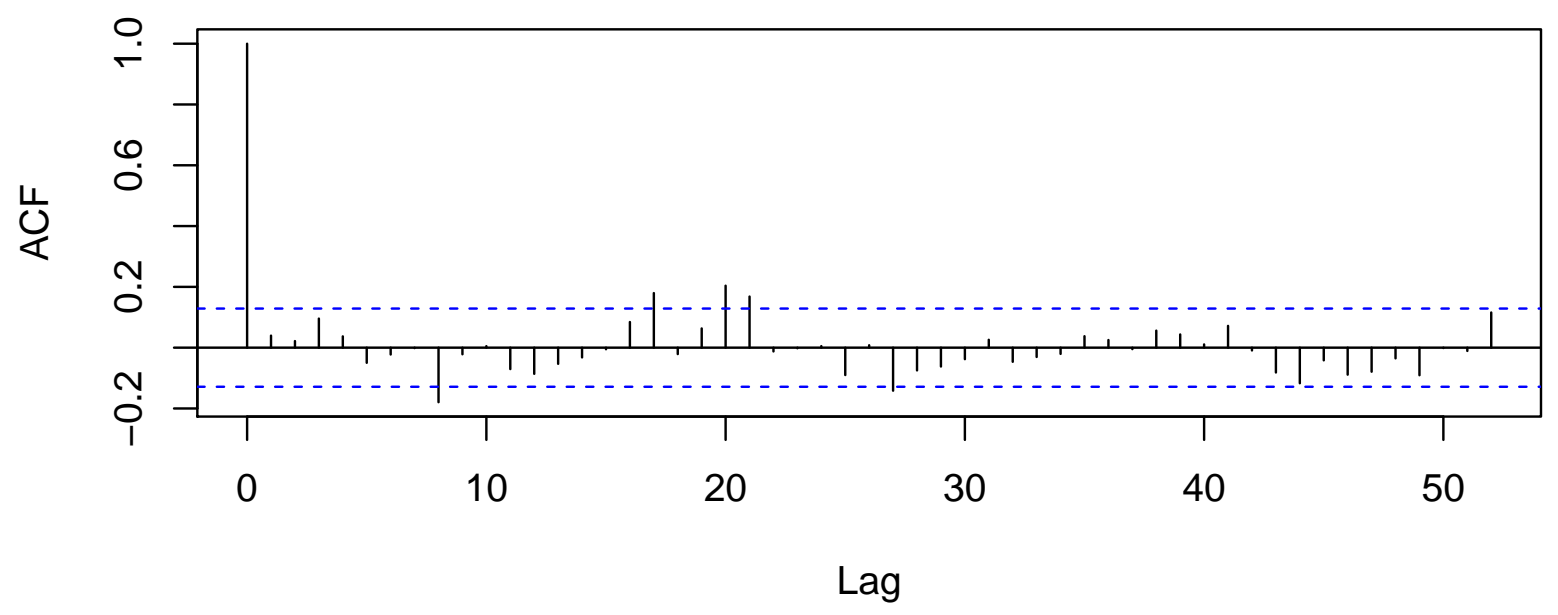

Autocorrelation Function for the Residuals from Updated Model (2)

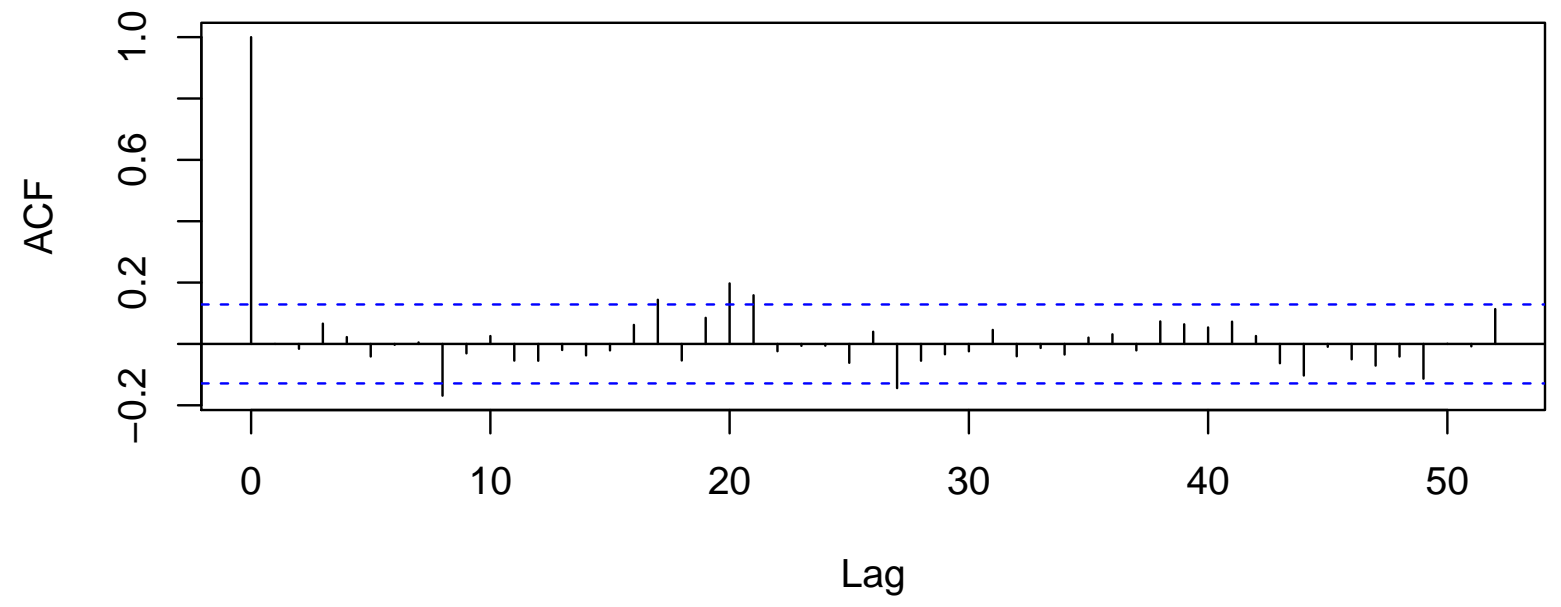




\subsection{A Poisson Regression Model as an Alternative to Model (2) for shooting.southeastern}

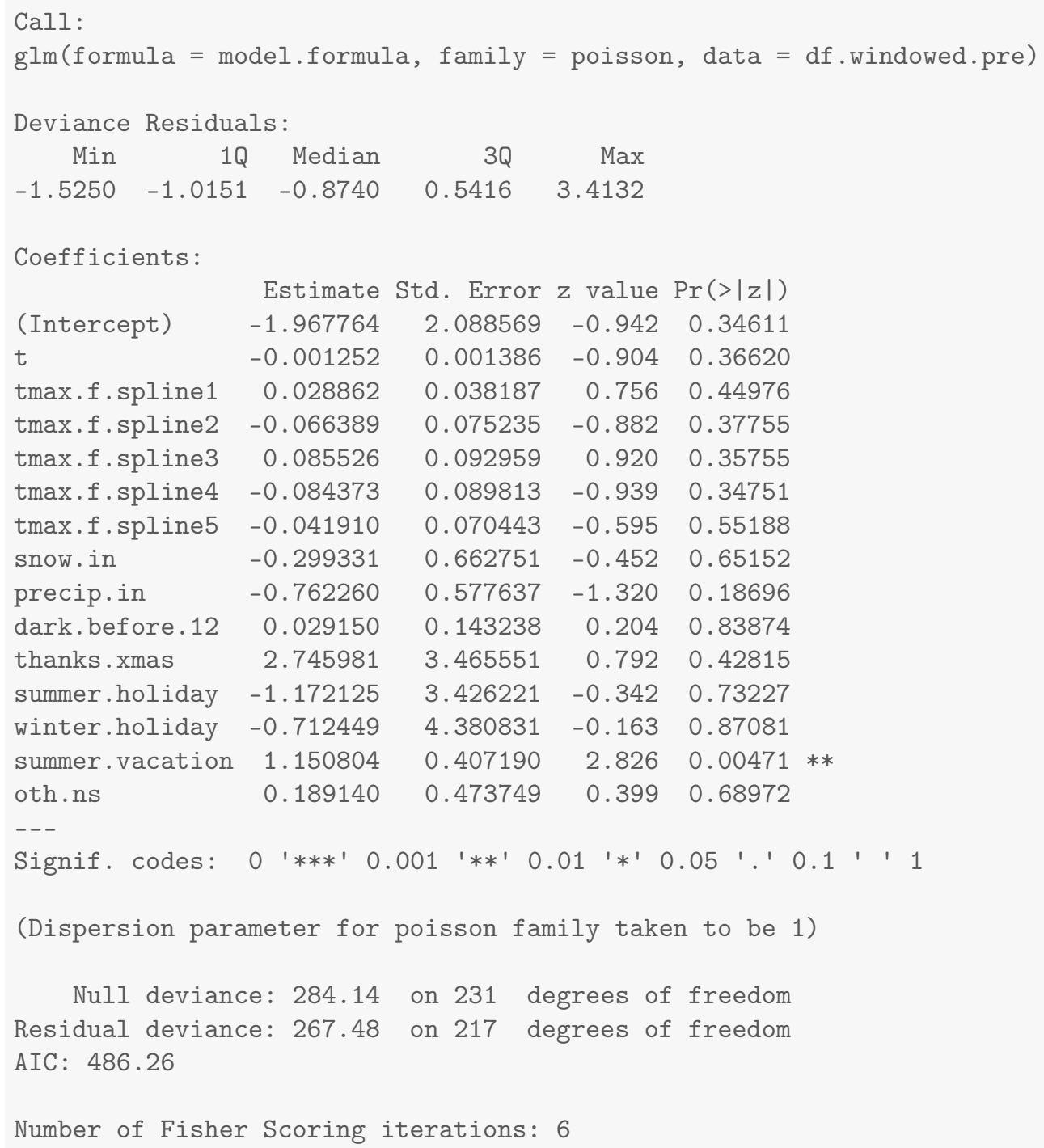




\section{Results for assault.by.threat}

\subsection{Summary Values for assault.by.threat}

Table 45.1: Descriptives for Outcome Before Ferguson Protests Begin

\begin{tabular}{lcccccccc}
\hline \hline Statistic & $\mathrm{N}$ & Mean & St. Dev. & Min & $\operatorname{Pctl}(25)$ & Median & $\operatorname{Pctl}(75)$ & Max \\
\hline assault.by.threat & 232 & 11.034 & 6.008 & 0 & 8 & 12 & 15 & 28 \\
\hline
\end{tabular}

Table 45.2: Descriptives for Outcome After Ferguson Protests Begin

\begin{tabular}{lcccccccc}
\hline \hline Statistic & $\mathrm{N}$ & Mean & St. Dev. & Min & $\operatorname{Pctl}(25)$ & Median & $\operatorname{Pctl}(75)$ & Max \\
\hline assault.by.threat & 290 & 0.000 & 0.000 & 0 & 0 & 0 & 0 & 0 \\
\hline
\end{tabular}




\subsection{Four Models for assault.by.threat}

Table 45.3: Four Models that Differ on the Specification of Adjustment and Intervention Variables

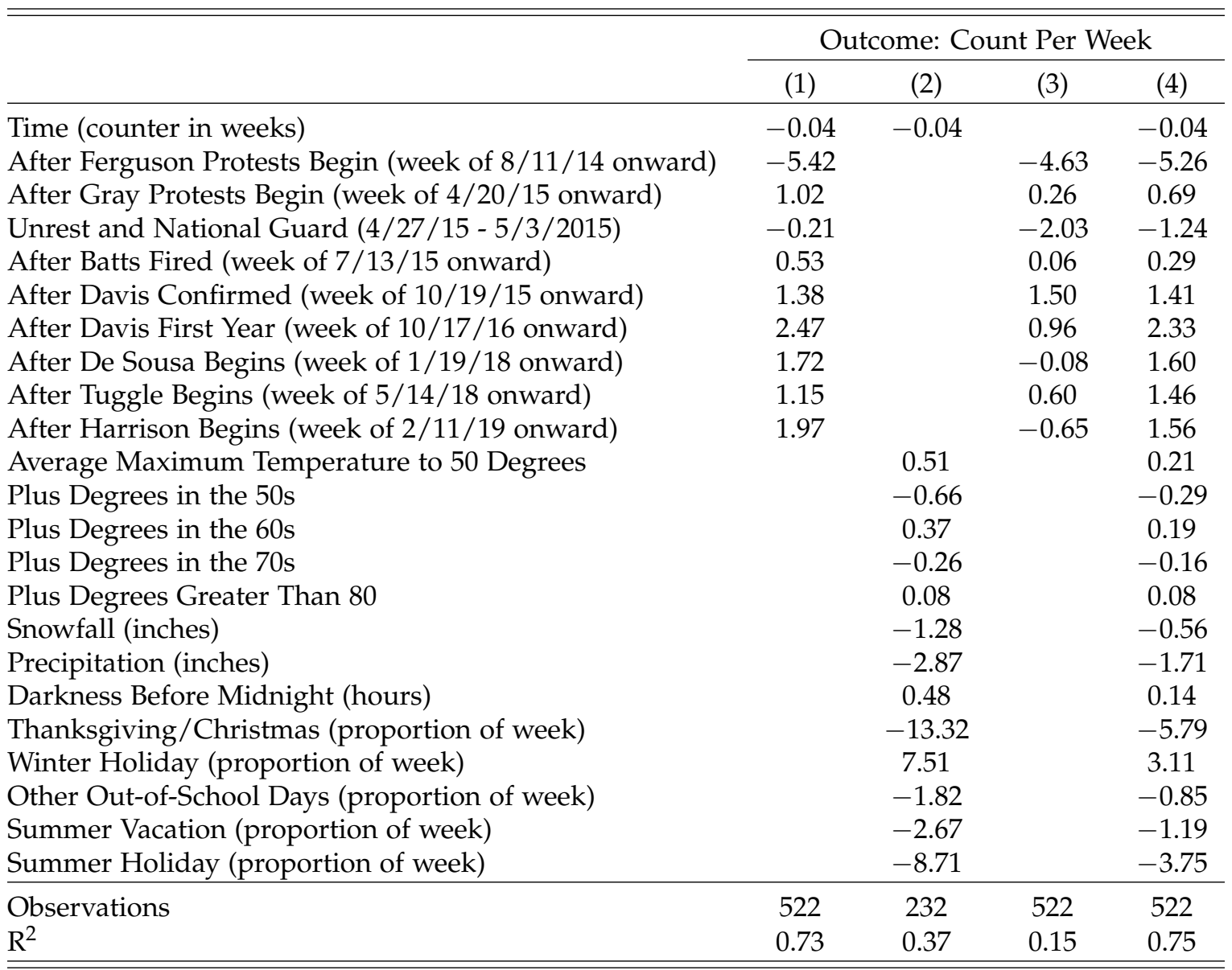


45.3 Least Squares Updated Model (2) for assault.by.threat assault.by.threat

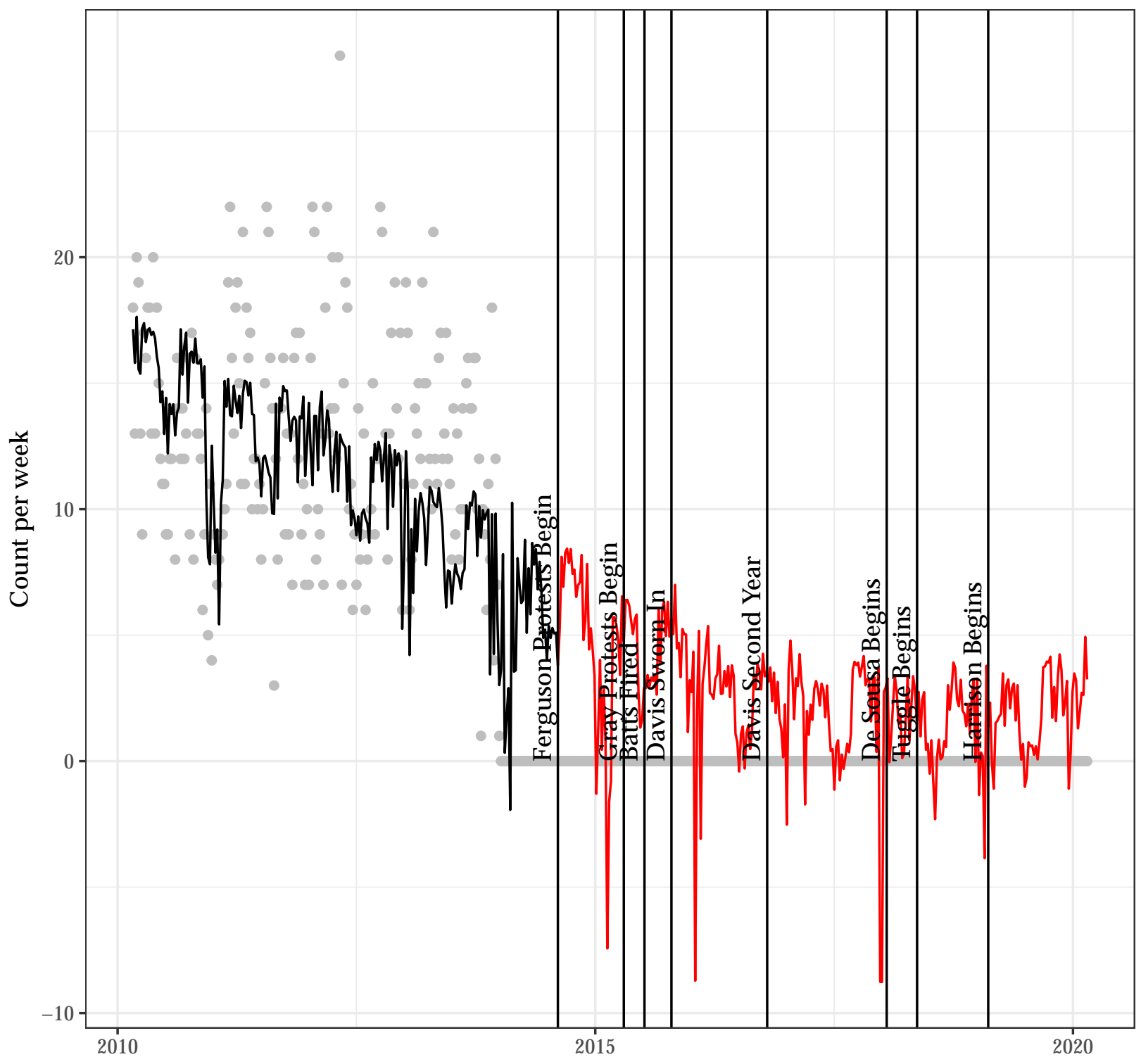




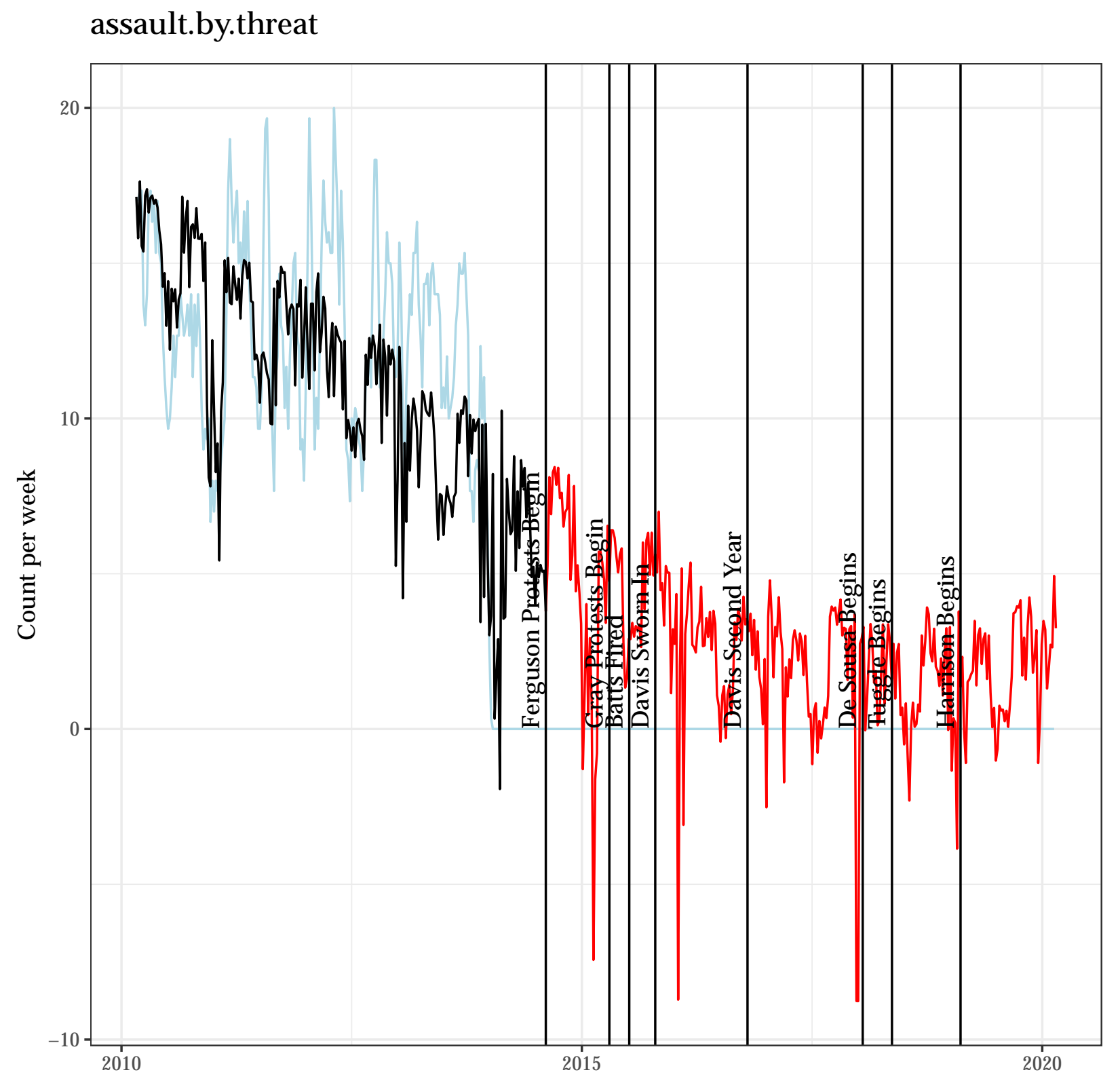


Autocorrelation Function for the Observed Outcome for Updated Model (2)

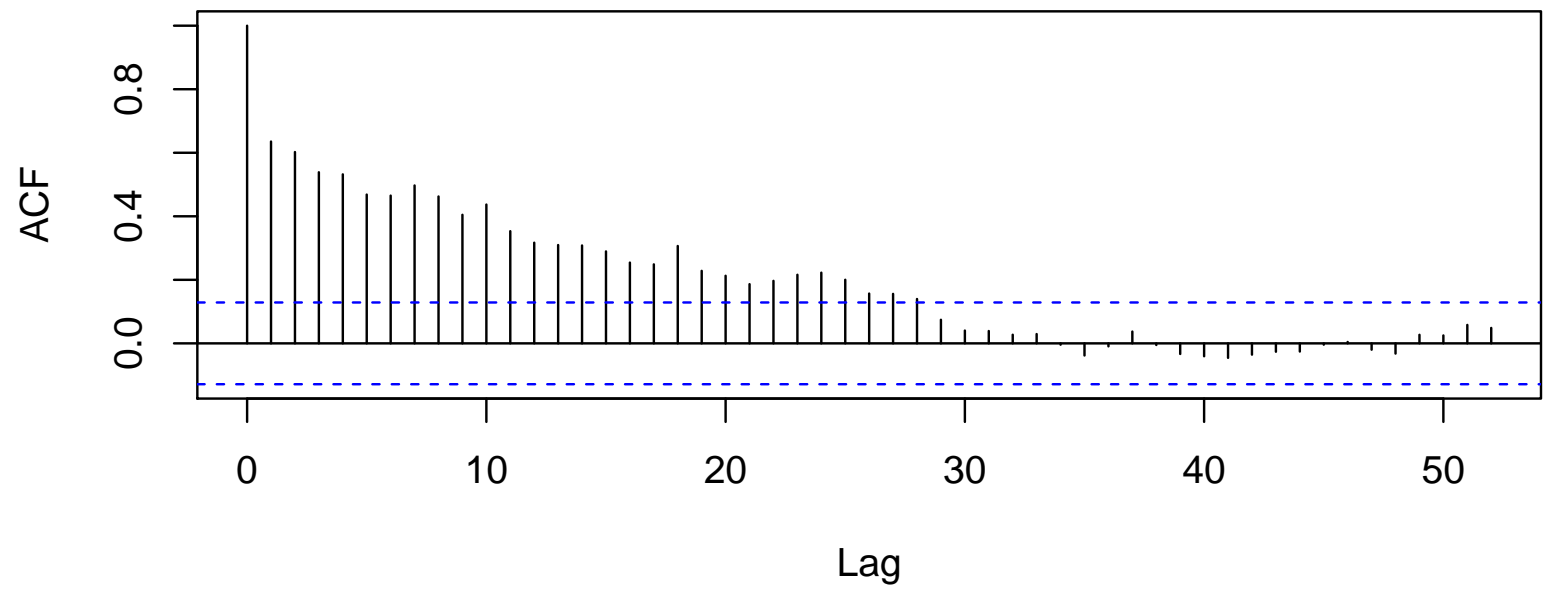

Autocorrelation Function for the Residuals from Updated Model (2)

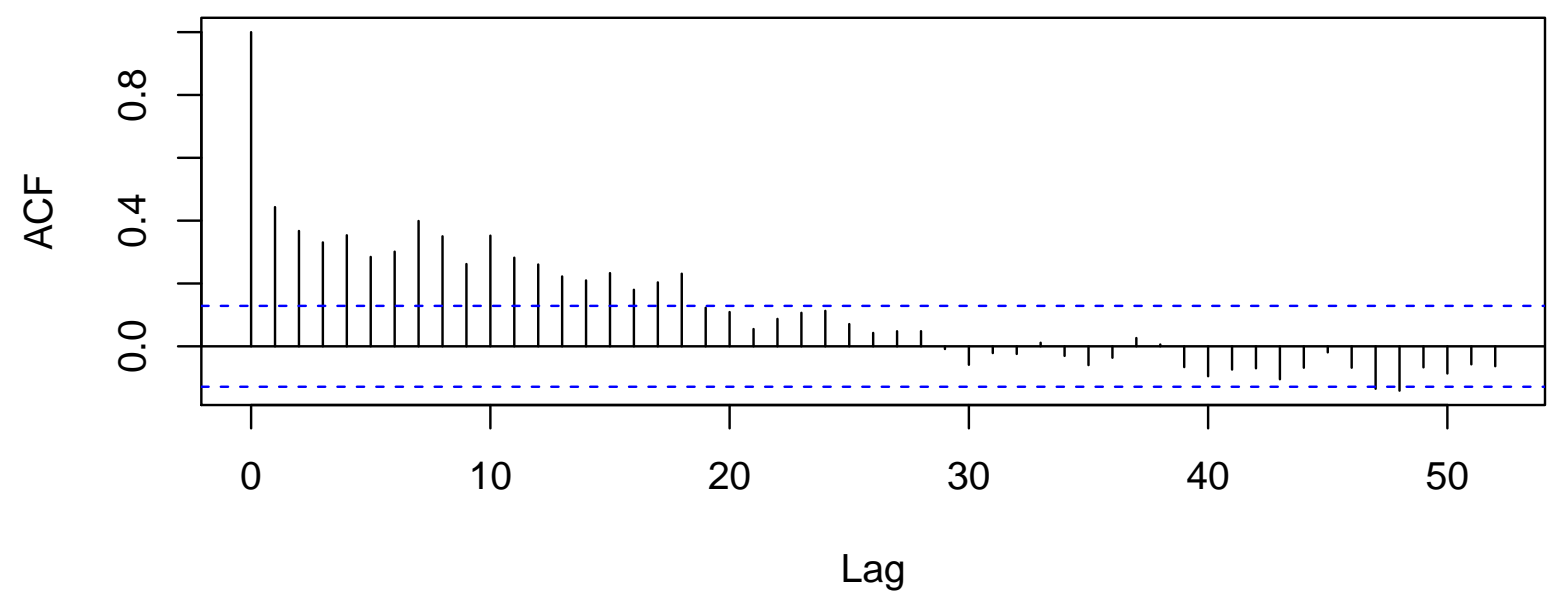




\subsection{A Poisson Regression Model as an Alternative to Model (2) for assault.by.threat}

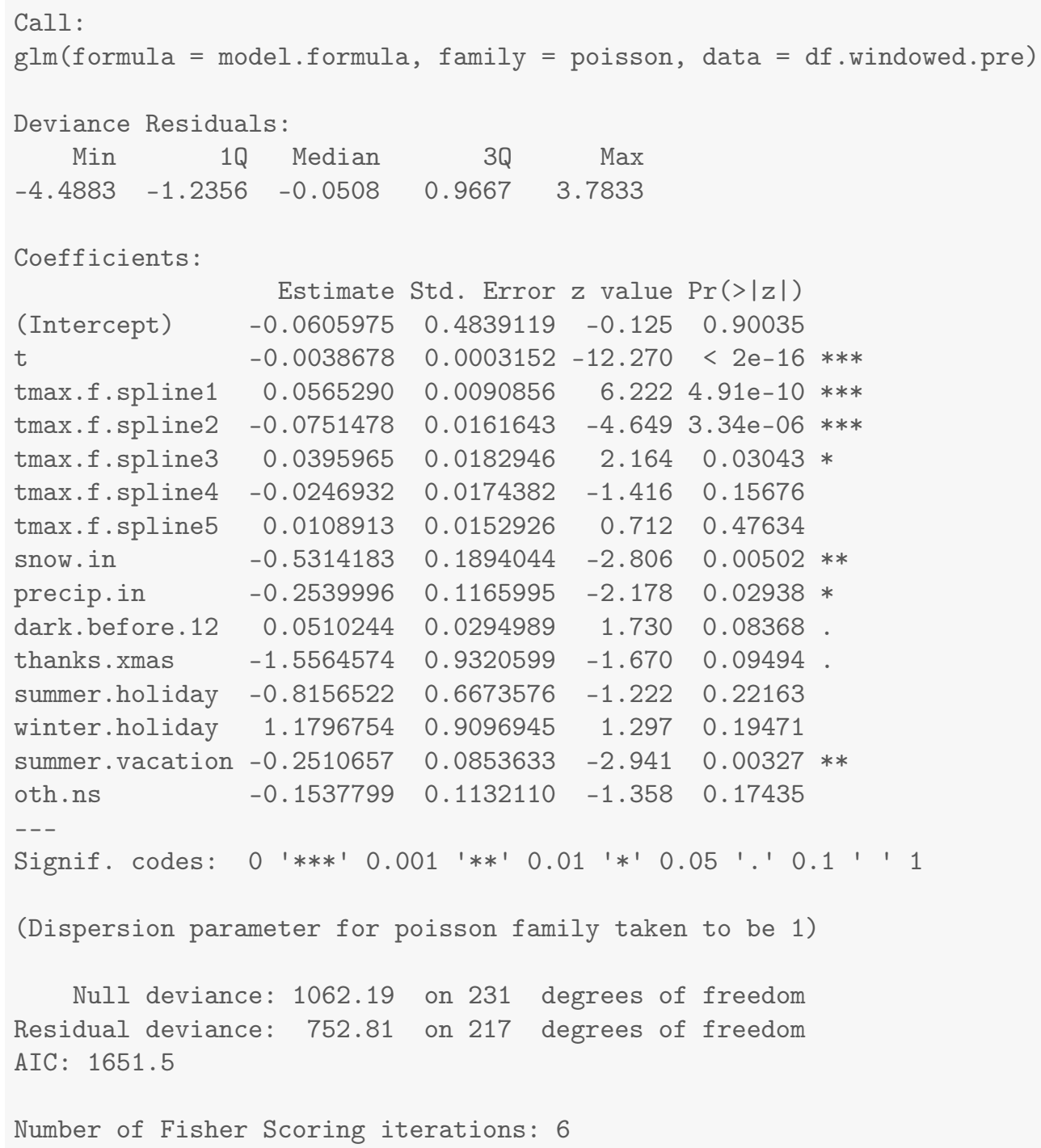




\section{Appendix 3}

\section{Comprehensive Analysis of BPD Arrest Data}

\author{
Stephen L. Morgan \\ Johns Hopkins University
}

\author{
Rhiannon N. Miller \\ Johns Hopkins University
}

May 11, 2020

\section{Navigation of this Document}

In order to jump to results of interest, display the Table of Contents for this pdf file in the left pane of your pdf viewer. The top level of the Table of Contents contains labeled sections for each outcome, within which subsections are nested for each piece of the analysis. Clicking the items in the pane will take you to the relevant set of results.

\section{Introduction}

For this updated analysis of BPD's arrest data, we offer comprehensive results that extend the results summarized in a Spring 2016 report and its Fall 2016 update:

- Morgan, Stephen L. and Joel A. Pally. 2016. "Ferguson, Gray, and Davis: An Analysis of Recorded Crime Incidents and Arrests in Baltimore City, March 2010 through December 2015." A Report Prepared for the 21st Century Cities Initiative at Johns Hopkins University. Baltimore, MD: Johns Hopkins University, March 15, 2016. (URL: https://osf.io/preprints/socarxiv/nshme/)

- Morgan, Stephen L. 2016. "Fall 2016 Update for Ferguson, Gray, and Davis: An Analysis of Recorded Crime Incidents and Arrests in Baltimore City, March 2010 through October 2016." Baltimore, MD: Johns Hopkins University, November 15, 2016. (URL: https://osf.io/preprints/socarxiv/6gdra/)

The results in the original report and its update were based on arrest data through December 2015 and October 2016, respectively. In this final report, we offer models that incorporate arrest data through Sunday, March 1, 2020.

The basic analysis strategy is the same as in the original report. We first model the prevailing cyclical arrest trend from Monday, January 7, 2013 through Sunday, August 10, 2014. Then, we use that estimated model to predict a counterfactual arrest trend, as if the protest events and any changes in police practice from August 11, 2014 onward had not occurred. We then estimate period effects as average differences between observed and counterfactual values from August 11, 2014 onward in distinct time intervals.

Unlike the original report, with the availability of additional data it is reasonable to now divide the interval after Police Commissioner Anthony Batts was fired into (1) a transition period while Kevin Davis was the Interim Police Commissioner and (2) two additional periods after Kevin Davis was sworn in as Batts' permanent replacement and with a five-year contract as the city's 38th Police Commmissioner. For the analysis reported here, the transition period begins with the week of Monday, July 13, 2015 and ends on Sunday, October 18, 2015. The first period for Davis as commissioner encompasses the first full year of his term, from the week of Monday, October 19, 2015 (10/19/15 to 10/25/15) through the week of Monday, October 10, 2016 (10/10/16 to 10/16/16). The second period for Davis begins with the second full year of his term, from the week of Monday, October 17, 2016 (10/17/16 to 10/23/16) through his final week as commissioner, Monday, January 15, 2018 (1/15/18 to 1/21/18).

More than three full years have passed since 2016, and the city has had three new police commissioners since then, necessitating three new periods for a parallel structure of models. The fourth period for De 
Sousa as commissioner commences with the week of Monday, January 22, 2018 (1/22/18 to 1/28/18) and ends with the week of Monday, May 7, 2018 (5/7/18 to 5/13/18). The fifth period for Tuggle as commissioner commences with the week of Monday, May 14, 2018 (5/14/18 to 5/20/18) and ends with the week of Monday, February 4, 2019 (2/4/19 to 2/10/19). Finally, the sixth period for Harrison as commissioner commences with the week of Monday, February 11, 2019 (2/11/19 to 2/17/19) and ends with the week of February 24, 2020 (2/24/20 to 3/1/20), which is the final full week before Governor Hogan declared a state of emergency because of the COVID-19 pandemic. Thus, the final period is approximately the first full year of Harrison's term as commissioner.

\section{Descriptive Summary of the Data}

The types of arrests analyzed in subsequent sections are listed and summarized in Table 2.1 in the Data section, after which a breakdown of types of arrests is provided by police district in Table 2.2. For this final report, we use an updated version of the keyword search routine in order to categorize arrests (see Appendix 1 for details).

Two figures then display the distributions of the variables utilized for subsequent models: (1) variables for weather and hours of darkness; (2) variables that parameterize types of days per week, such as holidays, vacations, and other days without school in the Baltimore City Schools. For this final report, we introduce a new set of variables for types of days within each week, separating holidays and vacation days from other types of non-school days.

\section{Results for Each Outcome}

For each outcome, we first offer a table with coefficients from four estimated models:

- Model 1 is a naive model with nine estimated coefficients for the period indicator variables for the full time series;

- Model 2 is a "pretreatment" model, estimated only for data prior to August 11, 2014, that fits coefficients for weather, hours of darkness, and types of days;

- Model 3 is analogous to Model 1 but utilizes an outcome variable for the full time series that has been de-trended using coefficients for weather, hours of darkness, and types of days estimated by Model 2 using data from the "pretreatment" period only;

- Model 4 is an alternative to Model 3 that fits coefficients for weather, hours of darkness, and types of days simultaneously with coefficients for the six period indicator variables.

The period effects estimated for Model 3 are our preferred estimates, and the period effects estimated for Model 4 are plausible alternatives.

We then offer two figures that depict the fit of Model 2 as well as the variation that generates the period effects estimated for the preferred Model 3. For both figures, the black line is the predicted time series from Model 2 while the red line is an extrapolated cyclical trend, generated by forming predictions that utilize the coefficients from Model 2 and the observed values for weather, hours of darkness, and school days from August 11, 2014 onward.

For this final report, we do not utilize a baseline time counter for the arrest models, unlike for the crime models. As a result, the counterfactual trend is determined only by weather, hours of darkness, and types of days. We made this change because the pre-Ferguson period for arrests is comparatively short, resulting in estimated underlying time trends that were more of a distraction than an aid to interpretation. In other words, with several more years of data, it has become clear that the pattern for arrests is easily interpretable without needing to fit an underlying time trend.

The first figure of each pair overlays gray dots for the observed weekly total for each outcome, and the second figure substitutes a light blue line for the three-week moving average of the observed weekly totals. The differences between the gray dots (or the light blue line) and the red line is the source of variation that 
generates the period effects estimated for the preferred Model 3. Additional details of Model 3 are offered in the main document for this updated report.

Finally, for each outcome a diagnostic model is also fit to assess the extent to which the least squares estimation of Model 2 adequately represents the underlying time series up through August 10, 2014. For this alternative model, a poisson distribution is assumed for the outcome because it is a weekly count bounded by zero. In general, these alternative models support the decision to utilize the simpler Model 2 to estimate the underlying time series that structures the extrapolated counterfactual trend. In this final report, we continue to offer the $\mathrm{R}$ output for the estimated poisson regression so that it can be checked against the similar results in the original report. However, we no longer offer analogous figures to demonstrate the near equivalence of results based on Model 2 and its poisson regression alternative. Interested readers can consult the close similarity from the original report, which holds for this updated analysis as well. 


\section{Data}

Table 2.1: Descriptive Statistics for Weekly Reported Arrests, with Breakdown by Type of Charge

\begin{tabular}{lccccccc}
\hline \hline Statistic & Mean & St. Dev. & Min & Pctl(25) & Median & Pctl(75) & Max \\
\hline murder.g & 6.0 & 2.6 & 0 & 4 & 6 & 8 & 15 \\
arson & 1.1 & 1.1 & 0 & 0 & 1 & 2 & 5 \\
robbery & 26.2 & 9.3 & 5 & 20 & 25 & 33 & 56 \\
aggravated.assault & 22.0 & 6.3 & 7 & 18 & 22 & 26 & 46 \\
deadly.weapon & 20.6 & 6.7 & 3 & 16 & 20 & 25 & 47 \\
common.assault & 81.5 & 13.3 & 36 & 72 & 81 & 90 & 123 \\
sex.offense.g & 10.3 & 5.5 & 0 & 7 & 9 & 13 & 35 \\
domestic & 2.8 & 2.0 & 0 & 1 & 3 & 4 & 11 \\
non.deadly.weapon & 1.1 & 1.1 & 0 & 0 & 1 & 2 & 7 \\
burglary & 11.7 & 5.1 & 1 & 8 & 11 & 14 & 52 \\
larceny & 29.8 & 10.4 & 7 & 22 & 28 & 36 & 63 \\
court.non.compliance & 86.3 & 21.4 & 40 & 72 & 82 & 98 & 165 \\
property.destruction & 5.0 & 2.6 & 0 & 3 & 5 & 7 & 14 \\
forgery.fraud.extortion & 1.4 & 1.3 & 0 & 0 & 1 & 2 & 6 \\
police.non.compliance & 5.0 & 4.5 & 0 & 2 & 3 & 7 & 22 \\
driving.violation & 8.7 & 5.9 & 0 & 4 & 7 & 13 & 26 \\
trespass & 6.4 & 5.0 & 0 & 3 & 5 & 9 & 25 \\
drug.g & 144.7 & 94.4 & 17 & 83 & 107 & 170 & 423 \\
loitering.vagabond & 0.4 & 1.1 & 0 & 0 & 0 & 0 & 8 \\
disorderly.conduct & 10.5 & 12.1 & 0 & 4 & 7 & 15 & 189 \\
prostitution & 6.7 & 5.6 & 0 & 2 & 5 & 10 & 27 \\
only.temp.detained & 7.9 & 3.5 & 1 & 5 & 8 & 10 & 20 \\
unknown & 28.3 & 16.6 & 4 & 16 & 24 & 38 & 121 \\
ungrouped & 5.7 & 3.9 & 0 & 3 & 5 & 8 & 28 \\
total & 530.0 & 170.8 & 219 & 410 & 471 & 641 & 928 \\
\hline
\end{tabular}

Table 2.2: Descriptive Statistics for Weekly Reported Arrests, with Breakdown by District

\begin{tabular}{lccccccc}
\hline \hline Statistic & Mean & St. Dev. & Min & Pctl(25) & Median & Pctl(75) & Max \\
\hline northwestern & 25.5 & 15.4 & 6 & 15 & 20 & 32 & 95 \\
northern & 19.3 & 9.5 & 1 & 12 & 18 & 25 & 56 \\
northeastern & 34.8 & 16.6 & 8 & 23 & 31 & 41 & 93 \\
western & 37.9 & 21.1 & 6 & 22 & 31 & 52 & 113 \\
central & 32.8 & 18.1 & 6 & 20 & 27 & 40 & 87 \\
eastern & 38.2 & 22.5 & 6 & 22 & 32 & 49 & 116 \\
southwestern & 29.1 & 11.0 & 8 & 20 & 27 & 36 & 65 \\
southern & 38.6 & 21.8 & 8 & 22 & 30 & 56 & 109 \\
southeastern & 36.9 & 18.9 & 8 & 23 & 30 & 47 & 93 \\
total & 530.0 & 170.8 & 219 & 410 & 471 & 641 & 928 \\
\hline
\end{tabular}




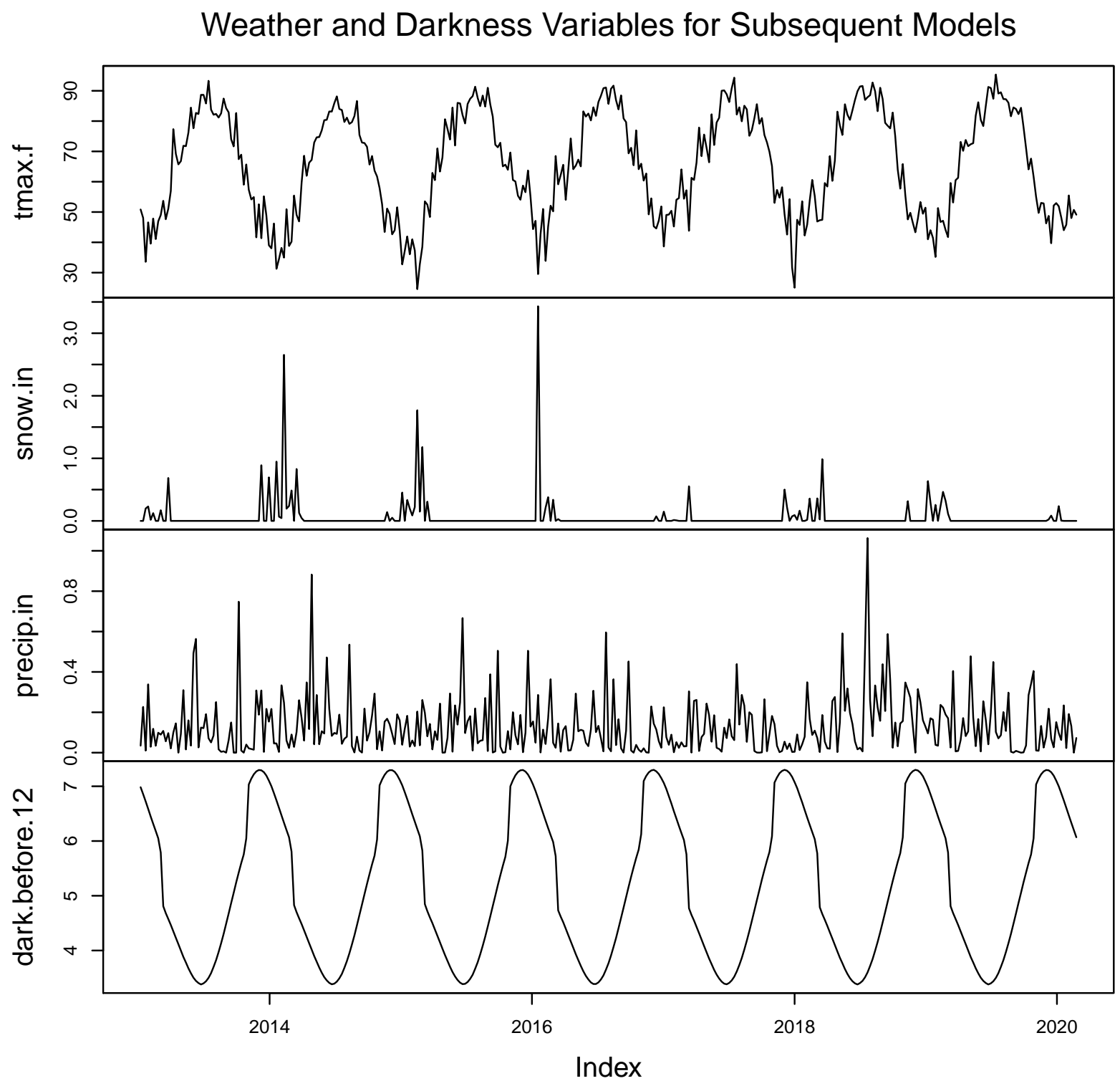

- tmax.f is the weekly average of the daily maximum temperature, measured in degrees Fahrenheit

- snow.in is the weekly average of total daily snowfall, measured in inches

- precip.in is the weekly average of total daily precipitation, measured in inches

- dark.before.12 is the weekly average of daily hours between sunset and midnight 


\section{Types of Day Variables for Subsequent Models}
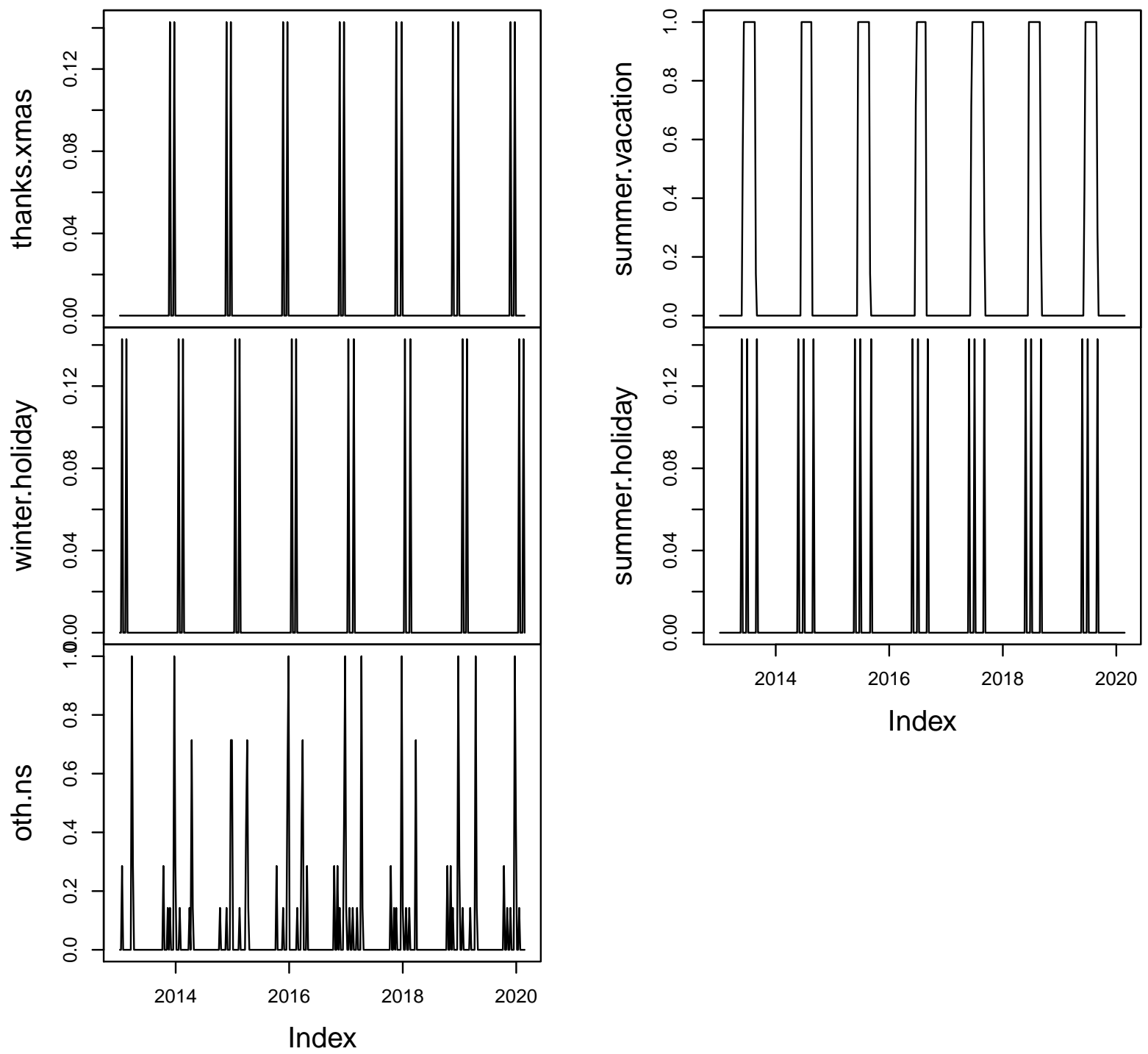

- thanks.xmas is the proportion of days of the week that are either Thanksgiving or Christmas

- winter.holiday is the proportion of days of the week that are a winter holiday other than Thanksgiving or Christmas

- other.ns is the proportion of days of the week that are other types of non-school days for the Baltimore City Schools (e.g., professional development days and winter vacation days)

- summer.vacation is the proportion of days of the week that are summer vacation for the Baltimore City Schools

- summer.holiday is the proportion of days of the week that are a summer holiday (e.g., 4th of July) 


\section{Results for total}

\subsection{Summary Values for total}

Table 3.1: Descriptives for Outcome Before Ferguson Protests Begin

\begin{tabular}{lcccccccc}
\hline \hline Statistic & $\mathrm{N}$ & Mean & St. Dev. & Min & Pctl(25) & Median & Pctl(75) & Max \\
\hline total & 83 & 800.952 & 73.407 & 565 & 772.5 & 807 & 855.5 & 928 \\
\hline
\end{tabular}

Table 3.2: Descriptives for Outcome After Ferguson Protests Begin

\begin{tabular}{lcccccccc}
\hline \hline Statistic & $\mathrm{N}$ & Mean & St. Dev. & Min & Pctl(25) & Median & Pctl(75) & Max \\
\hline total & 290 & 452.514 & 94.496 & 219 & 392.2 & 444 & 492 & 865 \\
\hline
\end{tabular}




\subsection{Four Models for total}

Table 3.3: Four Models that Differ on the Specification of Adjustment and Intervention Variables

\begin{tabular}{|c|c|c|c|c|}
\hline & \multicolumn{4}{|c|}{ Outcome: Count Per Week } \\
\hline & $(1)$ & $(2)$ & $(3)$ & $(4)$ \\
\hline After Ferguson Protests Begin (week of $8 / 11 / 14$ onward) & -188.42 & & -170.07 & -171.84 \\
\hline After Gray Protests Begin (week of 4/20/15 onward) & -205.16 & & -245.64 & -237.88 \\
\hline Unrest and National Guard (4/27/15 - 5/3/2015) & 185.64 & & 169.59 & 160.30 \\
\hline After Batts Fired (week of 7/13/15 onward) & 110.35 & & 127.73 & 119.17 \\
\hline After Davis Confirmed (week of 10/19/15 onward) & -43.79 & & -25.87 & -32.95 \\
\hline After Davis First Year (week of 10/17/16 onward) & -31.91 & & -37.50 & -30.51 \\
\hline After De Sousa Begins (week of 1/19/18 onward) & -3.08 & & -20.94 & -14.51 \\
\hline After Tuggle Begins (week of 5/14/18 onward) & -46.27 & & -15.30 & -21.99 \\
\hline After Harrison Begins (week of 2/11/19 onward) & -16.27 & & -37.18 & -32.82 \\
\hline Average Maximum Temperature to 50 Degrees & & 1.83 & & 2.55 \\
\hline Plus Degrees in the $50 \mathrm{~s}$ & & -9.51 & & -3.43 \\
\hline Plus Degrees in the 60s & & 9.32 & & 1.66 \\
\hline Plus Degrees in the 70s & & 3.27 & & -1.79 \\
\hline Plus Degrees Greater Than 80 & & -8.33 & & -1.73 \\
\hline Snowfall (inches) & & -88.87 & & -30.07 \\
\hline Precipitation (inches) & & -143.98 & & -91.09 \\
\hline Darkness Before Midnight (hours) & & -23.89 & & -13.17 \\
\hline Thanksgiving/Christmas (proportion of week) & & $-1,396.50$ & & -765.85 \\
\hline Winter Holiday (proportion of week) & & -1.88 & & 237.57 \\
\hline Other Out-of-School Days (proportion of week) & & -5.46 & & -38.26 \\
\hline Summer Vacation (proportion of week) & & -46.77 & & 10.16 \\
\hline Summer Holiday (proportion of week) & & -468.88 & & -148.99 \\
\hline Observations & 373 & 83 & 373 & 373 \\
\hline $\mathrm{R}^{2}$ & 0.86 & 0.66 & 0.87 & 0.91 \\
\hline
\end{tabular}




\subsection{Least Squares Updated Model (2) for total}

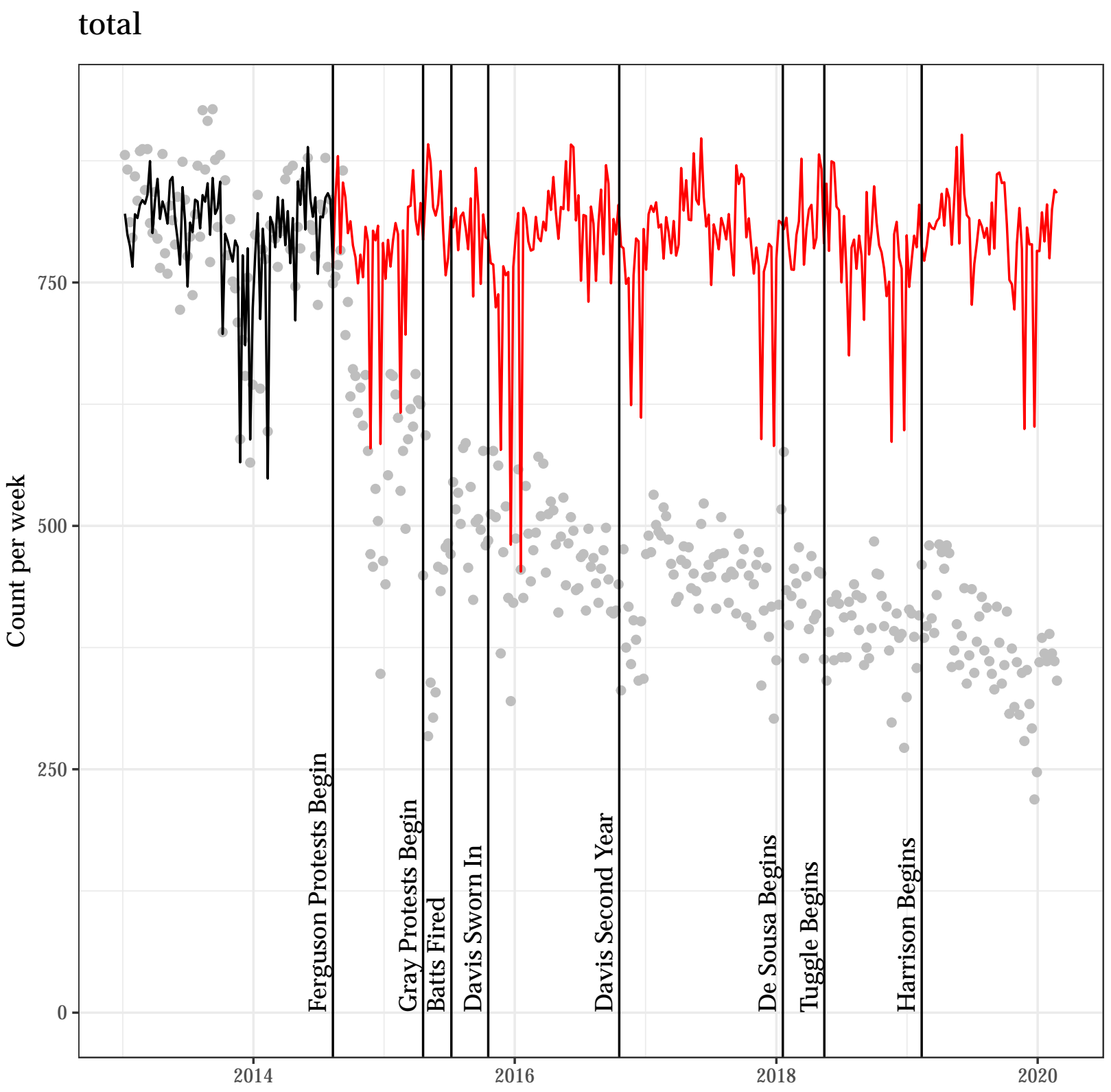




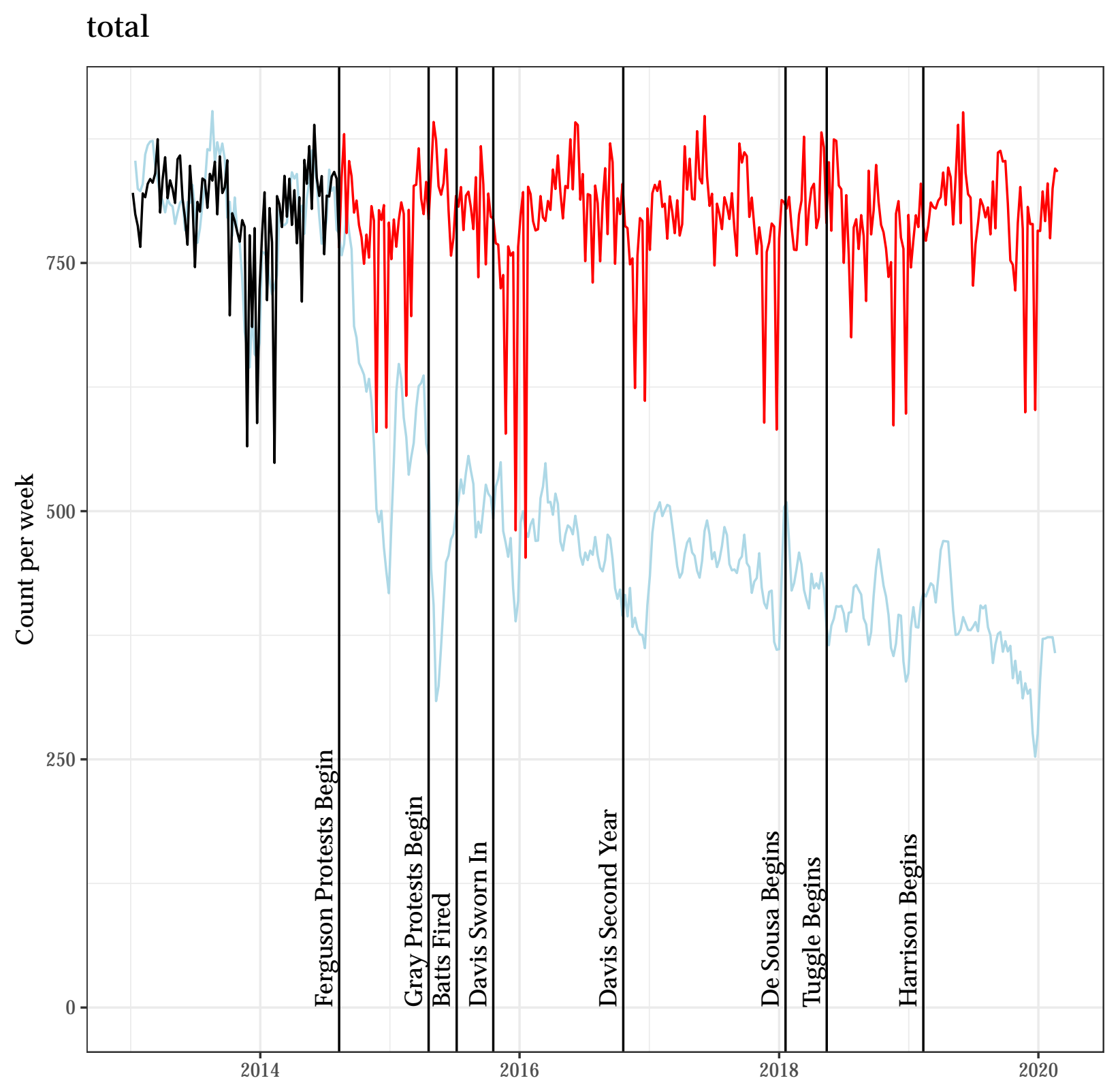


Autocorrelation Function for the Observed Outcome for Updated Model (2)

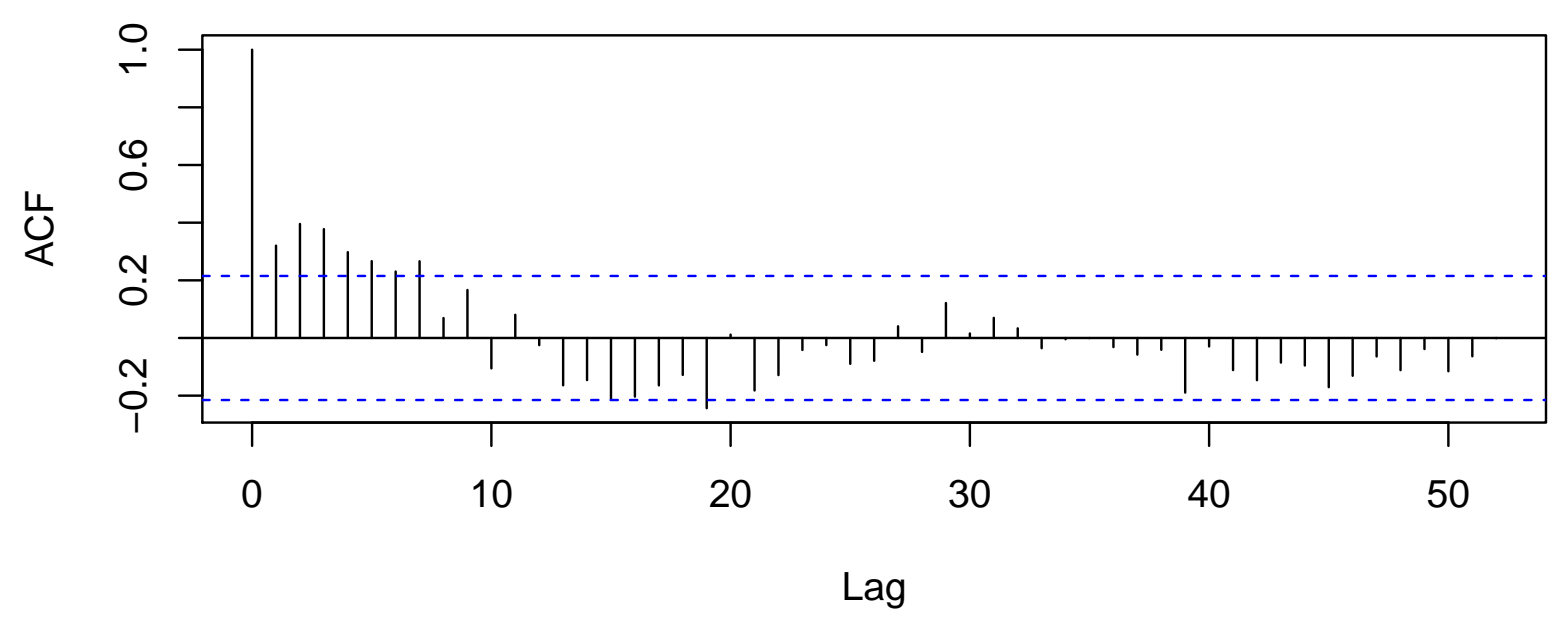

Autocorrelation Function for the Residuals from Updated Model (2)

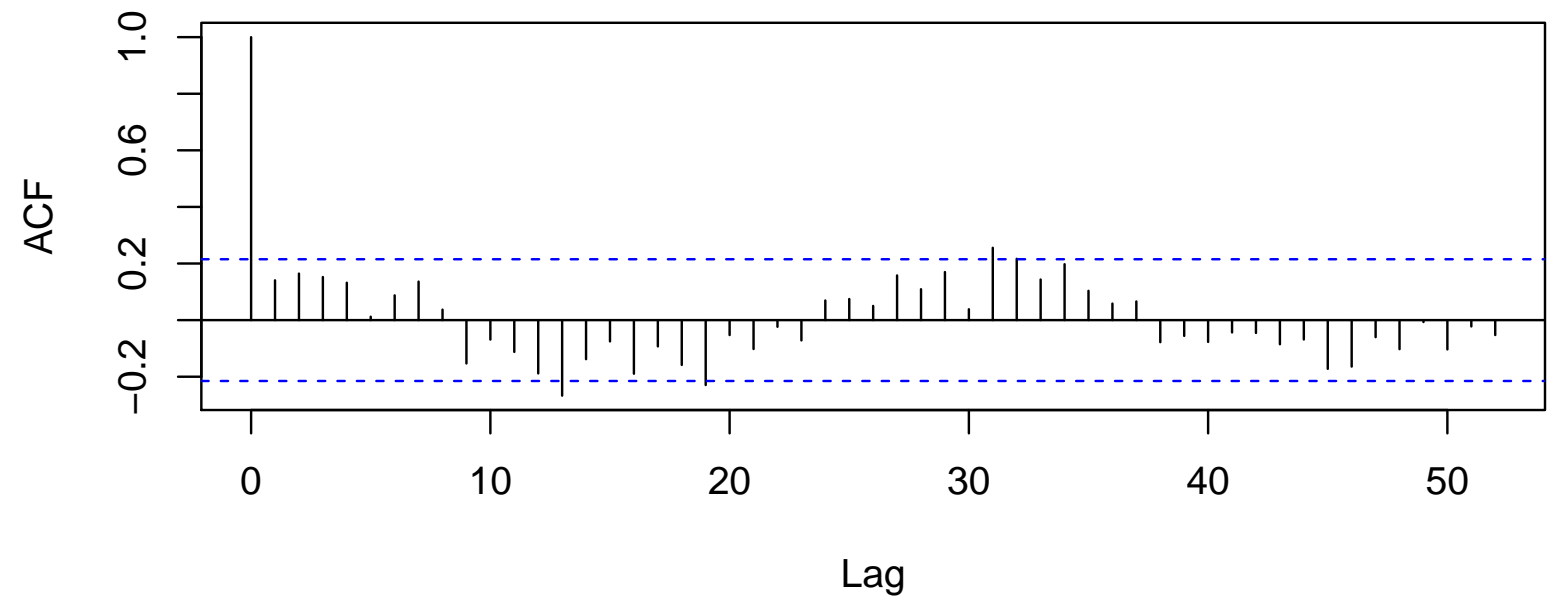




\subsection{A Poisson Regression Model as an Alternative to Model (2) for total}

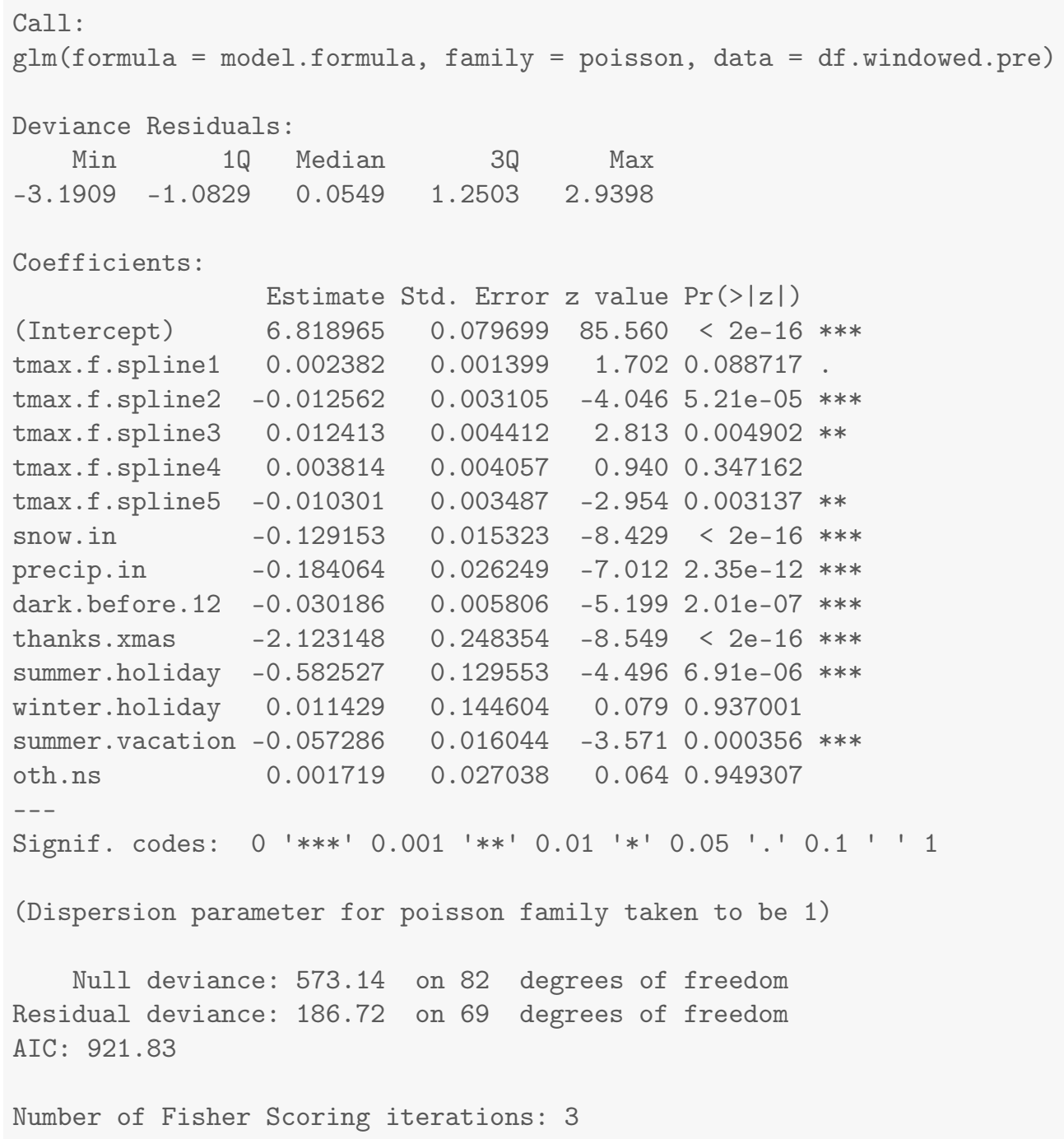




\section{Results for $b$}

\subsection{Summary Values for $b$}

Table 4.1: Descriptives for Outcome Before Ferguson Protests Begin

\begin{tabular}{lcccccccc}
\hline \hline Statistic & $\mathrm{N}$ & Mean & St. Dev. & Min & Pctl(25) & Median & Pctl(75) & Max \\
\hline $\mathrm{b}$ & 83 & 654.723 & 66.943 & 450 & 625 & 661 & 703 & 772 \\
\hline
\end{tabular}

Table 4.2: Descriptives for Outcome After Ferguson Protests Begin

\begin{tabular}{lcccccccc}
\hline \hline Statistic & $\mathrm{N}$ & Mean & St. Dev. & Min & Pctl(25) & Median & Pctl(75) & Max \\
\hline $\mathrm{b}$ & 290 & 371.410 & 75.813 & 187 & 327.2 & 365.5 & 410.8 & 676 \\
\hline
\end{tabular}




\subsection{Four Models for $b$}

Table 4.3: Four Models that Differ on the Specification of Adjustment and Intervention Variables

\begin{tabular}{|c|c|c|c|c|}
\hline & \multicolumn{4}{|c|}{ Outcome: Count Per Week } \\
\hline & (1) & $(2)$ & (3) & (4) \\
\hline After Ferguson Protests Begin (week of 8/11/14 onward) & -164.31 & & -147.16 & -150.73 \\
\hline After Gray Protests Begin (week of 4/20/15 onward) & -162.78 & & -197.48 & -188.67 \\
\hline Unrest and National Guard (4/27/15 - 5/3/2015) & 180.36 & & 164.31 & 156.70 \\
\hline After Batts Fired (week of 7/13/15 onward) & 91.51 & & 103.92 & 99.15 \\
\hline After Davis Confirmed (week of 10/19/15 onward) & -30.32 & & -10.34 & -21.77 \\
\hline After Davis First Year (week of 10/17/16 onward) & -19.89 & & -26.35 & -19.09 \\
\hline After De Sousa Begins (week of 1/19/18 onward) & -7.88 & & -22.19 & -18.98 \\
\hline After Tuggle Begins (week of 5/14/18 onward) & -38.04 & & -14.70 & -16.20 \\
\hline After Harrison Begins (week of 2/11/19 onward) & -11.57 & & -30.01 & -26.27 \\
\hline Average Maximum Temperature to 50 Degrees & & 2.38 & & 2.36 \\
\hline Plus Degrees in the 50s & & -12.50 & & -4.06 \\
\hline Plus Degrees in the 60s & & 14.43 & & 2.88 \\
\hline Plus Degrees in the 70s & & -1.09 & & -2.34 \\
\hline Plus Degrees Greater Than 80 & & -5.38 & & -0.92 \\
\hline Snowfall (inches) & & -78.91 & & -27.00 \\
\hline Precipitation (inches) & & -125.05 & & -77.39 \\
\hline Darkness Before Midnight (hours) & & -21.46 & & -11.61 \\
\hline Thanksgiving/Christmas (proportion of week) & & $-1,338.96$ & & -714.44 \\
\hline Winter Holiday (proportion of week) & & 72.71 & & 237.83 \\
\hline Other Out-of-School Days (proportion of week) & & 5.89 & & -32.74 \\
\hline Summer Vacation (proportion of week) & & -40.41 & & 4.57 \\
\hline Summer Holiday (proportion of week) & & -414.94 & & -122.92 \\
\hline Observations & 373 & 83 & 373 & 373 \\
\hline $\mathrm{R}^{2}$ & 0.84 & 0.63 & 0.84 & 0.90 \\
\hline
\end{tabular}




\subsection{Least Squares Updated Model (2) for b}

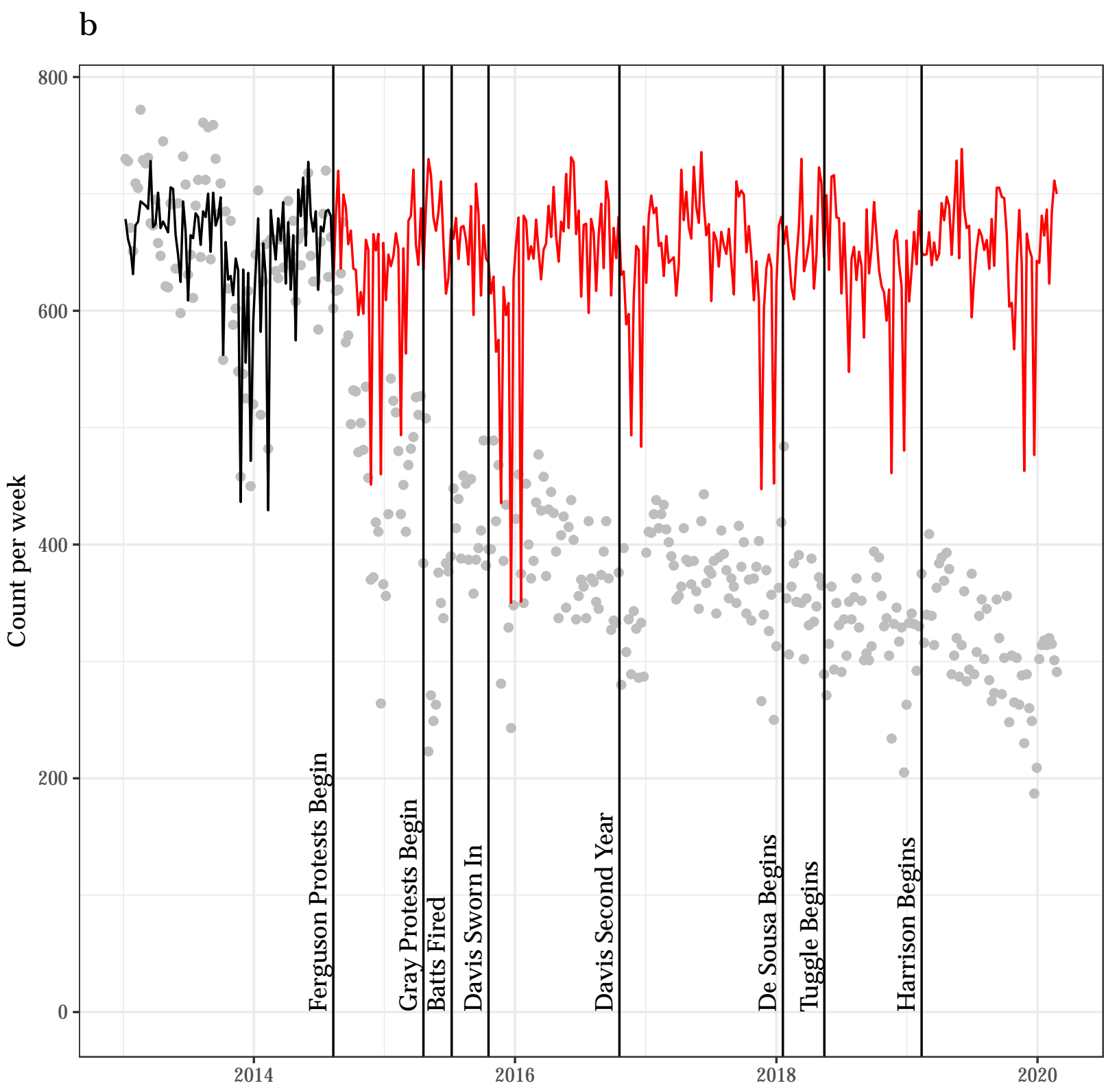




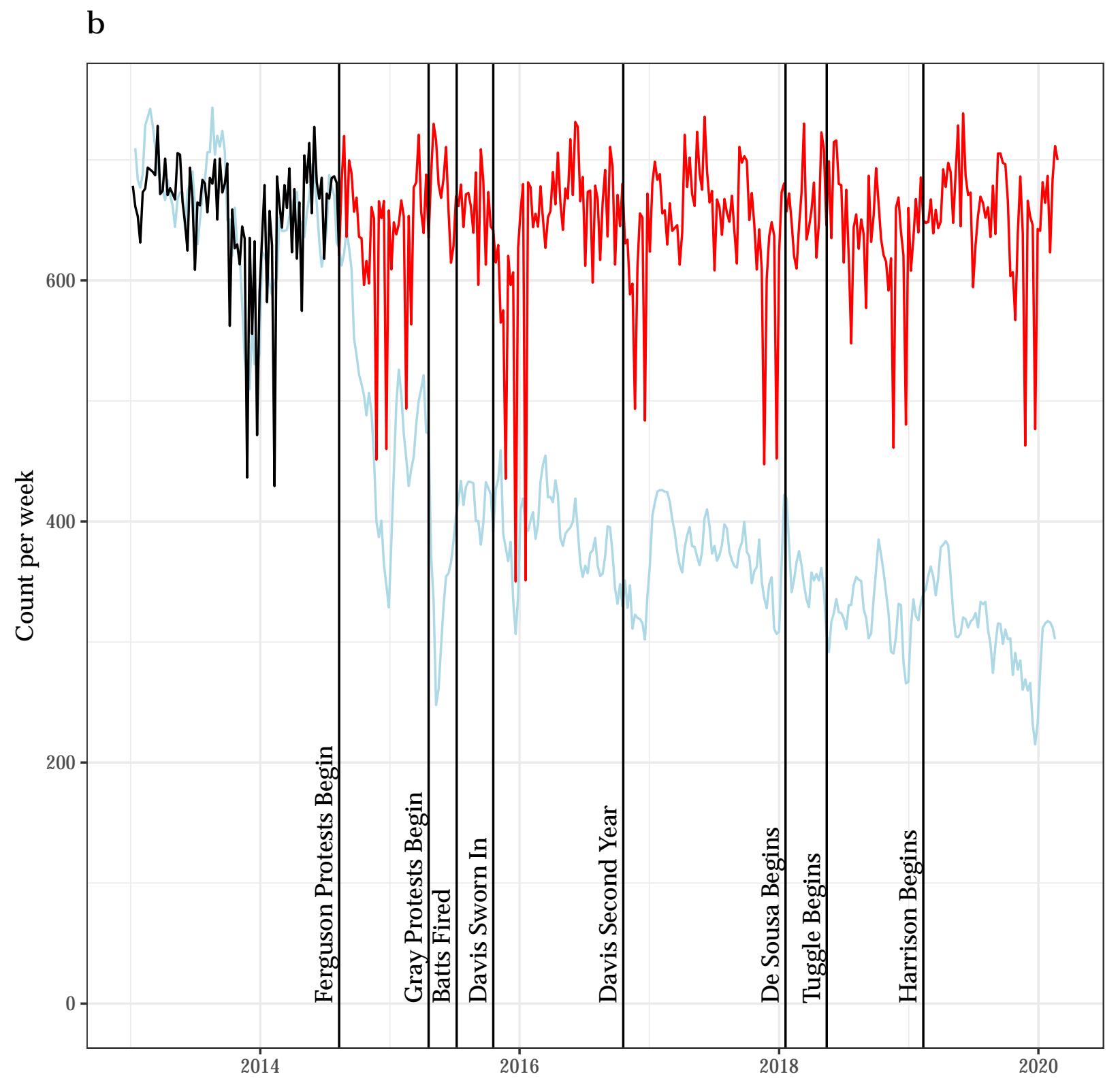


Autocorrelation Function for the Observed Outcome for Updated Model (2)

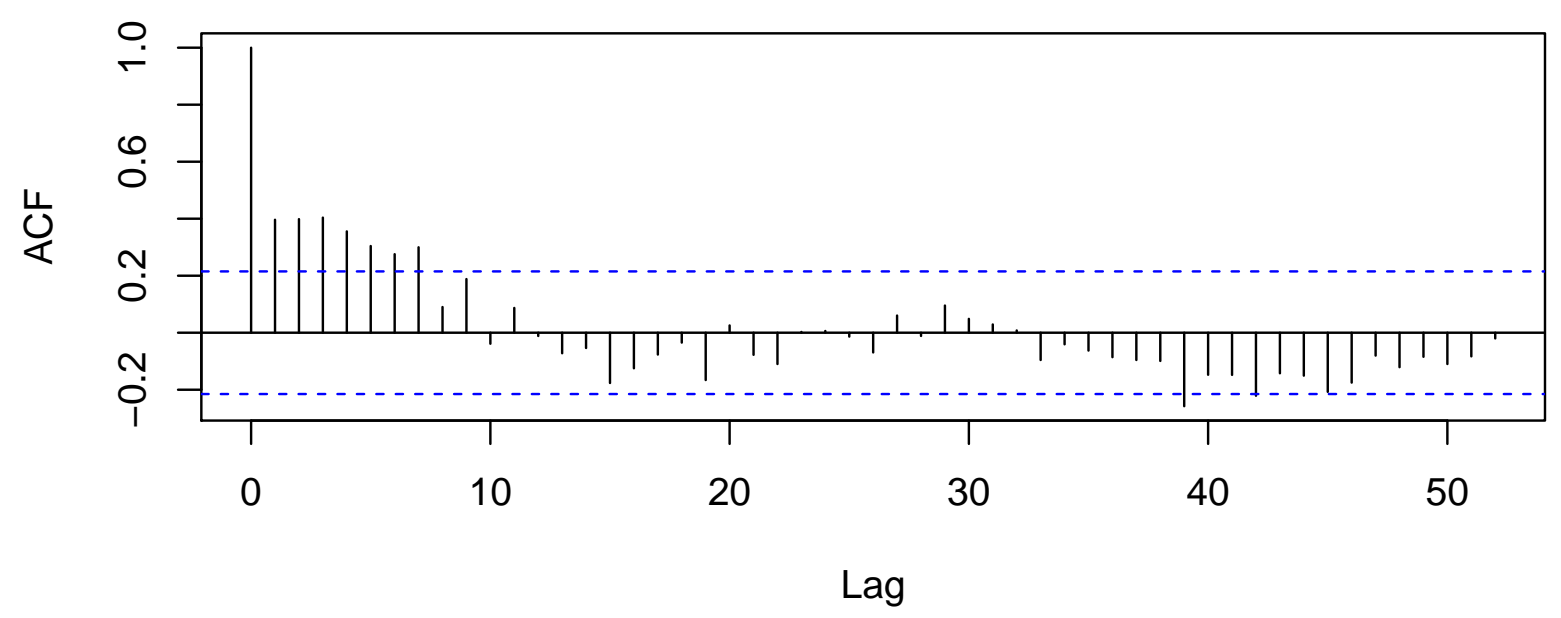

Autocorrelation Function for the Residuals from Updated Model (2)

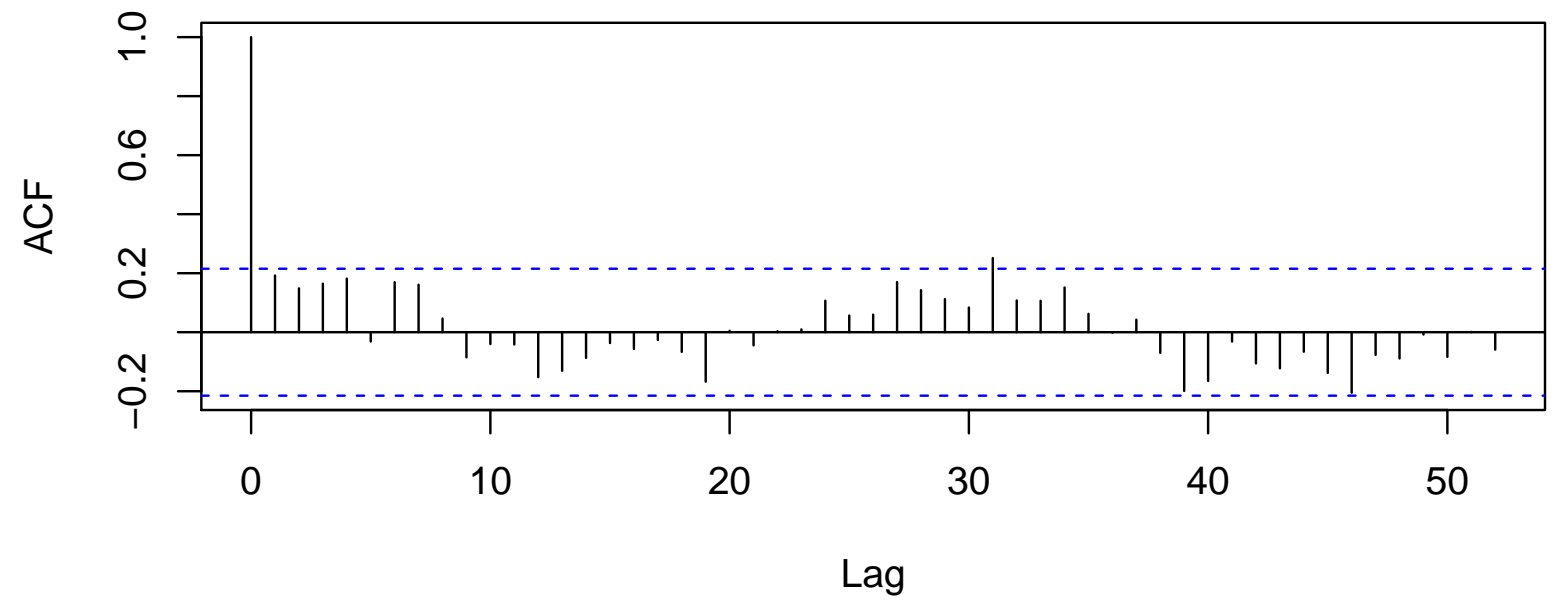




\subsection{A Poisson Regression Model as an Alternative to Model (2) for b}

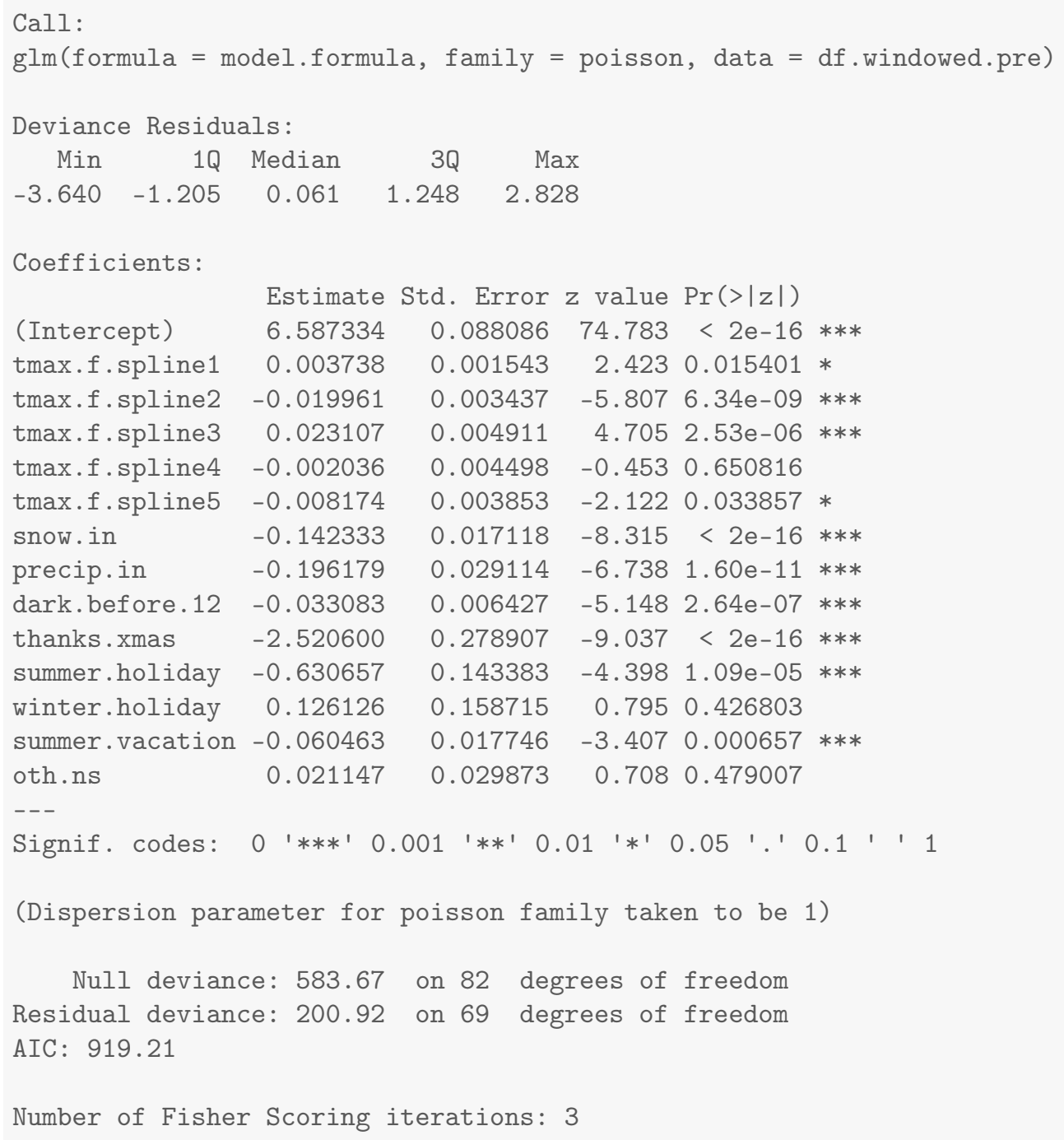




\section{Results for $w$}

\subsection{Summary Values for $w$}

Table 5.1: Descriptives for Outcome Before Ferguson Protests Begin

\begin{tabular}{lcccccccc}
\hline \hline Statistic & $\mathrm{N}$ & Mean & St. Dev. & Min & Pctl(25) & Median & Pctl(75) & Max \\
\hline $\mathrm{w}$ & 83 & 126.530 & 15.761 & 96 & 115 & 126 & 134 & 171 \\
\hline
\end{tabular}

Table 5.2: Descriptives for Outcome After Ferguson Protests Begin

\begin{tabular}{lcccccccc}
\hline \hline Statistic & $\mathrm{N}$ & Mean & St. Dev. & Min & Pctl(25) & Median & Pctl(75) & Max \\
\hline $\mathrm{w}$ & 290 & 69.152 & 20.425 & 25 & 56 & 65 & 78 & 174 \\
\hline
\end{tabular}




\subsection{Four Models for w}

Table 5.3: Four Models that Differ on the Specification of Adjustment and Intervention Variables

\begin{tabular}{|c|c|c|c|c|}
\hline & \multicolumn{4}{|c|}{ Outcome: Count Per Week } \\
\hline & (1) & $(2)$ & (3) & (4) \\
\hline After Ferguson Protests Begin (week of $8 / 11 / 14$ onward) & -20.53 & & -19.15 & -17.65 \\
\hline After Gray Protests Begin (week of 4/20/15 onward) & -37.00 & & -43.41 & -43.85 \\
\hline Unrest and National Guard (4/27/15 - 5/3/2015) & 8.00 & & 6.24 & 6.19 \\
\hline After Batts Fired (week of $7 / 13 / 15$ onward) & 13.79 & & 18.56 & 14.97 \\
\hline After Davis Confirmed (week of 10/19/15 onward) & -10.77 & & -11.36 & -8.17 \\
\hline After Davis First Year (week of 10/17/16 onward) & -9.59 & & -9.06 & -9.06 \\
\hline After De Sousa Begins (week of $1 / 19 / 18$ onward) & 3.83 & & 1.61 & 3.81 \\
\hline After Tuggle Begins (week of 5/14/18 onward) & -6.15 & & -0.04 & -4.16 \\
\hline After Harrison Begins (week of 2/11/19 onward) & -6.03 & & -8.44 & -7.78 \\
\hline Average Maximum Temperature to 50 Degrees & & -0.64 & & 0.08 \\
\hline Plus Degrees in the $50 \mathrm{~s}$ & & 2.51 & & 0.62 \\
\hline Plus Degrees in the $60 \mathrm{~s}$ & & -3.44 & & -0.88 \\
\hline Plus Degrees in the 70s & & 2.90 & & 0.30 \\
\hline Plus Degrees Greater Than 80 & & -2.68 & & -0.75 \\
\hline Snowfall (inches) & & -10.33 & & -3.48 \\
\hline Precipitation (inches) & & -18.26 & & -13.49 \\
\hline Darkness Before Midnight (hours) & & -2.52 & & -1.57 \\
\hline Thanksgiving/Christmas (proportion of week) & & -47.62 & & -52.12 \\
\hline Winter Holiday (proportion of week) & & -60.18 & & 6.00 \\
\hline Other Out-of-School Days (proportion of week) & & -11.03 & & -5.33 \\
\hline Summer Vacation (proportion of week) & & -6.06 & & 4.56 \\
\hline Summer Holiday (proportion of week) & & -72.71 & & -25.42 \\
\hline Observations & 373 & 83 & 373 & 373 \\
\hline $\mathrm{R}^{2}$ & 0.80 & 0.32 & 0.80 & 0.84 \\
\hline
\end{tabular}




\subsection{Least Squares Updated Model (2) for w}

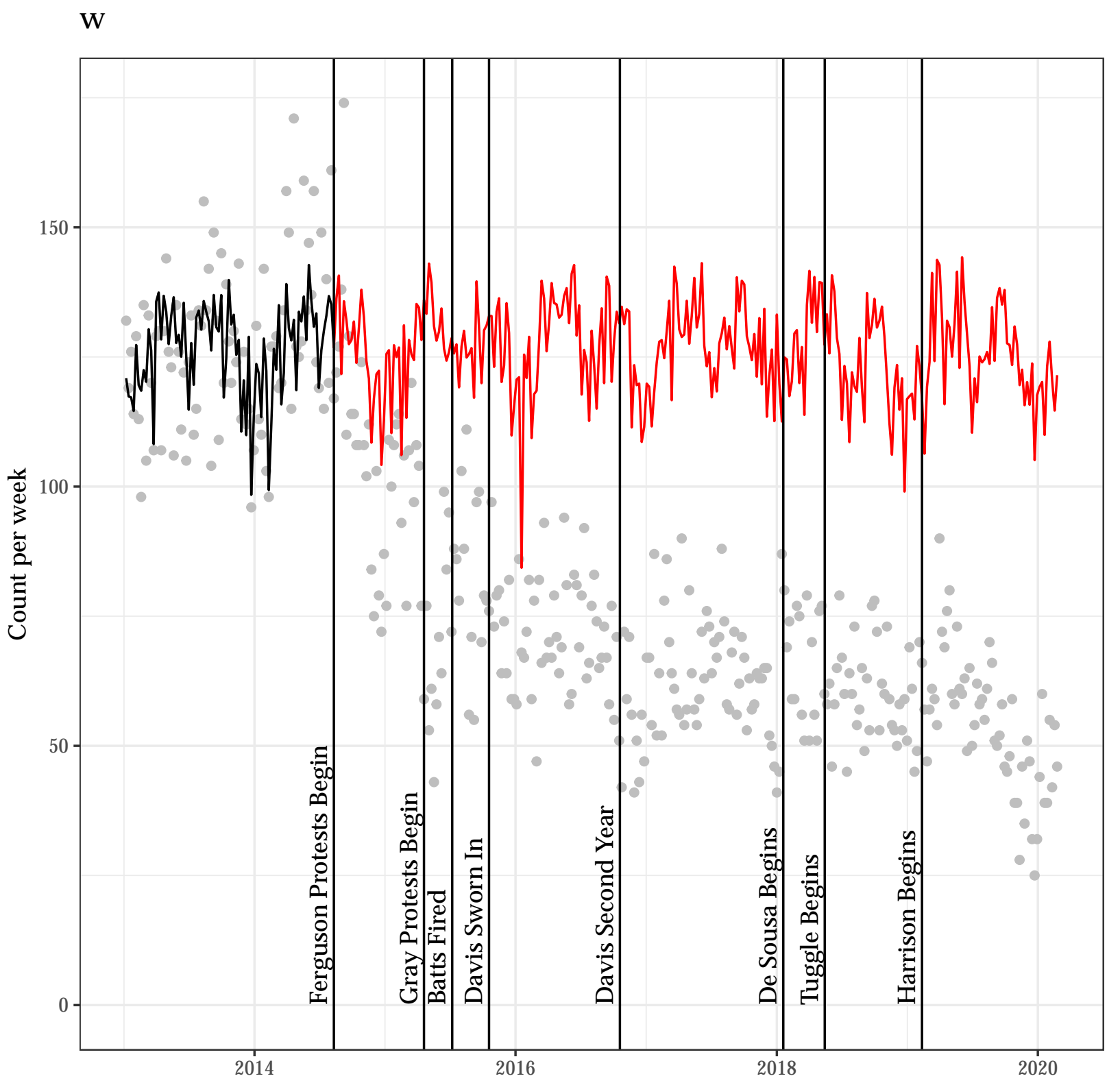




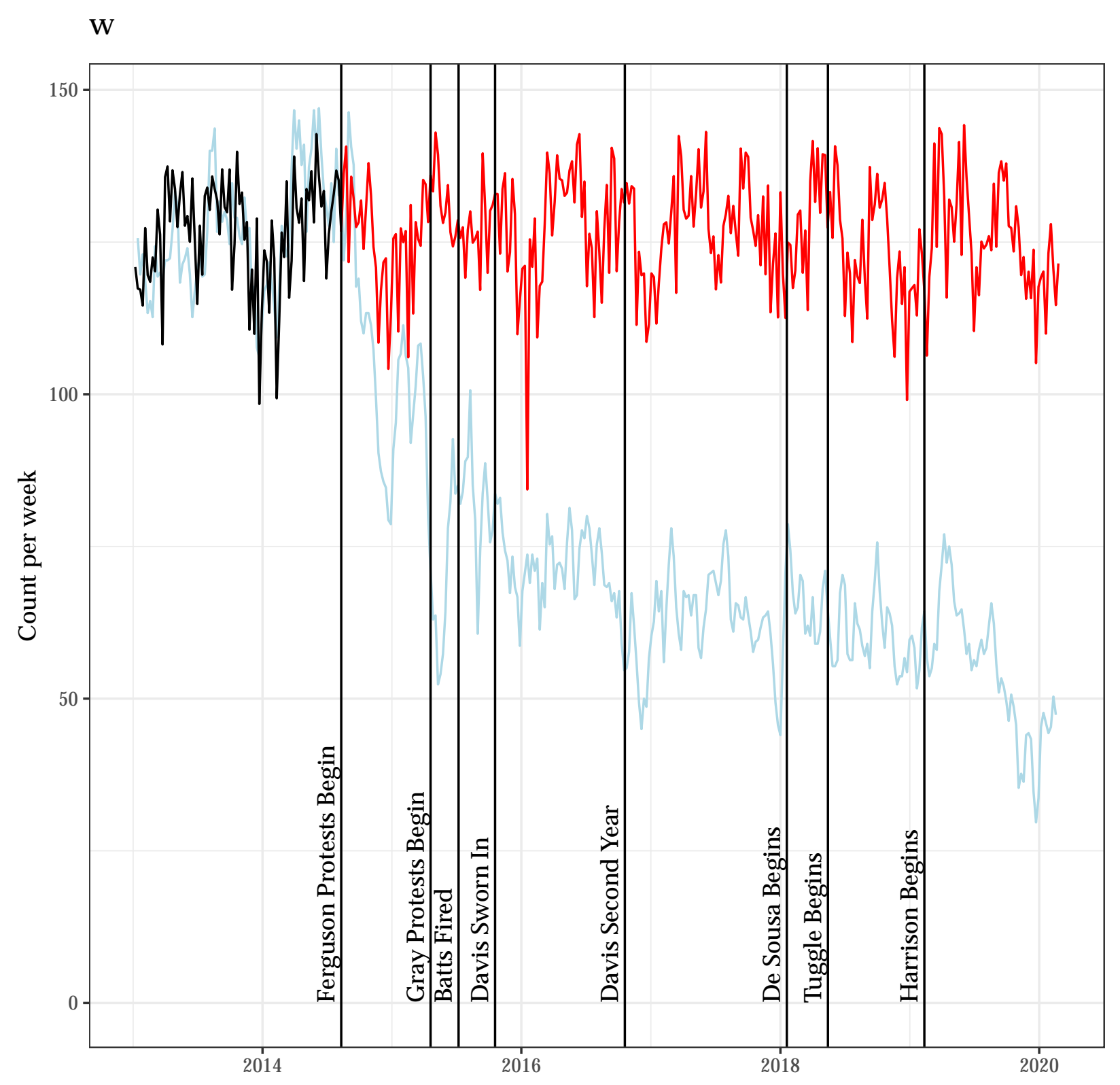


Autocorrelation Function for the Observed Outcome for Updated Model (2)

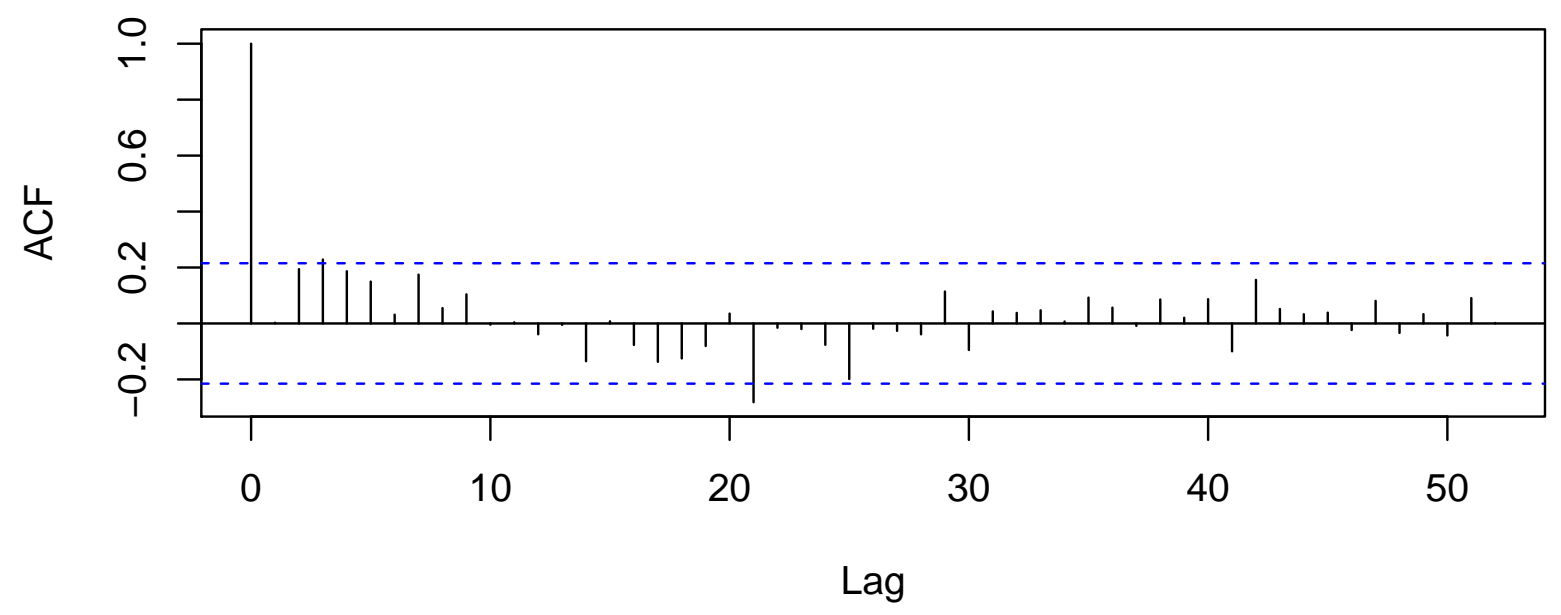

Autocorrelation Function for the Residuals from Updated Model (2)

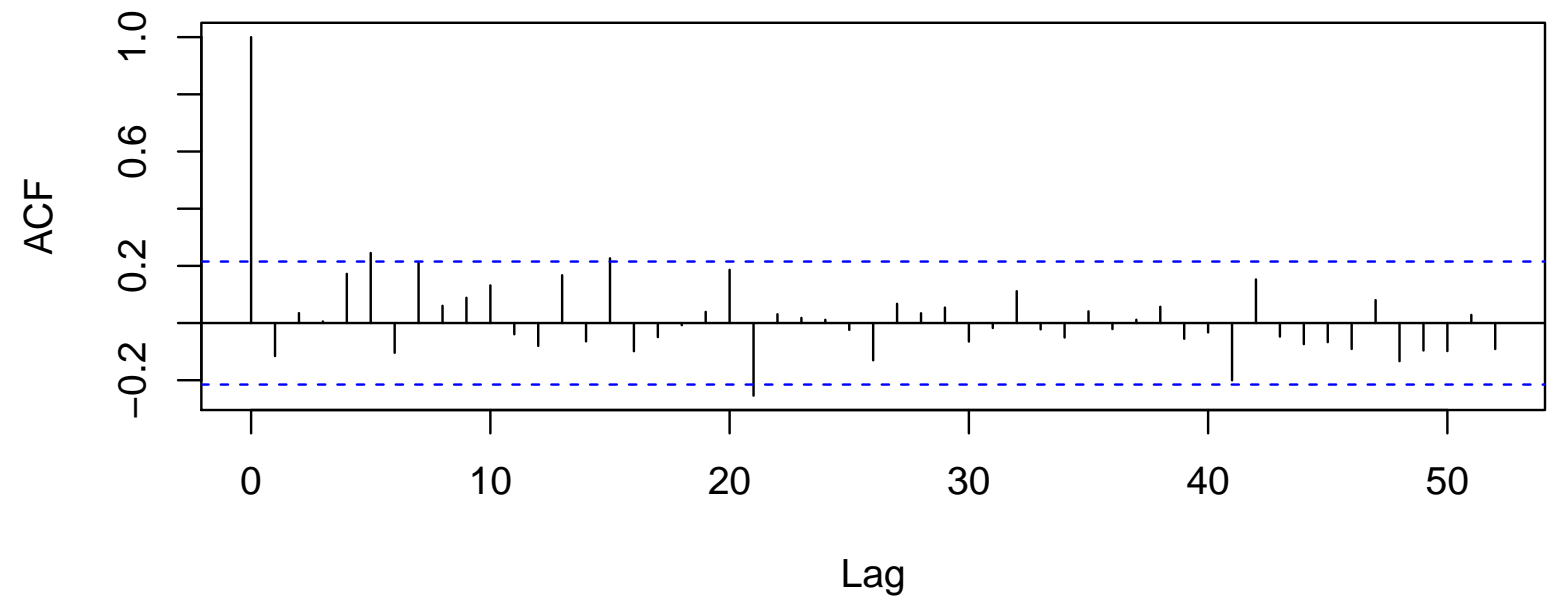




\subsection{A Poisson Regression Model as an Alternative to Model (2) for w}

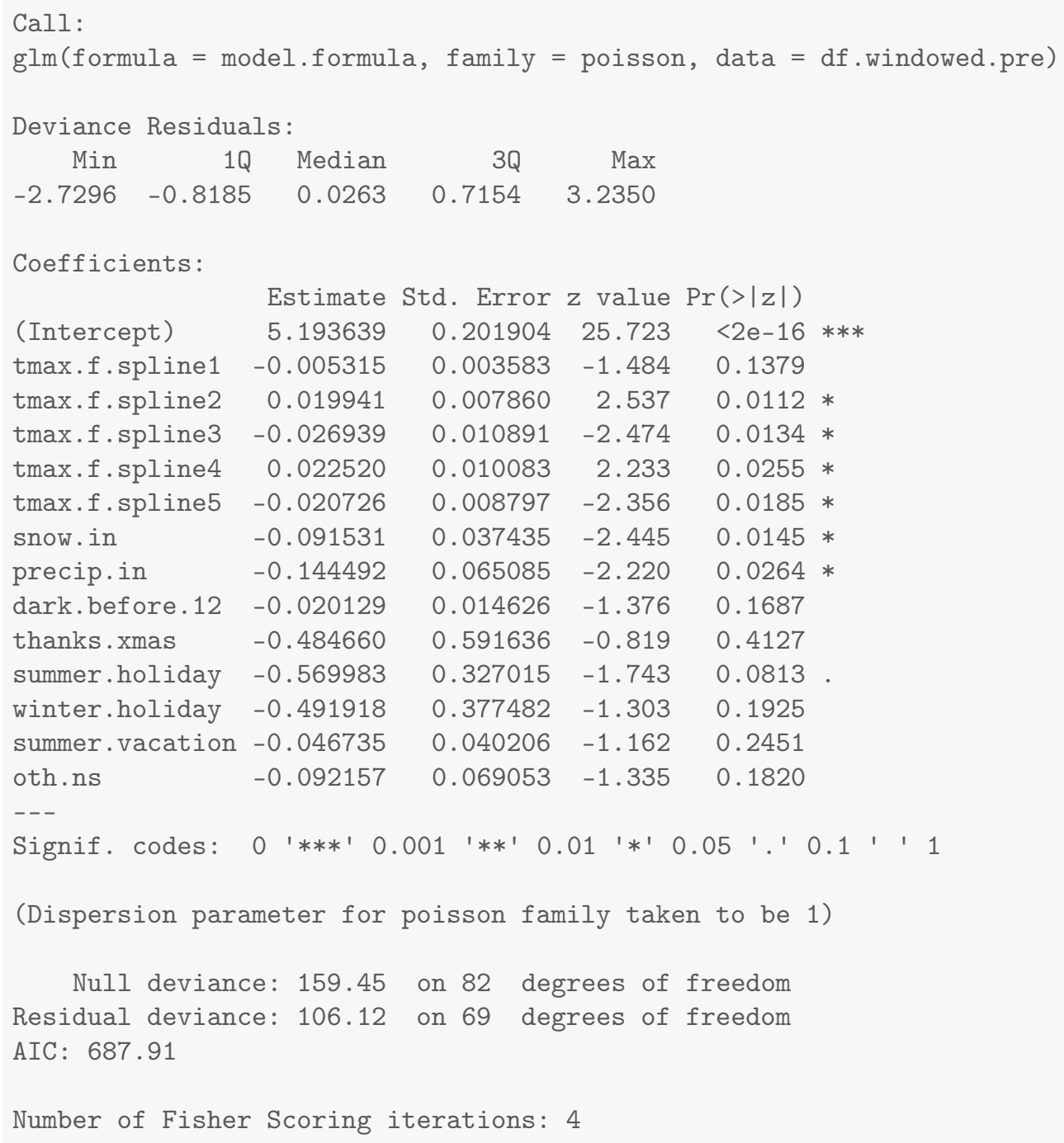




\section{Results for $u$}

\subsection{Summary Values for $\mathbf{u}$}

Table 6.1: Descriptives for Outcome Before Ferguson Protests Begin

\begin{tabular}{lcccccccc}
\hline \hline Statistic & $\mathrm{N}$ & Mean & St. Dev. & Min & $\operatorname{Pctl}(25)$ & Median & Pctl(75) & Max \\
\hline $\mathrm{u}$ & 83 & 15.940 & 5.285 & 5 & 13 & 15 & 18 & 32 \\
\hline
\end{tabular}

Table 6.2: Descriptives for Outcome After Ferguson Protests Begin

\begin{tabular}{lcccccccc}
\hline \hline Statistic & $\mathrm{N}$ & Mean & St. Dev. & Min & Pctl(25) & Median & Pctl(75) & Max \\
\hline $\mathrm{u}$ & 290 & 9.610 & 3.823 & 2 & 7 & 9 & 12 & 28 \\
\hline
\end{tabular}




\subsection{Four Models for $\mathrm{u}$}

Table 6.3: Four Models that Differ on the Specification of Adjustment and Intervention Variables

\begin{tabular}{|c|c|c|c|c|}
\hline & \multicolumn{4}{|c|}{ Outcome: Count Per Week } \\
\hline & $(1)$ & $(2)$ & (3) & $(4)$ \\
\hline After Ferguson Protests Begin (week of 8/11/14 onward) & -3.22 & & -3.56 & -3.07 \\
\hline After Gray Protests Begin (week of $4 / 20 / 15$ onward) & -4.72 & & -3.31 & -4.61 \\
\hline Unrest and National Guard (4/27/15 - 5/3/2015) & -4.00 & & -2.49 & -3.94 \\
\hline After Batts Fired (week of 7/13/15 onward) & 3.57 & & 3.16 & 3.45 \\
\hline After Davis Confirmed (week of 10/19/15 onward) & -1.30 & & -2.88 & -1.63 \\
\hline After Davis First Year (week of 10/17/16 onward) & -1.60 & & -1.47 & -1.63 \\
\hline After De Sousa Begins (week of $1 / 19 / 18$ onward) & -0.04 & & -1.02 & -0.23 \\
\hline After Tuggle Begins (week of 5/14/18 onward) & -0.37 & & 0.81 & 0.08 \\
\hline After Harrison Begins (week of 2/11/19 onward) & 1.00 & & 1.01 & 0.82 \\
\hline Average Maximum Temperature to 50 Degrees & & 0.26 & & 0.18 \\
\hline Plus Degrees in the $50 \mathrm{~s}$ & & 0.20 & & -0.12 \\
\hline Plus Degrees in the 60s & & -1.42 & & -0.25 \\
\hline Plus Degrees in the 70s & & 1.28 & & 0.24 \\
\hline Plus Degrees Greater Than 80 & & -0.28 & & -0.14 \\
\hline Snowfall (inches) & & 1.17 & & 0.90 \\
\hline Precipitation (inches) & & 0.13 & & -0.99 \\
\hline Darkness Before Midnight (hours) & & 0.76 & & 0.18 \\
\hline Thanksgiving/Christmas (proportion of week) & & -1.76 & & 3.64 \\
\hline Winter Holiday (proportion of week) & & -15.75 & & -5.76 \\
\hline Other Out-of-School Days (proportion of week) & & -1.50 & & -0.42 \\
\hline Summer Vacation (proportion of week) & & 0.55 & & 1.24 \\
\hline Summer Holiday (proportion of week) & & 11.11 & & -2.22 \\
\hline Observations & 373 & 83 & 373 & 373 \\
\hline $\mathrm{R}^{2}$ & 0.35 & 0.20 & 0.36 & 0.38 \\
\hline
\end{tabular}




\subsection{Least Squares Updated Model (2) for u}

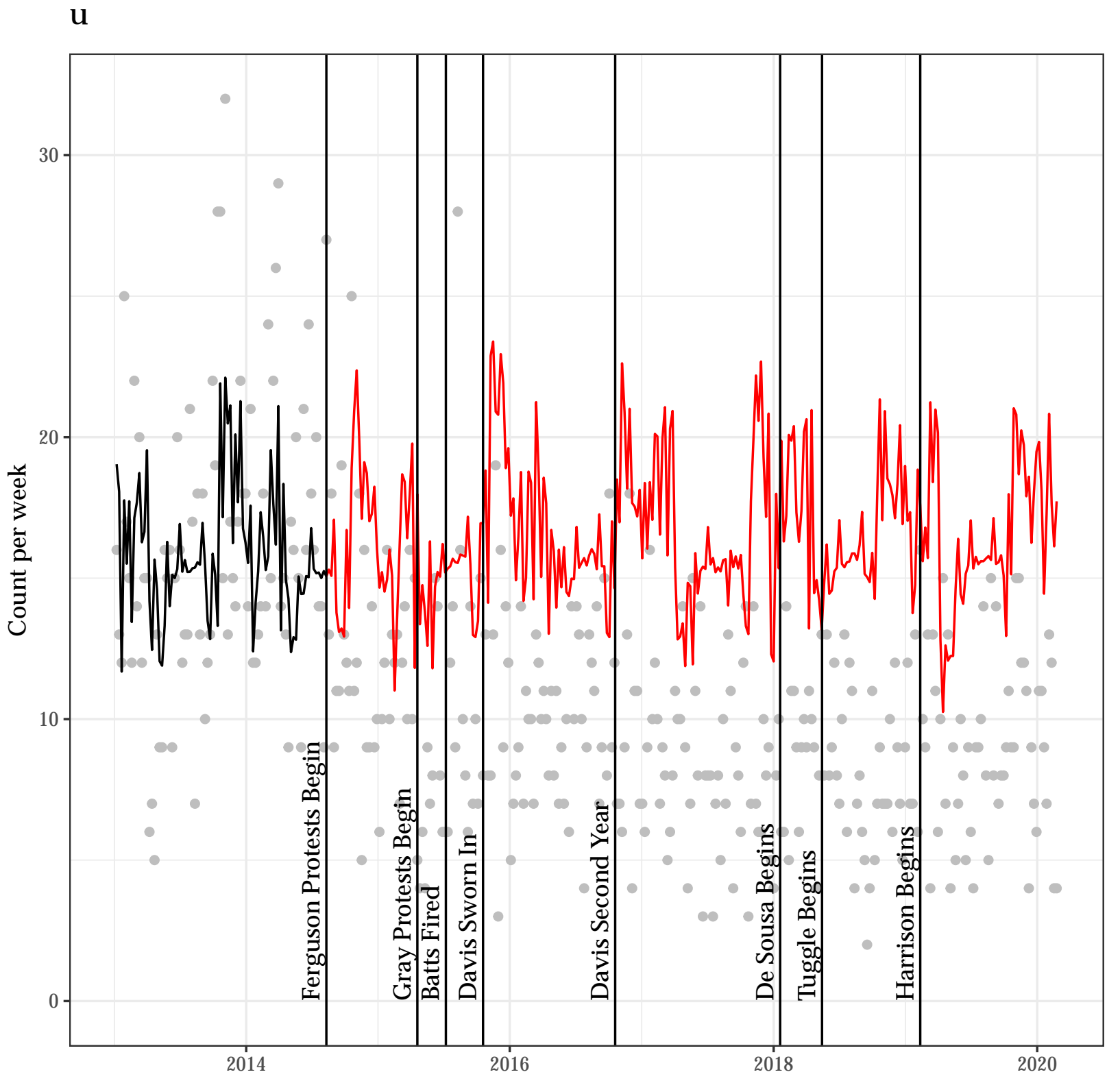




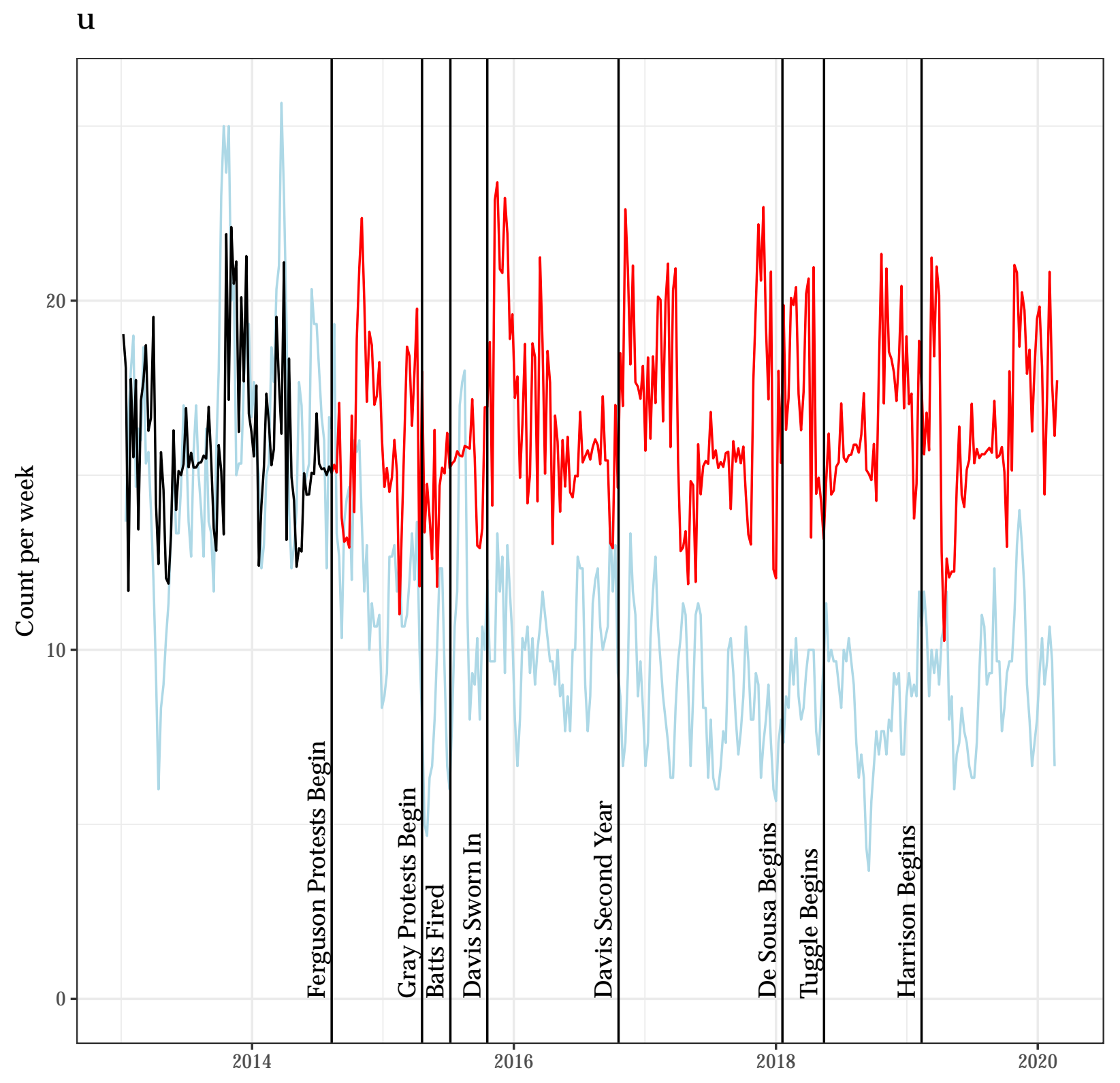


Autocorrelation Function for the Observed Outcome for Updated Model (2)

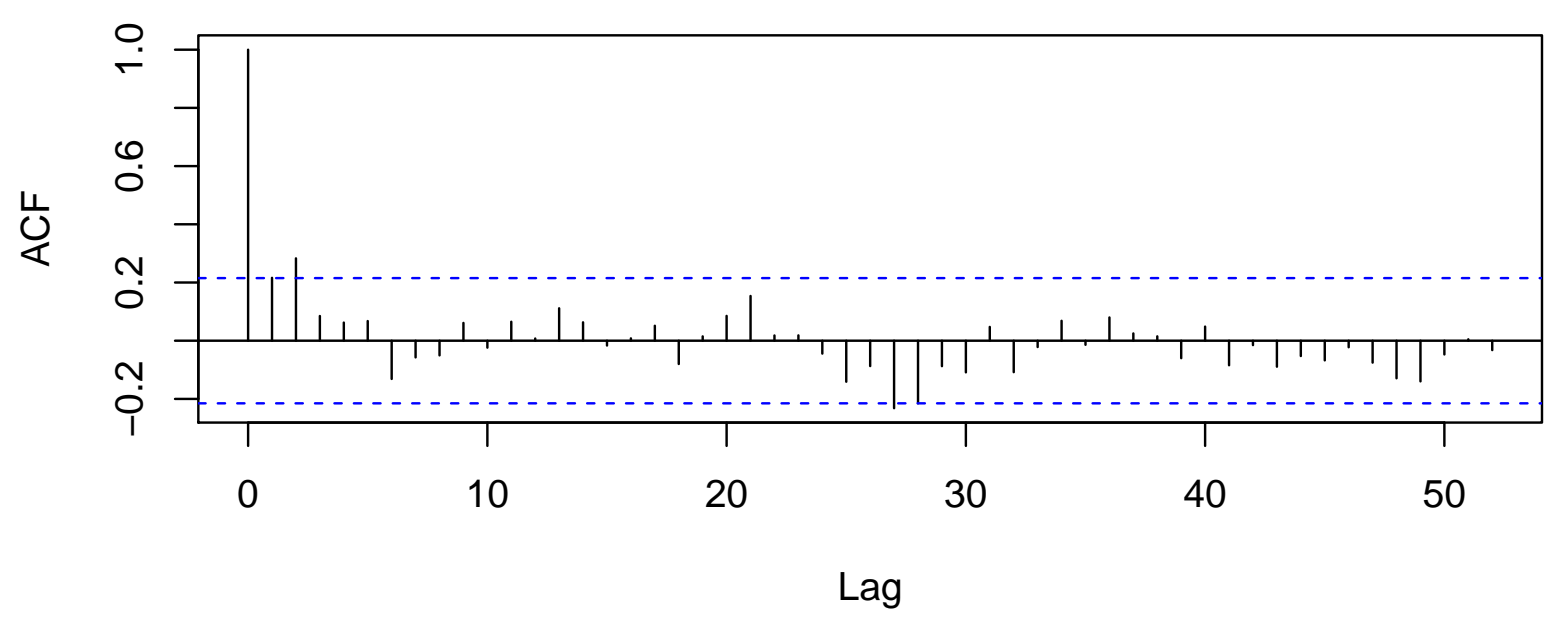

Autocorrelation Function for the Residuals from Updated Model (2)

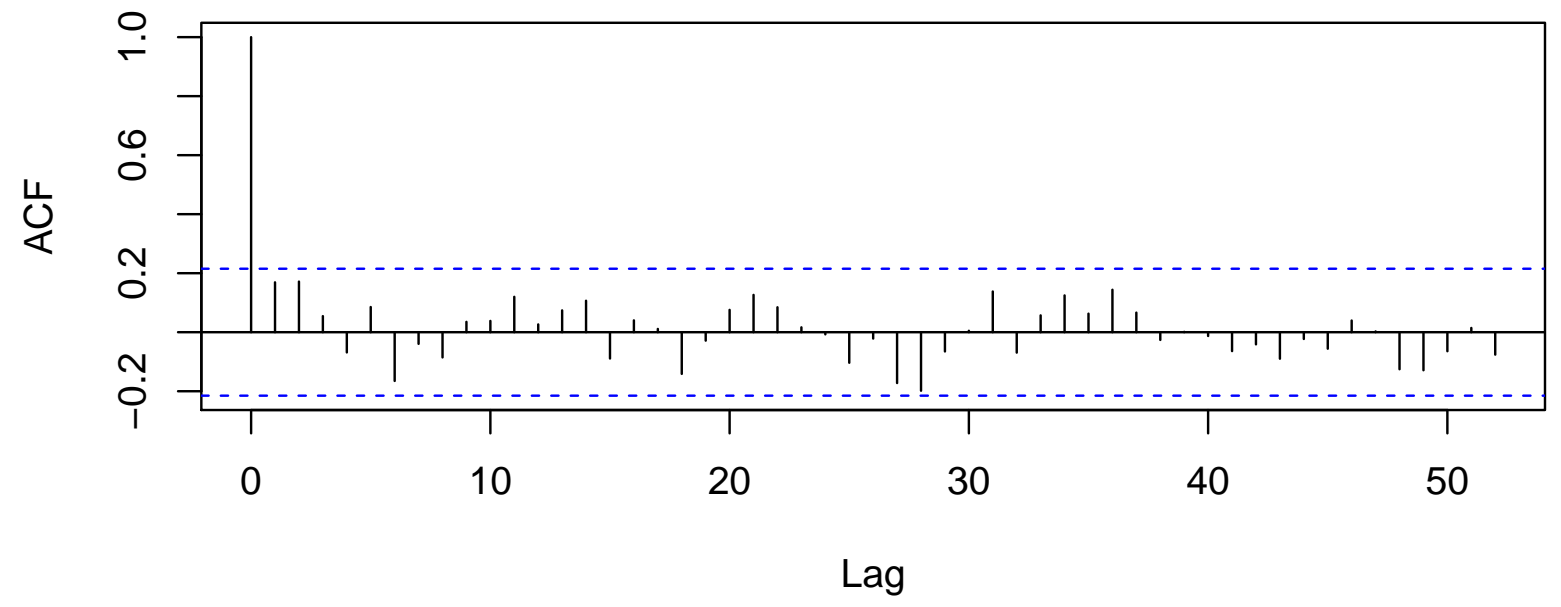




\subsection{A Poisson Regression Model as an Alternative to Model (2) for u}

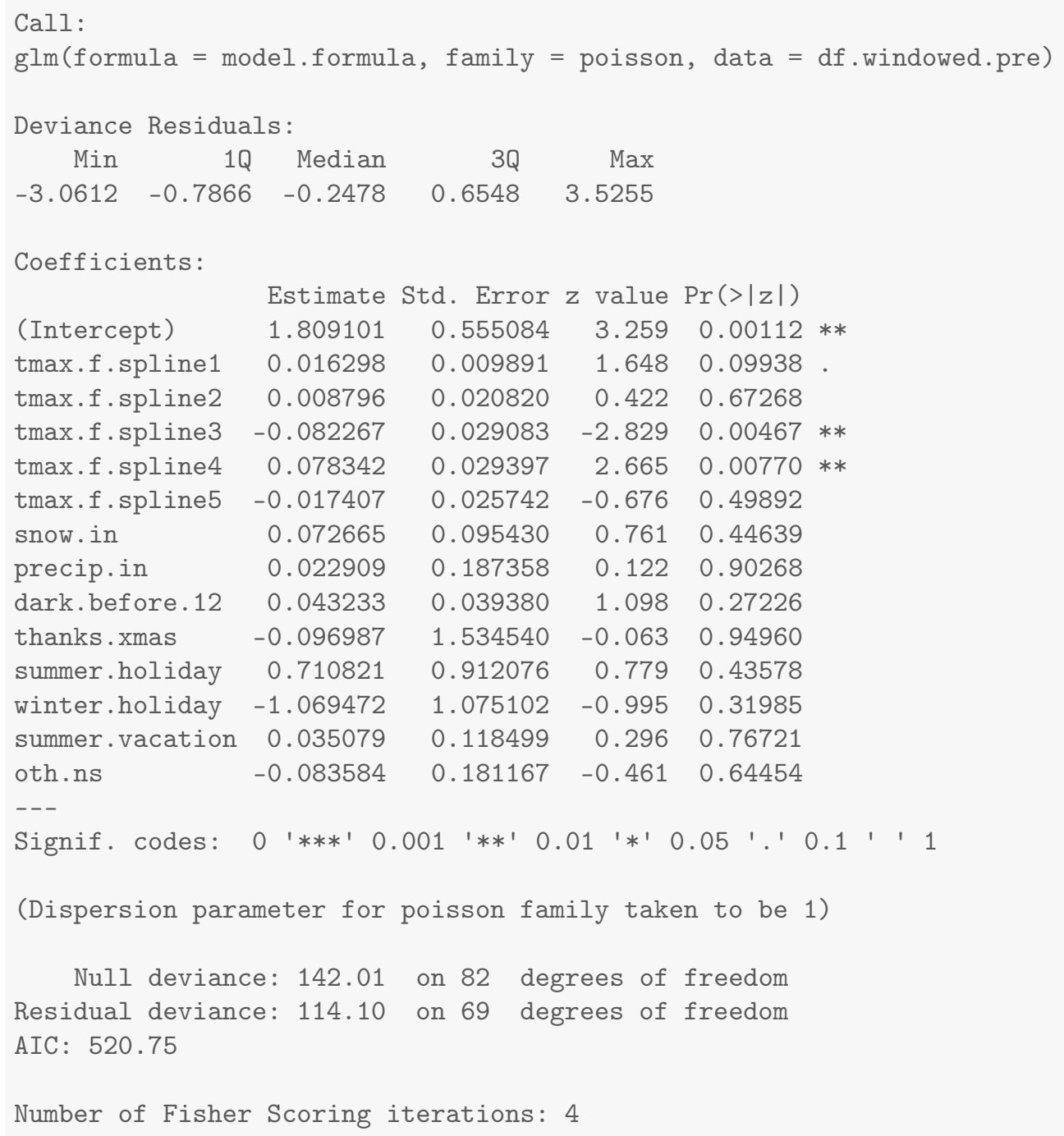




\section{Results for $\mathrm{i}$}

\subsection{Summary Values for $i$}

Table 7.1: Descriptives for Outcome Before Ferguson Protests Begin

\begin{tabular}{lcccccccc}
\hline \hline Statistic & $\mathrm{N}$ & Mean & St. Dev. & Min & Pctl(25) & Median & Pctl(75) & Max \\
\hline $\mathrm{i}$ & 83 & 1.759 & 1.303 & 0 & 1 & 2 & 2.5 & 6 \\
\hline
\end{tabular}

Table 7.2: Descriptives for Outcome After Ferguson Protests Begin

\begin{tabular}{lcccccccc}
\hline \hline Statistic & $\mathrm{N}$ & Mean & St. Dev. & Min & Pctl(25) & Median & Pctl(75) & Max \\
\hline $\mathrm{i}$ & 290 & 1.310 & 1.436 & 0 & 0 & 1 & 2 & 8 \\
\hline
\end{tabular}




\subsection{Four Models for i}

Table 7.3: Four Models that Differ on the Specification of Adjustment and Intervention Variables

\begin{tabular}{|c|c|c|c|c|}
\hline & \multicolumn{4}{|c|}{ Outcome: Count Per Week } \\
\hline & $(1)$ & $(2)$ & (3) & $(4)$ \\
\hline After Ferguson Protests Begin (week of $8 / 11 / 14$ onward) & -0.04 & & 0.10 & -0.09 \\
\hline After Gray Protests Begin (week of $4 / 20 / 15$ onward) & -0.09 & & -0.54 & 0.04 \\
\hline Unrest and National Guard (4/27/15 - 5/3/2015) & 1.36 & & 1.38 & 1.09 \\
\hline After Batts Fired (week of $7 / 13 / 15$ onward) & 1.36 & & 1.75 & 1.44 \\
\hline After Davis Confirmed (week of 10/19/15 onward) & -1.33 & & -1.45 & -1.43 \\
\hline After Davis First Year (week of 10/17/16 onward) & -0.75 & & -0.52 & -0.74 \\
\hline After De Sousa Begins (week of 1/19/18 onward) & 0.76 & & 0.29 & 0.63 \\
\hline After Tuggle Begins (week of 5/14/18 onward) & -0.94 & & -0.43 & -0.68 \\
\hline After Harrison Begins (week of 2/11/19 onward) & 0.18 & & 0.09 & 0.08 \\
\hline Average Maximum Temperature to 50 Degrees & & -0.09 & & -0.05 \\
\hline Plus Degrees in the $50 \mathrm{~s}$ & & 0.25 & & 0.06 \\
\hline Plus Degrees in the 60s & & -0.39 & & -0.05 \\
\hline Plus Degrees in the 70s & & 0.23 & & 0.01 \\
\hline Plus Degrees Greater Than 80 & & 0.07 & & 0.02 \\
\hline Snowfall (inches) & & -0.42 & & -0.08 \\
\hline Precipitation (inches) & & -0.36 & & -0.66 \\
\hline Darkness Before Midnight (hours) & & -0.60 & & -0.16 \\
\hline Thanksgiving/Christmas (proportion of week) & & 3.33 & & -2.25 \\
\hline Winter Holiday (proportion of week) & & 0.27 & & -2.16 \\
\hline Other Out-of-School Days (proportion of week) & & 0.62 & & 0.46 \\
\hline Summer Vacation (proportion of week) & & -0.25 & & 0.19 \\
\hline Summer Holiday (proportion of week) & & 2.60 & & -0.23 \\
\hline Observations & 373 & 83 & 373 & 373 \\
\hline $\mathrm{R}^{2}$ & 0.14 & 0.22 & 0.10 & 0.16 \\
\hline
\end{tabular}




\subsection{Least Squares Updated Model (2) for i}

i

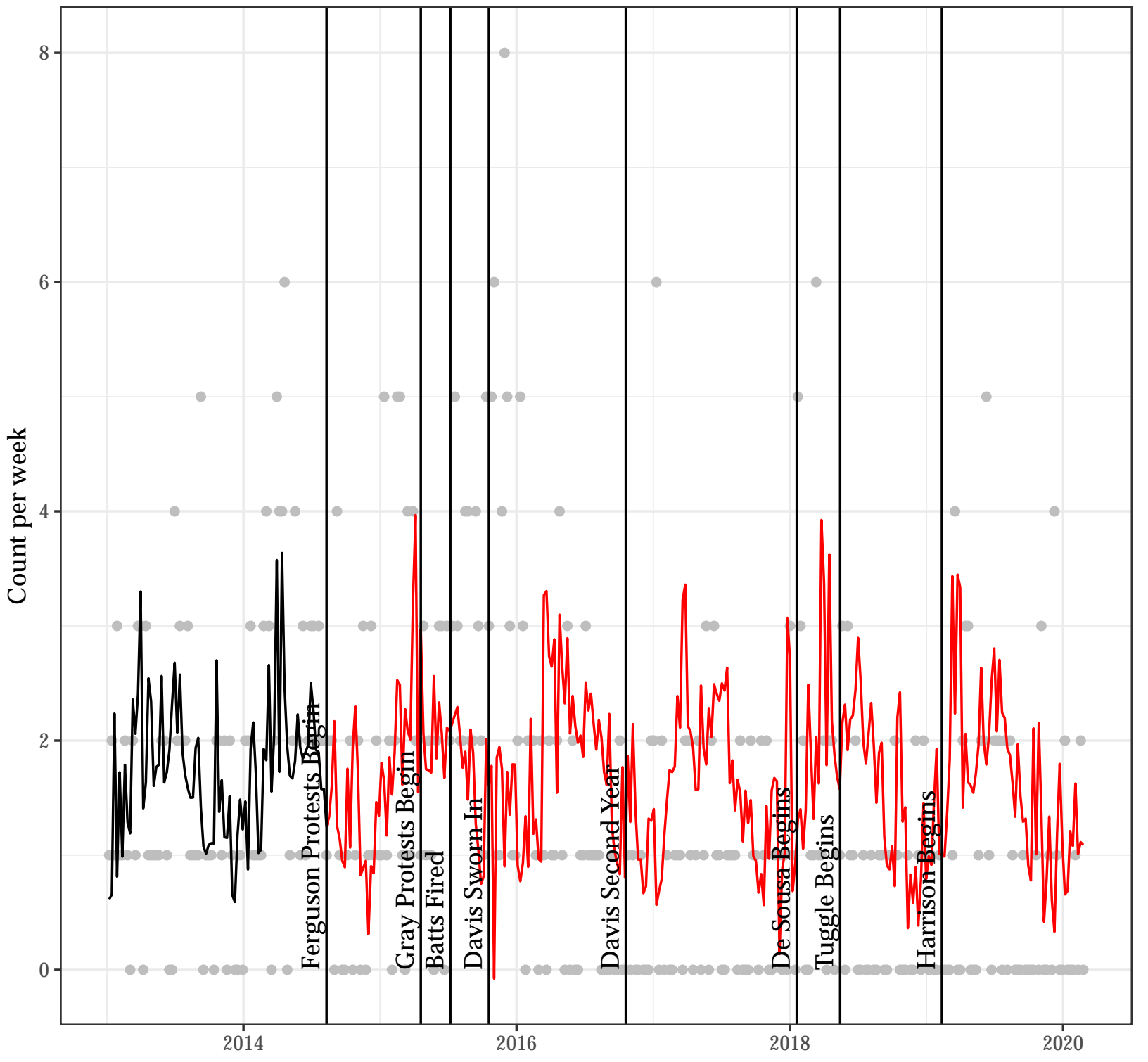




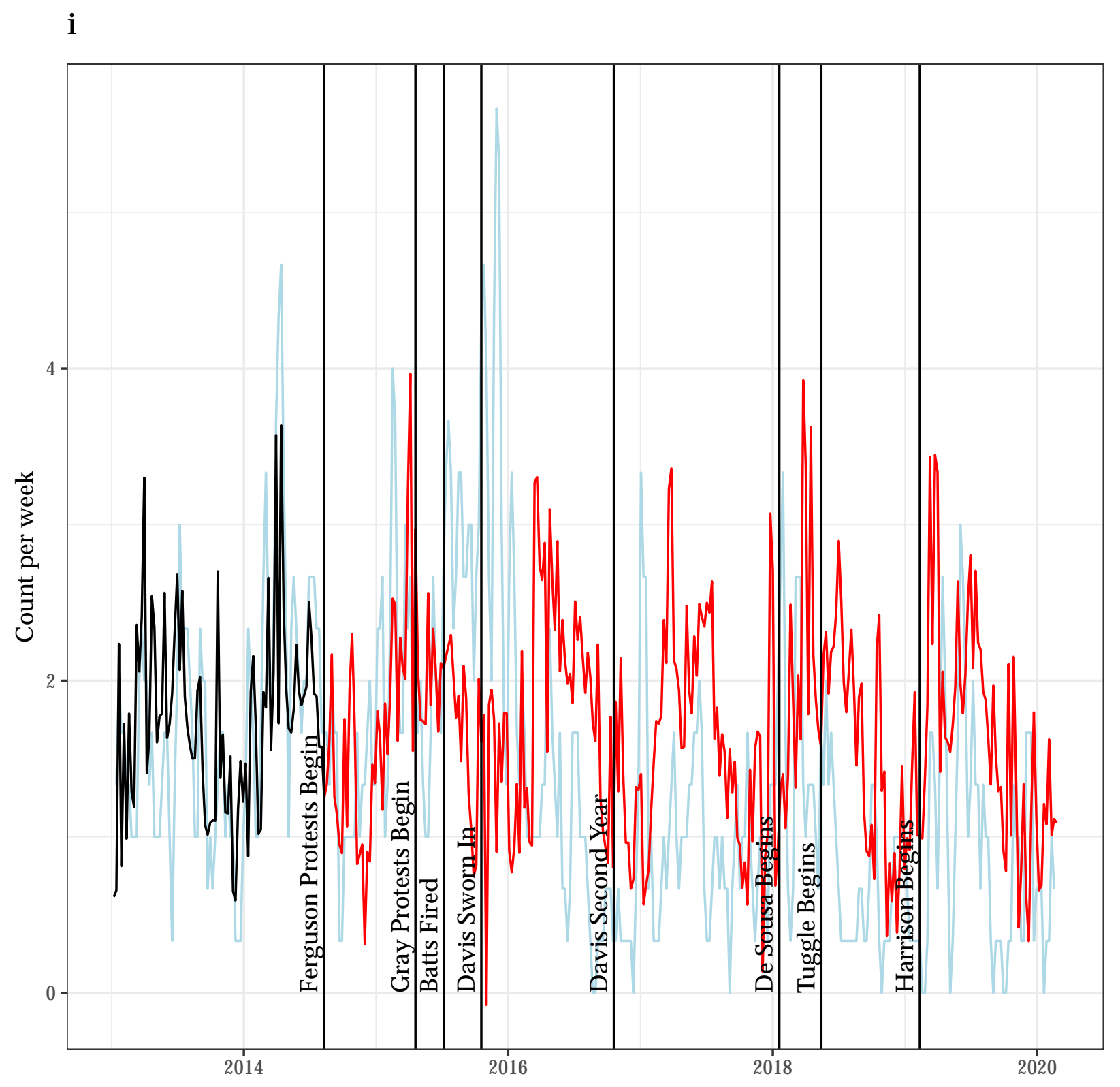


Autocorrelation Function for the Observed Outcome for Updated Model (2)

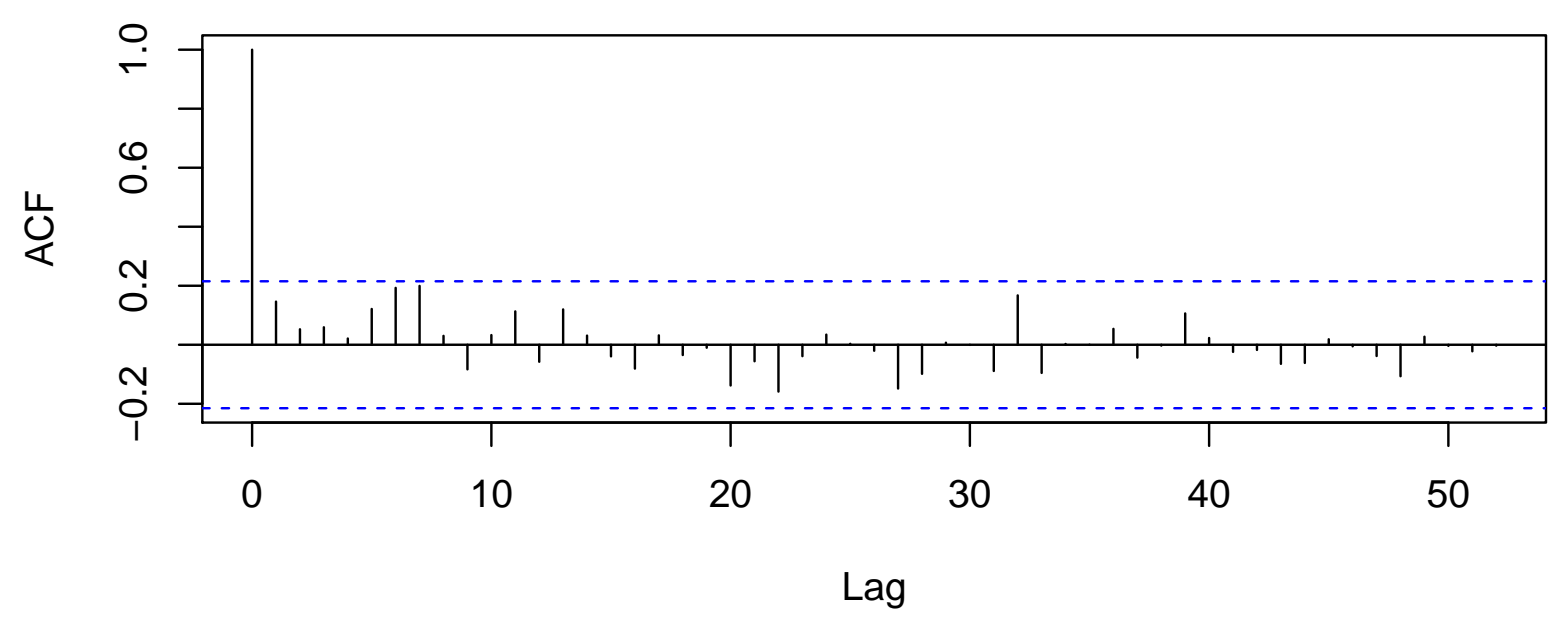

Autocorrelation Function for the Residuals from Updated Model (2)

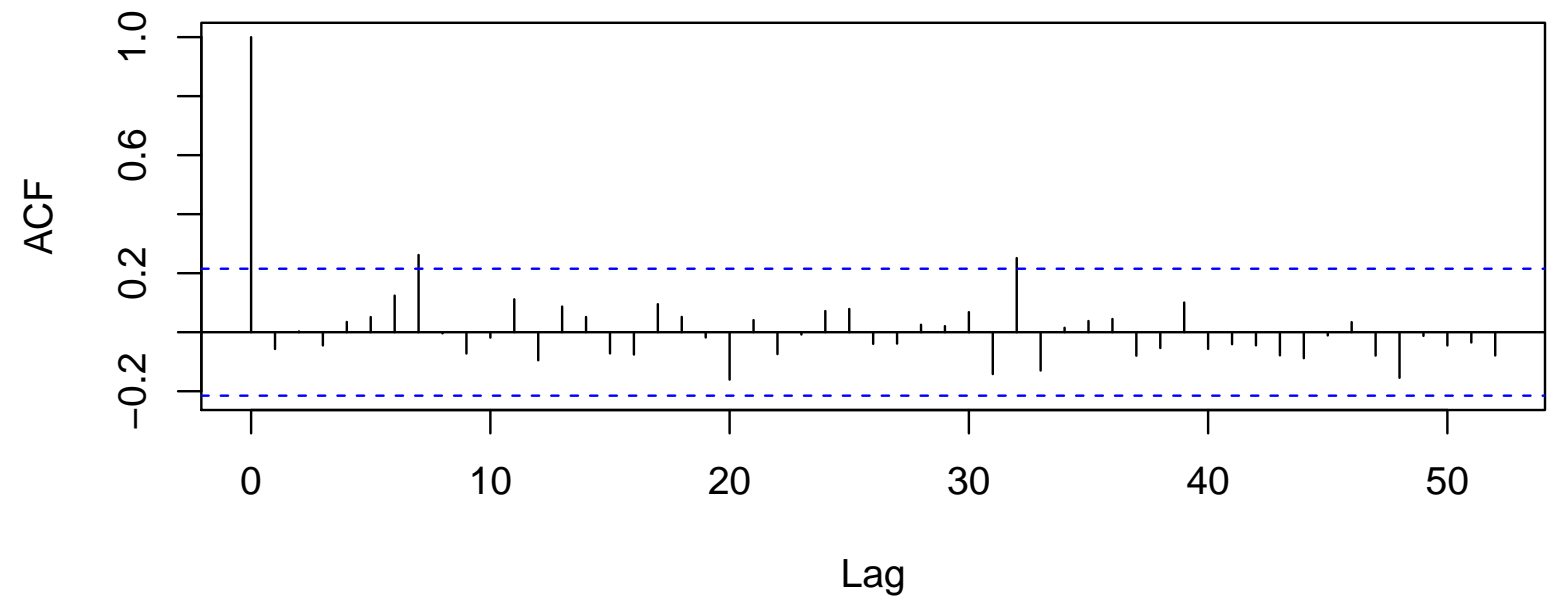




\subsection{A Poisson Regression Model as an Alternative to Model (2) for i}

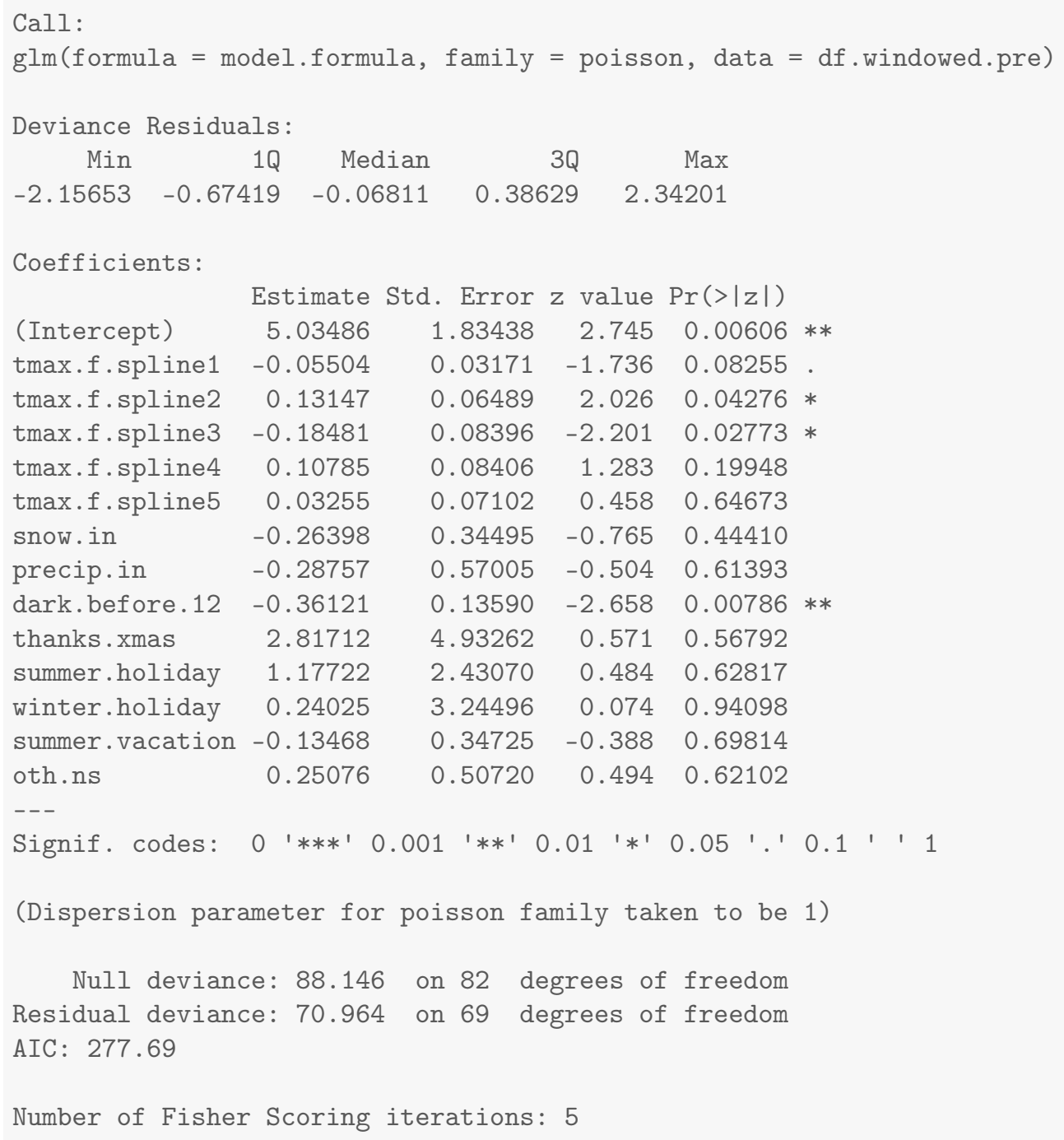




\section{Results for murder.g}

\subsection{Summary Values for murder.g}

Table 8.1: Descriptives for Outcome Before Ferguson Protests Begin

\begin{tabular}{lcccccccc}
\hline \hline Statistic & $\mathrm{N}$ & Mean & St. Dev. & Min & Pctl(25) & Median & Pctl(75) & Max \\
\hline murder.g & 83 & 6.506 & 2.864 & 1 & 4 & 6 & 8 & 15 \\
\hline
\end{tabular}

Table 8.2: Descriptives for Outcome After Ferguson Protests Begin

\begin{tabular}{lcccccccc}
\hline \hline Statistic & $\mathrm{N}$ & Mean & St. Dev. & Min & Pctl(25) & Median & Pctl(75) & Max \\
\hline murder.g & 290 & 5.821 & 2.533 & 0 & 4 & 6 & 7 & 13 \\
\hline
\end{tabular}




\subsection{Four Models for murder.g}

Table 8.3: Four Models that Differ on the Specification of Adjustment and Intervention Variables

\begin{tabular}{|c|c|c|c|c|}
\hline & \multicolumn{4}{|c|}{ Outcome: Count Per Week } \\
\hline & (1) & $(2)$ & $(3)$ & $(4)$ \\
\hline After Ferguson Protests Begin (week of $8 / 11 / 14$ onward) & -0.14 & & 0.26 & 0.09 \\
\hline After Gray Protests Begin (week of 4/20/15 onward) & -0.72 & & -1.78 & -1.13 \\
\hline Unrest and National Guard $(4 / 27 / 15-5 / 3 / 2015)$ & -4.64 & & -3.80 & -4.76 \\
\hline After Batts Fired (week of 7/13/15 onward) & 1.36 & & 2.12 & 1.37 \\
\hline After Davis Confirmed (week of 10/19/15 onward) & -1.17 & & -0.81 & -0.89 \\
\hline After Davis First Year (week of 10/17/16 onward) & 0.78 & & 0.52 & 0.76 \\
\hline After De Sousa Begins (week of 1/19/18 onward) & -1.79 & & -1.85 & -1.89 \\
\hline After Tuggle Begins (week of 5/14/18 onward) & 0.55 & & 0.82 & 0.65 \\
\hline After Harrison Begins (week of 2/11/19 onward) & -0.40 & & -0.72 & -0.53 \\
\hline Average Maximum Temperature to 50 Degrees & & 0.05 & & 0.06 \\
\hline Plus Degrees in the 50s & & -0.29 & & -0.16 \\
\hline Plus Degrees in the 60s & & 0.33 & & 0.14 \\
\hline Plus Degrees in the 70s & & 0.17 & & -0.04 \\
\hline Plus Degrees Greater Than 80 & & -0.47 & & -0.0002 \\
\hline Snowfall (inches) & & -1.18 & & -0.26 \\
\hline Precipitation (inches) & & -3.22 & & -1.31 \\
\hline Darkness Before Midnight (hours) & & -0.25 & & -0.10 \\
\hline Thanksgiving/Christmas (proportion of week) & & -34.06 & & -9.43 \\
\hline Winter Holiday (proportion of week) & & 4.09 & & -3.75 \\
\hline Other Out-of-School Days (proportion of week) & & 0.26 & & -2.02 \\
\hline Summer Vacation (proportion of week) & & -0.89 & & 0.16 \\
\hline Summer Holiday (proportion of week) & & 5.92 & & 0.25 \\
\hline Observations & 373 & 83 & 373 & 373 \\
\hline $\mathrm{R}^{2}$ & 0.07 & 0.23 & 0.07 & 0.14 \\
\hline
\end{tabular}




\subsection{Least Squares Updated Model (2) for murder.g murder.g}

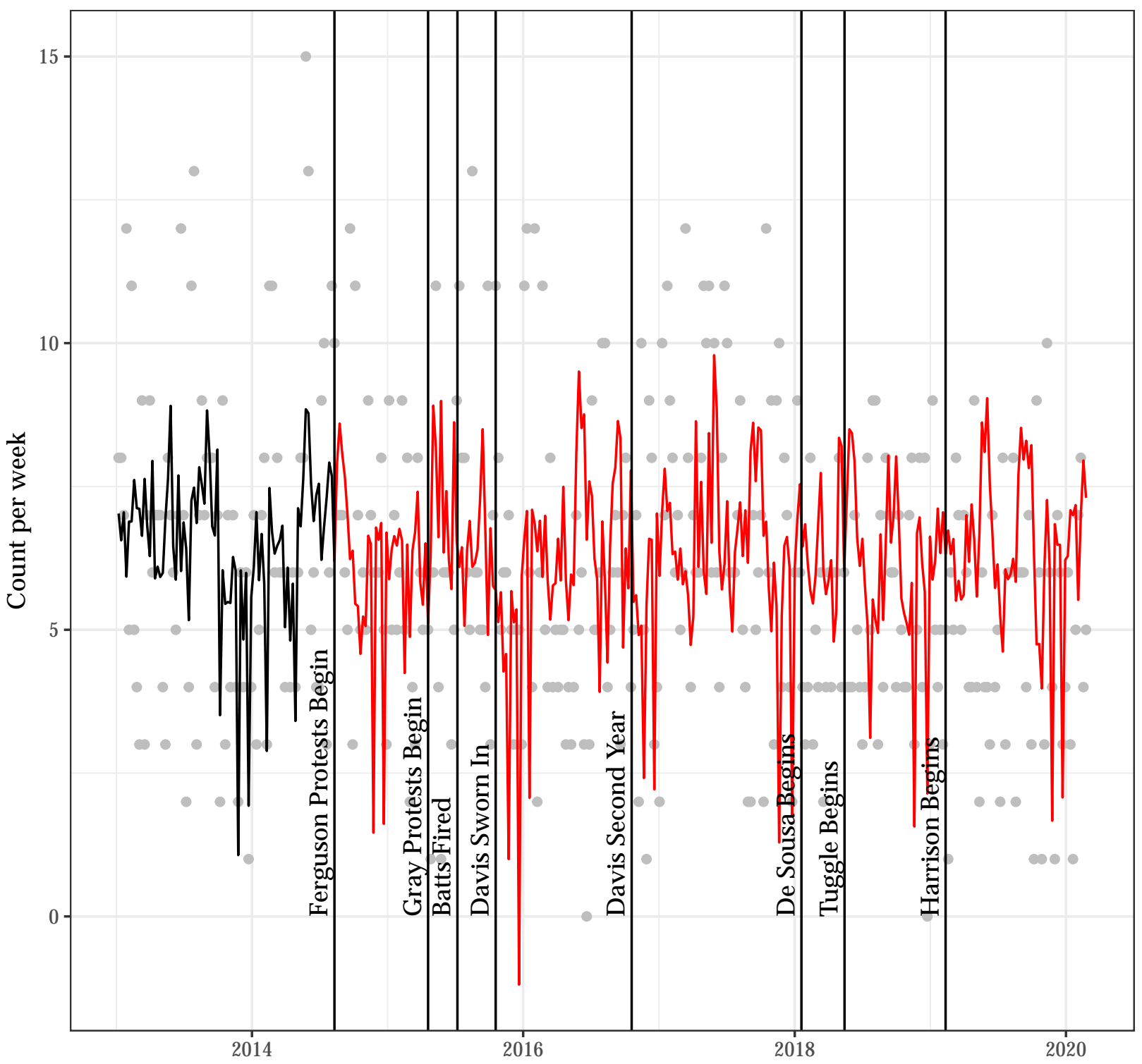




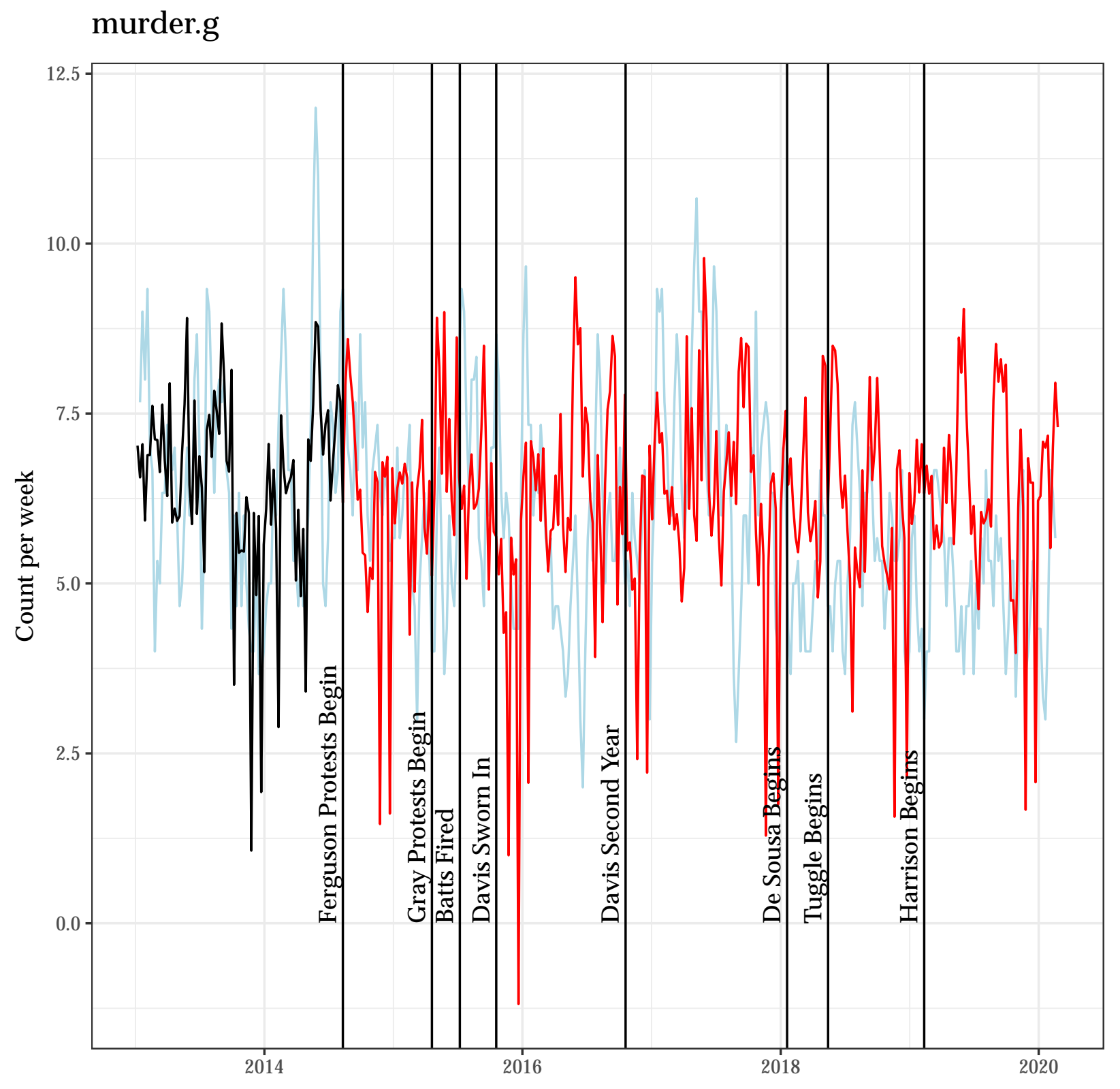


Autocorrelation Function for the Observed Outcome for Updated Model (2)

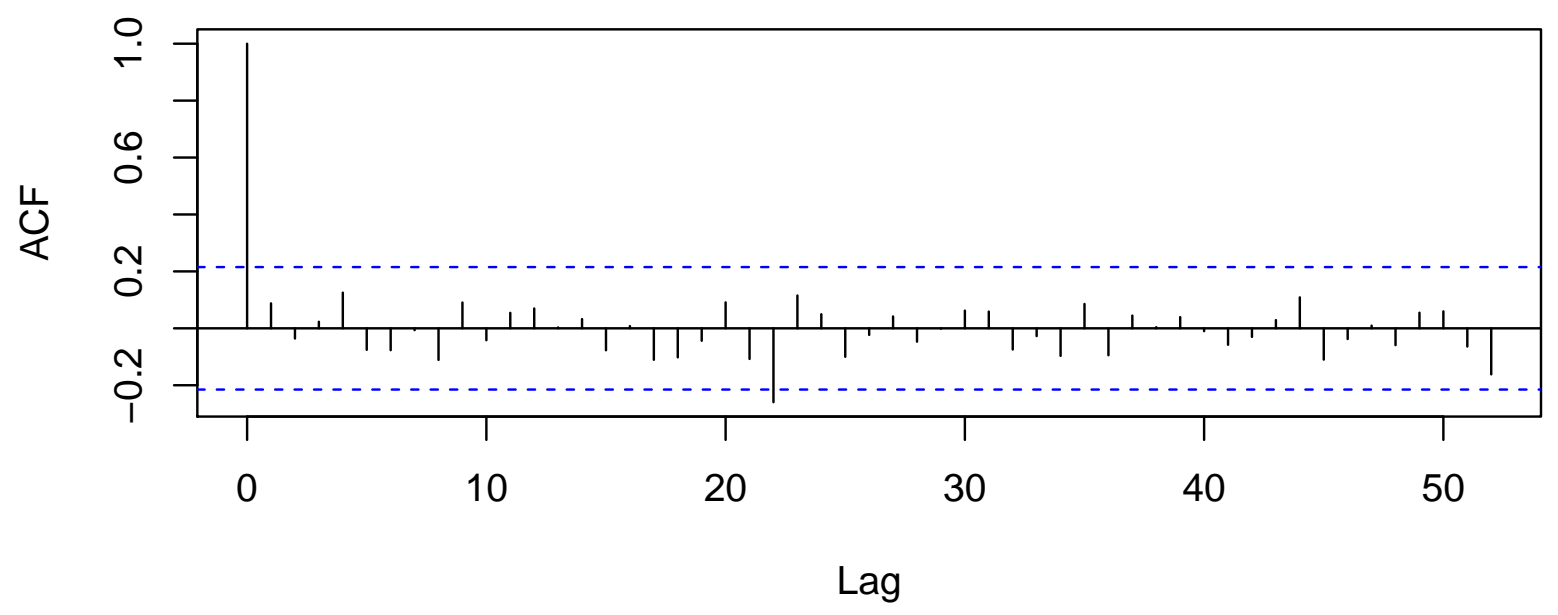

Autocorrelation Function for the Residuals from Updated Model (2)

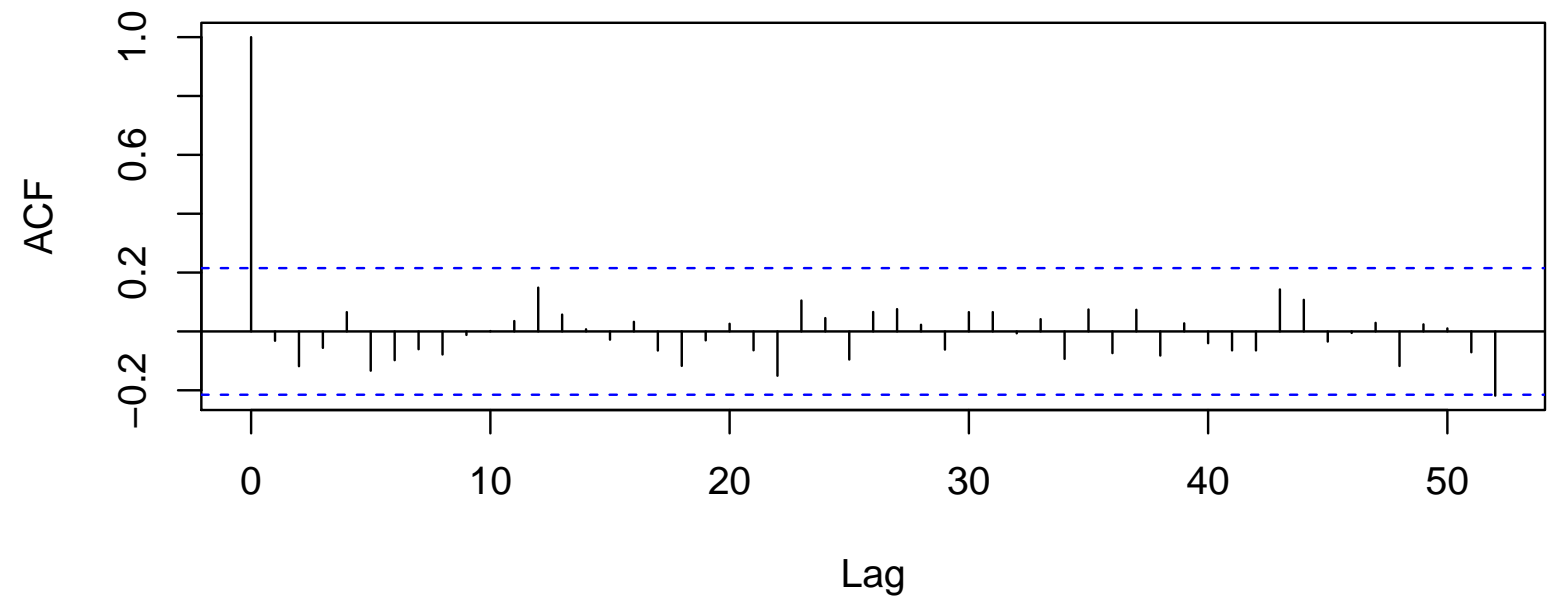




\subsection{A Poisson Regression Model as an Alternative to Model (2) for murder.g}

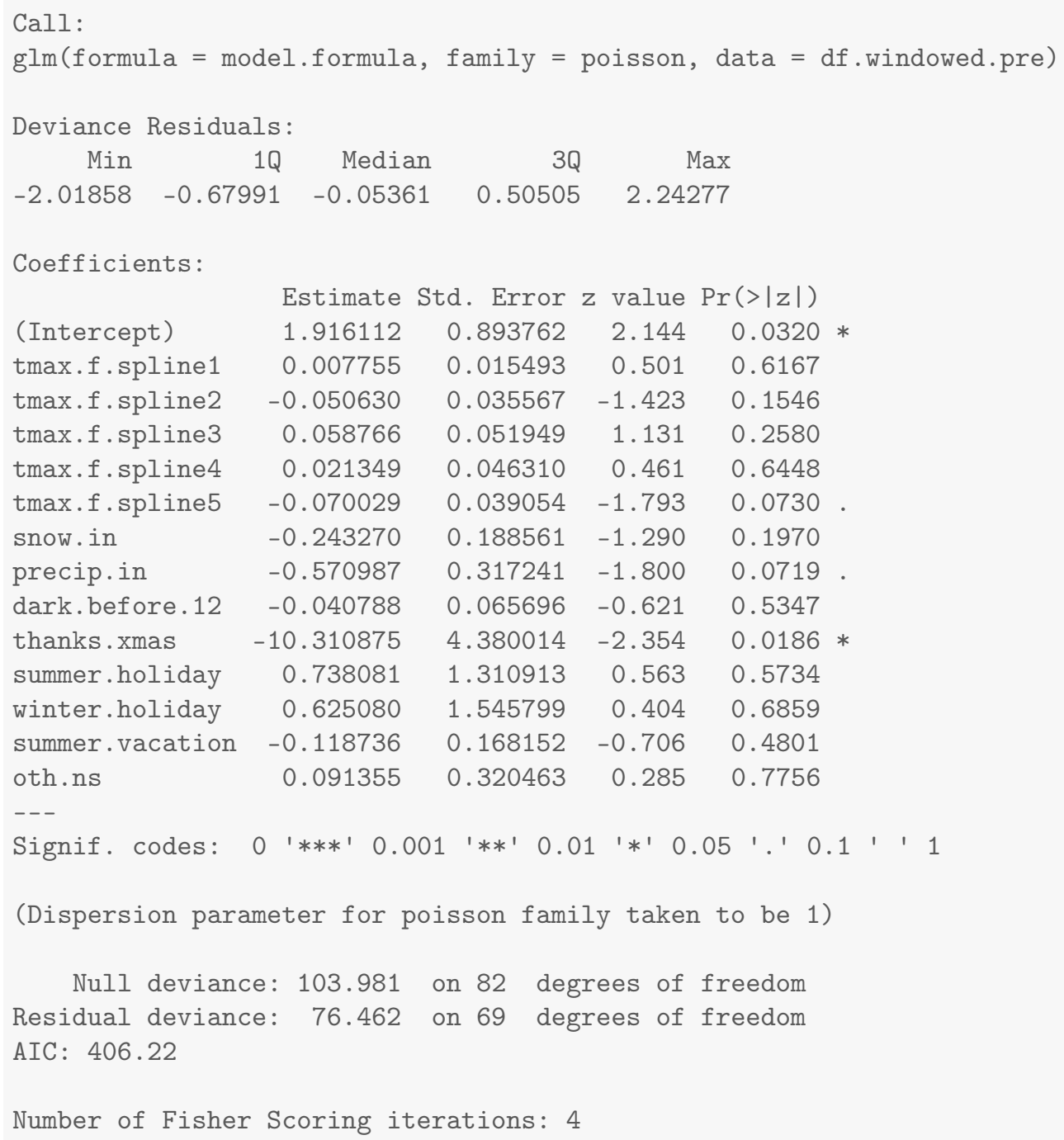




\section{Results for arson}

\subsection{Summary Values for arson}

Table 9.1: Descriptives for Outcome Before Ferguson Protests Begin

\begin{tabular}{lcccccccc}
\hline \hline Statistic & $\mathrm{N}$ & Mean & St. Dev. & Min & Pctl(25) & Median & Pctl(75) & Max \\
\hline arson & 83 & 1.241 & 1.043 & 0 & 0.5 & 1 & 2 & 5 \\
\hline
\end{tabular}

Table 9.2: Descriptives for Outcome After Ferguson Protests Begin

\begin{tabular}{lcccccccc}
\hline \hline Statistic & $\mathrm{N}$ & Mean & St. Dev. & Min & Pctl(25) & Median & Pctl(75) & Max \\
\hline arson & 290 & 1.072 & 1.081 & 0 & 0 & 1 & 2 & 5 \\
\hline
\end{tabular}




\subsection{Four Models for arson}

Table 9.3: Four Models that Differ on the Specification of Adjustment and Intervention Variables

\begin{tabular}{|c|c|c|c|c|}
\hline & \multicolumn{4}{|c|}{ Outcome: Count Per Week } \\
\hline & $(1)$ & (2) & (3) & $(4)$ \\
\hline After Ferguson Protests Begin (week of $8 / 11 / 14$ onward) & -0.27 & & -0.39 & -0.27 \\
\hline After Gray Protests Begin (week of 4/20/15 onward) & -0.24 & & -0.29 & -0.26 \\
\hline Unrest and National Guard (4/27/15 - 5/3/2015) & 0.27 & & 0.82 & 0.39 \\
\hline After Batts Fired (week of 7/13/15 onward) & 0.49 & & 0.71 & 0.46 \\
\hline After Davis Confirmed (week of 10/19/15 onward) & 0.13 & & -0.03 & 0.19 \\
\hline After Davis First Year (week of 10/17/16 onward) & -0.22 & & -0.25 & -0.26 \\
\hline After De Sousa Begins (week of 1/19/18 onward) & -0.31 & & -0.17 & -0.24 \\
\hline After Tuggle Begins (week of 5/14/18 onward) & 0.34 & & 0.22 & 0.28 \\
\hline After Harrison Begins (week of 2/11/19 onward) & -0.28 & & -0.17 & -0.26 \\
\hline Average Maximum Temperature to 50 Degrees & & -0.04 & & 0.0002 \\
\hline Plus Degrees in the $50 \mathrm{~s}$ & & 0.15 & & -0.01 \\
\hline Plus Degrees in the 60s & & -0.17 & & 0.02 \\
\hline Plus Degrees in the $70 \mathrm{~s}$ & & 0.17 & & 0.03 \\
\hline Plus Degrees Greater Than 80 & & -0.18 & & -0.07 \\
\hline Snowfall (inches) & & -0.48 & & 0.01 \\
\hline Precipitation (inches) & & 0.64 & & -0.11 \\
\hline Darkness Before Midnight (hours) & & 0.26 & & 0.10 \\
\hline Thanksgiving/Christmas (proportion of week) & & -3.01 & & -3.35 \\
\hline Winter Holiday (proportion of week) & & -0.07 & & -1.53 \\
\hline Other Out-of-School Days (proportion of week) & & -0.12 & & -0.25 \\
\hline Summer Vacation (proportion of week) & & -0.53 & & -0.06 \\
\hline Summer Holiday (proportion of week) & & 5.84 & & -1.80 \\
\hline Observations & 373 & 83 & 373 & 373 \\
\hline $\mathrm{R}^{2}$ & 0.03 & 0.18 & 0.03 & 0.05 \\
\hline
\end{tabular}




\subsection{Least Squares Updated Model (2) for arson}

arson

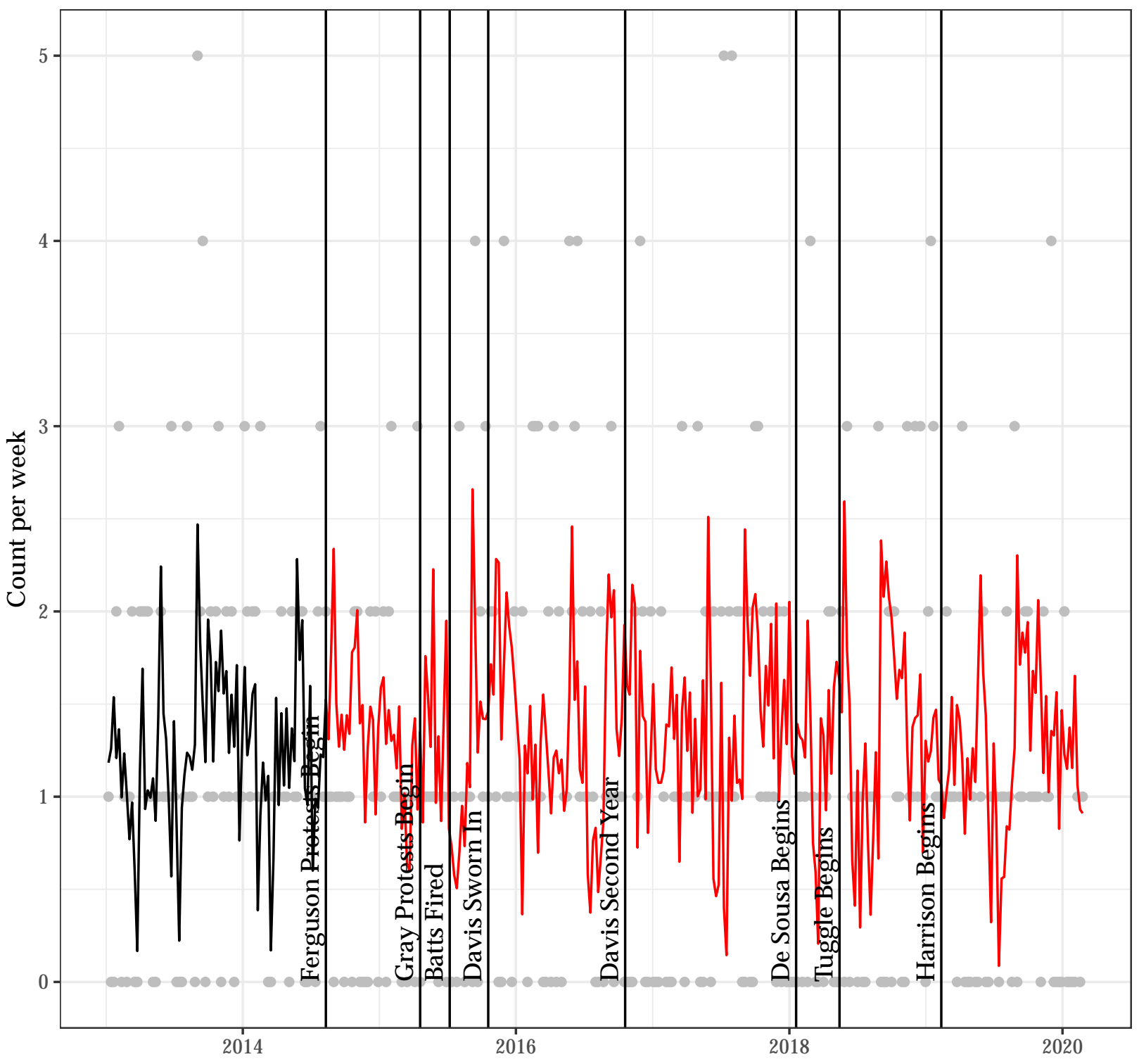




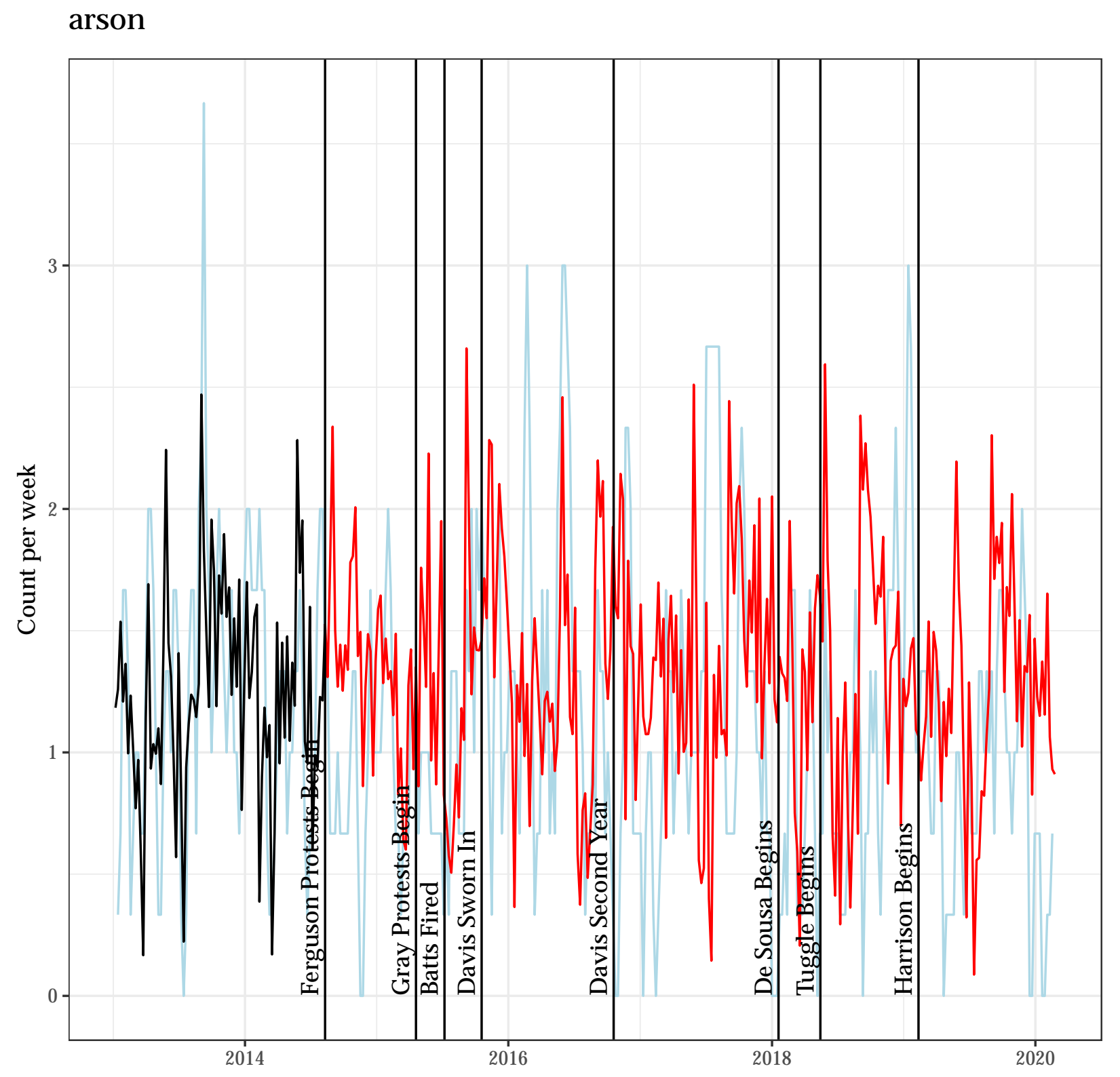


Autocorrelation Function for the Observed Outcome for Updated Model (2)

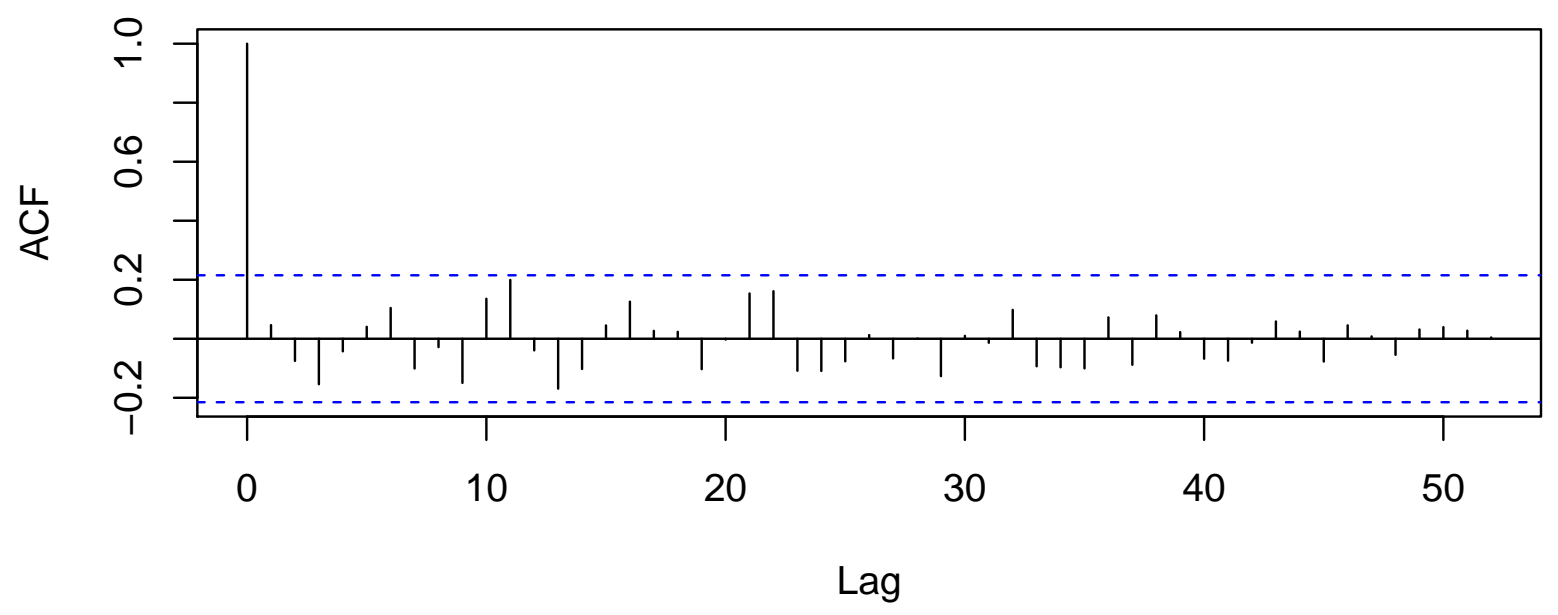

Autocorrelation Function for the Residuals from Updated Model (2)

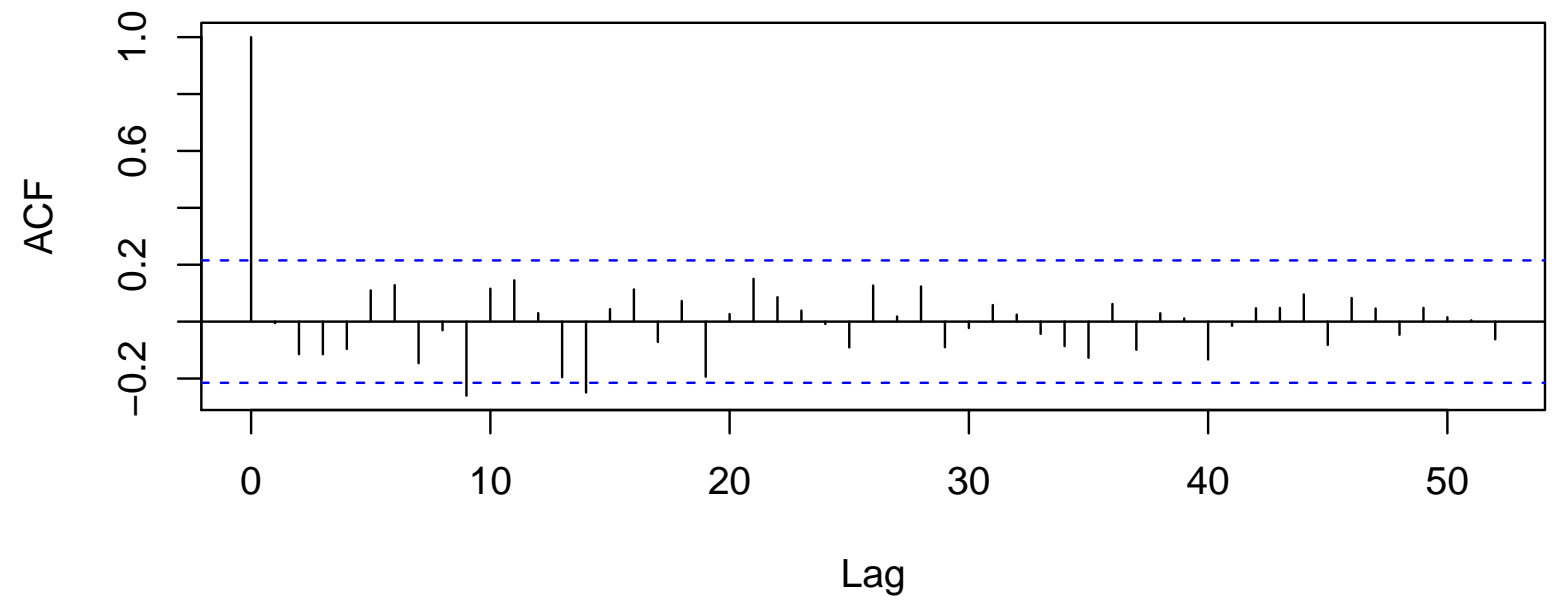




\subsection{A Poisson Regression Model as an Alternative to Model (2) for arson}

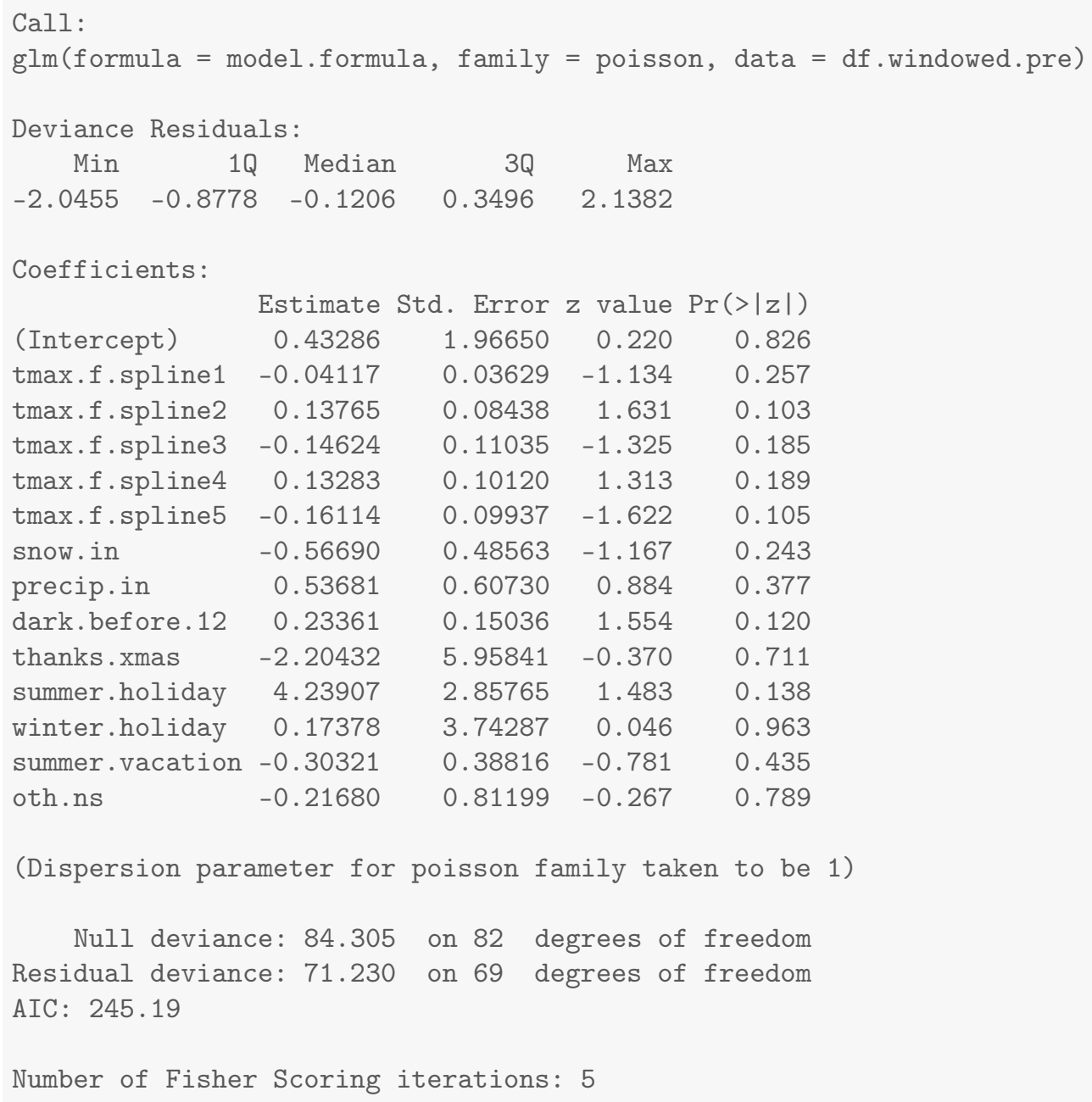




\section{Results for robbery}

\subsection{Summary Values for robbery}

Table 10.1: Descriptives for Outcome Before Ferguson Protests Begin

\begin{tabular}{lcccccccc}
\hline \hline Statistic & $\mathrm{N}$ & Mean & St. Dev. & Min & Pctl(25) & Median & Pctl(75) & Max \\
\hline robbery & 83 & 24.771 & 12.796 & 5 & 14.5 & 21 & 38 & 55 \\
\hline
\end{tabular}

Table 10.2: Descriptives for Outcome After Ferguson Protests Begin

\begin{tabular}{lcccccccc}
\hline \hline Statistic & $\mathrm{N}$ & Mean & St. Dev. & Min & Pctl(25) & Median & Pctl(75) & Max \\
\hline robbery & 290 & 26.583 & 7.980 & 6 & 21 & 26 & 32 & 56 \\
\hline
\end{tabular}




\subsection{Four Models for robbery}

Table 10.3: Four Models that Differ on the Specification of Adjustment and Intervention Variables

\begin{tabular}{|c|c|c|c|c|}
\hline & \multicolumn{4}{|c|}{ Outcome: Count Per Week } \\
\hline & (1) & $(2)$ & $(3)$ & $(4)$ \\
\hline After Ferguson Protests Begin (week of $8 / 11 / 14$ onward) & 9.34 & & 10.25 & 10.64 \\
\hline After Gray Protests Begin (week of 4/20/15 onward) & -3.20 & & -7.04 & -5.55 \\
\hline Unrest and National Guard (4/27/15 - 5/3/2015) & -12.91 & & -11.02 & -13.03 \\
\hline After Batts Fired (week of 7/13/15 onward) & 2.52 & & 10.95 & 3.20 \\
\hline After Davis Confirmed (week of 10/19/15 onward) & -4.39 & & -7.43 & -3.64 \\
\hline After Davis First Year (week of 10/17/16 onward) & -3.55 & & -2.56 & -3.14 \\
\hline After De Sousa Begins (week of 1/19/18 onward) & 0.02 & & -4.82 & -0.79 \\
\hline After Tuggle Begins (week of 5/14/18 onward) & -3.99 & & 2.54 & -2.85 \\
\hline After Harrison Begins (week of 2/11/19 onward) & 0.60 & & -0.89 & 0.01 \\
\hline Average Maximum Temperature to 50 Degrees & & -1.04 & & 0.12 \\
\hline Plus Degrees in the 50s & & 1.76 & & -0.02 \\
\hline Plus Degrees in the 60s & & -2.35 & & -0.25 \\
\hline Plus Degrees in the 70s & & 2.30 & & 0.14 \\
\hline Plus Degrees Greater Than 80 & & -2.03 & & -0.26 \\
\hline Snowfall (inches) & & 0.60 & & 1.74 \\
\hline Precipitation (inches) & & -6.36 & & -3.36 \\
\hline Darkness Before Midnight (hours) & & -5.56 & & -0.77 \\
\hline Thanksgiving/Christmas (proportion of week) & & 3.33 & & -35.87 \\
\hline Winter Holiday (proportion of week) & & -22.51 & & 8.02 \\
\hline Other Out-of-School Days (proportion of week) & & -8.27 & & -5.68 \\
\hline Summer Vacation (proportion of week) & & 1.42 & & 3.61 \\
\hline Summer Holiday (proportion of week) & & 19.75 & & -6.40 \\
\hline Observations & 373 & 83 & 373 & 373 \\
\hline & 0.18 & 0.20 & 0.14 & 0.25 \\
\hline
\end{tabular}


10.3 Least Squares Updated Model (2) for robbery robbery

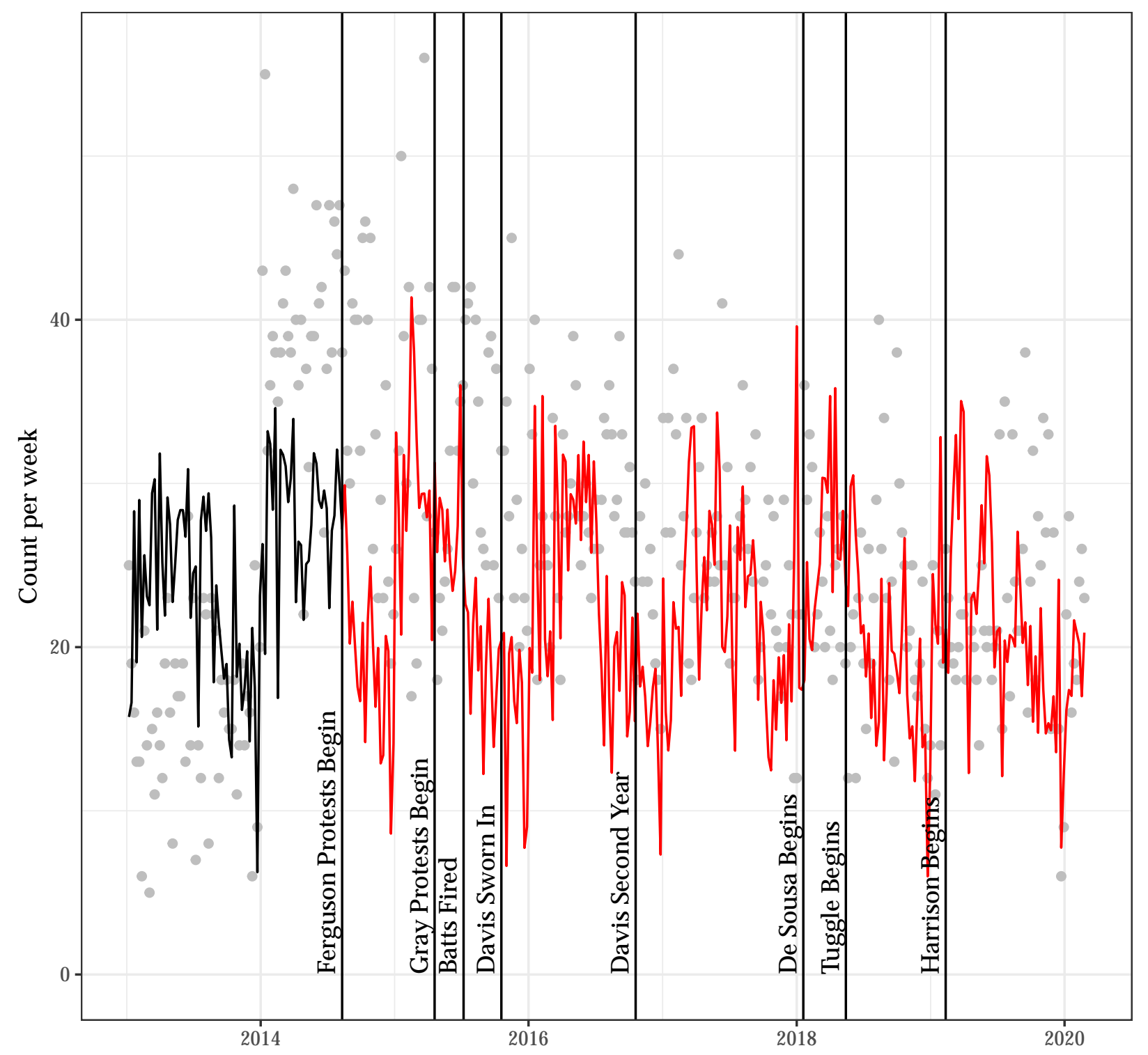




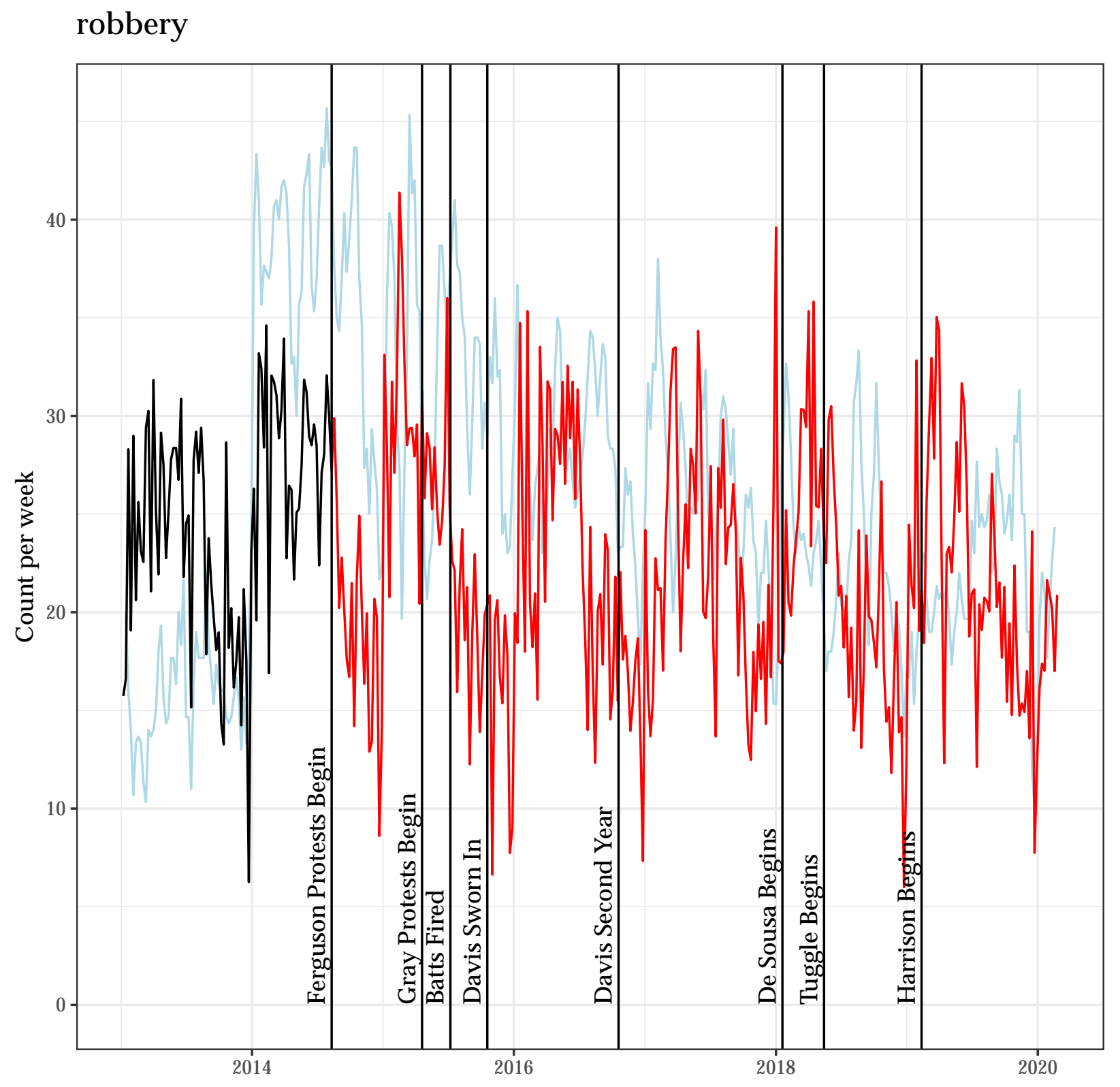


Autocorrelation Function for the Observed Outcome for Updated Model (2)

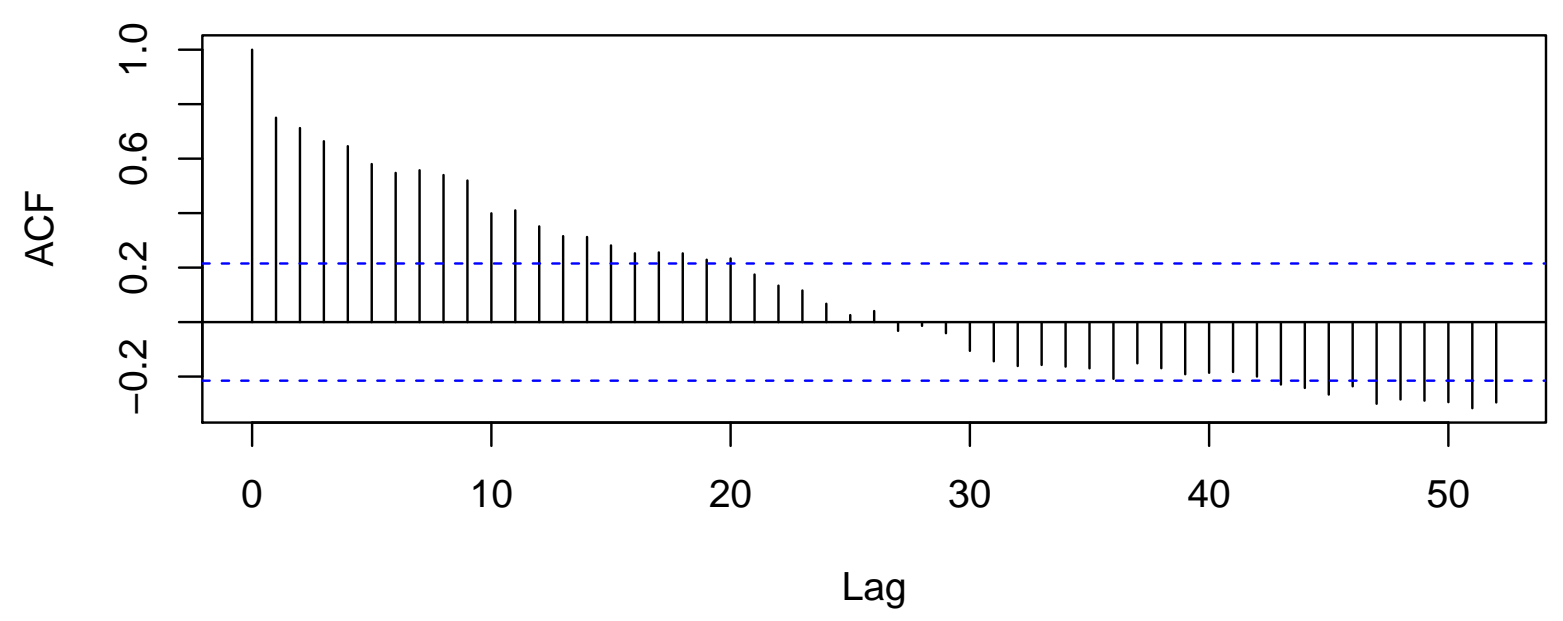

Autocorrelation Function for the Residuals from Updated Model (2)

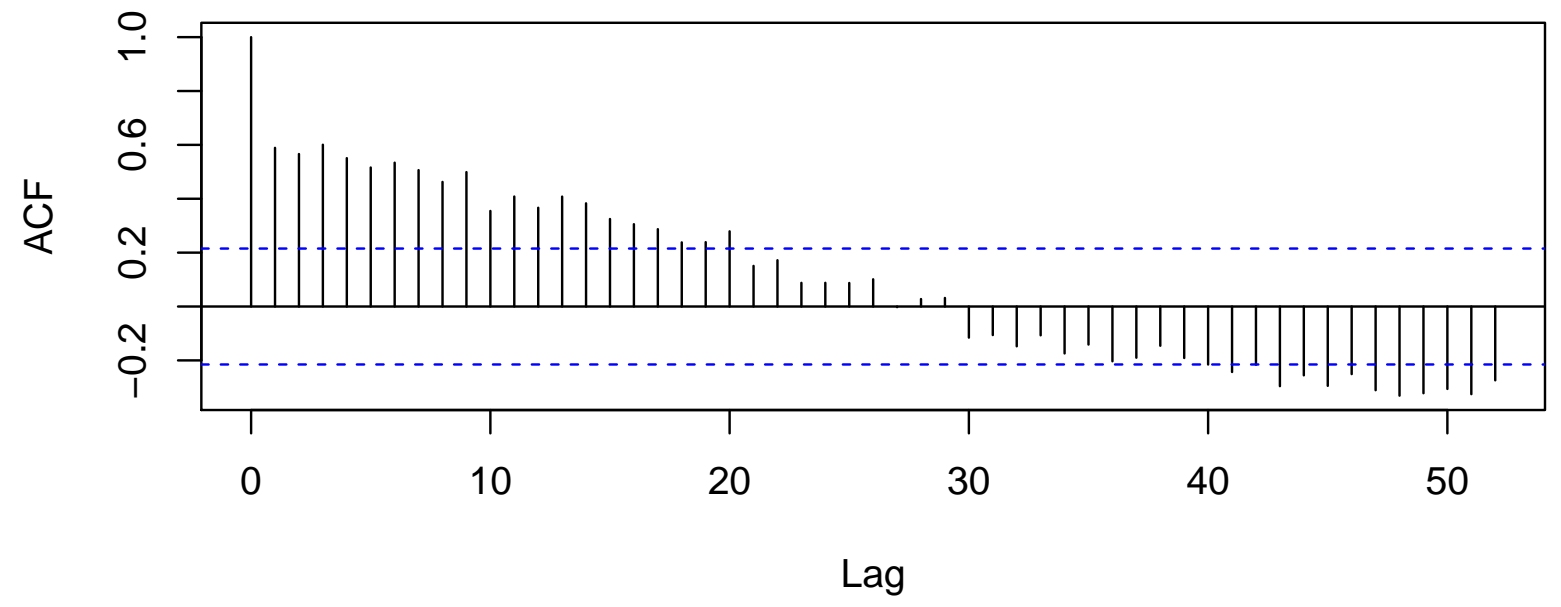




\subsection{A Poisson Regression Model as an Alternative to Model (2) for robbery}

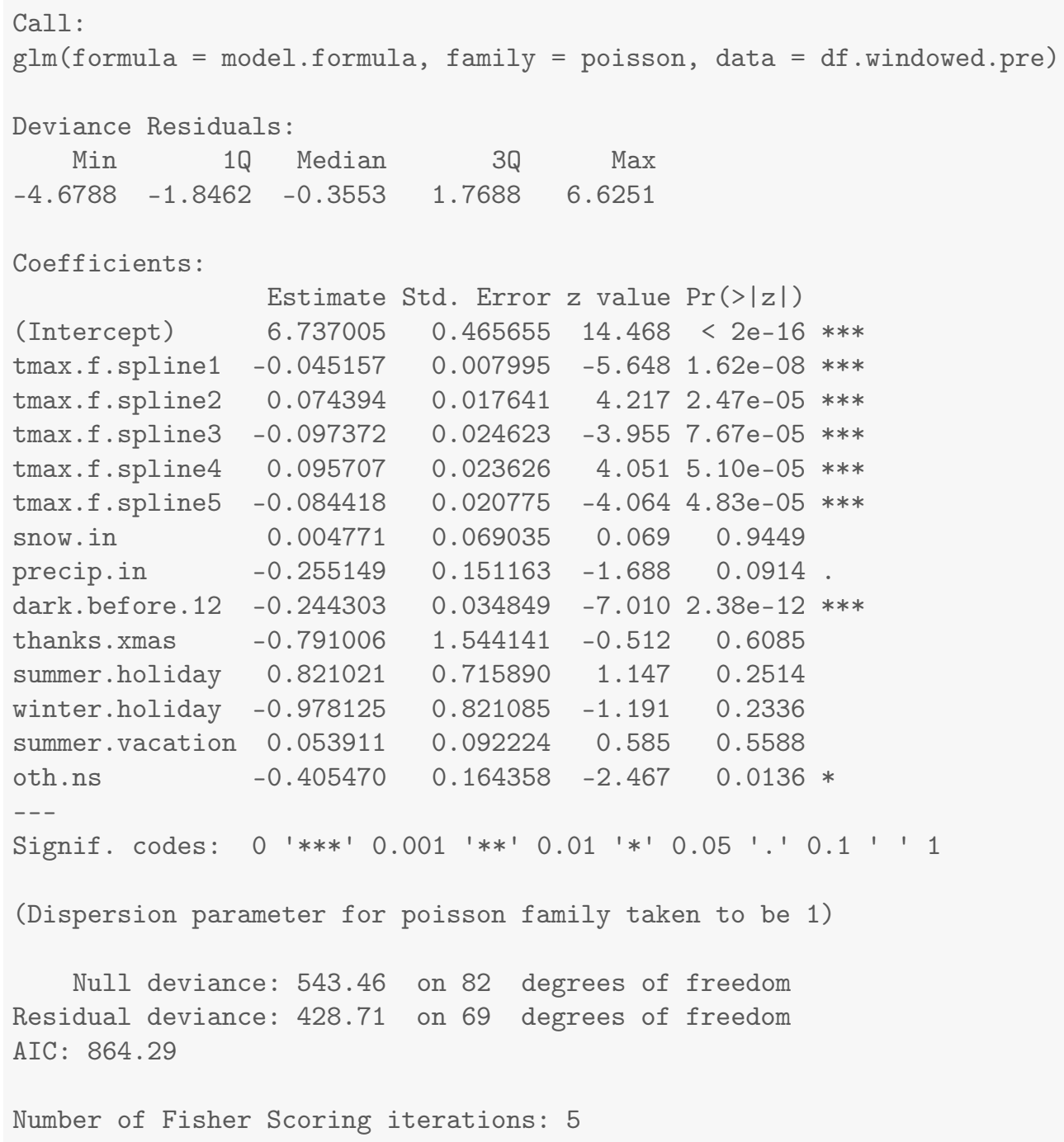




\section{Results for aggravated.assault}

\subsection{Summary Values for aggravated.assault}

Table 11.1: Descriptives for Outcome Before Ferguson Protests Begin

\begin{tabular}{lcccccccc}
\hline \hline Statistic & N & Mean & St. Dev. & Min & Pctl(25) & Median & Pctl(75) & Max \\
\hline aggravated.assault & 83 & 25.422 & 7.566 & 7 & 20 & 25 & 30.5 & 46 \\
\hline
\end{tabular}

Table 11.2: Descriptives for Outcome After Ferguson Protests Begin

\begin{tabular}{lcccccccc}
\hline \hline Statistic & $\mathrm{N}$ & Mean & St. Dev. & Min & Pctl(25) & Median & Pctl(75) & Max \\
\hline aggravated.assault & 290 & 21.014 & 5.582 & 7 & 17 & 21 & 24.8 & 40 \\
\hline
\end{tabular}




\subsection{Four Models for aggravated.assault}

Table 11.3: Four Models that Differ on the Specification of Adjustment and Intervention Variables

\begin{tabular}{|c|c|c|c|c|}
\hline & \multicolumn{4}{|c|}{ Outcome: Count Per Week } \\
\hline & $(1)$ & $(2)$ & $(3)$ & $(4)$ \\
\hline After Ferguson Protests Begin (week of 8/11/14 onward) & -4.87 & & -2.40 & -3.28 \\
\hline After Gray Protests Begin (week of 4/20/15 onward) & -0.10 & & -5.39 & -3.49 \\
\hline Unrest and National Guard (4/27/15 - 5/3/2015) & -8.45 & & -7.79 & -7.55 \\
\hline After Batts Fired (week of 7/13/15 onward) & 2.12 & & 2.32 & 2.09 \\
\hline After Davis Confirmed (week of 10/19/15 onward) & -2.71 & & -0.43 & -1.06 \\
\hline After Davis First Year (week of 10/17/16 onward) & 1.24 & & 1.76 & 1.61 \\
\hline After De Sousa Begins (week of 1/19/18 onward) & 0.14 & & -0.49 & -0.15 \\
\hline After Tuggle Begins (week of 5/14/18 onward) & -1.89 & & -1.36 & -1.91 \\
\hline After Harrison Begins (week of 2/11/19 onward) & 3.91 & & 3.16 & 3.61 \\
\hline Average Maximum Temperature to 50 Degrees & & 0.63 & & 0.36 \\
\hline Plus Degrees in the $50 \mathrm{~s}$ & & -0.88 & & -0.52 \\
\hline Plus Degrees in the $60 \mathrm{~s}$ & & 0.24 & & 0.17 \\
\hline Plus Degrees in the 70s & & 0.37 & & 0.26 \\
\hline Plus Degrees Greater Than 80 & & -0.07 & & -0.02 \\
\hline Snowfall (inches) & & 0.98 & & 0.87 \\
\hline Precipitation (inches) & & -8.42 & & -4.48 \\
\hline Darkness Before Midnight (hours) & & -1.52 & & -0.92 \\
\hline Thanksgiving/Christmas (proportion of week) & & -24.32 & & -13.71 \\
\hline Winter Holiday (proportion of week) & & 4.07 & & 13.24 \\
\hline Other Out-of-School Days (proportion of week) & & 0.48 & & -0.12 \\
\hline Summer Vacation (proportion of week) & & -3.72 & & -2.40 \\
\hline Summer Holiday (proportion of week) & & -8.40 & & -7.09 \\
\hline Observations & 373 & 83 & 373 & 373 \\
\hline $\mathrm{R}^{2}$ & 0.12 & 0.30 & 0.15 & 0.27 \\
\hline
\end{tabular}




\subsection{Least Squares Updated Model (2) for aggravated.assault}

aggravated.assault

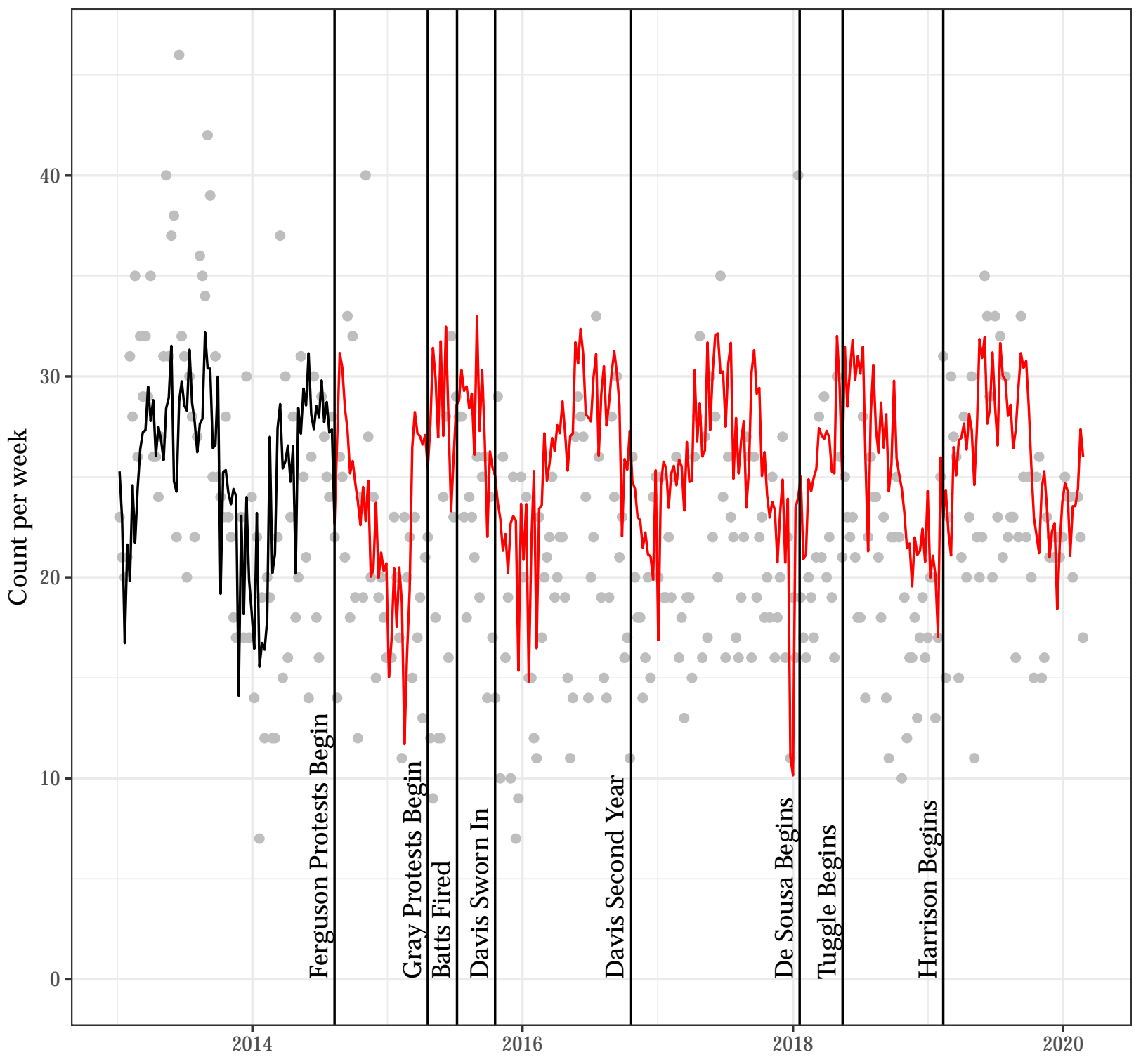




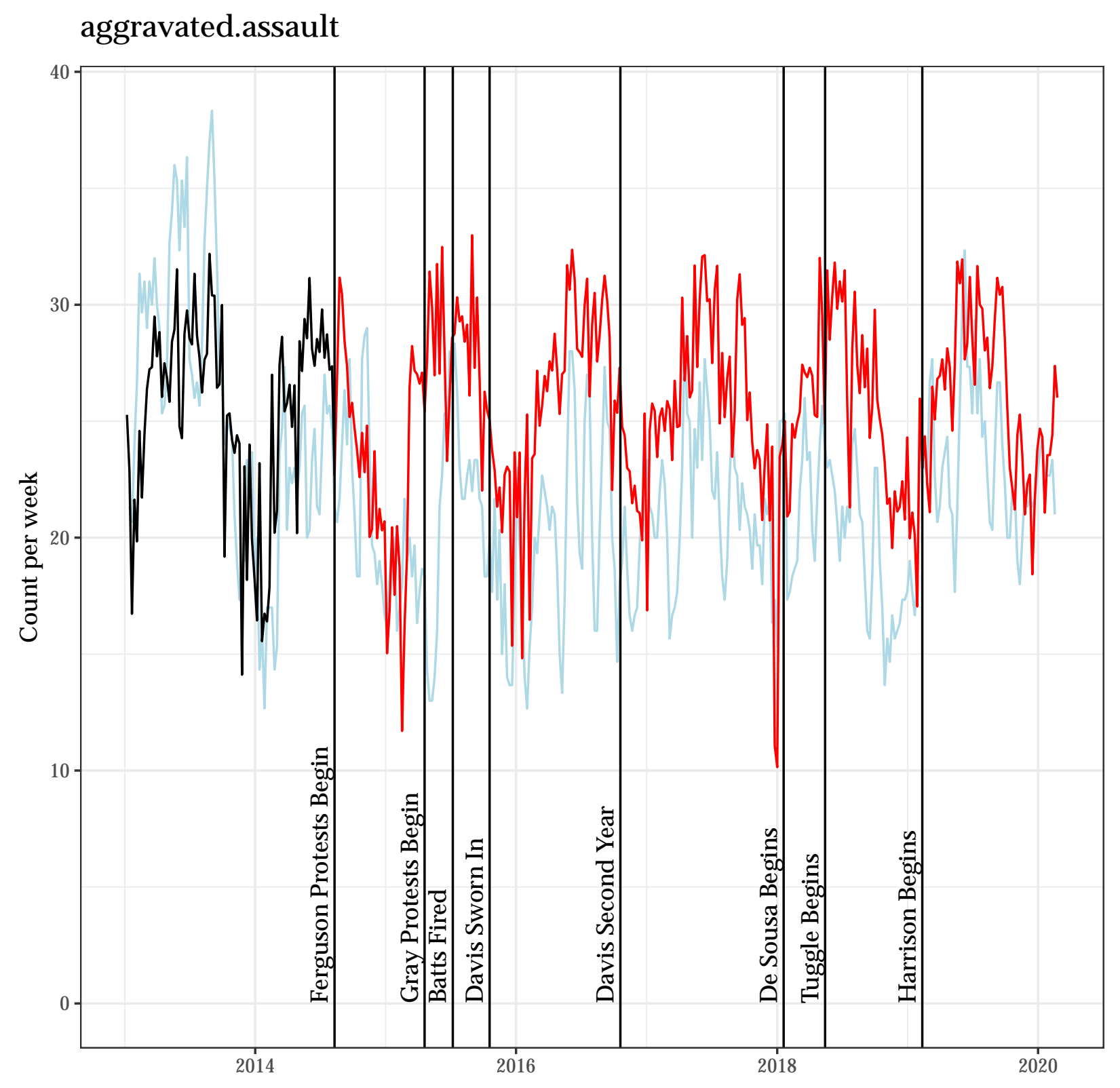


Autocorrelation Function for the Observed Outcome for Updated Model (2)

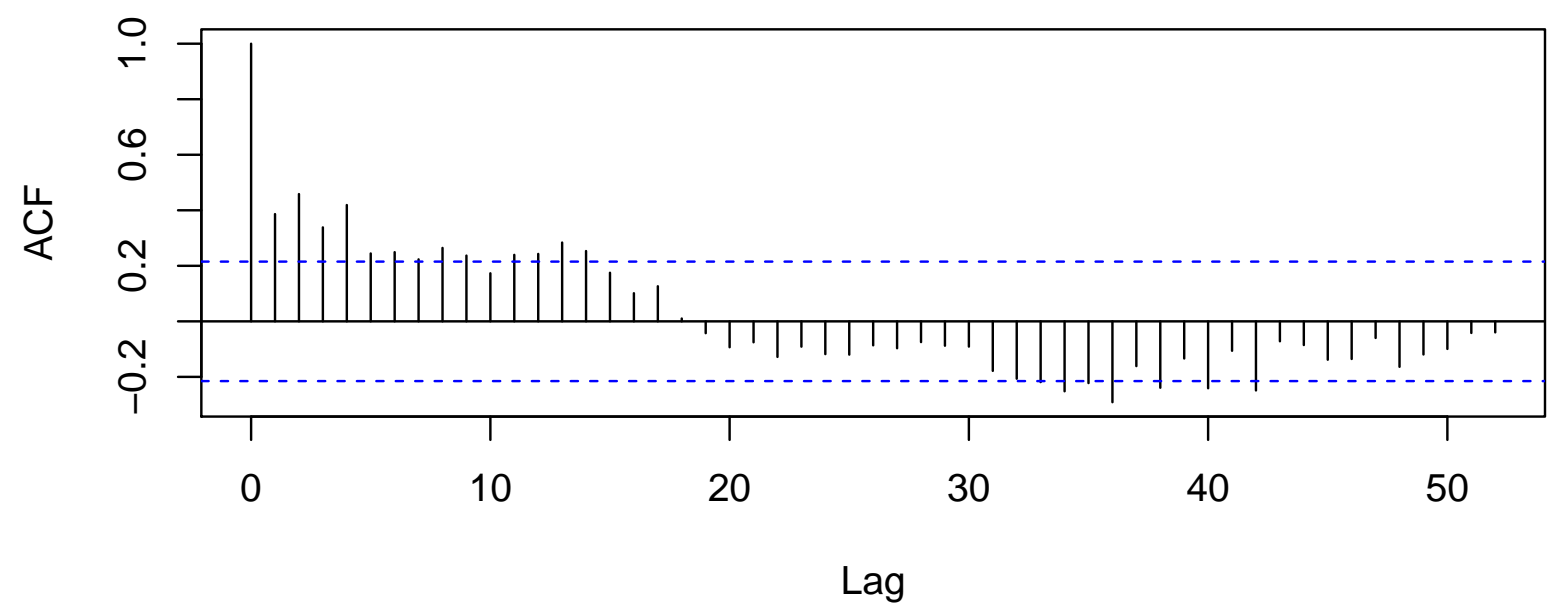

Autocorrelation Function for the Residuals from Updated Model (2)

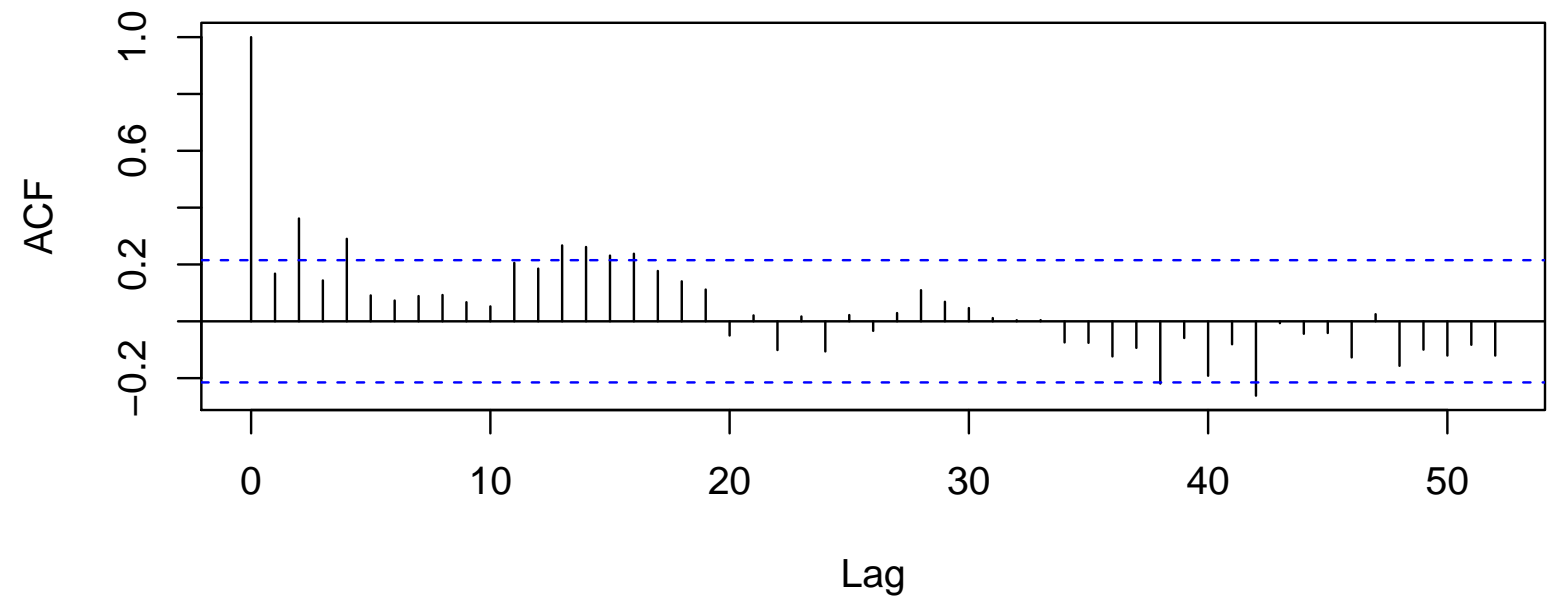




\subsection{A Poisson Regression Model as an Alternative to Model (2) for aggravated.assault}

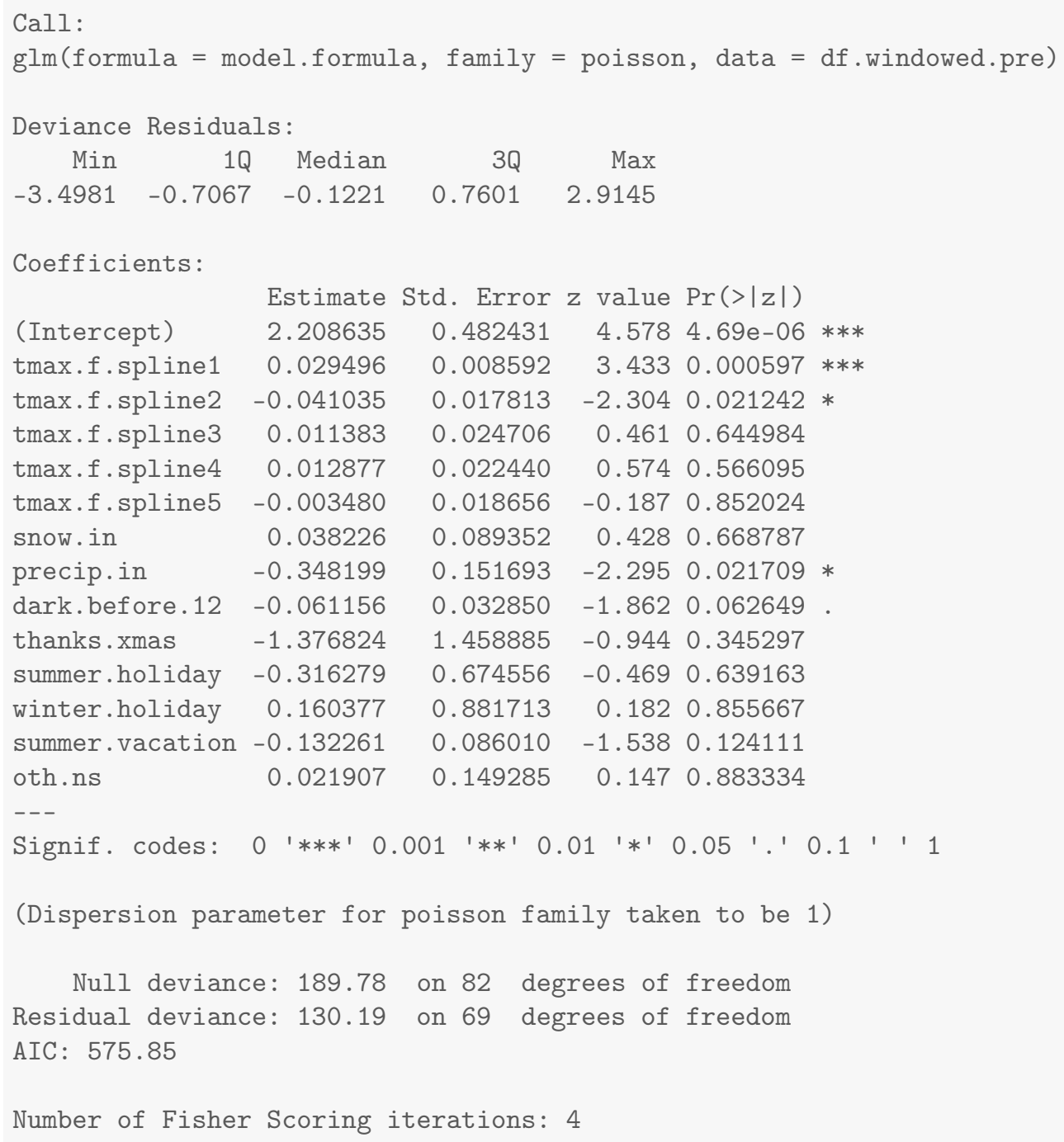




\section{Results for deadly.weapon}

\subsection{Summary Values for deadly.weapon}

Table 12.1: Descriptives for Outcome Before Ferguson Protests Begin

\begin{tabular}{lcccccccc}
\hline \hline Statistic & $\mathrm{N}$ & Mean & St. Dev. & Min & Pctl(25) & Median & Pctl(75) & Max \\
\hline deadly.weapon & 83 & 21.289 & 6.529 & 9 & 17 & 21 & 25 & 43 \\
\hline
\end{tabular}

Table 12.2: Descriptives for Outcome After Ferguson Protests Begin

\begin{tabular}{lcccccccc}
\hline \hline Statistic & N & Mean & St. Dev. & Min & Pctl(25) & Median & Pctl(75) & Max \\
\hline deadly.weapon & 290 & 20.390 & 6.804 & 3 & 16 & 20 & 24.8 & 47 \\
\hline
\end{tabular}




\subsection{Four Models for deadly.weapon}

Table 12.3: Four Models that Differ on the Specification of Adjustment and Intervention Variables

\begin{tabular}{|c|c|c|c|c|}
\hline & \multicolumn{4}{|c|}{ Outcome: Count Per Week } \\
\hline & $(1)$ & (2) & $(3)$ & (4) \\
\hline After Ferguson Protests Begin (week of $8 / 11 / 14$ onward) & -3.29 & & -3.93 & -3.26 \\
\hline After Gray Protests Begin (week of $4 / 20 / 15$ onward) & -2.36 & & -0.55 & -1.75 \\
\hline Unrest and National Guard $(4 / 27 / 15-5 / 3 / 2015)$ & -7.64 & & -5.93 & -8.48 \\
\hline After Batts Fired (week of $7 / 13 / 15$ onward) & 12.65 & & 9.94 & 11.67 \\
\hline After Davis Confirmed (week of 10/19/15 onward) & -5.67 & & -4.25 & -5.06 \\
\hline After Davis First Year (week of $10 / 17 / 16$ onward) & -2.99 & & -3.67 & -3.43 \\
\hline After De Sousa Begins (week of 1/19/18 onward) & 2.38 & & 3.48 & 2.67 \\
\hline After Tuggle Begins (week of 5/14/18 onward) & -0.90 & & -3.17 & -0.96 \\
\hline After Harrison Begins (week of 2/11/19 onward) & -2.14 & & -1.36 & -2.56 \\
\hline Average Maximum Temperature to 50 Degrees & & 0.10 & & 0.16 \\
\hline Plus Degrees in the $50 \mathrm{~s}$ & & -0.65 & & -0.59 \\
\hline Plus Degrees in the $60 \mathrm{~s}$ & & 1.06 & & 0.83 \\
\hline Plus Degrees in the 70s & & -0.10 & & -0.47 \\
\hline Plus Degrees Greater Than 80 & & 0.03 & & 0.12 \\
\hline Snowfall (inches) & & -2.97 & & -1.23 \\
\hline Precipitation (inches) & & 2.80 & & -3.02 \\
\hline Darkness Before Midnight (hours) & & 1.99 & & 0.55 \\
\hline Thanksgiving/Christmas (proportion of week) & & -87.82 & & -58.96 \\
\hline Winter Holiday (proportion of week) & & 18.93 & & 18.10 \\
\hline Other Out-of-School Days (proportion of week) & & 3.65 & & 2.20 \\
\hline Summer Vacation (proportion of week) & & -4.95 & & -0.09 \\
\hline Summer Holiday (proportion of week) & & -36.60 & & -4.77 \\
\hline Observations & 373 & 83 & 373 & 373 \\
\hline $\mathrm{R}^{2}$ & 0.12 & 0.21 & 0.12 & 0.19 \\
\hline
\end{tabular}


12.3 Least Squares Updated Model (2) for deadly.weapon deadly.weapon

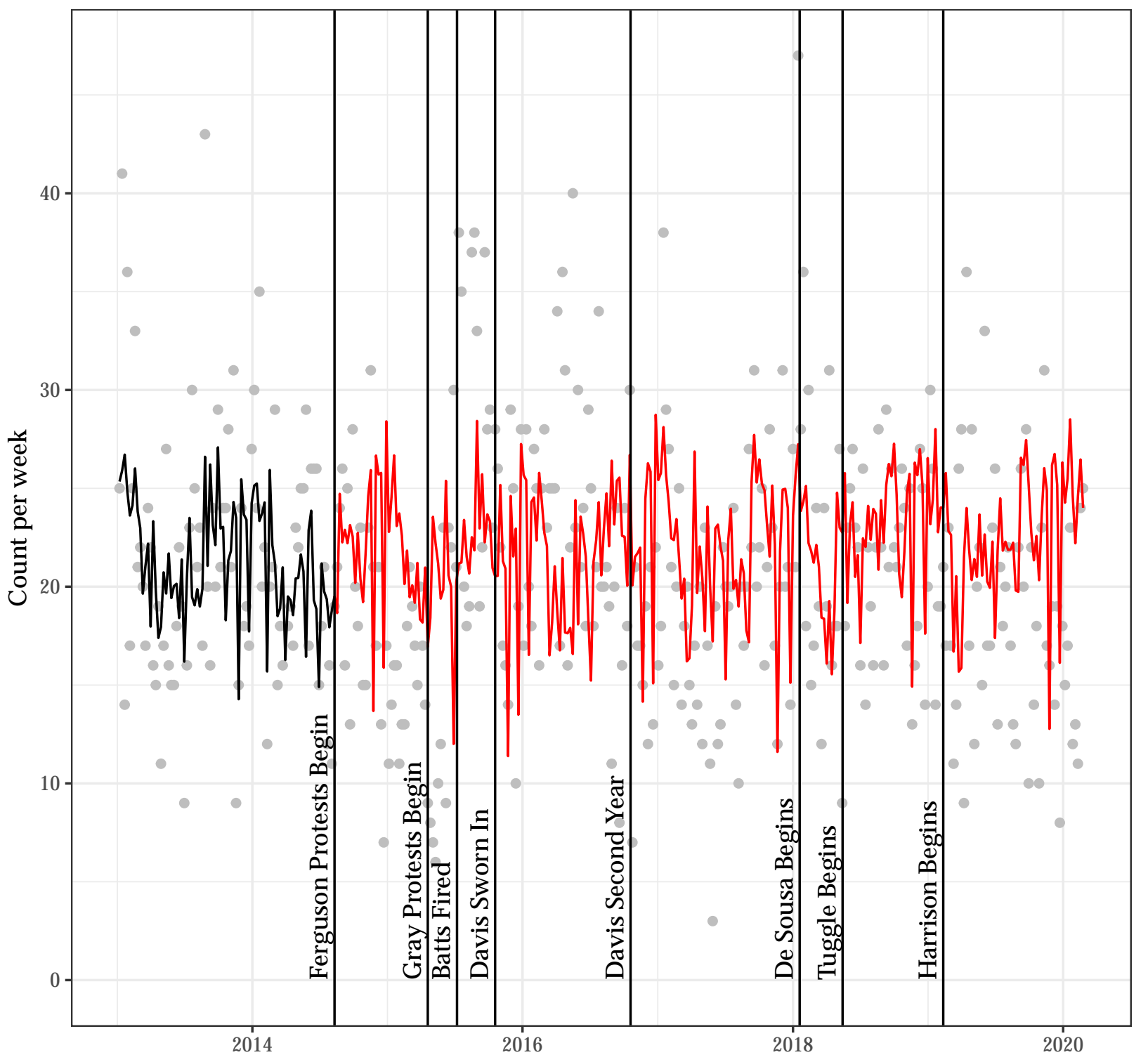




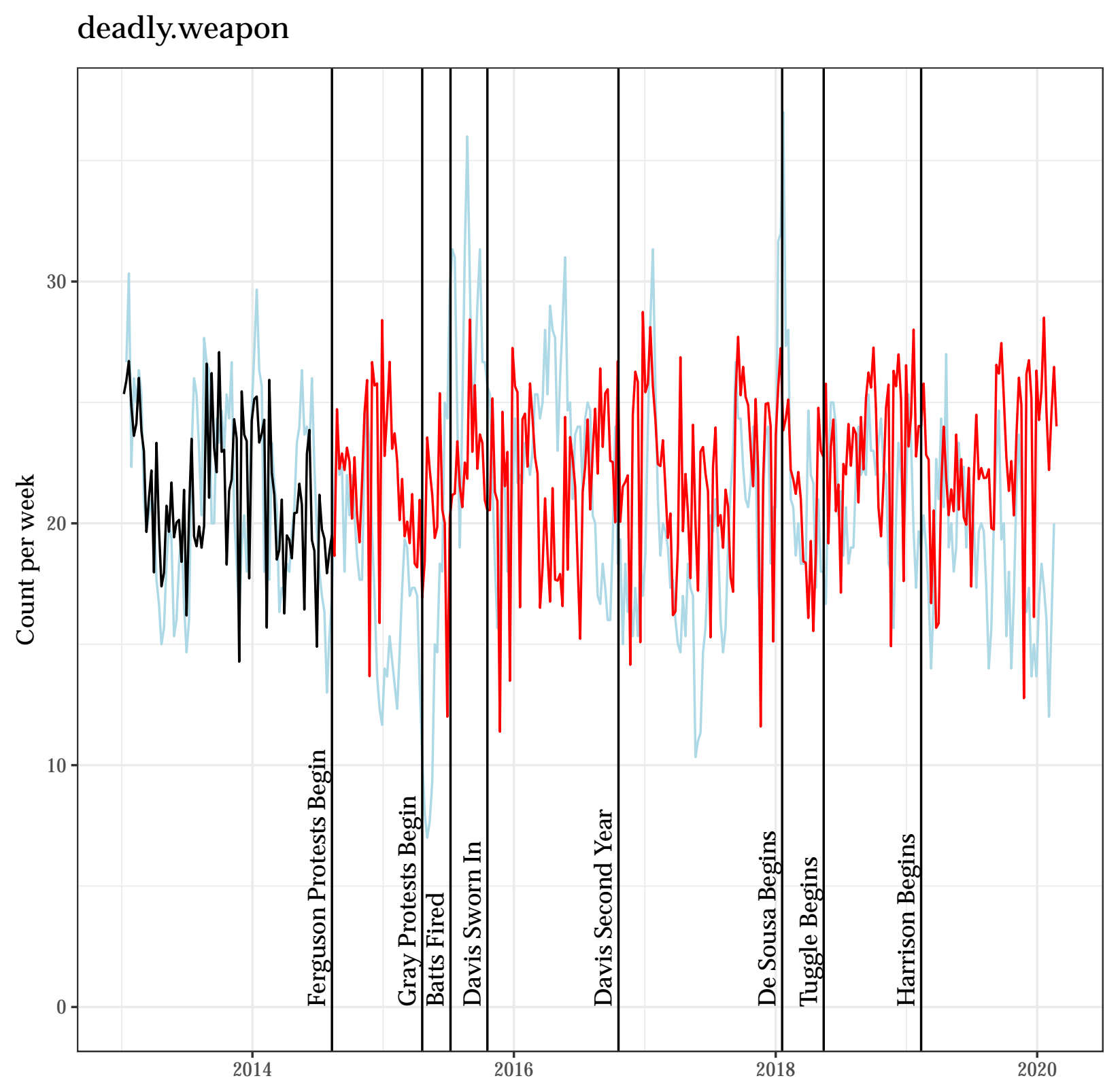


Autocorrelation Function for the Observed Outcome for Updated Model (2)

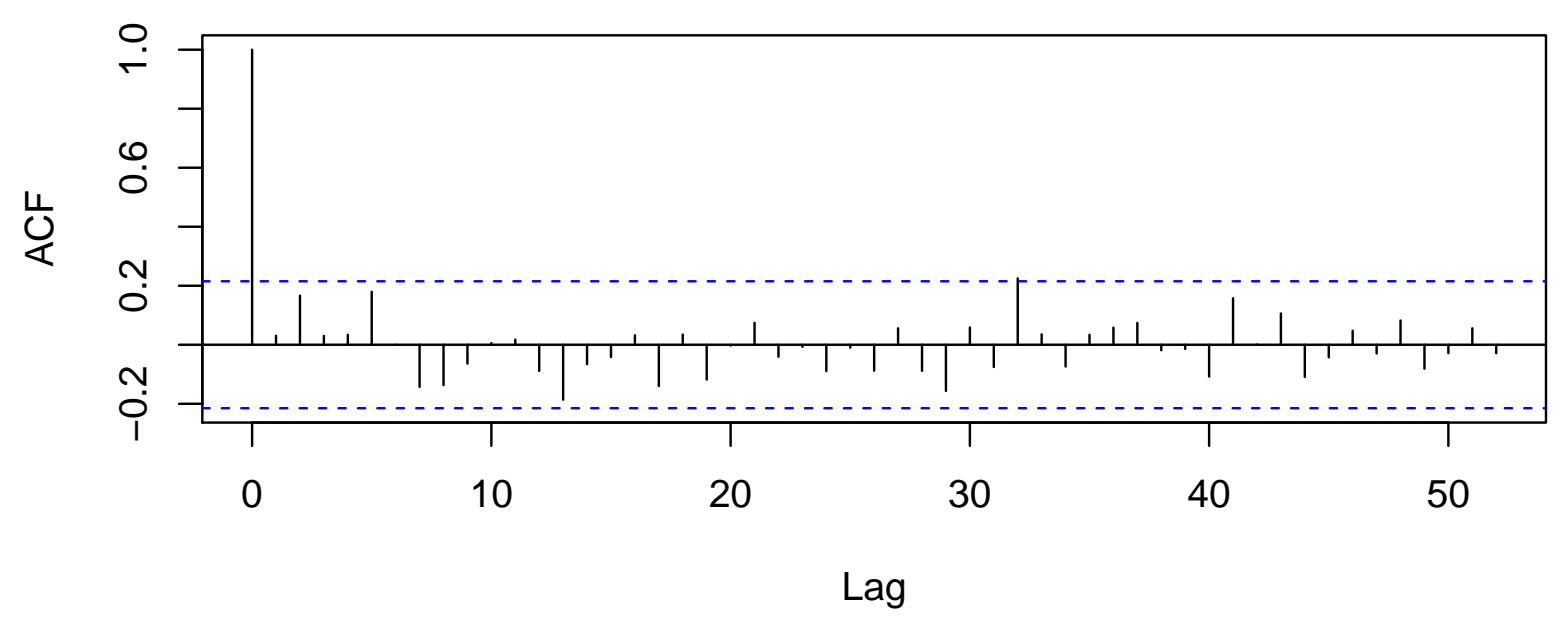

Autocorrelation Function for the Residuals from Updated Model (2)

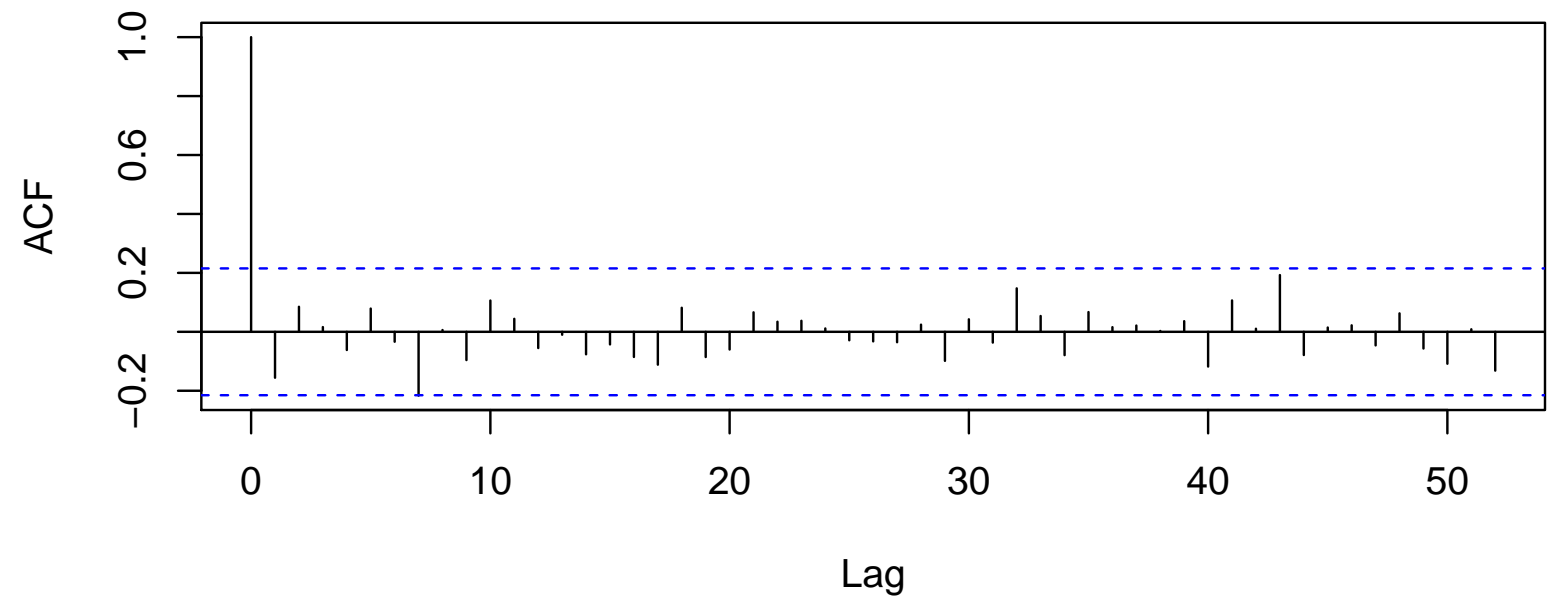




\subsection{A Poisson Regression Model as an Alternative to Model (2) for deadly.weapon}

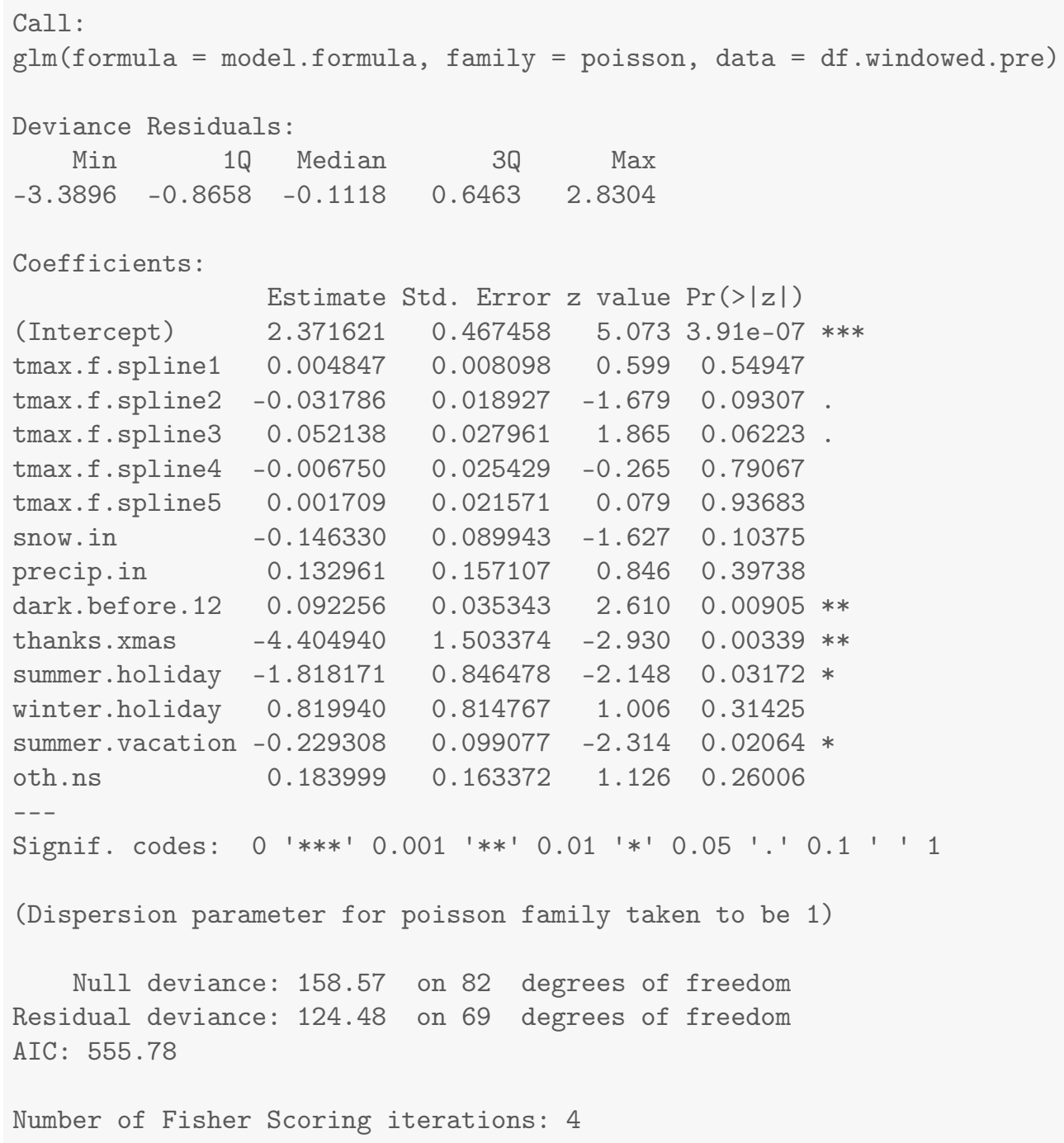




\section{Results for common.assault}

\subsection{Summary Values for common.assault}

Table 13.1: Descriptives for Outcome Before Ferguson Protests Begin

\begin{tabular}{lcccccccc}
\hline \hline Statistic & $\mathrm{N}$ & Mean & St. Dev. & Min & Pctl(25) & Median & Pctl(75) & Max \\
\hline common.assault & 83 & 91.434 & 12.240 & 52 & 83 & 90 & 101 & 117 \\
\hline
\end{tabular}

Table 13.2: Descriptives for Outcome After Ferguson Protests Begin

\begin{tabular}{lcccccccc}
\hline \hline Statistic & N & Mean & St. Dev. & Min & Pctl(25) & Median & Pctl(75) & Max \\
\hline common.assault & 290 & 78.714 & 12.217 & 36 & 71 & 79 & 87 & 123 \\
\hline
\end{tabular}




\subsection{Four Models for common.assault}

Table 13.3: Four Models that Differ on the Specification of Adjustment and Intervention Variables

\begin{tabular}{|c|c|c|c|c|}
\hline & \multicolumn{4}{|c|}{ Outcome: Count Per Week } \\
\hline & (1) & (2) & (3) & (4) \\
\hline After Ferguson Protests Begin (week of $8 / 11 / 14$ onward) & -14.91 & & -13.00 & -12.24 \\
\hline After Gray Protests Begin (week of 4/20/15 onward) & -1.07 & & -6.27 & -7.84 \\
\hline Unrest and National Guard $(4 / 27 / 15$ - 5/3/2015) & -37.45 & & -38.36 & -38.88 \\
\hline After Batts Fired (week of $7 / 13 / 15$ onward) & 6.12 & & 2.56 & 5.74 \\
\hline After Davis Confirmed (week of 10/19/15 onward) & -7.09 & & -0.49 & -2.56 \\
\hline After Davis First Year (week of 10/17/16 onward) & 8.50 & & 7.63 & 9.10 \\
\hline After De Sousa Begins (week of $1 / 19 / 18$ onward) & -4.86 & & -0.27 & -3.85 \\
\hline After Tuggle Begins (week of 5/14/18 onward) & -0.28 & & -5.44 & -1.62 \\
\hline After Harrison Begins (week of 2/11/19 onward) & 2.63 & & 2.78 & 2.11 \\
\hline Average Maximum Temperature to 50 Degrees & & 0.25 & & 0.09 \\
\hline Plus Degrees in the $50 \mathrm{~s}$ & & -1.07 & & -0.24 \\
\hline Plus Degrees in the $60 \mathrm{~s}$ & & 2.89 & & 1.02 \\
\hline Plus Degrees in the 70s & & -2.12 & & -1.29 \\
\hline Plus Degrees Greater Than 80 & & 0.46 & & 0.72 \\
\hline Snowfall (inches) & & -9.33 & & -5.25 \\
\hline Precipitation (inches) & & -5.53 & & -5.11 \\
\hline Darkness Before Midnight (hours) & & 1.40 & & -1.09 \\
\hline Thanksgiving/Christmas (proportion of week) & & -114.30 & & -97.98 \\
\hline Winter Holiday (proportion of week) & & 10.28 & & 11.10 \\
\hline Other Out-of-School Days (proportion of week) & & 15.22 & & 0.79 \\
\hline Summer Vacation (proportion of week) & & -3.85 & & 0.27 \\
\hline Summer Holiday (proportion of week) & & -41.98 & & 12.12 \\
\hline Observations & 373 & 83 & 373 & 373 \\
\hline $\mathrm{R}^{2}$ & 0.23 & 0.32 & 0.26 & 0.41 \\
\hline
\end{tabular}


13.3 Least Squares Updated Model (2) for common.assault common.assault

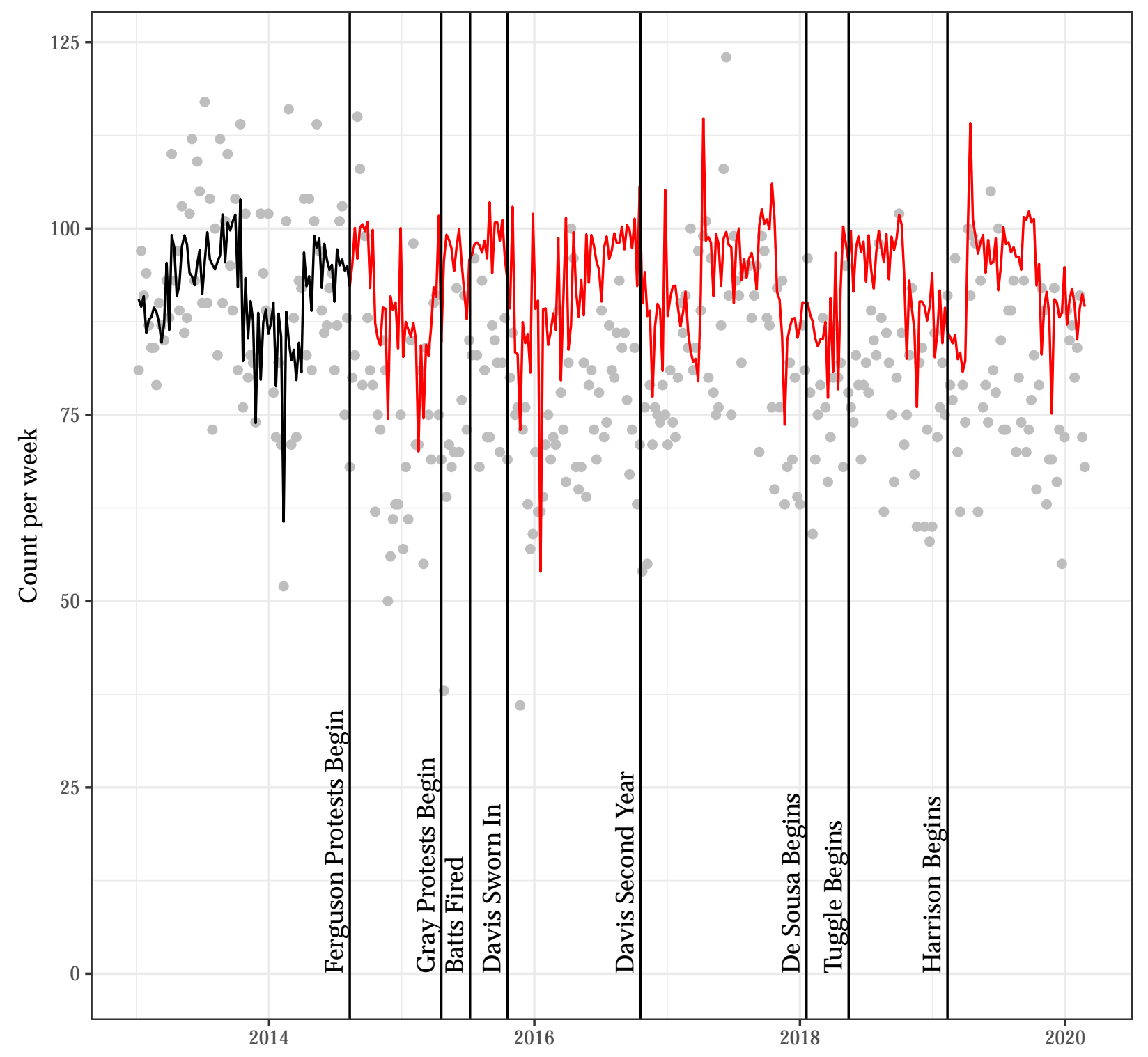




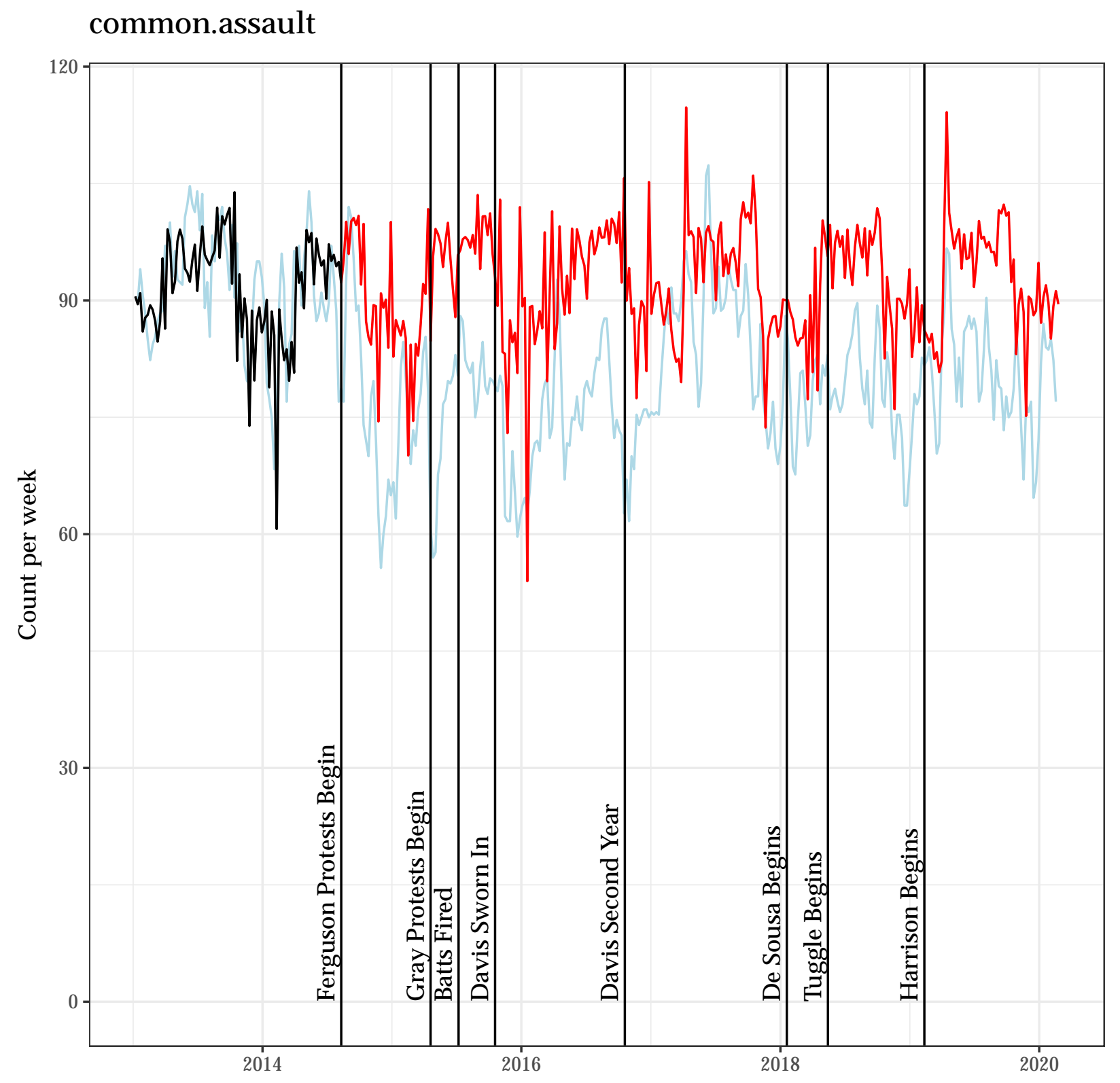


Autocorrelation Function for the Observed Outcome for Updated Model (2)

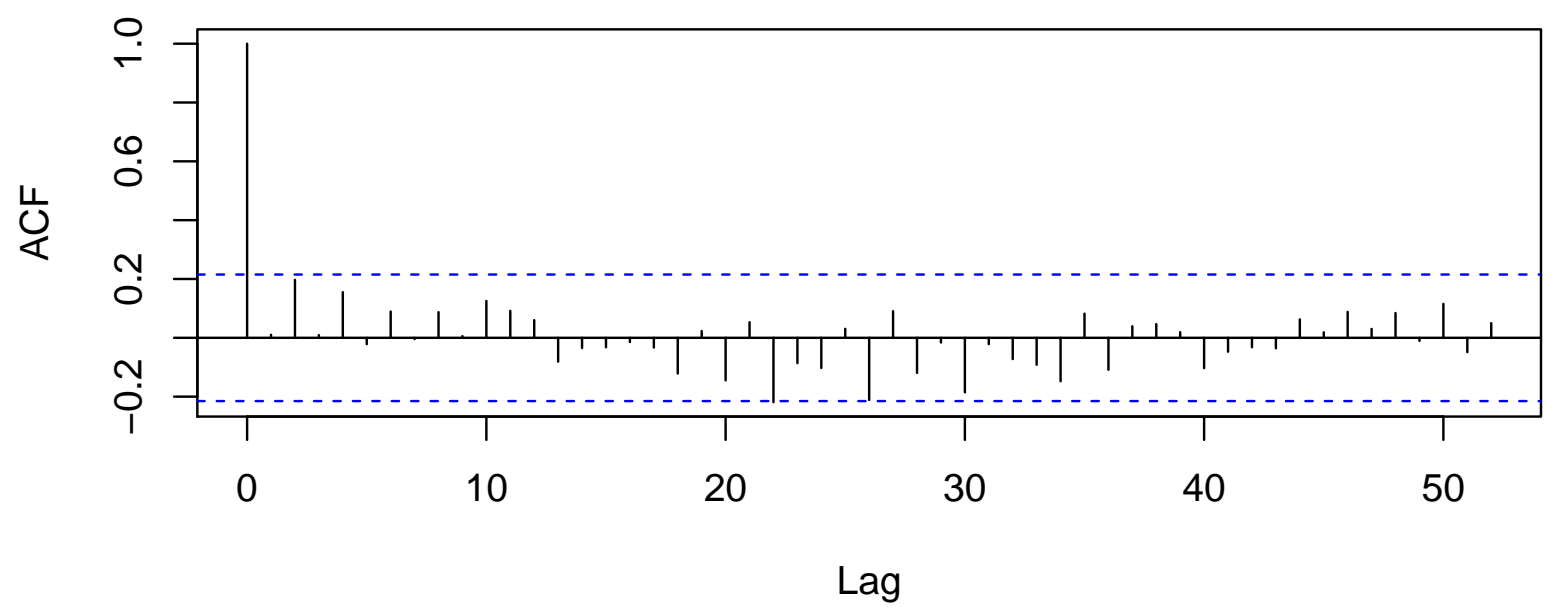

Autocorrelation Function for the Residuals from Updated Model (2)

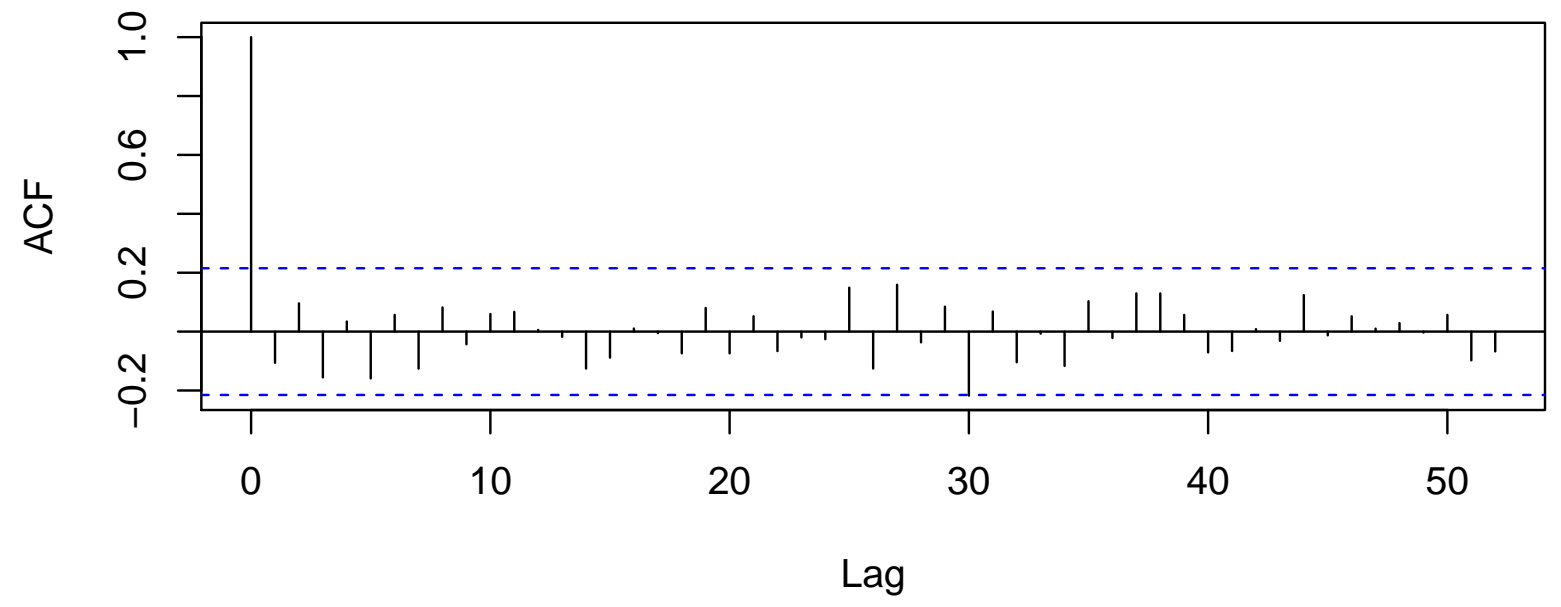




\subsection{A Poisson Regression Model as an Alternative to Model (2) for common.assault}

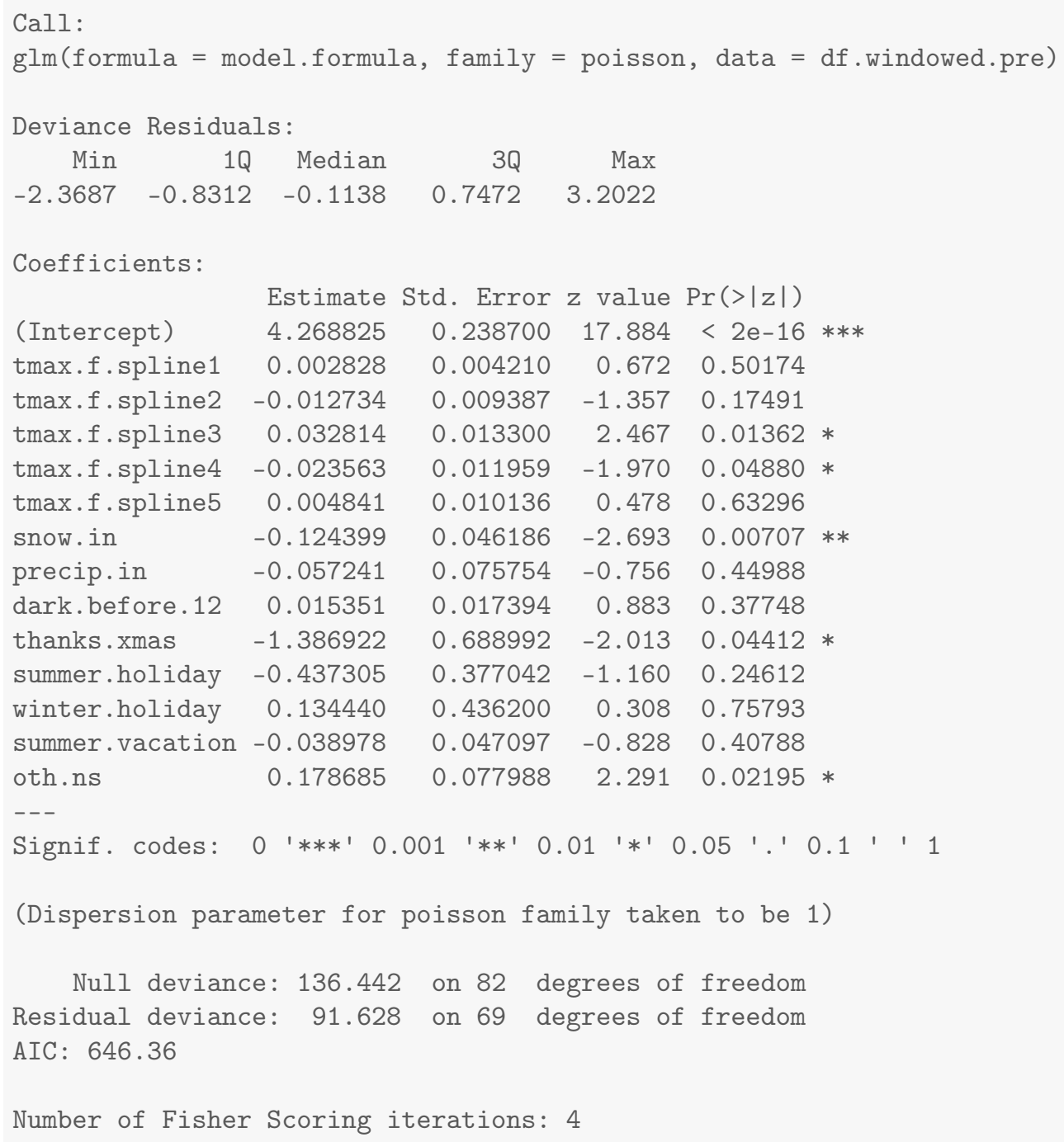




\section{Results for sex.offense.g}

\subsection{Summary Values for sex.offense.g}

Table 14.1: Descriptives for Outcome Before Ferguson Protests Begin

\begin{tabular}{lcccccccc}
\hline \hline Statistic & $\mathrm{N}$ & Mean & St. Dev. & Min & Pctl(25) & Median & Pctl(75) & Max \\
\hline sex.offense.g & 83 & 15.060 & 5.709 & 5 & 11 & 14 & 17 & 33 \\
\hline
\end{tabular}

Table 14.2: Descriptives for Outcome After Ferguson Protests Begin

\begin{tabular}{lcccccccc}
\hline \hline Statistic & $\mathrm{N}$ & Mean & St. Dev. & Min & Pctl(25) & Median & Pctl(75) & Max \\
\hline sex.offense.g & 290 & 8.997 & 4.683 & 0 & 6 & 8 & 12 & 35 \\
\hline
\end{tabular}




\subsection{Four Models for sex.offense.g}

Table 14.3: Four Models that Differ on the Specification of Adjustment and Intervention Variables

\begin{tabular}{|c|c|c|c|c|}
\hline & \multicolumn{4}{|c|}{ Outcome: Count Per Week } \\
\hline & $(1)$ & (2) & (3) & $(4)$ \\
\hline After Ferguson Protests Begin (week of 8/11/14 onward) & -1.31 & & -1.75 & -1.54 \\
\hline After Gray Protests Begin (week of $4 / 20 / 15$ onward) & -6.39 & & -5.79 & -5.90 \\
\hline Unrest and National Guard $(4 / 27 / 15-5 / 3 / 2015)$ & 27.64 & & 25.54 & 27.18 \\
\hline After Batts Fired (week of $7 / 13 / 15$ onward) & 3.85 & & 6.82 & 4.39 \\
\hline After Davis Confirmed (week of $10 / 19 / 15$ onward) & -1.89 & & -4.68 & -2.76 \\
\hline After Davis First Year (week of $10 / 17 / 16$ onward) & -0.36 & & 0.27 & -0.11 \\
\hline After De Sousa Begins (week of $1 / 19 / 18$ onward) & -1.47 & & -4.72 & -2.66 \\
\hline After Tuggle Begins (week of 5/14/18 onward) & -0.04 & & 3.90 & 1.16 \\
\hline After Harrison Begins (week of 2/11/19 onward) & -1.04 & & -1.82 & -1.17 \\
\hline Average Maximum Temperature to 50 Degrees & & -0.31 & & -0.10 \\
\hline Plus Degrees in the $50 \mathrm{~s}$ & & 0.73 & & 0.29 \\
\hline Plus Degrees in the 60s & & -1.30 & & -0.55 \\
\hline Plus Degrees in the 70s & & 0.72 & & 0.34 \\
\hline Plus Degrees Greater Than 80 & & -0.001 & & 0.06 \\
\hline Snowfall (inches) & & -1.41 & & -1.27 \\
\hline Precipitation (inches) & & 1.86 & & 2.15 \\
\hline Darkness Before Midnight (hours) & & -2.47 & & -0.51 \\
\hline Thanksgiving/Christmas (proportion of week) & & 35.51 & & -6.34 \\
\hline Winter Holiday (proportion of week) & & 2.82 & & 12.36 \\
\hline Other Out-of-School Days (proportion of week) & & -5.26 & & -1.93 \\
\hline Summer Vacation (proportion of week) & & -1.66 & & -1.15 \\
\hline Summer Holiday (proportion of week) & & -8.88 & & -9.69 \\
\hline Observations & 373 & 83 & 373 & 373 \\
\hline $\mathrm{R}^{2}$ & 0.39 & 0.22 & 0.33 & 0.43 \\
\hline
\end{tabular}




\subsection{Least Squares Updated Model (2) for sex.offense.g sex.offense.g}

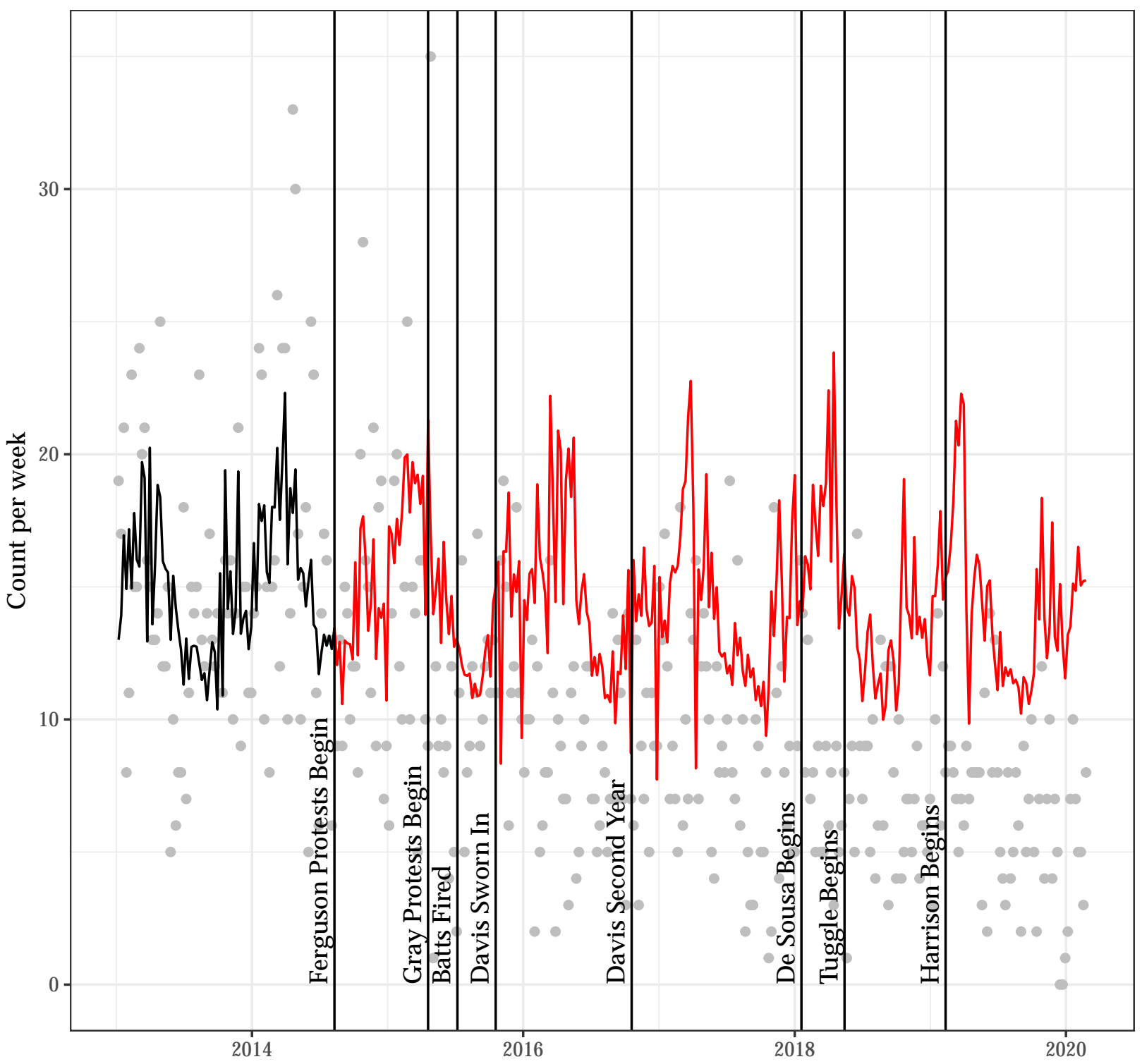




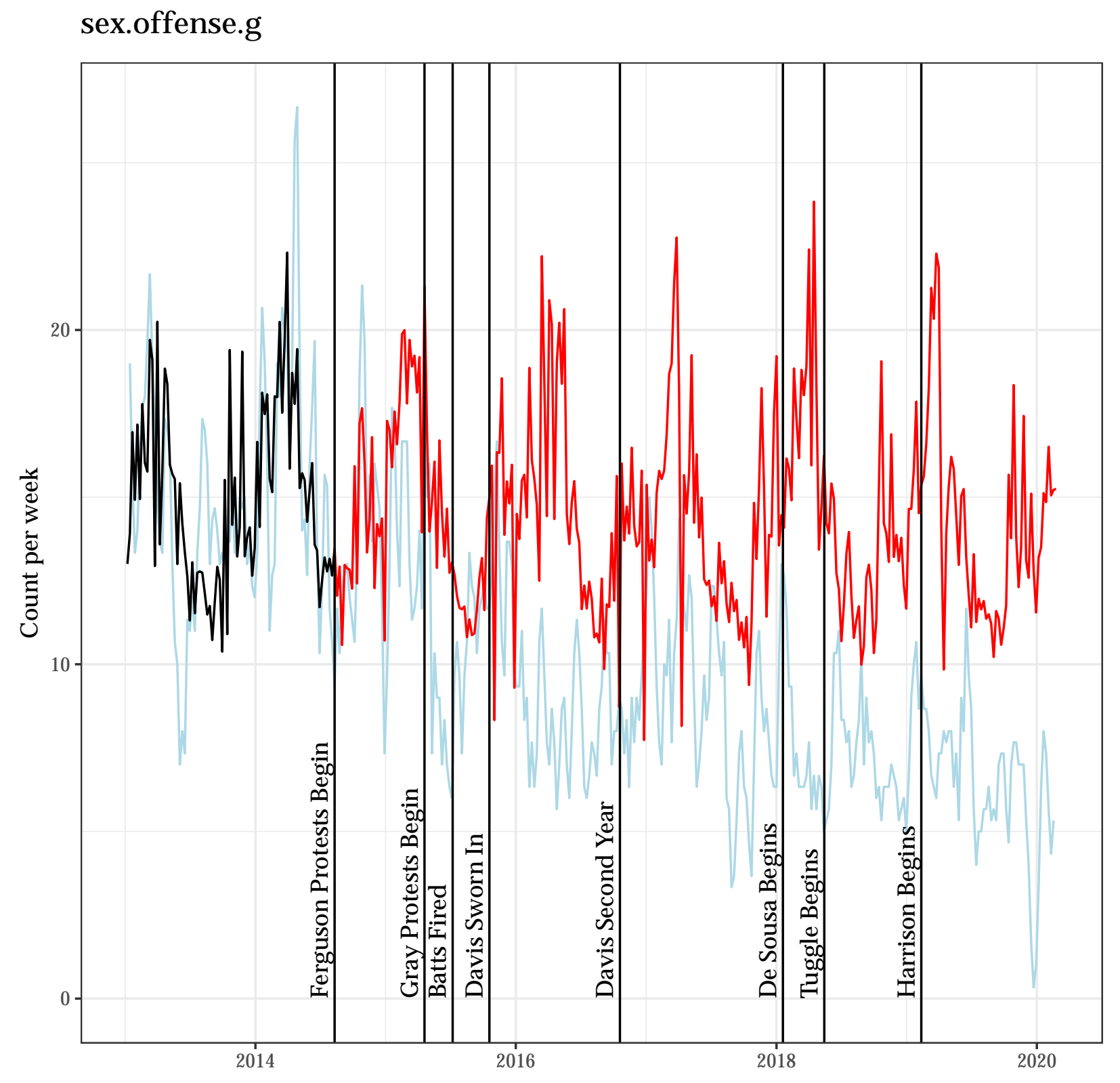


Autocorrelation Function for the Observed Outcome for Updated Model (2)

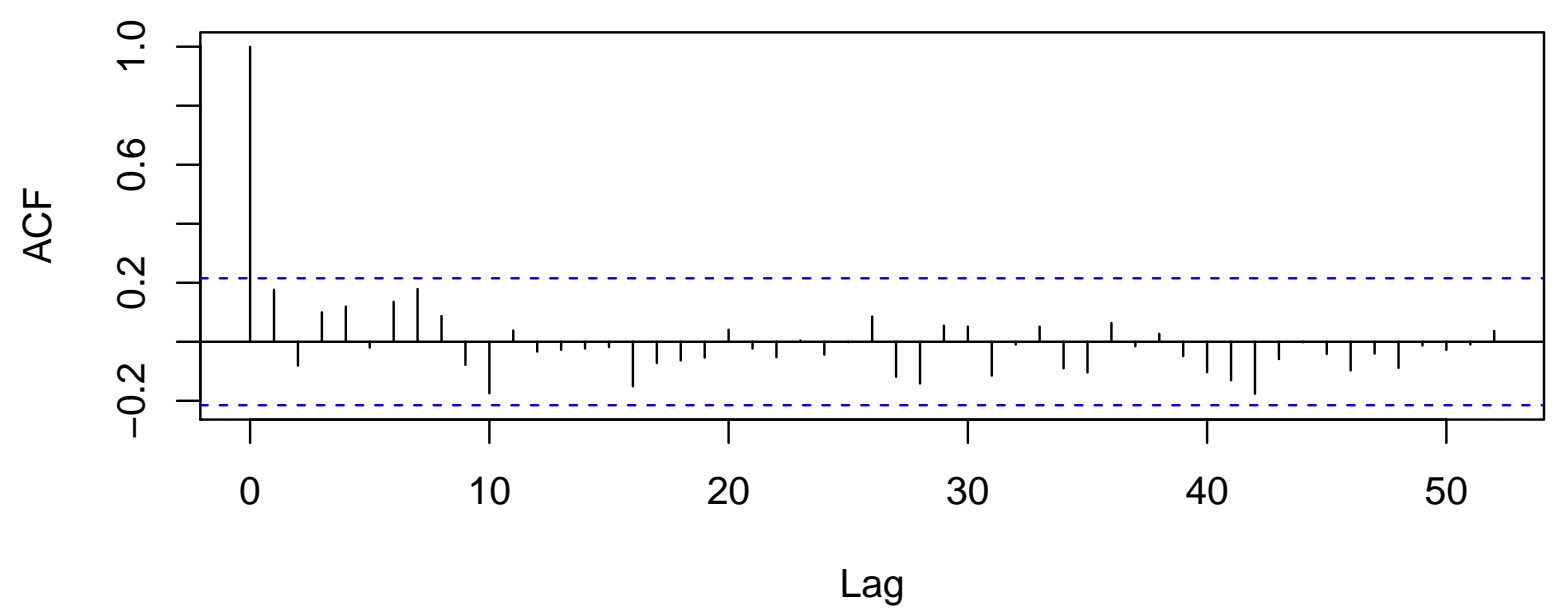

Autocorrelation Function for the Residuals from Updated Model (2)

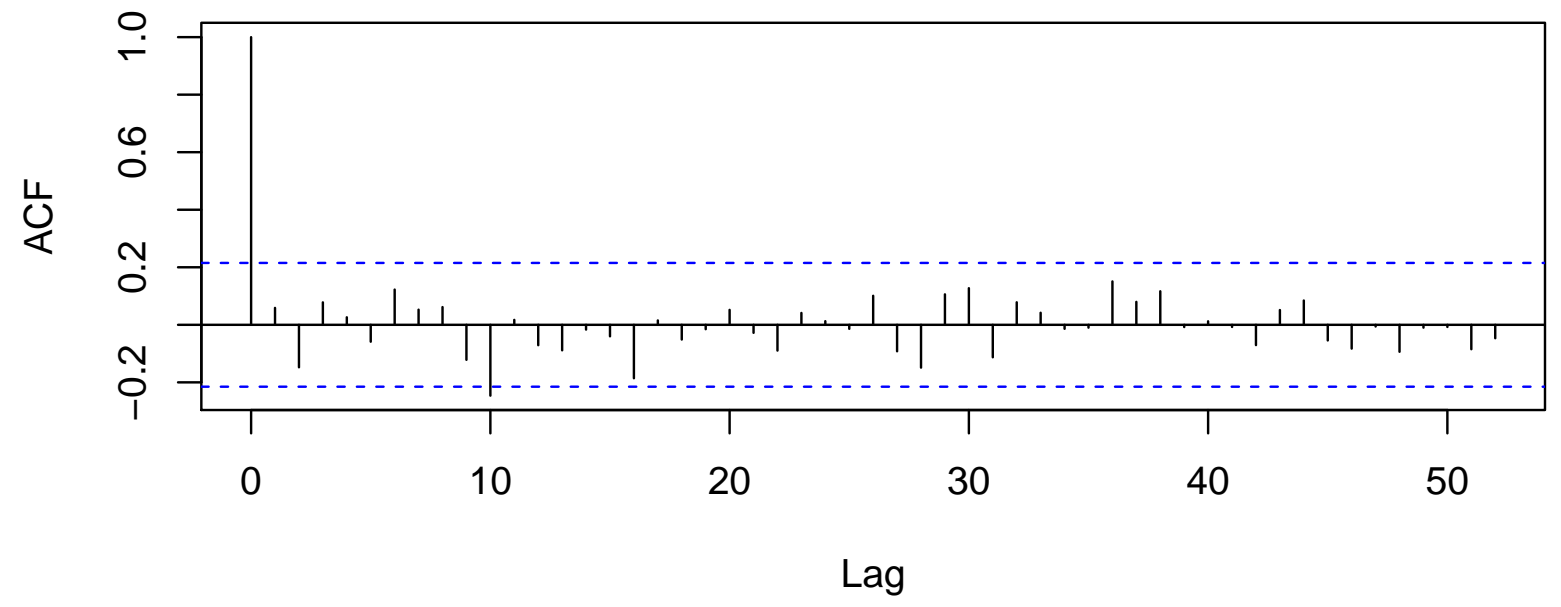




\subsection{A Poisson Regression Model as an Alternative to Model (2) for sex.offense.g}

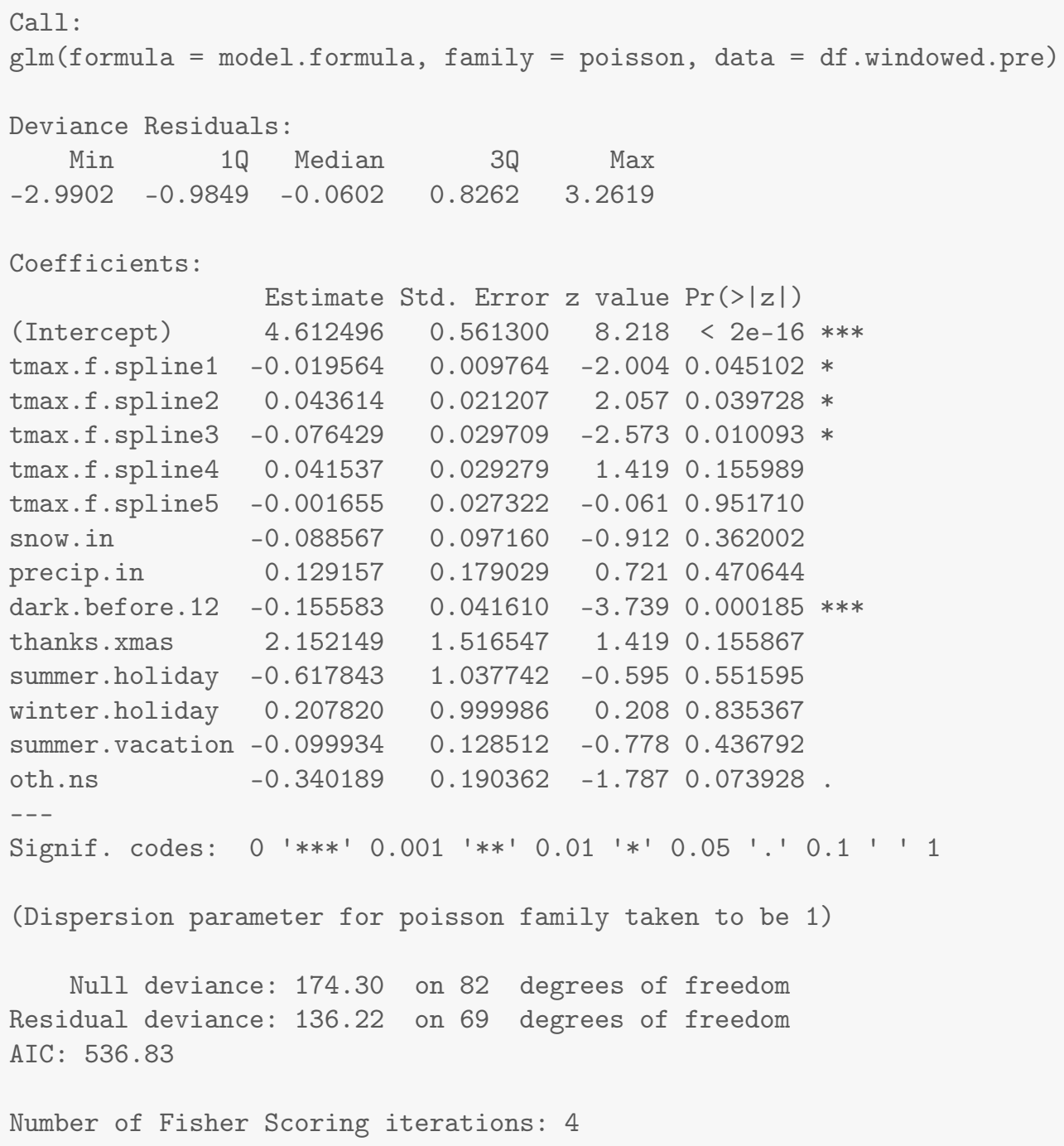




\section{Results for domestic}

\subsection{Summary Values for domestic}

Table 15.1: Descriptives for Outcome Before Ferguson Protests Begin

\begin{tabular}{lcccccccc}
\hline \hline Statistic & $\mathrm{N}$ & Mean & St. Dev. & Min & Pctl(25) & Median & Pctl(75) & Max \\
\hline domestic & 83 & 3.602 & 2.295 & 0 & 2 & 3 & 5 & 11 \\
\hline
\end{tabular}

Table 15.2: Descriptives for Outcome After Ferguson Protests Begin

\begin{tabular}{lcccccccc}
\hline \hline Statistic & $\mathrm{N}$ & Mean & St. Dev. & Min & Pctl(25) & Median & Pctl(75) & Max \\
\hline domestic & 290 & 2.569 & 1.905 & 0 & 1 & 2 & 4 & 9 \\
\hline
\end{tabular}




\subsection{Four Models for domestic}

Table 15.3: Four Models that Differ on the Specification of Adjustment and Intervention Variables

\begin{tabular}{|c|c|c|c|c|}
\hline & \multicolumn{4}{|c|}{ Outcome: Count Per Week } \\
\hline & $(1)$ & $(2)$ & $(3)$ & (4) \\
\hline After Ferguson Protests Begin (week of $8 / 11 / 14$ onward) & -1.49 & & -1.72 & -1.61 \\
\hline After Gray Protests Begin (week of 4/20/15 onward) & 0.34 & & 0.64 & 0.42 \\
\hline Unrest and National Guard (4/27/15 - 5/3/2015) & -0.45 & & -0.76 & -1.05 \\
\hline After Batts Fired (week of 7/13/15 onward) & -0.60 & & -0.99 & -0.33 \\
\hline After Davis Confirmed (week of $10 / 19 / 15$ onward) & 0.85 & & 0.74 & 0.56 \\
\hline After Davis First Year (week of 10/17/16 onward) & 0.59 & & 0.68 & 0.68 \\
\hline After De Sousa Begins (week of 1/19/18 onward) & -0.49 & & -0.37 & -0.90 \\
\hline After Tuggle Begins (week of 5/14/18 onward) & -1.25 & & -1.45 & -0.74 \\
\hline After Harrison Begins (week of 2/11/19 onward) & 1.14 & & 1.28 & 1.03 \\
\hline Average Maximum Temperature to 50 Degrees & & 0.02 & & -0.05 \\
\hline Plus Degrees in the $50 \mathrm{~s}$ & & 0.14 & & 0.13 \\
\hline Plus Degrees in the $60 \mathrm{~s}$ & & -0.17 & & -0.15 \\
\hline Plus Degrees in the 70s & & -0.03 & & 0.04 \\
\hline Plus Degrees Greater Than 80 & & 0.13 & & 0.05 \\
\hline Snowfall (inches) & & -0.49 & & -0.94 \\
\hline Precipitation (inches) & & 1.32 & & 0.29 \\
\hline Darkness Before Midnight (hours) & & 0.60 & & -0.17 \\
\hline Thanksgiving/Christmas (proportion of week) & & -3.11 & & -3.51 \\
\hline Winter Holiday (proportion of week) & & 2.90 & & -1.11 \\
\hline Other Out-of-School Days (proportion of week) & & -2.80 & & -1.91 \\
\hline Summer Vacation (proportion of week) & & -0.91 & & -0.81 \\
\hline Summer Holiday (proportion of week) & & 3.98 & & -2.20 \\
\hline Observations & 373 & 83 & 373 & 373 \\
\hline $\mathrm{R}^{2}$ & 0.10 & 0.15 & 0.13 & 0.19 \\
\hline
\end{tabular}


15.3 Least Squares Updated Model (2) for domestic domestic

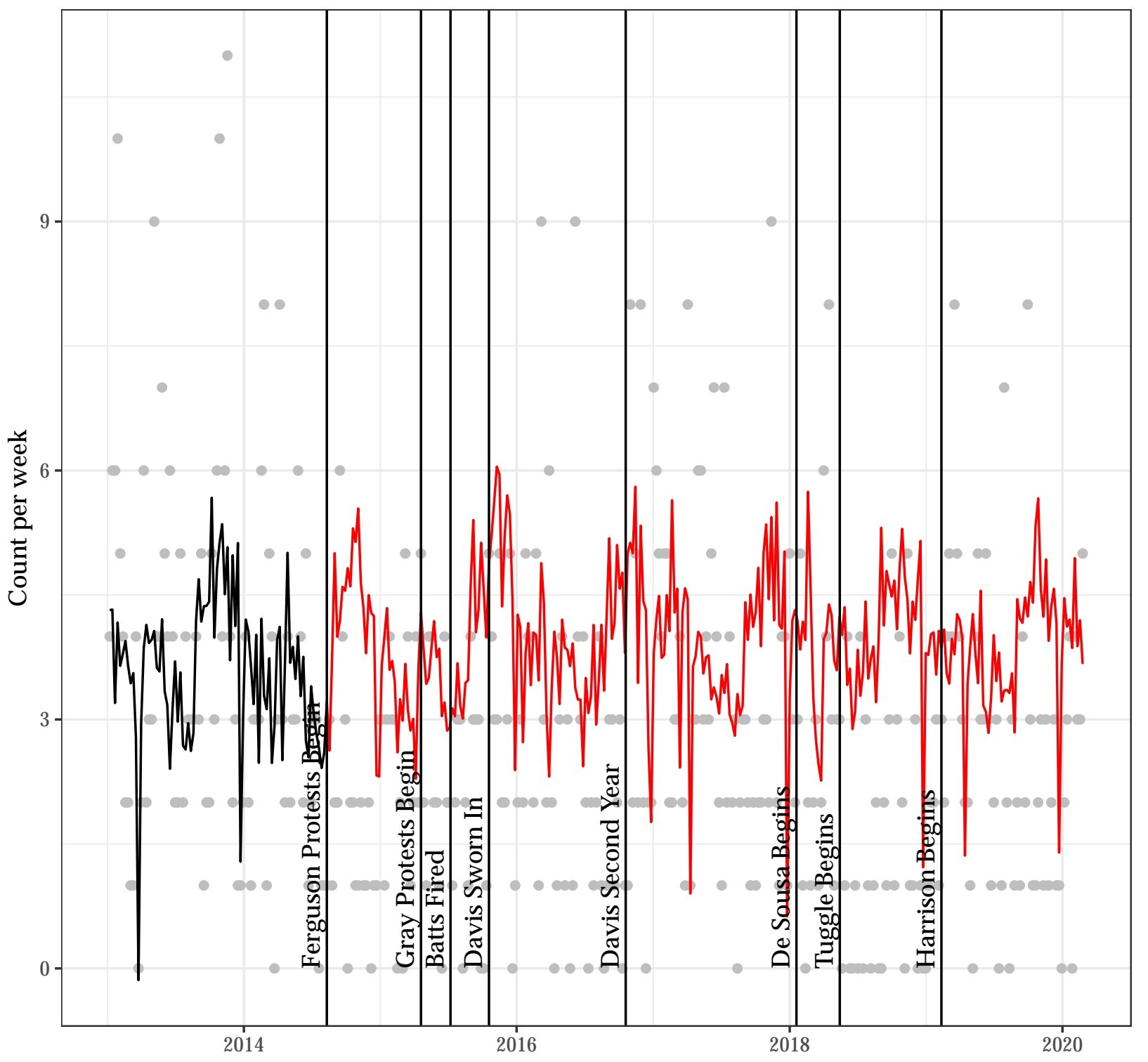




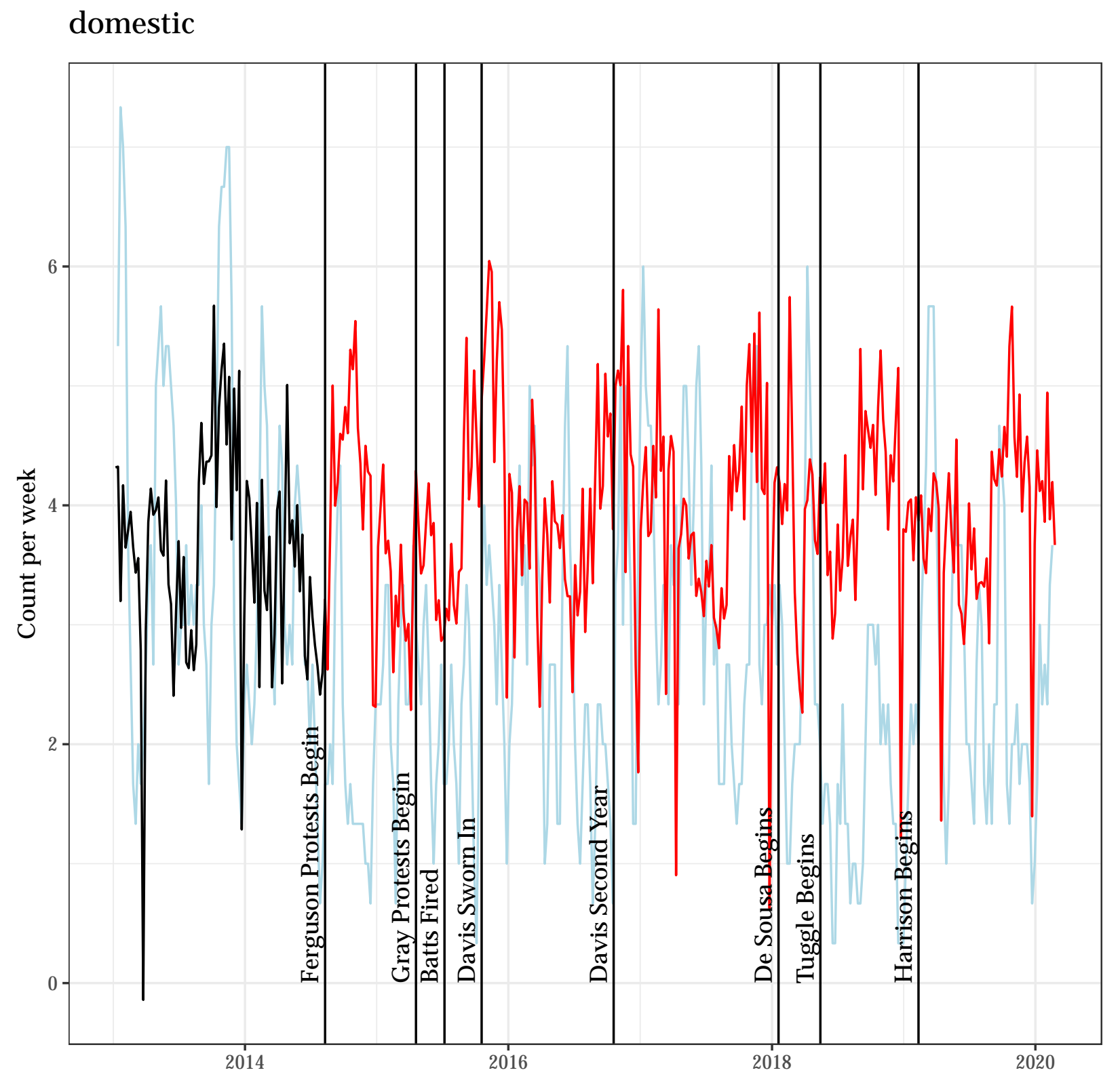


Autocorrelation Function for the Observed Outcome for Updated Model (2)

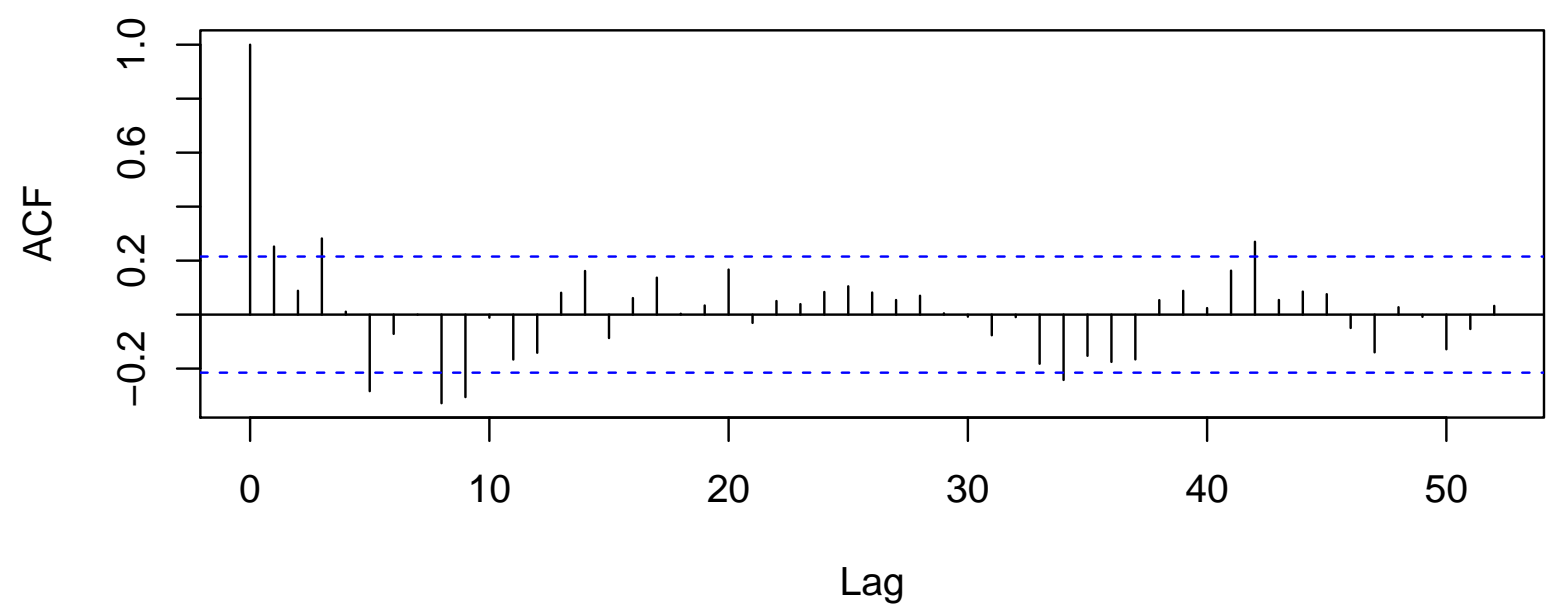

Autocorrelation Function for the Residuals from Updated Model (2)

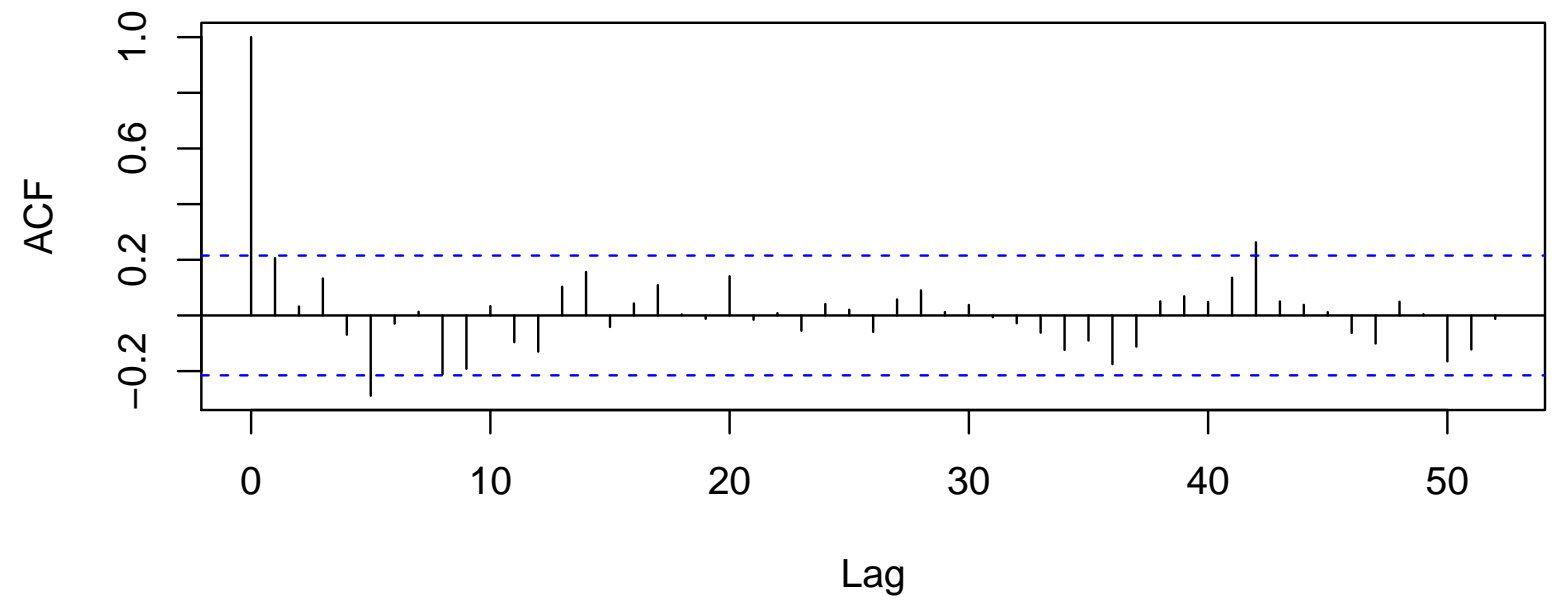




\subsection{A Poisson Regression Model as an Alternative to Model (2) for domestic}

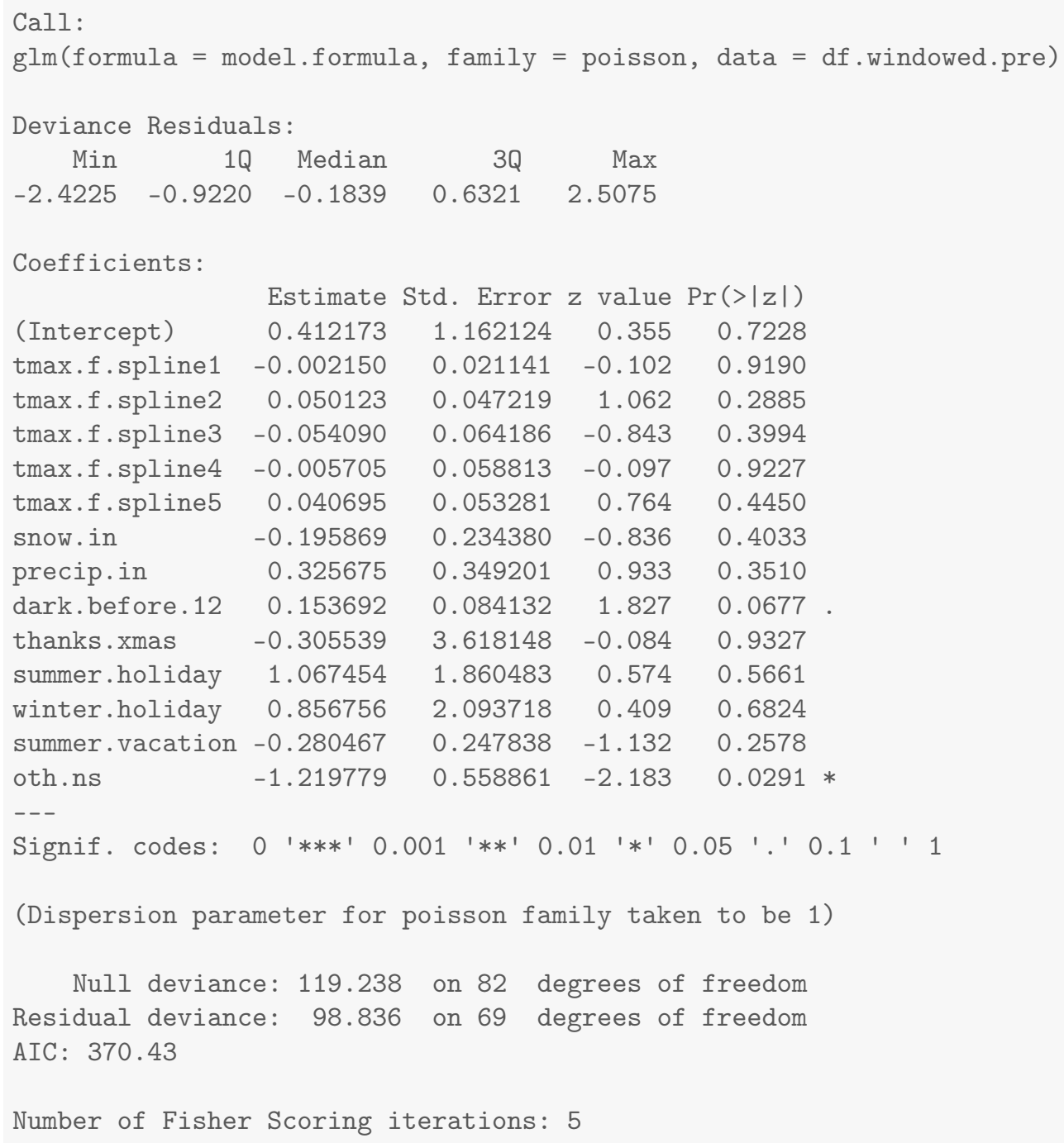




\section{Results for non.deadly.weapon}

\subsection{Summary Values for non.deadly.weapon}

Table 16.1: Descriptives for Outcome Before Ferguson Protests Begin

\begin{tabular}{lcccccccc}
\hline \hline Statistic & $\mathrm{N}$ & Mean & St. Dev. & Min & Pctl(25) & Median & Pctl(75) & Max \\
\hline non.deadly.weapon & 83 & 1.217 & 1.230 & 0 & 0 & 1 & 2 & 5 \\
\hline
\end{tabular}

Table 16.2: Descriptives for Outcome After Ferguson Protests Begin

\begin{tabular}{lcccccccc}
\hline \hline Statistic & $\mathrm{N}$ & Mean & St. Dev. & Min & Pctl(25) & Median & Pctl(75) & Max \\
\hline non.deadly.weapon & 290 & 1.086 & 1.108 & 0 & 0 & 1 & 2 & 7 \\
\hline
\end{tabular}




\subsection{Four Models for non.deadly.weapon}

Table 16.3: Four Models that Differ on the Specification of Adjustment and Intervention Variables

\begin{tabular}{|c|c|c|c|c|}
\hline & \multicolumn{4}{|c|}{ Outcome: Count Per Week } \\
\hline & $(1)$ & (2) & $(3)$ & (4) \\
\hline After Ferguson Protests Begin (week of 8/11/14 onward) & -0.02 & & 0.04 & -0.01 \\
\hline After Gray Protests Begin (week of $4 / 20 / 15$ onward) & -0.38 & & -0.48 & -0.37 \\
\hline Unrest and National Guard $(4 / 27 / 15-5 / 3 / 2015)$ & 0.18 & & 0.16 & 0.15 \\
\hline After Batts Fired (week of $7 / 13 / 15$ onward) & 0.11 & & 0.31 & 0.17 \\
\hline After Davis Confirmed (week of 10/19/15 onward) & 0.65 & & 0.49 & 0.60 \\
\hline After Davis First Year (week of $10 / 17 / 16$ onward) & -0.46 & & -0.43 & -0.46 \\
\hline After De Sousa Begins (week of 1/19/18 onward) & -0.12 & & -0.25 & -0.18 \\
\hline After Tuggle Begins (week of 5/14/18 onward) & -0.03 & & 0.27 & 0.06 \\
\hline After Harrison Begins (week of 2/11/19 onward) & -0.27 & & -0.38 & -0.30 \\
\hline Average Maximum Temperature to 50 Degrees & & 0.02 & & 0.01 \\
\hline Plus Degrees in the $50 \mathrm{~s}$ & & 0.03 & & -0.03 \\
\hline Plus Degrees in the 60s & & -0.11 & & 0.01 \\
\hline Plus Degrees in the $70 \mathrm{~s}$ & & 0.08 & & 0.01 \\
\hline Plus Degrees Greater Than 80 & & -0.07 & & 0.01 \\
\hline Snowfall (inches) & & 0.45 & & 0.15 \\
\hline Precipitation (inches) & & -0.73 & & -0.34 \\
\hline Darkness Before Midnight (hours) & & -0.10 & & -0.07 \\
\hline Thanksgiving/Christmas (proportion of week) & & -1.29 & & 1.60 \\
\hline Winter Holiday (proportion of week) & & -2.55 & & -3.49 \\
\hline Other Out-of-School Days (proportion of week) & & 0.73 & & -0.29 \\
\hline Summer Vacation (proportion of week) & & 0.27 & & 0.11 \\
\hline Summer Holiday (proportion of week) & & 3.34 & & 2.17 \\
\hline Observations & 373 & 83 & 373 & 373 \\
\hline $\mathrm{R}^{2}$ & 0.05 & 0.07 & 0.04 & 0.07 \\
\hline
\end{tabular}




\subsection{Least Squares Updated Model (2) for non.deadly.weapon}

non.deadly.weapon

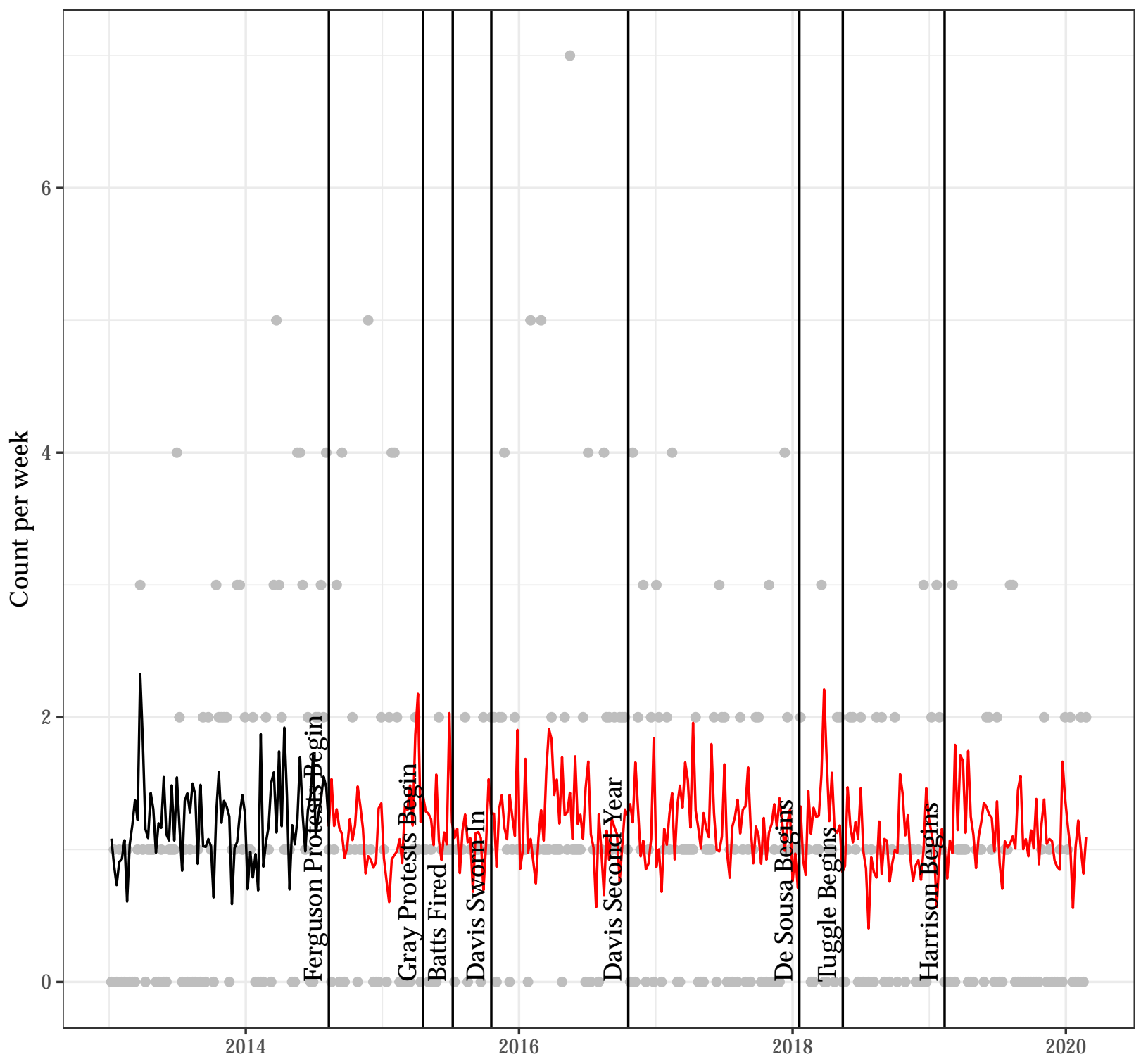




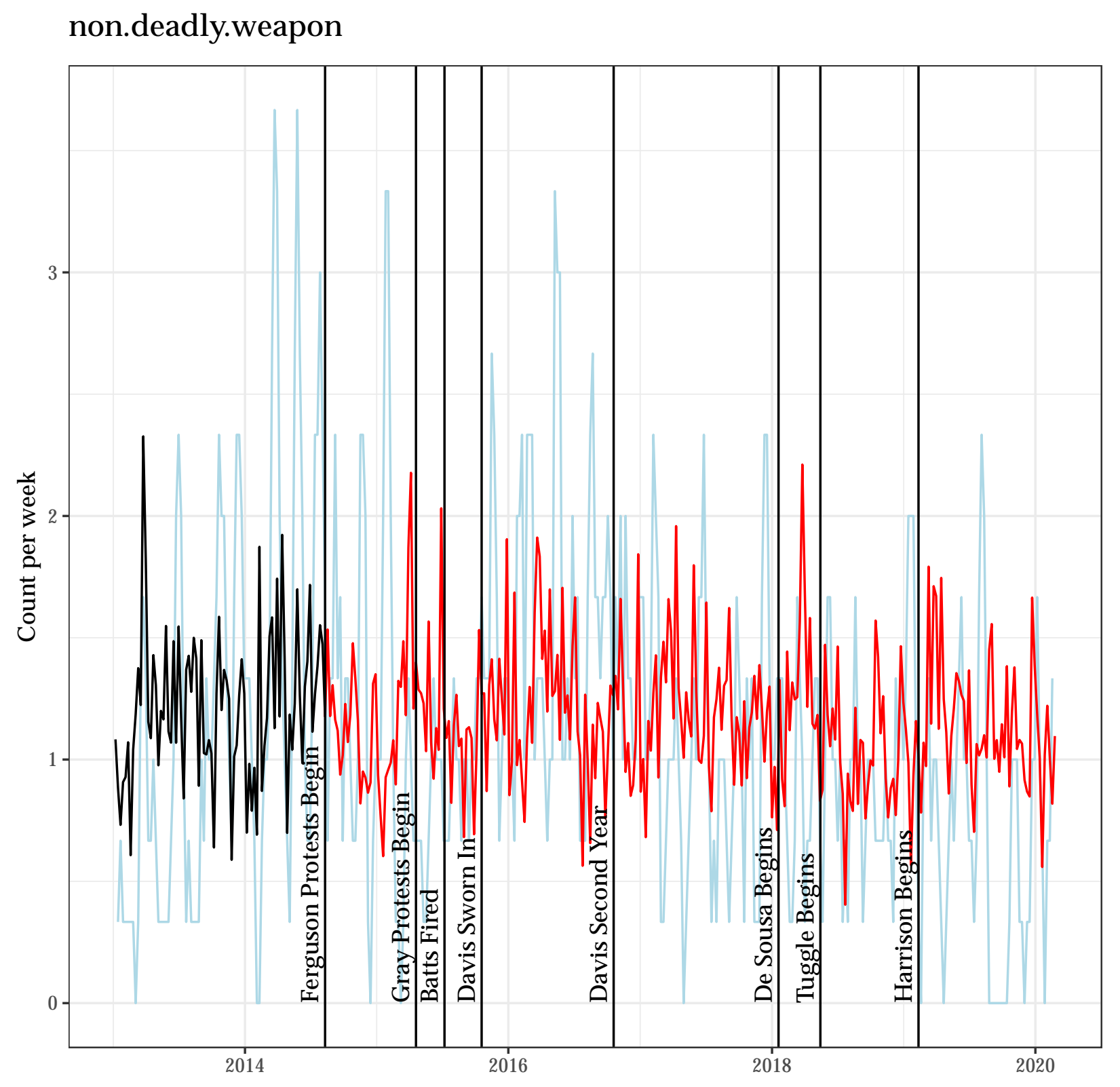


Autocorrelation Function for the Observed Outcome for Updated Model (2)

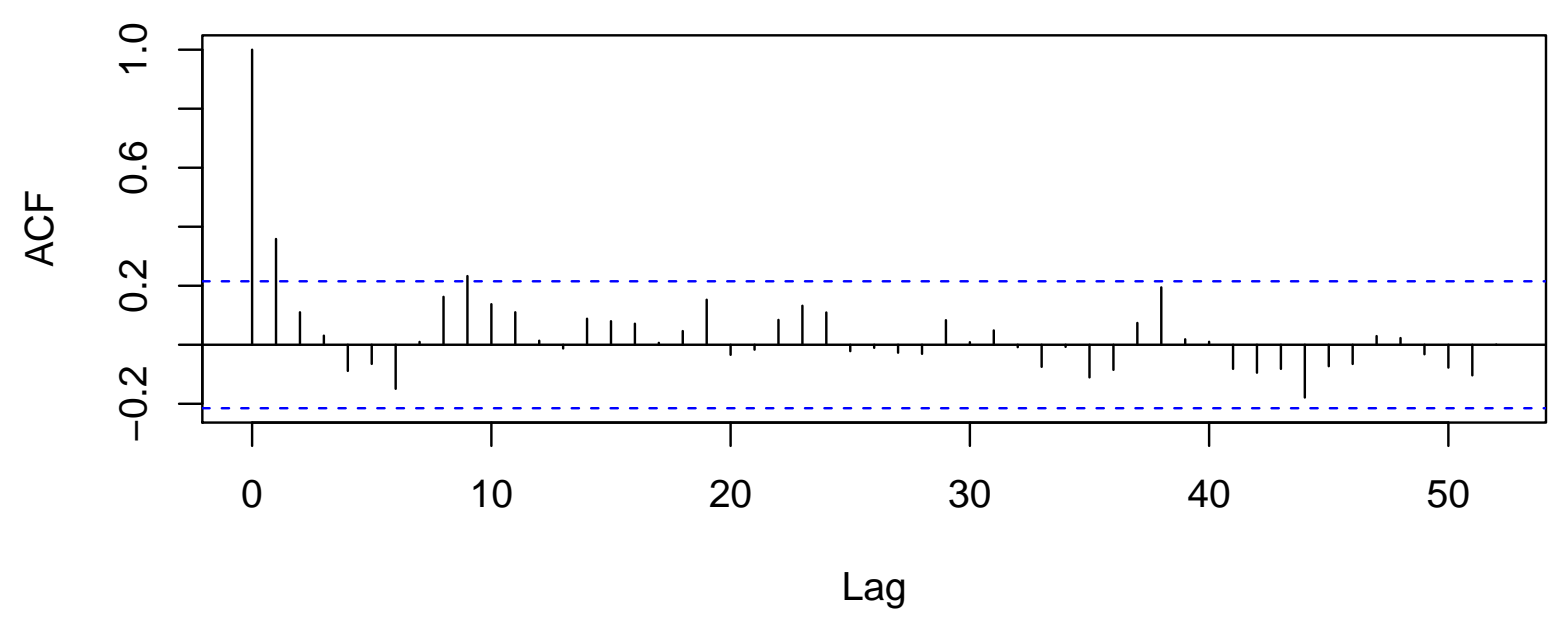

Autocorrelation Function for the Residuals from Updated Model (2)

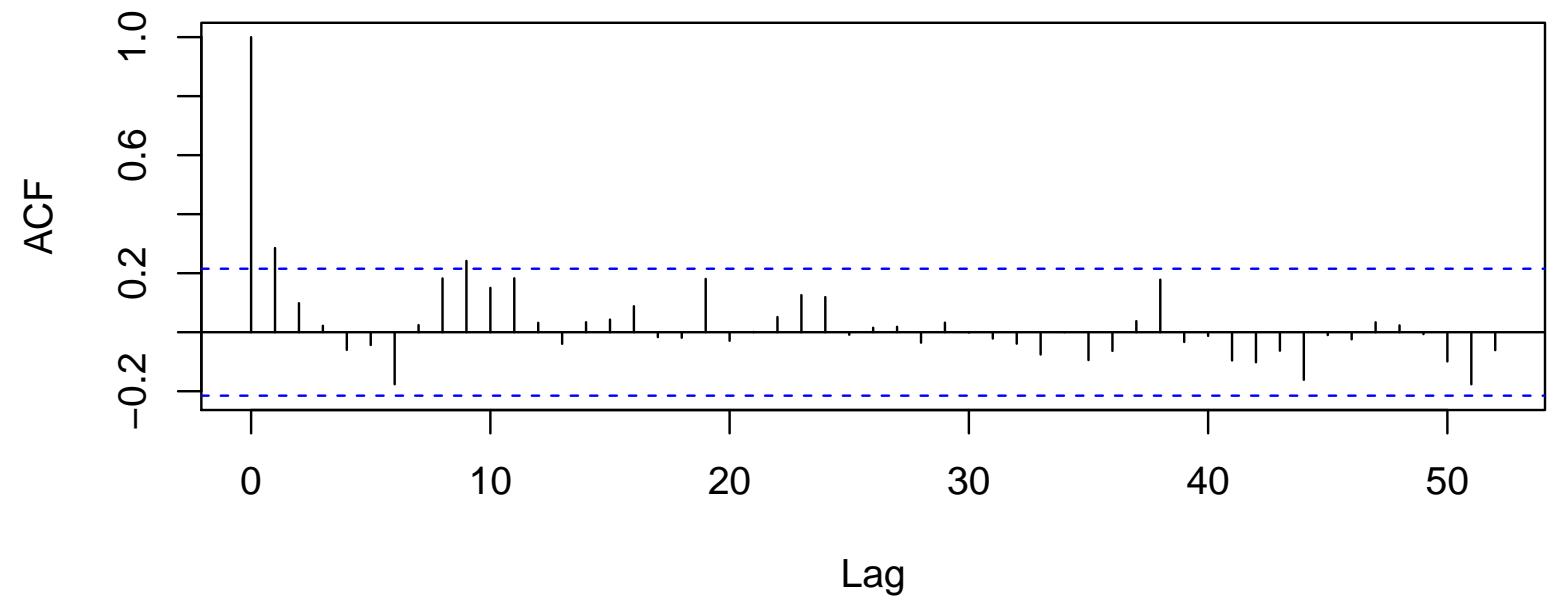




\subsection{A Poisson Regression Model as an Alternative to Model (2) for non.deadly.weapon}

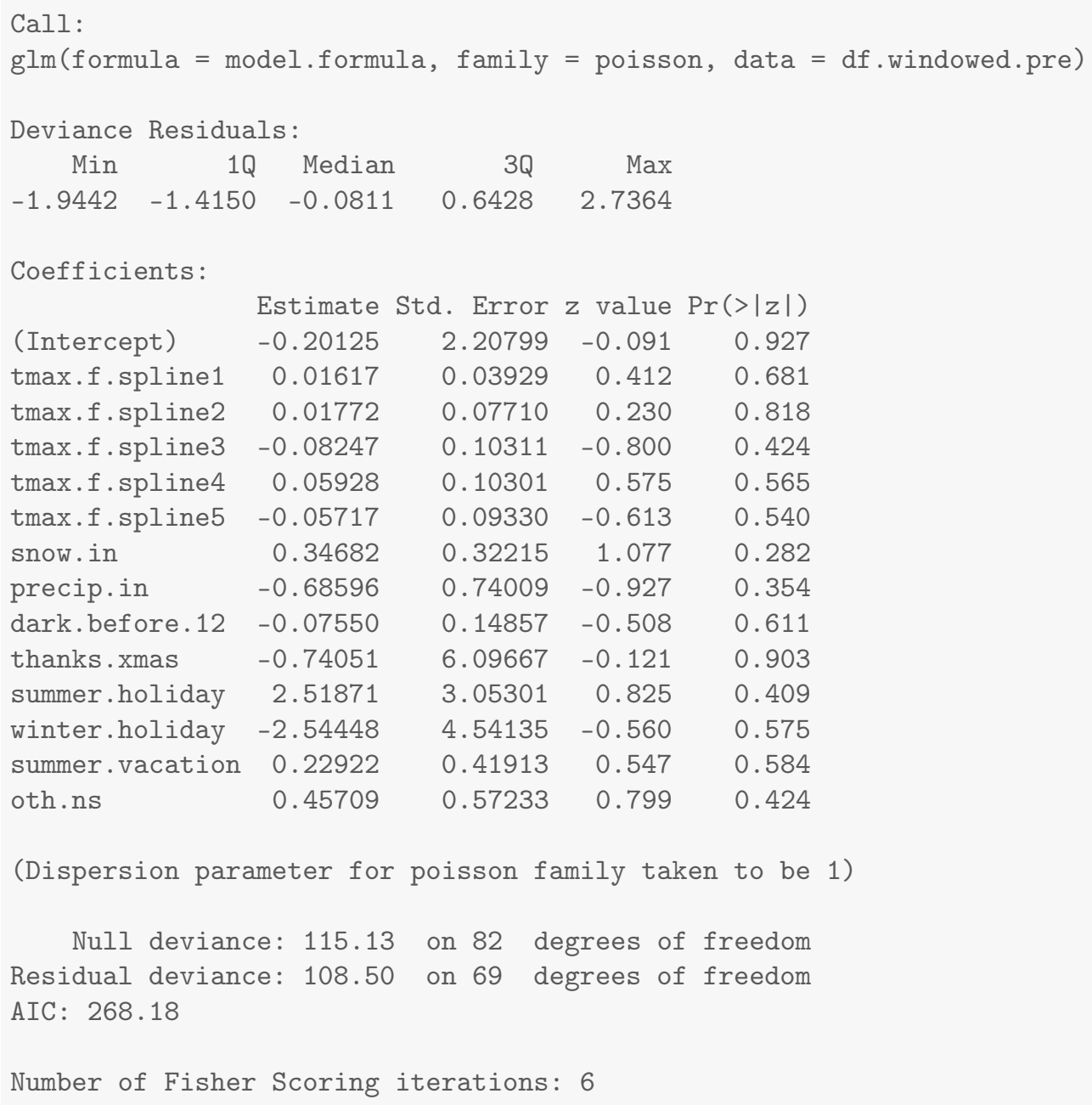




\section{Results for burglary}

\subsection{Summary Values for burglary}

Table 17.1: Descriptives for Outcome Before Ferguson Protests Begin

\begin{tabular}{lcccccccc}
\hline \hline Statistic & $\mathrm{N}$ & Mean & St. Dev. & Min & Pctl(25) & Median & Pctl(75) & Max \\
\hline burglary & 83 & 14.530 & 4.735 & 2 & 12 & 14 & 17 & 26 \\
\hline
\end{tabular}

Table 17.2: Descriptives for Outcome After Ferguson Protests Begin

\begin{tabular}{lcccccccc}
\hline \hline Statistic & $\mathrm{N}$ & Mean & St. Dev. & Min & Pctl(25) & Median & Pctl(75) & Max \\
\hline burglary & 290 & 10.897 & 4.974 & 1 & 8 & 10 & 13 & 52 \\
\hline
\end{tabular}




\subsection{Four Models for burglary}

Table 17.3: Four Models that Differ on the Specification of Adjustment and Intervention Variables

\begin{tabular}{|c|c|c|c|c|}
\hline & \multicolumn{4}{|c|}{ Outcome: Count Per Week } \\
\hline & $(1)$ & $(2)$ & $(3)$ & $(4)$ \\
\hline After Ferguson Protests Begin (week of $8 / 11 / 14$ onward) & -1.53 & & -0.69 & -1.22 \\
\hline After Gray Protests Begin (week of 4/20/15 onward) & -1.27 & & -2.74 & -1.67 \\
\hline Unrest and National Guard (4/27/15 - 5/3/2015) & 40.27 & & 37.78 & 38.33 \\
\hline After Batts Fired (week of $7 / 13 / 15$ onward) & 1.06 & & 1.56 & 0.23 \\
\hline After Davis Confirmed (week of $10 / 19 / 15$ onward) & -2.90 & & -2.90 & -2.32 \\
\hline After Davis First Year (week of 10/17/16 onward) & 0.90 & & 0.98 & 0.88 \\
\hline After De Sousa Begins (week of 1/19/18 onward) & 1.34 & & 0.75 & 1.59 \\
\hline After Tuggle Begins (week of 5/14/18 onward) & -2.48 & & -0.94 & -2.18 \\
\hline After Harrison Begins (week of 2/11/19 onward) & 0.10 & & -1.04 & -0.43 \\
\hline Average Maximum Temperature to 50 Degrees & & 0.29 & & 0.15 \\
\hline Plus Degrees in the $50 \mathrm{~s}$ & & -0.33 & & -0.15 \\
\hline Plus Degrees in the 60s & & 0.11 & & 0.19 \\
\hline Plus Degrees in the 70s & & -0.35 & & -0.36 \\
\hline Plus Degrees Greater Than 80 & & 0.22 & & 0.26 \\
\hline Snowfall (inches) & & -1.32 & & -0.80 \\
\hline Precipitation (inches) & & -4.83 & & -3.97 \\
\hline Darkness Before Midnight (hours) & & -0.92 & & 0.12 \\
\hline Thanksgiving/Christmas (proportion of week) & & 12.28 & & -4.51 \\
\hline Winter Holiday (proportion of week) & & 42.61 & & 10.58 \\
\hline Other Out-of-School Days (proportion of week) & & -1.79 & & -1.33 \\
\hline Summer Vacation (proportion of week) & & 0.49 & & -0.04 \\
\hline Summer Holiday (proportion of week) & & -0.87 & & -12.79 \\
\hline Observations & 373 & 83 & 373 & 373 \\
\hline $\mathrm{R}^{2}$ & 0.30 & 0.21 & 0.29 & 0.36 \\
\hline
\end{tabular}




\subsection{Least Squares Updated Model (2) for burglary burglary}

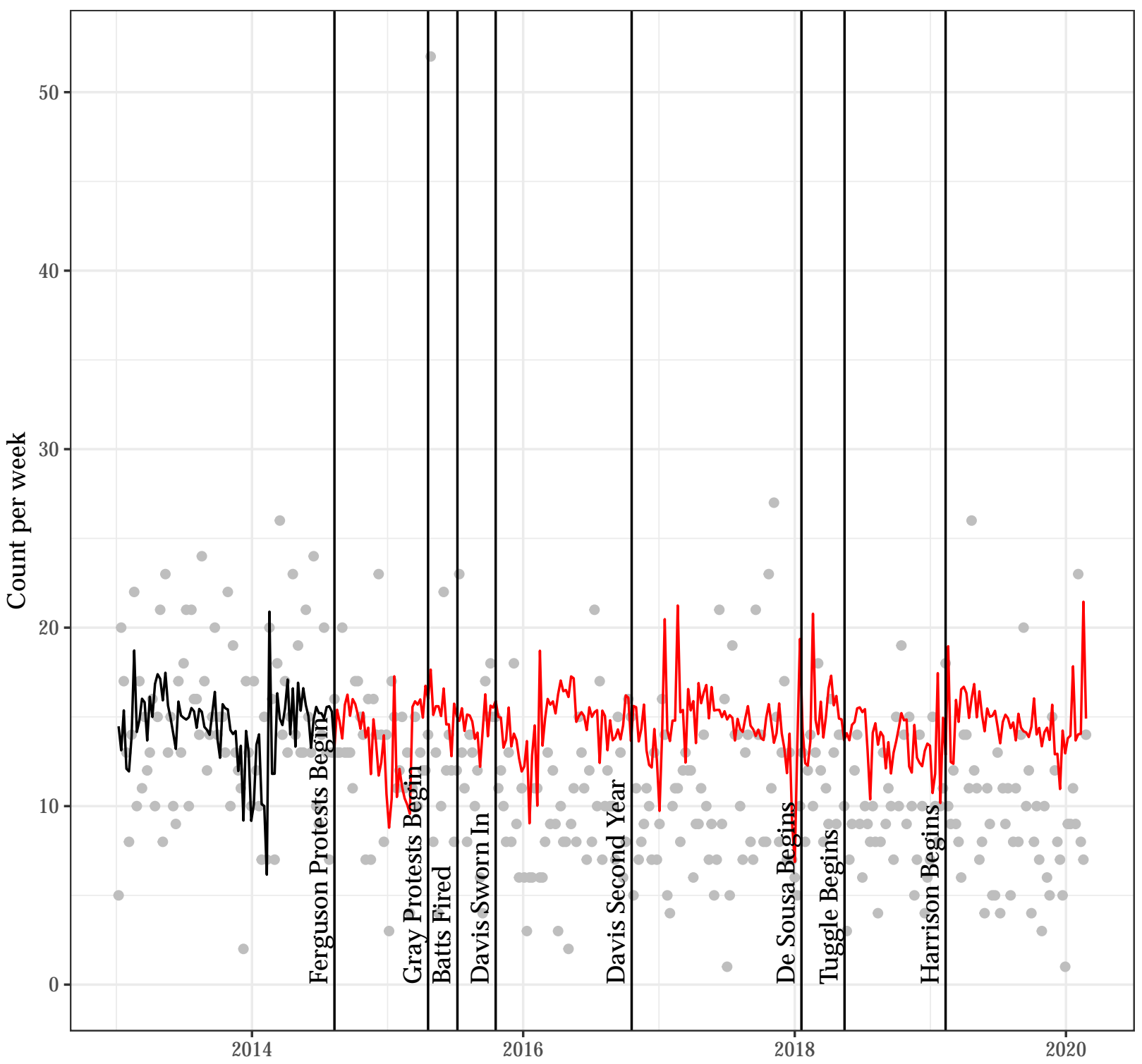




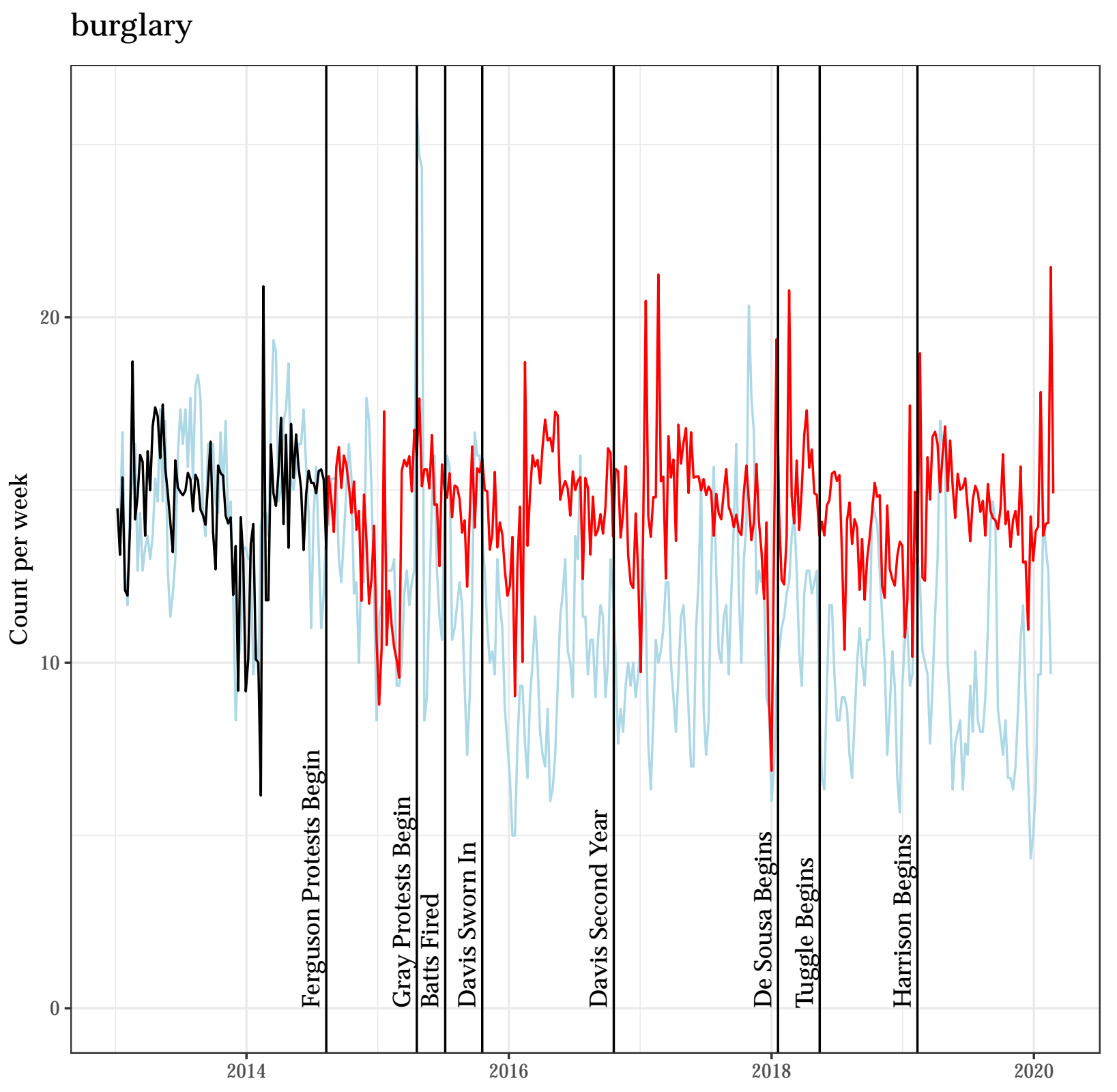


Autocorrelation Function for the Observed Outcome for Updated Model (2)

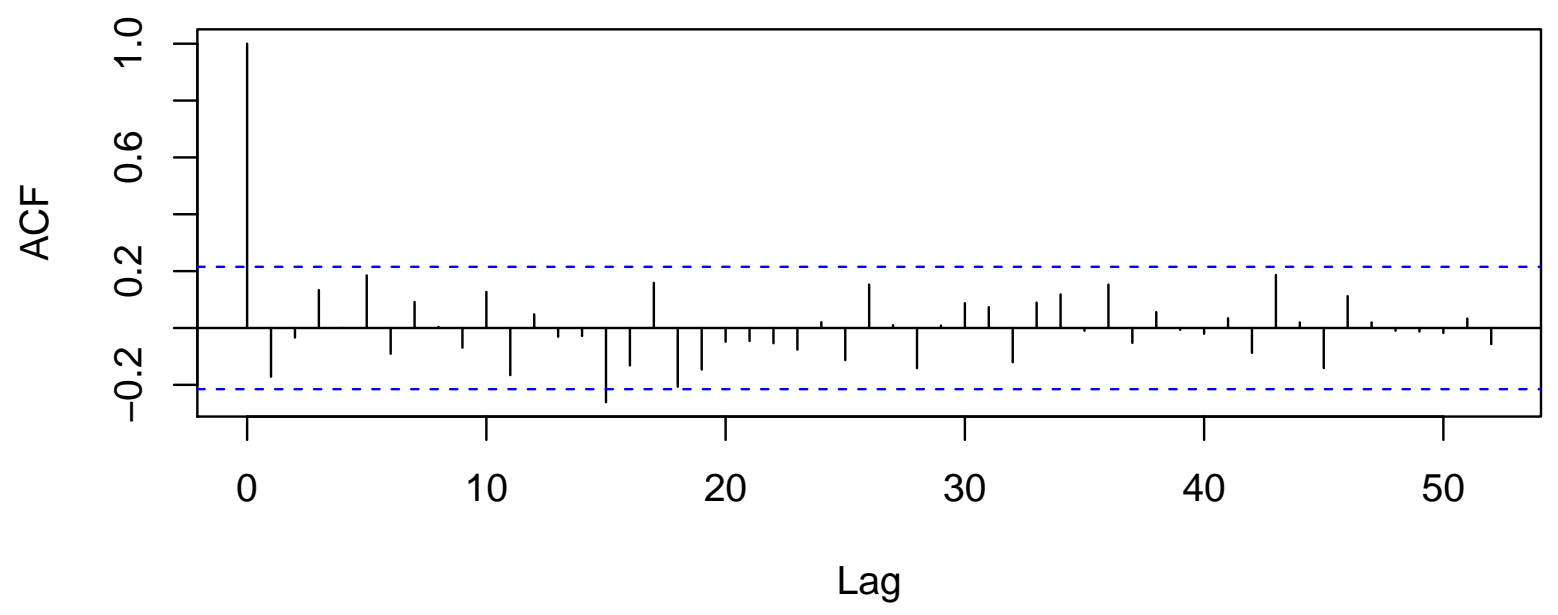

Autocorrelation Function for the Residuals from Updated Model (2)

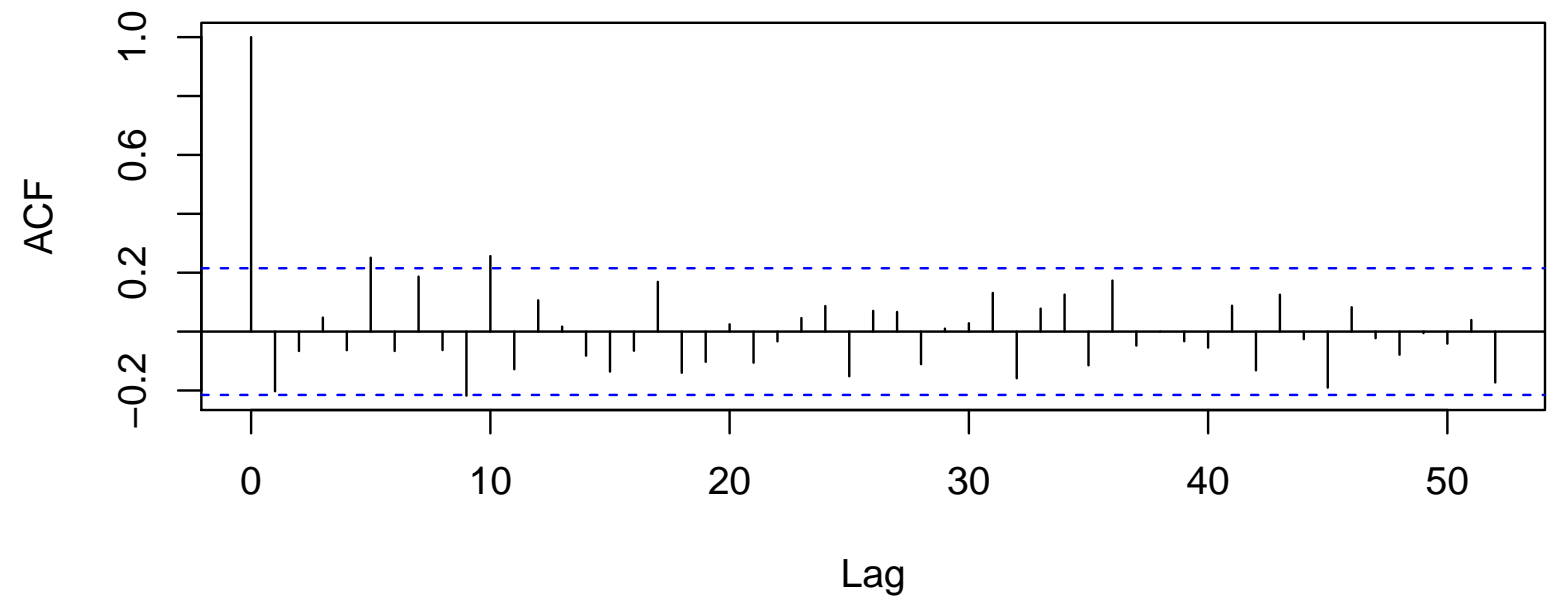




\subsection{A Poisson Regression Model as an Alternative to Model (2) for burglary}

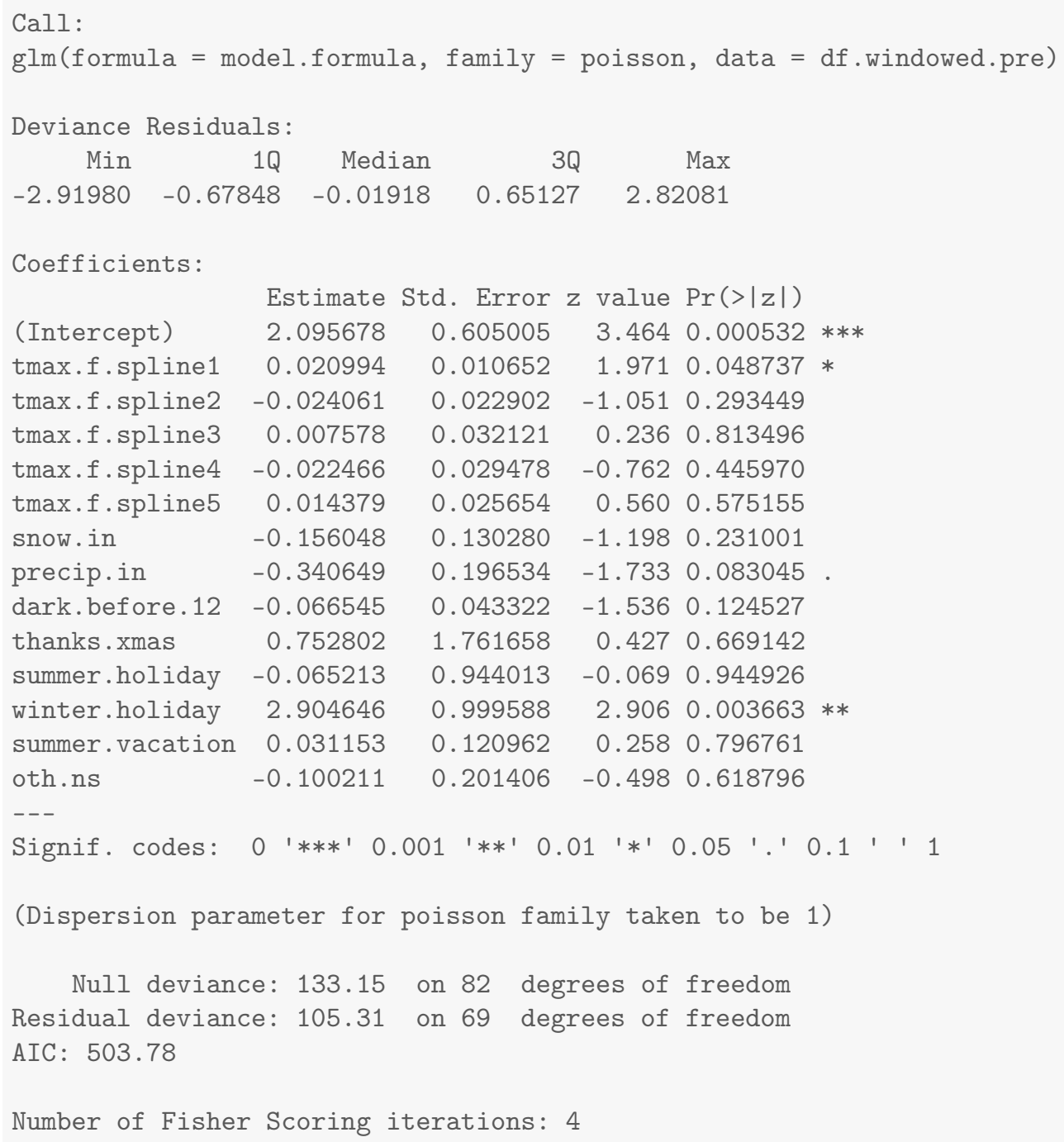




\section{Results for larceny}

\subsection{Summary Values for larceny}

Table 18.1: Descriptives for Outcome Before Ferguson Protests Begin

\begin{tabular}{lcccccccc}
\hline \hline Statistic & $\mathrm{N}$ & Mean & St. Dev. & Min & Pctl(25) & Median & Pctl(75) & Max \\
\hline larceny & 83 & 42.193 & 8.510 & 18 & 36 & 41 & 47 & 63 \\
\hline
\end{tabular}

Table 18.2: Descriptives for Outcome After Ferguson Protests Begin

\begin{tabular}{lcccccccc}
\hline \hline Statistic & $\mathrm{N}$ & Mean & St. Dev. & Min & Pctl(25) & Median & Pctl(75) & Max \\
\hline larceny & 290 & 26.190 & 7.924 & 7 & 20 & 25.5 & 30 & 54 \\
\hline
\end{tabular}




\subsection{Four Models for larceny}

Table 18.3: Four Models that Differ on the Specification of Adjustment and Intervention Variables

\begin{tabular}{|c|c|c|c|c|}
\hline & \multicolumn{4}{|c|}{ Outcome: Count Per Week } \\
\hline & (1) & (2) & (3) & (4) \\
\hline After Ferguson Protests Begin (week of $8 / 11 / 14$ onward) & -4.72 & & -2.30 & -3.37 \\
\hline After Gray Protests Begin (week of 4/20/15 onward) & -11.38 & & -17.05 & -14.46 \\
\hline Unrest and National Guard $(4 / 27 / 15-5 / 3 / 2015)$ & -3.09 & & 1.10 & -1.64 \\
\hline After Batts Fired (week of $7 / 13 / 15$ onward) & 4.84 & & 7.28 & 5.35 \\
\hline After Davis Confirmed (week of $10 / 19 / 15$ onward) & -3.43 & & -1.39 & -1.99 \\
\hline After Davis First Year (week of 10/17/16 onward) & -2.02 & & -2.84 & -2.21 \\
\hline After De Sousa Begins (week of 1/19/18 onward) & -0.55 & & -0.22 & -0.08 \\
\hline After Tuggle Begins (week of 5/14/18 onward) & -2.86 & & -3.17 & -3.46 \\
\hline After Harrison Begins (week of 2/11/19 onward) & -1.51 & & -2.06 & -1.68 \\
\hline Average Maximum Temperature to 50 Degrees & & 0.35 & & 0.20 \\
\hline Plus Degrees in the $50 \mathrm{~s}$ & & -0.85 & & -0.28 \\
\hline Plus Degrees in the 60s & & 0.71 & & 0.20 \\
\hline Plus Degrees in the 70s & & 0.84 & & 0.11 \\
\hline Plus Degrees Greater Than 80 & & -2.00 & & -0.56 \\
\hline Snowfall (inches) & & -7.04 & & -4.08 \\
\hline Precipitation (inches) & & -1.28 & & 0.41 \\
\hline Darkness Before Midnight (hours) & & -1.00 & & -0.09 \\
\hline Thanksgiving/Christmas (proportion of week) & & -42.21 & & -25.61 \\
\hline Winter Holiday (proportion of week) & & 82.65 & & 40.46 \\
\hline Other Out-of-School Days (proportion of week) & & 0.44 & & -2.34 \\
\hline Summer Vacation (proportion of week) & & -1.20 & & 1.34 \\
\hline Summer Holiday (proportion of week) & & -60.54 & & -16.97 \\
\hline Observations & 373 & 83 & 373 & 373 \\
\hline $\mathrm{R}^{2}$ & 0.59 & 0.45 & 0.59 & 0.65 \\
\hline
\end{tabular}


18.3 Least Squares Updated Model (2) for larceny larceny

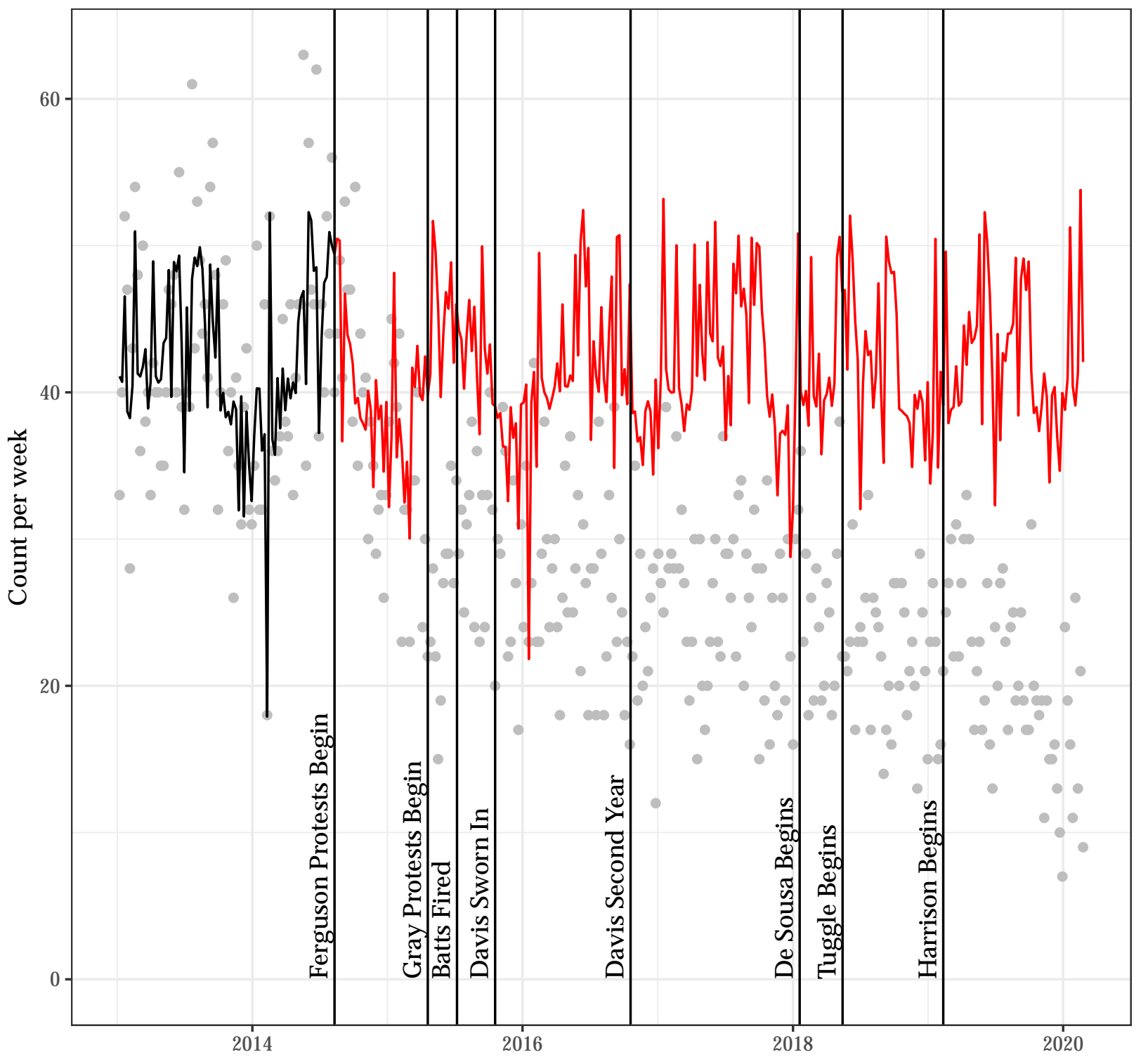




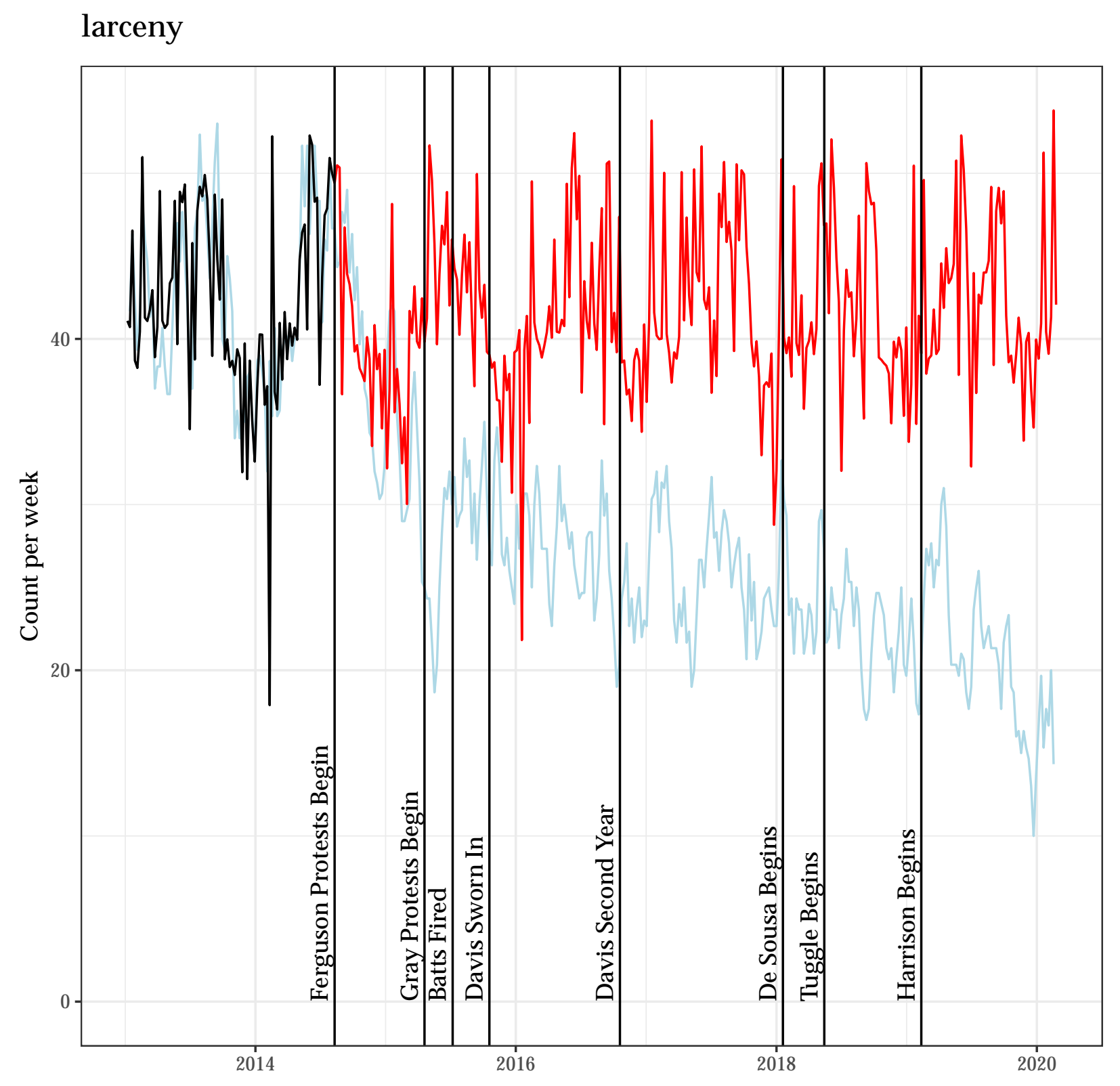


Autocorrelation Function for the Observed Outcome for Updated Model (2)

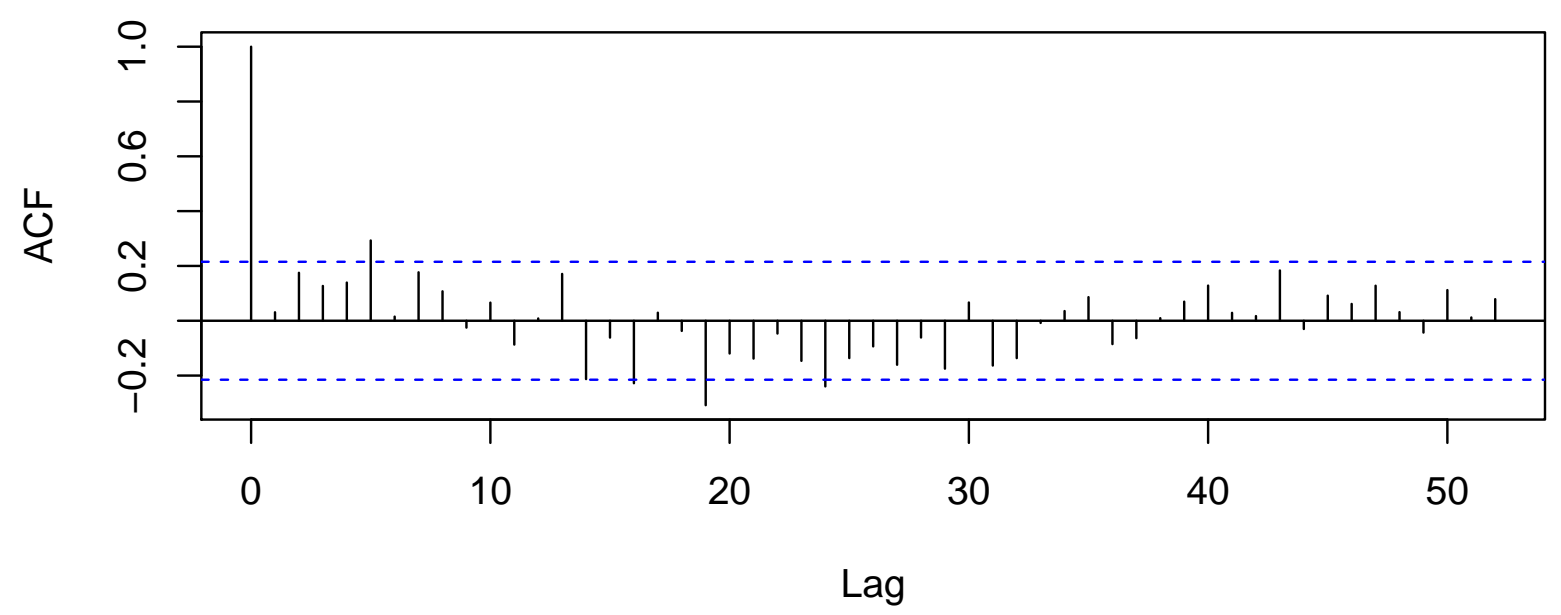

Autocorrelation Function for the Residuals from Updated Model (2)

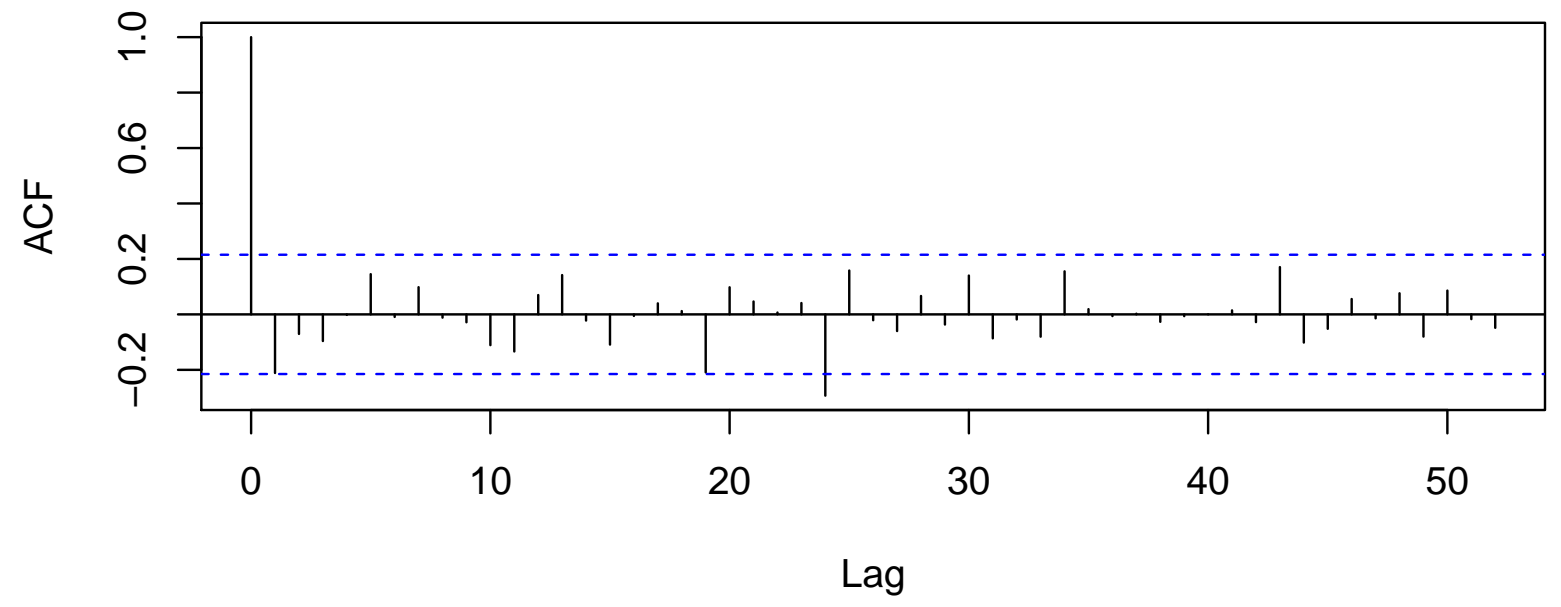




\subsection{A Poisson Regression Model as an Alternative to Model (2) for larceny}

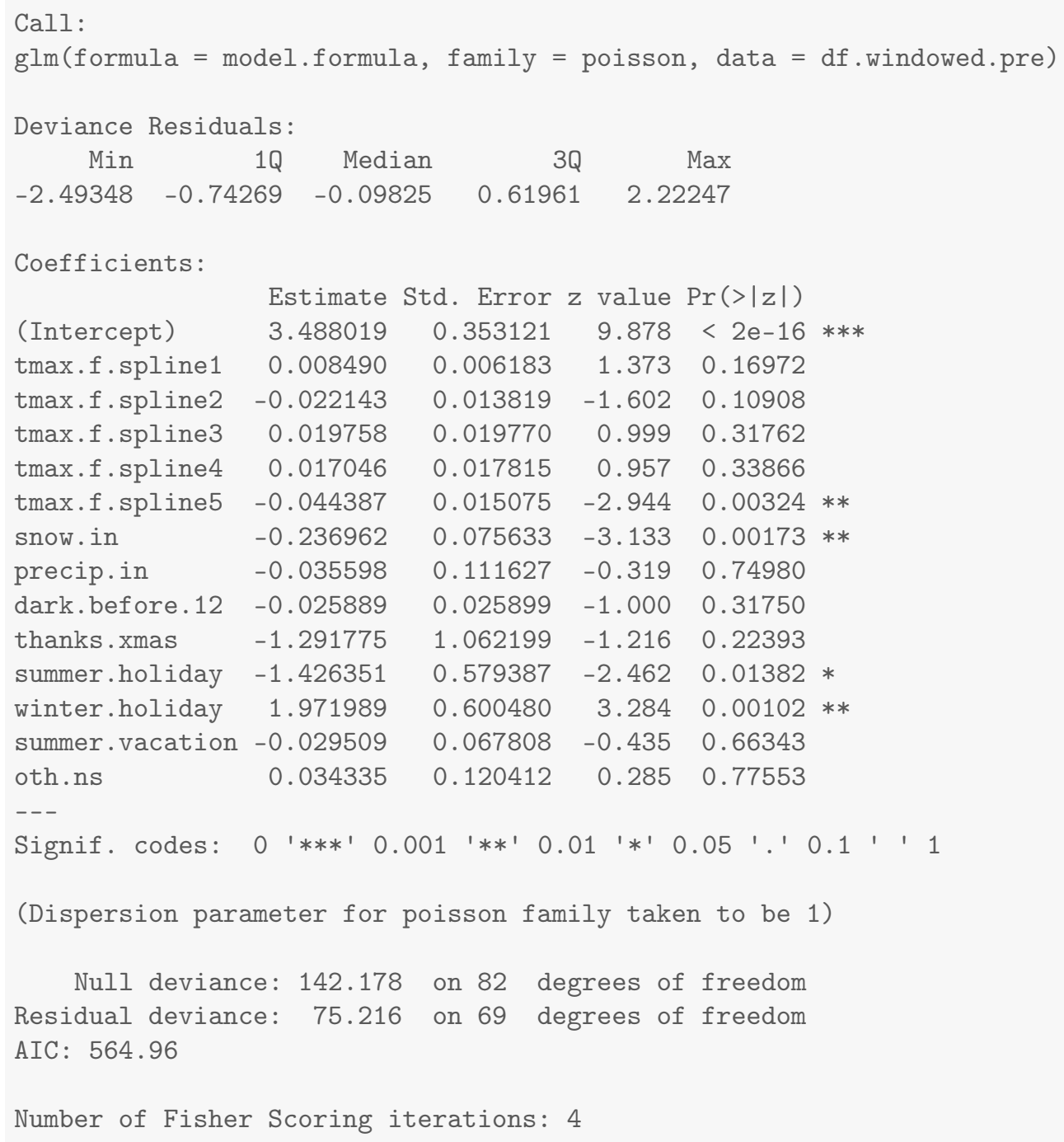




\section{Results for court.non.compliance}

\subsection{Summary Values for court.non.compliance}

Table 19.1: Descriptives for Outcome Before Ferguson Protests Begin

\begin{tabular}{lcccccccc}
\hline \hline Statistic & $\mathrm{N}$ & Mean & St. Dev. & Min & Pctl(25) & Median & Pctl(75) & Max \\
\hline court.non.compliance & 83 & 105.048 & 22.247 & 68 & 88 & 100 & 122 & 165 \\
\hline
\end{tabular}

Table 19.2: Descriptives for Outcome After Ferguson Protests Begin

\begin{tabular}{lcccccccc}
\hline \hline Statistic & $\mathrm{N}$ & Mean & St. Dev. & Min & Pctl(25) & Median & Pctl(75) & Max \\
\hline court.non.compliance & 290 & 80.921 & 17.930 & 40 & 70 & 78 & 88.8 & 143 \\
\hline
\end{tabular}




\subsection{Four Models for court.non.compliance}

Table 19.3: Four Models that Differ on the Specification of Adjustment and Intervention Variables

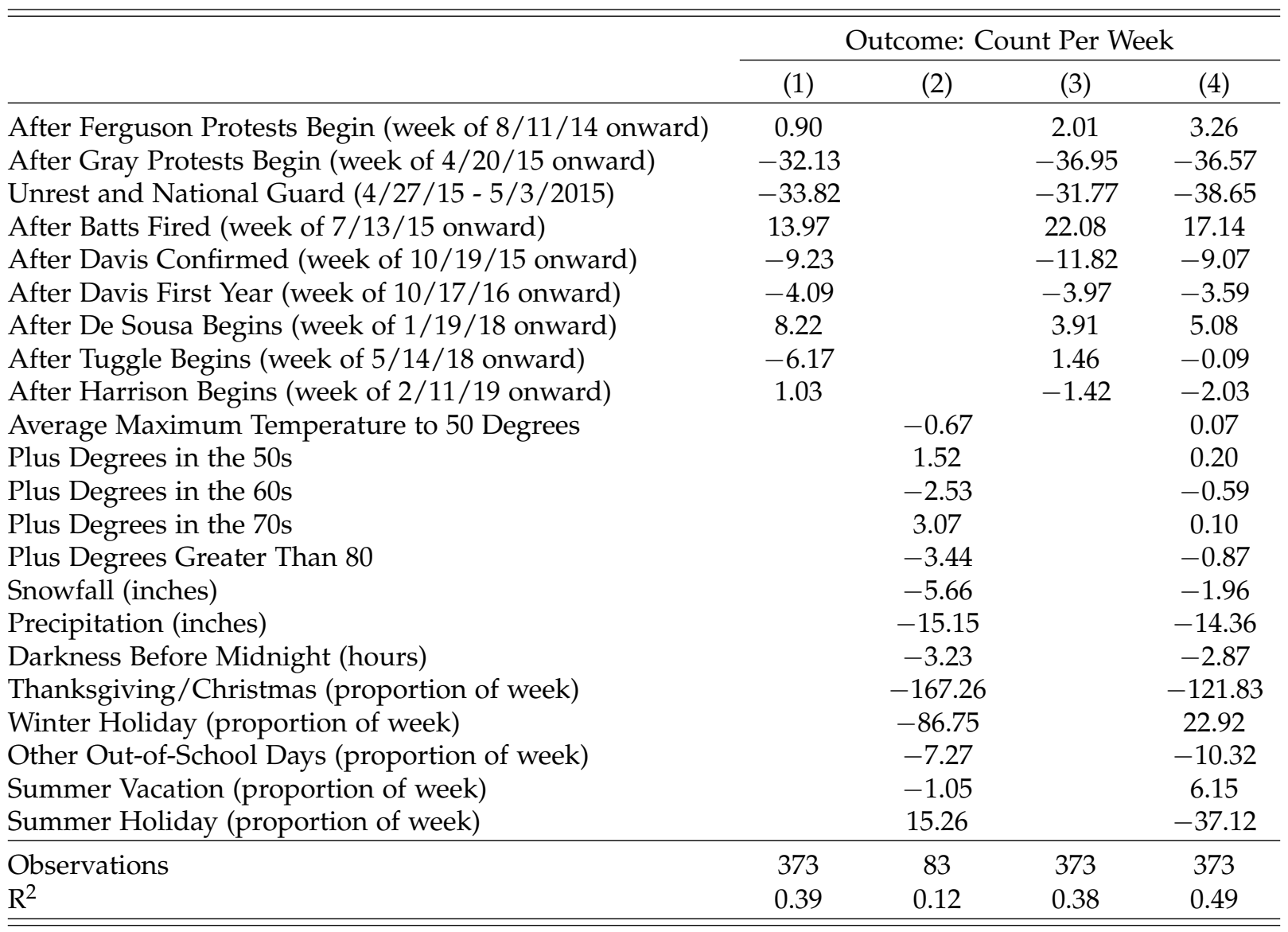


19.3 Least Squares Updated Model (2) for court.non.compliance court.non.compliance

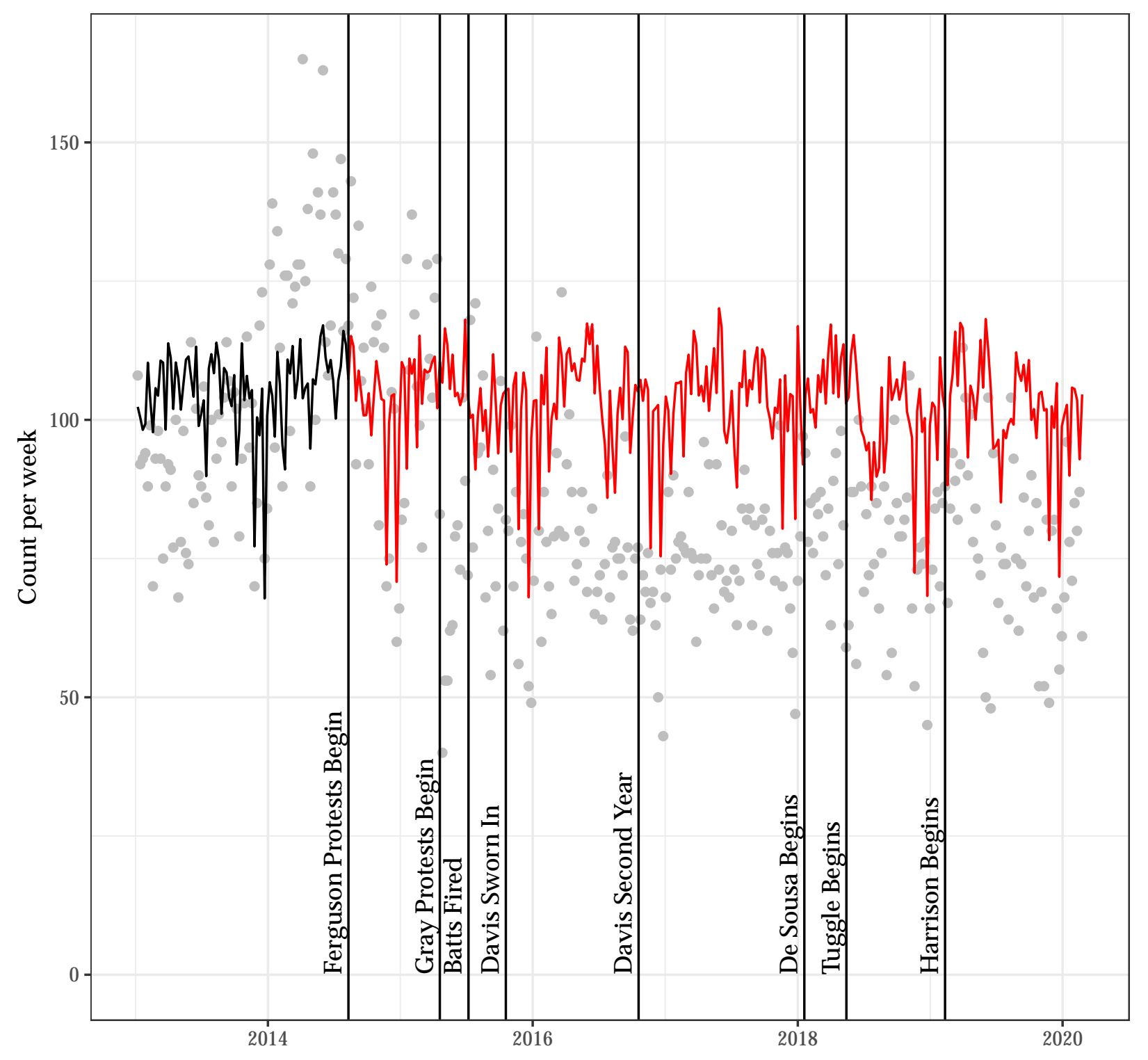




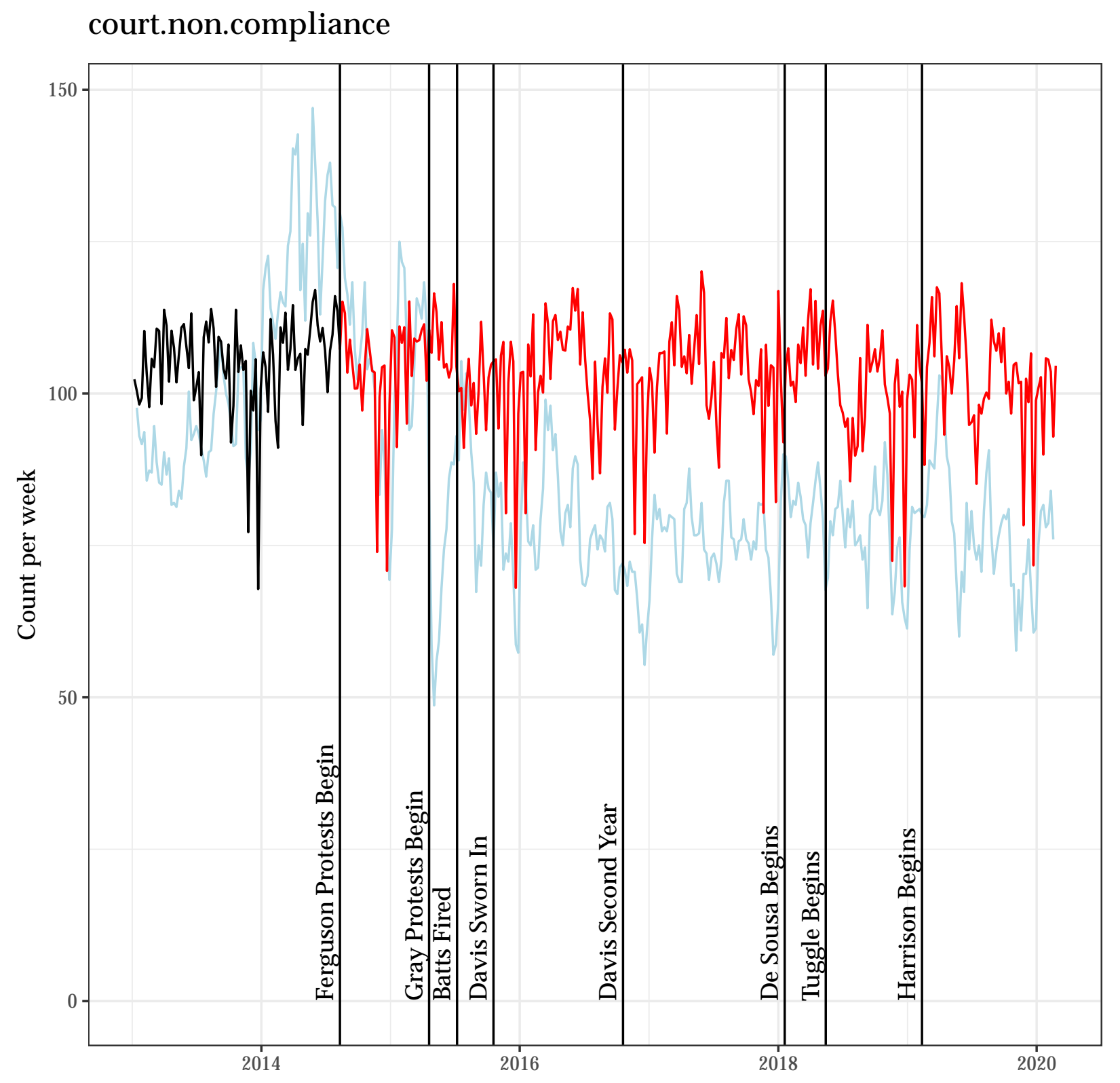


Autocorrelation Function for the Observed Outcome for Updated Model (2)

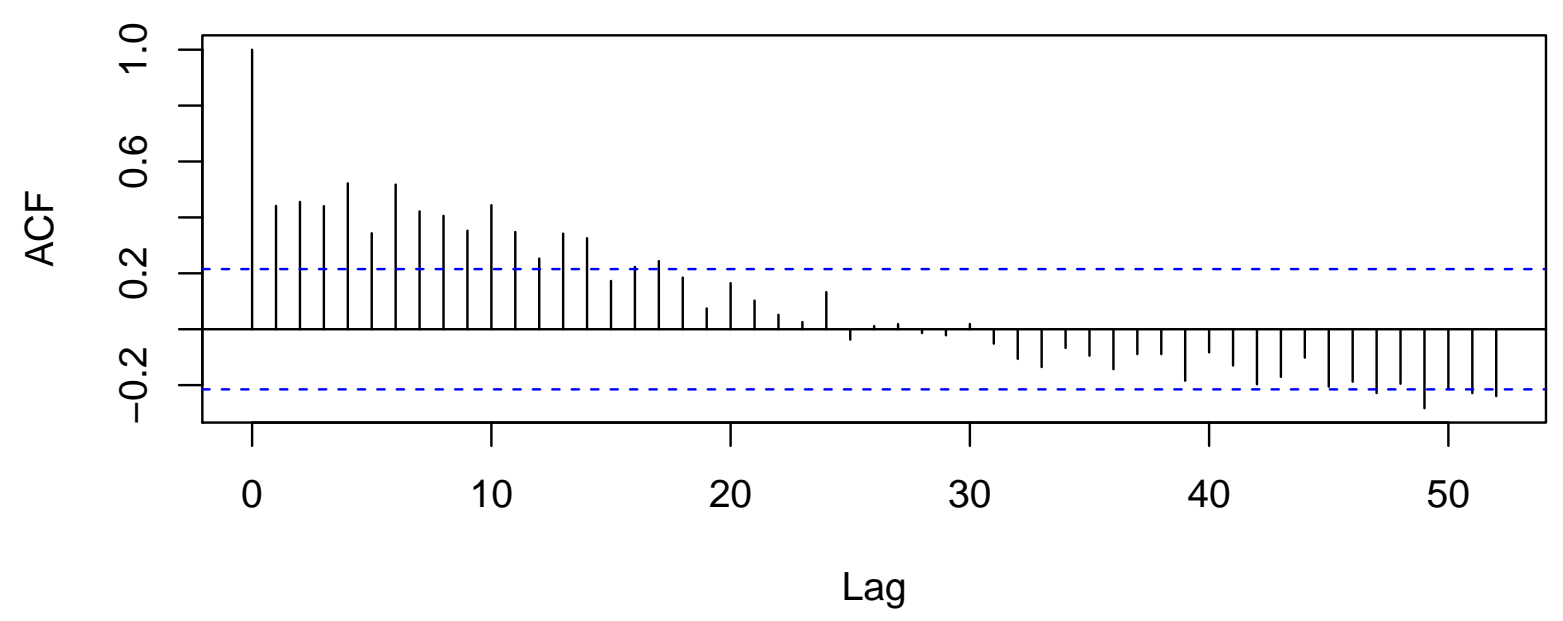

Autocorrelation Function for the Residuals from Updated Model (2)

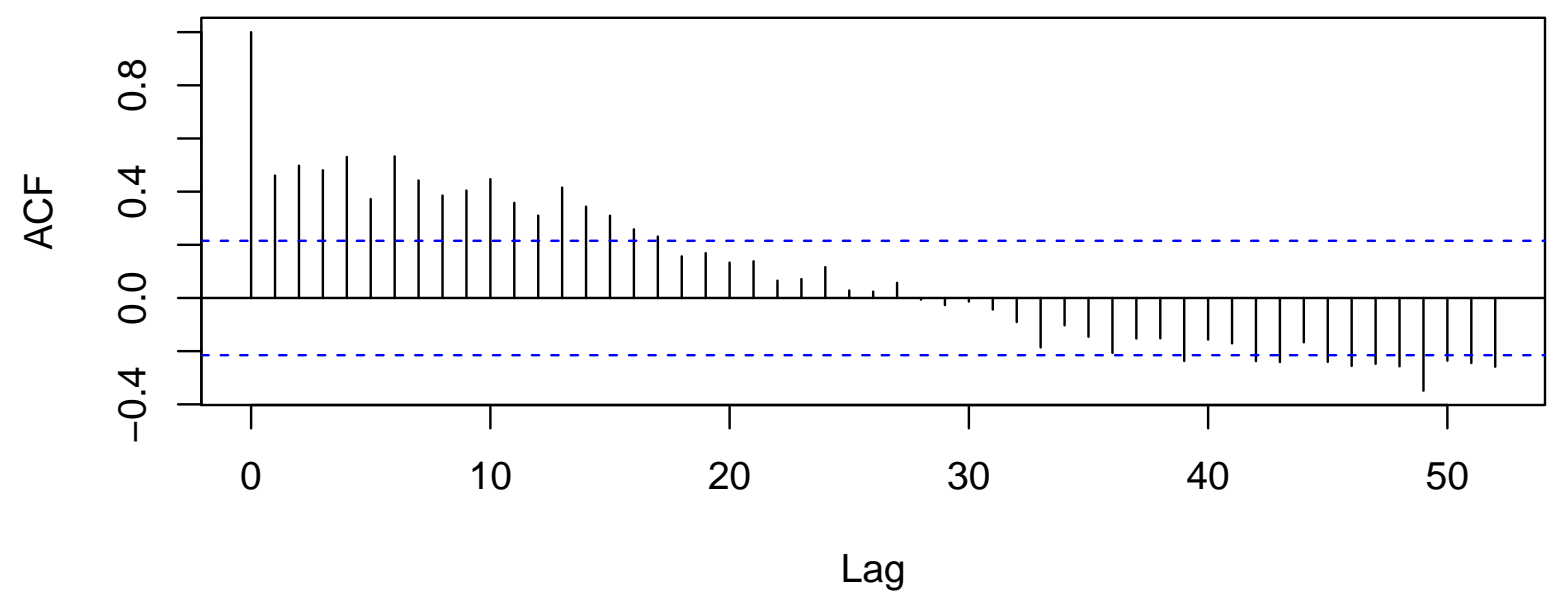




\subsection{A Poisson Regression Model as an Alternative to Model (2) for court.non.compliance}

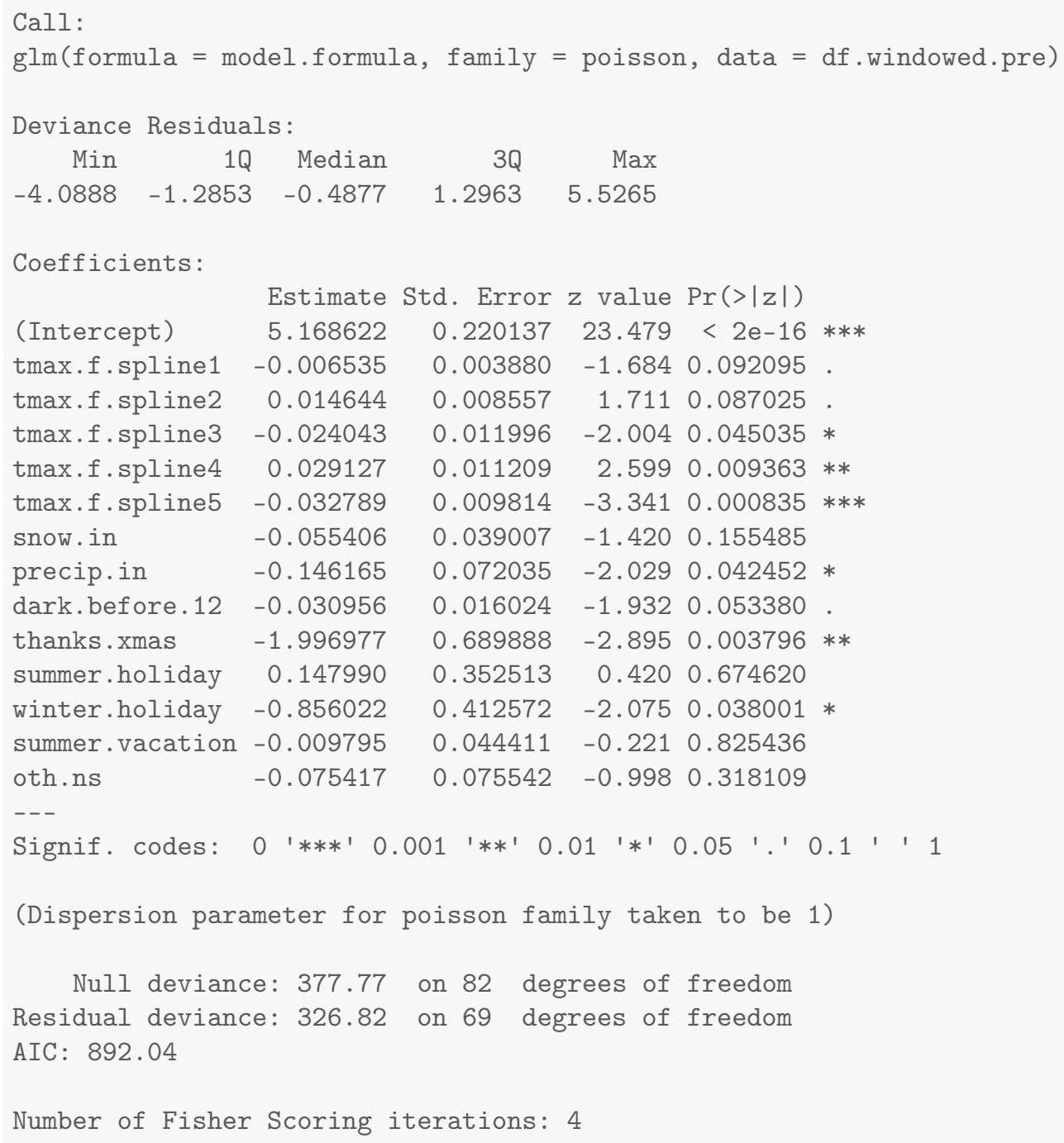




\section{Results for property.destruction}

\subsection{Summary Values for property.destruction}

Table 20.1: Descriptives for Outcome Before Ferguson Protests Begin

\begin{tabular}{lcccccccc}
\hline \hline Statistic & $\mathrm{N}$ & Mean & St. Dev. & Min & Pctl(25) & Median & Pctl(75) & Max \\
\hline property.destruction & 83 & 6.542 & 2.981 & 1 & 5 & 6 & 8 & 14 \\
\hline
\end{tabular}

Table 20.2: Descriptives for Outcome After Ferguson Protests Begin

\begin{tabular}{lcccccccc}
\hline \hline Statistic & $\mathrm{N}$ & Mean & St. Dev. & Min & Pctl(25) & Median & Pctl(75) & Max \\
\hline property.destruction & 290 & 4.531 & 2.286 & 0 & 3 & 4 & 6 & 12 \\
\hline
\end{tabular}




\subsection{Four Models for property.destruction}

Table 20.3: Four Models that Differ on the Specification of Adjustment and Intervention Variables

\begin{tabular}{|c|c|c|c|c|}
\hline & \multicolumn{4}{|c|}{ Outcome: Count Per Week } \\
\hline & $(1)$ & $(2)$ & $(3)$ & (4) \\
\hline After Ferguson Protests Begin (week of 8/11/14 onward) & -1.93 & & -1.73 & -1.81 \\
\hline After Gray Protests Begin (week of $4 / 20 / 15$ onward) & 0.03 & & -0.31 & -0.30 \\
\hline Unrest and National Guard $(4 / 27 / 15-5 / 3 / 2015)$ & 4.36 & & 3.12 & 4.05 \\
\hline After Batts Fired (week of $7 / 13 / 15$ onward) & 0.44 & & 0.78 & 0.55 \\
\hline After Davis Confirmed (week of 10/19/15 onward) & 0.02 & & -0.41 & 0.001 \\
\hline After Davis First Year (week of $10 / 17 / 16$ onward) & -0.58 & & -0.40 & -0.37 \\
\hline After De Sousa Begins (week of 1/19/18 onward) & -0.33 & & -1.00 & -0.85 \\
\hline After Tuggle Begins (week of 5/14/18 onward) & 0.15 & & 1.28 & 0.67 \\
\hline After Harrison Begins (week of 2/11/19 onward) & -0.37 & & -0.93 & -0.52 \\
\hline Average Maximum Temperature to 50 Degrees & & 0.12 & & -0.02 \\
\hline Plus Degrees in the $50 \mathrm{~s}$ & & -0.06 & & 0.10 \\
\hline Plus Degrees in the 60s & & -0.16 & & -0.21 \\
\hline Plus Degrees in the $70 \mathrm{~s}$ & & -0.01 & & 0.13 \\
\hline Plus Degrees Greater Than 80 & & 0.17 & & 0.15 \\
\hline Snowfall (inches) & & -0.01 & & -0.50 \\
\hline Precipitation (inches) & & -2.18 & & -0.61 \\
\hline Darkness Before Midnight (hours) & & -0.50 & & -0.50 \\
\hline Thanksgiving/Christmas (proportion of week) & & 4.68 & & -4.90 \\
\hline Winter Holiday (proportion of week) & & 21.90 & & 5.58 \\
\hline Other Out-of-School Days (proportion of week) & & -0.85 & & 0.63 \\
\hline Summer Vacation (proportion of week) & & -1.09 & & -1.33 \\
\hline Summer Holiday (proportion of week) & & 5.30 & & -2.28 \\
\hline Observations & 373 & 83 & 373 & 373 \\
\hline $\mathrm{R}^{2}$ & 0.13 & 0.12 & 0.13 & 0.19 \\
\hline
\end{tabular}




\subsection{Least Squares Updated Model (2) for property.destruction}

\section{property.destruction}

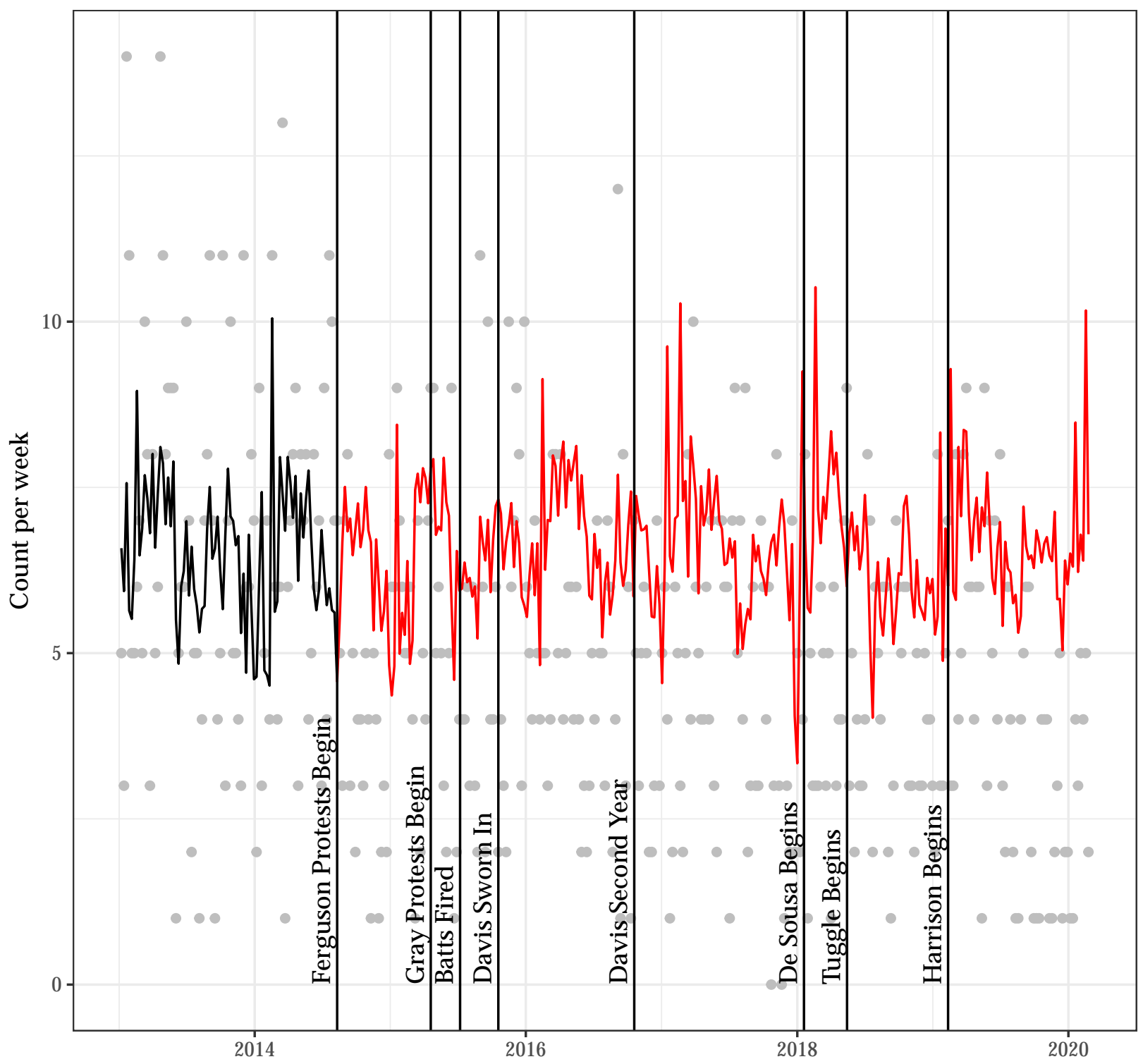




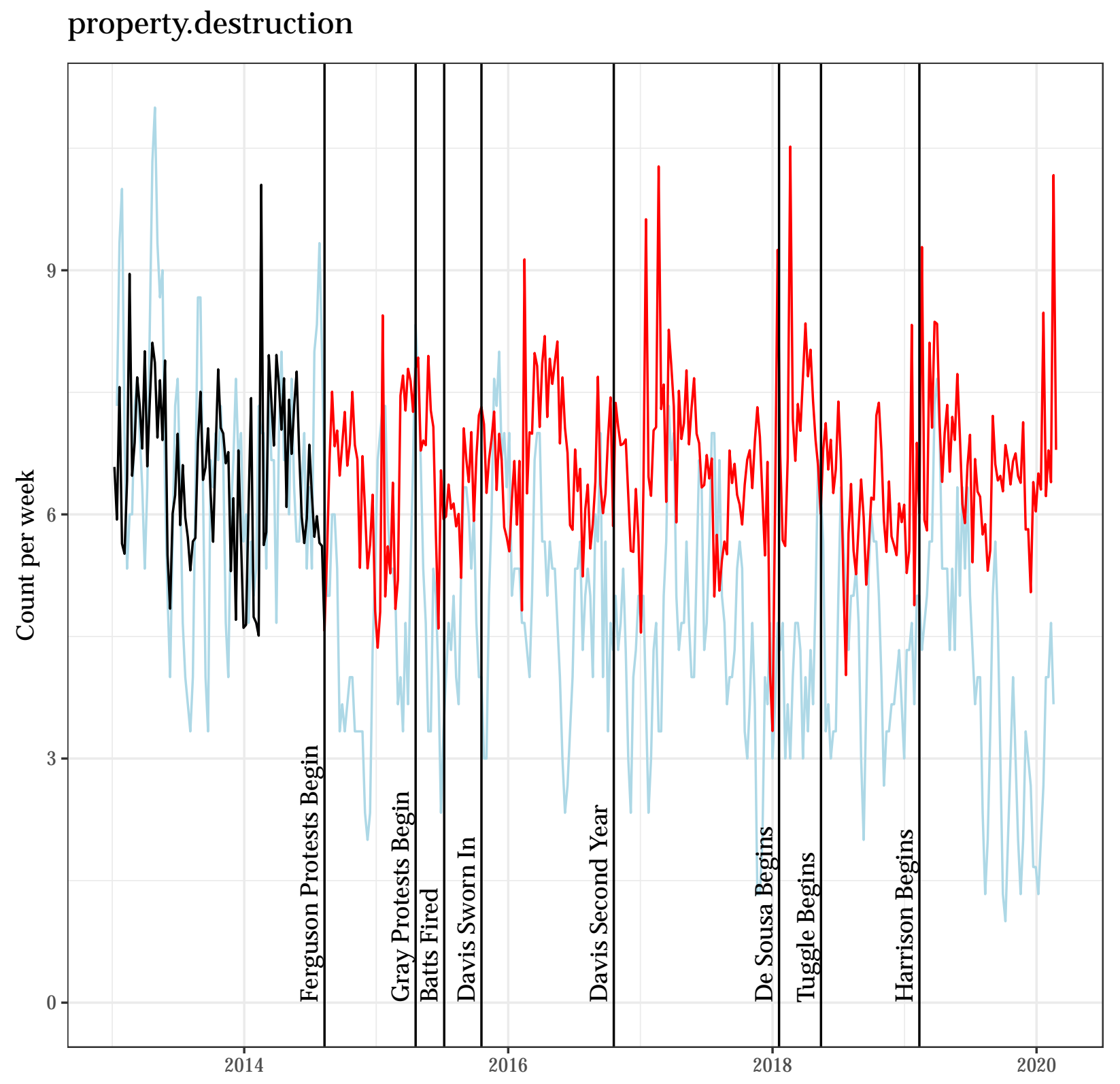


Autocorrelation Function for the Observed Outcome for Updated Model (2)

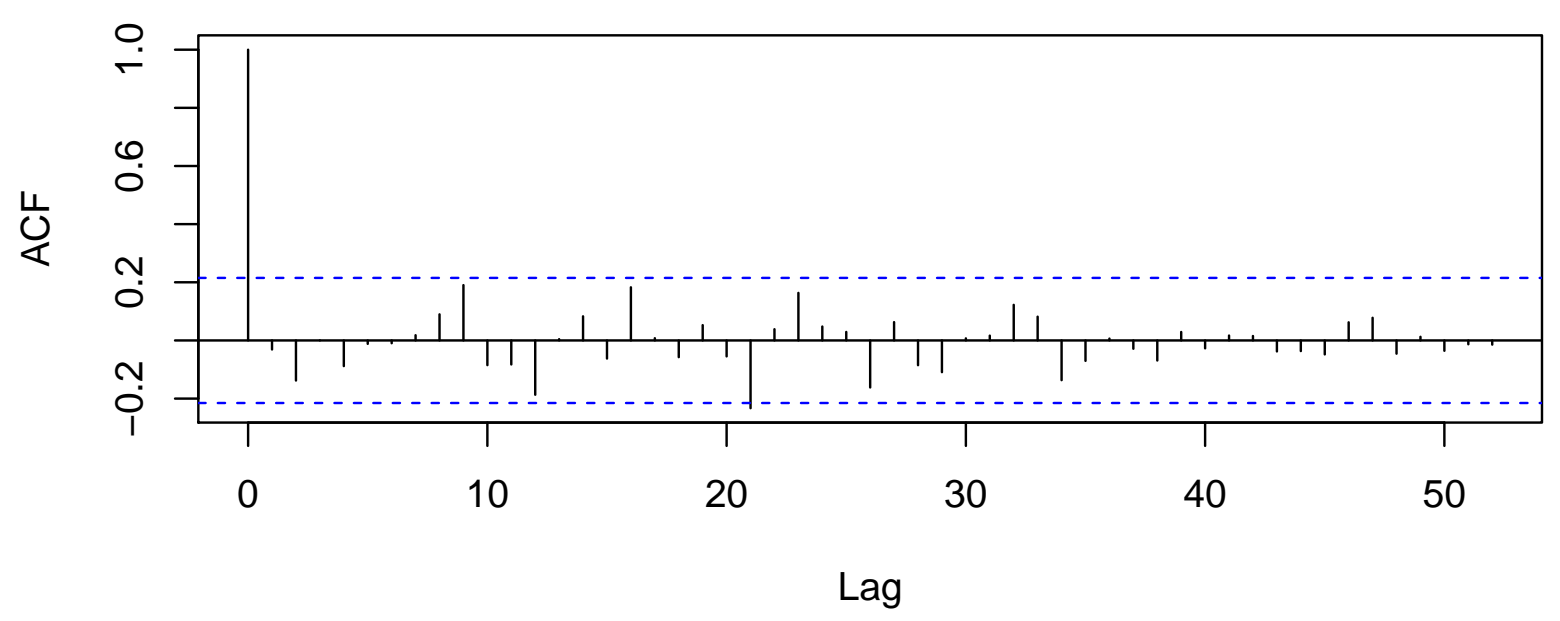

Autocorrelation Function for the Residuals from Updated Model (2)

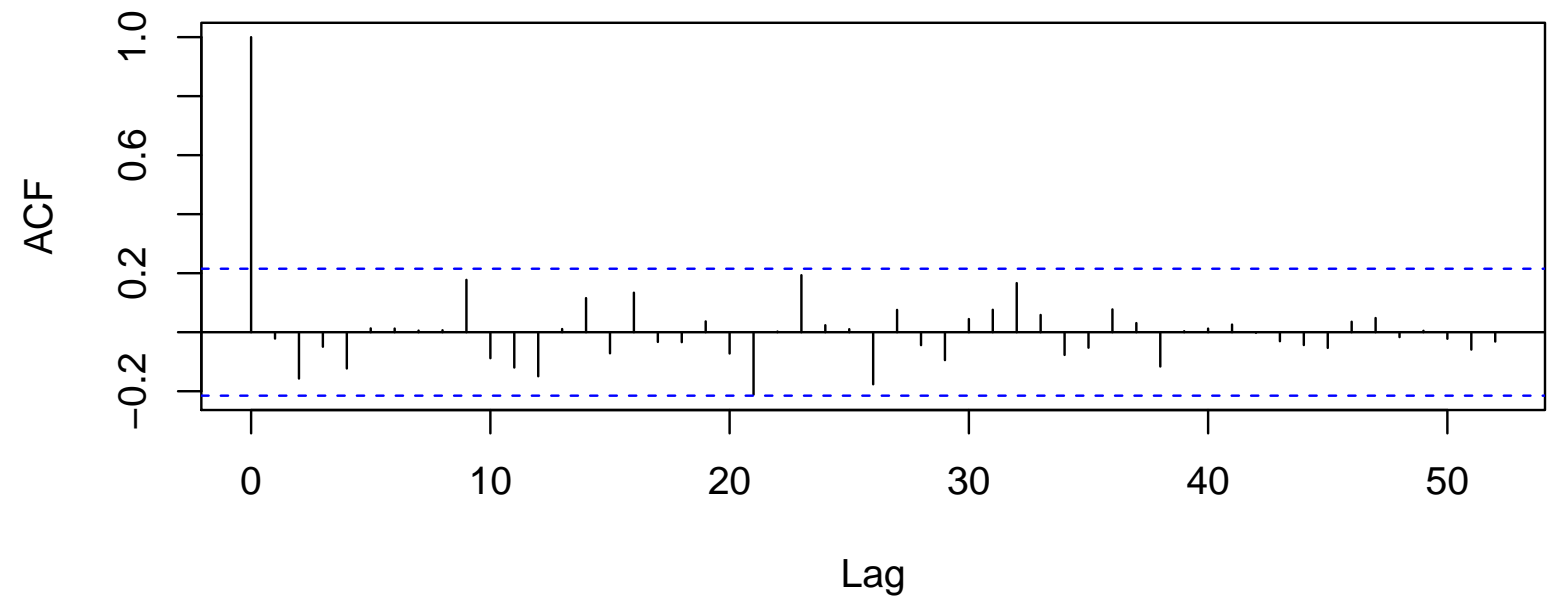




\subsection{A Poisson Regression Model as an Alternative to Model (2) for property.destruction}

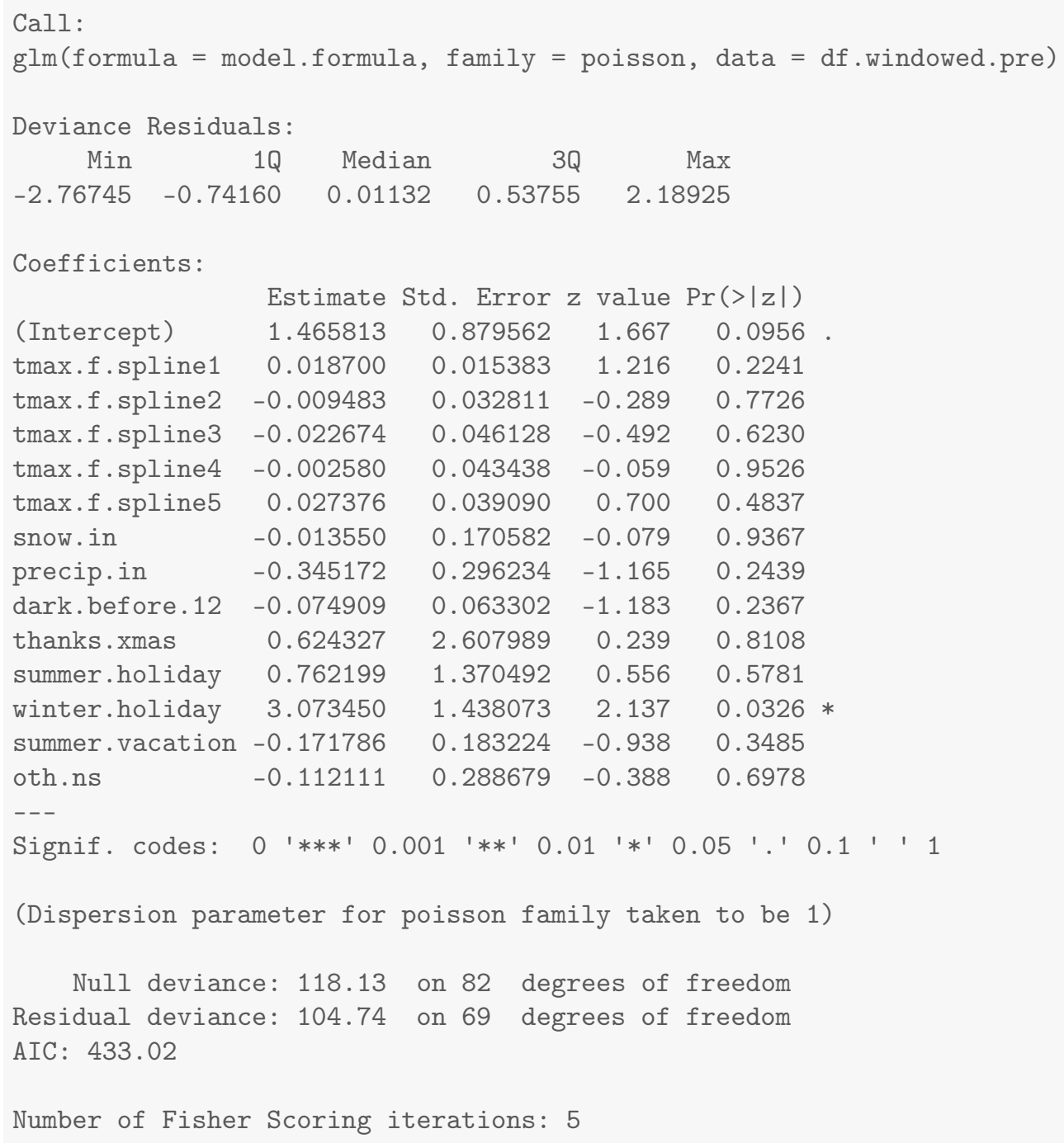




\section{Results for forgery.fraud.extortion}

\subsection{Summary Values for forgery.fraud.extortion}

Table 21.1: Descriptives for Outcome Before Ferguson Protests Begin

\begin{tabular}{lcccccccc}
\hline \hline Statistic & N & Mean & St. Dev. & Min & Pctl(25) & Median & Pctl(75) & Max \\
\hline forgery.fraud.extortion & 83 & 2.036 & 1.365 & 0 & 1 & 2 & 3 & 5 \\
\hline
\end{tabular}

Table 21.2: Descriptives for Outcome After Ferguson Protests Begin

\begin{tabular}{lcccccccc}
\hline \hline Statistic & $\mathrm{N}$ & Mean & St. Dev. & Min & Pctl(25) & Median & Pctl(75) & Max \\
\hline forgery.fraud.extortion & 290 & 1.186 & 1.170 & 0 & 0 & 1 & 2 & 6 \\
\hline
\end{tabular}




\subsection{Four Models for forgery.fraud.extortion}

Table 21.3: Four Models that Differ on the Specification of Adjustment and Intervention Variables

\begin{tabular}{|c|c|c|c|c|}
\hline & \multicolumn{4}{|c|}{ Outcome: Count Per Week } \\
\hline & $(1)$ & $(2)$ & (3) & $(4)$ \\
\hline After Ferguson Protests Begin (week of 8/11/14 onward) & 0.07 & & 0.35 & 0.14 \\
\hline After Gray Protests Begin (week of $4 / 20 / 15$ onward) & -0.75 & & -1.28 & -0.89 \\
\hline Unrest and National Guard $(4 / 27 / 15-5 / 3 / 2015)$ & -0.36 & & 0.02 & -0.22 \\
\hline After Batts Fired (week of 7/13/15 onward) & -0.01 & & 0.03 & 0.01 \\
\hline After Davis Confirmed (week of $10 / 19 / 15$ onward) & -0.11 & & 0.19 & -0.09 \\
\hline After Davis First Year (week of $10 / 17 / 16$ onward) & -0.17 & & -0.23 & -0.14 \\
\hline After De Sousa Begins (week of $1 / 19 / 18$ onward) & -0.14 & & 0.003 & -0.22 \\
\hline After Tuggle Begins (week of $5 / 14 / 18$ onward) & -0.07 & & -0.27 & -0.02 \\
\hline After Harrison Begins (week of 2/11/19 onward) & 0.001 & & -0.05 & 0.005 \\
\hline Average Maximum Temperature to 50 Degrees & & 0.12 & & 0.02 \\
\hline Plus Degrees in the $50 \mathrm{~s}$ & & -0.25 & & 0.005 \\
\hline Plus Degrees in the 60s & & 0.26 & & -0.07 \\
\hline Plus Degrees in the $70 \mathrm{~s}$ & & -0.04 & & 0.09 \\
\hline Plus Degrees Greater Than 80 & & -0.13 & & -0.03 \\
\hline Snowfall (inches) & & 0.63 & & -0.002 \\
\hline Precipitation (inches) & & -0.61 & & -0.002 \\
\hline Darkness Before Midnight (hours) & & 0.07 & & -0.02 \\
\hline Thanksgiving/Christmas (proportion of week) & & -3.82 & & 0.56 \\
\hline Winter Holiday (proportion of week) & & 12.23 & & 1.95 \\
\hline Other Out-of-School Days (proportion of week) & & -0.35 & & -0.72 \\
\hline Summer Vacation (proportion of week) & & -0.45 & & -0.16 \\
\hline Summer Holiday (proportion of week) & & -0.77 & & -1.26 \\
\hline Observations & 373 & 83 & 373 & 373 \\
\hline $\mathrm{R}^{2}$ & 0.15 & 0.20 & 0.15 & 0.17 \\
\hline
\end{tabular}


21.3 Least Squares Updated Model (2) for forgery.fraud.extortion forgery.fraud.extortion

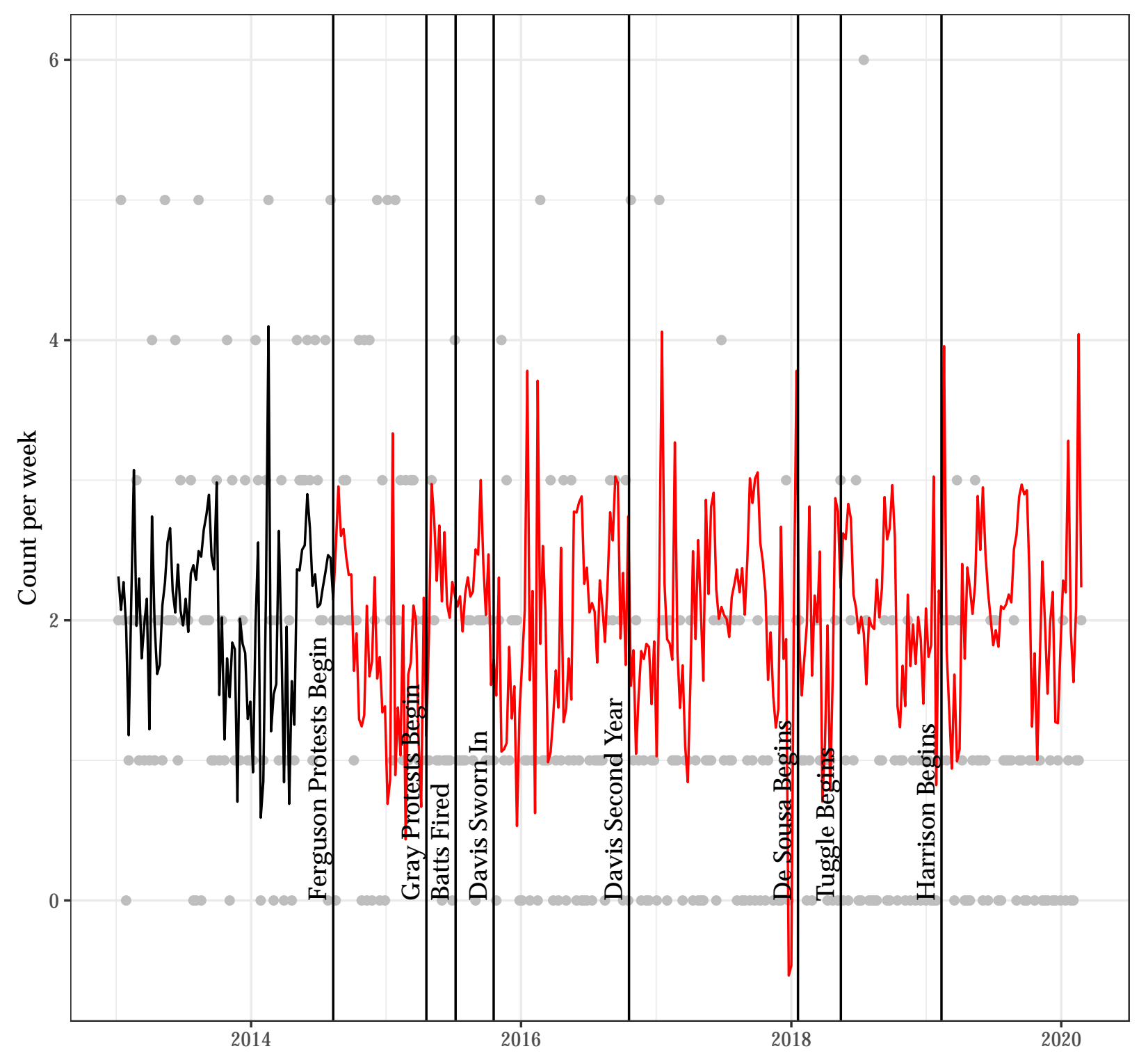




\section{forgery.fraud.extortion}

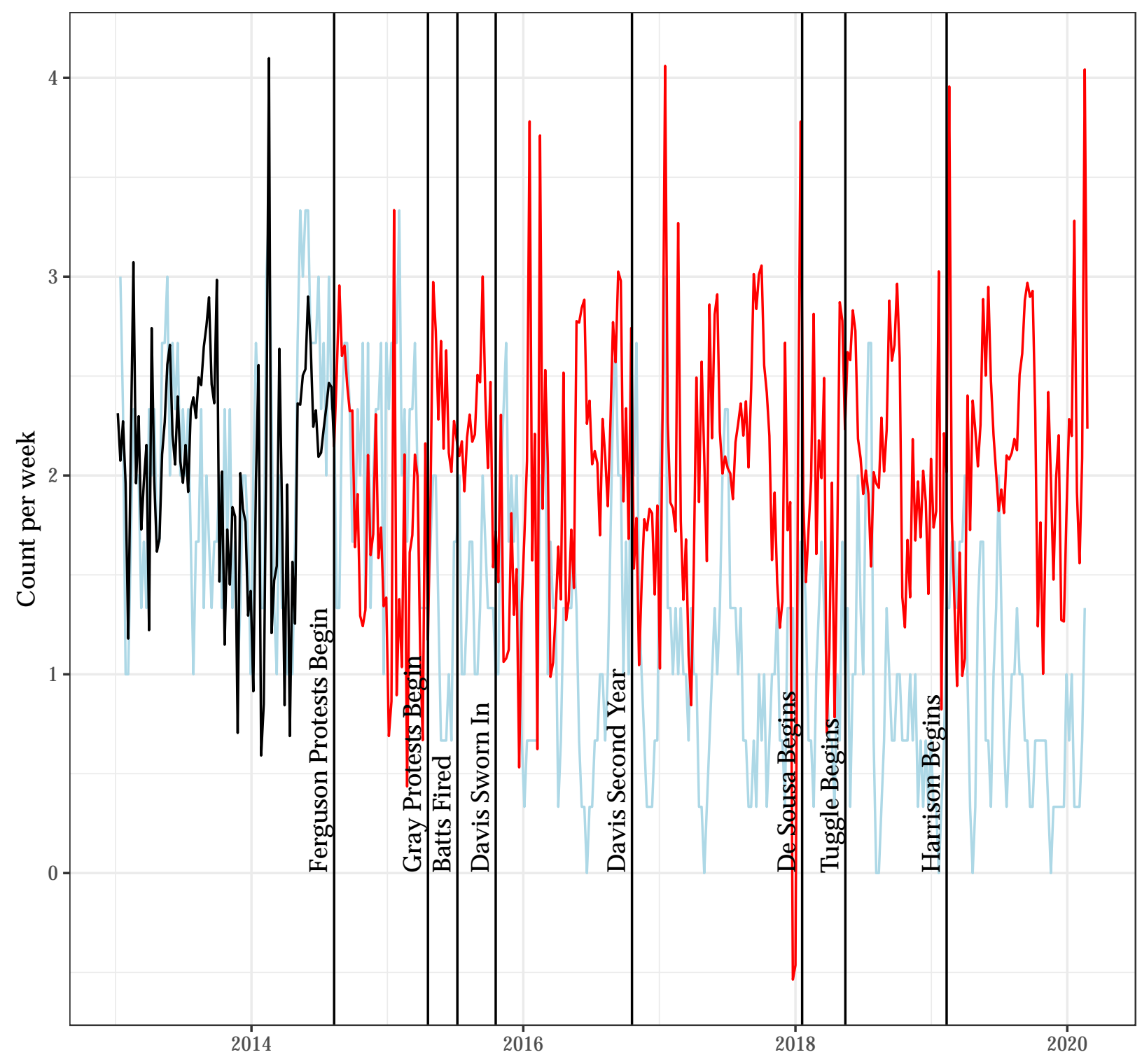


Autocorrelation Function for the Observed Outcome for Updated Model (2)

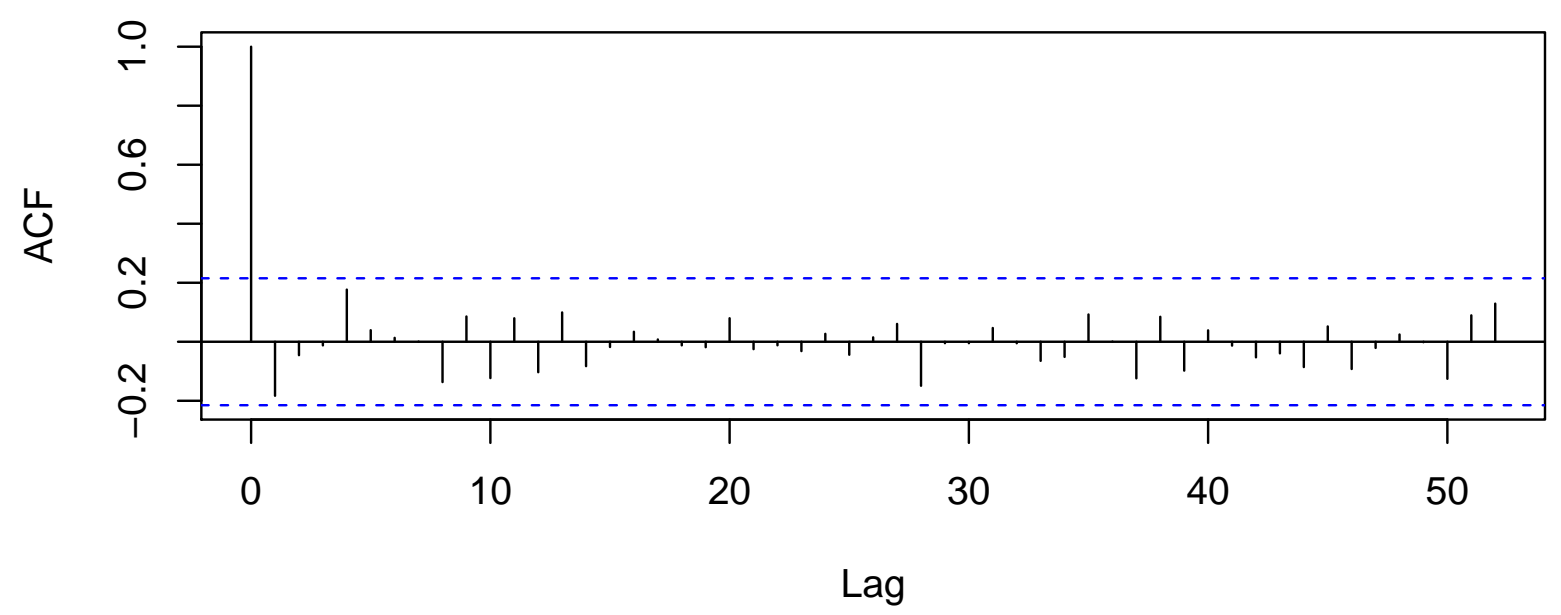

Autocorrelation Function for the Residuals from Updated Model (2)

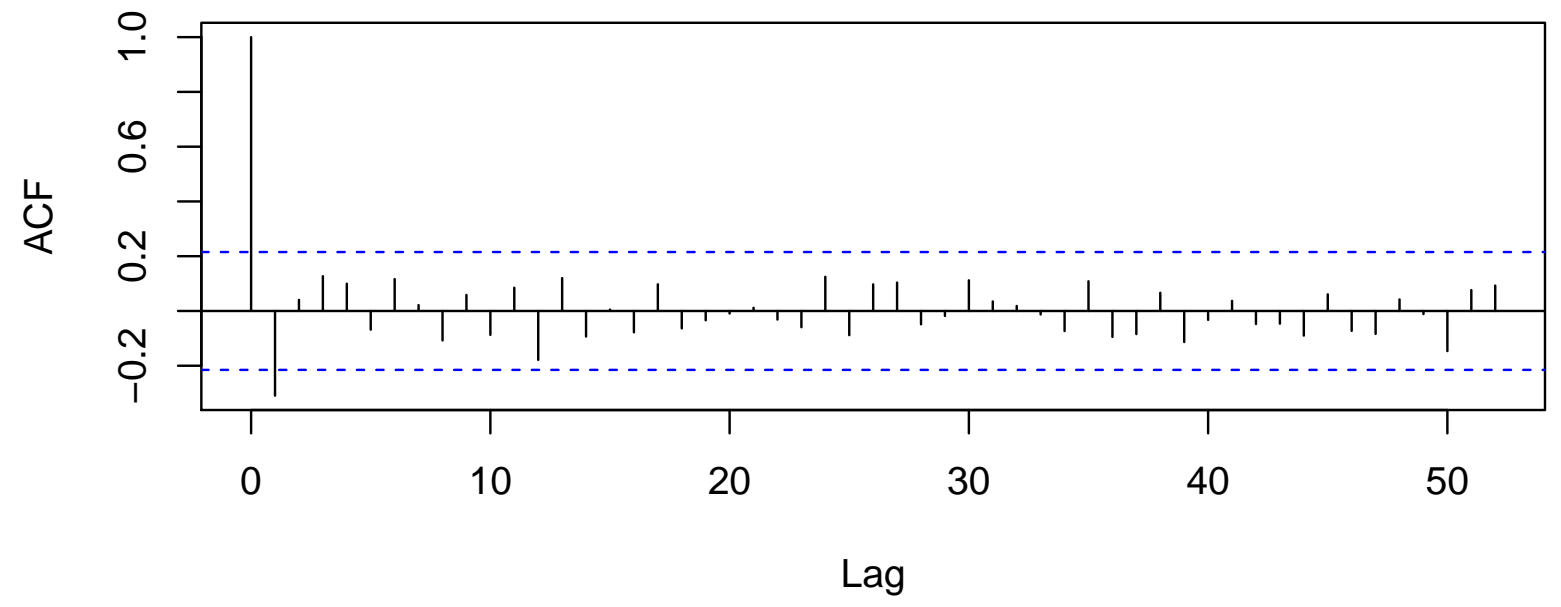




\subsection{A Poisson Regression Model as an Alternative to Model (2) for forgery.fraud.extortion}

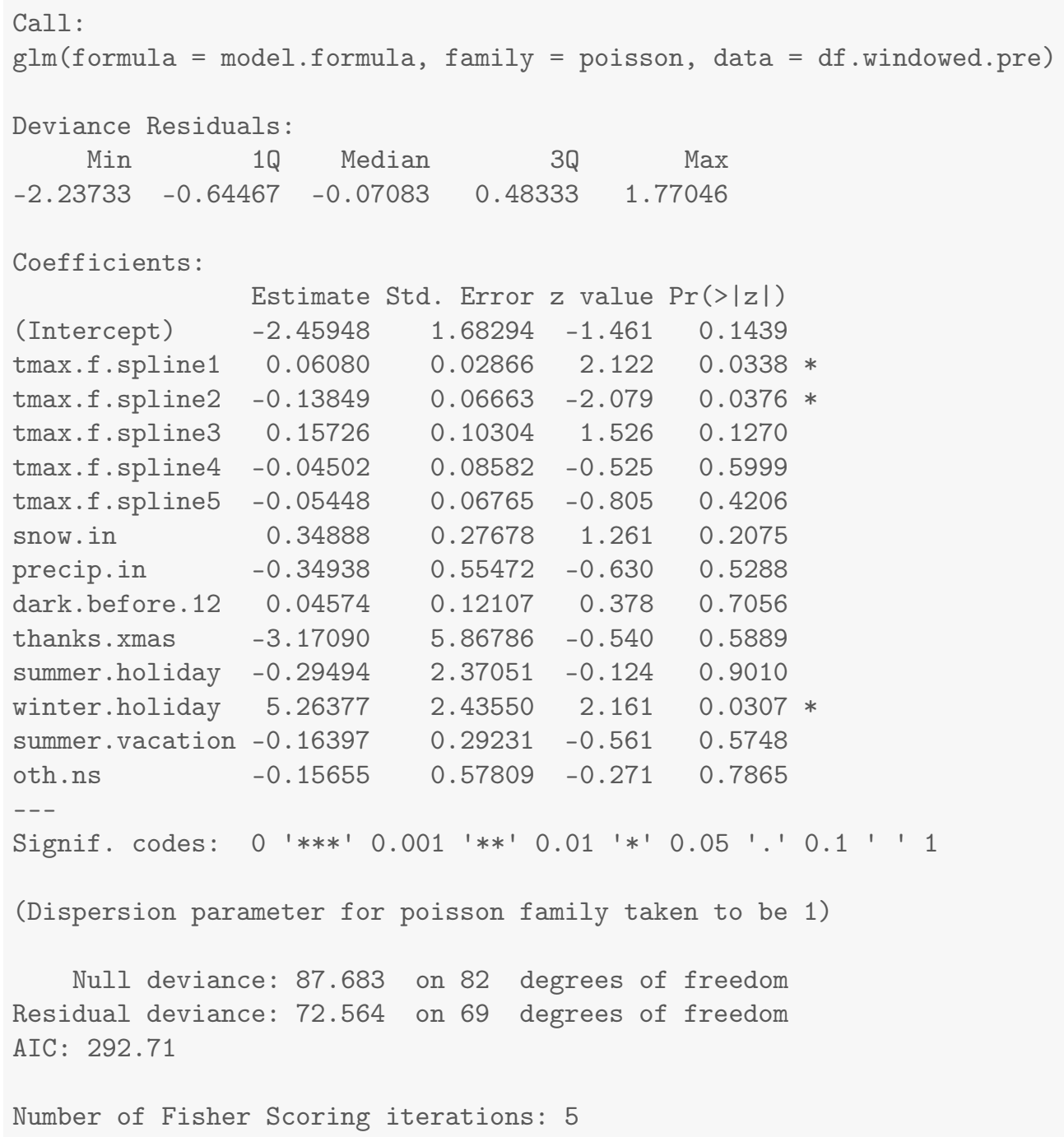




\section{Results for police.non.compliance}

\subsection{Summary Values for police.non.compliance}

Table 22.1: Descriptives for Outcome Before Ferguson Protests Begin

\begin{tabular}{lcccccccc}
\hline \hline Statistic & N & Mean & St. Dev. & Min & Pctl(25) & Median & Pctl(75) & Max \\
\hline police.non.compliance & 83 & 11.566 & 4.165 & 3 & 8.5 & 12 & 15 & 22 \\
\hline
\end{tabular}

Table 22.2: Descriptives for Outcome After Ferguson Protests Begin

\begin{tabular}{lcccccccc}
\hline \hline Statistic & $\mathrm{N}$ & Mean & St. Dev. & Min & Pctl(25) & Median & Pctl(75) & Max \\
\hline police.non.compliance & 290 & 3.128 & 2.362 & 0 & 1 & 3 & 4 & 13 \\
\hline
\end{tabular}




\subsection{Four Models for police.non.compliance}

Table 22.3: Four Models that Differ on the Specification of Adjustment and Intervention Variables

\begin{tabular}{|c|c|c|c|c|}
\hline & \multicolumn{4}{|c|}{ Outcome: Count Per Week } \\
\hline & $(1)$ & $(2)$ & (3) & (4) \\
\hline After Ferguson Protests Begin (week of $8 / 11 / 14$ onward) & -5.07 & & -4.28 & -4.74 \\
\hline After Gray Protests Begin (week of $4 / 20 / 15$ onward) & -3.41 & & -4.32 & -3.88 \\
\hline Unrest and National Guard $(4 / 27 / 15-5 / 3 / 2015)$ & -0.09 & & -1.53 & -0.42 \\
\hline After Batts Fired (week of $7 / 13 / 15$ onward) & 0.19 & & -0.80 & -0.25 \\
\hline After Davis Confirmed (week of 10/19/15 onward) & 0.06 & & 1.16 & 0.53 \\
\hline After Davis First Year (week of $10 / 17 / 16$ onward) & -1.03 & & -1.43 & -1.14 \\
\hline After De Sousa Begins (week of 1/19/18 onward) & 0.31 & & 1.05 & 0.63 \\
\hline After Tuggle Begins (week of 5/14/18 onward) & -0.19 & & -0.45 & -0.38 \\
\hline After Harrison Begins (week of 2/11/19 onward) & -0.14 & & -0.79 & -0.32 \\
\hline Average Maximum Temperature to 50 Degrees & & 0.42 & & 0.15 \\
\hline Plus Degrees in the $50 \mathrm{~s}$ & & -0.80 & & -0.20 \\
\hline Plus Degrees in the 60s & & 0.81 & & 0.17 \\
\hline Plus Degrees in the $70 \mathrm{~s}$ & & -0.65 & & -0.16 \\
\hline Plus Degrees Greater Than 80 & & 0.24 & & 0.05 \\
\hline Snowfall (inches) & & -1.37 & & -0.90 \\
\hline Precipitation (inches) & & -4.68 & & -1.15 \\
\hline Darkness Before Midnight (hours) & & 0.21 & & 0.23 \\
\hline Thanksgiving/Christmas (proportion of week) & & -8.69 & & -6.72 \\
\hline Winter Holiday (proportion of week) & & 12.55 & & 8.93 \\
\hline Other Out-of-School Days (proportion of week) & & 1.50 & & -0.17 \\
\hline Summer Vacation (proportion of week) & & 2.07 & & 0.77 \\
\hline Summer Holiday (proportion of week) & & -3.68 & & -3.09 \\
\hline Observations & 373 & 83 & 373 & 373 \\
\hline $\mathrm{R}^{2}$ & 0.67 & 0.24 & 0.67 & 0.70 \\
\hline
\end{tabular}




\subsection{Least Squares Updated Model (2) for police.non.compliance}

police.non.compliance

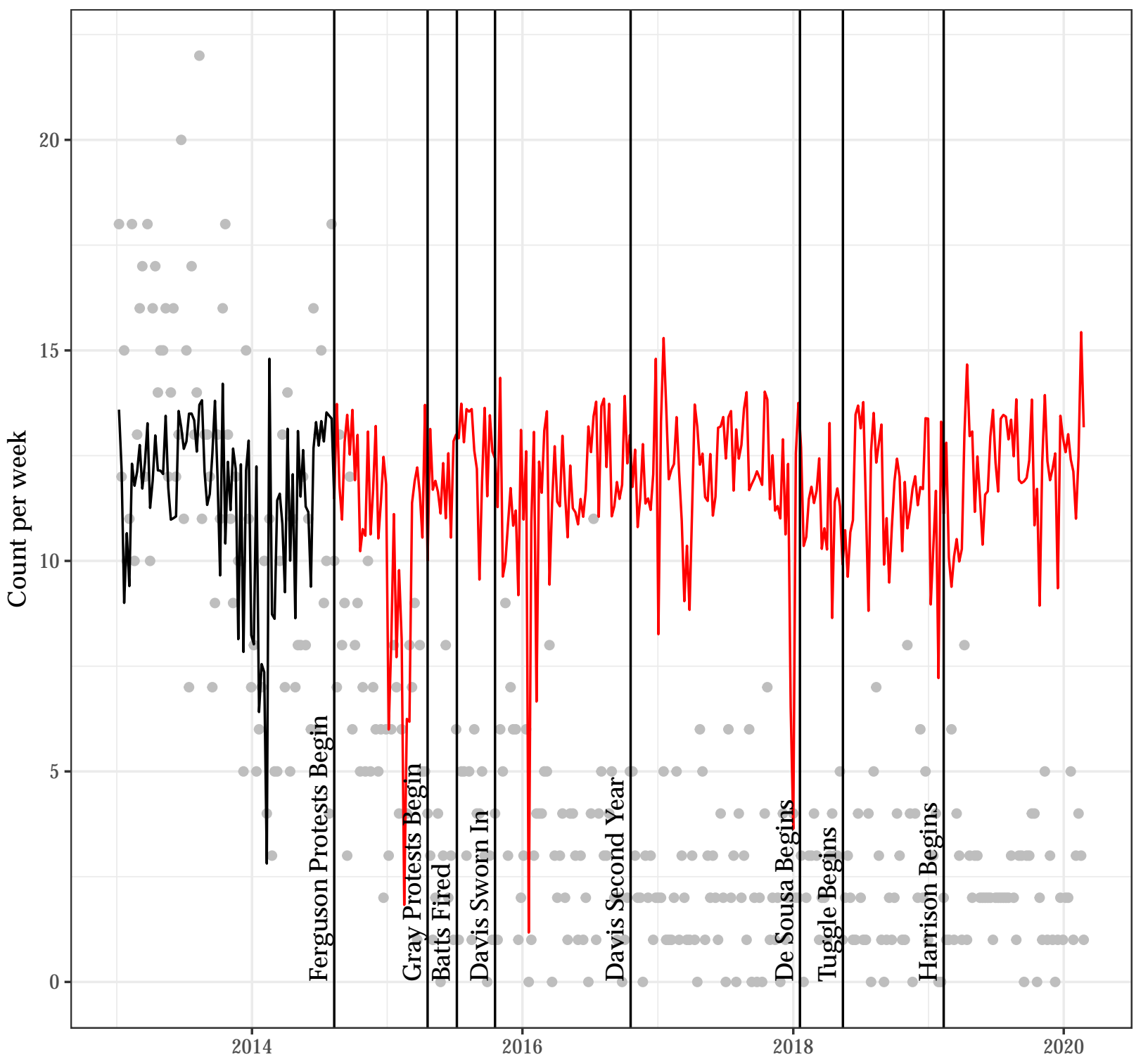




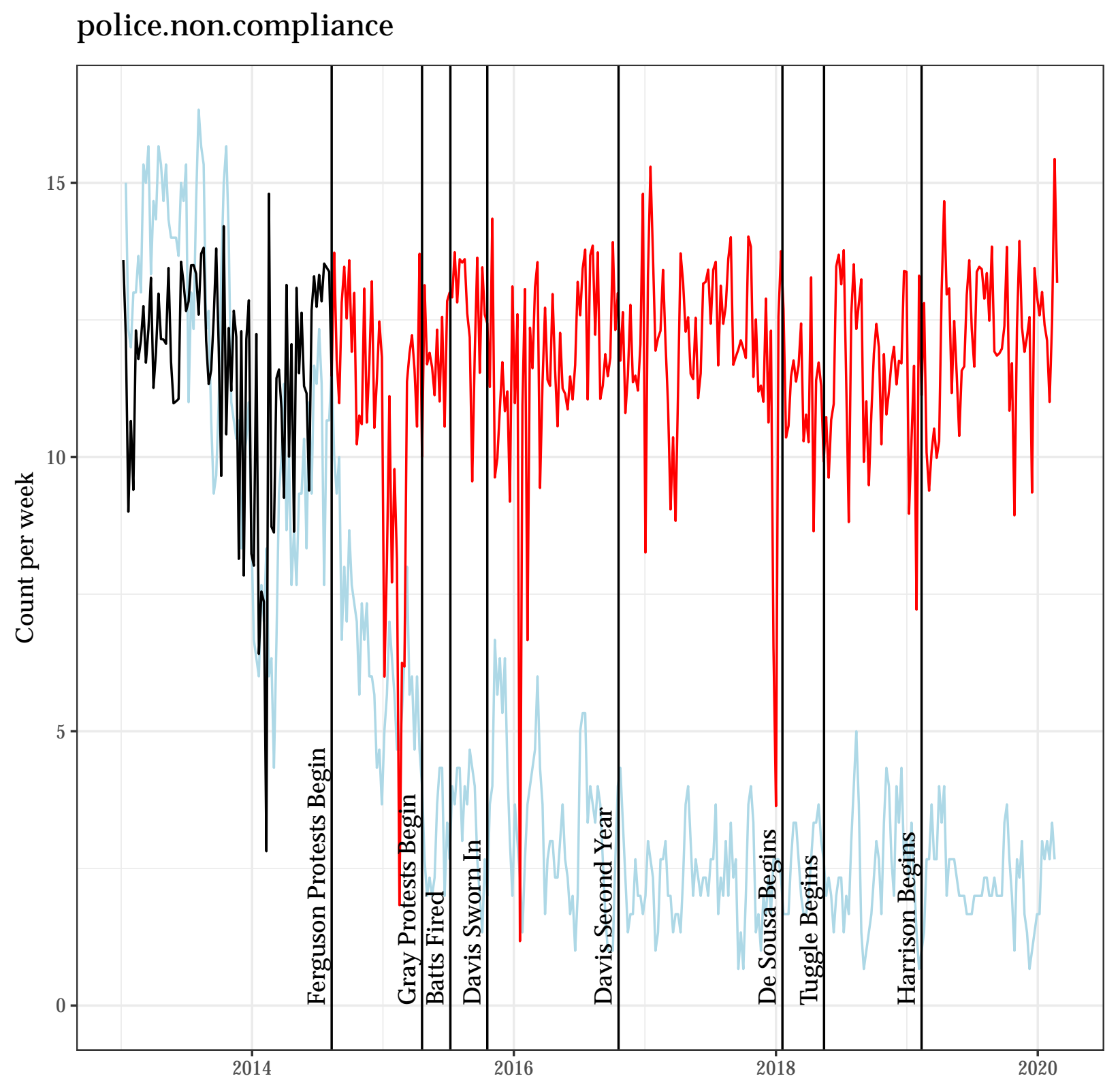


Autocorrelation Function for the Observed Outcome for Updated Model (2)

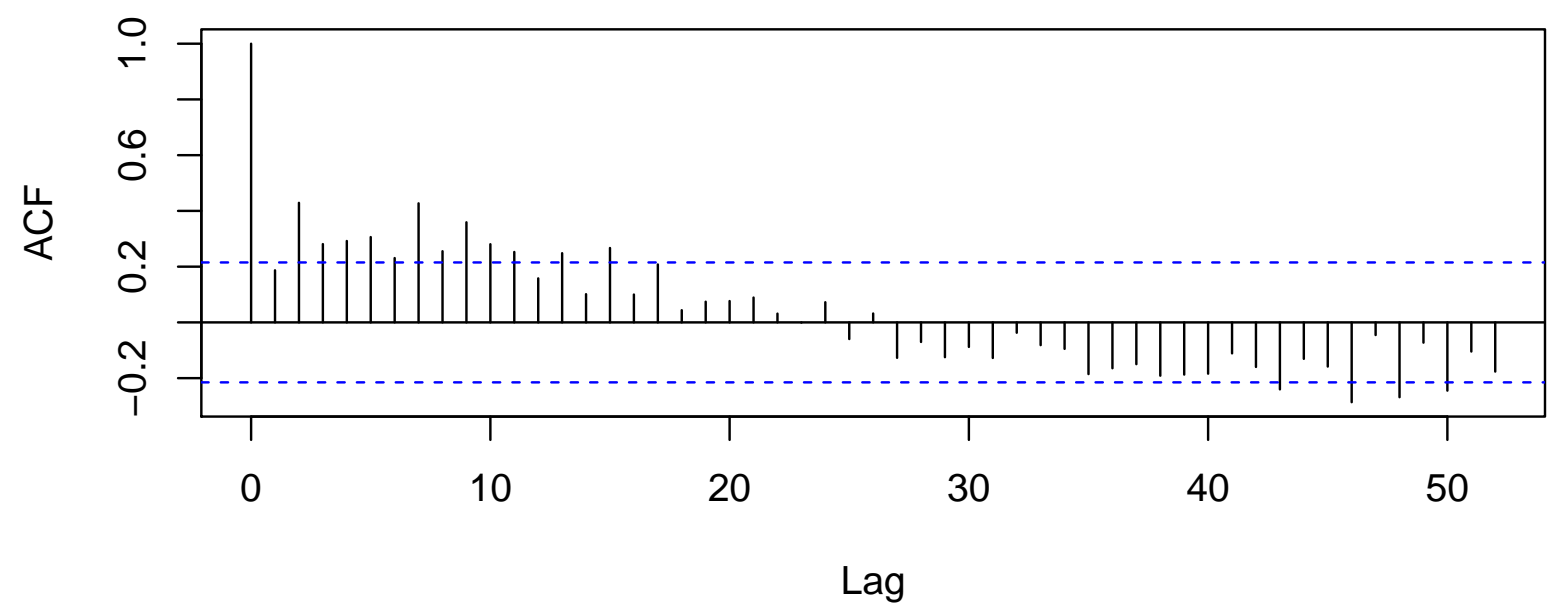

Autocorrelation Function for the Residuals from Updated Model (2)

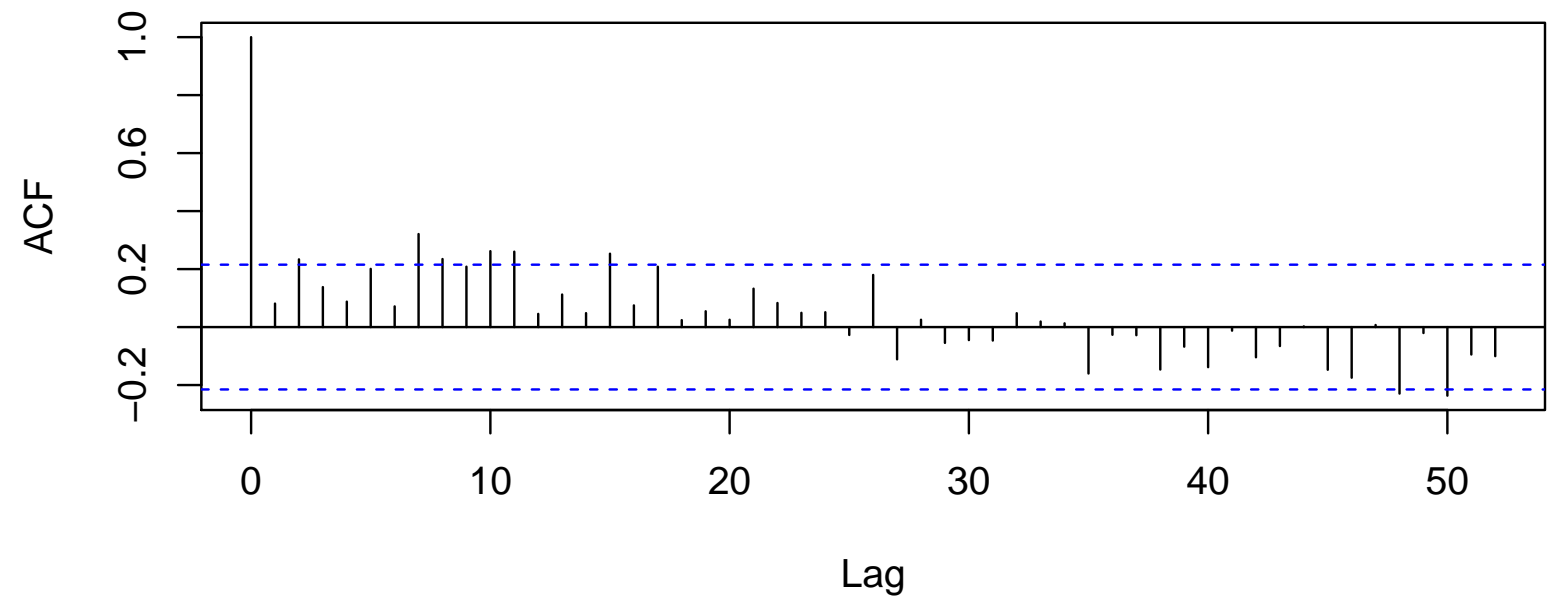




\subsection{A Poisson Regression Model as an Alternative to Model (2) for police.non.compliance}

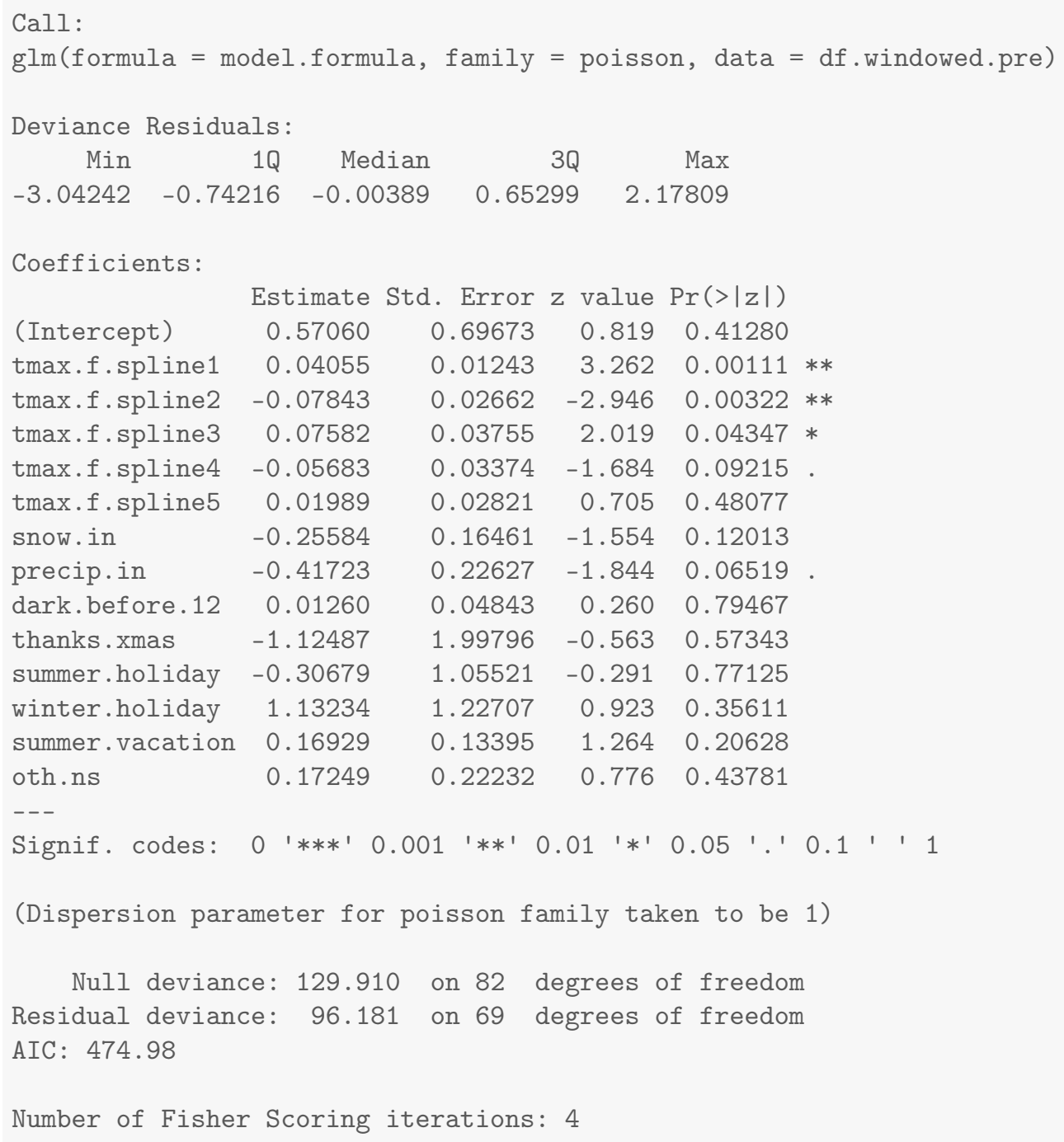




\section{Results for driving.violation}

\subsection{Summary Values for driving.violation}

Table 23.1: Descriptives for Outcome Before Ferguson Protests Begin

\begin{tabular}{lcccccccc}
\hline \hline Statistic & $\mathrm{N}$ & Mean & St. Dev. & Min & Pctl(25) & Median & Pctl(75) & Max \\
\hline driving.violation & 83 & 15.012 & 4.419 & 7 & 11.5 & 15 & 18 & 25 \\
\hline
\end{tabular}

Table 23.2: Descriptives for Outcome After Ferguson Protests Begin

\begin{tabular}{lcccccccc}
\hline \hline Statistic & $\mathrm{N}$ & Mean & St. Dev. & Min & Pctl(25) & Median & Pctl(75) & Max \\
\hline driving.violation & 290 & 6.907 & 4.955 & 0 & 3 & 6 & 9.8 & 26 \\
\hline
\end{tabular}




\subsection{Four Models for driving.violation}

Table 23.3: Four Models that Differ on the Specification of Adjustment and Intervention Variables

\begin{tabular}{|c|c|c|c|c|}
\hline & \multicolumn{4}{|c|}{ Outcome: Count Per Week } \\
\hline & $(1)$ & $(2)$ & (3) & (4) \\
\hline After Ferguson Protests Begin (week of 8/11/14 onward) & -0.15 & & -0.90 & -0.48 \\
\hline After Gray Protests Begin (week of $4 / 20 / 15$ onward) & -9.13 & & -7.04 & -8.33 \\
\hline Unrest and National Guard $(4 / 27 / 15-5 / 3 / 2015)$ & -2.73 & & -6.37 & -4.01 \\
\hline After Batts Fired (week of $7 / 13 / 15$ onward) & 5.13 & & 5.52 & 5.56 \\
\hline After Davis Confirmed (week of 10/19/15 onward) & -0.97 & & -3.33 & -1.83 \\
\hline After Davis First Year (week of $10 / 17 / 16$ onward) & -4.64 & & -4.37 & -4.63 \\
\hline After De Sousa Begins (week of 1/19/18 onward) & -0.62 & & -2.47 & -1.26 \\
\hline After Tuggle Begins (week of 5/14/18 onward) & -1.57 & & 1.94 & -0.33 \\
\hline After Harrison Begins (week of 2/11/19 onward) & 0.53 & & -0.65 & 0.08 \\
\hline Average Maximum Temperature to 50 Degrees & & -0.03 & & -0.07 \\
\hline Plus Degrees in the $50 \mathrm{~s}$ & & 0.64 & & 0.16 \\
\hline Plus Degrees in the 60s & & -1.28 & & -0.24 \\
\hline Plus Degrees in the $70 \mathrm{~s}$ & & 0.43 & & 0.05 \\
\hline Plus Degrees Greater Than 80 & & 0.11 & & 0.001 \\
\hline Snowfall (inches) & & -0.30 & & -0.07 \\
\hline Precipitation (inches) & & -3.87 & & -1.84 \\
\hline Darkness Before Midnight (hours) & & -0.24 & & -0.28 \\
\hline Thanksgiving/Christmas (proportion of week) & & -0.93 & & 0.22 \\
\hline Winter Holiday (proportion of week) & & 5.99 & & 1.35 \\
\hline Other Out-of-School Days (proportion of week) & & -5.93 & & -1.76 \\
\hline Summer Vacation (proportion of week) & & 0.64 & & 0.07 \\
\hline Summer Holiday (proportion of week) & & -8.26 & & 0.71 \\
\hline Observations & 373 & 83 & 373 & 373 \\
\hline $\mathrm{R}^{2}$ & 0.67 & 0.29 & 0.61 & 0.69 \\
\hline
\end{tabular}




\subsection{Least Squares Updated Model (2) for driving.violation}

driving.violation

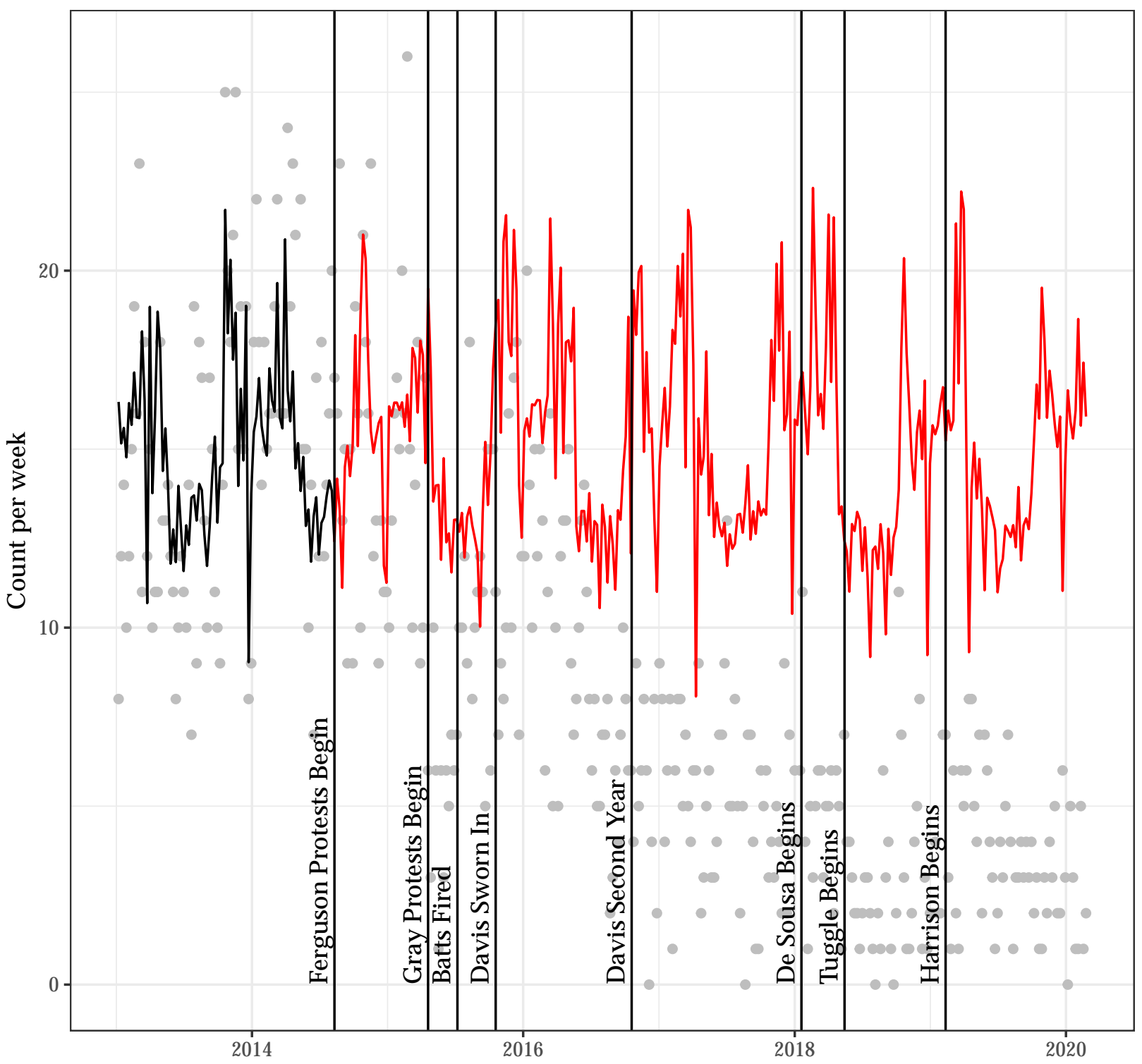




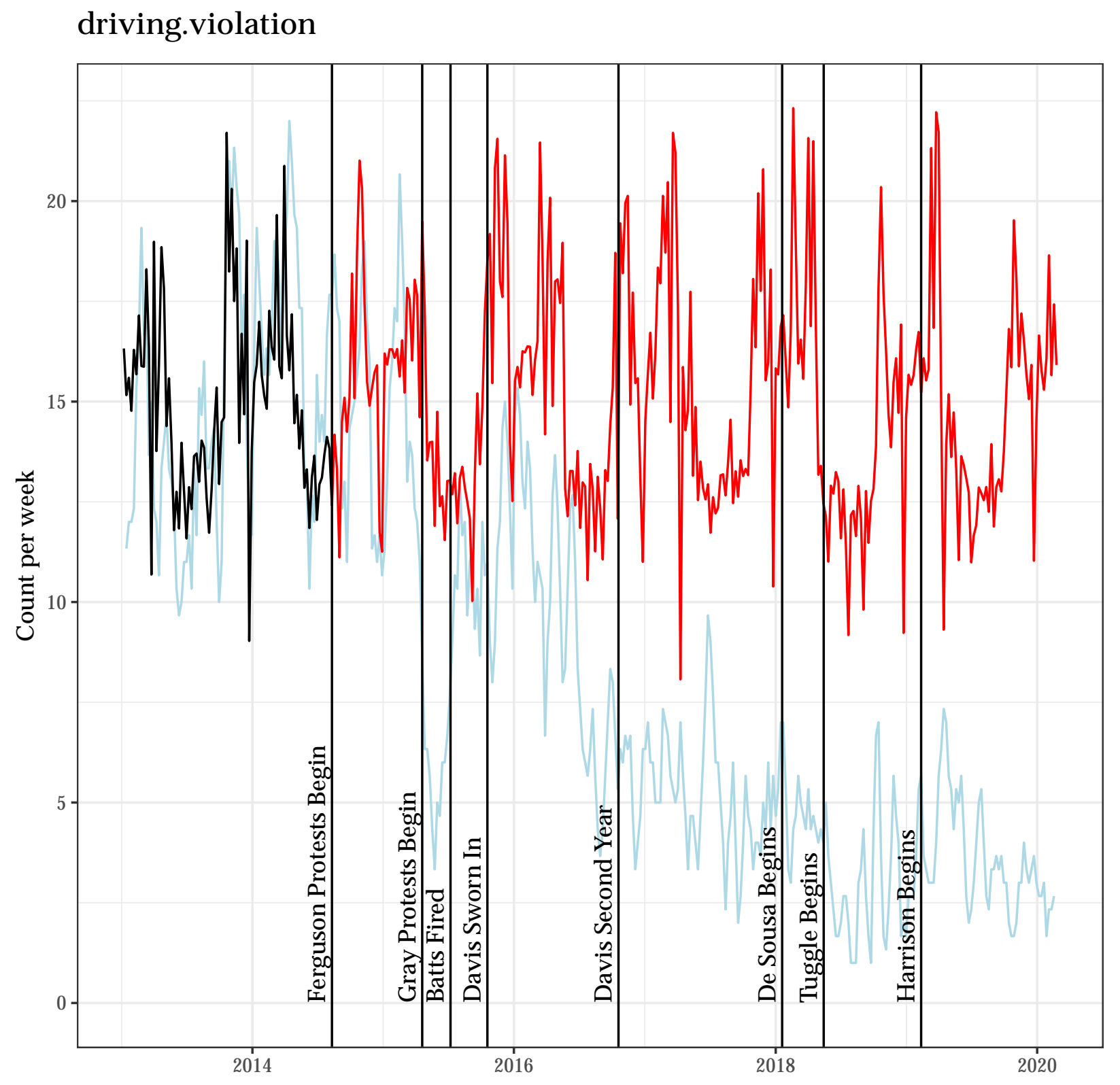


Autocorrelation Function for the Observed Outcome for Updated Model (2)

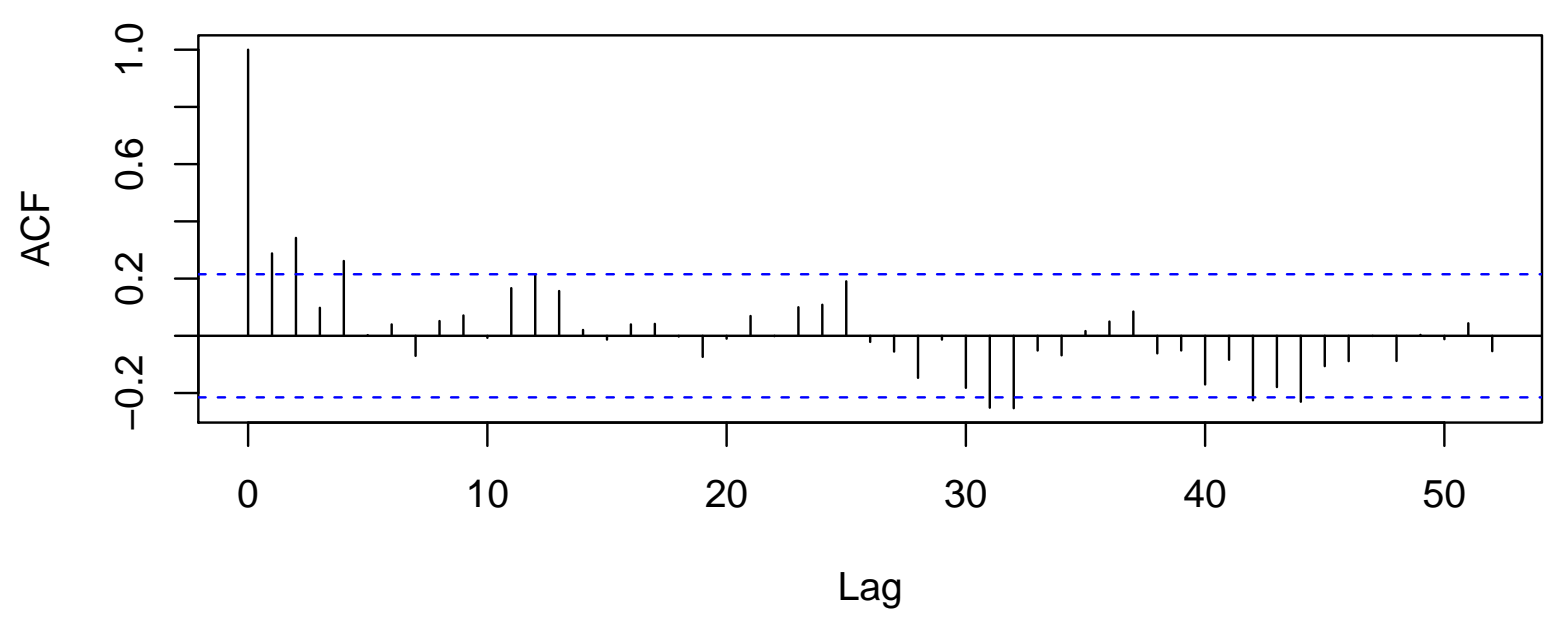

Autocorrelation Function for the Residuals from Updated Model (2)

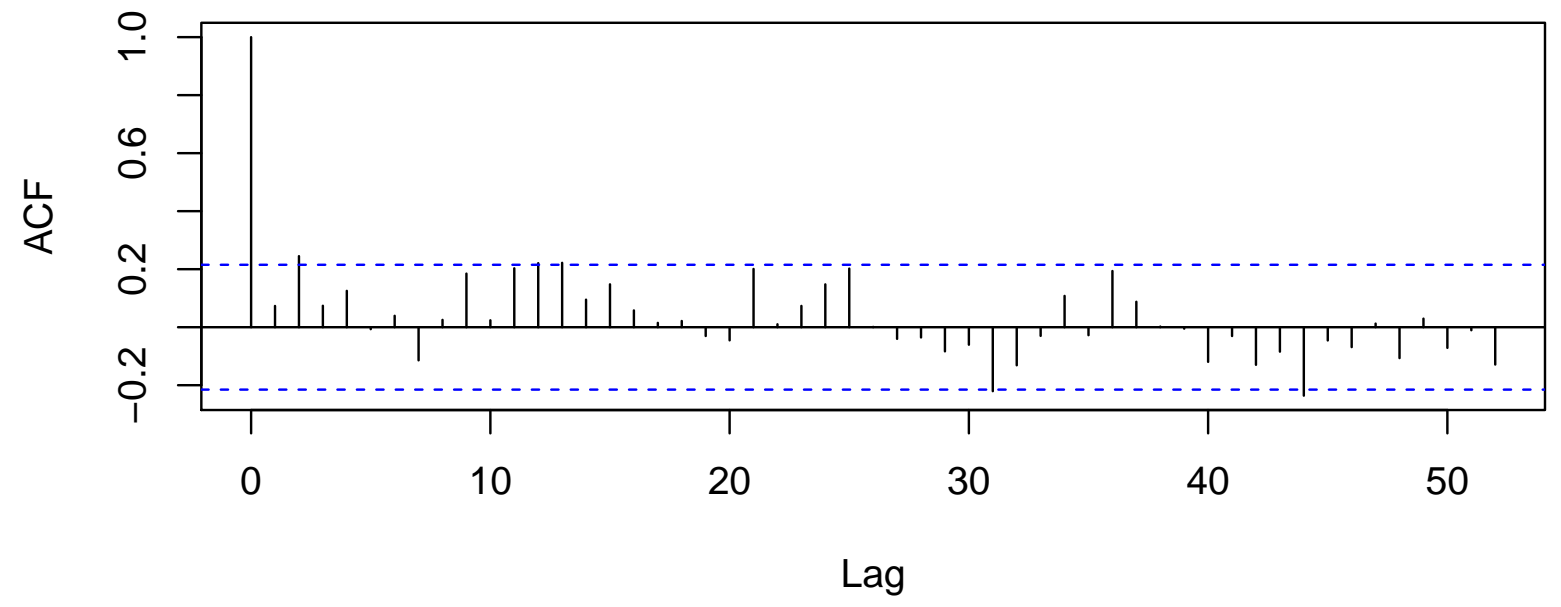




\subsection{A Poisson Regression Model as an Alternative to Model (2) for driving.violation}

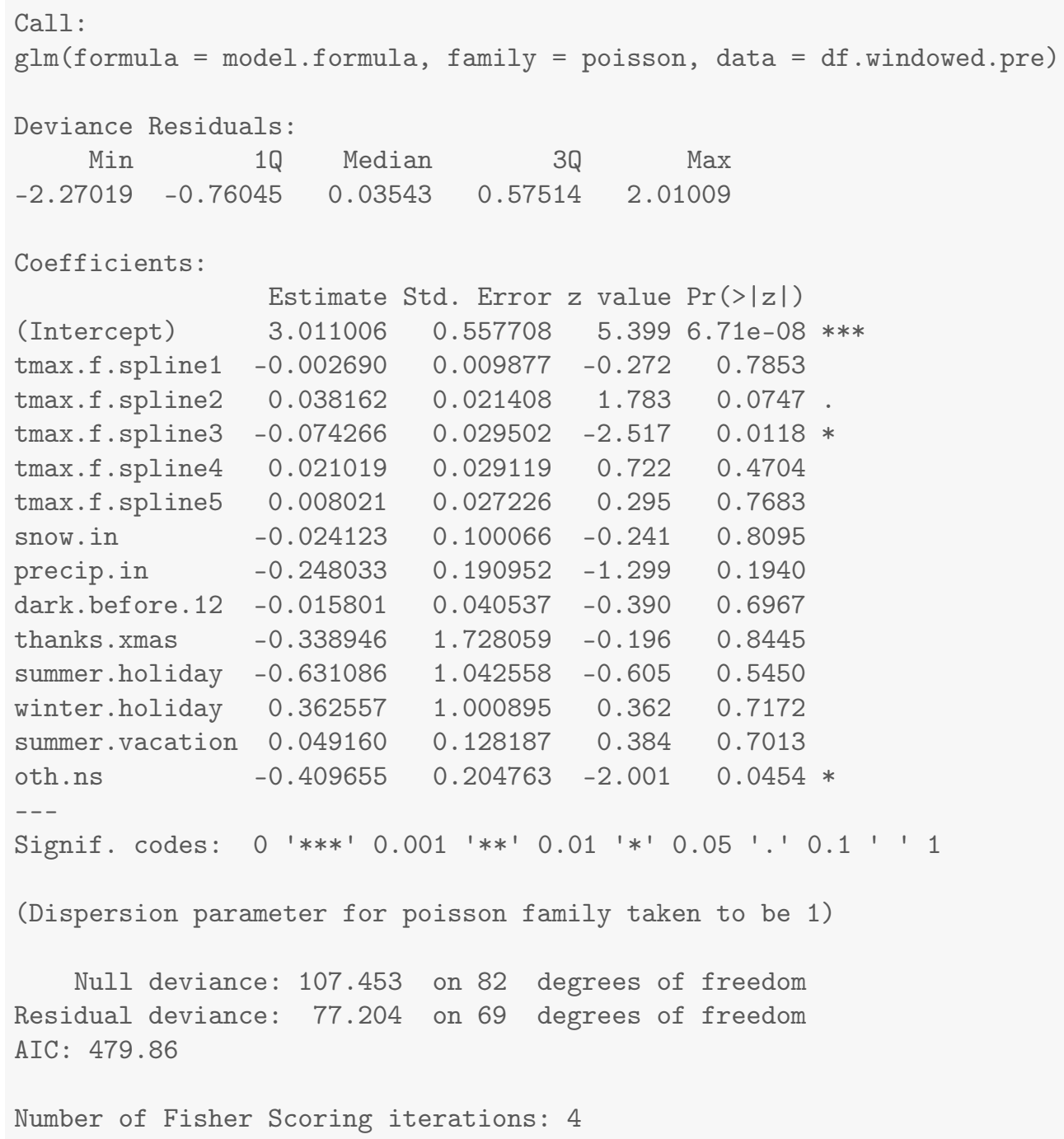




\section{Results for trespass}

\subsection{Summary Values for trespass}

Table 24.1: Descriptives for Outcome Before Ferguson Protests Begin

\begin{tabular}{lcccccccc}
\hline \hline Statistic & $\mathrm{N}$ & Mean & St. Dev. & Min & Pctl(25) & Median & Pctl(75) & Max \\
\hline trespass & 83 & 12.446 & 4.551 & 3 & 9 & 11 & 16 & 25 \\
\hline
\end{tabular}

Table 24.2: Descriptives for Outcome After Ferguson Protests Begin

\begin{tabular}{lcccccccc}
\hline \hline Statistic & $\mathrm{N}$ & Mean & St. Dev. & Min & Pctl(25) & Median & Pctl(75) & Max \\
\hline trespass & 290 & 4.641 & 3.585 & 0 & 2 & 4 & 6 & 22 \\
\hline
\end{tabular}




\subsection{Four Models for trespass}

Table 24.3: Four Models that Differ on the Specification of Adjustment and Intervention Variables

\begin{tabular}{|c|c|c|c|c|}
\hline & \multicolumn{4}{|c|}{ Outcome: Count Per Week } \\
\hline & $(1)$ & (2) & $(3)$ & (4) \\
\hline After Ferguson Protests Begin (week of 8/11/14 onward) & -1.83 & & -0.83 & -1.52 \\
\hline After Gray Protests Begin (week of $4 / 20 / 15$ onward) & -6.79 & & -8.73 & -7.29 \\
\hline Unrest and National Guard $(4 / 27 / 15-5 / 3 / 2015)$ & -2.82 & & -3.67 & -3.56 \\
\hline After Batts Fired (week of $7 / 13 / 15$ onward) & 2.82 & & 1.83 & 2.93 \\
\hline After Davis Confirmed (week of 10/19/15 onward) & -1.57 & & 0.62 & -1.44 \\
\hline After Davis First Year (week of $10 / 17 / 16$ onward) & -1.23 & & -1.19 & -1.29 \\
\hline After De Sousa Begins (week of 1/19/18 onward) & -0.41 & & -0.29 & -0.51 \\
\hline After Tuggle Begins (week of 5/14/18 onward) & -1.03 & & -2.01 & -0.59 \\
\hline After Harrison Begins (week of 2/11/19 onward) & 0.52 & & 0.44 & 0.09 \\
\hline Average Maximum Temperature to 50 Degrees & & 0.18 & & 0.13 \\
\hline Plus Degrees in the $50 \mathrm{~s}$ & & -0.79 & & -0.22 \\
\hline Plus Degrees in the $60 \mathrm{~s}$ & & 1.12 & & 0.20 \\
\hline Plus Degrees in the 70s & & -0.78 & & -0.22 \\
\hline Plus Degrees Greater Than 80 & & 0.86 & & 0.07 \\
\hline Snowfall (inches) & & -3.45 & & -0.79 \\
\hline Precipitation (inches) & & -1.31 & & -1.84 \\
\hline Darkness Before Midnight (hours) & & -0.95 & & -0.13 \\
\hline Thanksgiving/Christmas (proportion of week) & & -20.64 & & -17.08 \\
\hline Winter Holiday (proportion of week) & & 13.38 & & 13.72 \\
\hline Other Out-of-School Days (proportion of week) & & 0.89 & & 0.78 \\
\hline Summer Vacation (proportion of week) & & -1.99 & & 0.52 \\
\hline Summer Holiday (proportion of week) & & -10.61 & & 6.82 \\
\hline Observations & 373 & 83 & 373 & 373 \\
\hline $\mathrm{R}^{2}$ & 0.61 & 0.24 & 0.57 & 0.64 \\
\hline
\end{tabular}




\subsection{Least Squares Updated Model (2) for trespass}

\section{trespass}

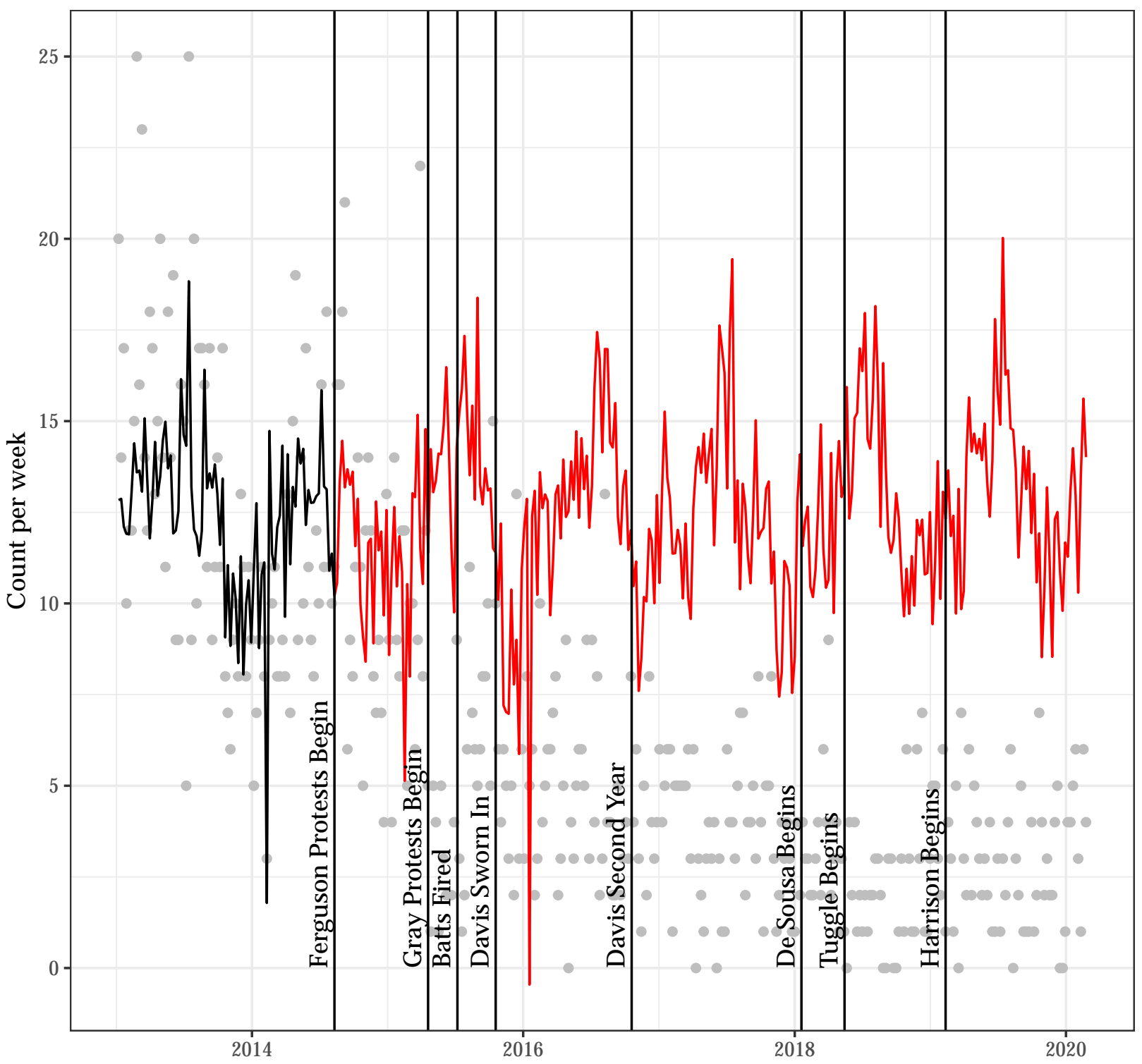




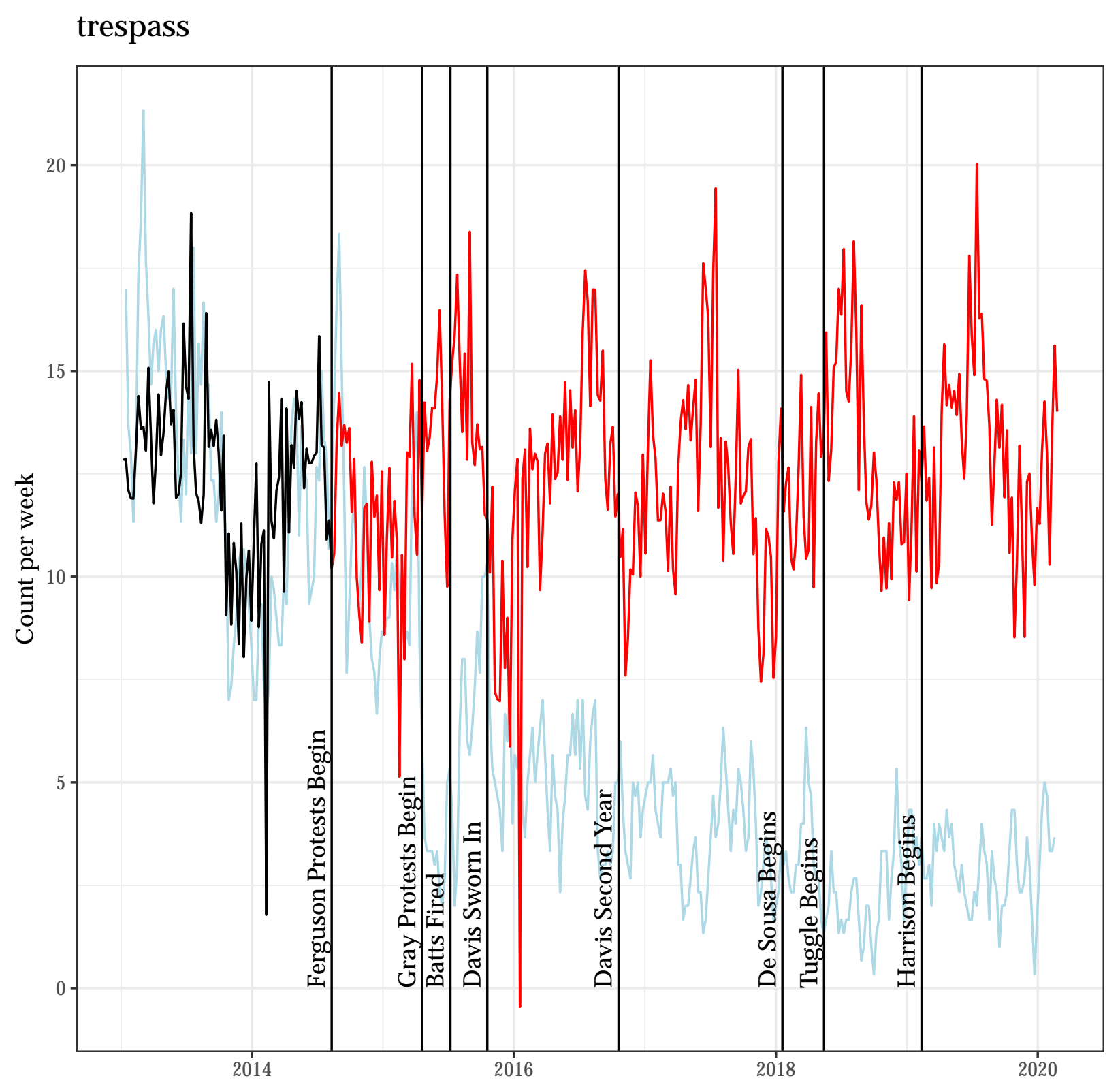


Autocorrelation Function for the Observed Outcome for Updated Model (2)

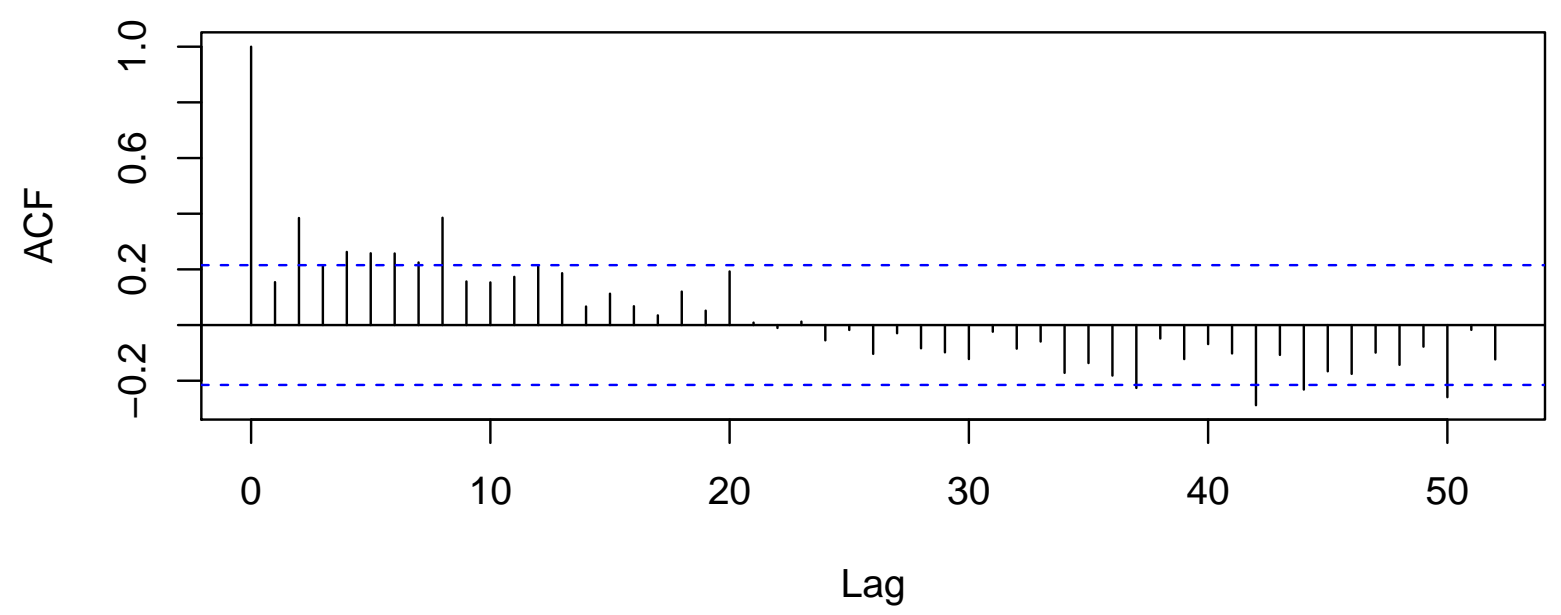

Autocorrelation Function for the Residuals from Updated Model (2)

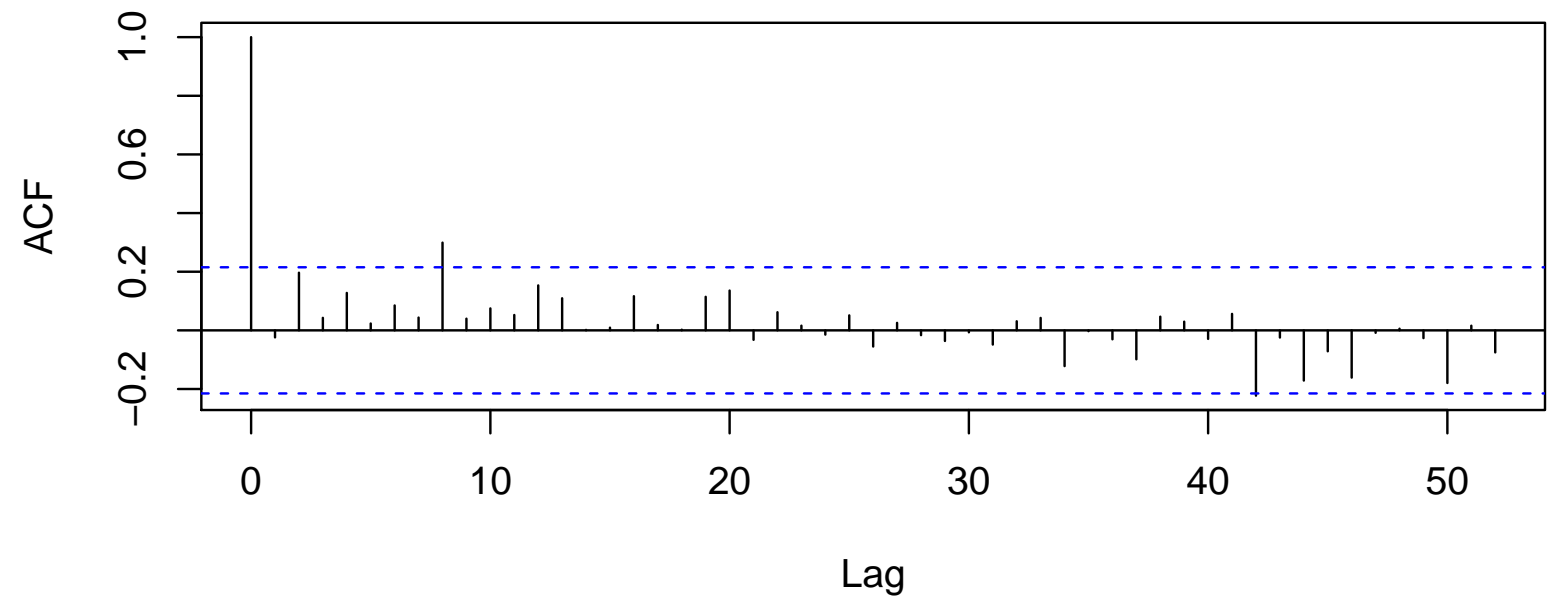




\subsection{A Poisson Regression Model as an Alternative to Model (2) for trespass}

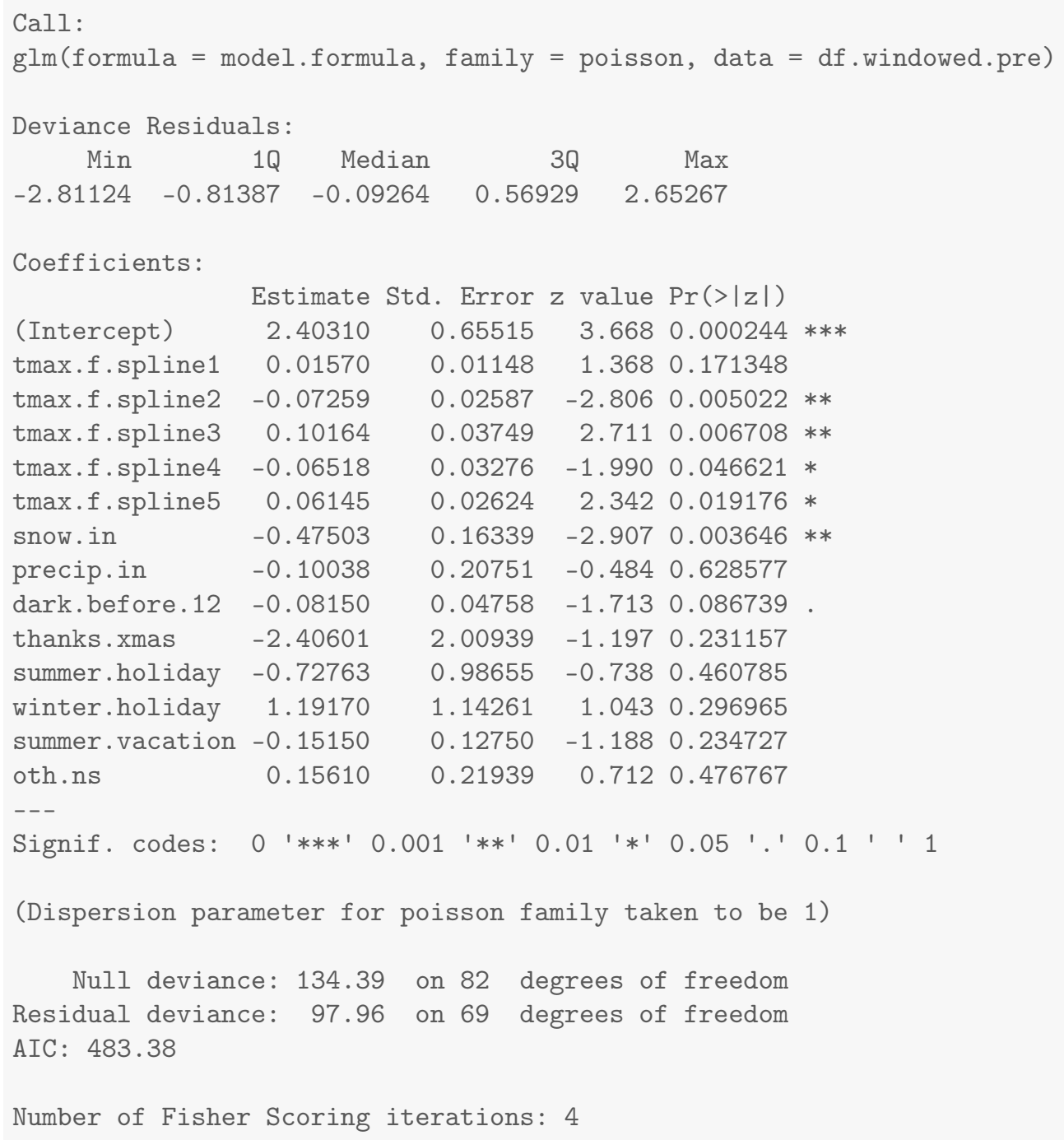




\section{Results for drug.g}

\subsection{Summary Values for drug.g}

Table 25.1: Descriptives for Outcome Before Ferguson Protests Begin

\begin{tabular}{lcccccccc}
\hline \hline Statistic & $\mathrm{N}$ & Mean & St. Dev. & Min & Pctl(25) & Median & Pctl(75) & Max \\
\hline drug.g & 83 & 300.916 & 55.036 & 179 & 260 & 296 & 337 & 423 \\
\hline
\end{tabular}

Table 25.2: Descriptives for Outcome After Ferguson Protests Begin

\begin{tabular}{lcccccccc}
\hline \hline Statistic & $\mathrm{N}$ & Mean & St. Dev. & Min & Pctl(25) & Median & Pctl(75) & Max \\
\hline drug.g & 290 & 99.962 & 39.986 & 17 & 76 & 94 & 116.8 & 283 \\
\hline
\end{tabular}




\subsection{Four Models for drug.g}

Table 25.3: Four Models that Differ on the Specification of Adjustment and Intervention Variables

\begin{tabular}{|c|c|c|c|c|}
\hline & \multicolumn{4}{|c|}{ Outcome: Count Per Week } \\
\hline & (1) & $(2)$ & (3) & (4) \\
\hline After Ferguson Protests Begin (week of $8 / 11 / 14$ onward) & -135.94 & & -129.46 & -132.15 \\
\hline After Gray Protests Begin (week of 4/20/15 onward) & -95.70 & & -104.74 & -100.88 \\
\hline Unrest and National Guard (4/27/15 - 5/3/2015) & -49.27 & & -66.33 & -63.16 \\
\hline After Batts Fired (week of 7/13/15 onward) & 44.51 & & 47.16 & 48.56 \\
\hline After Davis Confirmed (week of 10/19/15 onward) & -0.75 & & 8.67 & -0.86 \\
\hline After Davis First Year (week of 10/17/16 onward) & -10.90 & & -15.60 & -11.87 \\
\hline After De Sousa Begins (week of 1/19/18 onward) & -4.32 & & -14.81 & -9.92 \\
\hline After Tuggle Begins (week of 5/14/18 onward) & -17.53 & & -1.67 & -4.55 \\
\hline After Harrison Begins (week of 2/11/19 onward) & -19.19 & & -30.52 & -27.22 \\
\hline Average Maximum Temperature to 50 Degrees & & 1.38 & & 0.99 \\
\hline Plus Degrees in the $50 \mathrm{~s}$ & & -8.81 & & -2.11 \\
\hline Plus Degrees in the 60s & & 10.11 & & 1.37 \\
\hline Plus Degrees in the 70s & & -1.88 & & -0.97 \\
\hline Plus Degrees Greater Than 80 & & -0.73 & & -0.78 \\
\hline Snowfall (inches) & & -43.57 & & -10.70 \\
\hline Precipitation (inches) & & -81.84 & & -44.40 \\
\hline Darkness Before Midnight (hours) & & -12.50 & & -5.16 \\
\hline Thanksgiving/Christmas (proportion of week) & & -778.54 & & -268.80 \\
\hline Winter Holiday (proportion of week) & & -105.57 & & 72.04 \\
\hline Other Out-of-School Days (proportion of week) & & -3.47 & & -9.73 \\
\hline Summer Vacation (proportion of week) & & -24.01 & & 5.70 \\
\hline Summer Holiday (proportion of week) & & -274.26 & & -37.79 \\
\hline Observations & 373 & 83 & 373 & 373 \\
\hline $\mathrm{R}^{2}$ & 0.87 & 0.30 & 0.83 & 0.89 \\
\hline
\end{tabular}


25.3 Least Squares Updated Model (2) for drug.g drug.g

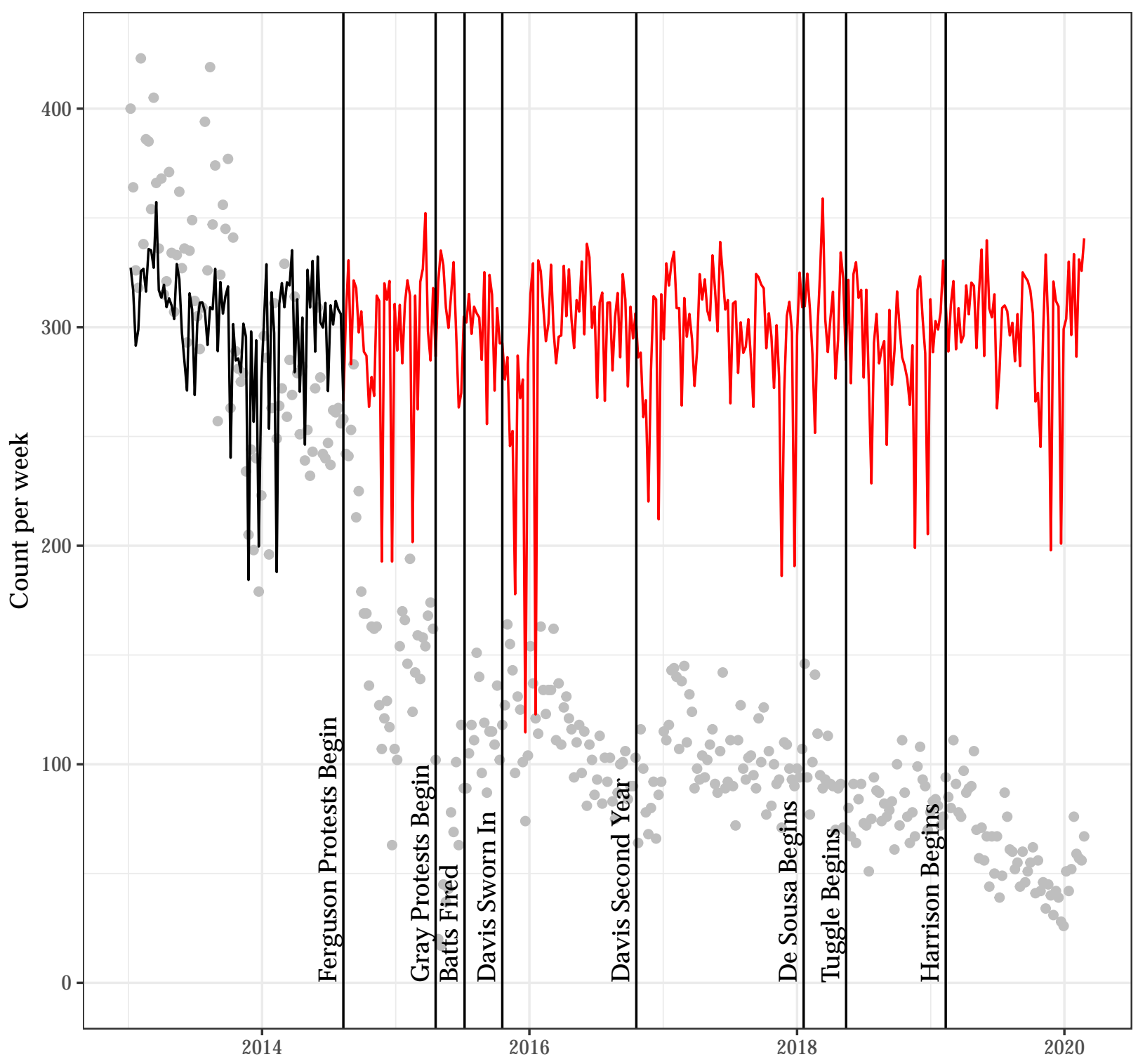




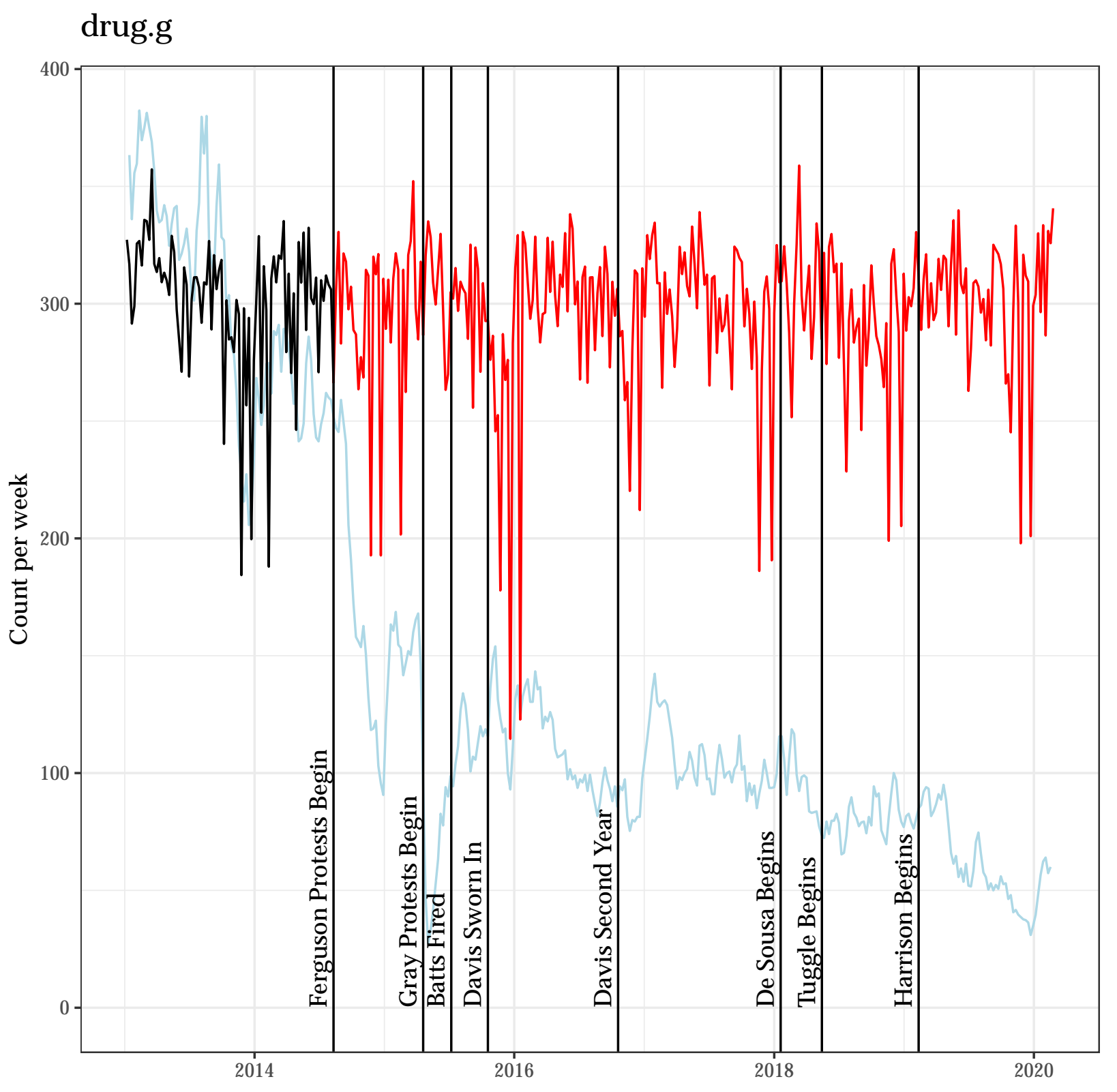


Autocorrelation Function for the Observed Outcome for Updated Model (2)

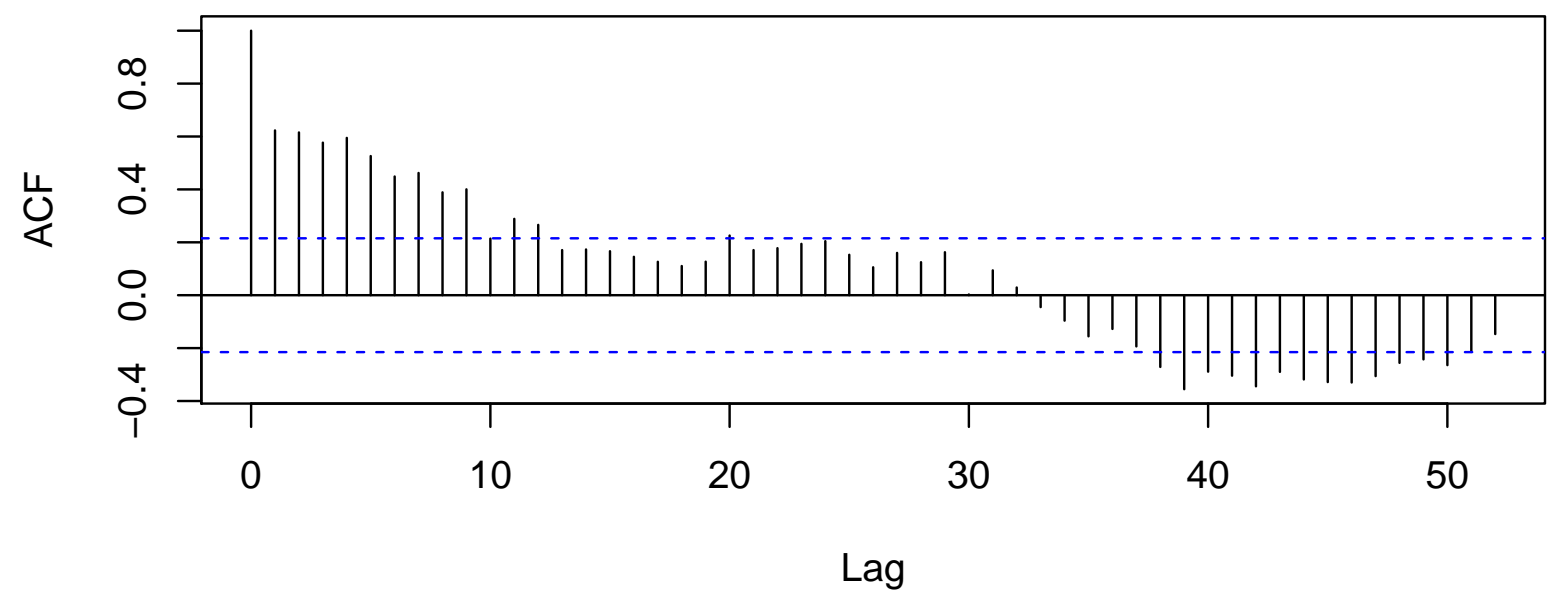

Autocorrelation Function for the Residuals from Updated Model (2)

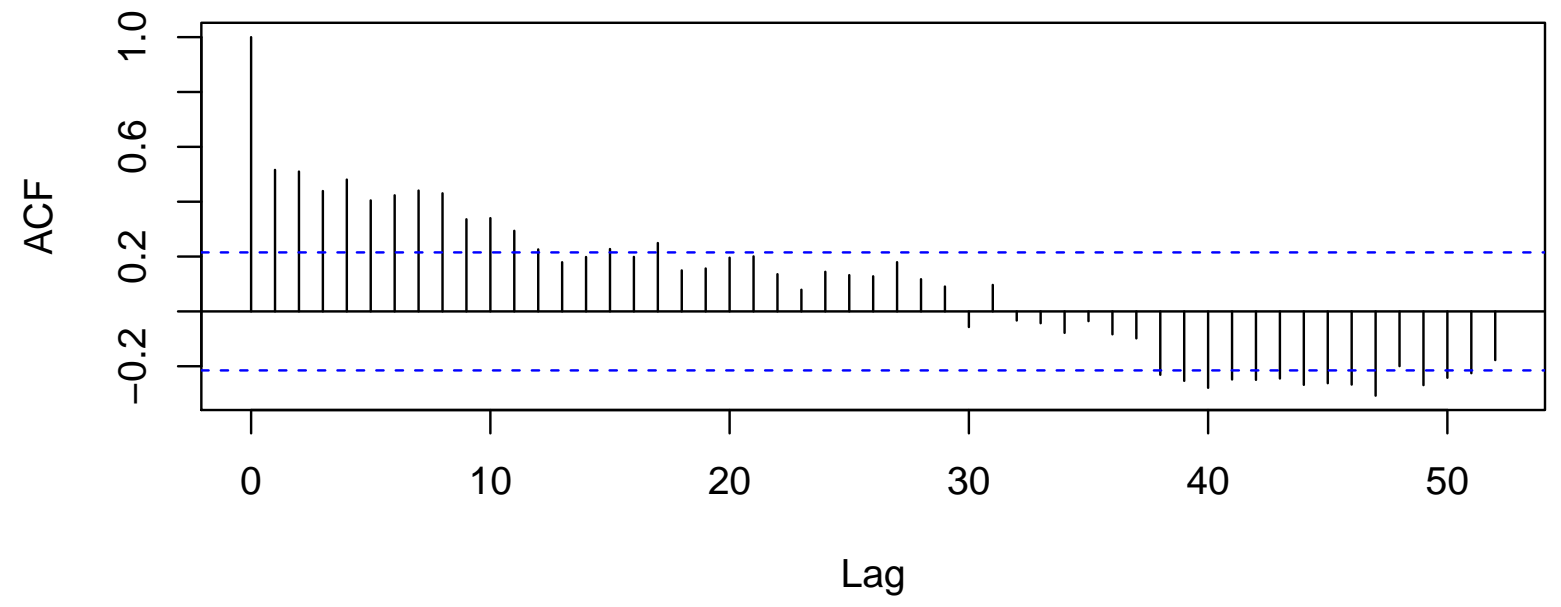




\subsection{A Poisson Regression Model as an Alternative to Model (2) for drug.g}

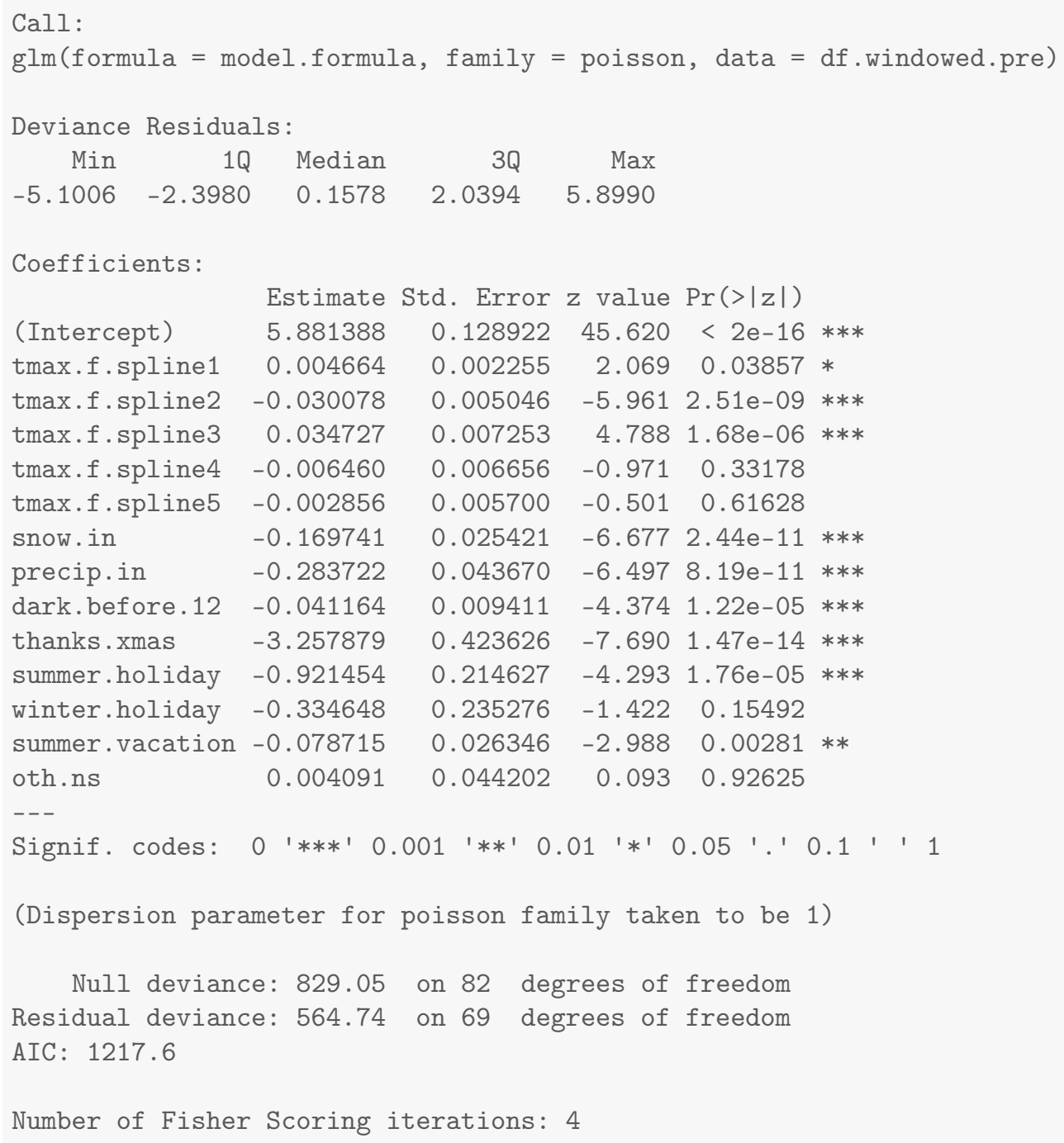




\section{Results for loitering.vagabond}

\subsection{Summary Values for loitering.vagabond}

Table 26.1: Descriptives for Outcome Before Ferguson Protests Begin

\begin{tabular}{lcccccccc}
\hline \hline Statistic & $\mathrm{N}$ & Mean & St. Dev. & Min & Pctl(25) & Median & Pctl(75) & Max \\
\hline loitering.vagabond & 83 & 1.422 & 1.754 & 0 & 0 & 1 & 2 & 8 \\
\hline
\end{tabular}

Table 26.2: Descriptives for Outcome After Ferguson Protests Begin

\begin{tabular}{lcccccccc}
\hline \hline Statistic & $\mathrm{N}$ & Mean & St. Dev. & Min & Pctl(25) & Median & Pctl(75) & Max \\
\hline loitering.vagabond & 290 & 0.169 & 0.480 & 0 & 0 & 0 & 0 & 4 \\
\hline
\end{tabular}




\subsection{Four Models for loitering.vagabond}

Table 26.3: Four Models that Differ on the Specification of Adjustment and Intervention Variables

\begin{tabular}{|c|c|c|c|c|}
\hline & \multicolumn{4}{|c|}{ Outcome: Count Per Week } \\
\hline & (1) & $(2)$ & $(3)$ & $(4)$ \\
\hline After Ferguson Protests Begin (week of $8 / 11 / 14$ onward) & -0.92 & & -0.80 & -0.87 \\
\hline After Gray Protests Begin (week of 4/20/15 onward) & -0.32 & & -0.42 & -0.39 \\
\hline Unrest and National Guard (4/27/15 - 5/3/2015) & 1.82 & & 1.70 & 1.77 \\
\hline After Batts Fired (week of 7/13/15 onward) & 0.03 & & -0.26 & 0.06 \\
\hline After Davis Confirmed (week of 10/19/15 onward) & -0.003 & & 0.39 & 0.04 \\
\hline After Davis First Year (week of 10/17/16 onward) & -0.11 & & -0.04 & -0.09 \\
\hline After De Sousa Begins (week of 1/19/18 onward) & -0.04 & & -0.001 & -0.07 \\
\hline After Tuggle Begins (week of 5/14/18 onward) & -0.04 & & -0.23 & -0.01 \\
\hline After Harrison Begins (week of 2/11/19 onward) & 0.05 & & 0.10 & 0.03 \\
\hline Average Maximum Temperature to 50 Degrees & & -0.06 & & 0.0004 \\
\hline Plus Degrees in the 50s & & 0.04 & & -0.01 \\
\hline Plus Degrees in the 60s & & 0.06 & & 0.02 \\
\hline Plus Degrees in the 70s & & -0.13 & & -0.04 \\
\hline Plus Degrees Greater Than 80 & & 0.17 & & 0.02 \\
\hline Snowfall (inches) & & -0.16 & & 0.06 \\
\hline Precipitation (inches) & & 0.21 & & 0.01 \\
\hline Darkness Before Midnight (hours) & & -0.20 & & -0.08 \\
\hline Thanksgiving/Christmas (proportion of week) & & -2.80 & & 0.03 \\
\hline Winter Holiday (proportion of week) & & 3.13 & & 0.71 \\
\hline Other Out-of-School Days (proportion of week) & & 0.52 & & 0.09 \\
\hline Summer Vacation (proportion of week) & & 0.94 & & 0.34 \\
\hline Summer Holiday (proportion of week) & & -5.55 & & -0.27 \\
\hline Observations & 373 & 83 & 373 & 373 \\
\hline $\mathrm{R}^{2}$ & 0.26 & 0.09 & 0.20 & 0.28 \\
\hline
\end{tabular}


26.3 Least Squares Updated Model (2) for loitering.vagabond loitering.vagabond

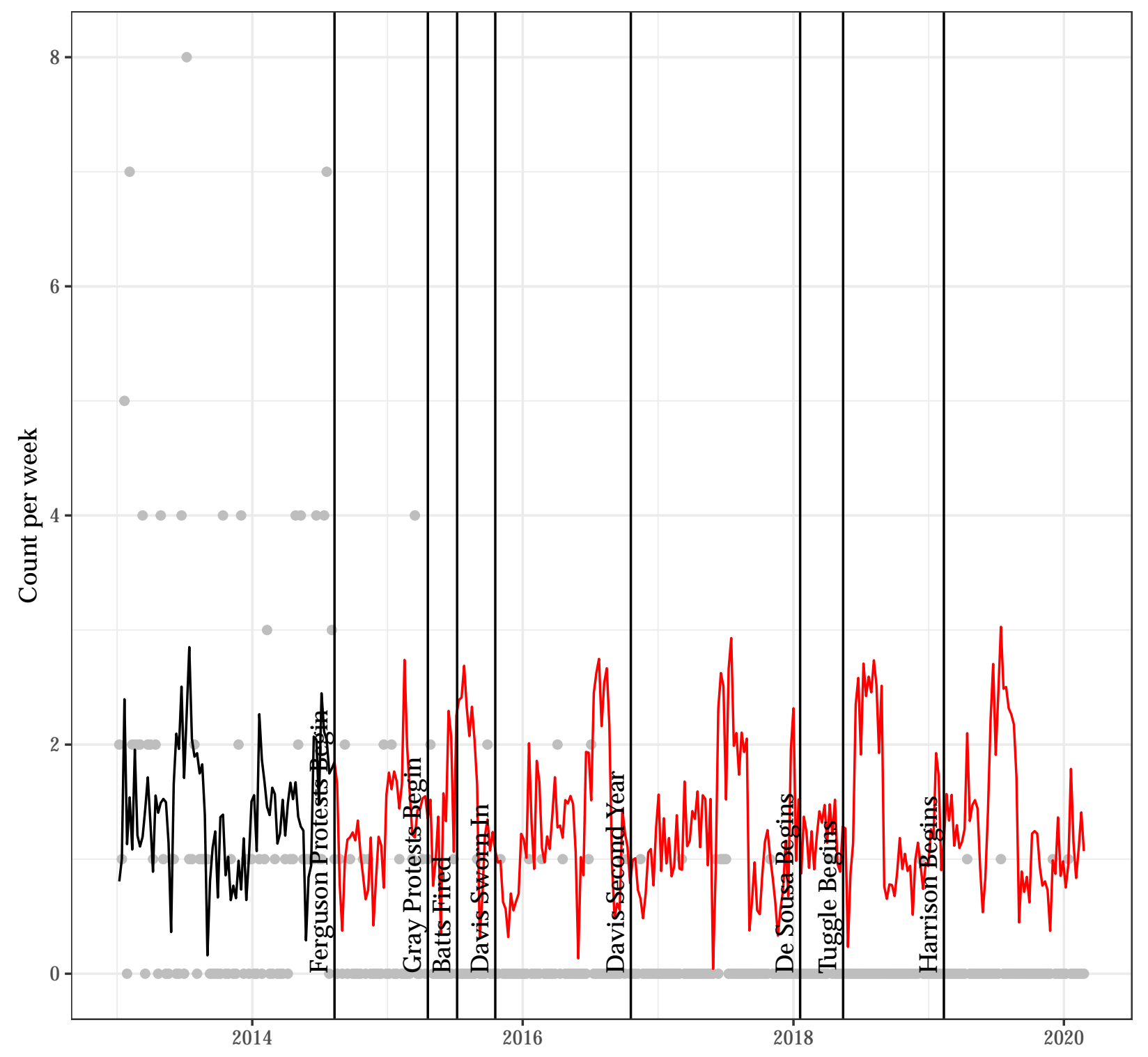




\section{loitering.vagabond}

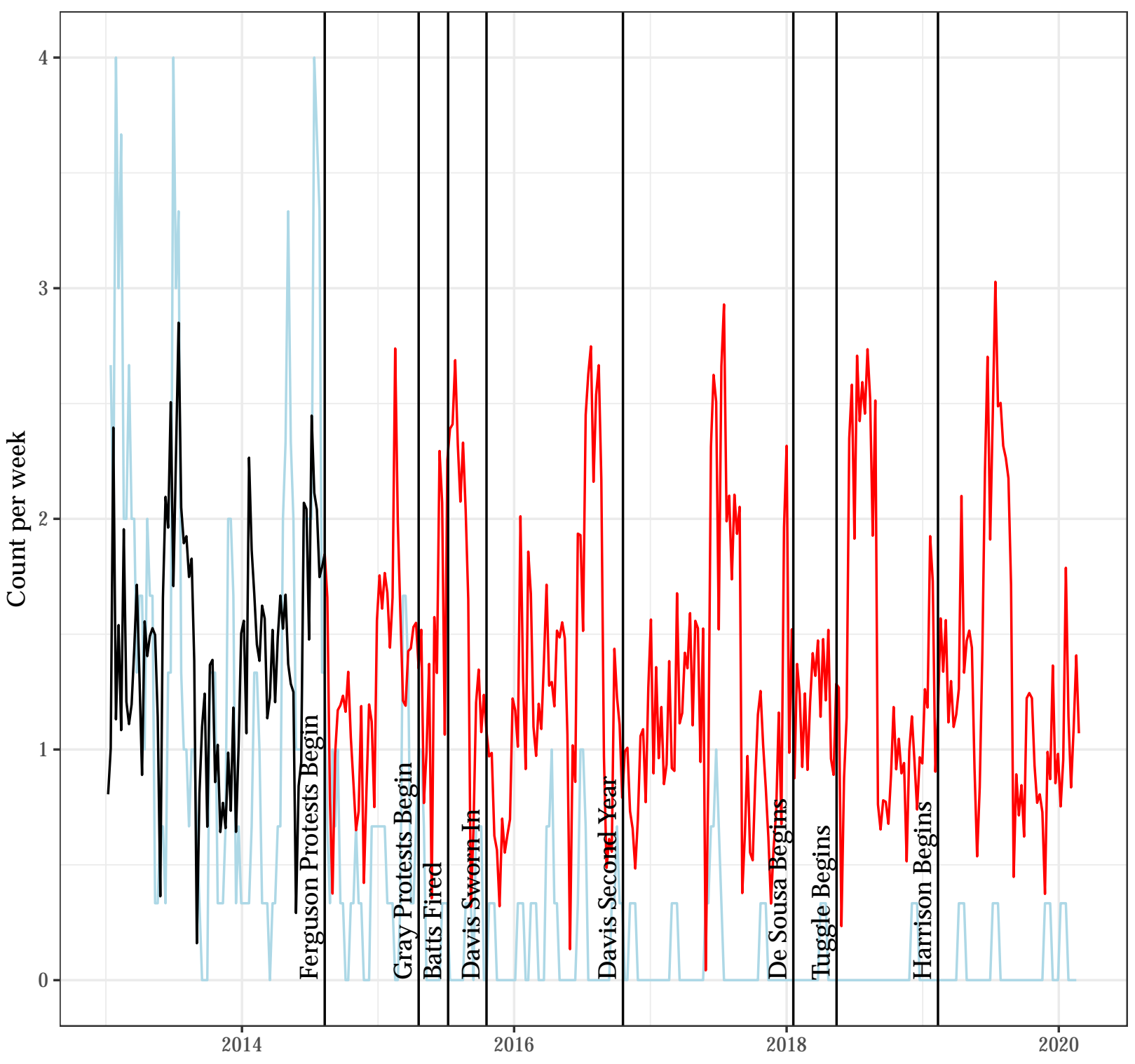


Autocorrelation Function for the Observed Outcome for Updated Model (2)

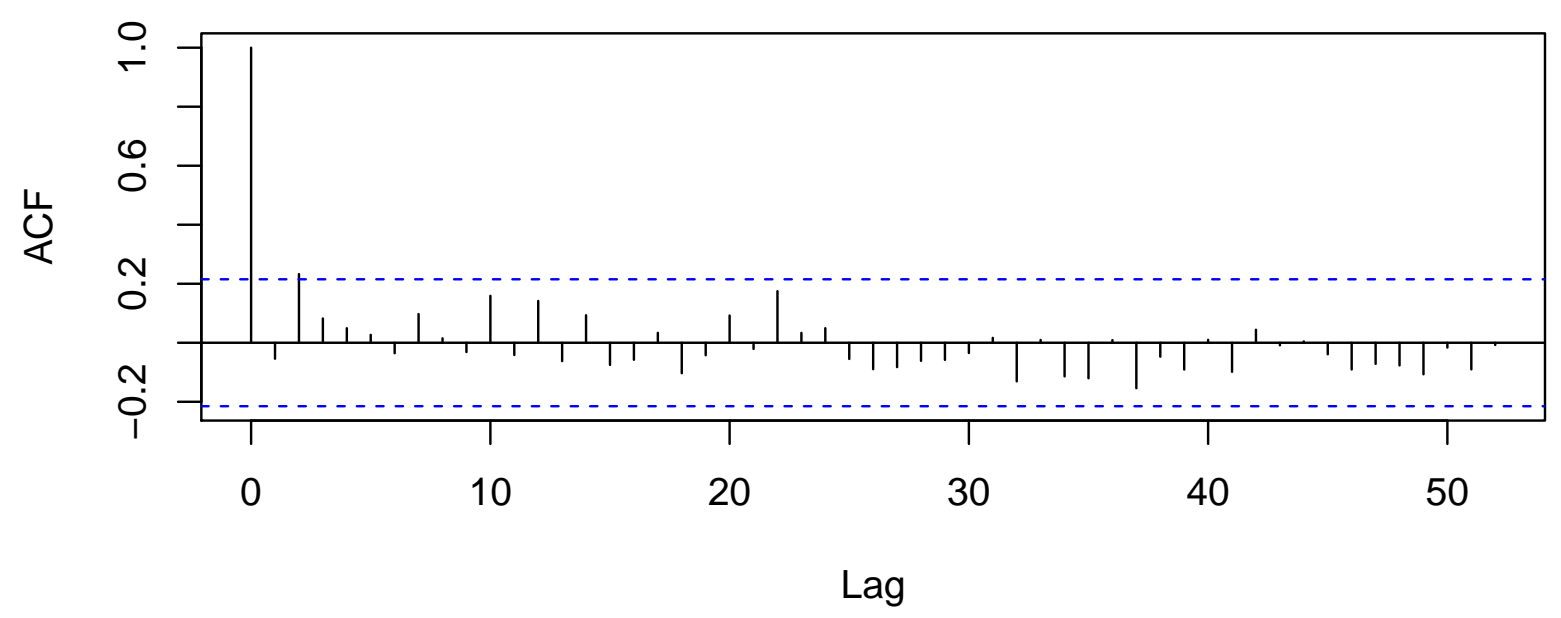

Autocorrelation Function for the Residuals from Updated Model (2)

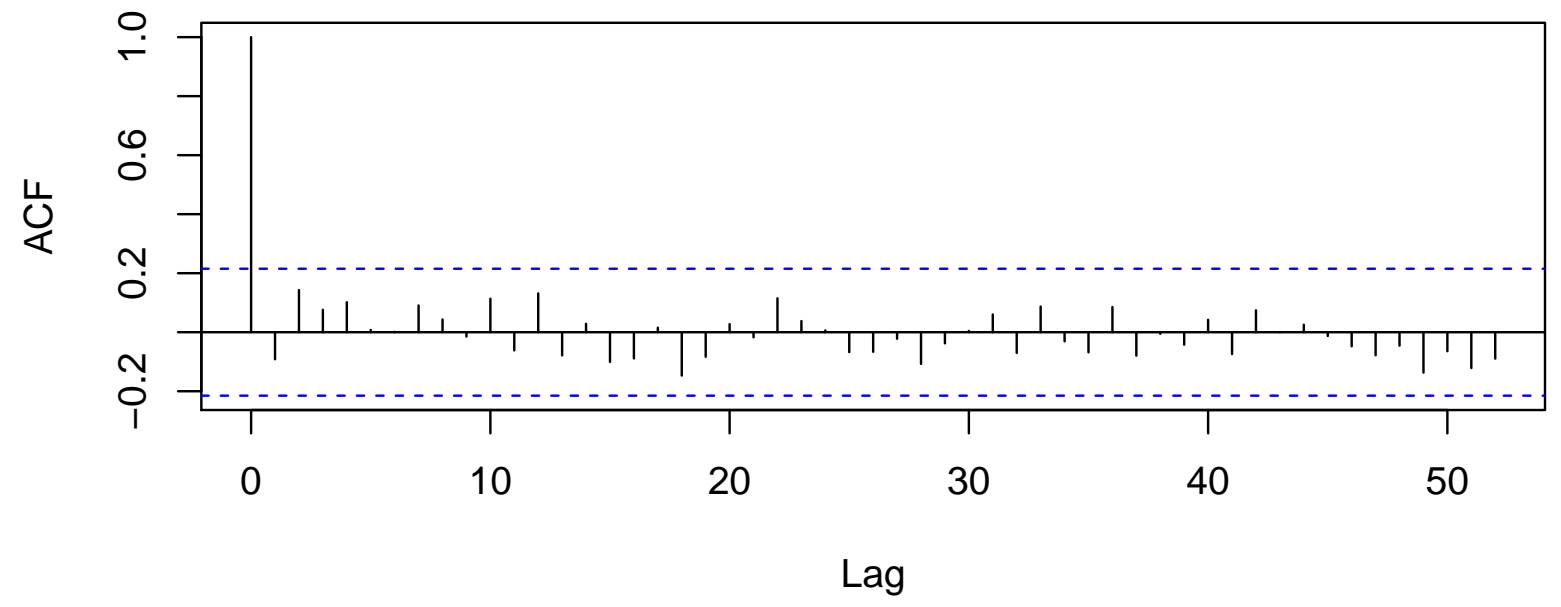




\subsection{A Poisson Regression Model as an Alternative to Model (2) for loitering.vagabond}

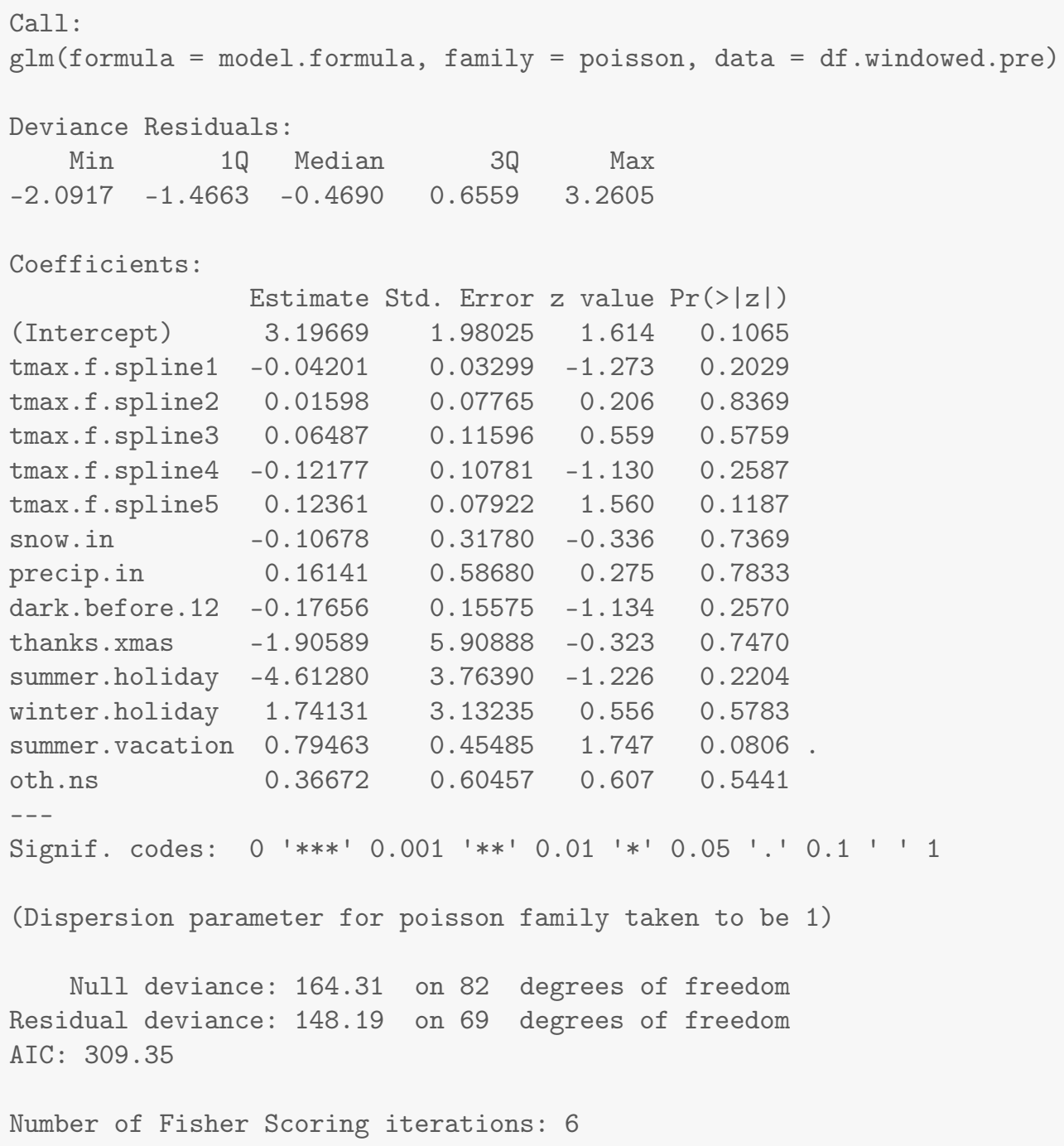




\section{Results for disorderly.conduct}

\subsection{Summary Values for disorderly.conduct}

Table 27.1: Descriptives for Outcome Before Ferguson Protests Begin

\begin{tabular}{lcccccccc}
\hline \hline Statistic & $\mathrm{N}$ & Mean & St. Dev. & Min & Pctl(25) & Median & Pctl(75) & Max \\
\hline disorderly.conduct & 83 & 20.783 & 6.002 & 9 & 17 & 21 & 24 & 42 \\
\hline
\end{tabular}

Table 27.2: Descriptives for Outcome After Ferguson Protests Begin

\begin{tabular}{lcccccccc}
\hline \hline Statistic & $\mathrm{N}$ & Mean & St. Dev. & Min & Pctl(25) & Median & Pctl(75) & Max \\
\hline disorderly.conduct & 290 & 7.597 & 11.815 & 0 & 4 & 6 & 9 & 189 \\
\hline
\end{tabular}




\subsection{Four Models for disorderly.conduct}

Table 27.3: Four Models that Differ on the Specification of Adjustment and Intervention Variables

\begin{tabular}{|c|c|c|c|c|}
\hline & \multicolumn{4}{|c|}{ Outcome: Count Per Week } \\
\hline & (1) & $(2)$ & $(3)$ & $(4)$ \\
\hline After Ferguson Protests Begin (week of $8 / 11 / 14$ onward) & -6.20 & & -4.32 & -5.06 \\
\hline After Gray Protests Begin (week of 4/20/15 onward) & -6.58 & & -11.88 & -9.41 \\
\hline Unrest and National Guard (4/27/15 - 5/3/2015) & 181.00 & & 181.36 & 180.43 \\
\hline After Batts Fired (week of 7/13/15 onward) & 2.57 & & 4.84 & 3.74 \\
\hline After Davis Confirmed (week of 10/19/15 onward) & -1.34 & & 0.32 & -0.73 \\
\hline After Davis First Year (week of 10/17/16 onward) & -3.99 & & -3.89 & -3.91 \\
\hline After De Sousa Begins (week of 1/19/18 onward) & -1.12 & & -1.40 & -1.28 \\
\hline After Tuggle Begins (week of 5/14/18 onward) & -0.54 & & 0.08 & 0.29 \\
\hline After Harrison Begins (week of 2/11/19 onward) & 0.43 & & -0.29 & -0.30 \\
\hline Average Maximum Temperature to 50 Degrees & & 0.21 & & 0.17 \\
\hline Plus Degrees in the 50s & & -0.51 & & -0.15 \\
\hline Plus Degrees in the 60s & & 0.65 & & 0.04 \\
\hline Plus Degrees in the 70s & & -0.02 & & -0.06 \\
\hline Plus Degrees Greater Than 80 & & -0.71 & & -0.28 \\
\hline Snowfall (inches) & & -2.29 & & -1.62 \\
\hline Precipitation (inches) & & -5.22 & & -3.87 \\
\hline Darkness Before Midnight (hours) & & -1.64 & & -0.89 \\
\hline Thanksgiving/Christmas (proportion of week) & & -45.37 & & -11.98 \\
\hline Winter Holiday (proportion of week) & & -20.35 & & 5.05 \\
\hline Other Out-of-School Days (proportion of week) & & 3.72 & & 1.13 \\
\hline Summer Vacation (proportion of week) & & -3.66 & & 0.53 \\
\hline Summer Holiday (proportion of week) & & -4.46 & & 4.78 \\
\hline Observations & 373 & 83 & 373 & 373 \\
\hline $\mathrm{R}^{2}$ & 0.88 & 0.46 & 0.87 & 0.91 \\
\hline
\end{tabular}


27.3 Least Squares Updated Model (2) for disorderly.conduct disorderly.conduct

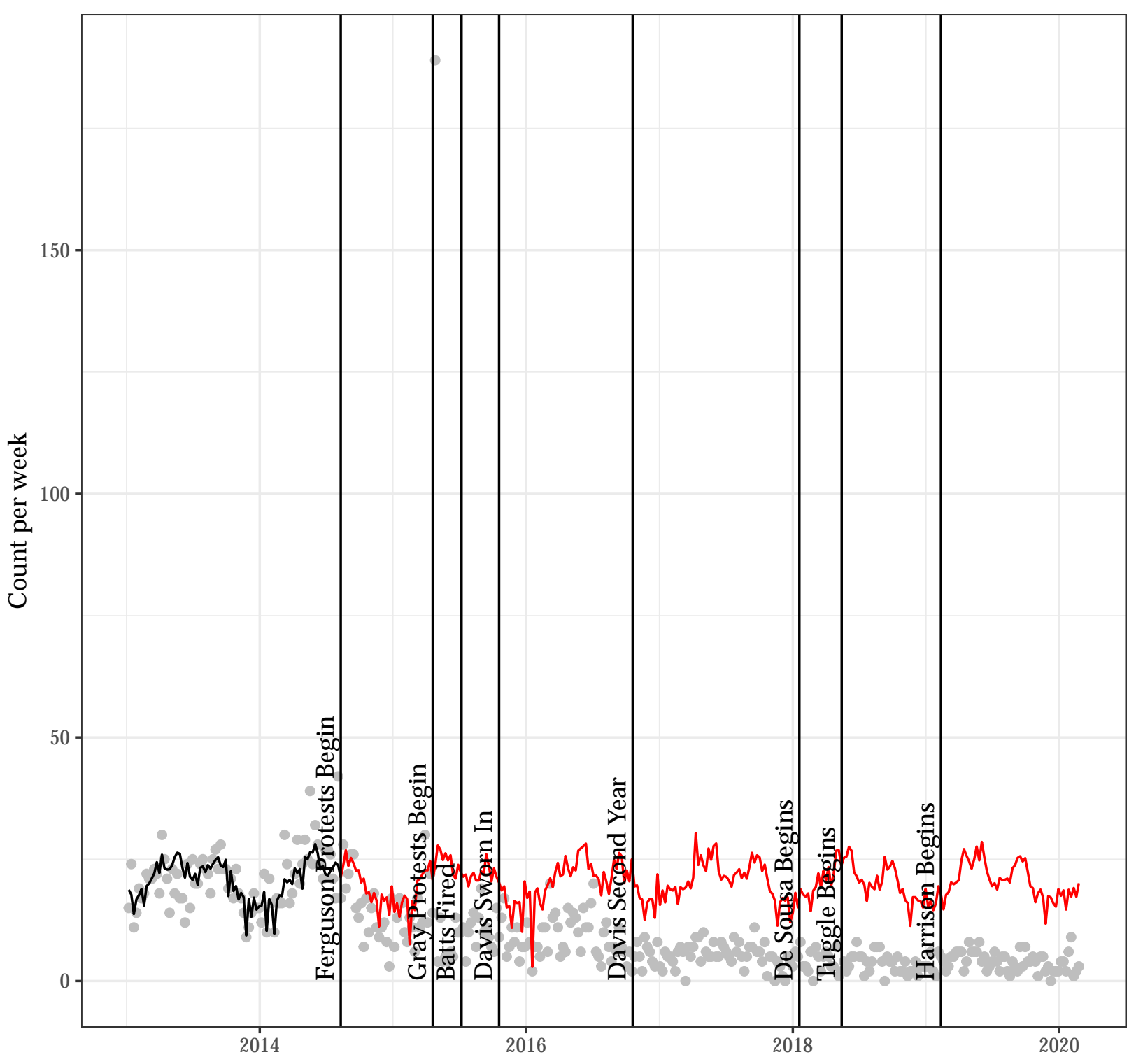




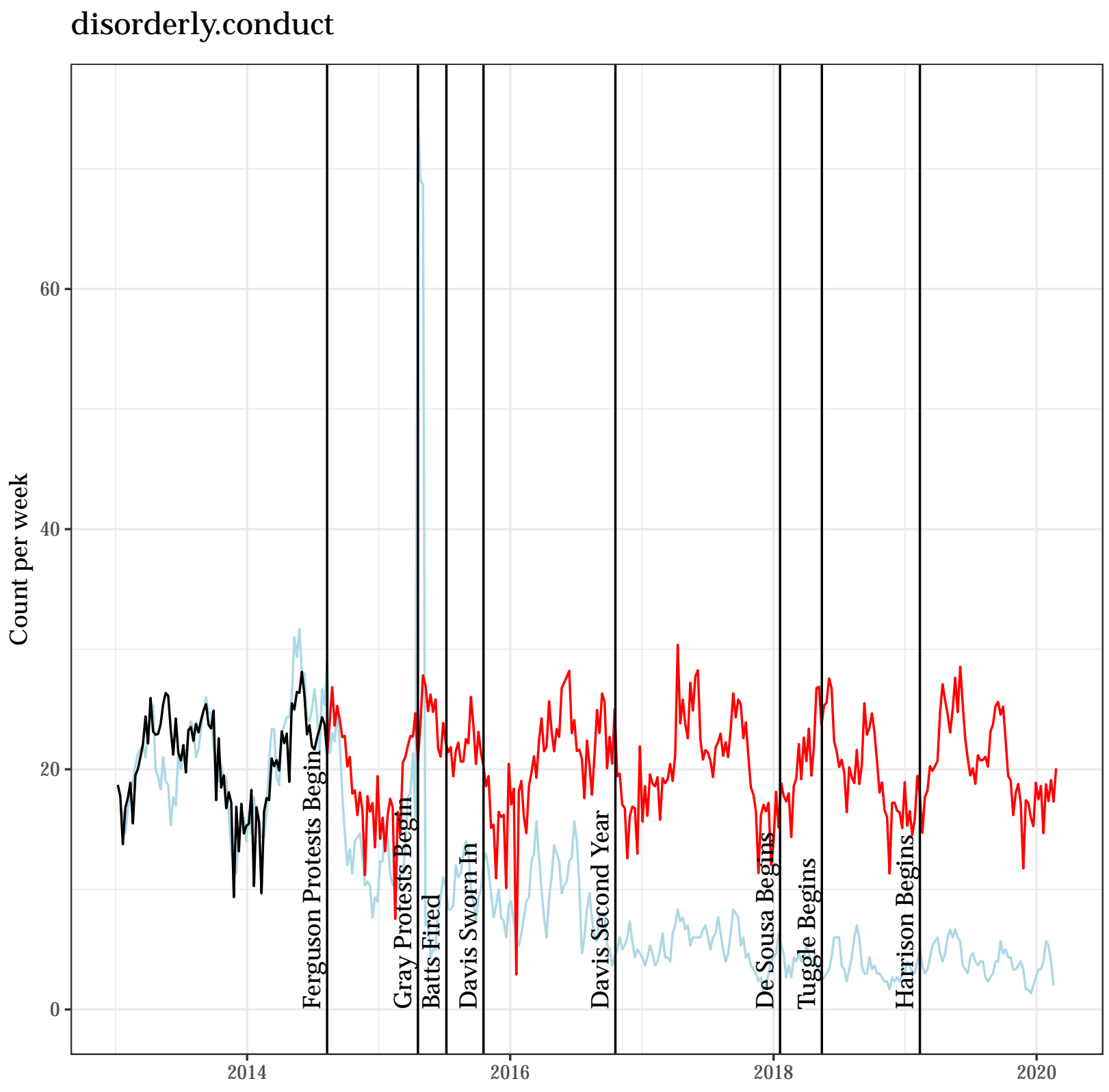


Autocorrelation Function for the Observed Outcome for Updated Model (2)

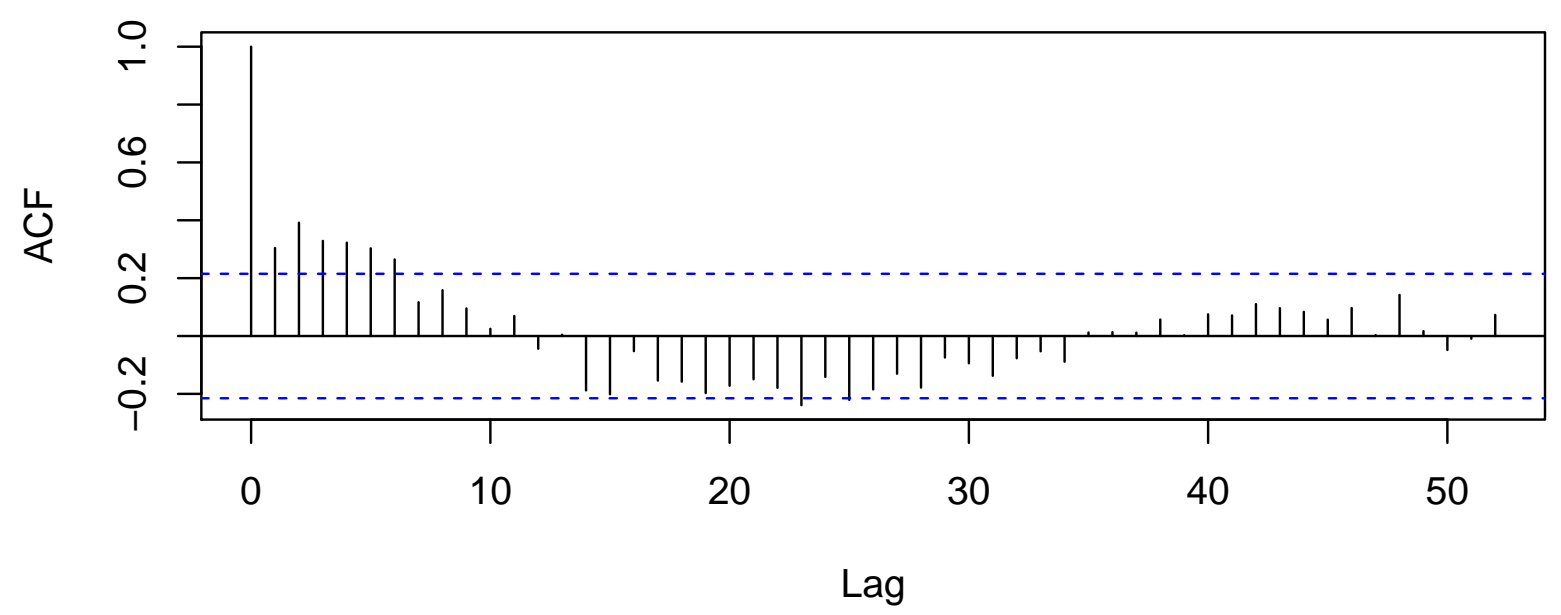

Autocorrelation Function for the Residuals from Updated Model (2)

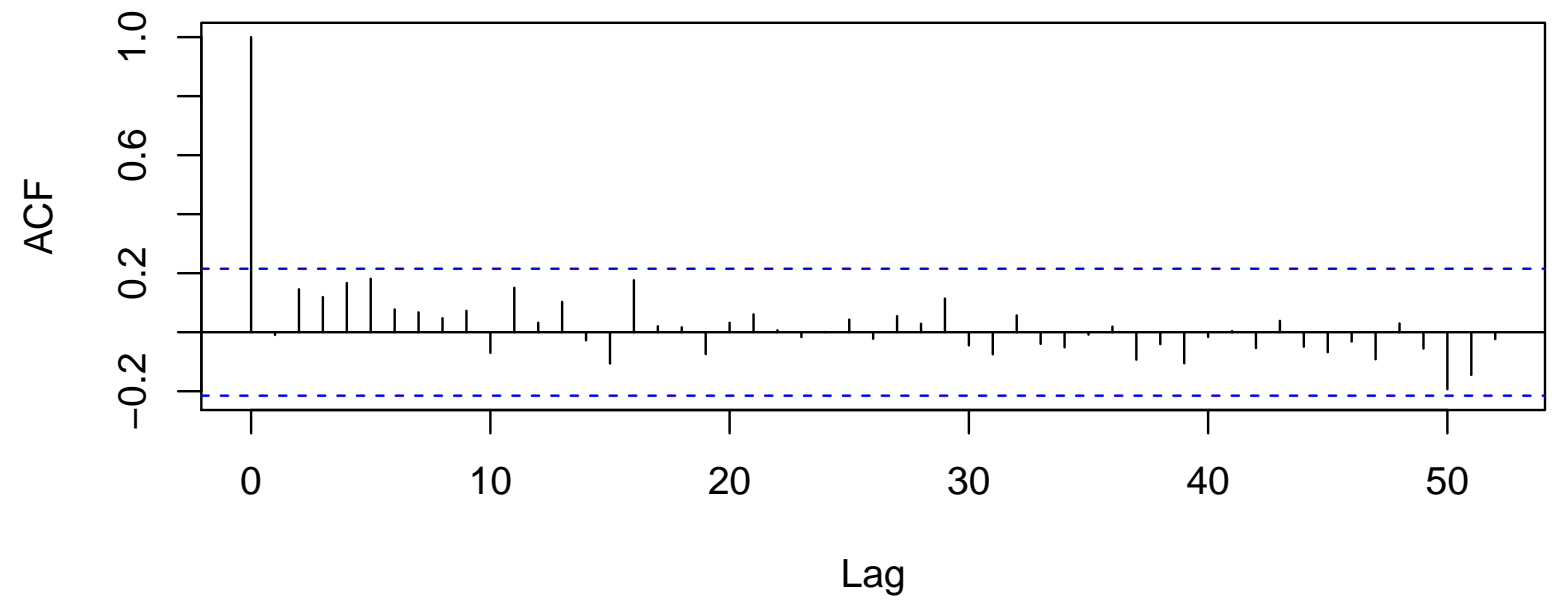




\subsection{A Poisson Regression Model as an Alternative to Model (2) for disorderly.conduct}

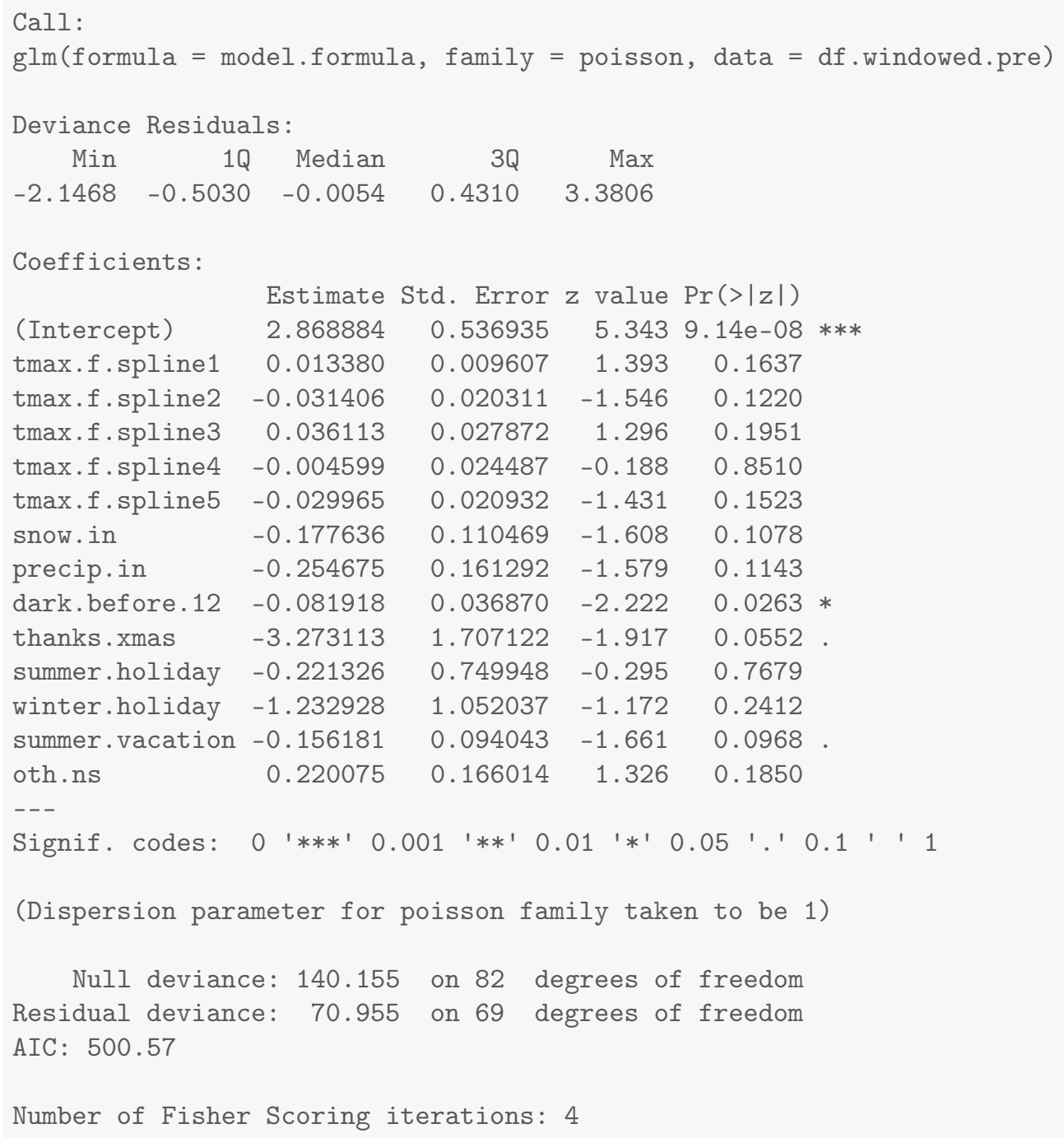




\section{Results for prostitution}

\subsection{Summary Values for prostitution}

Table 28.1: Descriptives for Outcome Before Ferguson Protests Begin

\begin{tabular}{lcccccccc}
\hline \hline Statistic & $\mathrm{N}$ & Mean & St. Dev. & Min & Pctl(25) & Median & Pctl(75) & Max \\
\hline prostitution & 83 & 12.084 & 5.912 & 1 & 7.5 & 11 & 17 & 27 \\
\hline
\end{tabular}

Table 28.2: Descriptives for Outcome After Ferguson Protests Begin

\begin{tabular}{lcccccccc}
\hline \hline Statistic & $\mathrm{N}$ & Mean & St. Dev. & Min & Pctl(25) & Median & Pctl(75) & Max \\
\hline prostitution & 290 & 5.172 & 4.413 & 0 & 2 & 4 & 8 & 20 \\
\hline
\end{tabular}




\subsection{Four Models for prostitution}

Table 28.3: Four Models that Differ on the Specification of Adjustment and Intervention Variables

\begin{tabular}{|c|c|c|c|c|}
\hline & \multicolumn{4}{|c|}{ Outcome: Count Per Week } \\
\hline & $(1)$ & (2) & $(3)$ & (4) \\
\hline After Ferguson Protests Begin (week of $8 / 11 / 14$ onward) & -4.53 & & -4.02 & -3.97 \\
\hline After Gray Protests Begin (week of $4 / 20 / 15$ onward) & -3.46 & & -4.78 & -5.08 \\
\hline Unrest and National Guard $(4 / 27 / 15-5 / 3 / 2015)$ & -3.09 & & -4.41 & -3.44 \\
\hline After Batts Fired (week of $7 / 13 / 15$ onward) & -0.66 & & -1.19 & -1.13 \\
\hline After Davis Confirmed (week of 10/19/15 onward) & 1.30 & & 2.79 & 2.43 \\
\hline After Davis First Year (week of $10 / 17 / 16$ onward) & -1.19 & & -1.23 & -1.10 \\
\hline After De Sousa Begins (week of 1/19/18 onward) & 7.52 & & 8.09 & 8.21 \\
\hline After Tuggle Begins (week of 5/14/18 onward) & -5.40 & & -5.43 & -5.77 \\
\hline After Harrison Begins (week of 2/11/19 onward) & -1.01 & & -1.41 & -1.18 \\
\hline Average Maximum Temperature to 50 Degrees & & -0.23 & & -0.01 \\
\hline Plus Degrees in the $50 \mathrm{~s}$ & & 0.37 & & 0.26 \\
\hline Plus Degrees in the $60 \mathrm{~s}$ & & 0.01 & & -0.16 \\
\hline Plus Degrees in the 70s & & -0.18 & & -0.01 \\
\hline Plus Degrees Greater Than 80 & & -0.09 & & -0.14 \\
\hline Snowfall (inches) & & -3.07 & & -1.58 \\
\hline Precipitation (inches) & & -5.02 & & -2.74 \\
\hline Darkness Before Midnight (hours) & & -0.17 & & 0.31 \\
\hline Thanksgiving/Christmas (proportion of week) & & -19.30 & & -13.51 \\
\hline Winter Holiday (proportion of week) & & 2.23 & & 6.58 \\
\hline Other Out-of-School Days (proportion of week) & & -5.97 & & -2.47 \\
\hline Summer Vacation (proportion of week) & & 1.58 & & 0.04 \\
\hline Summer Holiday (proportion of week) & & -30.39 & & -13.11 \\
\hline Observations & 373 & 83 & 373 & 373 \\
\hline $\mathrm{R}^{2}$ & 0.36 & 0.21 & 0.38 & 0.45 \\
\hline
\end{tabular}


28.3 Least Squares Updated Model (2) for prostitution prostitution

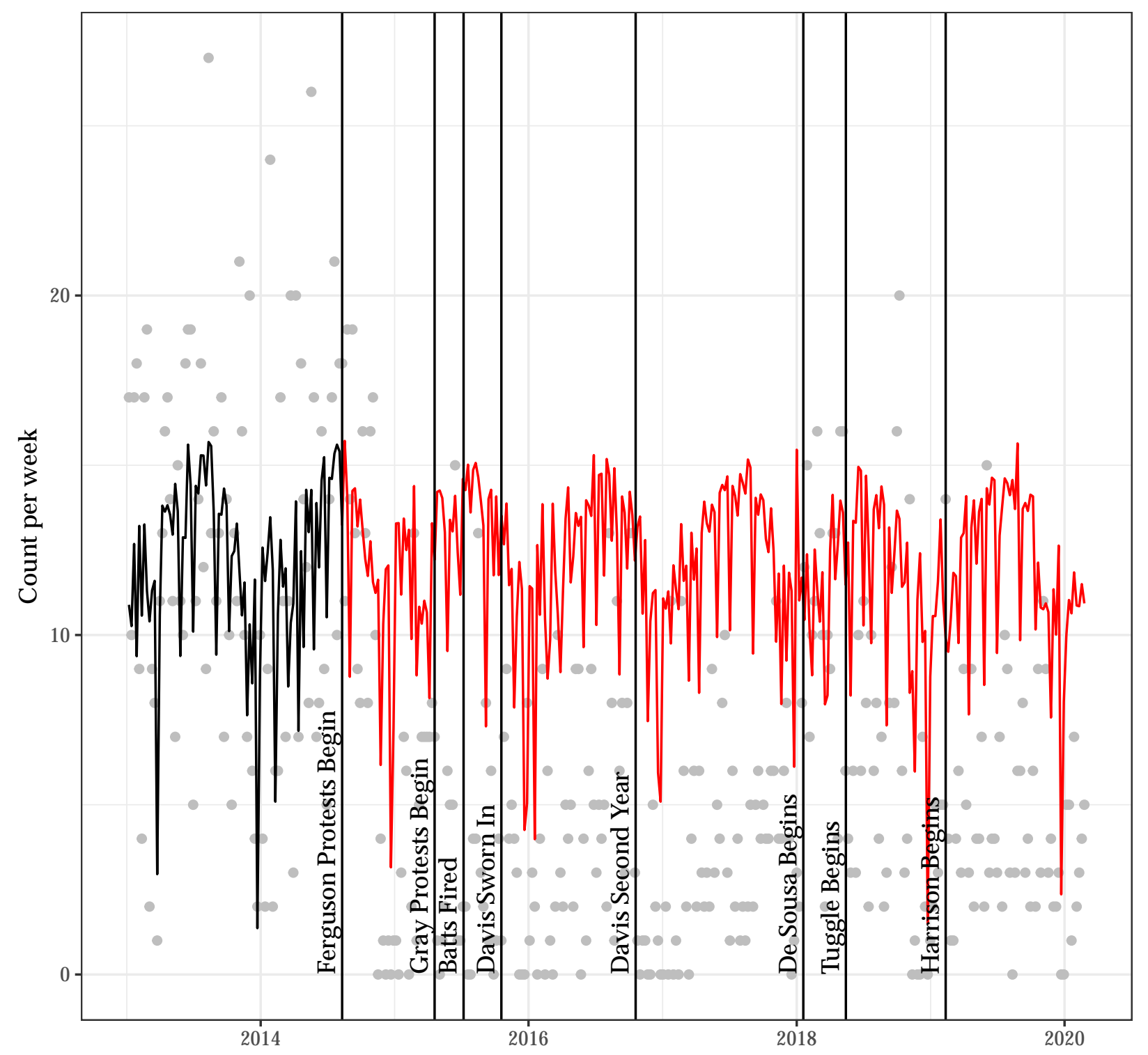




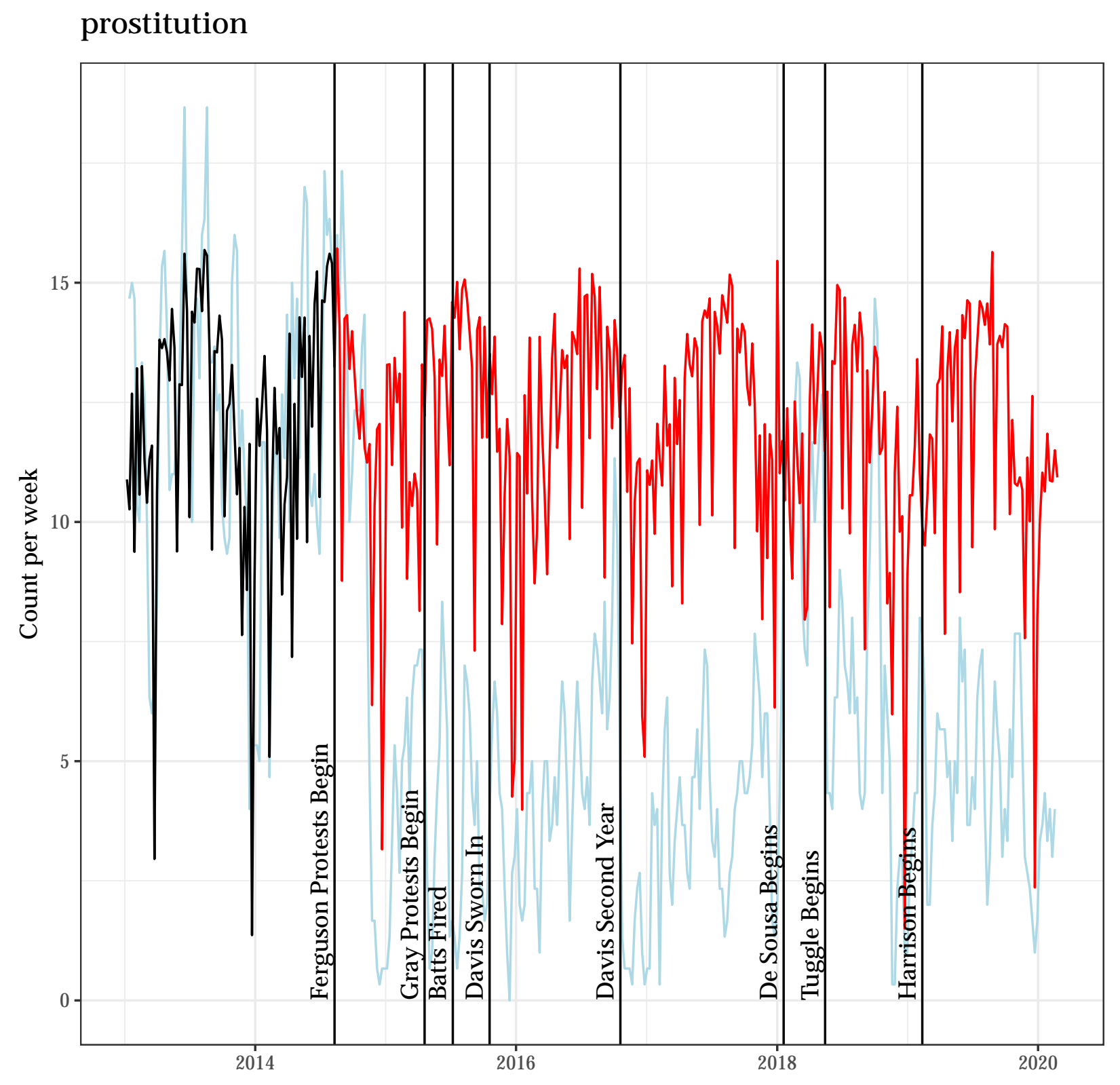


Autocorrelation Function for the Observed Outcome for Updated Model (2)

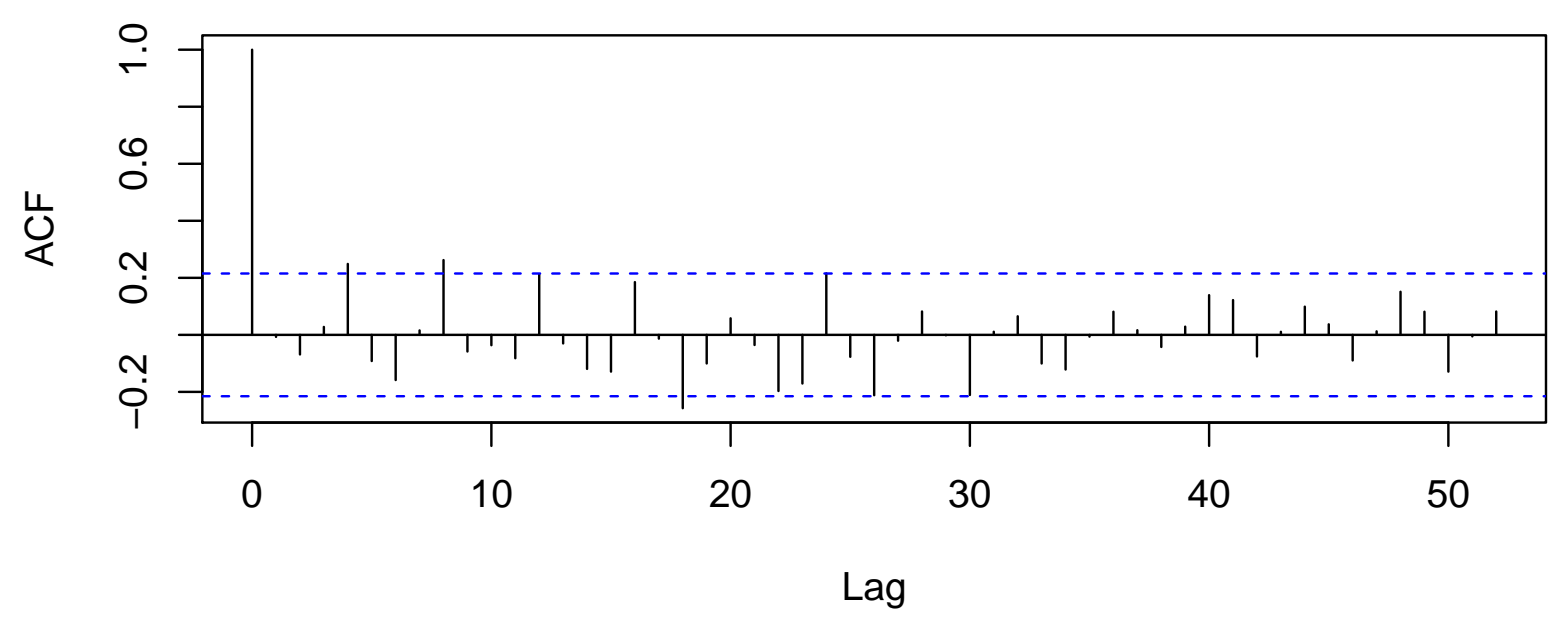

Autocorrelation Function for the Residuals from Updated Model (2)

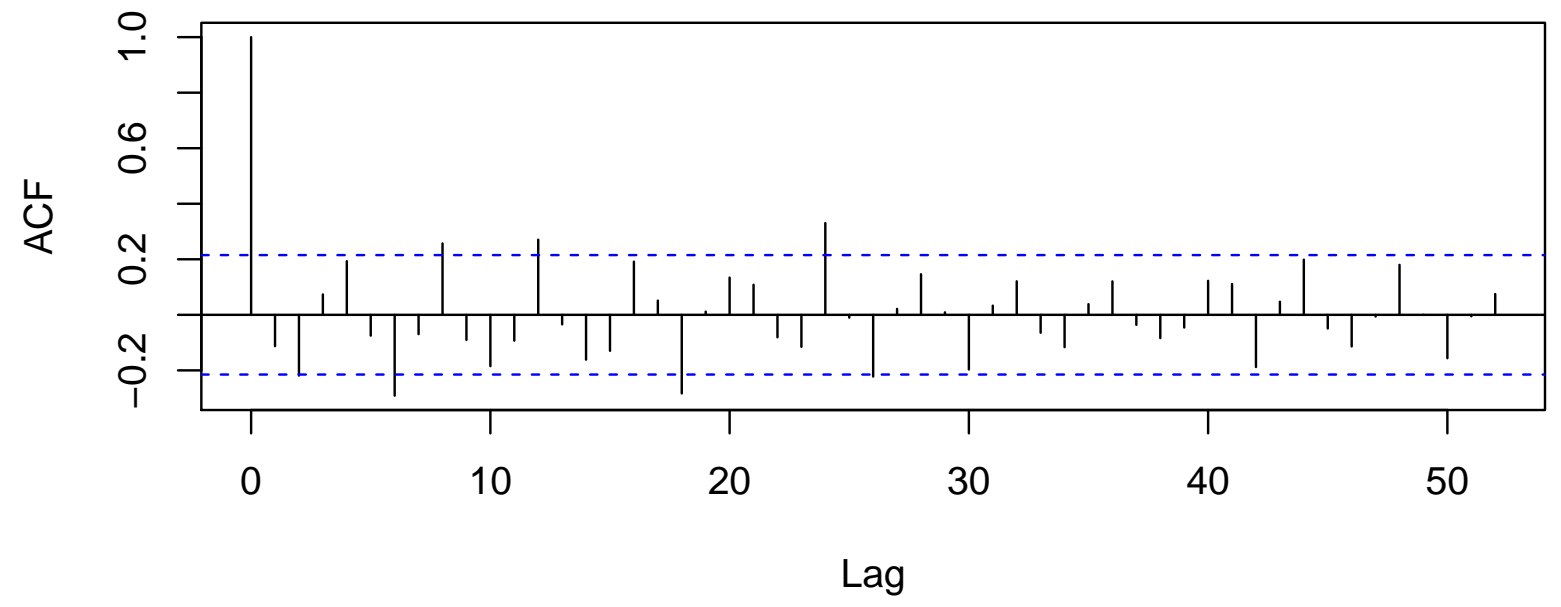




\subsection{A Poisson Regression Model as an Alternative to Model (2) for prostitution}

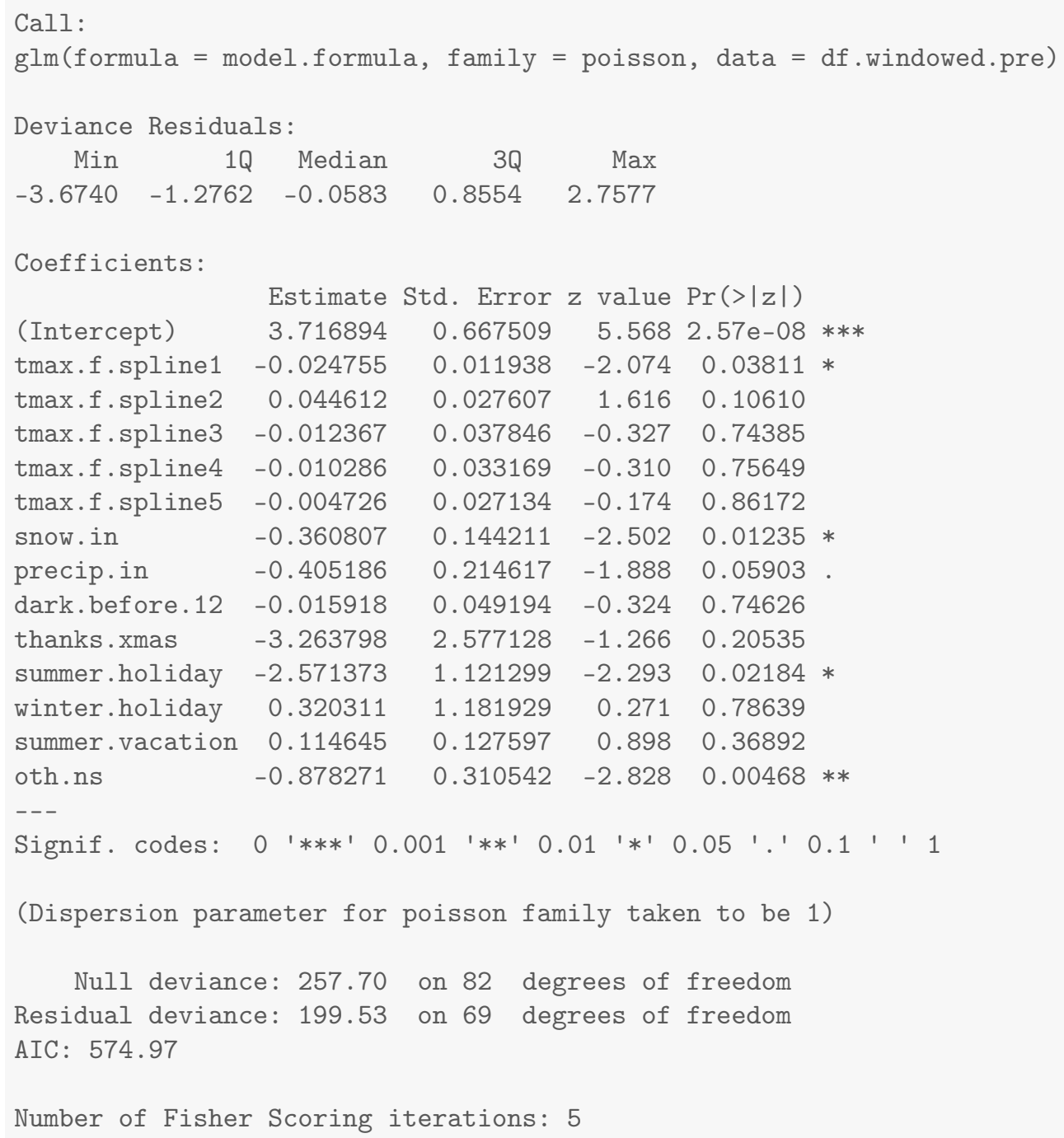




\section{Results for only.temp.detained}

\subsection{Summary Values for only.temp.detained}

Table 29.1: Descriptives for Outcome Before Ferguson Protests Begin

\begin{tabular}{lcccccccc}
\hline \hline Statistic & $\mathrm{N}$ & Mean & St. Dev. & Min & $\operatorname{Pctl}(25)$ & Median & $\operatorname{Pctl}(75)$ & Max \\
\hline only.temp.detained & 83 & 9.482 & 3.497 & 2 & 7 & 9 & 12 & 20 \\
\hline
\end{tabular}

Table 29.2: Descriptives for Outcome After Ferguson Protests Begin

\begin{tabular}{lcccccccc}
\hline \hline Statistic & $\mathrm{N}$ & Mean & St. Dev. & Min & Pctl(25) & Median & Pctl(75) & Max \\
\hline only.temp.detained & 290 & 7.434 & 3.400 & 1 & 5 & 7 & 10 & 20 \\
\hline
\end{tabular}




\subsection{Four Models for only.temp.detained}

Table 29.3: Four Models that Differ on the Specification of Adjustment and Intervention Variables

\begin{tabular}{|c|c|c|c|c|}
\hline & \multicolumn{4}{|c|}{ Outcome: Count Per Week } \\
\hline & (1) & $(2)$ & $(3)$ & $(4)$ \\
\hline After Ferguson Protests Begin (week of $8 / 11 / 14$ onward) & -1.34 & & -0.71 & -0.81 \\
\hline After Gray Protests Begin (week of 4/20/15 onward) & -1.32 & & -2.67 & -2.45 \\
\hline Unrest and National Guard $(4 / 27 / 15-5 / 3 / 2015)$ & -3.82 & & -4.51 & -3.90 \\
\hline After Batts Fired (week of 7/13/15 onward) & 1.18 & & -0.57 & 1.00 \\
\hline After Davis Confirmed (week of 10/19/15 onward) & 0.46 & & 2.04 & 1.14 \\
\hline After Davis First Year (week of 10/17/16 onward) & -1.34 & & -0.77 & -1.15 \\
\hline After De Sousa Begins (week of 1/19/18 onward) & 1.82 & & 1.76 & 1.70 \\
\hline After Tuggle Begins (week of 5/14/18 onward) & -2.01 & & -2.84 & -2.10 \\
\hline After Harrison Begins (week of 2/11/19 onward) & -0.56 & & -0.20 & -0.57 \\
\hline Average Maximum Temperature to 50 Degrees & & 0.15 & & 0.07 \\
\hline Plus Degrees in the 50s & & -0.03 & & -0.04 \\
\hline Plus Degrees in the 60s & & -0.14 & & -0.06 \\
\hline Plus Degrees in the 70s & & -0.09 & & -0.03 \\
\hline Plus Degrees Greater Than 80 & & 0.79 & & 0.14 \\
\hline Snowfall (inches) & & -1.03 & & -0.76 \\
\hline Precipitation (inches) & & 1.00 & & -0.07 \\
\hline Darkness Before Midnight (hours) & & -0.12 & & -0.20 \\
\hline Thanksgiving/Christmas (proportion of week) & & 1.76 & & -10.03 \\
\hline Winter Holiday (proportion of week) & & -9.33 & & -5.91 \\
\hline Other Out-of-School Days (proportion of week) & & -2.94 & & -2.19 \\
\hline Summer Vacation (proportion of week) & & -2.55 & & 0.63 \\
\hline Summer Holiday (proportion of week) & & -27.55 & & -4.63 \\
\hline Observations & 373 & 83 & 373 & 373 \\
\hline $\mathrm{R}^{2}$ & 0.11 & 0.32 & 0.16 & 0.19 \\
\hline
\end{tabular}




\subsection{Least Squares Updated Model (2) for only.temp.detained}

only.temp.detained

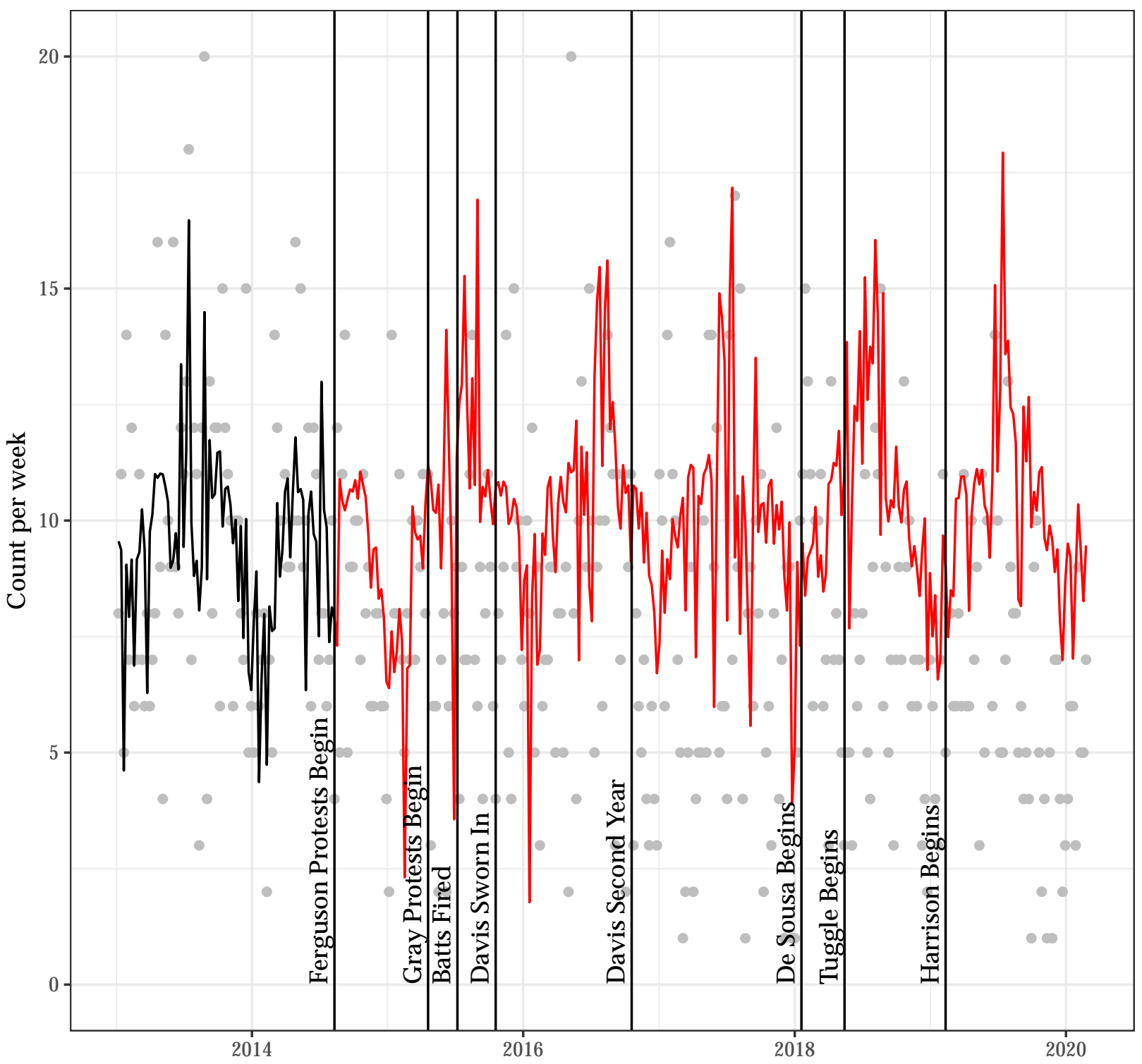




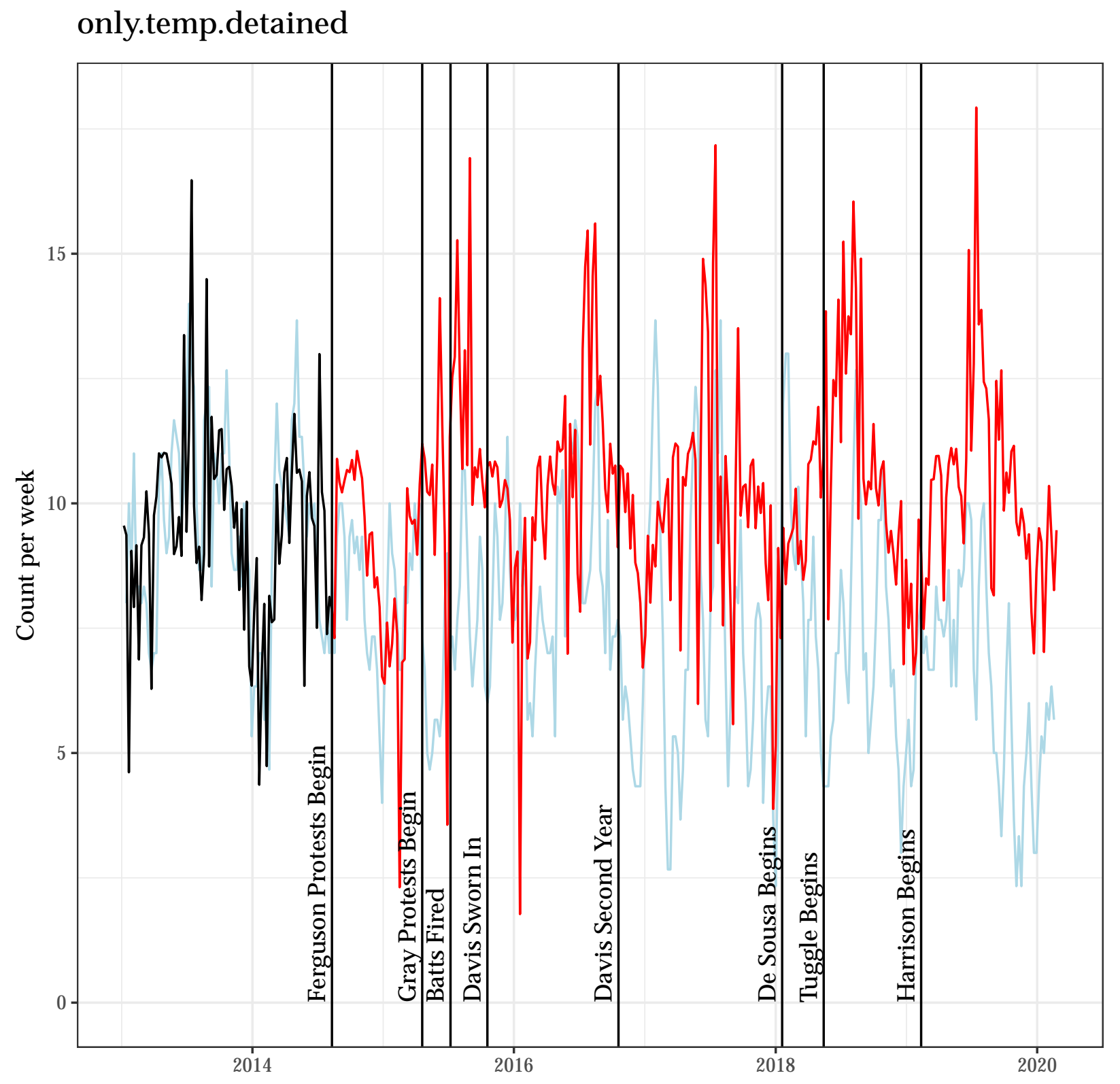


Autocorrelation Function for the Observed Outcome for Updated Model (2)

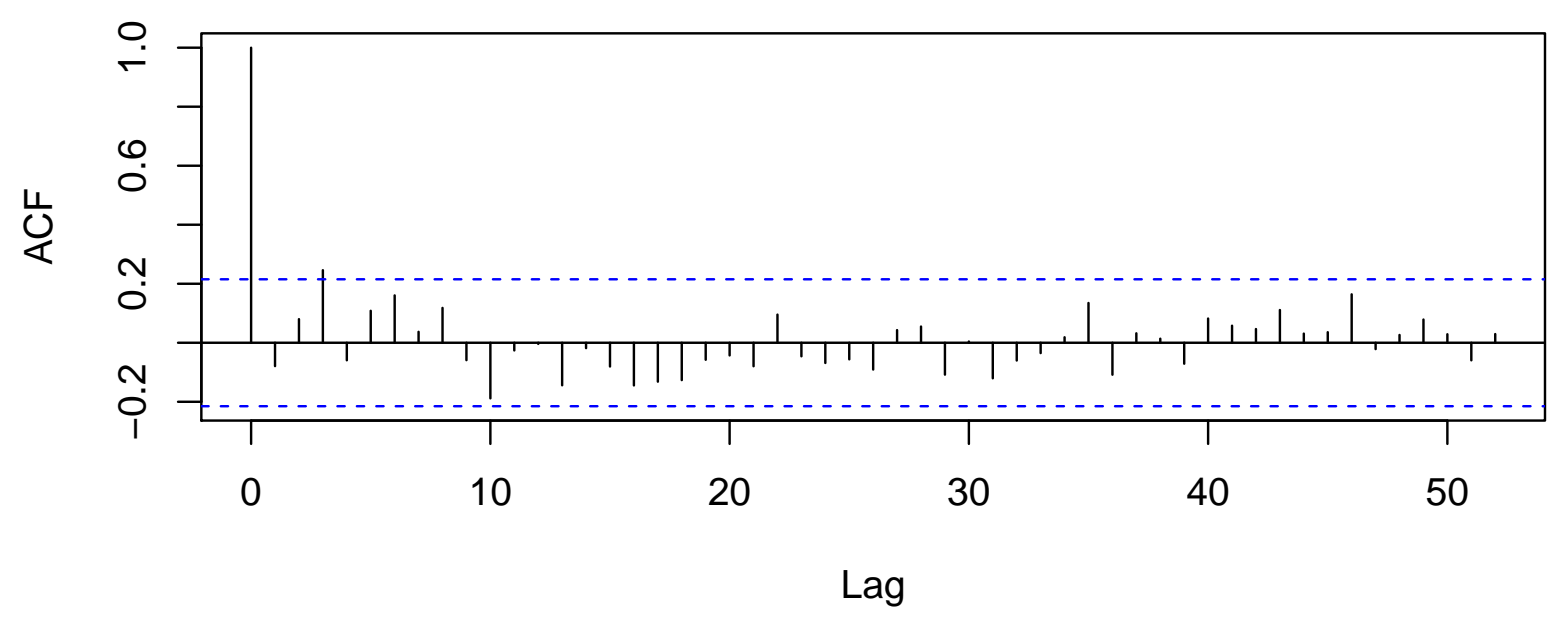

Autocorrelation Function for the Residuals from Updated Model (2)

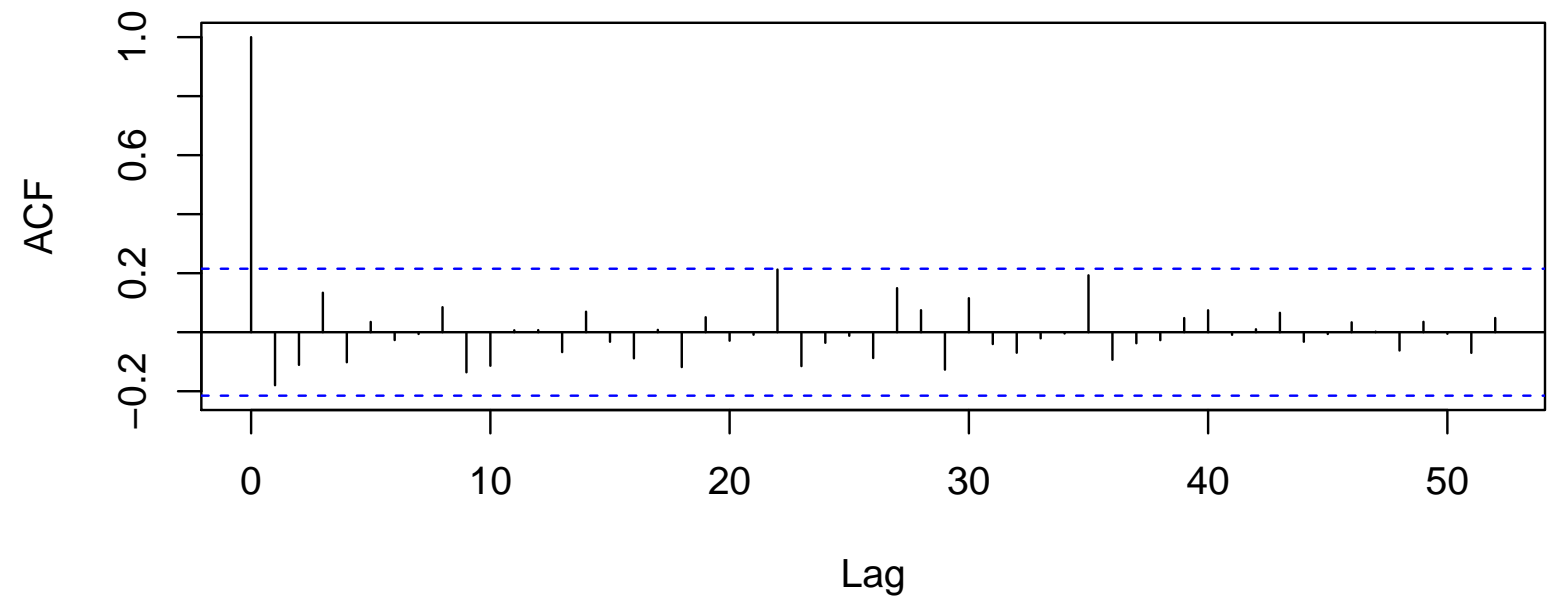




\subsection{A Poisson Regression Model as an Alternative to Model (2) for only.temp.detained}

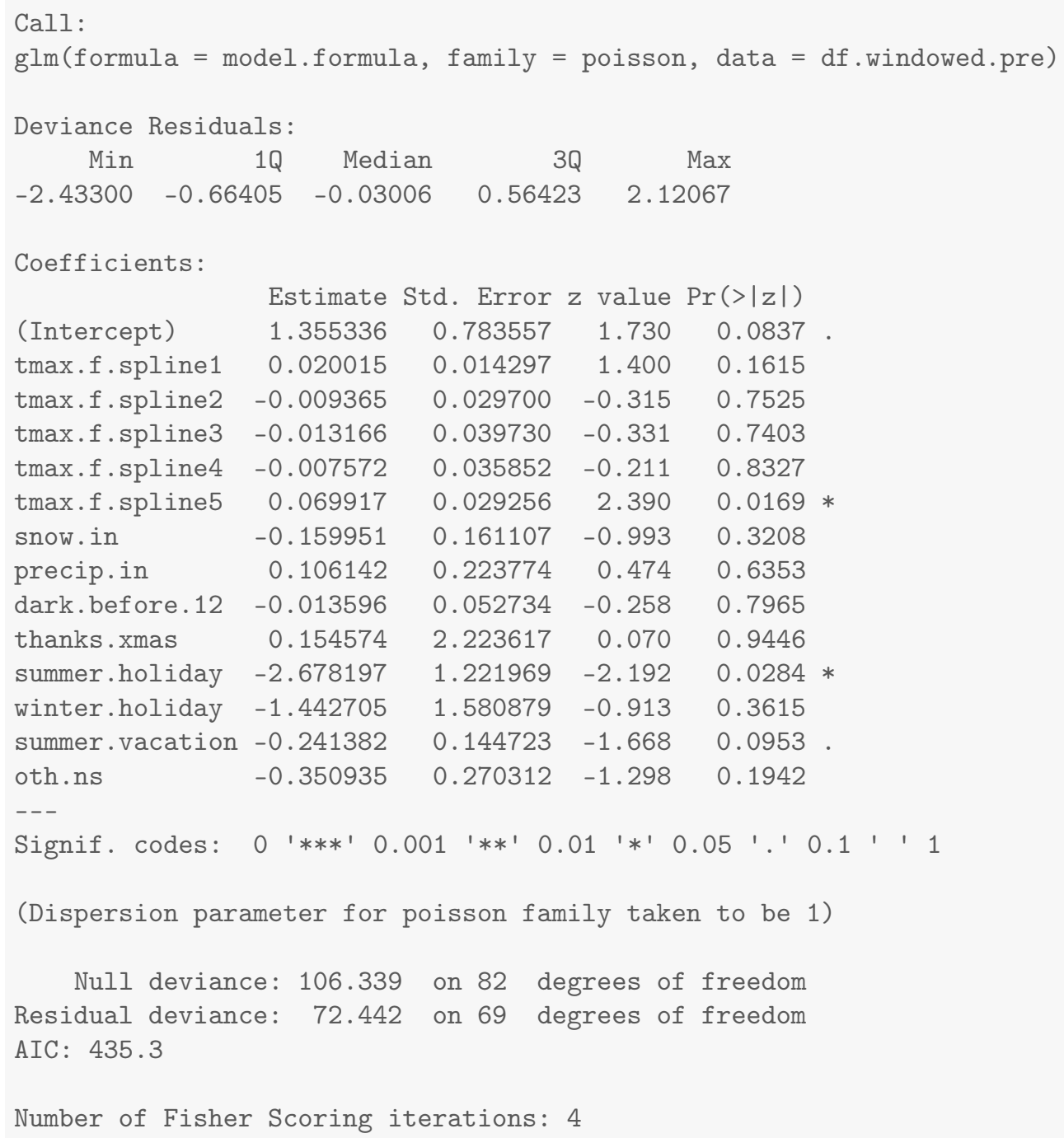




\section{Results for unknown}

\subsection{Summary Values for unknown}

Table 30.1: Descriptives for Outcome Before Ferguson Protests Begin

\begin{tabular}{lcccccccc}
\hline \hline Statistic & $\mathrm{N}$ & Mean & St. Dev. & Min & Pctl(25) & Median & Pctl(75) & Max \\
\hline unknown & 83 & 48.783 & 12.519 & 26 & 40.5 & 50 & 57 & 94 \\
\hline
\end{tabular}

Table 30.2: Descriptives for Outcome After Ferguson Protests Begin

\begin{tabular}{lcccccccc}
\hline \hline Statistic & $\mathrm{N}$ & Mean & St. Dev. & Min & Pctl(25) & Median & Pctl(75) & Max \\
\hline unknown & 290 & 22.379 & 12.473 & 4 & 14 & 19 & 28.8 & 121 \\
\hline
\end{tabular}




\subsection{Four Models for unknown}

Table 30.3: Four Models that Differ on the Specification of Adjustment and Intervention Variables

\begin{tabular}{|c|c|c|c|c|}
\hline & \multicolumn{4}{|c|}{ Outcome: Count Per Week } \\
\hline & $(1)$ & $(2)$ & (3) & (4) \\
\hline After Ferguson Protests Begin (week of $8 / 11 / 14$ onward) & -8.84 & & -11.16 & -8.87 \\
\hline After Gray Protests Begin (week of $4 / 20 / 15$ onward) & -17.22 & & -11.43 & -17.34 \\
\hline Unrest and National Guard $(4 / 27 / 15-5 / 3 / 2015)$ & 98.27 & & 98.21 & 97.38 \\
\hline After Batts Fired (week of $7 / 13 / 15$ onward) & 5.70 & & 2.63 & 5.97 \\
\hline After Davis Confirmed (week of 10/19/15 onward) & -3.27 & & -4.84 & -3.66 \\
\hline After Davis First Year (week of 10/17/16 onward) & -4.02 & & -5.43 & -3.59 \\
\hline After De Sousa Begins (week of 1/19/18 onward) & -7.64 & & -6.41 & -8.98 \\
\hline After Tuggle Begins (week of 5/14/18 onward) & 1.09 & & 0.77 & 2.52 \\
\hline After Harrison Begins (week of 2/11/19 onward) & -0.14 & & -0.20 & -0.45 \\
\hline Average Maximum Temperature to 50 Degrees & & 0.24 & & 0.01 \\
\hline Plus Degrees in the $50 \mathrm{~s}$ & & -0.29 & & 0.09 \\
\hline Plus Degrees in the 60s & & 0.06 & & -0.27 \\
\hline Plus Degrees in the 70s & & 0.52 & & 0.33 \\
\hline Plus Degrees Greater Than 80 & & -0.48 & & 0.01 \\
\hline Snowfall (inches) & & -4.10 & & 0.95 \\
\hline Precipitation (inches) & & -1.49 & & -1.12 \\
\hline Darkness Before Midnight (hours) & & 3.70 & & -0.45 \\
\hline Thanksgiving/Christmas (proportion of week) & & -77.08 & & -37.39 \\
\hline Winter Holiday (proportion of week) & & 11.80 & & 1.70 \\
\hline Other Out-of-School Days (proportion of week) & & 8.61 & & -1.27 \\
\hline Summer Vacation (proportion of week) & & -4.81 & & -4.78 \\
\hline Summer Holiday (proportion of week) & & -23.44 & & -17.68 \\
\hline Observations & 373 & 83 & 373 & 373 \\
\hline $\mathrm{R}^{2}$ & 0.72 & 0.13 & 0.69 & 0.73 \\
\hline
\end{tabular}




\subsection{Least Squares Updated Model (2) for unknown}

unknown

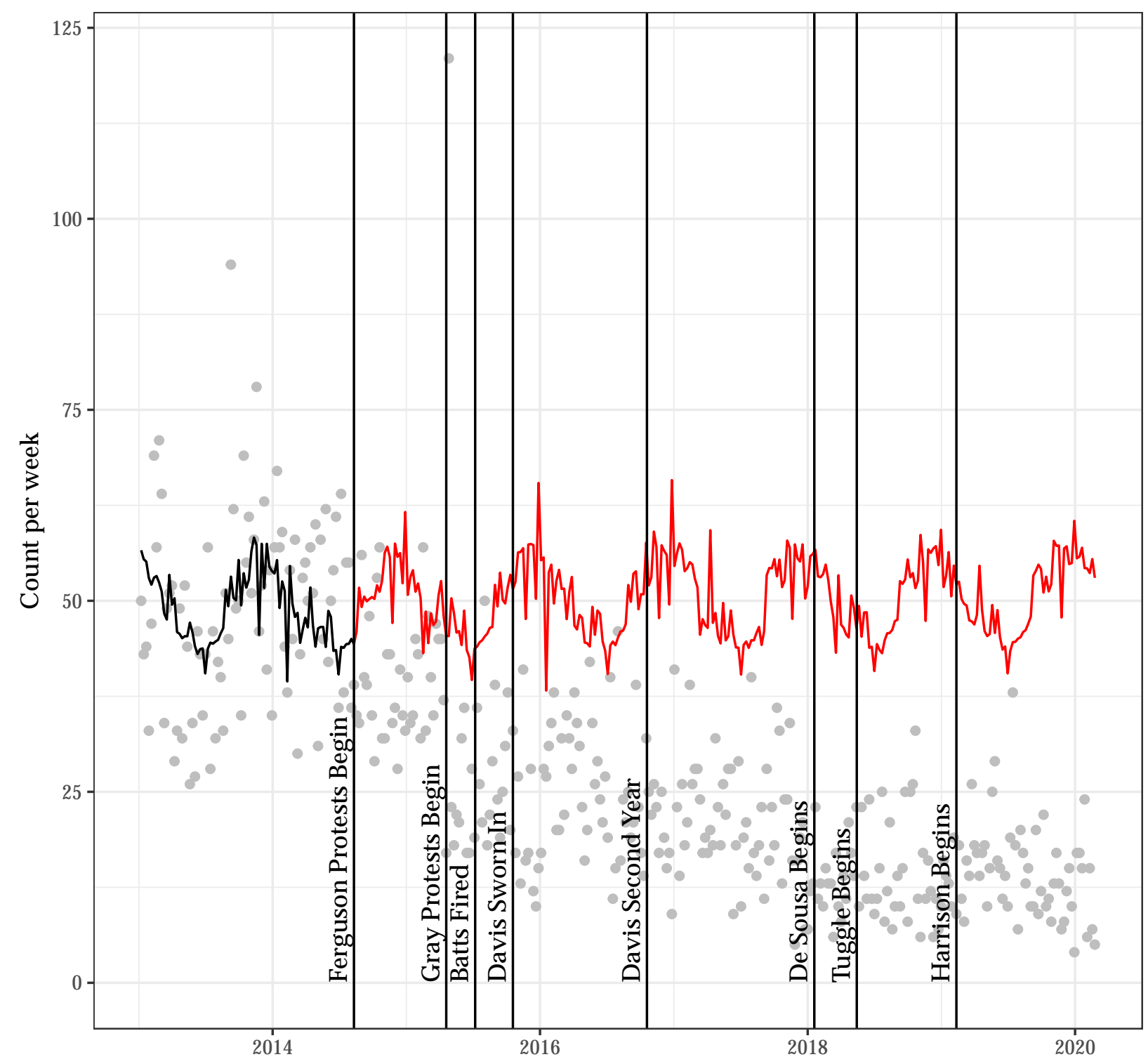




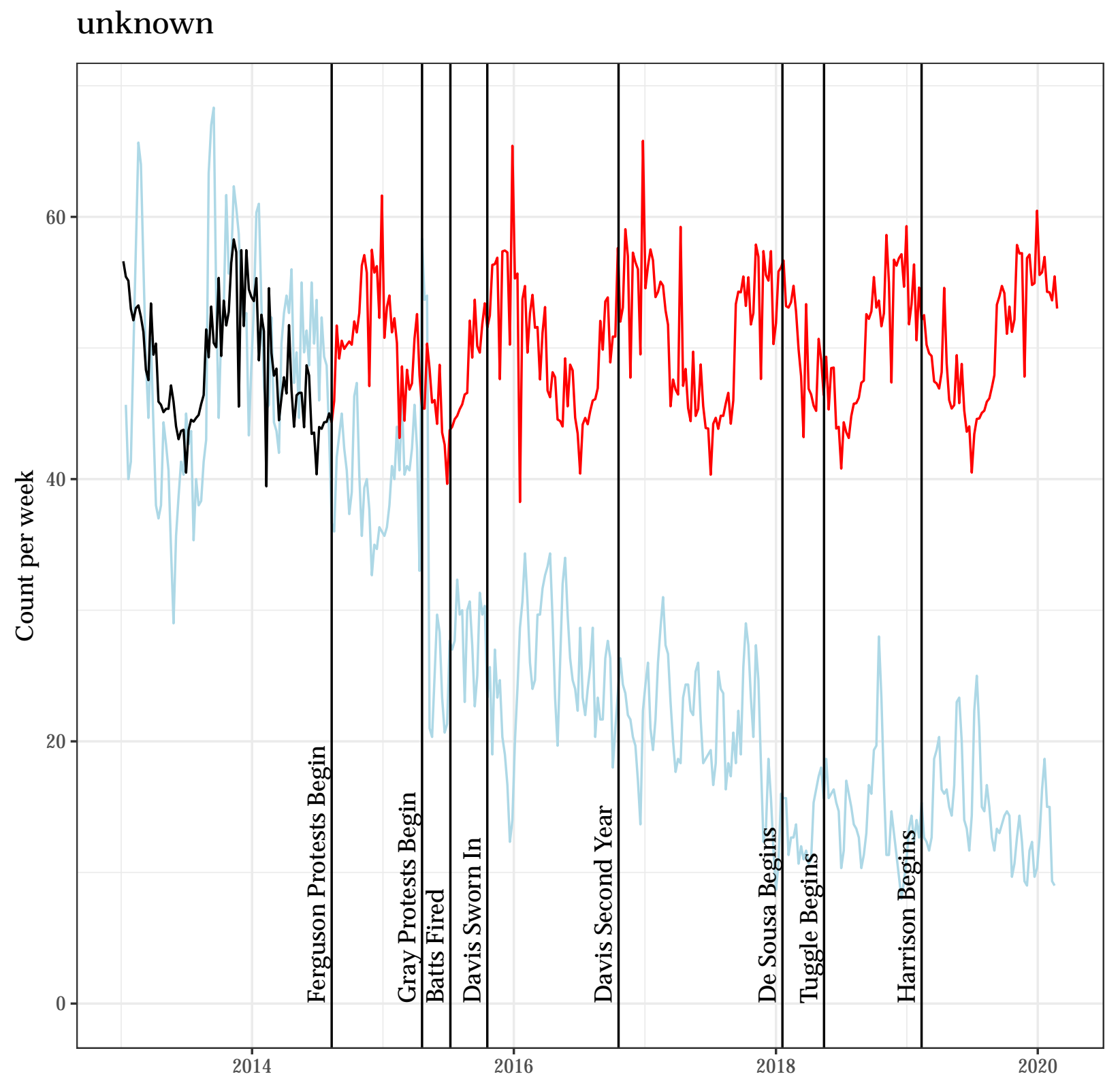


Autocorrelation Function for the Observed Outcome for Updated Model (2)

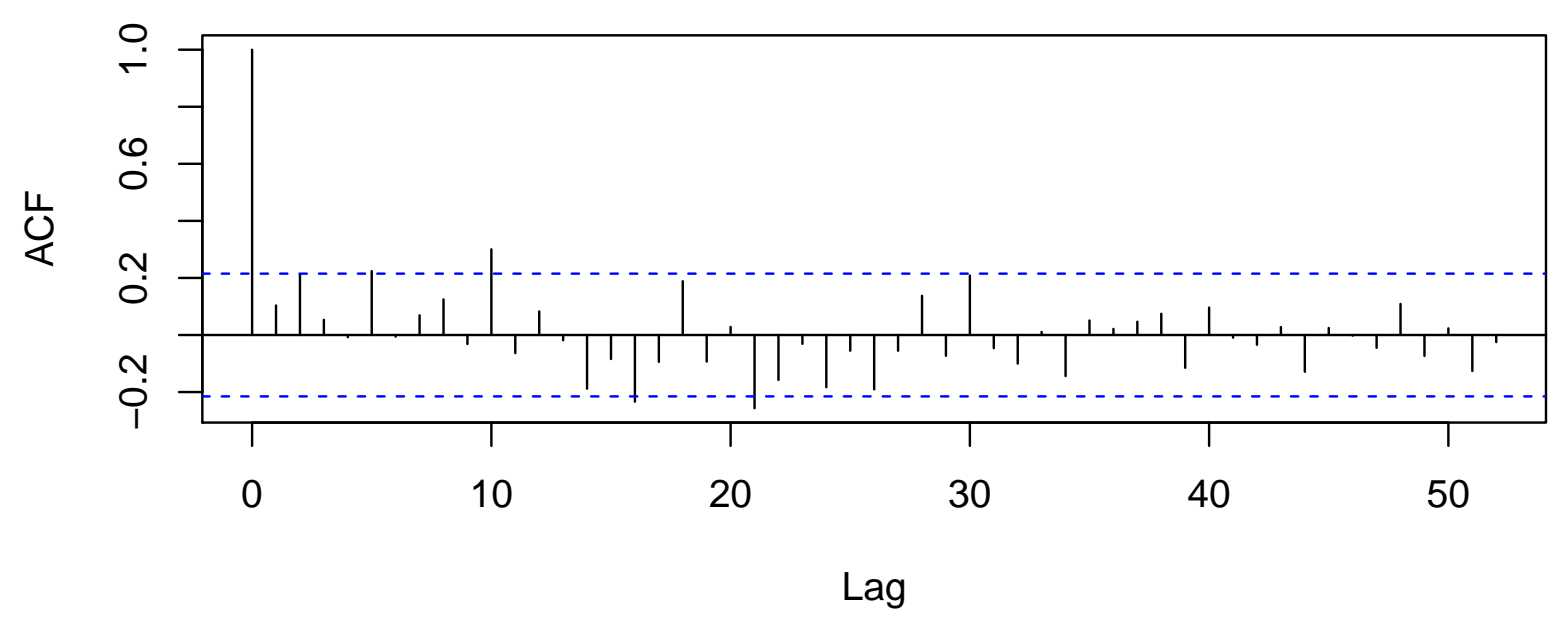

Autocorrelation Function for the Residuals from Updated Model (2)

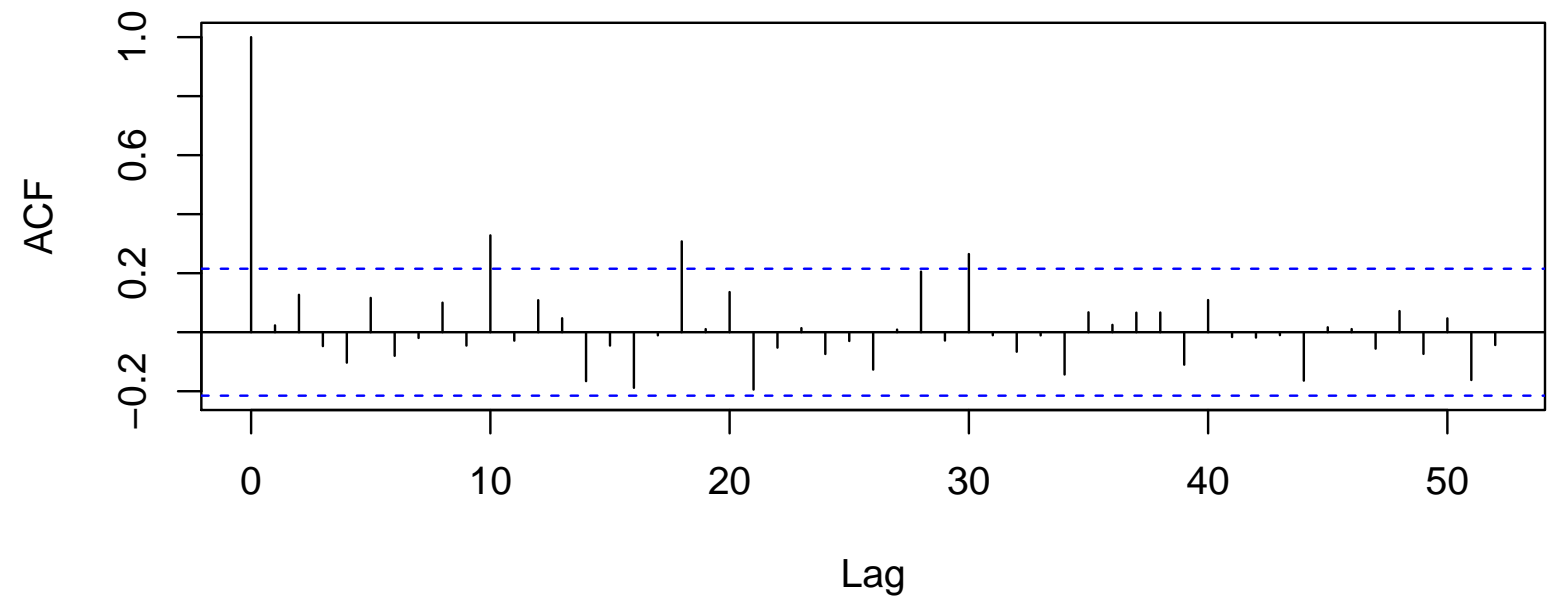




\subsection{A Poisson Regression Model as an Alternative to Model (2) for unknown}

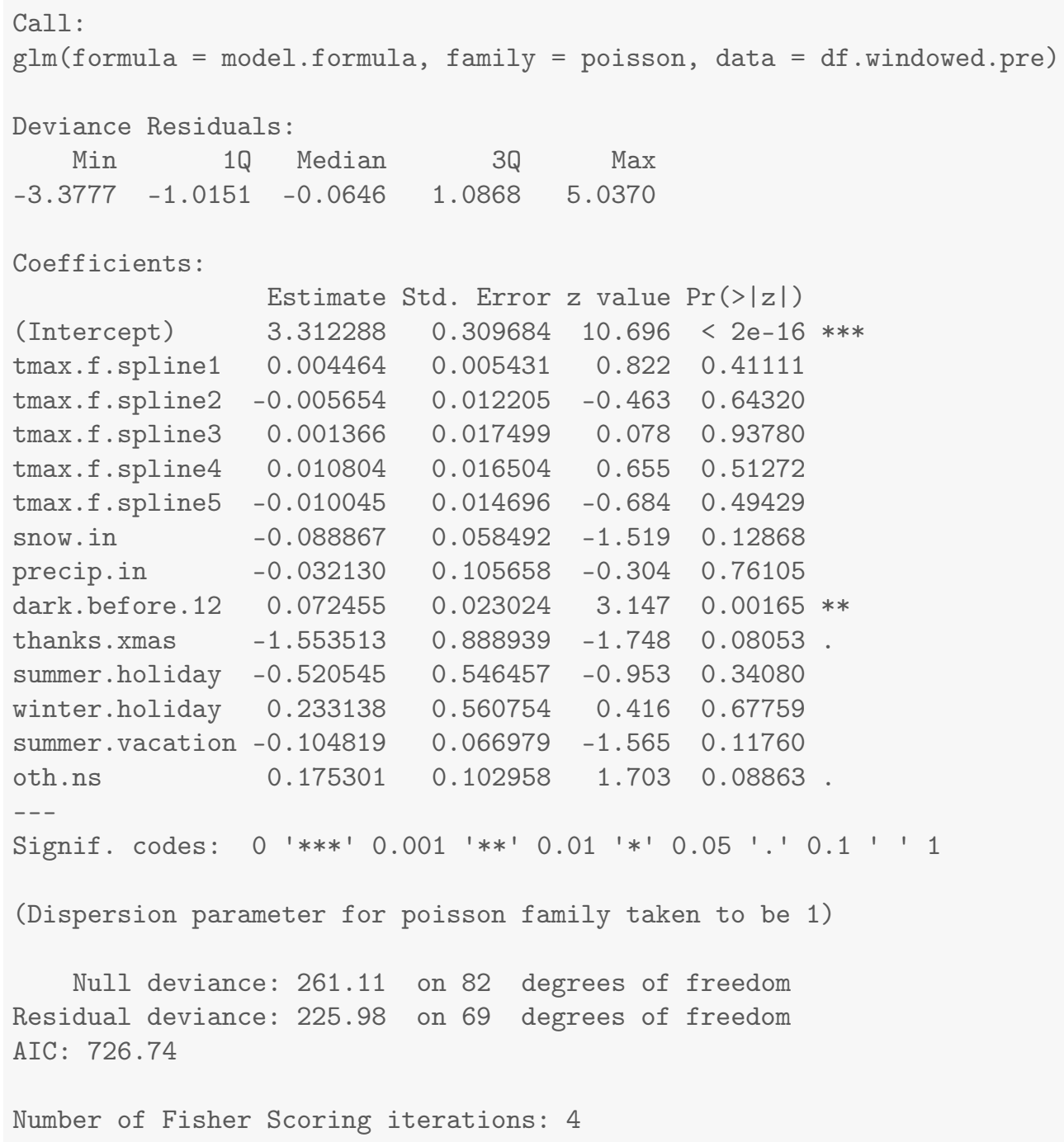




\section{Results for ungrouped}

\subsection{Summary Values for ungrouped}

Table 31.1: Descriptives for Outcome Before Ferguson Protests Begin

\begin{tabular}{lcccccccc}
\hline \hline Statistic & $\mathrm{N}$ & Mean & St. Dev. & Min & Pctl(25) & Median & Pctl(75) & Max \\
\hline ungrouped & 83 & 7.566 & 4.776 & 1 & 4 & 7 & 9 & 28 \\
\hline
\end{tabular}

Table 31.2: Descriptives for Outcome After Ferguson Protests Begin

\begin{tabular}{lcccccccc}
\hline \hline Statistic & $\mathrm{N}$ & Mean & St. Dev. & Min & Pctl(25) & Median & Pctl(75) & Max \\
\hline ungrouped & 290 & 5.155 & 3.484 & 0 & 3 & 5 & 6 & 25 \\
\hline
\end{tabular}




\subsection{Four Models for ungrouped}

Table 31.3: Four Models that Differ on the Specification of Adjustment and Intervention Variables

\begin{tabular}{|c|c|c|c|c|}
\hline & \multicolumn{4}{|c|}{ Outcome: Count Per Week } \\
\hline & $(1)$ & $(2)$ & $(3)$ & (4) \\
\hline After Ferguson Protests Begin (week of 8/11/14 onward) & 0.57 & & 1.40 & 1.10 \\
\hline After Gray Protests Begin (week of $4 / 20 / 15$ onward) & -1.59 & & -4.35 & -3.06 \\
\hline Unrest and National Guard $(4 / 27 / 15-5 / 3 / 2015)$ & 2.45 & & 6.03 & 3.34 \\
\hline After Batts Fired (week of $7 / 13 / 15$ onward) & -0.05 & & 2.11 & 0.68 \\
\hline After Davis Confirmed (week of 10/19/15 onward) & -0.79 & & -0.46 & -0.49 \\
\hline After Davis First Year (week of $10 / 17 / 16$ onward) & -1.04 & & -1.02 & -1.03 \\
\hline After De Sousa Begins (week of 1/19/18 onward) & -0.60 & & -0.44 & -0.58 \\
\hline After Tuggle Begins (week of 5/14/18 onward) & -0.14 & & -0.12 & -0.04 \\
\hline After Harrison Begins (week of 2/11/19 onward) & -0.16 & & -0.03 & -0.25 \\
\hline Average Maximum Temperature to 50 Degrees & & -0.32 & & 0.02 \\
\hline Plus Degrees in the $50 \mathrm{~s}$ & & 0.74 & & 0.08 \\
\hline Plus Degrees in the $60 \mathrm{~s}$ & & -0.91 & & -0.18 \\
\hline Plus Degrees in the 70s & & 0.98 & & 0.24 \\
\hline Plus Degrees Greater Than 80 & & -1.11 & & -0.39 \\
\hline Snowfall (inches) & & -2.28 & & -1.12 \\
\hline Precipitation (inches) & & -0.06 & & -0.25 \\
\hline Darkness Before Midnight (hours) & & -0.75 & & -0.17 \\
\hline Thanksgiving/Christmas (proportion of week) & & -19.50 & & -16.76 \\
\hline Winter Holiday (proportion of week) & & -6.31 & & -1.01 \\
\hline Other Out-of-School Days (proportion of week) & & 3.55 & & 0.62 \\
\hline Summer Vacation (proportion of week) & & 3.12 & & 0.71 \\
\hline Summer Holiday (proportion of week) & & 17.97 & & 3.09 \\
\hline Observations & 373 & 83 & 373 & 373 \\
\hline $\mathrm{R}^{2}$ & 0.17 & 0.33 & 0.13 & 0.24 \\
\hline
\end{tabular}




\subsection{Least Squares Updated Model (2) for ungrouped}

ungrouped

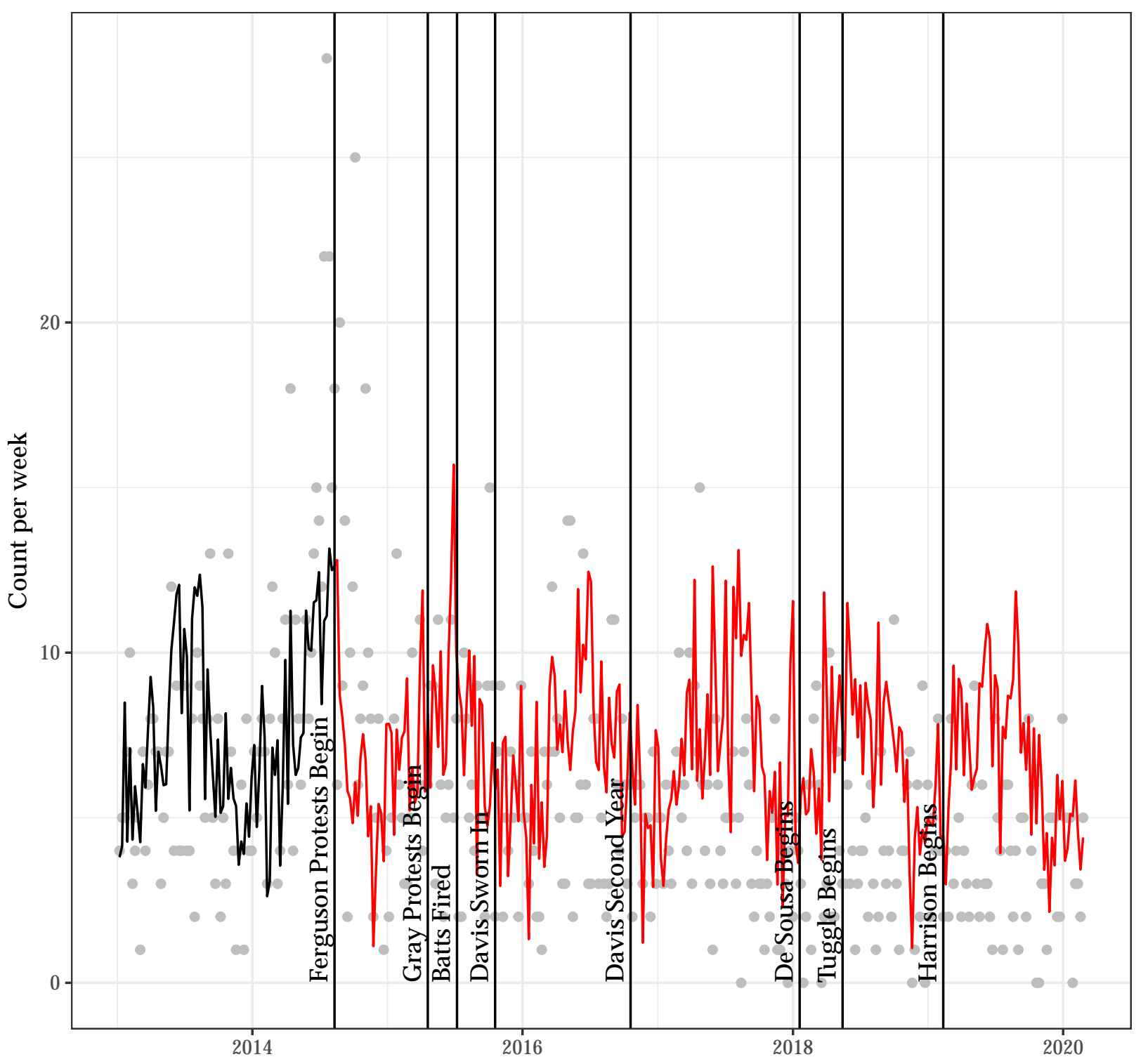




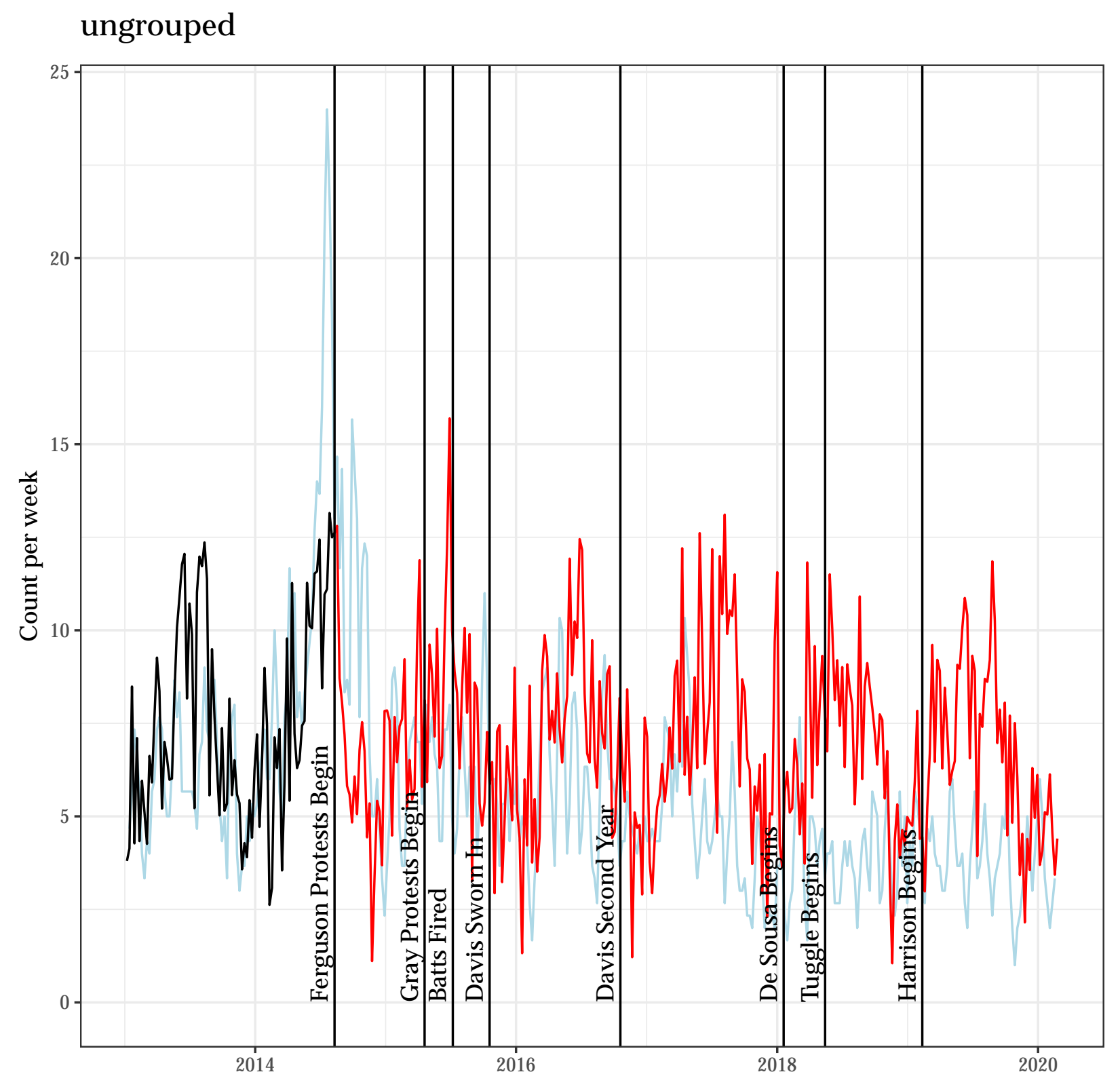


Autocorrelation Function for the Observed Outcome for Updated Model (2)

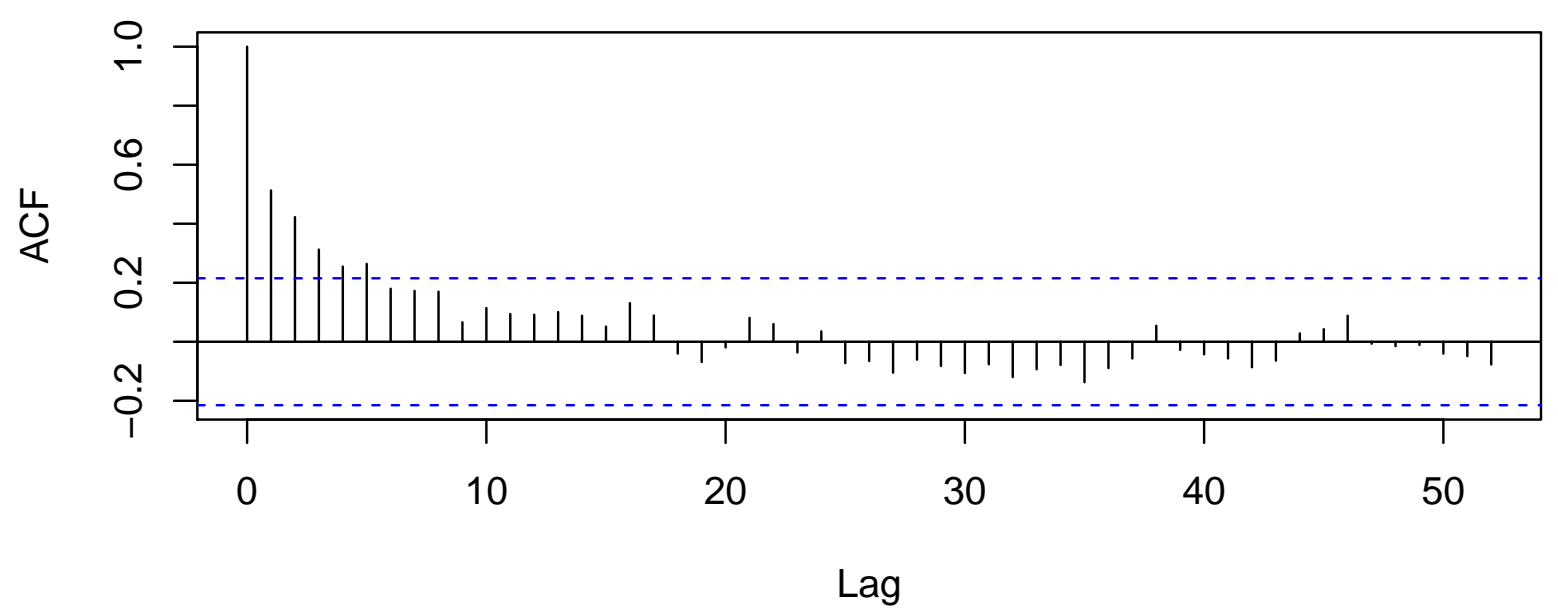

Autocorrelation Function for the Residuals from Updated Model (2)

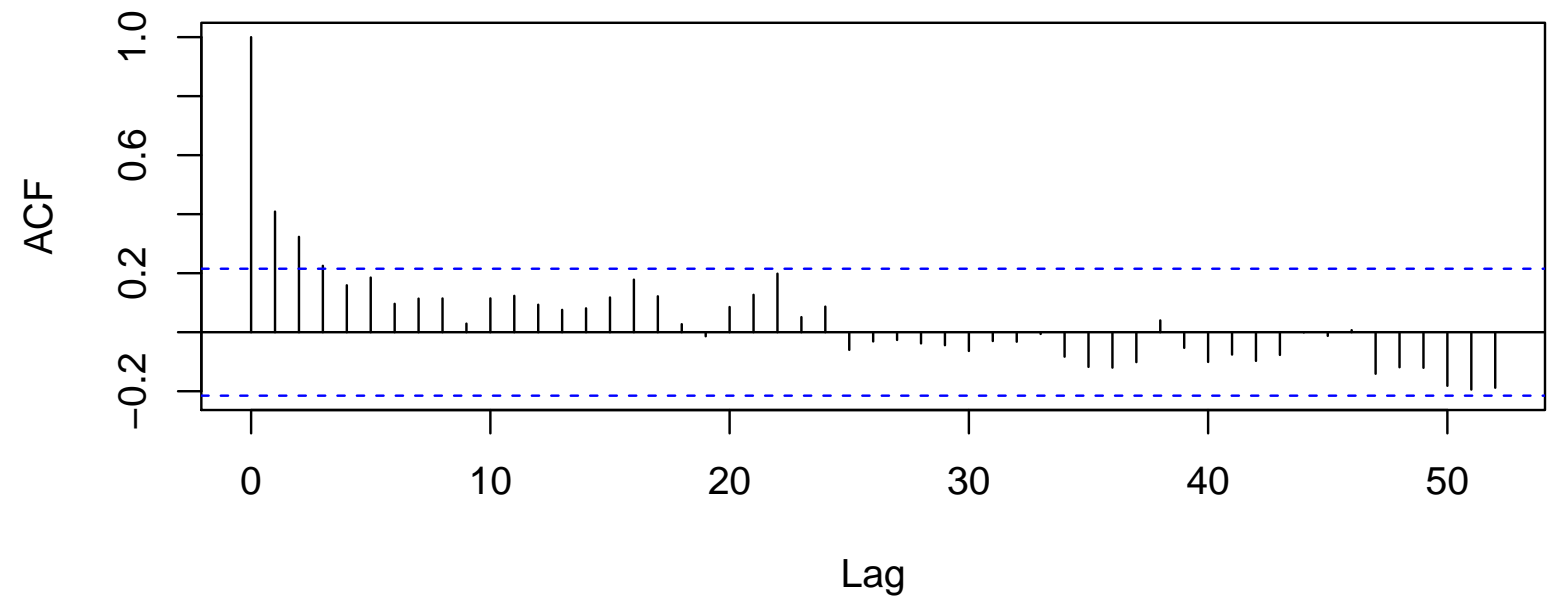




\subsection{A Poisson Regression Model as an Alternative to Model (2) for ungrouped}

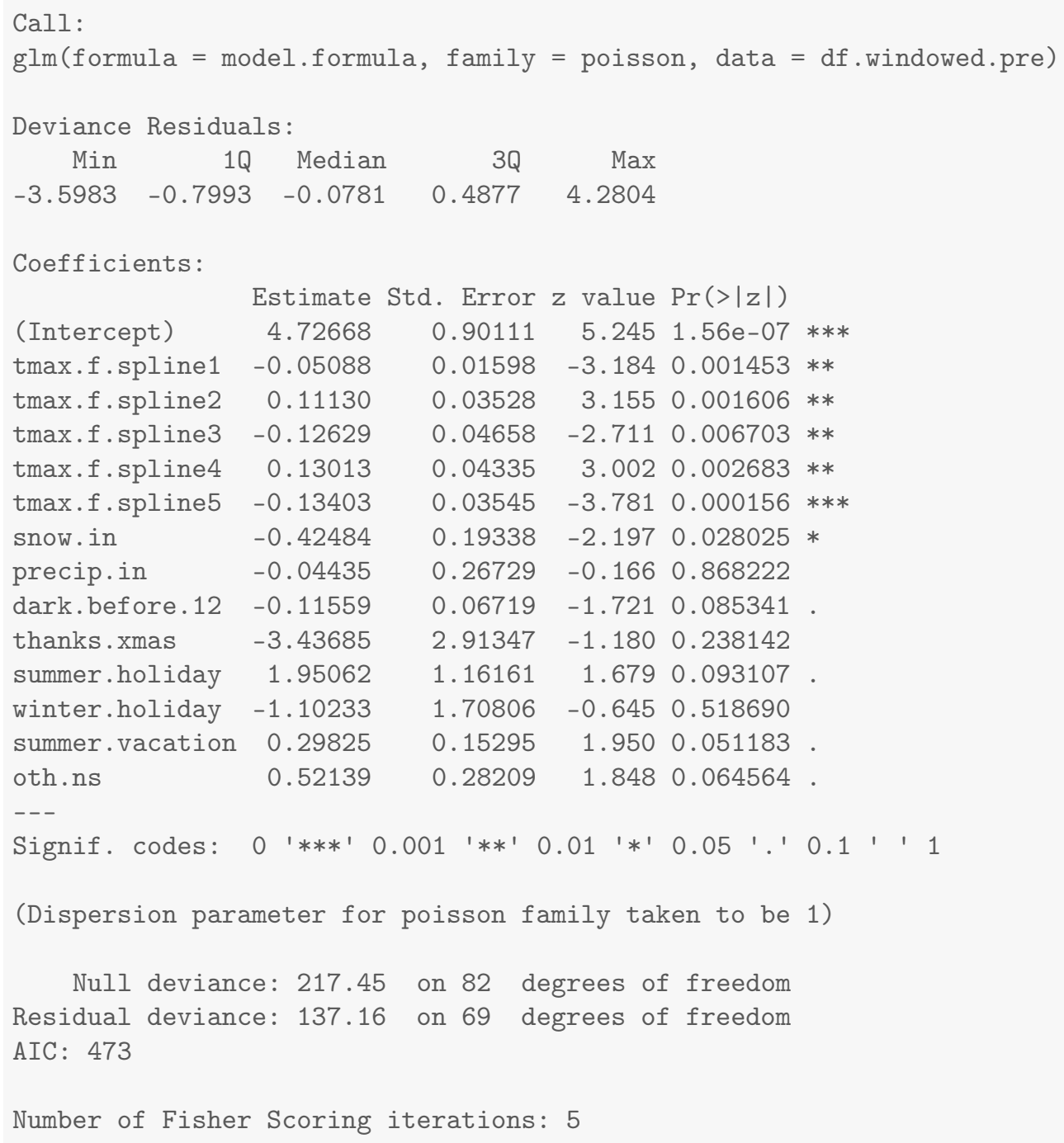




\section{Results for northwestern}

\subsection{Summary Values for northwestern}

Table 32.1: Descriptives for Outcome Before Ferguson Protests Begin

\begin{tabular}{lcccccccc}
\hline \hline Statistic & $\mathrm{N}$ & Mean & St. Dev. & Min & Pctl(25) & Median & Pctl(75) & Max \\
\hline northwestern & 83 & 48.253 & 13.548 & 26 & 38 & 47 & 57 & 95 \\
\hline
\end{tabular}

Table 32.2: Descriptives for Outcome After Ferguson Protests Begin

\begin{tabular}{lcccccccc}
\hline \hline Statistic & N & Mean & St. Dev. & Min & Pctl(25) & Median & Pctl(75) & Max \\
\hline northwestern & 290 & 19.038 & 7.925 & 6 & 14 & 18 & 23.8 & 56 \\
\hline
\end{tabular}




\subsection{Four Models for northwestern}

Table 32.3: Four Models that Differ on the Specification of Adjustment and Intervention Variables

\begin{tabular}{|c|c|c|c|c|}
\hline & \multicolumn{4}{|c|}{ Outcome: Count Per Week } \\
\hline & (1) & $(2)$ & (3) & (4) \\
\hline After Ferguson Protests Begin (week of $8 / 11 / 14$ onward) & -18.00 & & -18.05 & -17.79 \\
\hline After Gray Protests Begin (week of 4/20/15 onward) & -14.34 & & -10.62 & -14.03 \\
\hline Unrest and National Guard $(4 / 27 / 15$ - 5/3/2015) & 19.09 & & 13.48 & 16.37 \\
\hline After Batts Fired (week of $7 / 13 / 15$ onward) & 4.38 & & -0.97 & 3.33 \\
\hline After Davis Confirmed (week of 10/19/15 onward) & 0.14 & & 3.12 & 0.40 \\
\hline After Davis First Year (week of 10/17/16 onward) & -2.76 & & -5.67 & -3.32 \\
\hline After De Sousa Begins (week of $1 / 19 / 18$ onward) & 1.40 & & 4.39 & 1.95 \\
\hline After Tuggle Begins (week of 5/14/18 onward) & -3.24 & & -4.17 & -2.20 \\
\hline After Harrison Begins (week of 2/11/19 onward) & -1.49 & & -3.65 & -2.79 \\
\hline Average Maximum Temperature to 50 Degrees & & 0.76 & & 0.28 \\
\hline Plus Degrees in the $50 \mathrm{~s}$ & & -2.75 & & -0.35 \\
\hline Plus Degrees in the 60s & & 3.63 & & 0.25 \\
\hline Plus Degrees in the 70s & & -2.27 & & -0.40 \\
\hline Plus Degrees Greater Than 80 & & 0.63 & & 0.07 \\
\hline Snowfall (inches) & & -10.09 & & -2.98 \\
\hline Precipitation (inches) & & -20.56 & & -9.44 \\
\hline Darkness Before Midnight (hours) & & 2.30 & & 0.75 \\
\hline Thanksgiving/Christmas (proportion of week) & & -124.28 & & -37.37 \\
\hline Winter Holiday (proportion of week) & & -6.41 & & 19.30 \\
\hline Other Out-of-School Days (proportion of week) & & 2.77 & & -2.26 \\
\hline Summer Vacation (proportion of week) & & 7.91 & & 2.72 \\
\hline Summer Holiday (proportion of week) & & -35.76 & & 8.67 \\
\hline Observations & 373 & 83 & 373 & 373 \\
\hline $\mathrm{R}^{2}$ & 0.70 & 0.26 & 0.64 & 0.72 \\
\hline
\end{tabular}


32.3 Least Squares Updated Model (2) for northwestern northwestern

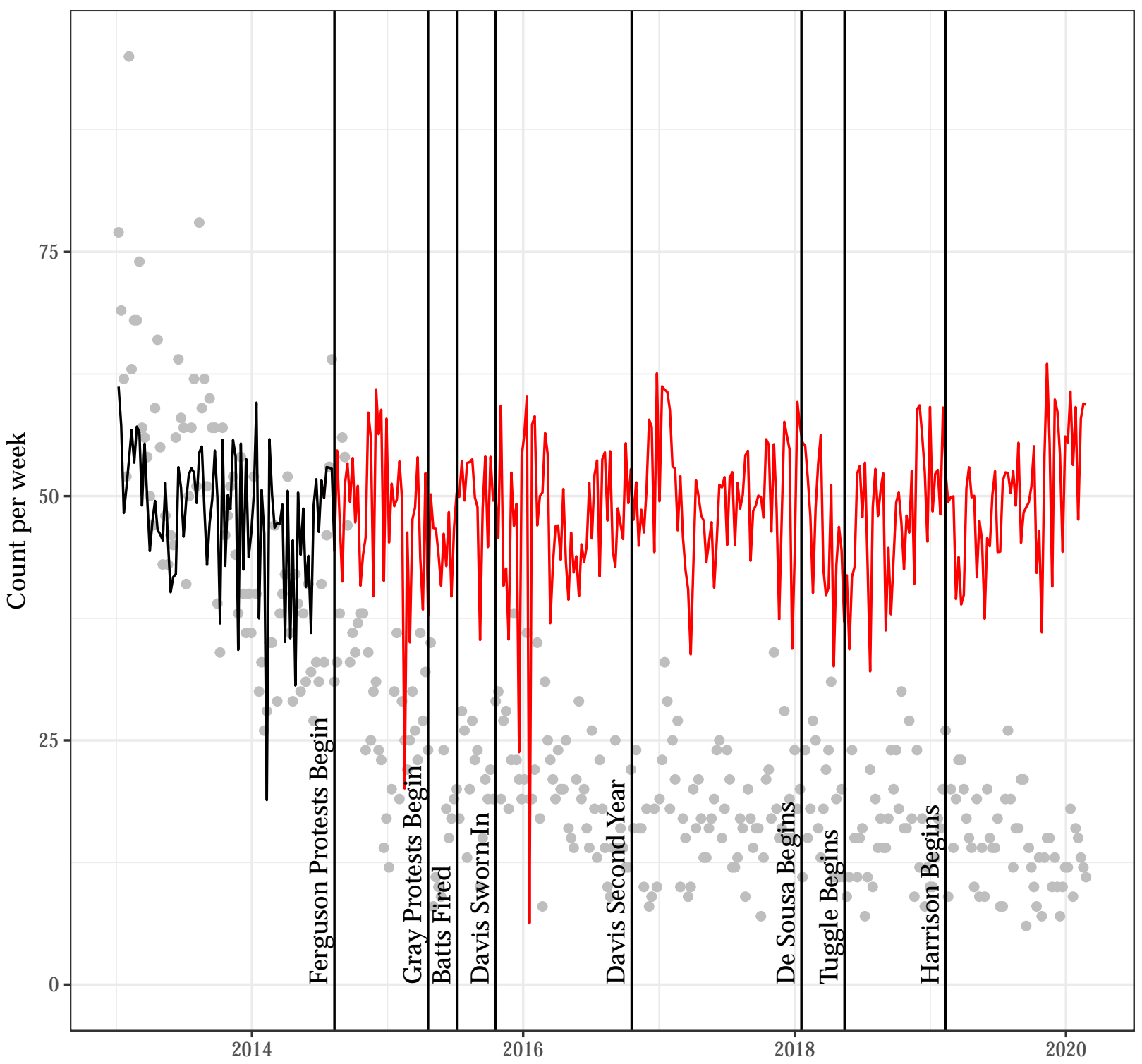




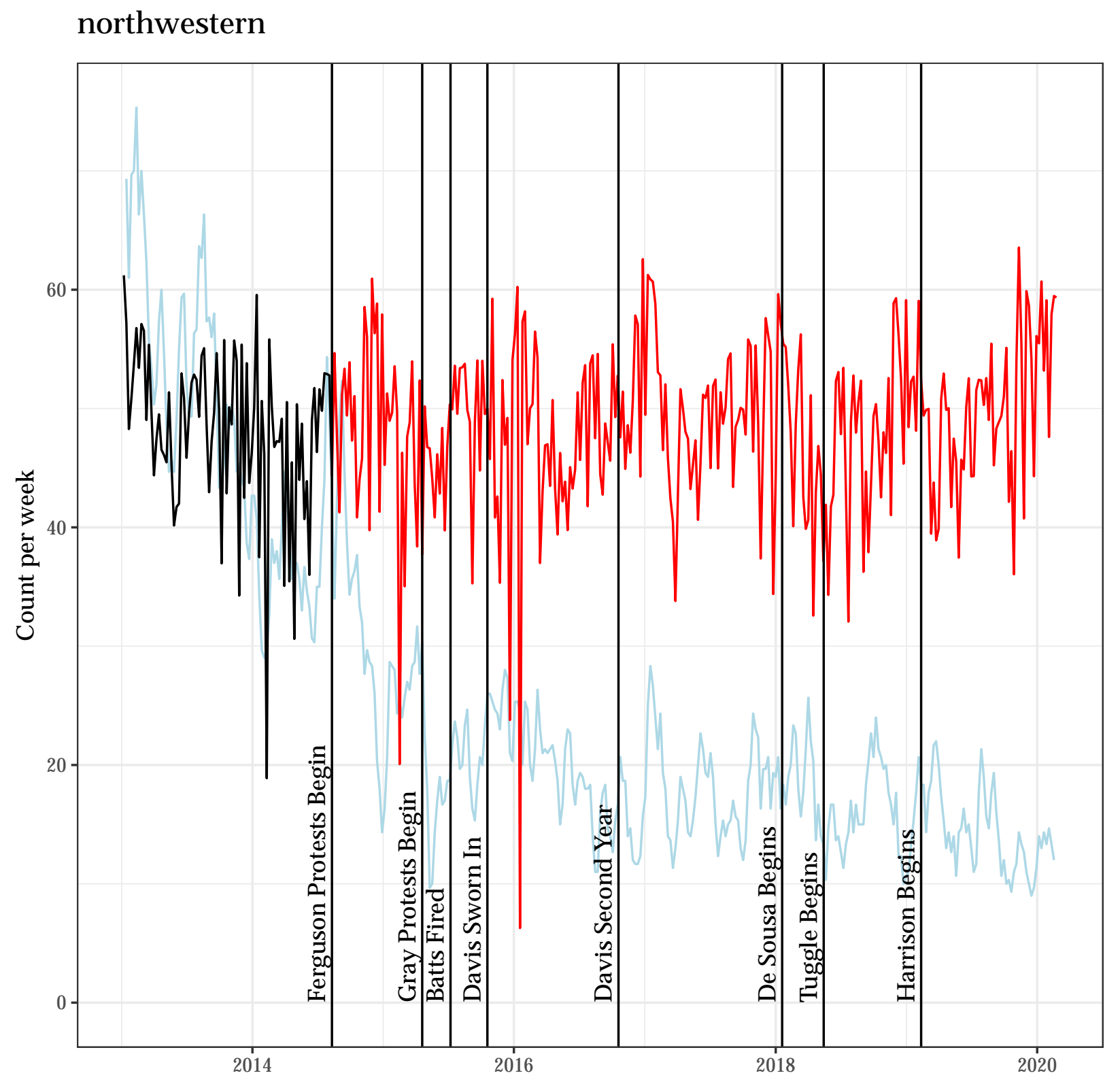


Autocorrelation Function for the Observed Outcome for Updated Model (2)

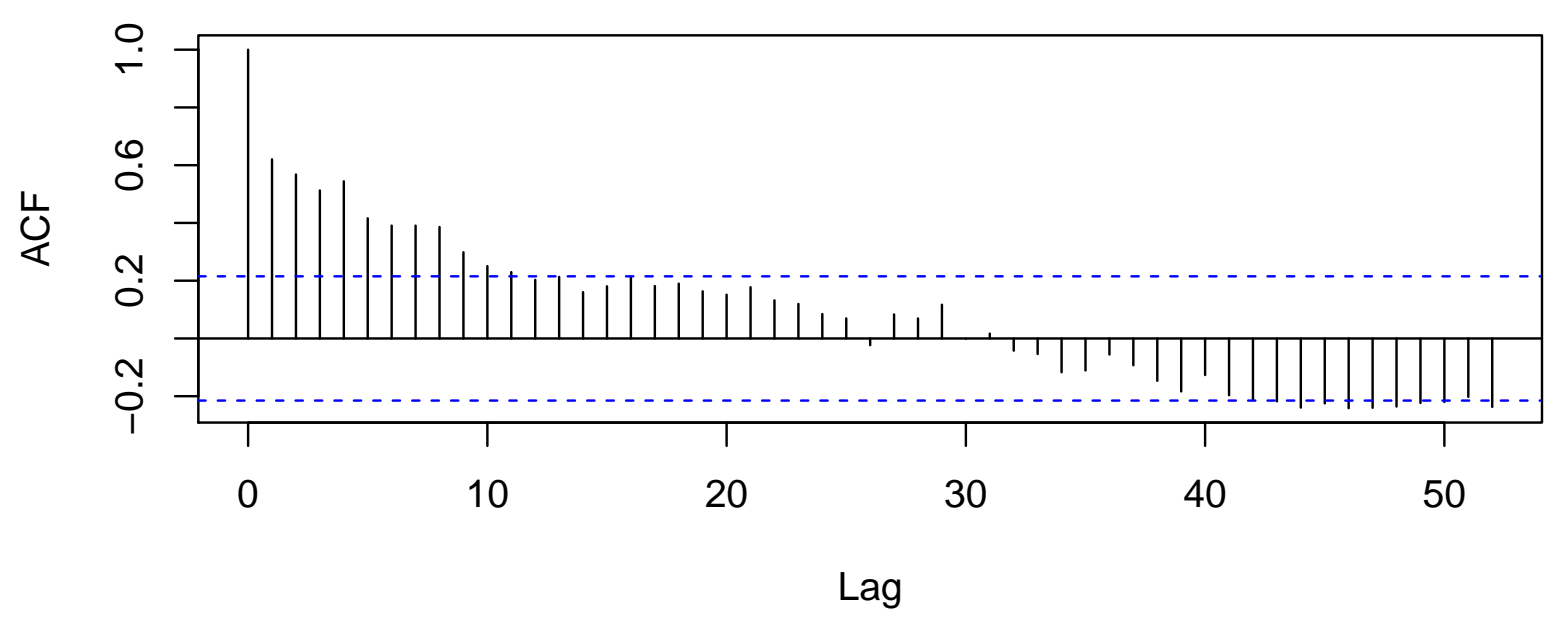

Autocorrelation Function for the Residuals from Updated Model (2)

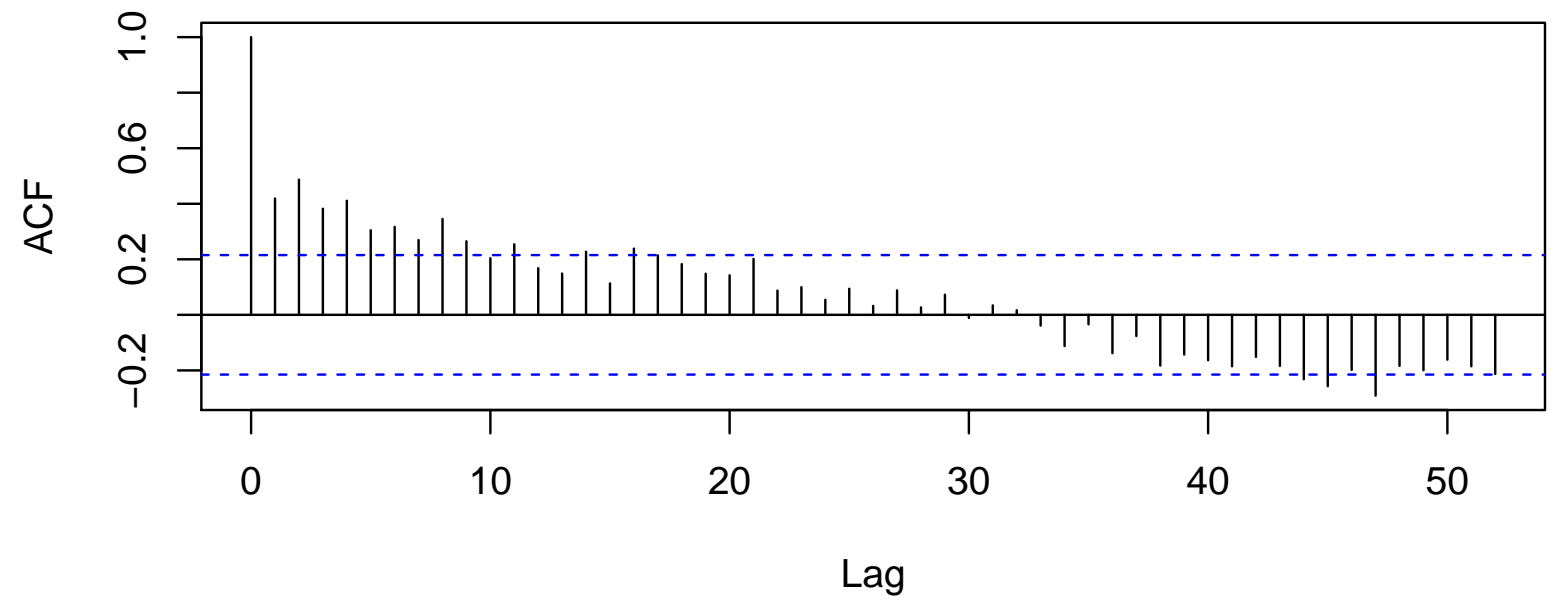




\subsection{A Poisson Regression Model as an Alternative to Model (2) for northwestern}

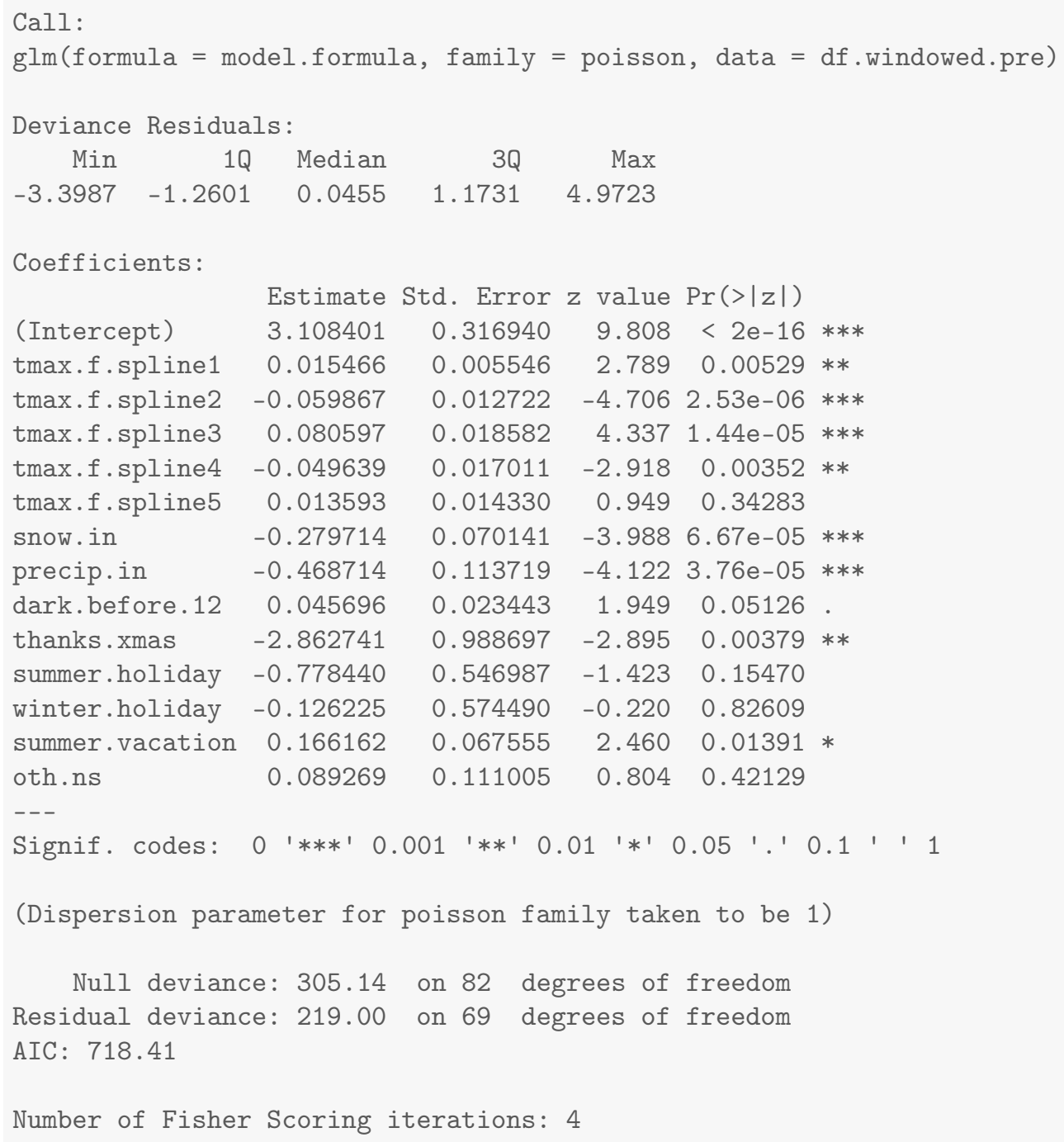




\section{Results for northern}

\subsection{Summary Values for northern}

Table 33.1: Descriptives for Outcome Before Ferguson Protests Begin

\begin{tabular}{lcccccccc}
\hline \hline Statistic & $\mathrm{N}$ & Mean & St. Dev. & Min & Pctl(25) & Median & Pctl(75) & Max \\
\hline northern & 83 & 30.771 & 8.284 & 15 & 25 & 30 & 36 & 56 \\
\hline
\end{tabular}

Table 33.2: Descriptives for Outcome After Ferguson Protests Begin

\begin{tabular}{lcccccccc}
\hline \hline Statistic & $\mathrm{N}$ & Mean & St. Dev. & Min & Pctl(25) & Median & Pctl(75) & Max \\
\hline northern & 290 & 15.997 & 6.851 & 1 & 11 & 15 & 20 & 40 \\
\hline
\end{tabular}




\subsection{Four Models for northern}

Table 33.3: Four Models that Differ on the Specification of Adjustment and Intervention Variables

\begin{tabular}{|c|c|c|c|c|}
\hline & \multicolumn{4}{|c|}{ Outcome: Count Per Week } \\
\hline & $(1)$ & (2) & (3) & $(4)$ \\
\hline After Ferguson Protests Begin (week of $8 / 11 / 14$ onward) & -8.33 & & -5.79 & -6.87 \\
\hline After Gray Protests Begin (week of $4 / 20 / 15$ onward) & -8.08 & & -15.68 & -11.62 \\
\hline Unrest and National Guard $(4 / 27 / 15-5 / 3 / 2015)$ & 0.64 & & 6.78 & 1.41 \\
\hline After Batts Fired (week of 7/13/15 onward) & 10.42 & & 14.70 & 11.45 \\
\hline After Davis Confirmed (week of 10/19/15 onward) & -5.15 & & -3.77 & -4.07 \\
\hline After Davis First Year (week of 10/17/16 onward) & -5.06 & & -4.43 & -4.70 \\
\hline After De Sousa Begins (week of 1/19/18 onward) & 0.99 & & -1.38 & 0.27 \\
\hline After Tuggle Begins (week of 5/14/18 onward) & -2.28 & & -1.04 & -1.52 \\
\hline After Harrison Begins (week of 2/11/19 onward) & -3.08 & & -3.17 & -3.57 \\
\hline Average Maximum Temperature to 50 Degrees & & 0.06 & & 0.12 \\
\hline Plus Degrees in the $50 \mathrm{~s}$ & & -0.12 & & -0.01 \\
\hline Plus Degrees in the 60s & & -0.86 & & -0.33 \\
\hline Plus Degrees in the $70 \mathrm{~s}$ & & 2.31 & & 0.41 \\
\hline Plus Degrees Greater Than 80 & & -1.51 & & -0.22 \\
\hline Snowfall (inches) & & -5.32 & & -2.61 \\
\hline Precipitation (inches) & & -3.09 & & -2.88 \\
\hline Darkness Before Midnight (hours) & & -3.07 & & -1.17 \\
\hline Thanksgiving/Christmas (proportion of week) & & -44.26 & & -37.05 \\
\hline Winter Holiday (proportion of week) & & 6.10 & & 15.78 \\
\hline Other Out-of-School Days (proportion of week) & & -6.84 & & -1.02 \\
\hline Summer Vacation (proportion of week) & & -9.73 & & -0.71 \\
\hline Summer Holiday (proportion of week) & & 3.47 & & 2.57 \\
\hline Observations & 373 & 83 & 373 & 373 \\
\hline $\mathrm{R}^{2}$ & 0.59 & 0.41 & 0.56 & 0.66 \\
\hline
\end{tabular}


33.3 Least Squares Updated Model (2) for northern northern

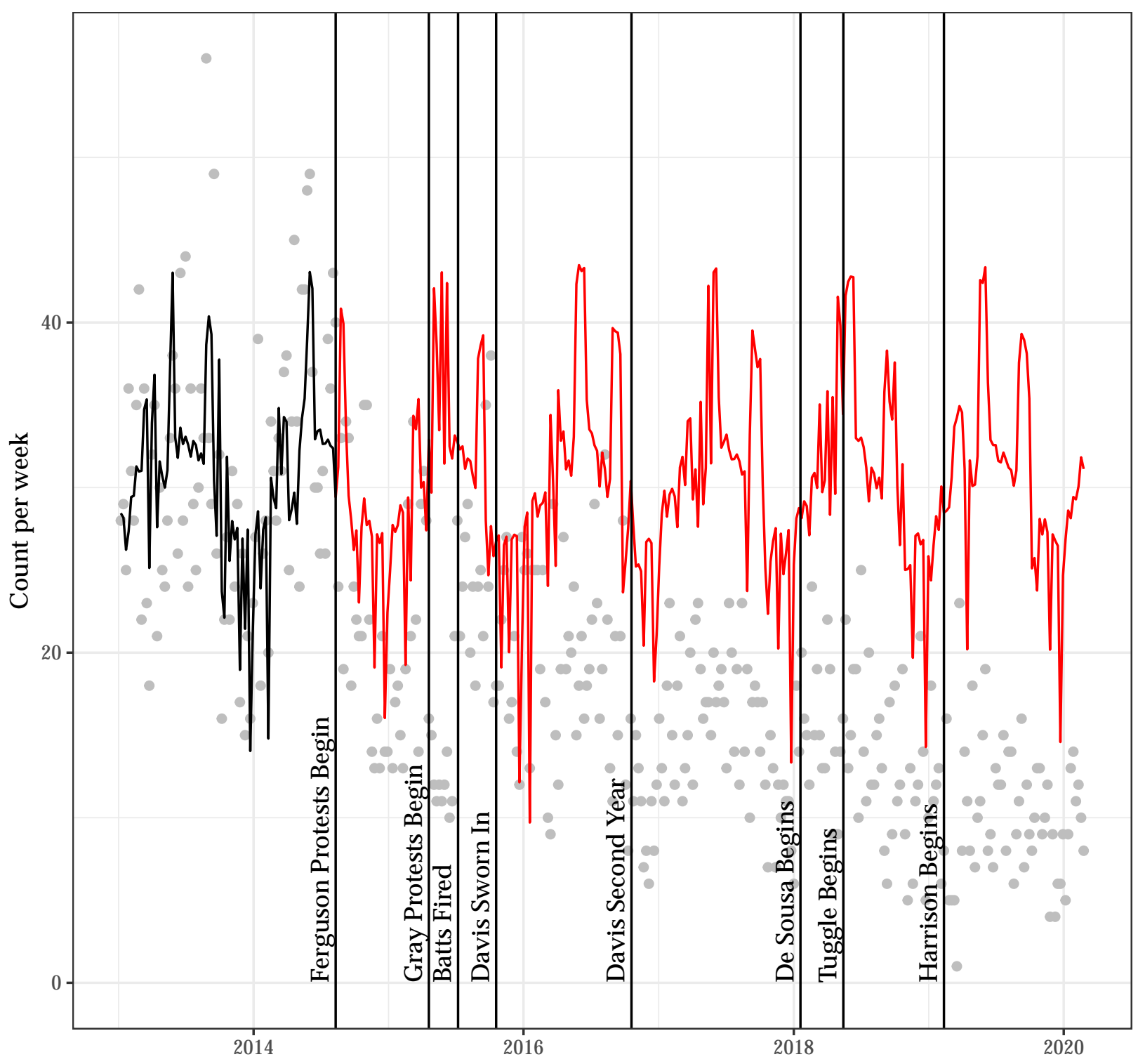




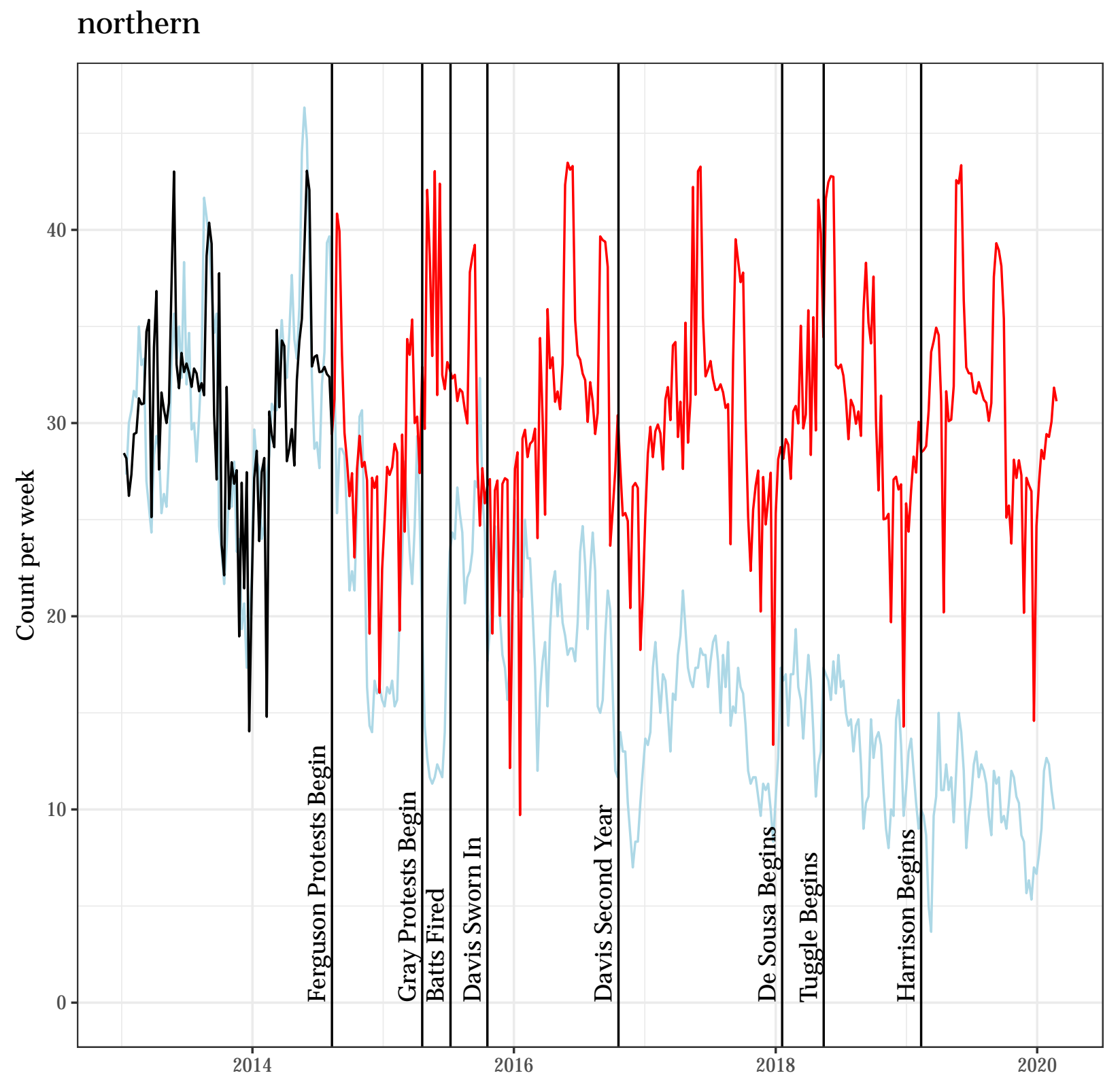


Autocorrelation Function for the Observed Outcome for Updated Model (2)

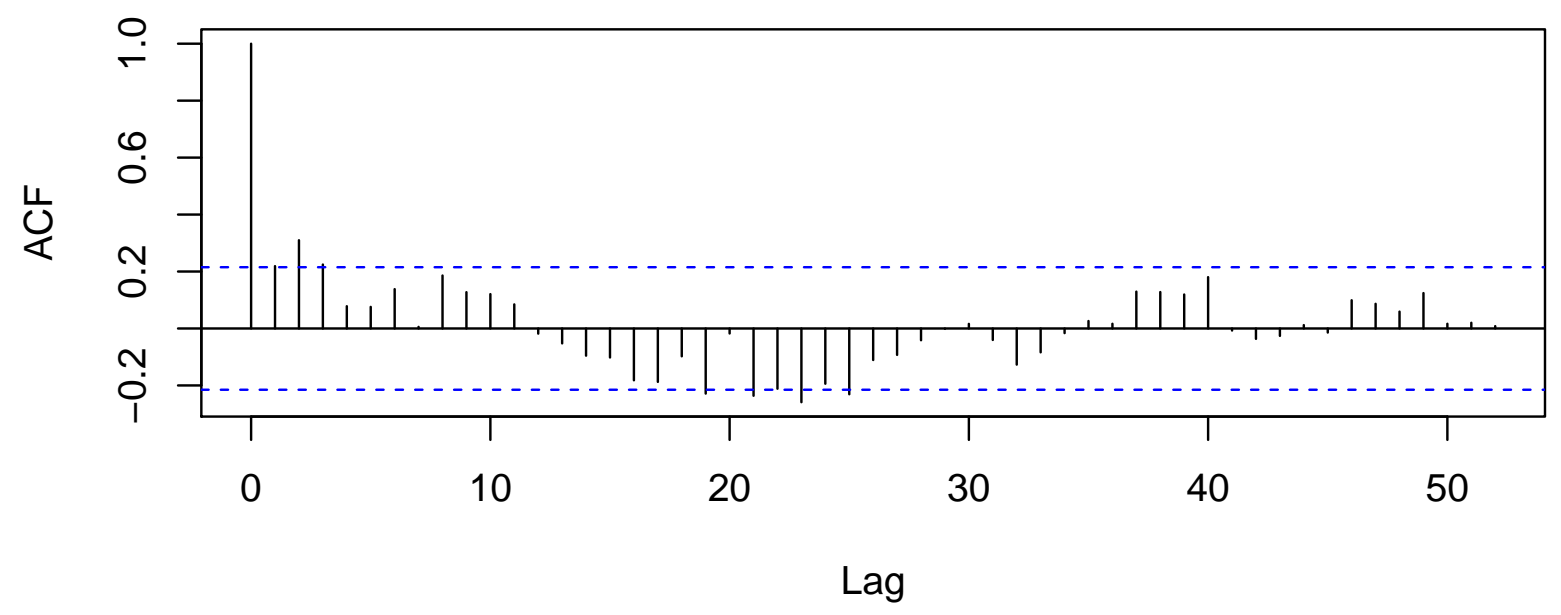

Autocorrelation Function for the Residuals from Updated Model (2)

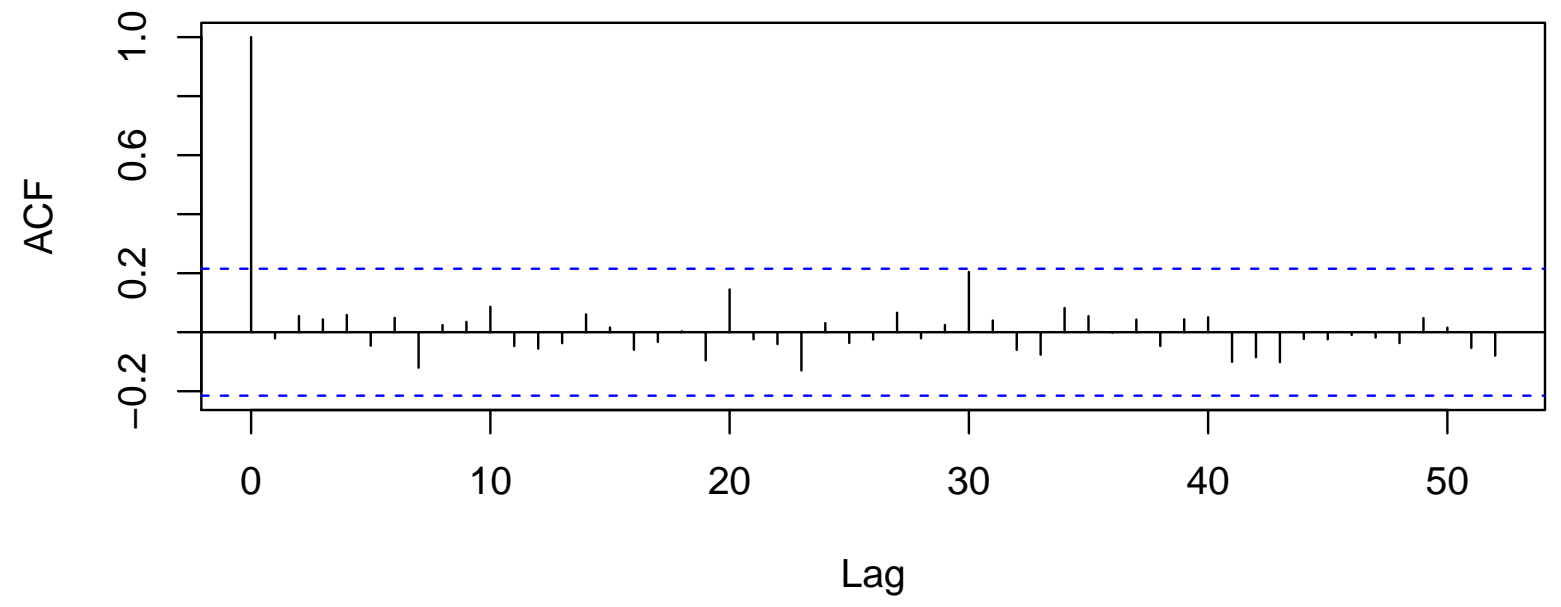




\subsection{A Poisson Regression Model as an Alternative to Model (2) for northern}

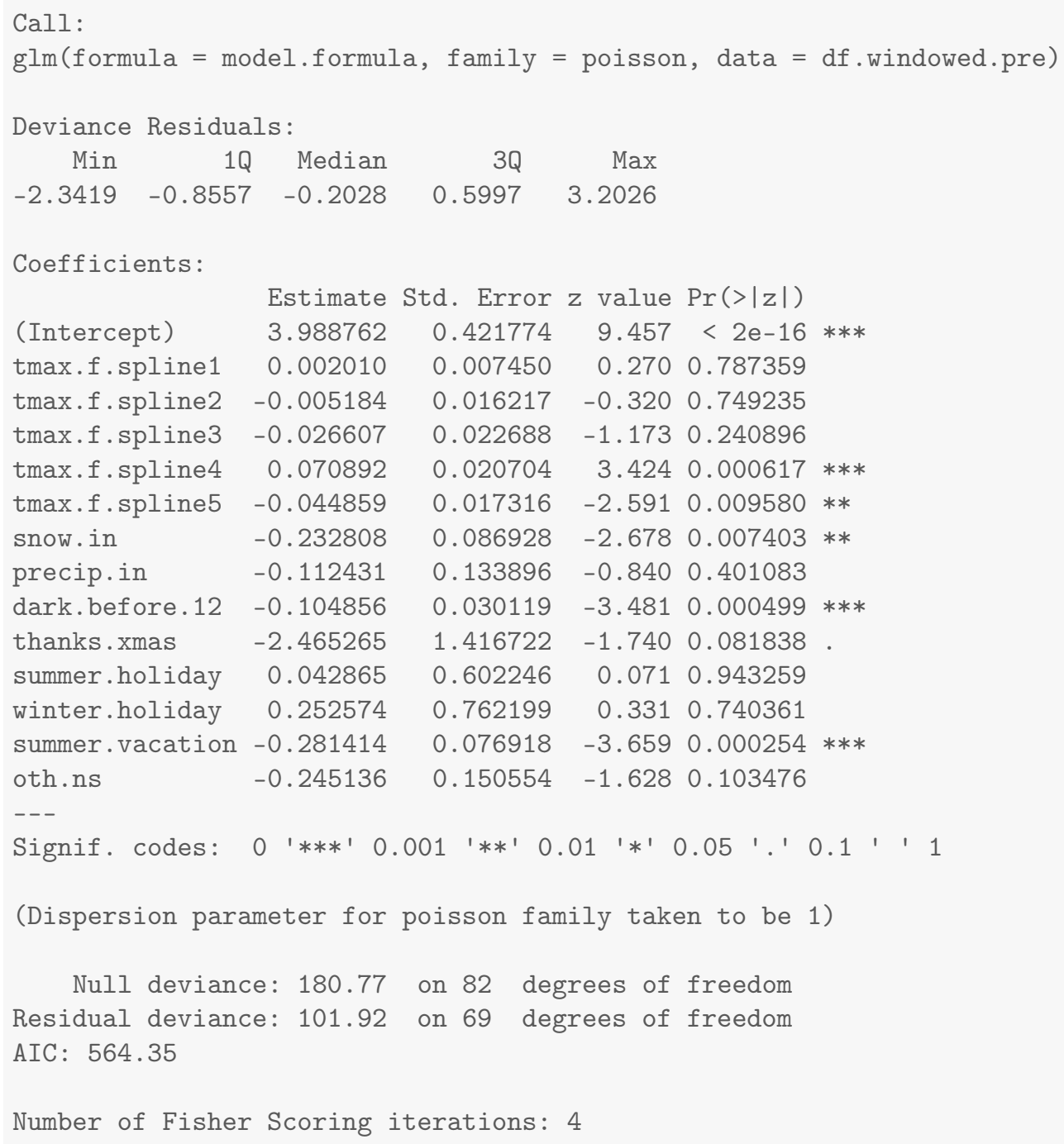




\section{Results for northeastern}

\subsection{Summary Values for northeastern}

Table 34.1: Descriptives for Outcome Before Ferguson Protests Begin

\begin{tabular}{lcccccccc}
\hline \hline Statistic & $\mathrm{N}$ & Mean & St. Dev. & Min & Pctl(25) & Median & Pctl(75) & Max \\
\hline northeastern & 83 & 59.072 & 14.665 & 26 & 50 & 57 & 66 & 93 \\
\hline
\end{tabular}

Table 34.2: Descriptives for Outcome After Ferguson Protests Begin

\begin{tabular}{lcccccccc}
\hline \hline Statistic & $\mathrm{N}$ & Mean & St. Dev. & Min & Pctl(25) & Median & Pctl(75) & Max \\
\hline northeastern & 290 & 27.841 & 8.601 & 8 & 22 & 28 & 33 & 58 \\
\hline
\end{tabular}




\subsection{Four Models for northeastern}

Table 34.3: Four Models that Differ on the Specification of Adjustment and Intervention Variables

\begin{tabular}{|c|c|c|c|c|}
\hline & \multicolumn{4}{|c|}{ Outcome: Count Per Week } \\
\hline & $(1)$ & $(2)$ & (3) & (4) \\
\hline After Ferguson Protests Begin (week of $8 / 11 / 14$ onward) & -21.93 & & -19.41 & -20.73 \\
\hline After Gray Protests Begin (week of 4/20/15 onward) & -14.23 & & -19.17 & -17.01 \\
\hline Unrest and National Guard (4/27/15 - 5/3/2015) & 10.09 & & 6.00 & 9.23 \\
\hline After Batts Fired (week of $7 / 13 / 15$ onward) & 4.88 & & 6.41 & 5.70 \\
\hline After Davis Confirmed (week of 10/19/15 onward) & 1.27 & & 3.85 & 2.23 \\
\hline After Davis First Year (week of 10/17/16 onward) & 1.58 & & 1.75 & 2.02 \\
\hline After De Sousa Begins (week of 1/19/18 onward) & 1.36 & & -2.88 & -0.03 \\
\hline After Tuggle Begins (week of 5/14/18 onward) & -8.92 & & -4.68 & -7.52 \\
\hline After Harrison Begins (week of 2/11/19 onward) & -2.75 & & -5.16 & -3.49 \\
\hline Average Maximum Temperature to 50 Degrees & & 0.21 & & 0.10 \\
\hline Plus Degrees in the $50 \mathrm{~s}$ & & -1.72 & & -0.28 \\
\hline Plus Degrees in the 60s & & 1.67 & & 0.11 \\
\hline Plus Degrees in the 70s & & -0.12 & & 0.13 \\
\hline Plus Degrees Greater Than 80 & & 0.96 & & 0.22 \\
\hline Snowfall (inches) & & -10.44 & & -1.81 \\
\hline Precipitation (inches) & & -15.02 & & -4.70 \\
\hline Darkness Before Midnight (hours) & & -6.48 & & -1.98 \\
\hline Thanksgiving/Christmas (proportion of week) & & -72.78 & & -27.13 \\
\hline Winter Holiday (proportion of week) & & 31.35 & & 12.79 \\
\hline Other Out-of-School Days (proportion of week) & & 5.66 & & -0.16 \\
\hline Summer Vacation (proportion of week) & & -12.18 & & -3.54 \\
\hline Summer Holiday (proportion of week) & & -85.95 & & -12.99 \\
\hline Observations & 373 & 83 & 373 & 373 \\
\hline $\mathrm{R}^{2}$ & 0.70 & 0.25 & 0.64 & 0.72 \\
\hline
\end{tabular}


34.3 Least Squares Updated Model (2) for northeastern northeastern

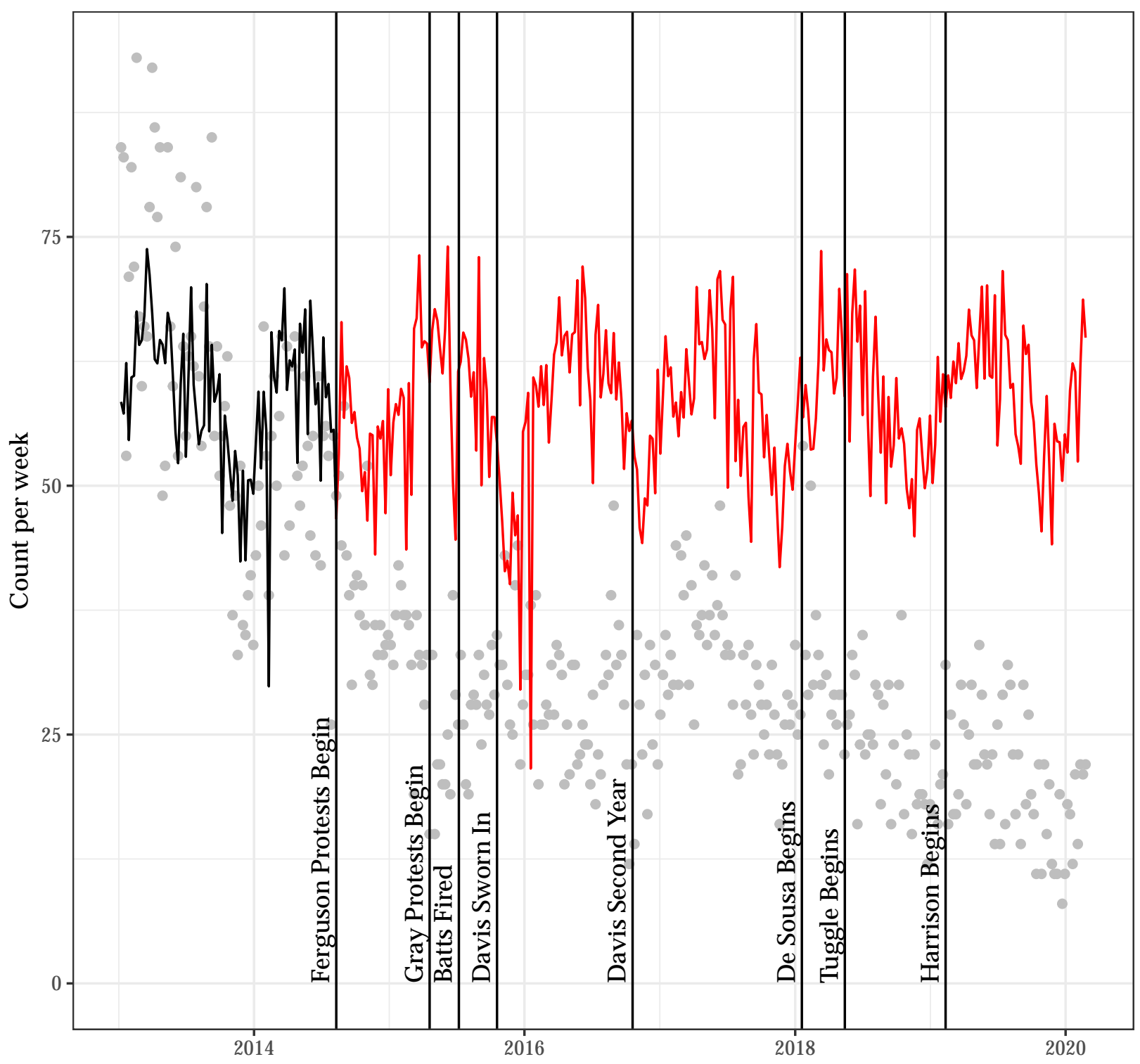




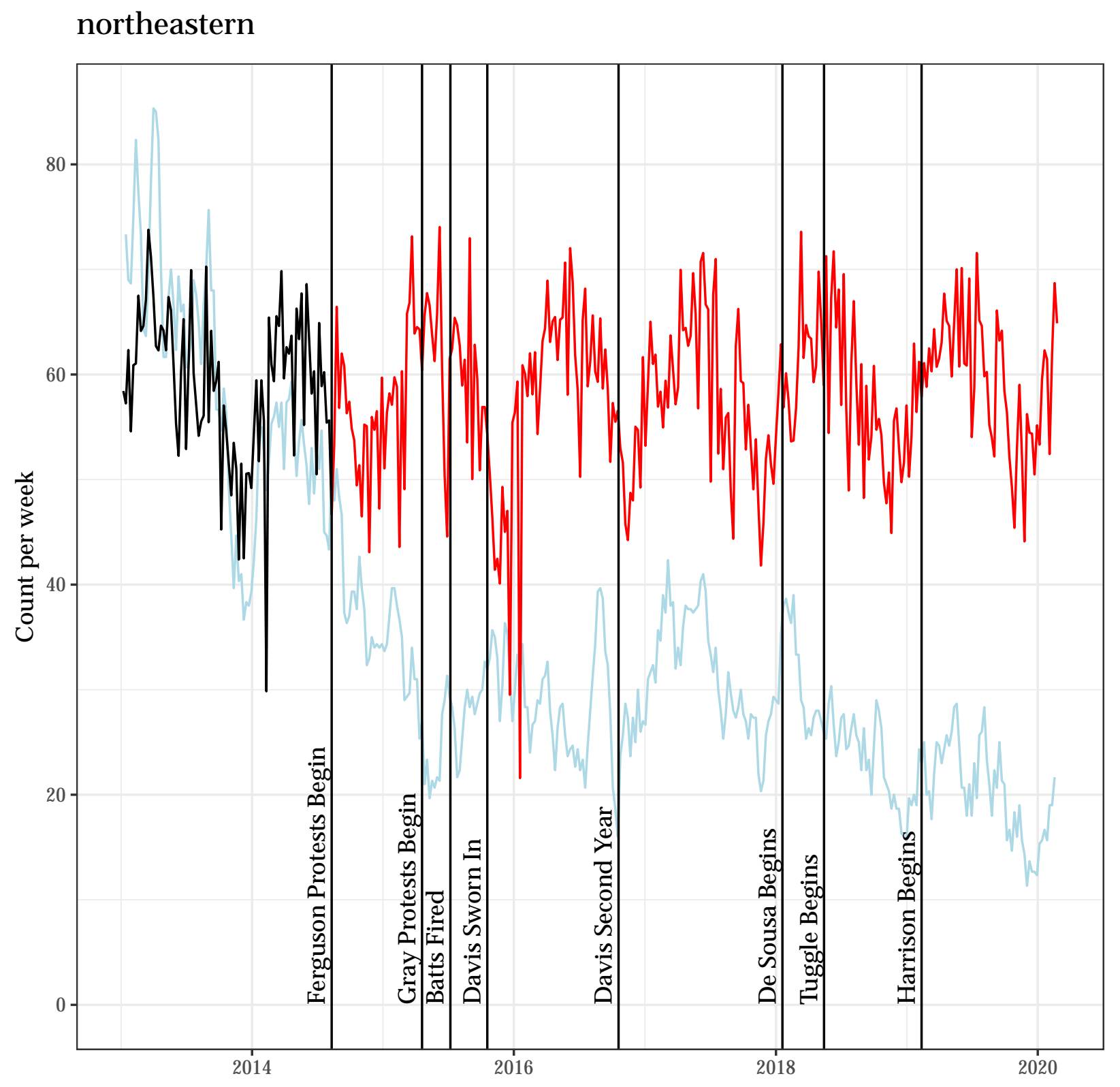


Autocorrelation Function for the Observed Outcome for Updated Model (2)

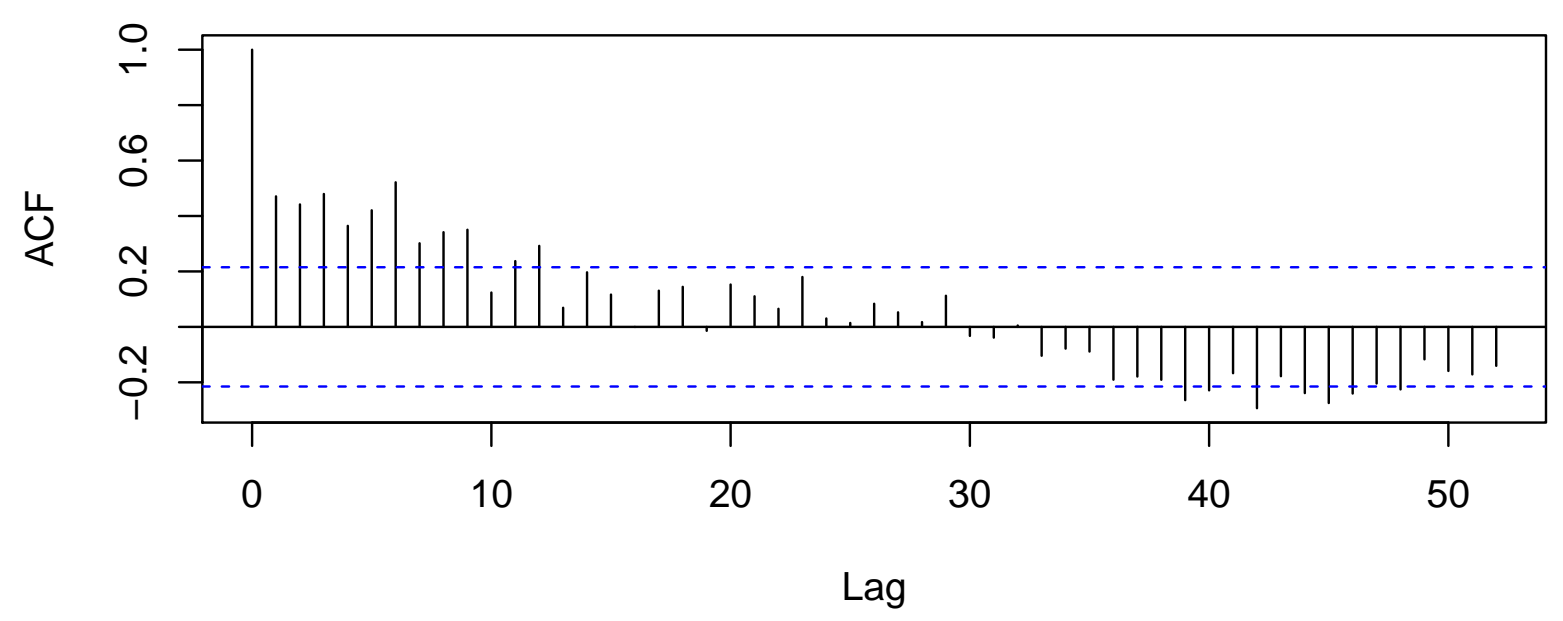

Autocorrelation Function for the Residuals from Updated Model (2)

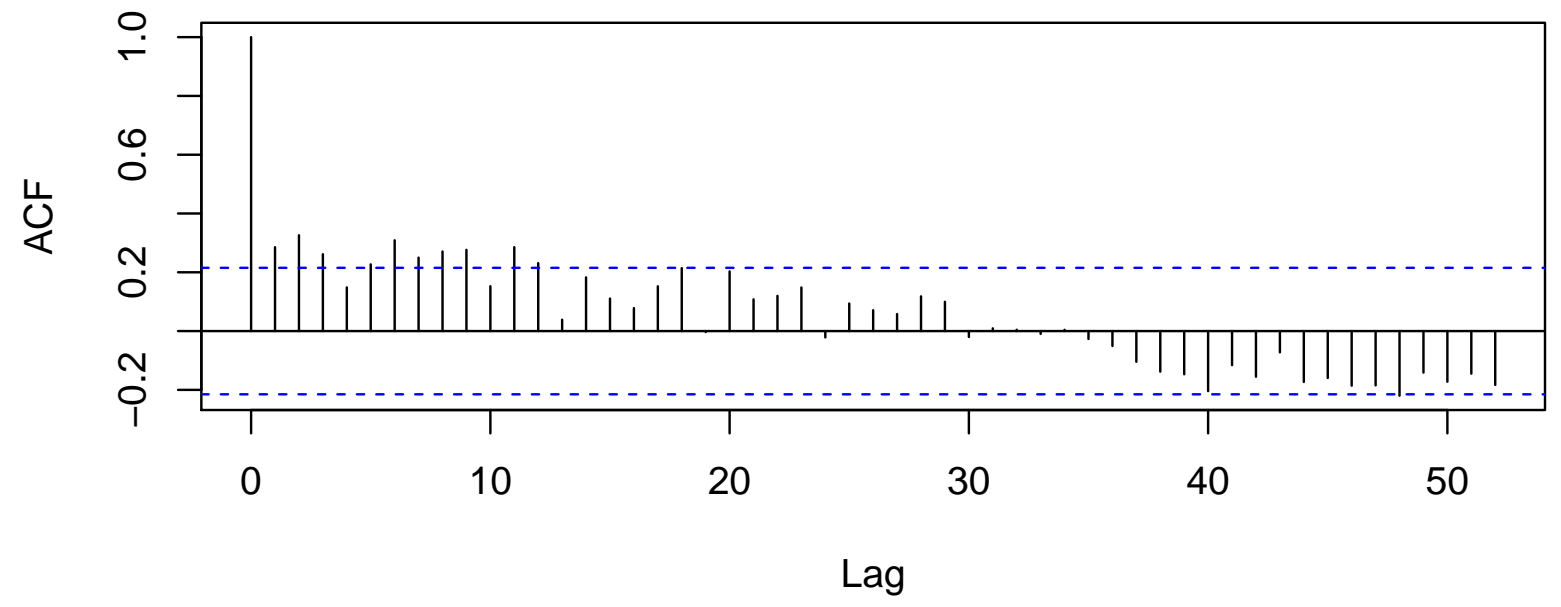




\subsection{A Poisson Regression Model as an Alternative to Model (2) for northeastern}

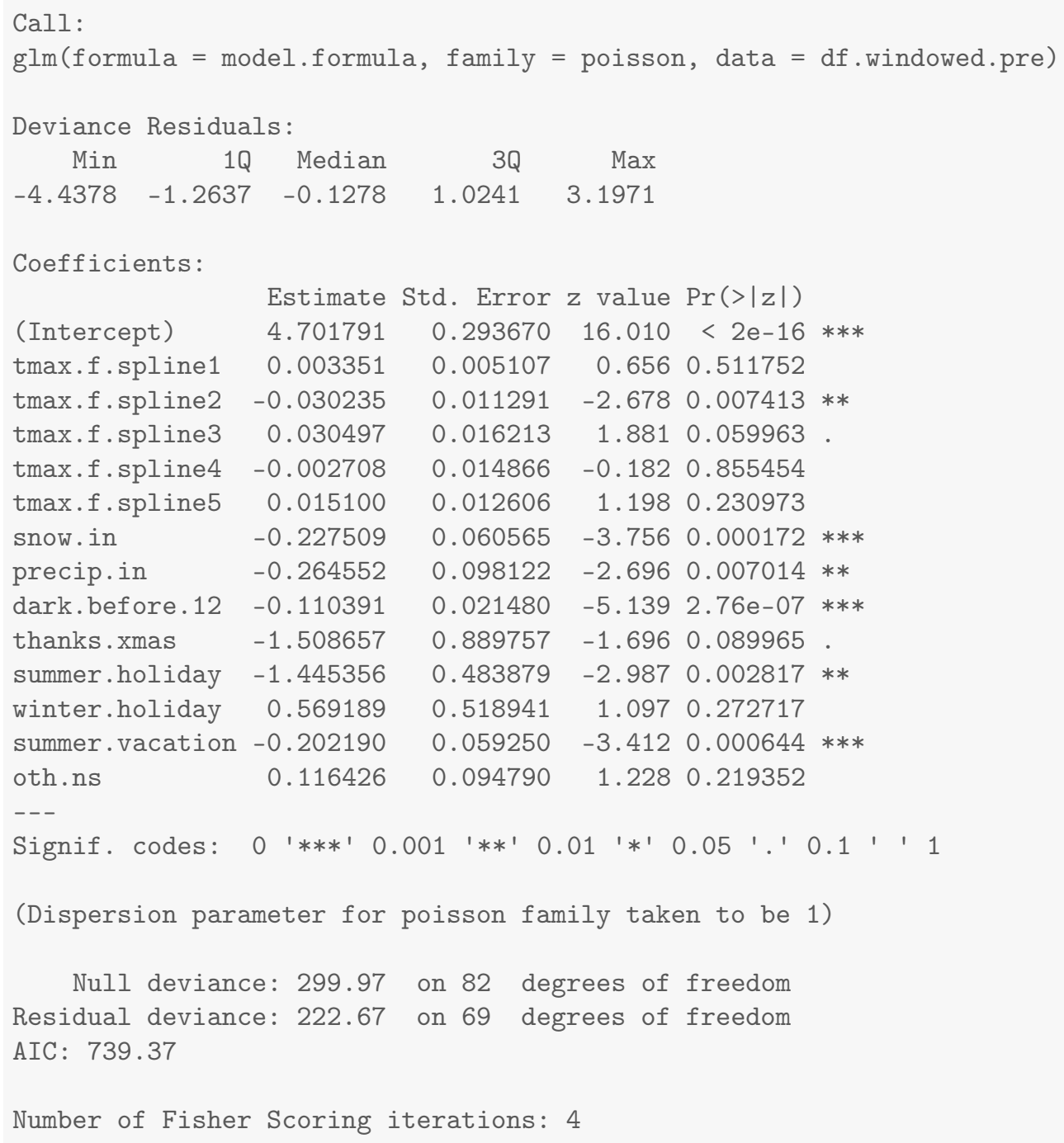




\section{Results for western}

\subsection{Summary Values for western}

Table 35.1: Descriptives for Outcome Before Ferguson Protests Begin

\begin{tabular}{lcccccccc}
\hline \hline Statistic & $\mathrm{N}$ & Mean & St. Dev. & Min & Pctl(25) & Median & Pctl(75) & Max \\
\hline western & 83 & 66.217 & 16.286 & 29 & 56 & 64 & 72.5 & 113 \\
\hline
\end{tabular}

Table 35.2: Descriptives for Outcome After Ferguson Protests Begin

\begin{tabular}{lcccccccc}
\hline \hline Statistic & $\mathrm{N}$ & Mean & St. Dev. & Min & Pctl(25) & Median & Pctl(75) & Max \\
\hline western & 290 & 29.810 & 14.168 & 6 & 20 & 27 & 37 & 93 \\
\hline
\end{tabular}




\subsection{Four Models for western}

Table 35.3: Four Models that Differ on the Specification of Adjustment and Intervention Variables

\begin{tabular}{|c|c|c|c|c|}
\hline & \multicolumn{4}{|c|}{ Outcome: Count Per Week } \\
\hline & (1) & (2) & (3) & (4) \\
\hline After Ferguson Protests Begin (week of $8 / 11 / 14$ onward) & -12.86 & & -9.09 & -11.05 \\
\hline After Gray Protests Begin (week of 4/20/15 onward) & -30.27 & & -37.01 & -33.27 \\
\hline Unrest and National Guard $(4 / 27 / 15-5 / 3 / 2015)$ & 27.91 & & 27.22 & 25.46 \\
\hline After Batts Fired (week of 7/13/15 onward) & 12.12 & & 17.48 & 14.31 \\
\hline After Davis Confirmed (week of 10/19/15 onward) & -2.14 & & -0.20 & -1.99 \\
\hline After Davis First Year (week of 10/17/16 onward) & -4.56 & & -5.91 & -4.67 \\
\hline After De Sousa Begins (week of 1/19/18 onward) & -1.77 & & -6.12 & -4.31 \\
\hline After Tuggle Begins (week of 5/14/18 onward) & -5.47 & & -1.67 & -1.95 \\
\hline After Harrison Begins (week of 2/11/19 onward) & -1.90 & & -4.37 & -3.82 \\
\hline Average Maximum Temperature to 50 Degrees & & 1.05 & & 0.46 \\
\hline Plus Degrees in the $50 \mathrm{~s}$ & & -3.95 & & -1.11 \\
\hline Plus Degrees in the 60s & & 3.72 & & 0.52 \\
\hline Plus Degrees in the 70s & & -0.73 & & -0.11 \\
\hline Plus Degrees Greater Than 80 & & -0.83 & & -0.04 \\
\hline Snowfall (inches) & & -14.23 & & -5.05 \\
\hline Precipitation (inches) & & -5.74 & & -7.69 \\
\hline Darkness Before Midnight (hours) & & -5.06 & & -2.64 \\
\hline Thanksgiving/Christmas (proportion of week) & & -148.35 & & -57.17 \\
\hline Winter Holiday (proportion of week) & & 12.30 & & 8.84 \\
\hline Other Out-of-School Days (proportion of week) & & -12.77 & & -4.85 \\
\hline Summer Vacation (proportion of week) & & -2.09 & & 1.59 \\
\hline Summer Holiday (proportion of week) & & -42.40 & & -4.29 \\
\hline Observations & 373 & 83 & 373 & 373 \\
\hline $\mathrm{R}^{2}$ & 0.70 & 0.32 & 0.66 & 0.74 \\
\hline
\end{tabular}


35.3 Least Squares Updated Model (2) for western western

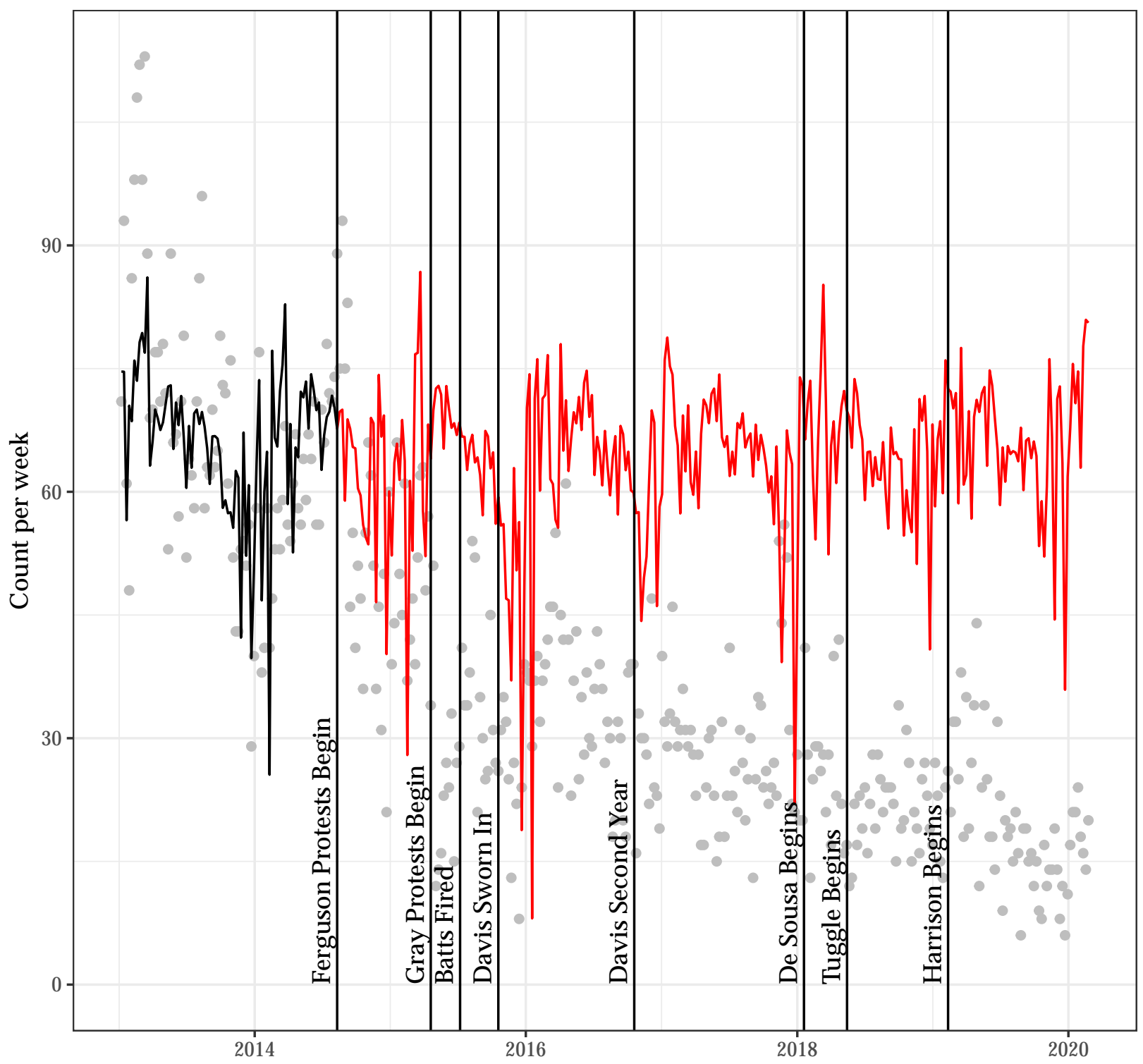




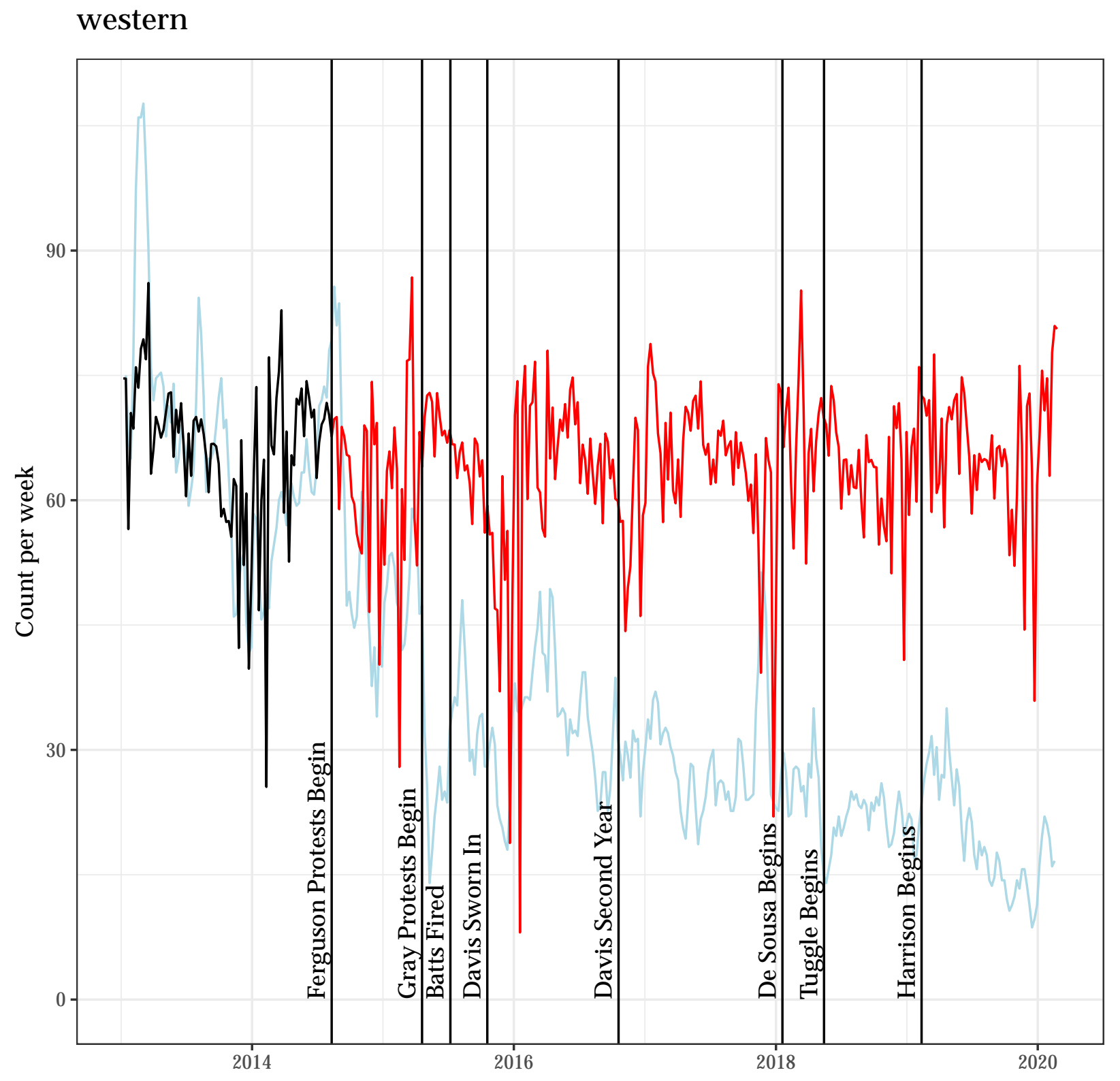


Autocorrelation Function for the Observed Outcome for Updated Model (2)

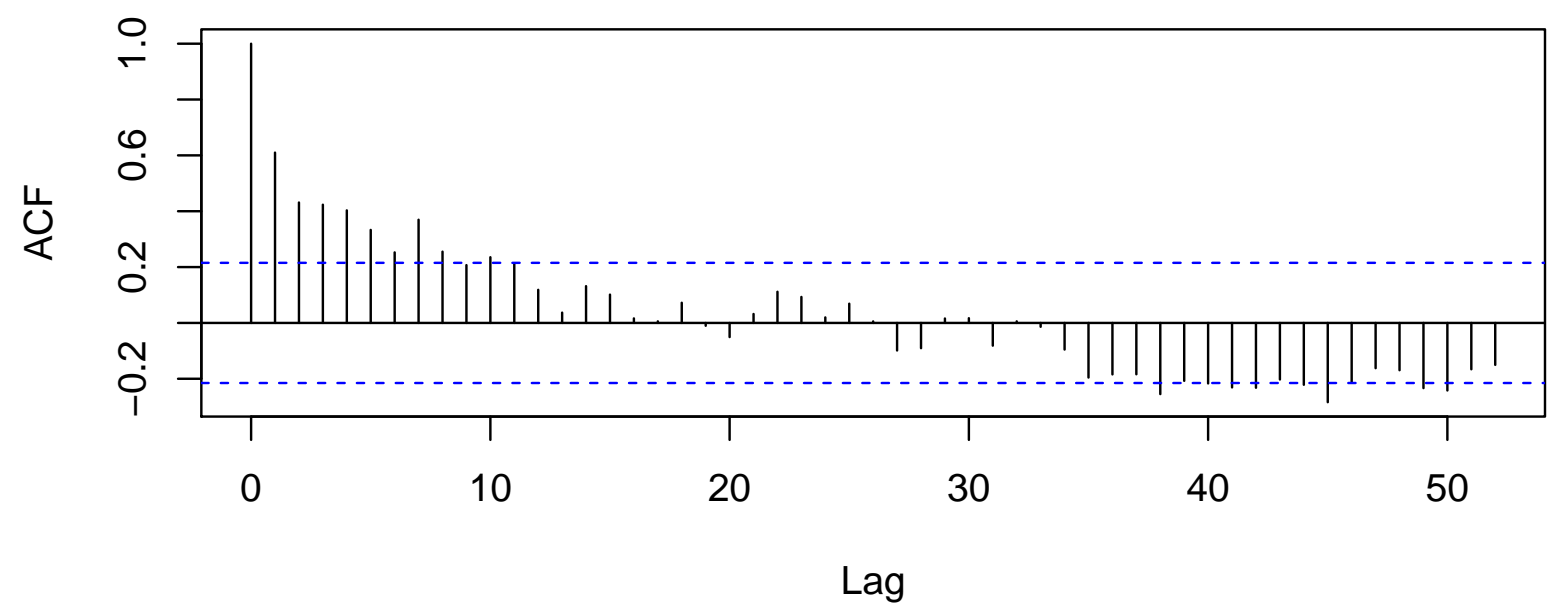

Autocorrelation Function for the Residuals from Updated Model (2)

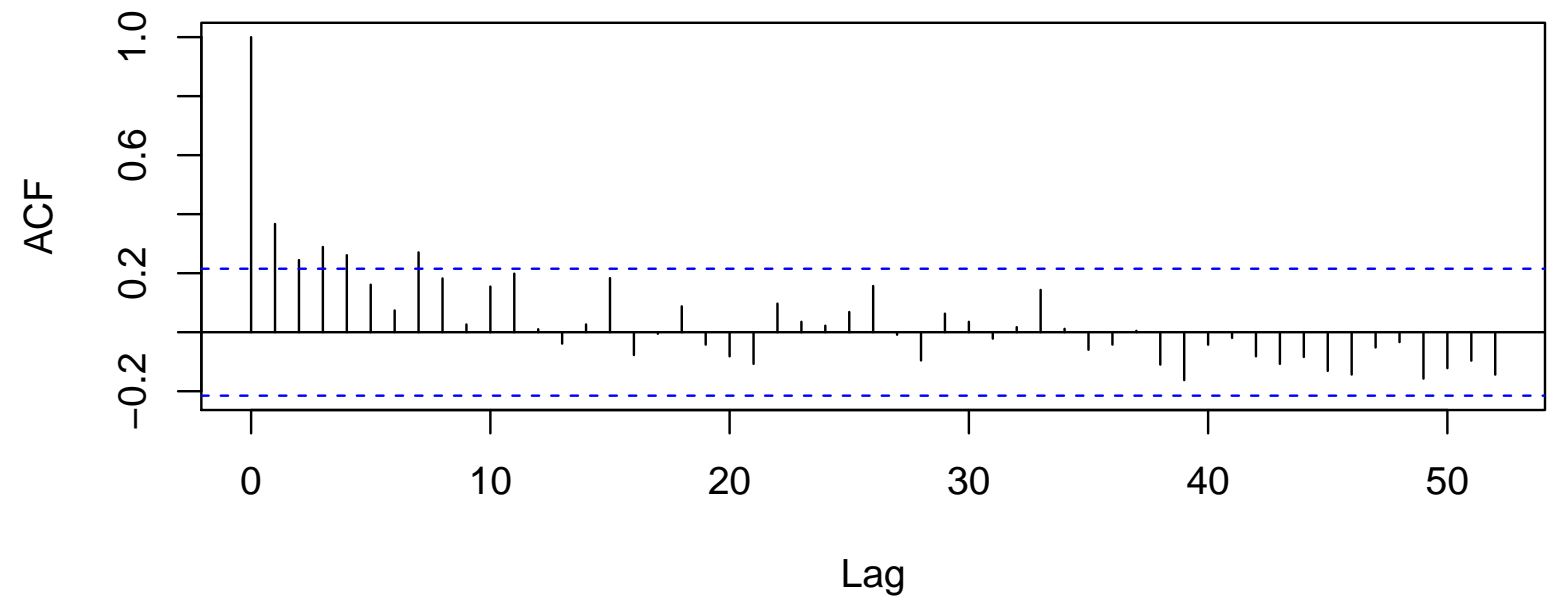




\subsection{A Poisson Regression Model as an Alternative to Model (2) for western}

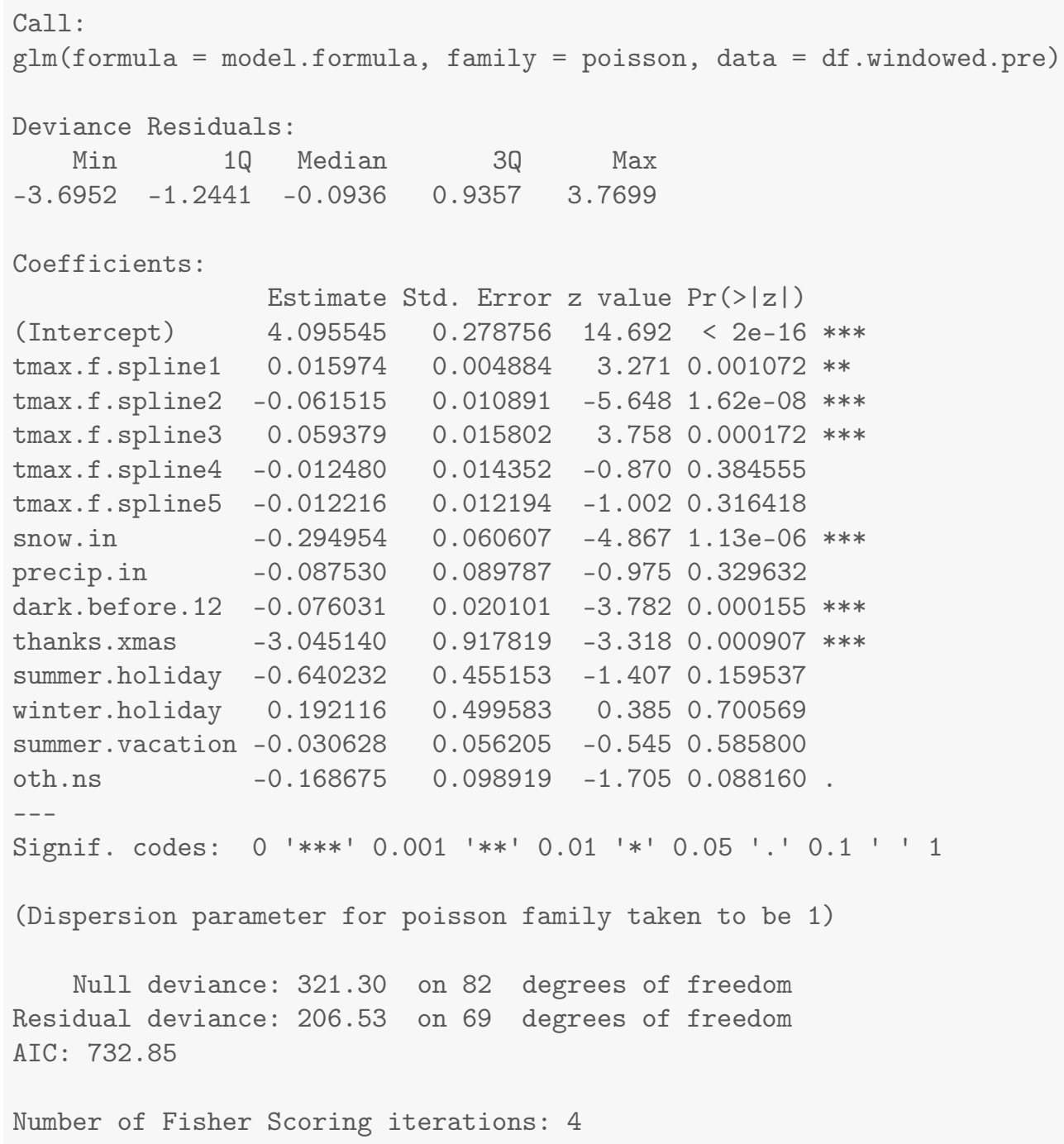




\section{Results for central}

\subsection{Summary Values for central}

Table 36.1: Descriptives for Outcome Before Ferguson Protests Begin

\begin{tabular}{lcccccccc}
\hline \hline Statistic & $\mathrm{N}$ & Mean & St. Dev. & Min & Pctl(25) & Median & Pctl(75) & Max \\
\hline central & 83 & 61.072 & 12.740 & 27 & 54 & 61 & 69.5 & 87 \\
\hline
\end{tabular}

Table 36.2: Descriptives for Outcome After Ferguson Protests Begin

\begin{tabular}{lcccccccc}
\hline \hline Statistic & $\mathrm{N}$ & Mean & St. Dev. & Min & Pctl(25) & Median & Pctl(75) & Max \\
\hline central & 290 & 24.683 & 8.935 & 6 & 18 & 24 & 30 & 85 \\
\hline
\end{tabular}




\subsection{Four Models for central}

Table 36.3: Four Models that Differ on the Specification of Adjustment and Intervention Variables

\begin{tabular}{|c|c|c|c|c|}
\hline & \multicolumn{4}{|c|}{ Outcome: Count Per Week } \\
\hline & $(1)$ & $(2)$ & (3) & $(4)$ \\
\hline After Ferguson Protests Begin (week of $8 / 11 / 14$ onward) & -27.43 & & -26.72 & -26.19 \\
\hline After Gray Protests Begin (week of 4/20/15 onward) & -11.64 & & -14.30 & -14.10 \\
\hline Unrest and National Guard $(4 / 27 / 15-5 / 3 / 2015)$ & 63.00 & & 57.41 & 59.66 \\
\hline After Batts Fired (week of $7 / 13 / 15$ onward) & 6.50 & & 7.50 & 6.57 \\
\hline After Davis Confirmed (week of 10/19/15 onward) & -1.19 & & 1.19 & 0.15 \\
\hline After Davis First Year (week of 10/17/16 onward) & -4.03 & & -5.29 & -3.98 \\
\hline After De Sousa Begins (week of $1 / 19 / 18$ onward) & -2.84 & & -2.69 & -3.31 \\
\hline After Tuggle Begins (week of 5/14/18 onward) & -1.33 & & 0.64 & 0.22 \\
\hline After Harrison Begins (week of 2/11/19 onward) & 2.59 & & 0.22 & 1.17 \\
\hline Average Maximum Temperature to 50 Degrees & & 0.02 & & 0.25 \\
\hline Plus Degrees in the $50 \mathrm{~s}$ & & -1.30 & & -0.54 \\
\hline Plus Degrees in the 60s & & 3.04 & & 0.75 \\
\hline Plus Degrees in the 70s & & -1.73 & & -0.74 \\
\hline Plus Degrees Greater Than 80 & & -0.43 & & 0.28 \\
\hline Snowfall (inches) & & -10.59 & & -1.89 \\
\hline Precipitation (inches) & & -14.15 & & -8.75 \\
\hline Darkness Before Midnight (hours) & & -1.42 & & -1.13 \\
\hline Thanksgiving/Christmas (proportion of week) & & -213.58 & & -62.55 \\
\hline Winter Holiday (proportion of week) & & -10.72 & & 8.37 \\
\hline Other Out-of-School Days (proportion of week) & & 22.10 & & 2.50 \\
\hline Summer Vacation (proportion of week) & & -10.14 & & -0.66 \\
\hline Summer Holiday (proportion of week) & & -55.33 & & -12.67 \\
\hline Observations & 373 & 83 & 373 & 373 \\
\hline $\mathrm{R}^{2}$ & 0.78 & 0.36 & 0.70 & 0.81 \\
\hline
\end{tabular}




\subsection{Least Squares Updated Model (2) for central}

central

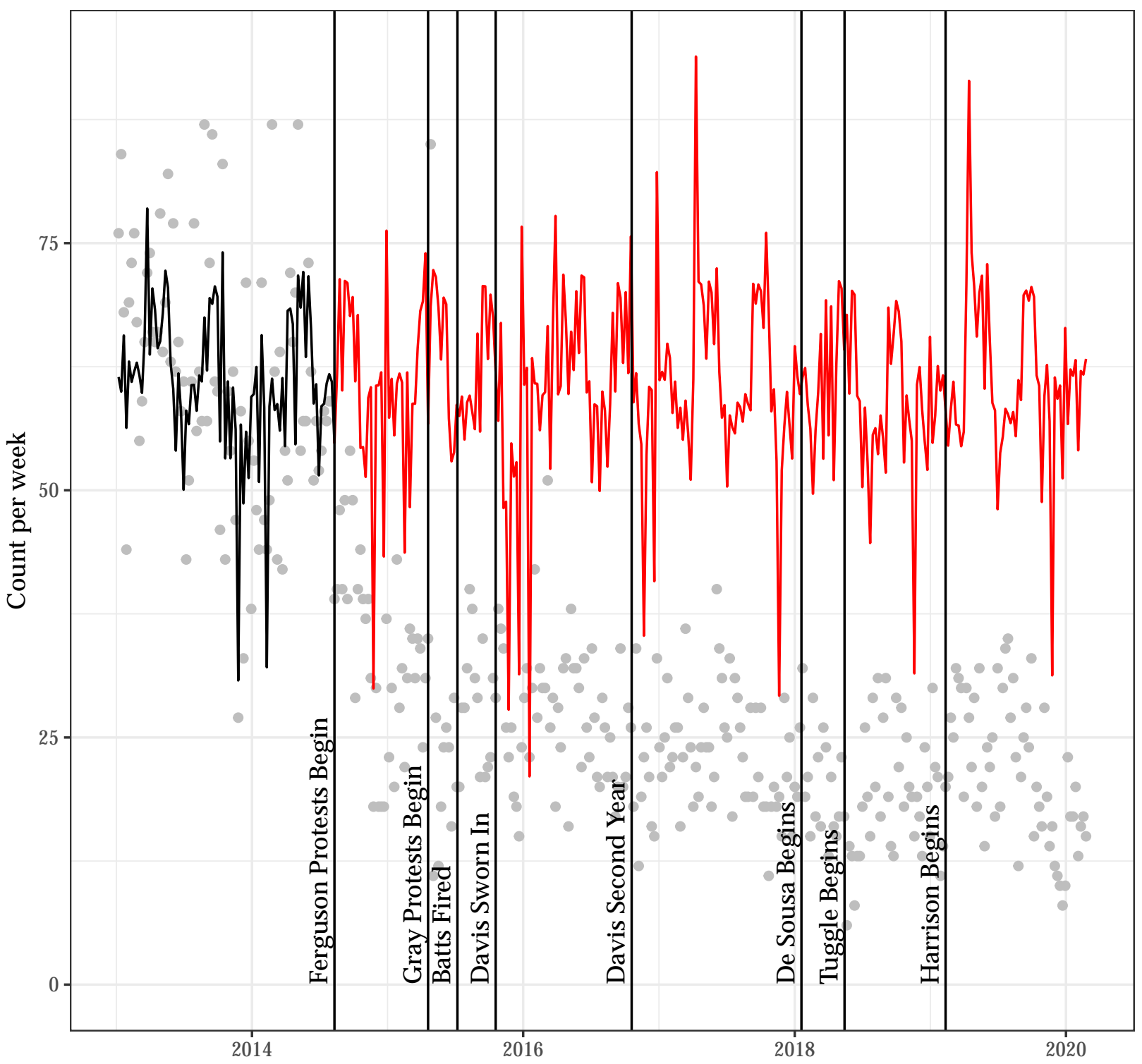




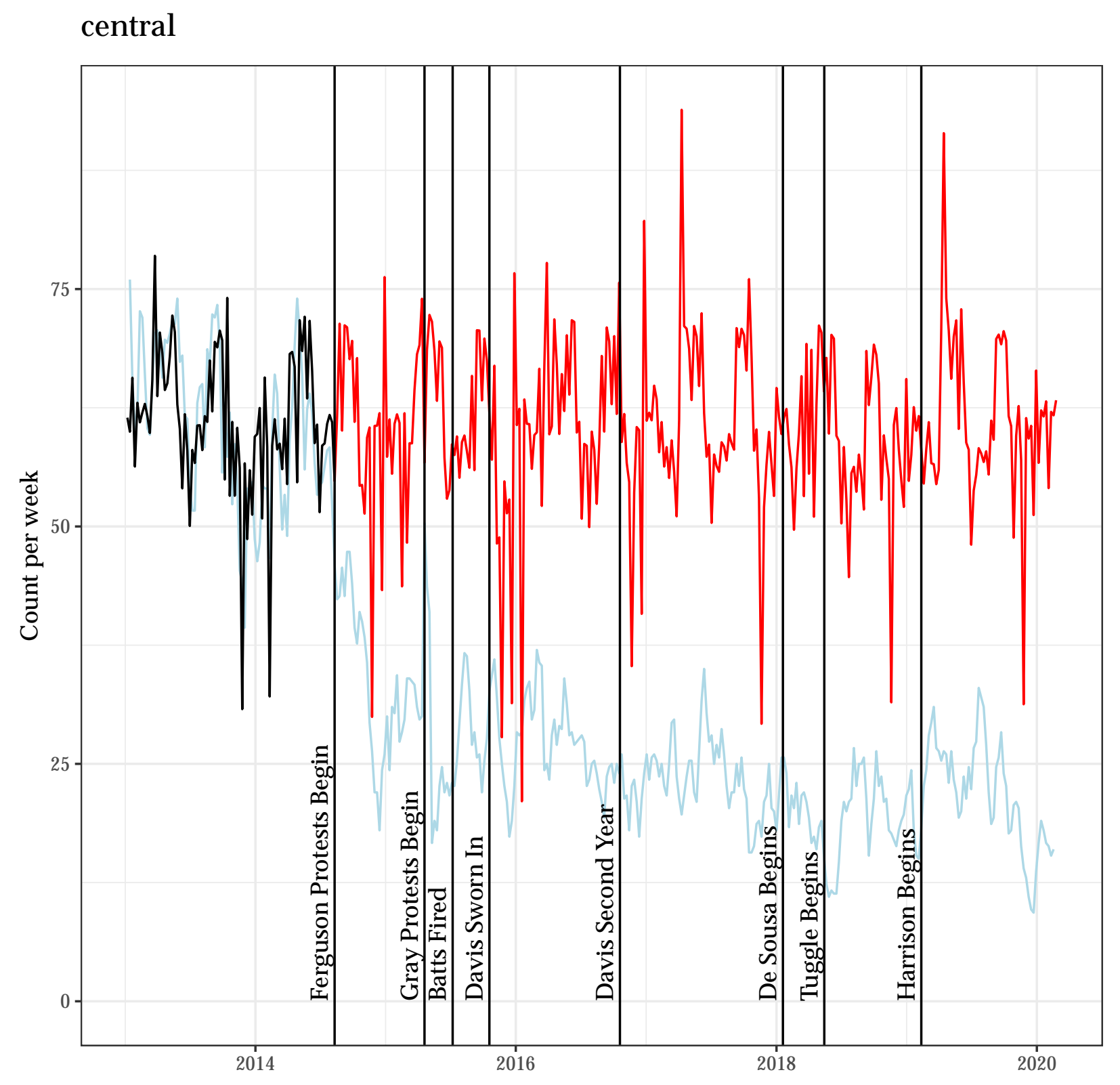


Autocorrelation Function for the Observed Outcome for Updated Model (2)

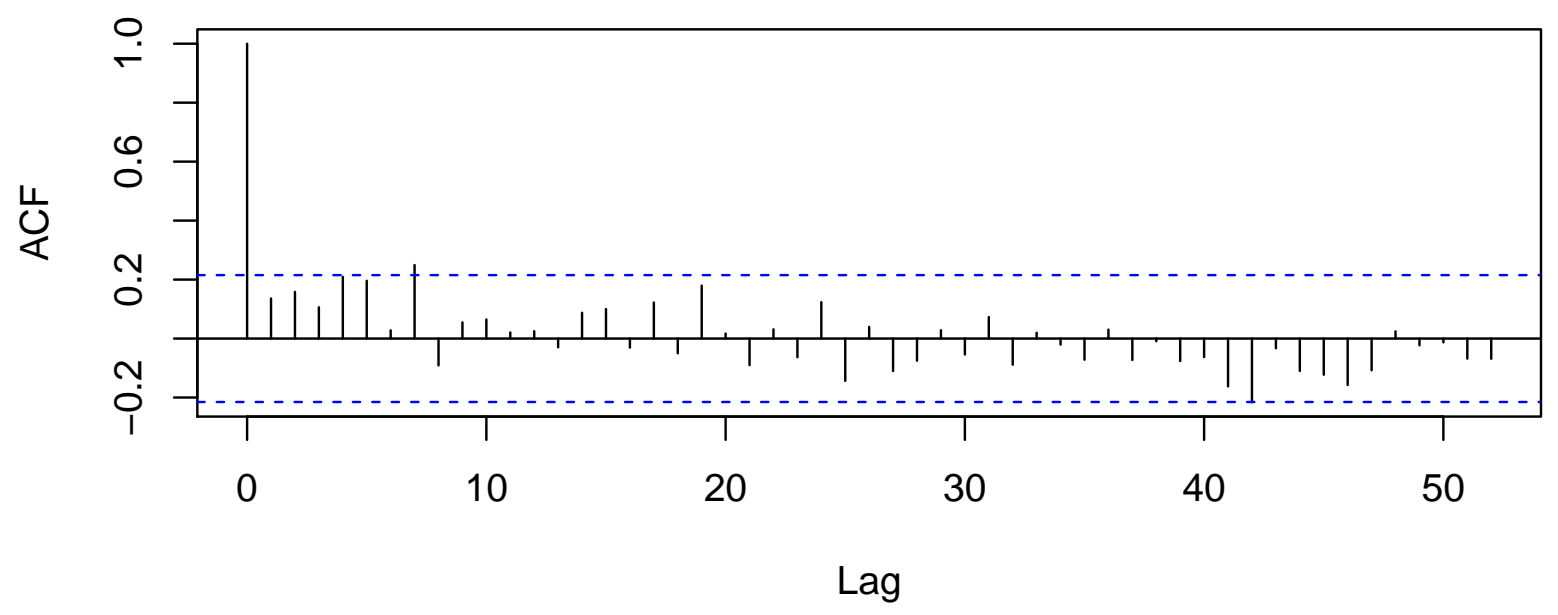

Autocorrelation Function for the Residuals from Updated Model (2)

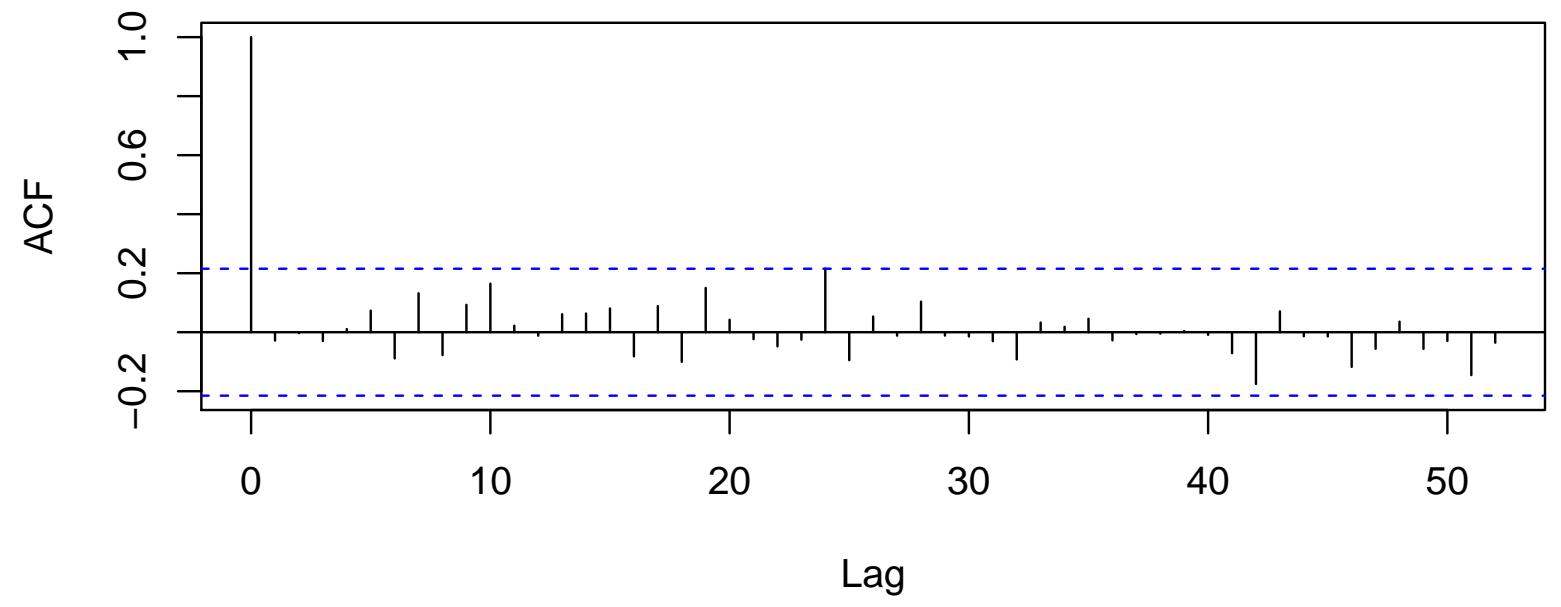




\subsection{A Poisson Regression Model as an Alternative to Model (2) for central}

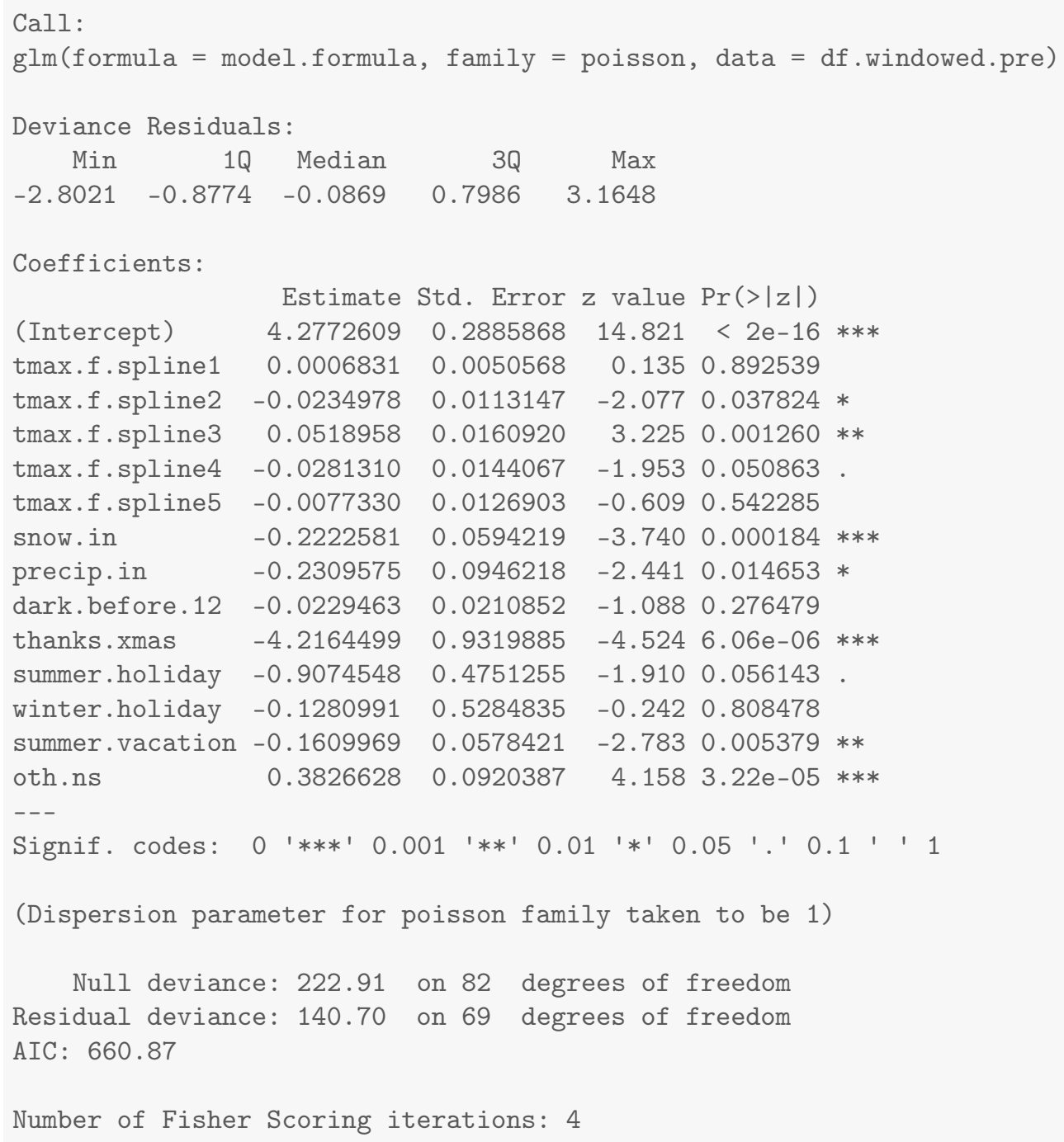




\section{Results for eastern}

\subsection{Summary Values for eastern}

Table 37.1: Descriptives for Outcome Before Ferguson Protests Begin

\begin{tabular}{lcccccccc}
\hline \hline Statistic & $\mathrm{N}$ & Mean & St. Dev. & Min & Pctl(25) & Median & Pctl(75) & Max \\
\hline eastern & 83 & 73.253 & 15.432 & 42 & 62 & 70 & 84.5 & 116 \\
\hline
\end{tabular}

Table 37.2: Descriptives for Outcome After Ferguson Protests Begin

\begin{tabular}{lcccccccc}
\hline \hline Statistic & $\mathrm{N}$ & Mean & St. Dev. & Min & Pctl(25) & Median & Pctl(75) & Max \\
\hline eastern & 290 & 28.169 & 11.496 & 6 & 20.2 & 27 & 35 & 73 \\
\hline
\end{tabular}




\subsection{Four Models for eastern}

Table 37.3: Four Models that Differ on the Specification of Adjustment and Intervention Variables

\begin{tabular}{|c|c|c|c|c|}
\hline & \multicolumn{4}{|c|}{ Outcome: Count Per Week } \\
\hline & $(1)$ & $(2)$ & (3) & (4) \\
\hline After Ferguson Protests Begin (week of $8 / 11 / 14$ onward) & -31.31 & & -30.35 & -30.42 \\
\hline After Gray Protests Begin (week of $4 / 20 / 15$ onward) & -16.31 & & -17.05 & -17.63 \\
\hline Unrest and National Guard $(4 / 27 / 15-5 / 3 / 2015)$ & 47.36 & & 39.13 & 44.66 \\
\hline After Batts Fired (week of 7/13/15 onward) & 7.65 & & 2.46 & 7.05 \\
\hline After Davis Confirmed (week of 10/19/15 onward) & -2.96 & & -0.26 & -2.44 \\
\hline After Davis First Year (week of 10/17/16 onward) & 1.14 & & 0.45 & 1.03 \\
\hline After De Sousa Begins (week of 1/19/18 onward) & -0.66 & & 1.06 & -0.93 \\
\hline After Tuggle Begins (week of 5/14/18 onward) & -9.22 & & -7.95 & -7.37 \\
\hline After Harrison Begins (week of 2/11/19 onward) & -6.15 & & -8.94 & -7.72 \\
\hline Average Maximum Temperature to 50 Degrees & & 1.20 & & 0.49 \\
\hline Plus Degrees in the 50s & & -1.59 & & -0.54 \\
\hline Plus Degrees in the 60s & & 1.44 & & 0.21 \\
\hline Plus Degrees in the 70s & & -1.14 & & -0.13 \\
\hline Plus Degrees Greater Than 80 & & 0.66 & & -0.11 \\
\hline Snowfall (inches) & & -1.38 & & -0.13 \\
\hline Precipitation (inches) & & -27.43 & & -11.32 \\
\hline Darkness Before Midnight (hours) & & 2.52 & & 0.17 \\
\hline Thanksgiving/Christmas (proportion of week) & & -70.34 & & -55.63 \\
\hline Winter Holiday (proportion of week) & & 26.94 & & 25.56 \\
\hline Other Out-of-School Days (proportion of week) & & -11.26 & & -1.70 \\
\hline Summer Vacation (proportion of week) & & -6.86 & & -1.12 \\
\hline Summer Holiday (proportion of week) & & -45.91 & & -5.61 \\
\hline Observations & 373 & 83 & 373 & 373 \\
\hline $\mathrm{R}^{2}$ & 0.81 & 0.26 & 0.80 & 0.82 \\
\hline
\end{tabular}




\subsection{Least Squares Updated Model (2) for eastern}

\section{eastern}

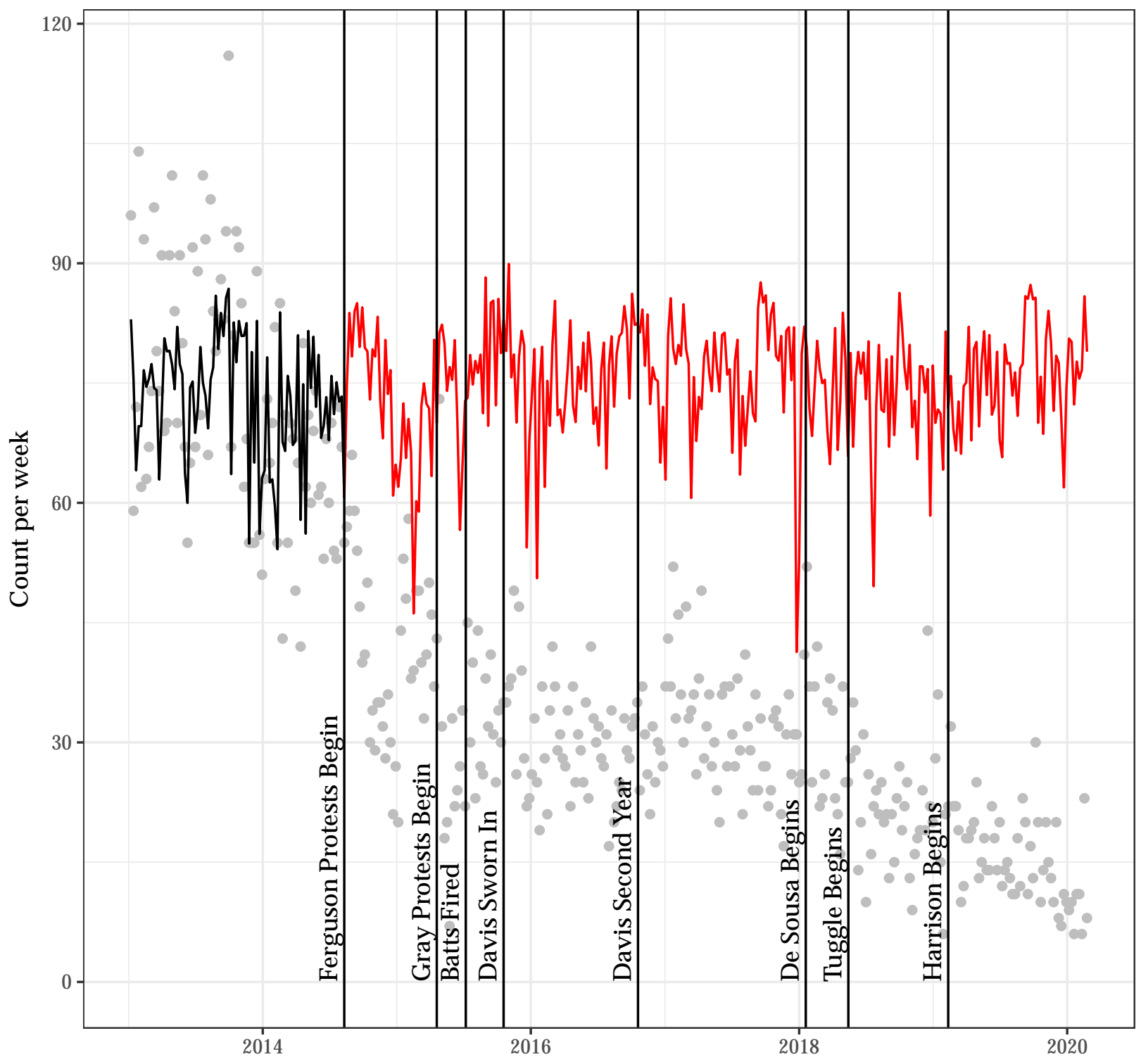




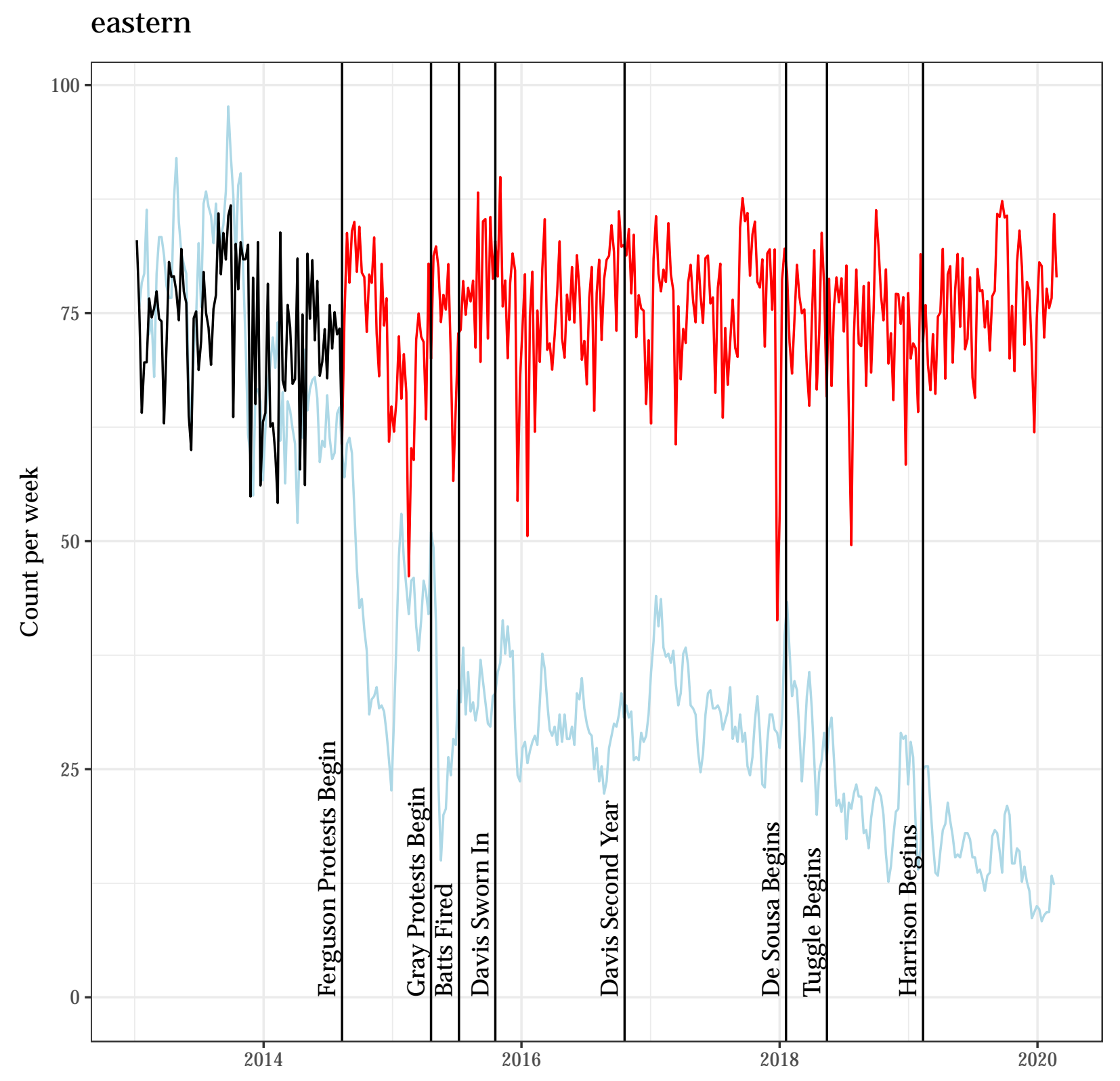


Autocorrelation Function for the Observed Outcome for Updated Model (2)

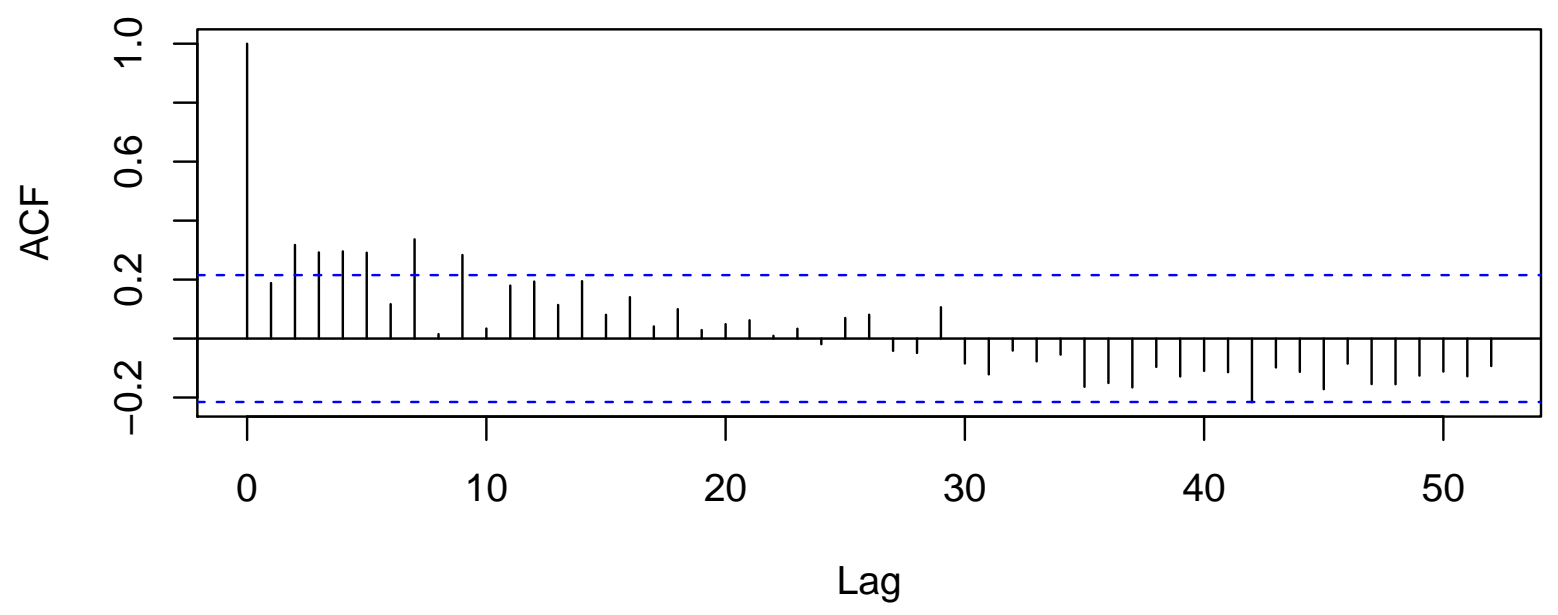

Autocorrelation Function for the Residuals from Updated Model (2)

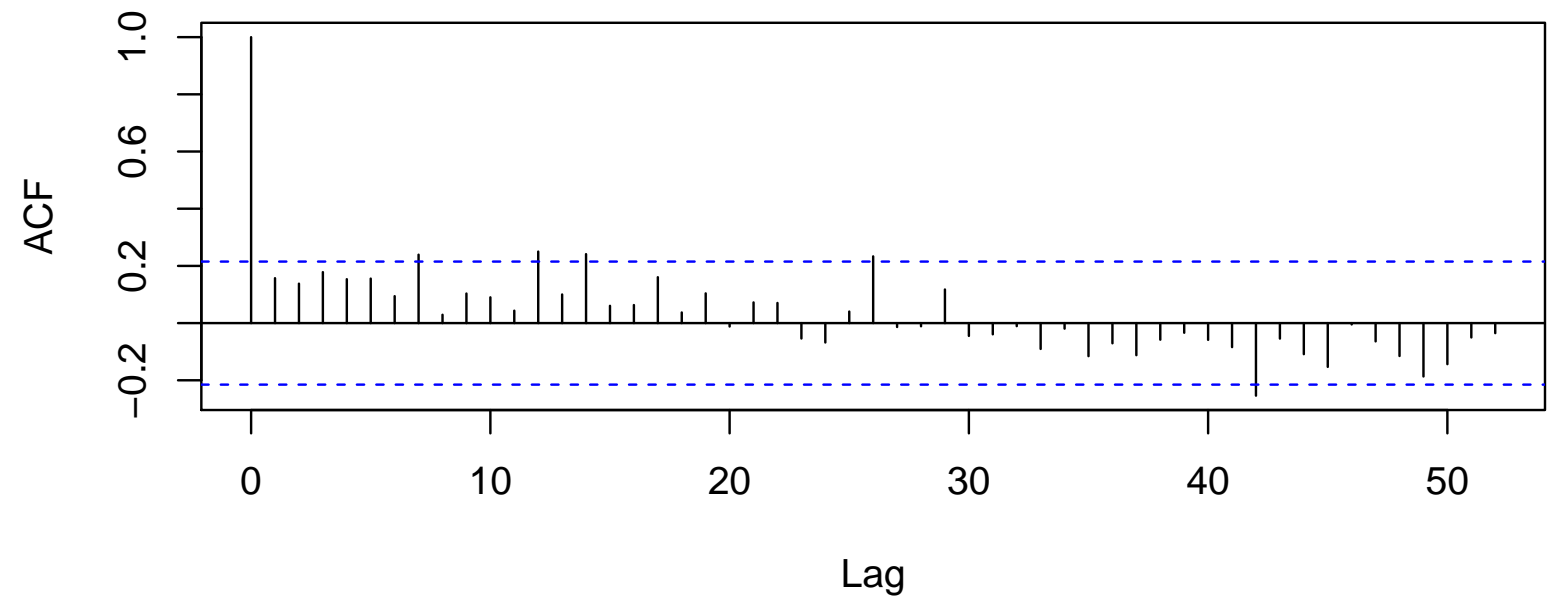




\subsection{A Poisson Regression Model as an Alternative to Model (2) for eastern}

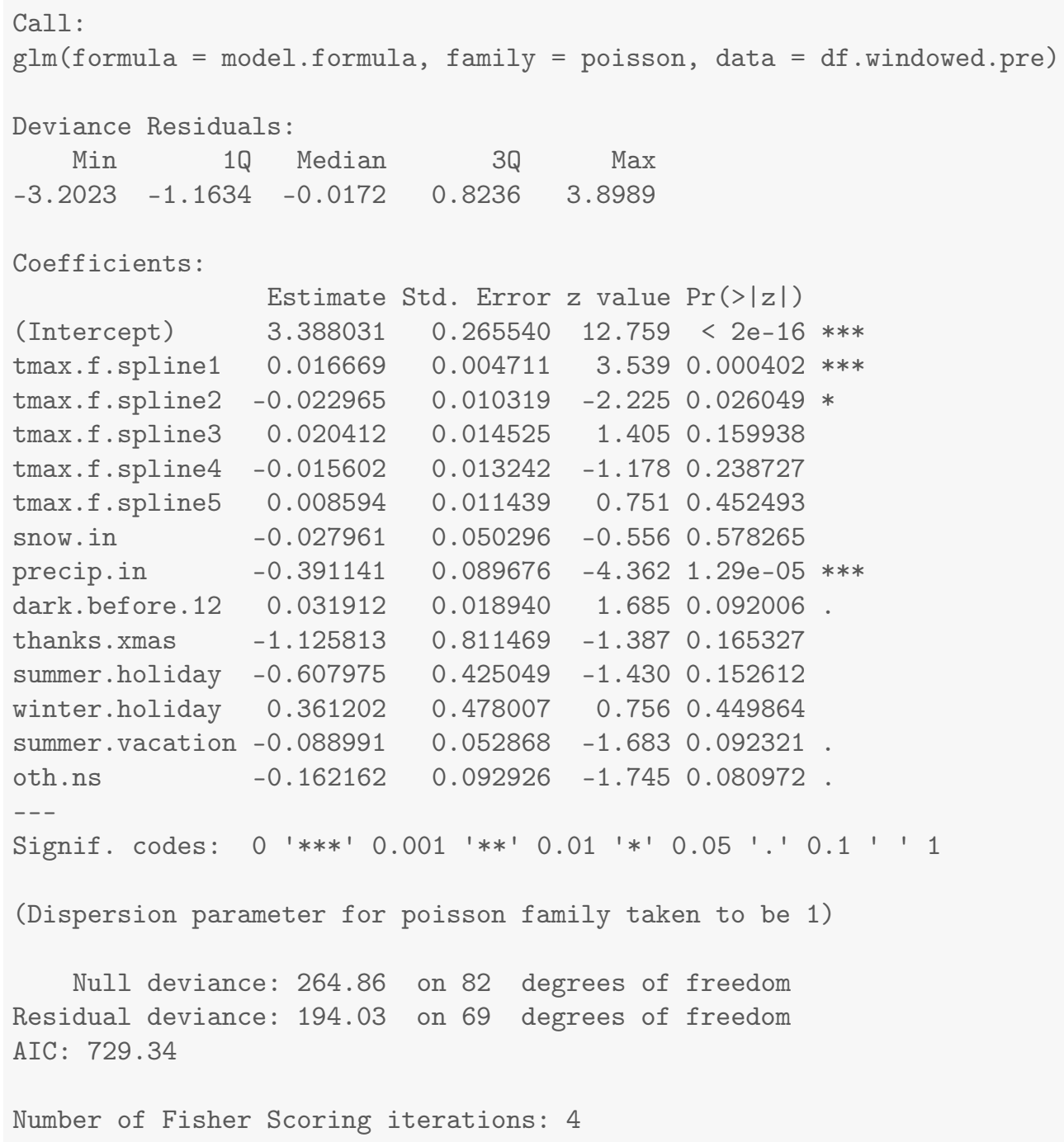




\section{Results for southwestern}

\subsection{Summary Values for southwestern}

Table 38.1: Descriptives for Outcome Before Ferguson Protests Begin

\begin{tabular}{lcccccccc}
\hline \hline Statistic & $\mathrm{N}$ & Mean & St. Dev. & Min & Pctl(25) & Median & Pctl(75) & Max \\
\hline southwestern & 83 & 42.639 & 8.467 & 23 & 36.5 & 42 & 47.5 & 65 \\
\hline
\end{tabular}

Table 38.2: Descriptives for Outcome After Ferguson Protests Begin

\begin{tabular}{lcccccccc}
\hline \hline Statistic & $\mathrm{N}$ & Mean & St. Dev. & Min & $\operatorname{Pctl}(25)$ & Median & $\operatorname{Pctl}(75)$ & Max \\
\hline southwestern & 290 & 25.183 & 8.225 & 8 & 19 & 24 & 30 & 57 \\
\hline
\end{tabular}




\subsection{Four Models for southwestern}

Table 38.3: Four Models that Differ on the Specification of Adjustment and Intervention Variables

\begin{tabular}{|c|c|c|c|c|}
\hline & \multicolumn{4}{|c|}{ Outcome: Count Per Week } \\
\hline & $(1)$ & (2) & (3) & $(4)$ \\
\hline After Ferguson Protests Begin (week of $8 / 11 / 14$ onward) & -9.06 & & -8.47 & -7.92 \\
\hline After Gray Protests Begin (week of $4 / 20 / 15$ onward) & -15.67 & & -17.34 & -18.20 \\
\hline Unrest and National Guard (4/27/15 - 5/3/2015) & 10.09 & & 12.35 & 9.28 \\
\hline After Batts Fired (week of $7 / 13 / 15$ onward) & 12.23 & & 10.26 & 13.02 \\
\hline After Davis Confirmed (week of 10/19/15 onward) & -4.89 & & -3.11 & -4.17 \\
\hline After Davis First Year (week of 10/17/16 onward) & -2.78 & & -2.59 & -2.80 \\
\hline After De Sousa Begins (week of 1/19/18 onward) & 3.41 & & 5.39 & 3.57 \\
\hline After Tuggle Begins (week of 5/14/18 onward) & 0.71 & & -0.73 & 1.58 \\
\hline After Harrison Begins (week of 2/11/19 onward) & -4.77 & & -4.59 & -5.77 \\
\hline Average Maximum Temperature to 50 Degrees & & -0.25 & & 0.17 \\
\hline Plus Degrees in the $50 \mathrm{~s}$ & & 1.27 & & -0.15 \\
\hline Plus Degrees in the 60s & & -1.56 & & 0.15 \\
\hline Plus Degrees in the 70s & & 0.93 & & -0.18 \\
\hline Plus Degrees Greater Than 80 & & -0.27 & & -0.30 \\
\hline Snowfall (inches) & & -1.95 & & -0.72 \\
\hline Precipitation (inches) & & -6.01 & & -6.44 \\
\hline Darkness Before Midnight (hours) & & 1.14 & & -0.56 \\
\hline Thanksgiving/Christmas (proportion of week) & & -11.63 & & -23.91 \\
\hline Winter Holiday (proportion of week) & & 23.04 & & 34.15 \\
\hline Other Out-of-School Days (proportion of week) & & 3.13 & & 1.27 \\
\hline Summer Vacation (proportion of week) & & 3.46 & & 1.29 \\
\hline Summer Holiday (proportion of week) & & 8.98 & & 10.81 \\
\hline Observations & 373 & 83 & 373 & 373 \\
\hline $\mathrm{R}^{2}$ & 0.54 & 0.19 & 0.55 & 0.58 \\
\hline
\end{tabular}


38.3 Least Squares Updated Model (2) for southwestern southwestern

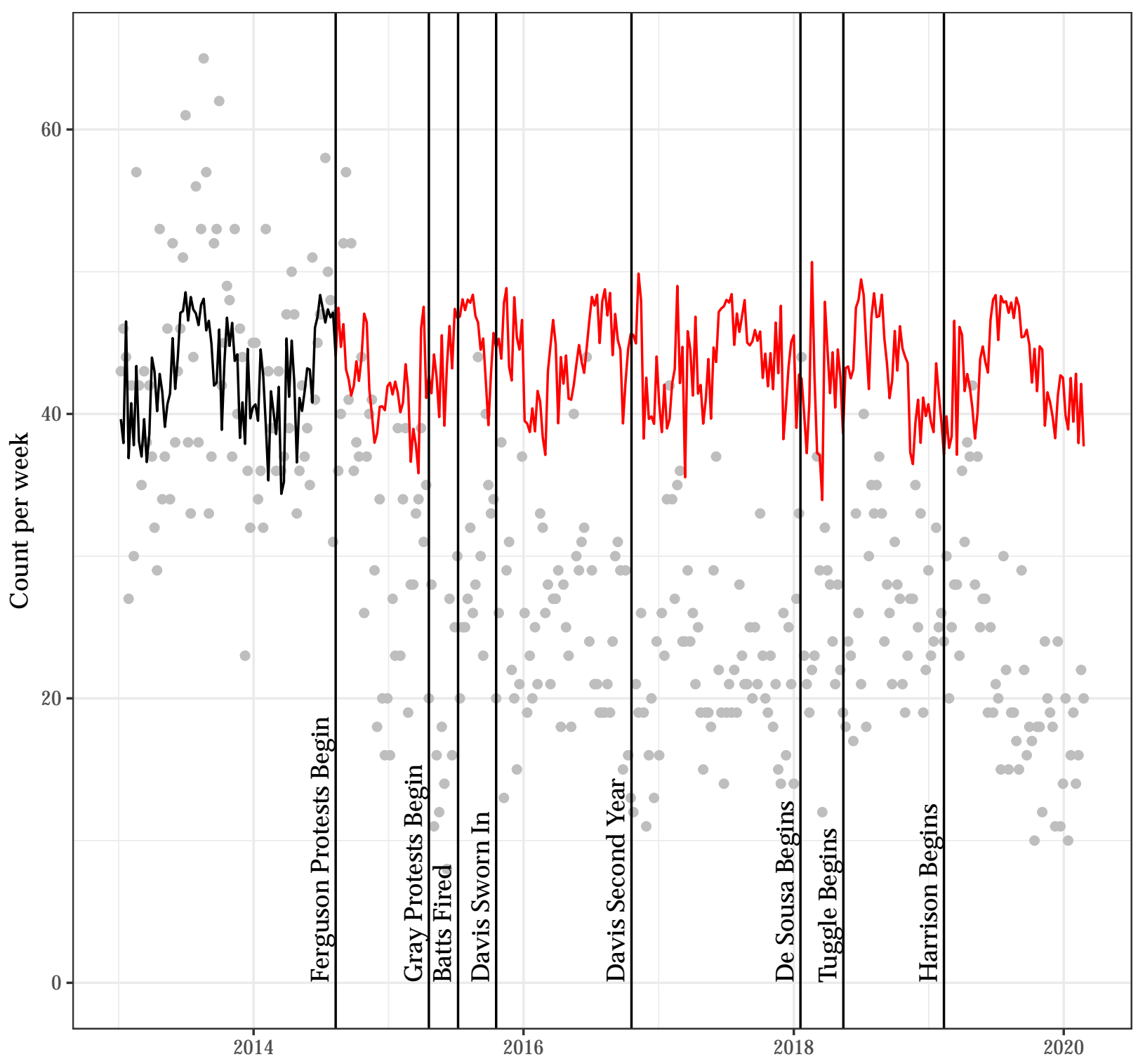




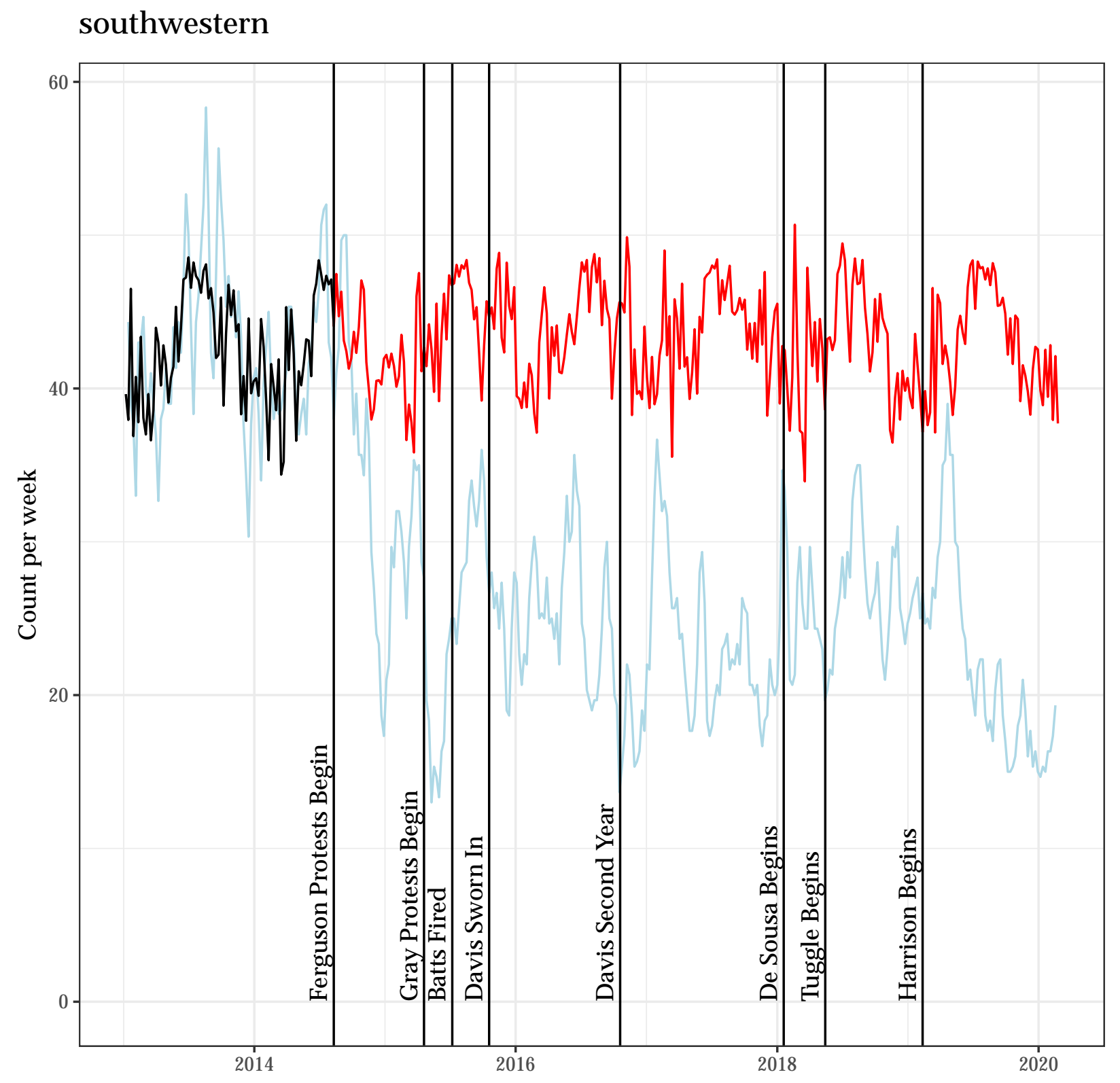


Autocorrelation Function for the Observed Outcome for Updated Model (2)

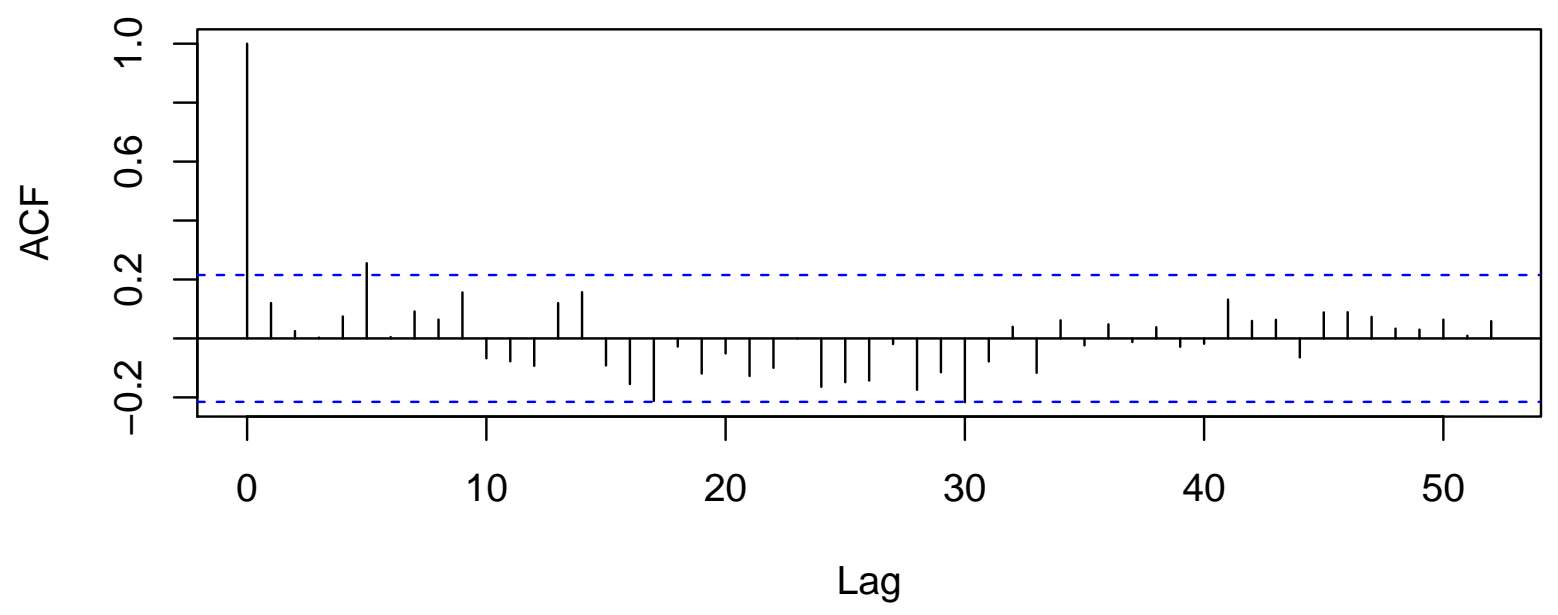

Autocorrelation Function for the Residuals from Updated Model (2)

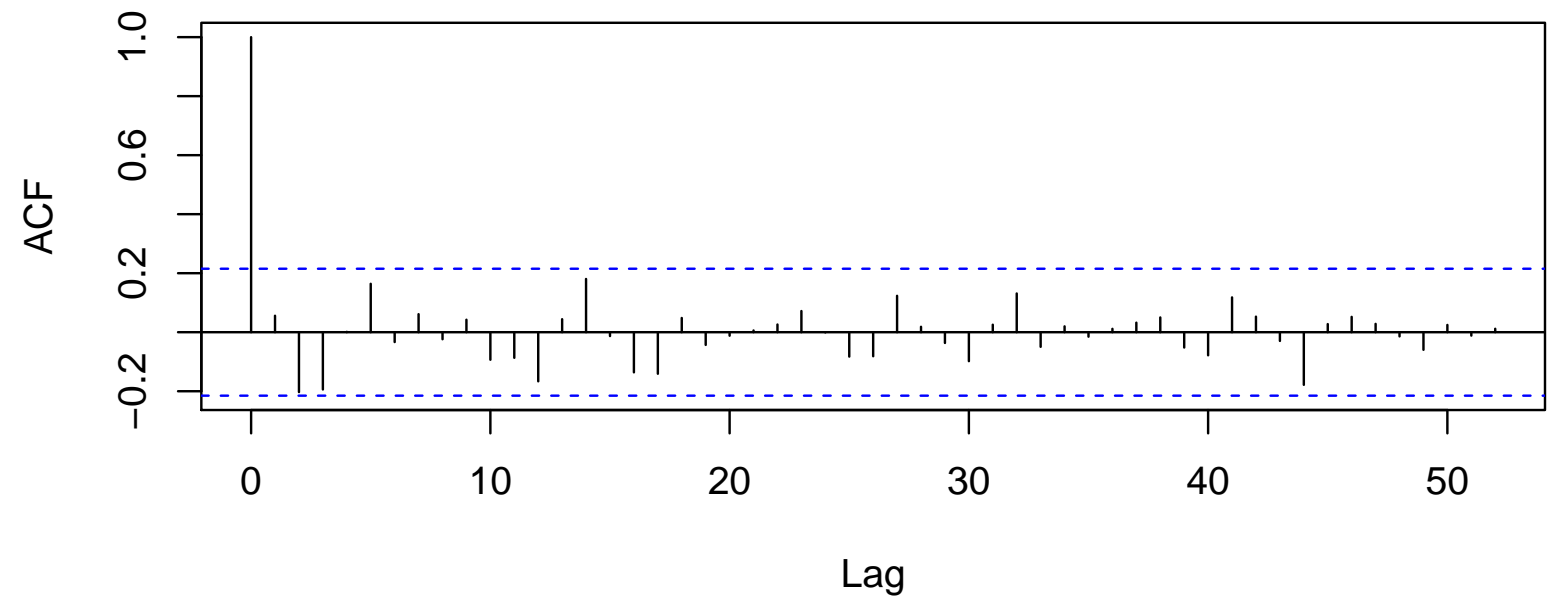




\subsection{A Poisson Regression Model as an Alternative to Model (2) for southwestern}

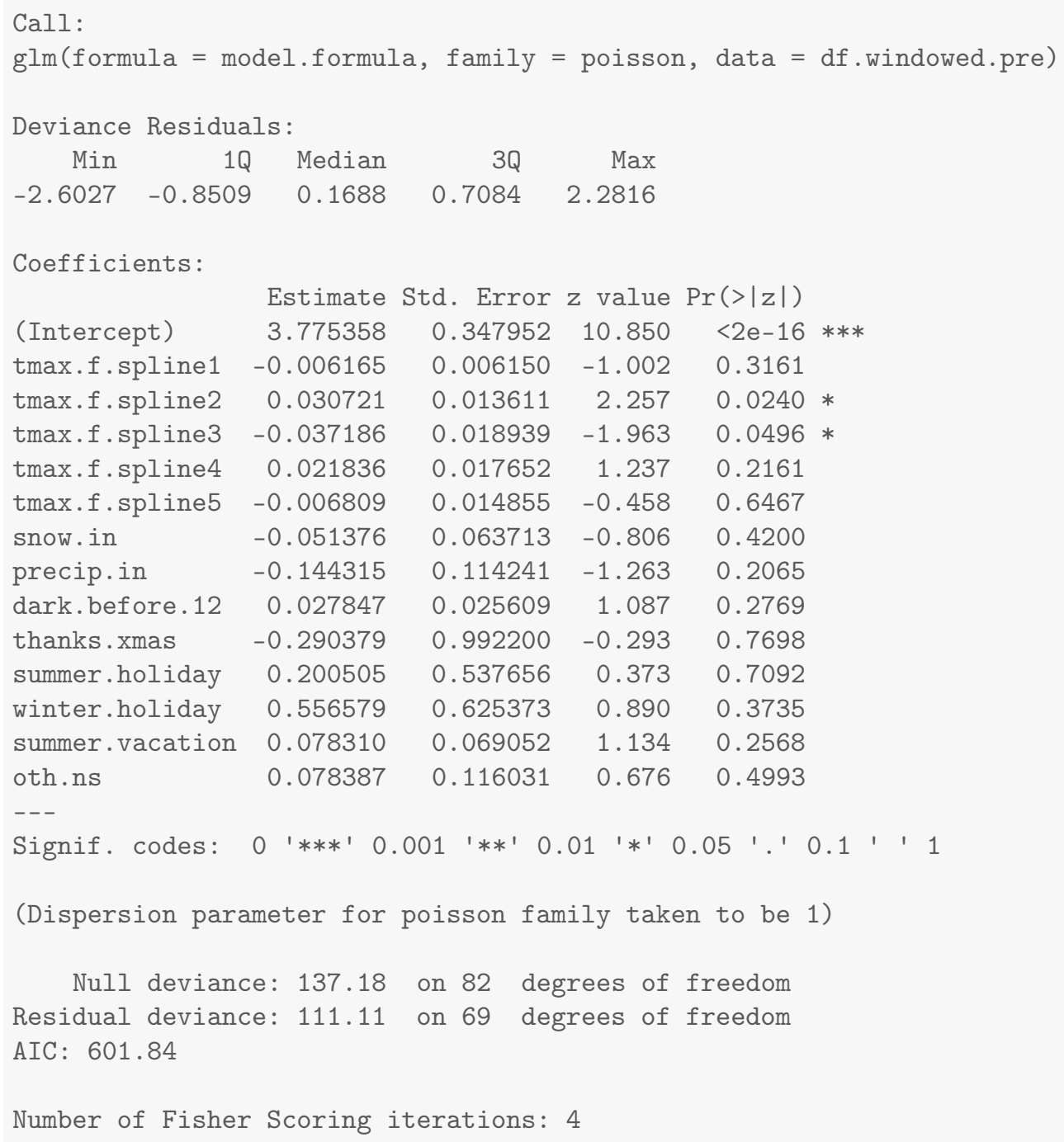




\section{Results for southern}

\subsection{Summary Values for southern}

Table 39.1: Descriptives for Outcome Before Ferguson Protests Begin

\begin{tabular}{lcccccccc}
\hline \hline Statistic & $\mathrm{N}$ & Mean & St. Dev. & Min & Pctl(25) & Median & Pctl(75) & Max \\
\hline southern & 83 & 72.265 & 11.562 & 40 & 64.5 & 72 & 77.5 & 109 \\
\hline
\end{tabular}

Table 39.2: Descriptives for Outcome After Ferguson Protests Begin

\begin{tabular}{lcccccccc}
\hline \hline Statistic & $\mathrm{N}$ & Mean & St. Dev. & Min & Pctl(25) & Median & Pctl(75) & Max \\
\hline southern & 290 & 29.017 & 12.448 & 8 & 20 & 26 & 35 & 83 \\
\hline
\end{tabular}




\subsection{Four Models for southern}

Table 39.3: Four Models that Differ on the Specification of Adjustment and Intervention Variables

\begin{tabular}{|c|c|c|c|c|}
\hline & \multicolumn{4}{|c|}{ Outcome: Count Per Week } \\
\hline & $(1)$ & $(2)$ & (3) & $(4)$ \\
\hline After Ferguson Protests Begin (week of $8 / 11 / 14$ onward) & -21.40 & & -22.41 & -20.64 \\
\hline After Gray Protests Begin (week of 4/20/15 onward) & -29.22 & & -27.20 & -30.47 \\
\hline Unrest and National Guard $(4 / 27 / 15-5 / 3 / 2015)$ & 13.36 & & 12.12 & 11.05 \\
\hline After Batts Fired (week of $7 / 13 / 15$ onward) & 15.15 & & 17.76 & 16.07 \\
\hline After Davis Confirmed (week of 10/19/15 onward) & -2.80 & & -5.23 & -2.99 \\
\hline After Davis First Year (week of $10 / 17 / 16$ onward) & -8.07 & & -8.45 & -7.94 \\
\hline After De Sousa Begins (week of $1 / 19 / 18$ onward) & -4.35 & & -7.64 & -5.26 \\
\hline After Tuggle Begins (week of 5/14/18 onward) & -1.10 & & 4.27 & 1.35 \\
\hline After Harrison Begins (week of 2/11/19 onward) & 0.92 & & -0.75 & -0.43 \\
\hline Average Maximum Temperature to 50 Degrees & & -0.68 & & 0.07 \\
\hline Plus Degrees in the $50 \mathrm{~s}$ & & 0.85 & & 0.12 \\
\hline Plus Degrees in the 60s & & -0.91 & & -0.20 \\
\hline Plus Degrees in the 70s & & 1.55 & & -0.06 \\
\hline Plus Degrees Greater Than 80 & & -1.42 & & -0.38 \\
\hline Snowfall (inches) & & -4.62 & & 0.64 \\
\hline Precipitation (inches) & & -6.63 & & -6.06 \\
\hline Darkness Before Midnight (hours) & & -2.51 & & -0.91 \\
\hline Thanksgiving/Christmas (proportion of week) & & -113.16 & & -41.43 \\
\hline Winter Holiday (proportion of week) & & 24.48 & & 31.20 \\
\hline Other Out-of-School Days (proportion of week) & & 15.67 & & 2.23 \\
\hline Summer Vacation (proportion of week) & & -6.69 & & 1.92 \\
\hline Summer Holiday (proportion of week) & & -89.38 & & -22.48 \\
\hline Observations & 373 & 83 & 373 & 373 \\
\hline $\mathrm{R}^{2}$ & 0.84 & 0.24 & 0.80 & 0.85 \\
\hline
\end{tabular}




\subsection{Least Squares Updated Model (2) for southern}

southern

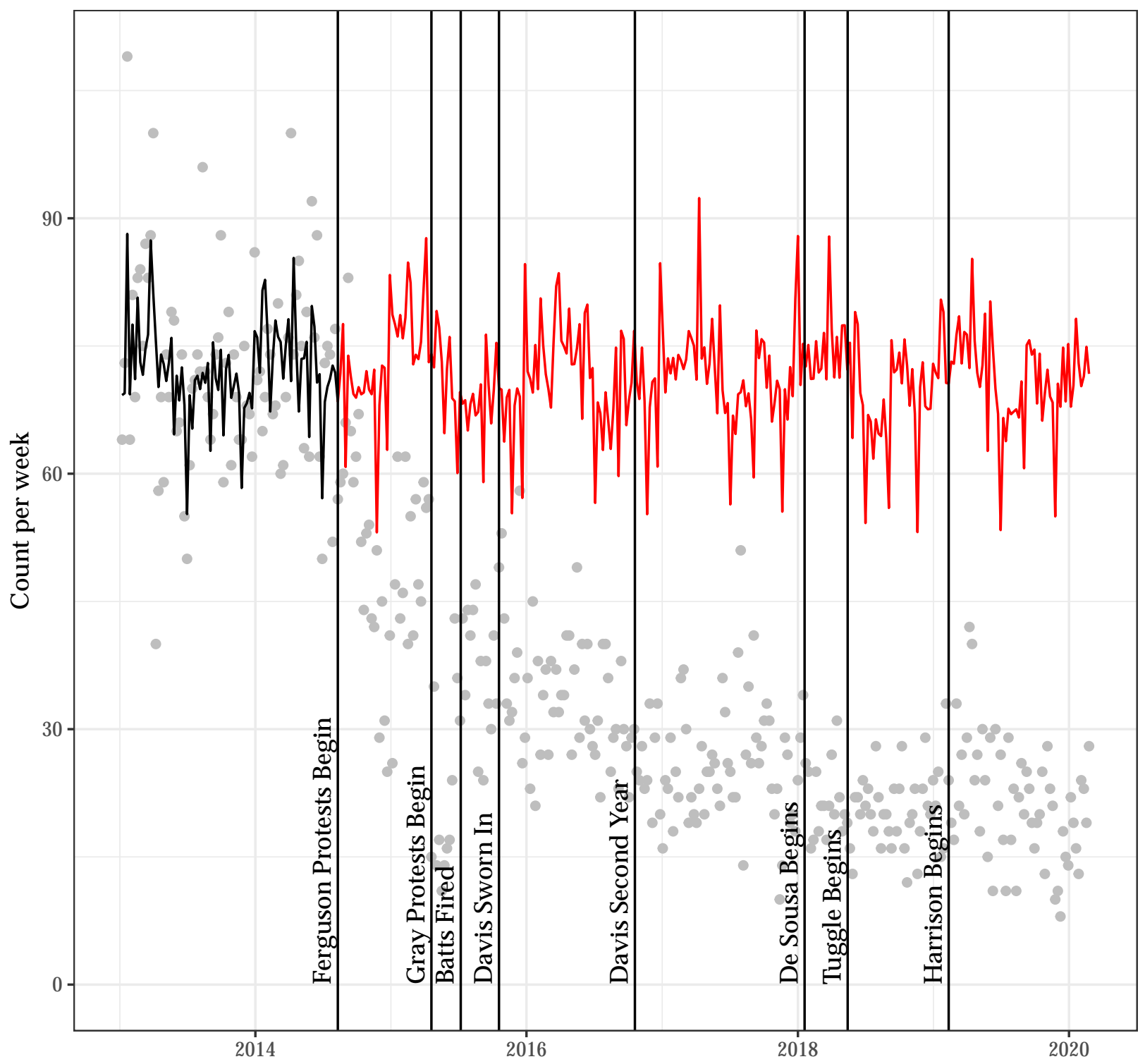




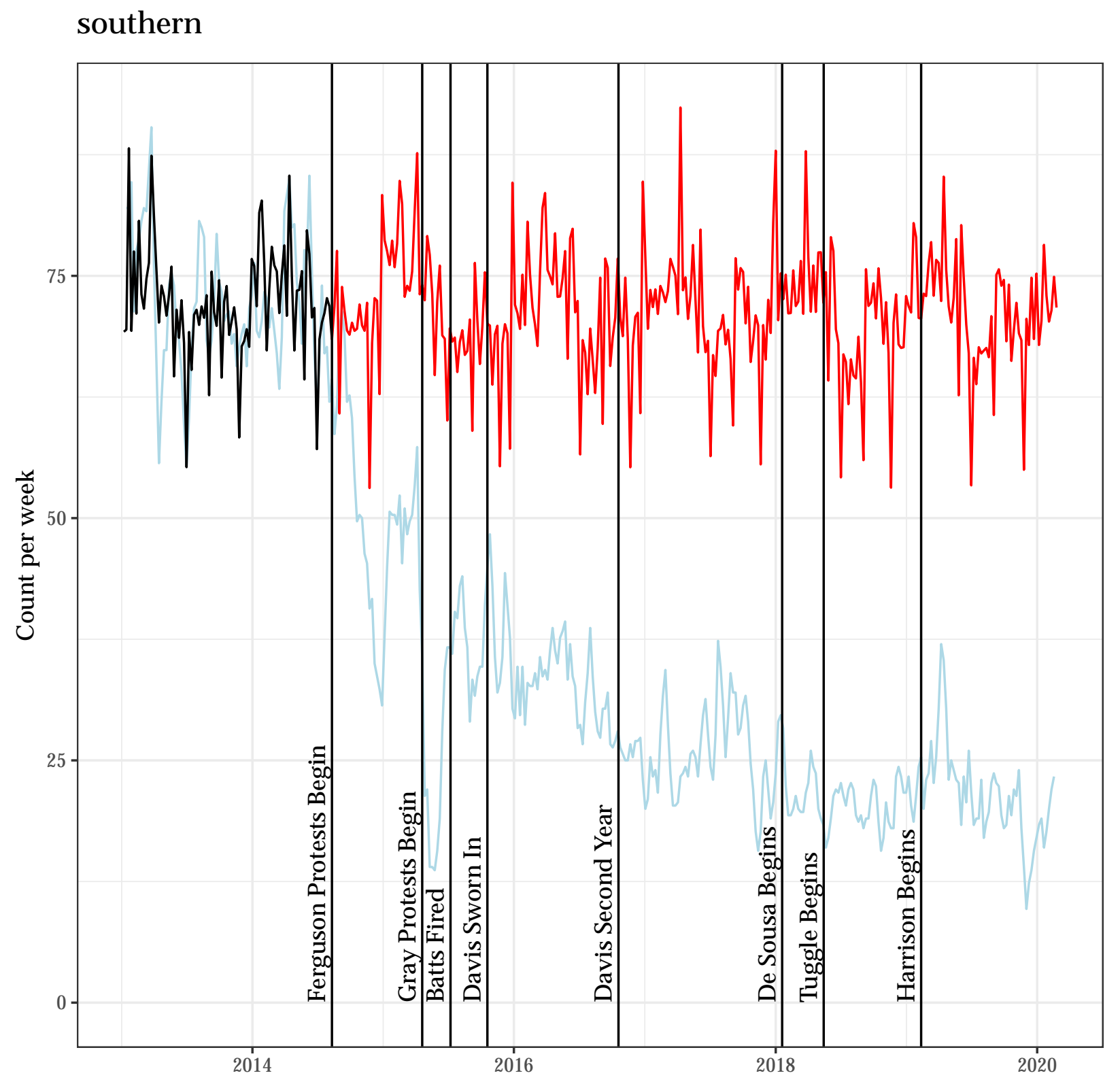


Autocorrelation Function for the Observed Outcome for Updated Model (2)

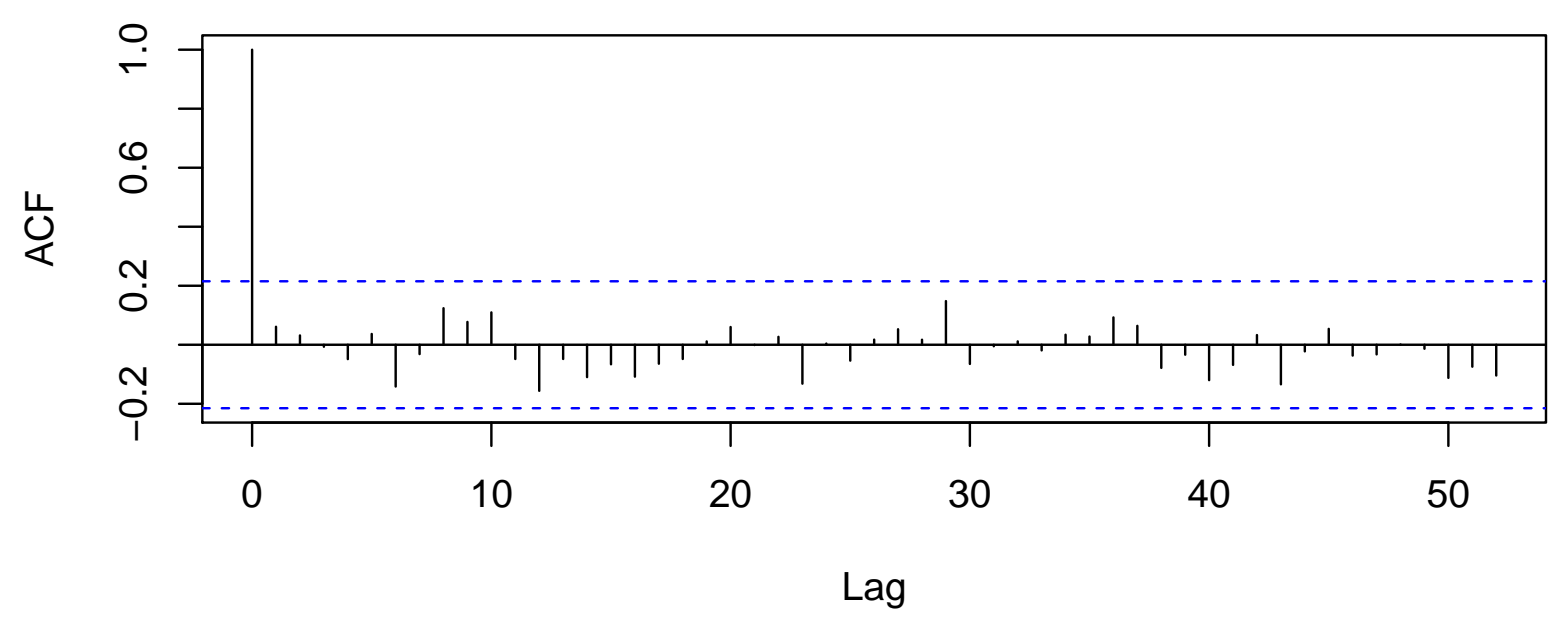

Autocorrelation Function for the Residuals from Updated Model (2)

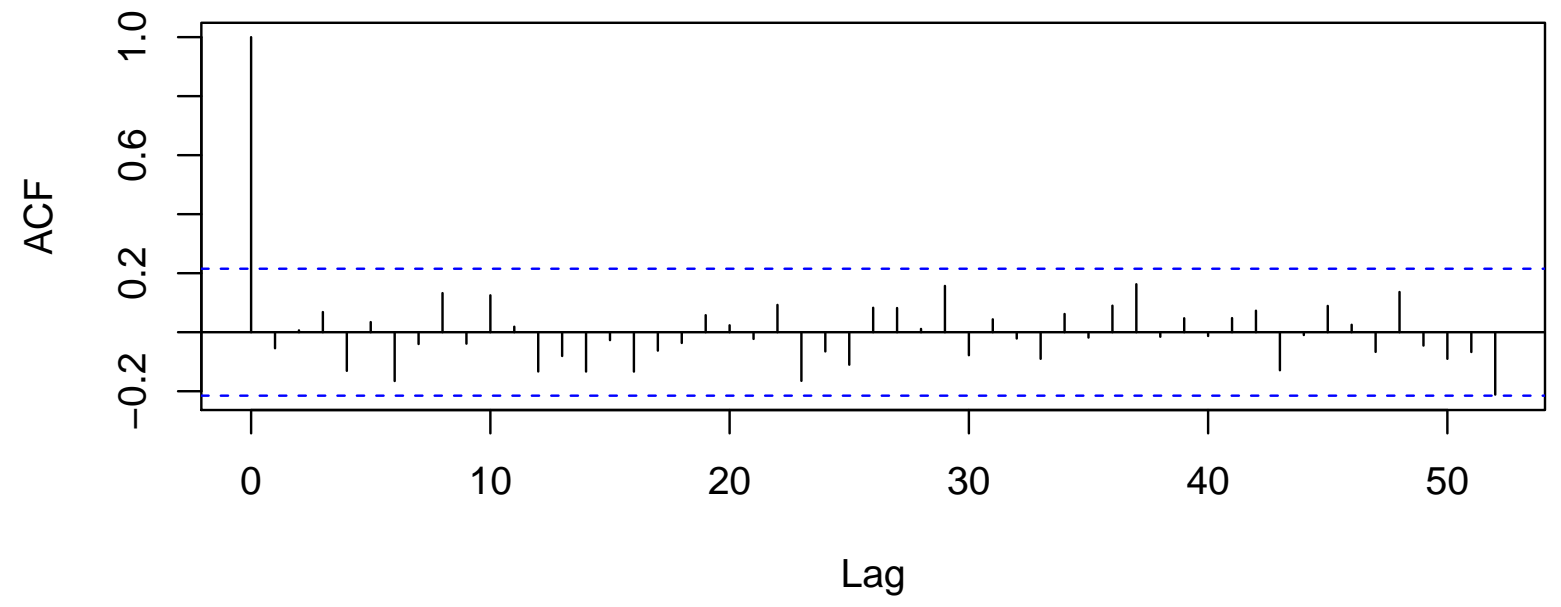




\subsection{A Poisson Regression Model as an Alternative to Model (2) for southern}

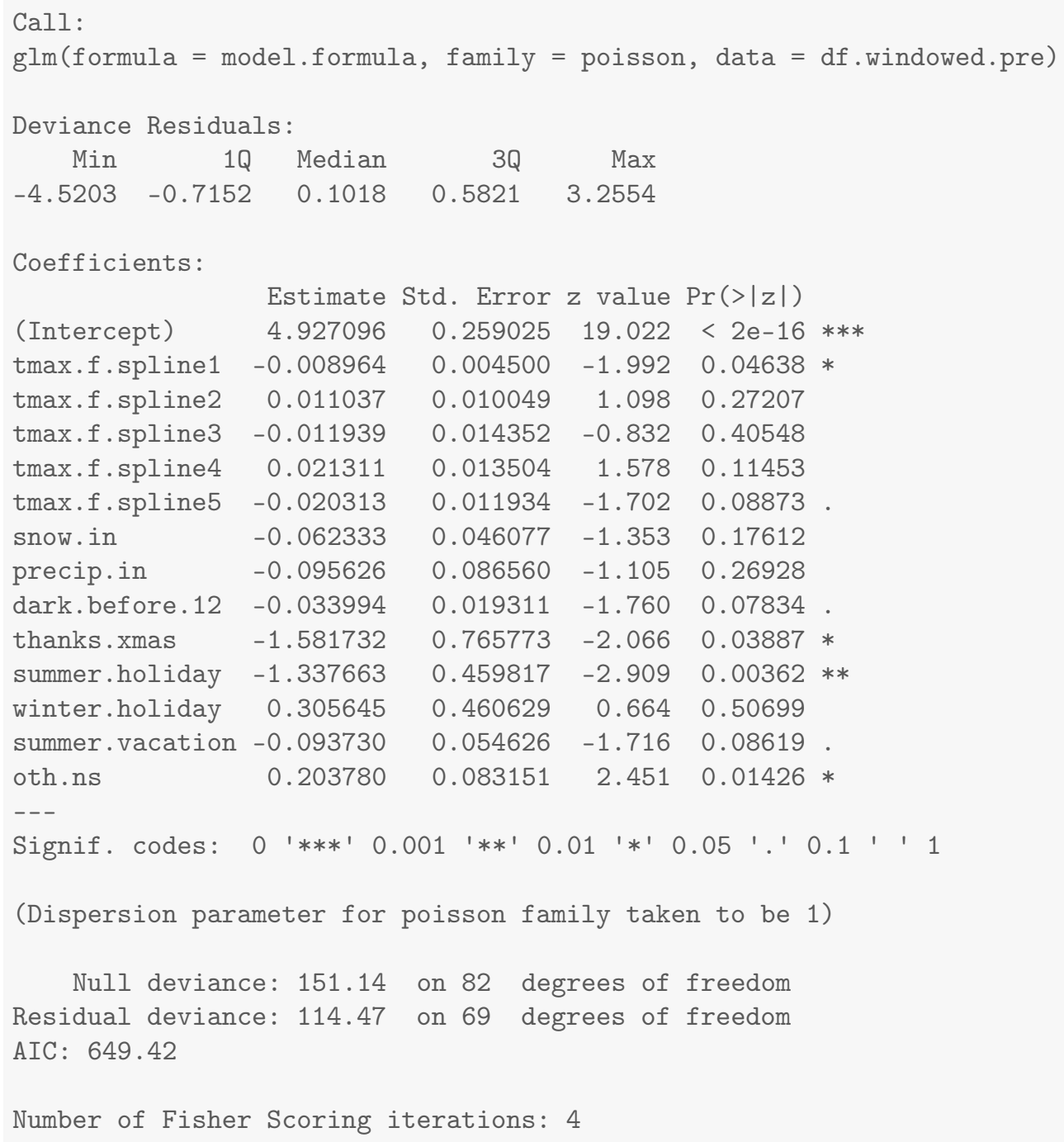




\section{Results for southeastern}

\subsection{Summary Values for southeastern}

Table 40.1: Descriptives for Outcome Before Ferguson Protests Begin

\begin{tabular}{lcccccccc}
\hline \hline Statistic & $\mathrm{N}$ & Mean & St. Dev. & Min & Pctl(25) & Median & Pctl(75) & Max \\
\hline southeastern & 83 & 65.735 & 11.248 & 43 & 58.5 & 64 & 71 & 93 \\
\hline
\end{tabular}

Table 40.2: Descriptives for Outcome After Ferguson Protests Begin

\begin{tabular}{lcccccccc}
\hline \hline Statistic & $\mathrm{N}$ & Mean & St. Dev. & Min & $\operatorname{Pctl}(25)$ & Median & $\operatorname{Pctl}(75)$ & Max \\
\hline southeastern & 290 & 28.607 & 10.751 & 8 & 21 & 27 & 34 & 71 \\
\hline
\end{tabular}




\subsection{Four Models for southeastern}

Table 40.3: Four Models that Differ on the Specification of Adjustment and Intervention Variables

\begin{tabular}{|c|c|c|c|c|}
\hline & \multicolumn{4}{|c|}{ Outcome: Count Per Week } \\
\hline & (1) & (2) & (3) & (4) \\
\hline After Ferguson Protests Begin (week of $8 / 11 / 14$ onward) & -23.32 & & -23.18 & -22.65 \\
\hline After Gray Protests Begin (week of 4/20/15 onward) & -10.14 & & -9.03 & -11.13 \\
\hline Unrest and National Guard (4/27/15 - 5/3/2015) & -16.27 & & -21.65 & -19.86 \\
\hline After Batts Fired (week of 7/13/15 onward) & 4.01 & & 1.94 & 3.22 \\
\hline After Davis Confirmed (week of $10 / 19 / 15$ onward) & -4.57 & & -4.67 & -3.92 \\
\hline After Davis First Year (week of 10/17/16 onward) & -5.00 & & -5.42 & -5.50 \\
\hline After De Sousa Begins (week of 1/19/18 onward) & -6.09 & & -5.79 & -4.80 \\
\hline After Tuggle Begins (week of 5/14/18 onward) & 5.14 & & 8.18 & 6.40 \\
\hline After Harrison Begins (week of 2/11/19 onward) & -4.99 & & -7.21 & -6.66 \\
\hline Average Maximum Temperature to 50 Degrees & & 0.13 & & 0.25 \\
\hline Plus Degrees in the $50 \mathrm{~s}$ & & 0.56 & & 0.04 \\
\hline Plus Degrees in the 60s & & -1.15 & & -0.02 \\
\hline Plus Degrees in the 70s & & -0.005 & & -0.50 \\
\hline Plus Degrees Greater Than 80 & & 0.18 & & -0.31 \\
\hline Snowfall (inches) & & -4.04 & & -1.76 \\
\hline Precipitation (inches) & & -17.46 & & -13.03 \\
\hline Darkness Before Midnight (hours) & & 0.75 & & 0.83 \\
\hline Thanksgiving/Christmas (proportion of week) & & -56.05 & & -27.01 \\
\hline Winter Holiday (proportion of week) & & -30.59 & & 6.86 \\
\hline Other Out-of-School Days (proportion of week) & & -1.52 & & -0.83 \\
\hline Summer Vacation (proportion of week) & & 8.60 & & 5.31 \\
\hline Summer Holiday (proportion of week) & & -1.56 & & 0.56 \\
\hline Observations & 373 & 83 & 373 & 373 \\
\hline $\mathrm{R}^{2}$ & 0.77 & 0.18 & 0.78 & 0.80 \\
\hline
\end{tabular}


40.3 Least Squares Updated Model (2) for southeastern southeastern

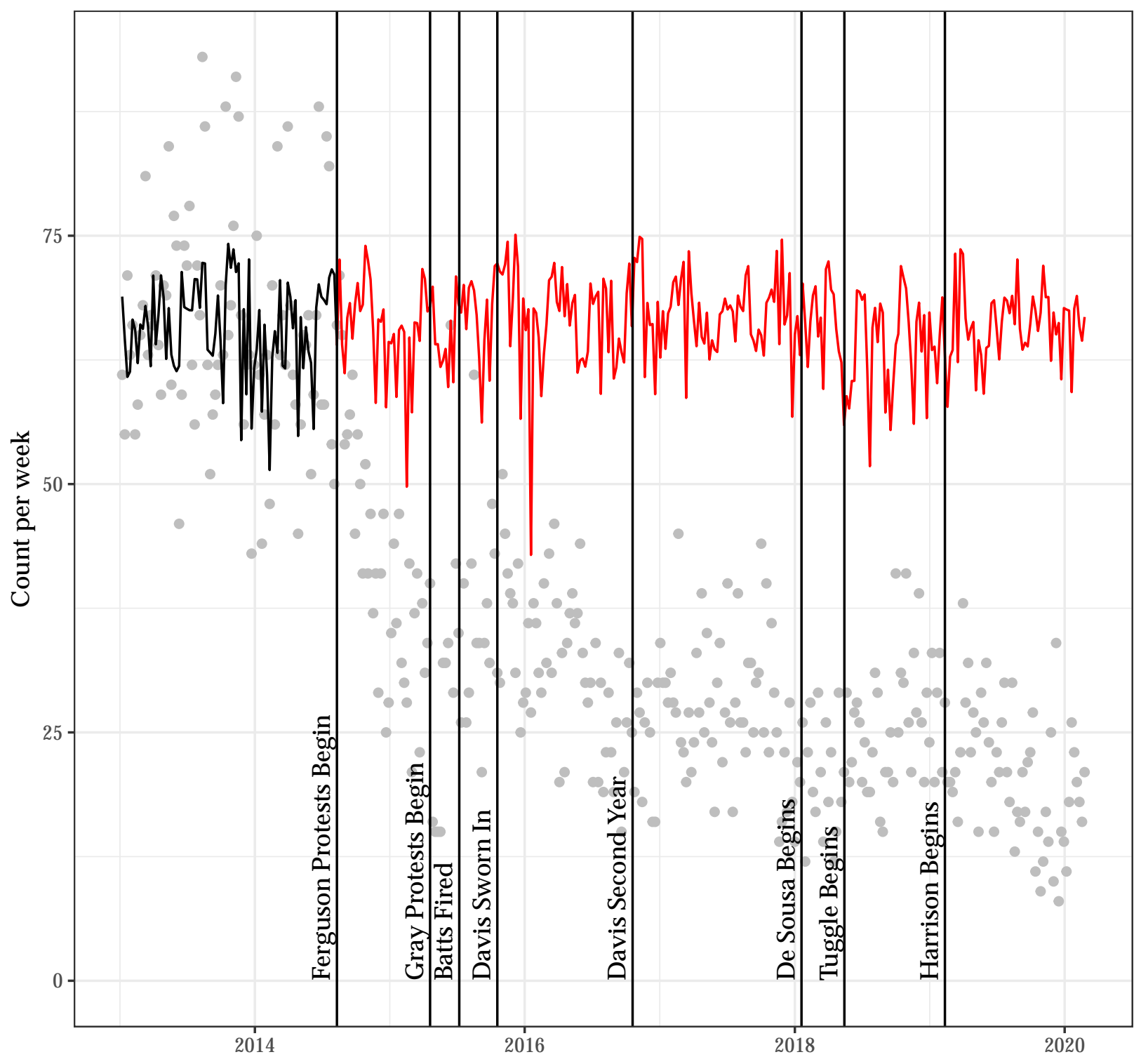




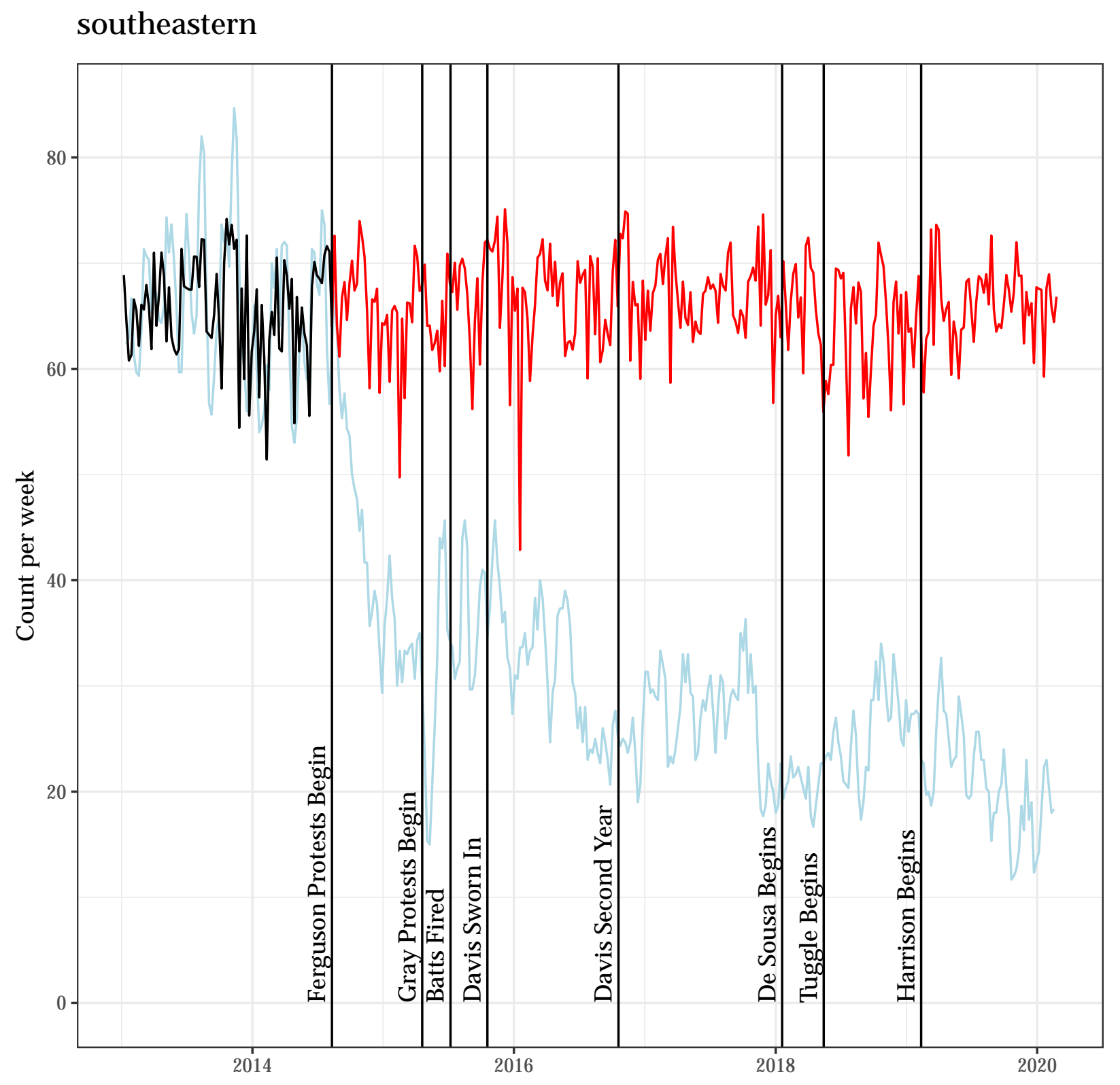


Autocorrelation Function for the Observed Outcome for Updated Model (2)

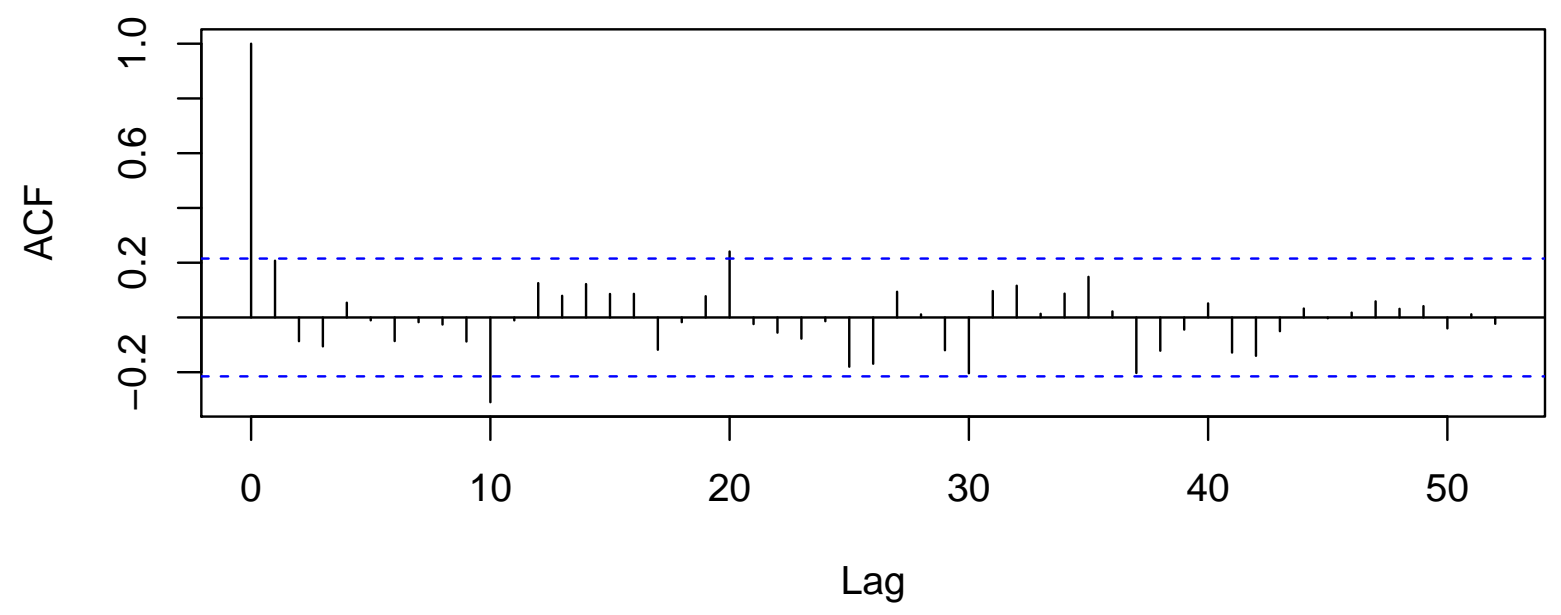

Autocorrelation Function for the Residuals from Updated Model (2)

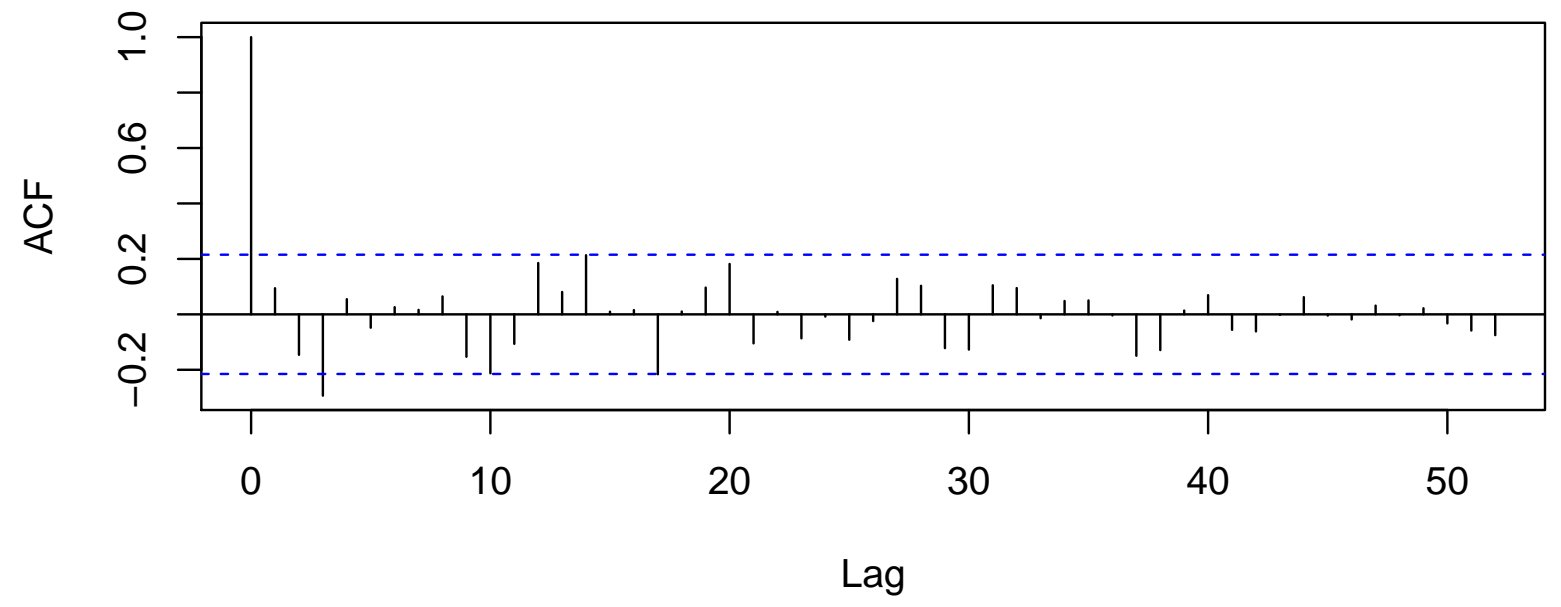




\subsection{A Poisson Regression Model as an Alternative to Model (2) for southeastern}

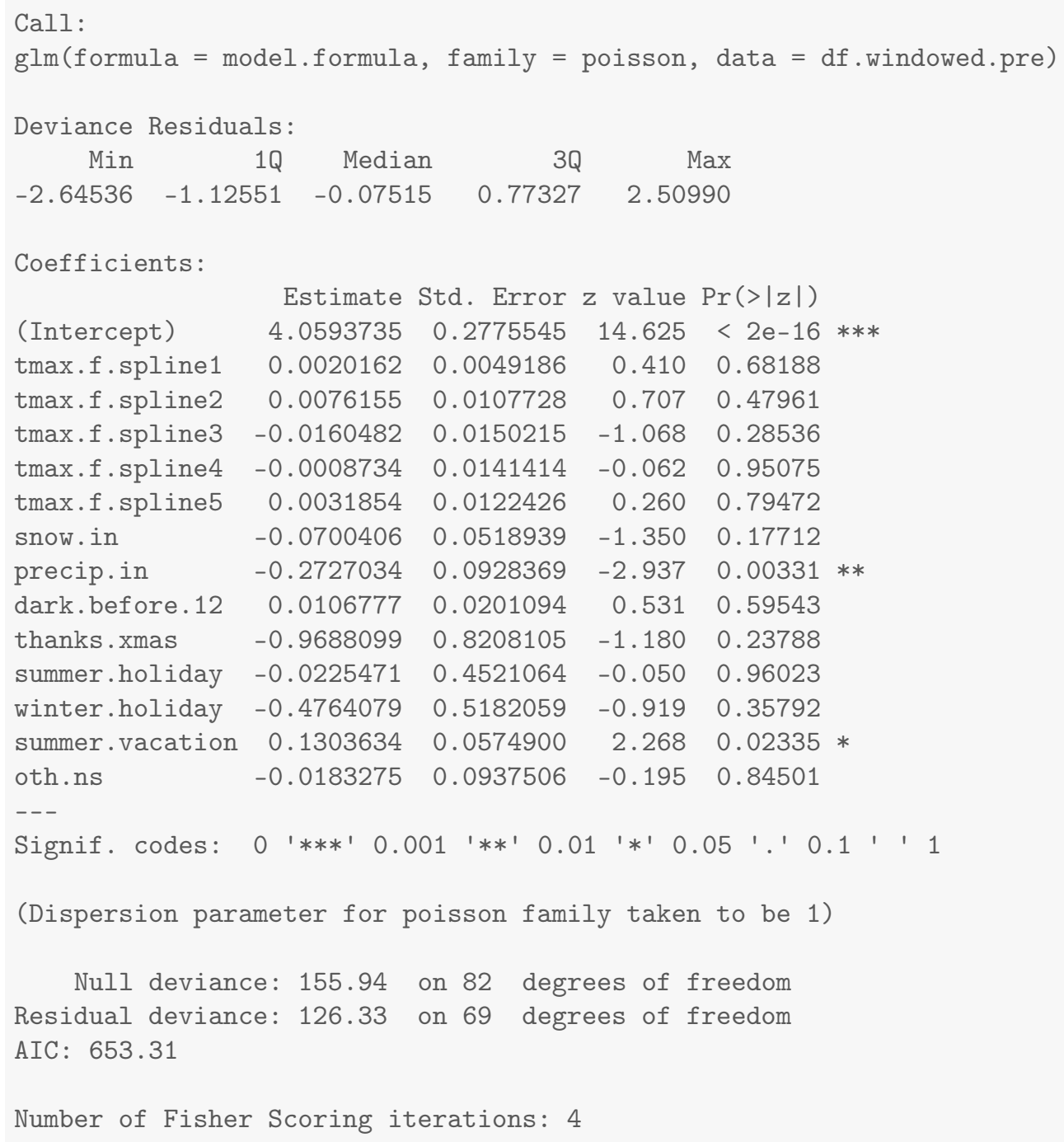

